Dirk Beier

\title{
Informationsmanagement aus Sicht der Betriebswirtschaftslehre
}




\section{Dirk Beier}

\section{Informationsmanagement aus Sicht der Betriebswirtschaftslehre}

Das Informationsmanagement hat seinen Status als modisches Schlagwort inzwischen überwunden und stellt ein etabliertes Themengebiet mit immer noch steigender Relevanz für Theorie und Praxis der Unternehmungsführung dar. Trotz zahlreicher Veröffentlichungen liegt eine umfassende theoretische Fundierung dieses Problembereichs jedoch bisher nicht vor. Die Arbeit konzipiert das Informationsmanagement als spezielles Untersuchungsfeld der Betriebswirtschaftslehre und leitet aus bestehenden Forschungsansätzen dieser Disziplin Anhaltspunkte für die Gestaltung und Steuerung der betrieblichen Informationsverarbeitung ab. Die entwickelten Theorieansätze werden am konkreten Anwendungsbeispiel Mobile Business zusammengeführt.

Dirk Beier wurde 1971 geboren. Von 1991 bis 1996 studierte er Wirtschaftswissenschaft mit den Schwerpunkten Wirtschaftsinformatik und Controlling an der Ruhr-Universität Bochum. Danach arbeitete er von 1997 bis 2001 als Wissenschaftlicher Mitarbeiter am Institut für Unternehmungsführung und Unternehmensforschung und am Lehrstuhl für Wirtschaftsinformatik der Ruhr-Universität Bochum. Seit dem Frühjahr 2002 ist er bei der Brenntag AG als Inhouse Consultant im Bereich Corporate Development tätig. 
Informationsmanagement aus Sicht der Betriebswirtschaftslehre 


\section{BOCHUMER BEITRÄGE ZUR UNTERNEHMUNGSFÜHRUNG UND UNTERNEHMENSFORSCHUNG}

Herausgegeben von Prof. Dr. Michael Abramovici,

Prof. Dr. Dr. h.c. mult. Walther Busse von Colbe, Prof. Dr. Dr. h.c. Werner H. Engelhardt,

Prof. Dr. Roland Gabriel, Prof. Dr. Arno Jaeger, Prof. Dr. Gert Laßmann,

Prof. Dr. Wolfgang Maßberg, Prof. Dr. Bernhard Pellens, Prof. Dr. Miarion Steven,

Prof. Dr. Rolf Wartmann, Prof. Dr. Brigitte Werners

Band 66

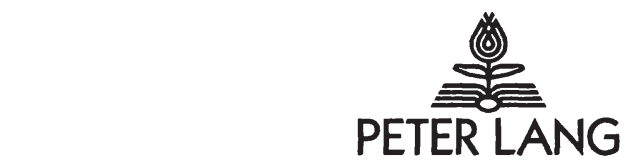

Frankfurt am Main - Berlin - Bern - Bruxelles - New York - Oxford - Wien 


\section{Dirk Beier}

\section{Informationsmanagement aus Sicht der Betriebswirtschaftslehre}

Theoretische Ansätze und das Beispiel Mobile Business

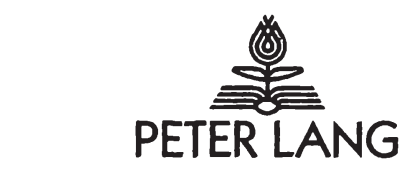

Europäischer Verlag der Wissenschaften 
Die Deutsche Bibliothek - CIP-Einheitsaufnahme

Beier, Dirk:

Informationsmanagement aus Sicht der Betriebswirtschaftslehre : theoretische

Ansätze und das Beispiel Mobile Business / Dirk Beier. - Frankfurt am Main ;

Berlin ; Bern ; Bruxelles ; New York ; Oxford ; Wien : Lang, 2002

(Bochumer Beiträge zur Unternehmensführung und Unternehmensfor-

schung ; Bd. 66)

Zugl.: Bochum, Univ., Diss., 2001

ISBN3-631-39697-X

Open Access: The online version of this publication is published on www.peterlang.com and www.econstor.eu under the international Creative Commons License CC-BY 4.0. Learn more on how you can use and share this work: http://creativecommons. org/licenses/by/4.0.

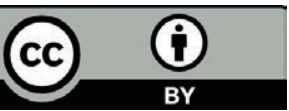

This book is available Open Access thanks to the kind support of ZBW - Leibniz-Informationszentrum Wirtschaft.

\title{
Gedruckt auf alterungsbeständigem, säurefreiem Papier.
}

\author{
D294 \\ ISSN 0175-7105 \\ ISBN 3-631-39697-X \\ ISBN 978-3-631-75500-6 (eBook) \\ (C) Peter Lang $\mathrm{GmbH}$ \\ Europäischer Verlag der Wissenschaften \\ Frankfurt am Main 2002 \\ Alle Rechte vorbehalten.
}

Das Werk einschließlich aller seiner Teile ist urheberrechtlich geschützt. Jede Verwertung außerhalb der engen Grenzen des

Urheberrechtsgesetzes ist ohne Zustimmung des Verlages unzulässig und strafbar. Das gilt insbesondere für

Vervielfältigungen, Übersetzungen, Mikroverfilmungen und die

Einspeicherung und Verarbeitung in elektronischen Systemen.

Printed in Germany 123467

www.peterlang.de 
Wissenschaft ist nicht ... Isolierung, Auflösung, Abstraktion oder gar Verarmung und Verfälschung des Weltbildes. Sie ist gerade aus Zusammenschau, Synthese des Gesamtbildes, ein Schauen höherer Ordnung mit wohlgeprüften Mitteln der Schau; sie ist eine gewaltige Bereicherung der Weltauffassung, Aufdeckung des sonst Verborgenen, Faßbarmachen des sonst Unfaßbaren. Sie ist das auch da noch, wo sie einseitig vorgeht. Denn sie ist grundsätzlich an keine ihrer Einseitigkeiten gebunden, kann sie in voller Freiheit alle wieder überschreiten. 
Dirk Beier - 978-3-631-75500-6

Downloaded from PubFactory at 01/11/2019 04:07:38AM 


\section{Geleitwort}

Die Notwendigkeit eines funktionsfähigen und erfolgreichen Informationsmanagements, das die Nutzung von computergestützten Informations- und Kommunikationssystemen (IuK-Systeme) in Unternehmungen systematisch plant, steuert und kontrolliert, ist heute unbestritten. Der seit Jahren zu beobachtende Trend zu einer fortschreitenden Durchdringung des Wirtschaftsgeschehens mit modernen IuKTechniken ist nach wie vor ungebrochen und steigt eher noch an. Aufgaben und Problemstellungen des Informationsmanagements stellen somit einen wichtigen Gegenstand der Wirtschaftsinformatik und immer mehr auch der Betriebswirtschaftslehre dar. Das Themengebiet bildet daher einen besonderen Arbeitsschwerpunkt in Forschung und Lehre des Lehrstuhls für Wirtschaftsinformatik an der RuhrUniversität Bochum, an dem in den letzten Jahren zahlreiche wissenschaftliche Beiträge entstanden sind.

Herr Dr. Dirk Beier, wissenschaftlicher Mitarbeiter am Lehrstuhl für Wirtschaftsinformatik, setzt sich in seiner Dissertationsschrift in sehr grundsätzlicher Form mit dem Informationsmanagement auseinander und konzipiert dieses als speziellen Untersuchungsbereich der Betriebswirtschaftslehre. Sein anspruchsvolles Ziel ist es, das Aufgabenfeld Informationsmanagement in bestehende Forschungsansätze der Betriebswirtschaftslehre einzubauen, wissenschaftlich zu analysieren, kritisch zu bewerten und die Erkenntnisse zur Ableitung von Aussagen über die Gestaltung und Steuerung der betrieblichen Informationsverarbeitung zu nutzen. Damit will Herr Dr. Beier für Theorie und Praxis eine größere Unabhängigkeit von tagesaktuellen Modeströmungen, die bestehende Ergebnisse mit immer neuen Schlagworten bereits nach kurzer Zeit wieder in Frage stellen, erreichen. Er erarbeitet zunächst einen eigenen Bezugsrahmen für den Theorieeinstieg, der seine im Anschluss entwickelten Theorieansätze für das Informationsmanagement miteinander verbindet. Mit dem Faktortheoretischen Ansatz (Gutenberg-Ansatz), dem Systemorientierten Ansatz und der Neuen Institutionenökonomie bezieht er sich auf weithin anerkannte Forschungsansätze der Betriebswirtschaftslehre, die ein besonders großes Erkenntnispotenzial für den Bereich der computergestützten Informationsverarbeitung aufweisen.

Die Zusammenführung der entwickelten Theorieansätze erfolgt in einem weiteren Kapitel an einem aktuellen Gestaltungsbeispiel. Die Nutzung der mobilen Datenübertragung steckt noch in den Kinderschuhen, und der Aufbau von Mobile BusinessAnwendungen wird die Unternehmungen noch längere Zeit beschäftigen. Hier demonstriert Herr Dr. Beier die konkrete Anwendbarkeit und die praktische Relevanz der auf den ersten Blick abstrakt wirkenden Aussagekomplexe. Er leitet nicht nur direkte Gestaltungsempfehlungen für die Praxis ab, sondern verdeutlicht zudem die Interdependenzen zwischen den verschiedenen Theorieansätzen. Damit belegt er zugleich die Notwendigkeit einer multiperspektivischen Analyse, die den Kern seines gesamten Vorgehens darstellt.

Die stilistisch sehr ansprechende Arbeit zeichnet sich durch eine ungewöhnlich hohe Eigenständigkeit und Intensität der Argumentation aus. Die hier vorgestellten Ideen sind neu, zum Teil überraschend, jedoch jederzeit nachvollziehbar und auf einem starken theoretischen betriebswirtschaftlichen Fundament basierend. An vielen Stellen 
weist die Arbeit, die von der Ruhr-Universität Bochum mit dem Gebrüder DeschauerPreis 2002 ausgezeichnet wurde, weit über sich selbst hinaus, indem sie den Weg für detailliertere Einzeluntersuchungen oder die Einbeziehung von Erkenntnissen benachbarter Wissenschaftsdisziplinen vorzeichnet. Sie ist geeignet, auch praxisorientierten Lesern interessante Denkanstöße und Hinweise zur Realisierung auf Dauer tragfähiger Lösungen zu geben. Ich wünsche, dass sich die Arbeit weit verbreitet und Anlass für weitere Diskussionen schafft.

Prof. Dr. Roland Gabriel 


\section{Vorwort}

Der zunehmende Einsatz moderner Informations- und Kommunikationstechniken stellt viele Unternehmungen vor große Herausforderungen und führte vor einigen Jahren dazu, dass das Informationsmanagement als neues betriebliches Aufgabenfeld mehr und mehr Beachtung fand. Dabei war zunächst nicht klar, ob es sich lediglich um eine vorübergehende Modewelle oder einen dauerhaften Wandel des gesamten Geschäftslebens handelte. Inzwischen hat das Informationsmanagement seinen Status als modisches Schlagwort überwunden und stellt ein etabliertes Themengebiet mit immer noch steigender Relevanz für Theorie und Praxis dar.

Trotz zahlreicher theoretischer Auseinandersetzungen mit der Problematik und einer inzwischen nahezu unüberschaubaren Vielzahl von Fachbeiträgen liegt eine umfassende Fundierung des Informationsmanagements bisher noch nicht vor. Aus dieser unbefriedigenden Situation heraus entstand die Motivation zur Erstellung der vorliegenden Dissertationsschrift. Die Arbeit will nicht nur bestehende Erkenntnisdefizite durch eine bessere theoretische Basis überwinden, sondern auch den konzeptionellen Bezugsrahmen für tiefergehende Analysen zu einzelnen Fragestellungen im Bereich des Informationsmanagements schaffen.

Für das hohe $\mathrm{Ma}$ an intellektuellem Freiraum, das notwendig war, um diese Zielsetzung zu erreichen, gebührt Herrn Professor Dr. Roland Gabriel als meinem akademischen Lehrer größter Dank und Respekt. Ohne die Möglichkeit, sich auch auf bisher weniger ausgetretenen wissenschaftlichen Pfaden zu bewegen, könnte eine Arbeit dieser Art nicht zu Stande kommen. Professor Gabriel hat diesen Weg aufmerksam begleitet und mit sicherem Gespür dafür, wenn es in meinem eigenen Interesse erforderlich war, steuernd eingegriffen. Professor Dr. Dr. h.c. Werner Hans Engelhardt hat sich als Korreferent intensiv mit dem Konzept und den Inhalten auseinander gesetzt und war durch seine konstruktiven Verbesserungsvorschläge ebenfalls erheblich am Gelingen der Arbeit beteiligt. Auch Ihm gilt mein besonderer Dank.

Erfolgreiches wissenschaftliches Arbeiten ist ohne den Austausch von Ideen und die kritische Diskussion neuer Gedankengänge nicht denkbar. Dazu braucht man Gesprächspartner, die bereit sind, sich auf ungewöhnliche und zunächst abwegig erscheinende Vorstellungen einzulassen. Hier ist an erster Stelle meine ehemalige Kollegin Dr. Frauke Streubel zu nennen, die auch mein ursprüngliches Interesse für das Thema Informationsmanagement geweckt hat. Kein Gedanke war seltsam genug, um von ihr nicht unvereingenommen diskutiert zu werden. Darüber hinaus hat sie mit ihrer eigenen Arbeit als Vorbild für meine persönlichen Anstrengungen gedient. Als weitere bedeutende Korrektive möchte ich hier Dr. Ane-Kristin Reif-Mosel, Carsten Dittmar und Dr. Martin Gersch aufführen, die mir mit Ihren Anmerkungen und Stellungnahmen wichtige Orientierungen auf unbekanntem Gebiet gegeben haben. Sonja Labusch hat die Arbeit in der Endphase vollständig durchgearbeitet und wertvolle Beiträge zur Feinabstimmung geleistet.

Ein ganz besonderer Dank gilt nicht zuletzt den Personen, von denen ich abseits des fachlichen Bereichs jede Unterstützung erfahren habe. Gerade in schwierigen Phasen, in 
denen der Fortschritt der Arbeit kaum noch erkennbar war, hat meine Partnerin Melanie Jellinghaus mich immer wieder aufgebaut und zur Veränderung der Perspektive bewegt. Erst diese Motivation hat mir den Ansporn gegeben, die Arbeit in der vorliegenden Form abzuschließen. Meine Eltern Renate und Karl Heinz Beier haben mich stets gefördert und überhaupt erst zu dem Punkt gebracht, an dem ich mit dieser Arbeit beginnen konnte. Meiner Mutter kommt ferner das Verdienst $\mathrm{zu}$, sich in kürzester Zeit in die neue Rechtschreibung eingearbeitet zu haben und die entsprechenden Korrekturen an meinen Entwürfen, die zunächst in alter Rechtschreibung vorlagen, vorgenommen zu haben. Meinen Eltern und Melanie möchte ich diese Arbeit widmen.

Dirk Beier 
Inhaltsverzeichnis

$\begin{array}{ll}\text { Abbildungsverzeichnis } & \text { XXI }\end{array}$

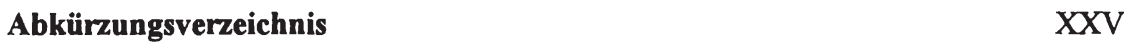

1 Einführung 1

$\begin{array}{ll}\text { 1.1 Motivation und Zielsetzung } & 1\end{array}$

$\begin{array}{ll}\text { 1.2 Aufbau der Arbeit } & 2\end{array}$

\section{Informationsmanagement als Gegenstand} betriebswirtschaftlicher Untersuchungen

2.1 Ausgangssituation: Ungelöste Problemstellungen des Informationsmanagements in Theorie und Praxis

2.1.1 Theoriedefizite des „State of the Art“ im Bereich des Informationsmanagements

2.1.2 Offene Probleme des Informationsmanagements in der Praxis $\quad 11$

2.1.2.1 Effektivität der betrieblichen Informationsverarbeitung $\quad 12$

2.1.2.2 Effizienz der Informationsverarbeitung 13

2.1.2.3 Organisatorische Zuordnung informationswirtschaftlicher Aufgaben

2.1.2.4 Rolle des Menschen in der Informationsverarbeitung

2.2 Wissenschaftstheoretische Grundlagen einer realwissenschaftlichen Untersuchung des Informationsmanagements

2.2.1 Informationsmanagement als realwissenschaftlicher Erfahrungsund Erkenntnisgegenstand

2.2.2 Überwindung bisheriger Theoriedefizite durch Bezugnahme auf bestehende Wissenschaftsprogramme

2.2.3 Dreistufiger Theorieeinstieg und weiteres Vorgehen zur Analyse des Informationsmanagements 
2.3 Konstruktion des Informationsmanagements als Erfahrungsgegenstand wissenschaftlicher Analysen

2.3.1 Der Begriffsbestandteil Information 25

2.3.1.1 Syntaktische Ebene in der Semiotik 26

2.3.1.2 Semantische Ebene in der Semiotik 27

2.3.1.3 Pragmatische Ebene in der Semiotik 28

2.3.1.4 „Information“ als Modell mit materiellen und immateriellen Bestandteilen $\quad 30$

2.3.1.5 Subjektbezogenheit versus Objektivität von „Information“ 31

2.3.2 Der Begriffsbestandteil Management 33

2.3.2.1 Begriffsvarianten $\quad 34$

2.3.2.2 Sachbezogene Funktionen des Managements 35

2.3.2.3 Personale Funktionen des Managements 36

2.3.3 Der Begriff Informationsmanagement 38

2.3.3.1 Zur Verwendung des Begriffsbestandteils „Management“: Informationsmanagement als funktional-sachorientiert abgrenzbarer Spezialbereich der Unternehmungsführung $\quad 38$

2.3.3.2 Zur Verwendung des Begriffsbestandteils „Information“: Bedeutung und Aktivitäten der Informationsverarbeitung durch das technikunterstützte Informationssystem der Unternehmung 40

2.3.3.3 Arbeitsdefinition: „Informationsmanagement“ 44

2.4 Die Betriebswirtschaftslehre als Referenzdisziplin für eine wissenschaftliche Analyse des Informationsmanagements 45

2.4.1 Zum Erfahrungsgegenstand der Betriebswirtschaftslehre 45

2.4.2 Standortbestimmung der Betriebswirtschaftslehre als Wissenschaft 49

2.4.2.1 Disziplinäre Abgrenzung der Betriebswirtschaftslehre 50

2.4.2.2 Stellung der Betriebswirtschaftslehre gegenüber anderen wissenschaftichen Disziplinen

2.4.3 Auswahl der in die Analyse des Informationsmanagements

einbezogenen Wissenschaftsprogramme der Betriebswirtschafslehre 


\section{Erkenntnispotenziale und Lösungsbeiträge des Faktor-} theoretischen Ansatzes für das Informationsmanagement

3.1 Der Faktortheoretische Ansatz der Betriebswirtschaftslehre

3.1.1 Erkenntnisinteressen des Faktortheoretischen Ansatzes

3.1.2 Inhaltliche Leitideen des Faktortheoretischen Ansatzes

3.1.3 Methodische Leitideen des Faktortheoretischen Ansatzes

3.2 Konstruktion des Informationsmanagements als Erkenntnisgegenstand aus der Perspektive des Faktortheoretischen Ansatzes

3.2.1 Information als Produktionsfaktor und als Produktionsergebnis

3.2.2 Der Bereich der Leistungserstellung bei der Herstellung informationeller Produkte

3.2.2.1 Objektbezogene menschliche Arbeitsleistungen in Prozessen der Informationsverarbeitung

3.2.2.2 Arbeits- und Betriebsmittel in Prozessen der Informationsverarbeitung

3.2.2.3 Werkstoffe in Prozessen der Informationsverarbeitung

3.2.2.4 Die dispositiven Faktoren in Prozessen der Informationsverarbeitung

3.2.3 Der Bereich der Leistungsverwertung und die finanzielle Sphäre aus Sicht des Informationsmanagements

3.2.3.1 Berührungspunkte zwischen Leistungsverwertung und technikunterstützter Informationsverarbeitung

3.2.3.2 Berührungspunkte zwischen finanzieller Sphäre und technikunterstützter Informationsverarbeitung

\subsection{Produktionsorientierte Analyse des Informationsmanagements}

3.3.1 Vorkombination von Elementarfaktoren im Bereich der technikunterstützten Informationsverarbeitung

3.3.1.1 Ergiebigkeit objektbezogener menschlicher Arbeitsleistungen bei der Erstellung informationeller Produkte

3.3.1.2 Ergiebigkeit von Betriebsmitteln bei der Erstellung informationeller Produkte

3.3.1.3 Ergiebigkeit von Werkstoffen bei der Erstellung informationeller Produkte

3.3.1.4 Anordnung der Elementarfaktoren zur Vorbereitung von Produktionsprozessen 
3.3.2 Endkombination von Elementarfaktoren im Bereich der technikunterstützten Informationsverarbeitung

3.3.2.1 Erkenntnispotenziale der Produktionstheorie für die technikunterstützte Informationsverarbeitung

3.3.2.2 Erkenntnispotenziale der Kostentheorie für die technikunterstützte Informationsverarbeitung

3.3.3 Informationsmanagement im System der dispositiven Faktoren

3.3.3.1 Planung der technikunterstützten Informationsverarbeitung

3.3.3.2 Organisation von Prozessen der Informationsverarbeitung

\subsection{Aussagekraft des Produktionsorientierten Ansatzes für eine} Theorie des Informationsmanagements

3.4.1 Lösungsansätze für offene Probleme in der Praxis

3.4.1.1 Effektivität der Informationsverarbeitung

3.4.1.2 Effizienz der Informationsverarbeitung

3.4.1.3 Organisatorische Zuordnung informationswirtschaftlicher Aufgaben

3.4.1.4 Rolle des Menschen in der Informationsverarbeitung

3.4.2 Erkenntnisgrenzen

3.4.2.1 Stark einschränkende Prämissen und eingeengtes

Erkenntnisinteresse

\section{Erkenntnispotenziale und Lösungsbeiträge des System- orientierten Ansatzes für das Informationsmanagement}

4.1.2 Inhaltliche Leitideen des Systemorientierten Ansatzes

4.1.2.1 Entwicklung und Verwendung einer einheitlichen Systemsprache

4.1.2.2 Lenkbarkeit von Systemen

4.1.3 Methodische Leitideen des Systemorientierten Ansatzes

4.1.3.1 Black box-Technik

4.1.3.2 Wechsel von Betrachtungsebenen 
4.2 Konstruktion des Informationsmanagements als Erkenntnisgegenstand aus der Perspektive des Systemorientierten Ansatzes

4.2.1 Abgrenzung des technikunterstützten Informationssystems als Subsystem der Unternehmung

4.2.2 Unterschiedliche Betrachtungsebenen bei der Untersuchung des technikunterstützten Informationssystems

4.2.3 Komplexität des technikunterstützten Informationssystems

4.2.4 Lenkung des technikunterstützten Informationssystems durch das Informationsmanagement

4.3 Systemorientierte Analyse des Informationsmanagements

4.3.1 Kybernetik I: Konservatives Informationsmanagement

4.3.1.1 Passive Bewältigung von Störungen durch Abschirmung des Systems

4.3.1.2 Aktive Bewältigung von Störungen durch Steuerung

4.3.1.3 Aktive Bewältigung von Störungen durch Regelung

4.3.1.4 Kombination verschiedener Maßnahmentypen zur Abwehr von Störungen

4.3.1.5 Subsystembildung, Ultra- und Multi-Stabilität

4.3.2 Kybernetik II: Innovatives Informationsmanagement

4.3.2.1 Geänderte Zielsetzung: Innovative Anpassungsfähigkeit statt konservativer Lenkung im Detail

4.3.2.2 Förderung der Anpassungsfähigkeit des technikunterstützten Informationssystems der Unternehmung

4.3.2.3 Symbolische Ebene des Informationsmanagements

4.3.2.4 Anpassungsvorgänge als Veränderungen der Wissensbasis: Lernende Informationssysteme

4.4 Aussagekraft des Systemorientierten Ansatzes für eine Theorie des Informationsmanagements

4.4.1 Lösungsansätze für offene Probleme in der Praxis

4.4.1.1 Effektivität der Informationsverarbeitung

4.4.1.2 Effizienz der Informationsverarbeitung

4.4.1.3 Organisatorische Zuordnung informationswirtschaftlicher Aufgaben

4.4.1.4 Rolle des Menschen in der Informationsverarbeitung 
4.4.2 Erkenntnisgrenzen $\quad 228$

4.4.2.1 Stark verallgemeinernde Betrachtungsweise 229

4.4.2.2 Fragwürdigkeit von Analogien 231

4.4.2.3 Verhältnis von konservativ und innovativ orientierten Maßnahmen

\section{Erkenntnispotenziale und Lösungsbeiträge der Neuen} Institutionenökonomie für das Informationsmanagement

5.1 Der Ansatz der Neuen Institutionenökonomie

5.1.1 Erkenntnisinteressen der Neuen Institutionenökonomie 236

5.1.2 Inhaltliche Leitideen der Neuen Institutionenökonomie 239

5.1.3 Methodische Leitideen der Neuen Institutionenökonomie 246

5.2 Konstruktion des Informationsmanagements als Erkenntnisgegenstand aus der Perspektive der Neuen Institutionenökonomie

5.2.1 Grundfragestellungen des Informationsmanagements aus Sicht der Neuen Institutionenökonomie

5.2.2 Informationsmanagement aus Sicht der Transaktionskostentheorie

5.2.2.1 Informationsverarbeitung in den verschiedenen Transaktionsphasen

5.2.2.2 Berührungspunkte zwischen den Charakteristika der Transaktion und der technikunterstützten Informationsverarbeitung

5.2.2.3 Zusammenhang zwischen technikunterstützter Informationsverarbeitung und institutioneller Koordination von Transaktionen

5.2.3 Informationsmanagement aus Sicht der Agency-Theorie

5.2.3.1 Anknüpfungspunkte für eine Theorie des Informationsmanagements in der Agency-Theorie

5.2.3.2 Hidden characteristics: Informationsunvollkommenheiten vor Vertragsabschluss

5.2.3.3 Hidden intention: Informationsunvollkommenheiten nach Vertragsschluss mit Ex post-Kenntnis des Principal

5.2.3.4 Hidden action: Informationsunvollkommenheiten nach Vertragsabschluss ohne Ex post-Kenntnis des Principal 
5.2.4 Berührungspunkte zwischen Transaktionskostentheorie und Agency-Theorie als Ausgangspunkt für die Institutionenökonomische Analyse des Informationsmanagements

5.2.4.1 Gemeinsame Problemkreise des Informationsmanagements aus Sicht von Transaktionskostentheorie und Agency-Theorie 278

5.2.4.2 Transaktionen als grundlegende Bezugseinheiten des Informationsmanagements

5.3 Institutionenökonomische Analyse des Informationsmanagements

5.3.1 Informationsmanagement vor Vertragsschluss

5.3.1.1 Charakterisierung der Informationsverarbeitung vor Vertragsschluss

5.3.1.2 Anwendungssysteme bei der Auswahl von Vertragspartnern: Die Sicht des Leistungsnachfragers

5.3.1.3 Anwendungssysteme bei der Auswahl von Vertragspartnern: Die Sicht des Leistungsanbieters

5.3.2 Informationsmanagement nach Vertragsschluss

5.3.2.1 Charakterisierung der Informationsverarbeitung nach Vertragsschluss

5.3.2.2 Operative Funktionalitäten von Anwendungssystemen bei der Erfüllung vertraglicher Pflichten

5.3.2.3 Analytische Funktionalitäten von Anwendungssystemen bei der Erfüllung vertraglicher Pflichten

5.3.3 Informationsmanagement bei der Festlegung des Vertragsdesigns

5.3.3.1 Gestaltung von Vorleistungen und Transaktionsspezifität durch das Informationsmanagement

5.3.3.2 Veränderte Kostenstrukturen der Koordination des Leistungsaustausches

5.3.3.3 Auswirkungen auf die Vorteilhaftigkeit der Koordinationsform 344

\subsection{Aussagekraft des Institutionenökonomischen Ansatzes für eine}

Theorie des Informationsmanagements

5.4.1 Lösungsansätze für offene Probleme in der Praxis

5.4.1.1 Effektivität der Informationsverarbeitung

5.4.1.2 Effizienz der Informationsverarbeitung und organisatorische Zuordnung informationswirtschaftlicher Aufgaben

5.4.1.3 Rolle des Menschen in der Informationsverarbeitung 
5.4.2 Erkenntnisgrenzen 364

5.4.2.1 Defizite beim bisherigen Theorieausbau 365

5.4.2.2 Verwendung von Hybridmodellen 369

5.4.2.3 Reduzierung des menschlichen Verhaltens auf ökonomische Gesichtspunkte

\section{Zusammenführung der entwickelten Theorieansätze} am Beispiel Mobile Business

\subsection{Mobile Business: Abgrenzung, technische Hintergründe und} Anwendungspotenziale

6.1.1 Mobile Business als Fortsetzung der „Internet-Revolution“

6.1.1.1 Das Internet als Auslöser wirtschaftlichen Wandels

6.1.1.2 Veränderung der Internet-Welt durch mobile Anwendungen

6.1.2 Begriffliche Abgrenzung und Erscheinungsformen des Mobile Business

6.1.2.1 Definition von Mobile Business

6.1.2.2 Klassifizierung unterschiedlicher Nutzungsformen des Mobile Business

6.1.3 Technische Grundlagen des Mobile Business

6.1.3.1 Übertragungsstandards

6.1.3.2 Verbindungs- und Darstellungsstandards

6.1.3.3 Endgeräte

6.1.4 Innovative Leistungsmerkmale und Anwendungspotenziale des Mobile Business

6.1.4.1 Überblick

6.1.4.2 Begleitende Leistungsmerkmale der nächsten Mobilfunkgenerationen

6.1.4.3 Kernmerkmale für den Aufbau innovativer Anwendungen mit zusätzlichem Kundennutzen

\subsection{Berührungspunkte zwischen Mobile Business und}

6.2.2 Gestaltungsfelder des Informationsmanagements beim Aufbau von Mobile Commerce-Anwendungen 
6.2.2.1 Unterstützung ortsbezogener Leistungsangebote durch das Informationsmanagement

6.2.2.2 Unterstützung zeitbezogener Leistungsangebote durch das Informationsmanagement

6.2.2.3 Unterstützung interessenbezogener Leistungsangebote durch das Informationsmanagement

6.2.2.4 Unterstützung ubiquitärer Leistungsangebote durch das Informationsmanagement

6.2.3 Gestaltungsfelder des Informationsmanagements beim Aufbau von Mobile Work-Anwendungen

6.2.3.1 Technische Aspekte: Ubiquitärer und kontextspezifischer Zugang der Beschäftigten zu unternehmungsinternen Informationen

6.2.3.2 Organisatorische Aspekte: Neugestaltung von Prozessen auf Basis der mobilen Datenübertragung

\subsection{Anwendung der entwickelten Theorieansätze und Untersuchung ihrer Beziehungen}

6.3.1 Effektivität der technikunterstützten Informationsverarbeitung im Bereich des Mobile Business

6.3.2 Effizienz der technikunterstützten Informationsverarbeitung im Bereich des Mobile Business

6.3.3 Organisatorische Zuordnung informationswirtschaftlicher Aufgaben im Bereich des Mobile Business

6.3.4 Rolle des Menschen bei der Informationsverarbeitung im Bereich des Mobile Business

7 Schlussbetrachtung 
Dirk Beier - 978-3-631-75500-6

Downloaded from PubFactory at 01/11/2019 04:07:38AM 


\section{Abbildungsverzeichnis}

Abb. 1/1: Aufbau der Untersuchung 3

Abb. 2/1: Multiperspektivische Analyse des Informationsmanagements 18

Abb. 2/2: Informationsmanagement als Teiluntersuchungsbereich unterschiedlicher Wissenschaftsdisziplinen

Abb. 2/3: Dreistufiger Theorieeinstieg für die Untersuchung des Informationsmanagements

Abb. 2/4: Information als Modell 30

Abb. 2/5: Verwendung von Informationen in Unternehmungen 41

Abb. 2/6: Vorgehen bei der Entwicklung betriebswirtschaftlicher Ansätze für eine Theorie des Informationsmanagements 59

Abb. 3/1: Konzeptioneller Aufbau von Betrieben im Faktortheoretischen Ansatz

Abb. 3/2: Produktionsfaktorenschema des Faktortheoretischen Ansatzes 65

Abb. 3/3: Systematisierung von Produkten $\quad 72$

Abb. 3/4: Bedingungen der Ergiebigkeit objektbezogener menschlicher $\begin{array}{ll}\text { Arbeitsleistungen } & 86\end{array}$

Abb. 3/5: Bedingungen der Ergiebigkeit von Betriebsmitteln 89

Abb. 3/6: Bedingungen der Ergiebigkeit von Werkstoffen 95

Abb. 3/7: Gestaltungsprinzipien für Produktionsprozesse 98

Abb. 3/8: Bestimmung von Informationsart, Einheitselement und Informationstyp (Beispiele) 105

Abb. 3/9: Aufgabentypen der Informationsverarbeitung 106

Abb. 3/10: Faktordiagramm mit zwei linear-limitationalen Prozessen 109

Abb. 3/11: Kostenfunktion mit linearem und nicht-linearem Kostenverlauf 112

Abb. 3/12: Expansionslinie bei einem limitationalen Prozess 114

Abb. 3/13: Expansionslinie zwei linear-limitationalen Prozessen 114

Abb. 3/14: Anpassung an Beschäftigungsschwankungen 119

Abb. 3/15: Einzelfelder der Planung nach Gutenberg 124

Abb. 3/16: Substitutionsprinzip der Organisation 129

Abb. 4/1: Black box-Methode und Regelkreissystem 161

Abb. 4/2: Wechsel von Betrachtungsebenen 166

$\begin{array}{ll}\text { Abb. 4/3: Bildung von Subsystemen des technikunterstützten } & \\ & \text { Informationssystems }\end{array}$ 
Abb. 4/4: Grundorientierungen des Informationsmanagements aus Sicht der Kybernetik

Abb. 4/5: Das Steuerungsprinzip

Abb. 4/6: Das Regelungsprinzip

Abb. 4/7: Mischlenkung

Abb. 4/8: Zweidimensionale Klassifikation von statischen Merkmalen der Organisationalen Wissensbasis

Abb. 5/1: Einflussgrößen auf die Höhe der Transaktionskosten

Abb. 5/2: Zusammenhang zwischen Transaktionseigenschaften und Koordinationsform

Abb. 5/3: Grundfragestellungen der Neuen Institutionenökonomie

Abb. 5/4: Grundfragestellungen des Informationsmanagements aus der Sicht der Neuen Institutionenökonomie

Abb. 5/5: Ansatzpunkte des Informationsmanagements aus Sicht der Transaktionskostentheorie

Abb. 5/6: Ansatzpunkte des Informationsmanagements aus Sicht der Agency-Theorie

Abb. 5/7: Kostenarten in den verschiedenen Transaktionsphasen

Abb. 5/8: Maßnahmen des Informationsmanagements zur Reduzierung des hidden action-Problems

Abb. 5/9: Gemeinsame Problemkreise von Transaktionskostentheorie und Agency-Theorie

Abb. 5/10: Begleitung von Transaktionen durch koordinierende Informationsverarbeitungsprozesse

Abb. 5/11: Aufbau der institutionenökonomischen Analyse des Informationsmanagements

Abb. 5/12: Ansatzpunkte des Informationsmanagements zur Erhöhung der Koordinationseffizienz nach Vertragsabschluss

Abb. 5/13: Verknüpfungsebenen des unternehmungsübergreifenden Informationsverarbeitung

Abb. 5/14: Raum-Zeit-Klassifikation von CSCW-Techniken

Abb. 5/15: Reduzierung des hidden action-Problems mit Hilfe von Executive Information Systemen

Abb. 5/16: Verdrängungseffekt zwischen Produktivität und Flexibilität im Bereich der unternehmungsübergreifenden Informationsverarbeitung

Abb. 5/17: Kostenstrukturen verschiedener Koordinationsformen aus Sicht von Transaktionskostentheorie und Agency-Theorie

Abb. 5/18: Einfluss moderner Informations- und Kommunikationstechnik auf den Verlauf der Transaktionskosten 
Abb. 5/19: Asymmetrische Reduzierung von Agency-Kosten durch die Mediatisierung von Koordinationsprozessen

Abb. 5/20: Effektivität und Effizienz der technikunterstützten Informationsverarbeitung aus Sicht der Neuen Institutionenökonomie

Abb. 6/1: Erscheinungsformen des Mobile Business

Abb. 6/2: Verknüpfung des leitungsgebundenen Internet mit Mobilfunknetzen

Abb. 6/3 Entwicklungsperspektiven für die Zusammenführung von leitungsgebundenem und mobilem Internet

Abb. 6/4: Leistungsmerkmale und Anwendungspotenziale des Mobile Business

Abb. 6/5: Kernmerkmale und begleitende Merkmale des Mobile Business

Abb. 6/6: Wertschöpfungskette der zweiten Mobilfunkgeneration

Abb. 6/7: Mögliche Wertschöpfungsketten im Mobile Business

Abb. 6/8: Bezugsrahmen des Mobile Business und Beispiele

Abb. 6/9: Zentralisierte Datenbank für ortsbezogene Leistungsangebote

Abb. 6/10: M-CRM als Teil eines umfassenden Customer Relationship Managements 
Dirk Beier - 978-3-631-75500-6

Downloaded from PubFactory at 01/11/2019 04:07:38AM 


\section{Abkürzungsverzeichnis}
$\mathrm{AA}_{\mathrm{i}} \quad$ Aktivität i des Anbieters
AM Amplitudenmodulation
$\mathrm{AN}_{\mathrm{i}} \quad$ Aktivität i des Nachfragers
B2B Business-to-Business
B2C Business-to-Consumer
B2D Business-to-Device
B2P Business-to-Professional
CERN Conseil Européen pour la Recherche Nucléaire
cHTML Compact HTML
CDMA Code Division Multiple Access
COO Cell-of-Origin
CRM Customer Relationship Management
CPU Central Processing Unit
CSCW Computer Supported Cooperative Work
C2C Consumer-to-Consumer
DA Directory Agent
DCS Digital Cellular System
DHTML Dynamic HTML
DV Datenverarbeitung
D2C Device-to-Consumer
EDGE Enhanced Data Rates for GSM-Evolution
EDI Electronic Data Interchange
EDV Elektronische Datenverarbeitung
E-OTD Enhanced Observed Time Difference
ERP Enterprise Resource Planning
$F_{i} \quad$ Frequenz der Funkzelle i
FM Frequenzmodulation
$\mathrm{F}+\mathrm{E} \quad$ Forschung + Entwicklung
GPRS General Packet Radio Services
GSM Global System for Mobile Communication
$\mathrm{H}_{\mathrm{E}} \quad$ Varietät des Systemoutput 


\begin{tabular}{|c|c|}
\hline $\mathrm{H}_{\mathrm{R}}$ & Varietät des Reglers \\
\hline $\mathrm{H}_{\mathrm{s}}$ & Varietät der durch die Situation verursachten Störungen \\
\hline HSCSD & High Speed Circuit Switched Data \\
\hline $\mathrm{H}_{\mathrm{SR}}$ & Innere Unsicherheit des Systemverhaltens \\
\hline HTML & Hypertext Markup Language \\
\hline IETF & Internet Engineering Task Force \\
\hline IP & Internet Protocol \\
\hline $\mathrm{K}$ & Kosten \\
\hline $\mathrm{k}_{\mathrm{g}}$ & Gesamte Stückkosten \\
\hline $\mathrm{K}_{\mathrm{v}}$ & Variable Kosten \\
\hline $\mathrm{k}_{\mathrm{v}}$ & Variable Stückkosten \\
\hline $\mathrm{K}^{\prime}$ & Grenzkosten \\
\hline M-CRM & Mobile Customer Relationship Management \\
\hline OS & Operating System \\
\hline PDA & Personal Digital Assistant \\
\hline PPS & Produktionsplanung und -steuerung \\
\hline$r_{i}$ & Menge des Faktors i \\
\hline SA & Service Agent \\
\hline$S_{\mathrm{i}}$ & Spezifitätsniveau i \\
\hline SIM & Subscriber Identification Module \\
\hline SLP & Service Location Protocol \\
\hline SMS & Short Message Service \\
\hline TCP & Transmission Control Protocol \\
\hline TDCDMA & Time Division Code Division Multiple Access \\
\hline TDMA & Time Division Multiple Access \\
\hline UA & User Agent \\
\hline UDP & User Datagram Protocol \\
\hline $\mathrm{UM}$ & Unified Messaging \\
\hline UMTS & Universal Mobile Telecommunications System \\
\hline VRML & Virtual Reality Markup Language \\
\hline WAE & Wireless Application Environment \\
\hline WAP & Wireless Application Protocol \\
\hline WDP & Wireless Datagram Protocol \\
\hline WML & Wireless Markup Language \\
\hline WSP & Wireless Session Protocol \\
\hline
\end{tabular}


WTLS Wireless Transport Layer Security

WTP Wireless Transaction Protocol

$\mathrm{x}_{\mathrm{i}} \quad$ Menge des Produkts $\mathrm{i}$

XML Extendes Markup Language 
Dirk Beier - 978-3-631-75500-6

Downloaded from PubFactory at 01/11/2019 04:07:38AM 


\section{Einführung}

Die vorliegende Arbeit untersucht die Erkenntnispotenziale der Betriebswirtschaftslehre für das Informationsmanagement. Es werden verschiedene Ansätze für eine Theorie des Informationsmanagements entwickelt, die anschließend am Beispiel des Mobile Business zusammengeführt werden. Im folgenden Abschnitt 1.1 werden zunächst die Motivation und die Zielsetzung der Arbeit näher erläutert. Abschnitt 1.2 verdeutlicht davon ausgehend den Gang der Untersuchung und gibt einen kurzen Überblick über die einzelnen Kapitel.

\subsection{Motivation und Zielsetzung}

Vor dem Hintergrund des stark gestiegenen Stellenwerts, der dem Einsatz von Informations- und Kommunikationstechniken für den Erfolg von Unternehmungen zukommt, haben Fragen des Informationsmanagements in den letzten Jahren mehr und mehr Beachtung in Theorie und Praxis gefunden. Die Diskussion in diesem Bereich wird durch auffällig viele, immer neue Modeströmungen bestimmt, die von unterschiedlichen Seiten unter werbewirksamen Schlagwörtern vermarktet werden und sich in schneller Folge abwechseln. Noch bevor das jeweils neueste Konzept vollständig umgesetzt ist, tritt häufig schon die nächste Modewelle auf, sodass kaum Gelegenheit zu einer Konsolidierung der realisierten Lösungen besteht. Eine kritische Auseinandersetzung mit den einzelnen Konzepten findet nur selten statt, obwohl sie in vielen Fällen dringend geboten wäre. Diese Situation ist auch durch das weitgehende Fehlen von grundlagenbezogenen theoretischen Erkenntnissen bedingt. Die bisher vorgelegten wissenschaftlichen Arbeiten beschäftigen sich vorwiegend mit speziellen Einzelfragen, ohne dass jedoch ein übergreifendes Gesamtkonzept für das Informationsmanagement entwickelt wird. Dies führt dazu, dass kaum fixe Orientierungspunkte gegeben sind, an denen die Gestaltungs- und Steuerungsmaßnahmen im konkreten Einzelfall ausgerichtet werden können.

Die hier vorgelegte Arbeit hat das Ziel, diese Defizite zu überwinden und das Informationsmanagement als betriebliches Aufgabenfeld auf eine bessere theoretische Basis zu stellen. Die Untersuchung geht von der Überzeugung aus, dass bestehende theoretische Ansätze bisher zu wenig im Umfeld der technikunterstützten Informationsverarbeitung genutzt wurden. Durch die Bezugnahme auf allgemeine wissenschaftliche Aussagesysteme, die für die Anwendung auf Problemstellungen des Informationsmanagements zu spezifizieren sind, soll ein Theoriegerüst geschaffen werden, dem unabhängig von tagesaktuellen Entwicklungen richtungweisende Funktion zukommt. Die Orientierung an den durch dieses Theoriegerüst gelieferten Anhaltspunkten für konkrete Gestaltungsmaßnahmen könnte zu einer größeren Stabilität der in den Unternehmungen eingerichteten Lösungen bei der Nutzung moderner Informations- und Kommunikationstechniken beitragen.

Als Referenzdisziplin für die Ableitung von Aussagen zum Informationsmanagement wird in dieser Arbeit die Betriebswirtschaftslehre gewählt. Diese Wahl ist keineswegs darauf zurückzuführen, dass andere Wissenschaftsdisziplinen als irrelevant für Probleme des Informationsmanagements angesehen werden. Tatsächlich kann eine umfassende Untersuchung dieses vielschichtigen Aufgabenfelds nur durch eine multiperspektivische Analyse geleistet werden, die auch die Erkenntnisse anderer Wissen- 
schaften (z.B. der Volkswirtschaftslehre, der Informatik und der Sozialpsychologie) einbezieht. Einzelne Beiträge zu dieser multiperspektivischen Analyse sollten sich jedoch disziplinär beschränken, um durch eine Spezialisierung des wissenschaftlichen Arbeitens einen erfolgreichen Erkenntnisfortschritt zu erzielen.

Im Einzelnen werden hier der Faktortheoretische Ansatz, der Systemorientierte Ansatz und der Ansatz der Neuen Institutionenökonomie aus dem breiten Spektrum betriebswirtschaftlicher Forschungsansätze aufgegriffen. Basierend auf den Erkenntnisinteressen sowie den inhaltlichen und methodischen Leitideen der genannten Wissenschaftsprogramme erfolgt jeweils eine Konzeptionalisierung des Informationsmanagements aus der speziellen Betrachtungsperspektive dieser Ansätze. Damit werden die Grenzen für die nachfolgende eigentliche Theoriebildung abgesteckt, in deren Rahmen die Untersuchung einzelner Teilprobleme aus Sicht des betreffenden Ansatzes stattfindet. Auf diese Weise werden unterschiedliche Ansätze für eine Theorie des Informationsmanagements entwickelt, deren Aussagekraft abschließend stets kritisch überprüft wird.

$\mathrm{Da}$ die verschiedenen Ansätze für sich genommen nur jeweils einen kleinen Teilausschnitt der mit dem Informationsmanagement verbundenen Problemstellungen beleuchten, ist ferner eine Zusammenführung erforderlich, um zu einem vollständigeren Bild zu gelangen. Diese Zusammenführung kann jedoch nicht auf dem Wege einer Synthese zu einer Art Gesamtansatz erfolgen, weil die in den Erkenntnisinteressen und Leitideen enthaltenen grundsätzlichen Annahmen der einzelnen Ansätze zum Teil voneinander abweichen, sodass Inkonsistenzen in der Argumentation unvermeidlich wären. Der Idee der multiperspektivischen Analyse entsprechend werden die einzelnen Ansätze stattdessen exemplarisch auf ein bestimmtes Gestaltungsproblem angewendet. Die exemplarische Anwendung macht zugleich deutlich, dass sich die zuvor abgeleiteten Aussagen, die auf Grund des angestrebten hohen Allgemeinheitsgrads relativ abstrakt angelegt sind, so weit konkretisieren lassen, dass sie auch für empirisch vorzufindende Problemstellungen tragfähige Anhaltspunkte für Gestaltungsmaßnahmen bieten.

Die Zusammenführung der verschiedenen Theorieansätze erfolgt in dieser Arbeit am Beispiel aktueller Entwicklungen im Bereich der Konvergenz von leitungsgebundenen und mobilen Informations- und Kommunikationstechniken. Der Aufbau von Anwendungssystemen des Mobile Business ist unmittelbar mit dem Gestaltungsbereich des Informationsmanagements verknüpft, da diese Systeme in technischer und organisatorischer Hinsicht mit den leitungsgebundenen Anwendungssystemen der Unternehmung zu integrieren sind. Die hier entwickelten Ansätze für eine Theorie des Informationsmanagements werden abschließend nicht nur auf den Bereich des Mobile Business angewendet, sondern auch hinsichtlich ihrer Interdependenzen untersucht. Da die jeweils zu Grunde liegenden Erkenntnisinteressen und Leitideen nicht vollständig übereinstimmen, sind dabei keineswegs ausschließlich komplementäre, sondern zum Teil auch konkurrierende Aussagen zu erwarten.

\subsection{Aufbau der Arbeit}

Nach der Entwicklung eines Bezugsrahmens für die betriebswirtschaftliche Analyse des Informationsmanagements werden jeweils isoliert voneinander die Erkenntnispotenziale 
der hier aufgegriffenen Wissenschaftsprogramme der Betriebswirtschaftslehre untersucht. Im Anschluss daran werden die verschiedenen Argumentationsstränge wieder zusammengeführt, indem die abgeleiteten Aussagen auf das Beispiel Mobile Business angewendet werden. Abschließend erfolgt eine Schlussbetrachtung, die eine kritische Würdigung vornimmt und einen kurzen Ausblick gibt. Dieses Konzept ist in Abbildung 1/1 grafisch verdeutlicht.

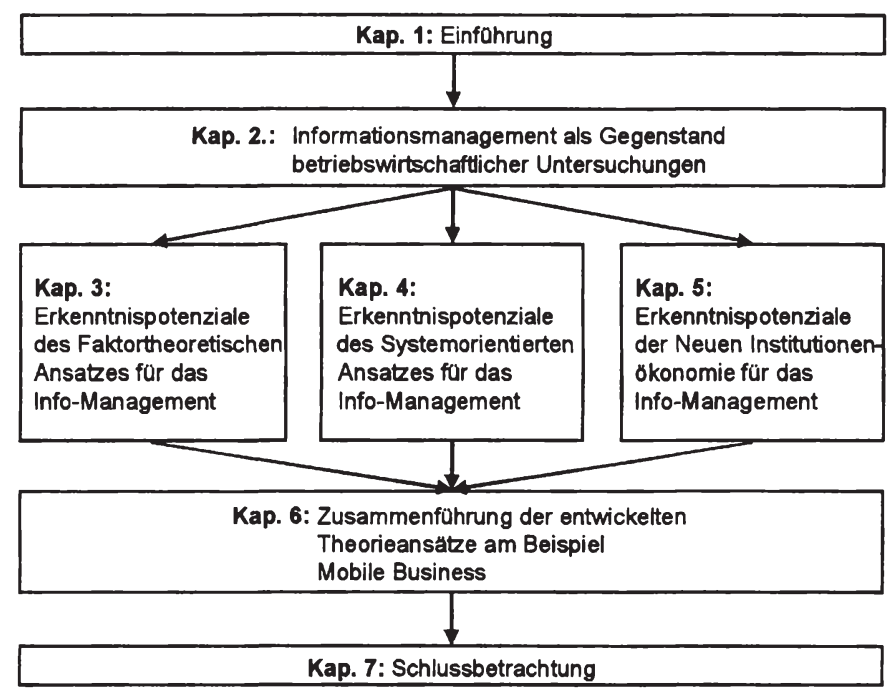

Abb. 1/1: Aufbau der Untersuchung

Nach dieser Einleitung beschreibt das zweite Kapitel zunächst die aktuelle Ausgangssituation, in der das Informationsmanagement durch gravierende ungelöste Problemstellungen in Theorie und Praxis gekennzeichnet ist. Die hier erarbeiteten Ergebnisse sollen einen Beitrag zur Lösung dieser Problemstellungen leisten. Im Rahmen der anschließenden Erläuterung der wissenschaftstheoretischen Grundlagen für eine realwissenschaftliche Untersuchung des Informationsmanagements wird ein Konzept für einen dreistufigen Theorieeinstieg entwickelt, das als Bezugsrahmen für die weitere Untersuchung dient. Die darauf folgenden Überlegungen des zweiten Kapitels bewegen sich auf der ersten Stufe des Theorieeinstiegs. Sie grenzen zum einen das Informationsmanagement als Erfahrungsobjekt $a b$ und stellen zum anderen die Betriebswirtschaftslehre als Referenzdisziplin der nachfolgenden Analyse vor. Neben einer Abgrenzung des Erfahrungsgegenstands der Betriebswirtschaftslehre erfolgen eine Standortbestimmung im Vergleich $\mathrm{zu}$ anderen Wissenschaftsdisziplinen und die Auswahl derjenigen Wissenschaftsprogramme, auf die in den anschließenden Kapiteln Bezug genommen wird.

Die drei nachfolgenden Kapitel stehen nebeneinander und sind analog zueinander strukturiert. Ihre Reihenfolge in dieser Arbeit ist allein durch die wissenschaftshistorische Reihenfolge der referenzierten Forschungsansätze bedingt. Im ersten und zweiten Abschnitt dieser drei Kapitel werden zunächst jeweils die zweite und die dritte Stufe des Theorieeinstiegs durch eine Konstruktion des allgemeinen und des speziellen 
Erkenntnisgegenstands bewältigt. Danach erfolgt im jeweils dritten Abschnitt die eigentliche Theoriebildung aus der Perspektive des betreffenden Ansatzes. Der vierte Abschnitt nimmt eine kritische Würdigung der Aussagekraft des entwickelten Ansatzes für eine Theorie des Informationsmanagements vor, die zum einen die Lösungsbeiträge zur Überwindung der im zweiten Kapitel beschriebenen Problemstellungen und zum anderen die Erkenntnisgrenzen betrachtet.

Das dritte Kapitel nimmt auf den Faktortheoretischen Ansatz der Betriebswirtschaftslehre Bezug. Dabei wird das Informationsmanagement als spezieller Teil des dispositiven Faktors konzeptionalisiert, der die Elementarfaktoren im Bereich der technikunterstützten Informationsverarbeitung bei der Herstellung informationeller Produkte miteinander kombiniert. Die abgeleiteten Aussagen beziehen sich auf drei spezielle Untersuchungsbereiche. Dabei handelt es sich um die Vorkombination und um die Endkombination von Elementarfaktoren im Gestaltungsbereich des Informationsmanagements sowie um das Informationsmanagement selbst, das im System der dispositiven Faktoren der rationalen sowie der gestaltend-vollziehenden Schicht zugeordnet wird.

Das vierte Kapitel bezieht sich auf den Systemorientierten Ansatz der Betriebswirtschaftslehre und fasst Informationsmanagement als Lenkung von Systemen auf. Das durch das Informationsmanagement zu lenkende System stellt aus dieser Perspektive ein Subsystem des Gesamtsystems Unternehmung dar. Im Zentrum der Argumentation stehen hier die Möglichkeiten zur Anpassung des Systems an veränderte System/Umweltsituationen. Die Beurteilung und Gestaltung dieser Möglichkeiten erfolgt mit Hilfe des Gesetzes der erforderlichen Varietät. Lenkungsmaßnahmen des Informationsmanagements können einerseits konservativ ausgerichtet sein und die Erkenntnisse der Kybernetik erster Ordnung nutzen. Sie können andererseits innovativ ausgerichtet sein und auf den Erkenntnissen der Kybernetik zweiter Ordnung aufbauen.

Das fünfte Kapitel untersucht, welche Erkenntnispotenziale und Lösungsbeiträge die Neue Institutionenökonomie für das Informationsmanagement bietet, und stützt sich dabei insbesondere auf die Ergebnisse der Transaktionskostentheorie und der AgencyTheorie. Aus dieser Sicht sollten moderne Informations- und Kommunikationstechniken dazu verwendet werden, die Koordinationseffizienz von Transaktionen als grundlegenden Bezugsobjekten dieses Ansatzes zu erhöhen. Die Ausführungen untersuchen zum einen die Gestaltungsoptionen des Informationsmanagements vor und nach Abschluss des Vertrags, auf dessen Basis die Transaktion abgewickelt wird. Zum anderen wird überprüft, inwiefern sich durch die Nutzung der aufgezeigten Gestaltungsoptionen Auswirkungen auf die Wahl des Vertragsdesigns ergeben.

Das sechste Kapitel führt die bis dahin getrennt voneinander entwickelten Theorieansätze zusammen, indem es diese exemplarisch auf die Gestaltung von Anwendungssystemen im Bereich des Mobile Business anwendet. Dazu ist zunächst eine Bestandsaufnahme erforderlich, in deren Rahmen die technischen Hintergründe und Anwendungspotenziale der mobilen Informationsverarbeitung erläutert werden. Davon ausgehend werden die Berührungspunkte zwischen dem Bereich des Mobile Business und dem Gestaltungsbereich des Informationsmanagements dargelegt. Auf dieser Basis erfolgt anschließend die exemplarische Anwendung in den vorangegangenen Kapiteln entwickelten Theorieansätze. Dabei wird auch überprüft, inwiefern die gewonnen 
Erkenntnisse einander ergänzen, sich neutral verhalten oder untereinander in einem konkurrierenden Verhältnis stehen.

Die Schlussbetrachtung im siebten Kapitel nimmt abschließend eine kritische Würdigung vor, die sich sowohl auf das Vorgehen als auch auf die Ergebnisse der Arbeit bezieht. Ferner wird ein Ausblick auf mögliche künftige Forschungsarbeiten zur weiteren theoretischen Durchdringung des Informationsmanagements gegeben. 
Dirk Beier - 978-3-631-75500-6

Downloaded from PubFactory at 01/11/2019 04:07:38AM 


\section{Informationsmanagement als Gegenstand betriebswirt- schaftlicher Untersuchungen}

Die Überlegungen in diesem Kapitel schaffen den Rahmen für die nachfolgende Analyse des Informationsmanagements. In Abschnitt 2.1 wird zunächst die Ausgangssituation betrachtet, in der ungelöste Problemstellungen in Theorie und Praxis bestehen. Der Abschnitt 2.2 erläutert die epistemologische Grundposition der Arbeit und stellt die wissenschaftstheoretischen Grundlagen dar, die der nachfolgenden Entwicklung unterschiedlicher Ansätze für eine Theorie des Informationsmanagements zu Grunde liegen. In Abschnitt 2.3 wird das Aufgabenfeld Informationsmanagement als Erfahrungsgegenstand der weiteren Untersuchung abgegrenzt. Abschnitt 2.4 schließlich stellt die Betriebswirtschaftslehre als Referenzdisziplin für eine wissenschaftiche Analyse des Informationsmanagements vor und die wählt diejenigen ihrer Wissenschaftsprogramme, auf die in den weiteren Kapiteln Bezug genommen wird, aus.

\subsection{Ausgangssituation: Unge/öste Problemstellungen des Informa- tionsmanagements in Theorie und Praxis}

Im Folgenden werden zunächst in Abschnitt 2.1.1 die Theoriedefizite herausgearbeitet, durch die der "State of the Art" im Bereich des Informationsmanagements gekennzeichnet ist und auf deren (teilweise) Überwindung die vorliegende Arbeit abzielt. Die unzureichende theoretische Durchdringung des Informationsmanagements hängt eng mit offenen Problemen in der Praxis zusammen, die im Abschnitt 2.1.2 erläutert werden.

\subsubsection{Theoriedefizite des „State of the Art" im Bereich des Informations- managements}

Informationsmanagement stellt im Vergleich zu anderen betrieblichen Problembereichen (z.B. dem Rechnungswesen, der Produktionswirtschaft oder der Personalwirtschaft) ein vergleichsweise junges Themengebiet dar, mit dem sich Theorie und Praxis der Unternehmungsführung erst seit relativ kurzer Zeit befassen. Es hat als betriebliches Aufgabenfeld seit etwa Mitte der 1970er Jahre mehr und mehr Beachtung in der betrieblichen Praxis gefunden. ${ }^{1}$ Die fortschreitende Verbreitung der Informations- und Kommunikationstechnik machte seinerzeit eine systematische Auseinandersetzung mit ihrer zielgerichteten Nutzung in den verschiedenen Unternehmungsbereichen erforderlich. ${ }^{2}$ Der Terminus "Informationsmanagement" geht in diesem Zusammenhang auf den amerikanischen Begriff „Information Management“ zurück, der Ende der 1970er Jahre von der US-amerikanischen Bundesverwaltung und in privatwirtschaftlichen Unternehmungen geprägt wurde. ${ }^{3}$

Vor dem Hintergrund der weiteren Diffusion der Informations- und Kommunikationstechnik in die verschiedenen betrieblichen Funktionsbereiche gewann die Informationsverarbeitung immer mehr an Bedeutung für das gesamte Unternehmungs-

\footnotetext{
Vgl. Brenner (1994), S. 5.

Vgl. Fank (1996), S. 15.

Vgl. Marchand (1989), S. 27ff.
} 
geschehen. Zugleich entstand eine Reihe unterschiedlichster Problemstellungen, deren Lösung infolge dieser gestiegenen Bedeutung immer dringender für den Geschäftserfolg wurde. Dazu zählen beispielsweise die ungebremste Steigerung der Kosten der Informationsverarbeitung, ein als zu gering empfundener Nutzen der verwendeten Systeme, der viel beklagte Stau bei der Entwicklung neuer Systeme in innovativen Anwendungsfeldern und eine häufig nur geringe Akzeptanz der technischen Systeme bei den Nutzern. ${ }^{4}$ In den 1980er Jahren entwickelte sich als Reaktion auf diese Probleme eine Diskussion um die Wettbewerbsrelevanz sowohl einzelner Anwendungssysteme als auch der gesamten Informationsverarbeitung von Unternehmungen. ${ }^{5}$ Es wurde ein ganzheitliches Konzept gefordert, das die (strategische) Unternehmungsführung mit der Planung, Steuerung und Kontrolle der Informationsverarbeitung verknüpfte. Etwa zu diesem Zeitpunkt fand auch im deutschsprachigen Raum eine „Entdeckung“ des Informationsmanagements statt. In der ersten Hälfte der 1990er Jahre entwickelte sich das Thema sowohl in der wissenschaftlichen Forschung als auch in der betrieblichen Praxis zu einer regelrechten Modewelle. ${ }^{6}$

Im Jahr 1987 erschien das von HEINRICH/BURGHOLZER ${ }^{7}$ verfasste, erste deutschsprachige Lehrbuch des Informationsmanagements, das sich in der Folgezeit zu einer Art Standardwerk entwickelte. In den 1990er Jahren folgten weitere Lehrbuchveröffentlichungen, von denen an dieser Stelle exemplarisch die Werke von BIETHAHN/MUCKSCH/RUF, HIDEBRAND, KRCMAR, SCHWARZE sowie VOß/GUTENSCHWAGER erwähnt seien. ${ }^{8}$ Neben dieser Behandlung im Rahmen von Lehrbüchern wurden Fragen des Informationsmanagements im letzten Jahrzehnt auch im Rahmen einer Vielzahl von Dissertationen untersucht. Als Beispiele für diese Kategorie seien hier die Arbeiten von PFEIFFER, BROCKHAUS, TROTT ZU SOlZ, NAWATZKI, SchWARZER, NUUTTGENS, SCHELLMANN und STREUBEL genannt. ${ }^{9} \mathrm{Zu}$ den Forschungsbeiträgen zählen auch die Arbeiten von PFAU und SCHLÖGL, die sich als Habilitationsschriften mit Fragen des betrieblichen Informationsmanagements auseinander setzen. ${ }^{10}$ Das Spektrum der Diskussionsbeiträge wird ferner durch eine nahezu unüberschaubare Vielzahl von Aufsätzen in diversen Fachzeitschriften ergänzt, die mal stärker praxisorientiert, mal stärker theorieorientiert einzelne Fragen des Informationsmanagements aufarbeiten.

Obwohl es sich beim Informationsmanagement erst um eine relativ junge Disziplin handelt, hat also bereits eine umfangreiche Aufarbeitung des Problembereiches stattgefunden. Trotz dieser starken Beachtung des Informationsmanagements konnte allerdings in grundsätzlichen Punkten, die für nachfolgende Forschungsaktivitäten wesentlich sind, noch keine Einigkeit erzielt werden. Die Diskussion ist vielmehr von einem Nebeneinander vielfältiger, zum Teil sehr unterschiedlicher Begriffs-

4 Für eine Übersicht über typische Probleme, die zur Auslösung der vertieften Auseinandersetzung mit dem Informationsmanagement führten, vgl. z.B. Schwarze (1998), S. 15ff.

5 Vgl. Porter/Millar (1986), S. 26ff.; Brenner (1994), S. 5.

6 Vgl. Streubel (2000), S. 47 f.

7 Heinrich/Burgholzer (1987).

8 Biethahn/Mucksch/Ruf (1990) und (1991), Hildebrand (1995), Krcmar (1997), Schwarze (1998), Voß/Gutenschwager (2001). Die genannten Jahreszahlen beziehen sich jeweils auf die erste Auflage des betreffenden Lehrbuches.

9 Pfeiffer (1990), Brockhaus (1992), Trott zu Solz (1992), Nawatzki (1994), Schwarzer (1994), Nüttgens (1995), Schellmann (1997), Streubel (2000).

${ }^{10}$ Pfau (1997), Schlögl (2001). 
auffassungen und entsprechender Abgrenzungen des Aufgabenfeldes geprägt. ${ }^{11}$ Die vor gut einem Jahrzehnt von ZAHN und RUTTLER aufgestellte These, das Informationsmanagement befinde sich als Forschungsrichtung in einem frühen Stadium der Entwicklung, in dem sich noch kein allgemein anerkanntes Konzept habe entwickeln können, ${ }^{12}$ kennzeichnet auch derzeit noch die Situation. Nach wie vor existieren verschiedene Ansätze, die sich hinsichtlich Zielsetzung, Fragestellung und der Wahl des Bezugsrahmens erheblich voneinander unterscheiden.

Ferner ist anzumerken, dass trotz der Vielzahl von Arbeiten bisher keine umfassende theoretische Fundierung des Informationsmanagements insgesamt vorliegt. Eine wesentliche Ursache für diese Problematik ist zunächst darin $\mathrm{zu}$ sehen, dass vielen Arbeiten eine präzise Definition des von ihnen betrachteten Untersuchungsgegenstands fehlt. ${ }^{13}$ Auf Grund der fehlenden Begriffsschärfe wird die inhaltliche Strukturierung des Aufgabenfeldes erschwert, da kein klarer Bezugspunkt für die Identifikation und Analyse einzelner Teilprobleme des Informationsmanagements besteht. Auch die Verknüpfung dieser Teilprobleme zu einem Gesamtkonzept kann sich infolgedessen nicht an der dafür erforderlichen grundlegenden Begriffsabgrenzung, auf deren Basis die gesamte Untersuchung erfolgt, orientieren. Auf diese mangelnde Strukturierung ist es zurückzuführen, dass sich das Informationsmanagement dem Leser häufig als Sammlung unverbundener Aufgaben darstellt. ${ }^{14}$ Aufgaben werden dabei gemäß durch den Autor mehr oder weniger willkürlich gewählter Kriterien wie z.B. „Gestaltungsobjekt", „Zeithorizont“, „Nähe zur Technik“ oder "Wettbewerbsrelevanz der Aufgabenstellung" gruppiert und sequenziell abgearbeitet. Solche Problem- und Aufgabenlisten erweisen sich für die Entwicklung eines Gesamtkonzeptes als untauglich, da sie Grundprobleme des Informationsmanagements einfach umgehen und sich auf die Untersuchung von Einzelfragen beschränken. ${ }^{15}$

Das Nicht-Vorhandensein eines Gesamtkonzepts stellt allerdings nur einen Aspekt der unzureichenden theoretischen Fundierung des Informationsmanagements dar. Diese wird dadurch verstärkt, dass auch im Rahmen der eigentlichen Untersuchungen nur selten eine Bezugnahme auf größere theoretische Zusammenhänge stattfindet. Bestehende Erkenntnisse aus den verschiedenen wissenschaftlichen Disziplinen, die auf Grund ihrer inhaltlichen und methodischen Ausrichtung im Prinzip für eine Anwendung auf den Bereich der technikunterstützten Informationsverarbeitung in Unternehmungen in Frage kämen, werden im Umfeld des Informationsmanagements bisher weitgehend ignoriert. Die Argumentation stützt sich stattdessen häufig auf Plausibilitätsüberlegungen, die keines tiefergehenden theoretischen Hintergrunds bedürfen. Typisch für eine derartige Vorgehensweise sind mehr oder weniger einfach strukturierte Vor-/Nachteilsbetrachtungen und nicht über das Exemplarische hinausreichende Darstellungen von Fallstudien, aus denen ohne weitere Reflexion auf zu Grunde liegende allgemeingültige Zusammenhänge geschlossen wird.

Problematisch an der Verwendung solcher Fallstudien ist nicht die Praxisorientierung der Forschung an sich. Tatsächlich gewährleistet allein ein erheblicher Praxisbezug des

${ }^{11}$ Vgl. Streubel (2000), S. 48; Schlögl (2001), S. 1.

12 Vgl. Zahn/Rüttler (1990), S. 5.

13 Vgl. Streubel (2000), S. 48.

${ }^{14} \mathrm{Vgl}$. Schwarzer (1994), S. 107.

15 Vgl. Krcmar (2000), S. 33. 
wissenschaftlichen Arbeitens im Umfeld des Informationsmanagements, dass aktuelle Entwicklungen im intendierten Anwendungsbereich der Theorie nicht unbemerkt bleiben und immer neue Impulse für eine weitere Ausdifferenzierung des theoretischen Bezugsrahmens ausgelöst werden. Praxis und Praxiskonzepte bleiben aber trotzdem "nur" das notwendige empirische Feld, an dem die Theorie gemessen werden muss. ${ }^{16}$ Eine fehlgerichtete Konzentration auf Fallbeispiele birgt deshalb die Gefahr der zu engen Anbindung an tagesaktuelle Modeströmungen in der Praxis, die schon nach kurzer Zeit durch neue Trends abgelöst werden. Aufgabe der wissenschaftlichen Auseinandersetzung mit derartigen Modeströmungen ist es vielmehr, „das Vorliegende und Offensichtliche mit ihrem Methodenschatz zu bewerten, grundlegende neue Wege aufzuzeigen und ihre Kontextbedingungen zu erschließen. “17 Dementsprechend ist der von ROLF erhobenen Forderung zuzustimmen, dass sich die Wirtschaftsinformatik, beziehungsweise die wissenschaftiche Untersuchung des Informationsmanagements als Teilbereich der Wirtschaftsinformatik, auf die sich die Überlegungen in dieser Arbeit beschränken, in verstärktem Maße als Grundlagenforschung verstehen sollte. ${ }^{18}$

Zwar ist häufig zu beobachten, dass in Arbeiten zum Informationsmanagement an einzelnen Stellen eine Bezugnahme auf bereits bestehende größere Theoriekomplexe erfolgt. Diese beschränkt sich jedoch auf das jeweilige Teilproblem, für das sich der betreffende Ansatz nach Auffassung des Autors besonders gut eignet. Dagegen wird die Untersuchung anderer Teilprobleme entweder gar nicht oder mit Hilfe anderer Ansätze, die an der entsprechenden Stelle als besser geeignet angesehen werden, theoretisch fundiert. Die Beziehungen der verschiedenen referenzierten Ansätze untereinander, die möglicherweise auf unterschiedlichen, zum Teil kaum miteinander vereinbaren Prämissen beruhen, werden dabei üblicherweise nicht thematisiert. Der Theoriebezug bleibt bei einem derartigen, eklektischen Vorgehen facettenhaft, teilweise widersprüchlich und ohne zusammenhängendes Gesamtkonzept für das Informationsmanagement. Diese Kritik ist dahingehend zu relativieren, dass sehr wohl Arbeiten vorliegen, die auf einer gut ausgebauten theoretischen Basis eine detaillierte Analyse einzelner Themenstellungen vornehmen und keine eklektisch bedingten Widersprüche beinhalten. Die entsprechenden Arbeiten richten sich jedoch schwerpunktmäßig auf die Untersuchung des betreffenden Teilgebietes und zielen regelmäßig nicht auf ein umfassendes Gesamtkonzept ab.

Die vorliegende Arbeit nimmt die beschriebenen Theoriedefizite des "State of the Art" im Bereich des Informationsmanagement zum Anlass, eine umfassendere theoretische Fundierung $\mathrm{zu}$ entwickeln, die auf verschiedenen Wissenschaftsprogrammen der Betriebswirtschaftslehre basiert. Die Untersuchungen gehen von der Überzeugung aus, dass die bereits bestehenden Forschungsrichtungen der Betriebswirtschaftslehre erhebliche Erkenntnispotenziale für das Informationsmanagement besitzen, die bisher nur ansatzweise erschlossen wurden. Die Zielsetzung der Arbeit liegt darin, diese Erkenntnispotenziale aufzuzeigen und Anhaltspunkte für die Ableitung von erklärenden und gestaltenden Aussagen im konkreten Einzelfall zu bieten.

\footnotetext{
${ }^{16}$ Vgl. Rolf (1998), S. 7. Dieser bezieht sich mit seinen Aussagen allerdings auf den gesamten Bereich der Wirtschaftsinformatik.

17 Rolf (1998), S. 7.

18 Vgl. Rolf (1998), S. 7.
} 


\subsubsection{Offene Probleme des Informationsmanagements in der Praxis}

Empirische Untersuchungen sowohl der 1980er als auch der 1990er Jahre zeigen, dass eine Vielzahl von Unternehmungen von der Ausschöpfung der Möglichkeiten moderner Informations- und Kommunikationstechnik noch weit entfernt ist. ${ }^{19}$ Die oben angesprochenen Ausgangsprobleme als Auslöser des Informationsmanagements konnten nur teilweise gelöst werden und die zwischenzeitlichen Entwicklungen im organisatorischen, technischen und personalen Umfeld $^{20}$ führen $\mathrm{zu}$ weiteren Herausforderungen. Die Ausführungen in diesem Abschnitt wenden sich der Situation der Informationsverarbeitung zu, der das Informationsmanagement vor diesem Hintergrund in vielen Unternehmungen gegenübersteht. Das Ziel der nachfolgenden Situationsbeschreibung ist es, ungelöste Problemstellungen in der Praxis herauszuarbeiten, für die eine anwendungsorientierte wissenschaftliche Auseinandersetzung mit dem Informationsmanagement Lösungsansätze entwickeln sollte. Entsprechend den oben geäußerten Vorbehalten gegenüber der Überbetonung von Fallstudien, gehen die folgenden Überlegungen nicht von der konkreten Situation eines oder weniger einzelner Unternehmungen aus. Sie richten sich vielmehr auf die Beschreibung von archetypischen Problemstellungen, die auf Grund eines hohen Abstraktionsgrades eine große Zahl von Unternehmungen betreffen, im einzelnen Fall jedoch unterschiedliche konkrete Ausprägungsformen annehmen können. Die Orientierung an idealtypischen Problemstellungen trägt der Annahme Rechnung, dass theoretische Aussagen einen um so höheren Wert besitzen, je höher ihr Grad der Allgemeingültigkeit ist. ${ }^{21}$

Vor dem Hintergrund der im vorigen Abschnitt aufgezeigten Theoriedefizite des „State of the Art" im Bereich des Informationsmanagements wird hier die These vertreten, dass die offenen Probleme des Informationsmanagements in der Praxis zumindest zum Teil auch eine Folge der bisher fehlenden theoretischen Fundierung bei der wissenschaftlichen Erforschung dieses Aufgabenfeldes sind. Die nachfolgend beschriebenen ungelösten Probleme in der Praxis stellen das erkenntnisleitende Kriterium dar, an dem die Aussagekraft der theoretischen Erkenntnisse zum Informationsmanagement, die durch eine Bezugnahme auf vorliegende Wissenschaftsprogramme der Betriebswirtschaftslehre gewonnen werden können, zu messen ist. Von einer Erschließung der Erkenntnispotenziale, die die bereits bestehenden Wissenschaftsprogramme der Betriebswirtschaftslehre für das Informationsmanagement besitzen, ist zu erhoffen, dass sich neue Vorschläge entwickeln lassen, die wirksam zur Lösung der hier skizzierten Probleme in der Praxis beitragen können. Am Ende der nachfolgenden drei Kapitel wird daher jeweils überprüft, inwiefern der zuvor entwickelte Ansatz für eine Theorie des Informationsmanagements in der Lage ist, einen Beitrag zur Lösung dieser Probleme zu leisten.

Auf Grund des hohen Allgemeingültigkeitsanspruchs kann die hier vorgenommene, idealtypische Situationsbeschreibung zwar nicht alle denkbaren Einzelprobleme erfassen, die im konkreten Fall durch das Informationsmanagement in der Praxis zu

19 Vgl. dazu z.B. die publizierten Ergebnisse der Studien von Selig (1986), Pfeiffer (1990), Krcmar/ Federmann (1990), Wesseler (1992) sowie die umfassende Übersicht bei Schellmann (1997), S. 57ff.

${ }^{20}$ Für eine nähere Beschreibung von allgemeinen Entwicklungstendenzen in diesen Bereichen vgl. Streubel (1996), S. 3ff; Gabriel/Beier (2000), S. 3ff.

${ }^{21} \mathrm{Vgl}$. Schanz (1997a), S. 138. Die Allgemeingültigkeit von Aussagen darf allerdings nicht so weit gehen, dass empirisch nicht überprüfbare Tautologien entstehen. 
lösen sind. Die hier vorgenommene Auswahl erhebt jedoch den Anspruch, dass die dominierenden Fragestellungen, mit denen sich das Informationsmanagement als betriebliches Aufgabenfeld in der Praxis auseinander setzen muss, den aufgeführten Archetypen zugeordnet werden können. Entsprechend der allgemein anerkannten Forderung nach einer ganzheitlichen Denkweise im Bereich des Informationsmanagements ${ }^{22}$ ist ferner davon auszugehen, dass die hier erläuterten Problembereiche nicht vollkommen isoliert voneinander gesehen und behandelt werden dürfen, sondern in einer interdependenten Beziehung zueinander stehen.

\subsubsection{Effektivität der betrieblichen Informationsverarbeitung}

Die Informationsverarbeitung muss auf die Unterstützung der allgemeinen Unternehmungsziele ausgerichtet werden. ${ }^{23}$ Die verstärkte Unterstützung der Unternehmungsstrategie durch computergestützte Anwendungssysteme wurde in der Praxis zu Beginn der 1990er Jahre als eine der Hauptaufgaben des Informationsmanagements mit erheblichem Nachholbedarf angesehen. ${ }^{24}$ Dabei sind die prinzipiellen Anwendungspotenziale der Informations- und Kommunikationstechnik auf der einen Seite und die unternehmungsindividuellen Anforderungen, die sich aus der Markt- und Wettbewerbssituation im konkreten Einzelfall ergeben, auf der anderen Seite aufeinander abzustimmen. ${ }^{25}$ Empirische Studien zeigen, dass es in der Praxis bisher erst relativ selten gelungen ist, aus der Unternehmungsstrategie eine Strategie für die technikunterstützte Informationsverarbeitung in der Unternehmung abzuleiten. ${ }^{26}$ Die empirisch festgestellten, deutlichen Abweichungen zwischen Ist- und Sollzustand auf diesem Gebiet sind so zu interpretieren, dass die Bedeutung einer strategisch ausgerichteten Gestaltung von Informationssystemen zwar erkannt, aber die damit verbundenen Aufgaben noch nicht gelöst wurden. ${ }^{27}$ Nach wie vor ist die Situation häufig durch ein fehlendes informationswirtschaftliches Gesamtkonzept gekennzeichnet, ${ }^{28}$ ohne das eine funktionsübergreifende Orientierung bei der Unterstützung von Geschäftsprozessen nicht erreicht werden kann.

Eine mangelnde Effektivität der Informationsverarbeitung ist zudem nicht nur mit Blick auf das Gesamtsystem festzustellen, sondern auch in Bezug auf die konkrete Gestaltung einzelner Anwendungssysteme. Das wesentliche Problem liegt dabei darin, die richtige Information zur richtigen Zeit am richtigen Ort bereitzustellen. ${ }^{29}$ Durch die unbefriedigende Beurteilbarkeit des Nutzens, dessen Wert unter anderem von subjektiven Einflüssen abhängt, wird eine zielgerichtete Versorgung der Anwender mit den erforderlichen Informationen erschwert. Der von technikunterstützten Informationssystemen produzierte Output zielt deshalb immer noch häufig an den Anforderungen der Benutzer vorbei.

\footnotetext{
${ }^{22}$ Diese Forderung erheben z.B. Trott zu Solz (1992), S. 5; Biethahn/Mucksch/Ruf (1994), S. 11; Streubel (1996), S. 1; Schwarze (1998), S. 46; Krcmar (2000), S. 33.

${ }^{23} \mathrm{Vgl}$. Voß/Gutenschwager (2001), S. 66.

${ }^{24}$ Vgl. Brenner (1994), S. 31.

${ }^{25} \mathrm{Vgl}$. ähnlich Schwarze (1998), S. 21.

${ }^{26}$ Vgl. dazu Roithmayr/Wendner (1992), S. 472ff.; Brenner (1994), S. 49f.

27 Vgl. Krcmar (1997), S. 14.

${ }^{28}$ Vgl. Streubel (1996), S. 6.

${ }^{29}$ Vgl. Gabriel/Rohrs (1995), S. 4.
} 


\subsubsection{Effizienz der Informationsverarbeitung}

Während auf die Effektivität der Informationsverarbeitung vor allem in der Systementwicklungsphase Einfluss genommen werden kann, rückt bei bereits im Einsatz befindlichen Anwendungssystemen die Effizienz der Nutzung stärker in den Vordergrund. Nach wie vor steigen in vielen Unternehmungen die Kosten der Informationsverarbeitung überproportional $\mathrm{zu}$ dem diesen Kosten gegenüberstehenden, empfundenen Nutzen. ${ }^{30}$ Die Wirtschaftlichkeit der Informationsverarbeitung ist in der Praxis also häufig nicht gewährleistet. Ein ganzheitliches Konzept für eine effiziente Informationsverarbeitung lässt sich nur dann erreichen, wenn deren Kosten- und Leistungsaspekte vollständig berücksichtigt werden. ${ }^{31}$

Wie bereits im Rahmen der Ausführungen zur Effektivität angesprochen, stellt sich hier zum einen das Problem der Messung und Bewertung des Nutzens von Informationssystemen. Für Wirtschaftlichkeitsanalysen, die sich auf das Verhältnis von Output zu Input beziehen, sind zum anderen auch die für die Informationsverarbeitung entstehenden Kosten zu messen und zu bewerten. Da sich mit der Einführung neuer Anwendungssysteme häufig aufbau- und ablauforganisatorische Veränderungen ergeben, sind die Kostenwirkungen der Informationsverarbeitung im Vergleich zu anderen Objekten der Kostenrechnung besonders schwer zu erfassen. ${ }^{32}$ Zur Realisierung von Effizienzsteigerung müssen die Unternehmungen ferner nicht nur die Kosten der Informationsverarbeitung messen, sondern auch Anreize schaffen, diese zu senken. Bisher verfügt die Praxis nicht über geeignete Mess- und Anreizsysteme (z.B. auf die speziellen Charakteristika der Informationsverarbeitung abgestimmte Kostenrechnungssysteme, Systeme zur internen Leistungsverrechnung oder effizienzorientierte Entlohnungssysteme), die in der Lage sind, die Effizienz der betrieblichen Informationsverarbeitung $\mathrm{zu}$ gewährleisten. Information wurde im Gegenteil von den Anwendern sehr lange als freies Gut angesehen. ${ }^{33}$

\subsubsection{Organisatorische Zuordnung informationswirtschaftlicher Aufgaben}

In der Praxis stellt sich die Frage, wie die Führungs- und Durchführungsaufgaben der Informationsverarbeitung in der Unternehmung im konkreten Fall organisatorisch zuzuordnen sind. Auf der einen Seite wird vielfach die möglichst hohe, zentrale Ansiedlung von Aufgaben des Informationsmanagements gefordert. ${ }^{34}$ Auf der anderen Seite ist dagegen in vielen Wirtschaftsbereichen ein Trend zur organisatorischen Dezentralisierung zu beobachten, dessen grundsätzliche Relevanz für das Informationsmanagement in der Praxis durch viele empirische Untersuchungen belegt wird. ${ }^{35}$ Die organisatorische Zuordnung informationswirtschafticher Aufgaben bewegt sich deshalb im Spannungsfeld zwischen zentralen und dezentralen Lösungen.

Dabei stellt sich in der Regel nicht die Frage nach vollständiger Zentralisierung oder Dezentralisierung. Vielmehr ist jeweils aufgabenspezifisch über die organisatorische

\footnotetext{
${ }^{30} \mathrm{Vgl}$. Angermeyer (1993), S. 235.

${ }^{31}$ Vgl. Biethahn/Mucksch/Ruf (1994), S. 53.

${ }^{32}$ Vgl. Schwarze (1998), S. 212.

${ }^{33} \mathrm{Vgl}$. Schwarze (1998), S. $37 \mathrm{f}$.

${ }^{34}$ Vgl. z.B. Schwarze (1990), S. 113; Angermeyer (1993), S. 236.

${ }^{35}$ Vgl. dazu die Übersicht bei Krcmar (1997), S. 9ff.; Schellmann (1997), S. 68ff.
} 
Zuordnung zu entscheiden. ${ }^{36}$ Das Informationsmanagement ist gefordert, organisatorische Dezentralisierungskonzepte mit erhöhter Autonomie der dezentralen Einheiten zu unterstützen oder sogar erst zu ermöglichen, und zugleich die Gefahren einer zu starken Heterogenität bei der dezentralen Lösung informationswirtschaftlicher Probleme $\mathrm{zu}$ vermeiden. Solche Gefahren können beispielsweise in Kompetenzüberschneidungen auf Grund unklarer Zuständigkeiten zwischen den an der Erstellung der Gesamtleistung beteiligten Einheiten und in aus Gesamtsicht suboptimalen Insellösungen liegen, die zu Redundanzen in der Informationsverarbeitung führen. ${ }^{37}$ Durch einen parallel zum organisatorischen Trend auftretenden technischen Trend zur Dezentralisierung, der ebenfalls die Verlagerung von Zuständigkeiten in die Fachabteilungen begünstigt, ${ }^{38}$ rücken mehr und mehr die Schnittstellen zwischen verschiedenen (organisatorischen oder technischen) Teilsystemen in den Blickpunkt des Informationsmanagements. ${ }^{39}$

\subsubsection{Rolle des Menschen in der Informationsverarbeitung}

Dem Verhalten von Menschen wurde bisher in der Praxis und der Theorie des Informationsmanagements nur wenig Beachtung geschenkt. ${ }^{40} \mathrm{Im}$ Zuge der allgemein $\mathrm{zu}$ beobachtenden "Wiederentdeckung menschlicher Arbeit als primärer Unternehmensressource"41 findet jedoch die These, dass auch der Erfolg des Einsatzes von Informationssystemen maßgeblich durch die Mitarbeiter der Unternehmung bestimmt wird, mehr und mehr Zustimmung. Deshalb wird in immer stärkerem Maße versucht, das menschliche Verhalten bei der Gestaltung computergestützter Informationssysteme in angemessener Weise zu berücksichtigen. Die Betrachtung geht mittlerweile weit über die ursprünglichen Themen Akzeptanz und Partizipation hinaus und befasst sich mit grundlegenderen Fragen hinsichtlich der persönlichen Bedürfnisse von Menschen, von deren Erfüllung die Motivation der Mitarbeiter bei der Ausübung der ihnen übertragenen Aufgaben abhängt. Die Diskussion wird dabei einerseits durch gestiegene Qualitätsansprüche der Mitarbeiter an die eigene Arbeit sowie andererseits durch Forderungen nach einem stärker generalistisch orientierten Qualifikationsprofil und höherer sozialer Kompetenz der Mitarbeiter bestimmt.

Ferner wird nicht nur das Ergebnis der Systementwicklung verstärkt an den menschlichen Benutzern ausgerichtet, sondern die Gestaltung des gesamten Entwicklungsprozesses orientiert sich stärker an den Aktivitäten der beteiligten Akteure. Neue Modellierungssprachen zur Beschreibung von Geschäftsprozessen sollen eine systematische Vorgehensweise bei der Gestaltung von Informationssystemen fördern und die Verständigungsschwierigkeiten zwischen beteiligten Personen mit unterschiedlichem Ausbildungshintergrund, die in fachlich gemischten Teams zusammenarbeiten, verringern. Die Feststellung, dass verschiedene Interessengruppen als Akteure im Prozess der Entwicklung von technikunterstützten Informationssystemen miteinander in Interaktion treten, macht zudem eine Veränderung der bisherigen Sichtweise erforderlich. Der Entwicklungsprozess ist als komplexes Phänomen mit vielfachen

36 Vgl. Voß/Gutenschwager (2001), S. 98.

${ }^{37}$ Vgl. Wolfram (1990), S. 4.

38 Vgl. Schwarze (1998), S. 284.

$39 \mathrm{Vgl}$. Biethahn/Mucksch/Ruf (1994), S. 97ff.

${ }^{40}$ Vgl. Fank (1996), S. 272; Streubel (1996), S. 1.

${ }^{41}$ Picot/Reichwald/Wigand (1996), S. 443. 
Rückkopplungen aufzufassen.$^{42}$ Durch die verstärkte Akteursorientierung wird die Vorstellung von einem "Konstruktionskorridor" überwunden, die in der Vergangenheit häufig dem Systementwicklungsprozess zu Grunde lag und den Benutzern die rein passive Rolle von Vollzugsinstanzen zuwies. ${ }^{43}$

\subsection{Wissenschaftstheoretische Grundlagen einer realwissenschaft- lichen Untersuchung des Informationsmanagements}

In diesem Abschnitt werden die wissenschaftstheoretischen Grundüberlegungen für eine Untersuchung des Informationsmanagements aus der Perspektive verschiedener Wissenschaftsprogramme der Betriebswirtschaftslehre angestellt. Zunächst erfolgen einige Vorüberlegungen zur Abgrenzung des Informationsmanagements als Erfahrungsgegenstand und als Erkenntnisgegenstand wissenschafticher Untersuchungen (Abschnitt 2.2.1). Im Anschluss daran wird in Abschnitt 2.2.2 die Idee, die zu Beginn dieses Kapitels beschriebenen Theoriedefizite durch eine Bezugnahme auf vorliegende Wissenschaftsprogramme (der Betriebswirtschaftslehre) zu überwinden, näher vorgestellt. Abschnitt 2.2.3 entwickelt ein Konzept für einen dreistufigen Theorieeinstieg, auf dessen Basis in den nachfolgenden Kapiteln eine theoretisch fundierte Analyse des Informationsmanagements vorgenommen werden kann.

\subsubsection{Informationsmanagement als realwissenschaftlicher Erfahrungs- und Erkenntnisgegenstand}

Die Untersuchung des Informationsmanagements bezieht sich auf eine tatsächlich in der (Unternehmungs-) Wirklichkeit vorliegende Problemstellung und hat somit realwissenschaftlichen Charakter. Realwissenschaften sind dadurch gekennzeichnet, dass sie sich mit in der Wirklichkeit vorhandenen, individuellen, raum-zeitlich feststellbaren Tatsachen und Problemen befassen. ${ }^{44}$ Beim Informationsmanagement als Feld von Aufgaben, deren Erfullung in jeder real existierenden Unternehmung eine der Voraussetzungen für die Erstellung ihrer Leistungen darstellt, handelt es sich um einen solchen realen Sachverhalt. Die Entwicklung theoretisch fundierter Aussagen erfordert in den Realwissenschaften stets eine genaue Bestimmung des Objektbereiches, auf den sich die Untersuchung bezieht. Dabei ist zwischen dem Erfahrungsgegenstand und dem Erkenntnisgegenstand der jeweiligen Disziplin zu unterscheiden.

Der Erfahrungsgegenstand entspricht dem empirischen Phänomen, auf das sich die wissenschaftichen Betrachtungen beziehen. ${ }^{45}$ Die Annahmen über die allgemeine Beschaffenheit dieses Erfahrungsgegenstandes hängen von der epistemologischen Grundauffassung des Betrachters ab. Geht man von einer naiv realistischen Auffassung aus, dann wird angenommen, dass eine objektive Wirklichkeit existiert, die der Mensch so wahrnehmen und erkennen kann, wie sie ist. ${ }^{46}$ Das Erkennen des Menschen im Rahmen wissenschaftlichen Arbeitens unterliegt nach dieser Vorstellung keinen subjektiven Einflüssen. Der Erfahrungsgegenstand einer Wissenschaft stellt dann etwas

\footnotetext{
${ }^{42}$ Vgl. Rolf (1998), S. 19.

${ }^{43}$ Zur Sicht des Konstruktionskorridors und zur Kritik daran vgl. Rolf (1998), S. 15ff.

${ }^{44}$ Vgl. Schweitzer (1997), S. 27; Stein (1993), Sp. 479.

45 Vgl. Neus (1998), S. 2.

${ }^{46} \mathrm{Vgl}$. Streubel (2000), S. 19.
} 
unmittelbar Gegebenes, eine von allem Denken unberührte Wirklichkeit dar, die dem Wissenschaftler unmittelbar vorliegt. ${ }^{47}$ Diese Eigenschaften des Erfahrungsgegenstandes gelten im Prinzip auch dann, wenn man von einer kritisch rationalistischen Grundposition ausgeht. In diesem Fall wird angenommen, dass eine (objektive) "Wirklichkeit an sich" existiert, deren subjektive Erkenntnis jedoch einen Bewusstseinszustand des erkennenden Menschen darstellt und somit nicht frei von subjektiven Einflüssen ist. ${ }^{48}$

Dagegen bildet Erkenntnis nach radikal konstruktivistischer Grundauffassung nicht eine objektive Wirklichkeit ab, sondern sie konstruiert etwas, das der Betrachter erkennend als Wirklichkeit akzeptiert. ${ }^{49}$ Die Welt, so wie sie vom Betrachter wahrgenommen wird, ist dementsprechend die Erfindung des Betrachters. ${ }^{50}$ Folgt man dieser Auffassung, dann entsteht der Erfahrungsgegenstand einer Wissenschaft erst durch Konstruktion des Beobachters. Er stellt nach diesem Verständnis ein Objekt dar, welches auf Basis von theoretischem Vorverständnis, Alltagswissen, Erfahrung und Interessen ermittelt wird. ${ }^{51}$ Das Erfahrungsobjekt unterscheidet sich von dem „Gegebenen“ dadurch, dass es selbst den Charakter einer Repräsentation besitzt. Es wird von der wissenschaftlichen Gemeinschaft nicht gefunden, sondern von ihr erfunden bzw. bestimmt. ${ }^{52}$ Dieser Auffassung wird hier gefolgt.

Der Erkenntnisgegenstand entspricht dagegen dem Blickwinkel und der speziellen Fragestellung, aus der heraus der Erfahrungsgegenstand betrachtet wird. ${ }^{53}$ Er stellt ein abstraktes Denkobjekt dar, das aus dem Erfahrungsobjekt gedanklich abgeleitet und für sich vergegenständlicht ist. ${ }^{54}$ Der Erkenntnisgegenstand präzisiert den Erfahrungsgegenstand und ersetzt ihn im jeweiligen Kontext. Um den Erfahrungsgegenstand in seiner Gesamtheit zu erfassen, zu analysieren und zu gestalten, müssen mehrere Einzelwissenschaften sich mit dem entsprechenden Bereich befassen. ${ }^{55}$ Zur Konstruktion des jeweiligen Erkenntnisgegenstandes müssen die verschiedenen Einzelwissenschaften ihre individuellen Grundfragestellungen herausarbeiten, auf die sich ihre speziellen Untersuchungen richten. Während es sich bei dem Erfahrungsgegenstand also um ein empirisches Konstrukt handelt, handelt es sich beim Erkenntnisgegenstand um ein theoretisches Konstrukt. ${ }^{56}$ Das theoretische Konstrukt entsteht dadurch, dass die Repräsentation des empirischen Konstruktes zum Zweck der Entwicklung einer konkreten Theorie „zurechtgemacht" wird. Dies entspricht einem zweistufigen Theorieeinstieg, ${ }^{57}$ da zunächst eine allgemeine, "unpräparierte“ Beschreibung des Untersuchungsbereiches $\mathrm{zu}$ entwickeln ist, die anschließend durch eine vor dem Hintergrund des jeweiligen Erkenntnisinteresses „präparierte“ Beschreibung abgelöst wird.

${ }^{47}$ Diese Auffassung geht auf Ammon (1927), S. 21ff. zurïck.

48 Vgl. Popper (1995), S. 112.

${ }^{49}$ Vgl. Schmidt (1982), S. 357.

${ }^{50} \mathrm{Vgl}$. Foerster (1988), S. 10.

${ }^{51}$ Vgl. Haase (2000), S. 14.

52 Vgl. Haase (2000), S. 15.

${ }^{53}$ Vgl. Neus (1998), S. 2.

54 Vgl. Kosiol (1961), S. 131.

55 Vgl. ähnlich Schweitzer (1997), S. 24.

56 Vgl. dazu Haase (2000), S. $15 f$.

$57 \mathrm{Vgl}$. Cartwright (1983), S. 15. 
Überträgt man diese grundsätzlichen Überlegungen auf den Bereich des Informationsmanagements, so ist ebenfalls zwischen dem Informationsmanagement als Erfahrungsobjekt und dem Informationsmanagement als Erkenntnisobjekt realwissenschaftlicher Forschungsbemühungen zu unterscheiden. Dabei stellt das Erfahrungsobjekt Informationsmanagement ein empirisches Konstrukt dar, das auf der Basis eines allgemeinen (im wissenschaftichen Sinne „adisziplinären“) Vorverständnisses zu beschreiben ist, um die zweite Stufe des Theorieeinstiegs vorzubereiten. Dieses Erfahrungsobjekt kann anschließend zum Untersuchungsgegenstand unterschiedlicher wissenschaftlicher Disziplinen gemacht werden, die es entsprechend ihrer speziellen Untersuchungsperspektive für eine eingehende einzelwissenschaftiche Untersuchung „zurechtmachen“. So können beispielsweise Probleme des Informationsmanagements, das mit Hilfe einer allgemeinen begrifflichen Abgrenzung des Untersuchungsfeldes als Erfahrungsobjekt konstruiert wurde, aus Sicht der Informatik, der Psychologie, der Soziologie oder eben auch der Betriebswirtschaftslehre analysiert werden. Dazu ist jeweils zunächst die theoriegeleitete Konstruktion des individuellen Erkenntnisobjekts Informationsmanagement notwendig, das dann mit den Mitteln der einzelnen Disziplin weiter erforscht werden kann.

Durch eine multiperspektivische Analyse des Informationsmanagements aus Sicht mehrer Wissenschaftsdisziplinen würde ein umfassendes Bild entstehen, das mehr Details und Facetten des Erfahrungsobjekts beinhaltet als eine einzelwissenschaftliche Untersuchung zu zeichnen in der Lage wäre. Der gemeinsame Erfahrungsgegenstand stellt dabei das verbindende Element der unterschiedlichen Annäherungen an das Informationsmanagement dar, während die individuelle Konstruktion des Erkenntnisobjekts unterschiedliche Schwerpunktsetzungen durch die jeweiligen Einzeldisziplinen erlaubt. Es ist darauf hinzuweisen, dass die hier vertretene Position im Widerspruch zu einigen Lehrbüchern des Informationsmanagements steht, die ihr Untersuchungsgebiet als einzelwissenschaftlichen Teilbereich verorten, der allein der Betriebswirtschaftslehre $^{58}$ oder der Wirtschaftsinformatik ${ }^{59}$ zuzurechnen ist. Die hier vertretene Position weist dagegen größere Gemeinsamkeiten mit einer Sichtweise auf, die das Informationsmanagement an der Schnittstelle zwischen verschiedenen realwissenschaftlichen Disziplinen ansiedelt und allen diesen Disziplinen jeweils eigene Erkenntnispotenziale zuspricht. ${ }^{60}$

Trotz dieser Forderung nach einer multiperspektivischen Analyse konzentrieren sich die weiteren Überlegungen in dieser Arbeit darauf, das Informationsmanagement aus dem speziellen Blickwinkel der Betriebswirtschaftslehre zu untersuchen. Diesem Vorgehen liegt die Überzeugung zu Grunde, dass die Betriebswirtschaftslehre als Wissenschaft ein erhebliches Erkenntnispotenzial für die durch das Informationsmanagement zu lösenden Probleme aufweist, da ein besonders starker Zusammenhang zum Untersuchungsbereich dieser Wissenschaftsdisziplin besteht. Die soeben angestellten Überlegungen haben allerdings deutlich gemacht, dass von einem Rückgriff auf die Betriebswirtschaftslehre nicht die Entwicklung einer allgemeingültigen, generellen

58 Vgl. z.B. Brenner (1994), S. 5f.; Fank (1996), S. 22; Voß/Gutenschwager (2001), S. 85.

59 Vgl. Heinrich (1999), S. 11f.

${ }^{60}$ Vgl. zu dieser Position Schwarze (1998), S. 45. Auch Peterhans geht von der Vorteilhaftigkeit der Bezugnahme auf mehrere Forschungsdisziplinen aus. Vgl. Peterhans (1996), S. 48. Seine Begriffsabgrenzung für das Informationsmanagement beinhaltet allerdings nicht nur Führungsaufgaben, sondern auch Durchfühungsaufgaben der Informationsverarbeitung und entspricht damit nach der hier verwendeten Terminologie eher dem Begriff „Informationswirtschaft“. 
Theorie des Informationsmanagements erwartet werden darf. Ein vielschichtigeres Verständnis ließe sich vielmehr erst dadurch erreichen, dass zu den hier entwickelten betriebswirtschaftlich fundierten Aussagen die Erkenntnisse anderer Wissenschaftsdisziplinen hinzugefügt würden. Das in dieser Arbeit gezeichnete Bild des Informationsmanagements muss also infolge der hier vorgenommenen Beschränkung auf nur eine einzelwissenschaftliche Disziplin unvollständig bleiben.

Ferner ist auch die Betriebswirtschaftslehre selbst nicht als homogene Disziplin zu sehen, die nur eine alleingültige Betrachtung des Informationsmanagements zulässt. Tatsächlich existieren auch im Bereich der Betriebswirtschaftslehre unterschiedliche Ansätze, die jeweils eigene Konstruktionen ihres Erkenntnisobjekts vornehmen und auf diese Weise auch zu unterschiedlichen Aussagesystemen für das Informationsmanagement gelangen können. Durch die unterschiedlichen Sichtweisen entstehen verschiedene betriebswirtschaftstheoretisch fundierte Ansätze des Informationsmanagements, die das Erfahrungsobjekt Informationsmanagement aus unterschiedlichen Perspektiven beleuchten und dadurch zu einem betriebswirtschaftlichen Gesamtbild dieses Untersuchungsbereichs kommen. Die hier entwickelten Aussagesysteme werden im Weiteren jeweils als Ansatz für eine Theorie des Informationsmanagements bezeichnet. Diese Begriffswahl macht den Aspekt, dass eine Mehrzahl von Ansätzen nebeneinander bestehen können, auch terminologisch deutlich.

Abbildung $2 / 1$ verdeutlicht die soeben beschriebenen Zusammenhänge. Die Beziehung zwischen den verschiedenen Ansätzen für eine Theorie des Informationsmanagements ist nicht eindeutig vorherzusagen. Es ist zu erwarten, dass unterschiedliche Sichtweisen zum Teil zu einander ergänzenden (komplementären), zum Teil aber auch zu untereinander in Widerspruch stehenden (konkurrierenden) Aussagen führen können.

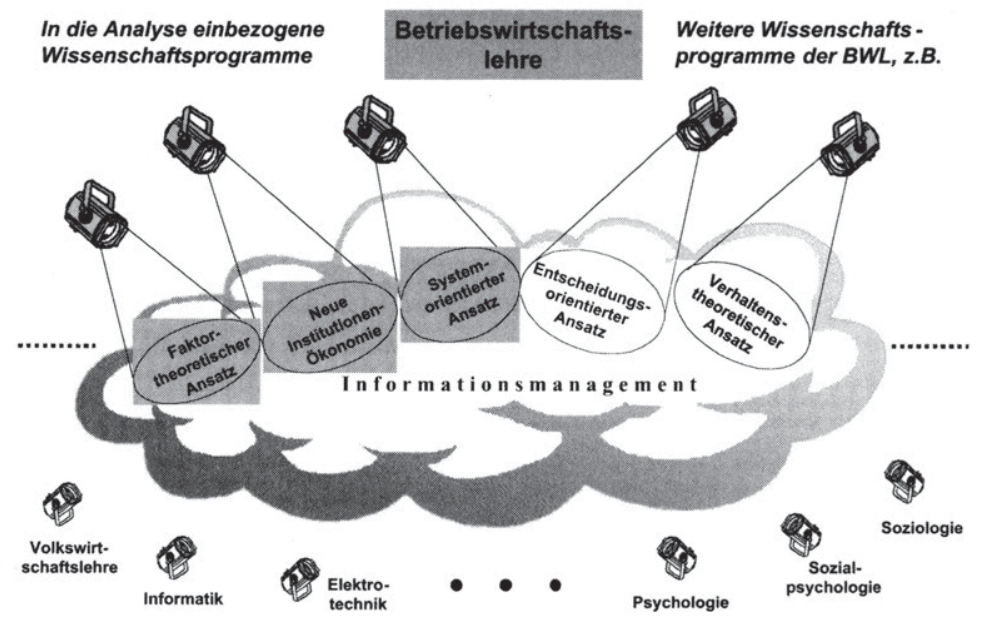

Abb. 2/1: Multiperspektivische Analyse des Informationsmanagements

In Abbildung 2/1 sind diejenigen Ansätze der Betriebswirtschaftslehre, auf die im Rahmen der vorliegenden Arbeit Bezug genommen wird, optisch hervorgehoben. Dabei handelt es sich um den Faktortheoretischen Ansatz, den Systemorientierten Ansatz und die Neue Institutionenökonomie. Im dritten, vierten und fünften Kapitel der 
Arbeit werden die Erkenntnispotenziale und Lösungsbeiträge untersucht, die jeweils einer dieser Ansätze für Fragen des Informationsmanagements bietet. Die Auswahl der hier referenzierten Ansätze erfolgt in Abschnitt 2.4.

\subsection{2 Überwindung bisheriger Theoriedefizite durch Bezugnahme auf bestehende Wissenschaftsprogramme}

Die weiteren Untersuchungen in dieser Arbeit gehen von der Annahme aus, dass die Bezugnahme auf bestehende Forschungsansätze ein geeignetes Vorgehen zur Überwindung der in Abschnitt 2.1.1 angesprochenen Theoriedefizite bisheriger Auseinandersetzungen mit dem Informationsmanagement darstellt. Im Folgenden werden die verschiedenen Bestandteile solcher Ansätze, die deren jeweilige Eigenständigkeit als Forschungskonzeption begründen, erläutert. Diese Bestandteile stellen stets den Ausgangspunkt für die Untersuchung spezieller Problemstellungen dar, durch die das Aussagesystem des betreffenden Ansatzes weiter ausgebaut wird. Forschungskonzeptionen, die sich durch die nachfolgend erläuterten grundsätzlichen Bestandteile konstituieren, werden als Wissenschaftsprogramme bezeichnet. ${ }^{61}$

Wissenschaftsprogramme bauen auf zentralen Gedanken auf, auf deren Basis ausgehend von den jeweils verfolgten Erkenntnisinteressen mosaikartig einzelne Erklärungsversuche und Gestaltungsempfehlungen gemacht werden. Bei diesen konstituierenden Grundgedanken handelt es sich um Leitideen, ${ }^{62}$ die sowohl methodische als auch inhaltliche Implikationen haben. Leitideen stellen die „harten Kerne ${ }^{\text {“63 }}$ von Wissenschaftsprogrammen dar, um die herum die eigentliche Theoriebildung stattfindet. Dabei muss es sich nicht immer nur um ausgefeilte, gut bewährte Theorien handeln. Vielmehr können in diesem Zusammenhang auch metaphysische Ideen eine große Rolle spielen, die etwas über die Realität behaupten, aber zu wenig spezifiziert sind, als dass sie tatsächlich überprüft werden könnten. ${ }^{64}$ Häufig werden diese bei der Theoriebildung nicht offen erläutert, weil sie dem Forschenden selbst nicht vollständig bewusst sind. ${ }^{65}$ In solchen Fällen ist für die Entwicklung neuer Aussagen im Rahmen des entsprechenden Wissenschaftsprogramms, die konform zu dessen bestehendem Aussagespektrum sein sollen, eine Rekonstruktion der Leitideen erforderlich, da diese teilweise nur implizit in die bisherige Theoriebildung eingeflossen sind.

Leitideen von Wissenschaftsprogrammen haben erhebliche normative Nebenbedeutung, wenn nicht im Sinne praktischer Maximen, so doch durch die Auszeichnung theoretisch

${ }^{61} \mathrm{Vgl.} \mathrm{Schanz} \mathrm{(1997a),} \mathrm{S.} \mathrm{81f.} \mathrm{Diesen} \mathrm{Begriff} \mathrm{verwendet} \mathrm{auch} \mathrm{Stein} \mathrm{(1993),} \mathrm{Sp.} 472$.

62 Vgl. Schanz (1997a), S. 93.

${ }^{63}$ Der Begriff "harter Kern" als Zentrum des jeweiligen wissenschaftlichen Ansatzes wurde von Lakatos (1974), S. 129ff. vorgeschlagen.

${ }^{64} \mathrm{Vgl}$. Schanz (1997a), S. 93. Es sei darauf hingewiesen, dass die Unterscheidung, ob es sich in einem konkreten Fall um die Leitidee eines Aussagesystems handelt oder nicht, nicht immer eindeutig zu treffen ist. Dennoch wird hier am Konzept der „Leitidee“ festgehalten, da damit sehr anschaulich beschrieben wird, wie sich die einzelnen Wissenschaftsprogramme der Betriebswirtschaftslehre konstituieren und voneinander abgrenzen. In diesem Zusammenhang wird häufig auch der Begriff „Paradigma“ gebraucht. Ein Paradigma umfasst die theoretischen Annahmen und Gesetze sowie Techniken ihrer Anwendung, die die wissenschaftliche Gemeinschaft anerkennt. Vgl. Chalmers (1989), S. 91f.. Der Paradigmenbegriff, der selber wiederum mit Problemen behaftet ist, wird im Folgenden jedoch nicht weiter verwendet. Vgl. dazu Engelhardt (1997), S. $5 f$.

${ }^{65} \mathrm{Vgl}$. Schneider (1997), S. 5. 
privilegierter Zustände. ${ }^{66}$ Damit beinhalten sie eine Art "Vor-Urteil"67, mit dem der wissenschaftliche Erkenntnisprozess in Angriff genommen wird. Leistungsfähigen VorUrteilen wohnt ein erhebliches heuristisches Potenzial inne, das bei der Hinzufügung neuer Aussagen zu dem bereits bestehenden System - auch durch nachfolgende Forschergenerationen - als Orientierungshilfe immer wieder neu zum Ausdruck kommt. Zwangsläufig begrenzen die zu Grunde liegenden Leitideen aber auch die möglichen Resultate, die aus einem Erkenntnisprozess gewonnen werden können. Aus einer Menge von Leitideen, die auf andere wissenschaftliche Disziplinen Bezug nehmen können, entsteht ein Wissenschaftsprogramm, das sich dem Untersuchungsgegenstand auf eine bestimmte Art und Weise nähert und dadurch eine jeweils eigene Perspektive im Hinblick auf diesen Untersuchungsgegenstand einnimmt. Die ausgehend von den Leitideen verfolgte Sichtweise legt den zu untersuchenden Wirklichkeitsausschnitt und den Aspekt, unter dem die Untersuchung stattfinden soll, fest. ${ }^{68}$ Darauf basiert das Vorgehen bei der Theoriebildung.

Häufig beabsichtigen Leitideen die Anwendung von bildhaften Vergleichen auf die zu untersuchende Fragestellung. ${ }^{69}$ Die Verwendung von bildhaften Vergleichen erfolgt in der Erwartung, dass zwischen unterschiedlichen Forschungsbereichen Analogien bestehen, durch deren Identifikation und Nutzung Rückschlüsse für die eigenen Zwecke gezogen werden können. Dabei kann es sich um inhaltliche Analogien in Bezug auf den Untersuchungsgegenstand sowie um methodische Analogien in Bezug auf die Vorgehensweise bei dessen Untersuchung handeln. Zwar kann die unreflektierte Verwendung von Analogien auch gefährlich sein, da die verschiedenen Betrachtungsgegenstände genau genommen nicht wirklich gleich geartet sind, sondern in ihrer konkreten Erscheinungsform wesentliche Unterschiede aufweisen. ${ }^{70}$ Eine sorgfaltig konzipierte Analogiebildung erkennt jedoch diese Unterschiede an und beschränkt sich auf die von der konkreten Erscheinungsform des Objekts unabhängigen zu Grunde liegenden Zusammenhangsmuster. Deshalb ist das Bilden von Analogien zwischen konkret verschiedenen Objekten, die von verschiedenen Disziplinen untersucht werden, schwierig und „voller Fallstricke“"71, es kann aber äußerst fruchtbar sein. Bei der Untersuchung sozialer Zusammenhänge sollte die Analogiebildung zu den Naturwissenschaften beispielsweise nicht als Gleichsetzen des sozialen Geschehens mit der Natur geschehen, ${ }^{72}$ sondern vielmehr in Form von Metaphern erfolgen, die bestimmte Einstellungen zur Beschreibung, Erklärung und Gestaltung des Untersuchungsgegenstandes beinhalten. Der Wert der Verwendung von Analogien liegt also in ihrem erheblichen heuristischen Potenzial für die Forschung, ${ }^{73}$ denn Analogien als solche können die Richtigkeit einer Theorie für den Bereich, in den sie übertragen wurden, nicht beweisen. ${ }^{74}$

66 Vgl. Müller (1992), S. 350.

${ }^{67}$ Ulrich (1971), S. 43.

${ }^{68} \mathrm{Vgl}$. Schneider (1997), S. 4.

${ }^{69} \mathrm{Vgl}$. Schneider (1996), S. 1098.

${ }^{70}$ Zur Diskussion über die Zulässigkeit von Analogiebildungen vgl. den Dialog zwischen Sandner (1982), Malik (1982) und Probst/Dyllick (1982).

71 Vgl. Ulrich (1988), S. 184.

72 Vgl. Müller (1992), S. 351.

${ }^{73}$ Vgl. Probst/Dyllick (1982), S. 83.

${ }^{74}$ Vgl. Sandner (1982), S. 83. 


\subsubsection{Dreistufiger Theorieeinstieg und weiteres Vorgehen zur Analyse des Informationsmanagements}

Wird die Betriebswirtschaftslehre als Referenzdisziplin zur Analyse des Informationsmanagements gewählt, so stellen die zu untersuchenden Probleme einen Teil des insgesamt umfassenderen Untersuchungsbereichs der Betriebswirtschaftslehre dar. Die Analyse hat aus diesem Grund den Charakter einer Speziellen Betriebswirtschaftslehre, da die Speziellen Betriebswirtschaftslehren sich auf wirtschaftszweig- oder funktionsspezifische Besonderheiten des allgemeinen Untersuchungsbereichs beziehen. ${ }^{75} \mathrm{Die}$ klassische Funktionengliederung der Betriebswirtschaftslehre wird folglich um eine neue Funktion erweitert, die sich mit der Informationsverarbeitung und Kommunikation in Unternehmungen befasst und gleichrangig neben die bereits bisher betrachteten Funktionen zu stellen ist. ${ }^{76}$ Dieser Funktionsbereich enthält die als „Informationswirtschaft" zu bezeichnenden Aufgaben der technikunterstützten Informationsverarbeitung, von denen das Informationsmanagement, das ausschließlich Führungsaufgaben umfasst, ein Teilgebiet darstellt. Die Informationswirtschaft ist von ihrer Stellung her mit anderen betriebswirtschaftlichen Funktionslehren wie etwa der Personalwirtschaft oder der Finanzwirtschaft vergleichbar. ${ }^{77}$

Folgt man dieser Auffassung, so gilt die typische Beziehung zwischen der Allgemeinen Betriebswirtschaftslehre und ihren Spezialdisziplinen auch für den Bereich der Informationswirtschaft bzw. des Informationsmanagements. Die Allgemeine Betriebswirtschaftslehre richtet sich auf die Untersuchung von Tatbeständen, die für alle Wirtschaftseinheiten (mit unterschiedlichem Gewicht) Gültigkeit besitzen. Sie bildet das Fundament, auf dem die Speziellen Betriebswirtschaftslehren aufbauen. ${ }^{78}$ Metaphorisch gesprochen hat die Allgemeine Betriebswirtschaftslehre „die Aufgabe, einen Baumstamm zu entwickeln, einen Wissensstamm zu lehren, aus dem die Speziellen Betriebswirtschaftslehren sich verästeln." ${ }^{\text {79 }}$ Wissenschaftstheoretisch fundierte Aussagen zum Informationsmanagement können sich also einerseits auf die allgemeingültigen, funktionsübergreifenden Erkenntnisse des jeweiligen Wissenschaftsprogramms stützen und müssen diese andererseits um eigene Aussagen zum spezifischen Untersuchungsbereich des Informationsmanagements erweitern.

Aus diesen Zusammenhängen ergeben sich bestimmte Konsequenzen für die Argumentationslinie bei der Entwicklung betriebswirtschaftlich fundierter Ansätze für eine Theorie des Informationsmanagements. Der im vorigen Abschnitt erläuterte zweistufige Theorieeinstieg ist zu modifizieren und um eine weitere Stufe zu einem dreistufigen Theorieeinstieg zu erweitern. Die erste Stufe der Theoriebildung, auf der die (adisziplinäre) Abgrenzung des Erfahrungsgegenstands als empirisches Konstrukt auf der Basis eines allgemeinen Vorwissens erfolgt, bleibt dabei im Wesentlichen unverändert. Neben dem Informationsmanagement ist ferner auch das Erfahrungsobjekt der Betriebswirtschaftslehre, das deren verschiedene Wissenschaftsprogramme mit ihren jeweiligen Mitteln analysieren, abzugrenzen. In Abbildung 2/2 sind die Erfahrungsobjekte so dargestellt, dass das Aufgabenfeld Informations-

\footnotetext{
${ }^{75} \mathrm{Vgl}$. Stein (1993), Sp. 470.

${ }^{76}$ Diese Position vertritt auch Schwarze (1998), S. 22.

77 Vgl. Weber (1993), S. $15 f$.

${ }^{78}$ Vgl. Schierenbeck (1995), S. 9.

79 Schneider (1990a), S. 25.
} 
management keine reine Teilmenge des Erfahrungsobjekts der Betriebswirtschaftslehre darstellt. Dies hebt die Auffassung hervor, dass das Informationsmanagement auch Probleme enthält, die mit den Mitteln der Betriebswirtschaftslehre nicht untersucht werden können, sondern ausschließlich mit Hilfe anderer Wissenschaftsdisziplinen einer Analyse zugänglich sind (z.B. rein technische Fragestellungen im Bereich der Hardund Software). Die erste Stufe des Theorieeinstiegs durch die Abgrenzung der empirischen Konstrukte erfolgt in den Abschnitten 2.3 und 2.4 dieser Arbeit.

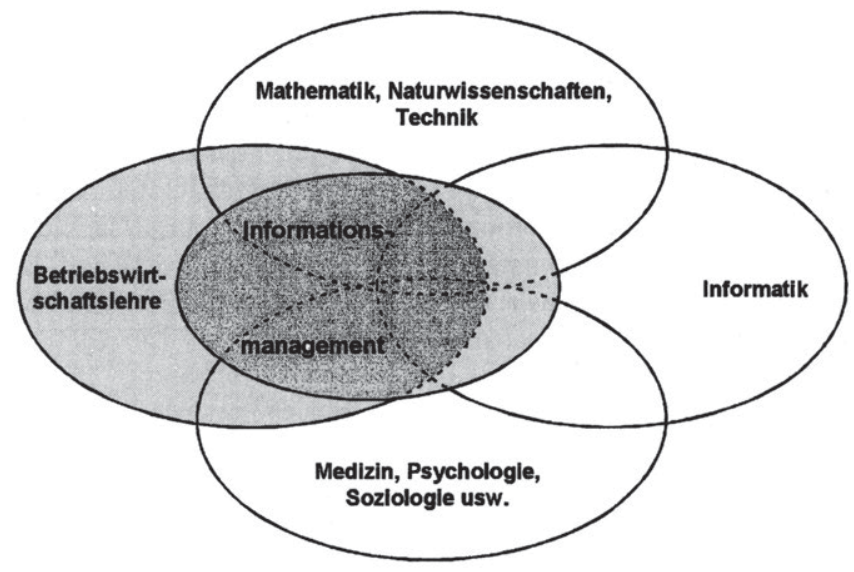

Abb. 2/2: Informationsmanagement als Teiluntersuchungsbereich unterschiedlicher
Wissenschaftsdisziplinen
Quelle: in Anlehnung an Schwarze (1998), S. 45.

Auf der zweiten Stufe des Theorieeinstiegs wird das Erkenntnisobjekt des jeweiligen Wissenschaftsprogramms der Betriebswirtschaftslehre als theoretisches Konstrukt entwickelt. Dies geschieht durch die Herausarbeitung der Erkenntnisinteressen sowie der inhaltlichen und methodischen Leitideen des betreffenden Wissenschaftsprogramms. Da das Vorgehen auf dieser Stufe sich auf den gesamten Erkenntnisbereich des jeweils referenzierten Wissenschaftsprogramms bezieht, wird das Ergebnis hier als allgemeines theoretisches Konstrukt bezeichnet. Die nachfolgenden Kapitel dieser Arbeit beginnen stets mit der zweiten Stufe des Theorieeinstiegs. Im ersten Abschnitt der einzelnen Kapitel wird also das allgemeine theoretische Konstrukt des Faktortheoretischen Ansatzes, des Systemorientierten Ansatzes bzw. der Neuen Institutionenökonomie abgegrenzt.

Auf der dritten und letzten Stufe des Theorieeinstiegs ist schließlich aus dem zuvor konstruierten allgemeinen Erkenntnisobjekt des betriebswirtschaftlichen Ansatzes das Informationsmanagement als spezielles Erkenntnisobjekt abzuleiten. Das Ergebnis dieses Schritts wird hier als spezielles theoretisches Konstrukt bezeichnet. Entsprechend der eingangs angesprochenen „Baummetapher“, die die Beziehung zwischen der Allgemeinen Betriebswirtschaftslehre und ihren Spezialdisziplinen beschreibt, kann sich diese Konstruktion des Erkenntnisobjektes für die Spezialdisziplin auf die allgemeinen Erkenntnisinteressen und Leitideen des betreffenden Wissenschaftsprogramms stützen. Zur Konstruktion des durch die Spezielle Betriebswirtschaftslehre Informationsmanagement $\mathrm{zu}$ analysierenden Erkenntnisobjektes ist es erforderlich, die konsti- 
tuierenden Bestandteile des Wissenschaftsprogramms auf den im ersten Schritt des Theorieeinstiegs grundsätzlich abgegrenzten Erfahrungsgegenstand Informationsmanagement zu übertragen. Die Zielsetzung liegt dabei in einer ,präparierten“ Beschreibung des Untersuchungsbereiches, durch die der Boden für eine tiefergehende theoretische Analyse des Informationsmanagements mit den Mitteln des betreffenden Wissenschaftsprogramms der Betriebswirtschaftslehre bereitet wird. Die dritte Stufe des Theorieeinstiegs wird jeweils im zweiten Abschnitt der nachfolgenden Kapitel bewältigt. Dort erfolgt eine Konstruktion des Informationsmanagements als Erkenntnisgegenstand aus Sicht des jeweils referenzierten Wissenschaftsprogramms.

Abbildung 2/3 fasst das beschriebene dreistufige Vorgehen zum Theorieeinstieg für die Analyse des Informationsmanagements zusammen:

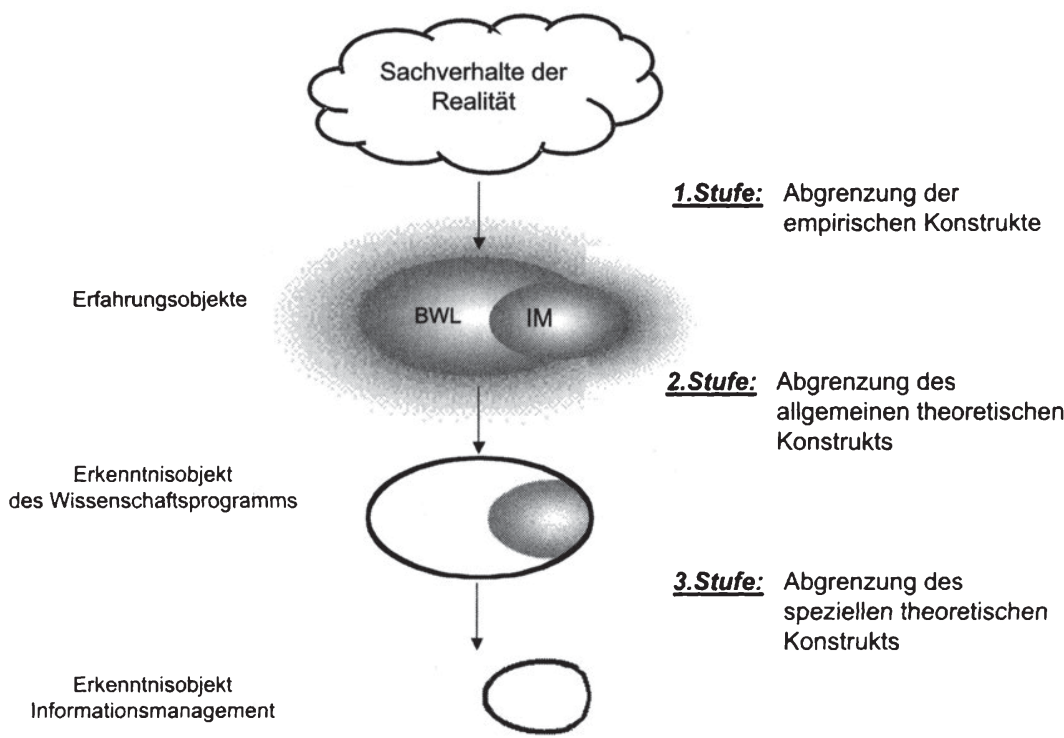

Abb. 2/3: Dreistufiger Theorieeinstieg für die Untersuchung des Informationsmanagements

Ausgehend von dem Ergebnis der dritten Stufe des Theorieeinstiegs kann die eigentliche Theoriebildung vorgenommen werden. Diese erfolgt jeweils im dritten Abschnitt der nachfolgenden Kapitel. Dort werden die Anknüpfungspunkte, die die einzelnen Ansätze für eine Untersuchung des Informationsmanagements bieten, zur Ableitung theoretisch fundierter Aussagesysteme genutzt.

\subsection{Konstruktion des Informationsmanagements als Enfahrungs- gegenstand wissenschaftlicher Analysen}

Die nachfolgenden Überlegungen konstruieren das Informationsmanagement als Erfahrungsgegenstand wissenschafticher Analysen und sind damit auf der ersten Stufe 
des soeben beschriebenen Vorgehens für einen dreistufigen Theorieeinstieg zur Untersuchung des Informationsmanagements angesiedelt. Unter dem Schlagwort Informationsmanagement werden derzeit in Theorie und Praxis vielfältige Bemühungen um einen effektiveren und effizienteren Einsatz moderner Informationstechniken in Unternehmungen zusammengefasst. ${ }^{80}$ Die Gemeinsamkeiten der verschiedenen Beiträge gehen allerdings kaum über diese grundsätzliche Zielsetzung hinaus. Die Diskussion ist im Gegenteil durch eine Fülle unterschiedlicher Auffassungen darüber, was genau unter „Informationsmanagement“ zu verstehen ist, geprägt. ${ }^{81}$

Die Transparenz der jeweiligen Standpunkte wird zudem häufig dadurch reduziert, dass das einem Beitrag zu Grunde liegende Begriffsverständnis nicht hinreichend deutlich gemacht wird. ${ }^{82}$ Die in der Literatur vertretenen Positionen unterscheiden sich, sofern sie überhaupt explizit dargelegt werden, durch unterschiedliche Akzentuierungen, die insbesondere in der jeweiligen Weite des Begriffsumfanges zum Ausdruck kommen. $^{83} \mathrm{Je}$ nach gewählter Definition des Informationsmanagements werden entweder institutionale oder funktionale, vorgehens- oder ergebnisorientierte, technische oder betriebswirtschaftliche, unternehmungsinterne oder unternehmungsexterne Aspekte betont.

Das Ziel der nachfolgenden Überlegungen ist weniger eine Rekonstruktion der unterschiedlichen Standpunkte, die bereits von verschiedenen anderen Autoren geleistet wurde. ${ }^{84}$ Es geht an dieser Stelle vielmehr darum, einen Argumentationsrahmen zu entwickeln, innerhalb dessen es im Weiteren möglich sein wird, entsprechend den unterschiedlichen Erkenntnisinteressen und Leitideen der verschiedenen Wissenschaftsprogramme der Betriebswirtschaftslehre jeweils unterschiedliche Facetten des Informationsmanagements näher $\mathrm{zu}$ betrachten und theoretische Aussagesysteme aufzubauen. Daraus folgt, dass eine zu enge Abgrenzung des Informationsmanagements auf der hier betrachteten Ebene nicht zielführend ist, da sie nicht genug Raum für ein flexibles Herausgreifen spezieller Aspekte durch die verschiedenen Wissenschaftsprogramme lassen würde.

Zur ersten Annäherung an das Informationsmanagement wird an vielen Stellen eine sprachliche Zerlegung des Begriffes in seine Bestandteile "Information“ und „Management" vorgeschlagen. ${ }^{85}$ Folgt man diesem Vorschlag, so stellt das Informationsmanagement in einer stark vereinfachenden ersten Annäherung das Management von Informationen dar. ${ }^{86}$ Demnach handelt es sich um eine spezielle Ausprägung des Managements, deren Bezugsobjekt Informationen sind. ${ }^{87}$ Die nachfolgende Entwicklung des empirischen Konstrukts greift die Idee der Begriffszerlegung auf. Aus diesem Grund wird zunächst eine allgemeine Abgrenzung des Informationsbegriffs (Abschnitt 2.3.1) und des Managementbegriffs (Abschnitt 2.3.2) vorgenommen. Anschließend

${ }^{80}$ Vgl. Nawatzki (1994), S. 6.

81 Vgl. Nüttgens (1995), S. 6.

82 Vgl. Streubel (2000), S. 48.

83 Vgl. Schotters (1992), S. 15.

${ }^{84}$ Vgl. z.B. Krcmar (2000), S. 31ff.; Streubel (2000), S. 48ff.; Nawatzki (1994), S. 6ff.; Schotters (1992), S. 15ff.; Seibt (1993), S. 6ff.; Brockhaus (1992), S. 42ff.

85 Vgl. z.B. Pfeiffer (1990), S. 18f;; Krcmar (1991), S. 165; Schwarzer (1994), S. 89; Fank (1996), S. 19; Streubel (2000), S. 60.

${ }^{86}$ Vgl. Fank (1996), S. 19.

87 Vgl. Streubel (1996), S. 14. 
erfolgt eine Synthese der beiden Begriffsbestandteile (Abschnitt 2.3.3), die zu einer weit gefassten Arbeitsdefinition des Begriffs Informationsmanagement führt, auf deren Basis einzelne Wissenschaftsprogramme ihrem jeweiligen Erkenntnisinteresse entsprechend eine Präzisierung vornehmen können.

\subsubsection{Der Begriffsbestandteil Information}

Bei dem Wort Information handelt es sich um einen besonders schillernden Begriff, der nicht nur immer häufiger im Alltag, sondern auch in vielen verschiedenen wissenschaftlichen Disziplinen verwendet wird. In den Wissenschaften wird er in sehr unterschiedlichen, aus dem jeweiligen Forschungsinteresse der Disziplin resultierenden Zusammenhängen benutzt. Vor diesem Hintergrund wird der Begriff „Information” zum Teil sogar als ,gemeinsamer Grundbegriff”, der zahlreiche Wissenschaften mit unterschiedlichstem Erkenntnisinteresse verbindet, bezeichnet. ${ }^{88}$

Diese Vielfalt der Verwendung des Informationsbegriffs in den unterschiedlichen disziplinären Zusammenhängen führt dazu, dass eine allgemeinverbindliche Definition weder möglich noch zweckmäßig ist. ${ }^{89}$ Selbst innerhalb der einzelnen Disziplinen ist es bisher in der Regel trotz vieler Bemühungen nicht gelungen, eine weithin akzeptierte Definition für den Begriff "Information“ zu entwickeln. ${ }^{90}$ Die einzelnen Begriffsauffassungen innerhalb einer Disziplin weisen einerseits zwar unübersehbare Ähnlichkeiten, andererseits jedoch erhebliche Unterschiede im Detail auf. Viele betriebswirtschaftliche Arbeiten nehmen diese Situation zum Anlass, bei der Abgrenzung des Begriffes eine Ansammlung immer wieder ähnlicher Definitionsversuche nebeneinander $\mathrm{zu}$ stellen, um davon ausgehend ein Begriffsverständnis entweder auszusuchen oder modular aus unterschiedlichen Definitionsversuchen zusammenzufügen. ${ }^{91}$ KIRSCH kommt sogar zu dem Ergebnis, dass der Begriff undefinierbar sei. ${ }^{92}$ Gerade im Rahmen einer breit angelegten Untersuchung ist es in der Tat fraglich, ob eine exakte Definition überhaupt erforderlich ist. ${ }^{93}$ Für die hier geforderte multiperspektivische Analyse des Informationsmanagements erscheint es stattdessen sinnvoller, einen groben Rahmen abzustecken, innerhalb dessen der Begriff im jeweiligen Kontext mit leicht unterschiedlichen Akzentuierungen verwendet werden kann. Im Folgenden werden deshalb die wichtigsten Dimensionen, in denen der Informationsbegriff untersucht werden kann, beschrieben, ohne den Versuch einer auf einen oder maximal zwei Sätze reduzierten, verbindlichen Definition zu unternehmen.

Ein erstes Gefühl für die empirischen Sachverhalte, die mit dem Begriff „Information” belegt werden, gibt eine Bezugnahme auf die sprachlichen Wurzeln dieses Begriffs. Das Wort „Information” leitet sich ethymologisch vom lateinischen Wort „informatio” ab,

${ }^{88} \mathrm{Vgl}$. Schneider (1990b), S. 145.

${ }^{89} \mathrm{Vgl}$. ăhnlich Maier/Lehner (1995), S. 167.

$90 \mathrm{Vgl}$. diese Aussage für die Disziplinen Wirtschaftsinformatik, Betriebswirtschaftslehre und Informatik bei Maier/Lehner (1995), S. 165 ff. Einen Überblick über die verschiedenen Verwendungen des Informationsbegriffes in der Betriebswirtschaftslehre und den Nachbardisziplinen geben z.B. folgende Autoren: Bode (1997), S. 449ff;; Maier/Lehner (1995), 165ff.; Krcmar (1991), S. 168ff; Schneider (1990b), S. 148ff.

91 Vgl. Schneider (1990b), S. 145.

92 Vgl. Kirsch (1971), S. 82.

${ }^{93}$ Zur Frage der Sinnhaftigkeit einer präzisen Definition des Informationsbegriffes vgl. Schneider (1990b), S. 146. 
welches wiederum auf einer Zusammensetzung der Bestandteile „in" und "formare” beruht. ${ }^{94}$ "Informare” bedeutet wörtlich „eine Form geben” oder „eine Gestalt geben”. Auch die Übersetzungen ,sich etwas im Geiste vorstellen”, „sich denken”, „darstellen”, „schildern" sowie „unterrichten”, „unterweisen” sind möglich. Die jeweils etwas andere Akzentuierung der verschiedenen Übersetzungen spiegelt sich in den verschiedenen nachfolgend erläuterten Betrachtungsdimensionen wieder.

In fast allen wissenschaftlichen Disziplinen wird zur Klärung des Begriffes „Information" auf eine oder mehrere Ebenen der Semiotik, der Lehre von den Zeichen, Bezug genommen. ${ }^{95}$ Deshalb gehen die folgenden Ausfürungen in den Abschnitten 2.3.1.1, 2.3.1.2 und 2.3.1.3 zunächst auf die drei Ebenen der Semiotik, also die Syntaktik, die Semantik und die Pragmatik ein. In Abschnitt 2.3.1.4 wird der Modellcharakter von Informationen, die stets materielle und immaterielle Bestandteile enthalten, erläutert. Abschnitt 2.3.1.5 schließlich wendet sich der Frage zu, inwiefern von einer Objektivität bzw. von einer Subjektbezogenheit von Informationen auszugehen ist.

\subsubsection{Syntaktische Ebene in der Semiotik}

„Information ist Formgebung des menschlichen Denkens durch Sprache."96 Dieser Definitionsversuch legt die besondere Betonung auf den Aspekt, dass es sich bei Information um einen Ausdruck handelt, der in irgendeiner Sprache, die syntaktischen Regeln gehorcht, formuliert sein muss. Auf der syntaktischen Ebene der Semiotik wird die Kenntnis über Sachverhalte der realen Welt mit Hilfe von Zeichen (z.B. Buchstaben, Schallwellen oder elektronischen Impulsen) und deren Verknüpfungen ausgedrückt. ${ }^{97}$ Die Syntaktik untersucht diese Problemstellung und stellt dabei allein auf die formalen Regeln zur Darstellung und Kombination der Zeichen ab. Sender und Empfänger einer Zeichenfolge müssen auf der syntaktischen Ebene über den gleichen Zeichenvorrat verfügen, um einander zu verstehen. Dagegen werden die inhaltliche Bedeutung der Zeichen und ihr Zweck furr den Benutzer in diesem Zusammenhang nicht näher untersucht.

Auf der syntaktischen Ebene erfolgt in betriebswirtschaftichen Arbeiten häufig die Unterscheidung der Begriffe „Daten” und „Information”, die in der Informatik häufig synonym benutzt oder zumindest nicht explizit voneinander abgegrenzt werden. Es wird dann vorgeschlagen, von „Daten” in solchen Fällen zu sprechen, in denen die Zeichen in maschinenverarbeitbarer Form, also in einer Maschinen verständlichen Sprache vorliegen. Die maschinelle Verarbeitbarkeit stellt dagegen kein Kriterium zur Abgrenzung des Informationsbegriffs dar. ${ }^{98}$ Folgt man dieser Auffassung, so können Informationen sowohl in Sprachen, die eine maschinenverarbeitbare Form haben, als auch in Sprachen, die eine nicht-maschinenverarbeitbare Form haben, ausgedrückt sein.

\footnotetext{
${ }_{94}^{94}$ Vgl. dazu Krcmar (1991), S. 168. Strombach (1986), S. 3.

95 Vgl. Schneider (1990b), S. 151.

${ }^{96}$ Gipper (1986), S. 257. Die Verwendung des Begriffes "Sprache" in dieser Aussage ist nicht unproblematisch, da Information nicht nur durch gesprochene Sprache übertragen werden kann, sondern auch durch andere Ausdrucksformen wie Mimik, Gestik o.ă. ("Körpersprache", "Gebärdensprache"). Auch diese Ausdrucksformen sind jedoch einer syntaktischen Analyse zugänglich.

${ }^{97}$ Vgl. Trott zu Solz (1992), S. 42.

98 Vgl. dazu Streubel (2000), S. 63.
} 
Daten hingegen sind physisch immer an ein maschinell lesbares Trägermedium gebunden. Daten existieren völlig unabhängig vom Bedeutungsgehalt des durch die jeweiligen Zeichen Dargestellten.

Mit dem Informationsgehalt auf der syntaktischen Ebene beschäftigt sich die Nachrichtentheorie. Die Theorie führt den Informationsgehalt auf den Überraschungswert eines Zeichens oder einer Zeichenfolge zurück. ${ }^{99}$ Information wird dabei verstanden als "diejenige Unsicherheit, die durch Erscheinen des betreffenden Zeichens beseitigt wird." 100 Das Maß für diese Unsicherheit ist die relative Häufigkeit der Selektion aus einem gegebenen Zeichenvorrat. Mit abnehmender Auftrittswahrscheinlichkeit nimmt die Überraschung durch das Erscheinen des Zeichens, also auch sein Informationsgehalt, zu. ${ }^{101}$ Auf dieser Ebene ist es irrelevant, welche Bedeutung diese Überraschung für den Empfänger der Information hat.

\subsubsection{Semantische Ebene in der Semiotik}

Die semantische Ebene der Semiotik befasst sich mit den Beziehungen zwischen den sprachlichen Zeichen und dem bezeichneten realen Objekt sowie zwischen Aussagen (Begriffen, Ausdrücken) und ihrer inhaltlichen Bedeutung. ${ }^{102}$ Der Fokus der Betrachtung verschiebt sich hier von den formalen Fragen der syntaktischen Ebene hin zum Sinn der Aussagen, die die Zeichen über die Realität machen. Der Übergang von der syntaktischen zur semantischen Ebene erfordert ein reflektierendes Bewusstsein, ${ }^{103}$ denn auf der Ebene der Semantik wird die Welt abgebildet, so wie sie vom Menschen denkend erfasst wird.

Auf dieser Ebene erfolgt die Anwendung von Interpretationsvorschriften auf die vorliegenden Zeichen. ${ }^{104}$ Für den Austausch von Informationen zwischen Menschen (Kommunikation) ist es von zentraler Bedeutung, dass Sender und Empfänger einer übertragenen Zeichenfolge denselben Ausschnitt der Wirklichkeit beziehungsweise einer Aussage denselben Sinnzusammenhang zuordnen. ${ }^{105}$ Die Realität selbst und der beschriebene Realitätsausschnitt befinden sich nach dieser Auffassung außerhalb der Sprache und sind durch die Ausdrucksmöglichkeiten der Sprache möglichst differenziert abzubilden. ${ }^{106}$ Semantische Probleme sind in der Betriebswirtschaftslehre und

\footnotetext{
99 Vgl. dazu Shannon/Weaver (1976), S. 8. Von einigen Autoren wird die Nachrichtentheorie in einer noch differenzierteren Betrachtungsweise auf der Ebene der Statistik als vor-syntaktischer Ebene angesiedelt, vgl. z.B. Hildebrand (1995), S. 6, der sich auf Gitt (1989), S. 9 bezieht. Zumeist wird in der Literatur aber nicht zwischen Syntaktik und Statistik unterschieden.

${ }^{100}$ Gitt (1989), S. 4.

${ }^{101} \mathrm{Vgl}$. Krcmar (1991), S. 169.

${ }^{102}$ Vgl. Maier/Lehner (1995), S. 173. Einige Autoren folgen der Unterscheidung von Klaus, der eine Trennung zwischen Semantik und Sigmatik vornimmt, vgl. Klaus (1969), S. 565. Dabei beinhaltet die Sigmatik die Beziehung zwischen den Zeichen und den durch sie bezeichneten realen Sachverhalten. Diese Unterscheidung wird jedoch in der sprachphilosophischen Literatur stark kritisiert und konnte sich auch innerhalb der Betriebswirtschaftlehre nicht durchsetzen.

${ }^{103}$ Vgl. Hildebrand (1995), S. 7.

${ }^{104}$ Vgl. Ferstl/Sinz (1993), S. 89ff;; Maier/Lehner (1995), S. 218.

${ }^{105}$ Vgl. ăhnlich Gebert (1992), Sp. 1110.

${ }^{106}$ In Abschnitt 2.2.1 wurde erläutert, dass die Realităt im ontologischen Weltbild des radikalen Konstruktivismus überhaupt erst mit Hilfe der Sprache konstruiert wird. Die Schwierigkeiten, einen Wirklichkeitsausschnitt mit den Mitteln der Sprache moglichst differenziert zu beschreiben, sind allerdings sehr ähnlich, egal ob diese Welt außerhalb der Sprache bereits existiert oder durch die Konstruktion des Betrachters erst geschaffen wird.
} 
der Wirtschaftsinformatik bei der Entwicklung und Verwendung von Modellierungssprachen zu beachten, die nach formalen Ausdrucksmöglichkeiten zur Beschreibung der betrieblichen Realität (z.B. der Geschäftsprozesse der Unternehmung) suchen.

Die Messung des Informationsgehaltes auf der semantischen Ebene gestaltet sich weitaus schwieriger als auf der syntaktischen Ebene. In semantischer Hinsicht bedeutet Information die Reduktion von Unsicherheit ${ }^{107}$ und der Informationsgehalt hängt von der Menge ausgeschlossener Möglichkeiten in der Realität ab, die einer Aussage zu entnehmen ist. ${ }^{108}$ Mit dem Ziel einer solchen Messung des semantischen Informationsgehaltes wurden mehrere Versuche zur Erweiterung der Informationstheorie von SHANNON um semantische und pragmatische Aspekte unternommen, die jedoch insgesamt als erfolglos zu bewerten sind. ${ }^{109}$ Die Aussage von WחD, die mathematische Informationstheorie lasse keine Aussage über den Informationsgehalt von semantischen Informationen $z u,{ }^{110}$ besitzt daher nach wie vor Gültigkeit.

Durch die Betriebswirtschaftslehre wird von diesen Überlegungen häufig die Vorstellung von der Überraschungsqualität von Information übernommen, die in viele betriebswirtschaftliche Definitionen (zumindest implizit) eingegangen ist. ${ }^{111}$ In der entscheidungslogischen Theorie schlägt sich dies in der gedanklichen Konstruktion eines Zustands vollkommener Information nieder, in dem alle Informationen bekannt sind und weitere Nachrichten beim Empfänger keinerlei Überraschungspotenzial aufweisen. Dieser Zustand wird als Maßstab für die Analyse realistischerer Informationssituationen verwendet, in denen zusätzliche Informationen normalerweise über ein solches Überraschungspotenzial verfügen. Liegt eine Situation mit vollkommener Information vor, so ist genau genommen gar keine Entscheidung notwendig, da die Situation keinerlei Freiheitsgrade enthält. Auf der anderen Seite wird durch jede Entscheidung, der eine Handlung folgt, neue Information erzeugt, da eine Auswahl aus einer nicht-atomaren Menge von Handlungsmöglichkeiten vorgenommen und somit eine Kontingenz erzeugt wird. Damit erweitert sich der Informationsstand für nachfolgende Entscheidungen. ${ }^{112}$

\subsubsection{Pragmatische Ebene in der Semiotik}

Die pragmatische Ebene der Semiotik knüpft an die Beziehung zwischen den Inhalten einer Zeichenfolge und ihrem Empfänger an. ${ }^{113}$ Es werden die verhaltensbestimmenden Wirkungen auf den Empfänger der Nachricht betrachtet. Die Inhalte der Nachricht werden auf dieser Ebene erst dann zur Information, wenn der Empfänger sie im Rahmen seiner Entscheidungsfindung verwendet. ${ }^{114}$ In der Regel stellt dieser Verwendungszusammenhang den Anknüpfungspunkt für betriebswirtschaftliche Unter-

\footnotetext{
${ }^{107}$ Vgl. Capurro (1987), S. 110.

${ }^{108}$ Vgl. Pfau (1997), S. 7.

${ }^{109}$ Vgl. dazu Schneider (1990b), S. 149; Zemanek (1986), S. 23.

${ }^{110}$ Vgl. Wild (1971), S. 321.

${ }^{111}$ Vgl. Schneider (1990b), S. 149.

${ }^{112}$ Vgl. Schneider (1990b), S. 163.

${ }^{113}$ Vgl. Wolfram (1990), S. 38.

${ }^{114}$ Vgl. Pfau (1997), S. 7.
} 
suchungen dar, die zumeist an der Zweckbezogenheit des übertragenen Wissens ansetzen. ${ }^{115}$

Der Verwendungszusammenhang steht auch im Mittelpunkt der Begriffsbildung von WITTMANN, die maßgeblichen Einfluss auf den Informationsbegriff und den Umgang mit Informationsproblemen in der Betriebswirtschaftslehre gewinnen konnte. Danach handelt es sich bei Informationen um ,zweckorientiertes Wissen, also solches Wissen, das zur Erreichung eines Zweckes, nämlich einer möglichst vollkommenen Disposition eingesetzt wird." 116 Dieser Definitionsversuch wurde vor allem deswegen kritisiert, weil er eine Definition auf der Basis zweier selbst wiederum undefinierter Begriffe vornimmt. Es bleibt einerseits unklar, was unter „Wissen” zu verstehen ist und andererseits, worauf die Zweckorientierung des Wissens zurückzuführen ist. ${ }^{117}$ Bei dieser Art der Begriffsbildung wird die eigentliche Definitionsarbeit lediglich auf den Begriff „Wissen" verlagert ${ }^{118}$ und die Frage aufgeworfen, ob und wie der Zweckbezug (objektiv oder subjektiv) ermittelbar ist. Festzuhalten bleibt, dass aus dieser Perspektive nicht jede sprachliche Aussage an sich zweckorientiert ist. Von Information wird nach dieser Auffassung vielmehr erst dann gesprochen, wenn eine zweckorientierte Verwendung der Aussage im konkreten Entscheidungsfall stattfindet. ${ }^{119}$

Da auf der pragmatischen Ebene die Person des Empfängers ausschlaggebend für das Vorliegen von Informationen ist, stellt sich die Frage, ob die durch einen Übertragungsprozess empfangenen Inhalte für den Empfänger neu sein müssen, um in die Kategorie Information zu fallen. Während in vielen Fällen über den Neuigkeitsgrad keine Aussage gemacht wird, besteht in den Fällen, in denen die Autoren explizit Stellung beziehen, keine einheitliche Auffassung darüber, ob nur dann von Informationen gesprochen werden kann, wenn die übermittelten Kenntnisse für den Empfänger bisher unbekannt waren. ${ }^{120}$ Liegt die Betonung bei der Begriffsabgrenzung auf der Entscheidungsrelevanz, so müssen die betreffenden Aussagen für eine Person nicht unbedingt neu sein, um sich auf ihre Handlungen auszuwirken. Tatsächlich können auch Kenntnisse, die bereits seit langem vorliegen, das Verhalten in konkreten Entscheidungssituationen bestimmen. Eine Negierung der Neuigkeitsanforderung widerspricht auf der anderen Seite dem syntaktischen Informationsbegriff. Damit ergeben sich zugleich Widersprüche gegenüber der oben erläuterten in der Betriebswirtschaftslehre weit verbreiteten Auffassung, dass Informationen Überraschungsqualität aufweisen müssen.

\footnotetext{
${ }^{115}$ Vgl. Krcmar (1991), S. 170.

${ }^{116}$ Wittmann (1959), S. 14. Im Original keine Hervorhebung.

${ }^{117}$ Vgl. Schneider (1990b), S. 158; Krcmar (1991), S. 170.

${ }^{118} \mathrm{Vgl}$. Maier/Lehner (1995), S. 215.

${ }^{119}$ In Arbeiten, die sich mit dem aktuell stark beachteten Konzept des Wissensmanagements beschăftigen, wird zum Teil das Vorliegen von Wissen erst auf der pragmatischen Ebene bestimmt während der Begriff der Information als dem Wissensbegriff vorgelagert gesehen wird. Vgl. z.B. Eulgem (1998), S. 20f. sowie die dort zitierten Quellen. Die Behandlung pragmatischer Gesichtspunkte ist, wie die Ausführungen dieses Abschnitts zeigen, jedoch nicht neu und findet in der Betriebswirtschaftslehre bereits seit langem auch im Zusammenhang mit dem Informationsbegriff statt.

${ }^{120} \mathrm{Vgl}$. dazu die Übersicht bei Maier/Lehner (1995), S. 252.
} 


\subsubsection{4 „Information" als Modell mit materiellen und immateriellen Bestandteilen}

STEINMULLER fasst die drei Ebenen der Semantik in seiner Konzeptionalisierung von Information als Modell, also als Abbildung eines Realitätsausschnittes, zusammen. ${ }^{121}$ Er schlägt vor, „Information” als „immaterielles Modell eines Originals für Zwecke eines Subjektes" zu definieren. ${ }^{122}$ Bei einer Information handelt es sich demnach, wie in Abbildung 2/4 dargestellt, um ein System aus drei Elementen, nämlich das durch das Modell abgebildete Original, das Subjekt, welches das Modell verwendet sowie das Modell selbst.

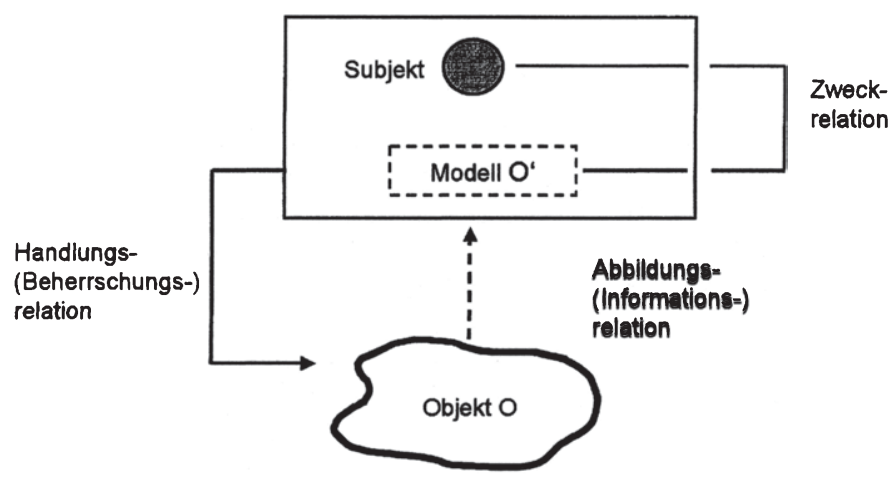

Abb. 2/4: Information als Modell

Quelle: Steinmüller (1981), S. 74.

Folgende Beziehungen bestehen zwischen den Elementen dieses Systems: ${ }^{123}$

- die Abbildungs- und Informationsrelation zwischen dem Original und dem abbildenden Modell,

- die Zweckrelation zwischen dem Modell und den Handlungszwecken des Subjektes

- und die Handlungsrelation zwischen den durch das Subjekt vorgenommenen, auf dem Modell basierenden Handlungen und den Handlungsergebnissen im Original.

Dieses - sozialwissenschaftlich ausgerichtete - Begriffsverständnis von „Information” macht besonders deutlich, dass eine Information, "weil Modell, zugleich eine radikale Vereinfachung der abgebildeten sozialen Objekte [...] darstellt, die sie für andere Zwecke [...] nur höchst bedingt oder überhaupt nicht verwendbar sein lässt". ${ }^{124}$ Dadurch wird der pragmatische Aspekt von Informationen in besonderem $\mathrm{Maße}$ in den Vordergrund gerückt. Die Qualität des Modells wiederum, das die Realität stark vereinfachend abbildet, wird durch Ähnlichkeitseigenschaften, die sich auf struk-

\footnotetext{
${ }^{121}$ Vgl. dazu Steinmüller (1981), S. $72 \mathrm{ff}$.

${ }^{122}$ Steinmüller (1981), S. 73.

${ }^{123}$ Vgl. Maier/Lehner (1995), S. 168.

${ }^{124}$ Steinmüller (1981), S. 74.
} 
turelle Ähnlichkeit, funktionelle Ähnlichkeit und Verhaltensähnlichkeit beziehen, ${ }^{125}$ bestimmt.

Die von STEINMULLLR vorgeschlagene Definition weist ein hohes heuristisches Potenzial auf, da es damit gelingt, die syntaktische, semantische und pragmatische Ebene der Untersuchung von Informationen zu einem Gesamtkonzept zu integrieren. Allerdings wird durch die Einschränkung der Betrachtung auf die immateriellen Eigenschaften von Informationen der materielle Aspekt vernachlässigt. ${ }^{126}$ Auch bei diesem Begriffsverständnis wird daher die Betrachtung verengt, sodass der Definitionsversuch nicht für alle Anwendungszwecke brauchbar ist.

Tatsächlich benötigt Information immer ein Trägermedium, umfasst also neben der immateriellen auch eine materielle Komponente. Es kann daher festgestellt werden: "Information is always born on a marker". ${ }^{127}$ Die jeweilige inhaltliche Aussage ist zwar nicht gegenständlicher Natur, sie kann jedoch nicht von den sie darstellenden physischen Zeichen getrennt werden. ${ }^{128}$ Es gelten also stets beide Aspekte zugleich: Information ist Modell, und Information ist an ein Trägermedium gebunden. ${ }^{129}$ Dieser Zusammenhang erschwert einerseits die Diskussion und bietet andererseits Raum furr unterschiedliche Schwerpunktsetzungen bei der Untersuchung von Problemen der technikunterstützten Informationsverarbeitung. Häufig beschränkt sich die Analyse entweder auf den materiellen oder auf den immateriellen Aspekt, da die jeweils zu betrachtenden Fragestellungen sehr unterschiedlichen Charakter haben. ${ }^{130}$

\subsubsection{Subjektbezogenheit versus Objektivität von "Information“}

Die erläuterte Abgrenzung des Informationsbegriffes auf der pragmatischen Ebene stellt auf die Zweckbezogenheit beim Entscheidungsträger ab. Wenn dabei nicht von einem objektiv ermittelbaren Zweck oder zumindest einer objektiv ermittelbaren Zweckeignung ausgegangen wird, ${ }^{131}$ dann impliziert dieses Begriffsverständnis die Subjektbezogenheit von Information. Bei der Übertragung von Nachrichten wird nach dieser Vorstellung allein durch die Person des Empfängers bedingt, welche Aussagen Informationen darstellen und welche nicht. Eine objektiv überprüfbare Existenz von Informationen ist dagegen nicht gegeben.

Allerdings ist bei vielen Autoren im Bereich der Betriebswirtschaftslehre eine ambivalente Position zu dieser Frage erkennbar. Diese kommt beispielsweise darin zum Ausdruck, dass eine begriffliche Unterscheidung von Information und Informationswert vorgenommen wird. ${ }^{132}$ Wenn zwischen diesen beiden Kategorien unterschieden wird,

\footnotetext{
${ }^{125}$ Vgl. Lehner (1995), S. 27.

${ }^{126}$ Steinmüller hălt seine Annahmen der Immaterialităt und der Subjektbezogenheit selbst nicht konsequent durch, wenn er von Wissen als speziellem Bündel von Informationen spricht, und "Wissen als Anhăufung von Informationsmodellen in einem Speicher, also einem anderen System" bezeichnet, vgl. Steinmüller (1981), S. 75. Zur Kritik vgl. Maier/Lehner (1995), S. 169.

${ }^{127}$ Miller (1978), S. 15.

${ }^{128} \mathrm{Vgl}$. Streubel (2000), S. 66.

${ }^{129}$ Vgl. Krcmar (1991), S. 175

${ }^{130}$ Eine Ausnahme stellt die Arbeit von Streubel dar, die bei ihrer Untersuchung gerade auf den Zusammenhang zwischen der immateriellen und der materiellen Ebene der technikunterstützten Informationsverarbeitung abstellt. Vgl. Streubel (2000), S. 281ff.

${ }^{131} \mathrm{Zu}$ diesem Punkt nimmt Wittmann nicht explizit Stellung. Vgl. Schneider (1990b), S. 158.

${ }^{132} \mathrm{Vgl}$. Schneider (1990b), S. 159.
} 
dann stellt der Informationswert eine subjektbezogene Größe dar und hängt von dem Vorwissen des Empfängers, das den Überraschungswert der Information bestimmt, sowie seiner individuellen Verarbeitung des informatorischen Inputs ab. ${ }^{133}$ Gleichzeitig deutet diese Unterscheidung jedoch auf die Annahme hin, dass es sich bei der Information selbst um eine objektiv vorhandene Größe handelt, die lediglich ihren Wert durch die Interpretationsleistung eines Entscheidungsträgers erhält. Es handelt sich allerdings nicht um einen zwingenden Zusammenhang, da die Unterscheidung von „Information" und „Informationswert" zumindest im Prinzip auch mit der Annahme der Subjektbezogenheit von Informationen vereinbar ist.

Die Auffassung, dass es sich bei Information um eine objektive Größe handelt, ist insbesondere in naturwissenschaftichen und technischen Zusammenhängen vorherrschend. Wenn von der Objektivität von Informationen ausgegangen wird, sind diese „real“ und nicht an ein menschliches Gehim gebunden. ${ }^{134}$ So sieht etwa der Ansatz der Information Physics Information als eigenständige dritte Komponente des Universums neben Materie und Energie. Ausgehend von der Annahme, dass alle „organisierten Strukturen" Informationen beinhalten werden durch diesen Ansatz drei Grundpositionen vertreten: ${ }^{135}$

- Physical information is reflected by organization. ${ }^{136}$

- Physical information is a function of improbability.

- Physical information is a function of the amount of work required to create it.

Für betriebswirtschaftliche Fragestellungen ist daran interessant, dass Information als Funktion des Erstellungsaufwandes des Senders, präziser gesagt des Erstellers, gesehen wird. Aus dieser Perspektive ist Information in jedem wie auch immer organisierten System vorhanden, ohne dass ein Empfänger überhaupt zu existieren braucht. Daraus kann für betriebswirtschaftliche Zwecke die Anregung zu einer stärkeren Beachtung der Person des Senders gewonnen werden, die bei einer rein pragmatischen Definition des Informationsbegriffes vollkommen vernachlässigt wird. Tatsächlich ist in der jüngsten Zeit in Theorie und Praxis eine teilweise Verschiebung der Perspektive hin zu einer verstärkten Fokussierung der intersubjektiven Komponente von Informationen erkennbar. Diese Vorstellung erweist sich auch für die Betrachtung von Information als wirtschaftlichem Gut und als Ressource im Leistungserstellungsprozess als hilfreich, da diese von den Eigenschaften der zeitlichen und räumlichen Konstanz, der Speicherbarkeit und der Übertragbarkeit von Informationen ausgehen muss. ${ }^{137}$

Festzuhalten bleibt die objektiv/subjektive Janusköpfigkeit des Informationsbegriffes, die auch als "Dualität" von Informationen bezeichnet wird. Eine Information enthält sowohl eine subjektiv-individuelle Komponente, durch die unter anderem der Wert der Information bestimmt wird, als auch eine vom einzelnen Empfänger unab-

\footnotetext{
${ }^{133}$ Vgl. Österle (1981), S. 13.

${ }^{134}$ Vgl. Maier/Lehner (1995), S. 169.

${ }^{135} \mathrm{Vgl}$. Stonier (1986), S. 224.

${ }^{136}$ Der Begriff Organisation wird hier in einem anderen Zusammenhang verwendet als in der Betriebswirtschaftslehre. Er stellt hier nicht auf arbeitsteilige Leistungserstellung ab, sondern auf die beobachtbare Ordnung eines Systems.

${ }^{137} \mathrm{Vgl}$. ăhnlich Schneider (1990b), S. 162.
} 
hängige, intersubjektive Komponente. ${ }^{138}$ Information ist Abbild der Wirklichkeit und Teil der Wirklichkeit zugleich und je nach Untersuchungsziel ist die eine oder die andere Eigenschaft von größerer Relevanz für die Analyse.

\subsubsection{Der Begriffssestandteil Management}

Der Begriff „Management” als zweiter Begriffsbestandteil von „Informationsmanagement" wird zwar ebenfalls in verschiedenen Bedeutungsvarianten verwendet, diese liegen jedoch erheblich näher zusammen als es beim Begriff „Information” der Fall ist. Da zudem weitgehend Einigkeit über die verschiedenen Verwendungsmöglichkeiten des Begriffes besteht, gelingt die Abgrenzung des Erfahrungsgegenstands in diesem Punkt deutlich präziser als bei der soeben vorgenommenen Abgrenzung des Informationsbegriffs.

Eine erste Annäherung an den Begriff ist auch hier über eine ethymologische Herleitung möglich. Diese gelingt allerdings nicht eindeutig, da unterschiedliche Auffassungen über die lateinischen Wurzeln des Wortes „Management” bestehen, die von ,manu agere" (mit der Hand arbeiten) über "manus agere” (an der Hand führen) bis hin zu „mansionem agere" (das Haus für einen Eigentümer bestellen) als Wortherkunft reichen. ${ }^{139}$ In vielen praxisorientierten Veröffentlichungen weist die Interpretation des Managementbegriffs große Ähnlichkeiten mit dem Begriff der „Verwaltung“ auf, wenn beispielsweise von „Datenmanagement", „Datenbankmanagement" oder „Netzwerkmanagement" gesprochen wird. Dagegen werden in weiten Teilen der theoretisch ausgerichteten betriebswirtschaftlichen Literatur die Begriffe "Management" und „Führung” synonym verwendet. Unter Führung wird die „zielorientierte Gestaltung und Steuerung sozialer Systeme" verstanden. ${ }^{140}$ Dazu gehören die Festlegung von Zielen, die Systemgestaltung und die Steuerung der Prozesse der Zielerreichung. Das zentrale Merkmal der Führung ist ganz allgemein die Beeinflussung menschlichen Verhaltens, das auf die Erreichung bestimmter Ziele hingelenkt werden soll.

Für eine vollständige Entwicklung des empirischen Konstrukts müsste an dieser Stelle also sowohl eine nähere Betrachtung des Führungsbegriffs als auch des Verwaltungsbegriffs erfolgen. Die nachfolgenden Überlegungen gehen jedoch nicht näher auf den Verwaltungsbegriff ein und konzentrieren sich allein auf den Führungsbegriff, da dieser der in den nachfolgenden Kapiteln vorgenommenen betriebwirtschaftstheoretisch ausgerichteten Untersuchung stärker entspricht. Auf der hier betrachteten Ebene des Erfahrungsbereichs ist keinerlei Eingrenzung in Bezug darauf vorzunehmen, welche Ziele bei der Wahrnehmung von Führungsaufgaben verfolgt werden und wie diese zu Stande kommen. Eine dahingehende Präzisierung sollte stattdessen bei der Entwicklung des theoretischen Konstrukts auf der zweiten Stufe des Theorieeinstiegs aus der Perspektive einzelner Wissenschaftsprogramme an Hand der jeweils verfolgten Erkenntnisinteressen vorgenommen werden.

Im Folgenden werden in Abschnitt 2.3.2.1 zunächst verschiedene Varianten des Managementbegriffs unterschieden. Abschnitt 2.3.2.2 stellt davon ausgehend die sach-

\footnotetext{
${ }^{138} \mathrm{Vgl}$. Schneider (1990b), S. 164.

${ }^{139}$ Vgl. Staehle (1999), S. 71.

${ }^{140}$ Vgl. dazu Wild (1982), S. 32.
} 
bezogenen Funktionen des Managements dar und beschreibt kurz die verschiedenen Phasen des Managementzyklusses. Abschnitt 2.3.2.3 schließlich erläutert die personenbezogenen Funktionen des Managements.

\subsubsection{Begriffsvarianten}

In der betriebswirtschaftlichen Literatur werden häufig zwei verschiedene Varianten des Managementbegriffs verwendet. Management wird einerseits als Institution verstanden und andererseits als Komplex von Aufgaben, die zur Steuerung eines Systems erfüllt werden müssen. ${ }^{141}$ Dementsprechend sind ein institutionaler und ein funktionaler Managementbegriff zu unterscheiden.

Bei der institutionalen Begriffsvariante (managerial roles approach) bezeichnet Management diejenigen Personen oder Personengruppen, die Führungsaufgaben wahrnehmen. ${ }^{142}$ Zum Management gehören demnach alle Mitglieder einer Organisation, die Vorgesetztenfunktionen wahrnehmen. ${ }^{143}$ In diesem Zusammenhang werden die Aktivitäten von Managern und ihre sozialen Rollen zumeist in einem handlungsorientierten Ansatz untersucht. ${ }^{144}$ Die funktionale Begriffsverwendung (managerial functions approach) bezieht sich auf die Aufgaben und Prozesse, deren Gegenstand die zielgerichtete Beeinflussung der Leistungserstellung in der gesamten Unternehmung bzw. in dem zu führenden System ist. ${ }^{145}$ Die funktionale Begriffsvariante ist prinzipiell unabhängig von einer Fixierung der Aufgaben auf bestimmte Positionen oder Führungsebenen. In aller Regel werden zwar spezielle Leitungspositionen geschaffen, die mit der Erfüllung von Führungsfunktionen betraut werden. ${ }^{146}$ Die Inhaber dieser Positionen haben jedoch in mehr oder weniger großem Umfang auch Durchführungsaufgaben zu erfüllen. Analysen, die von dieser Begriffsvariante ausgehen, untersuchen auf analytisch-funktionsorientiertem Wege das zweckmäßige Vorgehen zur Erfüllung der Management-Funktionen.

Im Folgenden wird der funktionale Managementbegriff noch näher betrachtet. Dieser lässt sich weiter in einen sachbezogenen und einen personenbezogenen Bereich einteilen. ${ }^{147}$ Die sachbezogenen Management-Funktionen umfassen die verschiedenen Tätigkeiten des Managements, die sich nicht unmittelbar auf die Führung von Mitarbeitern in einer direkten Interaktion beziehen. Dazu gehören die Planung und Entscheidung, Steuerung und Kontrolle von Prozessen zur Zielerreichung. Dagegen betreffen die personenbezogenen Funktionen die Führung von Personen und Kleingruppen, also die zielorientierte Beeinflussung menschlichen Verhaltens durch die unmittelbare Interaktion zwischen Individuen. Von manchen Autoren wird als dritter Bereich des Managements ferner die Unternehmensforschung genannt, die mit formalwissenschaftlichen Erkenntnissen die optimale Zielerreichung bei vollständig strukturierbaren Problemstellungen anstrebt. ${ }^{148}$ Diese Begriffsvariante wird hier nicht

\footnotetext{
${ }^{141} \mathrm{Vgl}$. Steinmann/Schreyogg (2000), S. 5f.

${ }^{142}$ Vgl. Staehle (1999), S. 71.

${ }^{143} \mathrm{Vgl}$. Steinmann/Schreyögg (2000), S. 6.

${ }^{144}$ Staehle formuliert pointiert: „Die Vertreter eines handlungsorientierten Ansatzes (work activity school) fragen konkret: Was tun Manager eigentlich den ganzen Tag?" Staehle (1999), S. 82.

${ }^{145} \mathrm{Vgl}$. Kuhn (1990), S. 2.

${ }^{146} \mathrm{Vgl}$. Steinmann/Schreyögg (2000), S. 6.

${ }^{147}$ Vgl. Staehle (1999), S. 72.

${ }^{148}$ Vgl. Staehle (1999), S. 72.
} 
näher betrachtet, da nur ein relativ geringer Bezug zu den in dieser Arbeit fokussierten realwissenschaftlichen Fragestellungen besteht.

\subsubsection{Sachbezogene Funktionen des Managements}

Bei der Betrachtung der sachbezogenen Funktionen wird Management in der Regel als Prozess mit zyklischen Eigenschaften aufgefasst. ${ }^{149}$ Die Autoren sprechen dann vom Managementzyklus und entwickeln verschiedene Phasenschemata, die sich durch unterschiedliche Detaillierungsgrade und geringfugige Abweichungen in der Terminologie voneinander unterscheiden. Diese Phasenschemata bringen die ManagementFunktionen in eine zeitliche Reihung und interpretieren sie als aufeinander folgende Stadien in einem Zyklus, ${ }^{150}$ in dem jeweils der Abschluss einer vorausgegangenen Phase den Beginn der nachfolgenden Phase auslöst.

In der Regel wird von einem (mindestens) dreiteiligen Managementzyklus ausgegangen, der sich aus den Phasen Planung und Entscheidung, Steuerung und Kontrolle zusammensetzt. Planungsaktivitäten stellen den logischen Ausgangspunkt des Managementprozesses dar. ${ }^{151}$ Hierunter fallen insbesondere die Bestimmung der grundsätzlich verfolgten Ziele, die Formulierung möglicher Maßnahmen zur Erreichung dieser Ziele und die Auswahl einer Handlungsoption im Rahmen der Entscheidungsfindung. Planung kann als gedankliche Vorbereitung (Vorstrukturierung) von Entscheidungen sowie der sich daraus ergebenden Handlungen verstanden werden. ${ }^{152}$ Die Entscheidung als Auswahl einer Handlungsalternative stellt den Abschluss des Planungsprozesses dar. ${ }^{153}$

An die Planung und Entscheidung schließt sich die Durchsetzung der Entscheidung an. Diese Phase des Managementprozesses, in deren Rahmen eine zielorientierte Einflussnahme auf das laufende Geschehen erfolgt, wird als Steuerungsphase bezeichnet. $\mathrm{Zu}$ den Steuerungsaufgaben im Rahmen des Führungsprozesses zählen die konkrete Veranlassung der Arbeitsausfuhrung und ihre zieladäquate Feinsteuerung im Rahmen der Managementphase der Steuerung. Häufig wird diese Phase des Managementprozesses als „Führung im engeren Sinne“ bezeichnet und durch das operative Management ausgeführt. Die Steuerung zielt darauf ab, die Vorgaben der Planungsund Entscheidungsphase tatsächlich zu erreichen.

Die Kontrolle stellt den letzten logischen Schritt des skizzierten Managementprozesses dar. Hier werden die realisierten Ergebnisse mit den ursprünglichen Planwerten verglichen, eventuelle Abweichungen zwischen Ist und Soll festgestellt und auf ihre Ursachen untersucht. ${ }^{154}$ Mögliche Maßnahmen zur Anpassung von Soll und Ist können einerseits aus Korrekturmaßnahmen bestehen, die zu einer Anpassung der realisierten an die geplanten Werte führen, andererseits können mittels Planrevisionen die

\footnotetext{
${ }^{149}$ Vgl. z.B. Terry/Franklin (1982), S. 38; Wild (1982), S. 33ff.; Mag (1995), S. 47ff.; Gluchowski/Gabriel/Chamoni (1997), S. 13ff.

${ }^{150} \mathrm{Vgl}$. Gluchwoski/Gabriel/Chamoni (1997), S. 13.

${ }^{151}$ Vgl. Steinmann/Schreyőgg (2000), S. 8.

${ }^{152}$ Mag (1995), S. 2.

${ }^{153}$ Mag (1995), S. 8. Planung und Entscheidung werden in der Literatur nicht immer zu einer Phase zusammengefasst. An dieser Stelle wird jedoch der Auffassung von Mag gefolgt, der in der Entscheidung den Abschluss der Planung sieht.

${ }^{154} \mathrm{Vgl}$. Steinmann/Schreyögg (2000), S. 10.
} 
ursprünglichen Planwerte verändert werden. Die letztgenannte Variante bedeutet ein Wiedereintreten des Managementprozesses in die Planungsphase. Somit stellt die Kontrollphase nicht nur den logischen Endpunkt des Managementprozesses dar, sondern ihre Ergebnisse bilden zugleich den Ausgangspunkt für die Entwicklung Neuplanung und einen neuen Managementzyklus.

Von einigen Autoren wird auch die Organisation als Management-Funktion gesehen und in das Phasenschema (üblicherweise zwischen Entscheidung und Steuerung) eingebaut. Diese Einordnung ist jedoch nicht eindeutig, da organisatorische Regeln zur Gestaltung arbeitsteiliger Prozesse auch als eigenständiges Bezugsobjekt des Managements angesehen werden können. Organisatorische Aktivitäten stellen dann einen Teilbereich der gesamten Aktivitäten des Managements dar, der selbst wiederum durch einen Phasenzyklus beschrieben werden kann. ${ }^{155}$ Daher wird hier nur zwischen der Planung und Entscheidung, Steuerung und Kontrolle als Funktionen des Managements unterschieden. Auch detailliertere Phaseneinteilungen werden an dieser Stelle nicht in die allgemeine Betrachtung des Managementbegriffs einbezogen. ${ }^{156}$

\subsubsection{Personale Funktionen des Managements}

Die personenbezogene Komponente des Managements betriff die Personal- bzw. Mitarbeiterführung. Ziel der Mitarbeiterführung ist die Fremdbestimmung des Verhaltens der Mitarbeiter durch den oder die Manager. Dazu gehören alle im Rahmen von direkten Interaktionen stattfindenden Aktivitäten der unmittelbaren, persönlichen Beeinflussung menschlichen Verhaltens sowie sämtliche damit zusammenhängenden Aspekte, wie Motivation, Gruppenführung, Machtausübung, Konfliktlösung oder soziale Kontrolle. ${ }^{157}$ Derartige Probleme entstehen dadurch, dass zwischen den beteiligten Personen auf den verschiedenen Ebenen der Unternehmungshierarchie ein Vorgesetztenverhältnis besteht, in dem der Manager gegenüber seinen Mitarbeitern in irgendeiner Form befugt ist, Weisungen zu erteilen. ${ }^{158}$

Zur Untersuchung von Führungssachverhalten sind zahlreiche Ansätze entstanden, die sich entweder stärker auf die Person des Führenden beziehen oder vermehrt auf die Interaktion zwischen Führer und Geführten abstellen und Führerschaft als sozialen Beeinflussungsprozess auffassen. ${ }^{159}$ Ansätze zur Analyse von Führungseigenschaften sind individualistisch orientiert und nehmen an, dass bestimmte Persönlichkeitsmerkmale den Führer vor den Geführten auszeichnen. ${ }^{160}$ Die Untersuchung richtet sich davon ausgehend auf die Identifikation von relevanten Eigenschaften, die die Person des Führers von den Geführten unterscheiden. Diese Eigenschaften können angeboren sein, sie können teilweise aber auch durch Erfahrungen im Laufe der Zeit erworben werden. Theorien, die diesem Muster entsprechen, konnten allerdings empirisch kaum belegt werden, da ein direkter Zusammenhang zwischen den geforderten Persönlich-

\footnotetext{
${ }^{155} \mathrm{Vgl}$. ăhnlich Streubel (2000), S. 42.

${ }^{156}$ Vgl. dazu z.B. Mag (1992), S. $60 \mathrm{ff}$.

${ }^{157}$ Vgl. Staehle (1991), S. 77.

${ }^{158}$ Vgl. Gluchowski/Gabriel/Chamoni (1997), S. 11.

${ }^{159}$ Vgl. Steinmann/Schreyögg (2000), S. 573.

${ }^{160}$ Vgl. Staehle (1999), S. 331.
} 
keitszügen und der Bewährung von Personen in Führungspositionen kaum festgestellt werden konnte. ${ }^{161}$

Etwas stärker auf empirisch beobachtbares Beeinflussungsverhalten beziehen sich Ansätze, die den Führungsstil in den Mittelpunkt der Betrachtung stellen. Als Führungsstil wird ein in wechselnden Situationen relativ konstantes, sinnvoll strukturiertes Verhaltensmuster, das als Konkretisierung einer verhaltensorganisierenden Einstellung oder Grundhaltung aufzufassen ist, bezeichnet. ${ }^{162}$ Dieses Untersuchungskonzept basiert auf der Annahme, dass auf der Grundlage letztlich eigenschaftsorientierter Typologien generell Zusammenhänge über die situationsinvariante Effizienzwirkung solcher Verhaltensmuster erkannt werden können. ${ }^{163}$ Das situationsinvariante Muster des Führungsverhaltens ist demnach die entscheidende Determinante für die Einstellungen der Mitarbeiter zur Organisation, zu deren Zielen, Aufgaben und letztlich auch für deren Zielereichung. ${ }^{164}$

Problematisch an den auf die Person des Führenden konzentrierten Ansätzen ist insgesamt, dass der eigentliche Prozess der Beeinflussung in direkter Interaktion mit den Geführten zu wenig betrachtet wird. Diese Betrachtungsweise ist verkürzt, da es nicht nur von der Führungsperson abhängt, ob in der Praxis Führung gelingt. Im Hinblick auf die Adressaten der Führung ist es von entscheidender Bedeutung zu erkennen, dass Menschen nicht bereit sind, sich unbesehen jedem Versuch der äußeren Einflussnahme zu beugen. ${ }^{165}$ Aus diesem Grund beziehen sich Führungstheorien auf die Zusammenhänge zwischen Führer, Geführten, organisatorischen Anforderungen und Führungserfolg. ${ }^{166} \mathrm{Je}$ nach Untersuchungsschwerpunkt werden dabei stärker die Merkmale der einzelnen Situation, die Identität der Führer/Geführten-Beziehung insgesamt, der unmittelbare Prozess der Interaktion oder weitere Aspekte von empirisch vorfindbaren Führungsproblemen fokussiert.

Die gedankliche Trennung zwischen der sach- und der personenbezogenen Komponente des funktional verstandenen Managements wirkt etwas künstlich, da in der Realität stets eine enge Verwobenheit der Sach- und Personenorientierung festzustellen ist. ${ }^{167}$ Dies hat bereits in den 1970er Jahren zur Kritik an ausschließlich auf die sachbezogene Begriffsvariante bezogenen Betrachtungen geführt. Tatsächlich hat eine solche Trennung rein analytischen Charakter, kann aber für die Eingrenzung von zu untersuchenden Problemstellungen für einzelne Untersuchungen sinnvoll sein. Dabei darf allerdings der Gesamtzusammenhang, in dem Interdependenzen zwischen sachbezogener und personenbezogener Führung bestehen, nicht vollständig aus den Augen verloren werden.

\footnotetext{
${ }^{161} \mathrm{Vgl}$. Stogdill (1974), S. 62.

${ }^{162} \mathrm{Vgl}$. Neuberger (1977), S. 97.

${ }^{163}$ Vgl. Staehle (1999), S. 334f.

${ }^{164} \mathrm{Vgl}$. Steinmann/Schreyögg (2000), S. 588.

${ }^{165} \mathrm{Vgl}$. Steinmann/Schreyögg (2000), S. 580.

${ }^{166}$ Vgl. Staehle (1999), S. 347.

${ }^{167}$ Vgl. Staehle (1991), S. 77.
} 


\subsubsection{Der Begriff Informationsmanagement}

Die Abgrenzung des Informationsmanagements als Erfahrungsgegenstand wissenschaftlicher Analysen kann an die Ausführungen zu den Begriffsbestandteilen „Information" und „Management” anknüpfen, sie ergibt sich jedoch nicht durch eine einfache Summierung der beiden Komponenten. Es ist zunächst zu präzisieren, welche Verwendung des Managementbegriffs für eine Analyse des Informationsmanagements zweckmäßig ist. Diese Präzisierung erfolgt in Abschnitt 2.3.3.1. Ferner ist eine genauere Betrachtung des Gestaltungsgegenstands, der bisher sehr allgemein als „Information" abgegrenzt wurde, erforderlich. Die Ausführungen in Abschnitt 2.3.3.2 zeigen, dass es sich nicht um Information an sich, sondern um den Umgang mit Informationen, d.h. ihre Verarbeitung durch ein bestimmtes System handelt. Abschnitt 2.3.3.3 nimmt abschließend eine allgemeine Arbeitsdefinition des Begriffs Informationsmanagement vor, die zum Ausgangspunkt der Betrachtungen in den nachfolgenden Kapiteln gemacht wird.

\subsubsection{Zur Verwendung des Begriffsbestandteils „Management“: Informations- management als funktional-sachorientiert abgrenzbarer Spezialbereich der Unternehmungsfuhrung}

Im Folgenden wird der in Abschnitt 2.3.2 allgemein abgegrenzte Managementbegriff für die nachfolgende Untersuchung des Informationsmanagements präzisiert. Folgt man dem dort einleitend angesprochenen Begriffsverständnis, das Management als „Verwaltung“ auffasst, so wäre eine Erklärung des Begriffs „Informationsmanagement“ als „Verwalten von Informationen“ möglich. Für die in dieser Arbeit verfolgten Zwecke eines betriebswirtschaftstheoretisch fundierten Informationsmanagements ist dagegen eine Sichtweise zweckmäßiger, die Management mit „Führung“ gleichsetzt. Darüber hinaus lässt sich auch diese Einschränkung noch weiter präzisieren, da nicht alle Varianten des Führungsbegriffs gleich gut für eine Analyse des Informationsmanagements geeignet sind.

Ein institutionales Verständnis des Informationsmanagementbegriffs würde implizieren, dass Informationsmanagement diejenigen Aufgaben umfasst, die von so genannten Informationsmanagern ausgeübt werden. Ein solcher positivistisch ausgerichteter Ansatz wäre auf Grund der inflationären und heterogenen Verwendung der Begriffe "Informationsmanagement" und Informationsmanager" in der Praxis mit erheblichen Problemen verbunden. Dies hängt insbesondere damit zusammen, dass eine große Zahl von Unternehmungen in der jüngeren Vergangenheit ihre frühere Abteilung „(Organisation und) Datenverarbeitung“ mit dem modischen Namen „Informationsmanagement" versehen hat. Allein wegen dieser Änderung der Deklaration werden viele ehemalige DV-Leiter inzwischen häufig als „Informationsmanager“ bezeichnet. In vielen Fällen geschieht dies jedoch, ohne dass sich die fachliche Abgrenzung ihres Zuständigkeitsbereichs verändert hat.

Dementsprechend erscheint eine Orientierung an der funktionalen Variante des Managementbegriffs angemessener für eine Analyse des Informationsmanagements. ${ }^{168}$

\footnotetext{
${ }^{168}$ Dies geschieht in Übereinstimmung mit weiten Teilen der Literatur zum Informationsmanagement. Eine institutionale Begriffsverwendung findet sich dagegen z.B. bei Klutmann (1992), S. 70.
} 
In der allgemeinen Abgrenzung dieser Begriffsvariante wurde keinerlei Abgrenzung hinsichtlich der Art des zu führenden Systems gemacht. Demnach könnte sich die Bezeichnung Informationsmanagement im Prinzip nicht nur auf Aufgabenstellungen in Unternehmungen, sondern beispielsweise auch auf solche in privaten Haushalten, öffentlichen Betrieben, Vereinen oder sonstigen Institutionen beziehen. Die weitere Untersuchung konzentriert sich zwar auf Unternehmungen, viele der entwickelten Aussagen lassen sich jedoch mit geringfugigen Anpassungen auch auf andere Arten sozialer Systeme anwenden.

Das Informationsmanagement gehört bei dieser Abgrenzung zur Gesamtmenge der Unternehmungsführungsaufgaben und unterscheidet sich von den übrigen Aufgaben der Unternehmungsführung durch seinen spezifischen Gestaltungsbereich. Zum Informationsmanagement zählen alle Aktivitäten, die auf die zielgerichtete Beeinflussung der (technikunterstützten) Informationsverarbeitung in der Unternehmung gerichtet sind. Dabei ist es nicht notwendig, dass das Informationssystem als Gegenstand des Informationsmanagements eigenständig als reales Subsystem einer Unternehmung institutionalisiert ist. Die Führung durch das Informationsmanagement bezieht sich vielmehr auf einen Funktionsbereich, der durch eine gedankliche Aufgliederung der Unternehmung abgegrenzt wird.

Entsprechend der hier vorgenommenen Gleichsetzung der Begriffe „Management” und „Führung" beinhaltet das Aufgabenfeld ausschließlich Führungsaufgaben. In der Literatur hingegen rechnen die Autoren zum Teil auch Durchführungsaufgaben zum Informationsmanagement hinzu. ${ }^{169}$ Dieser Auffassung, die in der Regel mehr oder weniger implizit vertreten wird und selten auch explizit deutlich zum Ausdruck kommt, wird hier nicht gefolgt. Diese definitorische Beschränkung des Informationsmanagements auf Führungsaufgaben erfordert allerdings eine sowohl theoretisch als auch in der Realität nur schwer vorzunehmende klare Trennung zwischen Führungs- und Durchführungsaufgaben. Unter Durchfürungsaufgaben, die selbst nicht Teil des Informationsmanagements, sondern vielmehr dessen Gestaltungsobjekt sind, werden im Folgenden solche Aufgaben verstanden, bei denen es nicht um die Beeinflussung fremden Verhaltens, sondern um die Verfolgung einer gegebenen Zielsetzung im Rahmen eines fest definierten Entscheidungsrahmens geht.

Es erscheint ferner zweckmäßig, innerhalb der funktionalen Begriffsauffassung ausschließlich sachbezogene Managementaufgaben zum Bereich des Informationsmanagements zu zählen. Zwar ist die personenbezogene Komponente des Managements auch im Bereich der Informationsverarbeitung in einer Unternehmung relevant. Dies gilt speziell in Bezug auf die Führung der einer informationswirtschaftlichen Abteilung angehörenden Mitarbeiter durch die jeweils vorgesetzte Person. Es sind jedoch keine spezifischen Eigenschaften der Personalführung in informationswirtschaftlichen Abteilungen erkennbar, die von der allgemeinen Personalführung in den anderen Bereichen der Unternehmung abweichen und damit eine Sonderbehandlung unter dem Begriff „Informationsmanagement” rechtfertigen würden. Deshalb werden die personenbezogenen Führungsaufgaben einer mit Aufgaben des Infor-

\footnotetext{
${ }^{169}$ Vgl. z.B. Schotters (1992), S. 18; Nawatzki (1994), S. 19; Peterhans (1996), S. 35. Dies konnte damit im Zusammenhang stehen, dass der Begriff „Management” in der Informatik in der Regel nicht als „Führung”, sondern als „Verwaltung” interpretiert wird und dann auch Durchführungsaufgaben umfasst, vgl. Streubel (1996), S. 52.
} 
mationsmanagements betrauten Person, die zugleich Vorgesetztenfunktionen gegenüber bestimmten Mitarbeitern ausübt, hier nicht weiter betrachtet. Diese Linienaufgaben gehören in das Aufgabenfeld des allgemeinen Managements und fallen nach der hier vertretenen Begriffsauffassung nicht unter die Bezeichnung „Informationsmanagement".

Es sei erwähnt, dass zu den personenbezogenen Führungsaufgaben insofern ein Bezug besteht, als deren Unterstützung sehr wohl in den Gestaltungsbereich des sachbezogenen Informationsmanagements fallen kann. Dies ist immer dann der Fall, wenn zur Erfüllung einzelner Teilaufgaben der allgemeinen Personalführung informations- und kommunikationstechnische Lösungen eingesetzt werden. Die Gestaltung und Steuerung derartiger Anwendungssysteme gehört eindeutig zum Aufgabengebiet des Informationsmanagements. Somit zählt auch die Suche nach Innovationsmöglichkeiten im Bereich der allgemeinen Personalführung, die mit Hilfe moderner Informations- und Kommunikationstechniken realisiert werden können, zum Gegenstand des Informationsmanagements.

\subsubsection{Zur Verwendung des Begriffsbestandteils „Information“: Bedeutung und Aktivitäten der Informationsverarbeitung durch das technikunterstützte Informationssystem der Unternehmung}

In der ersten Annäherung zu Beginn des Abschnitts 2.3 wurde Informationsmanagement stark vereinfachend als spezielle Ausprägung des Managements bezeichnet, deren Bezugsobjekt Informationen darstellen. Die anschließenden Ausführungen in Abschnitt 2.3.1 haben deutlich gemacht, dass es sich bei diesem Bezugsobjekt um einen ausgesprochen facettenreichen Gegenstand handelt, der aus unterschiedlichsten Perspektiven betrachtet werden kann. Ausgehend von den im vorigen Abschnitt vorgenommenen Überlegungen zur Verwendung des Begriffsbestandteils „Management“ ist an dieser Stelle eine Präzisierung hinsichtlich der weiteren Verwendung des Begriffsbestandteils „Information“ erforderlich. Im Folgenden wird zunächst kurz die Bedeutung von Informationen für das Geschehen in Unternehmungen verdeutlicht. Im Anschluss daran erfolgt eine nähere Beschreibung des technikunterstützten Informationssystems der Unternehmung, in dem die Verarbeitung von Informationen stattfindet.

\section{a) Funktion von Informationen im Unternehmungsgeschehen}

Die essenzielle Bedeutung von Informationen, die eine zielgerichtete Informationsverarbeitung häufig zur Existenzfrage machen, basiert im Wesentlichen auf drei Aspekten, die die Verwendungszwecke von Informationen in Unternehmungen betreffen. Wie Abbildung $2 / 5$ zeigt, ist zwischen der unternehmungsinternen und der unternehmungsexternen Verwendung von Informationen zu unterscheiden. Zum Bereich der unternehmungsinternen Verwendung gehören der Aufbau von Informationsvorsprüngen gegenüber anderen Akteuren im Wirtschaftsgeschehen sowie die Koordination von Aktivitäten im Rahmen der Leistungserstellung. In die Kategorie der unternehmungsexternen Verwendung fallt die Herstellung von Informationen als Produktbestandteil. Diese Verwendungszwecke werden nachfolgend kurz erläutert. 


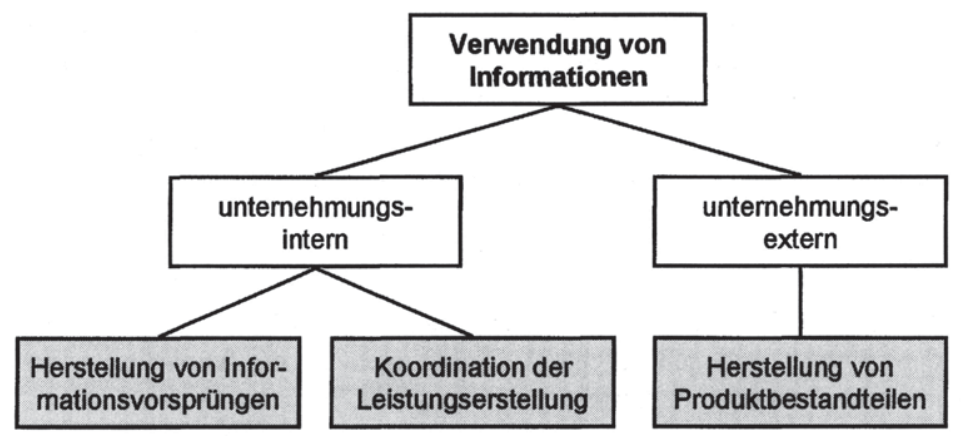

Abb. 2/5: Verwendung von Informationen in Unternehmungen

In einer Gesellschaft verfügt nicht jedes einzelne Individuum über eine vollständige Kenntnis aller für die Befriedigung seiner und anderer Bedürfnisse relevanten Sachverhalte. Aufgrund dieser über die verschiedenen Teilnehmer am Wirtschaftsgeschehen ungleichmäßigen Verteilung von Wissen eröffnet sich die Möglichkeit, durch unternehmerisches Handeln Informationsvorsprünge zu erkennen oder herzustellen und anschließend ökonomisch auszunutzen. ${ }^{170}$ Solche Informationsvorsprünge können sich beispielsweise auf (regionale, produkt- oder kundenspezifische) Märkte oder auf bestimmte Produktionsverfahren beziehen. Information wird deshalb zum Teil als erster, allen anderen Produktionsfaktoren vorgeschalteter Produktionsfaktor bezeichnet. ${ }^{171}$ Dieser erste Aspekt betrifft Informationsasymmetrien zwischen Mitgliedern der Unternehmung und anderen, unternehmungsexternen Akteuren.

Der zweite Aspekt, der die Bedeutung der Informationsverarbeitung für Unternehmungen begründet, betrifft die Koordination von Aktivitäten im Rahmen der eigentlichen Leistungserstellung. Die Ziele von Unternehmungen werden in arbeitsteiligen Prozessen durch ein Zusammenwirken von Menschen und Maschinen erreicht, deren Aktivitäten in geeigneter Weise aufeinander abzustimmen sind. Der Informationsfluss kann in diesem Zusammenhang als Spiegelbild des originären Leistungserstellungsprozesses interpretiert werden. ${ }^{172}$ Dies bedeutet, dass sämtliche Aktivitäten zur Erstellung von Leistungen in einer Unternehmung durch Informationsbeziehungen miteinander verbunden sind. ${ }^{173}$

Schließlich können Informationen als Bestandteil in die Produkte eingehen oder auch selbst das Produkt sein. ${ }^{174}$ In einem solchen Fall werden die betreffenden Informationen unternehmungsextern verwendet, und die Informationsverarbeitung stellt eine originäre Aufgabe im Prozess der Leistungserstellung dar. Während die oben angesprochenen Informationsvorsprünge und die Koordination von Aktivitäten der Leistungserstellung durch informationelle Steuerungsprozesse in allen Unternehmungen von hoher Relevanz sind, ist dies bei Informationen als Produktbestandteil nicht immer der Fall. Dieser Aspekt spielt insbesondere für solche Unternehmungen eine exponierte Rolle,

\footnotetext{
${ }^{170}$ Vgl. Huber/Maier (1991), S. 6; Picot (1990), S. 296.

${ }^{171}$ Vgl. Picot (1990), S. 6.

${ }^{172} \mathrm{Vgl}$. Streubel (1996), S. 35

${ }^{173} \mathrm{Vgl}$. Angermeyer (1990), S. 177.

${ }^{174}$ Vgl. Bode (1997), S. 449; Schüler (1989), S. $181 \mathrm{ff}$.
} 
die einerseits als intelligent $\mathrm{zu}$ bezeichnende materielle Produkte (z.B. Gebrauchsgegenstände mit elektronisch gesteuerten Funktionserweiterungen wie Haushalts- und Elektrogeräte, Autos, Hardware o.ä.) oder andererseits informationelle Produkte (z.B. Nachrichten, Beratungsleistungen aller Art, Software o.ä.) herstellen. Information als Produktbestandteil rückte in der öffentlichen Diskussion der 1980er Jahre in den Blickpunkt des Interesses. ${ }^{175}$

Die Unterscheidung zwischen diesen Verwendungszwecken von Informationen wird in den nachfolgenden Kapiteln an verschiedenen Stellen aufgegriffen, da sich je nach Verwendungszweck unterschiedliche Anforderungen an die Maßnahmen des Informationsmanagements ergeben können.

\section{b) Das technikunterstützte Informationssystem der Unternehmung als Gestal- tungsgegenstand des Informationsmanagements}

Das soziale - genauer genommen, das sozio-technische - System, in dem die Verarbeitung von Informationen stattfindet und das den Gegenstand des Informationsmanagements bildet, wird im Folgenden als technikunterstütztes ${ }^{176}$ Informationssystem der Unternehmung bezeichnet. Durch diese Begriffswahl kommen zwei Dinge zum Ausdruck:

Zum einen wird der Gestaltungsgegenstand des Informationsmanagements auf solche Aktivitäten eingegrenzt, die durch informations- und kommunikationstechnische Hilfsmittel, zu denen sowohl Hardwarekomponenten als auch Softwarekomponenten gehören, ${ }^{177}$ unterstützt werden. Ohne diese Einschränkung würden sich die Grenzen des Untersuchungsbereichs nahezu ins Uferlose ausdehnen, da sich praktisch jede Aktivität in einer Unternehmung (z.B. Unterhaltungen, jede Art des Denkens, Lesens, Schreibens usw.) dem Gestaltungsgegenstand des Informationsmanagements subsumieren ließe. Es sei darauf hingewiesen, dass es als genuine Aufgabe des Informationsmanagements gesehen werden kann, durch die Verwendung innovativer Techniken immer mehr Aktivitäten durch informations- und kommunikationstechnische Hilfsmittel zu unterstützen, wenn dies zur Effektivität und Effizienz der Informationsverarbeitung beiträgt. Dadurch werden die Grenzen des technikunterstützten Informationssystems der Unternehmung immer weiter ausgedehnt. Aus diesem Grund sollte der Gestaltungsbereich des Informationsmanagements auch solche Aktivitäten der Informationsverarbeitung umfassen, die zwar bisher nicht durch informations- und kommunikationstechnische Hilfsmittel unterstützt wurden, für die sich durch den Einsatz innovativer Techniken jedoch die Möglichkeit zur technischen Unterstützung konkret abzeichnet.

\footnotetext{
${ }^{175} \mathrm{Vgl}$. Streubel (2000), S. 65.

${ }^{176}$ Streubel spricht in diesem Zusammenhang vom computergestützten Informationssystem, vgl. Streubel (2000), S. 70. Dieser Begriff wird hier durch den des technikunterstutzten Informationssystems ersetzt, da er sich auf das technische Hilfsmittel „Computer” beschränkt, unter den bestimmte technische Geräte (z.B. Geräte zur Übertragung von Informationen) nicht direkt gefasst werden können. Ferner wird zum Teil vorgeschlagen, den Prozess der Kommunikation durch eine entsprechende Begriffswahl besonders hervorzuheben und von Informations- und Kommunikationssystemen zu sprechen. Diesem Vorschlag wird hier einerseits aus Gründen der sprachlichen Vereinfachung und andererseits deshalb, weil die Kommunikation genau genommen einen Teilbereich der Informationsverarbeitung darstellt, nicht gefolgt.

${ }^{177}$ Vgl. Hansen (1996), S. 28.
} 
Zum anderen bringt der Zusatz Informationssystem der Unternehmung die Unabdingbarkeit einer ganzheitlichen Sichtweise zum Ausdruck. Diese wird in vielen Veröffentlichungen der jüngeren Vergangenheit als Abwendung von der reinen Technikorientierung des Informationsmanagements gefordert. ${ }^{178}$ Das Informationssystem der Unternehmung besteht aus den Elementarten „Mensch” und „Technik”, die bei der technikunterstützten Informationsverarbeitung stets zusammenwirken. ${ }^{179}$ Für die Zwecke einer detaillierteren Betrachtung ist eine Zerlegung dieses Gesamtsystems in verschiedene Subsysteme auf dem Wege der hierarchischen Dekomposition sehr wohl möglich. Es können dann entweder einzelne Anwendungssysteme, die wiederum die Elementarten „Mensch" und „Technik” enthalten, oder rein technische bzw. rein menschliche Teilsysteme analysiert und gestaltet werden. Dabei ist jedoch stets darauf zu achten, dass der Gesamtzusammenhang in der Betrachtung erhalten bleibt, da eine isolierte Untersuchung einzelner Teilsysteme den realen Gegebenheiten nicht gerecht werden kann. Die durch das System zu erfüllenden Aufgaben stellen die zielsetzende Größe dar, an der sich die Gestaltung des technikunterstützten Informationssystems zu orientieren hat.

Nachdem geklärt wurde, um welche Art von System es sich bei dem Gestaltungsobjekt des Informationsmanagements handelt, sind nun noch die Aktivitäten zu beschreiben, die durch das Informationsmanagement beeinflusst werden sollen. Dabei handelt es sich um verschiedene Tätigkeiten der Informationsverarbeitung, die folgendermaßen eingeteilt werden können: ${ }^{180}$

- Generierung von Informationen

Dazu gehören die Gewinnung von Informationen aus externen Quellen sowie die Erstellung von Informationen (im Sinne der gedanklichen Entwicklung eines Modells zur Abbildung von Sachverhalten) durch den Menschen.

- Speicherung von Informationen

Die Speicherung umfasst die Ablage sowie das Wiederauffinden von Informationen und ist insbesondere dann mit Schwierigkeiten verbunden, wenn große Informationsmengen verarbeitet werden.

- Bearbeitung von Informationen

Dabei handelt es sich um Transformationsaktivitäten auf der syntaktischen Ebene, die die Informationsdarstellung durch ein Trägermedium betreffen, indem sie z.B. eine Vervielfältigung, Sortierung, Kombination mehrerer Informationen oder eine Änderung der Darstellungsart vornehmen.

- Übertragung von Informationen

Zur Informationsübertragung sind Aktivitäten des Sendens und des Empfangens von Signalen zur Überbrückung räumlicher und zeitlicher Distanzen erforderlich.

- Auswertung von Informationen

Die Auswertung von Informationen erfolgt durch gedankliche Interpretationsaktivitäten des Menschen im Rahmen seiner Entscheidungsfindung.

\footnotetext{
${ }^{178}$ Vgl. z.B. Zahn/Rüttler (1989), S. 25; Brockhaus (1992), S. 2; Schotters (1992), S. 14; Nawatzki (1994), S. 8; Streubel (1996), S. 81

${ }^{179}$ Vgl. Streubel (2000), S. 70 ff.

${ }^{180}$ Vgl. ăhnlich Gabriel et al. (1994), S. 15.
} 
Durch diese innerhalb von Informationssystemen stattfindenden Aktivitäten, die mit Ausnahme der Generierung und der Auswertung von Informationen sowohl durch Menschen als auch durch Maschinen erfolgen können, werden die Aufgaben des Systems erfüllt. Diese Aktivitäten sind durch das Informationsmanagement in zielorientierter Weise zu gestalten und zu steuern. Dabei sei darauf hingewiesen, dass sich der Begriff „Management” entsprechend der oben vorgestellten Definition genau genommen nur auf die Beeinflussung menschlichen Verhaltens bezieht. Die zum technikunterstützten Informationssystem der Unternehmung gehörenden Verhaltensaktivitäten der Menschen werden jedoch durch informationstechnische Hilfsmittel unterstützt und sind deshalb stets mit dazu gehörenden maschinellen Aktivitäten verknüpft. Deshalb ist es - auch vor dem Hintergrund der Forderung nach einer ganzheitlichen Sichtweise - konsequent, hier auch die Funktionalität der Informationstechnik in den Gestaltungsbereich des Informationsmanagements mit einzubeziehen. ${ }^{181}$ Im Folgenden wird daher von menschlichen Aufgaben- und maschinellen Aktionsträgern gesprochen.

\subsubsection{Arbeitsdefinition: „Informationsmanagement"}

Im Folgenden werden die Überlegungen des Abschnitts $2.3 \mathrm{zu}$ einer allgemeinen Arbeitsdefinition zusammengefuihrt, die das Informationsmanagement als Erfahrungsobjekt der weiteren Überlegungen abgrenzt. Die hier vorgenommene Definition lässt Raum für eine Präzisierung, die bei der Bezugnahme auf verschiedene Wissenschaftsprogramme den jeweiligen Erkenntnisinteressen und Leitideen entsprechend vorgenommen werden kann. Bei der Interpretation des Begriffes in den nachfolgenden Kapiteln werden sich also jeweils etwas andere Akzentuierungen ergeben. Diese sind auf Grund der unterschiedlichen Erkenntnisziele der einzelnen Wissenschaftsprogramme nicht nur unvermeidbar, sondern im Rahmen einer möglichst vielschichtigen Untersuchung, die Ergebnisse aus verschiedenen Betrachtungsperspektiven liefert, sogar erwünscht.

Die weiteren Untersuchungen dieser Arbeit gehen von folgender Arbeitsdefinition aus, die das Informationsmanagement als Erfahrungsgegenstand wissenschaftlicher Untersuchungen abgrenzt:

Informationsmanagement umfasst die Gestaltung und Steuerung des technikunterstützten Informationssystems der Unternehmung sowie seiner verschiedenen Teilsysteme. Dies geschieht mit der Absicht der zielorientierten Beeinflussung von Aktivitäten der Informationsverarbeitung, die durch menschliche Aufgaben- und maschinelle Aktionsträger ausgefuihrt werden.

\footnotetext{
${ }^{181}$ Vgl. ähnlich Reif-Mosel (2000), S. 21.
} 


\subsection{Die Betriebswirtschafts/ehre als Referenzdisziplin für eine wissen- schaftliche Analyse des Informationsmanagements}

Nachfolgend wird die Betriebswirtschaftslehre als die in dieser Arbeit gewählte Referenzdisziplin für eine wissenschaftiche Analyse des Informationsmanagements vorgestellt. Abschnitt 2.4.1 konzentriert sich zunächst auf eine Abgrenzung des empirischen Konstrukts, das den Erfahrungsgegenstand der Betriebswirtschaftslehre darstellt. Diese Ausführungen sind auf der ersten Stufe des hier gewählten dreistufigen Verfahrens für den Theorieeinstieg zur Untersuchung des Informationsmanagements angesiedelt. In Abschnitt 2.4.2 erfolgen einige grundsätzliche Überlegungen zur disziplinären Abgrenzung der Betriebswirtschaftslehre und ihrer Stellung gegenüber anderen Wissenschaften. Diese Einordnung ist deshalb erforderlich, weil sich daraus generelle Vorgaben dafür ergeben, mit welcher Art von Fragestellungen sich betriebswirtschaftlich fundierte Analysen des Informationsmanagements auseinander setzen sollten. Abschnitt 2.4.3 nimmt eine Auswahl derjenigen Wissenschaftsprogramme aus dem Gesamtspektrum betriebswirtschaftlicher Forschungsansätze vor, auf die in den nachfolgenden Kapiteln Bezug genommen wird.

Vorweg sei angemerkt, dass die Auffassungen, die von verschiedenen Vertretern der Betriebwirtschaftslehre hinsichtlich Gegenstand, Methoden und Konzepten ihrer Disziplin vertreten werden, ausgesprochen kontrovers sind. ${ }^{182}$ Die Auffassungsunterschiede sind so tiefgreifend, dass sogar die These, von einem Grundkonsens über Ziele, Inhalte und Methoden des Fachs könne kaum noch gesprochen werden, diskutiert wird. ${ }^{183}$ Die Betriebswirtschaftslehre existiere allenfalls als Institution in der Bezeichnung von Lehrstühlen, Verbänden und anderen professionellen Einrichtungen. Eine gemeinsame Vorstellung über die Grundlagen des Fachs wäre allerdings wünschenswert, da dies eine wichtige Orientierungsfunktion erfullt und den Wissenschaftsprozess wesentlich prägt. ${ }^{184}$ Die Erzielung eines Minimalkonsenses könnte daher die Effektivität betriebswirtschaftlicher Forschung und Lehre steigern. Dies gilt selbst dann, wenn die fachliche Identität nur einen relativ lockeren Rahmen vorgibt, in dem ein pluralistisches Arbeiten stattfindet und die verschiedenen Konzepte miteinander in einem flexiblen Wettbewerb um das leistungsfähigste Aussagesystem stehen. ${ }^{185}$ Die nachfolgenden Überlegungen konzentrieren sich daher auf die Gemeinsamkeiten, die verschiedenen Forschungsansätzen des Faches zu Grunde liegen.

\subsubsection{Zum Erfahrungsgegenstand der Betriebswirtschaftslehre}

Der Untersuchungsbereich der Betriebswirtschaftslehre ist Gegenstand einer langen Diskussion, die sich sowohl auf den Erfahrungsgegenstand als auch auf den Erkenntnisgegenstand der Disziplin bezieht. Analog zum Vorgehen bei der Abgrenzung des Informationsmanagements als empirischem Konstrukt bietet sich auch hier zunächst einmal eine Begriffszerlegung, insbesondere in die beiden ersten Bestandteile „Betrieb“ und „Wirtschaft" bzw. „wirtschaften“ an. Bei diesem Vorgehen wird häufig der Begriffsbestandteil „Betrieb“ als Erfahrungsbereich und der Begriffsbestandteil „Wirt-

\footnotetext{
${ }^{182}$ Vgl. Raffée (1993), S. 3.

${ }^{183}$ Vgl. Bleicher (1988), S. 110; ähnlich Schanz (1997a), S. 81.

${ }^{184}$ Vgl. Raffée (1993), S. 3.

${ }^{185}$ Vgl. Bleicher (1988), S. 115; ähnlich Schanz (1997a), S. 81.
} 
schaft" als Erkenntnisbereich der Betriebswirtschaftslehre gesehen. ${ }^{186}$ Dieser Schritt führt allerdings noch nicht zu einem präzisen Ergebnis, da beide Begriffe semantisch nicht eindeutig belegt sind, ${ }^{187}$ sodass eine weitere inhaltliche Klärung erforderlich ist. Ferner ist umstritten, ob eine Fokussierung auf Betriebe als Erfahrungsbereich der Betriebswirtschaftlehre zielführend ist, oder ob sie eine unzulässige Verengung der Perspektive darstellt. ${ }^{188}$

Eine besonders breite Abgrenzung des Erfahrungsbereichs wird damit begründet, dass auch außerhalb von Betrieben Sachverhalte vorliegen, die Relevanz für das Betriebsgeschehen besitzen. Die Vertreter dieser Auffassung argumentieren, dass alle wirtschaftlichen Sachverhalte mit einer Fülle weiterer Sachverhalte (z.B. religiöser, künstlerischer, rechtlicher, technischer, medizinischer, sozialer, ökologischer Art) eng verknüpft sind. ${ }^{189}$ Die volle Erfahrungswelt und empirische Grundlage der Betriebswirtschaftslehre müsse daher die gesamte Wirklichkeit menschlichen Handelns und Verhaltens mit all seinen Auswirkungen und Gestaltungen in der Realität umgreifen und somit die gesamte Kulturwelt des Menschen beinhalten. ${ }^{190}$ Auf eine ähnlich weite Abgrenzung läuft auch der Vorschlag hinaus, als Ausgangstatbestand der Forschung das „Handeln einzelner Menschen in Märkten, Unternehmungen und weiteren Organisationen (wie Parlamenten, Verbänden) sowie die Regeln für das Handeln des Einzelnen und das möglichst konfliktarme Zusammenleben mit anderen " zu wählen. ${ }^{191} \mathrm{Um}$ die betriebswirtschaftlichen Zusammenhänge aus dieser vielschichtigen Erfahrungswelt gedanklich abzusondern, ist ein Auswahlkriterium zu verwenden, das dann den Erkenntnisgegenstand der Betriebswirtschaftslehre darstellt. Die Vorteilhaftigkeit einer derart weiten Abgrenzung des Untersuchungsfeldes liegt darin begründet, dass ein breites Spektrum an Wissenschaftsprogrammen aus diesem Bereich ihr spezielles Erkenntnisobjekt selektieren kann und dabei der gemeinsame Erfahrungsgegenstand der verschiedenen Ansätze als verbindendes Element erhalten bleibt.

Das hier gewählte dreistufige Vorgehen für einen Theorieeinstieg zur Analyse des Informationsmanagements, das von der radikal-konstruktivistischen Grundposition ausgeht, macht allerdings bereits auf der ersten Stufe eine intensivere Auseinandersetzung mit dem Erfahrungsgegenstand notwendig. Da der Erfahrungsgegenstand nach dieser Vorstellung selbst den Charakter einer Repräsentation besitzt, muss er zunächst durch den Betrachter erfunden werden. Erst auf dieser Grundlage kann der Erkenntnisgegenstand als abstraktes Denkobjekt gedanklich abgeleitet und für sich vergegenständlicht werden. Auf dieser Ebene ist daher auch bereits das wirtschaftliche Handeln von Menschen näher zu betrachten. Ferner ist das Phänomen der Betriebe, in denen dieses Handeln stattfindet, auf Basis eines allgemeinen Vorverständnisses abzugrenzen. Beide Begriffsbestandteile werden hier also dem Erfahrungsbereich der Betriebswirtschaftslehre zugeordnet, der die verschiedenen Wissenschaftsprogramme dieser Disziplin miteinander verbindet. Erst auf der zweiten Stufe des Theorieeinstiegs entstehen die Unterschiede zwischen den Forschungsansätzen, wenn das Erfahrungs-

\footnotetext{
${ }^{186}$ Für diese Position vgl. z.B. Neus (2001), S. 2; Zelewski (1999), S. 11 ff.

${ }^{187} \mathrm{Vgl}$. Neus (2001), S. 2.

${ }^{188}$ Für diese Position vgl. z..B. Kosiol (1961), S. 132; Schweitzer (1997), S. $27 f$.

${ }^{189}$ Vgl. Schweitzer (1997), S. 28.

${ }^{190}$ Vgl. Kosiol (1961), S. 130.

${ }^{191}$ Vgl. Schneider (1995), S. 25.
} 
objekt den jeweiligen Erkenntnisinteressen entsprechend für die weitere Analyse präpariert wird.

Unter dem Begriff „Wirtschaften“ werden ganz allgemein sämtliche Tätigkeiten des Menschen zusammengefasst, die unter Einsatz knapper Güter unmittelbar oder mittelbar der Bedürfnisbefriedigung dienen. ${ }^{192}$ In der Literatur wird der Begriff vielfach an ein planvolles Vorgehen geknüpft, sodass nur rational beabsichtigtes Verhalten zu den wirtschaftlichen Handlungen gehört. ${ }^{193}$ Dieser Auffassung wird hier nicht gefolgt, da damit von vornherein eine Analyse jener Spielarten menschlicher Handlungen ausgeschlossen wäre, die zwar keine Rationalität, wohl aber wirtschaftlichen Inhalt beanspruchen können. ${ }^{194}$ Eine derartige Verengung des Blickfeldes sollte frühestens auf der zweiten Stufe des Theorieeinstiegs, also bei der Konstruktion des Erkenntnisgegenstands eines einzelnen Forschungsansatzes vorgenommen werden. Mit der gleichen Begründung wird hier eine dahingehende Einengung des Begriffs abgelehnt, dass nur die Versorgung mit knappen Gütern dem Begriff des „Wirtschaftens" subsumiert wird. ${ }^{195}$ Die hier verwendete Definition schließt vielmehr auch die Verwendung von Gütern ein. Dies lässt ebenfalls für die zweite Ebene des Theorieeinstiegs die Möglichkeit zur Beschränkung der Betrachtung offen, durch die ein einzelnes Wissenschaftsprogramm sich allein auf Fragen der Güterversorgung konzentrieren kann.

Wirtschaftliches Handeln erstreckt sich nicht auf die Befriedigung von Bedürfnissen schlechthin, sondern nur auf jene Handlungen, die Bedürfnisse durch die Verwendung knapper Güter zu stillen versuchen. ${ }^{196}$ Aus diesem Grund ist eine Präzision in Bezug darauf erforderlich, was unter dem Begriff des knappen Guts zu verstehen ist. Wird in diesem Punkt eine weite Begriffsabgrenzung gewählt, so ist dann von knappen Gütern zu sprechen, wenn eine nicht mit einer Gegenleistung verbundene Erhöhung der verfügbaren Gütermenge eine Nutzensteigerung herbeiführt. ${ }^{197}$ Dies erweist sich allerdings als problematisch, weil auch spezielle immaterielle Werte wie Solidarität, Liebe usw. knapp sind und damit zum Untersuchungsbereich der Betriebswirtschaftslehre gehören würden. ${ }^{198}$ Aus diesem Grund sollte die Untersuchung auf solche Güter eingegrenzt werden, die geeignet sind, als Tauschobjekt produziert und verwertet zu werden. Damit werden Tauschprozesse zu einem zentralen Kennzeichen wirtschaftlichen Handelns. ${ }^{199}$

Wirtschaftliche Probleme treten auf verschiedenen Ebenen des menschlichen Daseins auf. ${ }^{200}$ Die Betriebswirtschaftslehre erhebt keineswegs den Anspruch, wirtschaftliche Handlungen auf allen diesen Ebenen zu untersuchen. ${ }^{201}$ Der Ausgangspunkt der Betriebswirtschaftslehre ist vielmehr die Erforschung der wirtschaftlichen Erschei-

\footnotetext{
${ }^{192}$ Vgl. Endes (1991), S. 1.

${ }^{193}$ Vgl. z.B. Wöhe (2000), S. 2; Zelewski (1999), S. 11 .

${ }^{194}$ Vgl. Raffée (1993), S. 8.

${ }^{195}$ Vgl. z.B. Kosiol (1961), S. 130.

${ }^{196}$ Vgl. Zelewski (1999), S. 12.

${ }^{197}$ Vgl. Neus (2001), S. 6.

${ }^{198}$ Vgl. Raffée (1993), S. 9.

${ }^{199}$ Vgl. Raffée (1993), S. 9f. Dieser weist darauf hin, dass auch bei kollektiven Gütern, die wegen ihrer speziellen Merkmale nicht einzeln zwischen Individuen ausgetauscht werden können, von irgendeiner Art von Austauschprozessen ausgegangen werden kann.

${ }^{200} \mathrm{Vgl}$. Weber (1993), S. 2.

${ }^{201}$ Vgl. Zelewski (1999), S. 20.
} 
nungen an der Stelle ihrer Entstehung, also in den Betrieben ${ }^{202}$ Diese Einschränkung erfordert eine nähere Auseinandersetzung mit dem Begriff des Betriebs, über dessen inhaltliche Bedeutung keine vollständige Einigkeit besteht. In der allgemeinsten Form handelt es sich bei Betrieben um soziale Gebilde, die mit menschlichem Zweckhandeln erfüllt sind. ${ }^{203}$ Nimmt man eine erste Einschränkung der Definition auf wirtschaftliche Zusammenhänge vor, so stellt ein Betrieb die kleinste Einheit dar, in der sich durch Zusammenfassung von Menschen und Sachen wirtschaftliche Handlungen vollziehen lassen. ${ }^{204}$ Dieser Definition wird hier gefolgt. Alles (unmittelbar) wirtschaftliche Handeln vollzieht sich demnach in Betrieben, bei denen es sich um spezielle Objekte des menschlichen Kulturbereichs handelt. ${ }^{205}$

Eine bedeutende Kontroverse zwischen verschiedenen Vertretern des Fachs entzündet sich daran, ob in das Betrachtungsfeld neben den ausschließlich für den Fremdbedarf produzierenden Betrieben auch die privaten Haushalte und die öffentlichen Betriebe einbezogen werden sollen. ${ }^{206}$ Häufig wird die Untersuchung lediglich auf Betriebe bezogen, die mit der Herstellung von Gütern befasst sind. In diesem Fall ist zu entscheiden, ob auch die produzierenden Aktivitäten in privaten Haushalten in die Analyse aufgenommen werden sollen. Diese sind dadurch gekennzeichnet, dass die Verwendung der Güter durch die gleichen Personen erfolgt, die diese auch erstellt haben. In der Regel werden private Haushalte aus der Untersuchung ausgeklammert, sodass der Begriff des Betriebs auf „Stätten der Güterausbringung für den fremden Bedarf $^{6207}$ eingeschränkt wird. Es wird also erst dann von Betrieben gesprochen, wenn Sachgüter und/oder Dienstleistungen erstellt und abgesetzt werden. ${ }^{208}$ Aus gesamtwirtschaftlicher Sicht besteht die Aufgabe dieser Betriebe in der Deckung des Bedarfs an wirtschaftlichen Leistungen einer Gesellschaft. ${ }^{209}$ Der einzelne Betrieb ist dann eine ökonomische, technische, soziale und umweltbezogene Einheit mit der Aufgabe der Bedarfsdeckung mit selbstständigen Entscheidungen und eigenen Risiken. ${ }^{210}$ Vertreter dieser engen Begriffsfassung erkennen zwar an, dass eine umfassende Erkenntnisgewinnung über wirtschaftliche Zusammenhänge dadurch gefördert wird, dass diese aus unterschiedlichen Perspektiven untersucht werden. ${ }^{211}$ Diese Feststellung reicht nach dieser Auffassung jedoch nicht aus, um die privaten Haushalte in die Untersuchung einzubeziehen. Öffentliche Institutionen sollten aus dieser Sicht ebenfalls nur berücksichtigt werden, wenn und so weit diese mit privatwirtschaftlichen Betrieben vergleichbar sind.

Eine derart enge Begriffsfassung passt allerdings nicht zu der oben vorgenommenen weiten Abgrenzung des Begriffs "Wirtschaft" bzw. „wirtschaften". Wirtschaftliches Handeln wurde dort so weit abgegrenzt, dass dazu jegliches Handeln gehört, das sich darauf richtet, menschliche Bedürfnisse unter Einsatz knapper (wirtschaftlicher) Güter zu befriedigen. Dies beinhaltet nicht nur die Herstellung von Gütern, sondern auch

\footnotetext{
${ }^{202} \mathrm{Vgl}$. Seyffert (1957), S. 7.

${ }^{203} \mathrm{Vgl}$. Seyffert (1956), Sp. 736.

${ }^{204}$ Vgl. Zelewski (1999), S. 20.

${ }^{205}$ Vgl. Schweitzer (1997), S. 23.

${ }^{206}$ Vgl. Stein (1993), Sp. 471.

${ }^{207}$ Endres (1991), S. 1.

${ }^{208}$ Vgl. Wöhe (2000), S. 2; Stein (1993), Sp. 472. Hervorhebung durch den Verfasser.

${ }^{209}$ Vgl. Weber (1993), S. 1.

${ }^{210}$ Vgl. Schweitzer (1997), S. 30.

${ }^{211}$ Vgl. Stein (1993), Sp. 471.
} 
deren Verwertung im Rahmen der Bedürfnisbefriedigung. Konsistenter ist es daher, zu den Betrieben auch diejenigen Wirtschaftseinheiten zu zählen, die einen besonderen Schwerpunkt in der Konsumtion haben. Die Betriebswirtschaftslehre wird damit als Lehre von den Einzelwirtschaften konzipiert. ${ }^{212}$ Dieser Position wird hier gefolgt, da sie als Basis für einen Minimalkonsens, von dem aus einzelne Wissenschaftsprogramme der Disziplin ihren speziellen Erkenntnisgegenstand flexibel präparieren können, besser geeignet ist als eine Beschränkung auf produzierende Wirtschaftseinheiten.

Abschließend ist hier noch auf den Begriff der Unternehmung einzugehen, der den Ausgangspunkt zahlreicher Analysen in der Betriebswirtschaftslehre darstellt. Häufig werden die Termini „Betrieb“ und „Unternehmung“ als synonyme Bezeichnungen für denselben Begriffsinhalt verwendet. ${ }^{213}$ Tatsächlich ist für viele betriebswirtschaftstheoretische Betrachtungen eine Trennung zwischen Betrieben und Unternehmungen nicht zwingend erforderlich. ${ }^{214}$ Dennoch findet ebenfalls an vielen Stellen eine Unterscheidung zwischen Unternehmungen und Betrieben statt. Dabei wird der Begriff „Unternehmung beispielsweise dahingehend ausgelegt, dass er sich auf spezielle Betriebsarten bezieht. ${ }^{215}$ In diesem Fall werden Unternehmungen als derivative Betriebe konzipiert und entsprechen damit dem oben erläuterten engen Betriebsbegriff, der vielen Arbeiten der Betriebswirtschafslehre zu Grunde liegt. ${ }^{216}$ Zum Teil wird der Unternehmungsbegriff auch mit dem marktwirtschaftlichen System so fest verknüpft, dass es in planwirtschaftlichen Systemen keine Unternehmungen (wohl aber Betriebe) gibt. $^{217}$ Schließlich ist auch vorstellbar, den Unternehmungsbegriff auf einer vollständig anderen Ebene als den Betriebsbegriff anzusiedeln und damit die rechtliche, organisatorische und finanzielle Einheit zu bezeichnen, während der Betrieb als technische Einheit aufgefasst wird. ${ }^{218}$ Die Erstellung von Leistungen innerhalb einer Unternehmung kann nach dieser Auffassung innerhalb eines einzigen Betriebs oder aber auch innerhalb mehrerer Betriebe, die dem gleichen rechtlichen, organisatorischen und finanziellen Gebilde angehören, stattfinden. Diese Auffassung, die sich mit den genannten speziellen Anforderungen des Unternehmungsbegriffs an das Wirtschaftssystem verknüpfen lässt, wird hier gefolgt.

\subsubsection{Standortbestimmung der Betriebswirtschaftslehre als Wissenschaft}

Nachdem der Erfahrungsgegenstand der Betriebswirtschaftslehre konstruiert wurde, erfolgen an dieser Stelle einige Grundüberlegungen zu ihrer disziplinären Abgrenzung (Abschnitt 2.4.2.1) und zur Ansiedlung des Fachs als wissenschaftlicher Disziplin (Abschnitt 2.4.2.2). Diese Standortbestimmung verdeutlicht die Leistungsmöglichkeiten, die die Betriebswirtschaftslehre insgesamt als Referenzdisziplin für eine wissenschaftliche Analyse des Informationsmanagements aufweist. Die Bezugnahme auf einzelne Wissenschaftsprogramme bei der Entwicklung verschiedener Ansätze für eine

\footnotetext{
${ }^{212}$ Vgl. Raffée (1993), S. 5.

${ }^{213}$ Vgl. Zelewski (1999), S. 21.

${ }^{214} \mathrm{Vgl}$. Busse v. Colbe/Laßmann (1991), S. 19.

${ }^{215}$ Vgl. Zelewski (1999), S. 21.

${ }^{216}$ Vgl. z.B. Weber (1993), S. 2.

${ }^{217}$ Vgl. Busse v. Colbe/Laßmann (1991), S. 18; Weber (1993), S. 2. Diese Auffassung geht auf den von Gutenberg entwickelten Faktortheoretischen Ansatz zurück, der im nächsten Kapitel ausfuihrlich dargestellt wird.

${ }^{218} \mathrm{Vgl}$. Zelewski (1999), S. 22.
} 
Theorie des Informationsmanagements in den nachfolgenden Kapiteln muss sich innerhalb des hier abgesteckten generellen Rahmens bewegen.

\subsubsection{Disziplinäre Abgrenzung der Betriebswirtschaftslehre}

Eine Abgrenzung von Wissenschaften ist im Allgemeinen schwierig, weil es vielfach zu Überlappungen der Problemfelder unterschiedlicher Disziplinen kommt. ${ }^{219}$ Die Frage nach der disziplinären Abgrenzung der Betriebswirtschaftslehre gegenüber anderen wissenschaftlichen Fächern impliziert ferner die Auffassung, dass eine solche Standortbestimmung überhaupt zweckmäßig ist. ${ }^{220}$ Tatsächlich kann die Suche nach einem Identität vermittelnden Untersuchungsgegenstand nur dann erfolgreich sein, wenn dieser Gegenstand ein Herausschälen und Isolieren fachspezifischer Fragestellungen ermöglicht. ${ }^{221}$ Bezogen auf die Betriebswirtschaftlehre ist die Zweckmäßigkeit einer solchen Isolierung fachspezifischer Problemstellungen nicht unbestritten, da viele Probleme sich nur durch die Integration einzeldisziplinärer Perspektiven lösen lassen. ${ }^{222}$ Weite Kreise von Fachvertretern gehen deshalb davon aus, dass eine disziplinäre Abgrenzung der Betriebswirtschaftslehre weder möglich noch zweckmäßig ist. ${ }^{223}$

Diese Ablehnung wird damit begründet, dass bei der Untersuchung wirtschaftlichen Handelns in Betrieben ein so enger Problemverbund vorliegt, dass ein Herausgreifen einzelner disziplinspezifischer Teilprobleme bzw. Einzelfragen weder möglich noch opportun ist. Aus diesem Grund wird die Ansicht geäußert, die meisten Probleme ließen sich nur durch die Integration einzeldisziplinärer (z.B. wirtschaftlicher, psychologischer und technischer) Perspektiven lösen. ${ }^{224}$ Nach dieser Ansicht müsste sich das forschende Fragen auf den einheitlichen und komplexen Gegenstand Betrieb beziehen. ${ }^{225}$ Es müsste also eine Einheitswissenschaft ${ }^{226}$ betrieben werden, in der nicht mehr zwischen Erfahrungsgegenstand und Erkenntnisgegenstand unterschieden werden dürfte und könnte. Dieses Vorgehen wirft jedoch Probleme auf, da eine sich zunehmend öffnende Schere zwischen rapidem Wissenszuwachs und begrenzter persönlicher Forscherkapazität gegeben ist. ${ }^{227} \mathrm{Um}$ die drohende Überforderung zu vermeiden, muss die Komplexität des Untersuchungsgegenstands auf ein fassbares Maß von durch den Forschenden zu bewältigenden Problemstellungen reduziert werden. ${ }^{228}$ Die vollkommene Verschmelzung der fachlichen Disziplinen würde sonst zu einem generellen Wissenschaftsdilettantismus führen, ${ }^{229}$ mit dem der angestrebte Erkenntnisgewinn nicht erreicht werden könnte.

\footnotetext{
${ }^{219}$ Vgl. Weber (1993), S. 4.

${ }^{220}$ Vgl. Raffée (1993), S. 4.

${ }^{221}$ Vgl. Bleicher (1988), S. 114.

${ }^{222}$ Vgl. Raffée (1993), S. 4f.

${ }^{223}$ Vgl. Bleicher (1988), S. 114.

${ }^{224} \mathrm{Vgl}$. Ackoff (1977), S. 285.

${ }^{225}$ Vgl. Schweitzer (1997), S. 45.

${ }^{226}$ Schweitzer (1997), S. 45.

${ }^{227}$ Vgl. Schweitzer (1997), S. 46

${ }^{228}$ Vgl. Bleicher (1988), S. 113.

${ }^{229}$ Vgl. Chmielewicz (1994), S. 21.
} 
Das zu beobachtende hohe Tempo des wissenschaftlichen Fortschritts spricht daher für eine Arbeitsteilung in der Wissenschaft. ${ }^{230}$ Wollte man sämtliche Prozesse, die sich in Betrieben vollziehen, zum Gegenstandsbereich der Betriebswirtschaftslehre zählen, so bedeutete dies den Verzicht auf jegliche sinnvolle wissenschaftliche Spezialisierung und damit mit Sicherheit eine Beeinträchtigung des Erkenntnisfortschritts. ${ }^{231}$ Dieser Auffassung wird hier gefolgt, obwohl jede Abgrenzung des disziplinären Gegenstands eines Fachs einem Dilemma unterliegt. ${ }^{232}$ Die Vorteile einer disziplinären Arbeitsteilung schlagen dort in ihr Gegenteil um, wo im Zuge von Grenzziehungen das wissenschaftiche Problemlösungspotenzial vermindert wird und/oder lösungsbedürttige Probleme überhaupt nicht mehr bemerkt werden. Der als Konsequenz erhobenen Forderung nach so viel disziplinärer Abgrenzung wie nötig und so viel Durchlässigkeit wie möglich ist daher zuzustimmen. Es stellt sich allerdings die Frage, wie diese Durchlässigkeit bzw. Trennung konkret ausgestaltet werden sollte.

Häufig wird davon ausgegangen, dass die Identität der Betriebswirtschaftslehre durch ihr individuelles Erkenntnisobjekt, das aus dem Erfahrungsobjekt herauszugreifen ist, gestiftet wird. ${ }^{233}$ Das Erkenntnisobjekt, so diese Auffassung, bündelt isolierend und abstrahierend die Betrachtung auf einen begrenzten Kreis von Erscheinungen und grenzt diese damit zugleich von anderen disziplinären Erkenntnisbestrebungen $a b^{234}$ Diesem Vorgehen wird hier nicht gefolgt. Hier wird vielmehr die Auffassung vertreten, dass einzelne Wissenschaftsprogramme der Betriebswirtschaftslehre jeweils ein individuelles theoretisches Konstrukt erstellen, das sie aus dem gemeinsamen empirischen Konstrukt aller Forschungsansätze der Betriebswirtschaftslehre herausgreifen. Die Identität der Betriebswirtschaftslehre bestimmt sich somit durch das im vorigen Abschnitt konstruierte Erfahrungsobjekt. ${ }^{235}$ Diese Position lässt Raum für ein interdisziplinäres Arbeiten im Rahmen einzelner Wissenschaftsprogramme, das jedoch auf die Integration von Erkenntnissen einzelner Nachbardisziplinen beschränkt ist, ohne vor den oben angesprochenen Problemen zu stehen, die mit einer umfassenden Integration im Rahmen einer Einheitswissenschaft verbunden sind.

Als Schlussfolgerung für die weiteren Überlegungen dieser Arbeit ist daher festzuhalten, dass sich die Überlegungen der nachfolgenden Kapitel, die auf einzelne Wissenschaftsprogramme der Betriebswirtschaftslehre Bezug nehmen, im Rahmen der im vorigen Abschnitt abgesteckten Grenzen des Erfahrungsgegenstands bewegen müssen. Es ist also wirtschaftliches Handeln zu untersuchen, das in Betrieben stattfindet. Diese Grenzen lassen allerdings Raum für eine flexible Schwerpunktsetzung, die auf der Basis einer individuellen Präparierung des empirischen Konstrukts zu einem speziell auf das jeweilige Erkenntnisinteresse ausgerichteten theoretischen Konstrukt vorgenommen werden kann.

\footnotetext{
${ }^{230}$ Vgl. Chmielewicz (1994), S. 21.

${ }^{231} \mathrm{Vgl}$. Raffée (1993), S. 8.

${ }^{232} \mathrm{Vgl}$. zum Folgenden Raffée (1993), S. 5.

${ }^{233}$ Vgl. z.B. Schweitzer (1997), S. 47; Bleicher (1988), S. 114; Kosiol (1961), S. 131.

${ }^{234}$ Vgl. Bleicher (1988), S. 114.

${ }^{235}$ Eine verstärkte Erfahrungsobjektorientierung statt Erkenntnisobjektorientierung fordert auch Raffée (1974), S. 56.
} 


\subsubsection{Stellung der Betriebswirtschaftslehre gegenüber anderen wissenschaftlichen Disziplinen}

Ausgehend von der soeben vorgenommenen disziplinären Abgrenzung wird die Betriebswirtschaftslehre in diesem Abschnitt im Vergleich zu anderen Wissenschaften positioniert. Aus dieser Einordnung ergeben sich Vorgaben hinsichtlich der Ziele und der Methoden des Fachs, die sich auch auf die Analyse des Informationsmanagements in den nachfolgenden Kapiteln auswirken. Obwohl es nicht an Versuchen fehlt, Ordnung in die Vielfalt der Wissenschaften zu bringen, liegt keine allgemein akzeptierte Systematik vor, auf die an dieser Stelle zurückgegriffen werden könnte. ${ }^{236} \mathrm{Im}$ Folgenden wird auf die am weitesten verbreiteten Unterscheidungskriterien Bezug genommen, um den Standort des Faches zu bestimmen.

Da der Untersuchungsbereich der Betriebswirtschaftslehre selbst nicht zum Bereich der Wissenschaften gehört, handelt es sich nicht um eine Meta-Wissenschaft, sondern um eine Objektwissenschaft. ${ }^{237}$ Innerhalb der Objektwissenschaften wird in der Regel zwischen Realwissenschaften und Formalwissenschaften unterschieden. ${ }^{238}$ Dabei befassen sich die Realwissenschaften mit individuellen, raum-zeitlich feststellbaren Tatsachen, ${ }^{239}$ während die Formalwissenschaften sich mit „formalen“ Objekten auseinander setzen, die innerhalb wissenschaftlicher Sprachsysteme - z.B. logischmathematischer Kalküle - definiert sind. In jüngster Zeit gewinnt zudem die Kategorie der Strukturwissenschaften verstärkt an Bedeutung, die sich mit Strukturen befassen, die sich sowohl bei realen als auch bei formalen Objekten manifestieren können. ${ }^{240}$ Zwischen den verschiedenen Vertretern des Fachs besteht große Einigkeit darüber, dass die Betriebswirtschaftslehre der Kategorie der Realwissenschaften einzuordnen ist. $^{241}$

Die weitere Klassifizierung unterschiedlicher Disziplinen innerhalb der Realwissenschaften ist umstritten. Die Einteilung in Geistes- und Naturwissenschaften genießt zwar eine lange Tradition, sie hat sich jedoch - insbesondere für die als Geisteswissenschaften eingeordneten Fächer - als unfruchtbar herausgestellt. ${ }^{242}$ Die Unterscheidung beruht im Wesentlichen auf der Annahme, dass in Geistes- und Naturwissenschaften unterschiedliche Methoden verwendet werden. Demnach bedienen sich die Naturwissenschaften der Methode des Erklärens mit Hilfe von Gesetzen, während die Geisteswissenschaften auf die Methode des Beschreibens und des Verstehens (Hermeneutik) angewiesen sind. ${ }^{243}$ Tatsächlich streben jedoch auch zahlreiche Geisteswissenschaften die Aufstellung allgemeiner Gesetze an, ${ }^{244}$ sodass das zur Klassifikation verwendete Kriterium nicht tragfähig ist. Wegen dieser Schwierigkeiten erscheint es zweckmäßiger, innerhalb der Realwissenschaften nach dem Objektbereich zwischen Naturwissenschaften und Kulturwissenschaften zu unterscheiden. ${ }^{245}$ Die Kultur-

${ }^{236}$ Vgl. Weber (1993), S. 5.

${ }^{237}$ Vgl. Zelewski (1999), S. 5.

${ }^{238}$ Vgl. Raffée (1974), S. 21.

${ }^{239}$ Vgl. Schweitzer (1997), S. 27. Vgl. dazu auch die einleitenden Ausführungen in Abschnitt 2.2.1.

${ }^{240}$ Vgl. Zelewski (1999), S. 5.

${ }^{241}$ Vgl. z.B. Zelewski (1999), S. 5; Schweitzer (1997), S. 27; Stein (1993), Sp. 479; Raffée (1974), S. $22 \mathrm{f}$.

${ }^{242}$ Vgl. Zelewski (1999), S. 7.

${ }^{243}$ Vgl. Raffée (1974), S. 21.

${ }^{244}$ Vgl. Raffée (1974), S. 21.

${ }^{245}$ Vgl. Zelewski (1999), S. 7. 
wissenschaften, zu denen auch die Betriebswirtschaftslehre zu zählen ist, studieren die Verhaltensweisen von Menschen, die über rein biologische Verhaltensaspekte hinausreichen, sowie die Artefakte, die von Menschen künstlich erschaffen wurden. Dieser Einteilung, die für die Betriebswirtschaftslehre die Wahl der Methoden nicht von vornherein auf ein bestimmtes Repertoire einschränkt, wird hier gefolgt.

Da sowohl die Betriebswirtschaftslehre als auch die Volkswirtschaftslehre zum Gebiet der Wirtschaftswissenschaft, die sich in allgemeiner Abgrenzung mit wirtschaftichen Sachverhalten befasst, gehören, ${ }^{246}$ ist hier abschließend auf die Beziehung zwischen diesen beiden Disziplinen einzugehen. Sowohl die Betriebswirtschaftslehre als auch die Volkswirtschaftslehre untersuchen das Wirtschaften als speziellem Teilaspekt menschlichen Handelns. ${ }^{247}$ Die Unterschiede zwischen der Betriebswirtschaftslehre und der Volkswirtschaftslehre beruhen auf der Perspektive, aus der das Wirtschaftsgeschehen betrachtet wird. Die Volkswirtschaftslehre betrachtet wirtschaftliches Geschehen primär als Gesamtphänomen, als Wirkungszusammenhang aus einer totalen Perspektive heraus. ${ }^{248}$ Dagegen sieht die Betriebswirtschaftslehre wirtschaftliches Geschehen als gegliederten Komplex, als Zusammengesetztheit aus Wirtschaftseinheiten. Diese Abgrenzung entspricht der hier vorgenommenen Konzeptionalisierung der Betriebswirtschaftslehre als Einzelwirtschaftslehre.

Problematisch ist dabei allerdings, dass auch innerhalb der Volkswirtschaftslehre zwischen makro- und mikroökonomischen Fragestellungen unterschieden wird und im Rahmen der Mikroökonomie die Aktivitäten einzelner Wirtschaftseinheiten untersucht werden. Daraus ergeben sich Abgrenzungsprobleme zwischen der Mikroökonomie und der Betriebswirtschaftslehre. Diese Probleme sind Gegenstand einer langen Diskussion innerhalb der Wirtschaftswissenschaft, in der zum Teil vorgeschlagen wird, nicht mehr zwischen Mikroökonomie und Betriebswirtschaftslehre zu unterscheiden. ${ }^{249} \mathrm{Da}$ diese Diskussion hier nicht inhaltlich nachvollzogen werden kann, sei an dieser Stelle lediglich festgehalten, dass alle Probleme, die sich auf das wirtschaftliche Handeln in einzelnen Wirtschaftseinheiten beziehen, prinzipiell zum Gegenstand betriebswirtschaftlicher Analysen gemacht werden können. Die Untersuchung aggregierter Wirtschaftsbereiche ist dagegen eindeutig Gegenstand der Volkswirtschaftslehre.

Daraus ergeben sich folgende Schlussfolgerungen für die allgemeinen Erkenntnispotenziale der Betriebswirtschaftslehre bei der Analyse des Informationsmanagements: Wenn zur Überwindung der bestehenden Theoriedefizite auf Wissenschaftsprogramme der Betriebswirtschaftslehre Bezug genommen wird, dann erstrecken sich die Aussagen stets auf wirtschaftliche Probleme in einzelnen Betrieben. Das bedeutet einerseits, dass Probleme nicht-wirtschaftlicher (z.B. rein technischer, rein psychologischer, rein soziologischer) Art, die im Umfeld der technikunterstützten Informationsverarbeitung auftreten können, aus der Betrachtung ausgeschlossen sind. Es bedeutet andererseits, dass auch gesamtwirtschaftliche Fragestellungen, etwa die Veränderung von Branchenstrukturen durch den Einsatz moderner Informations- und Kommunikationstechnik oder deren Auswirkungen auf das Wirtschaftswachstum nicht in die Analyse einbezogen werden.

\footnotetext{
${ }^{246}$ Vgl. Kosiol (1961), S. 129.

${ }^{247}$ Vgl. Weber (1993), S. 1.

${ }^{248}$ Vgl. Kosiol (1961), S. 133

${ }^{249}$ Vgl. Raffée (1974), S. $24 f$.
} 


\subsubsection{Auswahl der in die Analyse des Informationsmanagements einbezogenen Wissenschaftsprogramme der Betriebswirtschafslehre}

Innerhalb der hier umrissenen fachlichen Identität, die gewissermaßen einen lockeren Rahmen für das betriebswirtschaftliche Forschen vorgibt, ${ }^{250}$ besteht Raum für eine vielschichtige Analyse mit unterschiedlichen Untersuchungsschwerpunkten. So sehr nämlich einerseits ein Minimalkonsens sinnvoll erscheint, so sehr sollte andererseits auch einem „gezähmten Pluralismus“ Raum gegeben werden. ${ }^{251}$ Dabei können verschiedene Wissenschaftsprogramme und ihre Vertreter in eine Ideenkonkurrenz treten, sodass ein flexibler Wettstreit um die leistungsfähigste Theorie des Wirtschaftens in Betrieben entsteht. ${ }^{252}$ Es dürfte auch dem wissenschaftlichen Fortschritt insgesamt nützen, wenn in Lehre und Forschung unterschiedliche Konzepte vertreten werden, die sich im Wettbewerb miteinander zu bewähren haben. ${ }^{253}$

Durch Ausrichtung der Forschungsbestrebungen auf einen bestimmten Teilaspekt des Erfahrungsbereichs sind nacheinander oder nebeneinander verschiedene Wissenschaftsprogramme der Betriebswirtschaftslehre entstanden. ${ }^{254}$ Bei diesen Wissenschaftsprogrammen handelt es sich selbst um vergleichsweise breit angelegte Entwürfe, die eine spezifische Einordnung von Einzelproblemen ermöglichen. ${ }^{255}$ Wie in Abschnitt 2.2.2 erläutert, wird jedes dieser Wissenschaftsprogramme durch eigene Erkenntnisinteressen sowie inhaltliche und methodische Leitideen konstituiert, die als Kerne bei der Theoriebildung fungieren. Diese Basisentscheidungen lassen sich disziplinimmanent nicht weiter rechtfertigen und stellen den normativen Rahmen dar, innerhalb dessen über zulässige und unzulässige wissenschaftliche Erkenntnisse geurteilt werden kann. ${ }^{256}$

Die durch die einzelnen Wissenschaftsprogramme aus der Gesamtheit des Erfahrungsbereichs ausgewählten Erkenntnisgegenstände können sich teilweise überdecken, aber auch sehr weitgehend unterscheiden. ${ }^{257}$ Die innere Spannweite des Fachs drückt sich in diesem Zusammenhang vor allem in der Orientierung an zwei divergierenden Polen aus. ${ }^{258}$ Die Untersuchung wirtschaftlichen Handelns konzentriert sich entweder auf nur einen einzigen - den ökonomischen - Aspekt. Dieses Vorgehen wird als „ökonomisches Basiskonzept ${ }^{\text {“259 }}$ bezeichnet und stellt das ökonomischen Rationalprinzip bei der Durchfuhrung wirtschaftlicher Handlungen in den Vordergrund der Betrachtung. Oder die Untersuchung zielt darauf $a b$, wirtschaftliches Handeln im mehrdimensionalen Zusammenhang menschlichen Verhaltens, das neben der ökonomischen weitere Dimensionen enthält, zu untersuchen. ${ }^{260}$ Eine derartige mehrdimensionale Analyse macht die Einbeziehung von Erkenntnissen anderer Wissenschaften und somit ein interdisziplinäres Arbeiten erforderlich. $\mathrm{Da}$ es sich dabei nicht

\footnotetext{
${ }^{250} \mathrm{Vgl}$. Bleicher (1988), S. 115.

${ }^{251}$ Vgl. Raffée (1993), S. 44.

${ }^{252}$ Vgl. Schanz (1997a), S. 81.

${ }^{253}$ Vgl. Raffée (1993), S. 44.

${ }^{254}$ Vgl. Stein (1993), Sp. 472.

${ }^{255}$ Vgl. Schanz (1997a), S. 82.

${ }^{256}$ Vgl. Zelewski (1999), S. 8. Dieser unterscheidet allerdings zwischen ontologischen, epistemologischen und methodologischen Basisentscheidungen und nicht, wie in dieser Arbeit, zwischen Erkenntnisinteressen sowie inhaltlichen und methodischen Leitideen. Vgl. Zelewski (1999), S. 9.

${ }^{257}$ Vgl. Stein (1993), Sp. 472.

${ }^{258}$ Vgl. Bleicher (1988), S. 111.

${ }^{259}$ Raffée (1993), S. 26.

${ }^{260}$ Vgl. Raffée (1993), S. 25.
} 
ausschließlich um sozialwissenschaftliche, sondern auch um naturwissenschaftliche oder formal- und strukturwissenschaftliche Erkenntnisse handeln kann, erscheint die in der Literatur vorgeschlagene Bezeichnung „,sozialwissenschaftliches Basiskonzept ${ }^{\text {“261 }}$ für die Antipode des ökonomischen Basiskonzepts zu eng. Hier wird daher der Begriff ,interdisziplinäres Basiskonzept" gewählt.

Die Ursache für die Kontroverse zwischen Vertretern des interdisziplinären und des ökonomischen Basiskonzepts geht letztlich auf die unterschiedliche Beurteilung der Leistungsfähigkeit eines engen, geschlossenen gegenüber der eines weiten, geöffneten Forschungsansatzes zurück. ${ }^{262}$ Vertreter des ökonomischen Basiskonzepts sind der Auffassung, dass nur eine strenge Begrenzung des Untersuchungsbereichs die notwendigen Spezialisierungs- und Vertiefungsmöglichkeiten bietet, um Erkenntnisfortschritt erfolgreich zu fördern. Dagegen sind Vertreter des interdisziplinären Basiskonzepts überzeugt, dass nur durch eine Öffnung der Betriebswirtschaftslehre empirisch tragfähiges Wissen erlangt werden kann.

Wegen der Vielfalt der vorliegenden Forschungsansätze kann in dieser Arbeit nicht auf jedes einzelne Wissenschaftsprogramm der Betriebswirtschaftslehre Bezug genommen werden, um ein vollständiges Bild des Informationsmanagements aus Sicht dieser Disziplin zu erhalten. Daher ist eine Beschränkung der Analyse erforderlich, die eine Auswahl der in den nächsten Kapiteln zu referenzierenden Wissenschaftsprogramme notwendig macht. Allerdings können die Grundkonzepte der verschiedenen bestehenden Forschungsansätze der Betriebswirtschaftslehre an dieser Stelle nicht im Detail inhaltlich erläutert und beurteilt werden. Die hier vorgenommene Selektion orientiert sich stattdessen an einer groben Abschätzung der Erkenntnispotenziale, die die einzelnen Wissenschaftsprogramme jeweils für Fragen des Informationsmanagements aufweisen.

In der Literatur besteht keine Einigkeit über eine Systematisierung der verschiedenen Forschungsrichtungen innerhalb der Betriebswirtschaftslehre. Die vorgeschlagenen Klassifikationen ${ }^{263}$ sind nicht nur unterschiedlich detailliert, sondern weichen zum Teil auch voneinander $\mathrm{ab}$. $\mathrm{Da}$ in den weiteren Kapiteln eine Bezugnahme auf Wissenschaftsprogramme der Betriebswirtschaftslehre als größere Forschungskomplexe angestrebt wird, ist eine zu detaillierte Aufgliederung an dieser Stelle nicht zweckmäßig. Wie gesehen, sind Wissenschaftsprogramme insgesamt sehr breit angelegt und lassen Raum für die Untersuchung unterschiedlicher Einzelprobleme, die aus dem jeweiligen Erkenntnisgegenstand des Ansatzes herausgehoben werden können. Mit der Ausarbeitung und Weiterentwicklung von Wissenschaftsprogrammen beschäftigt sich in der Regel eine kleinere oder größere Gemeinschaft von Wissenschaftlern. ${ }^{264}$ Einzelne Theorien sind daher an dieser Stelle nicht zu berücksichtigen. Die nachfolgenden Überlegungen orientieren sich deshalb an der von SCHANZ vorgeschlagenen Einteilung, die sich explizit auf gesamte Wissenschaftsprogramme der Betriebswirtschaftslehre bezieht. Als große Forschungslinien sind danach der Faktortheoretische Ansatz, der Entscheidungsorientierte Ansatz, der Systemorientierte Ansatz, der an der Neuen

\footnotetext{
${ }^{261}$ Raffée (1993), S. 26.

${ }^{262}$ Vgl. zum Folgenden Raffée (1993), S. 27.

${ }^{263}$ Vgl. z.B. Zelewski (1999), S. 10; Schanz (1997a), S. 99ff.; Stein (1993), Sp. 475ff.; Chmielewicz (1994), passim.

${ }^{264}$ Vgl. Schanz (1997a), S. $90 \mathrm{f}$.
} 
Institutionenökonomie orientierte Ansatz und der Verhaltenstheoretische Ansatz zu unterscheiden. ${ }^{265}$

Der Faktortheoretische Ansatz, der bis heute die betriebswirtschaftliche Forschung und Lehre stark beeinflusst, geht auf GUTENBERG zurück und wurde nach dem Zweiten Weltkrieg ausgearbeitet. ${ }^{266}$ Historisch gesehen handelt es sich um den ersten theoretisch geschlossenen Ansatz, der auf Grund seiner beträchtlichen intellektuellen Anziehungskraft zahlreiche Arbeiten inspirieren konnte, die zur Vervollständigung des Aussagesystems beigetragen haben. ${ }^{267}$ Obwohl insgesamt ein produktionstheoretischer Standpunkt eingenommen wird, betrachtet dieses Wissenschaftsprogramm auch den Absatz- und Finanzbereich von Betrieben und fügt diese drei Bereiche $\mathrm{zu}$ einem Gesamtkonzept zusammen. Der Faktortheoretische Ansatz wird insbesondere aus zwei Gründen in die weiteren Betrachtungen dieser Arbeit einbezogen: Er ist zum einen als vollständiges Konzept angelegt, das darauf abzielt, sämtliche Bereiche des betrieblichen Produktionsgeschehens zu erfassen. Tatsächlich konnten auf Basis der diesem Ansatz zu Grunde liegenden Leitideen unterschiedlichste Problemstellungen erfolgreich analysiert und gelöst werden, sodass auch von einer Untersuchung des Informationsmanagements aus der Perspektive dieses Ansatzes ein hohes Erkenntnispotenzial erwartet werden darf. Zum anderen liegen gerade zu Fragen des Informationsmanagements bisher nur wenige auf dem Faktortheoretischen Ansatz basierende Arbeiten vor, sodass der Ansatz, obwohl er nicht mehr ganz neu ist, immer noch Chancen zur Gewinnung echter neuer Erkenntnisse für diesen Bereich bietet.

Der Entscheidungsorientierte Ansatz, dessen Entwicklung auf HEINEN zurückgeht und Ende der 1960er Jahre begann, greift aus dem Erfahrungsbereich der Betriebswirtschaftslehre den Aspekt des Entscheidens als speziellen Erkenntnisgegenstand heraus. ${ }^{268}$ Innerhalb dieses Wissenschaftsprogramms sind eine entscheidungslogische und eine realtheoretische Variante zu unterscheiden. ${ }^{269}$ Während die entscheidungslogische Variante von einer unterstellten Zielfunktion sowie gegebenen einschränkenden Bedingungen ausgeht, untersucht die realtheoretische Variante das tatsächliche Entscheidungsverhalten von Wirtschaftssubjekten. Dieser Ansatz wird aus folgenden Gründen nicht mehr weiter in die Überlegungen dieser Arbeit einbezogen: Die beiden Varianten des Ansatzes sind so unterschiedlich angelegt, dass genau genommen nicht von einer geschlossenen Konzeption auf Basis gemeinsamer Erkenntnisinteressen und Leitideen gesprochen werden kann. Entscheidungslogische Fragen werden auch im Rahmen des Faktortheoretischen Ansatzes behandelt, sodass es sich nicht um ein wirklich neues Arbeitsgebiet der Betriebswirtschaftslehre handelt. Die realtheoretische Variante des Ansatzes wiederum greift US-amerikanische Vorarbeiten, insbesondere von SIMON, ${ }^{270}$ auf und leitete seinerzeit die verhaltenswissenschaftliche Öffnung der deutschsprachigen Betriebswirtschaftslehre ein. ${ }^{271}$ Der Entscheidungsorientierte Ansatz insgesamt geht jedoch nicht konsequent von dem oben angesprochenen interdisziplinären Basiskonzept aus. Die konzeptionelle Eigenständigkeit dieses Ansatzes als Wissenschaftsprogramm ist daher nicht klar erkennbar.

\footnotetext{
${ }^{265}$ Vgl. Schanz (1997a), S. $111 \mathrm{ff}$.

${ }^{266}$ Vgl. Stein (1993), Sp. 475.

${ }^{267}$ Vgl. Schanz (1997a), S. 112

${ }^{268}$ Vgl. Heinen (1969), S. 207ff.

${ }^{269}$ Vgl. Schanz (1997a), S. 120.

${ }^{270}$ Vgl. dazu Simon (1976), passim.

${ }^{271}$ Vgl. Schanz (1997a), S. $120 \mathrm{f}$.
} 
Der Systemorientierte Ansatz untersucht wirtschaftliches Handeln in Betrieben mit Hilfe von Ideen der allgemeinen Systemtheorie und der Kybernetik. ${ }^{272}$ Dieses Wissenschaftsprogramm ist stark interdisziplinär angelegt und zielt auf eine Öffnung der Betriebswirtschaftslehre ab. Auf der Basis einer allgemeinen Konzeptionalisierung von Betrieben als Systemen sollen die Erkenntnisse zahlreicher benachbarter Wissenschaftsdisziplinen für die Betriebswirtschaftslehre nutzbar gemacht werden. Dieses Wissenschaftsprogramm wird in die weiteren Untersuchungen dieser Arbeit einbezogen, da der Gestaltungsbereich des Informationsmanagements als System eines bestimmten Typs abgegrenzt wurde und sich somit eine Bezugnahme auf das Systemkonzept geradezu anbietet. Von einer Spezifizierung von allgemeinen Aussagen der Systemorientierten Betriebswirtschaftslehre sind daher große Erkenntnispotenziale zu erwarten. Diese Erkenntnisse dürften zudem einen hohen Neuigkeitsgrad aufweisen, da die vorliegenden Arbeiten zum Informationsmanagement bisher kaum auf die Systemtheorie, insbesondere auf deren neuere Variante, Bezug genommen haben. ${ }^{273}$

Der Ansatz der Neuen Institutionenökonomie stellt ein noch relativ junges Wissenschaftsprogramm der Betriebswirtschaftslehre dar, dessen Wurzeln in eher volkswirtschaftich ausgerichteten Arbeiten aus dem US-amerikanischen Raum liegen. ${ }^{274}$ Dieser Ansatz zielt darauf ab, das bis dahin bestehende institutionelle Vakuum der Betriebswirtschaftslehre zu überwinden und eine angemessenere Berücksichtigung empirisch gegebener Informations- und Interessensituationen bei der Bildung ökonomischer Modelle vorzunehmen. Die Neue Institutionenökonomie wird ebenfalls in dieser Arbeit aufgegriffen, da Informationsprobleme durch dieses Wissenschaftsprogramm explizit fokussiert werden. Somit besteht ein direkter Bezug zu Fragen des Informationsmanagements, dessen Gestaltungs- und Steuerungsmaßnahmen sich bei konkret vorliegenden Problemstellungen unmittelbar auf die modellmäßige Abbildung der Problemstellung auswirken können. Der Ansatz weist zudem auch deshalb besonders hohe Relevanz als Orientierungshilfe für das Informationsmanagement in der Praxis auf, weil immer weitere institutionelle Bereiche des Wirtschaftsgeschehens von dem fortschreitenden Einsatz von Informations- und Kommunikationstechniken betroffen sind.

Der Verhaltenstheoretische Ansatz setzt die interdisziplinäre Öffnung der Betriebswirtschaftslehre fort und strebt eine konsequente Integration sozialwissenschaftlicher Erkenntnisse an. Die Betriebswirtschaftslehre wird dabei als spezielle Sozialwissenschaft neben anderen Disziplinen wie der Soziologie, Sozialpsychologie usw. konzipiert. ${ }^{275}$ Wirtschaftliches Handeln soll aus dieser Perspektive nicht allein unter ökonomischen oder technischen Aspekten, sondern von der gesamten „menschlichen“ Seite betrachtet werden. Dieses Wissenschaftsprogramm wird nicht in die weiteren Überlegungen dieser Arbeit einbezogen. Dies geschieht insbesondere deshalb nicht, um nicht in die oben angesprochene Gefahr des Wissenschaftsdilettantismus, der mit dem Verzicht auf eine Spezialisierung des wissenschaftlichen Arbeitens verbunden ist, zu geraten. Der Verzicht auf eine differenziertere Analyse des menschlichen Verhaltens bedeutet allerdings keinesfalls, dass dessen grundsätzliche Relevanz für den

\footnotetext{
${ }^{272}$ Vgl. Stein (1993), Sp. 476.

${ }^{273}$ Ein Beispiel für eine systemtheoretisch fundierte Untersuchung des Informationsmanagements stellt die Arbeit von Streubel dar. Vgl. Streubel (2000), passim.

${ }^{274}$ Vgl. Schanz (1997a), S. 140.

${ }^{275}$ Vgl. Schanz (1997a), S. 152.
} 
Gestaltungsbereich des Informationsmanagements abgestritten wird. Es entspricht jedoch der in Abschnitt 2.2.1 erhobenen Forderung nach einer multiperspektivischen Analyse, wenn eine Untersuchung dieses Aspekts anderen, nicht-betriebswirtschaftlichen Ansätzen für eine Theorie des Informationsmanagements vorbehalten bleibt.

Damit bleibt festzuhalten, dass in den nachfolgenden Kapiteln auf den Faktortheoretischen Ansatz, den Systemorientierten Ansatz sowie auf die Neue Institutionenökonomie Bezug genommen wird, um betriebswirtschaftliche Ansätze für eine Theorie des Informationsmanagements zu entwickeln. Die dort entwickelten Ansätze gehen jeweils von dem als gemeinsamem Grundkonsens betriebswirtschaftlicher Wissenschaftsprogramme entwickelten Erfahrungsgegenstand aus und setzen mit ihren Überlegungen bei der Konstruktion des allgemeinen Erkenntnisgegenstands auf der zweiten Stufe des Theorieeinstiegs an. Anschließend wird das Informationsmanagement als spezieller Erkenntnisgegenstand des jeweiligen Ansatzes entwickelt. Im dritten Abschnitt der einzelnen Kapitel findet die eigentliche Theoriebildung statt, und im vierten Abschnitt erfolgt stets eine kritische Analyse der gewonnen Erkenntnisse sowie der Erkenntnisgrenzen des betreffenden Ansatzes für eine Theorie des Informationsmanagements. Dieses Vorgehen ist in Abbildung $2 / 6$ grafisch verdeutlicht. 


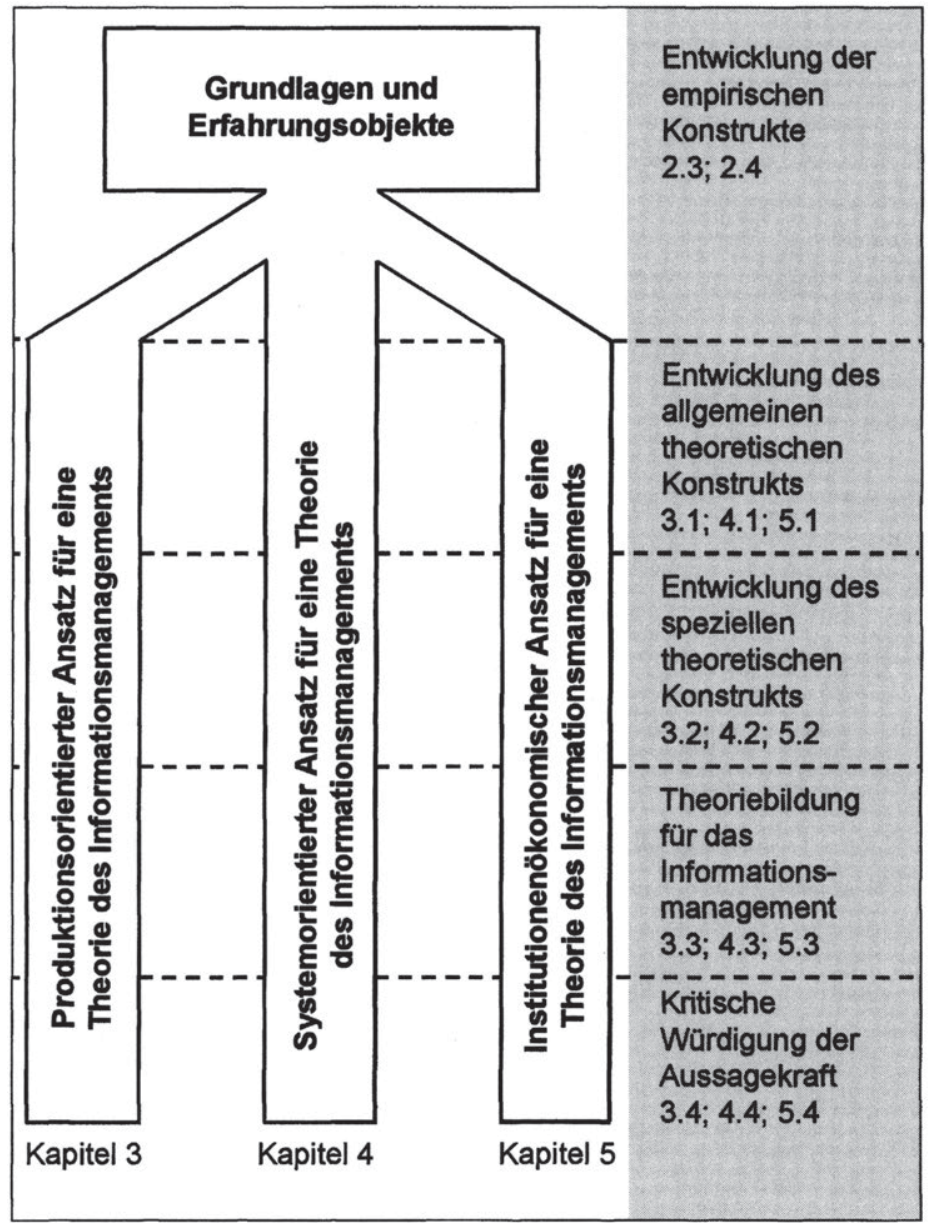

Abb. 2/6: Vorgehen bei der Entwicklung betriebswirtschaftlicher Ansätze für eine Theorie des Informationsmanagements 
Dirk Beier - 978-3-631-75500-6

Downloaded from PubFactory at 01/11/2019 04:07:38AM 


\section{Erkenntnispotenziale und Lösungsbeiträge des Faktor- theoretischen Ansatzes für das Informationsmanagement}

Die nachfolgende Untersuchung nimmt auf den Faktortheoretischen Ansatz als erstes Wissenschaftsprogramm der Betriebswirtschaftslehre Bezug, auf dessen Basis die im zweiten Kapitel festgestellten Theoriedefizite des Informationsmanagements überwunden werden können. Der Abschnitt 3.1 konstruiert den allgemeinen Erkenntnisgegenstand des Faktortheoretischen Ansatzes und enthält damit die zweite Stufe des Theorieeinstiegs, die auf der im zweiten Kapitel bewältigten ersten Stufe aufbaut. Die dritte Stufe des Theorieeinstiegs ist Gegenstand des Abschnitts 3.2, in dem das Informationsmanagement als spezielles Erkenntnisobjekt des Faktortheoretischen Ansatzes konstruiert wird. Nach Abschluss des Theorieeinstiegs nimmt Abschnitt 3.3 die eigentliche Theoriebildung für das Informationsmanagement vor. Es werden das Vorgehen und das angestrebte Ergebnis eines produktionsorientiert abgegrenzten Informationsmanagements untersucht. In Abschnitt 3.4 schließlich erfolgt eine kritische Würdigung der Aussagekraft des entwickelten Ansatzes für eine Theorie des Informationsmanagements. Diese bezieht sich einerseits auf die aus dem Ansatz ableitbaren Lösungsansätze für offene Problemstellungen in der Praxis und andererseits auf die Erkenntnisgrenzen des Ansatzes.

\subsection{Der Faktortheoretische Ansatz der Betriebswirtschafts/ehre}

Der von GUTENBERG in den 1950er, 1960er und 1970er Jahren entwickelte Faktortheoretische Ansatz stellte wissenschaftshistorisch gesehen ein erstes umfassendes und geschlossenes Gesamtkonzept betriebswirtschaftlicher Forschung und Lehre dar. ${ }^{1}$ Dieses Wissenschaftsprogramm sollte die Betriebswirtschaftslehre als eigenständige Disziplin mit eigenem Erkenntnisobjekt, nämlich der Produktivitätsbeziehung alternativer Faktorkombinationen, konzipieren. ${ }^{2}$ Es ist demnach dem ökonomischen Basiskonzept $^{3}$ zuzurechnen. Wegen seiner konzeptionellen Geschlossenheit und seines hohen heuristischen Potenzials fand dieses Wissenschaftsprogramm sowohl national als auch international große Anerkennung und dominierte über einen längeren Zeitraum das betriebswirtschaftliche Denken in Theorie und Praxis. Zwar ist die Dominanz des Faktortheoretischen Ansatzes mittlerweile überwunden, sein Einfluss auf die Arbeit betriebswirtschaftlicher Forscher ist aber immer noch erheblich, wenn er auch häufig nicht explizit zum Ausdruck gebracht wird. Aus diesem Grunde erscheint es inzwischen gerechtfertigt, GUTENBERG als Klassiker in zweierlei Sinne zu bezeichnen: Sein Werk gehört einerseits der Vergangenheit an, es bleibt andererseits aber Teil des geistigen Bezugssystems, das nachfolgenden (Forscher-)Generationen zur Überprüfung der Navigation auf ihrem eigenen Weg dienen kann. ${ }^{4}$

Die Untersuchungen in diesem Kapitel beziehen sich im Wesentlichen auf die dreibändigen „Grundlagen der Betriebswirtschaftslehre“, die die von GUTENBERG

1 Es sei darauf hingewiesen, dass Gutenberg selbst es abgelehnt hat, „schulebildend“ zu wirken. Für ihn war betriebswirtschaftliches Denken eine Einheit, von Paradigmata in der Betriebswirtschaftslehre hielt er nichts. Vgl. Albach (1988), S. 5.

2 Vgl. Ulrich/Hill (1979), S 171.

3 Zum ökonomischen Basiskonzept als Grundorientierung betriebswirtschaftlicher Wissenschaftsprogramme vgl. die Ausfuihrungen in Abschnitt 2.3.1.

4 Vgl. Dietz (1997), S. 1067. 
entwickelte ursprüngliche Konzeption des Faktortheoretischen Ansatzes enthalten. Die nahezu unüberschaubare Vielfalt weiterführender Arbeiten, die das Aussagesystem innerhalb des ursprünglichen Bezugsrahmens weiter ausdifferenzierten, wird nur insoweit berücksichtigt, als ein Zusammenhang zu Fragen der Informationsverarbeitung klar erkennbar ist oder eindeutig hergestellt werden kann.

\subsubsection{Erkenntnisinteressen des Faktortheoretischen Ansatzes}

Der Ausgangspunkt des Faktortheoretischen Ansatzes ist die Interpretation eines Betriebes als Einheit, die sich aus den Teilbereichen der Leistungserstellung, der Leistungsverwertung und der Finanzierung zusammensetzt ${ }^{5}$ und deren Ziel die Maximierung des erwirtschafteten Gewinns darstellt. GUTENBERG unterscheidet zwischen Betrieben und Unternehmungen. Als Betrieb bezeichnet er das wirtschaftssystemindifferente, „rein technische Gebilde“, mit dem die Faktorkombination nach dem Prinzip der Wirtschaftlichkeit vorgenommen wird. Unternehmungen dagegen stellen bei ihm Verknüpfungen wirtschaftssystem-indifferenter, genereller Merkmale von Betrieben (System produktiver Faktoren, Ökonomisches Prinzip) mit marktwirtschaftssystem-bezogenen Koordinationsmerkmalen (Autonomieprinzip, erwerbswirtschaftliches Prinzip, Alleinbestimmung) dar. ${ }^{6}$ GUTENBERG knüpft mit seinen Untersuchungen schwerpunktmäßig an die wirtschaftssystem-indifferenten Merkmale an. Darin schlägt sich das produktionsorientierte Erkenntnisinteresse seines Forschungsprogramms nieder, das von einem ,produktionstheoretischen Standpunkt ${ }^{47}$ ausgeht.

Zur Analyse dieses Untersuchungsgegenstandes überträgt GUTENBERG das in der volkswirtschaftlich ausgerichteten, neoklassischen Theorie verwendete methodische Instrumentarium, zu dessen Kern Partialanalysen und Grenzwertbetrachtungen gehören, auf einzelwirtschaftliche Fragestellungen. Seine originäre Leistung kann darin gesehen werden, dieses Instrumentarium in einer Weise zu benutzen, die der betriebswirtschaftlichen Problematik näher steht. ${ }^{8}$ Während die volkswirtschaftliche Blickrichtung sich auf eine einzige Umwandlungsbeziehung zwischen Beschaffungs- und Absatzmärkten beschränkt, erweitert die betriebswirtschaftliche Produktionsorientierung die Betrachtung auf eine Fülle einzelner Transformationsprozesse. ${ }^{9}$ Der Forschungskonzeption des Faktortheoretischen Ansatzes liegen die folgenden Postulate zu Grunde: ${ }^{10}$

1. Die Betriebswirtschaftslehre ist als ein wertfreies Forschungsunternehmen zu betreiben.

2. Es soll eine reine Theorie der Betriebswirtschaftslehre entwickelt werden. Praxisbezogene Forschung ist zwar grundsätzlich erwünscht, darf aber auf keinen Fall den theoretischen Bestrebungen im Wege stehen.

5 Vgl. Gutenberg (1980), S. 2.

Vgl. Gutenberg (1983), S. $510 \mathrm{ff}$.

Gutenberg (1956), S. 430.

Vgl. Schanz (1997a), S. 116.

Vgl. Schneider (1997), S. 12.

${ }^{10}$ Vgl. Jehle (1973), S. 170. 
3. Die betriebswirtschaftiche Forschung ist vorzugsweise im Sinne empirisch-realistischer Erkenntnisprogramme zu strukturieren.

4. Die Betriebswirtschaftslehre soll als eine nomologische, d.h. Gesetze formulierende, Wissenschaft verstanden werden.

5. Im Rahmen der betriebswirtschaftlichen Forschungsbemühungen soll in erster Linie die hypothetisch-deduktive Methode angewendet werden.

Aus diesen Postulaten geht hervor, dass der Faktortheoretische Ansatz als Wissenschaftsprogramm eindeutig an naturwissenschaftlichen Maßstäben ausgerichtet ist. ${ }^{11}$ Die Betriebswirtschaftslehre stellt sich hier als Wissenschaft dar, in der das Erklärungsziel unzweifelhaft im Vordergrund steht. Sie ist stark quantitativ auf die Untersuchung von Beziehungen zwischen Faktoreinsätzen und den damit erzielten Erträgen ausgerichtet. Ein wesentlicher Teil der auf dieser Konzeption basierenden Forschungsarbeiten hat die Analyse der Regeln und Gesetzmäßigkeiten, die dem Produktionsprozess zu Grunde liegen, zum Ziel. ${ }^{12}$

Diese Interpretation des GUTENBERG'schen Wissenschaftsprogramms ist allerdings nicht unumstritten, denn die tatsächlichen Forschungsaktivitäten innerhalb der skizzierten Grundkonzeption sind deutlich mehr anwendungsorientiert als es in den aufgeführten fünf Postulaten zum Ausdruck kommt. Das Interesse liegt nicht nur in der Beschreibung und Erklärung der betrieblichen Phänomene, sondern vielmehr auch in einer Analyse der Möglichkeiten, wie in der Realität vorgefundene Situationen im Sinne des Rationalprinzips verbessert und an sich ändernde Datenkonstellationen optimal angepasst werden können. ${ }^{13}$ Das Forschungsprogramm enthält daher eindeutig auch Elemente, die auf die Gestaltung der Realität abzielen. Allerdings liegt der Fokus im Vergleich zu den anderen in dieser Arbeit behandelten Wissenschaftsprogrammen, die die in Abschnitt 3.4.2 beschriebenen Erkenntnisgrenzen des Faktortheoretischen Ansatzes überwinden wollen, erheblich stärker auf erklärenden als auf gestaltenden Aspekten.

Der produktionstheoretische Standpunkt wird im Faktortheoretischen Ansatz um die Kostenorientierung und die Absatzorientierung ergänzt. Die auf der Produktionstheorie aufbauende Kostentheorie beschäftigt sich mit den durch alternative Faktorqualitäten, Faktoreinsatzmengen und Faktorpreise entstehenden Produktionskosten. ${ }^{14}$ Die Produktions- und Kostenorientierung des Faktortheoretischen Ansatzes dokumentiert sich in einem umfangreichen, zur Mathematisierung neigenden produktions- und kostentheoretischen Schriftum, dessen Ziel die Verfeinerung und Weiterentwicklung der GUTENBERG'schen Konzeption war. Die Absatztheorie stellte vor der Entwicklung des Faktortheoretischen Ansatzes ein nur wenig beachtetes Fachgebiet der Betriebswirtschaftslehre dar. ${ }^{15}$ Erst durch die Integration absatzwirtschaftlicher Betrachtungen entstand eine vollständige und geschlossene Konzeption einer Theorie der güterwirtschaftlichen Sphäre mit den Teilbereichen Leistungserstellung und Leistungsverwertung.

$11 \mathrm{Vgl}$. Ulrich/Hill (1979), S. 171.

12 Vgl. Raffée (1993), S. 30.

13 Vgl. Hundt (1977), S. 159.

14 Vgl. Raffée (1993), S. 30.

15 Vgl. Albach (1971), S. 138. 
Der güterwirtschaftlichen Seite von Betrieben steht die finanzwirtschaftliche Sphäre gegenüber. ${ }^{16}$ Der durch güterwirtschaftliche Gegebenheiten determinierte Kapitalbedarf wird durch einen Kapitalfonds gedeckt, der mit Hilfe der Kapitalmärkte gefuillt werden kann. Die finanzwirtschaftlichen Untersuchungen des Faktortheoretischen Ansatzes zielen auf die Herstellung eines finanziellen Gleichgewichtes ab, bei dem die Höhe des Kapitalbedarfes und des Kapitalfonds aufeinander abgestimmt sind. Abbildung 3/1 zeigt die angesprochenen Teilbereiche von Betrieben, die der Faktortheoretische Ansatz untersucht.

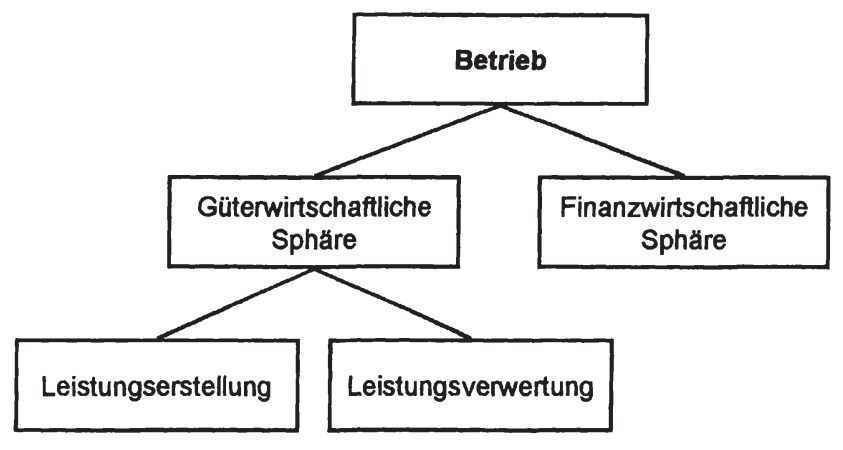

Abb. 3/1: Konzeptioneller Aufbau von Betrieben im Faktortheoretischen Ansatz

\subsubsection{Inhaltliche Leitideen des Faktortheoretischen Ansatzes}

Der Faktortheoretische Ansatz sucht nach den Grundlagen betrieblichen Handelns jenseits der Zeitläufe und politischen Systeme, also nach generellen Aussagen über allgemeine Gesetzmäßigkeiten in Betrieben. ${ }^{17} \mathrm{Zu}$ diesem Zweck wird die Unternehmung als Reaktionsgefüge aufgefasst, das ,auf Änderungen in den außerbetrieblichen Daten oder auf von der Unternehmung selbst initiierte Datenänderungen reagiert, derart, dass jeweils ein bestimmter Ausgangszustand [...] in einen anderen Zustand überführt wird." ${ }^{18}$ Dabei ist eine Prozessgestaltung zu realisieren, die angesichts der eingetretenen Lage als die Günstigste erscheint.

Zur Durchführung der verschiedenen Transformationsprozesse erfolgt in der Unternehmung eine Kombination von Produktionsfaktoren. Unter Produktionsfaktoren sind Güter zu verstehen, die in einem Produktionsprozess kombiniert werden, um andere Güter hervorzubringen. ${ }^{19}$ GUTENBERG entwickelt ein eigenes Klassifikationsschema für Produktionsfaktoren, in dem zwischen drei Arten von Elementarfaktoren und dem dispositiven Faktor als viertem Faktor unterschieden wird. ${ }^{20}$ Dieses Schema ist in Abbildung 3/2 grafisch dargestellt.

${ }^{16}$ Vgl. Gutenberg (1983), S. 12.

17 Vgl. Dietz (1997), S. 1068.

18 Vgl. Gutenberg (1984), S. 1159.

19 Vgl. Corsten (2000), S. 8.

${ }^{20}$ Vgl. Gutenberg (1958), S. 27; Gutenberg (1983), S. 3. 


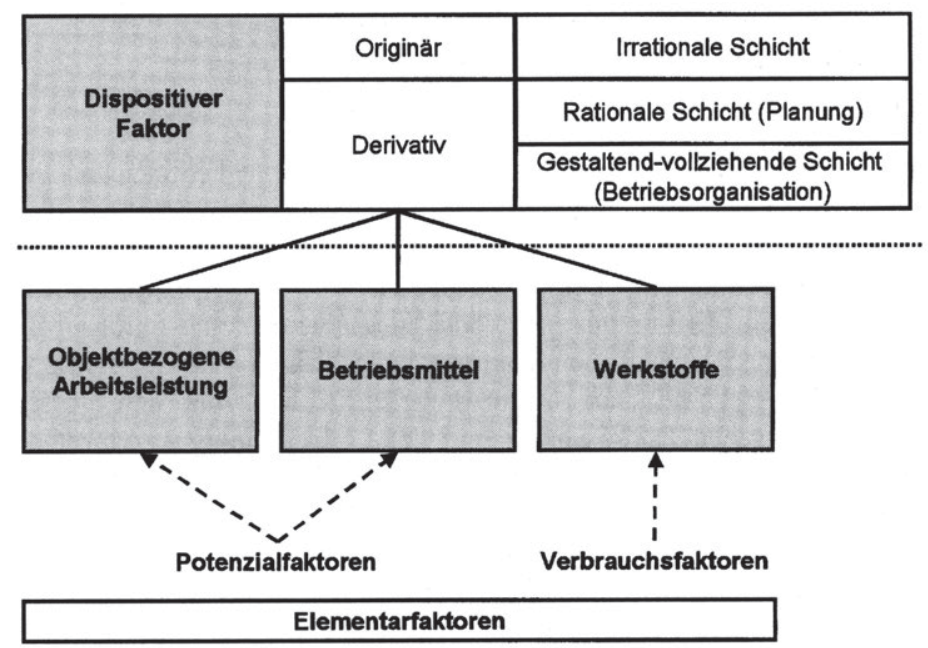

Abb. 3/2: Produktionsfaktorenschema des Faktortheoretischen Ansatzes

Bei den Elementarfaktoren handelt es sich um Werkstoffe, Arbeits- und Betriebsmittel sowie unmittelbar objektbezogene Arbeitsleistungen. Der Bereich der Werkstoffe umfasst Rohstoffe, Hilfsstoffe sowie Halb- und Fertigerzeugnisse, die als Grundmaterialien in die Herstellung der Enderzeugnisse einfließen. $\mathrm{Zu}$ den Arbeits- und Betriebsmitteln gehören Einrichtungen und Anlagen als diejenigen Sachmittel, die zur Leistungserstellung benutzt werden. Bei den objektbezogenen menschlichen Arbeitsleistungen kann es sich sowohl um geistige als auch um körperliche Tätigkeiten handeln, ${ }^{21}$ die sich im Rahmen der Produktion an den Werkstoffen vollziehen. Diese Elementarfaktoren werden durch die dispositiven Faktoren, bei denen es sich um die Geschäfts- und Betriebsleitung und von ihr autorisierte Führungsinstanzen handelt, in einem Kombinationsprozess miteinander verknüpft. Es ist darauf hinzuweisen, dass ein und dieselbe Arbeitskraft als Person zugleich sowohl objektbezogene als auch dispositive Arbeit - je nach hierarchischer Position in unterschiedlichen Relationen - erbringen kann. ${ }^{22}$ Vom originären dispositiven Faktor abgeleitet und weisungsgebunden sind die Planung und die Betriebsorganisation als derivative dispositive Faktoren. ${ }^{23}$

Bei den Elementarfaktoren objektbezogene menschliche Arbeitsleistungen sowie Arbeits- und Betriebsmittel handelt es sich um Potenzialfaktoren. ${ }^{24}$ Die Potenzialfaktoren stehen der Unternehmung über einen längeren Zeitraum zur Verfügung und wirken an der Produktion mit. Dies geschieht entweder durch die Abgabe von Werkverrichtungen oder durch statische Funktionen im Sinne einer „Ermöglichung des Betriebsgeschehens". ${ }^{25}$ Bei den Werkstoffen handelt es sich dagegen um Verbrauchsfaktoren. Verbrauchsfaktoren, die auch als Repetierfaktoren bezeichnet werden, gehen als selbstständige Güter im Produktionsgeschehen unter oder verändern

${ }^{21} \mathrm{Vgl}$. Nebl (1996), S. 49.

${ }^{22} \mathrm{Vgl}$. Kern (1992), S. 14.

${ }^{23}$ Vgl. Gutenberg (1983), S. $7 f$.

24 Vgl. Nebl (1996), S. 8.

${ }^{25}$ Vgl. Busse v. Colbe/Laßmann (1991), S. 80. 
ihre Eigenschaften im Produktionsprozess und werden dadurch zu Gütern anderer Art bzw. werden Bestandteil des produzierten Gutes. ${ }^{26}$ Sie werden für jeden Produktionsvorgang immer wieder neu beschafft und bereitgestellt.

Die Leistung der Elementarfaktoren stellt vom produktionstheoretischen Standpunkt aus gesehen immer zugleich eine Leistung der dispositiven Faktoren dar, da die dispositiven Faktoren die Elementarfaktoren miteinander kombinieren und somit über deren Ergiebigkeit bestimmen. ${ }^{27}$ Diese Ergiebigkeit wird in einer Produktionsfunktion abgebildet, die den Ertrag als Ergebnis des Faktorkombinationsprozesses in Abhängigkeit von den eingesetzten Faktormengen erfasst. Die Produktionsfunktion einer Unternehmung basiert auf Verbrauchsfunktionen, die den gesetzmäßigen Zusammenhang zwischen Faktoreinsatz und Faktorausbringung an einzelnen Aggregaten der Fertigung beschreiben. ${ }^{28}$ Verbrauchsfunktionen weisen spezifische Eigenschaften in Abhängigkeit von der konkreten Erscheinungsform des jeweiligen Produktionsprozesses auf. Dabei wird gegenüber der traditionellen Sichtweise in der mikroökonomischen Volkswirtschaftslehre die eingeschränkte oder fehlende Substituierbarkeit der einzelnen Produktionsfaktoren betont und der Blick auf limitationale Einsatzverhältnisse von Produktionsfaktoren gelenkt. ${ }^{29}$ Dazu stellt Gutenberg der traditionellen ertragsgesetzlichen Produktionsfunktion (Typ A) eine „betriebswirtschaftliche“ Produktionsfunktion (Typ B) gegenüber, bei der die Faktorproportionen nicht frei variierbar sind.

Aus der Produktionsfunktion wird die Kostenfunktion der Unternehmung abgeleitet, die neben der Umsatzfunktion die zweite Einflussgröße für die Höhe des erwirtschafteten Periodengewinnes darstellt. Insbesondere in der Verbindung von Produktionsund Kostentheorie hat der Faktortheoretische Ansatz wichtige neue Erkenntnisse geliefert und damit die Betriebswirtschaftslehre außerordentlich bereichert. ${ }^{30}$

Auf der Seite der Leistungsverwertung wird die Höhe des Umsatzes mit Hilfe absatztheoretischer Modelle analysiert. Diese beziehen im Hinblick auf den Einsatz eines „absatzpolitischen Instrumentariums“ (Vertriebspolitik, Preispolitik, Werbungspolitik und Produktpolitik) mehr Beobachtungssachverhalte ein als die angelsächsische Theorie der monopolistischen Konkurrenz zum Zeitpunkt der Entwicklung des Faktortheoretischen Ansatzes. ${ }^{31}$ In der Absatztheorie des Faktortheoretischen Ansatzes sind drei Ideen besonders hervorzuheben: ${ }^{32}$ Die Vorstellung einer doppelt geknickten PreisAbsatz-Funktion, die These des abnehmenden Grenzerfolgs der Werbung und die Idee des Produktlebenszyklus, die in der Folge eine Vielzahl weiterer Arbeiten angeregt hat.

Die Produktions- und Absatztheorie des Faktortheoretischen Ansatzes wird durch die Investitions- und Finanzierungstheorie zu einer geschlossenen betriebswirtschaftlichen Konzeption vervollständigt. Dabei bildet die Kapitalbedarfsfunktion den zukünftigen Kapitalbedarf in Abhängigkeit von der Zeit ab. Der Kapitalbedarf stellt die noch nicht durch Einnahmen gedeckten Ausgaben für die Produktion dar. Kapitalfondsprozesse

${ }^{26}$ Vgl. Busse v. Colbe/Laßmann (1991), S. 77.

${ }^{27}$ Vgl. Gutenberg (1983), S. 132.

${ }^{28}$ Vgl. Albach (1971), S. 136.

29 Vgl. Schneider (1997), S. 12.

${ }^{30} \mathrm{Vgl}$. Raffée (1993), S. 31.

${ }^{31}$ Vgl. Gutenberg (1979), S. 104ff. i.V.m. Schneider (1997), S. 13.

${ }^{32}$ Vgl. Albach (1971), S. 138. 
nehmen die in einer Periode angefallenen Einnahmenüberschüsse oder Ausgabenüberschüsse auf und decken einen eventuell anfallenden Kapitalbedarf durch Eigenoder Fremdkapital oder befriedigen mit dem Zahlungsüberschuss die Ansprüche von Eigen- und/oder Fremdkapitalgebern. Mit Hilfe der Finanzplanung ist das finanzielle Gleichgewicht unabhängig vom Wirtschaftssystem für jede Art von Unternehmen stets einzuhalten.

\subsubsection{Methodische Leitideen des Faktortheoretischen Ansatzes}

Das wichtigste methodische Merkmal des Faktortheoretischen Ansatzes liegt in der Orientierung an dem der Volkswirtschaftslehre entlehnten, neoklassischen Methodenarsenal. ${ }^{33}$ Aus diesem Grunde ist für eine Rekonstruktion der methodischen Leitideen auf die Leitideen des neoklassischen Wissenschaftsprogramms in der Volkswirtschaftslehre zurückzugreifen.

Die neoklassische Theorie übertrug Erkenntnisse aus der Physik, die etwa in der Mitte des 19. Jahrhunderts entstanden sind, in einem bildhaften Vergleich auf die Untersuchung von ökonomischen Sachverhalten. ${ }^{34}$ Dabei fand eine Analogiebildung statt, in deren Rahmen Konzepte wie „Präferenzen“ oder „Nutzen" methodisch in gleicher Weise wie potenzielle Energie behandelt wurden. Es wurde außerdem angenommen, dass sich die untersuchten Systeme in einer Weise verhalten, die eine analoge Anwendung der Gesetze der Mechanik erlaube. Die neoklassische Theorie hat daher eine lange Geschichte der Imitation verschiedener Teildisziplinen der Physik. ${ }^{35}$ Eine zentrale Stellung nehmen in diesem Zusammenhang Gleichgewichte ein, die als grundlegend für die ökonomische Analyse angesehen werden. ${ }^{36}$ Ein Gleichgewicht bezeichnet einen Ruhezustand, der durch das Einwirken von Kräften und Gegenkräften zustande gekommen ist. ${ }^{37}$ Ein ökonomisches System befindet sich zwar nicht zu jedem Zeitpunkt im Gleichgewicht, es strebt aber nach der neoklassischen Vorstellung durch das Zusammenwirken der verschiedenen Kräfte und Gegenkräfte immer auf ein Gleichgewicht zu. Die in der Physik vorgenommene Unterscheidung von stabilen, indifferenten und labilen Gleichgewichten wird allerdings nicht auf die neoklassische Theorie übertragen. Für die ökonomische Analyse wird vielmehr gefordert, dass ein Gleichgewicht stabil sein muss, wenn es ökonomische Bedeutung haben soll. ${ }^{38}$

Die Gleichgewichtsanalyse ökonomischer Systeme geht daher mit einer Stabilitätsanalyse einher, die die Bedingungen für ein stabiles Gleichgewicht aufzeigt. In der betriebswirtschaftlichen Theorie der Unternehmung kommt die Vorstellung vom stabilen Gleichgewicht vor allem in der Annahme über den Zustand von Märkten (Gleichgewicht von Angebot und Nachfrage), als Planungsgleichgewicht (Abgestimmtheit von Absatz-, Produktions-, Beschaffungs- und Finanzierungshandlungen) sowie als

\footnotetext{
${ }^{33} \mathrm{Vgl}$. Schanz (1997b), S. 556.

${ }^{34} \mathrm{Vgl}$. Mirowski (1990), S. 290.

35 Vgl. Mirowski (1990), S. 290.

${ }^{36}$ Vgl. Felderer/Homburg (1991), S. $12 f$.

37 Vgl. Schneider (1997), S. 7.

${ }^{38}$ Vgl. Felderer/Homburg (1991), S. 13f.
} 
finanzielles Gleichgewicht (Gleichheit von Ein- und Auszahlungen in einer Periode) zum Ausdruck. $^{39}$

Die Einführung von Grenzwertbetrachtungen zur Untersuchung stabiler Gleichgewichte stellt die in methodischer Hinsicht bedeutsamste Innovation der neoklassischen Theorie gegenüber ihren Vorläufern dar. Diese Methodik wird auch als Marginalismus oder als marginalistischer Ansatz bezeichnet. ${ }^{40}$ In einer Grenzwertbetrachtung wird in Form einer Partialanalyse die Auswirkung einer marginal kleinen Änderung einer oder mehrerer Einflussgrößen auf das untersuchte System betrachtet. Es wird also nur ein Teil der in einem Modell erfassten Variablen variiert, während alle anderen betrachteten Variablen mit Hilfe einer Ceteris-Paribus-Bedingung definitionsgemäß als konstant angenommen werden. Ein Beispiel dafür, wie ein solches Vorgehen im Rahmen des Faktortheoretischen Ansatzes zum Ausdruck kommt, stellt die partielle Faktorvariation in der Produktionstheorie dar. Dabei werden im betrachteten Produktionsprozess die Einsatzmengen mindestens einer, nicht aber aller Faktorarten geändert und die Auswirkungen auf die Ausbringungsmenge untersucht. ${ }^{41}$

Die Durchführung von Partial- und Grenzwertbetrachtungen entspricht der in der traditionellen NEWTON'schen Physik üblichen Methode, Systeme zur Untersuchung in ihre verschiedenen Einzelteile zu zerlegen. ${ }^{42}$ Im Rahmen einer solchen, reduktionistisch ausgerichteten Vorgehensweise werden schlecht zu fassende, komplizierte Systeme lokal betrachtet, um die in ihnen wirkenden Gesetzmäßigkeiten $\mathrm{zu}$ isolieren und $\mathrm{zu}$ erklären. Anschließend, so die Erwartung, können die gewonnenen Erkenntnisse wieder integriert und zu einer Erklärung des Gesamtsystems zusammengefügt werden. Diesem grundsätzlichen Schema entspricht in der neoklassischen Theorie und dem auf ihr basierenden Faktortheoretischen Ansatz die der Theorienbildung vorgelagerte Entscheidung, bei der Modellierung des Geschehens in einer Unternehmung die einzelne Person als Bezugspunkt zu nehmen. Wenn institutionale Strukturen und institutionales Handeln in einer Unternehmung beschrieben, erklärt und gestaltet werden sollen, so ist prinzipiell auf die Eigenschaften und das Verhalten der diese Institutionen konstituierenden Personen einzugehen. ${ }^{43}$ Hinter dieser, als methodologischer Individualismus ${ }^{44}$ bezeichneten Vor-Entscheidung, verbirgt sich die Auffassung, dass das Verhalten des gesamten Systems auf der Grundlage eines Verständnisses des Verhaltens der einzelnen Beteiligten erklärt und gestaltet werden kann. Der methodologische Individualismus steht demnach im Einklang mit dem der NEWTON'schen Physik zu Grunde liegenden reduktionistischen Weltbild und kann direkt aus diesem abgeleitet werden.

Bei GUTENBERG selbst finden sich kaum Hinweise auf das seinen Untersuchungen zu Grunde liegende Menschenbild. ${ }^{45}$ Auf Grund der Tatsache, dass das neoklassische Instrumentarium verwendet und auf die Betriebswirtschaftslehre übertragen wird, lässt sich dieses allerdings recht gut rekonstruieren. Die neoklassische Volkswirtschaftslehre arbeitet mit der Vorstellung eines repräsentativen, vollkommen rational handelnden

${ }^{39} \mathrm{Vgl}$. Schneider (1997), S. 7.

40 Vgl. Felderer/Homburg (1991), S. 25.

41 Vgl. Busse v. Colbe/Laßmann (1991), S 110.

${ }^{42}$ Vgl. Bertalanffy (1950), S. 134.

43 Vgl. Raffée (1993), S. 7.

${ }^{44}$ Rosen/Windisch (1997), S. 14; Blankart (1998), S. 10.

${ }^{45}$ Vgl. Schanz (1997a), S. 117. 
Wirtschaftssubjekts, das dort als Homo Oeconomicus bezeichnet wird. ${ }^{46}$ Dieses Wirtschaftssubjekt stellt eine Art Elementarteilchen in einem ökonomischen System dar, dessen Verhalten in vergleichbarer Weise vorhergesagt werden kann, wie das der Elementarteilchen in der Physik. Das Verhalten des Homo Oeconomicus ist durch die Maximierung seines Nutzens unter konsequenter Beachtung des ökonomischen Rationalprinzips bestimmt. Aus diesem Grund verzichtet der Faktortheoretische Ansatz auf eine Einbeziehung verhaltenswissenschaftlicher Erkenntnisse. ${ }^{47}$ Vielmehr wird - wenn auch zumeist implizit - ein vollkommenes Funktionieren der Menschen im Produktionsprozess unterstellt. ${ }^{48}$

\subsection{Konstruktion des Informationsmanagements als Erkenntnisgegen- stand aus der Perspektive des Faktortheoretischen Ansatzes}

In diesem Abschnitt wird das Informationsmanagement als spezielles theoretisches Konstrukt aus der Perspektive des Faktortheoretischen Ansatzes entwickelt. Dabei handelt es sich um die dritte und letzte Stufe des im zweiten Kapitel erläuterten dreistufigen Vorgehens zum Theorieeinstieg für das Informationsmanagement. Abschnitt 3.2.1 nimmt zunächst eine Einordnung des Wirtschaftsguts Information in das Analyseschema des Faktortheoretischen Ansatzes vor. Im Gegensatz zu bisherigen Konzepten zur Einbeziehung dieses Wirtschaftsguts in den Faktortheoretischen Ansatz wird Information hier nicht nur als Produktionsfaktor, sondern auch als Produktionsergebnis aufgefasst, das durch die Kombination geeigneter Elementarfaktoren hergestellt wird. Abschnitt 3.2.2 betrachtet das Produktionsfaktorsystem als Kern des Faktortheoretischen Ansatzes näher und erläutert die bei der Herstellung von Informationen verwendeten Produktionsfaktoren. Das Informationsmanagement selbst stellt aus dieser Perspektive einen Teil der dispositiven Faktoren dar. Abschnitt 3.2.3 vervollständigt das Bild und geht auf die Bedeutung von Informationen im Bereich der Leistungsverwertung und der finanziellen Sphäre von Unternehmungen ein.

\subsubsection{Information als Produktionsfaktor und als Produktionsergebnis}

Vor einer Anwendung der Grundgedanken des Faktortheoretischen Ansatzes auf Fragestellungen des Informationsmanagements ist zunächst anzumerken, dass sich das Erkenntnisinteresse GUTENBERGS in erster Linie auf Vorgänge der industriellen Fertigung richtete. ${ }^{49}$ Dabei handelt es sich aus heutiger Sicht um einen Spezialfall, der jedoch zum Zeitpunkt der Entwicklung des Faktortheoretischen Ansatzes und während seiner Blütephase das typische Anwendungsgebiet betriebswirtschaftlicher Analysen in Theorie und Praxis darstellte. Die technikunterstützte Informationsverarbeitung in Unternehmungen gehört also nicht zum originären Untersuchungsbereich dieses betriebswirtschaftlichen Wissenschaftsprogramms.

Aus dieser Tatsache könnte nun die Schlussfolgerung gezogen werden, dass der Faktortheoretische Ansatz auf Grund seines Erkenntnisinteresses gänzlich ungeeignet dazu ist, mit Hilfe seiner inhaltlichen und methodischen Leitideen Aussagen über die

\footnotetext{
${ }^{46}$ Vgl. Kirchgässner (2000), S. 2.

${ }^{47}$ Vgl. Raffée (1993), S. 31.

48 Vgl. Albach (1986), S. 589.

${ }^{49}$ Vgl. Gutenberg (1983), S. 8; Altenburger (1979), S. 863.
} 
technikunterstützte Informationsverarbeitung zu gewinnen. Tatsächlich kommen ältere Arbeiten, die sich mit der Beziehung zwischen der Informationsverarbeitung und den Erkenntnissen des Faktortheoretischen Ansatzes auseinander setzen, zu dem Schluss, das vorliegende Instrumentarium könne nicht zur Analyse von Prozessen der Informationsverarbeitung verwendet werden. Dies wird insbesondere mit Messproblemen begründet, die bei der Erfassung von Informationen und geistigen Arbeitsleistungen als zentralen Bestandteilen des Input und Output von Prozessen der Informationsverarbeitung bestehen. ${ }^{50}$ Ferner wird argumentiert, die Veränderlichkeit von Prozessen der Informationsverarbeitung stehe einer quantitativen Disposition und einer analogen Anwendung von Modellen der Produktionstheorie im Weg. ${ }^{51}$

Da Informationen jedes wirtschaftliche Handeln und Entscheiden zentral prägen, ${ }^{52}$ ist ihre fehlende explizite Betrachtung in der traditionellen Produktionstheorie jedoch unbefriedigend. Obwohl die zentrale Relevanz von Informationen für das Wirtschaftsgeschehen zwischenzeitlich allgemein erkannt wurde und Informationen in zahlreichen betriebswirtschaftlichen Arbeiten untersucht wurden, finden sich nach wie vor nur wenige produktionstheoretisch fundierte Beiträge zu ihrer Analyse. ${ }^{53}$ Verschiedentlich wird gefordert, der gestiegenen Bedeutung von Information dadurch Rechnung zu tragen, dass diese explizit als Produktionsfaktor berücksichtigt wird ${ }^{54}$ Die Notwendigkeit, Informationen zu den Produktionsfaktoren zu zählen, ist mittlerweile kaum noch umstritten, ihre Stellung im Faktorensystem und ihre Abgrenzung zu anderen Faktoren sind dagegen nach wie vor weitgehend ungeklärt. ${ }^{55}$

Grundsätzlich bieten sich zwei Alternativen an, um Informationen in das bestehende Produktionsfaktorsystem aufzunehmen. ${ }^{56}$ Einerseits ist vorstellbar, Information als Produktionsfaktor in die vorhandenen Kategorien einzufuigen und keine neue Faktorkategorie in das bestehende Faktorsystem einzubauen. Andererseits könnte Information eigenständig als Produktionsfaktorkategorie sui generis neben die bestehenden Faktorkategorien treten und das bisher bestehende Faktorsystem ergänzen. Beide Wege sind in der jüngeren Vergangenheit beschritten worden und unterscheiden sich im Ergebnis nicht wesentlich. Informationen lassen sich wegen ihrer vielfältigen Erscheinungsformen nicht einer einzigen Faktorkategorie generell zuordnen. Mit dieser Feststellung kann sowohl die Schaffung einer eigenen Kategorie, die gewissermaßen als Restposten alle Arten von Informationen enthält, ${ }^{57}$ als auch die jeweils im Einzelfall vorzunehmende individuelle Zuordnung einer Information zu einer bestimmten bereits bestehenden Faktorkategorie ${ }^{58}$ begründet werden. Die letztgenannte Variante ermöglicht eine etwas differenziertere Untersuchung von Informationen, da bei einer fallweisen Zuordnung zu Faktorkategorien die spezifischen Merkmale einer Information im Einzelfall berücksichtigt werden können. Entscheidend ist jedoch, dass beide Alternativen eine Überwindung der Gefahr, durch die nur implizite Einbeziehung von

${ }^{50} \mathrm{Vgl}$. Schweitzer/Küpper (1974), S. 45; Rehberg (1973), S. 86ff.

51 Vgl. Wild (1971), S. 319.

52 Vgl. Schwarze (1998), S. 30.

53 Vil. Bode (1993), S. 27.

${ }^{54}$ Vgl. z.B. Kern (1992), S. 15f.; Schwarze (1998), S. $29 f$

${ }^{55}$ Vgl. Bode/Zelewski (1992), S. 600.

56 Vgl. Streubel (1996), S. 35.

${ }^{57}$ Für dieses Vorgehen vgl. z.B. Kern (1992), S. 15ff.

${ }^{58}$ Für dieses Vorgehen vgl. z.B. Corsten (1990), S. 102; Busse v. Colbe/Laßmann (1991), S. 83; Bode (1993), S. 77ff.; Streubel (1996), S. 35ff. 
Informationen deren Bedeutung im Produktionsprozess zu vernachlässigen, möglich machen.

Die Anerkennung der Produktionsfaktoreigenschaft stellt einen bedeutenden Schritt zu einem wirtschaftlichen Umgang mit Informationen bei der Herstellung jeder Form von Produkten dar. Damit werden die Voraussetzungen für eine systematische Auseinandersetzung mit der zielgerichteten Nutzung von Informationen in den verschiedenen Unternehmungsbereichen geschaffen. Bisher ist allerdings zu beobachten, dass sich die Einbeziehung von Informationen weitgehend auf die soeben beschriebene Input-Seite der Leistungserstellung beschränkt. Für die Zwecke des Informationsmanagements stellt dies jedoch eine unzureichende Verkürzung dar, da bei diesem Vorgehen der Zugang zu einer Untersuchung des Wirtschaftlichkeitsprinzips im Bereich der technikunterstützten Informationsverarbeitung selbst verwehrt bleibt. Die Einengung der Betrachtung auf die Faktorseite ist deshalb für die Untersuchungsziele, die mit einem Produktionsorientierten Ansatz für eine Theorie des Informationsmanagements verfolgt werden, nicht adäquat. Vielmehr ist zusätzlich darauf abzustellen, dass die Leistungsergebnisse im Gestaltungs- und Steuerungsbereich des Informationsmanagements ebenfalls Informationen darstellen. Die Untersuchung ist also auf die Output-Seite von Produktionsprozessen auszudehnen.

Die Vielfältigkeit der Erscheinungsformen von Informationen bedingt, dass auch auf der Output-Seite allgemeine Aussagen über den Charakter informationeller Produkte nur auf sehr abstraktem Niveau getroffen werden können. In der Literatur wird häufig der von CORSTEN entwickelten Klassifikation gefolgt, nach der Informationen zu den immateriellen Realgütern gehören. Diese Klassifikation ist in Abbildung 3/3 grafisch dargestellt. Problematisch ist dabei allerdings, dass die Beziehung zwischen Informationen und Dienstleistungen unklar bleibt, da Informationen häufig Gegenstand und Ergebnis eines Dienstleistungsprozesses sind. ${ }^{59}$ Ferner sind Informationen nicht rein immateriell, sondern stets an ein materielles Trägermedium gebunden. ${ }^{60}$

${ }^{59}$ Vgl. Bode (1993), S. 64.

${ }^{60} \mathrm{Vgl}$. dazu die Ausführungen in Abschnitt 2.3.1.4, die zwischen einer materiellen und einer immateriellen Ebene von Informationen unterscheiden. 


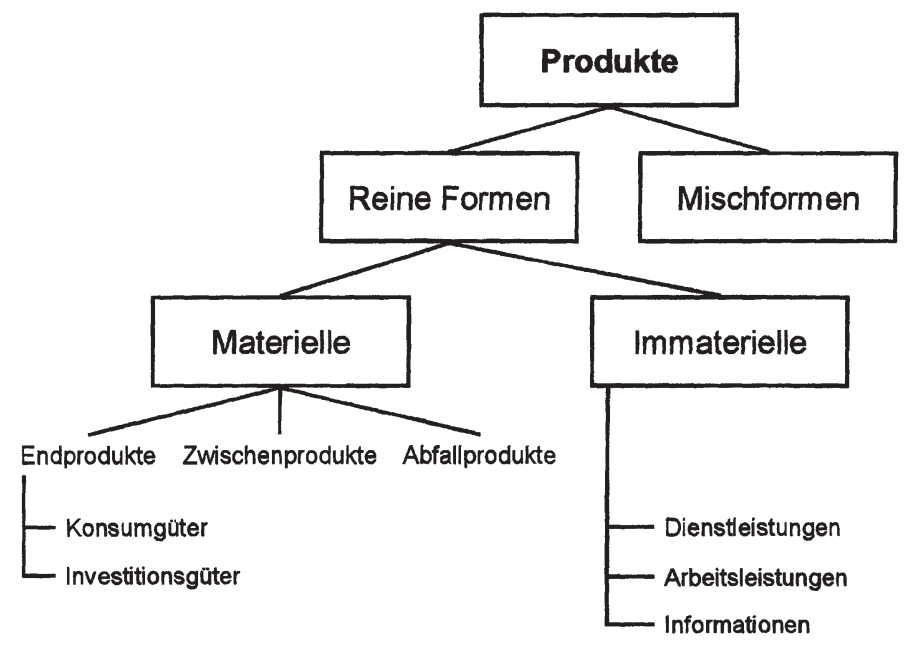

Abb. 3/3: Systematisierung von Produkten

Quelle: Corsten (2000), S. 12.

BODE schlägt vor, der Herstellung von Informationen begrifflich eine eigene Betriebsform zuzuordnen, die er als Informationsbetrieb bezeichnet. Informationsbetriebe stellen danach eine spezielle Ausprägung von Betrieben dar, die als Hauptprodukt Informationen produziert. ${ }^{61}$ Dieser Auffassung wird hier aus folgenden Gründen nicht gefolgt: Im zweiten Kapitel wurde erläutert, dass zwischen unterschiedlichen Verwendungszwecken von Informationen zu unterscheiden ist. Die produzierten Informationen können einerseits als Bestandteil in die Produkte eingehen. In diesem Fall erfolgt die Verwendung des Leistungsergebnisses unternehmungsextern durch die Kunden. Dabei kann es sich zum Beispiel um Beratungsleistungen, Nachrichtendienste oder Standardsoftware-Module handeln. Andererseits findet eine unternehmungsinterne Nutzung von Informationen zur Koordination arbeitsteiliger Prozesse sowie zur Verbesserung von Entscheidungen mit dem Ziel der Erreichung von Wettbewerbsvorteilen, d.h. für analytische Zwecke statt. In diesen Fällen findet die Informationserzeugung für den Eigengebrauch statt. $^{62}$ Dabei kann es sich etwa um logistische Planungen, Kostenanalysen oder um Koordinationsleistungen bei der Erfüllung individueller Kundenaufträge handeln. Die Erstellung und Verwendung unternehmungsintern genutzter Informationen begleitet in diesem Fall die Produktion der unternehmungsextern abgesetzten Güter. Durch die vorgeschlagene begriffliche Abgrenzung von Informationsbetrieben wird demnach nur ein Teil der Informationsverarbeitung in der Unternehmung erfasst. Eine Einschränkung der Betrachtung auf spezielle Abteilungen einer Unternehmung erscheint jedoch für die Zwecke des Informationsmanagements nicht adäquat.

Eine umfassende Analyse der Informationserzeugung aus Sicht der Produktionstheorie existiert bisher nicht. ${ }^{63}$ Aus produktionstheoretischer Perspektive findet in

$61 \mathrm{Vgl}$. Bode (1993), S. 31.

62 Vgl. ähnlich Bode (1993), S. 33.

${ }^{63} \mathrm{Vgl}$. Bode (1993), S. 30. 
Prozessen der Informationsverarbeitung eine Kombination von Elementarfaktoren statt, bei der Input-Informationen zu Output-Informationen umgewandelt werden. ${ }^{64}$ Prozesse der Informationsverarbeitung sind also von der Struktur her vergleichbar mit industriellen Fertigungsprozessen, in denen gleichfalls Input-Güter durch objektbezogene Tätigkeiten in Output-Güter transformiert werden. Wegen dieser strukturellen Ähnlichkeit ist grundsätzlich eine analoge Anwendung von bestehenden produktionstheoretischen Erkenntnissen auf den Bereich der Informationsverarbeitung vorstellbar. Dies steht im Einklang mit der programmatischen Aussage GUTENBERGs, das Ziel des Faktortheoretischen Ansatzes sei es, ,alle[n] Fragen, die die Grundlagen der Betriebswirtschaftslehre umschließen, eine erschöpfende systematische Behandlung " ${ }^{465}$ zukommen zu lassen. Das Betrachtungsfeld erstrecke sich nicht nur auf die Gewinnung von Rohstoffen und die Produktion von industriellen Fabrikaten, sondern es schließe die Herstellung von nicht-industriellen Produkten ein. ${ }^{66}$ Im Vorwort zur zehnten Auflage des ersten Bandes weist GUTENBERG darauf hin, die Grundkonzeption habe sich ,als so elastisch erwiesen, dass sich auch die neuesten Entwicklungen auf dem Gebiet der Betriebswirtschaftslehre ohne weiteres in [die] Systematik einfügten “67. Tatsächlich konnten immer wieder neue Erkenntnisse in das Aussagesystem integriert werden, ohne dabei von seiner grundsätzlichen Ausrichtung abzugehen. ${ }^{68}$

Die strukturelle Ähnlichkeit zwischen industriellen Fertigungsprozessen und Prozessen der Informationsverarbeitung ist allein allerdings noch keine hinreichende Voraussetzung für eine analoge Anwendung des produktionstheoretischen Instrumentariums. Die Anwendbarkeit hängt vielmehr davon ab, ob die produktionstheoretischen Modelle, die zur Untersuchung der industriellen Fertigung formuliert wurden, auch die Herstellung informationeller Produkte adäquat abbilden. Dabei kommt es darauf an, ob die durch die Modelle vorgenommenen Abstraktionen in gleichem, in geringerem oder in größerem Ausmaß ins Gewicht fallen, als dies bei industriellen Fertigungsprozessen der Fall ist. ${ }^{69}$ Wenn eine gleiche oder geringere Bedeutung der jeweiligen Abstraktionen gegeben ist, dann kann von der Anwendbarkeit des produktionstheoretischen Instrumentariums auf Prozesse der Informationsverarbeitung ausgegangen werden. Bei den weiteren Überlegungen dieses Kapitels wird deshalb stets überprüft, ob die allgemeinen Annahmen, die den jeweiligen Untersuchungen des Faktortheoretischen Ansatzes zu Grunde liegen, auch für Prozesse der Informationsverarbeitung zutreffen. Dabei sind insbesondere die oben angesprochenen Vorbehalte

\footnotetext{
${ }_{64}^{64}$ Vgl. ähnlich Schwarze (1998), S. 34.

${ }^{65}$ Gutenberg (1979), S. VI.

${ }^{66} \mathrm{Vgl}$. Gutenberg (1983), S. 1.

${ }^{67}$ Gutenberg (1983), S. VII.

${ }^{68}$ Vgl. Dietz (1997), S. 1066; Lücke (1997), S. 1249; Steven (1998), S. V, die sich exemplarisch auf den Bereich des Umweltschutzes bezieht und diesen durch eine Erweiterung der bestehenden Modelle berücksichtigt.

${ }^{69} \mathrm{Vgl}$. Altenburger (1980), S. 50, der sich mit dieser Aussage auf die Produktion von Dienstleistungen bezieht. Altenburger spricht allerdings von „Diskrepanz zwischen betriebswirtschaftlicher Produktionstheorie und Realität“. Die Aussage ist in dieser Form nicht mit der in Kapitel 2 vertretenen wissenschaftstheoretischen Position, dass das Erfahrungsobjekt einer Wissenschaft nach radikal-konstruktivistischer Auffassung selbst bereits eine Repräsentation darstellt, vereinbar. Angemessener aus Sicht der radikal-konstruktivistischen Position ist deshalb die hier gewăhlte Formulierung, bei der von Abstraktionen der Modelle gesprochen wird, durch die das Erfahrungsobjekt entsprechend dem Erkenntnisinteresse eines Forschungsansatzes in sein Erkenntnisobjekt umgewandelt wird.
} 
hinsichtlich der Messbarkeit und der Veränderlichkeit des Geschehens im Gestaltungsbereich des Informationsmanagements zu berücksichtigen.

Es bleibt insgesamt festzuhalten, dass Information zur Erfüllung der Gestaltungs- und Steuerungsaufgaben im Bereich der technikunterstützten Informationsverarbeitung sowohl als Produktionsfaktor (Input) als auch als Produktionsergebnis (Output) aufzufassen ist. Die weiteren Überlegungen dieses Kapitels stellen stark auf den objektbezogenen Charakter von Prozessen der Informationsverarbeitung ab. Dementsprechend werden auf der Input-Seite in erster Linie die Werkstoff-Eigenschaften von Information als Produktionsfaktor betrachtet. Auf der Output-Seite erfolgt an dieser Stelle keine generelle Einschränkung der Betrachtung, da im Prinzip jede Art von zu erzeugender Information zum Gegenstand produktionstheoretischer Analysen gemacht werden kann.

\subsubsection{Der Bereich der Leistungserstellung bei der Herstellung informationeller Produkte}

Den Leitideen des Faktortheoretischen Ansatzes folgend, ist zwischen der güterwirtschaftlichen und der finanzwirtschaftlichen Sphäre von Unternehmungen zu unterscheiden. ${ }^{70}$ Die güterwirtschaftliche Sphäre setzt sich aus den beiden Teilbereichen der Leistungserstellung und der Leistungsverwertung zusammen. Ihr steht eine finanzielle Sphäre mit Kapitaldispositionsvorgängen gegenüber, die die Voraussetzungen für den güterwirtschaftlichen Leistungsvollzug schaffen. Die nachfolgenden Überlegungen beziehen sich zunächst auf den Bereich der Leistungserstellung, in dem die größten Erkenntnispotenziale für Fragen des Informationsmanagements liegen. Dabei wird das produktionswirtschaftliche Untersuchungsschema des Faktortheoretischen Ansatzes auf die Herstellung informationeller Produkte übertragen. In den Abschnitten 3.2.2.1, 3.2.2.2 und 3.2.2.3 werden die verschiedenen Elementarfaktorarten in Prozessen der Informationsverarbeitung betrachtet. Abschnitt 3.2.2.4 wendet sich den dispositiven Faktoren zu, die die Elementarfaktoren in Prozessen der Informationsverarbeitung kombinieren. Weiter unten werden ergänzend der Bereich der Leistungsverwertung und die finanzielle Sphäre betrachtet.

Im Faktortheoretischen Ansatz wird die betriebliche Leistungserstellung als ein Kombinationsprozess aufgefasst, in dem die Elementarfaktoren Betriebsmittel, Werkstoffe und objektbezogene menschliche Arbeitsleistungen durch die dispositiven Faktoren, bei denen es sich um leitende und lenkende menschliche Arbeitsleistungen handelt, miteinander verbunden werden. Wenn die Systematik der Elementarfaktoren auch zum Zwecke der Untersuchung industrieller Produktionsprozesse entwickelt wurde, so lässt sich doch zeigen, dass sie grundsätzlich ebenfalls auf die technikunterstützte Informationsverarbeitung anwendbar ist. Im zweiten Kapitel wurde dargelegt, dass die Prozesse der Informationsverarbeitung durch das technikunterstützte Informationssystem der Unternehmung als Gestaltungsgegenstand des Informationsmanagements erfolgen. Dieses technikunterstützte Informationssystem der Unternehmung besteht aus menschlichen und technischen Elementen. Die Aktivitäten dieser

\footnotetext{
${ }^{70}$ Vgl. Gutenberg (1983), S. 2. Diese konzeptionelle Dreiteilung spiegelt sich auch in der dreibändigen Aufteilung von Gutenbergs Grundlagen der Betriebswirtschaftslehre wider, die mit „Die Produktion“, „Der Absatz“ und „Die Finanzen“ betitelt sind.
} 
Elemente richten sich auf die Verarbeitung informationeller Objekte. Zwischen dieser definitorischen Abgrenzung des Informationsmanagements und seines Gestaltungsgegenstands und dem Untersuchungsschema des Faktortheoretischen Ansatzes bestehen, wie im Folgenden erläutert wird, klare Parallelen.

\subsubsection{Objektbezogene menschliche Arbeitsleistungen in Prozessen der Informa- tionsverarbeitung}

Bei objektbezogenen menschlichen Arbeitsleistungen als erster Elementarfaktorart handelt es sich in der Systematik des Faktortheoretischen Ansatzes um solche Tätigkeiten, die unmittelbar mit der Leistungserstellung, der Leistungsverwertung und mit finanziellen Aufgaben im Zusammenhang stehen, ohne dispositiv-anordnender Natur zu sein. $^{71}$ Die Arbeitsleistungen beziehen sich auf Objekte, durch deren Verarbeitung das herzustellende Produkt entsteht. Diese definitorischen Merkmale treffen auch auf Prozesse der technikunterstützten Informationsverarbeitung zu. Innerhalb solcher Prozesse nehmen die menschlichen Elemente des technikunterstützten Informationssystems Aktivitäten vor, die sich auf die Transformation informationeller Eingangsobjekte in informationelle Ausgangsobjekte richten. Informationen werden in solchen Prozessen durch die menschlichen Aufgabenträger den im zweiten Kapitel erläuterten Einzelaktivitäten der Informationsverarbeitung, also der Generierung, Speicherung, Bearbeitung, Übertragung und Auswertung, unterzogen. Im Bereich der technikunterstützten Informationsverarbeitung stellen allerdings nicht, wie üblicherweise innerhalb des Faktortheoretischen Ansatzes unterstellt, industrielle Werkstoffe, sondern Informationen die Objekte dar, an denen sich objektbezogene menschliche Arbeitsleistungen vollziehen.

Tatsächlich besitzen alle Aktivitäten der Informationsverarbeitung objektbezogenen Charakter. Sie sind deshalb, sofern sie durch menschliche Aufgabenträger ausgeführt werden, der Elementarfaktorart objektbezogene menschliche Arbeitsleistungen hinzuzurechnen. Hinsichtlich der Art der informationsobjektbezogenen Tätigkeit und des zu erstellenden informationellen Produktes werden dabei keinerlei Einschränkungen gemacht. Das bedeutet, dass es sich dabei sowohl um unternehmungsextern genutzte, als auch um unternehmungsintern für Koordinations- oder für analytische Zwecke genutzte Informationen handeln kann. Dies hat zur Folge, dass für die Zwecke des Informationsmanagements grundsätzlich auch planende Aktivitäten, mit denen durch die Verarbeitung von Informationen Entscheidungen vorbereitet werden, als Elementarfaktoren aufzufassen sind.

Diese kontra-intuitive Einordnung planender Aktivitäten ist allerdings näher zu begründen, da die Planung entsprechend der ursprünglichen Logik des Faktortheoretischen Ansatzes zu den dispositiven Faktoren mit derivativem Charakter gehört. Zur Begründung ist es notwendig, den ursprünglichen Untersuchungsgegenstand und die Untersuchungsabsicht genauer zu betrachten. Im Bereich der Leistungserstellung wurden von den Vertretern des Faktortheoretischen Ansatzes industrielle Produktionsprozesse mit dem Ziel untersucht, eine möglichst hohe Produktivität der Produktionsverfahren herzustellen. Die analysierten Verfahren erfolgten an festen, flüssigen oder gasförmigen Stoffen und nahmen daran Formänderungen, Stoffumwand-

${ }^{71}$ Vgl. Gutenberg (1983), S. 3. 
lungen, Lageänderungen oder Messoperationen vor. ${ }^{72}$ Planende Aktivitäten gehörten aus dieser Perspektive nicht zum eigentlichen Produktionsprozess und wurden folgerichtig getrennt davon als (derivativer) dispositiver Faktor untersucht.

Eine Verschiebung des Untersuchungsschwerpunktes auf die Analyse von Prozessen der Informationsverarbeitung macht in diesem Punkt eine Neuinterpretation erforderlich. Die in Planungsprozessen hergestellten Produkte sind informationeller Art (z.B. Planungsszenarien im Absatzbereich, Produktionspläne, Kostenanalysen). Die objektbezogenen Tätigkeiten vollziehen sich in Prozessen der Planung an Informationen als Objekten und nehmen daran Veränderungen und Auswertungen vor. Damit werden aus dieser Perspektive alle Aktivitäten, die im Zusammenhang mit der Vorbereitung und Überprüfung von Entscheidungen stehen, zu objektbezogenen Arbeitsleistungen, die im Faktortheoretischen Ansatz als Elementarfaktoren aufgefasst werden. Dies gilt unabhängig davon, ob es sich aus der ursprünglichen Perspektive ebenfalls um Elementarfaktoren oder um dispositive Faktoren handelte. Aus diesem Grund ist es sinnvoll, aus Sicht des Informationsmanagements auch planende menschliche Aktivitäten (z.B. Aktivitäten im Bereich der Produktions- oder Absatzplanung), für deren informationstechnische Unterstützung ebenfalls Konzepte zu entwickeln sind, als zur eigentlichen Leistungserstellung gehörende Elementarfaktoren aufzufassen.

\subsubsection{Arbeits- und Betriebsmittel in Prozessen der Informationsverarbeitung}

Menschliche Arbeit ist in ihrer Ergiebigkeit stark begrenzt, solange nicht deren Unterstützung, Intensivierung oder Substitution durch den Einsatz von Hilfsmitteln stattfindet. ${ }^{73} \mathrm{Zu}$ diesem Zweck werden Arbeits- und Betriebsmittel eingesetzt. Bei Arbeits- und Betriebsmitteln handelt es sich in der Systematik des Faktortheoretischen Ansatzes um die gesamte technische Apparatur, derer sich eine Unternehmung bedient, um ihre Leistungen zu erstellen. ${ }^{74}$ Als Potenzialfaktoren stellen die Arbeits- und Betriebsmittel Nutzungspotenziale dar, die über einen längeren Zeitraum, der auch als Nutzungsdauer bezeichnet wird, im Rahmen der Leistungserstellung zum Einsatz gelangen. ${ }^{75}$ Arbeits- und Betriebsmittel finden sich grundsätzlich nicht nur im Bereich der Leistungserstellung, sondern auch der Leistungsverwertung und des Finanzwesens. Im Folgenden wird vereinfachend nur noch von Betriebsmitteln gesprochen. Während Betriebsmittel mit Abgabe von Werkverrichtungen zu einem Fertigungsfortschritt an den betreffenden Arbeitsobjekten führen, obliegt den Betriebsmitteln ohne Abgabe von Werkverrichtungen eine unterstützende Funktion im Rahmen des Produktionsprozesses. ${ }^{76}$ Werkverrichtungen gleichen in technischer Hinsicht den Verbrauchsfaktoren, sie werden jedoch am Beschaffungsmarkt nicht einzeln, sondern in Form längerfristiger Potenziale für die gesamte Nutzungsdauer des Betriebsmittels beschafft. ${ }^{77}$

In Prozessen der Informationsverarbeitung werden informations- und kommunikationstechnische Hilfsmittel eingesetzt. Dies geschieht, um die Kapazitätsgrenzen der menschlichen Informationsverarbeiter zu überwinden und um Informationen zwischen

${ }^{72}$ Vgl. Gutenberg (1983), S. 86f.

73 Vgl. Kern (1992), S. 194.

${ }^{74} \mathrm{Vgl}$. Gutenberg (1983), S. 70.

75 Vgl. Corsten (2000), S. 335.

76 Vgl. Corsten (2000), S. 336.

${ }^{77}$ Vgl. ähnlich Busse v. Colbe/Laßmann (1991), S. 80. 
den beteiligten menschlichen Aufgabenträgern zu übertragen ${ }^{78}$ Dabei handelt es sich ebenfalls um einen Teil der in der Unternehmung verwendeten technischen Apparatur, sodass die informationstechnischen Hilfsmittel dem Elementarfaktor Betriebsmittel zugeordnet werden können. Dazu zählen die hard- und softwaretechnischen Elemente des technikunterstützten Informationssystems der Unternehmung, also die Rechner und Netze sowie die System- und die Anwendungssoftware.

In produktionswirtschaftlichen Arbeiten, die sich auf die industrielle Fertigung konzentrieren, werden - ansonsten nicht näher betrachtete - „EDV-Anlagen“ typischerweise als Betriebsmittel aufgefasst, die zwar Werkverrichtungen abgeben, jedoch nur mittelbar am eigentlichen Produktionsprozess beteiligt sind. ${ }^{79}$ Im Folgenden ist zu überprüfen, inwiefern diese Einordnung für die Zwecke des Informationsmanagements adäquat ist. Von einer Abgabe von Werkverrichtungen lässt sich tatsächlich sprechen, da die im zweiten Kapitel genannten Aktivitäten der Informationsverarbeitung mit Ausnahme der Generierung und Auswertung von Informationen, die jeweils interpretative Leistungen von Menschen erfordern, auch maschinell durchgeführt werden können. Bei den Werkverrichtungen der informations- und kommunikationstechnischen Hilfsmittel kann es sich also um die Speicherung, Bearbeitung und Übertragung der in den Prozessen zu Output-Informationen umgewandelten informationellen Objekte handeln. Die Frage der direkten oder indirekten Beteiligung dieser Betriebsmittel am Produktionsprozess ist deshalb von Bedeutung, weil die unmittelbare Zurechenbarkeit von Potenzialfaktoren Voraussetzung für die eindeutige Aufstellung von Produktions- und Kostenfunktionen ist. ${ }^{80}$ Im Gestaltungs- und Steuerungsbereich des Informationsmanagements sind die verwendeten informations- und kommunikationstechnischen Hilfsmittel entgegen der traditionellen Sichtweise nicht nur mittelbar am Produktionsprozess beteiligt. Vielmehr tragen die Aktivitäten dieser Betriebsmittel direkt zur Erzeugung des informationellen Produkts bei, sodass eine Einordnung als Betriebsmittel mit unmittelbarer Beteiligung am Produktionsprozess angemessen ist.

\subsubsection{Werkstoffe in Prozessen der Informationsverarbeitung}

Nachdem aufgezeigt wurde, dass die Elementarten des technikunterstützten Informationssystems der Unternehmung in der Systematik des Faktortheoretischen Ansatzes als Elementarfaktoren der ersten beiden Elementarfaktorkategorien aufgefasst werden können, ist es eine konsequente Fortführung des Gedankengangs, die verarbeiteten Informationen dem Elementarfaktor Werkstoffe zuzuordnen. Unter Werkstoffen werden im Faktortheoretischen Ansatz alle Rohstoffe, Halb- und Fertigfabrikate verstanden, die als Ausgangs- und Grundstoffe für die Herstellung von Erzeugnissen zu dienen bestimmt sind. ${ }^{81}$ Sie sind dadurch gekennzeichnet, dass sie nach ihrer Verarbeitung Bestandteile der hergestellten Produkte werden. Während des Produktionsprozesses werden die Werkstoffe Formänderungen, Stoffumwandlungen, Lageänderungen und/oder Messoperationen unterzogen. ${ }^{82}$

${ }^{78} \mathrm{Vgl}$. Streubel (2000), S. 71.

79 Vgl. z.B. Corsten (2000), S. 336.

$80 \mathrm{Vgl}$. Busse v. Colbe/Laßmann (1991), S. 81.

81 Vgl. Gutenberg (1983), S. 122.

82 Vgl. Gutenberg (1983), S. 86f. 
Betrachtet man Prozesse der technikunterstützten Informationsverarbeitung, so werden die eingehenden Input-Informationen während des Produktionsprozesses Maßnahmen unterzogen, die deutliche Analogien zu den Verfahren der industriellen Fertigung aufweisen. Sie werden transformiert (Formänderung), aggregiert und manipuliert (Stoffumwandlung), übertragen (Lageänderung) und analysiert (Messoperation). Anschließend finden sie Eingang in das informationelle Endprodukt. Es bestehen also klare Parallelen zwischen Informationen in Prozessen der technikunterstützten Informationsverarbeitung sowie Rohstoffen, Halb- und Fertigfabrikaten in der industriellen Fertigung. ${ }^{83}$ Die innerhalb des Faktortheoretischen Ansatzes untersuchte Fragestellung, welche Eigenschaften Werkstoffe aufweisen müssen, wenn sie in der Gesamtkombination der Produktivfaktoren zu einem Höchstmaß an Ergiebigkeit gelangen sollen, ${ }^{84}$ kann deshalb im Grundsatz auch auf die Werkstoffart Information übertragen werden.

In diesem Punkt weicht die Argumentation dieser Arbeit gegenüber dem ursprünglichen Gedankengang innerhalb des Faktortheoretischen Ansatzes ab. GUTENBERG hebt hervor, Werkstoffe fänden sich nur in Fertigungsbetrieben, nicht aber in Gewinnungs-, Veredelungs- oder Dienstleistungsbetrieben. ${ }^{85}$ Diese Aussage besitzt jedoch nur Gültigkeit furr die von ihm betrachteten Arten von Werkstoffen, also Rohstoffe, Halb- und Fertigfabrikate, die in industriellen Fertigungsprozessen verwendet und erzeugt werden. Prozesse der Informationsverarbeitung dagegen, bei denen Informationen die Elementarfaktorart Werkstoffe darstellen, finden sich in jeder Art von Betrieb, da der eigentliche Prozess der Leistungserstellung stets von einem begleitenden Koordinationsprozess informationeller Art begleitet wird. Dieser beinhaltet das Erkennen von Zusammenhängen und Wechselbeziehungen ebenso wie Tätigkeiten des Vergleichens, des Bewertens und des Ableitens von Schlussfolgerungen und Urteilen. ${ }^{86}$ Ferner tritt auch die zweite unternehmungsinterne Verwendungsart von Informationen, also die Herstellung von Informationsasymmetrien gegenüber unternehmungsexternen Akteuren, in nahezu jedem Betrieb auf. Die Nutzung von Informationen als Werkstoffen zur Herstellung unternehmungsextern genutzter Informationen in Form von Bestandteilen der an die Kunden abgesetzten Produkte findet dagegen nicht in jeder Form von Betrieben, sondern nur bei der Herstellung entsprechender Produkttypen statt.

Im Abschnitt 3.2.1 wurde erläutert, dass Information als Produktionsfaktor nicht eindeutig einer bestimmten Faktorkategorie zugerechnet werden kann. Eine alleinige Klassifikation von Information als Werkstoff wäre deshalb verkürzt, obwohl diese an einigen Stellen in der Literatur vorgenommen wird. ${ }^{87}$ Im Gegensatz dazu wird zum Teil auch gefordert, Informationen als Betriebsmittel einzuordnen, da sie während des Produktionsprozesses erhalten bleiben. ${ }^{88}$ Tatsächlich haben Informationen, die der Unternehmung längerfristig zur Verfügung stehen und nicht unmittelbar in die zu erstellenden Produkte eingehen, etwa Informationen über bestimmte Produktionsverfahren oder generelle Informationen über Beschaffungs- und Absatzmärkte, eher

\footnotetext{
${ }^{83}$ Diese Position vertritt auch Bode (1993), S. 82.

84 Vgl. Gutenberg (1983), S. 122.

${ }^{85} \mathrm{Vgl}$. Gutenberg (1983), S. 122. Altenburger weist ebenfalls darauf hin, dass der Produktionsfaktor Werkstoffe bei der Produktion von Dienstleistungen kaum Verwendung findet. Vgl. Altenburger (1980), S. 55.

${ }^{86}$ Vgl. Nebl (1996), S. 49.

${ }^{87}$ Vgl. z.B. Schüler (1987), S. 61; Müller (1987), S. 128.

${ }^{88} \mathrm{Vgl}$. Zelewski (1987), S. 740.
} 
den Charakter von Betriebsmitteln als von Werkstoffen. Da es jedoch auf die Dauer der Nutzung ankommt und viele Informationen nur einmalig im Produktionsprozess genutzt werden, erscheint eine generelle Klassifikation von Informationen als Betriebsmittel ebenfalls nicht angemessen. Die weiteren Überlegungen dieses Kapitels konzentrieren sich auf den Werkstoffcharakter von Informationen, eine Erweiterung des Aussagesystems durch die Einbeziehung von Informationen als Betriebsmittel wäre aber grundsätzlich möglich.

\subsubsection{Die dispositiven Faktoren in Prozessen der Informationsverarbeitung}

Die beschriebenen drei Arten von Elementarfaktoren werden durch die dispositiven Faktoren miteinander in Produktionsprozessen kombiniert. Für eine vollständige Anwendung der Systematik des Faktortheoretischen Ansatzes auf die technikunterstützte Informationsverarbeitung ist daher noch eine nähere Betrachtung der dispositiven Faktoren erforderlich, die GUTENBERG selbst folgendermaßen beschreibt: „Die Geschäfts- und Betriebsleitung, der vierte, dispositive Faktor bildet das Zentrum, die eigentlich bewegende Kraft des betrieblichen Geschehens. Im Gesamtsystem der betrieblichen Willensbildung stellt sie diejenige Instanz dar, in der alle Anordnungs- und Entscheidungsbefugnis kulminiert. ${ }^{\text {c }}{ }^{89}$ Er unterscheidet zwischen einer irrationalen, einer rationalen und einer gestaltend-vollziehenden Schicht dieses vierten Faktors. ${ }^{90}$

Die irrationale Schicht des dispositiven Faktors ist nach seiner Vorstellung mit betriebswirtschaftlichen Methoden allein nicht erklärbar. Es handelt sich dabei um nicht quantifizierbare, individuelle Eigenschaften der Mitglieder der Unternehmungsleitung als viertem Faktor, die aus der Perspektive GUTENBERGS einen rational nicht auflösbaren Rest darstellen. ${ }^{91}$ An die Stelle der Analyse dieser Schicht tritt ein ,mystifikatorisches Substitut", ${ }^{92}$ das beispielsweise in folgender Formulierung zum Ausdruck kommt: Es „werden Grenzen wissenschaftlichen Bemühens sichtbar, die nicht in der Methodik oder in dem Vermögen des einzelnen Forschers, sondern in der Sache selbst liegen". ${ }^{93}$ Nach dieser Vorstellung sind lediglich die rationale und die gestaltendvollziehende Schicht des vierten Faktors einer betriebswirtschaftlichen Analyse zugänglich.

Bei der rationalen Schicht des dispositiven Faktors handelt es sich um die betriebliche Planung, durch die der Betriebs- und Vertriebsprozess sowie der finanzielle Bereich von Zufälligkeiten freigemacht werden sollen. ${ }^{94}$ Der gestaltend-vollziehenden Schicht, die als Betriebsorganisation bezeichnet wird, obliegt es, „das Geplante Wirklichkeit werden $\mathrm{zu}$ lassen, also dafür Sorge zu tragen, dass Hemmungen und Widerstände überwunden werden, die sich dem Gewollten entgegensetzen." ${ }^{\text {(95 }}$ Aufgaben der Planung und der Betriebsorganisation können nach dieser Auffassung an Personen übertragen werden, die nicht zur unmittelbaren Geschäftsleitung gehören. Diese beiden Schichten des dispositiven Faktors besitzen also derivativen Charakter. Dagegen

${ }^{89}$ Gutenberg (1983), S. 131.

90 Vgl. Gutenberg (1983), S. 132.

91 Vgl. Gutenberg (1983), S. 7.

92 Dietz (1997), S. 1070.

93 Gutenberg (1983), S. 133.

${ }_{94} \mathrm{Vgl}$. Gutenberg (1983), S. 7.

${ }_{95}$ Gutenberg (1983), S. 132. 
können „echte Führungsentscheidungen" nur durch die Geschäftsleitung selbst getroffen werden und entziehen sich einer Delegation an nachgeordnete Einheiten. Solche echten Führungsentscheidungen zeichnen sich dadurch aus, dass sie von lebenswichtiger Bedeutung für die Zukunt der Unternehmung sind und dass sie sich im Gegensatz zu „Ressortentscheidungen“ auf die Unternehmung als Ganzes beziehen. ${ }^{96}$

Für das Informationsmanagement können aus diesen Ausführungen folgende Schlussfolgerungen gezogen werden. Die Aufgaben des Informationsmanagements, nämlich die Gestaltung und Steuerung des technikunterstützten Informationssystems der Unternehmung, sind dispositiv-anordnender Natur und deshalb zu den dispositiven Faktoren zu rechnen. Das technikunterstützte Informationssystem als Gestaltungsgegenstand des Informationsmanagements stellt ein Teilsystem der Gesamtunternehmung dar. Bei Entscheidungen in Bezug auf dieses System handelt es sich also nicht um echte Führungsentscheidungen, sondern um delegierbare, der betriebswirtschaftlichen Analyse zugängliche Ressortaufgaben. Zum Informationsmanagement zählen sowohl Aufgaben, die zur rationalen Schicht (Planung) des dispositiven Faktors gehören als auch Aufgaben, die zur gestaltend-vollziehenden Schicht (Betriebsorganisation) gehören.

Bereits weiter oben wurde dargelegt, dass zum Gestaltungsgegenstand des Informationsmanagements auch Aufgaben der Informationsverarbeitung gehören, die planenden Charakter haben und als solche wiederum dispositiv-anordnende Funktion für andere Teilbereiche der Unternehmung besitzen. Aus der Sicht des Informationsmanagements handelt es sich dabei um objektbezogene Tätigkeiten. Die fachliche Autorität der mit solchen derivativen Führungsaufgaben betrauten Personen wird durch die Gestaltungs- und Steuerungsaufgaben des Informationsmanagements nicht angetastet. Die Dispositionskompetenz des Informationsmanagements beschränkt sich vielmehr allein auf die informationstechnische Unterstützung dieser Planungsaufgaben, also die Art und Weise, in der die informationsobjektbezogenen Aktivitäten bei der Erfüllung der Planungsaufgaben durchgeführt werden.

Die exklusive und originäre Aufgabe des Informationsmanagements als Teil des dispositiven Faktors besteht in der Kombination der Elementarfaktoren, durch die sich die Prozesse der technikunterstützten Informationsverarbeitung in der Unternehmung vollziehen. Entsprechend den inhaltlichen Leitideen des Faktortheoretischen Ansatzes ist das Ergebnis der Leistungserstellung zum einen von der Beschaffenheit der Elementarfaktoren selbst und zum anderen von ihrer Kombination im Produktionsprozess abhängig. ${ }^{97}$ Demnach hat das Informationsmanagement einerseits die größtmögliche Ergiebigkeit der einzelnen Elementarfaktoren, also der objektbezogenen menschlichen Arbeitsleistungen, der technischen Elemente des Informationssystems der Unternehmung und der eingesetzten Informationen als Werkstoffen, zu gewährleisten und andererseits eine möglichst günstige Kombination dieser Elementarfaktoren herzustellen.

96 Vgl. Gutenberg (1983), S. 134.

97 Vgl. Gutenberg (1983), S. 8. 


\subsubsection{Der Bereich der Leistungsverwertung und die finanzielle Sphäre aus Sicht des Informationsmanagements}

Der Bereich der Leistungserstellung, auf den sich die Ausführungen des vorangegangenen Abschnitts richteten, weist besonders große Erkenntnispotenziale für das Informationsmanagement auf, da eine Übertragung des durch den Faktortheoretischen Ansatz entwickelten Instrumentariums auf den Bereich der technikunterstützten Informationsverarbeitung sehr weitgehend möglich ist. Die Überlegungen in diesem Abschnitt wenden sich ergänzend der Leistungsverwertung (Abschnitt 3.2.3.1) und der finanziellen Sphäre (Abschnitt 3.2.3.2) als weiteren Untersuchungsobjekten des Faktortheoretischen Ansatzes zu und untersuchen, in welchem Zusammenhang diese zum Gestaltungs- und Steuerungsbereich des Informationsmanagements stehen.

\subsubsection{Berührungspunkte zwischen Leistungsverwertung und technikunterstützter Informationsverarbeitung}

Im Bereich der Leistungsverwertung spielen Fragen der technikunterstützten Informationsverarbeitung unter zweierlei Gesichtspunkten eine Rolle. Zum einen können Informationen als Bestandteil in die Produkte eingehen und damit zum Gegenstand der Produktpolitik als absatzpolitischem Instrument werden. Die durch die Prozesse der Informationsverarbeitung erzeugten Produkte werden in diesem Fall unternehmungsextern genutzt. Zum anderen werden im Bereich der Leistungsverwertung Informationen unternehmungsintern dazu verwendet, die absatzpolitische Entscheidungssituation zu verbessern und damit die Gefahr von Fehlentscheidungen über absatzpolitische Maßnahmen zu verringern.

Informationen zählen als Produktbestandteil zu den funktionalen Eigenschaften eines Produktes, von denen dessen Verwendungsmöglichkeiten abhängen. Die Produktgestaltung stellt im Faktortheoretischen Ansatz neben der Absatzmethode, der Preispolitik und der Werbung eines von vier absatzpolitischen Hauptinstrumenten ${ }^{98} \mathrm{dar}$, mit denen Unternehmungen Einfluss auf das Absatzgeschehen nehmen können. ${ }^{99}$ Die Ausstattung eines Produktes mit Informationen kann als Mittel der Qualitätskonkurrenz verwendet werden, das es ermöglicht, das Produkt gegenüber alternativen Angeboten zu differenzieren. Den methodischen Leitideen des Faktortheoretischen Ansatzes entsprechend, sind Maßnahmen der Qualitätspolitik in einer Marginalanalyse daraufhin zu analysieren, inwieweit sie Verschiebungen der Nachfragekurve für das betreffende Produkt auslösen, die wiederum Veränderungen des erzielbaren Preises und Umsatzes nach sich ziehen. ${ }^{100}$ Diesen Preis- und Erlösänderungen sind die veränderten Produktionskosten, die durch die Qualitätsvariation entstehen, gegenüberzustellen. Das optimale Qualitätsniveau des Produktes ist dann erreicht, wenn sich die Grenzerlöse und Grenzkosten einer Qualitätsvariation exakt zu Null ausgleichen.

\footnotetext{
${ }^{98}$ Erweiterungen des absatzpolitischen Instrumentariums, die in der Nachfolge Gutenbergs entwickelt wurden, werden hier nicht berücksichtigt, da es für die vorliegenden Zwecke nur auf die Leitideen, also die Kerngedanken des Wissenschaftsprogramms ankommt.

99 Vgl. Gutenberg (1979), S. 9.

${ }^{100}$ Zur Behandlung von Qualitătsproblemen in der quantitativ ausgerichteten Gutenberg'schen Produktions- und Absatztheorie vgl. Lücke (1973), S. 264ff.
} 
Damit sind zugleich die Interdependenzen zwischen der Leistungserstellung und der Leistungsverwertung innerhalb der güterwirtschaftlichen Sphäre von Unternehmungen angesprochen. Die Konzeption der zu produzierenden Güter hängt von den Möglichkeiten ihrer Verwertung am Markt ab und stellt damit zunächst einmal eine absatzwirtschaftliche Problemstellung dar. ${ }^{101}$ Bei der qualitativen Ausstattung von Produkten handelt es sich in fachlicher Hinsicht also um ein Problem der Absatzpolitik. Die Ausgestaltung des Produkts determiniert jedoch maßgeblich die Ausprägung der zu seiner Herstellung erforderlichen Faktoren und ihrer Kombination im Produktionsprozess und ist deshalb gleichermaßen produktionswirtschaftlich von Bedeutung. Generell ist dabei von einer beidseitigen Beeinflussung auszugehen, da umgekehrt auch veränderte produktionswirtschaftliche Möglichkeiten neue absatzwirtschaftliche Potenziale eröffnen können.

Für das Informationsmanagement, das im vorigen Abschnitt produktionsorientiert abgegrenzt wurde, ergibt sich daraus folgende Konsequenz: Die Frage, wie die herzustellenden Güter mit informationellen Produktbestandteilen auszustatten sind, hat absatzwirtschaftlichen Charakter und fällt damit nicht in den Zuständigkeitsbereich eines produktionsorientiert abgegrenzten Informationsmanagements. Allein die Herstellung des informationellen Produktbestandteils entsprechend den absatzpolitischen Vorgaben stellt ein produktionswirtschaftliches Problem dar, das durch das Informationsmanagement zu lösen ist. Das bedeutet, dass diejenigen Aussagen des Faktortheoretischen Ansatzes, die sich auf die absatzpolitischen Hauptinstrumente beziehen, nur mittelbar Relevanz für das Informationsmanagement besitzen.

Einen zweiten Verwendungszweck von Informationen im Bereich der Leistungsverwertung stellt die absatzpolitische Information zur Senkung des absatzpolitischen Risikos dar. Dieses Risiko liegt darin begründet, dass die Unternehmung keine Gewissheit über sämtliche für die Leistungsverwertung relevanten Tatbestände, z.B. Verhaltensweisen der Konkurrenten, der Kunden oder gesamtwirtschaftliche Trends, besitzt. ${ }^{102}$ Durch die Beschaffung von absatzpolitischen Informationen über derartige Tatbestände kann das absatzpolitische Risiko reduziert werden. $\mathrm{Zu}$ den $\mathrm{zu}$ beschaffenden Informationen gehören dabei Zielgruppen-, Instrumental-, Konkurrenzund Trendinformationen, ${ }^{103}$ die mit Instrumenten der Marktforschung gewonnen werden können. Hinsichtlich der Bedeutung für das Informationsmanagement ist der Bereich der absatzpolitischen Informationen ähnlich zu beurteilen wie die oben betrachtete Herstellung von Informationen als Produktbestandteil: Bei der Frage, welche absatzpolitischen Informationen mit Hilfe welcher Instrumente der Marktforschung beschafft werden sollen, handelt es sich in fachlicher Hinsicht zunächst einmal um eine absatzwirtschaftliche Problemstellung. Erst die produktionswirtschaftliche Realisierung der fachlichen Vorgaben unter Verwendung geeigneter informationstechnischer Hilfsmittel fällt in den Gestaltungsbereich des Informationsmanagements. Damit besitzen auch die Aussagen, die der Faktortheoretische Ansatz in Bezug auf die absatzpolitische Information entwickelt, nur mittelbar Relevanz für das Informationsmanagement.

\footnotetext{
${ }^{101}$ Vgl. Kern (1992), S. 96.

${ }^{102}$ Vgl. Gutenberg (1979), S. 16.

${ }^{103}$ Vgl. Gutenberg (1979), S. 36ff.
} 
Diese kurzen Überlegungen zeigen, dass es sich bei den beiden Verwendungszwecken von Informationen im Bereich der Leistungsverwertung um Anwendungsgebiete der technikunterstützten Informationsverarbeitung handelt. Die Erfüllung der angesprochenen fachlichen Aufgaben der Informationsverarbeitung erfolgt mit Hilfe von Anwendungssystemen (z.B. Finanzmarkt-Informationssystemen oder Desktop-PublishingSystemen), die aus der Sicht des Informationsmanagements jedoch nicht anders als Anwendungssysteme in anderen Bereichen der Unternehmung (z.B. im Bereich des Rechnungswesens oder der Produktionsplanung und -steuerung) zu behandeln sind. Entsprechend den im vorigen Abschnitt vorgenommenen, produktionsorientierten Abgrenzung des Informationsmanagements werden die Aussagen zur Leistungsverwertung im Folgenden nicht mehr näher betrachtet.

\subsubsection{Berührungspunkte zwischen finanzieller Sphäre und technikunterstützter Informationsverarbeitung}

Die finanzielle Sphäre der Unternehmung stellt genau wie der Bereich der Leistungsverwertung aus der Sicht des Informationsmanagements ein bestimmtes Anwendungsgebiet der technikunterstützten Informationsverarbeitung dar. In der finanziellen Sphäre ist der Kapitalbedarf durch Kapitalfonds zu decken, um das finanzielle Gleichgewicht der Unternehmung herzustellen. ${ }^{104}$ Dabei unterliegen sowohl die für den Kapitalbedarf maßgeblichen güterwirtschaftichen Prozesse als auch die für die Deckung dieses Bedarfs erforderlichen güterwirtschaftlichen und finanzwirtschaftlichen Aktivitäten der Unsicherheit. ${ }^{105}$ Die Reduzierung dieser Unsicherheit und die Gewährleistung des finanziellen Gleichgewichts ist Aufgabe einer integrierten Finanzplanung, die die Ein- und Auszahlungen der betrachteten Periode miteinander zum Ausgleich bringt. Bei der integrierten Finanzplanung handelt es sich, genau wie bei der Produktionsplanung und der Absatzplanung, um eine fachliche Problemstellung, die durch das technikunterstützte Informationssystem der Unternehmung unterstützt werden kann. Erst diese informationstechnische Unterstützung finanzwirtschaftlicher Aufgabenstellungen, die ein produktionswirtschaftliches und kein finanzwirtschaftliches Problem darstellt, fällt in den Gestaltungsbereich des Informationsmanagements.

\subsection{Produktionsorientierte Analyse des Informationsmanagements}

Ausgehend von dem im vorigen Abschnitt erfolgten Abschluss des Theorieeinstiegs durch Entwicklung des Informationsmanagements als speziellem theoretischen Konstrukt nehmen die nachfolgenden Ausführungen eine produktionsorientierte Analyse des Informationsmanagements vor. Durch die Übertragung und Anwendung bestehender Erkenntnisse über die Leistungserstellung auf den Bereich der technikunterstützten Informationsverarbeitung wird ein Produktionsorientierter Ansatz für eine Theorie des Informationsmanagements entwickelt.

Bei der Darstellung der methodischen Leitideen des Faktortheoretischen Ansatzes in Abschnitt 3.1.3 wurde erläutert, dass das in weiten Teilen der Physik übliche Vorgehen, Systeme zur Untersuchung in ihre verschiedenen Einzelteile zu zerlegen, über-

\footnotetext{
${ }^{104} \mathrm{Vgl}$. Gutenberg (1980), S. 3f.

${ }^{105}$ Vgl. Gutenberg (1980), S. 381ff.
} 
nommen wird. Überträgt man dieses reduktionistisch orientierte Vorgehen auf die Analyse produktionswirtschaftlicher Zusammenhänge, so folgt daraus, dass eine Desaggregation der Faktorkombination bis hin zu den einzelnen Elementarfaktoren erforderlich ist. Aus diesem Grund sind zunächst die verschiedenen Elementarfaktoren getrennt voneinander $\mathrm{zu}$ betrachten, um davon ausgehend eine Untersuchung ihres Zusammenwirkens im Produktionsprozess vorzunehmen.

Dieser Methodik folgend unterscheiden Vertreter des Faktortheoretischen Ansatzes häufig zwischen einer Vorkombination und einer Endkombination von Produktionsfaktoren. Dabei begründet die Vorkombination zunächst nur eine Leistungsbereitschaft und findet im Hinblick auf eine intendierte Endkombination statt. ${ }^{106}$ Der Vorkombination obliegt also der Aufbau des Leistungspotenzials, während es Ziel der Endkombination ist, durch die Nutzung des Leistungspotenzials absetzbare Leistungen $\mathrm{zu}$ erstellen. ${ }^{107}$ Die Untersuchung der Vorkombination konzentriert sich dabei weitgehend auf die einzelnen Elementarfaktoren, während deren Zusammenwirken in erster Linie im Rahmen der Endkombination betrachtet wird. Eine vergleichbare Unterscheidung, jedoch ohne Verwendung der Begriffe Vor- und Endkombination, findet sich bereits bei GUTENBERG. Dieser untersucht für die verschiedenen Faktorarten zunächst, ,welche Umstände es sind, die den produktiven Beitrag bestimmen, den sie im Rahmen einer Faktorkombination zu leisten imstande sind.“108 Erst danach wird nach den Grundsätzen des Zusammenspiels zwischen den verschiedenen Produktionsfaktoren gefragt.

Die nachfolgenden Überlegungen zur produktionsorientierten Analyse des Informationsmanagements übernehmen das beschriebene Vorgehen und beziehen sich auf drei große Aussagebereiche. Zunächst werden Zusammenhänge betrachtet, die den Gestaltungsgegenstand des Informationsmanagements, also das technikunterstützte Informationssystem der Unternehmung und die darin stattfindenden Prozesse, betreffen. Die entwickelten Aussagen beziehen sich dabei auf die durch die Gestaltungs- und Steuerungsaktivitäten des Informationsmanagements anzustrebenden Gestaltungsergebnisse. Zur Sicherstellung einer möglichst hohen Produktivität von Prozessen der Informationsverarbeitung ist zum einen in der Vorkombination die größtmögliche Ergiebigkeit der eingesetzten Elementarfaktoren als solcher herzustellen. Zum anderen ist in der Endkombination das entsprechend dem Wirtschaftichkeitsprinzip optimale Zusammenwirken dieser Elementarfaktoren zu gewährleisten. Dementsprechend richten sich die Überlegungen des Abschnitts 3.3.1 auf die einzelnen Elementarfaktoren und deren Ergiebigkeit in Prozessen der technikunterstützten Informationsverarbeitung, während im Abschnitt 3.3.2 das Zusammenwirken der Elementarfaktoren bei der Herstellung informationeller Produkte untersucht wird.

\footnotetext{
${ }^{106} \mathrm{Vgl}$. Kern (1992), S. 21.

${ }^{107}$ Vgl. Corsten (1988), S. 83. Die Vorkombination wird zum Teil auch als Potenzialgestaltung und die Endkombination als Prozessgestaltung bezeichnet. Einige Autoren nehmen zusätzlich noch die der Potenzialgestaltung vorgelagerte Produktgestaltung als eigenen Gestaltungsbereich der industriellen Produktion in die Analyse auf. Vgl. z.B. Kern (1992), S. 82ff;; Corsten (2000), S. 28ff. Diesem Vorgehen wird hier nicht gefolgt, da starke Interdependenzen der Produktgestaltung zum absatzwirtschaftlichen Bereich bestehen, der bereits im Abschnitt 3.2.3.1 wegen nur mittelbarer Relevanz aus der hier vorgenommenen produktionsorientierten Analyse des Informationsmanagements ausgeklammert wurde.

${ }^{108}$ Gutenberg (1983), S. 8.
} 
Im Anschluss an diese Untersuchung der Gestaltungsergebnisse erfolgt im Abschnitt 3.3.3 eine Betrachtung des dispositiven Faktors, aus der sich Aussagen über das zweckmäßige Vorgehen bei der Gestaltung und Steuerung des technikunterstützten Informationssystems der Unternehmung ergeben. Die Überlegungen betreffen dort nicht mehr den Gestaltungsgegenstand, sondern das Informationsmanagement als betriebliches Aufgabenfeld selbst. Dazu wird überprüf, inwieweit diejenigen Aussagen des Faktortheoretischen Ansatzes, die sich auf die dispositiven Faktoren mit derivativem Charakter, also auf die Planung und Betriebsorganisation, beziehen, auf das Informationsmanagement als speziellen Teilbereich der dispositiven Faktoren übertragen werden können.

\subsubsection{Vorkombination von Elementarfaktoren im Bereich der technikunter- stützten Informationsverarbeitung}

In diesem Abschnitt erfolgt die Betrachtung der Vorkombination der verschiedenen Elementarfaktoren in Prozessen der technikunterstützten Informationsverarbeitung. Es wird untersucht, wie das Informationsmanagement eine möglichst hohe Ergiebigkeit der einzelnen Faktorarten sicherstellen kann, um einen entsprechend hohen Potenzialbeitrag der jeweiligen Faktorart zu gewährleisten. In den Abschnitten 3.3.1.1, 3.3.1.2 und 3.3.1.3 wird nacheinander die Ergiebigkeit von objektbezogenen menschlichen Arbeitsleistungen, Betriebsmitteln und Werkstoffen bei der Erstellung informationeller Produkte untersucht. Abschnitt 3.3.1.4 unternimmt eine erste Betrachtung des Zusammenwirkens der verschiedenen Elementarfaktoren bzw. Elementarfaktorarten und wendet sich dem Potenzialbeitrag zu, der im Rahmen der Vorkombination durch die Anordnung der Faktoren zu Produktionssystemen erreicht werden kann.

\subsubsection{Ergiebigkeit objektbezogener menschlicher Arbeitsleistungen bei der Erstel- lung informationeller Produkte}

Der Mensch ist aus Sicht der Produktionstheorie Träger der von ihm zu erbringenden Arbeitsleistungen und somit ein personifizierter Produktionsfaktor. ${ }^{109}$ Der Faktortheoretische Ansatz beschränkt sich mit der Analyse auf diese versachlichte Rolle der Arbeitnehmer. In neueren Arbeiten zur Produktionswirtschaft werden in verstärktem Maße verhaltenswissenschaftliche Erkenntnisse übernommen und in das produktionsbezogene Aussagesystem eingebaut. ${ }^{110}$ Diese Erweiterungen gehören jedoch nicht mehr zum Kern des Ansatzes und werden daher an dieser Stelle nicht im Einzelnen berücksichtigt. ${ }^{111}$ Die folgenden Überlegungen konzentrieren sich statt dessen auf die Förderung der Ergiebigkeit objektbezogener menschlicher Arbeitsleistungen in Prozessen der Informationsverarbeitung unter Berücksichtigung des Rationalprinzips. Die Ergiebigkeit menschlicher Arbeitsleistungen im Betrieb hängt aus der Perspektive des Faktortheoretischen Ansatzes von subjektiven und objektiven Bedingungen sowie

\footnotetext{
${ }^{109} \mathrm{Vgl}$. Kern (1992), S. 159.

${ }^{110}$ Vgl. z.B. Kern (1992), S. 159ff.; Corsten (2000), S. 269ff.

${ }^{11}$ Dies betriff insbesondere die Frage, wie die individuelle Leistungsbereitschaft dahingehend gefordert werden kann, dass die individuelle Leistungsfahigkeit als maximal erreichbare Arbeitsleistung möglichst vollständig ausgeschopft wird. Vgl. dazu z.B. Corsten (2000), S. 275ff. Auch die Berücksichtigung unterschiedlicher Führungsstile in produktionswirtschaftlichen Analysen wird hier nicht năher thematisiert. Vgl. dazu z.B. Corsten (2000), S. 298.
} 
vom Entgelt ab, das für die Arbeit bezahlt wird. ${ }^{112}$ Diese Systematik ist in Abbildung 3/4 dargestellt. Sie ist grundsätzlich auf Prozesse der Informationsverarbeitung übertragbar, da die isoliert betrachtete Produktivität dieser Elementarfaktorart in solchen Prozessen prinzipiell den gleichen Einflussgrößen wie in industriellen Prozessen unterliegt.

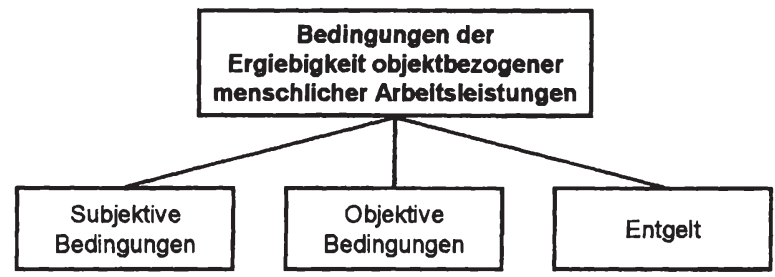

Abb. 3/4: Bedingungen der Ergiebigkeit objektbezogener menschlicher Arbeitsleistungen

\section{a) Subjektive Bedingungen}

Als subjektive Bedingungen der Ergiebigkeit menschlicher Arbeitsleistungen werden solche Umstände bezeichnet, die in der Person der Arbeitenden selbst liegen. ${ }^{113}$ Dabei handelt es sich um die Eigenschaften der arbeitenden Person, die ihre Eignung für eine bestimmte Arbeitsaufgabe determinieren. Sprachlich genauer wäre in diesem Zusammenhang eigentlich nicht von subjektiven, sondern von subjektbezogenen Bedingungen zu sprechen, da von einer objektiven Feststellbarkeit dieser Eigenschaften ausgegangen wird. ${ }^{114}$ Entsprechend der GUTENBERG'schen Auffassung gehören dazu die Antriebsstruktur, das persönliche Temperament, die Gesinnung, die Begabung sowie die körperliche Verfassung. ${ }^{115}$ Ein Optimum an Ergiebigkeit menschlicher Arbeitsleistungen wird dann erreicht, wenn die arbeitenden Menschen nur mit Aufgaben betraut werden, die ihrem Eignungstyp entsprechen. Sowohl ein Eignungsmangel als auch ein Eignungsüberschuss führen zur Minderleistung der arbeitenden Person.

Das Informationsmanagement hat die Aufgabe, das in der Unternehmung vorhandene, gesamte Eignungspotenzial für Prozesse der Informationsverarbeitung mit dem durch die Arbeitsanforderungen determinierten Bedarf an Arbeitskräften in Übereinstimmung zu bringen. ${ }^{116}$ Dazu sind eine quantitative und eine qualitative Personalplanung für die informationsverarbeitenden Tätigkeiten in der Unternehmung erforderlich. Die quantitative Personalplanung umfasst die Bedarfsplanung, die Beschaffungsplanung (im Falle der quantitativen Unterdeckung des Bedarfes) und die Freisetzungsplanung (im Falle der quantitativen Überdeckung des Bedarfes). Die qualitative Personalplanung dagegen legt fest, über welche Qualifikationen das Personal bis zum Planungs-

\footnotetext{
${ }^{112} \mathrm{Vgl}$. Gutenberg (1983), S. 40.

${ }^{113}$ Vgl. Gutenberg (1983), S. 14.

${ }^{114}$ Corsten (2000), S. 272, unterscheidet deshalb zwischen individuellen und situationsbedingten Einflussgrößen der menschlichen Arbeitsleistung.

${ }^{115}$ Vgl. Gutenberg (1983), S. 18.

${ }^{116}$ Diese Personalaufgabe bezieht sich lediglich auf Prozesse der Informationsverarbeitung. Personalmaßnahmen für alle anderen in der Unternehmung ablaufenden Prozesse sind nicht Gegenstand des Informationsmanagements.
} 
horizont verfuggen muss und $\mathrm{zu}$ welchen Stellen- bzw. Personalkategorien diese Qualifikationen gebündelt werden sollten. ${ }^{117}$ Dazu gehören insbesondere die Planungsfelder Personaleinsatz und -entwicklung. ${ }^{118}$

Insbesondere die Personalentwicklung als Teil der qualitativen Personalplanung gewinnt im Umfeld eines sich immer rascher vollziehenden technischen Wandels zunehmend an Bedeutung, da entwicklungsfähige Mitarbeiter frühzeitig auf den Umgang mit neuen Betriebsmitteln vorbereitet und an ihnen geschult werden müssen. ${ }^{119}$ Vor diesem Hintergrund ergibt sich auch für das Informationsmanagement beim Einsatz innovativer informationstechnischer Lösungen ein erheblicher Handlungsbedarf im qualitativen Bereich. Die für Aufgaben der technikunterstützten Informationsverarbeitung relevanten Qualifikationen umfassen fachliche und überfachliche Qualifikationsmerkmale. ${ }^{120}$ Fachliche Qualifikationsmerkmale können sich einerseits auf die Fähigkeit zum unmittelbaren Umgang mit der eingesetzten Hard- und Software beziehen oder andererseits ein Gesamtverständnis über die Funktionalität von Informationstechnik und ihre Anwendung im Wirtschaftsgeschehen betreffen. Überfachliche Qualifikationsmerkmale beinhalten Fähigkeiten $\mathrm{zu}$ abstraktem, planerischem und theoretischem Denken, zu Teamarbeit und Kommunikation sowie zum Erkennen übergreifender Zusammenhänge. Bei der überfachlichen Qualifikation können innovatorische Qualifikationsmerkmale, die sich auf die Veränderung der Arbeitssituation durch den Einsatz innovativer Informationstechnik beziehen, und soziale Qualifikationsmerkmale, die die Fähigkeit zur Konfliktbewältigung und Kooperation betreffen, unterschieden werden.

Die Einflussmöglichkeiten des Informationsmanagements beziehen sich auf fachliche Qualifikationsmerkmale, während es sich bei den überfachlichen Qualifikationsmerkmalen um einen allgemeinen personalwirtschaftlichen Aspekt handelt, der nicht originär in den Gestaltungsbereich des Informationsmanagements fallt. Mit Hilfe geeigneter Qualifizierungsmaßnahmen (z.B. Anwenderschulungen, Entwicklung und Bereitstellung von Benutzerhandbüchern, unternehmungsinterne Beratung) hat das Informationsmanagement sicherzustellen, dass das fachliche Know-how in der Unternehmung im benötigten Maße verfügbar ist.

Generell ist in der Literatur umstritten, wie sich die Anforderungen an die Qualifikation der Mitarbeiter in Folge des Einsatzes innovativer (Informations-)Technik ändern. Dabei stehen sich eine Höherqualifizierungs-, eine Dequalifizierungs- und eine Polarisierungsthese gegenüber. ${ }^{121}$ Die Dequalifizierungsthese nimmt an, dass es durch den Einsatz innovativer Informationstechnologie zu einer Verringerung der Qualifikationsanforderungen an die mit Aufgaben der Informationsverarbeitung betrauten Mitarbeiter kommt. Diese These wurde besonders in älteren Publikationen häufiger vertreten. ${ }^{122}$ Inzwischen setzt sich jedoch die Ansicht, dass höhere Qualifikationsanforderungen die Voraussetzung für die zukünftige Wettbewerbsfähigkeit von Unternehmungen darstellen, mehr und mehr durch. ${ }^{123}$ Dieser Gedanke spricht tenden-

\footnotetext{
${ }^{117}$ Vgl. Drumm (1992), Sp. 1763.

${ }^{118}$ Vgl. Gaugler (1989), Sp. 1356ff.

${ }^{119}$ Vgl. Kern (1992), S. 171.

${ }^{120} \mathrm{Vgl}$. zum folgenden Nawatzki (1994), S. 88ff.

${ }^{121}$ Vgl. Corsten (2000), S. $291 \mathrm{ff}$.

${ }^{122}$ Vgl. z.B. Baethge/Oberbeck (1986), S. 288.

${ }^{123}$ Vgl. dazu z.B. Pawlowsky (1992), S. 188ff.; Picot/Reichwald/Wigand (2001), S. 459.
} 
ziell eher für die Höherqualifizierungs- und die Polarisierungsthese. Geht man davon aus, dass keine Zwangsläufigkeit zwischen Automatisierung und Qualifikation besteht, so entstehen Gestaltungsspielräume für das Informationsmanagement, ${ }^{124}$ durch geeignete organisatorische Lösungen eine Passung zwischen den Anforderungen der Unternehmung und dem vorhandenen Arbeitskräftepotenzial herzustellen.

\section{b) Objektive Bedingungen}

Bei den objektiven Bedingungen der Ergiebigkeit menschlicher Arbeitsleistungen handelt es sich um solche Umstände, die nicht unmittelbar mit der Person des Aufgabenträgers zusammenhängen. Diese Bedingungen betreffen die äußeren Arbeitsbedingungen und erstrecken sich auf das Arbeitsverfahren, den Arbeitsplatz, die am Arbeitsplatz wirksamen Umwelteinflüsse sowie die Arbeitszeit. ${ }^{125}$ Im Rahmen der Gestaltung der objektiven Bedingungen wird das Ziel verfolgt, durch die Gestaltung des Arbeitsumfelds leistungsfördernde Verhältnisse zu schaffen, um eine möglichst hohe Produktivität der Aufgabenträger zu erreichen.

$\mathrm{Zu}$ den Arbeitsverfahren zählen die einzelnen Tätigkeiten bei der Ausführung der Arbeit, deren rationelle Gestaltung das Ergebnis fortdauernder Verbesserungsbemühungen der Aufgabenträger einerseits und arbeitswissenschaftlicher Untersuchungen andererseits ist. Allgemeine Voraussetzung für die Auswahl des arbeitswissenschaftlich gesehen geeignetesten Verfahrens sind Arbeitsablaufstudien und darauf aufbauende Bewegungs- und Zeitstudien. ${ }^{126}$ Die Gestaltung des Arbeitsplatzes und seines Umfeldes muss sich an den Erfordernissen der eingesetzten Arbeitsverfahren orientieren und den menschlichen Aufgabenträgern ausreichende Entfaltungsmöglichkeiten bieten. Durch eine entsprechende Arbeitszeitregelung kann schließlich gewährleistet werden, dass die Ermüdungserscheinungen während der Arbeitszeit so gering wie möglich gehalten werden. Dazu ist eine Zeitermittlung notwendig, die eine Systematisierung einzelner Tätigkeiten und Unterbrechungen der Arbeitskraft vornimmt. $^{127}$

Im Bereich der technikunterstützten Informationsverarbeitung gehört zu den äußeren Arbeitsbedingungen zunächst die möglichst rationelle Gestaltung der in diesen Prozessen eingesetzten Verfahren der Informationsverarbeitung. Durch die Auswahl der jeweils effizientesten Verfahrensalternative kann das Informationsmanagement eine hohe Ergiebigkeit der betreffenden objektbezogenen menschlichen Arbeitsleistungen erreichen. $\mathrm{Zu}$ diesem Zweck sind im konkreten Fall Arbeits- und Zeitanalysen durchzuführen, die Aufschluss über die unterschiedliche Produktivität alternativ möglicher informationstechnischer und organisatorischer Lösungen geben. $\mathrm{Zu}$ den durch das Informationsmanagement gestaltbaren äußeren Arbeitsbedingungen gehört ferner die Ergonomie der an Arbeitsplätzen, an denen Informationstechnik eingesetzt wird, verwendeten informationstechnischen Geräte. Dagegen ist die Arbeitszeitregelung Bestandteil genereller Vereinbarungen und entzieht sich somit der direkten Beeinflussbarkeit durch das Informationsmanagement. Bei der Regelung der allge-

\footnotetext{
${ }^{124}$ Für diese Position vgl. z.B. Fotilas (1983), S. 92; Kudera/Ruff/Schmidt (1982), S. 142.

${ }^{125}$ Vgl. Gutenberg (1983), S. 34.

${ }^{126}$ Vgl. Corsten (2000), S. 312.

${ }^{127}$ Vgl. Nebl (1996), S. 55.
} 
meinen Arbeitszeit sind allerdings stets die Erfordernisse, die sich aus Aufgaben im Bereich der Informationsverarbeitung ergeben, zu berücksichtigen.

\section{c) Arbeitsentgelt}

Der Vorstellung, dass das Arbeitsentgelt als dritte Einflussgröße maßgeblich für die Ergiebigkeit menschlicher Arbeitsleistungen ist, liegt die Überzeugung zu Grunde, dass auf die Produktivität menschlicher Arbeit durch Maßnahmen zur extrinsischen Motivation wirksam Einfluss genommen werden kann. Im Faktortheoretischen Ansatz werden das Problem der Lohngerechtigkeit und die Verhältnisse zwischen Arbeitsentgelt, Arbeitsschwierigkeit, individueller Arbeitsleistung und individueller Mehrleistung untersucht. ${ }^{128}$ Die Gestaltung des Entlohnungssystems und der Entgelthöhe sind allerdings ebenso wie die Arbeitszeitregelung Gegenstand genereller Vereinbarungen. Sie fallen deshalb nicht in den Aufgabenbereich des Informationsmanagements und werden hier nicht weiter untersucht.

\subsubsection{Ergiebigkeit von Betriebsmitteln bei der Erstellung informationeller Produkte}

Der produktive Beitrag, den Arbeits- und Betriebsmittel für technische und wirtschaftliche Aufgaben erbringen, hängt wie in Abbildung 3/5 dargestellt, von unternehmungsspezifischen und unternehmungsunspezifischen Faktoren $a b$.

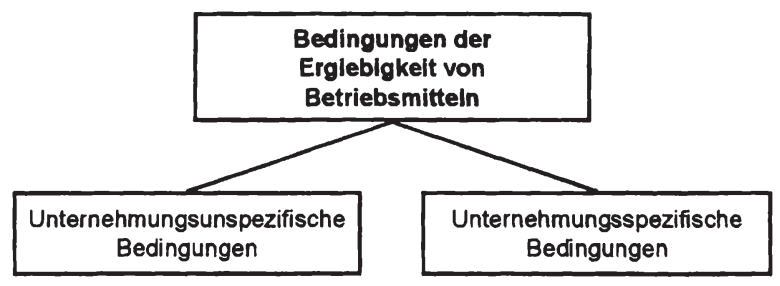

Abb. 3/5: Bedingungen der Ergiebigkeit von Betriebsmitteln

GUTENBERG nennt drei unternehmungsunspezifische Einflussgrößen, die ohne Rücksicht auf die besonderen Verhältnisse einer Unternehmung die allgemeine technische Leistungsfähigkeit der betrieblichen Apparatur bestimmen. Dabei handelt es sich um den Grad der Modernität, den Abnutzungsgrad und den Zustand an Betriebsfähigkeit, in dem sich der Betriebsmittelbestand befindet. ${ }^{129}$ Die Leistungsfähigkeit von Betriebsmitteln hängt aber nicht nur von ihrem technischem Leistungsstand als solchem, sondern auch von ihrer unternehmungsspezifischen Eignung für die konkrete Art der Leistungserstellung in der Unternehmung ab. Nachfolgend ist zu überprüfen, inwieweit diese für Arbeits- und Betriebsmittel aus dem Bereich der industriellen Fertigung aufgestellte Systematik auf informationstechnische Betriebsmittel übertragbar ist und welche Modifikationen erforderlich sind, um den besonderen Eigenschaften der Informationstechnik Rechnung zu tragen.

${ }^{128} \mathrm{Vgl}$. Gutenberg (1983), S. 40ff.

${ }^{129}$ Vgl. Gutenberg (1983), S. 71. 


\section{a) Unternehmungsunspezifische Einflussgrößen}

Der Grad der Modernität der Betriebsmittel ist deshalb von Relevanz, weil davon auszugehen ist, dass die Leistungsfáhigkeit neuerer technischer Anlagen größer ist als die älterer Betriebsmittel. Der Bestand an Betriebsmitteln veraltet häufig, noch bevor er technisch unbrauchbar wird. Dies gilt in besonderem Maße für Betriebsmittel aus dem Bereich der Informationstechnik, in dem in der jüngeren Vergangenheit eine besonders hohe Innovationsgeschwindigkeit zu beobachten war. In diesem Punkt ist das GUTENBERG'sche System von Einflussgrößen also auf den Gestaltungsgegenstand des Informationsmanagements übertragbar. Bei dem Problem der Ausmusterung alter und der Anschaffung neuer Anlagen handelt es sich um eine investitionstheoretische und nicht um eine produktionstechnische Fragestellung, ${ }^{130}$ da die technisch mögliche von der wirtschaftlich sinnvollen Lebensdauer eines Betriebsmittels abweichen kann. ${ }^{131}$ Die Frage der Neuanschaffung kann einerseits vor Beginn der eigentlichen Nutzung und andererseits während der Nutzungszeit gestellt werden. In der Investitionstheorie wird im ersten Fall vom Problem der optimalen Nutzungsdauer, im zweiten Fall vom Problem des optimalen Ersatzzeitpunkts, der sich während der ursprünglich geplanten Nutzungsdauer verschieben kann, gesprochen. ${ }^{132}$ Im Folgenden wird überprüft, inwieweit das von der Investitionstheorie entwickelte Instrumentarium zur Untersuchung der genannten Problemstellungen auf den Gestaltungsbereich des Informationsmanagements übertragbar ist, um die optimale Nutzungsdauer sowie den optimalen Ersatzzeitpunkt hard- und softwaretechnischer Betriebsmittel zu bestimmen.

Für die Ex ante-Entscheidung über die optimale Nutzungsdauer werden in der dynamischen Investitionstheorie zwei Fälle unterschieden: Im ersten Fall soll eine einmalige Investition vorgenommen werden, die am Ende nicht durch ein gleichartiges oder technisch verändertes Objekt ersetzt wird. Im zweiten Fall wird eine Investitionskette betrachtet, bei der das Investitionsobjekt am Ende seiner Nutzungsdauer einmal oder mehrmals gegen ein Ersatzobjekt ausgetauscht wird. Auf Grund der hohen Entwicklungsgeschwindigkeit des Hard- und Software-Marktes ergibt sich für das Informationsmanagement die Situation, dass der Planungshorizont für eine Ex anteBetrachtung mehrerer Investitionsobjekte nicht lang genug ist. Weder die technischen noch die wirtschaftlichen Eigenschaften zukünftig am Markt angebotener Betriebsmittel sind über einen ausreichend langen Zeitraum absehbar. ${ }^{133}$ Aus diesem Grunde ist eine Ex ante-Betrachtung von Investitionsketten für das Informationsmanagement nicht sinnvoll, es muss sich vielmehr auf die Betrachtung einer einmaligen Investition beschränken, die für sich genommen schon nur schwer prognostizierbar ist. Für die Betrachtung einmaliger Investitionen bietet die dynamische Investitionstheorie das Instrument der Kapitalwertmethode zur Ermittlung der optimalen Nutzungsdauer an, die dann erreicht ist, wenn der Kapitalwert des Objektes als Funktion seiner Nutzungszeit ein Maximum erreicht. ${ }^{134}$ Problematisch ist allerdings die ursächliche Zuord-

\footnotetext{
${ }^{130}$ Vgl. Kern (1992), S. $208 f$.

${ }^{131}$ Vgl. Corsten (2000), S. 354.

${ }^{132}$ Vgl. dazu die ausfuihrliche Darstellung bei Busse v. Colbe/Laßmann (1990), S. 131ff.

${ }^{133}$ So ist es bspw. nicht möglich, bei der Beschaffung eines Datenbankservers zugleich die Beschaffung eines zukünftigen Rechners zu planen, der den unmittelbar zu beschaffenden Rechner irgendwann ablösen wird. Das gleiche gilt im Software-Bereich bspw. für die Entscheidung über die Einfuihrung eines Vertriebs-Informationssystems, das zu einem späteren Zeitpunkt durch eine neue Software, deren Funktionalităt zum Entscheidungszeitpunkt noch unbekannt ist, ersetzt werden soll.

${ }^{134}$ Vgl. Busse v. Colbe/Laßmann (1990), S. 132.
} 
nung von Zahlungsgrößen zu einzelnen Investitionsobjekten. Einzahlungen können Investitionen in Informationstechnik häufig gar nicht unmittelbar zugeordnet werden, sodass sich der Vergleich von Alternativen auf die Auszahlungsseite beschränken muss.

Eine Ex post-Entscheidung über den optimalen Ersatzzeitpunkt ist dann zu treffen, wenn während des Gebrauchs einer Anlage neue Entwicklungen erkennbar werden, die zum Investitionszeitpunkt nicht in das ursprüngliche Kalkül eingeflossen sind. Bei Investitionen in informationstechnische Betriebsmittel dürfte dies wegen der großen Dynamik der Märkte den Normalfall darstellen. Es ist dann zu entscheiden, ob die alte Anlage zum gegenwärtigen Zeitpunkt ersetzt oder zunächst weiter betrieben werden soll, um nach Ablauf einer weiteren Planungsperiode erneut die Ersatzalternative zu überprüfen. ${ }^{135}$ Die Kapitalwertmethode stellt auch für diese Aufgabe ein geeignetes Mittel zum Vergleich der beiden Alternativen dar, wobei sich ebenfalls das Problem der im Regelfall fehlenden Zurechenbarkeit von Einzahlungen zu Alternativen stellt.

Als zweiten Faktor, der die Produktivität von Betriebsmitteln bestimmt, nennt GUTENBERG den Grad der Abnutzung, da die Arbeitsgenauigkeit und der Wirkungsgrad eines Betriebsteils mit zunehmender Benutzung nachlassen. ${ }^{136}$ Je weniger abgenutzte Betriebsmittel im gesamten Anlagenbestand enthalten sind, um so höher ist ceteris paribus dessen technische Ergiebigkeit. Diese Einflussgröße ist nicht analog von Betriebsmitteln für die industrielle Fertigung auf informationstechnische Betriebsmittel übertragbar, da bei der Nutzung von Hard- und insbesondere von Software kaum altersbedingter Verschleiß auftritt, der die technische Performance beeinträchtigt. Sie wird hier deshalb nicht weiter betrachtet.

Schließlich hängt die Produktivität der betrieblichen Apparatur davon ab, in welchem Zustand der Betriebsfähigkeit sich die technischen Einrichtungen befinden. Mängel in der Betriebsmittelpflege haben Störungen des Fertigungsablaufes und damit die Unwirtschaftlichkeit der Leistungserstellung zur Folge. ${ }^{137}$ Derartige Mängel sind allerdings keine unabänderlichen Konsequenzen der Betriebsmittelnutzung, da sowohl nachträgliche als auch vorbeugende Instandhaltungsmaßnahmen möglich sind. ${ }^{138}$ Diese Feststellung ist im Prinzip von Betriebsmitteln zur industriellen Fertigung auf informationstechnische Betriebsmittel übertragbar. Das Informationsmanagement hat deshalb mit Hilfe geeigneter Wartungsmaßnahmen sicherzustellen, dass die Ausfallzeiten der informationstechnischen Betriebsmittel (unter Einhaltung des Wirtschaftlichkeitsprinzips) so gering wie möglich bleiben. Diese Aufgabe betrifft nicht nur die Hardware, sondern auch die Software, bei der während des Nutzungszeitraums Mängel- und Fehlerbeseitigungen, Anpassungen und Aktualisierungen vorzunehmen sind. ${ }^{139}$

\section{b) Unternehmungsspezifische Einflussgrößen}

Außer durch prinzipielle Leistungseigenschaften wird die Leistungsfähigkeit von Betriebsmitteln auch durch ihre Eignung für die unternehmungsspezifischen Anforderungen der Leistungserstellung bestimmt. Als Betriebsmitteleignung wird das Verhältnis zwischen der von den Betriebsmitteln verlangten und der mit ihnen tatsächlich

\footnotetext{
${ }^{135}$ Vgl. Busse v. Colbe/Laßmann (1990), S. 144

${ }^{136} \mathrm{Vgl}$. Gutenberg (1983), S. 72.

${ }^{137}$ Vgl. Gutenberg (1983), S. 73.

${ }^{138}$ Vgl. Kern (1992), S. 209.

${ }^{139}$ Vgl. Schwarze (1998), S. 205.
} 
erzielbaren Leistung bezeichnet. ${ }^{140}$ Die Betriebsmitteleignung bestimmt sich durch die quantitative und qualitative Kapazität sowie durch die fertigungstechnische Elastizität der Betriebsmittel.

Im Bereich der quantitativen Kapazität als produktionsspezifischem Merkmal lassen sich die Maximal-, die Minimal- und die Optimalkapazität unterscheiden ${ }^{141}$ Eine Anlage wird dann besonders kostengünstig genutzt, wenn die durchschnittliche Inanspruchnahme im Rahmen des Leistungserstellungsprozesses ihrer optimalen Kapazität entspricht. Allerdings ist eine gewisse Kapazitätsreserve, also ein Spielraum zwischen Optimal- und Maximalkapazität, zu fordern, damit die Anlage auch vorübergehenden Spitzenbelastungen gewachsen ist. Das Betriebsmittel ist dann für die Unternehmung überdimensioniert, wenn die betriebsgewöhnliche Inanspruchnahme zu nah an seiner Mindestkapazität liegt und nicht mit einer nachhaltigen Erhöhung der Kapazitätsausnutzung zu rechnen ist.

Quantitative Kapazitätsprobleme treten auch bei der Nutzung von Hardware als informationstechnischem Betriebsmittel auf. Merkmale der Hardware-Kapazität sind bei Prozessoren beispielsweise die Rechnerleistung im engeren Sinne (Anzahl der verarbeiteten Befehle je Zeiteinheit) und die Taktfrequenz, bei Speichern beispielsweise die Größe und die Zugriffszeit. ${ }^{122}$ Für die Hardware gelten die gleichen Aussagen zur Kapazität wie zu Betriebsmitteln im Bereich der industriellen Fertigung. Eine zu hohe Auslastung nahe an der Maximalkapazität von Prozessoren, von Speichermedien und auch von Übertragungsmedien führt zu unakzeptablen Verarbeitungs- und Antwortzeiten. Dagegen lässt eine Nutzung der Hardware an der unteren Kapazitätsgrenze vermuten, dass die entsprechenden Geräte für die Unternehmung überdimensioniert sind. Im Vergleich zu Kapazitätsentscheidungen bei industriellen Produktionsanlagen unterscheiden sich Kapazitätsentscheidungen im HardwareBereich durch zwei Aspekte: Auf der einen Seite ist eine Überdimensionierung wegen der anhaltenden rapiden Verbesserung des Preis/Leistungsverhältnisses der am Markt angebotenen Informationstechnik, die eine kostengünstigere Beseitigung von Kapazitätsengpässen in der Zukunft erlaubt, in besonderem Maße unwirtschaftlich. Auf der anderen Seite jedoch fällt eine Überdimensionierung wegen der absolut gesehen geringen Kosten der Hardware im Vergleich zu industrieller Produktionstechnik weniger ins Gewicht.

Die Software-Kapazität ist schwieriger $\mathrm{zu}$ definieren und $\mathrm{zu}$ messen als die Hardware-Kapazität. Kapazitätsmerkmale bei Betriebssystemen sind die Zahl (unterschiedlicher) Peripheriegeräte, die damit betrieben werden können, die möglichen Betriebsarten und der verfügbare Befehlsvorrat. ${ }^{143}$ Die Kapazität von Anwendungsprogrammen hängt von ihrer hardware- und aufgabenbezogenen Optimierung und der Zahl der möglichen Anwender ab. Das Informationsmanagement hat die kapazitätsmäßige Ausstattung der softwaretechnischen Elemente des technikunterstützten Informationssystems der Unternehmung so auszulegen, dass die betreffende Software

\footnotetext{
${ }^{140}$ Vgl. Gutenberg (1983), S. 73.

${ }^{141}$ Vgl. zum Folgenden Gutenberg (1983), S. 73ff.

${ }^{142} \mathrm{Vgl}$. Hansen (1996), S. 568f.

${ }^{143}$ Vgl. Hansen (1996), S. 49.
} 
durch die abzuwickelnden Informationsverarbeitungsprozesse möglichst entsprechend ihrer Optimalkapazität ausgelastet wird. ${ }^{144}$

Die qualitative Kapazität als zweites produktionsprozessspezifisches Merkmal der Leistungsfähigkeit von Betriebsmitteln bezieht sich auf die Eigenschaften und die Güte der Leistungen, die sie abzugeben in der Lage sind. ${ }^{145}$ Analog zur quantitativen Kapazität ist auch für die qualitative Kapazität eines Betriebsmittels die Vermeidung von Unter- oder Überbeanspruchung durch den Prozess der Leistungserstellung zu fordern. Der Eignungswert eines Betriebsmittelbestandes ist um so höher, je mehr seine betriebliche Inanspruchnahme seinem qualitativen Potenzial entspricht. In Prozessen der Informationsverarbeitung beruht die Qualität des Output weniger auf der qualitativen Kapazität der Hardware sowie der System- und der systemnahen Software, als auf der Qualität der eingesetzten Anwendungssoftware. Bei der hier besonders betrachteten betriebswirtschaftlichen Anwendungssoftware als speziellem Anwendungssoftwaretyp hängt die Genauigkeit und Zuverlässigkeit der produzierten Ergebnisse von der Möglichkeit zur Parametrisierung, d.h. zur Anpassung der Software an die Gegebenheiten der Unternehmung, ab. Das Informationsmanagement hat die diesbezüglichen Anforderungen (z.B. Anforderungen an die Genauigkeit der Abbildung von Produktionsprozessen zum Zwecke der Kalkulation, Anforderungen an die Flexibilität von Auswertungstools) zu bestimmen und die qualitative Kapazität der Software entsprechend auszulegen.

Die fertigungstechnische Elastizität stellt gemäß der GUTENBERG'schen Systematik das dritte Element der Eignung von Betriebsmitteln für die unternehmungsspezifische Art der Leistungserstellung dar. Damit ist die Möglichkeit gemeint, durch geänderte Absatzverhältnisse oder andere Veränderungen erforderliche Anpassungen des Produktionsprogramms mit Hilfe von Umgestaltungsmaßnahmen im Betriebsmittelbestand vorzunehmen. ${ }^{146}$ Die Häufigkeit und Tiefe der vorzunehmenden Umstellungen und damit die Anforderungen an die fertigungstechnische Elastizität der Produktionsmittel unterscheidet sich von Unternehmung zu Unternehmung entsprechend der unternehmungsspezifischen Wettbewerbssituation. GUTENBERG unterscheidet zwischen Universal- und Sondermaschinen als Betriebsmitteltypen mit unterschiedlicher fertigungstechnischer Elastizität. Während an Universalmaschinen sämtliche Tätigkeiten einer bestimmten Arbeitsart an verschiedenartigen Werkstücken durchgeführt werden können, sind Sondermaschinen dadurch gekennzeichnet, dass sie sich entweder nur für einen ganz bestimmten Arbeitsgang oder für ganz wenige Arbeitsgänge grundsätzlich gleicher Art verwenden lassen. Universalmaschinen sind flexibler einsetzbar als Sondermaschinen. Ihre hohe betriebstechnische Elastizität wird aber in der Regel durch eine verhältnismäßig ungünstige Kostensituation erkauft. Umgekehrt besteht die kostenmäßige Vorteilhaftigkeit von Sondermaschinen gegenüber Universalmaschinen jedoch nur dann, wenn die Sondermaschinen auch tatsächlich gemäß ihres ursprünglichen Konstruktionszweckes genutzt werden. Daraus folgt, dass die Eignung von Betriebsmitteln für die unternehmungsspezifischen Anforderungen mit ihrem Speziali-

\footnotetext{
${ }^{144}$ Diese Aussage impliziert die Annahme, dass der Betrieb einer Anlage bei Optimalkapazität auch gegenüber anderen, alternativen Anlagen vorteilhaft ist, die entweder höhere variable oder höhere fixe Kosten aufweisen.

${ }^{145} \mathrm{Vgl}$. Gutenberg (1983), S. 77.

${ }^{146}$ Vgl. zum Folgenden Gutenberg (1983), S. 81ff.
} 
sierungsgrad steigt, wenn die technischen und wirtschaftichen Gegebenheiten der Produktion ein erhöhtes $\mathrm{Ma} ß$ an Spezialisierung zulassen und umgekehrt. ${ }^{147}$

Bezogen auf den Gestaltungsbereich des Informationsmanagements lassen sich in diesem Zusammenhang deutliche Parallelen zur Entscheidung über die Verwendung von Individual- oder Standardsoftware herstellen. Individualsoftware ist dabei mit Sondermaschinen, die auf eine ganz bestimmte Art von Prozessen spezialisiert sind, vergleichbar. Bei der Entwicklung von Individualsoftware für eine Unternehmung können deren besondere Anforderungen an die Prozesse der Informationsverarbeitung berücksichtigt werden. Deshalb wird eine hohe Spezialisierung auf die Unternehmung und damit eine besondere unternehmungsspezifische Eignung des Betriebsmittels erreicht. Dagegen ist Standardsoftware, die analog zu Universalmaschinen behandelt werden kann, weniger auf die speziellen Verhältnisse einer bestimmten Unternehmung als auf die unabhängig von der Unternehmungssituation bestehenden Grundzüge der zu unterstützenden betrieblichen Prozesse ausgerichtet. Durch die vom Hersteller vorgesehenen Möglichkeiten zur Parametrisierung, die auch als Customizing bezeichnet wird, kann sie (in gewissen Grenzen) an die besonderen Gegebenheiten einer Unternehmung angepasst werden.

Die Schlussfolgerung über die Vorteilhaftigkeit von Standard- oder Individualsoftware ergibt sich analog zur Schlussfolgerung über die unternehmungsspezifische Eignung von Universal- oder Sondermaschinen: Wenn die technischen und wirtschaftichen Gegebenheiten einer Unternehmung eine hohe betriebstechnische Elastizität der verwendeten Betriebsmittel verlangen, dann weist Standardsoftware tendenziell eine höhere Eignung als Individualsoftware auf, da sie mittels Customizing-Aktivitäten oder Austausch gegen andere Software-Produkte leichter an veränderte Anforderungen angepasst werden kann. Befindet sich die Unternehmung jedoch in einer Situation, in der die funktionalen Anforderungen an die verwendete Software im Zeitablauf nur wenigen Änderungen unterworfen sind, so sind die Voraussetzungen günstig für den Einsatz von Individualsoftware. Wenn die zu unterstützenden Prozesse der Informationsverarbeitung stark spezialisiert sind, dann ist es in diesem Fall günstiger, individuell ausgestaltete Anwendungssoftware einzusetzen.

Es ist darauf hinzuweisen, dass neuere Ansätze zur objektorientierten Programmierung, bei denen die Anwendungssoftware aus weitgehend unabhängig voneinander funktionierenden kleineren Modulen zusammengesetzt wird, die Flexibilität von individuell programmierter Software erhöhen können. Dadurch lässt sich der Vorteil verringern, den Standardsoftware in dynamischen Situationen mit hohen Anforderungen an die Elastizität der Betriebsmittel gegenüber Individualsoftware aufweist. Generell ist allerdings anzunehmen, dass es nicht zu einer vollständigen Auflösung des beschriebenen Zusammenhangs kommt, da auch die Hersteller von Standardsoftware bei der Entwicklung ihrer Produkte objektorientierte Konzepte zu Grunde legen können, um ein noch höheres $\mathrm{Maß}$ an Flexibilität zu erreichen.

\footnotetext{
${ }^{147}$ Den methodischen Leitideen des Faktortheoretischen Ansatzes folgend wird in der Literatur zum Teil versucht, durch die Angabe von Marginalbedingungen ein optimales Flexibilitätsniveau anzugeben, bei dem Grenznutzen und Grenzkosten der Flexibilităt übereinstimmen. Vgl. z.B. Reichwald/Behrbohm (1983), S. 831ff.; Mössner (1982), S. 334. Diese Ansätze werden hier wegen der bestehenden Operationalisierungsprobleme und wegen der in Abschnitt 3.4.2.1 erlảuterten grundsătzlichen Vorbehalte gegenüber einer Überbetonung des Gleichgewichtsdenkens nicht näher betrachtet.
} 
Im Hardwarebereich sowie bei der System- und systemnahen Software besteht die Option zum Einsatz unternehmungsspezifisch konstruierter Betriebsmittel praktisch nicht. Hier ist die Unternehmung im Normalfall auf die am Markt angebotenen Informationstechniken angewiesen. Nur in Ausnahmefällen ist auch im Hardware-Bereich die Möglichkeit zur Entwicklung und Verwendung spezieller Komponenten zu überprüfen. Dabei sind dann die gleichen Kriterien anzuwenden wie bei der Entscheidung zwischen individuellen oder standardisierten Software-Systemen.

\subsubsection{Ergiebigkeit von Werkstoffen bei der Erstellung informationeller Produkte}

Die Werkstoffe als dritter Elementarfaktor beeinflussen die Produktivität von Produktionsprozessen insofern, als Unterschiede in ihrer Beschaffenheit unterschiedlichen Werkstoffverbrauch und unterschiedlichen Einsatz der übrigen Elementarfaktoren zur Folge haben. Das fertigungstechnische Optimum wird dann erreicht, wenn die fertigungstechnischen Eigenschaften des Werkstoffes ein Minimum an Werkstoffverbrauch und an Einsatz der übrigen Elementarfaktoren bewirken.

\section{a) Anwendbarkeit von Kriterien für das fertigungstechnische Optimum in Prozessen der industriellen Produktion}

GUTENBERG nennt drei Bedingungen für die Erreichung des fertigungstechnischen Optimums: ${ }^{148}$ Die Werkstoffersparnis ist erstens um so größer, je geringer der Unterschied zwischen eingesetzter Rohmenge und Fertiggewicht, je geringer also der Materialverlust ist. Der Werkstoffverbrauch ist zweitens um so geringer, je mehr von der Möglichkeit Gebrauch gemacht wird, standardisiertes oder genormtes Material zu verwenden. Drittens schließlich gestalten sich der Verbrauch und die Bearbeitung von Werkstoffen um so günstiger, je mehr die Werkstoffe den spezifischen Anforderungen der Fertigung im Einzelfall entsprechen. Diese Bedingungen sind in Abbildung 3/6 zusammengefasst.

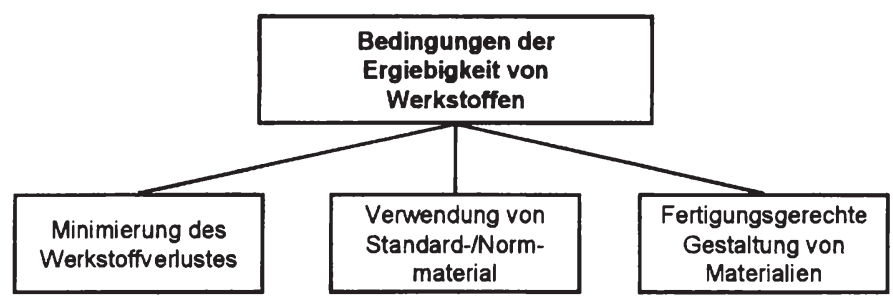

Abb. 3/6: Bedingungen der Ergiebigkeit von Werkstoffen

Betrachtet man diese Bedingungen näher, so tritt besonders deutlich zu Tage, dass sich das Erkenntnisinteresse des Faktortheoretischen Ansatzes in erster Linie auf industrielle Fertigungsprozesse und nicht auf die Erzeugung informationeller Produkte richtet. Obwohl der entwickelte Bedingungskatalog nicht unmittelbar auf Prozesse der Informationsverarbeitung übertragbar ist, können allerdings doch einige Anhaltspunkte für die Verwendung von Informationen als Werkstoffen abgeleitet werden.

\footnotetext{
${ }^{148}$ Vgl. Gutenberg (1983), S. 123.
} 
Insbesondere die erste und die dritte Bedingung, also die Minimierung des Materialverlustes und die fertigungsgerechte Gestaltung der Werkstoffe ergeben im Hinblick auf die Verarbeitung von Informationen nur wenig Sinn. Werkstoffverluste entstehen durch Abfälle, Nebenprodukte und Ausschuss. In Prozessen der Informationsverarbeitung ist jedoch kein Werkstoffverlust feststellbar, da die ursprünglichen Informationen als Einsatzstoffe nicht untergehen und somit nicht verbraucht werden. ${ }^{149}$ Allenfalls kann das Kriterium abgeleitet werden, in Informationsverarbeitungsprozessen zur Minimierung des Werkstoffeinsatzes möglichst wenig „Datenabfall“, der für die zu erstellende Leistung eigentlich nicht benötigt wird (z.B. zu stark detaillierte Auflistungen oder von den Empfängern unbeachtete Standardberichte), zu produzieren. Eine fertigungsgerechte Gestaltung von Materialien soll nicht nur die Materialabfälle gering halten, sondern auch die Bearbeitungszeit verringern und dadurch die kapazitätsmäßige Auslastung der übrigen Elementarfaktoren reduzieren. ${ }^{150}$ Aus dieser Bedingung ergibt sich für die syntaktische Ebene der Informationsverarbeitung, dass Informationen bereits in einer unmittelbar verarbeitbaren Form in Informationsverarbeitungsprozesse einfließen sollen, damit sie vor ihrer Verarbeitung nicht noch umgewandelt werden müssen (z.B. durch die Konvertierung in andere Dateiformate, die Extraktion aus umfangreichen Listen o.ä.).

GUTENBERG stellt fest, dass die Forderung nach minimalem Werkstoffverlust, minimalem Aufwand an Arbeitsleistung, Betriebsmittelnutzung und Bearbeitungszeit in ganz besonderem Maße durch standardisiertes oder genormtes Material erfüllt wird. ${ }^{151}$ Die Normung kann sich auf quantitative und auf qualitative Eigenschaften des zu verwendenden Werkstoffes beziehen. Der Rationalisierungseffekt hängt nicht nur von der Zahl der Gegenstände ab, die durch Normung erfasst werden, sondern auch von der Größe des Geltungsbereiches der Normung. Er beruht auf dem Wegfall von Umstellungen, Entwürfen, Werkstattzeichnungen, Mustern usw., die mit einem häufigen Wechsel der eingesetzten und der hergestellten ${ }^{152}$ Gegenstände verbunden sind. Durch eine zu weitgehende Normung besteht allerdings die Gefahr der Erstarrung des Produktionsprozesses.

Diese Überlegungen können im Grundsatz auf die Verarbeitung von Informationen übertragen werden. Die Normung bezieht sich dabei auf die Art und Weise, in der informationelle Objekte zwischen den am Informationsverarbeitungsprozess Beteiligten übergeben werden. Die Übergabe dieser Objekte zwischen den Teilsystemen bzw. Systemelementen des technikunterstützten Informationssystems der Unternehmung erfolgt über Schnittstellen, die die Verbindungen zwischen den verschiedenen Akteuren herstellen. ${ }^{153}$ Durch eine geeignete Schnittstellenverwaltung wird es möglich, auf der Basis festgelegter Eigenschaften, über die die zu übertragenden Informationen verfugen sollen, den Informationsaustausch zwischen unterschiedlichen Aufgabenträgern auf der syntaktischen Ebene zu automatisieren und damit zu rationalisieren. Durch festgelegte Eigenschaften der zu übergebenden informationellen Objekte werden Aktivitäten zur Umwandlung der Information in die benötigte Form reduziert und zudem auf der semantischen Ebene Unklarheiten über die richtige Interpretation

\footnotetext{
${ }^{149} \mathrm{Vgl}$. Streubel (1996), S. 43.

${ }^{150} \mathrm{Vgl}$. Gutenberg (1983), S. 129f.

${ }^{151} \mathrm{Vgl}$. dazu Gutenberg (1983), S. 127ff.

${ }^{152}$ Die Normung von herzustellenden Gegenständen wird als Typung bezeichnet.

${ }^{153} \mathrm{Vgl}$. Biethahn/Mucksch/Ruf (1994), S. 97.
} 
beseitigt. Die Normung von Informationen kann sich beispielsweise auf Eigenschaften wie den Aggregationsgrad, die Darstellungsweise und die Aktualität beziehen.

\section{b) Überprüfung spezieller Werkstoffeigenschaften von Informationen}

Es ist zu überprüfen, ob sich Informationen als Werkstoffe möglicherweise durch typische Merkmale von Werkstoffen in industriellen Fertigungsprozessen unterscheiden und das fertigungstechnische Optimum der Informationsverarbeitung deshalb an weitere Bedingungen geknüpft ist. Die Eigenschaften, die Information als Wirtschaftsgut auszeichnen, sind in der Vergangenheit im Zusammenhang mit der „Entdeckung" des Informationsmanagements in der wissenschaftlichen Diskussion bereits mehrfach untersucht worden. ${ }^{154}$ Dies geschah jedoch nicht unter produktionswirtschaftlichen Gesichtspunkten, sondern mit der Zielsetzung, eine gesonderte Behandlung der Ressource Information durch eine eigene Disziplin Informationsmanagement zu rechtfertigen. Ein informationswirtschaftliches Optimum in Analogie zum produktionswirtschaftlichen Optimum wird zwar an einigen Stellen gefordert, ${ }^{155}$ was darunter zu verstehen ist, bleibt bisher jedoch unklar.

Aus der Perspektive eines Produktionsorientierten Ansatzes für eine Theorie des Informationsmanagements unterscheiden die meisten angefürten Eigenschaften Informationen nicht von Werkstoffen, die in industriellen Fertigungsprozessen verwendet werden. Dies gilt beispielsweise für folgende Feststellungen: ${ }^{156}$

- Bei Informationen handelt es sich um knappe Güter,

- deren Verarbeitung mit Kosten verbunden ist

- und Nutzen stiftet,

- die sich durch unterschiedliche Qualitäten auszeichnen,

- deren Bestandsbewertung problematisch ist,

- deren Wert von der kontextspezifischen sowie der zeitlichen Verwendung abhängt,

- deren Wert durch Hinzufügen, Selektieren, Konkretisieren und Weglassen (also allgemein: durch Bearbeitung) verändert werden kann.

Bei dem ebenfalls angeführten Merkmal, dass die Vervielfältigungskosten von Informationen niedrig sind, ${ }^{157}$ handelt es sich zwar um einen Aspekt, der Informationen von den meisten anderen Werkstoffen unterscheidet. Für die hier untersuchte Frage des fertigungstechnischen Optimums besitzt dieser Punkt jedoch keine Relevanz. Die Vervielfältigungskosten betreffen die wertmäßige Betrachtung von Informationsverarbeitungsprozessen, während sich die fertigungstechnische Analyse ausschließlich auf den mengenmäßigen Verbrauch des Werkstoffes und der anderen Elementarfaktoren richtet und deshalb der wertmäßigen Analyse vorgelagert ist. Die Kostenbetrachtung erfolgt im Faktortheoretischen Ansatz erst nach der Produktionsbetrachtung durch die Bewertung der bei der fertigungstechnisch optimierten Produktion verbrauchten

\footnotetext{
${ }^{154}$ Vgl. z.B. Martiny/Klotz (1998), S. 18f.; Lehner/Maier (1994), S. 12f;; Picot/Franck (1988), S. 545; Krcmar (1991), S. 172; Streubel (1996), S. $41 \mathrm{ff}$.

${ }^{155} \mathrm{Vgl}$. Schwarzer (1994), S. 96; ähnlich auch Link (1982), S. $265 \mathrm{f}$.

${ }^{156} \mathrm{Vgl}$. ähnlich Martiny/Klotz (1998), S. 23.

${ }^{157} \mathrm{Vgl}$. Martiny/Klotz (1998), S. 23.
} 
Faktormengen mit Faktorpreisen. Auch aus der Möglichkeit des vielfachen Besitzes einer Information, aus der u.a. die Unmöglichkeit einer exklusiven Übertragung der Information, bei der der Käufer das Original erwirbt, resultiert, kann keine neue Bedingung der optimalen Ergiebigkeit des Werkstoffes Information in Informationsverarbeitungsprozessen abgeleitet werden.

Die Aussagen des Faktortheoretischen Ansatzes zur Ergiebigkeit des Werkstoffeinsatzes eignen sich also nur sehr eingeschränkt für eine Übertragung auf den Bereich der Informationsverarbeitung. Es ist lediglich festzustellen, dass durch die Festlegung von Standards für den Informationsaustausch zwischen den beteiligten Aufgabenträgern der Prozess der Informationsverarbeitung auf Grund der reduzierten Inanspruchnahme der Elementarfaktoren menschliche Arbeitsleistungen und Betriebsmittel rationalisiert und auf diesem Wege die Ergiebigkeit des Werkstoffes Information erhöht werden kann.

\subsubsection{Anordnung der Elementarfaktoren zur Vorbereitung von Produktions- prozessen}

Vor der tatsächlichen Durchführung von Produktionsprozessen im Rahmen der Endkombination ist es erforderlich, die bisher jeweils isoliert betrachteten Elementarfaktoren zu Produktionssystemen zusammenzufassen. In der Literatur werden verschiedene Kriterien verwendet, um die unüberschaubare Vielfalt realer Produktionssysteme zu strukturieren. ${ }^{158}$ Die Ausführungen beschränken sich an dieser Stelle auf die prozessorientierte Art der Typisierung. Andere Klassifikationsmerkmale wie etwa die vorherrschende Technologie oder die Art der Stoffverwertung dienen zum einen vorwiegend beschreibenden Untersuchungszielen und besitzen zum anderen keinen bzw. kaum Bezug zur technikunterstützten Informationsverarbeitung. In Bezug auf die Art der Produktionsprozesse werden bei der Anordnung der Elementarfaktoren unterschiedliche Gestaltungsprinzipien unterschieden. Auf der obersten Abstraktionsebene handelt es sich dabei um das Verrichtungsprinzip und das Prozessfolgeprinzip. ${ }^{159}$ Diese Unterscheidung ist in Abbildung 3/7 dargestellt. Die genannten Prinzipien sind grundsätzlich auf den Gestaltungsbereich des Informationsmanagements übertragbar, und ihre charakteristischen Merkmale bieten dort ebenfalls Anhaltspunkte für Gestaltungsmaßnahmen.

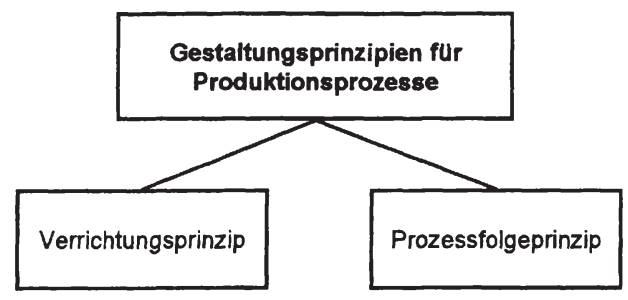

Abb. 3/7: Gestaltungsprinzipien für Produktionsprozesse

\footnotetext{
${ }^{158}$ Vgl. Corsten (2000), S. 31. Für einen Überblick über verschiedene Klassifikationsmerkmale vgl. Kern (1992), S. 94.

${ }^{159} \mathrm{Vgl}$. Corsten (2000), S. 33; Kern (1992), S. 91.
} 
Das Verrichtungsprinzip ist dadurch gekennzeichnet, dass gleichartige Tätigkeiten zu fertigungstechnischen Einheiten im Produktionsprozess zusammengefasst werden. ${ }^{160}$ Die wichtigste Erscheinungsform dieser fertigungstechnischen Einheiten wird auch als Werkstatt und die entsprechende Art des Herstellungsprozesses als Werkstattfertigung bezeichnet. ${ }^{161}$ Bei der Werkstattfertigung von industriellen Produkten passt sich der Materialfluss den Gegebenheiten einer räumlichen und kaum veränderlichen Werkstättenlokalisierung an. ${ }^{162}$ Diese Art der Anordnung von Elementarfaktoren wird dann als vorteilhaft angesehen, wenn eine ständig variierende Auftragsstruktur mit veränderlichen Materialflüssen gegeben ist. ${ }^{163}$ Die Spezialisierung von Personal und Betriebsmitteln richtet sich unter diesen Bedingungen auf die Art der Tätigkeit (Verrichtung), wobei die verschiedenen Tätigkeiten flexibel zur Herstellung unterschiedlicher Produkte miteinander kombiniert werden können. Die Werkstücke durchlaufen die verschiedenen Werkstätten also auf unterschiedlichen Fertigungspfaden. ${ }^{164}$ Auf Grund hoher Kosten des Materialtransports, langer Durchlaufzeiten, hohen Raumbedarfs und vergleichsweise intensiver Arbeitsvorbereitung stellt die Werkstattfertigung nur dann eine wirtschaftliche Lösung dar, wenn keine stärkere Standardisierung der Produktionsprozesse möglich erscheint. ${ }^{165}$

Ist durch eine höhere Gleichartigkeit der Produktionsanforderungen die Voraussetzung für eine stärkere Standardisierung der Produktionsprozesse gegeben, so können die Elementarfaktoren nach dem Prozessfolgeprinzip zusammengefasst werden. Die entsprechende Art des Herstellungsprozesses wird als FlieBfertigung bezeichnet. ${ }^{166}$ Bei der Fließfertigung werden die jeweiligen Arbeitsplätze in der Reihenfolge der an den Materialen zu verrichtenden Tätigkeiten angeordnet. Dies reduziert die Transportwege und ermöglicht eine schnellere Fertigstellung des Endprodukts. Da Änderungen der Prozesse größeren Umstellungsaufwand erfordern, ist eine solche Anpassung des Produktionssystems an die Reihenfolge der Tätigkeiten allerdings nur dann sinnvoll, wenn das Produktionsprogramm keinen kurzfristigen Veränderungen unterliegt. Wenn zusätzlich zur Anordnung nach der Reihenfolge eine feste zeitliche Abstimmung zwischen den Arbeitsplätzen vorgesehen ist, dann wird von Fließfertigung mit Zeitzwang gesprochen. Der Zeitzwang ermöglicht - zu Lasten der Flexibilität (Rückflüsse oder Überspringen von Produktionsstellen sind nicht möglich) - eine noch friktionsfreiere Abarbeitung der einzelnen Vorgänge.

Wendet man die beschriebenen Gestaltungsprinzipien analog auf Prozesse im Bereich der technikunterstützten Informationsverarbeitung an, so treten die erläuterten charakteristischen Merkmale in nahezu gleicher Form auf. Einschränkend ist lediglich festzustellen, dass die Übertragung von Informationen als Werkstoffen wegen der Verfügbarkeit leistungsfähiger Netzwerke auch über größere Strecken hinweg relativ

\footnotetext{
${ }^{160} \mathrm{Vgl}$. Gutenberg (1983), S. $96 \mathrm{f}$.

${ }^{161}$ Vgl. Gutenberg (1983), S. 97; Corsten (2000), S 33. Kern (1992), S. 91, nennt die Werkbankfertigung, bei der eine Person einen Einzelauftrag bis zu seiner Fertigstellung komplett bearbeitet, als weitere Erscheinungsform des Verrichtungsprinzips. Diese spielt in arbeitsteiligen industriellen Prozessen jedoch nur eine sehr untergeordnete Rolle und wird daher nachfolgend nicht weiter betrachtet.

${ }^{162}$ Vgl. Kern (1992), S. 91.

${ }^{163}$ Vgl. Corsten (2000), S. 34; Adam (1998), S. 5; Kern (1992), S. 92.

${ }^{164} \mathrm{Vgl}$. Nebl (1996), S. 235.

${ }^{165}$ Vgl. Gutenberg (1983), S. $97 f$.

${ }^{166}$ Vgl. zum Folgenden Corsten (2000), S. 34f.; Kern (1992), S. $92 f$.
} 
kostengünstig erfolgen kann. Folglich kommt reinen Transportproblemen weniger entscheidende Bedeutung zu, als dies bei der industriellen Fertigung der Fall ist. Die Frage der prozessbezogenen Anordnung ist also bei der Zusammenfassung von Elementarfaktoren der technikunterstützten Informationsverarbeitung weniger unter räumlichen, als unter logisch-inhaltlichen Gesichtspunkten zu betrachten. Die übrigen oben angesprochenen Charakteristika kennzeichnen jedoch auch im Gestaltungsbereich des Informationsmanagements das Verrichtungsprinzip und das Prozessfolgeprinzip.

Eine Anordnung der menschlichen Aufgabenträger und der informationstechnischen Betriebsmittel, die dem Grundkonzept der Werkstattfertigung entspricht, lässt wie bei der industriellen Fertigung auf der einen Seite eine flexible und kurzfristige Anpassung des Produktionsprogramms an situative Gegebenheiten zu. Auf der anderen Seite erfordert sie jedoch vor der Durchführung der einzelnen Prozessschritte im Vergleich zum Prozessfolgeprinzip intensivere Einarbeitungsphasen durch die beteiligten menschlichen Aufgabenträger und längere Wartezeiten an den einzelnen Bearbeitungsstellen. Dagegen ist die Anordnung der Elementarfaktoren nach dem Prozessfolgeprinzip auch im Gestaltungsbereich des Informationsmanagements durch kürzere Durchlaufzeiten sowie eine geringere Anpassungsflexibilität des Produktionssystems gekennzeichnet.

Für das Informationsmanagement kann deshalb die Schlussfolgerung gezogen werden, dass das im Rahmen der Vorkombination bereitgestellte Produktionspotenzial nicht allein von der Ergiebigkeit der jeweils isoliert betrachteten einzelnen Elementarfaktorarten im Bereich der technikunterstützten Informationsverarbeitung abhängt. Vielmehr besitzt auch die logisch-inhaltliche Anordnung der Potenzialfaktoren, aus der sich Restriktionen für die Art der im Rahmen der Endkombination durchführbaren Produktionsprozesse ergeben, Einfluss auf die Höhe dieses Produktionspotenzials. Im Rahmen der Vorkombination der Elementarfaktoren wird damit die bewusste Entscheidung zwischen Verrichtungsprinzip und Prozessfolgeprinzip zur wichtigen Gestaltungsvariablen für das Informationsmanagement.

Bei der Gestaltung von Informationsverarbeitungsprozessen, die sich häufig in gleicher Form wiederholen und für die nur selten größere Änderungen des Produktionsablaufs vorgenommen werden, bewirkt eine Orientierung am Prozessfolgeprinzip ein höheres Produktionspotenzial als eine Orientierung am Verrichtungsprinzip. Diese Voraussetzungen liegen in erster Linie bei den im nächsten Abschnitt noch näher betrachteten Routine- und Regelaufgaben vor. Die Umsetzbarkeit dieses Prinzips hängt im Einzelfall allerdings zusätzlich noch vom mengenmäßigen Prozessaufkommen ab. ${ }^{167}$ Wenn das Fertigungsvolumen nicht hoch genug für eine Spezialisierung der Elementarfaktoren nach dem Prozessfolgeprinzip ist, dann ist trotz des Vorliegens der genannten Voraussetzungen auch bei diesen Aufgabentypen das Verrichtungsprinzip vorzuziehen. Generell gewährleistet die Gestaltung nach dem Verrichtungsprinzip bei solchen Informationsverarbeitungsprozessen eine höhere Effizienz, die sich in ihren Ablaufeinzelheiten seltener wiederholen bzw. häufigeren Änderungen unterliegen. Diese Voraussetzungen liegen insbesondere bei den im nächsten Abschnitt ebenfalls noch näher betrachteten Projekt- und Einzelfallaufgaben vor, für die eine eindeutige Reihenfolge der abzuarbeitenden Aktivitäten nicht vorgegeben werden kann.

${ }^{167}$ Zur Beziehung zwischen Gestaltungsprinzip und mengenmäßigem Produktionsaufkommen vgl. allgemein Adam (1998), S. 5f. 
Neuere Konzepte der prozessbezogenen Anordnung von Elementarfaktoren zielen im Bereich der industriellen Fertigung darauf ab, die jeweiligen Vorteile der beschriebenen Gestaltungsprinzipien miteinander zu kombinieren und zugleich deren Nachteile zu vermeiden. ${ }^{168}$ An dieser Stelle sei exemplarisch das Konzept der Fertigungsinsel genannt, nach dem einzelne Arbeitsgruppen umfangreiche Aufgabenkomplexe ehemals hintereinander liegender Arbeitsstationen übernehmen und die dazu gehörenden dispositiven Aufgaben autonom lösen. ${ }^{169}$ Bei der Einrichtung von Fertigungsinseln werden die Elementarfaktoren so zusammengefasst, dass weitgehend unabhängige Fertigungsbereiche entstehen. Diese nehmen selbstständig die Herstellung einzelner Produkte bzw. Zwischenprodukte vor, die ähnliche Fertigungsanforderungen aufweisen und daher auch als Teilefamilien bezeichnet werden. Die verschiedenen Fertigungsinseln in einem Betrieb werden nur durch eine so genannte Rumpf-Fertigungssteuerung zentral koordiniert.

Das Fertigungsinselkonzept besitzt auch für den Gestaltungsbereich des Informationsmanagements erhebliches heuristisches Potenzial, da die Umsetzung des Prozessfolgeprinzips in der Praxis häufig am zu geringen Prozessvolumen scheitert. Durch eine geeignete Aufteilung des Produktionsprogramms mit weitgehend getrennten Zuständigkeiten für die Herstellung ähnlicher informationeller Produkte kann es gelingen, das Prozessfolgeprinzip innerhalb der Gruppe situationsspezifisch anwendbar zu machen. $\mathrm{Da}$ alle von der Gruppe hergestellten Produkte ähnliche Anforderungen an die durchzufuihrenden Prozesse der technikunterstützten Informationsverarbeitung aufweisen, ist die Anpassung der Gruppe an das jeweilige Produktionsprogramm der Periode ohne größeren Umstellungsaufwand möglich.

\subsubsection{Endkombination von Elementarfaktoren im Bereich der technikunter- stützten Informationsverarbeitung}

Durch die im vorigen Abschnitt untersuchte Vorkombination erstellt das Informationsmanagement ein Potenzial zur Erzeugung informationeller Produkte, das für die eigentliche Erstellung der Produkte, die erst nach der Vorkombination stattfindet, genutzt werden kann. Der Transformationsprozess zur Herstellung des Produktionsergebnisses wird auch als Endkombination oder Throughput bezeichnet. Dabei kommt es darauf an, die Einsatzmengen der Elementarfaktoren so zu verknüpfen, dass ein möglichst produktiver Effekt der Gesamtkombination zu Stande kommt. ${ }^{170}$ Während die Elementarfaktoren bisher also vor Aufnahme der eigentlichen Leistungserstellung betrachtet wurden, rückt nun ihr möglichst produktives Zusammenwirken im Rahmen von Produktionsprozessen in den Mittelpunkt der Analyse.

Der Faktortheoretische Ansatz führt die vielgestaltigen Formen und Arten betrieblicher Leistungserstellung auf die Beziehung zwischen Faktorertrag und Faktoreinsatz zurück, die als Grundbeziehung des industriellen Produktionsprozesses angesehen wird. Die wichtigsten Konzepte zur Untersuchung dieser Beziehung stellen die Produktionsfunktion und die daraus abgeleitete Kostenfunktion dar. Im Folgenden wird überprüft, ob und in welcher Weise eine Anwendung dieser Konzepte im Gestaltungs-

\footnotetext{
${ }^{168}$ Vgl. Corsten (2000), S. 36.

${ }^{169}$ Vgl. dazu Mónig (1985), S. 83ff.; Bohr/Eberwein (1989), S. 218ff.; Kern (1992), S. 93. Weitere Konzepte sind etwa das der Flexiblen Fertigungszelle und der Flexiblen Fertigungssysteme.

${ }^{170} \mathrm{Vgl}$. Gutenberg (1983), S. 299.
} 
bereich des Informationsmanagements möglich ist. Abschnitt 3.3.2.1 konzentriert sich zunächst auf produktionstheoretische Aspekte und analysiert demzufolge mengenmäßige Zusammenhänge zwischen Faktoreinsatz und Faktorertrag im Bereich der technikunterstützten Informationsverarbeitung. Der anschließende Abschnitt 3.3.2.2 geht von diesen mengenmäßigen Zusammenhängen aus und richtet sich auf die Untersuchung von Kosten, zu deren Zweck zusätzlich wertmäßige Gesichtpunkte zu berücksichtigen sind.

\subsubsection{Erkenntnispotenziale der Produktionstheorie für die technikunterstützte Informationsverarbeitung}

Die Produktionstheorie strebt nach formalen Erkenntnissen über die quantitativen Beziehungen zwischen Faktoreinsatz und Faktorertrag bei der Kombination von Elementarfaktoren in Produktionsprozessen. ${ }^{171} \mathrm{Im}$ Folgenden werden die Erkenntnispotenziale untersucht, die das innerhalb des Faktortheoretischen Ansatzes verwendete produktionstheoretische Instrumentarium für den Gestaltungsbereich des Informationsmanagements aufweist. Nach einer grundsätzlichen Überprüfung der Anwendbarkeit des Produktionsfunktionskonzeptes wird die Frage untersucht, welcher Produktionsfunktionstyp sich zur Abbildung bestimmter Prozesse der technikunterstützten Informationsverarbeitung am besten eignet. Anschließend wenden sich die Überlegungen dem Problem des fertigungstechnischen Optimums im Bereich der technikunterstützten Informationsverarbeitung $\mathrm{zu}$.

\section{a) Anwendungsmöglichkeiten des Produktionsfunktionskonzeptes in Prozessen der Informationsverarbeitung}

Zur Untersuchung produktionstheoretischer Zusammenhänge wurden verschiedene Ansätze entwickelt, wobei als Basisvarianten die aktivitätsanalytische und die funktionalistische Produktionstheorie zu unterscheiden sind. ${ }^{172}$ Der aktivitätsanalytische Ansatz leitet, ausgehend von den formalen Eigenschaften einer Technologie, die Eigenschaften von Produktionsfunktionen und Entscheidungsempfehlungen her. ${ }^{173}$ Dagegen setzt der funktionalistische Ansatz bestimmte Eigenschaften der Produktionsfunktion voraus ${ }^{174}$ und konzentriert sich auf die Untersuchung der Relationen zwischen Faktoreinsatz und Faktorertrag, die unter den angenommenen Bedingungen gegeben sind. Die nachfolgenden Überlegungen beschränken sich auf den funktionalistischen Ansatz, dem GUTENBERG bei der Analyse von produktionstheoretischen Zusammenhängen folgt.

Wie in der Einleitung zu Abschnitt 3.3.2 bereits angesprochen, bildet im Rahmen dieses Ansatzes das als Produktivitätsbeziehung bezeichnete Verhältnis zwischen Faktorertrag und Faktoreinsatz die Grundbeziehung industrieller Produktionsprozesse. ${ }^{175}$ Faktoreinsatz und Faktorertrag stehen in einem funktionalen Zusammenhang, der zum Ausgangspunkt der Analyse von Produktionsprozessen gemacht und in Form einer (statischen) Produktionsfunktion

\footnotetext{
${ }^{171} \mathrm{Vgl}$. Kern (1992), S. 25.

${ }^{172}$ Vgl. Corsten (2000), S. 57.

${ }^{173}$ Vgl. Kistner (1993), S. 54.

${ }^{174}$ Vgl. Steven (1998), S. 62.

${ }^{175}$ Vgl. zum Folgenden Gutenberg (1983), S. 298ff.; Busse v. Colbe/Laßmann (1991), S. 89ff.
} 


$$
x=f\left(r_{1}, r_{2}, \ldots, r_{n}\right)
$$

notiert wird, wobei mit $\mathrm{x}$ die produzierte Gütermenge und mit $\mathrm{r}_{\mathrm{i}}$ die eingesetzten Elementarfaktoren beschrieben werden. ${ }^{176}$ Bei der Formulierung der Produktionsfunktion wird unterstellt, dass die Entscheidung über alternative Produktionsprozesse bereits gefallen ist. Es werden also nur die zulässigen Zuordnungsalternativen in einer konstanten Bezugsperiode bei einem gegebenen verfugbaren Faktorbündel betrachtet. Für diese Situation zeigt die Produktionsfunktion an, wie sich der Ertrag ändert, wenn die Einsatzmengen der produktiven Faktoren variiert werden.

GUTENBERG unterscheidet grundsätzlich zwei Arten von Produktionsfunktionen. ${ }^{177}$ Dabei handelt es sich zum einen um Funktionen, bei denen die Faktorproportionen zumindest in gewissen Grenzen variierbar sind (substitutionale Produktionsfunktionen vom Typ A), und zum anderen um solche Funktionen, bei denen die Faktorproportionen nicht variiert werden können (limitationale Produktionsfunktionen vom Typ B). ${ }^{178}$ Die substitutionale Produktionsfunktion vom Typ A hat ihren Ursprung in der ertragsgesetzlichen Produktionsfunktion der mikroökonomischen Theorie. Ihr stellt GUTENBERG die limitationale Produktionsfunktion vom Typ B gegenüber, der die Annahme zu Grunde liegt, dass ein einziges effizientes Faktoreinsatzverhältnis existiert, bei dem es zu keiner Faktorverschwendung kommt. Er argumentiert, dass eine derartige Produktionsfunktion den Gegebenheiten in industriellen Produktionsprozessen besser entspreche, da der Mehrertrag bei einer Änderung der Faktoreinsatzmengen nur der Gesamtheit der zusätzlich eingesetzten Faktoren zugerechnet werden könne.

Während die Produktionsfunktion vom Typ A sich global auf den Gesamtbetrieb bezieht, ermöglicht der Funktionstyp B ein System von Teilfunktionen, das den Produktionsbereich analytisch detaillierter durchdringt. ${ }^{179}$ Durch die detailliertere Betrachtung wird es ferner möglich, nicht nur unmittelbare, sondern auch mittelbare Input/Output-Beziehungen abzubilden. Geht man von einer mittelbaren Input/OutputBeziehung aus, dann hängt der Verbrauch an Werkstoffen nicht nur von der OutputMenge, sondern auch von den situationsbezogenen Eigenschaften der eingesetzten Potenzialfaktoren ab. GUTENBERG unterscheidet in diesem Zusammenhang zwischen der Intensität, mit der der jeweilige Potenzialfaktor genutzt wird, und weiteren technischen Eigenschaften, die er zur so genannten z-Situation zusammenfasst. ${ }^{180}$ Die Intensität und die $\mathrm{z}$-Situation werden im Folgenden aus Gründen der Vereinfachung nicht näher betrachtet, eine Erweiterung der hier abgeleiteten Aussagen zum Informationsmanagement um diese Gesichtspunkte wäre jedoch zur Ergänzung des Ansatzes möglich.

Diese beschriebene Systematik der Produktionsfunktionen wurde später verfeinert und durch Produktionsfunktionen weiterer Typen ergänzt. Die von HEINEN entwickelte Produktionsfunktion vom Typ C greift ebenfalls auf Verbrauchsfunktionen zur Erfassung von Input-Output-Beziehungen zurück. ${ }^{181}$ Damit wird die von GUTENBERG

\footnotetext{
${ }^{176}$ Vgl. Gutenberg (1983), S. 302.

${ }^{177}$ Vgl. Gutenberg (1983), S. 303.

${ }^{178}$ Vgl. auch Busse v. Colbe/Laßmann (1991), S. 101.

${ }^{179}$ Vgl. Corsten (2000), S. 86.

${ }^{180}$ Vgl. Gutenberg (1983), S. 329ff.

${ }^{181}$ Vgl. Heinen (1983), S. 244ff.
} 
eingeleitete Tendenz zur besseren betriebswirtschaftlichen Fundierung und stärkeren Detaillierung der Darstellung in der Produktionstheorie fortgesetzt. ${ }^{182}$ Der Grundgedanke dabei ist die Aufspaltung des Produktionsgeschehens in kleinste Teileinheiten, die als Elementarkombinationen bezeichnet werden. ${ }^{183}$ Diese Elementarkombinationen sind einer getrennten Modellierung und Analyse zugänglich. Produktionsfunktionen vom Typ $C$ werden nachfolgend nicht weiter betrachtet, da es sich um eine Verfeinerung des Faktortheoretischen Ansatzes handelt, die dessen Erkenntnisinteressen sowie inhaltlichen und methodischen Leitideen unverändert zu Grunde legt. Für die Frage der prinzipiellen Anwendbarkeit auf den Gestaltungsbereich des Informationsmanagements ergeben sich daher keine neuen Aspekte. Bei der von KLOOCK entwickelten Input/Output-Analyse mit Hilfe der Produktionsfunktion vom Typ D handelt es sich um eine Verallgemeinerung, die die Produktionsfunktionen der Typen A, B und C als Spezialfall enthältt. ${ }^{184}$ KÜUPER erweitert die Untersuchung um dynamische Aspekte und entwickelt zu diesem Zweck die Produktionsfunktion vom Typ E. ${ }^{185}$ Diese Erweiterungen bewegen sich ebenfalls innerhalb des konzeptionellen Bezugsrahmens des Faktortheoretischen Ansatzes und werden daher nachfolgend nicht im Einzelnen betrachtet.

Für eine Anwendung der funktionalistischen Produktionstheorie als Teilgebiet des Faktortheoretischen Ansatzes auf die durch das Informationsmanagement gestalteten und gesteuerten Prozesse der Informationsverarbeitung ist zu überprüfen, ob sich auch für solche Prozesse Produktionsfunktionen aufstellen lassen. Sollte dies der Fall sein, so sind die Eigenschaften derartiger Produktionsfunktionen zu untersuchen, um im Anschluss daran mit dem Instrumentarium des Faktortheoretischen Ansatzes Aussagen über eine möglichst ökonomische Kombination der Elementarfaktoren in Informationsverarbeitungsprozessen abzuleiten. Die Anwendbarkeit des Produktionsfunktionskonzeptes hängt davon $a b$, ob die für die industrielle Fertigung zu Grunde gelegten Annahmen auch für die technikunterstützte Informationsverarbeitung getroffen werden können.

Bei der Aufstellung von Produktionsfunktionen wird davon ausgegangen, dass die Art und die Menge der verwendeten Produktionsfaktoren sowie der Zusammenhang zwischen Faktoreinsatz und Faktorertrag vollständig bekannt sind. Die Existenz von Unsicherheiten wird zum Teil durch die Verwendung von Zufallsvariablen berücksichtigt. Obwohl eine derart konstruierte Theorie die tatsächlichen Verhältnisse sowohl bei der Produktion von industriellen Gütern als auch bei der Herstellung von Dienstleistungen nur stark vereinfacht abbildet, ${ }^{186}$ basieren weite Teile des Aussagesystems der funktionalistischen Produktionstheorie auf dieser Sicherheitsprämisse. Die Übertragung des Produktionsfunktionskonzeptes auf den Gestaltungsbereich des Informationsmanagements ist daher nicht bereits deshalb abzulehnen, weil bei der Herstellung informationeller Produkte wie bei der Herstellung anderer Produkte auch Informationsunvollkommenheiten auftreten.

\footnotetext{
${ }^{182}$ Vgl. Steven (1998), S. 173.

${ }^{183}$ In diesem Punkt wird die stark reduktionistisch orientierte Methodik, die dem Faktortheoretischen Ansatz zu Grunde liegt, besonders deutlich.

${ }^{184}$ Vgl. Kloock (1969), S. 126ff.; Kloock (1993), S. 289ff.

${ }^{185}$ Vgl. Küpper (1979), S. 93 ff.

${ }^{186}$ Vgl. ähnlich Altenburger (1980), S. 65.
} 
Bestimmte einschränkende Annahmen werden allerdings nicht nur für die Input-Seite, sondern auch für die Output-Seite von Produktionsprozessen getroffen. Bei der Formulierung einer Produktionsfunktion wird angenommen, dass durch die Variation der Faktoreinsatzmengen allein die Menge der produzierten Leistungen variiert wird. Mit anderen Worten: Es wird die Produktion eines mehr oder weniger homogenen Gutes betrachtet. Die Übertragbarkeit des Produktionsfunktionskonzeptes auf Prozesse der technikunterstützten Informationsverarbeitung hängt von der Gültigkeit dieser Prämisse für die in diesen Prozessen erstellten Produkte ab. Die in Informationsverarbeitungsprozessen hergestellten Produkte müssen nicht nur erfasst werden können, sondern es ist auch zu überprüfen, inwiefern von einer Gleichartigkeit dieser informationellen Produkte ausgegangen werden kann.

Die Erfassung von informationellen Produkten nach Art und Menge ist im betriebswirtschaftlichen Schriftum bisher kaum untersucht worden. ${ }^{187}$ Zum Teil wird darauf hingewiesen, die Informationsmenge könne nicht präzise gemessen werden, insbesondere deshalb, weil Information als Produkt sich nicht in allen semantischen Dimensionen vollständig beschreiben lasse. ${ }^{188}$ Auch bei industriellen Gütern, für deren Untersuchung das produktionstheoretische Instrumentarium entwickelt wurde, ist jedoch im Allgemeinen keine Erfassung sämtlicher Eigenschaftsdimensionen möglich. ${ }^{189}$ BODE schlägt vor, zur Erfassung von Informationen auf der semantischen Ebene die Merkmale Informationsart, Einheitselement und Informationstyp zu verwenden, die sich auch zur Erfassung inhaltlich nicht ex ante bestimmbarer, neuer Informationen eigne. ${ }^{190}$ Die Informationsart bestimmt, welche Inhalte durch die zu erstellende Information beschrieben werden sollen. Das Einheitselement wird als nicht weiter teilbar angesehen und stellt somit die Bezugsgröße für die Ermittlung des mengenmäßigen Outputs dar. Der Informationstyp wiederum bezieht sich auf die formalen Anforderungen an die Darstellung der Informationsart. Abbildung 3/8 verdeutlicht dieses Vorgehen zur Bestimmung des informationellen Output von Produktionsprozessen:

\begin{tabular}{|l|l|l|}
\hline Informationsart & Einheitselement & Informationstyp \\
\hline $\begin{array}{l}\text { Vorjahresvergleich von } \\
\text { Kostenstellen }\end{array}$ & $\begin{array}{l}\text { Vorjahresvergleich einer } \\
\text { Kostenstelle }\end{array}$ & $\begin{array}{l}\text { Prozentzahl mit zwei } \\
\text { Nachkommastellen }\end{array}$ \\
\hline $\begin{array}{l}\text { Bestellung von Rohstoffen bei } \\
\text { den Teilelieferanten }\end{array}$ & Eine Bestellposition & $\begin{array}{l}\text { Datensatz mit mehreren ge- } \\
\text { nau definierten Datenfeldern } \\
\text { (z.B. Bestellnummer, Menge, } \\
\text { Wert) }\end{array}$ \\
\hline $\begin{array}{l}\text { Strategische Situations- } \\
\text { analyse }\end{array}$ & $\begin{array}{l}\text { Situationsanalyse für ein } \\
\text { Strategisches Geschäftsfeld }\end{array}$ & $\begin{array}{l}\text { Natürlichsprachlicher Text, } \\
\text { ergänzt durch grafische } \\
\text { Abbildungen }\end{array}$ \\
\hline
\end{tabular}

Abb. 3/8: Bestimmung von Informationsart, Einheitselement und Informationstyp (Beispiele)

\footnotetext{
${ }^{187} \mathrm{Vgl}$. Bode (1993), S. 139.

${ }^{188}$ Vgl. z.B. Rehberg (1973), S. 87; Schweitzer/Küpper (1974), S. 44.

${ }^{189}$ Vgl. Bode (1993), S. 142.

${ }^{190}$ Vgl. Bode (1993), S. 143f.
} 
Bei der Frage, inwiefern es sich bei den erzeugten Informationen um homogene Güter handelt, kommt es darauf an, ob die Gleichartigkeit der Einheitselemente als gegeben angesehen werden kann. Zur Überprüfung dieser Voraussetzung ist es hilfreich, an die von NIPPA entwickelte Klassifikation von Informationsverarbeitungsaufgaben anzuknüpfen, die im vorigen Abschnitt bei der Behandlung der Gestaltungsprinzipien bereits kurz angesprochen wurde. Dabei werden an Hand der Kriterien Aufgabenkomplexität, Aufgabendynamik, Aufgabendeterminiertheit, Aufgabentragweite und Aufgabenemotionalität idealtypische Aufgabenprofile im Bereich der Informationsverarbeitung identifiziert und gegeneinander abgrenzt. ${ }^{191}$ Abbildung 3/9 zeigt diese Unterscheidung und die zu Grunde gelegten Kriterien.

\begin{tabular}{|c|c|c|c|c|}
\hline \multirow[b]{2}{*}{$\begin{array}{l}\text { formale } \\
\text { Aufgaben- } \\
\text { merkmale }\end{array}$} & \multirow{2}{*}{$\begin{array}{l}\text { Aufgaben- } \\
\text { typ } 1 \\
\text { Elnzelfall- } \\
\text { aufgaben }\end{array}$} & \multicolumn{2}{|c|}{$\begin{array}{c}\text { Aufgabentyp } 2 \\
\text { Sachbezogener Fall }\end{array}$} & \multirow{2}{*}{$\begin{array}{c}\text { Aufgaben- } \\
\text { typ } 3 \\
\text { Routine- } \\
\text { aufgaben }\end{array}$} \\
\hline & & $\begin{array}{c}\text { Typ 2a } \\
\text { Projekt- } \\
\text { aufgaben }\end{array}$ & $\begin{array}{c}\text { Typ 2b } \\
\text { Regel- } \\
\text { aufgaben }\end{array}$ & \\
\hline Aufgabenkomplexitat & hoch & & & niedrig \\
\hline Aufgabendynamik & hoch & & & nledrig \\
\hline Aufgabendeterminierthelt & niedrig & & & hoch \\
\hline Aufgabentragweite & hooh & & & niedrig \\
\hline Aufgabenemotionalităt & hoch & & & niedrig \\
\hline
\end{tabular}

Abb. 3/9: Aufgabentypen der Informationsverarbeitung

Quelle: Nippa (1988), S. 90.

Die Extremfälle dieses Schemas stellen die Kategorien Einzelfallaufgaben und Routineaufgaben dar. Einzelfallaufgaben (Aufgabentyp 1) zeichnen sich durch hohe Komplexität und Dynamik, geringe Determiniertheit, eine große Tragweite der Ergebnisse der Aufgabenerfüllung und eine starke Personenbezogenheit der Problemstellung aus. Sie sind auf Grund ihrer hohen Individualität nicht für eine Formalisierung zugänglich. Dagegen weisen Routineaufgaben (Aufgabentyp 3) eine einfache Struktur auf und wiederholen sich in geringen Abständen. Sie sind durch gleichbleibende Problemstellungen gekennzeichnet, deren Lösungsweg nahezu vollständig formalisierbar ist. Zwischen den beschriebenen Extremfallen liegen sachbezogene Aufgaben (Aufgabentyp 2), bei denen Projektaufgaben und Regelaufgaben zu unterscheiden sind. Projektaufgaben (Aufgabentyp 2a) spielen sich zwar in wiederkehrenden Rahmenbedingungen $a b$, erfordern jedoch in jedem Fall eine neue Vorgehensweise zur möglichst zielgerechten Aufgabenerfüllung. Vorangegangene Problemlösungen können nur grobe Anhaltspunkte über erfolgversprechende Arbeitsfolgen für die vorliegende Aufgabenstellung geben. Im Gegensatz dazu weisen Regelaufgaben (Aufgabentyp 2b) eine geringere Komplexität und eine höhere Wiederholungsrate auf. Regelaufgaben können stärker typisiert und standardisiert werden, beinhalten jedoch im Vergleich zu Routineaufgaben eine höhere Zahl von Sonderfällen.

${ }^{191} \mathrm{Vgl}$. zum folgenden Nippa (1988), S. 88ff.; Rau (1991), S. 21ff.; Gabriel/Begau/Knittel/Taday (1994), S. 15f., 31ff. 
Das beschriebene Klassifikationsschema bezieht sich zwar auf die Art der Aufgabe und nicht auf die produzierte Leistung, es lassen sich jedoch Rückschlüsse auf die durch die Lösung der jeweiligen Aufgabenstellungen produzierten Ergebnisse ziehen. Aus der Bezeichnung des Aufgabentyps Einzelfallaufgaben geht bereits hervor, dass es sich bei der bearbeiteten Aufgabenstellung jeweils um individuelle Probleme handelt, die sich deutlich von vorhergegangenen und nachfolgenden Problemstellungen unterscheiden. Dieses Merkmal trifft auch auf die erstellte Leistung zu, bei der es sich z.B. um eine Produkt/Markt-Strategie oder ein neues Konzern-Führungskonzept handeln kann. Die produzierten Ergebnisse sind also sehr vielfältig und heterogen, sodass das Konzept der Produktionsfunktion für Einzelfallaufgaben nicht anwendbar ist, da ein homogenes Einheitselement nicht mit genügender Genauigkeit festgelegt werden kann. Die gleiche Schlussfolgerung ergibt sich auch für Projektaufgaben, die beispielsweise die Vorbereitung einer Messe oder die Entwicklung eines neuen Software-Moduls zur Ergänzung des internen Berichtswesens zum Gegenstand haben.

Bei der Betrachtung von Routineaufgaben und Regelaufgaben ergibt sich dagegen eine günstigere Beurteilung für die Aufstellung von Produktionsfunktionen. Durch die Erfullung solcher Aufgaben werden nahezu gleichartige Leistungen produziert. Diese können zwar bei den Regelaufgaben in gewissen Grenzen variieren, sie sind dennoch im Prinzip gut vergleichbar und können deshalb nach der Bildung von Durchschnitten auf einem aggregierten Niveau sinnvoll als gleichartig aufgefasst werden. ${ }^{192}$ Das Ergebnis von Routineaufgaben sind beispielsweise ausgestellte Rechnungen im Anschluss an erfolgte Lieferungen (Fakturierung) oder die Annahme von Bestellungen im telefonischen Vertrieb, während durch Regelaufgaben beispielsweise Beschwerden bearbeitet oder Einkaufsanforderungen abgegeben werden. Als Konklusion der angestellten Überlegungen ergibt sich, dass das Konzept der Produktionsfunktion zwar nicht auf Einzelfallaufgaben und Projektaufgaben der Informationsverarbeitung angewendet werden kann, dass die Anwendung auf Regelaufgaben und auf Routineaufgaben jedoch im Prinzip zulässig ist.

\section{b) Substitutionalität versus Limitationalität in Prozessen der Informations- verarbeitung}

Nachdem die grundsätzliche Anwendbarkeit des Vorgehens zur Analyse der Faktorkombination auf Prozesse der Informationsverarbeitung aufgezeigt wurde, stellt sich im zweiten Schritt die Frage, durch welchen Typ von Produktionsfunktion diese Prozesse am besten beschrieben werden. Als Grundformen zur modellmäßigen Abbildung von Produktionsprozessen stehen sich, wie bei der Darstellung des Produktionsfunktionskonzeptes erläutert, substitutionale und limitationale Produktionsfunktionen gegenüber. GUTENBERG nimmt an, dass Produktionsfunktionen vom Typ A industrielle Produktionsprozesse nicht adäquat abbilden, da sie die Konstanz der Einsatzmengen und Leistungsabgaben der anderen Faktoren bei Variation eines einzigen betrachteten

\footnotetext{
${ }^{192}$ Es sei angemerkt, dass genau genommen zwischen dem Determiniertheitsgrad der jeweiligen Aufgabe und ihrem tatsächlichen organisatorischen Standardisierungsgrad zu unterscheiden ist. Vgl. dazu Gersch (1998), S. 213. Diese Unterscheidung wird hier nicht weiter verfolgt, sondern es wird angenommen, dass im Falle einer hohen Determiniertheit die grundsătzliche Möglichkeit zur Standardisierung vollstăndig durch entsprechende organisatorische Gestaltungsmaßnahmen ausgenutzt wird. Der Determiniertheitsgrad und der Standardisierungsgrad einer Aufgabe stimmen dann überein.
} 
Faktors verlangen. ${ }^{193}$ Gerade dies ist bei industriellen Produktionsprozessen aber nicht der Fall, da in diesen die Einsatzmengen der Elementarfaktoren gemeinsam variieren und ihr Verbrauch durch technische Umstände determiniert wird. Folglich ist es auch nicht möglich, für jeden einzelnen Elementarfaktor isoliert den Beitrag zu bestimmen, den er im Produktionsprozess leistet. Mit anderen Worten: Es lassen sich keine partiellen Grenzproduktivitäten ermitteln. ${ }^{194}$

Substitutionalität als Beziehung zwischen den verschiedenen Elementarfaktoren innerhalb eines Produktionsprozesses ist für industrielle Prozesse also nur schwer vorstellbar. Unter der soeben begründeten Annahme, dass die Erfüllung von Routine- und Regelaufgaben der Informationsverarbeitung aus produktionstechnischer Sicht der industriellen Fertigung stark ähnelt, besitzen substitutionale Faktorbeziehungen auch innerhalb der hier betrachteten Prozesse der Informationsverarbeitung keine Relevanz. Vielmehr kann angenommen werden, dass in solchen Prozessen limitationale Produktionsbedingungen vorliegen. In Routine- und Regelprozessen, die durch einen hohen Strukturiertheitsgrad gekennzeichnet sind, wird eine einmal festgelegte Folge von Arbeitsschritten immer wieder durchlaufen, um ein gleichartiges informationelles Produkt herzustellen. Die isolierte Variation eines einzigen Faktors (z.B. die Bereitstellung eines zusätzlichen Arbeitsplatzrechners) bewirkt also keine Änderung der Ertragsmenge, wenn die Einsatzmengen der übrigen Faktoren (z.B. geleistete Arbeitsstunden, lizenzierte Installationen der benötigten Anwendungsprogramme) konstant gehalten werden. Erst die simultane Variation aller Faktoreinsatzmengen bewirkt hier eine Änderung der hergestellten Produktmenge. Die partielle Grenzproduktivität eines Faktors ist lediglich dann größer als Null, wenn es sich um den Engpassfaktor handelt und die Kapazitäten der übrigen Elementarfaktoren noch nicht vollständig ausgenutzt $\operatorname{sind}^{195}$ (beispielsweise bei Variation der geleisteten Arbeitsstunden und noch freien Kapazitäten der verfügbaren Arbeitsplatzrechner).

Substitutionale Faktorbeziehungen bestehen allerdings auch dann, wenn zur Herstellung eines Produktes verschiedene limitationale Produktionsverfahren miteinander kombiniert werden können. ${ }^{196}$ Sofern die Verfahren sich nicht gegenseitig ausschließen, können sie (unter der Voraussetzung der Teilbarkeit der Elementarfaktoren und produzierten Güter) beliebig miteinander kombiniert werden, sodass eine kontinuierliche Substitutionsbeziehung zwischen den Faktoren entsteht. Trägt man zwei linearlimitationale Prozesse wie in Abbildung 3/10 in ein Faktordiagramm ein, so ergeben sich zwei Prozessstrahlen, die die alternativen Faktorkombinationen zur Herstellung des Produktes angeben. Die Punkte gleicher Ausbringung zwischen den Prozessstrahlen werden als Isoquanten bezeichnet. Die Gesamtausbringungsmenge $Q_{\text {ges }}$ ergibt sich hier als Summe der mit Prozess I hergestellten Menge $Q_{I}$ und der mit Prozess II hergestellten Menge $Q_{\mathbb{I}}$.

\footnotetext{
${ }^{193}$ Vgl. Gutenberg (1983), S. 325.

${ }^{194}$ Vgl. Gutenberg (1983), S. 329.

${ }^{195}$ Vgl. Busse v. Colbe/Laßmann (1991), S. 120f.

${ }^{196}$ Vgl. dazu Busse v. Colbe/Laßmann (1991), S. 126ff.
} 


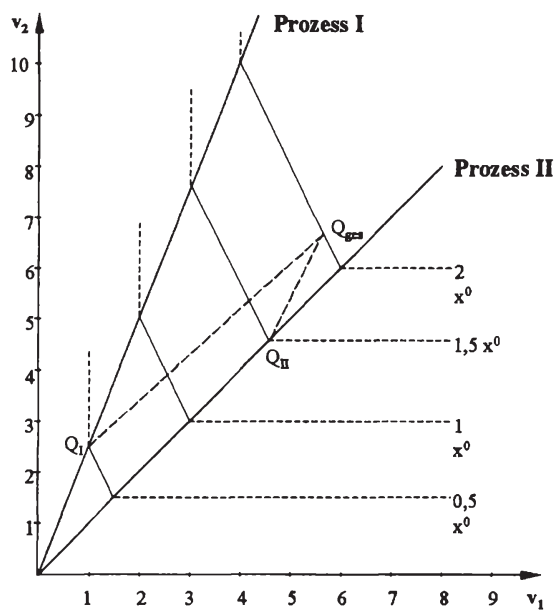

Abb. 3/10: Faktordiagramm mit zwei linear-limitationalen Prozessen Quelle: Busse v. Colbe/Laßmann (1991), S. 128.

Als Beispiel für eine derartige Situation im Bereich der Informationsverarbeitung sei hier die Bearbeitung von Eingangsrechnungen genannt. Es sei angenommen, dass diese entweder durch manuelle Erfassung bei Nutzung des herkömmlichen Postweges oder durch die automatische Datenübernahme vom Lieferanten auf der Basis eines elektronischen Datenaustausches erfolgen kann. Durch die Möglichkeit zur Kombination beider Verfahren werden die Faktoren menschliche Arbeitszeit und Rechnerzeit substituierbar, obwohl innerhalb beider Prozesse jeweils linear-limitationale Faktorbeziehungen vorliegen.

Beschränkt man die Betrachtung wie in diesem Beispiel auf nur zwei alternative linearlimitationale Produktionsverfahren, so verlaufen die Isoquanten im Faktordiagramm linear. Erst wenn davon ausgegangen wird, dass unendlich viele linear-limitationale Prozesse miteinander kombiniert werden können, erhält man den in der mikroökonomischen Theorie angenommenen nicht-linearen Isoquantenverlauf zwischen substitutiven Produktionsfaktoren. In Bezug auf Prozesse der Informationsverarbeitung wird diese Annahme der Realität jedoch nicht gerecht. Eine Unternehmung verfügt zu einem bestimmten Entscheidungszeitpunkt im Allgemeinen nicht über eine sehr große Auswahl realisierbarer Alternativen zur Erstellung eines informationellen Produktes. Vielmehr ist die Anzahl der möglichen Verfahren zur Erstellung des Produktes stark beschränkt. ${ }^{197}$ So könnte in dem oben angeführten Beispiel der Bearbeitung von Eingangsrechnungen neben den genannten Alternativen der herkömmlichen manuellen Verbuchung und dem elektronischen Datenaustausch als drittes Verfahren noch das Einscannen mit automatisierter Weiterverarbeitung (Image Processing) in Frage kommen. Selbst wenn weitere, grundsätzlich vorstellbare Prozessvarianten berücksichtigt werden, sind die alternativ möglichen Prozesse vollständig aufzählbar, sodass

${ }^{197}$ In einer längerfristigen Perspektive ist die Beschrănkung auf nur wenige Prozesse aufgehoben, da die Möglichkeit zur Entwicklung innovativer Verfahren besteht, die im Einzelnen sehr unterschiedlich ausgeprägt sein können. Für kurzfristig angelegte Probleme der Produktionsplanung ist die Auswahl zwischen nur wenigen Alternativen jedoch realistischer. 
es sich bei der Annahme einer unbegrenzten Anzahl alternativer Prozesse um eine nicht zulässige Näherung handelt. Es bleibt deshalb festzuhalten, dass für die betrachteten Routine- und Regelaufgaben der Informationsverarbeitung eine Limitationalität der Elementarfaktoren innerhalb der einzelnen Produktionsprozesse und eine Substitutionalität zwischen einer überschaubaren Anzahl verfügbarer Produktionsverfahren realistisch erscheint.

\section{c) Das fertigungstechnische Optimum in Prozessen der Informationsverarbeitung}

Aus den genannten Gründen besitzen für das Informationsmanagement nur die Bedingungen für ein fertigungstechnisches Optimum in limitationalen Produktionsprozessen Relevanz. Bei Betrachtung von zwei Faktorarten können für die partielle Faktorvariation in solchen Prozessen zwei einfache Fälle unterschieden werden. ${ }^{198}$ In dem Fall, dass das effiziente Faktoreinsatzmengenverhältnis noch nicht erreicht ist, weil die Kapazität des jeweils anderen Faktors noch nicht vollständig ausgelastet ist, ist entweder die Einsatzmenge der betrachteten Faktorart zu erhöhen oder die der anderen, überschüssigen Faktorart zu verringern. Wenn auf der anderen Seite das effiziente Faktoreinsatzmengen-Verhältnis bereits überschritten ist, so ist die Einsatzmenge der betrachteten Faktorart zu verringern oder die der jeweils anderen Faktorart zu erhöhen. Bei der Frage, welches von mehreren limitationalen Produktionsverfahren, die alternativ zur Herstellung eines bestimmten Produktes angewendet werden können, vorteilhaft ist, handelt es sich um ein Problem der Kostentheorie, das im folgenden Abschnitt behandelt wird.

Die in diesem Abschnitt angestellten Überlegungen betreffen die Herstellung nur eines (informationellen) Produktes auf nur einer Produktionsstufe. Sie sind grundsätzlich erweiterbar, sodass auch fur die mehrstufige Produktion (Stufenproduktion) sowie die Produktion mehrerer Produkte (verbundene Produktion mit dem Spezialfall der Kuppelproduktion) Aussagen entwickelt werden können, die auf den dargestellten Leitideen des Faktortheoretischen Ansatzes basieren. Bei der Analyse der Stufenproduktion werden modellmäßig mehrere limitationale Produktionsverfahren hintereinandergeschaltet und mit Hilfe der Vektorrechnung untersucht. ${ }^{199}$ Die verbundene Produktion ist dadurch gekennzeichnet, dass die gleichen Produktionsfaktoren zur Herstellung unterschiedlicher Produkte eingesetzt werden. In diesem Bereich sind die alternative Produktion, bei der mehrere Produkte abwechselnd gefertigt werden, und die Kuppelproduktion, bei der auf Grund der technischen Bedingungen mehrere Produkte notwendigerweise gleichzeitig in einem Produktionsverfahren erzeugt werden, $\mathrm{zu}$ unterscheiden. ${ }^{200}$ Bei den Untersuchungen von Stufenproduktion und verbundener Produktion handelt es sich um Verfeinerungen des Aussagesystems, die in dieser Arbeit nicht in die Betrachtung einbezogen werden, da sie der Frage der grundsätzlichen Anwendbarkeit und der generellen Erkenntnispotenziale der Leitideen des Faktortheoretischen Ansatzes für das Informationsmanagement nachgelagert sind.

Die Anwendbarkeit bestimmter Spezialbereiche der Produktionstheorie, die beispielsweise die Frage der Produktionselastizität und der Homogenität von Produk-

\footnotetext{
${ }^{198}$ Vgl. Busse v. Colbe/Laßmann (1991), S. $110 \mathrm{f}$.

${ }^{199}$ Vgl. dazu Busse v. Colbe/Laßmann (1991), S. 178ff.

${ }^{200}$ Vgl. Steven (1998), S. 14f.
} 
tionsfunktionen betreffen, ${ }^{201}$ hängt im Wesentlichen von den empirischen Gegebenheiten des jeweils betrachteten Informationsverarbeitungsprozesses ab. Diese speziellen Teilprobleme werden hier ebenfalls nicht näher betrachtet. Wenn im konkreten Einzelfall die grundsätzliche Anwendbarkeit des Produktionsfunktionskonzeptes gegeben ist, dann können die genannten Fragen untersucht und die diesbezüglichen Aussagesysteme der Produktionstheorie angewendet werden.

\subsubsection{Erkenntnispotenziale der Kostentheorie für die technikunterstützte Informa- tionsverarbeitung}

Die im vorigen Abschnitt betrachteten mengenmäßigen Produktionsbeziehungen erhalten ihre wirtschaftliche Bedeutung erst dadurch, dass sie als Kern in eine erweiterte Betrachtung einfließen, die im Rahmen der Kostentheorie die Einkaufspreise der Produktionsfaktoren einbezieht und damit ökonomische Aussagen liefert. ${ }^{202} \mathrm{Die}$ Kosten der Herstellung von Produkten können ermittelt werden, indem die mit Hilfe der Produktionsfunktion ermittelten, eingesetzten Faktoreinsatzmengen mit ihren Faktorpreisen bewertet werden. ${ }^{203}$ Durch kostentheoretische Aussagen sollen die Einflussgrößen auf die Kosten identifiziert und systematisiert, ihre gegenseitigen Abhängigkeiten ermittelt und die Abhängigkeit der Kosten von diesen Einflussgrößen festgestellt werden. ${ }^{204}$ GUTENBERG nennt als Hauptdeterminanten der Kosten die Faktorqualitäten, die Faktorproportionen und die Faktorpreise. Diese sind zum Teil selbst wiederum von der Beschäftigung, der Betriebsgröße und dem Fertigungsprogramm als weiteren Einflussgrößen abhängig. ${ }^{205}$ Die folgende Darstellung beschränkt sich auf die drei Hauptdeterminanten der Kosten, da dies ausreicht, um die Aussagepotenziale der Leitideen des Faktortheoretischen Ansatzes für das Informationsmanagement aufzuzeigen. Die abschließenden Überlegungen gehen auf das Problem ein, auf welche Weise unter Kostengesichtspunkten eine Anpassung an Beschäftigungsschwankungen erfolgen sollte.

\section{a) Kostenfunktionen als Instrument zur Analyse der technikunterstützten Infor- mationsverarbeitung}

Das wichtigste Instrument zur Analyse der Kosten ist die Kostenfunktion, die aus einer limitationalen Produktionsfunktion durch Umkehrung und Bewertung der Faktorverbräuche abgeleitet werden kann. ${ }^{206}$ Eine Kostenfunktion zeigt in statischer Form die funktionale Abhängigkeit der im Betrachtungszeitraum entstehenden Kosten von der hergestellten Produktmenge. Sie kann sowohl als Gesamtkostenfunktion als auch als

\footnotetext{
${ }^{201}$ Vgl. dazu z.B. Corsten (2000), S. 60ff.; Busse v. Colbe/Laßmann (1991), S. 113ff.

${ }^{202} \mathrm{Vgl}$. Busse v. Colbe/Laßmann (1991), S. 201, die auch die Absatztheorie als Teil der Gesamtbetrachtung nennen. Wie in Abschnitt 3.2.3.1 begründet, besitzt die Absatztheorie fuir das Informationsmanagement jedoch nur indirekt Relevanz und wird hier deshalb nicht betrachtet.

${ }^{203} \mathrm{Vgl}$. Gutenberg (1983), S. 338. Gutenberg führt an, dass zu den gesamten Kosten eines Betriebes auch weitere Kosten für Dienstleistungen, bestimmte Steuern und offentliche Abgaben gehören, die in der Produktionsfunktion nicht enthalten sind. Bei den Produktionskosten handele es sich jedoch "weitgehend" um die Kosten des Faktoreinsatzes.

${ }^{204} \mathrm{Vgl}$. Heinen (1983), S. 140ff.

${ }^{205} \mathrm{Vgl}$. Gutenberg (1983), S. 347. Diese Systematik von Kosteneinflussgroßen ist in der Folge erheblich erweitert und verfeinert worden. Vgl. dazu z.B. Heinen (1983), S. 481ff., Schweitzer/Küpper (1986), S. 245ff.; Hummel/Männel (1986), S. 101ff.

${ }^{206}$ Vgl. Busse v. Colbe/Laßmann (1991), S. $222 \mathrm{f}$.
} 
Stückkostenfunktion vorliegen. Durch die Unterscheidung fixer und variabler Kosten entstehen weitere Varianten, die den Verlauf der Grenzkosten, der variablen Gesamtund Stückkosten und der Fixkosten je Stück abbilden. ${ }^{207}$ Die Kostentheorie beschäftigt sich mit dem Verlauf dieser Kostenfunktionen und ihrer Abhängigkeit von den genannten Kosteneinflussgrößen.

Je nach Verlauf der zu Grunde liegenden Produktionsfunktion können Kostenfunktionen unterschiedliche Charakteristika aufweisen. Verläuft die Produktionsfunktion linear, so weist auch die Kostenfunktion lineare Eigenschaften auf. Sie läuft dann entweder durch den Ursprungspunkt des Kosten/Mengendiagrammes oder schneidet, bei Vorhandensein von Fixkosten, die y-Achse in Höhe der Fixkosten. Für den Fall, dass es sich bei der zu Grunde liegenden Produktionsfunktion um eine nicht-linear limitationale ${ }^{208}$ Funktion handelt, werden von GUTENBERG drei Varianten unterschieden. Die Grenzkosten können ansteigen, abnehmen oder zunächst abnehmen und dann ansteigen. Im letzten Fall weisen die Grenzkosten, die variablen Stückkosten und die gesamten Stückkosten Minimalstellen auf. Die Zusammenhänge sind in Abbildung 3/11 grafisch illustriert. Die linke Kostenfunktion zeigt einen linearen Kostenverlauf, während die rechte Kostenfunktion die Variante mit zunächst sinkenden und dann steigenden Grenzkosten darstellt. Die Gesamtkosten sind jeweils mit $\mathrm{K}(\mathrm{x})$, die Grenzkosten mit $\mathrm{K}^{\prime}(\mathrm{x})$, die variablen Stückkosten mit $\mathrm{k}_{\mathrm{v}}(\mathrm{x})$ und die gesamten Stückkosten mit $\mathrm{k}_{\mathrm{g}}(\mathrm{x})$ bezeichnet.

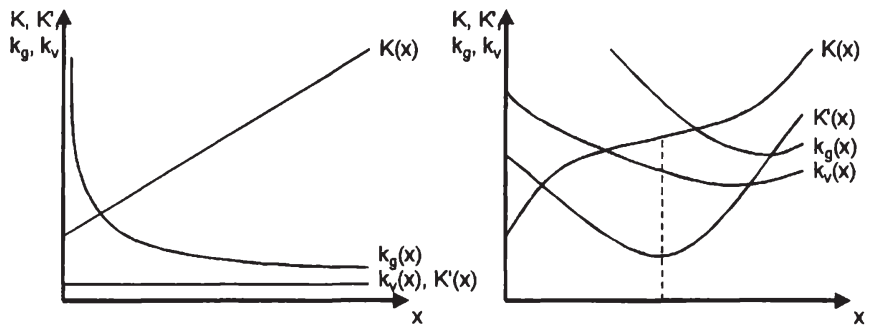

Abb. 3/11: Kostenfunktionen mit linearem und nicht-linearem Kostenverlauf Quelle: in Anlehnung an Gutenberg (1983), S. 340, S. 341.

Im Folgenden wird der Einfluss der Hauptdeterminanten der Kosten auf die Lage der Kostenfunktion und die Relevanz dieses Teilgebiets des Faktortheoretischen Ansatzes für das Informationsmanagement untersucht. Da die Kostenfunktion aus der Produktionsfunktion abgeleitet wird, stellt die Möglichkeit zur Anwendung des Produktionsfunktionskonzeptes die Voraussetzung zur Anwendung von Kostenfunktionen dar. Aus diesem Grund erstreckt sich die weitere Untersuchung in diesem Abschnitt nur auf Routine- und Regelprozesse, für die die Tragfahigkeit des Produktionsfunktionskonzeptes im vorigen Abschnitt begründet wurde. Entsprechend den methodischen Leitideen des Faktortheoretischen Ansatzes wird nachfolgend eine Partialanalyse durchgeführt. Dabei werden jeweils alle bis auf die zu analysierende Einflussgröße

\footnotetext{
${ }^{207}$ Vgl. dazu Gutenberg (1983), S. 338ff.

${ }^{208}$ Entsprechend den Ausfiihrungen im vorangegangenen Abschnitt werden hier nur noch limitationale Produktionsfunktionen betrachtet, da substitutionale Funktionen als unrealistisch verworfen wurden.
} 
konstant gehalten, und es wird die Wirkung einer isolierten Änderung der betrachteten Einflussgröße untersucht.

\section{b) Einfluss der Faktorproportionen auf die Kosten der technikunterstützten Informationsverarbeitung}

Die Faktorproportionen beeinflussen die Kostenfunktion durch die unterschiedliche Produktivität, die auf Grund der produktionstechnischen Bedingungen aus unterschiedlichen Faktoreinsatzverhältnissen resultiert. Das ökonomische Prinzip verlangt, dass die Gesamtkosten der für die Erzeugung einer bestimmten Produktionsmenge eingesetzten Faktoren ein Minimum erreichen. Diejenige Faktorproportion, die diese Forderung erfült, wird als Minimalkostenkombination bezeichnet. ${ }^{209}$ Das Vorgehen zur Bestimmung der Minimalkostenkombination hängt davon ab, ob limitationale oder substitutionale Produktionsbedingungen vorliegen. ${ }^{210} \mathrm{Da}$ in Routine- und Regelprozessen der technikunterstützten Informationsverarbeitung von limitationalen Produktionsbedingungen auszugehen ist, sind hier nur diejenigen Bedingungen der Minimalkostenkombination zu betrachten, die limitationale Produktionsprozesse betreffen.

Bei limitationalen Produktionsprozessen existiert für eine bestimmte Produktionsmenge nur eine einzige Einsatzmenge eines jeden Faktors, die den ineffizienten Faktoreinsatz vermeidet. $^{211}$ Folglich sind in Routine- und Regelprozessen im Bereich der technikunterstützten Informationsverarbeitung die wirtschaftlich günstigsten Faktorproportionen vollständig technisch determiniert. Wie Abbildung 3/12 zeigt, fällt die in das Faktordiagramm eingetragene Expansionslinie, auf der alle zulässigen Minimalkostenkombinationen liegen, in diesem Fall mit dem technisch-effizienten Prozessstrahl zusammen. Für das Informationsmanagement folgt daraus, dass das unter mengenmäßigen Gesichtspunkten bestimmte fertigungstechnische Optimum zur Erfüllung einer bestimmten Menge von Routine- oder Regelaufgaben zugleich das Optimum unter Kostengesichtspunkten darstellt. Die Kostenfunktion verläuft bei limitationalen Produktionsprozessen analog zur Produktionsfunktion entweder linear oder nichtlinear mit steigenden oder sinkenden Grenzkosten.

\footnotetext{
${ }^{209}$ Vgl. Busse v. Colbe/Laßmann (1991), S. 221.

${ }^{210}$ Vgl. Corsten (2000), S. 115.

${ }^{211}$ Vgl. Busse v. Colbe/Laßmann (1991), S. 231.
} 


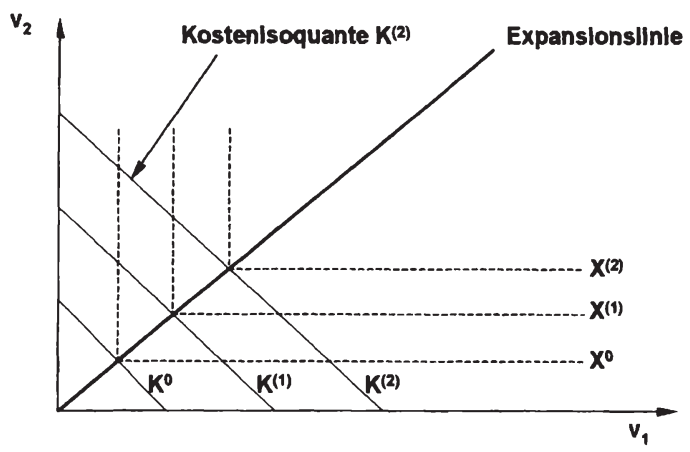

Abb. 3/12: Expansionslinie bei einem limitationalen Prozess Quelle: Busse v. Colbe/Laßmann (1991), S. 231.

Stehen zur Herstellung eines informationellen Produktes unterschiedliche Produktionsverfahren zur Verfügung, so ist unter den fertigungstechnisch optimalen Alternativen das kostenminimale Verfahren der Informationsverarbeitung zu bestimmen. ${ }^{212}$ Betrachtet man dazu zunächst die Herstellung eines Produktes mit zwei kontinuierlich variierbaren Faktorarten und zwei alternativen linear-limitationalen Prozessen, so ergibt sich das in Abbildung 3/13 dargestellte Faktordiagramm. Die Kurven gleicher Kostenhöhe (Kostenisoquanten) berühren die Kurven gleicher Ertragshöhe (Ertragsisoquanten) auf dem Strahl des fertigungstechnisch optimierten Prozesses II. Dies bedeutet, dass der Prozess II unter Kostengesichtspunkten dem Prozess I vorzuziehen ist, da mit ihm eine festgelegte Produktmenge zu den geringeren Kosten produziert werden kann.

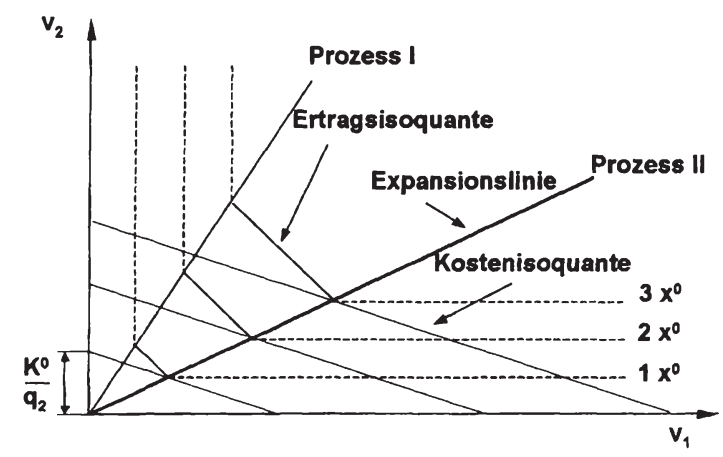

Abb. 3/13: Expansionslinie bei zwei linear-limitationalen Prozessen Quelle: Busse v. Colbe/Laßmann (1991), S. 235.

Dieses einfache Modell kann durch die Berücksichtigung mehrerer Prozesse, mehrerer Faktoren und die Einführung von Restriktionen erweitert werden. Als Aussage für das

${ }^{212}$ Vgl. dazu Busse v. Colbe/Laßmann (1991), S. $234 \mathrm{f}$. 
Informationsmanagement folgt aus diesem Modell, dass exakt ein Prozess unter der Voraussetzung der linearen Limitationalität kostenminimal ist und deshalb den anderen vorzuziehen ist. Für das im vorigen Abschnitt zur Illustration herangezogene Beispiel der Bearbeitung von Eingangsrechnungen folgt daraus, dass das kostengünstigste Verfahren unter mehreren Alternativen exakt bestimmt werden kann. Eine gegebene Menge von Eingangsrechnungen erfordert also beispielsweise den geringsten Faktoreinsatz, wenn die Rechnungsdaten elektronisch zwischen Lieferant und Kunde ausgetauscht werden. Folglich ist diese Prozessvariante den alternativen Verfahren der manuellen Verbuchung bzw. dem Einscannen mit automatisierter Weiterverarbeitung eindeutig überlegen. Zur Erfüllung dieser Routineaufgabe sollte dann - so weit möglich - unter Kostengesichtspunkten ausschließlich dieses Verfahren eingesetzt werden.

Eine Mischung verschiedener Prozessvarianten oder der Wechsel zwischen verschiedenen Prozessvarianten $a b$ einer bestimmten Ausbringungsmenge ist dann effizient, wenn bei mindestens einer Prozessvariante nichtlinear-limitationale Produktionsbedingungen gegeben sind. ${ }^{213}$ Unter diesen Voraussetzungen kann es ab einer bestimmten Ausbringungsmenge sinnvoll sein, die verschiedenen Prozessvarianten miteinander zu kombinieren, um kostenminimale Faktorproportionen einzusetzen. Es ist eine empirische Frage, ob diese Bedingungen bei einem konkreten Gestaltungsproblem des Informationsmanagements vorliegen. Ist dies der Fall, so können die entsprechenden Gestaltungsempfehlungen des Faktortheoretischen Ansatzes durch das Informationsmanagement angewendet werden.

\section{c) Einfluss der Faktorqualität auf die Kosten der technikunterstützten Infor- mationsverarbeitung}

Die Qualität der Elementarfaktoren in Prozessen der Informationsverarbeitung wurde bereits als Qualifikation der Mitarbeiter, als qualitative Kapazität der Betriebsmittel und als Qualität der Werkstoffe unter dem Aspekt der produktionstechnischen Ergiebigkeit der Elementarfaktoren behandelt. ${ }^{214}$ Es wurde ebenfalls bereits erläutert, welche Ausprägungen diese Größen in Prozessen der Informationsverarbeitung annehmen. Änderungen der Qualität können einerseits als stetige Änderungen in Form von Oszillationen um einen bestimmten Durchschnitt oder kontinuierlichen trendartigen Produktivitätsveränderungen auftreten. Sie können andererseits die Form von mutativen Änderungen bei der Einführung neuer Produktionsverfahren haben. ${ }^{215}$

Es wird angenommen, dass sich die Oszillationen der Qualität im Laufe der Zeit ausgleichen und deshalb insgesamt keinen Einfluss auf die Produktionskosten haben. Oszillative Qualitätsunterschiede der Elementarfaktoren haben daher keinen andauernden Einfluss auf den Verlauf der Kostenfunktion. Solche oszillativen Veränderungen der Faktorqualität treten im technikunterstützten Informationssystem nicht anders als in industriellen Fertigungsprozessen auf und können zu Schwankungen der Produktivität aller Elementarfaktorarten und auch des dispositiven Faktors, im hier betrachteten Fall also des Informationsmanagements, führen. So können beispielsweise die Antwort-

\footnotetext{
${ }^{213} \mathrm{Vgl}$. Busse v. Colbe/Laßmann (1991), S. 235.

${ }^{214}$ Gutenberg verwendet den Qualitätsbegriff allerdings nicht einheitlich. Mit qualitativen Änderungen der Produktionsbedingungen bezeichnet er in diesem Zusammenhang Änderungen der Produktionsfaktoren, die nicht-quantitativer Natur sind und eine Verănderung der Faktorproduktivităt zur Folge haben. Vgl. Gutenberg (1983), S. 394ff.

${ }^{215}$ Vgl. Gutenberg (1983), S. 394f.
} 
zeiten des Großrechners im Zeitablauf um einen bestimmten Durchschnitt schwanken, da die Performance unter anderem von der je nach Tageszeit unterschiedlichen Auslastung abhängt. Ähnliches gilt für das in der Unternehmung vorhandene Eignungspotenzial im Bereich der informationsobjektbezogenen menschlichen Arbeitsleistungen, das auf Grund der Mitarbeiterfluktuation typischerweise nicht vollständig konstant gehalten werden kann.

Im Gegensatz zu oszillativen Qualitätsschwankungen kann es durch kontinuierliche oder mutative Verbesserungen der Qualität im Zeitablauf gelingen, Einfluss auf die Kostenfunktion zu nehmen und die Produktionskosten dauerhaft zu senken ${ }^{216}$ Kontinuierliche Qualitätsverbesserungen bewirken eine stetige Steigerung der Ergiebigkeit der einzelnen Elementarfaktoren und auf diesem Wege eine höhere Ausbringungsmenge bei gleichem Faktoreinsatz, die zur Veränderung ${ }^{217}$ der Produktionsfunktion führt. Bei gleicher Produktionsmenge kommt es dadurch zu einer Senkung der Kosten, also zu Auswirkungen auf die Kostenfunktion. Im technikunterstützten Informationssystem der Unternehmung können solche Veränderungen beispielsweise in Form von Verbesserungen der Leistungsmerkmale der verwendeten Hard- und Software oder Erhöhungen der fachlichen Qualifikation der Mitarbeiter unter grundsätzlicher Beibehaltung der bisherigen Verfahren der Informationsverarbeitung auftreten.

Mutative Änderungen liegen dann vor, wenn das gesamte Produktionsverfahren durch ein neues Verfahren ersetzt wurde. Die alten Produktions- und Kostenfunktionen werden in diesem Fall vollständig durch neue ersetzt. Zur Überprüfung der Vorteilhaftigkeit sind dabei die durch die Produktionsfunktionen determinierten gesamten Produktionskosten des alten Verfahrens mit denen des neuen Verfahrens zu vergleichen. Ein Fall der mutativen Änderung liegt im Bereich der Informationsverarbeitung zum Beispiel vor, wenn große Teile der Auftragsbearbeitung durch die Einführung integrierter betriebswirtschaftlicher Anwendungssoftware automatisiert werden.

Die Festlegung der Faktorqualität richtet sich allerdings nicht nur nach den durch die Produktion verursachten Kosten, sondern auch nach den Anforderungen auf dem Absatzmarkt, sodass die ökonomisch optimale Faktorqualität keinesfalls immer mit der technisch perfekten übereinstimmen muss. ${ }^{218}$

\section{d) Einfluss der Faktorpreise auf die Kosten der technikunterstützten Infor- mationsverarbeitung}

Neben der Qualität und der Menge der eingesetzten Produktionsfaktoren stellen auch die Faktorpreise eine Größe dar, die die Höhe der entstehenden Produktionskosten beeinflusst. Bei den bisherigen Überlegungen wurden die Faktorpreise als gegeben aufgefasst, sodass die Kostenfunktion allein das Mengengerüst der Produktion widerspiegelt. Wird dagegen angenommen, dass auch die Faktorpreise mit der Pro-

\footnotetext{
${ }^{216} \mathrm{Vgl}$. Gutenberg (1983), S. 397ff.

${ }^{217}$ Um welche Art der Veränderung (Drehung, Verschiebung, Streckung usw.) es sich handelt, ist eine empirische Frage und kann deshalb hier nicht allgemein beantwortet werden. Deshalb wird hier nur von „Veränderung“ der Produktionsfunktion gesprochen.

${ }^{218}$ Vgl. Busse v. Colbe/Laßmann (1991), S. 214. Die qualitative Ausgestaltung informationeller Produkte als absatzpolitisches Instrument wird hier, wie oben bereits begründet, nicht weiter betrachtet.
} 
duktionsmenge variieren, so hängt die Gestalt der Kostenfunktion zusätzlich von dieser Einflussgröße $a b^{219}$ GUTENBERG unterscheidet drei Fälle der Einflüsse von Faktorpreisen auf die Kostenhöhe.

Den ersten Fall stellt die Variante dar, dass sich die Preise nicht mit der in Anspruch genommenen Menge ändern. Unter diesen Bedingungen bewirken Preisänderungen einen steileren oder flacheren Verlauf der Kurve der variablen Kosten. Die Gesamtlage der dazu gehörenden Gesamtkosten-, Grenzkosten- und Durchschnittskostenkurven ändert sich dadurch im Vergleich zum Verlauf unter der Annahme konstanter Faktorpreise nicht. ${ }^{220}$ Im zweiten Fall hängen die Faktorpreise von der Höhe der durch die Unternehmung entfalteten Nachfrage ab (z.B. durch die Erzielung von Preisvorteilen bei größeren Beschaffungsmengen). Unter diesen Voraussetzungen ist der Verlauf der Kostenkurve nicht mehr allein durch die Produktionsfunktion bestimmt. Vielmehr ergeben sich die variablen Kosten als

$$
\mathrm{K}_{\mathrm{v}}=\sum \mathrm{r}_{\mathrm{i}}(\mathbf{x}) \cdot \mathrm{p}_{\mathrm{i}}(\mathbf{x})
$$

wobei $x$ die produzierte Gütermenge, $r_{i}$ die jeweilige Einsatzmenge des Faktors $i$ und $p_{i}$ den entsprechenden Faktorpreis bezeichnet. Durch diese Abhängigkeit kann es zu Veränderungen in der Gesamtlage der Kostenkurve im Vergleich zum Kurvenverlauf bei Annahme der Konstanz der Faktorpreise kommen. ${ }^{221}$ Der dritte Fall liegt dann vor, wenn der Preis des Faktors sich ab dem Erreichen einer bestimmten Einsatzmenge ändert, aber nur für den Teil der Einsatzmenge, der diese Grenze überschreitet (z.B. bei der Inanspruchnahme von Überstunden). Unter dieser Voraussetzung wird die Kurve der variablen Kosten vor Erreichen dieser Grenze allein durch die technischen Bedingungen der Produktion determiniert. Oberhalb der Grenze verändert sich die Steigerung der Kostenkurve durch die Preisänderung, ihre Gesamtlage bleibt jedoch unverändert, wenn davon ausgegangen wird, dass der Faktorpreis oberhalb der Grenze zwar höher, aber dennoch konstant ist.

Die beschriebenen Auswirkungen der Faktorpreise sind grundsätzlich von industriellen Fertigungsprozessen auf Prozesse der Informationsverarbeitung zur Erfüllung von Routine- und Regelaufgaben übertragbar. Schwierigkeiten ergeben sich in diesem Zusammenhang jedoch durch die Notwendigkeit zur Bewertung des Verbrauchs von Informationen als Werkstoffen. Die Bewertung des Verbrauchs von Produktionsfaktoren mit Preisen stellt ein eigenständiges betriebswirtschaftliches Problem dar, wenn keine Marktpreise furr die in Anspruch genommenen Leistungen bestimmbar sind. ${ }^{222}$

Gerade bei Routine- und Regelaufgaben sind die als Werkstoffe eingehenden Informationen häufig nicht mit einem beobachtbaren Preis versehen, da sie zwischen den Beteiligten nicht als einzelnes ökonomisches Gut ausgetauscht werden. Dies ist in der Regel dann der Fall, wenn es sich um Informationen zur Koordination arbeitsteiliger Prozesse der Leistungserstellung handelt. Unter diesen Bedingungen stellt der Infor-

\footnotetext{
${ }^{219}$ Vgl. Gutenberg (1983), S. 415.

${ }^{220}$ Dieser Punkt betrifft die Lage von Wendepunkten der Kurve der variablen Gesamtkosten und von Extrempunkten der Grenz- und Durchschnittskostenkurve. Solche Punkte können allerdings nur bei nicht-linear limitationalen und bei substitutionalen Produktionsfunktionen vorkommen.

${ }^{221} \mathrm{Vgl}$. Gutenberg (1983), S. $417 \mathrm{ff}$.

${ }^{222}$ Vgl. Busse v. Colbe/Laßmann (1991), S. 214.
} 
mationsaustausch einen Teil der Abwicklung des gesamten Geschäftsprozesses dar (z.B. im Fall der Bearbeitung von Eingangsrechnungen, der Bestellabwicklung oder der Beschwerdebearbeitung) und die zwischen den Beteiligten übergebene Information wird nicht als Werkstoff vergütet. Die bei fehlendem Marktpreis zu lösende Frage der Bewertung informationeller Güter wird hier nicht näher behandelt, da es sich im Kern nicht um ein produktions- und kostentheoretisches Problem handelt. Wenn die Informationen dagegen als Werkstoffe extern über einen Beschaffungsmarkt bezogen werden (z.B. aus Online-Datenbanken oder -Archiven), wie es bei der Herstellung von Informationen als Produktbestandteil häufig der Fall ist, dann existiert ein eindeutig feststellbarer Preis, sodass die oben beschriebene Systematik auf die betreffende Elementarfaktorart übertragbar ist.

\section{e) Anpassung an Beschäftigungsschwankungen}

Die bisher behandelten Modelle nehmen bei der Beschreibung einer konkret vorliegenden Kostenfunktion in einer Unternehmung starke Abstraktionen vor und sind für praktische Planungszwecke in den meisten Fällen zu grob. ${ }^{223}$ Für eine differenziertere Abbildung des tatsächlichen Kostenverlaufes sind sie um Annahmen darüber zu ergänzen, auf welche Weise die Anpassung an unterschiedliche Herstellungsmengen erfolgt, wie die Unternehmung also auf Beschäftigungsschwankungen reagiert. Dabei ist zu untersuchen, auf welche Weise Einsatzmengen aus Werkstoffen und Potenzialfaktoren mit einem konstanten Bestand an Potenzialfaktoren kombiniert werden, um alternative Produktmengen $\mathrm{zu}$ erzeugen. ${ }^{224}$ Während der Bestand an Betriebsmitteln innerhalb eines kurzfristigen Zeitraums als konstant angesehen wird, kann ihre Leistungsabgabe innerhalb gewisser Grenzen schwanken, um eine Anpassung des Betriebs an schwankende Leistungsanforderungen $\mathrm{zu}$ ermöglichen. ${ }^{225}$ Durch die unterschiedliche Fahrweise der Betriebsmittel können mit einer bestimmten Kombination von Faktoreinsatzmengen mehrere Ausbringungsmengen verbunden sein, ohne dass gegen das Wirtschaftlichkeitsprinzip verstoßen wird. Aus diesem Grund ist von einer mittelbaren Beziehung zwischen Faktoreinsatz und Faktorertrag auszugehen, die unter anderem von den variierbaren technischen Einsatzbedingungen der Betriebsmittel abhängt.

GUTENBERG unterscheidet als Reaktion auf Beschäftigungsschwankungen ohne Änderung der Qualität der Einsatzfaktoren die intensitätsmäßige, die quantitätsmäßige und die zeitliche Anpassung. ${ }^{226}$ Bei der intensitätsmäßigen Anpassung werden die Betriebsmittel zeitlich unverändert in Betrieb gehalten, während ihre Inanspruchnahme variiert wird. Quantitative Anpassung bedeutet dagegen, dass Teile der Betriebsanlagen vollständig stillgelegt bzw. wieder in Betrieb genommen oder vollständig neu angeschafft werden, um die Ausbringungsmenge anzupassen. Bei der zeitlichen Anpassung schließlich wird die Betriebszeit der Potenzialfaktoren verlängert oder verkürzt, um auf Veränderungen der Beschäftigungslage zu reagieren. Abbildung 3/14 zeigt diese Systematik von Maßnahmen zur Anpassung an Beschäftigungsschwankungen.

\footnotetext{
${ }^{223}$ Vgl. ähnlich Busse v. Colbe/Laßmann (1991), S. 255.

${ }^{224}$ Vgl. Busse v. Colbe/Laßmann (1991), S. 255.

${ }^{225}$ Vgl. Steven (1998), S. 127.

${ }^{226}$ Vgl. Gutenberg (1983), S. 355f.
} 


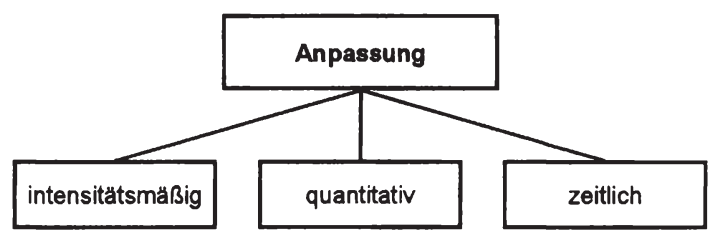

Abb. 3/14: Anpassung an Beschäftigungsschwankungen

In der Regel wird ein Betrieb auf Beschäftigungsschwankungen nicht ausschließlich mit einer Anpassungsform reagieren, sondern diese unter Kostengesichtspunkten auswählen und kombinieren. ${ }^{227}$ Aus der Kombination von Anpassungsformen ergibt sich der kostenminimale Anpassungspfad, der diejenige Fahrweise der Betriebsmittel angibt, die die Ausweitung oder Einschränkung der Beschäftigung zu den jeweils geringsten Kosten ermöglicht. Auf dem Anpassungspfad werden ausschließlich reine Anpassungsformen eingesetzt, zwischen denen immer dann ein Wechsel erfolgt, wenn entweder die bislang genutzte Anpassungsmöglichkeit ausgeschöpft ist, oder wenn eine Break-Even-Analyse den Wechsel aus Kostengründen nahe legt. ${ }^{228}$

Dieser Aussagekomplex des Faktortheoretischen Ansatzes ist zwar prinzipiell von der industriellen Fertigung auf den Gestaltungsbereich des Informationsmanagements übertragbar, die Gegebenheiten weichen jedoch von denen der industriellen Fertigung substanziell $a b$. Bereits für industrielle Fertigungsprozesse gilt, dass die intensitătsmäßige Anpassung in der Praxis nur seltener auftreten wird und kann, nämlich nur dort, wo die Art des Produktionsprozesses es überhaupt in wirtschaftlichem Umfang gestattet. ${ }^{229}$ Diese Anpassungsform kommt vor allem dort in Frage, wo es sich um Anlagen oder Anlagenkomplexe handelt, die nicht in mehrere selbstständige technische Teileinheiten zerlegt werden können. ${ }^{230}$ Tatsächlich können derartige Bedingungen innerhalb des technikunterstützten Informationssystems der Unternehmung auftreten, etwa bei Großrechnern oder bei Netzwerken, deren Übertragungskapazität auf die Bewältigung von Beschäftigungsspitzen ausgelegt ist. Bei der intensitätsmäßigen Anpassung im industriellen Bereich liegt das charakteristische Merkmal der intenstitätsmäßigen Anpassung allerdings in einer mit der Intensität schwankenden Variation des spezifischen Werkstoffverbrauchs, die zu einem konvexen Verlauf der Kostenfunktion führt. ${ }^{231}$ Dieses Merkmal ist im Bereich der technikunterstützten Informationsverarbeitung nicht gegeben, da zur Herstellung informationeller Produkte nicht mehr oder weniger Informationen als Werkstoffe verbraucht werden, wenn die intensitätsmäßige Inanspruchnahme des Großrechners oder des unternehmungsinternen Netzwerks variiert wird. Es ist deshalb anzunehmen, dass im Gestaltungsbereich des Informationsmanagements ein Spezialfall vorliegt, bei dem konstante Produktionskoeffizienten des Werkstoffverbrauchs unabhängig von der Intensität gegeben sind.

Zur Variation der Menge produzierter Informationen kommen ebenfalls die zeitliche und die quantitative Anpassung in Frage. Die zeitliche Anpassung ist dadurch gekenn-

\footnotetext{
${ }^{227} \mathrm{Vgl}$. Steven (1998), S. 150.

${ }^{228}$ Vgl. Steven (1998), S. 158.

${ }^{229}$ Vgl. Kern (1992), S. 43.

${ }^{230}$ Vgl. Gutenberg (1983), S. 355.

${ }^{231}$ Vgl. ahnlich Steven (1998), S. 146.
} 
zeichnet, dass bei konstanter Intensität die Betriebsdauer der Potenzialfaktoren verändert wird. ${ }^{232}$ Bei den hier betrachteten Routine- und Regelprozessen im Gestaltungsbereich des Informationsmanagements wird dies durch die Verkürzung der Arbeitszeiten bzw. durch die Aufnahme von Überstunden erreicht. Für das mehrfach in diesem Kapitel angesprochene Beispiel der Bearbeitung von Eingangsrechnungen bedeutet das, dass auf vorübergehende mengenmäßige Schwankungen durch längere oder kürzere Arbeitszeiten reagiert wird. Wenn diese Schwankungen so groß werden, dass sie durch zeitliche Anpassungsmaßnahmen nicht mehr zu bewältigen sind, ist grundsätzlich auch im Gestaltungsbereich des Informationsmanagements eine quantitative Anpassung vorstellbar. Wie bei der intensitätsmäßigen Anpassung weichen in diesem Punkt allerdings die Gegebenheiten im Bereich der technikunterstützten Informationsverarbeitung von denen der industriellen Fertigung ab. Wegen der hohen Geschwindigkeit der technischen Entwicklung und der häufig gegebenen Unteilbarkeiten bei den technischen Kapazitäten werden in der Regel keine gleichartigen Aggregate zu- bzw. abgeschaltet. Es ist statt dessen anzunehmen, dass bei der Neuanschaffung von Betriebsmitteln die jeweilige Vorgängergeneration vollständig ersetzt wird. ${ }^{233}$ Ist dies nicht der Fall, so kann es zum Spezialfall der selektiven Anpassung kommen. Dabei werden mit steigender Ausbringungsmenge zunächst diejenigen Anlagen mit den kostengünstigsten Produktionsbedingungen genutzt, während die kostenintensiveren Betriebsmittel nach und nach zugeschaltet werden. ${ }^{234}$

Insgesamt sind also Erweiterungen des oben erläuterten Grundmodells möglich, um eine differenziertere Abbildung der Kostenverhältnisse zu erreichen. Die Kostenwirkungen der einzelnen Anpassungsformen und die Entscheidung zwischen verschiedenen Anpassungsalternativen werden hier nicht im Einzelnen behandelt. Die Einzelheiten der kostentheoretischen Modelle sind für die Untersuchung der generellen Erkenntnispotenziale des Faktortheoretischen Ansatzes für das Informationsmanagement von untergeordneter Bedeutung. Entscheidend ist hier vielmehr die grundsätzliche Übertragbarkeit des Instrumentariums auf den Bereich der technikunterstützten Informationsverarbeitung. Die durch den Faktortheoretischen Ansatz entwickelten Modelle bieten für das Informationsmanagement in der Praxis Unterstützung bei der Aufgabe, die Kostensituation von Routine- und Regelaufgaben zu analysieren und zu gestalten.

\subsubsection{Informationsmanagement im System der dispositiven Faktoren}

Die bisher angestellten Überlegungen bezogen sich auf das technikunterstützte Informationssystem der Unternehmung, also den Gestaltungsgegenstand des Informationsmanagements. In diesem Abschnitt wendet sich die Untersuchung dem Vorgang der Gestaltung und Steuerung dieses Systems und somit dem Informationsmanagement selbst zu. Das Informationsmanagement stellt aus der Perspektive des Faktortheoretischen Ansatzes einen Teil der dispositiven Faktoren dar, die die Art und Weise bestimmen, mittels derer im Produktionsprozess Elementarfaktorkombinationen zur

\footnotetext{
${ }^{232}$ Vgl. Gutenberg (1983), S. 371.

${ }^{233}$ Genau genommen ist dies allerdings nicht als echte quantitative Anpassung einzuordnen, da die Untersuchung von Beschäftigungsschwankungen innerhalb des Faktortheoretischen Ansatzes von der Konstanz der Betriebsmittel innerhalb des betrachteten Zeitraums ausgeht. Vgl. Steven (1998), S. 127.

${ }^{234}$ Vgl. dazu Steven (1998), S. 141.
} 
Herstellung von Produkten erzeugt werden. ${ }^{235}$ Bei dem Gestaltungsgegenstand des Informationsmanagements handelt es sich um einen Unternehmungsteilbereich, vergleichbar mit anderen Teilbereichen wie beispielsweise dem Absatz und der Produktion. Die Kombination der Elementarfaktoren in solchen Teilbereichen der Unternehmung erfolgt, wie in Abschnitt 3.2.2.4 erläutert, durch die ressortbezogene Planung und Organisation als Teil der dispositiven Faktoren mit derivativem Charakter. Nachfolgend wird überprüft, welches Aussagepotenzial der Faktortheoretische Ansatz für die Planung und Betriebsorganisation des technikunterstützten Informationssystems der Unternehmung aufweist. Abschnitt 3.3.3.1 beleuchtet zunächst die Planung der technikunterstützten Informationsverarbeitung, während der anschließende Abschnitt 3.3.3.2 sich der Organisation dieser Prozesse zuwendet.

Ausgehend von den Erkenntnisinteressen und Leitideen des Faktortheoretischen Ansatzes ist im Laufe der Zeit ein umfangreiches Schriftum entstanden, das sich mit den dispositiven Faktoren auseinander setzt. Da an dieser Stelle kein umfassender Überblick über die verschiedenen Entwicklungen und ihre Übertragbarkeit auf den Bereich des Informationsmanagements gegeben werden kann, konzentrieren sich die nachfolgenden Überlegungen im Vergleich zu den vorangegangenen Abschnitten dieses Kapitels noch stärker auf die Kernpunkte des Faktortheoretischen Ansatzes. Aus diesem Grund beschränken sich die folgenden Überlegungen im Wesentlichen auf die von GUTENBERG getroffenen Aussagen zu den dispositiven Faktoren und überprüfen deren Erkenntnispotenziale für Fragen des Informationsmanagements.

\subsubsection{Planung der technikunterstützten Informationsverarbeitung}

Planung bedeutet eine gedankliche Vorwegnahme zukünftigen Handelns unter Beachtung des Rationalprinzips. ${ }^{236}$ GUTENBERG sieht das charakteristische Merkmal der Planung im Entwerfen einer Ordnung, in der ein bestimmter Prozess als sich vollziehend vorausgedacht wird. ${ }^{237}$ Die Planung gestaltet, indem sie eine Ordnung entwirf, in der sich der Betriebsprozess vollziehen kann. Die der Planung nachgelagerte Organisation $^{238}$ hat die Aufgabe, die durch Planung vorgegebene Ordnung im Betrieb

\footnotetext{
${ }^{235}$ Vgl. Nebl (1996), S. 187.

${ }^{236}$ Vgl. Grochla (1964), S. 314. Mit Fragen der betrieblichen Planung hat sich in der Folge Gutenbergs ein umfangreiches Schriftum befasst, sodass eine Vielfalt begrifflicher Varianten und inhaltlicher Konzepte entstanden ist. Vgl. stellvertretend die Arbeiten von Hruschka (1958), Koch (1961), Grochla (1964), Gälweiler (1974), Koch (1977), Zahn (1979), Wild (1982), Klausmann (1983), Mag (1995). Die Untersuchung beschränkt sich an dieser Stelle weitgehend auf das Verständnis der Planung, wie es von Gutenberg entwickelt wurde, da dieses die nachfolgenden Untersuchungen maßgeblich (wenn auch nicht ausschließlich) beeinflusst hat.

${ }^{237}$ Vgl. Gutenberg (1983), S. 148.

${ }^{238}$ Es ist darauf hinzuweisen, dass der Begriff „Organisation“ im Faktortheoretischen Ansatz abweichend von dem Begriffsverständnis der „organisatorischen Gestaltung“, das im zweiten Kapitel dieser Arbeit herausgearbeitet wurde, verwendet wird. Mit „Organisation“ wird von Gutenberg eine Managementfunktion bezeichnet, die der Realisierung unmittelbar vorgelagert ist. Der Akzent liegt dabei auf der Konkretisierung der Planung zur Vorbereitung der Realisierung, während die Betonung in dieser Arbeit auf der Gestaltung des arbeitsteiligen Zusammenwirkens liegt. Im Faktortheoretischen Ansatz werden sowohl generelle als auch fallweise Regelungen als Organisation bezeichnet, wenn der Tatbestand der Fremdregelung zur Durchsetzung des Geplanten vorliegt. In dieser Arbeit bedeutet organisatorische Gestaltung dagegen ausschließlich die Aufstellung genereller Regeln in Bezug auf arbeitsteilige Prozesse der Leistungserstellung, ohne dass es sich zwingend um Fremdregelung handeln muss, vgl. ähnlich Steinmann/Schreyögg (2000),
} 
zu realisieren. Planung und Organisation des Betriebes stehen aus dieser Perspektive in einem komplementären Verhältnis zueinander, da die Planung die Voraussetzung und Bedingung der Betriebsorganisation und diese wiederum die Bedingung dafür ist, dass das Geplante betriebliche Wirklichkeit werden kann. Daraus folgt, dass die Organisation immer nur dienenden oder instrumentalen Charakter hat, also Mittel zum Zweck ist. ${ }^{239}$

Die Vertreter des Faktortheoretischen Ansatzes hängen prinzipiell der Idee einer umfassenden Planung an, der die sogenannte synoptische Planungsrationalität zu Grunde liegt. ${ }^{240}$ Bei GUTENBERG kommt dies besonders deutlich in der Formulierung zum Ausdruck, die Planung schirme gegen Unordnung ab, ,indem sie den Betriebsprozess vorausbedenkt und versucht, ihn soweit als möglich von allen Zufälligkeiten und Unzulänglichkeiten frei zu halten. “241 Daraus leitet sich die Forderung ab, alles im Voraus zu berechnen, aufzulisten, zu selektieren, festzulegen und schließlich mit Hilfe organisatorischer Anweisungen umzusetzen. ${ }^{242}$

Das Ziel der Planung ist ein ruhiger, geordneter, abgesicherter Gang des betrieblichen Geschehens, das gegen Störungen durch vom einzelnen Betrieb aus gesehen unregelmäßig und zufällig erscheinende Ereignisse und durch alles Unvorhergesehene im technisch-organisatorischen Bereich abgesichert ist. ${ }^{243}$ Diese Denkweise findet sich auch bei späteren Autoren wieder, die davon sprechen, dass Planung erfolgt, um das eigene Handeln auf ein gewolltes Ziel hin "möglichst reibungslos“, also „ohne unerwünschte Nebenwirkungen irgendwelcher Art" gestalten zu können, ${ }^{244}$ und dass sie die Chance bietet, „vor unliebsamen Überraschungen in der Zukunft gefeit" zu sein. ${ }^{245}$ Der Erfolg der Planung ist nach dieser Vorstellung von ihrer Vollständigkeit abhängig, die allerdings nicht nur durch eine detaillierte Einzelplanung, sondern auch durch eine umrissbezogene Generalplanung sichergestellt werden kann. ${ }^{246}$ Diese Lückenlosigkeit im Sinne der Berücksichtigung aller entscheidenden Tatbestände außer- und innerbetrieblicher Art macht den Wert der Planung aus. ${ }^{247}$

\section{a) Anwendbarkeit der synoptischen Planungskonzeption auf das Informations- management}

Als typisches Bezugsobjekt der Planung werden üblicherweise die Prozesse in Betrieben mit industrieller Produktion betrachtet. ${ }^{248}$ Die Industrieunternehmung stellt also nicht nur bei der Untersuchung der Elementarfaktoren und ihres Zusammenwirkens, sondern auch bei der Betrachtung der dispositiven Faktoren den Modellfall dar.

S. 402. In Abschnitt 2.3.2.2 wurde zudem erläutert, dass die organisatorische Gestaltung selbst dem Managementzyklus mit den Phasen Planung, Steuerung und Kontrolle unterliegt.

${ }^{239}$ Vgl. Gutenberg (1983), S. 236.

${ }^{240}$ Vgl. Dietz (1997), S. 1077; Schreyögg (1984), S. 133ff.; S. $251 \mathrm{ff}$.

${ }^{241}$ Gutenberg (1983), S. 145.

${ }^{242}$ Vgl. Dietz (1997), S. 1077.

${ }^{243}$ Vgl. Gutenberg (1952), S. 672.

${ }^{244}$ Gălweiler (1974), S. 15.

${ }^{245} \mathrm{Mag}$ (1995), S. 1. Mag schränkt allerdings zum einen ein, dass diese Sicherheit vor Überraschungen zwar nicht generell, aber doch bis zu einem gewissen Grad erreichbar ist, zum anderen, dass Planen mit Aufwand verbunden ist, der eine Abwägung der Vor- und Nachteile erfordert. Der Gedanke der synoptischen Planungsrationalität bleibt dennoch deutlich erkennbar.

${ }^{246} \mathrm{Vgl}$. Gutenberg (1983), S. 150 i.V.m. Koch (1961), S. $29 \mathrm{ff}$.

${ }^{247} \mathrm{Vgl}$. Gutenberg (1983), S. 150f.

${ }^{248}$ Vgl. Koch (1961), S. 5; Mag (1995), S. 5. 
Nachdem in den vorigen Abschnitten bereits aufgezeigt werden konnte, dass eine Übertragung der Erkenntnisinteressen und Leitideen des Faktortheoretischen Ansatzes auf den Bereich der technikunterstützten Informationsverarbeitung unter bestimmten Voraussetzungen möglich ist, ist hier nun zu überprüfen, inwiefern dies auch für diejenigen Aussagen, die sich auf die Planung als Teil der dispositiven Faktoren beziehen, sinnvoll sein kann.

Voraussetzung für eine möglichst vollständige und genaue Planung ist eine hohe Gleichförmigkeit des Betriebsgeschehens, die von GUTENBERG allerdings nicht im Zusammenhang mit der Planung, sondern als ausschlaggebend für einen möglichst hohen Erfolg organisatorischer Regelungen genannt wird. ${ }^{249}$ Diese Bedingung gilt jedoch auch fur die synoptische Planung - zumindest in Form der als Einzelplanung bezeichneten Detailplanung -, da die Wahrscheinlichkeit von Überraschungen mit der Variabilität des zu planenden Objektes steigt. Es kommt deshalb darauf an, inwiefern Prozesse der Informationsverarbeitung für die Zwecke der Planung als gleichartig aufgefasst werden können, ohne zu starke Vereinfachungen in Kauf zu nehmen.

Zur Beantwortung dieser Frage ist es erneut hilfreich, auf die oben bereits verwendete Systematik der Informationsverarbeitungsaufgaben zurückzugreifen. In Abschnitt 3.3.1 wurde dargelegt, dass die durch die Erfullung von Routine- und Regelaufgaben produzierten Ergebnisse eine hohe Homogenität aufweisen, sodass die Voraussetzungen für eine Anwendung des Produktionsfunktionskonzepts gegeben sind. Dies wurde mit einer hohen Strukturiertheit und Wiederholbarkeit der Aufgabenstellung begründet. Der Prozess der Erfullung von Routine- und Regelaufgaben weist also eine hohe Gleichförmigkeit des Betriebsgeschehens auf. Demzufolge kann auch davon ausgegangen werden, dass die notwendige Bedingung für eine umfassende Planung, die dem Postulat der synoptischen Planungsrationalität entspricht, vorliegt. Routine- und Regelaufgaben sind daher einer detaillierten Einzelplanung sowohl des einzusetzenden Verfahrens als auch von Art, Umfang und Zeitpunkt der zu erbringenden Leistung zugänglich.

Dagegen sind die Prozesse und die produzierten Ergebnisse bei der Erfüllung von Einzelfall- und Projektaufgaben so heterogen und schlecht strukturiert, dass das Konzept der Produktionsfunktion nicht anwendbar ist. Da das betriebliche Geschehen zur Erfüllung von Einzelfall- und Projektaufgaben eine sehr hohe Variabilität aufweist, entziehen sich diese Aufgabentypen auch einer vollständigen ${ }^{250}$ und detaillierten Planung und können nur umrissbezogen geplant werden. Als Konsequenz ergibt sich, dass aus dem Faktortheoretischen Ansatz, in dem die Organisation als der Planung und Entscheidung nachgelagerte Führungsfunktion aufgefasst wird, auch nur grobe Aussagen zur Organisation von Einzelfall- und Projektaufgaben ableitbar sind. Die Organisation als Führungsfunktion hat die Durchführung der Planung zu gewährleisten, ${ }^{251}$

\footnotetext{
${ }^{249} \mathrm{Vgl}$. Gutenberg (1983), S. 240.

${ }^{250}$ Vollständigkeit der Planung von Einzelfall- und Projektaufgaben würde bedeuten, dass alle denkbaren Ergebnisse bei der Planung berücksichtigt werden. Da die Lösung derartiger Aufgaben jedoch ein hohes Maß an Kreativität erfordert, werden die moglichen Ergebnisse erst während der Aufgabenerfüllung erkennbar. Sie können deshalb ex ante bei der Planung noch nicht berücksichtigt werden, da es sich sonst um einen logischen Fehler handeln würde. Als Beispiel sei die Neuentwicklung eines Software-Moduls genannt, dessen genaue Funktionalităt erst im Laufe des Systementwicklungsprozesses immer detaillierter festgelegt wird.

${ }^{251}$ Vgl. Hruschka (1958), S. 429.
} 
ohne eigenständige Zwecke zu verfolgen. Da der Faktortheoretische Ansatz keine sinnvollen Aussagen zur Planung dieser Aufgabentypen ermöglicht, fehlt die erforderliche Grundlage für organisatorische Betrachtungen.

Als Zwischenergebnis ist somit festzuhalten, dass diejenigen Leitideen des Faktortheoretischen Ansatzes, die sich auf die dispositiven Faktoren beziehen, auf die Planung und Organisation von Routine- und Regelaufgaben, nicht aber auf die von Einzelfallund Projektaufgaben angewendet werden können. In diesem Punkt sind deutliche Parallelen zu den in den vorigen Abschnitten herausgearbeiteten Anwendungsmöglichkeiten der Produktions- und Kostentheorie auf den Bereich der technikunterstützten Informationsverarbeitung erkennbar.

\section{b) Einzelfelder der Planung von Prozessen der Informationsverarbeitung}

Als Ebenen der auf die Produktion bezogenen Planung unterscheidet GUTENBERG die Planung des Produktionsprogramms, die Bereitstellungsplanung sowie die Planung des Produktionsprozesses. ${ }^{252}$ Diese Unterscheidung ist in Abbildung 3/15 dargestellt.

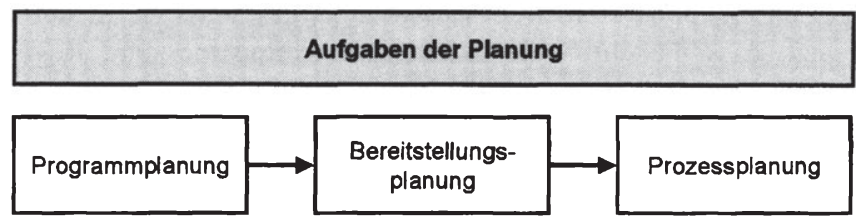

\section{Abb. 3/15: Einzelfelder der Planung nach Gutenberg}

Die Programmplanung legt fest, welche Erzeugnisarten und -mengen im betrachteten Zeitraum herzustellen sind. Das Produktionsprogramm weicht insofern vom Absatzprogramm ab, als inhaltliche Differenzen (durch den Zukauf von Handelswaren und durch die Erstellung selbstgenutzter Leistungen) und zeitliche Differenzen zwischen Produktion und Absatz auftreten können. ${ }^{253}$ An die Programmplanung schließt sich die Bereitstellungsplanung an, die die zur Herstellung des Produktionsprogramms erforderlichen Elementarfaktoren nach Art, Menge und Zeit ermittelt und deren Verfuggbarkeit gewährleistet. Die Prozessplanung bezieht sich auf den Produktionsprozess selbst und legt die Reihenfolge der Produktionsaufträge, die Losgrößen und die genaue Terminierung fest. Alle drei Planungsebenen bilden eine Einheit, da die Planerreichung sowohl von der Qualität der vorgelagerten als auch der der nachgelagerten Pläne abhängt.

In neueren Arbeiten zur Produktionsplanung wird häufig zwischen einer strategischen, einer taktischen und einer operativen Ebene der Produktionsplanung unterschieden. ${ }^{254}$ Dabei hat die strategische Planung die Aufgabe, längerfristige Rahmenbedingungen für

\footnotetext{
${ }^{252} \mathrm{Vgl}$. Gutenberg (1983), S. 149. Diese Systematik ist in der Folge erheblich verfeinert worden, vgl. z.B. Jacob (1996), Sp. 1468ff.; Hahn/Lassmann (1989), S. 7ff. Hier werden jedoch nur die von Gutenberg entwickelten Leitideen betrachtet, um die Erkenntnispotenziale für das Informationsmanagement zu überprüfen. Die Verfeinerung der abgeleiteten Aussagen stellt eine nachgelagerte Fragestellung dar, die hier nicht behandelt wird.

${ }^{253} \mathrm{Vgl}$. Corsten (2000), S. 242.

${ }^{254}$ Vgl. z.B. Kern (1992), S. 73f;; Nebl (1996), S. 284ff.; Corsten (2000), S. $241 \mathrm{ff}$.
} 
das Bestehen der Unternehmung im Wettbewerb $\mathrm{zu}$ schaffen. Die taktische Produktionsplanung konkretisiert die strategischen Vorgaben und macht grobe inhaltliche Spezifikationen. Die operative Planung bezieht sich innerhalb des taktischen Rahmens auf die tatsächlich innerhalb der Planungsperiode stattfindenden Produktionsvorgänge. Die methodische Unterstützung, die der Faktortheoretische Ansatz für die Erfüllung von Planungsaufgaben zur Verfugung stellt, bezieht sich allerdings im Wesentlichen auf operative Fragestellungen. ${ }^{255}$ Aus diesem Grund konzentrieren sich auch die folgenden Überlegungen ebenfalls auf den operativen Planungsbereich. Die genannten drei Planungsebenen sind mit geringfugigen Modifikationen auf die Verarbeitung von Informationen durch das technikunterstützte Informationssystem der Unternehmung übertragbar.

Programmplanung bedeutet für das Informationsmanagement die Planung von Art und Anzahl der herzustellenden informationellen Produkte durch die Erfüllung von Routine- und Regelaufgaben. Da die Informationsverarbeitung aus produktionstheoretischer Sicht einen Teil der Abwicklung übergeordneter Prozesse (z.B. informationstechnisch unterstützte Koordination der Ausführung von Kundenaufträgen, Abwicklung der Lohn- und Gehaltszahlung, Erstellung informationeller Produktbestandteile) darstellt, ist das Informationsmanagement bei der Programmplanung an die fachlichen Vorgaben anderer Teilplanungen (z.B. der Absatzplanung oder der Personalplanung) gebunden. Das Informationsmanagement verfügt daher nur über geringe gestalterische Freiheiten bei der Programmplanung.

Bereitstellungsplanung als Planung der einzelnen Elementarfaktoren bedeutet bezogen auf die technikunterstützte Informationsverarbeitung die Planung der Hard- und Softwarekapazitäten, der benötigten menschlichen Arbeitskräfte und der erforderlichen Werkstoffinformationen bei der Erfullung der nach Art und Menge geplanten Routineund Regelaufgaben. Die Planung der Hard- und Softwarekapazitäten wurde bereits oben unter dem Aspekt der optimalen Ergiebigkeit der entsprechenden Betriebsmittel behandelt. Im Hinblick auf die Bereitstellungsplanung objektbezogener menschlicher Arbeitsleistungen weist GUTENBERG auf größere Schwierigkeiten hin, wenn die erforderlichen Arbeitsleistungen nicht gleichartiger Natur sind, da diese Elementarfaktorart in solchen Fällen nicht beliebig teilbar ist. ${ }^{256} \mathrm{Da}$ hier jedoch nur Routine- und Regelaufgaben betrachtet werden, die durch eine hohe Gleichförmigkeit der erforderlichen Arbeitsleistungen gekennzeichnet sind, kann eine diskrete ${ }^{257}$ Teilbarkeit dieser Elementarfaktorart angenommen werden. Die Planung der Elementarfaktorart Werkstoffe kann nur zum Teil von der industriellen Fertigung auf die Erfullung von Routine- und Regelaufgaben der Informationsverarbeitung übertragen werden. In industriellen Fertigungsprozessen sind im Rahmen der Bereitstellungsplanung für Werkstoffe logistische Probleme im Bereich der Materialbeschaffung und der Lagerhaltung zu lösen, die in gut strukturierten Prozessen der Informations-

\footnotetext{
${ }^{255}$ Tatsächlich behandelt Gutenberg bei der Untersuchung von Planungsaufgaben nahezu ausschließlich Problemstellungen der Programm-, Bereitstellungs- und Prozessplanung, die innerhalb des angesprochenen Klassifikationsschemas als operativ einzuordnen sind. Vgl. Gutenberg (1983), S. 151ff. Auch Kern (1992), S. 76, weist darauf hin, dass viele der entwickelten Methoden gerade bei kurzfristigen (operativen) Planungen industrieller Produktion große Bedeutung erlangt haben.

${ }^{256}$ Vgl. Gutenberg (1983), S. 185.

${ }^{257}$ Diskret bedeutet hier, dass die Menge der verfügbaren Arbeitsleistungen ganzzahlig mit der Kapazität einer einzigen Arbeitskraft variierbar ist.
} 
verarbeitung nicht in ähnlicher Form anfallen. Wenn es sich um Informationen zur Unterstützung der Koordination handelt, fallen die zu verarbeitenden Informationen automatisch während der Abwicklung der übergeordneten Prozesse (z.B. Auftragsabwicklung, Lohn- und Gehaltsabrechnung) an, ohne dass eine Beschaffung ${ }^{258}$ oder Lagerhaltung erforderlich ist. Die Art der Bereitstellung der Werkstoffe wird bei diesen Aufgabentypen durch die Wahl des Informationsverarbeitungsverfahrens festgelegt. Bei Informationen als Produktbestandteilen kann im Gegensatz dazu eine Bereitstellungsplanung erforderlich sein, wenn die entsprechenden Werkstoffe unternehmungsextern beschafft werden.

Die tatsächlich stattfindenden Prozesse der Informationsverarbeitung bestehen aus einer Abfolge von menschlichen und maschinellen Aktivitäten, die sich an den informationellen Werkstoffen vollziehen. Prozessplanung bedeutet für das Informationsmanagement die genaue Festlegung von Zeit und Art der Inanspruchnahme menschlicher und maschineller Kapazitäten zur Durchführung der betreffenden Aktivitäten. Im Hardwarebereich ist beispielsweise die Belegung des Großrechners für unterschiedliche Rechenaufgaben (mit Online- oder Batch-Input-Schnittstellen) zu planen oder die Inanspruchnahme von Leitungen zur Datenfernübertragung festzulegen. Wenn menschliche Aufgabenträger im Planungszeitraum unterschiedliche objektbezogene Arbeitsleistungen erbringen sollen, so sind dafür die genaue Reihenfolge und die Termine zu planen. Auf der mengenmäßigen Planung des Produktionsprozesses basiert die Kostenplanung, die den Abschluss der gesamten Produktionsplanung darstellt. ${ }^{259}$ Die Planung der Produktionskosten ist für Routine- und Regelaufgaben der Informationsverarbeitung in analoger Weise zur industriellen Fertigung nach der Feinplanung der einzusetzenden Elementarfaktoren möglich.

\section{c) Planungsinstrumente für die Informationsverarbeitung}

Die im vorigen Abschnitt gegebene Übersicht benennt die einzelnen Planungsfelder, auf die sich die Aktivitäten eines produktionsorientiert abgegrenzten Informationsmanagements in der Praxis beziehen müssen. In diesem Abschnitt wird ein kurzer Ausblick darüber gegeben, welche Instrumente der Faktortheoretische Ansatz zur Unterstützung der Produktionsplanung bietet. Eine detaillierte Erläuterung und Würdigung dieser Instrumente kann an dieser Stelle nicht gegeben werden und ist auch nicht Aufgabe dieser Arbeit. Die hier verfolgte Zielsetzung lag vielmehr darin, die grundsätzliche Übertragbarkeit von Erkenntnisinteressen und Leitideen des Faktortheoretischen Ansatzes auf den Bereich der technikunterstützten Informationsverarbeitung zu untersuchen. Nachdem begründet wurde, dass dieses Wissenschaftsprogramm für bestimmte Teilbereiche der technikunterstützten Informationsverarbeitung erhebliche Erkenntnispotenziale aufweist, dient der nachfolgende Überblick über die zur Verfügung gestellten Instrumente allein der Vervollständigung der Argumentation. Hinsichtlich der konkreten Anwendung der Instrumente durch das Infor-

\footnotetext{
${ }^{258}$ Beschaffungsprobleme ergeben sich dagegen bei der Erfüllung von Projekt- und Einzelfallaufgaben, in denen die benötigten Informationen nicht quasi automatisch anfallen, sondern eine externe Beschaffung oder eine fallbezogene Analyse interner Sachverhalte notwendig sein kann. Projektund Einzelfallaufgaben werden an dieser Stelle jedoch nicht mehr behandelt, da sich der Faktortheoretische Ansatz nicht für die Ableitung von Aussagen zur Planung dieser Aufgabentypen eignet.

${ }^{259}$ Vgl. Gutenberg (1983), S. 233.
} 
mationsmanagement in der Praxis können hier keine weiter gehenden, allgemeingültigen Aussagen abgeleitet werden.

Es sei zunächst kurz daran erinnert, dass die methodischen Leitideen des Ansatzes in der Vorstellung von Gleichgewichten wurzeln. Zur Herstellung eines optimalen Gleichgewichtes fordert GUTENBERG, dass die Planung simultan vorgenommen wird, da alle Variablen in gegenseitiger Abhängigkeit voneinander stehen. ${ }^{260} \mathrm{Zu}$ diesem Zweck können mathematische Optimierungsmodelle aufgestellt werden, die die Optimierung (Maximierung oder Minimierung) einer Zielfunktion unter Nebenbedingungen als allgemeines Problem mit unterschiedlichen Varianten lösen. ${ }^{261}$ Solche Modelle der linearen und nicht-linearen Programmierung eignen sich in unterschiedlichen Ausdifferenzierungen nicht nur für Zwecke der Programmplanung, sondern auch für die Bereitstellungs- und die Prozessplanung. Sie erlauben die Verwendung einwertiger und mehrwertiger Koeffizienten, sodass auch Situationen der Unsicherheit abgebildet werden können, wenn zumindest die Wahrscheinlichkeiten der Koeffizienten (Risikosituationen) bekannt sind. ${ }^{262}$

Optimierungsmodelle, die das Postulat der synoptischen Planungsrationalität erfüllen, setzen voraus, dass eine vollständig strukturierte Entscheidungssituation vorliegt, in der es sich bei der Suche nach der optimalen Alternative um ein rein mathematisches Problem handelt. Gerade in dieser Voraussetzung wird von Vertretern des Faktortheoretischen Ansatzes ein großer Vorteil der betreffenden Modelle gesehen, da die Strukturierung der Problemstellung durch den Planer ein systematisches Planungsverhalten mit einer differenzierten Problemanalyse und der Erfassung aller relevanten Einflussgrößen erzwingt. ${ }^{263}$ Als Instrumente in diesem Bereich sind insbesondere die lineare und die nicht-lineare Optimierung, Differentiationskalküle und Simulationsansätze zu nennen.

Im Gegensatz zu optimierenden gehen prognostizierende Modelle von einer bereits existierenden, fixierten Situation aus und machen das Zusammenwirken vieler Elemente im Zeitablauf erkenn- und interpretierbar. ${ }^{264}$ Die wichtigste Erscheinungsform dieses Modelltyps stellen Netzplanmodelle dar. ${ }^{265}$ Netzpläne sind grafische Veranschaulichungen umfangreicher Prozesse, die in eine Vielzahl von Einzelaktivitäten zerlegt werden können. Sie erlauben die Struktur- und Zeitanalyse größerer Mengen vollständig bestimmter, aber in ihrer Gesamtheit nicht mehr unmittelbar überschaubarer Einzelaktivitäten mit graphentheoretischen Hilfsmitteln. Bei der Verwendung von Netzplänen werden die logischen Verknüpfungen zwischen den einzelnen Aktivitäten im Planungsbereich besonders hervorgehoben. ${ }^{266}$

\footnotetext{
${ }^{260}$ Vgl. Gutenberg (1983), S. 163.

${ }^{261} \mathrm{Vgl}$. Steinmann/Schreyogg (2000), S. 288.

${ }^{262} \mathrm{Vgl}$. Steinmann/Schreyogg (2000), S. 299.

${ }^{263}$ Vgl. Kern (1992), S. 76.

${ }^{264} \mathrm{Vgl}$. Meyer (1996), S. $72 \mathrm{f}$.

${ }^{265}$ Vgl. dazu Steinmann/Schreyögg (2000), S. 301ff.; Schwarze (1994), passim; Zimmermann (1971), passim.

${ }^{266} \mathrm{Vgl}$. Kern (1992), S. 310
} 


\subsubsection{Organisation von Prozessen der Informationsverarbeitung}

An die Planung schließt sich nach dem Konzept des Faktortheoretischen Ansatzes die Organisation des Betriebes an, die als weiterer dispositiver Faktor mit derivativem Charakter aufgefasst wird und den Vollzug der Planung sicherstellen soll. ${ }^{267}$ Die Organisation wird als Instrument verstanden, dessen man sich bedient, um vorgegebene Ziele und Ordnungen Gestalt werden zu lassen. Die Festlegung von Zielsetzungen und die Abgrenzung von Verhaltensmöglichkeiten ist dagegen Sache derjenigen Personen, die sich der Organisation bedienen, um ihre Zielsetzungen und Planungen Gestalt werden zu lassen. Das Wesen der Organisation besteht also darin, aus verschiedenartigen Elementen eine produktive Einheit zu machen. Nachfolgend wird untersucht, inwiefern die Aussagen des Faktortheoretischen Ansatzes zur Organisation des Betriebes für das Informationsmanagement Relevanz besitzen.

Für die Untersuchung der Organisation aus der Perspektive des Faktortheoretischen Ansatzes können neuere Auflagen des ersten Bandes der Grundlagen der Betriebswirtschaftslehre von GUTENBERG nur bedingt zu Grunde gelegt werden. Der Rückgriff auf ältere Auflagen ist deshalb notwendig, weil GUTENBERG in diesem Teil seines Werkes im Laufe der Zeit besonders viele zwischenzeitlich entstandene, neuere Erkenntnisse aufgegriffen und in sein Werk eingebaut hat (z.B. in Bezug auf Entscheidungsverhalten oder auf informelle Organisationsstrukturen). ${ }^{268}$ Der Einbau dieser Ideen stellt zwar die Flexibilität des Ansatzes unter Beweis, die Ideen sind jedoch nicht als Kernaussagen des Faktortheoretischen Ansatzes anzusehen, die seine ursprünglichen Erkenntnisinteressen ausdrücken und seine Eigenständigkeit als Wissenschaftsprogramm begründen. Es handelt sich stattdessen um Erweiterungen des Aussagesystems, die mit den ursprünglichen Kernaussagen (weitgehend) vereinbar sind.

\section{a) Fallweise und generelle Regelungen im Bereich der technikunterstützten Infor- mationsverarbeitung}

Die wesentliche Fragestellung, die GUTENBERG im Bereich der Betriebsorganisation ursprünglich untersucht hat, betrifft die Vorziehenswürdigkeit fallweiser oder genereller Regelungen des Betriebsablaufes. ${ }^{269}$ Zwischen fallweisen und generellen Regelungen besteht eine Konkurrenzsituation, da eine generelle Regelung fallweise Anordnungen überflüssig macht. ${ }^{270} \mathrm{Je}$ mehr die Form der generellen Regelung an Bedeutung gewinnt, um so mehr wird der Lenkungs- und Arbeitsprozess entindividualisiert. ${ }^{271}$ Bezüglich der Einsatzbedingungen organisatorischer Regelungen kann festgestellt werden, dass überall da, wo betriebliche Vorgänge ein hohes $\mathrm{Ma} ß$ an Gleichartigkeit und Periodizität aufweisen, die Tendenz wirksam wird, fallweise Regelungen durch gene-

\footnotetext{
${ }^{267}$ Vgl. dazu Gutenberg (1983), S. 235ff.

${ }^{268}$ Auf größere Änderungen in diesem Bereich wird auch explizit im Vorwort zur achtzehnten Auflage hingewiesen, vgl. Gutenberg (1983), S. VII.

${ }^{269}$ Vgl. Gutenberg (1951), S. 175ff. Fallweise Regelungen stellen nach der in dieser Arbeit vertretenen Auffassung keine organisatorischen Regelungen, sondern unmittelbare Führungsanweisungen dar. Vgl. dazu auch Steinmann/Schreyögg (2000), S. 405. Unabhăngig von den begrifflichen Differenzen wird die von Gutenberg betrachtete Fragestellung hier dennoch aufgegriffen und berücksichtigt, da es sich um einen Aspekt handelt, der - ganz gleich unter welchem Oberbegriff - das Informationsmanagement als Teil der dispositiven Faktoren betrifft.

${ }^{270} \mathrm{Vgl}$. Steinmann/Schreyögg (2000), S. 405.

${ }^{271}$ Vgl. Gutenberg (1951), S. 177.
} 
relle Regelungen zu ersetzen. ${ }^{272}$ Umgekehrt nimmt die Tendenz zur generellen Regelung mit steigender Variabilität der betrieblichen Tatbestände ab. Dieser Sachverhalt ist in Abbildung 3/16 optisch verdeutlicht und wird als Substitutionsprinzip der Organisation bezeichnet.

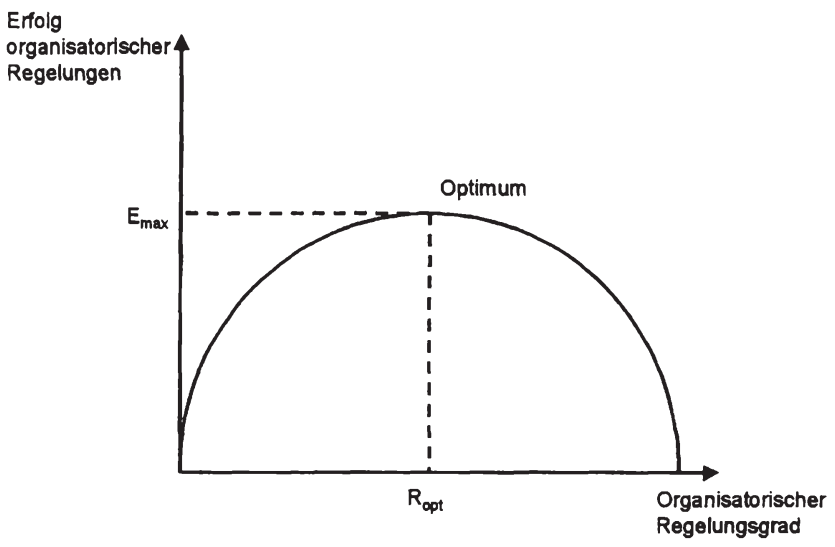

Abb. 3/16: Substitutionsprinzip der Organisation

Quelle: Steinmann/Schrøyögg (2000), S. 404.

Nach diesem Konzept wird angenommen, dass ein optimaler organisatorischer Regelungsgrad besteht, bei dem der Erfolg genereller Regelungen ein Maximum erreicht. ${ }^{273}$ In diesem Punkt tritt erneut das Denken in Gleichgewichten, das den Faktortheoretischen Ansatz in methodischer Hinsicht kennzeichnet, deutlich zu Tage. Überorganisation liegt dann vor, wenn zu viele Vorgänge generell geregelt sind, sodass der Variabilität des Betriebsgeschehens nicht in angemessenem Maße Rechnung getragen wird. Auf der anderen Seite besteht der Fall der Unterorganisation, wenn Vorgänge, die sich gleichförmig wiederholen, fallweise geregelt werden. In diesem Fall könnte die Effizienz der Produktionsprozesse durch die Einführung genereller Regelungen gesteigert werden.

GUTENBERG favorisiert die generelle gegenüber der fallweisen Regelung. Dies kommt darin zum Ausdruck, dass er die Anordnung ad hoc zur organisatorischen Anpassung an Sondersituationen (z.B. auf Grund von Terminänderungen) als Provisorium bezeichnet. ${ }^{274}$ Der auf lange Sicht unwirtschaftliche Zustand des Improvisierens soll möglichst bald beendet und ein geregelter Betriebsablauf wieder hergestellt werden. Er nimmt außerdem an, dass in großen Betrieben die generelle gegenüber der fallweisen Regelung an Gewicht gewinnt. Diesen Abdrängungsprozess sieht er als Symptom fur die ungeheure Wirksamkeit der Substitution der fallweisen durch die generelle

\footnotetext{
${ }^{272}$ Vgl. Gutenberg (1951), S. 179.

${ }^{273}$ Vgl. Steinmann/Schreyögg (2000), S. 404, i.V.m. Gutenberg (1983), S. 239ff. Methodische Probleme ergeben sich dabei durch die geringe Operationalisierbarkeit des Konzeptes, also durch die Schwierigkeiten bei der Messung der Gleichförmigkeit der Situation, des organisatorischen Regelungsgrades und des Erfolges der organisatorischen Regelungen. Das Substitutionsprinzip der Organisation überzeugt daher mehr durch sein hohes heuristisches Potenzial als durch seine unmittelbare praktische Anwendbarkeit.

${ }^{274}$ Vgl. Gutenberg (1951), S. 180.
} 
Regelung. ${ }^{275}$ An anderer Stelle bezeichnet er fallweise Regelungen als Ventile, die in Funktion treten, wenn generelle Regelungen dem Druck sich ändernder Tatsachen nicht mehr gewachsen sind. ${ }^{276}$ Diese Ventile sollen aber nur in nach Art und Umfang begrenztem Maße zum Einsatz kommen. Dieser Haltung liegt die Auffassung zu Grunde, dass die Voraussetzungen für eine Vorziehenswürdigkeit der generellen gegenüber der fallweisen Regelung in großem Umfang gegeben sind.

Die Möglichkeit zur Übertragung der beschriebenen Leitideen auf Prozesse der technikunterstützten Informationsverarbeitung hängt also auch bei dieser Problemstellung davon ab, inwiefern die Gleichartigkeit der Prozesse gegeben ist. Diese Frage wurde in den vorangegangenen Abschnitten bereits an zwei Stellen (bei der Überprüfung der Anwendbarkeit des Produktionsfunktionskonzeptes und bei der Überprüfung der Möglichkeit zur synoptischen Planung von Prozessen der Informationsverarbeitung) mit dem Ergebnis beantwortet, dass bei Routine- und Regelaufgaben eine Gleichartigkeit realitätsgerecht angenommen werden kann. Demnach ist bei Routine- und Regelaufgaben die generelle Regelung der fallweisen Regelung vorzuziehen. Kehrt man die Argumentation um, so lässt sich feststellen, dass die Variabilität des Betriebsgeschehens bei der Erfüllung von Projekt- und in noch stärkerem Maße von Einzelfallaufgaben so hoch ist, dass fallweise Regelungen gegenüber generellen Regelungen vorziehenswürdig erscheinen.

\section{b) Kompetenzzuordnung für Aufgaben des Informationsmanagements}

Neben der Vorziehenswürdigkeit fallweiser oder genereller Regelungen beschäftigte sich GUTENBERG in den frühen Auflagen des ersten Bandes seiner Grundlagen der Betriebswirtschaftslehre außerdem noch mit Fragen der Abteilungsbildung, die dementsprechend ebenfalls zum originären Erkenntnisbereich des Faktortheoretischen Ansatzes gezählt werden können. ${ }^{277}$ Dabei geht es in erster Linie um Fragen der Zuordnung von Entscheidungskompetenzen. GUTENBERG nimmt an, dass die fachlichen Anforderungen an die Leitung einer organisatorischen Einheit, die notwendigerweise mit entsprechenden Entscheidungskompetenzen ausgestattet werden muss, um so höher sind, je höher der Anteil an fallweisen Regelungen am gesamten Regelungsumfang ist. Er zieht außerdem das FAYOL'sche Prinzip der Einheit der Auftragserteilung dem Prinzip der Mehrfachunterstellung vor und weist auf Probleme hin, die im Funktionsmeistersystem, wie es TAYLOR vorgeschlagen hat, entstehen.

In späteren Auflagen befasst GUTENBERG sich im Zusammenhang mit der Abteilungsbildung zusätzlich noch mit der Delegation von Entscheidungen und der daraus folgenden Zentralisation oder Dezentralisation organisatorischer Kompetenzen. ${ }^{278}$ Als Maßstäbe, die der Entscheidung über die Zentralisation oder Dezentralisation von Entscheidungen zu Grunde gelegt werden sollten, nennt er mit der Tragweite der Entscheidung sowie der Interdependenz zu anderen Unternehmungsteilbereichen zwei Kriterien, die er auch für die Abgrenzung echter Führungsaufgaben anwendet. ${ }^{279}$ Dies entspricht dem grundlegenden Postulat der Kongruenz zwischen der Bedeutung einer Aufgabe für

\footnotetext{
${ }^{275}$ Vgl. Gutenberg (1951), S. 181.

${ }^{276}$ Vgl. Gutenberg (1951), S. 185.

${ }^{277}$ Vgl. dazu Gutenberg (1951), S. 184ff.

${ }^{278} \mathrm{Vgl}$. Gutenberg (1983), S. 244ff.

${ }^{279}$ Vgl. Gutenberg (1983), S. $249 f$.
} 
die Unternehmung und der Stellung ihrer Träger im Leitungssystem. ${ }^{280}$ Das zu Grunde liegende Konzept, das Eingriffe der oberen Hierarchieebenen einer Unternehmung nur in Ausnahmefällen vorsieht, während das Tagesgeschäft durch die unteren Hierarchieebenen abgewickelt wird, wurde später auch als Management-by-Exception bezeichnet. Bei der Delegation von Kompetenzen entsteht generell ein organisatorisches Risiko, das sich durch die Loslösung der Anordnungsbefugnis von der delegierenden Person ergibt. Daraus resultiert die Gefahr von Fehlentscheidungen auf Grund mangelnder Sachkenntnis oder von abweichenden Intentionen bei der mit der Entscheidung betrauten Stelle. ${ }^{281}$

Für das Informationsmanagement ergibt sich aus diesen Überlegungen die Empfehlung, möglichst die Anordnungsbefugnis für Routine- und Regelaufgaben an die unteren Hierarchieebenen zu delegieren. Routine- und Regelaufgaben haben zum einen eine geringe Tragweite und zum anderen spielen bei der Erfüllung dieser Aufgaben Interdependenzen zu anderen Unternehmungsbereichen nur eine untergeordnete Rolle. Nur in Ausnahmefällen sollte sich die Unternehmungsleitung mit solchen Aufgabenstellungen beschäftigen. Auf der anderen Seite sind die Voraussetzungen für eine Dezentralisation von Projekt- und Einzelfallaufgaben weniger günstig, da sowohl eine höhere Bedeutung für die Gesamtunternehmung als auch die Notwendigkeit zur Berücksichtigung übergeordneter Zusammenhänge vorliegt.

Die von GUTENBERG geforderte Einheit der Auftragserteilung ist auf dem Gebiet der technikunterstützten Informationsverarbeitung deshalb schwierig zu realisieren, weil Aufgaben der Informationsverarbeitung in allen Teilbereichen der Unternehmung anfallen. Das Informationsmanagement als (zum Teil) zentral wahrgenommene, fachbezogene Führungsaufgabe konkurriert folglich häufig mit den fachlichen und disziplinarischen Führungskompetenzen anderer Instanzen (z.B. der Fachbereichsleitungen) in der Unternehmung. Tatsächlich ist die Einheit der Auftragserteilung nur in solchen organisatorischen Einheiten erreichbar, die ausschließlich mit Aufgaben der Informationsverarbeitung betraut sind (z.B. Rechenzentrum, Software-Entwicklung, Benutzer-Service).

Abschließend ist noch kurz darauf einzugehen, dass dem Thema "Informationssysteme“ in neueren Auflagen des ersten Bandes von GUTENBERGs Grundlagen der Betriebswirtschaftslehre ein eigener Abschnitt im Kapitel zur Betriebsorganisation gewidmet ist. ${ }^{282}$ Darin werden die nachrichtentechnische Informationstheorie, Informationsstrukturen, Kommunikationswege, die Kommunikation in Gruppen und Fragen der normativen und deskriptiven Entscheidungstheorie behandelt. Dabei handelt es sich jedoch nicht um Leitideen, sondern um Erweiterungen des Faktortheoretischen Ansatzes, in den neue Erkenntnisse anderer Wissenschaftsprogramme eingebaut werden. Darüber hinaus fallen die meisten dieser Problemstellungen fachlich nicht unmittelbar in den Gestaltungs- und Steuerungsbereich des Informationsmanagements. Vielmehr folgen erst aus der gewählten organisatorischen Lösung die Vorgaben für die

\footnotetext{
${ }^{280}$ Vgl. Corsten (2000), S. 40.

${ }^{281}$ Vgl. Gutenberg (1983), S. 257. In diesem Punkt ist bereits der Einfluss der Neuen Institutionenökonomie erkennbar. Zu dem Zeitpunkt, als die spăten Auflagen der dreibändigen Grundlagen der Betriebswirtschaftslehre erschienen, fand die Neue Institutionenokonomie in der wissenschaftlichen Diskussion mehr und mehr Beachtung.

${ }^{282} \mathrm{Vgl}$. Gutenberg (1983), S. $267 \mathrm{ff}$.
} 
Gestaltung des technikunterstützten Informationssystems der Unternehmung. Aus diesen Gründen sind die betreffenden Ausführungen für einen Produktionsorientierten Ansatz zu einer Theorie des Informationsmanagements nur mittelbar relevant und werden hier nicht im Detail behandelt.

\subsection{Aussagekraft des Produktionsorientierten Ansatzes für eine Theorie des Informationsmanagements}

Das Ziel dieses Abschnittes ist es, die Aussagekraft des in diesem Kapitel entwickelten Produktionsorientierten Ansatzes für eine Theorie des Informationsmanagements kritisch zu überprüfen. Im Abschnitt 3.4.1 werden zunächst Lösungsansätze aufgezeigt, die der Ansatz für die im zweiten Kapitel beschriebenen, idealtypischen Problemstellungen der Informationsverarbeitung in der Praxis bietet. Anschließend werden im Abschnitt 3.4.2 die Erkenntnisgrenzen des Ansatzes dargelegt, die zugleich die Notwendigkeit zur Analyse des Informationsmanagements aus weiteren Perspektiven, also zur Entwicklung weiterer Theorieansätze, begründen.

\subsubsection{Lösungsansätze für offene Probleme in der Praxis}

Dem allgemeinen Vorgehen des Faktortheoretischen Ansatzes folgend nähert sich der hier entwickelte Produktionsorientierte Ansatz für eine Theorie des Informationsmanagements den zu erfüllenden Gestaltungs- und Steuerungsaufgaben unter dem Gesichtspunkt der Produktivitätssteigerung bzw. der Kostensenkung. Die Produktivität und die aus ihr folgende Kostensituation stellen im Faktortheoretischen Ansatz den Maßstab dar, an dem die Maßnahmen der dispositiven Faktoren auszurichten sind. Übertragen auf den Bereich des Informationsmanagements besteht das Erkenntnispotenzial dementsprechend in Aussagen zur Planung, Steuerung und Kontrolle von Produktivität und Kosten der technikunterstützten Informationsverarbeitung.

Die Aussagekraft der in dieser Arbeit entwickelten Theorieansätze für das Informationsmanagement ist generell daran zu messen, inwiefern diese Ansätze Möglichkeiten zur Lösung der im zweiten Kapitel beschriebenen idealtypischen Problemstellungen in der Praxis aufzeigen. Aus diesem Grund wird in den Abschnitten 3.4.1.1, 3.4.1.2, 3.4.1.3 und 3.4.1.4 untersucht, welchen Beitrag zur Lösung dieser Problemstellungen die in diesem Kapitel auf Basis des Faktortheoretischen Ansatzes abgeleiteten Anhaltspunkte für produktivitäts- und kostenorientierte Maßnahmen leisten können. Vorweg kann bemerkt werden, dass der Ansatz zwar in vielen Punkten abstrakte Kriterien liefert, an denen sich Gestaltungsmaßnahmen in der Praxis orientieren können. Diese sind jedoch größtenteils auf Grund von Problemen bei der Operationalisierung nicht unmittelbar praktisch umsetzbar.

\subsubsection{Effektivität der Informationsverarbeitung}

Mangelnde Effektivität der technikunterstützten Informationsverarbeitung kommt in einer fehlenden oder zu geringen Ausrichtung auf die (strategischen) Unternehmungsziele zum Ausdruck. Um den in solchen Fällen auftretenden, fehlgerichteten Ressourceneinsatz zu vermeiden, ist sicherzustellen, dass die betreffenden Anwendungssysteme und Prozesse auf die Erreichung der allgemeinen Unternehmungsziele 
ausgerichtet sind. Geht man, wie in diesem Kapitel, bei der Abgrenzung des Informationsmanagements von der Systematik des Faktortheoretischen Ansatzes aus, so handelt es sich bei den allgemeinen Unternehmungszielen um inhaltliche Vorgaben, die selbst nicht im Gestaltungs- und Steuerungsbereich des Informationsmanagements liegen. Diese Vorgaben sind daher als gegeben aufzufassen und zum Ausgangspunkt für die Planung des informationswirtschaftlichen Produktionsprogramms zu nehmen. In der Programmplanung ist deshalb die Schnittstelle des Informationsmanagements zur allgemeinen Unternehmungsführung mit den allgemeinen Unternehmungszielen als Ergebnis zu sehen. Aus der Perspektive des Faktortheoretischen Ansatzes kann die Effektivität der technikunterstützten Informationsverarbeitung also durch die Programmplanung als Planungsfeld des Informationsmanagements beeinflusst werden.

In Abschnitt 3.3.3.1 wurde erläutert, dass das durch den Faktortheoretischen Ansatz bereit gestellte Instrumentarium zur Unterstützung der Programmplanung sich im Wesentlichen auf operative Problemstellungen beschränkt. Um innerhalb des vorgegebenen strategischen Rahmens das gewinnmaximale Produktionsprogramm bestimmen zu können, müssen folgende Größen bekannt sein: Die technisch determinierten Produktionskoeffizienten, die zur Verfugung stehenden Elementarfaktorkapazitäten und die Stückgewinne je Produktart. ${ }^{283}$ Die im Planungszeitraum je Produktart herzustellenden Mengen können dann (insbesondere mit Mitteln der linearen Programmierung) ermittelt werden. Dieses Grundmodell der Produktionsplanung ist um weitere Einflussgrößen, z.B. eine variable Zahl von Erzeugnisarten, Variabilität der Faktorpreise u.ä., erweiterbar, um größere Gestaltungsfreiheiten bei der Programmplanung abzubilden.

Die genannten Voraussetzungen zur Bestimmung des gewinnmaximalen Produktionsprogramms sind für das Informationsmanagement bei der Produktion von unternehmungsintern genutzten Informationen, also Informationen zur Koordination arbeitsteiliger Aktivitäten sowie Informationen zur Herstellung von Informationsvorsprüngen gegenüber Wettbewerbern, nicht gegeben. Dies gilt selbst dann, wenn angenommen wird, dass sich die Kapazitäten der Potenzialfaktoren zuverlässig bestimmen lassen. Technisch determinierte Produktionskoeffizienten liegen lediglich bei solchen Prozessen vor, bei denen das Konzept der Produktionsfunktion anwendbar ist. Für Projekt- und Einzelfallaufgaben können folglich keine eindeutigen Verbrauchsfunktionen zur Abbildung des Zusammenhangs zwischen Faktoreinsatz und Faktorertrag aufgestellt werden. Für Routine- und Regelprozesse dagegen ist diese Problemstellung im Prinzip lösbar. Allerdings lässt sich auch bei diesen Aufgabentypen in der Regel kein Stückgewinn ermitteln, da die Informationsverarbeitung zur Unterstützung übergeordneter Prozesse dient, ohne dass die produzierte Information selber einen Erlös erzielen würde.

Bei der Produktion von unternehmungsextern genutzten Informationen (Informationen als Produktbestandteil) ist die Bestimmung des gewinnmaximalen Produktprogramms unter der Annahme möglich, dass der Zustand vollkommener Information über Absatzmöglichkeiten, Produktions- und Kostenfunktionen und sämtliche weiteren relevanten Einflussgrößen vorliegt. In der Realität ist diese Voraussetzung nicht erfüllt, da ungelöste Operationalisierungsprobleme für diese Größen bestehen. Daher kann das

${ }^{283} \mathrm{Vgl}$. Gutenberg (1983), S. $152 \mathrm{f}$. 
Gewinnmaximum auch für die Produktion unternehmungsextern genutzter Informationen nicht präzise bestimmt werden. ${ }^{284}$ Allerdings bietet der Ansatz in diesem Bereich zumindest ein abstraktes theoretisches Kriterium dafür, welche Eigenschaften ein optimales Produktionsprogramm aufweisen sollte. Dieses Kriterium besteht, den methodischen Leitideen des Ansatzes folgend, in der Angabe von Marginalbedingungen, durch die ein optimaler, anzustrebender Gleichgewichtszustand beschrieben wird. Die informationelle Ausstattung eines Produktes soll demnach exakt so beschaffen sein, dass die Grenzerlöse und die Grenzkosten des jeweiligen Qualitätsparameters zum Ausgleich kommen.

Als Schlussfolgerung ergibt sich, dass der Faktortheoretische Ansatz nur wenig Ansatzmöglichkeiten bietet, um in der Praxis die Effektivität der Informationsverarbeitung zu erhöhen. Die strategische Ausrichtung der technikunterstützten Informationsverarbeitung kann mit den Mitteln des Ansatzes nicht unterstützt werden. Im operativen Bereich liegt die Schnittstelle zwischen der allgemeinen Unternehmungsführung und einem produktionsorientiert aufgefassten Informationsmanagement in der Produktionsprogrammplanung. Bei Bekanntheit der produktionstechnischen und absatzpolitischen Einflussgrößen kann das gewinnmaximale Produktionsprogramm mit Hilfe von Optimierungsmethoden eindeutig bestimmt werden. Für unternehmungsintern genutzte Informationen besteht jedoch nur ein sehr mittelbarer Zusammenhang zur Absatzsphäre der Unternehmung. Bei unternehmungsextern genutzten Informationen liegt ein derartiger Zusammenhang, wenn die informationelle Ausstattung von Produkten als Mittel der Produktpolitik aufgefasst wird, zwar vor. Im praktischen Fall bestehen jedoch erhebliche Unsicherheiten, an denen die genaue Bestimmung des gewinnmaximalen Produktionsprogrammes auch in diesem Bereich scheitert. ${ }^{285}$

\subsubsection{Effizienz der Informationsverarbeitung}

Die Effizienz der Informationsverarbeitung stellt den Problembereich dar, in dem der Faktortheoretische Ansatz die größten Lösungspotenziale aufweist, da sich die Erkenntnisinteressen des Ansatzes auf die Anwendung des ökonomischen Prinzips im Wirtschaftsgeschehen und somit unmittelbar auf Effizienzfragen richten. Der hier entwickelte Produktionsorientierte Ansatz für eine Theorie des Informationsmanagements liefert sowohl für gut strukturierte als auch für weniger gut strukturierte Prozesse der Informationsverarbeitung Anhaltspunkte und Instrumente zur Erhöhung und Überwachung der Effizienz. Die Effizienzanalyse beschränkt sich allerdings sehr weitgehend auf die Input-Seite. Auf der Input-Seite werden zum einen die Faktor-

\footnotetext{
${ }^{284}$ Gutenberg geht in diesem Zusammenhang auf Spannungen zwischen produktionstechnischen und absatzpolitischen Überlegungen und Zielen sowie auf verschiedene Möglichkeiten zur Festlegung von Priorităten ein, vgl. Gutenberg (1983), S. 154ff. Diese Spannungen sind nichts anderes als Folge der in der Praxis vorliegenden Unsicherheitssituation, die eine gewinnmaximale Produktionsplanung verhindert, obwohl sie bei Vorliegen vollständiger Information rechentechnisch ermittelt werden konnte.

${ }^{285}$ Grundsătzlich lässt sich das Vorhandensein von Unsicherheit durch eine entsprechende Modifizierung der verwendeten Planungsmodelle berücksichtigen. Dies erfordert allerdings eine geeignete modellmäßige Abbildung der Unsicherheitssituation, die wiederum einschränkende Annahmen über zukünftige Zustände notwendig macht. Insbesondere bei hoch ausgeprägter Unsicherheit, bei der keine Kenntnis über sämtliche alternativ möglichen Entwicklungen besteht, lässt sich die Planungssituation auch durch eine Modifizierung der Modelle nicht adäquat abbilden.
} 
einsatzmengen und zum anderen die durch den Faktoreinsatz entstehenden Kosten untersucht. $^{286}$

Im Rahmen der Betrachtung der Vorkombination wurden zunächst jeweils für sich genommen die Bedingungen für die optimale Ergiebigkeit der Elementarfaktoren formuliert. Die Bedingungen für die größtmögliche Ergiebigkeit der Betriebsmittel und der objektbezogenen menschlichen Arbeitsleistungen können ohne größere Modifikationen auf den Bereich der technikunterstützten Informationsverarbeitung übertragen werden. Sie betreffen dann die in diesen Prozessen verwendete Hard- und Software sowie die an den informationellen Werkstoffen durch die menschlichen Aufgabenträger vollzogenen objektbezogenen Arbeitsleistungen. Im Bereich der Betriebsmittel hängt die Ergiebigkeit von der allgemeinen technischen Leistungsfähigkeit (Grad der Modernität, Grad der Abnutzung, Zustand der Betriebsfähigkeit) und von der unternehmungsspezifischen Eignung (quantitative und qualitative Kapazität, erforderliche fertigungstechnische Elastizität) $\mathrm{ab}$. Zur Sicherstellung einer optimalen Ergiebigkeit objektbezogener menschlicher Arbeitsleistungen sind die in Abschnitt 3.3.1.1 beschriebenen subjektiven und objektiven Bedingungen herzustellen. Die Bedingungen hoher Ergiebigkeit gelten sowohl für schlecht strukturierte als auch für gut strukturierte Prozesse der technikunterstützten Informationsverarbeitung. Deshalb liefert der im vorigen Abschnitt entwickelte Ansatz sowohl bei Prozessen zur Erfüllung von Routine- und Regelaufgaben als auch bei Prozessen zur Erfullung von Projekt- und Einzelfallaufgaben Hinweise für die Praxis zur Steigerung der faktorbezogenen Effizienz im Rahmen der Vorkombination.

Dagegen sind die Bedingungen furr eine optimale Ergiebigkeit des Werkstoffeinsatzes, wie in Abschnitt 3.3.1.3 gesehen, nur in sehr eingeschränktem Maße auf Informationen als Werkstoffe übertragbar. Allerdings sind die Forderung, standardisiertes Material zu verwenden, und die entsprechende Begründung auch im Gestaltungsbereich des Informationsmanagements prinzipiell tragfähig. Die Effizienz der Informationsverarbeitung kann im Rahmen der Vorkombination ferner auch durch die Anordnung der Elementarfaktoren beeinflusst werden. Für die Erfüllung dieser Aufgabe liefert der Faktortheoretische Ansatz mit den in Abschnitt 3.3.1.4 erläuterten Gestaltungsprinzipien ebenfalls einige Anhaltspunkte. Es wurden Bedingungen für die Entscheidung zwischen dem Verrichtungsprinzip und dem Prozessfolgeprinzip genannt und deren Bedeutung für den Gestaltungsbereich des Informationsmanagements erläutert.

An die Vorkombination schließt sich die Endkombination der Elementarfaktoren, von der ebenfalls Effizienzwirkungen ausgehen, an. Im Abschnitt 3.3.2.1 wurde begründet, dass die Anwendung derjenigen Aussagen des Faktortheoretischen Ansatzes, die die Endkombination von Elementarfaktoren betreffen, auf Grund der generellen Annahmen des Ansatzes lediglich für Routine- und Regelaufgaben zulässig ist. Für diese Aufgabentypen können Wirtschaftlichkeitsbetrachtungen auf Basis der Anwendung des Produktionsfunktionskonzeptes und mit Hilfe von aus den Produktionsfunktionen abgeleiteten Kostenfunktionen erfolgen. Ineffizienzen können während des Planungs-

\footnotetext{
${ }^{286}$ Allgemein kann zwischen bereitstellungsorientierten und verwendungsorientierten Wirtschaftlichkeitsbetrachtungen von Anwendungssystemen unterschieden werden, vgl. Dobschütz (1992), S. 44. Effizienzbetrachtungen, die auf Produktions- und Kostenfunktionen basieren, fokussieren die Aufwandsseite des jeweiligen Analyseobjektes und sind somit den bereitstellungsorientierten Wirtschaftichkeitsbetrachtungen zuzuordnen.
} 
zeitraums entstehen, falls die tatsächlichen Inputs und/oder Outputs von den geplanten Mengen abweichen. Solche Ineffizienzen lassen sich nicht nur feststellen, sondern ihre Ursachen können leichter aufgedeckt und beseitigt werden, wenn sich die Wirtschaftlichkeitskontrolle auf eine präzise Planung stützen kann, die auf Produktions- und Kostenfunktionen basiert. Zur Analyse derartiger Abweichungen ist in der Kostentheorie ein hoch entwickeltes Instrumentarium entstanden. ${ }^{287}$ Produktions- und Kostenfunktionen ermöglichen darüber hinaus den Vergleich alternativer Verfahren zur Herstellung eines informationellen Produktes und die auf einen längeren Zeitraum bezogene Beobachtung, wie sich die Produktions- und Kostenverhältnisse entwickeln. Die Untersuchung in diesem Kapitel beschränkte sich auf Produktionsfunktionen vom Typ B. Die Berücksichtigung weiterer Typen von Produktionsfunktionen wäre innerhalb des hier entwickelten konzeptionellen Bezugsrahmens möglich. Sie würde eine noch tiefergehende Analyse und Gestaltung von Prozessen im Bereich der technikunterstützten Informationsverarbeitung erlauben, ohne jedoch grundlegend neue Erkenntnispotenziale für das Informationsmanagement aufzuweisen.

In Bezug auf Fragen der Effizienz als einem der im zweiten Kapitel dargestellten idealtypischen Problemfelder des Informationsmanagements in der Praxis kann deshalb festgehalten werden, dass der Faktortheoretische Ansatz beträchtliche Erkenntnispotenziale für die Untersuchung und Gestaltung von Prozessen der technikunterstützten Informationsverarbeitung aufweist. Ausgehend von einer verbesserten Kostenverfolgung im Gestaltungsbereich des Informationsmanagements können ferner nicht nur einmalige Erhöhungen der Wirtschaftlichkeit erreicht werden, sondern es können auch Anreizsysteme geschaffen werden, die auf eine fortgesetzte Effizienzverbesserung der Prozesse abzielen.

Die Anhaltspunkte zur Steigerung der Effizienz betreffen ferner im Wesentlichen die Betriebsphase von Anwendungssystemen, nicht jedoch die Phase der Systementwicklung. Allerdings stellt die Idee der synoptischen Planungsrationalität, die nach Vorstellung des Faktortheoretischen Ansatzes Ausgangspunkt aller Planungsaktivitäten ist, auch für das Management von Systementwicklungsprojekten eine Orientierungsgröße dar. Die Qualität der Projektplanung und die Effizienz des Projektes sind aus dieser Perspektive wie andere Teilplanungen auch an dem allgemeinen Kriterium der Planungsgenauigkeit zu messen. Eine hohe Qualität der Projektplanung resultiert dementsprechend aus einem lückenlosen Vorausdenken aller im Rahmen des Projektes durchzuführenden Aktivitäten. Die Projektplanung kann im Prinzip durch die gleichen Instrumente (Optimierungsmodelle und Prognosemodelle) unterstützt werden, wie die allgemeine Produktionsplanung.

\subsubsection{Organisatorische Zuordnung informationswirtschaftlicher Aufgaben}

Die organisatorische Zuordnung von Aufgaben und Entscheidungskompetenzen wird durch den Faktortheoretischen Ansatz in erster Linie unter dem Aspekt der Delegation betrachtet. Der hier entwickelte Produktionsorientierte Ansatz für eine Theorie des Informationsmanagements blendet organisatorische Fragen also nicht vollständig aus. Er ist daher im Prinzip geeignet, Aussagen über die Vorziehenswürdigkeit zentraler bzw. dezentraler Lösungen bei der Zuordnung informationswirtschaftlicher Aufgaben

${ }^{287}$ Vgl. z.B. Coenenberg (1999), S. 372ff;; Haberstock (1999), S. 359ff. 
und Entscheidungskompetenzen zu treffen. Diese Aussagen basieren auf einer Beurteilung der mit der Delegation verbundenen Risiken und Chancen. Es werden außerdem Fragen der Leitungsspanne und der Gliederungstiefe behandelt.

Als Kriterien, nach denen sich die organisatorische Zuordnung von Anordnungs- und Entscheidungsbefugnissen richten sollte, führt GUTENBERG die Tragweite der Entscheidung sowie die Interdependenz $z u$ anderen Unternehmungsteilbereichen an. ${ }^{288}$ In Abschnitt 3.3.3.2 wurde daraus bereits die Schlussfolgerung gezogen, dass entsprechend dem Management-by-exception-Konzept die Anordnungsbefugnis für Routine- und Regelaufgaben tendenziell dezentral zugeordnet werden sollte, während bei Projekt- und Einzelfallaufgaben eher die Voraussetzungen für eine zentrale organisatorische Lösung vorliegen. In Bezug auf die Zuordnung von Aufgaben, die GUTENBERG als Arbeitsverteilung bezeichnet, wird angenommen, dass diese getrennt von der Zuordnung von Anordnungs- und Entscheidungsbefugnissen behandelt werden kann. ${ }^{289}$ Bei der Lösung beider Problemstellungen, die er ,auf zwei völlig verschiedenen Ebenen" ansiedelt, sind Kapazitäts-, Qualitäts- und Konformitätsbedingungen zu berücksichtigen. ${ }^{290}$

Die Aussagen zur Leitungsspanne und Gliederungstiefe sind für das Informationsmanagement insofern relevant, als sie Ansatzpunkte für die Konfiguration solcher organisatorischer Einheiten bieten, die sich ausschließlich mit Aufgaben der Informationsverarbeitung befassen (also z.B. Rechenzentrum, Benutzer-Service, SoftwareEntwicklung u.ä.). Für diese Abteilungen sollte so weit wie möglich die Einheit der Auftragserteilung gewährleistet werden, um Kompetenzstreitigkeiten und damit verbundenen Reibungen und Unzulänglichkeiten vorzubeugen. In Bezug auf die Leitungsspanne wird angenommen, dass in Situationen, in denen ein hohes $\mathrm{Ma} \beta$ an genereller Regelung realisierbar ist, eine hohe Leitungsspanne möglich ist, da sich die Arbeit der Führungsinstanz weitgehend auf disponierende und anordnende Aufgaben beschränkt. $^{291}$ Dagegen ist ein stärkerer Anteil fallweiser Regelungen mit einem erhöhten Ausmaß an fachlichen Aufgaben der Führungsinstanz verbunden, das eine niedrigere Leitungsspanne mit einer geringeren Zahl der unterstellten Personen erforderlich macht. Die Gliederungstiefe hängt eng mit der Leitungsspanne zusammen ${ }^{292}$ und kann als abhängige Variable aufgefasst werden. Niedrige Leitungsspannen führen zu hohen Gliederungstiefen und umgekehrt.

Der Wert der Aussagen zu Leitungsspanne und Gliederungstiefe liegt genau genommen mehr in ihrem heuristischen Potenzial, als in der unmittelbaren Umsetzbarkeit in praktischen Problemstellungen. Relativierend ist ferner darauf hinzuweisen, dass das Konzept des Faktortheoretischen Ansatzes in diesem Punkt weniger überzeugend ist als in anderen Bereichen. Dies hängt insbesondere mit der einseitig negativen Beurteilung dezentraler organisatorischer Lösungen zusammen. GUTENBERG liefert kein stichhaltiges Argument für die Delegation von Entscheidungsbefugnissen und begründet diese lediglich damit, dass die „Größe und Kompliziertheit des

\footnotetext{
${ }^{288}$ Vgl. Gutenberg (1983), S. 247ff.

${ }^{289}$ Vgl. Gutenberg (1983), S. 256.

${ }^{290}$ Vgl. Gutenberg (1983), S. 244ff.; S. 257.

${ }^{291} \mathrm{Vgl}$. Gutenberg (1983), S. 254.

${ }^{292} \mathrm{Vgl}$. Gutenberg (1983), S. 256.
} 
Unternehmens ${ }^{\text {c } 293}$ verlangen, dass Führungsgehilfen in den Steuerungsprozess eingefügt werden. Das Motiv für die Delegation von Entscheidungskompetenzen liegt demnach ausschließlich in einer kapazitätsmäßigen Entlastung der Unternehmungsleitung. Folglich wird das Delegationsrisiko stark betont, mit der Konsequenz, dass nur solche Entscheidungskompetenzen für eine Delegation empfohlen werden, bei denen Fehlentscheidungen auf Grund der geringen Bedeutung für die Gesamtunternehmung unproblematisch sind. Die mit einer Delegation von Entscheidungen verbundenen Chancen werden nicht in angemessener Weise deutlich, da der Analyse die Auffassung zu Grunde liegt, dass es sich bei der dezentralen Zuordnung von Entscheidungskompetenzen stets um eine Ersatzlösung auf Grund begrenzter Kapazitäten der zentralen Aufgabenträger handelt.

Eng mit dem Problem der Delegation hängt auch das Substitutionsprinzip der Organisation bzw. die Entscheidung zwischen dem Einsatz fallweiser oder genereller Regelungen zusammen. Ein hoher Anteil an fallweisen Regelungen erfordert bei gegebenen Kapazitäten der jeweils übergeordneten Instanz ${ }^{294}$ generell eine stärkere Delegation von Entscheidungskompetenzen. Wenn die Gesamtsituation einer Unternehmung in einem Umfeld von sich stark verändernden Marktbedingungen eine hohe Flexibilität der Informationsverarbeitung erfordert, dann hat dies zur Folge, dass die Gleichartigkeit und Periodizität der betrieblichen Vorgänge sinkt. In diesem Fall steigt aus Sicht des Faktortheoretischen Ansatzes die Vorteilhaftigkeit fallweiser gegenüber generellen Regelungen, sodass ohne Veränderung des generellen Regelungsgrads eine Situation der Überorganisation entsteht. Dieser Gefahr der Überorganisation lässt sich durch eine Erhöhung des Anteils fallweiser Regelungen, die die Reaktionsfähigkeit auf geänderte Marktbedingungen verbessert, begegnen. Damit wird zugleich eine verstärkte Delegation von Entscheidungskompetenzen notwendig, um die übergeordneten Instanzen zu entlasten.

Die Aussagekraft des Ansatzes für das Informationsmanagement liegt auch hier in erster Linie in seinem heuristischen Potenzial, da es sich bei dem optimalen organisatorischen Regelungsgrad um ein kaum operationalisierbares Konzept handelt. Es kann deshalb lediglich die abstrakte Empfehlung abgeleitet werden, auf steigende Flexibilitätsanforderungen mit der Substitution von generellen durch fallweise Regelungen zu reagieren und zugleich eine verstärkte Delegation von Entscheidungskompetenzen vorzunehmen. Die Aussagen lassen sich auch umkehren und auf eine Situation mit sinkender Dynamik des Unternehmungsumfelds anwenden. In einem solchen Fall kommt der Faktortheoretische Ansatz zu dem Ergebnis, dass der Tendenz zur Unterorganisation durch ein höheres Maß an generellen Regelungen und eine stärkere Zentralisation von Entscheidungskompetenzen zu begegnen ist. Generelle Regelungen sind längerfristig stets anzustreben, da diese nach Vorstellung des Faktortheoretischen Ansatzes insgesamt Effizienzvorteile gegenüber fallweisen Regelungen aufweisen.

\footnotetext{
${ }^{293}$ Gutenberg (1983), S. 246.

${ }^{294}$ Die Annahme von gegebenen Kapazitäten entspricht methodisch dem Vorgehen der Partialanalyse, bei der nur einzelne Einflussgrößen variiert werden, während alle anderen Einflussgrößen als unverändert angenommen werden.
} 


\subsubsection{Rolle des Menschen in der Informationsverarbeitung}

Der Mensch nimmt aus der Perspektive des Faktortheoretischen Ansatzes im Rahmen der technikunterstützten Informationsverarbeitung die Rolle eines Produktionsfaktors ein. Menschliche Arbeitsleistungen können entweder dispositiv-anordnender Natur sein und sind dann zu den dispositiven Faktoren, bezogen auf die Gestaltung und Steuerung der technikunterstützten Informationsverarbeitung zum Informationsmanagement, zu rechnen, oder sie sind objektbezogener Natur und stellen damit Elementarfaktoren dar.

Das Informationsmanagement selbst wurde in dieser Arbeit den dispositiven Faktoren mit derivativem Charakter zugeordnet, die eine rationale Schicht (Planung) und eine gestaltend-vollziehende Schicht (Betriebsorganisation) umfassen. Bei der Analyse dieser beiden Schichten wird im Faktortheoretischen Ansatz nahezu vollständig von Einflüssen abstrahiert, die mit der Person der Aufgabenträger zusammenhängen. Vielmehr wird angenommen, dass die Planung und Organisation der technikunterstützten Informationsverarbeitung als derivative dispositive Faktoren einer Analyse zugänglich sind, die sich allein am ökonomischen Prinzip orientiert. Daher ist es nicht erforderlich, bei der Lösung dieser Aufgabenstellungen persönliche Merkmale der Aufgabenträger zu berücksichtigen. Der Produktionsorientierte Ansatz für eine Theorie des Informationsmanagements triff folglich keinerlei Aussagen, die sich direkt auf die menschlichen Träger von Aufgaben des Informationsmanagements beziehen.

Die objektbezogenen menschlichen Arbeitsleistungen als Elementarfaktoren sind aus der Perspektive des Faktortheoretischen Ansatzes so zu gestalten, dass eine möglichst hohe Produktivität, die eine hohe Ergiebigkeit der Faktoren erfordert, erzielt werden kann. Der Produktionsorientierte Ansatz für eine Theorie des Informationsmanagements beleuchtet die Rolle des Menschen als Elementarfaktor in Prozessen der Informationsverarbeitung also unter dem oben bereits behandelten Gesichtspunkt der Effizienz. Einflussgrößen, die in der Person der Aufgabenträger selbst liegen, werden dabei als subjektive Bedingungen der Ergiebigkeit menschlicher Arbeitsleistungen bezeichnet. Bezogen auf die einzelne Person machen diese subjektiven Bedingungen in ihrer Gesamtheit das individuelle Eignungspotenzial dieser Person aus. Fasst man die individuellen Eignungspotenziale wiederum zusammen, so erhält man das gesamte Eignungspotenzial eines Betriebes.

Die Herstellung der subjektiven Bedingungen für die optimale Ergiebigkeit menschlicher Arbeitsleistungen wird im Faktortheoretischen Ansatz als Problem der Personalplanung aufgefasst. Sowohl für die einzelnen Personen als auch über den gesamten Betrieb hinweg ist eine optimale Eignung der Aufgabenträger für die ihnen übertragenen Arbeitsleistungen herbeizufüren, die sowohl einen Eignungsmangel als auch einen Eignungsüberschuss vermeidet und somit ein Gleichgewicht (!) herstellt. Den Ausgangspunkt stellen dabei die zu erfüllenden Aufgabenstellungen dar, für deren Erfullung der benötigte Personalbedarf zu ermitteln und bereitzustellen ist. Die Anpassung des verfügbaren Eignungspotenzials an das benötigte Eignungspotenzial kann sowohl auf dem Wege der Personalbeschaffung als auch auf dem Wege der Personalfreisetzung erfolgen. Die Beschäftigung der vorhandenen Arbeitskräfte erreicht 
dann ihren Bestwert, wenn das vorhandene Eignungspotenzial vollständig ausgeschöpft ist. $^{295}$

Die persönlichen Eigenschaften der in der Unternehmung beschäftigten Menschen, ihre Antriebsstruktur, ihr persönliches Temperament, die Gesinnung, die Begabung und die körperliche Verfassung, werden hier also nur insofern betrachtet, als sie Relevanz für die Ergiebigkeit der objektbezogenen menschlichen Arbeitsleistungen besitzen. Exemplarisch wird dies bei der Analyse des Verrichtungsprinzips und des Prozessfolgeprinzips deutlich, die allein unter dem Gesichtspunkt der unmittelbaren Effizienzwirkungen untersucht werden. Eventuelle Motivationswirkungen dieser Prinzipien werden durch den Faktortheoretischen Ansatz nicht betrachtet bzw. nur zur Ergänzung der Argumentation verwendet. Das betriebswirtschaftiche Problem im Bereich der objektbezogenen menschlichen Arbeitsleistungen besteht allein darin, Menschen mit dem richtigen Eignungsprofil für die in der Unternehmung zu erfüllenden Aufgaben als Mitarbeiter zu finden. Der Ansatz trifft dagegen keine Aussagen über den genauen Zusammenhang zwischen den persönlichen Charaktereigenschaften und der individuellen Eignung für bestimmte Aufgabentypen. Auch Probleme der Mitarbeiterführung, das heißt der zielorientierten persönlichen Beeinflussung der in der Unternehmung beschäftigten Personen unter Berücksichtigung ihrer individuellen Verhaltensmerkmale, werden nicht behandelt.

Der Produktionsorientierte Ansatz für eine Theorie des Informationsmanagements kann auf Grund dieser Reduzierung der Menschen auf ihre Funktion als Produktionsfaktoren keinen Beitrag zur Überwindung der Konstruktionskorridor-Vorstellung leisten, die die Anwender als rein passive Vollzugsinstanzen sieht. Auch für eine verstärkte Einbeziehung der späteren Anwender in den Prozess der Entwicklung von technikunterstützten Informationssystemen werden keine Anhaltspunkte geliefert, da die Konstruktion von Mensch-Maschine-Systemen nicht betrachtet wird. Das Aussagepotenzial des Ansatzes für eine angemessenere Berücksichtigung des Menschen in der Theorie und Praxis des Informationsmanagements ist deshalb relativ gering. Es beschränkt sich auf die Postulierung eines Gleichgewichtes zwischen der erforderlichen und der verfügbaren Qualifikation menschlicher Aufgabenträger in Prozessen der Informationsverarbeitung.

\subsubsection{Erkenntnisgrenzen}

Der in diesem Kapitel vorgestellte Produktionsorientierte Ansatz für eine Theorie des Informationsmanagements basiert auf den Erkenntnisinteressen und Leitideen des Faktortheoretischen Ansatzes. Damit wurde ein Wissenschaftsprogramm der deutschsprachigen Betriebswirtschaftslehre zum Ausgangspunkt genommen, das sich auf der einen Seite durch eine erhebliche inhaltliche Geschlossenheit und ein hoch entwickeltes methodisches Instrumentarium auszeichnet. Auf der anderen Seite enthält dieser Forschungsansatz jedoch auch einige Schwachpunkte, die von den Vertretern späterer Wissenschaftsprogramme stark angegriffen wurden. Die unbestrittene konzeptionelle Geschlossenheit des Ansatzes wurde mit der Beschränkung auf eine ganz bestimmte Klasse von Problemstellungen erkauft. ${ }^{296}$ Dabei handelt es sich um die einer

${ }^{295} \mathrm{Vgl}$. Gutenberg (1983), S. 13.

${ }^{296}$ Vgl. Schanz (1997a), S. 118. 
quantitativen Analyse zugänglichen Probleme, insbesondere im Bereich der Produktions-, Kosten-, Finanzierungs- und Investitions-, der Lagerhaltungs- sowie der Beschaffungstheorie. ${ }^{297}$

Die am Faktortheoretischen Ansatz geübte Kritik bezieht sich zum einen auf die Begrenzung des Untersuchungsgegenstandes durch stark einschränkende Prämissen, die in den Erkenntnisinteressen und Leitideen des Ansatzes zum Ausdruck kommen. Zum anderen werden diejenigen Aussagen innerhalb des durch die Prämissen abgegrenzten Bezugsrahmens besonders angegriffen, die die Führung von Unternehmungen durch die dispositiven Faktoren betreffen. Diese allgemeinen Erkenntnisgrenzen des Wissenschaftsprogramms wirken sich auch auf die aus dem Faktortheoretischen Ansatz abgeleiteten Aussagen zum Informationsmanagement als betrieblichem Aufgabenfeld aus. Nachfolgend werden die Auswirkungen der genannten Kritikpunkte auf den Produktionsorientierten Ansatz für eine Theorie des Informationsmanagements beleuchtet.

\subsubsection{Stark einschränkende Prämissen und eingeengtes Erkenntnisinteresse}

Die Orientierung am ökonomischen Basiskonzept innerhalb des Faktortheoretischen Ansatzes bedeutet eine Einengung des Untersuchungsgegenstandes auf das Rationalprinzip unter Verzicht auf die Einbeziehung verhaltenswissenschaftlicher Erkenntnisse. Die Untersuchungen gehen von vergleichsweise stark einschränkenden Voraussetzungen aus, die von Kritikern des Ansatzes zum Anlass genommen wurden, den Vorwurf des „Modell-Platonismus“ zu erheben. ${ }^{298}$ Bezogen auf das im zweiten Kapitel vorgeschlagene dreistufige Vorgehen zum Theorieeinstieg für das Informationsmanagement betriff dieser Kritikpunkt die zweite Stufe. Bei der Entwicklung des allgemeinen theoretischen Konstrukts werden Annahmen getroffen, die dem Erfahrungsgegenstand der Betriebswirtschaftslehre als empirischem Konstrukt nur in begrenztem Maße gerecht werden. Die theoretischen Überlegungen erstrecken sich dementsprechend weitgehend auf idealtypische Situationen und haben dabei axiomatisch-deduktiven Charakter, sind also entscheidungslogisch ausgerichtet. ${ }^{299} \mathrm{Das}$ hat zur Folge, dass die theoretischen Untersuchungsergebnisse kaum in die Praxis übertragbar sind, da die betreffenden Bedingungen dort in der Regel nicht gegeben sind. Mit der fortschreitenden Verfeinerung der Modelle entsteht somit zunehmend die Gefahr der Tautologisierung, da die aus den Modellen abgeleiteten Aussagen sich einer empirischen Überprüfbarkeit immer mehr entziehen.

Für den Produktionsorientierten Ansatz für eine Theorie des Informationsmanagements hat dies zur Folge, dass sich häufig nur Aussagen in nicht operationalisierter Form ableiten lassen. Es werden Bedingungen für die Existenz von Gleichgewichten im Gestaltungsbereich des Informationsmanagements aufgestellt, die nicht unmittelbar in praktische Gestaltungs- und Steuerungsmaßnahmen umgesetzt werden können. Vielmehr entziehen sich die theoretisch begründbaren Bedingungen im konkreten Fall einer nachprüfbaren Beobachtung. Bei der Überprüfung der Aussagekraft des Ansatzes im vorigen Abschnitt wurde deshalb mehrfach auf das heuristische Potenzial

\footnotetext{
${ }^{297} \mathrm{Vgl}$. Ulrich/Hill (1979), S. 171.

${ }^{298} \mathrm{Vgl}$. Ulrich/Hill (1979), S. 171.

${ }^{299}$ Vgl. Raffée (1993), S. 31.
} 
hingewiesen, das den eigentlichen Wert der Aussagen ausmacht. Die Aussagen selbst sind so abstrakt, dass sie in praktischen Problemstellungen nur Anhaltspunkte furr Maßnahmen des Informationsmanagements, nicht aber konkrete Handlungsempfehlungen bieten.

Die weitgehende Ausklammerung des menschlichen Verhaltens aus der Untersuchung führt außerdem $\mathrm{zu}$ einer weitgehenden Beschränkung der Betrachtung auf die syntaktische Ebene der Informationsverarbeitung. Information wird dabei als Werkstoff im Produktionsprozess, also als objektiv vorhandene und beobachtbare Größe, aufgefasst. Auf der syntaktischen Ebene beleuchtet der Produktionsorientierte Ansatz für eine Theorie des Informationsmanagements Fragen der Bearbeitung, Speicherung und Übertragung von Informationen unter Einhaltung des Rationalprinzips. Die Einbeziehung der semantischen und der pragmatischen Ebene würde dagegen eine Auseinandersetzung mit der Interpretation und Nutzung von Informationen durch den Menschen erfordern. Die immaterielle Ebene der Informationsverarbeitung, die die inhaltliche Ausgestaltung und die zweckmäßige Aufbereitung von Informationen für die menschlichen Aufgabenträger umfasst, wird somit durch den Faktortheoretischen Ansatz nicht untersucht.

Ferner stellen der Faktortheoretische Ansatz und die in diesem Rahmen entwickelten Modelle das Zielkriterium der Gewinnmaximierung sehr stark in den Vordergrund. Sie sind also insofern partikularistisch ausgerichtet, als sie sich im Wesentlichen an den Interessen der Unternehmungseigner orientieren. ${ }^{300}$ Damit wird vernachlässigt, dass in der Realität weitere Zielsetzungen Einfluss auf das Geschehen in der Unternehmung besitzen. Einerseits verfolgen auch die Unternehmungseigner üblicherweise weitere Ziele neben der Gewinnerzielung, andererseits spielen zusätzlich die Interessen weiterer Akteursgruppen eine Rolle. Tatsächlich ist die Situation durch ein Nebeneinander verschiedenster Interessenlagen und dementsprechend durch die Existenz von Interessenkonflikten gekennzeichnet. ${ }^{301}$ Die Erkenntnisgrenzen dieser einseitigen Orientierung am Ziel der Gewinnmaximierung ergeben sich weniger aus den zweifellos vorhandenen normativen Implikationen, als aus der nicht angemessenen Vereinfachung der Zusammenhänge im Erfahrungsbereich der Theorie bei der Abgrenzung ihres Erkenntnisgegenstands.

Die Effektivität der Informationsverarbeitung wird ausschließlich unter dem Aspekt der Gewinnmaximierung durch die Wahl des optimalen Produktionsprogrammes betrachtet. Für die Wirksamkeit der Informationsverarbeitung im Hinblick auf weitere in der Unternehmung verfolgte Zielsetzungen und die Bewältigung von Zielkonflikten können keine Aussagen abgeleitet werden, da diese nicht zum Untersuchungsbereich des Faktortheoretischen Ansatzes gehören. Bei der Effizienzbetrachtung steht die Erfüllung gegebener Aufgabenstellungen durch objektbezogene menschliche Arbeitsleistungen im Vordergrund. Es werden also nicht die Aufgabenträger selbst, sondern nur die von ihnen abgegebenen, objektbezogenen Leistungen betrachtet, die tatsächlich jedoch nur einen Teil ihrer gesamten Aktivitäten in der Unternehmung darstellen. Die Vernachlässigung anderer Zielsetzungen neben der Gewinnmaximierung beeinträchtigt außerdem in besonders starkem Maße eine angemessenere Beachtung der Rolle des Menschen in der Informationsverarbeitung durch eine akteursorientierte Analyse.

${ }^{300}$ Vgl. Raffée (1993), S. 31.

${ }^{301}$ Vgl. Steinmann/Schreyögg (2000), S. 75f.; Eberhardt (1998), S. $41 f$. 


\subsubsection{Konzept der Führung durch die dispositiven Faktoren}

Eine wesentliche Unzulänglichkeit des Ansatzes besteht - selbst bei Festhalten am ökonomischen Basiskonzept - in der Konzeptionalisierung der Unternehmung als Reaktionsgefüge, das sich an Änderungen in den außerbetrieblichen Daten oder an innerbetriebliche Situationsänderungen anpasst. Diese Modellkonzeption eines in seiner Grundstruktur primär auf Datenveränderungen nur reagierenden Systems ist ungeeignet zur Erklärung und Beurteilung der für seine Daseinserhaltung notwendigen, auf seine Um- und Innenwelt gerichteten durchsetzenden Aktionen. ${ }^{302}$ Den dispositiven Faktoren wird damit eine sehr passive Rolle zugewiesen, die ihrer tatsächlichen Funktion für das Betriebsgeschehen nicht gerecht wird.

Bezogen auf das Informationsmanagement bedeutet dies, dass der hier entwickelte produktionsorientierte Theorieansatz keine Erklärungs- und Gestaltungsaussagen für die Entwicklung langfristig angelegter Strategien der technikunterstützten Informationsverarbeitung liefert. Die oben beschriebene Produktionsprogrammplanung als Teilgebiet des Informationsmanagements, das die Effektivität der Informationsverarbeitung gewährleisten soll, ist auf die kurzfristige Erzielung optimaler Deckungsbeiträge entsprechend der gegebenen Marktsituation gerichtet. Der Gesichtspunkt der Einflussnahme auf die Produktionsbedingungen selbst, also die aktive Positionierung der Unternehmung in einer dynamischen Umwelt, spielt keine Rolle.

Auch in den anderen oben angesprochenen Problemfeldern wirkt sich das passive Grundkonzept, wenn auch weniger deutlich, aus. Die organisatorische Zuordnung informationswirtschaftlicher Aufgaben etwa erfolgt aus dieser Perspektive zum Zwecke der Entlastung zentraler Aufgabenträger, nicht aber zur systematischen Ausnutzung von Informationsvorteilen der dezentralen Aufgabenträger, die für den Verbleib der Unternehmung im Wettbewerb ausschlaggebend sein kann. Die Effizienzanalyse als weiteres Problemfeld bezieht sich in erster Linie auf die Auswahl zwischen gegebenen Alternativen bei der Abwicklung von Prozessen zur Erzeugung informationeller Produkte und weniger auf die aktive Gestaltung der Prozesse selbst. Diese kann zwar sehr wohl Gegenstand von Gestaltungsmaßnahmen sein, der Ansatz liefert jedoch kaum Anhaltspunkte dafür, wie diese Maßnahmen im Einzelnen ausgestaltet werden sollten.

Auch die Idealvorstellung der synoptischen Planungsrationalität ist zum Gegenstand der Kritik geworden. ${ }^{303}$ Diese Kritik bezieht sich zum einen auf einen logischen Widerspruch im Konzept des Faktortheoretischen Ansatzes. Die synoptische Planungsrealität steht in diametralem Gegensatz zum Phänomen der Irrationalität, das nach Auffassung des Ansatzes für echte Führungsentscheidungen der Geschäftsleitung maßgebliche Bedeutung besitzt. Ließe sich die irrationale Schicht des dispositiven Faktors in der Planung rational auflösen, dann würde es sich dabei nur um ein rhetorisches Element handeln, das die Bedeutung kreativer Ideen in der Planung betont. Zum anderen richtet sich die Kritik gegen die Vorstellung des „wohl bestellten Hauses", die dem synoptischen Planungsideal zu Grunde liegt. Eine zu detaillierte Planung beeinträchtigt die Möglichkeiten zur situationsabhängigen Reaktion auf unvorhergesehene Entwicklungen. Dadurch werden zum einen unnötige Ressourcen für die Planungsaktivitäten verschwendet, zum anderen besteht die Gefahr von Kon-

\footnotetext{
${ }^{302} \mathrm{Vgl}$. Dietz (1997), S. 1081.

${ }^{303}$ Vgl. z.B. Dietz (1997), S. 1077 f.
} 
flikten zwischen den Planungsinstanzen und den von der Planung betroffenen Einheiten, deren Handlungsspielraum durch die Vorgaben der Planung stark eingeschränkt wird. Die umstrittene Idee vom "wohl bestellten Haus" kommt auch in der Favorisierung genereller gegenüber fallweisen Regelungen im Rahmen der Organisation als Teil der dispositiven Faktoren zum Ausdruck.

Für das Informationsmanagement, das selbst nicht zur irrationalen Schicht der dispositiven Faktoren gehört, sondern in dieser Arbeit der rationalen und der gestaltend-vollziehenden Schicht zugeordnet wurde, ist in diesem Zusammenhang insbesondere der zweite Kritikpunkt von Relevanz. Auswirkungen des synoptischen Planungsideals sind in den abgeleiteten Aussagen zu allen oben angesprochenen Problembereichen erkennbar. Bei unternehmungsextern verwendeten Informationen verlangt die Gewährleistung der Effektivität durch eine genaue Produktionsprogrammplanung einen in der Praxis nicht zu realisierenden Kenntnisstand über die Absatzmöglichkeiten der betreffenden Produkte sowie die Produktions- und Kostenbedingungen der Unternehmung. Im Bereich der organisatorischen Zuordnung informationswirtschaftlicher Aufgaben wird durch den Faktortheoretischen Ansatz zwar anerkannt, dass in besonders dynamischen Umfeldern die fallweise Regelung mit hohem Delegationsgrad gegenüber der generellen Regelung mit stärkerer Zentralisierung von Entscheidungskompetenzen vorzuziehen ist. Ein möglichst hohes Ausmaß an genereller Regelung wird dennoch langfristig als vorteilhafter angesehen, da fallweise Regelungen aus dieser Perspektive lediglich ein Provisorium darstellen, das so weit wie möglich beseitigt werden sollte. Auch die Aussagen, die die Rolle des Menschen in der Informationsverarbeitung betreffen, werden durch das Ideal der synoptischen Planungsrationalität beeinflusst. Die menschlichen Aufgabenträger werden hier als Objekte detailgenauer Planungsaktivitäten gesehen, die nur ausführende Instanz in vollständig vorstrukturierten Prozessen der Informationsverarbeitung sind, ohne selber Einfluss auf die Leistungserstellung zu nehmen. Schließlich wirkt sich das synoptische Planungsideal in der Praxis auch auf die Vorstellungen über das Management von Projekten zur Entwicklung technikunterstützter Informationssysteme aus. Hier kommt die Kritik, dass eine zu detaillierte Planung die Reaktionsfähigkeit beeinträchtigt und in vielen Fällen zu Konflikten mit den Betroffenen führt, ebenfalls voll zum Tragen. 


\section{Erkenntnispotenziale und Lösungsbeiträge des System- orientierten Ansatzes für das Informationsmanagement}

Das Kapitel 4 untersucht die Erkenntnispotenziale, die der Systemorientierte Ansatz der Betriebswirtschaftlehre für das Informationsmanagement aufweist. Analog zum Vorgehen im vorangegangenen dritten Kapitel wird in Abschnitt 4.1 zunächst die zweite Stufe des Theorieeinstiegs bewältigt, indem der allgemeine Erkenntnisgegenstand des Systemorientierten Ansatzes konstruiert wird. Abschnitt 4.2 schließt den Theorieeinstieg ab und konstruiert das Informationsmanagement als spezielles Erkenntnisobjekt des Systemorientierten Ansatzes. Die eigentliche Theoriebildung erfolgt in Abschnitt 4.3, der den beiden großen Forschungsrichtungen der Kybernetik folgt und zwischen einem konservativen und einem innovativen Informationsmanagement unterscheidet. In Abschnitt 4.4 wird eine kritische Würdigung der gewonnenen Erkenntnisse vorgenommen, die sowohl auf die Lösungsansätze für offene Probleme in der Praxis als auch auf die Erkenntnisgrenzen des Ansatzes eingeht.

\subsection{Der Systemorientierte Ansatz der Betriebswirtschafts/ehre}

Der Systemorientierte Ansatz der Betriebswirtschaftslehre wurde im deutschsprachigen Raum ausgehend von der 1968 von ULRICH vorgestellten Arbeit „Die Unternehmung als produktives soziales System" zunächst an der Hochschule St. Gallen konzipiert. ${ }^{1}$ Er wurde mit der Zielsetzung entwickelt, bestimmte Schwächen des seinerzeit als "State of the Art" etablierten Faktortheoretischen Ansatzes zu überwinden. ULRICH ging davon aus, dass die Betriebswirtschaftslehre in ihrem damaligen Verständnis als Schwesterdisziplin der Volkswirtschaftslehre zu stark auf rein ökonomische Fragestellungen beschränkt und damit für in der betrieblichen Praxis vorliegende Problemstellungen nur eingeschränkt aussagekräftig war. Der Systemorientierte Ansatz strebt eine Öffnung der Betriebswirtschaftslehre als wissenschaftlicher Disziplin an. $\mathrm{Zu}$ diesem Zweck erfolgt eine Zuwendung zu Ideen und Modellen der Allgemeinen Systemtheorie und der Kybernetik.

Es können zwei wesentliche Merkmale des Systemorientierten Ansatzes festgestellt werden, die seine Eigenständigkeit begründen und dem betriebswirtschaftlichen Denken eine Neuorientierung gegeben haben, die bis in die Gegenwart fortwirkt. Zum einen stehen offene Systeme im Mittelpunkt der Allgemeinen Systemtheorie, ${ }^{2}$ die sich nicht nur mit den Systemen selbst, sondern auch mit deren Beziehungen zur Systemumwelt befasst. Deshalb lenkt der Systemorientierte Ansatz der Betriebswirtschaftslehre den Blick auf die Offenheit von Unternehmungen und dehnt die Analyse im Vergleich zu vorherigen Denkschulen auf ihre Umweltbeziehungen aus. ${ }^{3}$ Zum anderen stellt die These, es gebe zwar disziplinäre Wissenschaften, nicht aber disziplinäre Probleme in der Realität, ${ }^{4}$ einen Kern des Systemdenkens dar. Aus diesem Grund wird ein interdisziplinäres Vorgehen ${ }^{5}$ gefordert, das Erkenntnisse aus sozial-,

\footnotetext{
Vgl. Malik (1993), S. 49.

Vgl. Fuchs (1973), S. 1.

Vgl. Probst (1987), S. 54f.; Steinmann/Schreyøgg (1993), S. 63.

4 Vgl. ähnlich Ulrich (1970), S. 18f.; Probst (1987), S. 42; S. 294; Malik (1993), S. 49.

5 Probst spricht in diesem Zusammenhang nicht von einem interdisziplinären Vorgehen, sondern einem adisziplinären Abgrenzen und Behandeln von Kontexten. Vgl. Probst (1987), S. 43.
} 
natur- und ingenieurwissenschaftlichen Disziplinen aufgreift und für betriebswirtschaftliche Zwecke fruchtbar macht. Um den anspruchsvollen Forderungen einer ganzheitlichen und vieldimensionalen Betrachtung genügen zu können, war es im Laufe der Zeit erforderlich, immer mehr wissenschaftliche Gebiete einzubeziehen, die bis zur Entwicklung des Systemorientierten Ansatzes in keinerlei Zusammenhang zur Betriebswirtschaftslehre gestanden hatten. ${ }^{6}$

Die folgenden Ausfuihrungen erläutern die Erkenntnisinteressen und Leitideen der Systemorientierten Betriebswirtschaftslehre. Es handelt sich dabei um die zweite Stufe des Theorieeinstiegs zum Informationsmanagement, auf der das allgemeine theoretische Konstrukt des Wissenschaftsprogramms konstruiert wird, das anschließend für die Analyse des Informationsmanagements näher zu spezifizieren ist. Abschnitt 4.1.1 geht zunächst auf die Erkenntnisinteressen der Systemorientierten Betriebswirtschaftslehre ein. In den darauffolgenden Abschnitten werden anschließend die inhaltlichen (Abschnitt 4.1.2) und die methodischen (Abschnitt 4.1.3) Leitideen des Ansatzes erläutert.

\subsubsection{Erkenntnisinteressen des Systemorientierten Ansatzes}

Da die Erkenntnisinteressen der Systemorientierten Betriebswirtschaftslehre sich aus den Erkenntnisinteressen der Allgemeinen Systemtheorie ableiten, ist bei der nachfolgenden Darstellung auf diese Bezug zu nehmen. Die Systemtheorie insgesamt stellt kein geschlossenes und einheitliches Theoriegebäude dar. ${ }^{7}$ Vielmehr setzt sie sich aus vielfältigen Forschungsrichtungen zusammen, die ihre Wurzeln in sehr unterschiedlichen Wissenschaftsdisziplinen haben. Einige Grundgedanken und Zielsetzungen lassen sich jedoch in allen systemtheoretischen Arbeiten finden, die im Detail allerdings recht unterschiedlich ausgeprägt sein können. Der Systemansatz befasst sich auf formal-analytischer Ebene mit Prinzipien und Gesetzmäßigkeiten, die für alle Untersuchungsobjekte, die sich als System fassen lassen, Geltung besitzen sollen. Dabei geht es weniger um die Untersuchung spezifischer, konkreter Fragestellungen im Einzelfall als um die allgemeine Analyse der Funktionsweise und der Erhaltung von Systemen insgesamt. Die Kybernetik als für die Betriebswirtschaftslehre besonders relevanter Teilbereich der allgemeinen Systemtheorie befasst sich mit Fragen der Lenkung von zielorientierten, dynamischen Systemen. ${ }^{8}$

Das wesentlichste Merkmal der Systemtheorie liegt in der Art und Weise, wie Systeme aufgefasst werden. ${ }^{9}$ Die Systemtheorie nimmt eine ganzheitliche Perspektive ein und postuliert, dass das System als Ganzes mehr als die Summe seiner Teile darstellt. Die funktionalen Eigenschaften von Systemen resultieren nach dieser Auffassung nicht allein aus den lokal analysierbaren Eigenschaften der Systemelemente, sondern ganz wesentlich auch aus der spezifischen Verknüpfung dieser Elemente. ${ }^{10}$ Der damit angesprochene Sachverhalt, dass die Eigenschaften der Gesamtheit nicht vollständig

6 Vgl. Malik (1993), S. 52

7 Vgl. zum Folgenden Stünzner (1996), S. 39ff.

$8 \mathrm{Vgl}$. Ashby (1974), S. 282. Auch über das Wesen und den Inhalt der Kybernetik bestehen unterschiedlich akzentuierte Auffassungen. Für eine Übersicht vgl. Krieg (1971), S. 26ff., Malik (1992), S. 26ff.

9 Vgl. Stünzner (1996), S. 39.

${ }^{10}$ Vgl. Bertalanffy (1969), S. 45; Malik (1992), S. 80. 
aus den Merkmalen der einzelnen Teile abgeleitet werden können, da auf der höheren Betrachtungsebene neue Eigenschaften hinzutreten, wird als Emergenz bezeichnet. ${ }^{11}$

Als zentrale Problemstellung, mit der sich die Kybernetik und folglich auch die Systemorientierte Betriebswirtschaftslehre auseinander setzen, kann das Problem der Beherrschung von Komplexität aufgefasst werden. ${ }^{12}$ Die Kybernetik akzeptiert die Komplexität und Unbestimmtheit der untersuchten Systeme und Prozesse nicht nur vorbehaltlos, sondern sie sieht darin geradezu eine notwendige Voraussetzung für bestimmte Verhaltensweisen von Systemen. ${ }^{13}$ Dementsprechend geht die Systemorientierte Betriebswirtschaftslehre davon aus, dass für das Management sozialer Systeme die Bewältigung von Komplexität von entscheidender Bedeutung ist. ${ }^{14}$ Kybernetisches Management beruht daher auf der expliziten Anerkennung und Einbeziehung der Komplexität realer sozialer Systeme. ${ }^{15}$ Dabei existiert jedoch keine einheitliche Auffassung darüber, was unter dem Phänomen der Komplexität genau zu verstehen ist. Das nachfolgend dargelegte Verständnis des Komplexitätsphänomens lehnt sich an die führenden Vertreter der St. Galler Schule an. Komplexität entsteht im Gegensatz zu Kompliziertheit, unter der eine statisch gesehen große Anzahl von Elementen des Systems mit großer Unterschiedlichkeit verstanden werden kann, ${ }^{16}$ auf Grund der Dynamik des Systems. Sie äußert sich in der Zahl unterschiedlicher Zustände, die ein System im Zeitablauf annehmen kann. Die Ursache der Komplexität kann einerseits in den Interaktionen der Elemente, ${ }^{17}$ andererseits in einer sich schnell wandelnden Systemumwelt ${ }^{18}$ gesehen werden. Als Folge von Komplexität geht der Zusammenhang zwischen Ursache und Wirkung, also die Kausalität des Systemverhaltens, verloren. Dadurch wird die Wirkung von Gestaltungs- und Steuerungsmaßnahmen in Bezug auf das System schwer berechenbar bzw. vorhersehbar. ${ }^{19}$

Im Vergleich zu vorangegangenen Forschungsbestrebungen thematisieren die Systemtheorie und die Kybernetik in besonderer Weise die Beziehungen zwischen dem System und seiner Umwelt. ${ }^{20}$ In älteren Ansätzen standen dabei hauptsächlich die Offenheit des Systems und die einzelnen Input- und Outputbeziehungen, die das System mit der Umwelt verbinden, im Vordergrund, während in neueren Ansätzen die Gesamtpositionierung des Systems in der Umwelt und die Anpassung an wechselnde Umweltsituationen betrachtet werden. Dieser Wechsel des Betrachtungsschwerpunktes geht insbesondere in sozialwissenschaftlich orientierten Ansätzen mit einer Abwendung von der Offenheit und einer Zuwendung zur operationellen Geschlossenheit ${ }^{21}$ des Systems einher. Dabei wird untersucht, wie es sozialen Systemen gelingt, ihre Grenzen

11 Vgl. Bertalanffy (1969), S. 55; Stünzner (1996), S. 40

12 Vgl. Malik (1992), S. 77; Malik (1993), S. 54ff.

13 Vgl. Krieg (1971), S. 35.

${ }_{14}^{14}$ Vgl. Probst (1987), S. 30. Malik (1993), S. 58.

15 Vgl. Malik (1993), S. 186.

${ }^{16}$ Vgl. Probst (1987), S. 29.

17 Vgl. Malik (1992), S. 186.

18 Vgl. Stünzner (1996), S. 40.

19 Vgl. Malik (1993), S. 185; Parker/Stacey (1995), S. 23.

${ }^{20}$ Vgl. Bertalanffy (1969), S. 139ff.; Probst (1987), S. 54.

${ }^{21}$ Operationelle Geschlossenheit bedeutet, dass ein System in seinem Verhalten nicht durch die Umwelt determiniert wird. Vgl. Hejl (1982), S. 52. Zustandsveränderungen des Systems werden aus dieser Perspektive durch die Umwelt ausgelöst, jedoch nicht verursacht. Vgl. Streubel (2000), S. 182. 
gegenüber der Umwelt durch operationelle Schließung ständig zu reproduzieren und sich auf diese Weise selbst zu erhalten. ${ }^{22}$

Entsprechend der unterschiedlichen Untersuchungsschwerpunkte können entwicklungsgeschichtlich zwei Phasen der Kybernetik unterschieden werden, die auch in der Systemorientierten Betriebswirtschaftslehre erkennbar sind. In der ersten Phase, für die sich mittlerweile die Bezeichnung Kybernetik I. Ordnung (im Folgenden: Kybernetik I) durchgesetzt hat, ${ }^{23}$ standen gleichgewichtserhaltende Prozesse in Systemen jeglicher Art im Vordergrund der Betrachtung. Dementsprechend wurden in erster Linie Phänomene der Rückführung eines gestörten Systems in einen stabilen Zustand analysiert. $^{24}$ Die später entstandene Kybernetik II. Ordnung (im Folgenden: Kybernetik II) befasst sich dagegen mit der Anpassung komplexer Systeme an geänderte Situationen und beleuchtet dabei Phänomene wie Instabilität, Flexibilität, Wandel. ${ }^{25}$ Diese Art der Anpassung wird durch positive Rückkopplungsprozesse ermöglicht, die Abweichungen nicht beseitigen, sondern im Gegenteil verstärken. Gleichgewicht und Stabilität spielen also sowohl in der Kybernetik I als auch in der Kybernetik II eine zentrale Rolle. Während es jedoch in der Kybernetik I um die Bewahrung einmal erreichter Zustände, d.h. um Gleichgewichte in einem mehr konservativen Sinne geht, wird das Gleichgewicht in der Kybernetik II als innovatives dynamisches Gleichgewicht in einer sich wandelnden Umwelt aufgefasst.

Kybernetik I und II stehen untereinander in einem komplementären Verhältnis. ${ }^{26}$ Viele der in der Kybernetik I getroffenen Aussagen hängen nicht von der Größe und Komplexität des Systems $a b,{ }^{27}$ sodass sie auch für die durch die Kybernetik II untersuchten sehr komplexen Systeme Gültigkeit besitzen. Die beiden Forschungsrichtungen unterscheiden sich durch die jeweilige Ausrichtung der Untersuchung, wobei die Schwerpunkte der Kybernetik II ergänzend zu denen der Kybernetik I zu sehen sind. Ferner ist zu betonen, dass die Trennung zwischen den beiden Varianten der Kybernetik mehr analytisch begründbar als tatsächlich beobachtbar ist. Auch in älteren Beiträgen, die eher der Kybernetik I zuzuordnen sind, wurde bereits erkannt, dass kybernetische Systeme eine alternierende Folge von Anpassung und Konstanthaltung durchlaufen und in einer sich wandelnden Umwelt nur dann bestehen können, wenn sie ihr Verhalten nicht nur stabilisieren, sondern gegebenenfalls auch verändern können. ${ }^{28}$ Besondere Anstrengungen für die Entwicklung theoretischer Aussagen zur

${ }^{22} \mathrm{Vgl}$. Kasper/Mayrhofer/Meyer (1998), S. 608.

${ }^{23}$ Der Begriff der Kybernetik I. Ordnung geht auf Maruyama zurück, der erstmals die Entwicklung einer Kybernetik II. Ordnung forderte. Vgl. Maruyama (1963), S. 164ff.

${ }^{24}$ Vgl. Probst (1987), S. 18f., der in diesem Zusammenhang von konservativer Selbstorganisation spricht.

${ }^{25}$ Vgl. Probst (1987), S. 19. In diesem Zusammenhang spricht Probst von innovativer Selbstorganisation.

${ }^{26}$ Vg1. Probst (1987), S. 65.

27 Vgl. Ashby (1974), S. 353.

${ }^{28}$ Vgl. Krieg (1971), S. 42f. Ulrich erläutert bereits in einem älteren Beitrag, philosophisch gesehen betrachte die Kybernetik die Welt als etwas Werdendes, sich stets Veränderndes und nie Vollendetes, eine Umschreibung, die sicher auf die Unternehmung zutreffe. Vgl. Ulrich (1972), S. 19. Auch hier ist deutlich erkennbar, dass sich die Entwicklung einer neuen Variante der Kybernetik, die sich stärker mit dem Wandel von System und Umwelt auseinander setzt, anbahnt. Staehle weist darauf hin, dass Beer, dessen Arbeiten richtungsweisend für die systemtheoretischkybernetische Managementforschung gewesen sind, zunächst ganz in der Tradition der Kybernetik I gestanden hat und erst in seinen spăteren Arbeiten als Vertreter der Kybernetik II zu sehen ist. 
Anpassung an geänderte Umweltsituationen werden jedoch erst in neueren Ansätzen gemacht, die eindeutig der Kybernetik II zuzuordnen sind. ${ }^{29}$

Ein wesentlicher Ausgangspunkt systemtheoretisch-kybernetischen Denkens ist die Annahme, dass die Eigenschaften, Zustände und Verhaltensweisen unterschiedlicher realer Systeme durch formal isomorphe Systemeigenschaften erklärt werden können. ${ }^{30}$ Dieser Grundgedanke wird als Invarianztheorem bezeichnet. Das Invarianztheorem besagt, dass alle (komplexen) Systeme ungeachtet ihrer substanziellen Manifestation isomorphe Lenkungsstrukturen aufweisen. ${ }^{31}$ Isomorphie bedeutet dabei Formgleichheit und ist dann gegeben, wenn zwei Systeme zumindest in formaler Hinsicht austauschbar sind. ${ }^{32}$ Die Konstruktion isomorpher Modelle von Systemen eröffnet die Möglichkeit, die formal austauschbaren Aspekte dieser Systeme im Modell näher zu untersuchen. ${ }^{33}$ Diese Konzentration auf die Formgleichheit von Systemen wird allerdings mit einem relativ hohen Abstraktionsgrad der entwickelten Aussagen erkauft, sodass die Zusammenhänge zwischen den abstrakten systemtheoretisch-kybernetischen Aussagen und ihrer konkreten Anwendung auf praktische Problemstellungen nicht ohne weiteres offenkundig sind. ${ }^{34}$ Die entwickelten Aussagen betreffen häufig nicht präzise und numerisch spezifizierte Größen im Einzelfall, sondern sie richten sich auf bestimmte Klassen, Typen oder Muster zur Analyse der betrachteten Systeme und ordnen den involvierten Variablen ganze Bereiche möglicher Werte zu. ${ }^{35}$ Der Fokus der Betrachtung liegt folglich auf der Meta-Ebene von Systemen. Die verschiedenen Forschungsrichtungen der Kybernetik, die in diesem Rahmen entstanden sind, betrachten unterschiedliche real vorliegende Systemtypen. Die Ansätze konzentrieren sich mit im Einzelnen unterschiedlichen Schwerpunkten auf physikalisch-chemische, biologische oder soziale Fragestellungen. ${ }^{36}$

Als oberstes Ziel komplexer Systeme wird in kybernetischen Untersuchungen zumeist das Überleben des Systems angenommen. ${ }^{37}$ Entgegen der umgangsprachlichen Verwendung dieses Begriffes ist damit nicht das pure Überleben am Rande der Existenzmöglichkeiten, sondern die Stabilität der Menge der Systemzustände gemeint. ${ }^{38}$ Es handelt sich mit anderen Worten also um die „Sicherstellung der

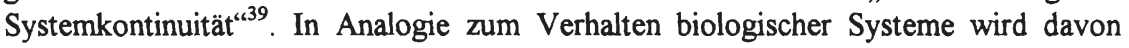

Vgl. Staehle (1999), S. 43. Die Grenze zwischen Kybernetik I und II verlăuft also fließend, und eine Trennung kann nur gedanklich vorgenommen werden.

${ }^{29}$ Vgl. ăhnlich Probst (1987), S. 65.

30 Vgl. Bertalanffy (1950), S. 136.

${ }^{31}$ Vgl. Malik (1992), S. 77f. Es handelt sich hierbei um eine metaphysische Annahme, also um eine philosophische Annahme, die nicht beweisbar ist, sondern den Ausgangspunkt der darauf aufbauenden Theoriebildung darstellt.

32 Vgl. Beer (1967), S. 59f; Ashby (1974), S. 143.

${ }^{33} \mathrm{Vgl}$. Ashby (1974), S. 147. Da es unwahrscheinlich, streng genommen sogar unmöglich, ist, dass von komplexen Systemen isomorphe Modelle gebildet werden können, beschrănkt sich die Kybernetik bei solchen Systemen auf die Verwendung homomorpher Modelle. Diese zeichnen sich dadurch aus, dass sie komplexe Systeme durch eine nicht-umkehrbar-eindeutige Transformation auf einfachere Modelle reduzieren, die anschließend auf isomorphe Aspekte hin untersucht werden können. Vgl. Ashby (1974), S. 158, Beer (1967), S. 60.

34 Vgl. Malik (1992), S. 177.

35 Vgl. Malik (1992), S. 202.

${ }^{36}$ Vgl. dazu ausfuihrlich die Übersichten bei Probst (1987), S. 19ff.; Staehle (1999), S. 41 ff.; Stünzner (1996), S. 41ff.; Göbel (1998), S. 39ff.

37 Vgl. Malik (1992), S. 112; Göbel (1998), S. 212

${ }^{38} \mathrm{Vgl}$. Ashby (1974), S. 284f.

${ }^{39}$ Krieg (1971), S. 43. 
ausgegangen, dass für das betrachtete System angestrebt wird, nur solche Zustände einzunehmen, die die fortdauernde Existenz des Systems gewährleisten. Diese Idee des Überlebens als Endziel wird durch die Systemorientierte Betriebswirtschaftslehre auf das System Unternehmung übertragen. ${ }^{40}$ Gegenüber anderen Wissenschaftsprogrammen der Betriebswirtschaftslehre, insbesondere gegenüber dem Faktortheoretischen Ansatz, wird hier eine wesentliche Verschiebung der Perspektive deutlich: Es geht nicht mehr um Gewinnmaximierung, Kostenminimierung o.ä., sondern es geht nun im Prinzip darum, im Geschäft zu bleiben. ${ }^{41}$ Als Erkenntnisinteresse der Systemorientierten Betriebswirtschaftslehre folgt daraus, dass Aussagen darüber zu gewinnen sind, wie die Lebensfähigkeit des Systems Unternehmung (im hier dargelegten begrifflichen Sinne) erhöht oder wiederhergestellt werden kann.

Im Vergleich zum Faktortheoretischen Ansatz zeichnet sich dieses Wissenschaftsprogramm durch eine verstärkte Betonung des pragmatischen Wissenschaftsziels aus. Als anwendungsorientierte Wissenschaft behandelt die Systemorientierte Betriebswirtschaftslehre alle Gestaltungs- und Führungsprobleme von produktiven sozialen Systemen (also nicht nur Unternehmungen, sondern auch öffentlichen Betrieben, Verwaltungen u.ä.). ${ }^{42}$ Das Ziel ist es dabei, den Führungskräften in der Praxis systemtheoretisch fundierte Anhaltspunkte zur Lösung ihrer Aufgabenstellungen zur Verfügung zu stellen. ${ }^{43}$ Die Abwendung vom erklärenden zum gestaltenden Ziel kann auch theoretisch begründet werden, da die betrachteten Systeme definitionsgemäß nicht vollständig beschreibbar und durch kausale Wirkungsketten determiniert sind. ${ }^{44}$ Dadurch bleiben sie einer Analyse des internen Geschehens durch eine erklärende Theorie weitgehend verschlossen. Dementsprechend wird durch kybernetische betriebswirtschaftliche Analysen nicht primär die theoretische Erklärung der untersuchten Systeme, sondern vielmehr die gestaltende und lenkende Beeinflussung der Wirklichkeit angestrebt. ${ }^{45}$

Die Betriebswirtschaftslehre wird dabei als eine den Ingenieurwissenschaften vergleichbare Disziplin aufgefasst, die sich von diesen „,nur dadurch unterscheidet, dass sie nicht technische, sondern soziale Systeme mit bestimmten Eigenschaften entwerfen will." ${ }^{46}$ Allerdings wird die anzustrebende Beziehung von erklärenden und gestaltenden Aussagen (nicht nur) von Vertretern der Systemorientierten Betriebswirtschaftslehre kontrovers diskutiert, ${ }^{47}$ und ULRICH als Begründer dieses Ansatzes hat seine diesbezügliche Position im Laufe der Zeit mehrfach geändert. ${ }^{48}$ Im Kern geht es dabei um die Frage, inwieweit Gestaltungs- und Steuerungsempfehlungen durch bewährte Hypothesen fundiert werden müssen. Die Extremposition eines „quasi theoriefreien Pragmatismus“49, der einen „Produktionsumweg über die Erfassung aller

\footnotetext{
${ }^{40}$ Vgl. z.B. Beer (1967), S. 172; Probst (1987), S. 59ff.; Malik (1992), S. 111ff. Gegen die Wahl des Überlebens als oberstes Unternehmungsziel und damit die a-priori-Festlegung auf einen bestimmten Systemzweck sprach sich zunächst Ulrich aus, vgl. Ulrich (1970), S. 115.

${ }^{41}$ Vgl. Malik (1992), S. 491.

${ }^{42} \mathrm{Vgl}$. Ulrich/Hill (1979), S. 172.

43 Vgl. Gomez (1998), S. 388.

${ }^{44}$ Vgl. Krieg (1971), S. 35. Göbel (1998), S. 25.

${ }^{45}$ Vgl. Krieg (1971), S. 37.

${ }^{46}$ Ulrich (1971), S. 47 (Hervorhebung im Original).

${ }^{47} \mathrm{Vgl}$. Hill (1988), S. 140.

${ }^{48}$ Vgl. Schanz (1997a), S. 134.

${ }^{49}$ Hill (1988), S. 141.
} 
Wirklichkeitszusammenhänge“s0 ablehnt, kann inzwischen als überholt gelten, da in neueren Quellen ausnahmslos anerkannt wird, dass Maßnahmen zur Einflussnahme auf das System der (zumindest prinzipiellen) Kenntnis der Natur des Systems und seiner Lenkungsmechanismen bedürfen. ${ }^{51}$ Die stärkere Ausrichtung auf das pragmatische Wissenschaftsziel bleibt trotz dieser Einschränkung allerdings auch in der modernen Systemorientierten Betriebswirtschaftslehre erhalten.

\subsubsection{Inhaltliche Leitideen des Systemorientierten Ansatzes}

Auch zur Darstellung der inhaltlichen Leitideen des Systemorientierten betriebswirtschaftlichen Ansatzes ist zunächst auf die Leitideen der Allgemeinen Systemtheorie zurückzugreifen, die durch den Systemorientierten Ansatz in der Betriebswirtschaftslehre zur Anwendung kommen sollen. Ausgehend von der Annahme der Gültigkeit des Invarianztheorems verfolgt die Allgemeine Systemtheorie die Zielsetzung, "Isomorphien in den Strukturen von Theorien über materiell unterschiedliche Sachverhalte aufzudecken, in einer einheitlichen Terminologie zu beschreiben und zu interdisziplinär verwendbaren, generalisierten Theoriensystemen zusammenzufassen".52 Derartige Isomorphien werden unabhängig von der konkreten Erscheinungsform des jeweils betrachteten Systems vermutet.

Als inhaltliche Leitideen der Systemorientierten Betriebswirtschaftslehre werden nachfolgend die Entwicklung und Verwendung einer einheitlichen Systemsprache (Abschnitt 4.1.2.1), die Annahmen zur Lenkbarkeit von Systemen (Abschnitt 4.1.2.2) sowie das Grundkonzept des systemischen Managements betrachtet (Abschnitt 4.1.2.3).

\subsubsection{Entwicklung und Verwendung einer einheitlichen Systemsprache}

Als Voraussetzung für die Identifikation von Gemeinsamkeiten in den Aussagesystemen unterschiedlicher Wissenschaftsdisziplinen wird die Formulierung der Aussagen in einer einheitlichen Sprache angesehen. Aus diesem Grund ist es die vorgelagerte Zielsetzung der Allgemeinen Systemtheorie, durch die Entwicklung eines gemeinsamen Begriffsystems die Grundlage für den Vergleich und die Übertragung von Aussagen unterschiedlicher Disziplinen zu schaffen. Zwangsläufig muss eine derartige Terminologie, wenn sie die gesamte Vielfalt von in der Realität zu beobachtenden konkreten Erscheinungsformen von Systemen erfassen und beschreiben soll, einen hohen Abstraktionsgrad aufweisen. Einen aus Sicht der Allgemeinen Systemtheorie wünschenswerten Nebeneffekt stellt dabei die Annäherung getrennter Einzeldisziplinen in Folge der Verwendung allgemeingültiger Begriffe dar. ${ }^{33}$ Im Folgenden werden kurz die Grundbegriffe der Allgemeinen Systemtheorie erläutert, die durch die Systemorientierte Betriebswirtschaftslehre für die Untersuchung des Systems Unternehmung und dessen Führung verwendet werden.

\footnotetext{
${ }^{50}$ Ulrich (1971), S. 46.

51 Vgl. z.B. Malik (1993), S. 56; Kasper et al. (1998), S. 603ff.; Gobbel (1998), S. 25.

52 Fuchs (1973), S. 25.

${ }^{53}$ Vgl. Bertalanffy (1969), S. 48f.; Fuchs (1973), S. 28.
} 
Der Begriff „System“ hat seine Wurzeln in der griechischen Sprache und bedeutet dort Zusammenstellung, Vereinigung oder Ganzes. ${ }^{54}$ In der Systemtheorie wird unter einem System eine geordnete Gesamtheit von Elementen verstanden, zwischen denen irgendwelche Beziehungen bestehen oder hergestellt werden können. ${ }^{55}$ Handelt es sich um ein offenes System, so sind auch die Beziehungen zur Umwelt zu berücksichtigen. ${ }^{56}$ Der Systembegriff ist also sehr formaler Art und seine wenigen konstitutiven Merkmale treffen auf überaus viele Sachverhalte $\mathrm{zu}^{57}$ Aus der vorgenommenen Definition von Systemen ergeben sich unmittelbar einige Folgefragen: Es ist zu erläutern, was unter einem Element und was unter einer Beziehung zu verstehen ist, und wie ein System gegenüber seiner Umwelt abgegrenzt wird, welche Elemente also zum System gehören und welche zu seiner Umwelt. Diese Fragen hängen unmittelbar zusammen und können nicht getrennt voneinander beantwortet werden.

Das Problem der Abgrenzung des Systems von seiner Umwelt wird von den verschiedenen Varianten der Systemtheorie unterschiedlich gesehen. In vielen Beiträgen wird davon ausgegangen, dass die Systemgrenzen sich nach der jeweiligen Fragestellung bestimmen, die durch systemtheoretische Analysen untersucht werden soll. ${ }^{58}$ Dementsprechend kann die Abgrenzung, welche Sachverhalte zum System gezählt werden und welche nicht, im jeweiligen Zusammenhang zielorientiert vorgenommen werden. Es kommt dann also auf die Perspektive der Betrachtung, die spezifische Zielsetzung der Untersuchung und das $\mathrm{zu}$ untersuchende Objekt im Einzelfall an. ${ }^{59}$ Ein System ist aus dieser Perspektive nicht ein gegebenes oder bestehendes Objekt, sondern es wird erst durch intensive Auseinandersetzung mit der vorliegenden Problemstellung als System konstituiert bzw. konstruiert.

Eine konträr entgegengesetzte Position nimmt die Theorie autopoietischer Systeme ein, die auf MATURANA/VARELA ${ }^{60}$ zurückgeht und zunächst ausschließlich dem biologischen Zweig der Systemtheorie zuzuordnen war. Autopoietische Systeme sind dadurch gekennzeichnet, dass sie sich nicht nur selbst erzeugen und andauernd reproduzieren, sondern auch ihre Grenzen gegenüber der Umwelt permanent durch die Interaktion ihrer Elemente selbst bestimmen. ${ }^{61}$ Aus dieser Perspektive werden Systeme (in der ursprünglichen Formulierung: lebende Systeme, also Lebewesen) nicht von

${ }^{54}$ Vgl. Flechtner (1966), S. 228; Ulrich (1970), S. 105.

55 Vgl. Ulrich (1970), S. 105. Wie viele andere Begriffe auch wird der Systembegriff in der Literatur nicht einheitlich verwendet, einen Überblick gibt Ulrich auf S. 105f. im Anschluss an seine eigene Systemdefinition. Für die Erläuterung der inhaltlichen Leitideen der Systemorientierten Betriebswirtschaftslehre ist an dieser Stelle keine detaillierte Betrachtung weiterer Begriffsvarianten erforderlich. Stattdessen wird hier die von Ulrich in seinem grundlegenden Werk, das den Ausgangspunkt für die nachfolgenden Beiträge zur Systemorientierten Betriebswirtschaftslehre darstellte, vorgenommene Definition übernommen.

${ }^{56}$ Genau genommen handelt es sich beim Begriff des geschlossenen Systems um ein gedankliches Konstrukt. Tatsächlich gibt es im gesamten Universum kein geschlossenes System. Vgl. dazu Hayek (1972), S. 14.

57 Vgl. Ulrich (1970), S. 106.

$58 \mathrm{Vgl} \mathrm{z.B.} \mathrm{Krieg} \mathrm{(1971),} \mathrm{S.} \mathrm{24;} \mathrm{Probst} \mathrm{(1987),} \mathrm{S.} 27$.

59 Vgl. Fuchs (1973), S. 40.

${ }^{60}$ Vgl. Maturana/Varela (1980), passim. Die Theorie autopoietischer Systeme beschäftigt sich mit der Erforschung des Gehirns und der Frage, wie es möglich ist, dass biologische Funktionen und Prozesse, Kognitionen, Bedeutungen und Emotion erzeugen können. Der Gegenstand des Ansatzes war also ursprünglich die Erklärung des Verhăltnisses von Körper als biologischem System, Geist als Bedeutungs- und Gedankensystem und Umwelt. Vgl. Stünzner (1996), S. 45.

${ }^{61} \mathrm{Vgl}$. Maturana (1981), S. 21. 
außen gemacht und in Gang gehalten, sondern es handelt sich dabei um Einheiten, die sich gleichsam von innen heraus produzieren und reproduzieren. ${ }^{62}$ Autopoietische Systeme stehen in Austausch mit ihrer Umwelt und sind dabei zwar nicht von dieser unabhängig, jedoch werden sie nicht durch die Umwelt definiert und determiniert. Es hat mehrere Versuche gegeben, die Theorie autopoietischer Systeme aus dem biologischen Umfeld heraus zu holen und auch für die Untersuchung sozialer Systeme, insbesondere Organisationen, fruchtbar zu machen. ${ }^{63}$ Durch solche Ansätze wird untersucht, inwiefern soziale Systeme - im hier interessierenden Falle Unternehmungen - als selbstreferenzielle Systeme aufgefasst werden können, die sich nicht nur autopoietisch selbst erzeugen und erhalten, sondern sich auch selbst beobachten und selbst beschreiben. ${ }^{64}$

Die Frage, worum es sich bei den Elementen eines Systems handelt, steht mit dem Problem der Systemabgrenzung in unmittelbarem Zusammenhang. Folgt man der ersten dargestellten Auffassung, nach der das System durch den Betrachter definiert und abgegrenzt wird, so können grundsätzlich sämtliche realen und gedachten Dinge und Sachverhalte als Elemente von Systemen oder als Systeme gedacht werden, ${ }^{65}$ da in der allgemeinen Systemdefinition keine Einschränkung hinsichtlich der Art des Systems und seiner Elemente vorgenommen wird. Elemente sind dann die einzelnen Teile eines Systems, die der Betrachter nicht weiter aufteilen kann oder will, also die kleinste interessierende Einheit im System. ${ }^{66}$ Elemente werden im Rahmen der jeweils betrachteten Problemstellung als atomar angesehen, d.h. sie werden nicht weiter aufgespalten. Die im gegebenen Zusammenhang relevanten Eigenschaften der Elemente werden durch Attribute beschrieben, die sich auf das Verhalten der Elemente oder ihren Zustand beziehen können.

Dagegen ist die Frage, was als Element des Systems aufzufassen ist, bei der Betrachtung autopoietischer Systeme nicht beliebig zu beantworten. Das typische Beispiel eines autopoietischen Systems im biologischen Bereich ist eine Zelle. ${ }^{67}$ Bei einer Zelle sind es chemische Reaktionen, die in einem Netzwerk Moleküle derart erzeugen, dass sie (1) durch ihre Interaktionen genau das Netzwerk an Reaktionen erzeugen bzw. an ihm rekursiv mitwirken, welches sie selbst erzeugte, und (2) die Zelle als materielle Einheit verwirklichen. Mithin sind in diesem Fall die chemischen Reaktionen die Elemente des Systems. Bei sozialen Systemen ist allerdings höchst umstritten, welches diejenigen Elemente sind, die das System erzeugen und gegenüber seiner Umwelt abgrenzen. ${ }^{68}$ Festzuhalten bleibt an dieser Stelle zunächst, dass bei autopoietischen Systemen die Art der Elemente nicht durch den Betrachter nach dessen Zwecken festgelegt wird, sondern dass es sich bei der Bestimmung der Elemente des Systems vielmehr um eine empirische Fragestellung handelt.

Die Beziehungen, die zwischen den Elementen eines Systems bestehen, machen das System erst für eine Untersuchung als Ganzheit interessant. Sie können grundsätzlich

\footnotetext{
$62 \mathrm{Vgl}$. Kirsch/Knyphausen (1991), S. 78.

${ }^{63} \mathrm{Vgl}$. für einen Überblick Kirsch/Knyphausen (1991), S. 82.

${ }^{64}$ Vgl. Kasper (1991), S. 9ff. Für einen Überblick über die Standpunkte hinsichtlich des Verhältnisses zwischen Autopoiese und Selbstreferenz vgl. Kirsch (1992), S. 195ff.

${ }^{65} \mathrm{Vgl}$. Kosiol/Szyperski/Chmielewicz (1965), S. 339.

66 Vgl. Ulrich (1970), S. 107.

${ }^{67} \mathrm{Vgl}$. dazu Maturana/Varela/Uribe (1982), S. 158.

${ }^{68}$ Vgl. Gobbel (1998), S. 73.
} 
gesehen materieller, immaterieller (informationeller) oder energetischer Natur sein. ${ }^{69}$ Beziehungen bestehen nicht nur zwischen den verschiedenen Elementen eines Systems, sondern auch zwischen den einzelnen Elementen und der Systemumwelt. Die Existenz von Beziehungen stellt eine gegenseitige Abhängigkeit her, sodass es zu einem interdependenten Verhalten zwischen den Elementen untereinander und zwischen dem System und seiner Umwelt kommt, welches in der Sprache der Systemtheorie als Rückkopplung bezeichnet wird. ${ }^{70}$ Während für gleichgewichtsbewahrende Prozesse die negative Rückkopplung ausschlaggebend ist, besitzt für die dynamische Anpassung an sich wandelnde Umweltsituationen die Form der sich selbst verstärkenden, positiven Rückkopplung hohe Bedeutung. ${ }^{71}$ Die Abhängigkeit zwischen den Elementen eines Systems braucht nicht unbedingt direkt zu sein, da nicht notwendigerweise jedes Element mit jedem anderen in Beziehung steht. ${ }^{72}$

Von der Art, der Anzahl und der Intensität der Beziehungen hängt es ab, wie leicht das System gedanklich zu erfassen und zu erklären ist. In der Kybernetik II wird besonders hervorgehoben, dass sich die Beziehungen im Laufe der Zeit verändern, oder anders ausgedrückt, dass die zu einem Betrachtungszeitpunkt bestehenden Beziehungen zwischen den Elementen des Systems das Resultat der Geschichte des Systems sind (history matters ${ }^{73}$ ). Dadurch wird betont, dass das Verhalten des Systems sich im Laufe der Zeit verändern kann, mit der Folge, dass das System auf gleiche Umweltzustände zu unterschiedlichen Zeitpunkten in verschiedener Weise reagiert. Die Folge dieser durch Rückkopplungsprozesse ausgelösten Dynamik ist die Auflösung des eindeutigen Zusammenhangs zwischen Ursachen und Wirkungen, da ein und dieselbe Ursache im Zeitablauf zu unterschiedlichen Wirkungen führen kann. ${ }^{74}$

\subsubsection{Lenkbarkeit von Systemen}

Aufbauend auf dem Fundament der allgemeinen Systemsprache befasst sich die Kybernetik mit der Möglichkeit der Einflussnahme auf Systeme zur Erreichung der Systemziele. Ganz allgemein geht es dabei darum, das System unter Kontrolle zu halten, um den Systemzweck so gut wie möglich zu erfüllen. Den wichtigsten Ausgangspunkt kybernetischer Aussagen über die Lenkung ${ }^{75}$ und Lenkbarkeit von Systemen stellt das bereits Mitte der fünfziger Jahre formulierte Gesetz der erforderlichen Varietät von ASHBY dar, das spätere systemtheoretische Arbeiten außerordentlich stark beeinflusst hat und den Dreh- und Angelpunkt vieler Beiträge zur Systemorientierten Betriebswirtschaftslehre darstellt. ${ }^{76}$

${ }^{69} \mathrm{Vgl}$. Baetge (1974), S. 37.

${ }^{70}$ Vgl. Ashby (1974), S. 88.

${ }^{71}$ Vgl. Probst (1987), S. 51.

${ }^{72}$ Vgl. Ulrich (1970), S. 109

${ }^{73}$ Vgl. ähnlich Probst (1987), S. 78

${ }^{74}$ In diesem Zusammenhang wird auch von der Nicht-Linearität von Systemen gesprochen, vgl. Parker/Stacey (1995), S. 23.

${ }^{75}$ Lenkung wird hier als Oberbegriff für verschiedene Formen der zielgerichteten Einflussnahme auf ein System verwendet. Dazu gehören die grundsătzliche Gestaltung des Systems und laufende Lenkungsmaßnahmen, die weiter unten noch detailliert behandelt werden. Bei dieser Art der Begriffsverwendung stellen alle Arten der zielorientierten Einflussnahme auf ein System Spezialfälle des allgemeinen Begriffes der Lenkung dar.

${ }^{76}$ Mirow bezeichnet das Gesetz der erforderlichen Varietät deshalb als das „Gesetz der Schwerkraft der Systemtheorie“, vgl. Mirow (1999), S. 17. 
Der Ausgangspunkt für die Formulierung des Gesetzes der erforderlichen Varietät ist die Feststellung, dass die Regelung eines Systems den Fluss der Vielfalt blockiert, dem das System in seiner Umwelt ausgesetzt ist. ${ }^{77}$ Als Vielfalt ${ }^{78}$ wird dabei die Anzahl der Elemente einer Menge (bzw. der Logarithmus zur Basis 2 dieser Anzahl), bezogen auf die Umwelt eines Systems also die Anzahl der unterscheidbaren möglichen Umweltzustände, bezeichnet. ${ }^{79}$ Die Vielfalt der möglichen System/Umweltzustände gibt die Menge der Verhaltensmöglichkeiten des Systems an, also den Umfang dessen, was das System tun könnte, wenn es durch keinerlei Regelungsmaßnahmen beeinflusst wird. Sie ist somit ein Maß für die Komplexität des Systems. ${ }^{80}$ Durch Regelung werden Begrenzungen ${ }^{81}$ für die Verhaltensvielfalt des Systems eingeführt und damit der Umfang des tatsächlichen Systemverhaltens gegenüber dem des potenziellen Systemverhaltens reduziert. Das Gesetz der erforderlichen Varietät macht Aussagen über die Beziehung zwischen der Vielfalt des Systemverhaltens, der Vielfalt der auf das System wirkenden Störungen aus der Systemumwelt und der Vielfalt des Reglers. ${ }^{82}$

Das Gesetz besagt, dass nur Vielfalt des Reglers die Vielfalt der Umwelt aufheben kann, um auf diese Weise die Vielfalt des Systemverhaltens zu reduzieren ${ }^{83}$ Die Varietät der tatsächlich auftretenden Systemzustände ist abhängig von der Umweltvarietät und der Varietät des Lenkungssystems. Anders ausgedrückt: Das steuernde System benötigt eine mindestens ebenso große Varietät wie die Umwelt, um das zu steuernde System im (immer gleichen) Gleichgewicht zu halten. Dieses Gesetz kann mathematisch bewiesen werden und ist in keiner Weise vom Experiment abhängig, ${ }^{84}$ sodass es für jede Art von System Gültigkeit besitzt. In konkreten Fällen ist es zwar nicht immer möglich und sinnvoll, alle möglichen Zustände der Einflussgrößen, die das Verhaltens des Systems bestimmen, anzugeben, um die exakte Varietät zu messen. ${ }^{85}$ Dennoch sind die Idee und der Begriff der erforderlichen Varietät für kybernetische Überlegungen unverzichtbar, ${ }^{86}$ da das Prinzip unabhängig von der konkreten Messbarkeit der Größen immer erfüllt ist. Für die Regelung und Steuerung von Systemen folgt aus dem Gesetz der erforderlichen Varietät, dass die zielgerichtete Verarbeitung von Umweltkomplexität immer eine entsprechende Systemkomplexität

${ }^{77} \mathrm{Vgl}$. Ashby (1974), S. 288.

78 Die Begriffe "Vielfalt" und „Varietät" werden hier synonym verwendet, da es sich um unterschiedliche Übersetzungen des von Ashby im englischsprachigen Original verwendeten Begriffes „variety“ handelt.

79 Vgl. Ashby (1974), S. 186.

80 Vgl. Malik (1992), S. 186.

${ }^{81}$ Eine Begrenzung stellt eine Beziehung zwischen zwei Mengen dar und ergibt sich, wenn die Vielfalt, die unter einer Bedingung existiert, geringer ist als die Vielfalt, die unter einer anderen Bedingung existiert. Vgl. Ashby (1974), S. 187.

82 Genau genommen beinhaltet das Gesetz in der von Ashby formulierten Form auch noch eine interne Storgroße, die die Unsicherheit des Beobachters über die richtige Reaktionsmaßnahme kennzeichnet, wenn die systemexterne Situation vollständig bekannt ist. Diese Größe ist ein Maß für die Determiniertheit des Systems. Vgl. Mirow (1969), S. 76. Für den vorliegenden Überblick ist es jedoch adäquat, nur von einer Störgröße auszugehen, die sämtliche auf das System wirkenden Störungen beinhaltet. Vgl. dazu Mirow (1969), S. 126f. Detailliertere Ausführungen dazu finden sich in Kapitel 4.3.

${ }^{83} \mathrm{Vgl}$. Ashby (1974), S. 299.

${ }^{84} \mathrm{Vgl}$. Ashby (1974), S. 302. Auf die Beweisfuihrung von Ashby, die er mit Hilfe spieltheoretischer Matrizen vornimmt, soll hier nicht eingegangen werden, da es für die weiteren Überlegungen allein auf das Ergebnis ankommt. Vgl. dazu im Einzelnen Ashby (1974), S. 293-302.

${ }^{85} \mathrm{Vgl}$. Probst (1987), S. 40.

${ }^{86}$ Vgl. Malik (1992), S. 197. 
erfordert. ${ }^{87}$ Die Lenkungsvarietät muss relativ zu den beabsichtigten Zielen mindestens so groß sein wie die Varietät des zu lenkenden Systems. ${ }^{88}$ Demnach sind die Kapazität des Reglers und die Umweltvarietät näher zu betrachten, um zu Aussagen über die Lenkbarkeit des Systems zu gelangen.

Neben der System/Umweltvielfalt, die die Kapazität der Lenkungsinstanz möglicherweise übersteigt, liefert die oben bereits angesprochene Tatsache, dass sich das Systemverhalten im Laufe der Zeit ändern kann, ein weiteres Argument gegen die exakte Steuerbarkeit von Systemen. Die Reaktion des Systems auf die Einflussnahme durch den Regler kann bei Systemen, die diese Eigenschaft aufweisen, nicht mehr vollständig ex ante prognostiziert werden. Dieser eng mit dem Verschwinden von Kausalität in komplexen Systemen verknüpfte Aspekt wurde von ASHBY, der betonte, dass die Schwierigkeit normalerweise in der Vielfalt der Störungen, nicht jedoch im Umfang des Systems, begründet liegt, ${ }^{89}$ noch nicht behandelt. ASHBY ging vielmehr davon aus, dass eine erfolgreiche „Verfolgung langer Ketten von Ursache und Wirkung ${ }^{\text {"90 }}$ es erlauben kann, das Systemverhalten erfolgreich $\mathrm{zu}$ prognostizieren. Neuere Arbeiten wenden dagegen gerade diese sich auflösende Kausalität in komplexen Systemen als Argument gegen die Lenkbarkeit solcher Systeme ein. ${ }^{91}$

\subsubsection{Systemisches Management}

Aus den bisher dargelegten Erkenntnisinteressen und Leitideen der Systemorientierten Betriebswirtschaftslehre ergeben sich sowohl für das Management im institutionalen Sinne als auch für das Management im funktionalen Sinne tiefgreifende Konsequenzen.

In Bezug auf das Management im institutionalen Sinne, also auf die Personen, die mit Aufgaben der Gestaltung und Steuerung betraut sind, sind diese Konsequenzen eher epistemologischer Art und besitzen nur mittelbare Relevanz furr die Formulierung pragmatischer Aussagen. Während Manager aus dem traditionellen Blickwinkel als außerhalb des zu gestaltenden Systems stehende Personen gesehen werden und damit eine Trennung zwischen dem Beobachter und dem Beobachtungsgegenstand impliziert wird, ${ }^{92}$ betont die neuere Systemtheorie, dass sie als Organisatoren Teil des organisierten Systems sind. ${ }^{93}$ Der lenkende Teil eines sozialen Systems ist damit im Gegensatz zu technischen Systemen nicht vom gelenkten Teil des Systems isolierbar. Dies trägt einerseits zur Auflösung von Ursache-Wirkungsketten bei, da das System als Ganzes nicht mehr von einem Außenstehenden überschaut und zweckrational geführt werden kann, und betont andererseits die Autonomie des Systems gegenüber seiner Umwelt. Fasst man eine Unternehmung allerdings als selbstreferenzielles soziales Handlungssystem auf, so wird ein erneuter Wechsel des Standpunktes erforderlich, da Manager als psychisch-physische Systeme außerhalb des sozialen Systems Unternehmung stehen und nur durch ihre sozialen Handlungen an dessen Konstruktion mitwirken, ohne dass die Person des Managers Teil des Systems sein kann.

${ }^{87} \mathrm{Vgl}$. Mirow (1999), S. 17.

${ }^{88}$ Vgl. Malik (1992), S. 194.

${ }^{89} \mathrm{Vgl}$. Ashby (1974), S. 353.

90 Ashby (1974), S. 363.

${ }^{91}$ Vgl. z.B. Parker/Stacey (1995), S. 21ff.

${ }^{92}$ Vgl. Kasper/Mayrhofer/Meyer (1998), S. 612.

93 Vgl. Probst (1987), S. 80. 
Bezüglich der Tätigkeiten des Managements, also des Managements im funktionalen Sinne, weisen Vertreter der Systemorientierten Betriebswirtschaftslehre darauf hin, dass das in der traditionellen Betriebswirtschaftslehre dominierende Prinzip der Zielrationalität voraussetzt, dass ausreichendes Wissen über die Kausalitäten, von denen die Zielerreichung abhängt, verfügbar ist. ${ }^{94}$ Die Vorstellung, dass eine zweckrationale Steuerung von Unternehmungen möglich ist, basiert also auf der Annahme, dass die dazu erforderliche Vollständigkeit der Information in der Praxis erreicht werden kann. Theoretische Aussagen, die von dieser Grundposition ausgehen, bezeichnet MALIK als „konstruktivistisch-technomorph“95. Die Funktionsweise einer Unternehmung ist aus der beschriebenen Perspektive mit der Funktionsweise einer Maschine im Sinne der klassischen Mechanik vergleichbar. Eine solche Maschine ist gemäß einer bewusst vorgefassten Zwecksetzung und einem Plan konstruierbar, ihre Funktion, Zuverlässigkeit und Effizienz hängt von den entsprechenden Funktionseigenschaften ihrer Einzelteile $a b .^{96}$ Die Maßnahmen des Managements betreffen dementsprechend den Entwurf und die Implementierung formaler Strukturen, die die Erreichung der anzustrebenden Ergebnisse gewährleisten sollen. ${ }^{97}$ Diese Sichtweise wird im Folgenden als „mechanistisch“ bezeichnet, da der von Malik gewählte Begriff „konstruktivistisch-technomorph“ die Gefahr von Verwechselungen mit der wissenschaftstheoretischen Grundposition des Konstruktivismus, die gerade auf eine Überwindung der traditionellen mechanistischen Sichtweise abzielt, birgt.

Der mechanistischen Perspektive stellt die auf der Kybernetik II basierende Variante der Systemorientierten Betriebswirtschaftslehre eine eigene Management-Konzeption gegenüber, die als „systemisch-evolutionär ${ }^{\text {“98 }}$ bezeichnet wird. Im Gegensatz zur traditionellen Position beruht die systemisch-evolutionäre Position auf der Annahme, dass soziale Institutionen nicht das Ergebnis einer planenden und gestaltenden Vernunft, sondern das Resultat von Wachstums- und Entwicklungsprozessen sind. ${ }^{99}$ Dieser Wechsel der Perspektive hat eine veränderte Auffassung darüber zur Folge, was als rational zu verstehen ist. Rationalität meint aus der systemisch-evolutionären Perspektive nicht mehr die Zielerreichung auf der Basis erkannter oder praktisch erkennbarer Kausalzusammenhänge, sondern sie bezeichnet ein auf die Erhaltung eines Ordnungstyps gerichtetes Verhalten, das die „größtmögliche Orientierbarkeit aller sich an diesem Ordnungstyp orientierenden Individuen “" ${ }^{\text {100 }}$ zum Ziel hat. Dies wird dadurch begründet, dass die hohen Anforderungen, die die mechanistische Konzeption an den Informationsstand der lenkenden Institution stellt, in der Realität nicht erfullt werden können und damit abzulehnen sind, obwohl sie im Falle der Realisierbarkeit zu besseren Ergebnissen führen würden. ${ }^{101}$

Das Management-Konzept der neueren Systemorientierten Betriebswirtschaftslehre sieht die Aufgabe des Gestalters in sozialen Systemen in der Schaffung und Erneuerung von Kontexten, welche das Verhalten des Systems in die gewünschte Richtung

\footnotetext{
${ }^{94}$ Vgl. Malik (1993), S. 66; ăhnlich Probst (1987), S. 93.

${ }_{95}$ Malik (1993), S. 63.

${ }^{96}$ Vgl. Malik (1993), S. 63.

97 Vgl. Probst (1987), S. 93

98 Malik (1993), S. 63.

99 Vgl. Malik (1992), S. 254.

${ }^{100}$ Malik (1993), S. 67.

${ }^{101}$ Vgl. Malik (1992), S. 255.
} 
lenken. ${ }^{102}$ Je höher der Grad der Komplexität einer Organisation ist, um so allgemeiner müssen die Verhaltensregeln formuliert sein, die die Organisation als System lenken. ${ }^{103}$ Mit anderen Worten: Unternehmungen, die ihre Lebensfähigkeit in sich dynamisch wandelnden Umweltsituationen aufrechterhalten wollen und dabei auf das verteilte Wissen vieler verschiedener Akteure angewiesen sind, müssen einen großen Handlungsspielraum vorsehen, innerhalb dessen diese Akteure in Interaktion mit der Umwelt treten können. Die Einflussnahme durch das Management richtet sich dann darauf, das System auf einen Entwicklungspfad zu bringen oder es auf einem solchen zu halten, wobei jedoch der Endzustand wie auch die Zwischenstufen der Entwicklung nicht vollständig ex ante bekannt sein können. ${ }^{104}$ Die Zielsetzung des systemischevolutionären Managements liegt dabei in der Kanalisierung der Systemvarietät. ${ }^{105}$

Die Einflussnahme des Managements auf das System muss nach dieser Vorstellung nicht nur auf der materiellen, sondern auch auf der immateriellen, geistig-sinnhaften Gestaltungsebene ansetzen. ${ }^{106}$ Die materielle Ebene, die auch als substanzielle Ebene bezeichnet wird, enthält die formalen Gebilde- und Prozessstrukturen des Systems, die auch von der traditionellen Betriebswirtschaftslehre als Gestaltungsgegenstand aufgefasst werden. Sie ist durch eine symbolische Systemgestaltung auf der immateriellen Ebene überlagert, die sich auf interpretative Aspekte bezieht und der Vermittlung oder Erfassung von Sinn dient. ${ }^{107}$ Auf dieser geistig-sinnhaften Ebene können symbolische Maßnahmen des Managements für die beteiligten Akteure die Möglichkeit schaffen, sich einzuordnen und zurechtzufinden. Durch solche Orientierungspunkte werden mittelbar die Handlungen der Akteure beeinflusst und in die gewünschte Richtung gelenkt. Mit der Zuwendung zur symbolischen Ebene soll dabei keineswegs die Bedeutung der substanziellen Ebene des Organisierens vernachlässigt werden. Vielmehr ergänzen sich beide Ebenen bei der zielorientierten Lenkung von Unternehmungen.

Statt der autoritär ausgerichteten Leitung durch die Formulierung von Zielen und die Ausübung der Managementfunktionen Planung, Steuerung und Kontrolle geht es bei der Lenkung von Systemen nunmehr um Anregung. ${ }^{108}$ An die Stelle der analytisch begründbaren Steuerung im Detail tritt in dieser Konzeption die Kunst der Intervention als zielgerichtete Kommunikation, die das Eigenleben des intervenierten Systems respektiert. ${ }^{109}$ Organisationen können lediglich mittels indirekter Interventionen, nicht jedoch durch direkte Steuerungsmaßnahmen zu den gewünschten Eigenaktivitäten angeregt werden. Der Verzicht auf detaillierte Führung ist aus dieser Perspektive nicht eine Frage der sozialen Akzeptanz, der Motivation und des Führungsstils, sondern vielmehr der faktischen Lenkungsmöglichkeit im Lichte der zu Grunde liegenden Komplexität des Systems. ${ }^{110}$ Geht man ferner von der oben

\footnotetext{
${ }^{102}$ Vgl. Probst (1987), S. 113.

${ }^{103}$ Vgl. Malik (1992), S. 229.

${ }^{104}$ Vgl. Malik (1992), S. 230.

${ }^{105}$ Vgl. Probst (1987), S. 113.

${ }^{106}$ Vgl. dazu Probst (1987), S. 91ff.

${ }^{107}$ Vgl. Probst (1987), S. 92. Probst führt aus, die symbolische Gestaltungsaufgabe liege darin, „Sprachspiele“, Mythen, Symbole, Etiketten, Denkmuster, Leitvorstellungen, Zeremonien, Riten usw. zu strukturieren, verändern, nutzen, fordern oder zu verhindern.

${ }^{108} \mathrm{Vgl}$. Kasper/Mayrhofer/Meyer (1998), S. 614.

${ }^{109}$ Zum Begriff der Intervention vgl. Willke (1987b), S. 333.

${ }^{110}$ Vgl. Malik (1993), S. 68.
} 
angesprochenen Annahme der operativen Geschlossenheit des sozialen Systems Unternehmung aus, dann muss dessen Steuerung definitionsgemäß immer und ausschließlich nur Selbststeuerung sein. ${ }^{111}$ Das Management muss sich dementsprechend darauf richten, die Selbststeuerung des Systems auszulösen, zu fördern und zu verstärken. ${ }^{112}$

\subsubsection{Methodische Leitideen des Systemorientierten Ansatzes}

Den im Folgenden zu erläuternden methodischen Kern des Systemorientierten Ansatzes stellen die in Abschnitt 4.1.3.1 erläuterte Black box-Technik und das in Abschnitt 4.1.3.2 betrachtete Verfahren der hierarchischen Dekomposition dar, die sich als Methoden ergänzen. Sie sind tendenziell eher der Kybernetik I zuzuordnen als der Kybernetik II, da diese weniger exakte Modellergebnisse im Einzelfall anstrebt, als sich vielmehr auf die Erkennung von gemeinsamen Grundmustern im Verhalten von konkret sehr unterschiedlich ausgeprägten komplexen Systemen konzentriert.

\subsubsection{Black box-Technik}

Bei Anwendung der Black box-Technik wird die Verhaltensweise bestimmter Systeme oder Systembestandteile auf einer gegebenen Abstraktionsebene als gegeben hingenommen und nicht im Einzelnen betrachtet. Es erfolgt also explizit keine Untersuchung der einzelnen darin vorhandenen Ursache-Wirkungs-Zusammenhänge. ${ }^{113}$ Vielmehr wird das Verhalten eines (Teil-) Systems mit Hilfe der von außen beobachtbaren Beziehungen zwischen seinem Input und Output beschrieben. Das System selbst wird als Black box aufgefasst, deren innere Vorgänge bei dem gegebenen Aggregationsniveau nicht erfasst und beobachtet werden können oder sollen. ${ }^{114}$ Durch die Manipulation des Systeminput und die Beobachtung des in Abhängigkeit dieses Input durch das System produzierten Systemoutput sollen Regelmäßigkeiten festgestellt werden, die Aussagen über das Verhalten des Systems machen, ohne seine Funktionsweise im Einzelnen zu kennen. Diese Black box-Technik stellt die bewusste und allgemeingültige Formulierung einer Methode zur Erforschung der Wirklichkeit dar, die sowohl im Alltag als auch in der Wissenschaft an vielen Stellen angewendet wird, ohne dass dies stets deutlich zum Ausdruck gebracht wird. ${ }^{115}$

Zur Untersuchung eines Systems ist das System nicht nur entsprechend der vorliegenden Fragestellung möglichst zweckmäßig gegenüber seiner Umwelt abzugrenzen, sondern es ist auch zu entscheiden, welche Sachverhalte im vorliegenden Fall als Black

\footnotetext{
${ }^{111}$ Operationelle Geschlossenheit ist als Voraussetzung für die vorangegangenen Ausführungen zur Lenkung von Systemen durch die Schaffung von Kontexten nicht unbedingt erforderlich, da diese das Phänomen der Komplexität zum Ausgangspunkt der Argumentation machen. Da komplexe Systeme nicht zwingend auch autopoietisch (und somit operationell geschlossen) sein müssen, stellt die Annahme der operationellen Geschlossenheit eine noch restriktivere Prämisse dar, vgl. dazu auch die Ausführungen im vorangegangenen Abschnitt zur Definition und Abgrenzung von Systemen. Liegt jedoch operationelle Geschlosssenheit vor, so ist jede Steuerung des Systems zugleich Selbststeuerung. Vgl. Kasper/Mayrhofer/Meyer (1998), S. 615.

${ }^{112}$ Vgl. Probst (1987), S. 88, der seine Ausführungen allerdings auf den Vorgang des Organisierens und nicht des Managements im Allgemeinen bezieht.

${ }^{113} \mathrm{Vgl}$. Beer (1967), S. 70; Ulrich (1970), S. 132.

${ }^{114}$ Vgl. Beer (1967), S. 70.

${ }^{115}$ Vgl. Ashby (1974), S. 133
} 
box aufzufassen und nicht weiter in ihre Bestandteile zu zerlegen sind. ${ }^{116}$ Eine Black box wird im Rahmen der jeweiligen Untersuchung wie ein atomares Element des Systems behandelt, dessen Verhalten durch seine Attribute beschrieben wird. Durch die Bestimmung dieser kleinsten Analyseeinheit wird das Aggregations- und Abstraktionsniveau der Untersuchung festgelegt. Jedes offene System stellt einen Teil eines Supersystems dar und kann auf der nächsthöheren Betrachtungsebene als Element dieses Supersystems aufgefasst werden. Auf der anderen Seite können die Elemente des betrachteten Systems, sofern es sich nicht um echte atomare Elemente handelt, zum Zwecke einer detaillierteren Analyse selber in ihre Bestandteile zerlegt und auf der dadurch geschaffenen niedrigeren Betrachtungsebene als System aufgefasst werden.

\subsubsection{Wechsel von Betrachtungsebenen}

Bei dem mit der Zerlegung von Systemen und Systembestandteilen bereits angesprochenen Prinzip der hierarchischen (De-) Komposition ${ }^{117}$ handelt es sich um die zweite bedeutsame methodische Leitidee der systemorientierten Vorgehensweise zur Untersuchung von realen Sachverhalten. Durch die sukzessive Auflösung von Black boxes im Rahmen der hierarchischen Dekomposition gelingt es, von einem relativ groben Überblick über das gesamte System beliebig ins Detail vorzudringen und die verschiedenen Bestandteile des Gesamtsystems zu betrachten, ohne den Zusammenhang $\mathrm{zu}$ den anderen Systembestandteilen und damit die vorhandenen Interdependenzen aus dem Auge zu verlieren. Umgekehrt können verschiedene Systembestandteile durch eine Bottom-up-Vorgehensweise $\mathrm{zu}$ übergeordneten Systemen zusammengefasst und damit die Voraussetzungen für eine Untersuchung ihres Zusammenwirkens geschaffen werden. Durch die Kombination dieser Verfahren wird im Rahmen des Systemorientierten Ansatzes angestrebt, abwechselnd auf unterschiedlichen Abstraktionsebenen zu arbeiten und so ein komplexes System bzw. einen Kontext in den Griff zu bekommen. Das ständige Hinauf- und Hinabgehen in verschiedene Ebenen, das Erkennen der Ebenen und die Schaffung von Verbindungen zwischen den Ebenen ist erforderlich, um auf höchstem Abstraktionsniveau ein System in seiner gesamten Komplexität abzubilden, gleichzeitig aber auch viele kleine Details und Teile im Lichte des Ganzen analysieren zu können. ${ }^{118}$

Die Black box-Technik und die hierarchische Dekomposition werden keinesfalls nur zur Unterstützung von systemtheoretischen Untersuchungen, die auf das erklärende Wissenschaftsziel ausgerichtet sind, angewendet. Vielmehr können auch pragmatisch orientierte Aufgaben der Lenkung von Systemen durch diese beiden Methoden unterstützt werden. Wenn das Verhalten eines Systems an Hand seiner Input/OutputBeziehungen beschrieben werden kann, so kann es auch gelingen, durch die Manipulation des Inputs das System in einer Weise zu beeinflussen, die es zur Produktion des gewünschten Outputs veranlasst.

\footnotetext{
${ }^{116}$ Aus den Ausfuihrungen des vorigen Abschnittes folgt, dass die Abgrenzung des Systems nicht immer durch den Beobachter erfolgt, sondern im Falle autopoietischer Systeme auch ein empirisches Problem sein kann. Die Black box-Technik kann dagegen in jedem Falle durch den Beobachter angewandt werden.

${ }^{117}$ Vgl. dazu Schwarze (1995), S. 679ff.

${ }^{118}$ Vgl. Probst (1987), S. 31.
} 
Die Lenkungsproblematik von dynamischen Systemen stellt den Kernbereich der Kybernetik I dar, die ein System (z.B. eine Unternehmung) gedanklich in drei institutionelle Bestandteile zerlegt ${ }^{119}$ und sich auf die Untersuchung von gleichgewichtserhaltenden Prozessen richtet. Bei den in Abbildung 4/1 dargestellten drei institutionellen Systembestandteilen handelt es sich um die zielsetzende Einheit (Sollwertgeber), die den Zweck des Systems definiert und den Sollwert für den Systemoutput festlegt, ${ }^{120}$ den Regler und die Regelstrecke, die den Systemoutput produziert. Auf die Regelstrecke wirkt der Systeminput ein, der auch als Störgröße bezeichnet wird. Die Aufgabe des Reglers ist es, mit Hilfe der bekannten Input/OutputBeziehungen der Regelstrecke den Input durch eine Stellgröße so zu modifizieren, dass der Systemoutput die durch den Sollwert vorgegebene gewünschte Größe erreicht. Die Regelstrecke selbst wird dabei als Black box aufgefasst, deren im Inneren stattfindende Vorgänge nicht im Einzelnen bekannt sind. Bei einer Unternehmung kann aus dieser Perspektive das mittlere Management als Regler aufgefasst werden, dessen Aufgabe es ist, durch Anweisungen an die Beschäftigten die Ziele des Top-Managements ${ }^{121}$ zu erreichen.

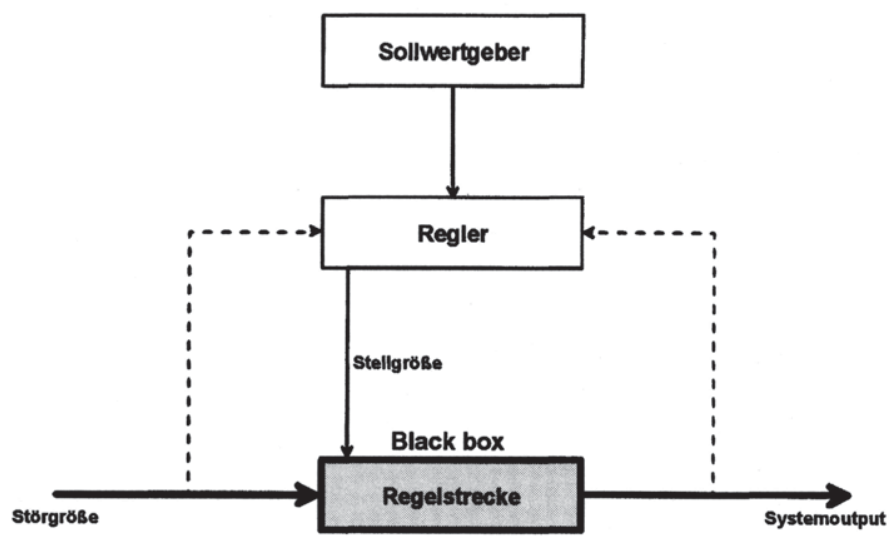

Abb. 4/1: Black box-Methode und Regelkreissystem

Quelle: in Anlehnung an Baetge (1974), S. 30.

Der als Regelgröße bezeichnete Output der Regelstrecke wird erfasst und zur Kontrolle an den Regler zurückgemeldet. Dieser kann auf Grund der Abweichung der Regelgröße vom Sollwert wiederum mit Hilfe geeigneter Maßnahmen die Stellgröße variieren, um eine noch größere Übereinstimmung von Regelgröße und Sollwert herbeizuführen. Die Steuerung und Regelung als komplementäre Lenkungsprinzipien der Kybernetik I werden weiter unten noch detaillierter betrachtet und in direkten Bezug

\footnotetext{
${ }^{119}$ Vgl. dazu Baetge (1974), S. 23 ff.

${ }^{120}$ Der Sollwertgeber gehört nicht zum eigentlichen System, das im Rahmen der systemtheoretischen Betrachtung untersucht wird. Ohne Sollwertgeber ist jedoch ein System nach der Vorstellung der Kybernetik I nicht denkbar, da dieser es ist, der ein zweckgerichtetes System konstituiert und konstruiert.

${ }^{121} \mathrm{Im}$ vorliegenden Zusammenhang ist es angemessen, die Ziele des Top-Managements als übereinstimmend mit den Zielen der Anteilseigner aufzufassen, da die Beziehung zwischen Anteilseignern und Top-Management durch die Kybernetik I nicht thematisiert wird.
} 
zum Informationsmanagement gesetzt. Kommt es zu einer gravierenden, dauerhaften Regelabweichung, so hat das zielsetzende System Anpassungsentscheidungen bezüglich des vorgegebenen Sollwertes oder strukturelle Änderungen im Systemaufbau vorzunehmen.

\subsection{Konstruktion des Informationsmanagements als Erkenntnisgegen- stand aus der Perspektive des Systemorientierten Ansatzes}

Die nachfolgenden Überlegungen nehmen eine Abgrenzung des Informationsmanagements aus Sicht der Systemorientierten Betriebswirtschaftslehre vor. Dabei handelt es sich um die letzte Stufe des im zweiten Kapitel beschriebenen dreistufigen Vorgehens zum Theorieeinstieg für das Informationsmanagement, auf der das spezielle theoretische Konstrukt als Erkenntnisgegenstand der Betrachtung entwickelt wird. Die Konstruktion des Erkenntnisgegenstandes bezieht sich zum einen auf den Gestaltungsgegenstand des Informationsmanagements, also das technikunterstützte Informationssystem der Unternehmung, und zum anderen auf die Aktivitäten der Lenkung dieses Systems, also auf das Informationsmanagement selbst. Dadurch wird die Grundlage geschaffen, auf der anschließend die eigentlichen theoretischen Aussagen für das Informationsmanagement abgeleitet werden können.

Eine Betrachtung des technikunterstützten Informationssystems als Gestaltungsgegenstand des Informationsmanagements muss sich entsprechend der methodischen Leitideen des Systemorientierten Ansatzes auf mehreren Aggregationsebenen bewegen. Dieses System stellt ein Subsystem des Gesamtsystems Unternehmung dar, das wiederum als Teil des gesamten Wirtschafts- und Gesellschaftssystems aufgefasst werden kann. Die grundsätzliche Abgrenzung des Systems wird in Abschnitt 4.2.1 behandelt, während im Fokus von Abschnitt 4.2.2 das Verhältnis zwischen dem technikunterstützten Informationssystem und der Gesamtunternehmung steht. Das System lässt sich selbst zudem entsprechend des jeweiligen Untersuchungszwecks in weitere Subsysteme dekomponieren. Aus systemtheoretischer Perspektive besitzen diejenigen Merkmale des technikunterstützten Informationssystems der Unternehmung besondere Bedeutung für das Informationsmanagement, die mit dessen hohem Komplexitätsniveau zusammenhängen. Deshalb schließt sich die Untersuchung von Charakteristika des Systemverhaltens, die durch die Komplexität des Systems hervorgerufen werden, an die Einordnung des Systems in den Gesamtzusammenhang an (Abschnitt 4.2.3).

Zuletzt wenden sich die Ausführungen in Abschnitt 4.2.4 der Lenkung dieses Systems durch das Informationsmanagement $\mathrm{zu}$. Das Informationsmanagement wird als Aufgabenfeld rekonstruiert, dessen Gegenstand die Lenkung eines speziellen Subsystems des komplexen Systems Unternehmung darstellt. Die Leitideen der Systemorientierten Betriebswirtschaftslehre, die allgemein die Gestaltung und Steuerung komplexer Systeme durch das Management betreffen, werden dabei auf das Informationsmanagement übertragen. 


\subsubsection{Abgrenzung des technikunterstïtzten Informationssystems als Subsystem der Unternehmung}

Bei dem durch das Informationsmanagement gelenkten technikunterstützten Informationssystem der Unternehmung handelt es sich um ein Subsystem des übergeordneten Gesamtsystems Unternehmung. Um die Definition und Abgrenzung des technikunterstützten Informationssystems aus der Perspektive der Systemorientierten Betriebswirtschaftslehre zu rekonstruieren, ist es deshalb erforderlich, zunächst auf das allgemeine Unternehmungsverständnis dieses Ansatzes einzugehen.

ULRICH als Begründer dieses Wissenschaftsprogrammes definiert das produktive soziale System Unternehmung als „von Menschen geschaffenes, reales Gebilde, welches u.a. aus Menschen besteht und den Zweck verfolgt, irgendwelche 'Leistungen' für die menschliche Gesellschaft bereitzustellen. "122 Menschen stellen nach dieser Sichtweise also eine, jedoch offensichtlich nicht die einzige Elementart des Systems Unternehmung dar. An anderer Stelle führt er aus, das Kommunikationssystem als Teilsystem der Unternehmung werde aus Menschen und Maschinen gebildet, welche für die Unternehmung produktiv tätig sind. Diese Klassifizierung von Menschen als Elemente der Unternehmung, die - häufig implizit - im Kern von den meisten nachfolgenden Vertretern der Systemorientierten Betriebswirtschaftslehre geteilt wird, basiert auf dem Grundgedanken der Partialinklusion der zum System gehörenden Individuen. Partialinklusion bedeutet, dass die Mitglieder einerseits Elemente einer Systemkonzeption sind, andererseits aber, dass der Mensch nie voll und ganz nur einem sozialen System zugehörig sein kann. ${ }^{123}$ Dementsprechend nimmt das System den ganzen Menschen als Mitglied auf, das Individuum selbst ist dem System aber nur mit einem Teil seiner Persönlichkeit verbunden, da es gleichzeitig auch einer Reihe anderer sozialer Systeme (z.B. Familie, Vereine, Kirche u.ä.) angehören kann. ${ }^{124}$

Mit der Frage, wie eine derartige Partialinklusion von Menschen in der Unternehmung entsteht, beschäftigt sich ULRICH nicht. Neuere systemtheoretische Arbeiten gehen davon aus, dass die Partialinklusion durch eine teilweise Parallelisierung der kognitiven Zustände der beteiligten Individuen erfolgt. Ein System, das auf diese Weise konstituiert und zusammengehalten wird, wird als synreferenzielles System bezeichnet. ${ }^{125}$ Synreferenz bedeutet, dass die Mitglieder eines sozialen Systems parallelisierte Zustände ihrer Gehirne aufweisen, auf die sie bei ihren Interaktionen mit anderen Mitgliedern des Systems Bezug nehmen. Bei Verfolgung dieser Sichtweise stellt das technikunterstützte Informationssystem der Unternehmung ein synreferenzielles Teilsystem des Gesamtsystems Unternehmung dar, das aus Menschen und Maschinen besteht, und dem die Menschen durch Partialinklusion angeschlossen werden. Das technikunterstützte Informationssystem der Unternehmung wird durch eine Parallelisierung der kognitiven Zustände der menschlichen Aufgabenträger in Bezug auf ihre Aktivitäten der technikunterstützten Informationsverarbeitung konstituiert. Diese Parallelisierung kognitiver Zustände wird über eine permanente

\footnotetext{
${ }^{122}$ Ulrich (1970), S. 134.

${ }^{123}$ Vgl. dazu ausfuihrlich Kasper (1991), S. 5, sowie die dort angegebenen Quellen.

${ }^{124}$ Vgl. Ulrich (1970), S. 156; Kasper (1991), S. 5f.

${ }^{125}$ Die Idee des synreferenziellen Systems geht zurïck auf Hejl (1982), S. 60ff.
} 
Selbstbeobachtung und Selbstbeschreibung der Systemmitglieder und somit auch des Systems als Ganzem erreicht. ${ }^{126}$

$\mathrm{Zu}$ den Mitgliedern, die als Elementart Mensch dem technikunterstützten Informationssystem der Unternehmung angehören, zählen unterschiedlichste Personengruppen. Dazu gehören beispielsweise die Anwender der Informations- und Kommunikationstechnik in den Fachabteilungen, Systementwickler, die sich mit der konzeptionellen und programmtechnischen (Weiter-) Entwicklung der Software befassen, sowie Hardware- und Netzwerktechniker, die mit der entsprechenden Pflege der technischen Komponenten befasst sind. Die Elementart Maschinen besteht aus den verwendeten hard- und softwaretechnischen Bestandteilen, mit denen die Menschen bei der Erfüllung ihrer Aufgaben unterstützt werden.

Die neuere sozialwissenschaftlich ausgerichtete Systemtheorie (insbesondere die von LUHMANN entwickelte soziologische Theorie autopoietischer sozialer Systeme) nimmt eine abweichende Position ein, da sie als Elemente des Systems nicht Menschen (und technische Anlagen), sondern Handlungen bzw. Entscheidungen ansieht. ${ }^{127}$ Organisationen sind danach Systeme, die „aus Entscheidungen, nichts als Entscheidungen “ ${ }^{128}$ bestehen. Nach dieser Auffassung bestehen soziale Systeme nicht aus einer Ansammlung von Menschen und Maschinen, sondern aus Handlungen, die zur Entscheidung werden, indem sie mit Verhaltenserwartungen der Beteiligten konform gehen oder von diesen abweichen. ${ }^{129}$ Um sich selbst $\mathrm{zu}$ erhalten und $\mathrm{zu}$ entfalten, müssen soziale Systeme eine Beschreibung ihres Selbst erzeugen und benutzen. ${ }^{130}$ Dieser Vorgang der andauernden Selbstbeobachtung und Selbstbeschreibung wird als Selbstreferenz bezeichnet. Die ein soziales System konstituierenden Entscheidungen sind über einen geschichtlichen Ablauf miteinander verbunden, da die Erwartungen bei späteren Entscheidungen durch die jeweils früheren Entscheidungen beeinflusst werden. Dadurch kommt es zu einer Verzeitlichung von Systemen, die nur in ihrem Verhältnis zur Zeitlichkeit zu erklären sind. ${ }^{131}$

Soziale Systeme sind aus dieser Perspektive Sinnsysteme, die durch sinnhaft aufeinander bezogene Handlungen, durch Kognitionen, Werte und Normen zusammengehalten werden. ${ }^{132}$ Die an diesem Sinnbildungsprozess beteiligten Menschen gehören dabei nicht selbst zum System, sondern zur Umwelt des Systems. ${ }^{133}$ Eine der Grundannahmen dieses Ansatzes ist es, dass sich soziales Geschehen nicht auf einzelne Subjekte reduzieren lässt. Daraus folgt in methodischer Hinsicht, dass ein methodologischer Individualismus abzulehnen ist, da bei einer Trennung von Sozialem und Psychischem emergente soziale Sachverhalte nicht durch die Untersuchung des

\footnotetext{
${ }^{126}$ Das System wird genau genommen durch eine Selbstbeobachtung und Selbstbeschreibung der Systemmitglieder, die sich auf das System bezieht, konstituiert. Von einer Selbstbeobachtung und Selbstbeschreibung des Systems kann in strengem Sinne nur dann gesprochen werden, wenn der methodologische Individualismus aufgegeben und das nachfolgend erlăuterte Konzept autopoietischer sozialer Systeme übernommen wird. Vgl. dazu ausführlich Kirsch/Knyphausen (1991), S. 89ff.

${ }^{127}$ Vgl. Kasper (1991), S. 5 f.

${ }^{128} \mathrm{Kirsch} / \mathrm{Knyphausen}$ (1991), S. 87.

${ }^{129} \mathrm{Vgl}$. Luhmann (1984b), S: 594; Kirsch (1992), S. 237; Gobel (1998), S. 74.

${ }^{130}$ Vgl. Luhmann (1984a), S. 25.

${ }^{131}$ Vgl. Stünzner (1996), S. 98.

${ }^{132}$ Vgl. Probst (1987), S. 50, der sich auf Luhmann bezieht, ohne eine exakte Quelle zu benennen.

${ }^{133}$ Vgl. Luhmann (1985), S. 40f.; Willke (1987a), S. 39; Kasper (1991), S. 6.
} 
Verhaltens einzelner Personen erklärt werden können. Legt man diese Position zu Grunde, so handelt es sich bei dem technikunterstützten Informationssystem der Unternehmung um ein dynamisches Gefüge von Handlungen der Unternehmungsmitglieder, die sich zur Ausführung dieser Handlungen informationstechnischer Hilfsmittel bedienen und mit den Handlungen den Erwartungen der anderen Beteiligten entsprechen oder widersprechen.

Die Frage, ob es sich bei Unternehmungen als Organisationen um autopoietische Systeme handelt oder nicht, und ob damit die Voraussetzungen für die Anwendbarkeit der Theorie autopoietischer Systeme vorliegen, wird kontrovers diskutiert. Sie wird in den meisten Fällen im strengen Sinne negativ beantwortet, wenn auch mit unterschiedlichen Begründungen. ${ }^{134}$ Im Folgenden wird dieser Forschungszweig der Systemorientierten Betriebswirtschaftslehre bei der Entwicklung von Aussagen zum Informationsmanagement aus zwei Gründen nicht mehr weiter berücksichtigt: Zum einen ist die Anwendbarkeit der Konzeption auf Unternehmungen generell fraglich, zum anderen besteht nur ein sehr indirekter Zusammenhang zur technikunterstützten Informationsverarbeitung als Gestaltungsgegenstand des Informationsmanagements. Das hier zu Grunde gelegte Konzept zeichnet sich dadurch aus, dass die Unternehmung und ihr technikunterstütztes Informationssystem nicht als autopoietisch aufgefasst wird. Zwar können die Unternehmungsmitglieder als autopoietische Systeme begriffen werden, die durch ihre spezifischen Interaktionen das soziale System Unternehmung konstituieren. Das Konzept der Autopoiese selbst wird jedoch in der Biologie belassen und bietet für theoretische Aussagen über soziale Systeme allenfalls heuristische Anhaltspunkte. $^{135}$

\subsubsection{Unterschiedliche Betrachtungsebenen bei der Untersuchung des technik- unterstützten Informationssystems}

Entsprechend der erläuterten Leitideen stellt der Wechsel zwischen unterschiedlichen Betrachtungsebenen ein spezielles methodisches Merkmal der Systemorientierten Betriebswirtschaftslehre dar, da die Beziehungen zwischen Teil und Ganzheit einen Schwerpunkt der Systemorientierten Analyse ausmachen. Das bedeutet, dass eine Untersuchung des technikunterstützten Informationssystems der Unternehmung sich an dieser Stelle nicht auf das System als solches beschränken darf, sondern dass dieses System als Subsystem des übergeordneten Gesamtsystems Unternehmung zu begreifen ist, das wiederum einen Teil des Wirtschafts- und Gesellschaftssystems darstellt. Umgekehrt kann das gesamte technikunterstützte Informationssystem der Unternehmung analytisch in verschiedene weitere Subsysteme zerlegt werden, die dann einer detaillierteren Betrachtung zugänglich sind. Abbildung $4 / 2$ verdeutlicht diesen Zusammenhang.

\footnotetext{
${ }^{134}$ Vgl. z.B. Heijl (1983), S. 56; Kirsch/Knyphausen (1991), S. 87; Stünzner (1996), S. 192ff.; Schneider (1997), S. 31; Göbel (1998), S. 77; Streubel (2000), S. 180. Göbel stellt fest, dass bei sozialen Systemen unklar ist „wer da was wie produziert", vgl. Göbel (1998), S. 73, (Hervorhebung im Original). Es könnte sich dabei um „von allen geteilte Wirklichkeitskonstrukte“ (Hejl (1992), S. 52), um eine geteilte Regelmenge (Kirsch (1992), S. 258), eine soziale Einheit, die der Ontogenese der Mitglieder dient (Maturana/Varela (1987), S. 209) oder noch etwas anderes handeln.

${ }^{135}$ Vgl. dazu auch Kirsch/Knyphausen (1991), S. 82ff.
} 

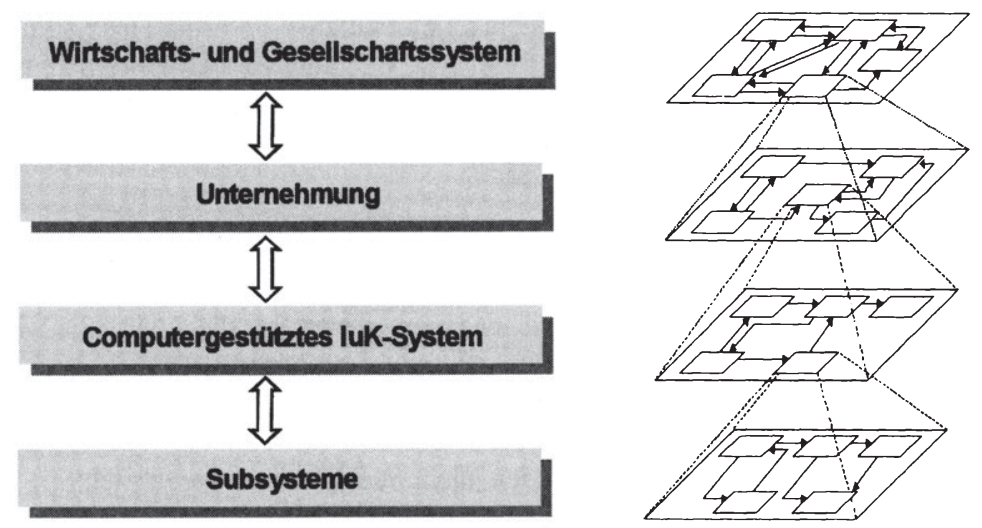

Abb. 4/2: Wechsel von Betrachtungsebenen

Dieses zuletzt angesprochene analytische Denken hat Wissenschaft und Wirtschaft vor der Entwicklung der Systemtheorie lange Zeit dominiert. ${ }^{136}$ Das weitaus bedeutendere Merkmal für einen Systemorientierten Ansatz für eine Theorie des Informationsmanagements stellt im Gegensatz dazu die Betonung des synthetischen Denkens durch die Systemorientierte Betriebswirtschaftslehre dar. Im Folgenden werden deshalb zunächst die Beziehungen zwischen dem durch das Informationsmanagement gestalteten und gesteuerten technikunterstützten Informationssystem und dem übergeordneten Gesamtsystem Unternehmung erläutert. Dabei sind insbesondere die Funktionen, die das technikunterstützte Informationssystem für die Gesamtunternehmung erbringt, für das Informationsmanagement von Relevanz.

Auf die funktionale Bedeutung der Informationsverarbeitung für die Gesamtunternehmung weist bereits ULRICH hin, der feststellt, dass ein zielorientiertes Handeln eines realen Systems ohne Kommunikation zwischen seinen Elementen und mit der Umwelt nicht denkbar ist. ${ }^{137}$ Er schlägt eine eigene Analysedimension ${ }^{138}$ zur Untersuchung der Informationsverarbeitung in der Unternehmung vor und bezeichnet diese als Kommunikationssystem. In dieser Analysedimension sollen die ,produktiven Elemente der Unternehmung als nachrichtenempfangende, -speichernde, -verarbeitende und -abgebende Einheiten, das Unternehmungsgeschehen als ebensolche Prozesse und die Struktur des Gebildes als Kommunikationsnetz ${ }^{\text {(139 }}$ aufgefasst werden. Unter diesem Blickwinkel erscheint das Geschehen „als ständiges Erfassen und Umsetzen von Nachrichten und als fortwährendes Erzeugen, Speichern und Verbrauchen von Informationen als den problemrelevanten Ergebnissen zielintendierter Nachrichtenverarbeitungsprozesse ${ }^{\text {c140 }}$. Folgt man dieser Überlegung, so ergibt sich, dass das

\footnotetext{
${ }^{136}$ Vgl. Probst (1987), S. 31f.

${ }^{137}$ Vgl. Ulrich (1970), S. 257.

${ }^{138}$ Daneben betrachtet er die materielle, die soziale und die wertmäßige Dimension als weitere Dimensionen des Unternehmungsgeschehens. Vgl. Ulrich (1970), S. $225 \mathrm{ff}$.

${ }^{139}$ Ulrich (1970), S. 257.

${ }^{140} \mathrm{Krieg}$ (1971), S. 104.
} 
Verhalten von (komplexen) Systemen im Wesentlichen durch Informationsströme bestimmt wird, ${ }^{141}$ an denen die Systemelemente ihre Handlungen ausrichten.

Der Zweck der Kommunikation besteht dabei in der Reduktion von Vielfalt und beseitigt Unsicherheit beim Empfänger der Information. Durch die Übertragung von Informationen ergeben sich Begrenzungen in den Erwartungen des Empfängers, durch die die Vorhersagbarkeit des System- und Umweltverhaltens erhöht wird. ${ }^{142}$ Eine gut funktionierende Informationsverarbeitung bewirkt aus dieser Perspektive, dass die beteiligten Individuen Entscheidungen treffen und darauf basierende Handlungen ausführen, die geeignet sind, das Überleben des Systems als oberstes Ziel sicherstellen. MALIK stellt deshalb fest, in letzter Konsequenz sei kybernetisches Management jede Beeinflussung eines Systems in Richtung auf ein Ziel durch Veränderung der Informationslage im System, da Menschen nicht auf Grund dessen handeln, was wirklich ist, sondern auf Grund dessen, was sie für wirklich halten. ${ }^{143}$

Mit diesen Erläuterungen ist zunächst die Bedeutung der Informationsverarbeitung für die Unternehmung skizziert, wenn die traditionelle Sichtweise verfolgt wird, bei der das technikunterstützte Informationssystem als Elemente Menschen und Maschinen enthält. Berücksichtigt man zusätzlich den oben angesprochenen Aspekt der Synreferenz, so wird durch die Informationsverarbeitung nicht nur das Überleben des Systems Unternehmung sichergestellt, sondern das System wird überhaupt erst durch synreferenzielle Informationsverarbeitung konstituiert. Diese Sichtweise betont, dass soziale Systeme und somit auch Unternehmungen laufend gezwungen sind, sich mit ihrer Umwelt und den von dieser ausgehenden Einflüssen auseinander zu setzen, um ihre Struktur, ihre Funktionsweise und ihre Identität aufrecht erhalten zu können. ${ }^{144}$ Die Identität des Systems gründet sich auf die permanente Reproduktion der Systemgrenzen gegenüber der Umwelt, ${ }^{145}$ auf die Konstruktion eines Bildes von sich selbst durch selbstbeobachtende und selbstbeschreibende Informationsverarbeitung. Auf dem Wege der Herstellung von System-Umwelt-Differenzen wird zugleich die Umwelt durch das System selbst konstruiert und ist nicht als etwas von vornherein Gegebenes anzusehen. ${ }^{146}$ Dementsprechend sind auch die Informationen aus der Umwelt nicht als außerhalb der Unternehmung liegende, gegebene Fakten zu verstehen, sondern sie werden erst zur Information, wenn das System sie wahrnimmt und auswertet. ${ }^{147}$ Informationen befinden sich also nicht außerhalb der Unternehmung und müssen gesammelt werden, sondern sie werden ,in-formiert", im Innern der Unternehmung geformt und erzeugt. ${ }^{148}$

Damit ist die Funktion beschrieben, die das technikunterstützte Informationssystem für das Gesamtsystem Unternehmung erbringt. Das technikunterstützte Informationssystem als Subsystem entlastet das übergeordnete Gesamtsystem, da es an dessen

\footnotetext{
${ }^{141}$ Vgl. Malik (1993), S. 229.

${ }^{142} \mathrm{Vgl}$. Ashby (1974), S. 194f.

${ }^{143} \mathrm{Vgl}$. Malik (1993), S. 186. Wenn die Aussage zumindest in dieser Form auch unter epistemologischen Gesichtspunkten problematisch ist, so wird doch die essenzielle Bedeutung deutlich, die das Informationsmanagement für das Verhalten der Systemmitglieder und damit für das Überleben des Gesamtsystems Unternehmung spielt.

${ }^{144}$ Vgl. Malik (1992), S. 170.

${ }^{145}$ Vgl. Kasper/Mayrhofer/Meyer (1998), S. 611.

${ }^{146}$ In diesem Sinne bedeutet Selbstreferenz immer zugleich Fremdreferenz. Vgl. Hahn (1987), S. 11.

${ }^{147} \mathrm{Vgl}$. Göbel (1998), S. 75.

${ }^{148}$ Vgl. Kasper/Mayrhofer/Meyer (1998), S. 604
} 
Konstituierung mitwirkt und zugleich dessen Komplexität reduziert, indem es spezifische Funktionen erbringt, die das Gesamtsystem Unternehmung wegen seiner hohen Komplexität nicht erfullen kann. Eine Funktion wird jedoch auch in umgekehrter Richtung durch das Supersystem Unternehmung für das technikunterstützte Informationssystem als dessen Subsystem erbracht. Das Gesamtsystem Unternehmung definiert für dieses Subsystem eine spezifische Umwelt und erhöht auf diese Weise das Ausmaß an Bestimmtheit und Sicherheit fur das Subsystem im Vergleich zur Umwelt des Gesamtsystems. Dadurch werden die Bedingungen für eine fortdauernde Existenz dieses Subsystems verbessert. Ein Beispiel dafür stellt die Marktwahlentscheidung auf der übergeordneten Ebene dar. Aus dieser ergeben sich für die untergeordnete Ebene einerseits Anforderungen zur Einbeziehung bestimmter Regionen, deren Markt bearbeitet wird, in die Informationssystemplanung, während andererseits die Sicherheit geschaffen wird, dass die Entwicklung eines Informationsverarbeitungskonzeptes für andere Regionen nicht erforderlich ist.

Die allgemeine Feststellung, dass zwischen den Subsystemen und dem Gesamtsystem ein Verhältnis der wechselseitigen Abhängigkeit auf Grund des gegenseitigen Entlastungspotenzials besteht, ${ }^{149}$ trifft also auch auf das technikunterstützte Informationssystem und die Unternehmung als übergeordnetes Supersystem zu. Es besteht ein Verhältnis der gegenseitigen Komplexitätsreduktion, wobei einerseits die Innenkomplexität (Informationssystem für Gesamtunternehmung) und andererseits die Außen- (=Umwelt-) Komplexität (Gesamtunternehmung für Informationssystem) reduziert wird.

Auch zwischen dem technikunterstützten Informationssystem der Unternehmung und seinen Subsystemen ist von dieser wechselseitigen Abhängigkeit zwischen Teil und Ganzheit auszugehen. Die Bildung von Subsystemen ist über mehrere Systemebenen hinweg möglich sodass eine vielschichtige Untergliederung des Gestaltungsgegenstandes des Informationsmanagements entsteht. Bei einer funktionalen Abgrenzung von Subsystemen, bei der die Subsysteme als Anwendungssysteme im engeren Sinne (bei der Beschränkung auf technische Komponenten) beziehungsweise Anwendungssysteme im weiteren Sinne (bei Einbeziehung der menschlichen Akteure) bezeichnet werden, wird die wechselseitige Abhängigkeit von Teil und Ganzheit besonders deutlich. Das Anwendungssystem erbringt eine Funktion für das übergeordnete Gesamtsystem, das wiederum die Umweltkomplexität für das Subsystem reduziert. Abbildung 4/3 zeigt ein Beispiel für die Untersuchung des technikunterstützten Informationssystems der Unternehmung auf mehreren Betrachtungsebenen.

\footnotetext{
${ }^{149}$ Vgl. dazu auch die allgemeinen Erörterungen bei Stünzner (1996), S. 85.
} 


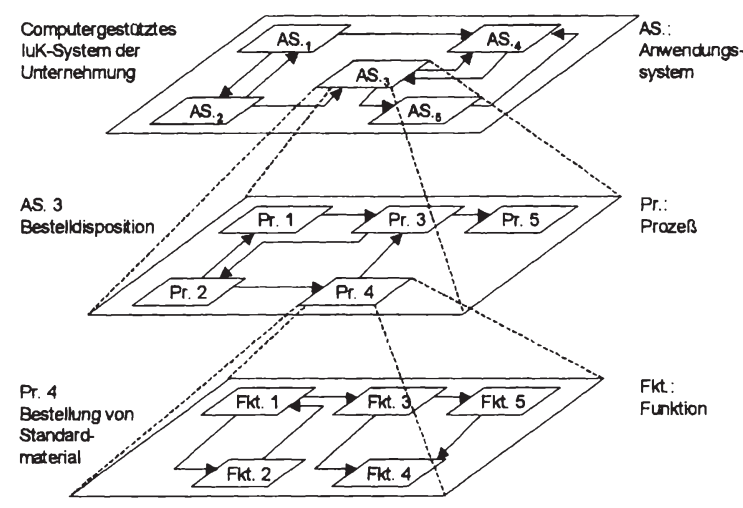

Abb. 4/3: Bildung von Subsystemen des technikunterstützten Informationssystems

\subsubsection{Komplexität des technikunterstützten Informationssystems}

$\mathrm{Zu}$ Beginn dieses Kapitels wurde dargelegt, dass die zentrale Problemstellung, auf die sich das Erkenntnisinteresse der Systemorientierten Betriebswirtschaftslehre richtet, in der Bewältigung von Komplexität liegt. ${ }^{150}$ Nachfolgend sind deshalb diejenigen Merkmale des im vorigen Abschnitt beschriebenen und in den Gesamtzusammenhang eingeordneten technikunterstützten Informationssystems der Unternehmung herauszuarbeiten, die die Komplexität dieses Systems ausmachen und folglich das System aus der Perspektive der Systemorientierten Betriebswirtschaftslehre besonders kennzeichnen.

Die Ursachen der Komplexität von Systemen werden allgemein darin gesehen, dass das Systemverhalten einerseits das Ergebnis der Interaktionen einer großen Zahl von Elementen ist und dass andererseits die Systemumwelt eine hohe Dynamik aufweist und Wandelprozessen unterliegt, die häufig diskontinuierlich verlaufen. ${ }^{151}$ Das Komplexitätsniveau von Unternehmungen wird in der Systemorientierten Betriebswirtschaftslehre als sehr hoch angesehen. ${ }^{152}$ Sowohl die unternehmungsintern stattfindenden Interaktionen zwischen den Mitarbeitern ${ }^{153}$ als auch die permanenten Wandelprozesse in der Unternehmungsumwelt machen eine sehr große Zahl an System/Umwelt-Konstellationen denkbar und bewirken somit eine extrem hohe Varietät des Systems Unternehmung. Dies gilt in analoger Weise für deren technikunterstütztes Informationssystem als Gestaltungsgegenstand des Informationsmanage-

\footnotetext{
${ }^{150} \mathrm{Vgl}$. dazu Kap. 4.1.

${ }^{151} \mathrm{Vgl}$. dazu Kap. 4.1.1. und die dort angegebenen Quellen. Zum Phänomen des Wandels vgl. Streubel (2000), S. 91ff.

${ }^{152}$ So ordnet bereits Beer die Unternehmung der Kategorie der äußerst komplexen Systeme zu, die sich dadurch auszeichnen, dass sie nicht vollständig beschreibbar sind, vgl. Beer (1967), S. 32f. Diese Auffassung wird von späteren Autoren explizit oder implizit übernommen. Vgl. z.B. Probst (1987), S. 9; Malik (1992), S. 24; Streubel (2000), S. 25.

${ }^{153}$ Auch wenn man von Entscheidungen als Systemelementen ausgeht, kann eine große Zahl von Beziehungen zwischen diesen Elementen, die in der Summe die Historie des Systems darstellen, konstatiert werden.
} 
ments. In diesem System wirkt ebenfalls eine Vielzahl von Mitarbeitern zusammen, die über einen unterschiedlichen fachlichen und persönlichen Hintergrund verfügen und ihr Verhalten in Folge neu hinzu kommender individueller Erfahrungen im Prinzip jederzeit ändern können. Ferner ist das technikunterstützte Informationssystem an seinen Grenzen genau wie das übergeordnete Gesamtsystem Unternehmung einer hohen Umweltdynamik ausgesetzt, die sich durch immer schneller werdende, allgemeine technische Entwicklungen sowie permanent verändernde Markt- und Wettbewerbssituationen ergibt.

Bei der Untersuchung des Verhältnisses zwischen dem technikunterstützten Informationssystem der Unternehmung und der Unternehmung als übergeordnetem Gesamtsystem wurde erläutert, dass die Unternehmung für das technikunterstützte Informationssystem als Subsystem eine Komplexitätsreduktion erzeugt, indem sie durch bestimmte Grundentscheidungen Begrenzungen in der Umwelt des Subsystems errichtet. Diese Begrenzungen erhöhen zwar in gewissem Maße die Sicherheit für das technikunterstützte Informationssystem der Unternehmung, die Varietät des Systems ist dennoch so hoch, dass sie in praktischen Fällen nicht gemessen werden kann. Dies liegt einerseits daran, dass die durch das Supersystem geleistete Komplexitätsreduktion sich nicht auf die Innenkomplexität des Subsystems auswirkt, die durch die Interaktion der innerhalb des Systems agierenden Akteure entsteht. Andererseits beschränkt sich die Reduktion der Außenkomplexität für das Subsystem nur auf solche Aspekte der Subsystemumwelt, die erfolgreich durch die Unternehmung als Supersystem beeinflussbar sind. Die Dynamik der Umwelt bleibt für dieses Subsystem dennoch sehr hoch.

Es kann deshalb festgestellt werden, dass das technikunterstützte Informationssystem der Unternehmung ein sehr hohes Komplexitätsniveau aufweist. Daraus resultieren für das Informationsmanagement zwei bedeutende Konsequenzen. Zum einen folgt aus der hohen System/Umwelt-Varietät eine sehr hohe erforderliche Varietät der Lenkungsinstanz als Voraussetzung für eine erfolgreiche Lenkung des Systems. Zum anderen muss für das Verhalten des technikunterstützten Informationssystems eine eingeschränkte Kausalität angenommen werden, wodurch wiederum die Lenkbarkeit des Systems reduziert wird. Die Notwendigkeit einer hohen Varietät der Lenkungsinstanz, also des Informationsmanagements, ergibt sich aus dem durch das Gesetz der erforderlichen Varietät begründeten Zusammenhang, dass nur Vielfalt des Reglers der Vielfalt der Umwelt entgegen wirken und auf diese Weise das Systemverhalten stabilisieren und lenken kann. Die Einschränkung der Kausalität folgt daraus, dass sich auf Grund zahlreicher Rückkopplungsprozesse zwischen den Beteiligten innerhalb des technikunterstützten Informationssystems der Unternehmung das Systemverhalten im Zeitablauf ändert, sodass die eindeutige Verbindung zwischen Ursachen und Wirkungen aufgelöst wird und das System auf bestimmte Gegebenheiten zu unterschiedlichen Zeitpunkten in unterschiedlicher Weise reagiert.

Diese Überlegungen zeigen, dass der Aspekt der begrenzten Lenkbarkeit im Falle des technikunterstützten Informationssystems der Unternehmung von erheblicher Bedeutung ist. Bei dem Gestaltungs- und Steuerungsgegenstand des Informationsmanagements handelt es sich um ein System, das zum einen hohe Anforderungen an die Lenkungskapazität des Reglers stellt und zum anderen wegen seiner hohen inneren Dynamik gewissermaßen „widerspenstig“ auf äußere Einflussnahmen reagiert. Hinsichtlich der zu bewältigenden Varietät trifft die von ASHBY gezogene 
Schlussfolgerung, dass dem Grad der erreichbaren Regelung bei sehr umfangreichen Systemen schon auf Grund der Vielfalt der Systeme eine absolute Grenze gesetzt ist, ${ }^{154}$ auf das technikunterstützte Informationssystem der Unternehmung zu. Zudem wurde gezeigt, dass der Hinweis von MALIK, dass die Gestaltung und Steuerung eines Systems, das eine hohe innere Dynamik aufweist, große Unterschiede gegenüber der in der traditionellen Betriebswirtschaftslehre herrschenden Vorstellung von Gestaltung und Steuerung der Unternehmung aufweisen muss, ${ }^{155}$ auch für das Informationsmanagement Relevanz besitzt. Die Lenkung eines solchen Systems besteht eher in dem Verhindern, dass sich das System in ungewünschte Bahnen bewegt, ${ }^{156}$ als in der präzisen Beeinflussung von Einzelheiten des Systemverhaltens. Diese Überlegungen haben erhebliche Folgen für die im Abschnitt 4.3 durchgeführte Systemorientierte Analyse des Informationsmanagements.

\subsubsection{Lenkung des technikunterstützten Informationssystems durch das Informationsmanagement}

Aus der Perspektive der Systemorientierten Betriebswirtschaftslehre kann der Begriff Management funktional aufgefasst und als der lenkende Teil eines Systems (Regler) verstanden werden, der den zu lenkenden Teil des Systems (Regelstrecke) beeinflusst und durch diese Einflussnahme das Überleben des Systems sicherstellt. Diesem allgemeinen Managementbegriff lässt sich auch das Informationsmanagement als spezielles Teilgebiet des Gesamtaufgabenfeldes Management, das durch seinen spezifischen Gestaltungsgegenstand konstituiert wird, subsumieren. Informationsmanagement stellt unter diesem Blickwinkel den Regler des technikunterstützten Informationssystems der Unternehmung dar, der die Regelstrecke beeinflusst, um das Überleben des Systems zu gewährleisten. Entsprechend der beiden Forschungsrichtungen der Kybernetik kann das Informationsmanagement entweder konservativ im Sinne der Bewahrung bereits erreichter Gleichgewichtszustände bzw. der Rückkehr zu diesen Zuständen oder innovativ im Sinne der flexiblen, adaptiven Anpassung an sich wandelnde Situationen durch die Entwicklung zu immer neuen Systemzuständen ausgerichtet sein. Diese beiden Grundorientierungen sind in Abbildung 4/4 dargestellt.

\footnotetext{
${ }^{154} \mathrm{Vgl}$. Ashby (1974), S. 353

${ }^{155}$ Vgl. Malik (1992), S. 83

${ }^{156}$ Vgl. Probst (1987), S. 40.
} 


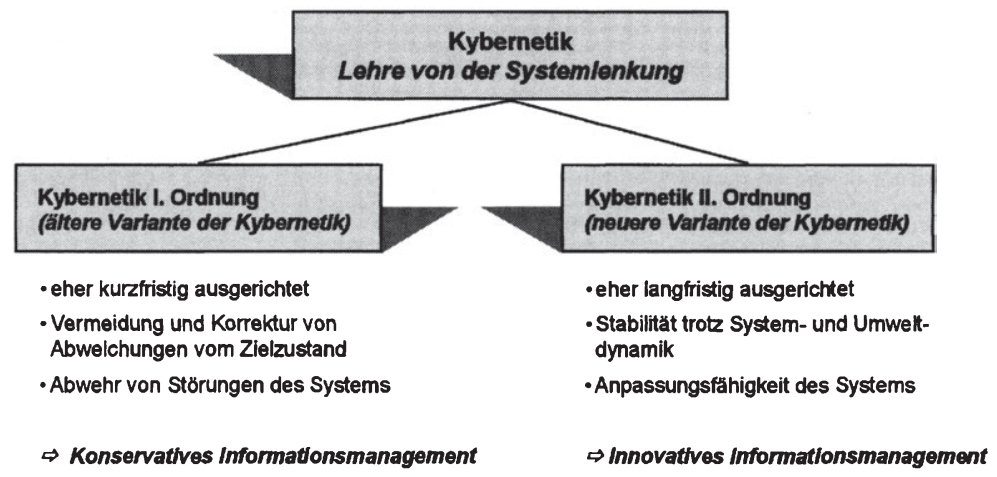

Abb. 4/4 Grundorientierungen des Informationsmanagements aus Sicht der Kybernetik

Im ersten Fall, in dem das Informationsmanagement eine Lenkung des Systems entsprechend der konservativ orientierten Zielsetzung der Kybernetik I anstrebt, geht es darum, vorgegebene Ziele des technikunterstützten Informationssystems der Unternehmung trotz vielfältiger Störeinflüsse $\mathrm{zu}$ erreichen. ${ }^{157}$ Formal ausgedrückt bedeutet dies, dass das Informationsmanagement beabsichtigt, die Varietät des Systemverhaltens so weit zu beschränken, dass nur die angestrebten Vorzugszustände des Systems eintreten. Die zentrale Aufgabe des Informationsmanagements ist bei dieser Ausrichtung darin zu sehen, die Übertragung der Störvarietät auf den Output des technikunterstützten Informationssystems der Unternehmung möglichst stark zu reduzieren und auf diese Weise das Systemverhalten innerhalb der gewünschten Grenzen zu halten.

Im Rahmen des konservativ ausgerichteten Informationsmanagements fallen beispielsweise Probleme wie schwankende Zahlen der durch das technikunterstützte Informationssystem zu bearbeitenden Kundenaufträge, Systemausfälle durch Hardoder Softwarestörungen oder Beeinträchtigungen der Datensicherheit auf Grund unbefugter Eingriffe an. Maßnahmen des Informationsmanagements zur Lösung derartiger Probleme können sich einerseits als Gestaltungsmaßnahmen auf die grundsätzliche Systemstruktur beziehen. In solchen Fällen wird die Regelstrecke durch eine geeignete Anordnung der Systemelemente und ihrer Beziehungen in die Lage versetzt, Vielfalt der Systemumwelt selbst auszugleichen, um die Stabilität des Systemverhaltens zu gewährleisten. Andererseits können die Maßnahmen des Informationsmanagements als Steuerungs- und Regelungsmaßnahmen die auf das System wirkenden Störungen derart modifizieren, dass das System in der bestehenden Systemstruktur den gewünschten Output produzieren kann.

Wenn das Informationsmanagement eine innovative Anpassungsfahigkeit des Systems entsprechend der Grundorientierung der Kybernetik II gewährleisten soll, dann geht es dagegen darum, die Flexibilität des Systemverhaltens zu fördern und das System auf wechselnde Umweltsituationen vorzubereiten. Das bedeutet formal gesehen, dass die

${ }^{157}$ Vgl. dazu Krieg (1970), S. $70 \mathrm{ff}$. 
Varietät des Systemverhaltens nicht in dem Maße reduziert wird, wie es bei der konservativ ausgerichteten Systemlenkung der Fall ist. Vielmehr wird die Überlebensfähigkeit des Systems gerade dadurch erhöht, dass die Möglichkeit geschaffen wird, durch die Variation der Funktionsweise des Systems den Output zu variieren, um auf diese Weise den Zielerreichungsgrad des technikunterstützten Informationssystems der Unternehmung zu verbessern. Zwar erfolgt auch in diesem Fall eine Varietätsreduktion durch das Informationsmanagement, diese ist jedoch weniger restriktiv, da eine höhere Varietät des Output durch das lenkende System akzeptiert wird. Das Lenkungssystem gibt dementsprechend durch den Ausschluss bestimmter Situationen lediglich eine grobe Richtung der Entwicklung und kein konkret anzustrebendes Ergebnis vor

Exemplarische Probleme in diesem Zusammenhang sind die Erschließung neuer Vertriebswege durch die Nutzung des Internet, die Einführung einer unternehmungsübergreifenden Vernetzung von Wertschöpfungsketten oder die Realisierung innovativer organisatorischer Konzepte (etwa im Bereich der Telearbeit) mit Hilfe moderner informationstechnischer Lösungen. In solchen Fällen geht es für das Informationsmanagement aus der Perspektive der Systemorientierten Betriebswirtschaftslehre weniger darum, die genaue Art der Informationsverarbeitung und die zu produzierenden Informationen detailliert und dauerhaft festzulegen, als darum, durch die Möglichkeit zu immer neuen kleineren und größeren innovatorischen Anpassungen die langfristige Überlebensfähigkeit des technikunterstützten Informationssystems und damit auch des übergeordneten Gesamtsystems Unternehmung zu erhöhen. Bei derartigen Problemstellungen steht für das Informationsmanagement also die Schaffung flexibler Prozess- und Gebildestrukturen für das technikunterstützte Informationssystem der Unternehmung im Vordergrund.

Die bisher angesprochenen Einflussnahmen des Informationsmanagements beziehen sich auf die formale Struktur des technikunterstützten Informationssystems der Unternehmung und sind damit auf der materiellen Ebene angesiedelt. Die materielle Management-Ebene wird entsprechend der in Abschnitt 4.1.2 erläuterten Leitideen der Systemorientierten Betriebswirtschaftslehre in komplexen sozialen Systemen durch eine geistig-sinnhafte, symbolische Ebene überlagert. ${ }^{158}$ Auch die materielle Ebene des Informationsmanagements ist folglich durch eine symbolische Ebene zu ergänzen. Diese symbolische Ebene der Gestaltung durch das Informationsmanagement dient der Vermittlung und Erfassung von Sinn und legitimiert dadurch die Gestaltungsmaßnahmen auf der materiellen Ebene. Die Aktivitäten der menschlichen Aufgabenträger in Prozessen der Informationsverarbeitung erfahren durch symbolische Gestaltungsmaßnahmen eine Orientierung und können auf diese Weise in die Richtung der Systemziele beeinflusst werden, ohne sie im Einzelnen zu determinieren. Gegenstand symbolischer Gestaltungsmaßnahmen können Handlungen, Sprache und materielle Objekte sein. ${ }^{159}$

Mit diesen Überlegungen ist auch die Frage angesprochen, wie der Begriff des Informationsmanagements aus der Perspektive der Systemorientierten Betriebswirtschaftslehre im institutionalen Sinne zu verstehen ist. Die Kybernetik I sieht das lenkende System (den Regler) als getrennt und eindeutig unterscheidbar vom zu

\footnotetext{
${ }^{158}$ Vgl. Probst (1987), S. 97 sowie die Ausführungen in Abschnitt 4.1.2.3.

${ }^{159}$ Vgl. Probst (1987), S. 100. Diese drei Bereiche der symbolischen Gestaltung werden später im Rahmen der eigentlichen Theoriebildung noch genauer untersucht.
} 
lenkenden System (der Regelstrecke). Aufgabenträger des Informationsmanagements nehmen aus diesem Blickwinkel also als externe Akteure Einfluss auf die Menschen und Maschinen, die die (Durchführungs-) Aufgaben der Informationsverarbeitung in der Unternehmung erfullen. Die Kybernetik II hebt dagegen die Bedeutung der Selbstregelung für die Überlebensfähigkeit des Systems hervor. Dies bedeutet einerseits, dass Aufgabenträger des Informationsmanagements Teil des technikunterstützten Informationssystems der Unternehmung sind und dieses nicht als unabhängige Beobachter und Konstrukteure nach ihren Wünschen lenken können. Es bedeutet andererseits, dass das zu lenkende System (die Regelstrecke) selbst ein hohes Gestaltungs- und Steuerungspotenzial besitzt, sodass Eingriffe von außen zum Teil überflüssig werden oder sogar kontraproduktiv wirken, da das zu lenkende System selbst zum lenkenden System wird. Der aktuelle Zustand und das Verhalten des technikunterstützten Informationssystems der Unternehmung sind demnach das Resultat vielfältiger Interaktionsprozesse zwischen den Elementen des Systems. Dies entspricht im Ergebnis der Sichtweise der sozialwissenschaftlichen Variante der Systemtheorie, die das System als soziales Entscheidungssystem auffasst, dass sich nur selbst beobachten und steuern kann. Der Mensch als physisch-psychisches System steht dabei allerdings außerhalb des betrachteten sozialen Systems.

\subsection{Systemorientierte Analyse des Informationsmanagements}

Nachdem die vorangegangenen Ausführungen den dreistufigen Theorieeinstieg für das Informationsmanagement vorgenommen haben, erfolgt in diesem Abschnitt die eigentliche Systemorientierte Analyse des Informationsmanagements. Dabei werden im Abschnitt 4.3.1 zunächst die Erkenntnispotenziale der Kybernetik I, die sich auf ein konservativ ausgerichtetes Informationsmanagement beziehen, beleuchtet. Im Abschnitt 4.3.2 werden dann basierend auf der Kybernetik II Erkenntnispotenziale für ein innovativ ausgerichtetes Informationsmanagement untersucht. Beide Ausrichtungen des Informationsmanagements sind praktisch relevant, da für ein dauerhaftes Überleben sowohl Stabilität als auch Wandel von Systemen erforderlich sind.

\subsubsection{Kybernetik I: Konservatives Informationsmanagement}

Systemorientiertes Management verfolgt in der konservativ ausgerichteten Variante der Kybernetik I das Ziel, das zu lenkende System in einem einmal definierten Gleichgewichtszustand $\mathrm{zu}$ halten oder es dahin zu bringen. In der Sprache der Systemtheorie formuliert geht es also darum, die Varietät des Systemoutputs in so engen Grenzen zu halten, dass die Systemziele in möglichst hohem Ausmaß erfüllt werden. Die nachfolgenden Ausführungen greifen die allgemeinen Überlegungen der Systemorientierten Betriebswirtschaftslehre zu diesem Punkt auf und konkretisieren diese durch die Übertragung auf die Problematik der Lenkung des technikunterstützten Informationssystems der Unternehmung durch das Informationsmanagement.

Die Ansatzpunkte für die Lenkung eines Systems ergeben sich aus dem Gesetz der erforderlichen Varietät, das formal ausgedrückt lautet: 


$$
\mathrm{H}_{\mathrm{E}} \geq \mathrm{H}_{\mathrm{S}}+\mathrm{H}_{\mathrm{SR}}-\mathrm{H}_{\mathrm{R}}{ }^{160}
$$

Dabei stellen $\mathrm{H}_{\mathrm{E}}$ die Varietät des Systemoutput, $\mathrm{H}_{\mathrm{S}}$ die Varietät der durch die Situation verursachten Störungen, $\mathrm{H}_{\mathrm{SR}}$ die innere Unsicherheit des Systemverhaltens für den Beobachter und $\mathrm{H}_{\mathrm{R}}$ die Varietät der Lenkungsmaßnahmen dar. ${ }^{161}$ Zur Stabilisierung des Verhaltens des technikunterstützten Informationssystems der Unternehmung kann das Informationsmanagement also an der Situation des Systems, an der inneren Ordnung des Systems oder an der eigenen Lenkungskapazität ansetzen. Aus der Perspektive der Kybernetik I sollte dabei im Idealfall die Varietät des Informationsmanagements die Varietät der Störvariablen übersteigen, sodass der Output des technikunterstützten Informationssystems der Unternehmung vollständig durch das Informationsmanagement bestimmt werden kann. ${ }^{162}$ Die Abwehr von Störungen kann durch passive und durch aktive Maßnahmen erfolgen. ${ }^{163}$

Passive Maßnahmen, die Gegenstand von Abschnitt 4.3.1.1 sind, zielen darauf ab, Störungen von vornherein vom technikunterstützten Informationssystem der Unternehmung fern zu halten, sodass es gar nicht erst zu einer Einwirkung auf das Systemverhalten kommen kann. Aktive Maßnahmen, die in den Abschnitten 4.3.1.2 und 4.3.1.3 behandelt werden, sind im Gegensatz dazu dadurch gekennzeichnet, dass wirksame Störungen des Systems durch gegenläufige Maßnahmen des Informationsmanagements abgewehrt werden sollen. Abschnitt 4.3.1.4 beleuchtet Möglichkeiten zur Kombination der verschiedenen Maßnahmetypen und Abschnitt 4.3.1.5 gibt einen Überblick über Ansätze zur weiteren Steigerung der Lenkungskapazität.

\subsubsection{Passive Bewältigung von Störungen durch Abschirmung des Systems}

Mit passiven Maßnahmen setzt das Informationsmanagement an der wahrgenommenen Umweltvarietät $\mathrm{H}_{S}$ an, um die Lenkbarkeit des technikunterstützten Informationssystems der Unternehmung zu erhöhen. Derartige Maßnahmen zur Reduzierung der Outputvarietät des technikunterstützten Informationssystems bewirken eine teilweise Isolierung dieses Systems von seiner Umwelt. ${ }^{164}$ Es kommt dadurch zu einer Abschirmung von externen Einflüssen, die unter sonst gleichen Umständen die relative Lenkungskapazität des Informationsmanagements gegenüber der Störvarietät der Umwelt erhöht und somit die Möglichkeiten des Informationsmanagements zur Sicherstellung des gewünschten Systemverhaltens verbessert. Dieses Mittel zur Störungsabwehr stellt den einfachsten Weg zur Stabilisierung von Systemen dar. ${ }^{165}$ Passive Maßnahmen können einerseits als Filter konstruiert werden, die Einflüsse aus der Systemumwelt aufhalten, sodass diese gar nicht erst dessen Umweltwahrnehmung erreichen. Andererseits kann die Bildung von Speichern oder Reserven bewirken, dass unvorhergesehene Störungen absorbiert werden und sich nicht unmittelbar auf das Systemverhalten auswirken. ${ }^{166}$

\footnotetext{
${ }^{160}$ Vgl. Ashby (1974), S. 300.

${ }^{161} \mathrm{Vgl}$. Ashby (1974), S. 300; Mirow (1969), S. $75 f$.

${ }^{162} \mathrm{Vgl}$. dazu allgemein Mirow (1969), S. 76.

${ }^{163} \mathrm{Vgl}$. Mirow (1969), S. 78; Krieg (1971), S. 71.

${ }^{164}$ Vgl. Mirow (1969), S. 79.

${ }^{165}$ Vgl. Krieg (1971), S. 71.

${ }^{166}$ Vgl. Klaus (1965), S. 198.
} 
Zur Verdeutlichung der konkreten Ausgestaltung passiver Abschirmungsmaßnahmen in der Form von Filtern werden häufig Beispiele aus der Tier- und Pflanzenwelt oder aus dem Bereich der technischen Systeme herangezogen. ${ }^{167}$ Demnach kann es sich dabei beispielsweise um Schalen oder Panzer sowie um Isolatoren oder Stoßdämpfer handeln. Im betriebswirtschaftlichen Bereich werden als Beispiele generelle Regelungen wie allgemeine Zahlungsbedingungen, Tarifverträge oder die Festlegung von Arbeitszeiten genannt, die die Unternehmungssituation in bestimmte Bahnen lenken. ${ }^{168}$ Übertragen auf die in dieser Arbeit betrachteten technikunterstützten Informationssysteme in Unternehmungen erfullen neben derartigen generellen Regelungen, die die Elementart Mensch betreffen, in Bezug auf die Elementart Technik z.B. auch Firewalls oder unternehmungsweite Hard- und Softwarestandards solche Filterfunktionen. Durch den Einsatz von Filtern für das technikunterstützte Informationssystem der Unternehmung wird verhindert, dass bestimmte unternehmungsexterne Entwicklungen oder Sachverhalte für dieses System relevant werden und dessen Lenkbarkeit reduzieren. Die hier genannten Beispiele im technischen Bereich schirmen das System im Falle der Firewalls vor externen Angriffen (etwa durch konkurrierende Unternehmungen) und im Falle der technischen Standards vor der großen Dynamik und der Gefahr von Fehlentwicklungen auf dem Hard- und Softwaremarkt ab.

Speicher bzw. Reserven haben in der Natur beispielsweise die Gestalt von Fettpolstern oder Nahrungsmittelvorräten bei bestimmten Tierarten und können in technischen Systemen die Form von Puffern oder Vorratstanks annehmen. Als Beispiele im betriebswirtschaftlichen Bereich werden Produktionslager in Industriebetrieben oder die Vorratskassenhaltung bei der Bank genannt. ${ }^{169}$ Übertragen auf technikunterstützte Informationssysteme in Unternehmungen sind vielfältige konkrete Ausgestaltungen solcher Speicher bzw. Reserven denkbar. In rein technischen Teilsystemen können etwa Pufferspeicher oder großzügig bemessene Übertragungskapazitäten in Netzwerken Funktionen zur passiven Störungsabwehr übernehmen. In ähnlicher Weise können Spitzenbelastungen durch eine über das unbedingt notwendige $\mathrm{Ma} ß$ hinaus gehende Ausstattung des Systems mit menschlichen Arbeitskapazitäten aufgefangen werden. Auch der Aufbau eines ganzen zweiten Rechenzentrums an einem anderen Standort als Ersatz furr Notfälle in Form von Feuer, Stromausfall o.ä. im ersten Rechenzentrum fällt in diese Kategorie von Maßnahmen. Im Gegensatz zur Funktionsweise von Filtern erreichen die Störungen in diesen Fällen tatsächlich das System. Sie werden jedoch durch das System absorbiert und können nicht zu unkontrollierten Ausbrüchen des Systemverhaltens führen.

Die erläuterten passiven Formen der Bewältigung von Komplexität sind mit gravierenden Nachteilen verbunden. ${ }^{170}$ Sie schützen das System zwar vor der unmittelbaren Zerstörung durch akute Störungen aus der Systemumwelt. Sie führen jedoch auf der anderen Seite zur Trägheit des Systems, da entweder (beim Einsatz von Filtern) bedeutsame Entwicklungen in der Systemumwelt zu spät wahrgenommen werden oder (bei der Einrichtung von Speichern bzw. Reserven) ein hohes Maß an Unbeweglichkeit des Systems die Folge ist. Soll eine Lähmung des technikunterstützten Informationssystems der Unternehmung verhindert werden, so sind

\footnotetext{
${ }^{167}$ Vgl. dazu Krieg (1971), S. 71 sowie die dort angegebenen Quellen.

${ }^{168} \mathrm{Vgl}$. Mirow (1969), S. 78.

${ }^{169}$ Vgl. Mirow (1969), S. 79.

${ }^{170}$ Vgl. Mirow (1969), S. 79f.; Krieg (1971), S. 71.
} 
deshalb aus der Sicht der Kybernetik I die passiven Schutzmaßnahmen des Informationsmanagements um aktive Maßnahmen zu ergänzen.

\subsubsection{Aktive Bewältigung von Störungen durch Steuerung}

Mit aktiven Maßnahmen setzt das Informationsmanagement an der Lenkungskapazität $\mathrm{H}_{\mathrm{R}}$ an, um die Möglichkeiten zur Lenkung des technikunterstützten Informationssystems der Unternehmung zu verbessern. Derartige Maßnahmen bewirken eine erhöhte Reaktionsfähigkeit gegenüber der auf das System wirkenden Komplexität der Systemumwelt. Wie bei den passiven Maßnahmen steigt auch bei den aktiven Maßnahmen unter sonst gleichen Umständen die relative Lenkungskapazität des Informationsmanagements, sodass der Zielerreichungsgrad des technikunterstützten Informationssystems der Unternehmung gesteigert werden kann. Aktive Maßnahmen können die Form der Steuerung und der Regelung haben. Die Analyse von Steuerung und Regelung stellt den Kern der dem Bereich der Kybernetik I zuzuordnenden Untersuchungen dar. ${ }^{171}$

Im Falle der Steuerung wird die Gegenmaßnahme des lenkenden Systems, das in diesem Zusammenhang als Steuerglied bezeichnet wird, durch die Störung selbst ausgelöst. ${ }^{172}$ Der für die Steuerung charakteristische offene Wirkungsweg basiert auf dem Prinzip der Vorwärtskopplung. ${ }^{173}$ Es wird dabei angenommen, dass das System frei von Rückwirkungen auf Lenkungsmaßnahmen ist. Die Steuerungsmaßnahmen beruhen in diesem Fall auf (tatsächlichen oder durch die Lenkungsinstanz angenommenen) Ursache-Wirkungsbeziehungen innerhalb des zu lenkenden Teils des Systems, der als Steuerstrecke bezeichnet wird. Das Ziel dabei ist es, eine der Störung entgegengesetzte Wirkung zu erzeugen, die die Auswirkungen der Störung exakt aufhebt. Das in Abbildung 4/5 dargestellte Prinzip der Steuerung ist inputorientiert, da die Grundlage der Verhaltensbeeinflussung Nachrichten über Inputveränderungen und daraus abgeleitete Erwartungen über die Veränderung des Outputs sind. ${ }^{174}$

\footnotetext{
${ }^{171}$ Vgl. dazu z.B. Mirow (1969), S. 91ff; Ulrich (1970), S. 120ff: Krieg (1971), S. 72ff.; Fuchs (1973), S. 167ff.; Baetge (1974), S. 23ff.; Ashby (1974), S. 282ff.; bezogen auf das Informationsmanagement Biethahn/Mucksch/Ruf (1994), S. 91 ff.

${ }^{172} \mathrm{Vgl}$. Mirow (1969), S. 92.

${ }^{173}$ Vgl. Krieg (1971), S. 72.

${ }^{174}$ Vgl. Krieg (1971), S. 73.
} 


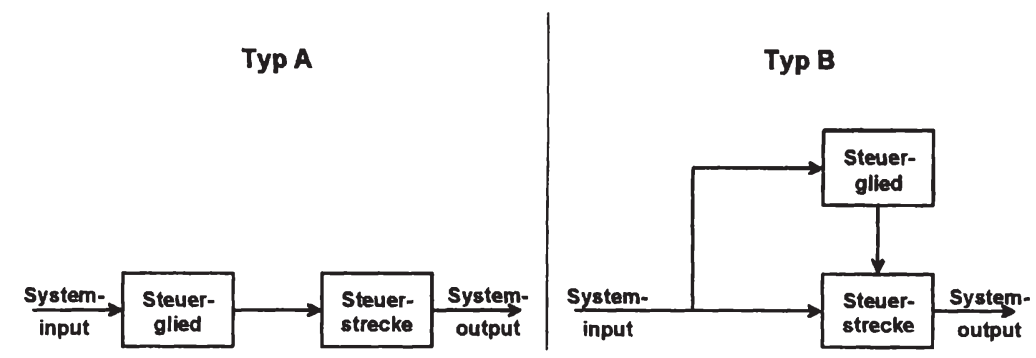

\section{Abb. 4/5: Das Steuerungsprinzip \\ Quelle: Baetge (1974), S. 23.}

Wie Abbildung 4/5 verdeutlicht können grundsätzlich zwei Typen der Steuerung unterschieden werden: ${ }^{175}$ Entweder findet eine Erfassung der Störgröße und die Gegenreaktion des Steuerglieds in der Weise statt, dass die Störgröße gar nicht erst die Steuerstrecke erreicht. Das Steuerglied funktioniert dann als Filter, der unerwünschte Einflüsse von der Steuerstrecke fernhält. Oder die Störung wirkt in vollem Umfang auf die Steuerstrecke und wird durch Einflussnahmen des Steuerglieds auf die Steuerstrecke kompensiert. Aus dem Gesetz der erforderlichen Varietät folgt, dass eine vollständige Stabilisierung des Outputs nur dann gelingen kann, wenn folgende Voraussetzungen erfüllt sind: ${ }^{176}$ Erstens müssen alle potenziellen Störungen bekannt sein und durch das Steuerglied erfasst werden können. Zweitens ist jeder Störung eine in ihrer Wirkung vollständig bekannte Steuerungsmaßnahme zuzuordnen. Drittens schließlich darf das System keine internen Unbestimmtheiten aufweisen, die interne Störungsgröße $\mathrm{H}_{S R}$ der Steuerstrecke muss mit anderen Worten gleich Null sein.

Auf Grund dieser hohen Anforderungen ist die Anwendbarkeit des Steuerungsprinzips in Unternehmungen als komplexen sozialen Systemen stark umstritten. Eine exakte Steuerung ist nur bei vollkommener Information und bei determinierten Systemen mit Prozessverläufen unter Sicherheit möglich. ${ }^{177}$ Äußerst komplexe Systeme können im Allgemeinen nicht durch Steuerketten stabilisiert werden, da auch Umwelteinflüsse auftreten, die nach Art, Umfang und zeitlichem Auftreten nicht bekannt sind. ${ }^{178}$ In Unternehmungen muss zudem auch mit internen Störungen gerechnet werden, deren Kompensation durch Steuerketten nicht sichergestellt werden kann. Auf der anderen Seite wird teilweise unterstellt, dass „es in vielen Organisationen Teilsysteme von geringer Varietät [gibt], deren Zustandsänderungen kausal-determiniert ablaufen ${ }^{\text {" }} 179$ mit der Folge, dass diese durch offene Wirkungsketten beherrscht werden können. Daraus könnte die Relevanz des Steuerungsprinzips in speziell abgegrenzten und überschaubaren Teilbereichen der Unternehmung abgeleitet werden.

Die Beantwortung der Frage, inwieweit das Steuerungsprinzip Relevanz für das Informationsmanagement besitzt, muss also an den Eigenschaften des technikunter-

\footnotetext{
${ }^{175} \mathrm{Vgl}$. Biethahn/Mucksch/Ruf (1994), S. 92.

${ }^{176}$ Vgl. Krieg (1971), S. 73, sowie die dort angegebenen Quellen.

${ }^{177}$ Vgl. Krieg (1971), S. 74.

${ }^{178} \mathrm{Vgl}$. Mirow (1969), S. 94.

${ }^{179}$ Mirow (1969), S. 100.
} 
stützten Informationssystems der Unternehmung ansetzen. BIETHAHN/MUCKSCH/RUF, die $\mathrm{zu}$ den wenigen Autoren gehören, die sich im Rahmen von Arbeiten zum Informationsmanagement mit der Systemtheorie beschäftigen, nehmen an, die Voraussetzungen für eine erfolgreiche Steuerung seien höchstens bei technischen und weniger bei ökonomischen Prozessen gegeben. Deshalb sei die Steuerung auch bei der Gestaltung von betrieblichen Informationssystemen selten anwendbar. ${ }^{180}$ Tatsächlich wurde im vorangegangenen Abschnitt bereits herausgearbeitet, dass das technikunterstützte Informationssystem der Unternehmung als sozio-technisches System ein hohes Komplexitätsniveau und eine eingeschränkte Kausalität aufweist. $\mathrm{Zu}$ diesem System gehören allerdings Subsysteme, die allein aus technischen Elementen bestehen und durch ein weitaus höheres $\mathrm{Ma} ß$ an Kausalität bei geringerem Komplexitätsniveau gekennzeichnet sind.

Für diese technischen Teilsysteme des technikunterstützten Informationssystems der Unternehmung haben vorwärtsgekoppelte offene Steuerketten gute Erfolgschancen. Hier ist insbesondere an schwankende Auslastungen bei Systemen mit weitgehend homogenem Output, z.B. bei Datenbanksystemen oder Internet-Servern, zu denken, die durch geeignete Maßnahmen zu kompensieren sind. Steuerungsmaßnahmen des Informationsmanagements beziehen sich bei Datenbanksystemen beispielsweise auf die Vermeidung von Spitzenbelastungen durch die bedarfsweise Umwandlung von OnlineTransaktionen in Batch-Transaktionen während wenig genutzter Betriebszeiten oder auf die Bereitstellung zusätzlicher Großrechnerkapazitäten im Bedarfsfall. In ähnlicher Weise können vorübergehende Überlastungen der unternehmungseigenen Internetanbindungen durch den zeitweisen Zugriff auf öffentliche Provider ausgeglichen werden.

Auch bei sozio-technischen Anwendungssystemen als Subsystemen des technikunterstützten Informationssystems der Unternehmung, deren Varietät weitaus höher und deren interne Abläufe weitaus weniger überschaubar sind als die von rein technischen Systemen, können vorwärtsgekoppelte Steuerungsmaßnahmen die Stabilität des Systems erhöhen. Der Einsatz von inputorientierten Steuerungsmaßnahmen ist bei diesem Systemtyp dann erfolgversprechend, wenn die durch das Anwendungssystem abgewickelten Informationsverarbeitungsprozesse ein hohes Maß an Gleichförmigkeit aufweisen und der dabei produzierte Output weitgehend homogen ist. ${ }^{181}$ Ein Beispiel für derartige Maßnahmen ist die Anpassung der Arbeitszeiten an das in Abhängigkeit von der Tageszeit schwankende Anrufvolumen in einem Call Center. In einer Industrieunternehmung stellt die Einstellung von Zeitarbeitskräften zum Zwecke der Bearbeitung von Eingangsrechnungen mit gleichzeitiger hard- und softwaremäßiger Ausstattung der entsprechenden Arbeitsplätze in hochkonjunkturellen Phasen mit guter Auftrags- und Produktionslage ein Beispiel für derartige Maßnahmen dar.

Allerdings tritt bereits bei diesen einfachen Beispielen die genaue Erfassung der auf das System wirkenden Einflussgrößen aus der Umwelt des Systems als nur näherungsweise zu lösende Problemstellung auf. Zudem besteht auch in vergleichsweise wenig komplexen sozio-technischen Systemen keine funktional determinierte

\footnotetext{
${ }^{180} \mathrm{Vgl.} \mathrm{Biethahn/Mucksch/Ruf} \mathrm{(1994),} \mathrm{S.} 93$.

${ }^{181}$ Dies entspricht den Kriterien, die im dritten Kapitel im Abschnitt 3.3.2 für die Anwendbarkeit des Produktionsfunktionskonzeptes in Prozessen der Informationsverarbeitung angeführt wurden. Es handelt sich also um Routine- und Regelprozesse der Informationsverarbeitung.
} 
Abhängigkeit zwischen dem Input des Systems und dessen Output. Es ist deshalb ULRICH zuzustimmen, der feststellt, dass der Steuerung im System Unternehmung vornehmlich akzessorische Bedeutung in Verbindung mit dem nachfolgend erläuterten Prinzip der Regelung zukommt. ${ }^{182}$

\subsubsection{Aktive Bewältigung von Störungen durch Regelung}

Wie die Steuerung richtet sich auch die Regelung als spezieller Typ aktiver Maßnahmen zur Abwehr von Störungen eines Systems auf eine Erhöhung der Lenkungskapazität $H_{R}$. Im Gegensatz zur Steuerung ist die Regelung jedoch outputorientiert und wird durch eine Abweichung der Zielvariablen vom vorgegebenen Sollwert ausgelöst. ${ }^{183}$ Die Beeinflussung eines Systems durch Regelung basiert auf dem Prinzip der Rückkopplung, ${ }^{184}$ das heißt, es besteht ein geschlossener Kreislauf mit einer Wechselwirkung zwischen dem Ausgang des Systems und dessen Eingang. Dabei handelt es sich um die Form der negativen Rückkopplung, da Abweichungen des Ergebnisses vom Sollwert zu entgegengerichteten Anpassungsmaßnahmen des Reglers führen.

Damit Rückkopplung möglich wird, muss der durch das System produzierte Output laufend erfasst und mit dem vorgegebenen Soll-Wert verglichen werden ${ }^{185}$ Erst auf der Basis einer festgestellten Abweichung des Ist-Wertes vom Soll-Wert wird der Regler aktiv. Diese Form der Verhaltensbeeinflussung auf der Basis tatsächlich wirksam gewordener Störungen hat zur Folge, dass es grundsätzlich unmöglich ist, eine Varietät des Ergebnisses von Null zu erzielen, das System also frei von Abweichungen im Gleichgewicht zu halten (es sei denn, es wirken keinerlei Störungen auf das System ein). ${ }^{186}$ Dieser geringeren Präzision der Einflussnahme steht der Vorteil gegenüber, dass die zwecks Zielerreichung zu bewältigende Varietät erheblich geringer ist als beim Prinzip der vorwärtsgekoppelten Steuerung, da unterschiedliche Störungen aus der Systemumwelt häufig die gleichen Abweichungen des Systemoutput auslösen. ${ }^{187}$ Dadurch wird es in vielen Fällen möglich, mit einem relativ einfach konstruierten Regler von geringer Varietät eine große Vielfalt von Störungen zu bewältigen, deren Ursache nicht im Einzelnen bekannt sein muss. ${ }^{188}$

Die Vorgänge in einem geregelten System werden sehr häufig wie in Abbildung 4/6 in Form eines sogenannten Regelkreises dargestellt. ${ }^{189}$ Bei der Anwendung des reinen Regelungsprinzips kommt es zunächst zu einer unmittelbaren Wirkung der Störgröße auf die Regelstrecke. Die Regelstrecke produziert unter diesen Bedingungen ungelenkt den Systemoutput mittels der im System stattfindenden Prozesse. Dieser Systemoutput wird durch eine geeignete Messstelle erfasst und an den Regler gemeldet. Der Regler vergleicht die gemessene Ist-Größe mit der Soll-Größe und stellt gegebenenfalls eine

\footnotetext{
${ }^{182}$ Vgl. Ulrich (1970), S. 124.

${ }^{183}$ Vgl. Mirow (1969), S. 92.

${ }^{184}$ Zum Prinzip der Rückkopplung vgl. Beer (1967), S. 44ff.

${ }^{185}$ Vgl. Ulrich (1970), S. 122.

${ }^{186} \mathrm{Vgl}$. Mirow (1969), S. 97.

${ }^{187}$ Vgl. Krieg (1971), S. 75. In mathematischen Begriffen gesprochen handelt es sich dabei um eine mehr-eindeutige Abbildung des Syteminputs auf den -output.

${ }^{188} \mathrm{Vgl}$. Mirow (1969), S. 98.

${ }^{189}$ Vgl. z.B. Mirow (1969), S. 96; Ulrich (1970), S. 123; Krieg (1971), S. 75; Biethahn/Mucksch/Ruf (1994), S. 94
} 
Abweichung fest. Basierend auf dieser Abweichung verändert der Regler die Stellgröße, die anschließend gemeinsam mit der (möglicherweise inzwischen veränderten) Störgröße auf die Regelstrecke wirkt.

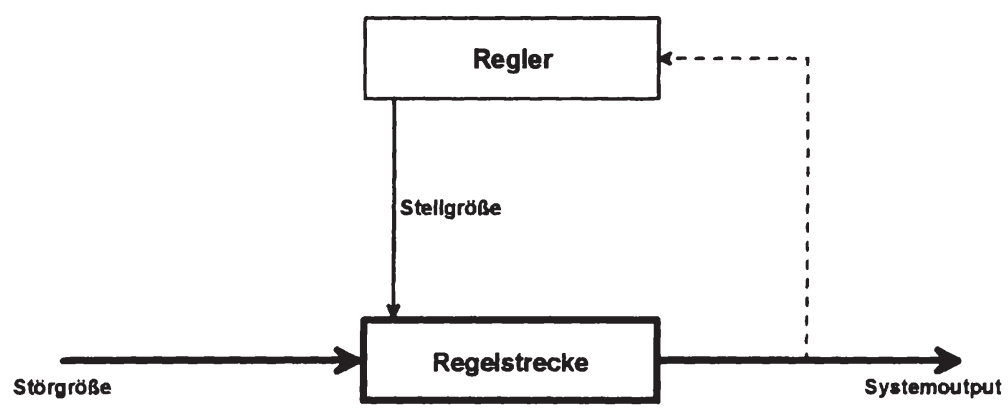

Abb. 4/6: Das Regelungsprinzip

Quelle: in Anlehnung an Baetge (1974), S. 27.

Die Anwendbarkeit des Regelungsprinzips in Unternehmungen als komplexen Systemen wird von den zum Bereich der Kybernetik I zu rechnenden Autoren als gegeben angesehen. Die Unternehmung wird als Regelsystem aufgefasst, das in die Unternehmungsumwelt eingebettet ist und auf Einflüsse aus dieser Umwelt reagieren muss. ${ }^{190}$ Mit Hilfe des Rückkopplungsprinzips wird es im Grundsatz für möglich gehalten, eine Unternehmung unter Kontrolle $\mathrm{zu}$ halten, auch wenn weder das Verhalten der Umwelt noch die internen Prozesse in der Unternehmung mit Sicherheit vorauszusehen sind. ${ }^{191}$ Allerdings erkennen auch bereits Vertreter der Kybernetik I, dass dem Verfahren der Regelung gewisse Instabilitätsgefahren innewohnen. Diese werden einerseits durch den Faktor Zeit begründet, da der gesamte Rückkopplungsvorgang so lange dauern kann, dass die Korrekturmaßnahmen zu spät erfolgen. ${ }^{192}$ Bei zyklischen Störungen kann die Schwingung des Systems in solchen Fällen verstärkt, statt gedämpft werden. Ferner steigen mit der Komplexität der Regelstrecke die Anforderungen an die Varietät des Regelsystems und damit die Gefahr des Versagens eines Regelkreises auf Grund zu geringer Lenkungskapazität.

Betrachtet man das technikunterstützte Informationssystem als sozio-technisches Subsystem, dessen Komplexität mit dem des übergeordneten Gesamtsystems Unternehmung vergleichbar ist, so können analoge Aussagen über die Relevanz des rückkopplungsgesteuerten Regelungsprinzips für das Informationsmanagement gemacht werden. Das Prinzip der Regelung stellt geringere Anforderungen an das lenkende System als das Prinzip der Steuerung. Die Abwehr von Störungen durch das Informationsmanagement wird daher durch die Konstruktion von Regelkreisen, deren Lenkungsmaßnahmen an der Erfassung und Auswertung der durch das technikunterstützte Informationssystem der Unternehmung produzierten Outputs ansetzen, erleichtert. Nicht nur rein technische Teilsysteme, sondern auch sozio-

\footnotetext{
${ }^{190}$ Vgl. z.B. Mirow (1969), S. 99; Ulrich (1970), S. 217ff.; Biethahn/Mucksch/Ruf (1994), S. 95.

${ }^{191}$ Vgl. Mirow (1969), S. 99.

${ }^{192}$ Vgl. dazu und zum Folgenden Ulrich (1970), S. 123f.
} 
technische Anwendungssysteme können dadurch besser unter Kontrolle gebracht werden.

Konkrete Ausprägungen von Regelsystemen im Bereich der Informationsverarbeitung können eine Vielzahl von Anwendungen im Bereich der Dispositions- und der Planungs- und Kontrollsysteme sein, bei denen bestimmte vorgegebene Zielgrößen einzuhalten sind. ${ }^{193}$ So handelt es sich beispielsweise bei der Durchlaufterminierung als Teil eines PPS-Systems um ein Regelsystem, das darauf abzielt, die Zahl der unmittelbar zur Produktion anstehenden Auftäge stets auf dem festgelegten Niveau zu halten. ${ }^{194}$ Ein Beispiel für ein echtes sozio-technisches Anwendungssystem als Regelkreis stellt die Bearbeitung von Routine- und Regelprozessen (in einer Versicherungsunternehmung etwa von einfachen Schadensmeldungen) dar, die fortlaufend durch ein Workflow Management-System als Regler überwacht und gelenkt wird. Bei der Gefahr der Überschreitung von Terminen erfolgt eine Warnmeldung durch das System, die zu einer beschleunigten Bearbeitung der jeweiligen Geschäftsvorfälle durch die Änderung der Prioritäten führt. Auch für rein technische Subsysteme des technikunterstützten Informationssystems der Unternehmung besitzt das Prinzip der rückwärtsgekoppelten Regelung hohe Relevanz, beispielsweise bei Großrechnern, bei denen die CPU-Auslastung fortlaufend gemessen und im Bedarfsfall durch Umschichtungsmaßnahmen des Betriebssystems reguliert wird.

Die oben genannten Grenzen des Regelungsprinzips gelten auch für die Anwendbarkeit im Bereich der Informationsverarbeitung. Die erforderliche Zeit für Gegenmaßnahmen durch den Regler kann zu lang sein, um Instabilitäten des Systems zu verhindern. Zudem ist die Varietät von sozio-technischen Anwendungssystemen so hoch, dass die Überforderung der Lenkungskapazität eines Reglers wahrscheinlich ist.

\subsubsection{Kombination verschiedener Maßnahmentypen zur Abwehr von Störungen}

Die bisher getrennt behandelten passiven und aktiven Einflussmöglichkeiten zur Abwehr von Störungen eines Systems können miteinander kombiniert werden, um die größtmögliche Lenkungskapazität zu erzielen. Die Kombination der Strukturprinzipien der Vorwärtskopplung, der negativen Rückkopplung sowie vorbeugender Maßnahmen zur Abschirmung des Systems wird als Mischlenkung bezeichnet. ${ }^{195}$ Bei der Mischlenkung kann die Bildung von Reserven als passive Maßnahme dazu dienen, Zeit zu gewinnen, bis aktive Maßnahmen zur Abwehr von Störungen einsetzen. ${ }^{196}$ Derartige Reserven dürfen nicht zu groß sein, damit die Reaktionsfähigkeit des Systems nicht übermäßig gemindert wird. Störungen, mit deren Auftreten gerechnet werden kann und deren Auswirkungen auf den Systemoutput bekannt sind, werden durch entgegengerichtete vorwärtsgekoppelte Steuerketten abgewehrt. Solche Steuerketten gewährleisten eine weitgehende Stabilität des Systemoutput, der zusätzlich durch rückwärtsgekoppelte Regelungsmaßnahmen gegen unvorhergesehene innere und äußere Störun-

\footnotetext{
${ }^{193} \mathrm{Vgl}$. Biethahn/Mucksch/Ruf (1994), S. 97.

${ }^{194}$ Dieses Beispiel nennen Biethahn/Mucksch/Ruf (1994), S. 96f. Genau genommen gehört die Regelstrecke dieses Systems allerdings nicht zum Bereich der Informationsverarbeitung, sondern zum Bereich der Fertigung, die mit Hilfe des PPS-Systems gelenkt wird.

${ }^{195}$ Vgl. Krieg (1971), S. 78.

${ }^{196}$ Vgl. zum Folgenden Mirow (1969), S. 100ff.
} 
gen abgesichert wird. Abbildung 4/7 verdeutlicht das Konzept der Mischlenkung für das Informationsmanagement.

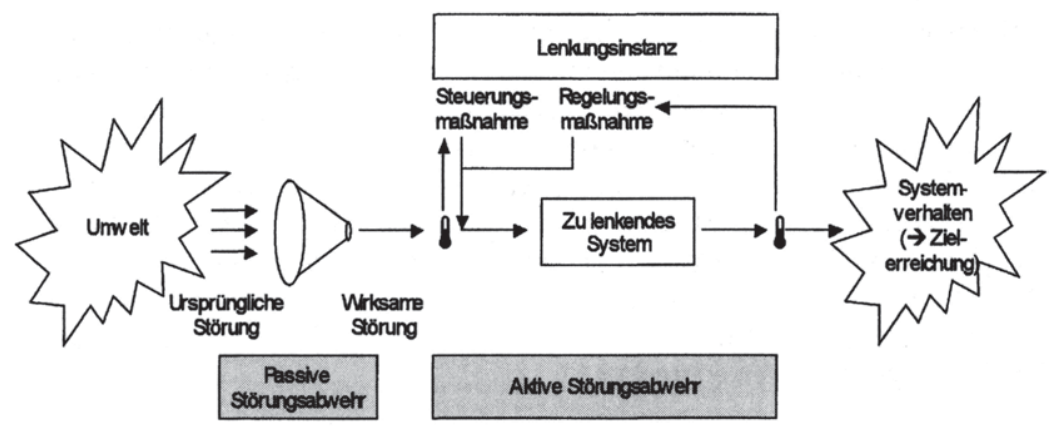

Abb. 4/7: Mischlenkung

In rein technischen Subsystemen des technikunterstützten Informationssystems der Unternehmung ist eine solche Mischlenkung beispielsweise für ein Datenbanksystem denkbar, das durch großzügig bemessene Kapazitäten gegen akute Überlastung, die zu nicht akzeptablen Antwortzeiten oder sogar zum völligen Ausfall des Systems führen kann, geschützt wird. Vorhersehbare Schwankungen der Systemauslastung werden durch die Verlagerung bestimmter Transaktionen in die Abend- und Nachtstunden als entgegengerichtete Steuerungsmaßnahme ausgeglichen. Zusätzlich zu diesen vorwärtsgekoppelten Maßnahmen wird die Auslastung des Systems durch einen Regelkreis überwacht. Bei einem unerwünschten Ansteigen der Antwortzeiten in Folge zu hoher Auslastung des Systems erfolgt schließlich eine Umleitung der Transaktionen auf dezentrale Datenbankserver und der Abgleich der Datenbestände zu einem späteren Zeitpunkt. Vorübergehend werden dabei Inkonsistenzen des zentralen und des dezentralen Datenbestandes in Kauf genommen.

Ein Beispiel für die Mischlenkung eines sozio-technischen Subsystems des technikunterstützten Informationssystems der Unternehmung ist ein Call-Center, in dem der After-Sales-Service einer Unternehmung mit Hilfe eines Help-Desk-Systems abgewickelt wird. Akute Schwankungen der Anrufzahl werden in einem solchen System durch die Einrichtung einer (nach Möglichkeit nicht zu langen) Warteschlange vom System ferngehalten, die als passive Abwehrmaßnahme aufgefasst werden kann. Vorhersehbaren Schwankungen der Anrufzahl zu unterschiedlichen Tageszeiten wird durch entsprechende Dienstpläne begegnet, die im vorliegenden Zusammenhang aktive Abwehrmaßnahmen in Form der vorwärtsgekoppelten Steuerung darstellen. Dieses Arrangement wird durch einen Regelkreis ergänzt, der dafür sorgt, dass eine Umleitung der Anrufe in die Vertriebsabteilung der Unternehmung erfolgt, falls die Warteschlange trotz der getroffenen Vorkehrungen das akzeptable Ausmaß überschreitet. 


\subsubsection{Subsystembildung, Ultra- und Multi-Stabilität}

Da der Stabilitätsbereich eines einfachen Regelkreises - selbst im Falle der Ergänzung durch Mischlenkungselemente - begrenzt ist, ${ }^{197}$ befasst sich die Kybernetik I auch mit Möglichkeiten zur Steigerung der Lenkungskapazität durch eine Erhöhung der Varietät des Verhaltens der Lenkungsinstanz. Dieses Ziel kann einerseits durch die Hintereinanderschaltung von (durch Mischlenkung ergänzten) Regelkreisen ${ }^{198}$ auf gleicher Ebene und andererseits durch Überlagerung solcher Regelkreise mit Lenkungssystemen höherer Ordnung erreicht werden. Der innere Aufbau eines komplexen Gesamtsystems ist also so zu gestalten, dass dieses aus mehreren, voneinander weitgehend getrennt operierenden Subsystemen auf gleicher Ebene besteht, die durch ein übergeordnetes Lenkungssystem miteinander verknüpft werden. Diese hierarchische Gliederung des Systems kann sich sowohl horizontal als auch vertikal über mehrere Stufen erstrecken.

Bei der horizontalen Hintereinanderschaltung handelt es sich um eine systemgestaltende Maßnahme, die mehrere Regelkreise in der Weise miteinander verknüpft, dass der Ausgang des einen jeweils den Eingang des nächsten darstellt. ${ }^{199} \mathrm{Da}$ sich die Varietät des Systemoutput aus der Differenz der auf ein System wirkenden Störungen und der Lenkungskapazität des Reglers ergibt, ergänzen sich in einer solchen Anordnung die Lenkungskapazitäten der Regler in den verschiedenen Subsystemen. Die gesamte Lenkungskapazität des Systems kann dadurch auf ein Vielfaches der Varietät der einzelnen Regler gesteigert werden. Während die Umwelteinflüsse auf die erste Stufe des Systems in vollem Umfang wirken, wird die Störvarietät innerhalb des Systems von Stufe zu Stufe verringert.

Dieses Konzept überzeugt zwar durch seine theoretische Klarheit, es kann für den Anwendungsbereich des Informationsmanagements allerdings lediglich gedanklich konstruiert werden. Die verschiedenen Subsysteme des technikunterstützten Informationssystems der Unternehmung können in der Realität nicht in der Weise errichtet werden, dass nur das jeweils erste einer in Reihe hintereinander geschalteter Teilsysteme in direktem Kontakt mit der Umwelt steht. Vielmehr verfugen die verschiedenen sozio-technischen Anwendungssysteme in einer Unternehmung allesamt über eigene Beziehungen zur Umwelt der Unternehmung. Darüber hinaus erfolgt der Austausch von Outputs zwischen den Anwendungssystemen, der im vorliegenden Zusammenhang als Störvarietät interpretiert werden kann, typischerweise in zweiseitiger Richtung, da die Systeme gegenseitig füreinander Leistungen erbringen. So erhält z.B. ein PPS-System Kundenauftragsdaten von einem Anwendungssystem im Bereich des Vertriebs, während es an dieses umgekehrt Daten über hergestellte und zur Auslieferung bereit stehende Produkte abgibt.

Das Prinzip der horizontalen Hintereinanderschaltung mehrerer Regelkreise ist trotz dieser fehlenden direkten Umsetzbarkeit in der Praxis von hoher Bedeutung für das Informationsmanagement, da damit nachgewiesen werden kann, dass die Bildung von weitgehend autonomen Subsystemen Komplexität reduziert und so die Lenkbarkeit

\footnotetext{
${ }^{197}$ Vgl. Ulrich (1970), S. 125.

${ }^{198}$ Im Folgenden werden diese durch Mischlenkung ergänzten Regelkreise aus Gründen der sprachlichen Vereinfachung nur noch als Regelkreise bezeichnet.

${ }^{199}$ Vgl. Mirow (1969), S. 127.
} 
des Gesamtsystems erhöht. Für eine effektive Komplexitätsreduktion sind bei diesem Vorgehen die Schnittstellen zwischen den verschiedenen Subsystemen des technikunterstützten Informationssystems der Unternehmung von entscheidender Bedeutung. ${ }^{200}$ Durch die Konstruktion geeigneter Schnittstellen können die verschiedenen Subsysteme voneinander getrennt und zugleich miteinander verbunden werden. Die Subsysteme können nur in der durch die Schnittstellen vorgesehenen Art und Weise Informationen austauschen. Dadurch werden die internen Prozesse und auch die spezifische Umwelt der jeweiligen Subsysteme für die benachbarten Subsysteme irrelevant. Die Störvarietät eines Subsystems, das aus der Perspektive eines anderen Subsystems zu dessen Umwelt zählt, wird so auf die Varietät der vordefinierten Schnittstelle reduziert.

Durch die vertikale Überlagerung mehrerer Regelkreise als systemgestaltende Maßnahme wird es möglich, dem System die Fähigkeit zur Reaktion auf größere Störungen zu verleihen, die die Lenkungskapazität des unmittelbar auf die Regelstrecke wirkenden Reglers übersteigen. ${ }^{201}$ Ein System, das durch zwei sich überlagernde Regelkreise gesteuert wird, wird als ultrastabiles System und die in diesem System stattfindende Regelung durch den höher gelagerten Regelkreis als Lenkung zweiter Ordnung bezeichnet. Durch die Lenkung zweiter Ordnung können neuartige, in ihrem Ausmaß nicht vorhergesehene oder das System als Ganzes betreffende Störungen bewältigt werden. Die Anpassung des Systems kann dabei entweder darin liegen, dass die Verhaltensweisen der Regelstrecke und die Regelungsvorgänge so lange geändert werden, bis die ursprünglich angestrebten Gleichgewichtszustände wieder realisiert werden, oder aber in einer Neubestimmung der anzustrebenden Zustände selbst.

Die erste Alternative stellt einen diskontinuierlichen Übergang zwischen unterschiedlichen Verhaltensweisen des Systems dar. ${ }^{202}$ Der Regler auf der untersten Systemebene versucht so lange, die Störung des Systems in der ursprünglich vorgesehenen Weise abzuwehren, bis ab einer gewissen Grenze die Regelung zweiter Ordnung einsetzt. Ab diesem Punkt bewirkt der Regler auf der nächsthöheren Ebene eine grundlegende Änderung des Systemverhaltens, die in einer Neuanordnung der Elemente und Beziehungen der Regelstrecke oder in neuen Vorgaben für das Verhalten des Reglers bestehen kann. Bei der zweiten Alternative werden die durch die Regelstrecke anzustrebenden Ziele neu definiert. ${ }^{203}$ Durch eine Anpassung der Ziele kann es gelingen, das System auf bleibende Veränderungen der Umwelt einzustellen, sodass das Überleben als oberstes Systemziel nicht gefährdet wird. Auf die Möglichkeit der Senkung der Anforderungen zur Erhöhung der Lenkbarkeit komplexer Systeme weist auch ASHBY hin, der allerdings der Ansicht ist, dass es sich hier um eine triviale Lösung des Problems handelt, die dennoch nicht außer Acht gelassen werden sollte. ${ }^{204}$

Lenkungsmaßnahmen zweiter Ordnung im Bereich des technikunterstützten Informationssystems der Unternehmung können sich beispielsweise auf die Anpassung technischer Kapazitäten (z.B. Übertragungskapazitäten von Netzwerken, Rechnerkapazitäten von Datenbankservern o.ä.) beziehen, wenn sich die verfügbaren Kapazi-

\footnotetext{
${ }^{200} \mathrm{Vgl}$. Biethahn/Mucksch/Ruf (1994), S. 97.

${ }^{201}$ Vgl. dazu Krieg (1971), S. 84.

${ }^{202}$ Vgl. Mirow (1969), S. 103; Krieg (1971), S. 82.

${ }^{203}$ Vgl. Ulrich (1970), S. 126; Mirow (1969), S. $105 f$.

${ }^{204}$ Vgl. Ashby (1974), S. 355f.
} 
täten dauerhaft als zu niedrig erweisen. Bei sozio-technischen Anwendungssystemen kommen neben technischen auch menschliche Arbeitskapazitäten als Objekt von Anpassungsmaßnahmen in Betracht. So kann die personelle Ausstattung von CallCentern zur Abwicklung des Vertriebs verbessert werden, wenn diese durch eine stetig steigende Zahl von Anrufen immer mehr überlastet werden. Hier sind auch technische Anpassungsmaßnahmen in Form von Zusatzprogrammierungen denkbar, wenn sich etwa herausstellt, dass regelmäßig bestimmte Kundenanfragen eingehen, die nicht standardisiert durch die verwendete Software beantwortet werden können. Bei diesen Fällen handelt es sich stets um diskontinuierliche Übergänge, die in Folge einer nachhaltigen Überforderung des Regelkreises erster Ordnung vorgenommen werden. Die Anpassung von Zielen als Lenkungsmaßnahme zweiter Ordnung kann im Bereich des Informationsmanagements beispielsweise in der Reduzierung des Anspruchsniveaus hinsichtlich der akzeptablen Antwortzeiten eines Datenbanksystems oder der Durchlaufzeiten von Aufträgen in einem Workflow-Management-System bestehen.

Durch die Kombination der vertikalen und der horizontalen Subsystembildung und die Ausstattung der einzelnen Subsysteme mit eigenen Regelkreisen wird ein Gesamtsystem konstruiert, das in der Lage ist, auf Störungen durch die partielle Anpassung von Gleichgewichtslagen zu reagieren. Dabei entsteht ein Netzwerk von Regelkreisen, das insgesamt letztendlich das Gesetz der erforderlichen Varietät erfuillen muss. ${ }^{205}$ Solche Systeme werden in der Kybernetik I als multistabile Systeme bezeichnet. ${ }^{206}$ Dabei verfolgt jedes Teilsystem ein Ziel, das als Unterziel des von der Gesamtunternehmung verfolgten Ziels (also des Überlebens der Unternehmung) angesehen werden kann. ${ }^{207}$ In der Kybernetik I wird dabei angenommen, dass es prinzipiell möglich sein müsste, die Unterziele so aufeinander abzustimmen, dass ein Optimum für das übergeordnete Gesamtziel erreicht wird, auch wenn es mit steigender Anzahl von Regelkreisen zunehmend schwierig wird, die einzelnen Unterziele in Bezug auf das Gesamtziel zu koordinieren. Die zu diesem Zweck genannten Instrumente (Sanktionen und Anreize) werden an dieser Stelle nicht näher behandelt, da sie nicht zum originären Forschungsgebiet der Kybernetik zählen.

Die Betrachtung multistabiler Systeme ist als Schnittstelle zwischen den beiden sich ergänzenden Forschungsrichtungen Kybernetik I und Kybernetik II zu sehen, da bei der Untersuchung multistabiler Systeme nicht mehr nur die Erreichung eines ex ante vordefinierten Gleichgewichtes, sondern auch die flexible Anpassung an sich ändernde Situationen in die Analyse einbezogen wird. Allerdings nehmen die der Kybernetik I zuzuordnenden Beiträge nach wie vor konstante Gebildestrukturen der Subsysteme und des Gesamtsystems an und betonen außerdem die Bewahrung des Gleichgewichts für das Gesamtsystem. Ferner analysieren sie die Anpassungsvorgänge der Subsysteme nicht im Einzelnen. An diesen Punkten setzen die Forschungsbestrebungen der nachfolgend für den Anwendungsbereich des Informationsmanagements aufgegriffenen Kybernetik II an.

\footnotetext{
${ }^{205}$ Vgl. Probst (1987), S. 39f.

${ }^{206}$ Vgl. Krieg (1971), S. 423.

${ }^{207}$ Vgl. Mirow (1969), S. 133.
} 


\subsubsection{Kybernetik II: Innovatives Informationsmanagement}

Entsprechend der Zweiteilung der Systemorientierten Betriebswirtschaftslehre ist dem an der Kybernetik I ausgerichteten konservativen Konzept des Informationsmanagements ein aus der Kybernetik II abzuleitendes innovatives Konzept gegenüberzustellen. Der in diesem Kapitel entwickelte Systemorientierte Ansatz für eine Theorie des Informationsmanagements ergibt sich in seiner Gesamtheit aus der Synthese dieser beiden sich ergänzenden Ansätze.

Im Folgenden (Abschnitt 4.3.2.1) wird zunächst die gegenüber der Kybernetik I veränderte Zielsetzung eines an der Kybernetik II orientierten Informationsmanagements beschrieben. Davon ausgehend wird in den Abschnitten 4.3.2.2 und 4.3.2.3 untersucht, welche Möglichkeiten das Informationsmanagement aus Sicht der Kybernetik II besitzt, um die Anpassungsfähigkeit des technikunterstützten Informationssystems der Unternehmung zu gewährleisten. Die Ausführungen richten sich zunächst auf die materielle Ebene des Informationsmanagements, die durch anschließend betrachtete Maßnahmen auf der symbolischen Ebene des Informationsmanagements zu ergänzen sind. Im Abschnitt 4.3.2.4 schließlich werden Möglichkeiten des Informationsmanagements zur Förderung von Anpassungsvorgängen erläutert.

\subsubsection{Geänderte Zielsetzung: Innovative Anpassungsfähigkeit statt konservativer Lenkung im Detail}

Im vorangegangenen Abschnitt wurde erläutert, über welche Möglichkeiten das Informationsmanagement verfügt, um das technikunterstützte Informationssystem der Unternehmung oder Teile davon mit Hilfe negativ rückgekoppelter (um Mischlenkungselemente erweiterter) Regelkreise trotz interner und externer Störeinflüsse im Gleichgewicht zu halten. Das Resultat einer derart konzipierten Lenkung ist im Erfolgsfall die Konstanz des Systemverhaltens in der gegenwärtig wahrgenommenen System- und Umweltsituation. Die Kritik, die aus der Perspektive der Kybernetik II gegen ein derartiges Konzept erhoben werden kann, richtet sich im Wesentlichen auf zwei Punkte, die für die insgesamt beschränkten Erkenntnismöglichkeiten der Kybernetik I stehen: Dabei handelt es sich zum einen um die fehlende Innovationsfähigkeit von regelkreisgesteuerten Systemen und zum anderen um die kritische Prämisse der Lenkbarkeit des komplexen Systems Unternehmung, auf der das aus der Kybernetik I abgeleitete Konzept basiert.

Aus der fortdauernden Bewahrung eines einmal erreichten Systemgleichgewichtes auf dem Wege der negativen Rückkopplung resultiert als Konsequenz die Unmöglichkeit der Innovation. ${ }^{208}$ Das dauerhafte Überleben des technikunterstützten Informationssystems der Unternehmung und somit auch des übergeordneten Gesamtsystems Unternehmung kann in einem solchen Falle nur basierend auf den bereits existierenden Fähigkeiten gewährleistet werden. Eine Anpassung des Systems an grundlegend veränderte Situationen ist nicht vorgesehen. In durch Wettbewerb mit konkurrierenden Systemen geprägten Umwelten muss die alleinige Orientierung am Status quo längerfristig gesehen nahezu zwangsläufig zu Fehlentwicklungen führen.

\footnotetext{
${ }^{208}$ Vgl. Parker/Stacey (1995), S. 62.
} 
Die Kybernetik II beschäftigt sich deshalb explizit mit der Anpassungsfähigkeit von Systemen an sich ändernde System/Umwelt-Konstellationen. Ausgangspunkt für diese Verschiebung des Erkenntnisinteresses auch im betriebswirtschaftlichen Zusammenhang ist die Feststellung, dass sich Unternehmungen als komplexe Systeme zunehmend dynamischeren Umwelten gegenübersehen, die durch schnelle, häufige und grundlegende Wandelprozesse gekennzeichnet sind. ${ }^{209}$ Es ist deshalb die Entwicklung einer Betrachtungsweise notwendig, die die Anpassung der Unternehmung und ihrer Subsysteme nicht als Ausnahme vom Regelfall der Stabilität, sondern als immer wieder erforderlichen Normalfall auffasst. Das technikunterstützte Informationssystem als Subsystem der Unternehmung steht zwar nicht in direkter Konkurrenz mit anderen Systemen in der Unternehmungsumwelt. Aus der hochdynamischen Wettbewerbssituation, in der sich eine Unternehmung als Ganzes befindet, können sich jedoch häufige Änderungen der Anforderungen an die Informationsverarbeitung ergeben, die jeweils Anpassungen des technikunterstützten Informationssystems der Unternehmung und/oder seiner Subsysteme notwendig machen. Das veränderte Erkenntnisinteresse der Kybernetik II besitzt deshalb auch für das Informationsmanagement Relevanz.

Die zweite wesentliche Kritikpunkt eines allein an den Erkenntnissen der Kybernetik I orientierten (Informations-) Managements läuft darauf hinaus, dass die Vorstellung der vollständigen Lenkbarkeit von sozialen bzw. sozio-technischen Systemen nicht realitätsgerecht ist. Vertreter der Kybernetik II vertreten die Auffassung, dass es sich bei den auf der Basis der Kybernetik I - wie auch den auf Basis der traditionellen Betriebswirtschaftslehre - gewonnenen Aussagen lediglich um realitätsferne gedankliche Konstrukte handelt. Diese Konstrukte können zwar definiert, diskutiert und beschrieben, nicht jedoch angewendet werden. ${ }^{210}$ MALIK bezeichnet Ansätze, die von dieser Grundvorstellung der Beherrschbarkeit des Gestaltungsgegenstandes ausgehen, als konstruktivistisch-technomorphen Theorietyp. ${ }^{211}$ In dieser Arbeit wird, wie in Kapitel 4.1.2 erläutert, die Bezeichnung „mechanistisch“ vorgezogen. Zur Begründung der für diesen Theorietyp aufgestellten Unmöglichkeitsbehauptung ist erneut auf das Gesetz der erforderlichen Varietät zurückzugreifen.

Um die richtigen Maßnahmen zur Beeinflussung des Systemverhaltens in der gewünschten Richtung ergreifen zu können, muss der Regler sowohl den aktuellen systeminternen Zustand, dessen Varietät oben als $\mathrm{H}_{\mathrm{SR}}$ bezeichnet wurde, als auch den Umweltzustand, dessen Varietät oben als $\mathrm{H}_{S}$ bezeichnet wurde, genau kennen und zudem in der Lage sein, seine Maßnahmen dem System mitzuteilen. ${ }^{212}$ Die Lenkungskapazität $\mathrm{H}_{R}$ des Reglers wird folglich nach oben hin durch seine Möglichkeit zur Wahrnehmung von Störungen und zur Mitteilung der Gegenmaßnahmen beschränkt. Aus diesen Überlegungen folgt, dass das Gesetz der erforderlichen Varietät dem Grad der erreichbaren Regelung eine absolute Grenze setzt, wenn die Kapazität des Reglers begrenzt ist. ${ }^{213}$ Wenn die System- und Umweltkomplexität im Vergleich zu der

\footnotetext{
${ }^{209} \mathrm{Vgl}$. Streubel (2000), S. 9lff., die darauf hinweist, dass das Phänomen des Wandels an sich nicht neu ist und bereits seit langem in der Betriebswirtschaftslehre diskutiert wird. Dennoch kann im Zeitablauf eine Verschärfung der Problematik für viele Unternehmungen festgestellt werden.

${ }^{210}$ Vgl. Malik (1992), S. 231; Lindblom (1959), S. 156.

${ }^{211}$ Vgl. Malik (1992), S. $37 \mathrm{f}$.

${ }^{212}$ Im Prinzip ist auch die Fähigkeit zur Durchsetzung der Maßnahmen erforderlich. Fragen der Durchsetzung von Managementmaßnahmen werden durch die Systemtheorie jedoch nicht behandelt.

${ }^{213}$ Vgl. Ashby (1974), S. 353.
} 
Kapazität des Reglers sehr hoch sind, dann kann der Regler also nur einen Bruchteil dessen erreichen, was er eigentlich zu erreichen anstrebt.

HAYEK argumentiert darüber hinaus, dass die Prognose zukünftiger Zustände komplexer Systeme ${ }^{214}$ selbst dann unmöglich ist, wenn die Wirkungszusammenhänge (innerhalb des betrachteten Systems) vollständig bekannt sind. ${ }^{215}$ Dies ist darauf zurückzuführen, dass trotz des Versuchs einer noch so genauen Erfassung der relevanten Einflussgrößen eine vollständige Kenntnis der Ausgangssituation nicht erreicht werden kann. ${ }^{216}$ Eine exakte Erfassung der Ausgangsgrößen ist jedoch notwendig, da kleinste Abweichungen der Ausgangssituation bei komplexen Systemen zu großen Veränderungen des Systemoutput führen können. Wenn, dieser Argumentation folgend, das Verhalten eines komplexen Systems nicht exakt prognostiziert werden kann, dann ist trotz der Kenntnis der internen Systemzusammenhänge keine exakte Lenkung des Systems durch geeignete Abwehrmaßnahmen gegen äußere und innere Störungen möglich.

Tatsächlich sind Unternehmungen als komplexe Systeme nicht nur einer sehr hohen Umweltvarietät ausgesetzt, sondern auch ihr interner Zustand verändert sich laufend und ist nur schwer beobachtbar, da er das Ergebnis der Geschichte des Systems ist. Diese Aussage triff auch auf das technikunterstützte Informationssystem als Subsystem der Unternehmung zu. Dessen Umweltkomplexität $\mathrm{H}_{\mathrm{R}}$ wird zwar durch die Einbettung in das übergeordnete Gesamtsystem reduziert, ${ }^{217}$ sie bleibt jedoch auf Grund der vielfältigen Kontakte dieses Systems mit seiner inneren ${ }^{218}$ und äußeren Umwelt sehr hoch. Darüber hinaus wurde oben bereits eine eingeschränkte Kausalität des technikunterstützten Informationssystems der Unternehmung, verbunden mit einer hohen systeminternen Störvarietät $\mathrm{H}_{\mathrm{SR}}$, festgestellt, da der Output des Systems von zahlreichen Interaktionen zwischen den beteiligten Individuen, die ihr Verhalten laufend ändern können, abhängig ist. ${ }^{219}$ Für eine erfolgreiche, präzise Lenkung des Systems müssten den Entscheidungsinstanzen, die in der Unternehmung die Aufgaben des Informationsmanagements erfüllen, alle diese Einflussgrößen sowie deren zukünftige Entwicklung bekannt sein. Die von Vertretern der Kybernetik II geäußerte Unmöglichkeitsbehauptung, dass die mechanistische Management-Konzeption zwar beschreibbar, aber nicht praktizierbar ist, ist daher auch für den Anwendungsbereich des Informationsmanagements als gerechtfertigt anzusehen. ${ }^{220}$

\footnotetext{
${ }^{214}$ Hayek selbst verwendet den Begriff ,komplexe Systeme“ nicht, sondern er wăhlt die Bezeichnung „komplexe Phänomene“ für seinen Untersuchungsgegenstand. Die Übertragung der Argumentation in die Sprache der Systemtheorie andert jedoch nichts am Inhalt der dargestellten Überlegung.

${ }^{215}$ Vgl. Hayek (1972), S. 29 f.

${ }^{216}$ Diese Gedankenführung von Hayek entspricht im Prinzip vollständig der Argumentationslinie von Ashby bei der Analyse des Gesetzes der erforderlichen Vielfalt, auch wenn beide Autoren in unterschiedlichen Begriffswelten argumentieren. Während Ashby auf die Übertragungskapazitat des Reglers beim Ausgleich von Stönungen als Eingangssignal abstellt, richtet sich Hayek auf die Unmöglichkeit der Erfassung von Einflussgrößen und die Prognose der zukünftigen Entwicklung. Das Ergebnis, dass eine vollständige Kenntnis aller wirkenden Variablen unmöglich ist, ist in beiden Fällen gleich.

${ }^{217}$ Vgl. dazu die Ausfuihrungen des Abschnittes 4.2.2

${ }^{218}$ Als innere Umwelt werden die Teile der Umwelt eines Subsystems bezeichnet, die als solche zum übergeordneten Gesamtsystem gehören.

${ }^{219} \mathrm{Vgl}$. dazu die Ausfuihrungen des Abschnittes 4.2.3.

${ }^{220}$ Aus diesen Überlegungen folgt auch, dass eine Verbesserung des Informationsstandes (z.B. durch den Einsatz moderner analyseorientierter Management Support Systeme), die die
} 
Aus den Vorbehalten gegenüber der Anwendbarkeit mechanistischer ManagementKonzepte und der Forderung nach innovativer Anpassungsfähigkeit als langfristiger Existenzvoraussetzung komplexer Systeme folgt eine veränderte Zielsetzung für das Informationsmanagement. Eine sinnvolle Zielsetzung bei der Lenkung komplexer Systeme kann sich nicht auf die konservativ ausgerichtete Lenkung des Systemverhaltens im Detail beziehen, sondern sie muss die Kanalisierung der Systemvarietät zum Gegenstand haben. ${ }^{221}$ Durch den Verzicht auf Detailbestimmungen wird der Gesamteinfluss der Lenkungsinstanz bei diesem Systemtyp nicht geringer, sondern im Gegenteil größer, ${ }^{222}$ da aktionistische Einflussnahmen ohne vollständige Kenntnis des System- und Umweltzustands häufig kontraproduktiv wirken und nicht das erreicht werden kann, was eigentlich beabsichtigt wird.

Es kommt also zu einer Abkehr von der voluntaristischen Vorstellung der vollständigen Beherrschbarkeit komplexer Systeme. ${ }^{223}$ Stattdessen erfolgt eine Hinwendung zu einer evolutionären Denkweise und somit eine innovative Ausrichtung der Lenkungsmaßnahmen. Aus der Sicht der Kybernetik II geht es darum, dem System langfristig Lebensfähigkeit ${ }^{224}$ zu verleihen, die durch die Anpassungsfähigkeit des Systems in sich wandelnden Situationen gewährleistet wird. Für das Informationsmanagement bedeutet das, dass das technikunterstützte Informationssystem der Unternehmung auf schnelle, häufige und grundlegende Änderungen vorzubereiten ist. ${ }^{225}$

\subsubsection{Förderung der Anpassungsfähigkeit des technikunterstützten Informationssystems der Unternehmung}

Im Folgenden wird untersucht, wie das Informationsmanagement durch Maßnahmen auf der materiellen Ebene die Anpassungsfähigkeit des technikunterstützten Informationssystems der Unternehmung gewährleisten kann.

\section{a) Komplexitätsbeherrschung durch Ordnung}

Die Überlegungen des vorangegangenen Abschnittes haben gezeigt, dass eine exakte Lenkung komplexer Systeme nicht möglich ist, da die Begrenztheit der Kenntnisse der Lenkungsinstanz über die Situation unvermeidlich und auch unaufhebbar ist. ${ }^{226}$ Rationale Entscheidungen im Sinne mechanistischer Konzepte können deshalb in Bezug auf komplexe Systeme nicht getroffen werden. Mechanistische Ansätze führen somit zu Empfehlungen, deren Umsetzung zwar in der Tat bessere Ergebnisse erzielen würde, die jedoch faktisch nicht realisierbar sind. ${ }^{227}$ Auf Grund dieses Befundes zielt das Lenkungskonzept der Kybernetik II bei komplexen Systemen nicht mehr wie das

Lenkungskapazität des Reglers vergrößert, das grundsätzliche Problem der unvollständigen

Lenkbarkeit auf Grund der Komplexität des Systems nicht überwinden kann.

${ }^{221}$ Vgl. Probst (1987), S. 113.

${ }^{222}$ Vgl. Malik (1992), S. 234.

${ }^{223}$ Kirsch bezeichnet diese Vorstellung als "Illusion der Machbarkeit“. Vgl. Kirsch (1992), S. 351.

${ }^{224}$ Vgl. Probst (1987), S. 115. Durch den Begriff der Lebensfahigkeit wird der in der Kybernetik I verwendete Begriff des Überlebens ersetzt, der aus der Sicht der Kybernetik II zu stark an kurzfristigen Reaktionen orientiert ist. Probst formuliert plakativ: „Überleben genügt nicht; langfristig muss ein System lebensfähig sein."

${ }^{225} \mathrm{Vgl}$. Streubel (2000), S. 96ff.

${ }^{226}$ Vgl. Malik (1992), S. 257.

${ }^{227}$ Vgl. Malik (1992), S. 255. 
der Kybernetik I auf die vollständige Beseitigung der Systemvarietät, sondern auf die Schaffung von Ordnung ab, innerhalb derer das System seine Varietät entfalten kann.

Als Ordnung wird ein Zustand oder Sachverhalt bezeichnet, der dadurch gekennzeichnet ist, dass eine Vielzahl von Elementen unterschiedlicher Art derart miteinander in Beziehung stehen, dass ein Beobachter auf Grund der Kenntnis eines räumlichen oder zeitlichen Teiles eines Ganzen in der Lage ist, korrekte Erwartungen bezüglich des Restes zu bilden, oder zumindest Erwartungen, welche eine gute Chance haben, sich als korrekt $\mathrm{zu}$ erweisen. ${ }^{228}$ Ordnung erlaubt es, etwas einzuordnen, zusammenzufügen, sich zurechtzufinden, Konsistenz und Kontinuität zu erfahren. ${ }^{229}$ Durch die Herstellung von Ordnung wird die potenzielle Komplexität des Systemverhaltens reduziert und das System auf diese Weise in eine bestimmte Richtung gelenkt. Die nachfolgenden Ausführungen erörtern die Frage, wie eine derartige Ordnung im Allgemeinen und für das technikunterstützte Informationssystem der Unternehmung im Besonderen geschaffen werden kann. Zu diesem Zweck werden verschiedene Arten der Ordnung bzw. der Ordnungsbildung unterschieden, die jeweils bestimmte Eigenschaften für die Lenkung komplexer Systeme aufweisen und deshalb gezielt eingesetzt werden können.

Den Ausgangspunkt der Analyse von Ordnung in der systemisch-evolutionären Betriebswirtschaftslehre stellen die Arbeiten von HAYEK dar, der zwischen bewusst geplanter Ordnung, die er mit dem griechischen Ausdruck „Taxis“ bezeichnet, und spontan entstandener, gewachsener Ordnung, die er mit dem Ausdruck „Kosmos“ bezeichnet, unterscheidet. ${ }^{230}$ Während bewusst geplante Ordnung im Sinne von „Taxis“ einem System exogen gegeben wird, entsteht spontane Ordnung im Sinne von "Kosmos" systemendogen von selbst durch Regelmäßigkeiten im Verhalten der Systemelemente. Bewusst geplante Ordnungen sind relativ simpel, da sie jeweils von einer einzigen Instanz geschaffen werden und somit deren Lenkungskapazität nicht übersteigen können. ${ }^{231}$ Spontane Ordnungen in sozialen Systemen sind dagegen das Ergebnis menschlichen Handelns, aber nicht menschlichen Entwurfs. ${ }^{232}$ Sie können im Prinzip jeden Komplexitätsgrad erreichen, da daran sehr viele Elemente mitwirken und somit ein erheblich höheres $\mathrm{Ma} ß$ an Wissen in ihre konkrete Ausgestaltung Eingang findet als dies bei bewusst geplanten Ordnungen möglich ist. Daraus wird gefolgert, dass spontane Ordnungskräfte eine um so größere Rolle spielen, je komplexer das betrachtete System ist. ${ }^{233}$ Durch bewusst geplante Ordnung kann die Bildung spontaner Ordnung angeregt werden, um das Systemverhalten in der gewünschten Richtung zu beeinflussen.

\section{b) Verlagerung des Informationsmanagements auf die Meta-Ebene}

Folgt man der vorangegangenen Argumentation, so geht es für die Lenkungsinstanz hier die zentrale Entscheidungsinstanz des Informationsmanagements - um die Schaffung von Rahmenbedingungen, die einen Verhaltensspielraum für das technikunterstützte Informationssystem der Unternehmung offen lassen, innerhalb dessen sich

\footnotetext{
${ }^{228}$ Vgl. Hayek (1969), S. 164; Hayek (1973), S. 36.

${ }^{229}$ Vgl. Probst (1987), S. 9.

${ }^{230}$ Vgl. Hayek (1969), S. 209.

${ }^{231}$ Vgl. Malik (1992), S. 216.

${ }^{232} \mathrm{Vgl}$. Hayek (1969), S. 97ff.

${ }^{233}$ Vgl. Hayek (1969), S. 216.
} 
das System selbstständig an neue Entwicklungen anpassen kann. Das Lenkungsproblem verschiebt sich damit von der konkreten Steuerungs- und Regelungsproblematik auf die Meta-Ebene. Aus dieser Perspektive stellt Informationsmanagement dann in erster Linie eine metasystemische Aktivität dar, ${ }^{234}$ die auf eine einzelfallbezogene Einflussnahme bei konkret vorliegenden Störungen des Systems verzichtet. Diese auf der Meta-Ebene angesiedelte indirekte Art der Systemlenkung kann vereinfacht dargestellt mit einem Vorgehen verglichen werden, bei dem gewissermaßen die Spielregeln für das Verhalten der Elemente definiert werden, ohne dieses Verhalten im Einzelnen zu bestimmen. ${ }^{235}$ Derartige Spielregeln stellen für das technikunterstützte Informationssystem der Unternehmung eine Ordnung her, ohne den informationellen Output des Systems vollständig festzulegen.

Dieser Verzicht auf Detailbestimmungen des Verhaltens, mit denen auf jede einzelne Störung reagiert wird, bedeutet eine Verlagerung der Maßnahmen der übergeordneten Lenkungsinstanz von der situativen auf die präsituative Regelungsebene. ${ }^{236}$ Präsituative Regelungsmaßnahmen stabilisieren ex ante die Aktivitäten innerhalb des Systems, indem sie eine Vereinheitlichung des Systemverhaltens beim Vorliegen bestimmter situativer Bedingungen bewirken. Da die auf komplexe Systeme einwirkenden Störungen im Einzelfall sehr unterschiedliche Erscheinungsformen annehmen können, besitzen präsituative Einflussnahmen allerdings nur dann Aussicht auf Erfolg, wenn sie sich auf bestimmte abstrakte Muster der System- und Umweltsituation beziehen, denen durch bestimmte, wiederum abstrakt formulierte Reaktionsmuster zu begegnen ist. Die situative Regelung durch die übergeordnete Lenkungsinstanz muss sich folglich auf die Schaffung von Kontexten richten, die den Akteuren innerhalb des Systems die Möglichkeit zur Orientierung bei der jeweils sehr unterschiedlich ausgestalteten Erfüllung ihrer Aufgaben im Einzelfall geben. Verhaltensregeln, die diesen Vorstellungen entsprechen, besitzen häufig negativen Charakter, indem sie nicht positiv vorgeben, was $\mathrm{zu}$ tun ist, sondern bestimmte Verhaltensweisen ausschließen. ${ }^{237}$ Die Aufgabenträger der Informationsverarbeitung verfügen bei dieser Art der Regelung über erhebliche Freiheitsgrade bei der Aufgabenerfüllung, da sie lediglich durch allgemeine Anhaltspunkte für ihre Aktivitäten beeinflusst werden, die ihnen einen vergleichsweise großen Handlungsspielraum offen lassen.

Auf diese Weise bringt das Informationsmanagement das technikunterstützte Informationssystem der Unternehmung auf einen Entwicklungspfad, ohne die Reaktion auf jede einzelne Störung des Systems vorwegzunehmen. Daraus ergibt sich die Notwendigkeit zur Selbstbeschränkung für das zentrale Informationsmanagement. Die direkte Einflussnahme auf die Aufgabenträger der Informationsverarbeitung durch eine zentrale Instanz im Einzelfall ist auf ein Minimum zu beschränken. Aufgabe des zentralen Informationsmanagements ist es aus dieser Perspektive stattdessen, das Verhalten der einzelnen Aufgabenträger für die übrigen beteiligten Akteure innerhalb des technikunterstützten Informationssystems der Unternehmung so weit berechenbar zu machen, dass diese verlässliche Verhaltenserwartungen bilden können, auf deren Basis sie ihre eigenen Aktivitäten ausüben können. Präsituative Regelungsmaßnahmen,

\footnotetext{
${ }^{234}$ Zum metasystemischen Charakter des Managements vgl. Malik (1992), S. 73.

${ }^{235}$ Vgl. Malik (1992), S. 216.

${ }^{236}$ Zur Unterscheidung situativer und präsituativer Regelung und zum Verhältnis dieser beiden

Regelungstypen vgl. Streubel (2000), S. 146ff., sowie die dort zitierten Quellen.

${ }^{237}$ Vgl. Malik (1992), S. 223.
} 
die diesen Zweck erfüllen, können in Form von Standards getroffen werden, die bei der Informationsverarbeitung in der Unternehmung einzuhalten sind. Derartige Standards können sich in technischer Hinsicht etwa auf die Verwendung bestimmter Betriebssysteme oder Übertragungsprotokolle, in fachlicher Hinsicht z.B. auf die Einhaltung von Reihenfolgen oder Bearbeitungshöchstdauern beziehen. Das Informationsmanagement setzt dadurch Restriktionen, die zwar nicht im Einzelnen präsituativ festlegen, was passieren wird, die jedoch Erwartungen darüber zulassen, was nicht passieren wird.

\section{c) Verstärkte Selbstregelung innerhalb des technikunterstützten Informations- systems der Unternehmung}

Die vorangegangenen Ausführungen haben dargelegt, dass sich eine zentrale Instanz als Aufgabenträger des Informationsmanagements auf abstrakte, präsituative Maßnahmen beschränken sollte, da sie im Vergleich zur Komplexität der Störungen des technikunterstützten Informationssystems der Unternehmung nur über eine geringe Lenkungskapazität verfuggt. Folgt man dieser Auffassung, so ergibt sich dementsprechend eine Verlagerung von Handlungs- und Entscheidungskompetenzen an die einzelnen Subsysteme bzw. Aufgabenträger innerhalb des technikunterstützten Informationssystems der Unternehmung, sodass es zu einem erhöhten Ausmaß an Selbstregelung innerhalb dieses Systems kommt. ${ }^{238}$ Das Informationsmanagement als Aufgabenfeld wird also aufgeteilt und teilweise dezentralisiert. Dabei ergibt sich zum einen die Frage, in welchem Umfang eine solche Dezentralisierung aus Sicht der Kybernetik II stattfinden sollte. Zum anderen ist furr die dezentrale Lenkungsinstanz das Verhältnis präsituativer und situativer Lenkungsmaßnahmen zu untersuchen, da die Konzentration auf präsituative Maßnahmen bisher nur für die zentrale Lenkungsinstanz begründet wurde.

Als erste Näherung bei der Beantwortung dieser Fragen kann der bereits angesprochene Vorschlag von MALIK aufgegriffen werden, dass die Lenkung komplexer Systeme durch eine Kombination von dezentraler Lösung auf der Objektebene bei zentraler Lenkung auf der Metaebene erfolgen sollte. ${ }^{239}$ Von der dezentralisierten Ausübung einzelfallbezogener Lenkungsaktivitäten, denen durch einen zentral hergestellten Kontext eine einheitliche Ausrichtung gegeben wird, wird die größtmögliche Ausschöpfung der Anpassungsfähigkeit und Flexibilität des Systems erwartet. Dabei kann jede Entscheidungsinstanz ihr unmittelbar vorliegendes Problem lösen, ohne die Vorgänge innerhalb der anderen Teile des Gesamtsystems im Einzelnen berücksichtigen zu müssen. ${ }^{240}$ Bei näherer Betrachtung ist eine starke Ähnlichkeit der Unterscheidungen zwischen Objekt- und Meta-Ebene sowie zwischen situativer und präsituativer Regelung festzustellen. Damit führen diese Überlegungen zu der Schlussfolgerung, dass die Lenkungskompetenz für situative Regelungsmaßnahmen generell dezentral angesiedelt werden sollte.

\footnotetext{
${ }^{238}$ Nach der relativen Position der Lenkungsinstanz und des zu lenkenden Systems können die Formen der Selbst- und der Fremdregelung unterschieden werden. Vgl. Streubel (2000), S. 152f. Als Fremdregelung wird dann die Form der Regelung bezeichnet, bei der die Person, die die Lenkungsaufgabe wahrnimmt, außerhalb des zu lenkenden (Sub-)Systems steht, wăhrend es sich bei der Selbstregelung um eine Form der Regelung handelt, bei der die lenkende Person Teil des zu lenkenden (Sub-)Systems ist.

${ }^{239}$ Vgl. Malik (1992), S. 240.

${ }^{240}$ Vgl. Malik (1992), S. 239.
} 
Allerdings bleibt dadurch ungeklärt, in welchen Fällen eine generelle präsituative Regelung gegenüber einer einzelfallbezogenen situativen Regelung vorteilhaft ist. Zudem ist vorstellbar, dass in bestimmten Fällen auch auf der Meta-Ebene dezentrale Regelungen Vorteile gegenüber zentralen Lösungen aufweisen. Auf diese Fragen gibt die Kybernetik II keine allgemeingültige Antwort, sondern sie liefert Tendenzaussagen, die bei der systemorientierten Lenkung von Unternehmungen im konkreten Anwendungsfall Anhaltspunkte für die Zuordnung von Kompetenzen geben können. ${ }^{241}$ Situative Regelungsmaßnahmen erweisen sich grundsätzlich als flexibler in dynamischen System- und Umweltsituationen, während präsituative Regelungsmaßnahmen zum einen Effizienzvorteile aufweisen, da sie über einen längeren Zeitraum gültig sind und zum anderen eine wichtige Stabilisierungsfunktion erfullen und das Verhalten des (Sub-) Systems sowohl für Außenstehende als auch für zum (Sub-) System gehörende Akteure besser vorhersehbar machen, also dessen Ordnung erhöhen. ${ }^{242}$

Präsituative fremdorganisatorische Maßnahmen können den Vorteil der höheren Methodenkompetenz und des besseren Überblickswissens bei der zentralen Entscheidungsinstanz haben. Zudem weisen sie möglicherweise eine höhere Transparenz für die nicht direkt betroffenen Subsysteme auf. Präsituative selbstorganisatorische Maßnahmen sind dagegen regelmäßig durch ein höheres Detailwissen der Entscheidungsinstanz, verbunden mit einer geringeren Tendenz zur Verharrung über die sinnvolle Geltungsdauer hinaus gekennzeichnet. ${ }^{243}$ Daraus kann die Empfehlung abgeleitet werden, dass fremdorganisatorische Maßnahmen immer dann tendenziell selbstorganisatorischen vorzuziehen sind, wenn die angestrebte Anpassung von Bedeutung für die Unternehmung als Ganzes ist. ${ }^{244}$ Umgekehrt sind selbstorganisatorische Maßnahmen dann vorteilhaft, wenn die Veränderungen die spezifische Situation eines einzelnen Subsystems betreffen. Diese abstrakten systemtheoretischen Überlegungen liefern im konkreten Anwendungsfall Denkanstöße und Argumentationshilfen, ohne die Entscheidung jedoch verbindlich festzulegen.

Die bisherige Argumentation betraf die Verteilung der Kompetenz zur Herstellung von Ordnung zwischen zwei Systemebenen. Sie ist grundsätzlich auf eine mehrstufige Betrachtung erweiterbar. Bei der Analyse mehrerer Systemebenen sollte aus der Perspektive der Kybernetik II die Kompetenz zur situativen Regelung stets auf der tiefstmöglichen Ebene, also möglichst nah bei den Aufgabenträgern der Informationsverarbeitung selbst, angesiedelt werden, da diese über die größtmöglichen Kenntnisse der im Einzelfall relevanten Einflussgrößen verfügen. Für die präsituative, generelle Regelung des Systemverhaltens begründen die vorangegangenen Ausführungen die Vorteilhaftigkeit des Subsidiaritätsprinzips: Demnach sollte die Regelungskompetenz so niedrig wie möglich in der (Sub-)Systemhierarchie verankert werden, damit einerseits die Lenkungskapazität der oberen Hierarchieebenen nicht überfordert wird, und andererseits in die jeweilige Maßnahme ein möglichst hohes Maß an Situationskenntnissen durch die Regelungsinstanz einfließt. Durch die Einhaltung des Subsidiaritätsprinzips wird ein möglichst hohes $\mathrm{Maß}$ an spontaner Ordnung

\footnotetext{
${ }^{241} \mathrm{Vgl}$. ăhnlich Streubel (2000), S. 157ff.

${ }^{242}$ Für eine ausführlichen Abwägung der präsituativen gegenüber der situativen Regelung vgl. Streubel (2000), S. 146ff.

${ }^{243}$ Für eine ausführliche Abwägung der Fremdorganisation gegenüber der Selbstorganisation vgl. Streubel (2000), S. 152ff.

${ }^{244}$ Vgl. Streubel (2000), S. 161.
} 
innerhalb des Systems erreicht, die entsprechend der eingangs erläuterten Ideen von HAYEK ein weitaus höheres Komplexitätsniveau bewältigen kann als die durch eine zentrale Instanz bewusst geplante Ordnung. ${ }^{245}$

Für die Verwirklichung des Subsidiaritätsprinzips ist eine möglichst umfassende Informationsversorgung der Entscheidungsträger auf der jeweils mit der Regelung betrauten Systemebene erforderlich. Eine zentrale Planung der Informationsversorgung für die dezentral angesiedelten Regelungsvorgänge ist dabei zum einen aus logischen, zum anderen aus epistemologischen Gründen abzulehnen. Zum einen erfolgt die Dezentralisierung der Regelungskompetenz aus der Sicht der Systemtheorie gerade deswegen, weil die dezentrale Instanz über bessere Kenntnisse des Entscheidungsfeldes verfügt als die zentrale Instanz. Die zentrale Festlegung der dezentralen Informationsversorgung wäre daher offensichtlich kontraproduktiv. Zum anderen spielen bei der Bestimmung des erforderlichen Informationsstandes auch Faktoren eine Rolle, die allein in der Person des Entscheidungsträgers begründet liegen. Der subjektive Informationsbedarf bei der dezentralen Regelung kann deshalb grundsätzlich der zentralen Instanz nicht im Einzelnen bekannt sein.

Es ist deshalb sinnvoll, dass die dezentralen Entscheidungsträger möglichst selbstständig darüber bestimmen, welche Informationen sie für eine erfolgreiche Regelung ihres Subsystems benötigen. In diesem Zusammenhang geben die von STREUBEL aus der Perspektive der Kybernetik II entwickelten „Gestaltungsempfehlungen für die immaterielle Ebene des computergestützten Informationssystems ${ }^{\text {‘246 }}$ Anhaltspunkte für Lenkungsmaßnahmen des Informationsmanagements auf der Meta-Ebene. Demnach sollte neben die Informationsversorgung oberer Hierarchieebenen des Systems die Fokussierung der Informationsversorgung der unteren Hierarchieebenen als weitere Gestaltungsperspektive treten. $\mathrm{Da}$ eine zentral ausgelöste Informationsversorgung der dezentralen Regelungsinstanzen (wie oben begründet) nicht sinnvoll ist, sollte die Informationsversorgung von Entscheidungsträgern insgesamt verstärkt am Holprinzip ausgerichtet werden. Zur Ermöglichung dieses Holprinzips ist ein unternehmungsweiter Informationspool einzurichten, auf den die Regelungsinstanzen zielgerichtet zugreifen können, wenn sie es für erforderlich halten. Durch die Einhaltung dieser Empfehlungen können Potenziale für ex ante nicht determinierte Regelungssituationen und damit Voraussetzungen für die Anpassungsfähigkeit des technikunterstützten Informationssystems der Unternehmung geschaffen werden. ${ }^{247}$

\footnotetext{
${ }^{245}$ In diesem Punkt ergeben sich allerdings begriffliche Probleme, da die Unterscheidung zwischen spontaner Ordnung und bewusst geplanter Ordnung durch Hayek, mit der die Dezentralisierung der Ordnungsbildung eingangs begründet wurde, zugleich auf das Kriterium des Bewusstseins bei der Ordnungsbildung und auf das Kriterium der Systemexogenităt abstellt. Eine bewusst geschaffene präsituative Ordnungsbildung durch die Akteure innerhalb des betroffenen Subsystems ist somit weder eindeutig als Ordnung im Sinne von „Kosmos" noch im Sinne von „Taxis" einzuordnen. Dennoch kann angenommen werden, dass die durch dezentrale Instanzen bewusst geplante Ordnung ein weitaus höheres $\mathrm{Maß}$ an Komplexităt erreichen kann als die zentrale, bewusst geplante Ordnung, da weitaus mehr Personen an der Bildung dieses Ordnungstyps beteiligt sind, sodass die relativen Vorteile der Dezentralisierung erhalten bleiben.

${ }^{246} \mathrm{Vgl}$. Streubel (2000), S. 320.

${ }^{247}$ Vgl. ahnlich Streubel (2000), S. 317.
} 


\subsubsection{Symbolische Ebene des Informationsmanagements}

Die vorangegangenen Überlegungen haben begründet, dass ein verstärktes Ausmaß an Selbstregelung erforderlich ist, um der Komplexität des technikunterstützten Informationssystems der Unternehmung eine möglichst hohe Lenkungskapazität gegenüberzustellen und auf diese Weise die Lenkbarkeit des Systems zu erhöhen. Durch die Verlagerung von Entscheidungskompetenzen auf eine Vielzahl dezentraler Instanzen entsteht allerdings die Möglichkeit, dass die dezentralen Instanzen (beabsichtigt oder unbeabsichtigt) Entscheidungen treffen, die den Gesamtzielen des Systems abträglich sind. Entsprechend der systemtheoretischen Grundannahmen ist dies durch die zentrale Instanz nicht unmittelbar erkennbar, da die Dezentralisierung von Entscheidungskompetenzen gerade auf Grund von Informationsunvollkommenheiten vorgenommen wird. Durch ein erhöhtes Ausmaß an Selbstregelung können deshalb zentrifugale Tendenzen entstehen, die den inneren Zusammenhalt des Systems gegenüber seiner Umwelt reduzieren und damit das langfristige Überleben des Systems gefährden.

\section{a) Vermittlung von Sinn als Ziel des Informationsmanagements auf der symbo- lischen Ebene}

Um diesen Selbstauflösungstendenzen entgegenzuwirken, ist es erforderlich, die im vorangegangenen Abschnitt erläuterten Maßnahmen, die auf der substanziellen Ebene angesiedelt sind, da sie konkrete Vorgaben über das Lenkungsverhalten innerhalb des Systems machen, durch eine symbolische Ebene des (Informations-) Managements zu ergänzen. Auf dieser symbolischen Ebene zielt die zentrale Lenkungsinstanz darauf ab, den dezentralen Entscheidungsträgern Orientierungen $\mathrm{zu}$ geben, durch die ein Auseinanderdriften der einzelnen Subsysteme verhindert wird. Symbolisches Management stellt in diesem Zusammenhang den Versuch des gezielten Aufbaus, der Pflege und der Vermittlung von Sinngemeinschaft durch bewusst gewählte und erfolgsrational eingesetzte Formen symbolischer Interaktion in der Unternehmung und ihren Subsystemen dar. ${ }^{248}$ Durch die explizite Berücksichtigung von „weichen“ Aspekten werden in diesem Rahmen Problembereiche thematisiert, die bis dahin in der betriebswirtschaftlichen Diskussion allenfalls am Rande Erwähnung gefunden haben. ${ }^{249}$ Symbolische Strukturen schaffen in der äußerst komplexen Realität Ordnung und erhöhen die Erwartbarkeit des Systemverhaltens. ${ }^{250}$

Die symbolische Ebene des Managements entfaltet eine legitimierende und orientierende Wirkung durch sinnmachende Prozesse ${ }^{251}$ und gibt dadurch den Maßnahmen auf der substanziellen Ebene eine Richtung. Durch die Vermittlung von Sinn werden die Handlungen von Menschen bedeutsam gemacht und können zur Identitätsfindung und zur Gemeinschaft mit anderen beitragen. ${ }^{252}$ Dadurch wird es möglich, die Zweckhaftigkeit von Handlungen zu erfassen und deren finalen Wert zu ermessen, ${ }^{253}$ sodass ein Maßstab bei der Beurteilung von Handlungsalternativen geschaffen wird. Die Vermittlung dieses Maßstabs erfolgt über Symbole, bei denen es sich um Zeichen

\footnotetext{
${ }^{248} \mathrm{Vgl}$. Ulrich (1990), S. 289.

${ }^{249}$ Vgl. Heinen (1987), S. $12 \mathrm{f}$.

${ }^{250}$ Vgl. ähnlich Ulrich (1990), S. 287; Probst (1987), S. 111.

${ }^{251}$ Vgl. Probst (1987), S. 97.

${ }^{252}$ Vgl. Ulrich (1990), S. 284.

${ }^{253}$ Vgl. Nagel (1995), S. 98.
} 
mit Bedeutungsinhalten handelt, die über ein bloßes denotatives Verständnis hinaus komplexere Kommunikationsinhalte vermitteln können. ${ }^{254}$ Symbole bringen „Ordnung ins Chaos der Komplexität “255 und schaffen überindividuell geteilten Sinn, da sie in erfahrungsreiche und bedeutungsschwere intersubjektive Zusammenhänge verwoben sind, die mit ihnen assoziiert werden. ${ }^{256}$ Aktivitäten des Managements auf der symbolischen Ebene verleihen den Handlungen der zu Führenden für diese selbst Sinn und Bedeutung. Sie geben Anhaltspunkte, welche Alternativen bei der Auswahl zwischen unterschiedlichen Entscheidungsmöglichkeiten durch die Gemeinschaft bevorzugt werden. Dem symbolischen Management kommt also eine „interpretative“ Funktion zu, da durch die Gestaltung geistig-sinnhafter Prozesse für die Mitarbeiter eines sozialen Systems Ungewissheit, Komplexität und Unsicherheit reduziert wird und das Geschehen sinnvoll erlebbar gemacht wird. ${ }^{257}$

Die symbolische Ebene des Managements ist in der Betriebswirtschaftslehre im Allgemeinen und der Systemorientierten Betriebswirtschaftslehre im Besonderen etwa seit Beginn der 80er Jahre in den Blickpunkt der Aufmerksamkeit gerückt und wird seitdem unter dem Stichwort Unternehmenskultur diskutiert. Durch die Betrachtung der Unternehmenskultur soll die Wirkung von Symbolen auf das Geschehen in Unternehmungen stärker hervorgehoben werden, deren Bedeutung in der bis dahin vorliegenden organisationstheoretischen Literatur weitgehend verkannt oder zumindest nicht in ausreichendem Maße gewürdigt wurde. ${ }^{258}$ Während die Relevanz symbolischer Aspekte für das Verhalten der Unternehmungsmitglieder inzwischen allerdings allgemein anerkannt ist, bleibt die Gestaltbarkeit einer Unternehmung und ihrer Subsysteme auf der symbolischen Ebene durch unternehmenskulturelle Maßnahmen umstritten. ${ }^{259}$ Im Prinzip stehen sich dabei zwei Grundpositionen gegenüber, die im Folgenden kurz umrissen werden, um davon ausgehend aus systemorientierter Perspektive zu einer realistischen Einschätzung der Einflussmöglichkeiten des Informationsmanagements auf der symbolischen Ebene zu gelangen.

Von weiten Teilen der deutschsprachigen Betriebswirtschaftslehre und auch von vielen Vertretern des Systemorientierten Ansatzes wird häufig eine relativ unkritische Sichtweise verfolgt, ${ }^{260}$ die die Unternehmungskultur als neu entdeckte weitere Variable der Unternehmungsgestaltung auffasst. Diese Position kann mit der Aussage „Die Unternehmung hat eine Kultur" treffend charakterisiert werden. Unternehmungskultur wird dann als empirisches Phänomen gesehen, dessen konkrete Erscheinungsform (Ist-Kultur) im Einzelfall erfasst werden kann und für das ein Ziel (in Form einer

\footnotetext{
${ }^{254}$ Vgl. Dill (1987), S. 70f.; Gussmann/Breit (1987), S. 109.

${ }^{255}$ Ulrich (1990), S. 284.

${ }^{256}$ Vgl. Ulrich (1990), S. 283.

${ }^{257}$ Vgl. Kasper (1990), S. 24.

${ }^{258}$ Vgl. Heinen (1987), S. 14f. Auf die in der Literatur vorzufindende Fülle unterschiedlicher Definitionsversuche zur Abgrenzung des Unternehmenskulturphänomens, für die bisher kein Konsens gefunden werden konnte, wird hier nicht eingegangen, da eine breit angelegte begriffliche Auseinandersetzung mit dem Phänomen der Unternehmenskultur für die vorliegenden Zwecke eines systemorientierten Ansatzes des Informationsmanagements nicht zielführend wäre. Für einen Überblick über verschiedene Definitionsansätze vgl. z.B. Drepper (1992), S. 23ff.; Nagel (1995), S. $16 \mathrm{ff}$.

${ }^{259}$ Für einen Überblick über die unterschiedlichen Positionen in diesem Punkt vgl. z.B. Heinen (1987), S. 15ff.; Ochsenbauer/Klofat (1987), S. 87ff.; Sackmann (1990), S. 155ff; Drepper (1992), S. $75 \mathrm{ff}$.; Nagel (1995), S. 20ff.

${ }^{260} \mathrm{Vgl}$. Heinen (1987), S. 16.
} 
Soll-Kultur) festgelegt und durch geeignete Maßnahmen des Managements auch tatsächlich erreicht werden kann. ${ }^{261}$ Eine gut funktionierende Unternehmungskultur wirkt aus dieser Perspektive gewissermaßen als "Autopilot" für die implizite Verhaltenssteuerung der Unternehmung als sozio-technischem System. ${ }^{262}$ Die Gefahr bei dieser Variante des Symbolischen Managements liegt allerdings darin, dass die sozial-technologische Vorstellung der vollständigen zentralen Lenkbarkeit des Systems Unternehmung und seiner Subsysteme nicht überwunden, sondern im Gegenteil bis ins Äußerste gesteigert wird. Es kommt dann zum Versuch eines „zweckrationalen Umgangs mit dem Vorrationalen “263.

Die konträr entgegengesetzte Position verwendet den Kulturbegriff als erkenntnisleitenden Grundbegriff (,root metaphor") und begreift Unternehmungen selbst als Kulturen: ${ }^{264}$ Die Unternehmung ist in diesem Fall eine Kultur. Dabei wird eine Unternehmung gleichsam als "Miniaturgesellschaft ${ }^{\text {265 }}$ aufgefasst und eine metaphorische Verwendung des Kulturbegriffs vorgenommen, um Erkenntnisse der ethnologischen und der anthropologischen Kulturforschung für das bessere Verständnis von Unternehmungen nutzbar zu machen. ${ }^{266}$ Bei dieser Betrachtungsweise liegt der Fokus auf den Prozessen, die die kulturelle Wirklichkeit einer Unternehmung ausmachen. Die Kultur wird hier als ein Medium betrachtet, in dem Bedeutungen permanent konstruiert, benutzt und auch geändert werden. ${ }^{267}$ Die Möglichkeiten einer geplanten Kulturgestaltung werden innerhalb dieses Ansatzes nicht diskutiert, da die Vertreter primär an einem besseren Verständnis von Organisationen (bzw. Unternehmungen) und zusätzlichen Erkenntnissen mit Hilfe der Kulturmetapher interessiert sind. ${ }^{268}$ Die Frage der Machbarkeit von Kulturen passt nicht zur subjektivistisch-interpretativen Ausrichtung dieses Forschungsprogramms, für das die teilnehmende Beobachtung die bedeutendste Forschungsmethode darstellt. Gerade diese Frage der Gestaltbarkeit von Kultur besitzt in einem pragmatisch orientierten Wissenschaftsprogramm wie der Systemorientierten Betriebswirtschaftslehre jedoch erhöhte Relevanz. Deshalb wird hier einer dritten, als dynamische Perspektive bezeichneten Variante gefolgt, die auf eine Synthese der zuvor beschriebenen Variablen- mit der Metapher-Position abzielt. Kultur wird dabei als dynamisches Konstrukt aufgefasst und Unternehmungen als sich entwickelnde Kultursysteme mit den ihnen eigenen ideellen und materiellen Wirklichkeiten gesehen. ${ }^{269}$ Unternehmungen sind aus diesem Blickwinkel Kulturen und haben zugleich kulturelle Aspekte. ${ }^{270}$

${ }^{261}$ Stellvertretend für diese Position vgl. Rühli (1990), S. 189ff.; Staerkle (1985), S. 529ff.; Matenaar (1983) passim.

${ }^{262}$ Bleicher (1986), S. 38.

${ }^{263}$ Ulrich (1990), S. 290.

${ }^{264} \mathrm{Vgl}$. Heinen (1987), S. 17f.

${ }^{265}$ Nagel (1995), S. 24.

${ }^{266}$ Für einen Überblick über Ansätze in diesem Bereich vgl. Dormayer/Kettern (1987), S. 49ff.;

${ }^{267}$ Vgl. Sackmann (1990), S. 161.

${ }^{268}$ Vgl. Sackmann (1990), S. 162.

${ }^{269}$ Vgl. Sackmann (1990), S. 162; Nagel (1995), S. 28. Ähnlich auch Klimecki/Probst (1990), S. 42ff.; Ochsenbauer/Klofat (1987), S. 96ff.; Dill/Hügler (1987), S. 145.

${ }^{270}$ Die dynamische Perspektive vermeidet Inkonsistenzen gegenüber der sonst in dieser Arbeit $\mathrm{zu}$ Grunde gelegten Sichtweise, dass das System Unternehmung aus den Elementarten Mensch und Technik besteht. Die Kultur stellt dabei einen Untersuchungsaspekt neben vielen anderen dar, unter dem das sozio-technische System Unternehmung betrachtet werden kann. 
Die Verknüpfung dieser beiden Ansätze entsteht daraus, dass Kultur, die sich aus der menschlichen Aktion und Interaktion heraus entwickelt und teilweise sichtbar oder spürbar, also teilweise materialisiert ist (Variablen-Perspektive), auch als ideelles System existiert (Metaphern-Perspektive) und als solches wiederum gewisse Funktionen erfullt (Variablen-Perspektive) ${ }^{271}$ Dieser doppelsinnige Charakter hat zur Folge, dass Gestaltungsempfehlungen nur mit großer Vorsicht und ohne die Erwartung, das Systemverhalten dadurch exakt lenken zu können, abgegeben werden können. ${ }^{272}$ Die Unternehmungskultur wird als eine Interpretation der Unternehmungsmitglieder aufgefasst (Metaphern-Perspektive). Jedoch können gezielt Interpretationen eingebracht werden, um die Kultur zu gestalten, und der gemeinsame Sinn kann auch funktional im Sinne des Managements sein (Variablen-Perspektive). ${ }^{273}$ An die Stelle eines Kulturmanagements tritt hier ein kulturbewusstes Management, ${ }^{274}$ das nicht von einer beliebigen (sozialtechnischen) Manipulierbarkeit der Kultur ausgeht, sondern von der Omnipräsenz der Unternehmungskultur, auf die durch Maßnahmen des Managements in begrenztem Maße Einfluss genommen werden kann. Kultur bleibt somit auch bei Nicht-Verfolgung der reinen Variablen-Perspektive ein Bezugsgegenstand von Aktivitäten des Managements. ${ }^{275}$

\section{b) Funktionen der Kultur und Symbole als Verkörperungen kultureller Inhalte}

Die Unternehmungskultur ist „inhärent funktional ${ }^{6276}$, das heißt, sie erfüllt sowohl für die Mitarbeiter als Individuen als auch für die Unternehmung als Ganzes bestimmte Funktionen. Für das einzelne Individuum liegen die wesentlichen Funktionen in der Reduktion der Umweltkomplexität und in einer Reduktion der Unsicherheit. ${ }^{277}$ Dem einzelnen Mitarbeiter wird durch die Unternehmungskultur ein fundamentaler Bezugsrahmen gegeben, der eine Selektion seiner Wahrnehmung bewirkt, die auf Grund der Vielzahl der auf das Individuum wirkenden Reize notwendig ist, und die emotionale Unsicherheit in neu eingetretenen Situationen verringert. Daneben vermittelt die Unternehmungskultur den Individuen Sinn, der sie die Wirklichkeit der Unternehmung wahrnehmen lässt und die Zweckhaftigkeit ihrer Handlungen verstehen lässt. ${ }^{278}$

Auf der überindividuellen Ebene bewirkt die Unternehmungskultur eine gleiche oder ähnliche Betrachtungsweise der Mitarbeiter. Zentrale Auswirkungen dieser Angleichung im Denken liegen im Bereich der Koordination, der Integration und der Motivation. ${ }^{279}$ Vor dem Hintergrund der im vorigen Abschnitt systemtheoretisch begründeten Notwendigkeit zur Dezentralisierung von Entscheidungskompetenzen sind hier insbesondere die Koordinations- und die Integrationsfunktion der Unternehmungskultur von Bedeutung. Koordination stellt das Komplement der sich aus der Systemdifferenzierung ergebenden Subsystembildung dar. ${ }^{280}$ Unternehmungskultur

\footnotetext{
${ }^{271}$ Vgl. Nagel (1995), S. 28.

${ }^{272}$ Vgl. Dill/Hügler (1987), S. 145.

${ }^{273}$ Vgl. Nagel (1995), S. 28.

${ }^{274}$ Vgl. Dill/Hügler (1987), S. 181; Ulrich (1990), S. 292.

${ }^{275}$ Vgl. ăhnlich Klimecki/Probst (1990), S. 58.

${ }^{276}$ Sackmann (1990), S. 164.

${ }^{277}$ Vgl. Nagel (1995), S. 97.

${ }^{278}$ Vgl. Nagel (1995), S. 98.

${ }^{279}$ Vgl. Dill/Hügler (1987), S. 147.

${ }^{280}$ Vgl. Dill/Hügler (1987), S. 147.
} 
ergänzt die auf der substanziellen Ebene des Managements angesiedelten formalen Koordinationsinstrumente auf der immateriellen Ebene, ${ }^{281}$ indem sie einen tragfähigen Basiskonsens herstellt und damit ein kommunikatives Verständigungspotenzial schaff, auf dessen Grundlage auch in Konfliktsituationen zwischen den einzelnen Subsystemen ein Konsens zwischen den einzelnen Beteiligten möglich wird. ${ }^{282}$ Neben der Koordination stellt auch die Integration aus systemtheoretischer Sicht eine entscheidende Funktion der Unternehmungskultur dar. ${ }^{283}$ Die Notwendigkeit zur Integration ergibt sich ebenfalls aus der Subsystembildung und der Dezentralisierung von Entscheidungskompetenzen. Der durch die Unternehmungskultur geschaffene Grundkonsens fördert den Zusammenhalt der einzelnen Subsysteme und verringert die Gefahr, dass sich einzelne Subsysteme verselbstständigen und es zu einem Auseinanderdriften der Bestandteile des Systems kommt. Der Unternehmungskultur wird schließlich auch motivationaler Charakter zugeschrieben, wenn die vermittelten Ziele und Perspektiven zu Engagement führen, wenn sie also von den Mitarbeitern als sinnvoll interpretiert werden und diese sich in hohem Maße damit identifizieren können. $^{284}$

Von Vertretern der Variablen-Perspektive der Unternehmungskultur werden verschiedene Typen von Symbolen genannt, die die Elemente der Unternehmungskultur und somit den Gegenstand von Gestaltungsmaßnahmen darstellen. Aus der dynamischen Perspektive handelt es sich bei solchen Symbolen allerdings nur um Materialisierungen der Kultur, die deren Inhalte als Bedeutungs- oder Sinnzuschreibungen transportieren, während die Unternehmungskultur selbst sich allein in den Köpfen der Mitarbeiter befindet. ${ }^{285}$ Auch in diesem Fall stellen die Symbole als Medien der Unternehmungskultur jedoch Ansatzpunkte für beeinflussende Maßnahmen durch das Management dar und werden deshalb nachfolgend kurz erörtert.

Wenn auch im Einzelnen unterschiedliche Begriffe gewählt werden, so werden doch im Wesentlichen drei Arten von Symbolen als Medien der Unternehmungskultur unterschieden. Dabei handelt es sich um Sprache, Handlungen und um materielle Objekte als Träger symbolischer Bedeutungsinhalte. ${ }^{286}$ Zentrales und bedeutendstes Medium zur Kommunikation unternehmenskultureller Werte und Normen ist die Sprache. $^{287}$ Sprachliche Symbole bieten die Möglichkeit einer Transformation von schwer fassbaren Bedeutungsinhalten in gemeinsames Wissen. Die innersystemische Kohäsionskraft einer gemeinsamen Sprache bewirkt auch eine trennende Funktion

\footnotetext{
${ }^{281}$ Formale Koordinationsinstrumente sind persőnliche Weisungen, Programme, Pläne und Selbstabstimmung. Vg1. Kieser/Kubicek (1992), S. 95; 103ff. Die Unternehmungskultur selbst sollte nicht als Koordinationsinstrument bezeichnet werden, da es sonst zu einer zu starken begrifflichen Nähe zur instrumentellen Verwendung der Kultur als Variablen kommt. Besser ist es deshalb in diesem Zusammenhang, von der Unternehmungskultur als informalem Koordinationsmedium zu sprechen.

${ }^{282} \mathrm{Vgl}$. Dill/Hügler (1987), S. 150f.

${ }^{283}$ Im Sinne der wörtlichen Übersetzung von Integration als (Wieder-) Herstellung eines Ganzen wird die Integrationsfunktion hier als Gewährleistung der Einheit des Systems trotz (mehrstufiger) Untergliederung des Systems Unternehmung in weitgehend selbstständig arbeitende Subsysteme verstanden.

${ }^{284}$ Vgl. Sackmann (1990), S. 164.

${ }^{285}$ Vgl. Nagel (1995), S. 103.

${ }^{286}$ Vgl. z.B. Probst (1987), S. 100ff; Bachinger (1990), S. 14ff.; Kasper (1990), S. 20ff.; Drepper (1992), S. 38ff.; Nagel (1995), S. 88ff.

${ }^{287} \mathrm{Vgl}$. Gussmann/Breit (1987), S. 111.
} 
zwischen der Unternehmung und ihrer Umwelt. ${ }^{288}$ Für die Unternehmungskultur wird häufig die Bedeutung von Mythen besonders hervorgehoben, die als "nicht wahrheitsfähige Wirklichkeitserklärungen “289 in bildhaft-symbolischer Form zentrale Werte und Überzeugungen übertragen und somit Orientierungen für das Verhalten der Mitarbeiter geben. Aber auch weitere sprachliche Elemente wie z.B. Leitsätze, Slogans und bestimmte Sprachregelungen können implizite Kommunikationselemente enthalten und damit große symbolische Überzeugungskraft gewinnen. Neben der Sprache können auch Handlungen als Medien der Unternehmungskultur fungieren, indem sie Bestätigungscharakter für die Inhalte der Unternehmungskultur aufweisen. ${ }^{290} \mathrm{Bei}$ derartigen Handlungen kann es sich beispielsweise um Riten, Rituale und Zeremonien handeln, ${ }^{291}$ die durch regelmäßige Wiederholung zur Verkörperung der Unternehmungskultur beitragen. Objekte stellen ebenfalls Träger der Sinnvermittlung dar, da sie durch ihre pure Existenz non-verbale Bedeutungsinhalte kommunizieren. Derartige Objekte, die auch als Kulturgüter bezeichnet werden können, sind etwa Firmenlogos, Firmenkleidung oder Statussymbole wie bestimmte Büroausstattungen, Privilegien oder Dienstfahrzeuge. ${ }^{292}$

Die angesprochenen Funktionen der Unternehmungskultur, die über die beschriebenen Symbole als Medien kommuniziert wird, sind auch für das Informationsmanagement und das technikunterstützte Informationssystem der Unternehmung als dessen Lenkungsgegenstand von großer Bedeutung. Damit das im vorangegangenen Abschnitt geforderte hohe $\mathrm{Ma} ß$ an Selbstregelung nicht letztlich die Auflösung des Systems zur Folge hat, die die Unternehmung als übergeordnetes System in ihrer Existenz gefährden würde, bedarf es eines gemeinsamen Grundkonsenses der dezentralen Entscheidungsträger hinsichtlich der Ziele der Informationsverarbeitung und der durch das technikunterstützte Informationssystem der Unternehmung zu erbringenden Leistungen für die Gesamtunternehmung. Die Unternehmungskultur kann diese Integrations- und Koordinationsfunktion erbringen, wenn die Entscheidungsträger die vermittelten Werte und Normen für sich selbst übernehmen und ihr Verhalten daran orientieren.

Die Annahme der vollständigen Beherrschbarkeit und exakten Lenkbarkeit des technikunterstützten Informationssystems der Unternehmung wurde auf Grund der hohen Komplexität des Systems in den vorangegangenen Abschnitten bereits abgelehnt. Die Unternehmungskultur bietet dem Informationsmanagement zwar einen weiteren Ansatzpunkt für Lenkungsmaßnahmen und dehnt dessen Einflussmöglichkeiten auf die symbolische Ebene des Unternehmungsgeschehens aus, damit darf jedoch realistischerweise keine Rückkehr zu einem mechanistischen Lenkungsverständnis verbunden sein. Kultur ist in den Köpfen aller Aufgabenträger innerhalb des technikunterstützten Informationssystems vorhanden und wird durch ihre Interaktionen auf allen Ebenen der Unternehmung permanent neu geschaffen. Sie unterliegt damit einem ständigen, nicht

\footnotetext{
${ }^{288}$ Vgl. Gussmann/Breit (1987), S. 112.

${ }^{289}$ Kasper (1990), S. 21.

${ }^{290} \mathrm{Vgl}$. Gussmann/Breit (1987), S. 116.

${ }^{291}$ Zur Abgrenzung dieser Begriffe vgl. Gussmann/Breit (1987), S. 116f; Bachinger (1990), S. 14f.; Kasper (1990), S. 22.

${ }^{292} \mathrm{Vgl}$. Bachinger (1990), S. 15; Kasper (1990), S. 23. Vordergründig ist die Zuordnung von materiellen Objekten zur symbolischen Ebene des Managements widersprüchlich. Tatsăchlich wird hier jedoch auf die den Objekten innewohnende Symbolik fokussiert, sodass auch materielle Objekte Gegenstand symbolischer Maßnahmen des Managements sein konnen.
} 
durch einzelne Personen (hier: Informationsmanager) steuerbaren Rekonstruktionsprozess. Dennoch kann das Informationsmanagement auf der symbolischen Ebene Einfluss auf das Verhalten des technikunterstützten Informationssystems der Unternehmung nehmen, wenn es in der Lage ist, die dezentralen Entscheidungsträger innerhalb dieses Systems von den über Symbole als Medien kommunizierten Sinn-Inhalten zu überzeugen. Die Interpretation der kommunizierten Bedeutungen verbleibt dabei jedoch stets bei den einzelnen Beteiligten, der Sinn muss von ihnen authentisch gefunden werden. ${ }^{293}$ Die Vermittlung von Sinn durch das Informationsmanagement erfolgt mit Hilfe der bereits angesprochenen Typen von Symbolen.

\section{c) Nutzung von Symbolen zur Vermittlung von Sinn durch das Informations- management}

Sprache als bedeutendstes Medium der Unternehmungskultur kann unternehmungsspezifisches Erfahrungswissen bezüglich der Informationsverarbeitung transportieren und dabei subtile Botschaften vermitteln. Mythen können im Hinblick auf den Bereich der Informationsverarbeitung beispielsweise die Entwicklung und den Einsatz innovativer technischer Lösungen (etwa mit Hilfe der Internet-Technologie und/oder neuester ERP-Software) betreffen, denen zugeschrieben wird, dass sie allein das Überleben der Unternehmung im immer härter werdenden Wettbewerb gewährleisten. Die Wirkung eines solchen Mythos beruht im Wesentlichen darauf, dass er die nicht durch den Einzelnen beherrschbare Unternehmungswirklichkeit (hier z.B. die hohe Belastung der Mitarbeiter in Folge der Beteiligung an mehreren Projekten neben dem Tagesgeschäft) erklärt und rechtfertigt. Auch Geschichten und Anekdoten können subtile Botschaften übertragen und damit zur Sinn-Schaffung im Bereich der Informationsverarbeitung beitragen. Sie schaffen beispielsweise Helden, wenn sie sich um verdiente Mitarbeiter (z.B. Systementwickler) ranken, die ihre Überzeugungen zum Wohle aller gegen große Widerstände in der Unternehmung durchgesetzt haben, oder sie erzählen von der gemeinsamen heroischen Bewältigung schwieriger Probleme in der Vergangenheit (z.B. von der Zusammenführung der Abrechnungssysteme bei der Fusion mit einer anderen Unternehmung). Derartige sprachliche Symbole kann das Informationsmanagement zwar nicht in der Art eines "symbolischen Apparats" einsetzen, es kann jedoch an ihrer Entstehung mitwirken und die Verbreitung fördern.

Handlungen, durch die der gemeinsame Grundkonsens der Mitarbeiter als Aufgabenträger innerhalb des technikunterstützten Informationssystems der Unternehmung immer wieder kommuniziert wird, können ebenfalls Gegenstand des Informationsmanagements auf der symbolischen Ebene sein. Als typisches Ritual ist hier etwa die monatliche Zusammenkunft der Entscheidungsträger der Informationsverarbeitung aus den einzelnen Fachbereichen im Rahmen eines ,jour fixe" zu nennen, bei der die Werte und Normen der Unternehmungskultur gegenseitig bestätigt werden. Den Beteiligten werden dadurch Anhaltspunkte zur Interpretation des aktuellen Geschehens vermittelt. und sie werden in ihren Überzeugungen immer wieder neu bestärkt. Auch andere Formen von schablonenhaft ablaufenden Handlungen sind dazu geeignet, als Medien der Unternehmungskultur zu dienen und zur Vermittlung von Sinnzusammenhängen genutzt zu werden. Ein besonders hervorzuhebendes Medium im Gestaltungsbereich des Informationsmanagements sind dabei Aus- und Weiterbildungsmaßnahmen, die

${ }^{293}$ Vgl. Ulrich (1990), S. 293. 
nicht nur die konkreten Inhalte weitergeben, sondern auch zur Sozialisation der Mitarbeiter in der Gemeinschaft beitragen und Orientierung hinsichtlich favorisierter Denk- und Verhaltensweisen geben. Die Betonung der symbolischen Dimension solcher und ähnlicher Handlungen (z.B. die Einführung neuer Mitarbeiter oder die Auszeichnung von Mitarbeitern für besondere Leistungen) bedeutet nicht, dass deren originäre Funktion in ihrer Bedeutung vernachlässigt oder abgewertet wird. Vielmehr geht es darum, die symbolische Relevanz dieser Handlungen zu erkennen und deren Interpretation im gewünschten Sinne zu fördern.

Schließlich stellen auch Objekte Träger symbolischer Bedeutungen dar und können durch das Informationsmanagement zur Vermittlung von kulturellen Inhalten genutzt werden. Objekte können zum einen Machtverhältnisse in subtiler Weise vergegenwärtigen und zum anderen gemeinsame Werte und Normen äußerlich verkörpern. Als Indikatoren der Wertschätzung ist im Bereich der Informationsverarbeitung an technische Statussymbole wie die besondere Hard- und Softwareausstattung des Arbeitsplatzes und an „Insignien von Macht und Status “294 wie Privilegien bezüglich des Zugriffs auf vertrauliche Informationen oder Titel in Form von Stellenbezeichnungen zu denken. Die Verkörperung von gemeinsamen Werten und Normen durch Objekte im Bereich der technikunterstützten Informationsverarbeitung erfolgt beispielsweise durch die optische Gestaltung unternehmungsindividueller Softwaresysteme (Benennung der Software, Verwendung von Firmenzeichen, Logos usw.) oder durch die konzeptionelle und optische Ausgestaltung eines Intranet. Die symbolische Bedeutung von Objekten ist nicht zu unterschätzen, da alles, was in einer Unternehmung sicht- und greifbar ist, entsprechend der oben angenommenen Allgegenwart der Unternehmungskultur der Interpretation durch die Mitarbeiter unterliegt und damit zum Ausgangspunkt für die individuelle Konstruktion von Sinnzusammenhängen wird.

\section{d) Zusammenfassendes Beispiel für die Förderung der Anpassungsfähigkeit des technikunterstützten Informationssystems durch das Informations- management}

In diesem Abschnitt werden die vorangegangenen theoretischen Überlegungen nach einer kurzen Zusammenfassung an einem ausführlichen Beispiel illustriert. Im Kern können folgende Gestaltungsempfehlungen zur Herstellung von Anpassungsfähigkeit des technikunterstützten Informationssystems der Unternehmung durch das Informationsmanagement festgehalten werden:

Die zentrale Entscheidungsinstanz des Informationsmanagements sollte mittels präsituativer kontextbezogener Regelungsmaßnahmen die verschiedenen Subsysteme des technikunterstützten Informationssystems der Unternehmung auf die Unternehmungsziele und die obersten Ziele der Informationsverarbeitung ausrichten und diese gewissermaßen „auf Kurs" halten. Von situativen Eingriffen sollte diese Instanz auf Grund ihrer unaufhebbaren Informationsdefizite so weit wie möglich absehen. Dementsprechend sollten Lenkungsmaßnahmen, die konkrete Vorgänge der Informationsverarbeitung im Einzelfall betreffen, dezentral erfolgen. Wenn in den einzelfallbezogenen Störungen des Subsystems bestimmte, immer wieder auftretende Muster erkennbar sind, dann empfiehlt sich tendenziell eine auf der Meta-Ebene angesiedelte, präsituative Regelung der Reaktion auf diesen Störungstyp. Diese Regelung auf der

\footnotetext{
${ }^{294}$ Kasper (1990), S. 23.
} 
Meta-Ebene sollte nur dann durch das zentrale Informationsmanagement erfolgen, wenn sie von Bedeutung für die Unternehmung und ihr technikunterstütztes Informationssystem als Ganzes ist, da zentral getroffene Regelungen eine größere Beharrungstendenz aufweisen. Ein zu hohes $\mathrm{Maß}$ an Einflussnahme durch das zentrale Informationsmanagement hat dementsprechend eine Minderung der zuküntigen Anpassungsfähigkeit des technikunterstützten Informationssystems der Unternehmung zur Folge.

Sowohl zentral als auch dezentral getroffene generelle Regelungen in Bezug auf einzelne Subsysteme sollten für die übrigen Subsysteme transparent sein, damit diese ihre Verhaltenserwartungen in Bezug auf das betroffene Subsystem anpassen können. Ist das betrachtete System in mehrere Subsystemebenen untergliedert, so empfiehlt sich für die Zuordnung von Regelungskompetenz das Subsidiaritätsprinzip. Eine möglichst zielgerichtete Informationsversorgung der Regelungsinstanzen auf den verschiedenen Subsystemebenen kann durch die Einrichtung eines unternehmungsweiten Informationspools, auf den die Entscheidungsträger nach dem Holprinzip fallweise zugreifen, gewährleistet werden.

Die beschriebenen Maßnahmen sind auf der substanziellen Ebene des Informationsmanagements angesiedelt und bedürfen einer Ergänzung auf der symbolischen Ebene. Symbolische Maßnahmen zielen darauf ab, den in Folge der Subsystembildung mit Dezentralisierung von Entscheidungskompetenzen entstehenden Desintegrationstendenzen entgegenzuwirken. Sprache, Handlungen und Objekte dienen dabei zur Vermittlung von Sinnzusammenhängen und erfüllen damit eine Integrations- und Koordinationsfunktion, ohne jedoch den Charakter der unvollständigen Systemlenkbarkeit vollständig zu beseitigen.

Als Beispiel zur praxisorientierten Verdeutlichung dieser Empfehlungen sei hier die Erschließung eines neuen Absatzweges mit Hilfe des Electronic Commerce in einer Unternehmung der Touristikbranche gewählt. Dabei handelt es sich möglicherweise um eine Aufgabenstellung, die zunächst nur die Fachabteilung Vertrieb betriff. Der Zentralbereich Informationswirtschaft in dieser Unternehmung könnte ein derartiges Projekt durch die Zurverfügungstellung von personellem Know-how unterstützen, z.B. durch die Beteiligung von internen Beratern an dem Projekt. Das zentrale Informationsmanagement definiert lediglich die Schnittstellen des neu zu entwickelnden Anwendungssystems, über die dieses System mit den anderen Anwendungssystemen in der Unternehmung verknüpft wird. In diesem Zusammenhang könnten beispielsweise die auszutauschenden Informationen in fachlicher Hinsicht sowie die zu verwendenden Übertragungsprotokolle und die Datentypen festgelegt werden. Auch weitere Rahmenbedingungen, die mehrere Teilsysteme der Unternehmung betreffen, wie etwa die Anwendung eines gegenüber dem üblichen Vorgehen in der Unternehmung abweichenden Mahnverfahrens, ${ }^{295}$ werden zentral festgesetzt.

Abgesehen von diesen, auf das notwendigste beschränkten, präsituativen Maßnahmen auf der Meta-Ebene wird die Handlungs- und Entscheidungsbefugnis in Bezug auf dieses System dezentral in der Vertriebsabteilung angesiedelt, die z.B. selbstständig

\footnotetext{
${ }^{295}$ Beim Mahnverfahren handelt es sich zwar nicht um eine originäre Aufgabenstellung der Informationsverarbeitung, ein geändertes Mahnverfahren schlägt sich jedoch in den Anforderungen an die Informationsverarbeitung nieder. Das Mahnverfahren betrifft nicht nur den Vertrieb, sondern es hat auch Auswirkungen auf das Rechnungswesen und den Finanzierungsbereich.
} 
über die personelle Ausstattung des neuen Vertriebsprozesses und die technische Ausrüstung der Arbeitsplätze entscheidet. Bei Belastungsspitzen oder bei der Einführung konkurrierender Angebote durch Anbieter der gleichen Branche entscheidet die Fachabteilung einzelfallbezogen selbst, wie auf diese Störungen des Anwendungssystems reagiert werden soll. Präsituative generelle Regelungen durch die Fachabteilung werden dann sinnvoll, wenn bestimmte Muster bei den auf das System wirkenden Umwelteinflüssen, z.B. systematische Kundenreaktionen auf die optische Ausgestaltung der Vertriebsseite im Internet oder auf bestimmte Internet-Werbemaßnahmen, erkennbar sind. Dabei wendet die Fachabteilung selbstständig DataMining-Methoden auf die Informationen in einem zentralen Data Warehouse der Unternehmung an. Außerdem greift sie auf Informationen über bestimmte Zielregionen zu, die durch die Mitarbeiter vor Ort in einem Intranet als unternehmungsweitem Informationspool allgemein verfügbar gemacht wurden.

Erst dann, wenn die Unternehmung als Ganzes von einer neuen Entwicklung betroffen ist, erscheint eine zentrale präsituative Regelung vorteilhaft. Dies könnte beispielsweise dann der Fall sein, wenn sich der neue Absatzweg als so erfolgreich erweist, dass insgesamt eine verstärkte Konzentration der Unternehmung auf den E-Commerce als neues Geschäftsfeld sinnvoll erscheint. Dies würde möglicherweise eine Anpassung des gesamten Geschäfts erforderlich machen, die sich auch in veränderten Anforderungen an das technikunterstützte Informationssystem der Unternehmung als Ganzes niederschlägt. So könnte die Konzentration auf den E-Commerce als Absatzweg beispielsweise durch die Einführung eines Helpdesk-Systems unterstützt werden, mit dessen Hilfe spezielle Fragen der Kunden, für die nun nicht mehr die Möglichkeit der persönlichen Betreuung im Reisebüro besteht, gelöst werden. Zudem könnte es zu Änderungen des Produktprogramms und zur Erschließung neuer, ebenfalls elektronischer Beschaffungsmärkte kommen, was sich wiederum auf die technikunterstützte Informationsverarbeitung auswirken würde.

Derartige Anpassungen sollten aus der Perspektive der Kybernetik II deshalb zentral eingeleitet werden, weil dadurch eine bessere Transparenz erreicht und den einzelnen Subsystemen verlässliche Verhaltenserwartungen bezüglich ihrer internen und externen Subsystemumwelt ermöglicht werden. Präsituative Maßnahmen des zentralen Informationsmanagements, die die Meta-Ebene der Informationsverarbeitung betreffen, könnten in diesem Zusammenhang z.B. die Einleitung von Systementwicklungsprojekten, die Festlegung veränderter Funktionszuordnungen zwischen den einzelnen Subsystemen und die Definition neuer technischer Schnittstellen sein. Die eigentliche Anpassung an die geänderte Gesamtsituation erfolgt innerhalb dieser Rahmenbedingungen dezentral durch die einzelnen Subsysteme, da diese über eine bessere Kenntnis der neuen Anforderungen an die technikunterstützte Informationsverarbeitung verfügen.

Durch die Unternehmungskultur kann ein derartiger Anpassungsprozess auf der symbolischen Ebene gefördert und in eine grundsätzliche Richtung gelenkt werden. Da die Wirkungszusammenhänge auf der symbolischen Ebene langfristig angelegt sind und Interpretationsspielräume offen lassen, steht dabei nicht die konkrete Einflussnahme auf einzelne Entscheidungen im Vordergrund, sondern das Setzen von Orientierungspunkten, in deren Rahmen die Mitarbeiter selbstständig handeln. Im vorliegenden Beispiel kann es sich dabei etwa um den Mythos handeln, dass das Internet eine tiefgreifende Veränderung des Wirtschaftslebens bewirken wird, deren Folgen nur mit 
denen der industriellen Revolution im 19. Jahrhundert vergleichbar sind. In Verbindung damit werden durch Erzählungen über die erfolgreiche Verwirklichung von Visionen durch die ersten Internet-Pioniere Helden geschaffen, deren Taten als Fixpunkte das Denken der Teammitglieder im Rahmen der Projektarbeit beeinflussen. Die Entstehung und Verbreitung solcher Mythen und Geschichten kann durch das Informationsmanagement initiiert und gefördert werden, sodass eine lenkende Beeinflussung des Systems auf der symbolischen Ebene möglich erscheint. Allerdings stellt die Entwicklung eines gemeinsamen Selbstverständnisses einen überindividuellen Prozess aller Beteiligten dar, der durch einzelne Personen nur angeregt, nicht aber im Ergebnis vorgegeben werden kann.

Der Stellenwert eines solchen Projektes kann auch durch Objekte symbolisiert werden, indem beispielweise ein hohes Budget für die technische Ausstattung der Projektarbeitsplätze bereitgestellt wird, sodass die Projektmitglieder über modernste Hardund Software verfügen können. Die hohe Wertschätzung der Projektmitglieder kann darüber hinaus durch weitere materielle Symbole wie beispielsweise die Wahl der Büroräumlichkeiten für das Projekt und besondere finanzielle Belohnungen dokumentiert werden. Rituelle Handlungen mit symbolischer Bedeutung, die der Bestätigung der geteilten Werte und Normen dienen, können etwa die Veranstaltung von Klausurtagungen in exklusiven Tagungshotels und die Berichterstattung an einen hochrangig besetzten Lenkungsausschuss beim Erreichen von Projektmeilensteinen sein.

Dieses ausführliche Beispiel demonstriert, wie ein systemorientiertes innovatives Informationsmanagement in der Praxis die Anpassungsfähigkeit des technikunterstützten Informationssystems der Unternehmung fördern kann. Damit zeigt sich, dass für praktische Problemstellungen eine Konkretisierung der sehr abstrakten Aussagen der Kybernetik II möglich ist und zu ebenso sinnvollen wie umsetzbaren Ergebnissen führt.

\subsubsection{Anpassungsvorgänge als Veränderungen der Wissensbasis: Lernende Informationssysteme}

Im vorigen Abschnitt wurde erläutert, wie das Informationsmanagement die Voraussetzungen für eine hohe Anpassungsfähigkeit des technikunterstützten Informationssystems der Unternehmung an sich wandelnde Situationen herstellen und dabei den inneren Zusammenhalt des Systems gewährleisten kann. Die Ausführungen dieses Abschnittes wenden sich nun den eigentlichen Anpassungsvorgängen innerhalb dieses Systems zu, die durch das Informationsmanagement zu fördern sind.

\section{a) Systemanpassungen als Lernprozesse}

Entsprechend den Erläuterungen des vorangegangenen Abschnittes sollte aus der Sicht der Systemorientierten Betriebswirtschaftslehre die Kompetenz zur Lenkung komplexer Systeme weitgehend dezentralisiert, also auf deren verschiedene Subsysteme übertragen werden. Die dezentralen Lenkungsinstanzen verfügen grundsätzlich über zwei alternative Möglichkeiten zur Reaktion auf Störungen des von ihnen zu lenkenden Subsystems. Einerseits können sie konservativ orientiert auf eine Stabilisierung des Systemoutputs trotz der aktuellen Störungen des Systems hinwirken. Dies entspricht der Zielsetzung der Kybernetik I, aus deren Sicht im Abschnitt 4.3.1 ausführlich 
mögliche Maßnahmen des Informationsmanagements erörtert wurden. Andererseits können sie innovativ orientiert auf eine grundlegendere Anpassung des Systems und/oder des durch das System produzierten Output hinwirken und dafür vorübergehende Instabilitäten in Kauf nehmen. Diese Art der Anpassung wird im Folgenden näher untersucht, da sich darauf das Erkenntnisinteresse der Kybernetik II richtet.

Die Anpassung von komplexen Systemen an sich wandelnde Situationen findet in jüngster Zeit verstärkt Beachtung in Theorie und Praxis und wird unter dem Stichwort des „Organisationalen Lernens" diskutiert. ${ }^{296}$ Aus der Perspektive der Systemorientierten Betriebswirtschaftslehre besitzt das Themengebiet Organisationales Lernen deshalb große Bedeutung, weil angenommen wird, dass unternehmungsexternen Störungen in Folge von Veränderungen der Unternehmungssituation mit unternehmungsinternen Veränderungen zu begegnen ist, die wiederum durch Organisationales Lernen ausgelöst werden. ${ }^{297}$ In Arbeiten zum Organisationalen Lernen wird - basierend auf einer begrifflichen Auseinandersetzung mit dem Phänomen des (individuellen) Lernens und seiner Übertragbarkeit auf Organisationen als multipersonelle Kollektive untersucht, wie Organisationales Lernen abläuft, welche Arten von Lernen die jeweils angemessene Reaktion auf unternehmungsexterne Veränderungen darstellen und auf welche Weise Organisationales Lernen durch die Lenkungsinstanzen des Systems gefördert werden kann.

Ein vollständiger Überblick über Lernvorgänge im Allgemeinen und Lernvorgänge in Unternehmungen als Organisationen im Besonderen kann an dieser Stelle nicht gegeben werden. Ein weithin akzeptiertes Konzept und eine entsprechende Definition Organisationalen Lernens liegen derzeit nicht vor. ${ }^{298}$ Die Lernvorgänge innerhalb des Systems weisen nicht den Charakter von Führungsaufgaben auf und sind damit selbst nicht Teil des Aufgabenfeldes Informationsmanagement. Bei der Untersuchung individueller und überindividueller Lernprozesse handelt es darüber hinaus um eine verhaltenstheoretische Fragestellung, die nicht mit den Mitteln der Systemtheorie analysiert werden kann. Deshalb wird hier auf eine ausfuhrliche Darstellung der Diskussion über die Existenz von Prozessen Organisationalen Lernens verzichtet und auch der Ablauf solcher Prozesse wird nicht im Einzelnen untersucht. ${ }^{299}$

Im Folgenden wird stattdessen die allgemeine definitorische Abgrenzung des Organisationalen Lernens als Veränderung der Organisationalen Wissensbasis übernommen, die in systemtheoretisch ausgerichteten Arbeiten häufig zu Gunde gelegt wird. $^{300}$ Geht man von der pragmatischen Zielsetzung der Systemorientierten Betriebswirtschaftslehre und der Black box-Technik als methodischer Leitidee aus, so ist eine genaue Kenntnis der systeminternen Lernvorgänge nicht zwingende Voraussetzung, um diese durch das Informationsmanagement zu fordern. Das Vorgehen in diesem Abschnitt entspricht also der allgemeinen Methodik der Systemtheorie, da das durch das Informationsmanagement zu lenkende System als Black box aufgefasst wird,

\footnotetext{
${ }^{296}$ Für eine Bestandsaufnahme über den Stand der Diskussion zum Organisationalen Lernen vgl. z.B. die Übersichten bei Wiegand (1996), S. 171ff.; Streubel (2000), S. $97 \mathrm{ff}$.

${ }^{297}$ Vgl. ăhnlich Wiegand (1996), S. 8; Streubel (2000), S. $134 f$.

${ }^{298}$ Vgl. Wiegand (1996), S. 309; Schreyögg/Noss (1997), S. 70.

${ }^{299}$ Vgl. dazu die sehr detaillierte Untersuchung bei Wiegand S. 323-531.

${ }^{300} \mathrm{Zu}$ dieser Definition vgl. Pautzke (1989), S. 44; Kirsch (1992), S. 316; Streubel (2000), S. 106.
} 
dessen intern stattfindende Lernprozesse für die Lenkungsinstanz nicht im Einzelnen erkennbar sind.

Für den hier zu entwickelnden Systemorientierten Ansatz für eine Theorie des Informationsmanagements sind daher insbesondere die beiden zuletzt genannten Aspekte der Erforschung Organisationalen Lernens, also die Art des Lernens und die Förderung von Lernprozessen, relevant. Das Informationsmanagement kann durch geeignete Maßnahmen die gewünschten Lernvorgänge innerhalb des technikunterstützten Informationssystems der Unternehmung und seiner Subsysteme auslösen und forcieren. Dementsprechend richtet sich das Erkenntnisinteresse der weiteren Betrachtungen auf Möglichkeiten zur Förderung bestimmter Arten der Veränderung von denjenigen Teilen der Organisationalen Wissensbasis, die die technikunterstützte Informationsverarbeitung in der Unternehmung betreffen. Es ist zunächst zu untersuchen, welche Art des Lernens aus Sicht der Kybernetik II anzustreben ist. Davon ausgehend werden Möglichkeiten zur Förderung von Lernprozessen dieses Typs durch das Informationsmanagement überprüft.

\section{b) Statische und dynamische Merkmale der Organisationalen Wissensbasis}

$\mathrm{Da}$ Organisationales Lernen durch eine Veränderung der Organisationalen Wissensbasis erreicht wird, werden hier zunächst die Merkmale dieser Wissensbasis näher betrachtet. Die Organisationale Wissensbasis enthält das individuelle und überindividuelle Wissen innerhalb der Unternehmung, das den Unternehmungsmitgliedern zur Lösung der ihnen übertragenen Teilaufgabenstellungen $\mathrm{zu}$ einem bestimmten Zeitpunkt zur Verfügung steht. ${ }^{301}$ Die Arbeiten zum Organisationalen Lernen unterscheiden verschiedene Kategorien von Wissen innerhalb dieser Organisationalen Wissensbasis und untersuchen, wie Wissen der jeweiligen Kategorien für die Unternehmung verfügbar gemacht, gespeichert und verändert werden kann. Die Veränderung des Wissens kann auch in einer Entfernung von Wissensbestandteilen aus der Organisationalen Wissensbasis bestehen, da auch das Ablegen bestimmter Verhaltensmuster zu einer Weiterentwicklung des Systems führen kann. ${ }^{302}$ Aus der Perspektive der Kybernetik $\Pi$ ist neben der Unterscheidung zwischen individuellem und überindividuellem Wissen besonders die Unterscheidung zwischen Oberflächenwissen und der Tiefenstruktur der Organisationalen Wissensbasis von Interesse. Wie im Folgenden erläutert wird, erfordert die Anpassung eines Systems an eine grundlegend veränderte Situation die Veränderung der Tiefenstruktur der Organisationalen Wissensbasis erfordert. Abbildung 4/8 zeigt die hier verwendete zweidimensionale Einteilung von statischen Merkmalen der Organisationalen Wissensbasis.

\footnotetext{
${ }^{301}$ Vgl. Streubel (2000), S. 108, die sich auf Oberschulte (1994), S. 62 und Pautzke (1989) S. $76 \mathrm{ff}$. bezieht.

${ }^{302}$ Vgl. Streubel (2000), S. 121ff.
} 


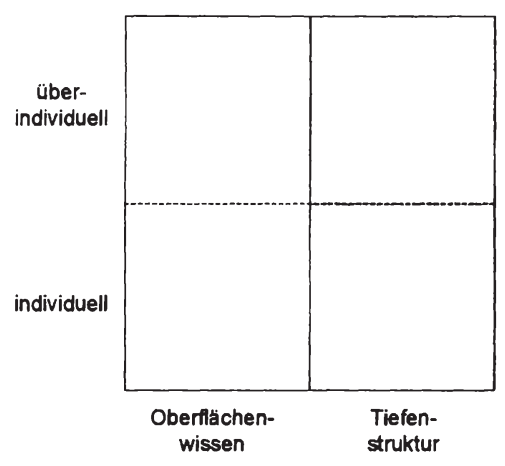

Abb. 4/8: Zweidimensionale Klassifikation von statischen Merkmalen der Organisationalen Wissensbasis

Die Unterscheidung zwischen Oberflächenwissen und Tiefenstruktur der Organisationalen Wissensbasis geht auf die kognitive Lerntheorie von PIAGET zurück, der annimmt, dass das Verhalten eines Menschen von bestimmten grundsätzlichen Verhaltenselementen abhängt, die in Form von kognitiven Strukturen vorliegen. ${ }^{303}$ Derartige kognitive Strukturen wurden im Lauf der individuellen Vergangenheit der betreffenden Person erworben und repräsentieren generalisierte Verhaltens- und Reaktionsmuster, ${ }^{304}$ die bei konkret vorliegenden Problemstellungen eine bestimmte grundsätzliche Vorgehensweise der jeweiligen Person bewirken. Kognitive Strukturen wirken als Wahrnehmungsfilter, mit deren Hilfe eine Person das neu Wahrgenommene erkennt, einordnet und interpretiert. ${ }^{305}$ In ihrer Gesamtheit stellen die kognitiven Strukturen ein mentales Modell der Umwelt dar, durch das die wahrgenommene Komplexität der Umwelt und die davon abhängende Varietät des eigenen Verhaltens auf ein handhabbares $\mathrm{Ma} ß$ reduziert wird.

Nach dieser Vorstellung sind zwei Arten von individuellen Lernprozessen zu unterscheiden, die als Assimilation und Akkomodation bezeichnet werden. ${ }^{306}$ Bei der Assimilation wird das bestehende mentale Modell um neue Wissensbestandteile ergänzt, ohne dass die bereits existierenden kognitiven Strukturen verändert werden müssen. Dagegen bedeutet Akkomodation die Veränderung der bestehenden kognitiven Strukturen und in Folge dessen auch eine Veränderung des bestehenden Verhaltensrepertoires, auf deren Grundlage anschließend neue Assimilationen möglich werden. $^{307}$ Dabei wird die Akkomodation durch erfolglose Assimilationsversuche ausgelöst, die zu Ungleichgewichten, etwa in Form von kognitiven Dissonanzen führen und dadurch die Akkomodation als Lernprozess höherer Ordnung anstoßen. Diese Bewegung von einem relativ dauerhaften Akkomodationszustand zum nächsten wird als Äquilibrationsprozess bezeichnet. ${ }^{308}$ Aus der Unterscheidung von zwei Arten von Lemprozessen folgt, dass das Individuum über zwei Arten von Wissen verfuggt. ${ }^{309}$ Auf

\footnotetext{
${ }^{303}$ Vgl. dazu Piaget (1976), S. 13ff.

${ }^{304} \mathrm{Vgl}$. Strauß (1996), S. 19.

${ }^{305}$ Vgl. Streubel (2000), S $103 \mathrm{f}$

${ }^{306}$ Vgl. Piaget (1976), S. 13ff.

${ }^{307}$ Vgl. Strauß (1996), S. 19.

${ }^{308}$ Vgl. Montada (1970), S. 21 ff.

${ }^{309} \mathrm{Vgl}$. Streubel (2000), S. 112.
} 
der einen Seite stehen die kognitiven Strukturen, die im Laufe der Zeit durch Akkomodation erworben wurden, und die als Tiefenstruktur des individuellen Wissens bezeichnet werden können. Auf der anderen Seite steht solches Wissen, das jeweils innerhalb bestehender kognitiver Strukturen durch Assimilation erworben wurde und das als Oberflächenwissen bezeichnet werden kann. Die Tiefenstruktur des Wissens strukturiert also das Oberflächenwissen und ermöglicht eine Einordnung neu hinzukommenden Wissens innerhalb der bestehenden generalisierten Interpretationsund Verhaltensmuster. ${ }^{310}$

Überträgt man diese Ideen auf diejenigen Teile der Organisationalen Wissensbasis, die das individuelle Wissen in der Unternehmung in Bezug auf die technikunterstützte Informationsverarbeitung beinhalten, so kann auch hier zwischen Oberflächenwissen und der Tiefenstruktur des Wissens unterschieden werden. Die Tiefenstruktur dieses Teils der Organisationalen Wissensbasis enthält die generalisierten Interpretations- und Verhaltensmuster, die einen generellen Rahmen für die Aktivitäten der Aufgabenträger innerhalb des technikunterstützten Informationssystems der Unternehmung bilden. Dabei kann es sich z.B. um individuelles Wissen über bestimmte Aktivitätsfolgen als Teil von übergeordneten Geschäftsprozessen oder um persönliche Kenntnisse über die allgemeine Funktionalität eines in der Unternehmung eingesetzten Software-Systems handeln. Auf der Basis dieser Tiefenstruktur wird neu hinzukommendes Oberflächenwissen interpretiert und das Verhalten im konkreten Einzelfall ausgebildet. Beispiele für solches Oberflächenwissen sind die genauen Bestelldaten eines konkreten Auftrags im Rahmen eines übergeordneten Auftragsabwicklungsprozesses oder die IstDaten für eine spezielle Kostenanalyse mit Hilfe des oben angesprochenen SoftwareSystems. Assimilationsprozesse betreffen hier etwa die Abwicklung weiterer Bestellvorgänge oder die Ergänzung der einzelnen Kostenanalyse durch eine Erlösanalyse, während Akkomodationsprozesse sich auf Änderungen der Grundstruktur der betreffenden Aufgabenstellung oder die Einführung neuer Arten der Ergebnisanalyse richten.

Die erläuterte Systematisierung von individuellem Wissen und individuellen Lernprozessen kann in ihren Grundzügen auch auf die überindividuellen Teile der Organisationalen Wissensbasis angewendet werden. ${ }^{311}$ Dabei lässt sich eine Analogie zwischen Organisationalen Handlungstheorien als überindividuellem Wissen und den kognitiven Strukturen des Individuums herstellen. ${ }^{312}$ Organisationale Handlungstheorien beinhalten verhaltensbezogene Hypothesen, die Erwartungen im Hinblick auf die Konsequenzen bestimmter Verhaltensweisen von Mitarbeitern der Unternehmung in bestimmten Situationen beinhalten. ${ }^{313}$ Organisationale Handlungstheorien sind mit Theorien im normalerweise verstandenen Sinne vergleichbar, da sie generelle Merkmale wie Allgemeinheit, Relevanz, Konsistenz, Vollständigkeit, empirische Überprüfbarkeit und Einfachheit aufweisen müssen. ${ }^{314}$ Sie stellen darüber hinaus die normative Handlungsgrundlage für die interagierenden Individuen innerhalb der Organisation

\footnotetext{
${ }^{310}$ Vgl. Oberschulte (1994), S. 61; Pautzke (1989), S. 82f., S. 86.

${ }^{311}$ Vgl. Pautzke (1989), S. 83ff.; Streubel (2000), S. 113.

${ }^{312}$ In diesem Punkt wird hier der Argumentation von Streubel gefolgt, die eine Verknüpfung der auf individuelles Lernen bezogenen Arbeiten Piagets mit den auf die Untersuchung Organisationalen Lernens gerichteten Arbeiten von Argyris et al. vornimmt. Vgl. Streubel (2000), S. 113ff.

${ }^{313}$ Vgl. Wolfsteiner (1995), S. 174.

${ }^{314} \mathrm{Vgl}$. Argyris/Schön (1978), S. 4 f.
} 
dar. ${ }^{315}$ Diese Handlungstheorien werden als "Organisational“ bezeichnet, da sie von allen oder zumindest von einem Teil der Mitarbeiter der Unternehmung ihrem Handeln zu Grunde gelegt werden. Organisationale Handlungstheorien leisten auf der überindividuellen Ebene die analoge Funktion zu kognitiven Strukturen auf der individuellen Ebene und stellen damit als überindividuelle Wissensbestandteile zusammen mit den kognitiven Strukturen als individuellen Wissensbestandteilen die Tiefenstruktur der Organisationalen Wissensbasis dar. ${ }^{316}$ Von dieser Tiefenstruktur hängt das Verhaltensmuster ab, das im konkreten Fall zusammen mit dem situationsbezogenen Oberflächenwissen das Verhalten der Mitarbeiter der Unternehmung bestimmt.

Die Analogie zwischen individuellem und überindividuellem Wissen lässt sich auch auf Prozesse der Wissensveränderung zwischen individuellem und überindividuellem Lernen ausdehnen. ${ }^{317}$ Die Einteilung in einfache und höhere Prozesse des Lernens, die auf der Ebene des Individuums als Assimilation und Akkomodation bezeichnet werden, ist auch auf der überindividuellen Ebene der Organisationalen Wissensbasis möglich. ${ }^{318}$ Auf der überindividuellen Ebene lassen sich als Lernniveaus das Single-loop Learning und das Double-loop Learning unterscheiden, die wiederum durch ein Deutero Learning auf der Meta-Ebene ergänzt und gefordert werden können. ${ }^{319}$ Beim SingleLoop Learning werden den Handlungen die Implikationen der organisationalen Handlungstheorie $\mathrm{zu}$ Grunde gelegt, sodass der durch diese definierte Zustand möglichst exakt hergestellt wird. ${ }^{320}$ Die Organisationalen Handlungstheorien selbst werden auf diesem Lernniveau nicht hinterfragt. Dagegen werden beim Double-Loop Learning als Lernniveau auf höherer Ebene gerade diese Organisationalen Handlungstheorien überprüft. ${ }^{321}$ Dies geschieht, da eine Lösung der vorliegenden Problemstellung mit den bestehenden Interpretations- und Verhaltensmustern nicht erreichbar erscheint. ${ }^{322}$ Das Deutero Learning richtet sich auf die Prozesse des SingleLoop Learning und des Double-Loop Learning selbst und ist damit auf der Meta-Ebene

${ }^{315}$ Die Organisationsmitglieder betätigen sich also als „naive Wissenschaftler“. Vgl. dazu Wiegand (1996), S. 208

${ }^{316} \mathrm{Vgl}$. Streubel (2000), S. $114 \mathrm{f}$.

${ }^{317} \mathrm{Vgl}$. Streubel (2000), S. 130.

${ }^{318}$ Eine Unterscheidung verschiedener Lernniveaus erfolgt in zahlreichen Arbeiten zum Organisationalen Lernen. Vgl. dazu die Übersichten bei Pawlowski (1992), S. 205f.; Streubel (2000), S. 130f. Die nachfolgenden Ausführungen beschränken sich auf eine exemplarische Darstellung der Argumentation von Argyris/Schön (1978), S. 18ff., da die Zielsetzung hier nicht in einer detaillierten Analyse von Prozessen des (Organisationalen) Lernens, sondern in dessen Förderung durch das Informationsmanagement liegt.

${ }^{319} \mathrm{Vgl}$. Argyris/Schön (1978), S. 18ff.

${ }^{320} \mathrm{Vgl}$. Wiegand (1996), S. 211.

${ }^{321} \mathrm{Vgl}$. Argyris/Schön (1978), S. 24ff.

${ }^{322}$ Wiegand (1996), S. 212, weist darauf hin, dass praktisch kein Unterschied zwischen Single-Loop und Double-Loop Learning erkennbar sei, da die Einordnung eines Lernprozesses in eine der beiden Kategorien lediglich von der subjektiv interpretierten Abweichung vom angestrebten Zustand abhănge. Dem kann entgegen gehalten werden, dass beim Single-Loop Learning lediglich eine Ergănzung der bestehenden Wissensbasis erfolgt, wăhrend sich das Double-Loop Learning gezielt auf die Veränderung oder Entfernung bereits bestehender Wissensbestandteile der Wissensbasis richtet. Die Unterscheidung zwischen Single-Loop Learning und Double-Loop Learning kann deshalb deutlich getroffen werden und wird in der vorliegenden Arbeit übernommen. 
des Lernens angesiedelt. Dazu ist eine Reflexion vergangener Lemprozesse mit einer Analyse von Lernhindernissen und Lernerleichterungen erforderlich. ${ }^{323}$

Diese allgemeinen Überlegungen zu den statischen und dynamischen Merkmalen der überindividuellen Teile der Organisationalen Wissensbasis können ebenfalls auf den Bereich der technikunterstützten Informationsverarbeitung übertragen werden. Bei den überindividuellen Teilen der Organisationalen Wissensbasis handelt es sich einerseits um kollektives Wissen als identisches oder äquifinales Wissen, das von allen Mitgliedern bzw. der überwiegenden Mehrheit eines Kollektivs geteilt wird und andererseits um personenungebundenes (institutionalisiertes) Wissen, das den Mitarbeitern der Unternehmung durch die Speicherung auf technischen Trägermedien zur Verfügung steht. ${ }^{324}$ Beispiele für personenungebundenes Wissen in Bezug auf die technikunterstützte Informationsverarbeitung in der Unternehmung sind etwa der Inhalt von Datenbanken oder die grafische Darstellung des Ablaufes bestimmter informationstechnisch unterstützter Geschäftsprozesse. Kollektives Wissen dieser Art stellen z.B. die allen Beteiligten eines Projektteams bewusste Rollenverteilung innerhalb des Teams zur Lösung einer schlecht strukturierten Problemstellung oder das Selbstverständnis dieses Teams hinsichtlich der Zielsetzung, die Wettbewerbsfähigkeit der Gesamtunternehmung durch die Einführung eines neuen Anwendungssystems zu erhöhen, dar.

Die einfachen und höheren Prozesse des überindividuellen Lernens können sowohl die kollektiven als auch die personenungebundenen Bestandteile der auf die technikunterstützte Informationsverarbeitung bezogenen überindividuellen Teile der Organisationalen Wissensbasis betreffen. Beispiele für Single-Loop Learning in diesem Bereich stellen die Ergänzung der Datenbank durch die Speicherung eines neuen Bestellvorgangs oder die Aufnahme eines neuen Mitglieds in das Projektteam mit entsprechender Änderung der Rollenzuweisung innerhalb des Teams dar. Das DoubleLoop Learning betrifft dagegen Änderungen der Tiefenstruktur des auf die technikunterstützte Informationsverarbeitung bezogenen überindividuellen Wissens. Dabei kann es sich etwa um die Änderung des der Datenbank zu Grunde liegenden Datenmodells auf Grund von Änderungen in der Aufbaustruktur der Unternehmung oder die Anpassung des Ablaufes von informationstechnisch unterstützten Geschäftsprozessen und deren grafische Dokumentation handeln. Als Deutero Learning sind in diesem Zusammenhang Maßnahmen zu verstehen, die die beschriebenen Prozesse des Single-Loop Learning und des Double-Loop Learning verbessern, z.B. die Zurverfügungstellung besser handhabbarer Tools als Hilfsmittel bei der Neugestaltung von Geschäftsprozessen.

\section{c) Lernen von Systemen aus der Sicht der Kybernetik II}

Aus der Perspektive der Systemorientierten Betriebswirtschaftslehre kann die vorgenommene Unterscheidung zwischen Oberflächenwissen und Tiefenstruktur des Wissens sowie zwischen verschiedenen Lernniveaus folgendermaßen interpretiert werden:

\footnotetext{
${ }^{323}$ Vgl. ähnlich Streubel (2000), S. 132.

${ }^{324}$ Vgl. Streubel (2000), S. $109 f f$.
} 
Beim Single-Loop Learning bzw. der Assimilation findet ein Prozess der negativen Rückkopplung mit dem Ziel der Stabilisierung des Systemverhaltens unter den gegebenen Rahmenbedingungen statt. ${ }^{325} \mathrm{Da}$ ein Hinterfragen der bestehenden kognitiven Strukturen bei diesem Lerntypus nicht erfolgt, handelt es sich hier um die Veränderung des Oberflächenwissens der Organisationalen Wissensbasis. Die Akteure innerhalb des Systems passen zur Erreichung der Systemziele ihr Verhalten in dem durch ihre aktuellen mentalen Modelle abgesteckten Rahmen an. Damit entsprechen das Single-Loop Learning und die Assimilation der konservativ orientierten Bewältigung von Komplexität, die im Zentrum des Erkenntnisinteresses der Kybernetik I steht. Die im Abschnitt 4.3.1 beschriebenen Maßnahmen zur Lenkung von Systemen, die auf die passive oder aktive Bewältigung von Störungen zielen, können deshalb als systemtheoretisch begründete Maßnahmen zur Förderung von Single-Loop Learning und Assimilation reinterpretiert werden. Passive Maßnahmen zielen in diesem Licht auf die Errichtung von Wahrnehmungsfiltern, die verhindern, dass das System überfordert wird, weil zu viele Lernprozesse erster Ordnung durch Entwicklungen in der Systemumwelt ausgelöst werden. Dagegen verbessern aktive Maßnahmen den Informationsstand der Lenkungsinstanz und ermöglichen dadurch eine Verstärkung der aktuell stattfindenden Assimilationen und Single-Loop Lernprozesse.

Bedeutsamer aus der Sicht der Kybernetik II sind die Prozesse des Double-Loop Learning bzw. der Akkomodation, bei denen das aktuelle mentale Modell selbst hinterfragt und verändert wird. Dazu ist zunächst eine positive Rückkopplung erforderlich, bei der eine auftretende Abweichung vom erwünschten Ergebnis durch die Lenkungsinstanz nicht verringert, sondern im Gegenteil verstärkt wird. Dem DoubleLoop Learning und der Akkomodation wohnt also ein destruktiver Aspekt inne, da überholte Verhaltensweisen innerhalb des Systems beseitigt werden. ${ }^{326}$ Es enthält darüber hinaus ein kreatives Element, da es zu neuen mentalen Modellen und neuen Verhaltensweisen des Systems führt. Das Lernen höherer Ordnung entspricht damit der innovativ orientierten Bewältigung von Komplexität, die im Zentrum des Erkenntnisinteresses der Kybernetik II steht. Die durch die Kybernetik II untersuchte Anpassung von Systemen an veränderte Gesamtsituationen stellt sich aus diesem Blickwinkel als Änderung des Systemverhaltens dar, die durch Lernprozesse höherer Ordnung in Form der Akkomodation oder des Double-Loop Learning ausgelöst wird. Die durch Lernprozesse höherer Ordnung veränderten Teile der Organisationalen Wissensbasis sind in Abbildung 4/8 optisch hervorgehoben.

Als normative Konsequenz furr die Lenkungsinstanz ergibt sich aus diesen Überlegungen, dass Lernprozesse höherer Ordnung zu fördern sind, da langfristig das Überleben eines Systems nicht allein auf der Basis der in der Kybernetik I fokussierten Prozesse des Single-Loop Learning bzw. der Assimilation gewährleistet werden kann. Dies führt zu einer Präzisierung der weiter oben angestellten Überlegungen zur Anpassungsfähigkeit komplexer Systeme. Die dort abgeleiteten Aussagen zur Lenkung und Lenkbarkeit komplexer Systeme beziehen sich allgemein auf die Bewältigung hoher System- und Umweltkomplexität und machen noch keine Aussagen zur Richtung der jeweiligen Anpassungsvorgänge. Sie betreffen deshalb sowohl konservativ ausgerichtete Lemprozesse erster Ordnung als auch innovativ ausgerichtete

${ }^{325} \mathrm{Vgl}$. Parker/Stacey (1995), S. 26.
${ }^{326}$ Vgl. Parker/Stacey (1995), S. $56 f$. 
Lernprozesse höherer Ordnung. Die grundlegenden Aussagen des vorigen Teilkapitels sind deshalb um Überlegungen darüber zu ergänzen, wie das Informationsmanagement speziell Lernprozesse höherer Ordnung auslösen und fördern kann. Das Informationsmanagement selbst kann aus dieser Sicht als Deutero Learning aufgefasst werden, da es Single-Loop und insbesondere Double-Loop Lernprozesse gezielt fördert und verbessert. Bei dieser Zielsetzung lässt sich auch der in jüngster Zeit in Theorie und Praxis verstärkt in Mode kommende Begriff des Wissensmanagements einordnen. Wissensmanagement stellt dann einen speziellen Teilbereich des Informationsmanagements dar, der im Vergleich zum bisherigen Gestaltungsschwerpunkt des Informationsmanagements einen neuen Aspekt, nämlich die Förderung von Lernprozessen höherer Ordnung betont. ${ }^{327}$

\section{d) Auslösung und Förderung von Lernprozessen höherer Ordnung durch das Informationsmanagement}

Die Ausführungen des vorangegangenen Abschnittes begründen, dass die durch die Kybernetik II angestrebten Anpassungen komplexer Systeme an veränderte interne und externe Situationen die Folge von Lernvorgängen höherer Ordnung innerhalb des Systems sind. Daraus ergibt sich für das innovativ ausgerichtete Informationsmanagement die Zielsetzung, die Möglichkeit für das Auftreten dieser Art von Lernvorgängen innerhalb des technikunterstützten Informationssystems der Unternehmung zu schaffen bzw. deren Wahrscheinlichkeit in Systemsituationen, die durch besondere Dynamik gekennzeichnet sind, zu erhöhen. Entsprechend der Black box-Methode ist es dazu nicht unbedingt erforderlich, den Ablauf der Lernvorgänge im Einzelnen zu kennen oder zu beobachten. Vielmehr kommt es allein darauf an, durch geeignete Maßnahmen die Eingangsgrößen (den Systeminput) des Systems so zu beeinflussen, dass Veränderungen in der Tiefenstruktur der informationssystembezogenen Organisationalen Wissensbasis stattfinden können. Im Wesentlichen geht es aus diesem Blickwinkel für die Lenkungsinstanz darum, eine schöpferische Atmosphäre innerhalb des Systems herzustellen, in der sich die Kreativität der Akteure (hier der menschlichen Aufgabenträger der technikunterstützten Informationsverarbeitung) entfalten kann. Durch eine derartige Atmosphäre, die z.T. auch als Lernkultur ${ }^{328}$ bezeichnet wird, werden die Voraussetzungen dafür geschaffen, dass sich Lern-Impulse als positive Rückkopplungen innerhalb des Systems besser ausbreiten können und Lernbarrieren, die die Inflexibilität des Systemverhaltens bewirken, leichter überwunden werden.

Bei der Beantwortung der Frage, wie diese Bedingungen durch Maßnahmen auf der substanziellen Ebene geschaffen werden können, spielt aus systemtheoretischer Sicht das zu Beginn dieses Kapitels angesprochene Invarianztheorem, nach dem bestimmte abstrakte Grundphänomene in allen Arten von Systemen zu beobachten sind, eine besondere Rolle. In diesem Punkt bezieht sich die betriebswirtschaftlich ausgerichtete Systemtheorie auf neuere Erkenntnisse in naturwissenschaftichen Forschungsgebieten der Systemtheorie, insbesondere auf die von PRIGOGINE entwickelte Theorie

\footnotetext{
${ }^{327}$ Probst/Romhardt bezeichnen das Wissensmanagement in diesem Zusammenhang als ,pragmatische Weiterentwicklung von Ideen Organisationalen Lernens". Probst/Romhardt (1997), S. 130.

${ }^{328}$ Vgl. Sonntag (1997), S. 51. Die Begriffswahl steht nicht im Widerspruch zu den obigen Ausführungen über das Thema Unternehmungskultur. Vielmehr trifft der Begriff der Lernkultur ziemlich genau den entscheidenden Punkt, dass Lernen von allen Beteiligten entsprechend der geteilten Werte und Normen als wünschens- und fordernswert betrachtet wird, sodass innerhalb des Systems immer neue Anregungen zur erfolgreichen Durchführung von Lernprozessen entstehen.
} 
dissipativer Systeme. ${ }^{329}$ Die Theorie dissipativer Systeme untersucht, wie in offenen Systemen trotz der allgegenwärtigen Zunahme von Entropie im Zeitablauf, die durch den zweiten Hauptsatz der Thermodynamik beschrieben wird, neue Ordnung entstehen kann. Dissipative Strukturen im naturwissenschaftlichen Bereich besitzen einige grundlegende Eigenschaften, die unter der Annahme der Invarianz auch für betriebswirtschaftliche Zusammenhänge interessant sind: ${ }^{330}$ Sie befinden sich weit entfernt vom (thermodynamischen) Gleichgewicht, das heißt, es findet zugleich eine beträchtliche externe Zufuhr von Energie in das System und eine Abgabe von Energie des Systems an die Umwelt (Dissipation) statt. Durch positive Rückkopplung werden neue Verhaltensmuster verstärkt, wobei große Unterschiede im individuellen Verhalten der Akteuere bestehen. Unter diesen Bedingungen wird ein gleichzeitiges Auftreten von Stabilität und Instabilität innerhalb des Systems möglich. An kritischen Punkten kann es bei kleinen Veränderungen der Systemsituation zu grundlegenden Änderungen des Systemverhaltens kommen. Bei solchen Änderungen handelt es sich zum Teil um Emergenzphänomene, da sie weder auf die Absichten einzelner Akteure zurückzuführen sind, noch allein aus deren isoliert betrachteten Verhaltenseigenschaften abgeleitet werden können, sondern nur im Zusammenhang mit der Geschichte des Systems erklärt werden können. Bei übermäßiger Energiezufuhr lösen sich die Stabilitäten innerhalb des Systems allerdings wieder auf und schlagen in instabiles Chaos um. ${ }^{331}$

Überträgt man diese abstrakten Merkmale auf soziale bzw. sozio-technische Systeme, so können daraus für das Informationsmanagement einige grundsätzliche Anhaltspunkte für die Lenkung des technikunterstützten Informationssystems der Unternehmung gewonnen werden. Lernprozesse höherer Ordnung können nur dann stattfinden, wenn das (Sub-) System in einem regen Austausch mit seiner Umwelt steht und sich damit fernab von einem stabilen Verhaltensgleichgewicht befindet. Aus der Umwelt nimmt es permanent Impulse entgegen, die zu Spannungen innerhalb des Systems, z.B. in Form kognitiver Dissonanzen führen. Diese Spannungen wiederum entlädt das System durch die Abgabe von Impulsen an die Umwelt (z.B. durch Umstrukturierungen der beschaffungs- oder absatzbezogenen Anwendungssysteme), die den Abbau der auslösenden kognitiven Dissonanzen bewirken. Innerhalb des zu lenkenden Systems muss den Akteuren die Möglichkeit zur Kommunikation und zur gegenseitigen Verhaltensbeobachtung gegeben werden, damit Änderungen des bisherigen Verhaltens wahrgenommen und in das eigene Verhaltensrepertoire aufgenommen werden können. Die Anpassung des Systems insgesamt schließlich ist sowohl die Folge erfolgreicher individueller als auch beabsichtigter und emergenter überindividueller Lernvorgänge. An den kritischen Punkten bewirken kleine Änderungen der Situation große Verhaltensänderungen des Systems, sodass ein gezieltes Eingreifen möglich erscheint.

Durch die vorgenommene Analogiebildung können einige Gestaltungsempfehlungen, die in der Literatur zum Organisationalen Lernen häufig ohne nähere Begründung abgegeben werden, auf eine bessere theoretische Basis gestellt werden. Funktions- und bereichsübergreifende Kommunikations- und Sozialisationsmöglichkeiten, die als wesentliche Instrumente zur Initiierung von anhaltenden Lern- und Veränderungs-

\footnotetext{
${ }^{329}$ Zur Theorie dissipativer Systeme vgl. Prigogine (1980), passim.

${ }^{330} \mathrm{Vgl}$. Parker/Stacey (1995), S. $38 \mathrm{f}$.

${ }^{331}$ Vgl. Göbel (1998), S. 42.
} 
prozessen genannt werden, ${ }^{332}$ stellen sich vor diesem Hintergrund als Voraussetzungen für die Ausbreitung neuer Verhaltensmuster innerhalb des zu lenkenden Systems dar, mit denen zunächst einzelne Akteure auf Impulse aus der Systemumwelt reagieren. Das Lernen im Team, dem entscheidende Bedeutung für das Organisationale Lernen zugeschrieben wird $^{333}$ und das beispielsweise durch die Durchfuhrung von problembezogenen Workshops oder die Bildung interdisziplinär besetzter Projektgruppen oder Task Forces gefördert werden kann, bildet aus dieser Sicht die Grundlage für das Auftreten emergenter Verhaltensanpassungen.

Auch die Förderung des individuellen Lernens, z.B. durch allgemeine oder problembezogene Weiterbildungsangebote, kann in diesem Rahmen begründet werden, da die Verhaltensänderungen Einzelner in Folge individueller Lernprozesse die Voraussetzung für die Ausbreitung neuer Verhaltensmuster und für kollektive Lernvorgänge darstellen. $\mathrm{Da}$ der Erfolg individueller und überindividueller Lernprozesse stark von Eigenschaften abhängt, die in der Person der Lernenden selbst liegen, kann das Informationsmanagement bereits bei der Personalauswahl von Aufgabenträgern der Informationsverarbeitung Einfluss auf die zukünftige Lernfähigkeit des Systems nehmen. Neben allgemeinen Persönlichkeitsmerkmalen wie Selbstbewusstsein, Spontaneität, Lern- und Aufnahmefähigkeit fördern auch Merkmale des sozialen Verhaltens wie Kontaktfreudigkeit und Aufgeschlossenheit gegenüber Neuem die Lernfähigkeit der Mitarbeiter ${ }^{334}$ und eignen sich daher als Auswahlkriterium unter dem Aspekt der Förderung von Lernprozessen höherer Ordnung.

Durch die angesprochenen Gestaltungsoptionen kann das Informationsmanagement die Voraussetzungen für das Auftreten von Lernprozessen höherer Ordnung innerhalb des technikunterstützten Informationssystems der Unternehmung verbessern, ohne die Lernprozesse selbst zu steuern. Ferner können punktuelle Steuerungseingriffe im richtigen Moment dem System entscheidende Impulse zur Anpassung an veränderte Situationen geben. Solche Maßnahmen können beispielsweise in dem gezielten Einsatz externer Berater für abgegrenzte Aufgabenbereiche oder in der temporären Einrichtung fachlich gemischter Teams zur Lösung konkreter Problemstellungen liegen. Nach dem Abschluss des jeweiligen Lernprozesses sind die Maßnahmen jedoch zu beenden, da es sonst entsprechend der Annahmen der Theorie dissipativer Systeme zur Überreizung des Systems kommt und das Systemverhalten insgesamt instabil wird.

Zur Verdeutlichung dieser Überlegungen sei hier erneut auf das Beispiel der Touristikunternehmung, die zunächst den E-Commerce als neuen Vertriebsweg erschließt und dann ihr gesamtes Geschäft auf E-Commerce umstellt, eingegangen. Für das technikunterstützte Informationssystem der Unternehmung als Ganzes ist durch das Informationsmanagement die Fähigkeit herzustellen, Impulse aus der Umwelt aufzunehmen und die Ergebnisse individueller Lernvorgänge unternehmungsweit überindividuell verfügbar zu machen. Die erste Zielsetzung kann beispielsweise durch das Entsenden von Aufgabenträgern der Informationsverarbeitung zu branchenbezogenen Workshops und zu IV-bezogenen Kongressen erreicht werden. Die betreffenden Mitarbeiter werden dadurch in die Lage versetzt, allgemeine Entwicklungen in der Umwelt des Systems zur Kenntnis zu nehmen und deren Chancen und Risiken für das

${ }^{332}$ Vgl. Emmrich (1997), S. 113f.; Steinmann/Hennemann (1997), S. 42.

${ }^{333}$ Vgl. Senge (1990), S. $19 f$.

${ }^{334}$ Vgl. Strauß (1996), S. 50, sowie die dort zitierten Quellen. 
eigene System kritisch zu überprüfen. Auf derartigen Veranstaltungen können sich die Mitarbeiter beispielsweise über allgemeine fachliche und technische Potenziale des Internet und speziell des E-Commerce informieren.

Neue Ideen als Ergebnis individueller Lernprozesse müssen die Möglichkeit haben, sich auf dem Wege des formalen und informalen funktions- und bereichsübergreifenden Gedankenaustausches innerhalb des technikunterstützten Informationssystems der Unternehmung zu verbreiten. Auf diese Weise könnte etwa der Vorschlag zur Durchführung eines Pilotprojekts mit dem Ziel, erste Erfahrungen im Bereich des ECommerce zu sammeln, Bekanntheit und Akzeptanz innerhalb des Systems gewinnen. Die zentrale Lenkungsinstanz des Informationsmanagements entscheidet schließlich über die Einrichtung eines solchen Projektes und besetzt dieses mit Mitarbeitern und zieht in begrenztem Umfang externe Berater hinzu, um das verfuggbare Know-how zu vergrößern und bessere Ergebnisse der überindividuellen Lernvorgänge innerhalb der Projektarbeit zu erzielen. Nach der Einführung des neuen Anwendungssystems findet eine laufende dezentrale Lenkung durch die Fachabteilung statt, die in diesem Zusammenhang als Single-Loop Learning interpretiert werden kann. Innerhalb der kreativen Atmosphäre des Systems können sich im Laufe der Zeit auf Grund des Erfolgs des neuen Vertriebswegs und der Fortentwicklung des am Markt verfügbaren informationstechnischen Angebotes Denkanstöße entwickeln, die in die Richtung einer Neugestaltung des Gesamtgeschäftes der Touristikunternehmung zielen. Eine zentrale Lenkungsinstanz des Informationsmanagements könnte davon ausgehend Vorstudien einleiten und schließlich ein größeres Projekt initiieren, das erneut in moderatem Umfang durch externe Berater unterstützt wird.

Durch ein derartiges Vorgehen kann das Informationsmanagement die Lernprozesse höherer Ordnung innerhalb des Systems fördern, ohne im Voraus die Lernergebnisse vorzugeben. Dieses Beispiel macht auch deutlich, dass der systeminterne Wandel in kleinen Schritten stattfinden kann, ohne dass radikale Umstrukturierungen innerhalb des Systems erforderlich werden. ${ }^{335}$

\subsection{Aussagekraft des Systemorientierten Ansatzes für eine Theorie des Informationsmanagements}

Der Systemorientierte Ansatz ist für die Betriebswirtschaftslehre von nachhaltigem Einfluss gewesen. ${ }^{336} \mathrm{Er}$ hat sie für Ideen und Erkenntnisse aus anderen Wissenschaften geöffnet. Dabei handelt es sich nicht nur um sozialwissenschaftiche, sondern auch um naturwissenschaftliche und logische Disziplinen. Durch die Entwicklung einer allgemeingültigen, disziplinenübergreifenden Sprache sollten in diesem

\footnotetext{
${ }^{335}$ Das Ausmaß der durch Lernprozesse höherer Ordnung anzustrebenden Veränderungen ist in der Diskussion zum Organisationalen Lernen umstritten. Während Argyris/Schön ursprünglich von der Möglichkeit des Double-Loop Learning in kleinen Schritten ausgingen (vgl. Argyris/Schön (1978), S. 25f.) und auch die Vertreter des evolutionären Managements die Notwendigkeit eines inkrementalen Vorgehens betonen (vgl. Göbel (1998), S. 112), wird in späteren Arbeiten häufig das Double-Loop Learning mit radikalen, diskontinuierlichen Wandelprozessen gleichgesetzt (vgl. Wiegand (1996), S. 214). Dem kann aber entgegen gehalten werden, dass die Beobachtung, dass in manchen Situationen radikale Veränderungen die einzig sinnvolle Lösung zu sein scheinen, hăufig dadurch erklärt werden kann, dass zuvor notwendige kleinere Anpassungen nicht stattgefunden haben. Vgl. Malik (1992), S. 327.

${ }^{336} \mathrm{Vgl}$. Steinmann/Schreyögg (1993), S. 64.
} 
Zusammenhang die Voraussetzungen für einen möglichst unverfälschten Erkenntnistransfer zwischen den einzelnen Wissenschaften geschaffen werden. ${ }^{337}$ Die Ausnutzung nachbarwissenschaftlicher Erkenntnisse bei der Lösung eigener Probleme birgt ein erhebliches heuristisches Potenzial, das zu einer Reihe von neuen und originellen Vorschlägen des Systemorientierten Ansatzes geführt hat.

Die nachfolgenden Ausführungen nehmen eine kritische Überprüfung des in diesem Kapitel entwickelten Systemorientierten Ansatzes für eine Theorie des Informationsmanagements vor. Dazu werden in Abschnitt 4.4.1 zunächst Lösungsansätze für die im dritten Kapitel beschriebenen idealtypischen Probleme der Informationsverarbeitung in der Praxis aufgezeigt, die aus den hier aufgestellten theoretischen Aussagen ableitbar sind. Anschließend erfolgt in Abschnitt 4.4.2 eine kritische Würdigung der Erkenntnisgrenzen eines sich auf die Systemorientierte Betriebswirtschaftslehre stützenden Ansatzes für eine Theorie des Informationsmanagements.

\subsubsection{Lösungsansätze für offene Probleme in der Praxis}

Der Systemorientierte Ansatz für eine Theorie des Informationsmanagements nähert sich den zu erfüllenden Gestaltungs- und Steuerungsaufgaben unter dem Aspekt der Bewältigung von Komplexität mit dem Ziel, das Überleben des Systems sicherzustellen. Es werden Möglichkeiten zur Steigerung der Lenkungskapazität gesucht, die sowohl in kurzfristiger als auch in langfristiger Perspektive die Stabilität des Systemverhaltens erhöhen. Dementsprechend steht die Frage der angemessenen Reaktion auf vorübergehende und dauerhafte Veränderungen im Vordergrund der Betrachtung. Das Informationsmanagement kann dabei die Stabilität des technikunterstützten Informationssystems der Unternehmung, das durch eine hohe System- und Umweltdynamik gekennzeichnet ist, einerseits durch konservativ orientierte, andererseits durch innovativ orientierte Maßnahmen erhöhen.

Analog zum Vorgehen im vorangegangenen dritten Kapitel wird auch in diesem Kapitel die Aussagekraft des Ansatzes daran gemessen, inwiefern Anhaltspunkte zur Lösung der im zweiten Kapitel beschriebenen idealtypischen Problemstellungen geliefert werden. Dementsprechend beziehen sich die nachfolgenden Abschnitte 4.4.1.1, 4.4.1.2, 4.4.1.3 und 4.4.1.4 auf die einzelnen idealtypischen Problemstellungen in der Praxis und beleuchten den Beitrag, den der Systemorientierte Ansatz für eine Theorie des Informationsmanagements zur Lösung dieser Problemstellungen liefern kann.

\subsubsection{Effektivität der Informationsverarbeitung}

Die Effektivität der Informationsverarbeitung im Hinblick auf das Überlebensziel steht in besonderem Maße im Blickpunkt eines systemtheoretisch ausgerichteten Informationsmanagements. Die sowohl aus der Perspektive der Kybernetik I als auch der der Kybernetik II begründbare Dezentralisierung der Lenkungskompetenzen entfaltet in diesem Zusammenhang eine ambivalente Wirkung. Einerseits verspricht die Verlagerung von Entscheidungszuständigkeiten auf die einzelnen Subsysteme sowohl insgesamt als auch bezüglich der einzelnen Subsysteme eine erhebliche Ausweitung der

${ }^{337}$ Vgl. Raffée (1993), S. 34. 
Lenkungskapazität, die die Voraussetzung für eine verbesserte Zielerreichung der Teilsysteme und des Gesamtsystems darstellt. Andererseits können gerade durch diese Dezentralisierung zentrifugale Tendenzen entstehen, die den Grad der Zielerreichung des Gesamtsystems in Folge eines nicht zu kontrollierenden Auseinanderdriftens der Subsysteme vermindern. Im Folgenden werden zunächst die Potenziale zur Steigerung der Effektivität, die sich aus den vorangegangenen theoretischen Ausfuhrungen ergeben, verdeutlicht und anschließend werden dysfunktionale Effekte und Möglichkeiten zur Dämpfung dieser Effekte erörtert.

Eine Erhöhung der Effektivität der Informationsverarbeitung kann aus Sicht der Systemorientierten Betriebswirtschaftslehre dadurch bewirkt werden, dass die zentrale Lenkungsinstanz des Informationsmanagements ihre Maßnahmen auf die Meta-Ebene verlagert. Obwohl es auf den ersten Blick so scheint, als ob durch ein derartiges Vorgehen Einflussmöglichkeiten der zentralen Instanz aus der Hand gegeben werden und deren Lenkungskapazität in Folge dessen reduziert wird, erhöht sich dadurch die Lenkbarkeit des technikunterstützten Informationssystems im Hinblick auf die übergeordneten Ziele der Unternehmung. Die Lenkungskapazität insgesamt vervielfacht sich durch die Einrichtung zusätzlicher Entscheidungsstellen nach dem Subsidiaritätsprinzip. Zugleich wird der Informationsstand der jeweils zuständigen Stelle, die „vor Ort" schneller über die subsystemspezifischen Informationen verfügen kann als dies einer zentralen Instanz möglich ist, verbessert. Die zentrale Instanz des Informationsmanagements beschränkt sich dabei auf solche Maßnahmen, die das Gesamtsystem betreffen und grundsätzlichen, vom Einzelfall abstrahierenden Charakter besitzen. Auf diese Weise wird die prinzipielle Unmöglichkeit der exakten Lenkung komplexer Systeme anerkannt und Fehlsteuerungen, die aus der illusionären Machbarkeitsvorstellung mechanistischer Ansätze des Managements resultieren, werden vermieden.

Die Kybernetik I zeigt Möglichkeiten auf, durch die die einzelnen Lenkungsinstanzen das Ausmaß der Erreichung vorgegebener Ziele für die von ihnen zu lenkenden Teilsysteme erhöhen und damit die Effektivität der Informationsverarbeitung durch diese Teilsysteme verbessern können. Durch passive und aktive Maßnahmen können Störungen abgewehrt und die Varietät des Systemoutputs, die in der Abweichung vom durch die Systemziele festgelegten Zustand zum Ausdruck kommt, reduziert werden. Die als Mischlenkung bezeichnete Kombination der Prinzipien von Steuerung und Regelung als aktiven Maßnahmentypen und der zusätzliche Schutz des Systems durch passive Maßnahmen, die durch dezentrale Instanzen des Informationsmanagements erfolgt, bewirkt aus Sicht der Kybernetik I die größtmögliche Effektivität der Informationsverarbeitung innerhalb der gegebenen System/Umweltsituation.

Ungeklärt bleibt in diesem Zusammenhang allerdings, auf welche Weise die Ziele für die einzelnen Teilsysteme festgelegt werden. Aus der Systemtheorie geht hier lediglich hervor, dass die Teilsysteme Leistungen für das technikunterstützte Informationssystem der Unternehmung als Ganzes erbringen, welches wiederum Funktionen für das übergeordnete Gesamtsystem Unternehmung erfullt. Daraus ergibt sich normativ, dass die jeweils zu verfolgenden Ziele aus den Zielen der Unternehmung abzuleiten sind, ohne dass jedoch Methoden zur Lösung dieser Problemstellung näher betrachtet werden. Es wird auch nicht darauf eingegangen, welches die übergeordneten Ziele der Unternehmung im Einzelnen sind. Da das oberste Unternehmungsziel systemtheoretisch äußerst abstrakt als Überleben der Unternehmung definiert wird, können hier 
keine allgemeinen Aussagen über die zu verfolgende Zielsetzung getroffen werden, die im praktischen Einzelfall unmittelbar durch das Informationsmanagement zu übernehmen wären. Die Bezugnahme auf den abstrakten Sachverhalt Überleben legt allerdings eine breite Orientierung des Informationsmanagements im Sinne eines Stakeholder-Ansatzes nahe, da vielfältige Ansprüche unterschiedlichster Interessengruppen zu erfüllen sind, um den dauerhaften Fortbestand der Unternehmung und ihres technikunterstützten Informationssystems zu gewährleisten. Aus der im Vergleich zu traditionellen Management-Ansätzen größeren Betonung einer ganzheitlichen Perspektive folgt außerdem die Notwendigkeit zur verstärkten Beachtung unternehmungsexterner Wettbewerbserfordernisse bei der Lenkung der verschiedenen Subsysteme.

In der längerfristigen Perspektive, die von der Kybernetik II verfolgt wird, reicht die Einhaltung von ex ante vorgegebenen Zielzuständen nicht aus, um das fortdauernde Überleben komplexer Systeme zu gewährleisten. Die dynamische Anpassung des Systemverhaltens und gegebenenfalls auch der Systemziele selbst gewährleisten aus dieser Sicht erst die Überlebensfähigkeit des Systems und werden damit zum eigenständigen Ziel höherer Ordnung, also zum Meta-Ziel, für das eigene Effektivitätskriterien gelten. Die Effektivität des technikunterstützten Informationssystems der Unternehmung ist unter diesem Gesichtspunkt dann gegeben, wenn das System in der Lage ist, erfolgreiche Lernprozesse höherer Ordnung durchzuführen und sich so an neue Entwicklungen anzupassen. Das Informationsmanagement kann auf dieser Betrachtungs- und Gestaltungsebene die Effektivität des von ihm gelenkten Systems erhöhen, indem es eine kreative Atmosphäre schaff, in der gute Voraussetzungen für die Entstehung und Verbreitung neuer Ideen bestehen, die das Verhaltensrepertoire des Gesamtsystems und seiner Subsysteme erhöhen. Die Lernvorgänge selbst müssen allerdings durch die menschlichen Aufgabenträger innerhalb des Systems durchgeführt werden und können nicht durch eine zentrale Lenkungsinstanz übernommen werden. Einige systemtheoretisch begründbare Maßnahmen zur Förderung von Lernprozessen höherer Ordnung, z.B. die Institutionalisierung von Gelegenheiten zum funktions- und bereichsübergreifenden Gedankenaustausch und die Förderung individueller Lernprozesse durch die Schaffung von Möglichkeiten zur Teilnahme an allgemeinen und fachlichen Aus- und Weiterbildungsmaßnahmen, wurden im vorangegangenen Teilkapitel genannt.

Dysfunktionale Effekte, die mit der Zuordnung von Lenkungskompetenzen nach dem Subsidiaritätsprinzip verbunden sind, ergeben sich aus den reduzierten Möglichkeiten der zentralen Instanz zur Einflussnahme im konkreten Einzelfall. Die Effektivität der Informationsverarbeitung wird dadurch gefährdet, dass die Subsysteme selbstständig Entscheidungen treffen und dabei über einen nicht zu überbrückenden Informationsvorsprung gegenüber der Zentrale verfügen. Eine zentrale Kontrolle dieser Entscheidungen ist somit nur in eingeschränktem Maße möglich, sodass es zu beabsichtigten oder unbeabsichtigten Fehlsteuerungen kommen kann, wenn die dezentralen Entscheidungsträger zu stark die Belange des jeweiligen Teilsystems und nicht die des Gesamtsystems und der Gesamtunternehmung im Blick haben.

In diesem Zusammenhang kommt der Unternehmungskultur eine bedeutende Rolle zu. Wenn die verschiedenen Entscheidungsträger innerhalb des technikunterstützten Informationssystems der Unternehmung einen gemeinsamen Basiskonsens über die innerhalb der Unternehmung geltenden Werte und Normen teilen, dann kann eine 
solche Unternehmungskultur eine Integrations- und Koordinationsfunktion erfullen, die den mit dem Subsidiaritätsprinzip verbundenen Differenzierungstendenzen entgegenwirkt. Die Effektivität der einzelnen Subsysteme wird in diesem Fall dadurch erhöht, dass die dezentralen Lenkungsinstanzen sich bei ihren Entscheidungen von den in der Unternehmungskultur enthaltenen Werten und Normen leiten lassen und somit verstärkt zur Erfüllung der Ziele des übergeordneten Gesamtsystems beitragen. Der Einfluss des Informationsmanagements auf dieses Instrument zur Effektivitätssteigerung ist begrenzt, da den Mitarbeitern die gemeinsamen Werte und Normen nicht unmittelbar von außen vorgegeben werden können. Durch die Beachtung der symbolischen Wirkungen von sprachlichen Artefakten, Handlungen und materiellen Objekten kann es dem (zentralen) Informationsmanagement dennoch gelingen, die Mitarbeiter von bestimmten Werten und Normen zu überzeugen und langfristig gesehen das Denken und Handeln der Menschen innerhalb des zu lenkenden Systems durch die Vermittlung von Sinn zu beeinflussen. Daraus ergeben sich für die Aufgabenträger der Informationsverarbeitung allerdings keine direkten Handlungsanweisungen im Einzelfall, sodass die Wirkung symbolischer Maßnahmen auf die Effektivität der Informationsverarbeitung eher langfristigen Charakter hat.

\subsubsection{Effizienz der Informationsverarbeitung}

Der Aspekt der Effizienz steht in der Systemorientierten Betriebswirtschaftslehre weniger im Vordergrund der Betrachtung als der der Effektivität. Im Hinblick auf das Überleben als oberstes Systemziel erscheinen vorübergehende und auch dauerhafte, jedoch vergleichsweise geringfügige Inefizienzen akzeptabel, wenn dadurch die Stabilität des Systemverhaltens insgesamt erhöht wird. In der Kybernetik I schlägt sich diese Abkehr von der reinen Produktivitätsorientierung in der positiven Beurteilung von passiven Maßnahmen zur Abwehr von Störungen, z.B. Kapazitätsreserven, nieder. Diese verursachen im Normalfall, d.h. im Fall des Nicht-Vorliegens größerer Störungen, lediglich Kosten, denen nicht unmittelbar ein quantitativ messbarer Nutzen gegenübersteht.

Diese relativ geringe Effizienzorientierung ist ebenfalls in der Kybernetik II in verschiedenen Punkten erkennbar. So impliziert etwa die empfohlene Steigerung des Selbststeuerungsanteils, dass Synergiepotenziale durch Economies of Scale zu Gunsten der höheren Selbstständigkeit der Subsysteme vielfach ungenutzt bleiben. Auch der langfristige Nutzen von symbolgeladenen Handlungen, z.B. regelmäßig wiederkehrenden Dienstbesprechungen oder unternehmungsinternen Aus- und Weiterbildungsangeboten, die zum einen der Pflege von Unternehmungskultur und zum anderen der Entstehung und Verbreitung von Lernimpulsen dienen, ist häufig nicht direkt erkennbar. Unter kurzfristigen Effizienzgesichtspunkten wirken sich deshalb viele der aus der Sicht der Kybernetik II theoretisch begründbaren Maßnahmen des Informationsmanagements ungünstig aus.

Allerdings ist das mittel- und langfristige Überleben von Unternehmungen in marktwirtschaftlichen Systemen mit wirksamen Wettbewerbselementen nur dann gesichert, wenn die Herstellung von am Markt absetzbaren Produkten ein konkurrenzfähiges Maß an Effizienz nicht unterschreitet. Dies gilt selbst dann, wenn die Unternehmung mit ihrer Strategie nicht auf Kostenführerschaft, sondern auf ein qualitativ hochwertiges Angebot, für das vergleichsweise hohe Preise am Markt durchsetzbar sind, abzielt. Effizienz wird somit auch aus der Perspektive der Systemorientierten Betriebswirt- 
schaftslehre zur Restriktion, die ein relevantes (Neben-) Ziel für die technikunterstützte Informationsverarbeitung in der Unternehmung darstellt. Von dieser Überlegung ausgehend können Maßnahmen zur Steigerung der Effizienz der Informationsverarbeitung aus den theoretischen Ausführungen dieses Kapitels rekonstruiert werden. Versteht man die Wirtschaftlichkeit der Informationsverarbeitung als Zielsetzung, deren Erreichen durch die Maßnahmen der Lenkungsinstanz sicherzustellen ist, so können sowohl Maßnahmen des konservativen als auch des innovativen Informationsmanagements zur Erfüllung des Effizienzziels beitragen. Das operative Informationsmanagement kann die abstrakten Prinzipien der Steuerung und der Regelung anwenden und kombinieren, um die Produktivität der Informationsverarbeitung zu erhöhen.

Allerdings gehen die Aussagen zur Steuerung und Regelung von gegebenen Zieldefinitionen aus. Das bedeutet, dass durch die Systemtheorie keine Anhaltspunkte dafür ableitbar sind, welche betriebswirtschaftlichen Kriterien zur Beurteilung der Wirtschaftlichkeit der Informationsverarbeitung zu Grunde gelegt werden sollen. Vielmehr beziehen sich die Überlegungen zum konservativen Informationsmanagement darauf, wie durch vorwärts- und rückwärtsgerichtete negative Rückkopplungsprozesse die Abweichung von den vorgegebenen Wirtschaftichkeitszielen möglichst gering gehalten werden kann. Bei rein technischen Prozessen und bei relativ gut strukturierten sozio-technischen Prozessen der Informationsverarbeitung, für die sich vergleichsweise genaue Input/Output-Relationen ermitteln lassen, kann auf Störungen des Systems so reagiert werden (z.B. durch die Nivellierung der technischen Auslastung oder die Anpassung von Dienstplänen), dass ein möglichst hohes Produktivitätsniveau gewährleistet wird. Für den laufenden Betrieb von Systemen lässt der Systemorientierte Ansatz für eine Theorie des Informationsmanagements außerdem die Aussage zu, dass unter Effizienzgesichtspunkten generelle Regelungen vorteilhafter sind als fallweise Regelungen, wenn über einen längeren Zeitraum hinweg unverändert gültige Muster des System/Umweltverhaltens zu erkennen sind. Dies ist damit zu begründen, dass generelle Regelungen wiederholt zum Einsatz kommen und damit die Kapazität der Lenkungsinstanz entlasten.

Die in der Unternehmung vorhandene Kenntnis über möglichst effiziente Verfahren der Informationsverarbeitung kann aus der Perspektive der Kybernetik II zudem als Teil der Tiefenstruktur der Organisatorischen Wissensbasis aufgefasst werden. So gesehen können Projekte zur Neugestaltung von Prozessen mit dem Ziel der Produktivitätserhöhung als Lernvorgänge höherer Ordnung aufgefasst werden, mit denen das technikunterstützte Informationssystem sich im Sinne der Kybernetik II innovativ an größere Veränderungen (etwa die Verfügbarkeit einer neuen Softwaregeneration am Markt oder den Druck zur Übernahme neuer organisatorischer Konzepte wie z.B. des Supply Chain-Managements) der Unternehmungssituation anpasst. Diese Lernprozesse höherer Ordnung können durch die im Abschnitt 4.3.2.4 angesprochenen Maßnahmen des Informationsmanagements, die sich auf die Schaffung von Möglichkeiten zu individuellem Lernen und die Begünstigung der Ausbreitung von Lernimpulsen innerhalb des technikunterstützten Informationssystems der Unternehmung richten, gefördert werden.

Die Suche nach immer neuen Effizienzsteigerungen kann außerdem auf der symbolischen Ebene des Informationsmanagements durch die Förderung einer Unternehmungskultur unterstützt werden, deren integraler Bestandteil eine positive 
Grundhaltung der Mitarbeiter als Aufgabenträger der Informationsverarbeitung gegenüber technischen und organisatorischen Innovationen ist. Dadurch kann die Richtung der angesprochenen Lernprozesse höherer Ordnung beeinflusst und das System somit auf einen Entwicklungspfad gebracht werden, auf dem die stetige Verbesserung der Wirtschaftlichkeit von Prozessen der Informationsverarbeitung einen hohen Stellenwert einnimmt.

\subsubsection{Organisatorische Zuordnung informationswirtschaftlicher Aufgaben}

Für die Frage der organisatorischen Zuordnung informationswirtschaftlicher Aufgaben weist der Systemorientierte Ansatz für eine Theorie des Informationsmanagements ein besonders hohes Aussagepotenzial auf. Schaut man zunächst auf die Führungsaufgaben der Informationsverarbeitung, also das Informationsmanagement selbst, so stellt das Gesetz der erforderlichen Varietät den Dreh- und Angelpunkt der Argumentation dar. Entsprechend des Gesetzes der erforderlichen Varietät kann eine zentrale Lenkungsinstanz die Aufgaben des Informationsmanagements immer dann erfolgreich erfüllen, wenn die Lenkungskapazität dieser Instanz so hoch ist, dass sie in der Lage ist, die äußere und innere Störvarietät des Systems auszugleichen und den Systemoutput innerhalb der durch die Systemziele festgelegten Grenzen zu halten. Die Lenkungskapazität einer Instanz bestimmt sich dabei durch ihre Möglichkeiten zur Wahrnehmung der externen und internen Systemsituation und zur Reaktion darauf durch wirksame Einflußnahmen auf das Systemverhalten.

Die Frage ist also, ob die Lenkungskapazität bei einer zentralen Zuordnung von Aufgaben des Informationsmanagements hoch genug ist, um Störungen des technikunterstützten Informationssystems der Unternehmung so weit auszugleichen, dass die Stabilität des Systemverhaltens gewährleistet werden kann. Zur Beantwortung dieser Frage ist das technikunterstützte Informationssystem der Unternehmung als Lenkungsgegenstand des Informationsmanagements näher zu betrachten. Dieses System wurde in Abschnitt 4.2.1 als sozio-technisches System mit Menschen und Maschinen als Elementen charakterisiert, die bei der Erstellung informationeller Produkte zusammenwirken. Die hohe Komplexität dieses Systems auf Grund der hohen Umweltvarietät und der vielfältigen internen Rückkopplungen des Verhaltens der einzelnen Akteure wurden ebenfalls herausgearbeitet. Das Verhalten des Systems ist von der Geschichte des Systems abhängig und kann deshalb nicht durch exakte Kausalzusammenhänge beschrieben werden. Damit stehen dem Informationsmanagement auch keine determinierten Input/Outputbeziehungen als Grundlage für Lenkungsmaßnahmen zur Verfügung. Vielmehr weist das System als Ganzes eine begrenzte Lenkbarkeit auf und kann deshalb nicht exakt in einem ex ante definierten Gleichgewichtszustand gehalten werden.

Aus diesem Grund ist die Bildung von Subsystemen erforderlich, die durch jeweils eigene Entscheidungsinstanzen dezentral gelenkt werden. Die mehrstufige Aufteilung des Systems bewirkt eine Verringerung der System- und Umweltvarietät der jeweils zu lenkenden Einheit. Gleichzeitig kommt es zu einer Vervielfachung der Zahl der Instanzen und allein schon dadurch zu einer Erhöhung der Lenkungskapazität. Die dezentralen Entscheidungsträger verfügen außerdem über einen weitaus besseren Informationsstand hinsichtlich der aktuellen System- und Umweltsituation der von ihnen zu lenkenden Subsysteme als eine zentrale Instanz. Eine hohe Lenkungskapazität 
erfordert ein hohes Maß an Varietät des Reglers, sodass den dezentralen Instanzen sowohl aus der Sicht der Kybernetik I als auch aus der Sicht der Kybernetik II eine hohe Entscheidungsfreiheit einzuräumen ist. Diese hohe Entscheidungsfreiheit der Teilsysteme stellt die Voraussetzung für die Stabilität aller Arten von Systemen dar, die einer hohen Störvarietät ausgesetzt sind.

Bis zu diesem Punkt kommen die beiden Forschungsrichtungen der Kybernetik also in der Frage der Zuordnung informationswirtschaftlicher Aufgaben zu einander entsprechenden Ergebnissen. Erst dann laufen die Argumentationslinien der Kybernetik I und II auseinander. Die Kybernetik I geht davon aus, dass die Bildung von Subsystemen so weit fortgesetzt werden kann, bis die Komplexität des jeweils zu lenkenden Teilsystems so weit reduziert ist, dass die zuständige Entscheidungsinstanz über eine ausreichend hohe Lenkungskapazität verfügt, um die auf das Teilsystem wirkende Störvarietät vollständig aufzuheben. Kommt es zu veränderten Anforderungen an das Subsystem durch Veränderungen der Gesamtsituation, so werden Eingriffe durch die jeweils höhere Lenkungsinstanz nötig, um das Gesamtverhalten des Subsystems an die neue System/Umweltsituation anzupassen. Durch die Kombination aktiver und passiver Lenkungsmaßnahmen und die Überlagerung von Regelkreisen auf unterschiedlichen Aggregationsniveaus innerhalb des Gesamtsystems ist in einem solchen Fall die exakte Bestimmung des Systemoutputs möglich. Dementsprechend ist das technikunterstützte Informationssystem der Unternehmung so weit in dezentral gelenkte Subsysteme zu zerlegen, bis die einzelnen Aufgabenträger des Informationsmanagements in der Lage sind, die von ihnen gelenkten Subsysteme vollständig zu kontrollieren. Die dezentralen Lenkungsinstanzen werden dabei selbst wiederum als Teil der Regelstrecke eines übergeordneten Regelkreises aufgefasst, der durch einen zentraleren Regler kontrolliert wird.

Dagegen geht die Kybernetik II von abweichenden Voraussetzungen aus. Eine vollständige Lenkbarkeit des technikunterstützten Informationssystems der Unternehmung ist auch durch eine fortgesetzte Subsystembildung nicht erreichbar, da die Komplexität durch Subsystembildung zwar reduziert, jedoch nicht so weit aufgelöst werden kann, dass die einzelnen Subsysteme vollständig beherrschbar werden. Demzufolge kann die Lenkungskapazität durch die Einrichtung dezentraler Entscheidungsinstanzen nicht in ausreichendem Maße erhöht werden, um das Verhalten des Systems als Ganzes endgültig zu stabilisieren. Die Anpassung an sich wandelnde Situationen wird aus dieser Perspektive nicht durch die übergeordneten Entscheidungsträger im Sinne eines mehrstufigen Regelkreises ausgelöst, sondern sie geht von den Teilsystemen selbst aus. Die Selbstständigkeit der Subsysteme, also der Grad der Dezentralisierung von Aufgaben des Informationsmanagements, geht in diesem Fall noch weiter als aus der Sicht der Kybernetik I.

Zentrale Eingriffe sind nach dieser Auffassung nur deshalb nötig, weil dadurch die Erwartbarkeit des Subsystemverhaltens für die anderen Subsysteme innerhalb des technikunterstützten Informationssystems erhöht wird. Durch zentrale Lenkungsmaßnahmen auf der Meta-Ebene reduziert sich die Störvarietät für die einzelnen Teilsysteme, da für das jeweils betrachtete Subsystem die anderen Subsysteme zur Umwelt gehören. Eine Zentralisierung von Aufgaben des Informationsmanagements ist dementsprechend nur dann sinnvoll, wenn es durch die betreffenden Lenkungsmaßnahmen zu Umgestaltungen an den Schnittstellen des Subsystems kommt, sodass weitere Subsysteme innerhalb des Gesamtsystems betroffen sind. In der Sprache der 
Systemtheorie bedeutet das, dass der Ausgang des jeweiligen Teilsystems, der zugleich den Eingang für weitere Teilsysteme darstellt, Änderungen unterworfen wird. Die Aufgabe der zentralen Instanz sollte sich dementsprechend auf die Abstimmung der Ziele der Informationsverarbeitung mit den allgemeinen Unternehmungszielen, die Bildung geeigneter Teilsysteme und die Kopplung dieser Teilsysteme zu einem funktionsfähigen Gesamtsystem beschränken. Dabei kann die Integration der Subsysteme durch zentral angesiedelte Maßnahmen auf der symbolischen Ebene unterstützt werden, die den inneren Zusammenhalt des Gesamtsystems erhöhen.

Die Auswirkungen des informationstechnischen Fortschrittes führen in diesem Zusammenhang dazu, dass die Lenkungskapazitäten der einzelnen Instanzen erhöht werden, da deren Informationsstand durch den Einsatz innovativer Informationstechnik (insbesondere im Bereich der Management Support Systeme) verbessert wird. Aus den in Kap. 4.3.2.1 dargestellten Überlegungen von HAYEK kann jedoch geschlussfolgert werden, dass die grundsätzlichen Informationsunvollkommenheiten einer zentralen Instanz, die die Motivation für eine weitgehend dezentrale Ansiedlung von Aufgaben des Informationsmanagements darstellen, dadurch prinzipiell nicht zu überwinden sind. Aus der Sicht des Systemorientierten Ansatzes für eine Theorie des Informationsmanagements beinhalten Weiterentwicklungen der Informationstechnik deshalb Potenziale für eine steigende Lenkungskapazität der dezentralen Entscheidungsinstanzen, ohne dadurch die grundsätzliche Vorteilhaftigkeit der dezentralen Zuordnung von Aufgaben des Informationsmanagements umzukehren.

Aus dem Systemorientierten Ansatz für eine Theorie des Informationsmanagements können auch Aussagen über die organisatorische Zuordnung von Durchführungsaufgaben der Informationsverarbeitung abgeleitet werden. Die organisatorische Zuordnung der Durchführungsaufgaben ist in den vorangegangenen Überlegungen zur Zuordnung von Führungsaufgaben bereits implizit enthalten. Die unter dem Aspekt der Lenkbarkeit von Systemen aus der Systemorientierten Betriebswirtschaftslehre ableitbare Empfehlung zur Bildung kleiner, weitgehend selbstständig agierender Subsysteme erstreckt sich auch auf die Durchführungsaufgaben der Informationsverarbeitung. Die dezentrale Ansiedlung dieser Aufgaben erhöht die Autonomie der Subsysteme, da sie die Beziehungen der einzelnen Subsysteme untereinander reduziert. Einzelne Subsysteme werden unabhängiger von den Vorgängen in den benachbarten Subsystemen, da sich Störungen der benachbarten Systeme nicht auf sie auswirken.

Diese Überlegung sei an einem Beispiel verdeutlicht. In einer Unternehmung, in der mehrere Divisionen eigenständig produzieren und ihre Produkte am Markt veräußern, ist es aus der Sicht der Systemorientierten Betriebswirtschaftslehre sinnvoll, wenn diese auch die dabei anfallenden Aufgaben der Informationsverarbeitung eigenständig übernehmen. Beispiele für dezentral anzusiedelnde Durchführungsaufgaben in diesem Zusammenhang sind etwa die Auftragsannahme im Vertrieb, der Betrieb eines Datenbanksystems, in dem die dabei anfallenden Transaktionsdaten gespeichert werden, oder die Abwicklung eines informationstechnischen Benutzerservice. Wenn diese Funktionen zentral durchgefürt werden, dann kommt es zu Interdependenzen mit den anderen Divisionen, sodass sich Störungen der einzelnen Divisionen auf die jeweils anderen Divisionen übertragen. Kurzfristige Überlastungen einer Division oder technisch bedingte Systemausfalle wirken sich in diesem Fall auf alle beteiligten Divisionen aus. Die Lenkbarkeit der Informationsverarbeitung wird deshalb erhöht, 
wenn diese Aufgaben getrennt werden und dezentral den einzelnen Divisionen zugeordnet werden.

Die Vorteilhaftigkeit der Dezentralisierung von Durchführungsaufgaben kann allerdings nicht nur für den laufenden Betrieb der Informationsverarbeitung (also aus Sicht der Kybernetik I) begründet werden, sondern auch (aus der Sicht der Kybernetik II) im Hinblick auf die Anpassungsfähigkeit der Systeme an sich ändernde System/Umweltsituationen. Anpassungserfordernisse werden schneller erkannt, da die Situation in dezentralisierten Teilsystemen unmittelbarer wahrgenommen wird. Zudem sind Anpassungsvorgänge weitaus leichter durchzuführen, da weniger Interdependenzen zu den anderen dezentralen Einheiten bestehen, sodass geringerer Abstimmungsbedarf erforderlich ist. Anpassungsvorgänge in Folge von Lernprozessen höherer Ordnung sind deshalb in kleineren Einheiten schneller und leichter durchsetzbar als in großen zentralisierten Systemen.

\subsubsection{Rolle des Menschen in der Informationsverarbeitung}

Menschen werden im Rahmen der hier vorgestellten begrifflichen Abgrenzung des technikunterstützten Informationssystems der Unternehmung als Elemente des durch das Informationsmanagement zu lenkenden sozio-technischen Systems aufgefasst. Aus der Perspektive der an der Kybernetik I orientierten Betriebswirtschaftslehre stellt der Mensch zunächst einmal eine systeminterne Quelle von Komplexität dar, durch deren hohe Verhaltensvarietät die Anforderungen an die Lenkungskapazität im Sinne des Gesetzes der erforderlichen Varietät erhöht werden. Die von der Kybernetik I ausgehende konservative Variante des Systemorientierten Informationsmanagements sieht deshalb die Rolle des Menschen in der Erfülung von Aufgaben der Informationsverarbeitung, bei der es zu unerwünschten Abweichungen vom geplanten Verhalten kommen kann, in deren Folge der Zielerreichungsgrad des technikunterstützten Informationssystems der Unternehmung reduziert wird. Die aus der Kybernetik I abgeleiteten Maßnahmen richten sich deshalb auf eine Erhöhung der relativen Lenkungskapazität der Entscheidungsinstanz, durch die die Möglichkeit des Auftretens solcher Verhaltensabweichungen eingeschränkt bzw. deren Auswirkungen auf den Systemoutput verringert werden können.

Die an der Kybernetik II orientierte innovative Variante des Systemorientierten Informationsmanagements fasst die Verhaltensvarietät der menschlichen Aufgabenträger dagegen nicht als reinen Störfaktor auf. Sie erkennt darin vielmehr auch positive Aspekte, da Anpassungen des Systemverhaltens insgesamt durch Verhaltensänderungen einzelner Personen (oder Gruppen) ausgelöst werden. Dementsprechend gilt es für das Informationsmanagement, die Komplexität des menschlichen Verhaltens nicht zu beseitigen, sondern sie entsprechend der Systemziele so zu kanalisieren, dass die Anpassungsfahigkeit als Voraussetzung für das langfristige Überleben des Systems erhöht wird. Ansatzpunkte für Lenkungsmaßnahmen in diesem Sinne stellen dabei die Unternehmungskultur und die in Abschnitt 4.3.2.4 beschriebenen Möglichkeiten zur Förderung von Lernprozessen höherer Ordnung dar. Allerdings stellen diese Konzepte vergleichsweise hohe Anforderungen an die fachlichen und persönlichen Kompetenzen der Beschäftigten, die im Rahmen der Kybernetik nicht näher betrachtet werden. Es wird nicht hinterfragt, inwiefern das empfohlene starke Dezentralisierungsniveau 
möglicherweise auch zur Überforderung der Mitarbeiter auf den unteren Hierarchieebenen der Unternehmung führen kann.

Insgesamt findet im Rahmen des Systemorientierten Ansatzes für eine Theorie des Informationsmanagements eine Abkehr von dem bis dahin in der Betriebswirtschaftslehre vorherrschenden, sehr mechanistischen Menschenbild statt. Der Mensch wird hier nicht mehr nur als reiner Produktionsfaktor aufgefasst, der sich analog zu anderen Produktionsfaktoren wie ein materieller Gegenstand im Leistungserstellungsprozess der Unternehmung einsetzen lässt. Das Verhalten der menschlichen Aufgabenträger innerhalb des Systems kann vielmehr nur in eingeschränktem Maße durch die Lenkungsinstanz prognostiziert und beherrscht werden. Das Hervorheben der Tatsache, dass das aktuelle Systemverhalten durch die Geschichte des Systems beeinflusst wird, bedeutet in sozialen bzw. sozio-technischen Systemen nichts anderes als die Anerkennung der Persönlichkeit der beteiligten Individuen, deren Verhalten nicht vollständig durch eine übergeordnete Instanz manipulierbar ist. Die menschlichen Aufgabenträger innerhalb des technikunterstützten Informationssystems der Unternehmung können im Zeitablauf aus Erfahrungen lernen und ihr persönliches Verhalten dementsprechend anpassen. In diesem Sinne liegt dem Systemorientierten Ansatz des Informationsmanagements ein differenzierteres Menschenbild zu Grunde als dem im dritten Kapitel dieser Arbeit entwickelten Produktionsorientierten Ansatz.

Allerdings entwickelt auch der Systemorientierte Ansatz für eine Theorie des Informationsmanagements kein tiefergehendes Verständnis der Zusammenhänge, von denen das Verhalten der Menschen als Aufgabenträger der Informationsverarbeitung im Einzelfall abhängt. Eine verbesserte Informationsverarbeitung durch die verstärkte Berücksichtigung der Bedürfnisse der Mitarbeiter im Rahmen des Informationsmanagements ist damit nicht originäre Zielsetzung dieses Ansatzes. Die aus der Kybernetik I ableitbaren Maßnahmen richten sich im Gegenteil auf eine Reduzierung der inneren Systemvarietät, was einer Eindämmung der Möglichkeiten der Mitarbeiter zur Störung des Systemoutputs entspricht. Das Verhalten der einzelnen Individuen wird systemtheoretisch als Black box aufgefasst, die mit den Mitteln der Systemtheorie nicht weiter analysiert werden kann. Für das Informationsmanagement geht es aus der Sicht der Kybernetik I gewissermaßen darum, trotz der „Widerspenstigkeit" der Systemelemente das System mit sozial-technisch ausgerichteten Maßnahmen erfolgreich zu lenken.

Nicht viel anders sind auch die aus der Kybernetik II ableitbaren Maßnahmen einzuordnen, die eine sehr weitgehende Dezentralisierung der Lenkungskompetenzen, die Koordination und Integration der Teilsysteme durch symbolische Maßnahmen und die Kanalisierung der längerfristigen Systementwicklung zum Gegenstand haben. Die erhebliche Ausweitung der Entscheidungskompetenzen der Mitarbeiter geschieht hier nicht etwa, um die Motivation und Zufriedenheit der Beteiligten zu erhöhen und in Folge dessen die Ergebnisse der Informationsverarbeitung zu verbessern. Die Absicht liegt allein in der Verbesserung der Lenkungsmöglichkeiten durch die Nutzung des Informationsvorsprungs dezentraler Stellen gegenüber zentralen Lenkungsinstanzen. Auch die Ausweitung der Lenkungsmaßnahmen auf die symbolische Ebene geschieht letzten Endes mit der Absicht einer sozial-technologischen Instrumentalisierung der Unternehmungskultur. Diese Zielsetzung wird auch von Vertretern der gemäßigten Sichtweise, die von einer eingeschränkten Beherrschbarkeit der Unternehmungskultur ausgehen, nicht abgestritten. Als letzter hier anzuführender Punkt erfolgt auch die 
systemtheoretisch fundierte Förderung von Lernprozessen höherer Ordnung durch das Informationsmanagement nicht auf der Basis einer genauen Kenntnis des Ablaufs von Lernvorgängen, sondern allein durch die Schaffung der Voraussetzungen entsprechend einer Input/Output-Betrachtung des Auftretens solcher Prozesse.

Es bleibt daher festzuhalten, dass sich der Systemorientierte Ansatz für eine Theorie des Informationsmanagements einer differenzierteren Berücksichtigung der Rolle des Menschen in der Informationsverarbeitung annähert. Sie unternimmt damit auch wichtige Schritte in die Richtung einer Überwindung der KonstruktionskorridorVorstellung, von der die Überlegungen zur Entwicklung technikunterstützter Informationssysteme in Theorie und Praxis lange Zeit gekennzeichnet waren. Die verstärkte Partizipation der Anwender und die höhere Akzeptanz technischer Neuerungen stellen dabei allerdings mehr oder weniger Nebenprodukte dar, die in Folge der Verlagerung von Verantwortungsbereichen mit dem Ziel der Erhöhung der Lenkungskapazität entstehen. Dies gilt nicht nur für den laufenden Betrieb von Anwendungssystemen, sondern auch für Systementwicklungsprojekte. Lernprozesse höherer Ordnung, mit denen in solchen Projekten eine Anpassung des Systems an veränderte Situationen erfolgt, müssen aus Sicht der Kybernetik II unter Federführung der dezentralen Einheiten erfolgen, da sie allein über die erforderliche Kenntnis der System/Umweltsituation des anzupassenden Subsystems verfügen. Zentral zur Verfügung gestellte Fachleute für Fragen der Informationsverarbeitung können dabei nur unterstützend wirken. Trotz dieser motivationsfördernden Nebeneffekte der aus dem Systemorientierten Ansatz für eine Theorie des Informationsmanagements abgeleiteten Maßnahmen findet im Rahmen dieses Ansatzes keine wirkliche Modellierung des menschlichen Verhaltens statt.

\subsubsection{Erkenntnisgrenzen}

Die durch den Systemorientierten Ansatz erfolgte Öffnung der Betriebswirtschaftslehre für die Erkenntnisse anderer Wissenschaftsdisziplinen führt zu einer Abwendung des Erkenntnisinteresses vom reinen ökonomischen Prinzip. Davon ausgehend kommt es bei der theoretischen Betrachtung von Unternehmungen zu einer stärkeren Orientierung an in der betrieblichen Praxis vorzufindenden Problemstellungen und in Folge dessen zu einer Verbreiterung des Untersuchungsfeldes. Unternehmungen werden nicht mehr allein unter dem Aspekt der möglichst effizienten Herstellung von Produkten, sondern unter dem Aspekt der dauerhaften Existenz in dynamischen Umwelten betrachtet. Diese Ausweitung des Untersuchungsinteresses wird allerdings mit einer Verlagerung der Forschungsaktivitäten auf systemtypübergreifende, allgemeingültige Gesichtspunkte erkauft. Daraus erwachsen bedeutende Erkenntnisgrenzen für den in dieser Arbeit entwickelten Systemorientierten Ansatz für eine Theorie des Informationsmanagements. Diese Problematik wird in Abschnitt 4.4.2.1 näher betrachtet. Weitere Erkenntnisgrenzen ergeben sich insbesondere aus der teilweise fragwürdigen Bildung von Analogien zu anderen Typen von Systemen (Abschnitt 4.4.2.2) und aus der unklaren Beziehung zwischen den beiden Forschungsrichtungen der Kybernetik (Abschnitt 4.4.2.3). 


\subsubsection{Stark verallgemeinernde Betrachtungsweise}

Das Ziel, einen einheitlichen Begriffsapparat für den Erkenntnisaustausch zwischen Wissenschaftsdisziplinen zu entwickeln und zu verwenden, wird von Gegnern des Systemorientierten Ansatzes angegriffen. Die Begründung liegt in der These, dass Theorien um so brauchbarer sind, je höher der Grad ihrer Allgemeingültigkeit ist, während bei Begriffen jedoch das Gegenteil der Fall ist. ${ }^{338}$ Je differenzierter ein Begriffssystem ausgestaltet ist, um so präziser lässt es sich bei der Formulierung von Theorien verwenden. Die Einführung einer einheitlichen Terminologie birgt die Gefahr der Verschleierung von Verschiedenheiten zwischen unterschiedlich funktionierenden realen Sachverhalten und der Überbetonung von Gemeinsamkeiten zwischen diesen.

Eng damit zusammen hängt der Vorwurf, dass der Systemorientierte Ansatz selber inhaltsleer ist und keine eigenen Aussagen über die betrachteten Systeme macht. ${ }^{339}$ Tatsächlich ist jede systemorientierte Untersuchung nur unter Hinzuziehung des jeweiligen spezifischen Fachwissens über das betrachtete System sinnvoll. In diesem Lichte erscheint der Systemorientierte Ansatz mehr als eine Technik zur zielorientierten Abgrenzung von Problemstellungen und weniger als ein eigenes Theoriegebäude zur Beschreibung, Erklärung und Gestaltung konkreter Systeme. Der Ansatz bietet die Möglichkeit, den Untersuchungsgegenstand entsprechend dem Untersuchungsziel zu definieren und ist somit geeignet, Probleme handhabbar zu machen und auf der anderen Seite die Erfassung von Gesamtzusammenhängen zu fördern. ${ }^{340}$ Bei der Anwendung der Systemtheorie auf den Erkenntnisgegenstand anderer wissenschafticher Disziplinen sollten allerdings die Besonderheiten des jeweiligen Untersuchungsgegenstandes nicht verdeckt werden. ${ }^{341}$ Eine Vereinigung von Systemtheorie und Betriebswirtschaftslehre muss dementsprechend in einer Weise stattfinden, die die Systemtheorie als theoretischen Rahmen verwendet, innerhalb dessen die Spezifika des Systems Unternehmung erhalten bleiben.

Diese allgemeinen Vorbehalte gegenüber der Systemorientierten Betriebswirtschaftslehre wirken sich auch auf den hier entwickelten Ansatz furr eine Theorie des Informationsmanagements aus. Die Verwendung einer allgemeinen Systemterminologie führt zur Angleichung der Analyse, Gestaltung und Steuerung von konkret sehr unterschiedlichen Systemen. Mit dem dadurch entstehenden hohen sprachlichen Allgemeinheitsgrad ist fur den Systemorientierten Ansatz des Informationsmanagements die Gefahr verbunden, dass die spezifischen Eigenschaften von technikunterstützten Informationssystemen, durch die diese sich von anderen Systemtypen unterscheiden, nicht richtig erkannt und die daraus resultierenden Anforderungen an die Gestaltung und Steuerung dieses speziellen Systemtyps nicht ausreichend durch das Systemorientierte Informationsmanagement berücksichtigt werden.

\footnotetext{
${ }^{338}$ Vgl. Schanz (1997a), S. 138.

${ }^{339}$ Vgl. dazu Picot (1975), S. 98ff; Schneider (1997), S. 31 ff.

${ }^{340}$ Vgl. Raffée (1993), S. 34.

${ }^{341} \mathrm{Vgl}$. Stünzner (1996), S. 178. Diese Überlegung führt zu der Frage, wie abstrakt eine wissenschaftliche Theorie sein darf, um noch genügend praktikable und begründbare Ansatzpunkte für konkrete Ableitungen zu bieten. Vgl. Stünzner (1996), S. 180. Eine zu hoher Anspruch auf Universalität wird mit dem Verlust von Anknüpfungspunkten für erklärende und gestaltende Aussagen im konkreten Einzelfall erkauft.
} 
Diese verallgemeinernde Betrachtungsweise kommt insbesondere in der großen Breite des Systemorientierten Ansatzes für eine Theorie des Informationsmanagements zum Ausdruck. Da keine Analyse von Besonderheiten vorgenommen wird, die speziell technikunterstützte Informationssysteme von Unternehmungen kennzeichnen, umfasst Informationsmanagement aus dieser Perspektive praktisch alle Bereiche, die ebenfalls Gegenstand der allgemeinen Unternehmungsfürung als generellem Aufgabenfeld der Lenkung des sozio-technischen Systemtyps Unternehmung sein können. Dazu zählen zum einen die grundsätzliche Gestaltung und die laufende Steuerung des soziotechnisch abgegrenzten technikunterstützten Informationssystems der Unternehmung. Zum anderen erstreckt sich die Einflussnahme eines systemtheoretisch ausgerichteten Informationsmanagements nicht nur auf die materielle, sondern auch auf die symbolische Ebene des Geschehens. Durch diesen hohen Allgemeinheitsgrad der aus der Systemorientierten Betriebswirtschaftslehre für das Informationsmanagement ableitbaren Aussagen entstehen Abgrenzungsschwierigkeiten gegenüber der Lenkung anderer Funktionsbereiche der Unternehmung und gegenüber der übergeordneten allgemeinen Unternehmungsführung.

Die stark verallgemeinernde Betrachtungsweise der Systemtheorie bedingt auch, dass systemtheoretische Überlegungen in der Betriebswirtschaftslehre mit der Bewältigung von Komplexität an einem sehr abstrakten Problem ansetzen, das grundsätzlich bei der Lenkung von Systemen aller möglichen Typen auftritt. Dementsprechend bezieht sich das Gesetz der erforderlichen Varietät, das den Ausgangspunkt zahlreicher Arbeiten der Systemorientierten Betriebswirtschaftslehre darstellt, auf das Auftreten von und den Umgang mit Informationsunvollkommenheiten durch die Lenkungsinstanz eines Systems. Dem spezifischen Systemtyp Unternehmung wird diese Vorgehensweise aber nur teilweise gerecht. Bei dem Auftreten von Situationen unvollkommener Information handelt es sich zwar um einen wichtigen, jedoch nicht um den einzigen Kritikpunkt gegen eine zu starke Konzentration der Betriebswirtschaftslehre auf das ökonomische Prinzip, deren Überwindung das erklärte Ziel des Systemorientierten Ansatzes ist.

Der zweite grundlegende Problembereich besteht in der Existenz divergierender Ziele bei multipersonalen Entscheidungen. ${ }^{342} \mathrm{Zu}$ dieser Problematik bietet der Systemorientierte Ansatz nur wenig Lösungsansätze. Die Ausdehnung der Lenkungsmaßnahmen auf die Symbolische Ebene wirkt in diesem Zusammenhang wie eine nachträgliche, etwas künstliche Ergänzung der ursprünglichen, am Problem der Komplexität ansetzenden Überlegungen der Systemorientierten Betriebswirtschaftslehre, die diese Lücke nur notdürftig schließen können. Grenzt man - wie in dieser Arbeit - den Gestaltungsgegenstand des Informationsmanagements als sozio-technisches System ab, so liegt in dem Auftreten von Zielkonflikten eine Besonderheit dieses Gestaltungsgegenstandes, auf den der Systemorientierte Ansatz für eine Theorie des Informationsmanagements nicht explizit eingeht. Auch eventuelle weitere Besonderheiten dieses Systemtyps (z.B. mögliche Emergenzphänomene beim Zusammenwirken von Menschen und Informationstechnik oder spezielle pragmatische Aspekte der Informationsverarbeitung in Unternehmungen) werden durch diesen Ansatz für eine Theorie des Informationsmanagements nicht näher betrachtet.

${ }^{342}$ Vgl. Göbel (1998), S. 111. 


\subsubsection{Fragwürdigkeit von Analogien}

Die soeben gewürdigte verallgemeinernde Betrachtungsweise der Systemorientierten Betriebswirtschaftslehre leistet unangemessenen Analogieschlüssen, die sich auf konkret sehr unterschiedliche Erscheinungsformen von Systemen beziehen, Vorschub. ${ }^{343}$ Das grundsätzliche Vorhandensein einer Gefahr der fehlerhaften Analogiebildung wird von Vertretern der Systemorientierten Betriebswirtschaftslehre nicht abgestritten. Ihre Bedeutung wird jedoch mit dem Hinweis darauf, es erfolge keine Gleichsetzung unterschiedlicher Systeme, sondern lediglich eine formal isomorphe Abbildung dieser Systeme entsprechend des jeweils verfolgten Erkenntnisinteresses (hier der Lenkung von Systemen), geringer bewertet als von Kritikern dieses Wissenschaftsprogrammes. ${ }^{344}$ Dieses Argument sei nachfolgend etwas näher beleuchtet.

Auf dem Gebiet der Analogiebildung sind materiale und formale Analogien zu unterscheiden. Durch die Bildung materialer Analogien in der Betriebswirtschaftslehre kommt es zu einer Ausdehnung naturwissenschaftlicher Gesetze auf das Wirtschaftsleben. ${ }^{345}$ Ein derartiges Vorgehen im Rahmen der Systemorientierten Betriebswirtschaftslehre entspräche vom Konzept her der Übertragung von Gesetzen der Mechanik auf ökonomische Fragen durch die Neoklassische Volkswirtschaftslehre und den Faktortheoretischen Ansatz der Betriebswirtschaftslehre. Der Rekurs auf benachbarte Wissenschaftsdisziplinen durch die Systemorientierte Betriebswirtschaftslehre stellt bei dieser Art der Analogiebildung eine „Naturalisierung ${ }^{\text {(346 }}$ wirtschaftlicher und gesellschaftlicher Verhältnisse unter Bezugnahme auf neuere Entwicklungen in diesen Wissenschaftsdisziplinen dar.

Dagegen erfolgt die Übertragung von naturwissenschaftlichen Erkenntnissen auf betriebswirtschaftliche Probleme bei der Bildung von formalen Analogien auf subtilere Weise. Es werden in diesem Fall keine Inhalte unmittelbar übertragen, sondern vielmehr wird nach Isomorphien in homomorphen Abbildungen der jeweils analysierten Systeme gesucht. ${ }^{347}$ Die inhaltlichen Schlussfolgerungen aus diesen formalen Analogien sind bei dieser Vorgehensweise durch die jeweiligen wissenschaftlichen Spezialdisziplinen zu ziehen. Diese Art der Analogiebildung streben methodisch fundierte Arbeiten der Systemorientierten Betriebswirtschaftslehre normalerweise an. Allerdings ist dagegen einzuwenden, dass die Bildung formaler Analogien die Existenz von miteinander vergleichbaren formalen Modellen erfordert, auf die die Erkenntnisse der jeweils anderen Disziplin angewendet werden können. Es kann angezweifelt werden, dass die Wissenschaftsdisziplinen, die sich mit der Analyse sozialer (bzw. soziotechnischer) Systeme befassen, über einen mit dem der Naturwissenschaften vergleichbaren Korpus von formalen Modellen verfügen, für die dieser Typ von Analogien hergestellt und angewendet werden kann. ${ }^{348}$ Ist dies tatsächlich nicht der Fall, so ist die Bildung formaler Analogien im Rahmen der Systemorientierten Betriebswirtschaftslehre wissenschaftstheoretisch gesehen unmöglich.

\footnotetext{
${ }^{343}$ Vgl. dazu Sandner (1982), S. $79 \mathrm{ff}$.

${ }^{344}$ Vgl. Malik (1982), S. 99f.; Probst/Dylli(1982), S. 108, sowie die dort zitierten Quellen.

${ }^{345}$ Vgl. Müller (1992), S. 347.

${ }^{346}$ Müller (1992), S. 370.

${ }^{347}$ Vgl. Malik (1982), S. 100.

${ }^{348}$ Vgl. ăhnlich Müller (1992), S. 365.
} 
Diese Überlegungen machen deutlich, dass die Absicht, durch die Bildung von Analogien zwischen physikalischen, chemischen, biologischen, sozialen und soziotechnischen Systemen einen Austausch wissenschaftlicher Erkenntnisse zu ermöglichen, auf einer zerbrechlichen Basis steht. Die Methoden der naturwissenschaftlich ausgerichteten älteren und jüngeren Systemtheorie sind nicht im strikten Sinn auf die Untersuchung sozialer und sozio-technischer Systeme übertragbar. ${ }^{349}$ Lehnt man eine Gleichsetzung von sozialem Geschehen mit Prozessen in der unbelebten Natur und der Biologie ab und erkennt außerdem an, dass die Voraussetzungen zur Bildung formaler Analogien nur im Ausnahmefall vorliegen, so bleibt allein die metaphorische Funktion solcher Analogien. Für ein systemorientiert ausgerichtetes Informationsmanagement liegt der Wert der Analogiebildung also in dem heuristischen Potenzial, das Analogien bei der Entwicklung von Aussagen, die sich auf die Lenkung technikunterstützter Informationssysteme beziehen, entfalten können. Der Anspruch eines echten Austausches von Forschungsergebnissen unterschiedlicher Wissenschaftsdisziplinen kann deshalb nur in erheblich reduzierter Form aufrecht erhalten werden.

\subsubsection{Verhältnis von konservativ und innovativ orientierten Maßnahmen}

Die hier entwickelte Systemorientierte Ansatz für eine Theorie des Informationsmanagements unterscheidet entsprechend der beiden Forschungsrichtungen der Kybernetik eine konservative und eine innovative Variante des Informationsmanagements. Konservative Maßnahmen zielen auf die Beibehaltung bereits erreichter Systemzustände $\mathrm{ab}$, während innovative Maßnahmen sich auf die grundsätzliche Anpassung des Systems an nachhaltig veränderte System/Umweltsituationen richten. Von Vertretern der neueren Systemorientierten Betriebswirtschaftslehre wird darauf hingewiesen, zwischen beiden Varianten der Kybernetik könne keine Wertung vorgenommen werden, da sie sich zueinander komplementär verhielten. ${ }^{350}$ Mit dieser relativ allgemeinen Aussage ist das Verhältnis zwischen konservativem und innovativem Informationsmanagement jedoch nicht hinreichend geklärt.

Tatsächlich stehen die konservativ und die innovativ orientierte Variante der Lenkung von Systemen untereinander auch in einer Konkurrenzsituation. Einerseits verhindert eine erfolgreiche Beibehaltung bereits erreichter Systemzustände das frühzeitige Erkennen von Anpassungsnotwendigkeiten und vermindert dadurch die Flexibilität des Systems. Andererseits durchbricht die Anpassung des Systems durch Lernprozesse höherer Ordnung die konservativ ausgerichteten Lenkungsversuche innerhalb der bereits etablierten Prozess- und Gebildestrukturen und weist so gesehen zerstörerische Merkmale auf. Der trotz dieser Konkurrenz bestehende komplementäre Charakter des Verhältnisses zwischen diesen beiden Varianten der Systemlenkung resultiert daraus, dass für die dauerhafte Existenz eines Systems in dynamischen Umwelten relativ stabile Phasen (entsprechend der Kybernetik I) und relativ instabile Phasen (entsprechend der Kybernetik II) einander abwechseln müssen.

In diesem Zusammenhang ist außerdem auf ein grundsätzliches konzeptionelles Dilemma der an der Kybernetik II orientierten Systemorientierten Betriebswirtschaftslehre hinzuweisen. Dieses liegt in dem Gegensatz zwischen dem Streben nach einer

\footnotetext{
${ }^{349}$ Vgl. ähnlich Müller (1992), S. 370.

${ }^{350}$ Vgl. Probst (1987), S. 65f.; Parker/Stacey (1995), S. $59 f$.
} 
bewussten Gestaltung und Steuerung von Unternehmungen einerseits sowie der Anerkennung systemtheoretischer Erkenntnisse, welche ein hohes $\mathrm{Ma}$ an Unbeeinflussbarkeit des Geschehens in Folge der Komplexität des Systems nachweisen, andererseits, begründet. ${ }^{351}$ Obwohl der Zweck-Mittel-Beziehung durch die an der neueren Systemtheorie orientierte Argumentation der Boden entzogen wird, wird gerade der pragmatische Anspruch von beiden Forschungszweigen der Systemorientierten Betriebswirtschaftslehre besonders betont. Trotz Ihres Eigenlebens werden Unternehmungen nicht als unbeeinflussbar, schicksalhaft, undurchdringlich und daher als unveränderbar gesehen, ${ }^{352}$ wenn im Vergleich zu traditionellen Ansätzen auch ein geringer Umfang an bewusst geplanter Gestaltbarkeit und Steuerbarkeit angenommen wird. ${ }^{353}$ Dieser konzeptionelle Konflikt stellt einen kaum zu überbrückenden inneren Widerspruch des Systemorientierten Ansatzes der Betriebswirtschaftslehre dar.

Insgesamt ist eine Kombination von negativen und positiven Rückkopplungsprozessen erforderlich, um das dauerhafte Überleben des Systems zu gewährleisen. ${ }^{354}$ Für ein theoretisch fundiertes Systemorientiertes Informationsmanagement werden deshalb Aussagen darüber benötigt, inwiefern der parallele Einsatz konservativer und innovativer Lenkungsmaßnahmen möglich und sinnvoll ist. Außerdem sind Kriterien dafür zu entwickeln, in welchen Fällen negative oder positive Rückkopplungsprozesse vorzuziehen sind, um die Systemziele so gut wie möglich zu erreichen. In diesem Punkt bietet die Systemtheorie bisher keine befriedigenden Anhaltspunkte, da die beiden Forschungsprogramme Kybernetik I und Kybernetik II auf ein Entweder/Oder bei der Lenkung von Systemen hinauslaufen. Eine Verknüpfung beider Varianten zu einem integrierten Konzept für eine Allgemeine Kybernetik steht bisher noch aus.

\footnotetext{
${ }^{351}$ Vgl. Stünzner (1996), S. 169.

${ }^{352}$ Vgl. Kasper (1991), S. 69.

${ }^{353}$ Vgl. Malik (1993), S. 110.

${ }^{354}$ Vgl. Parker/Stacey (1995), S. 63.
} 
Dirk Beier - 978-3-631-75500-6

Downloaded from PubFactory at 01/11/2019 04:07:38AM 


\section{Erkenntnispotenziale und Lösungsbeiträge der Neuen Institutionenökonomie für das Informationsmanagement}

Das Kapitel 5 wendet sich der Neuen Institutionenökonomie als weiterem Wissenschaftsprogramm der Betriebswirtschaftslehre zu, das eine potenzielle Basis zur Überwindung der im zweiten Kapitel festgestellten Theoriedefizite des Informationsmanagements bietet. Abschnitt 5.1 bewältigt die zweite Stufe des hier gewählten Vorgehens für einen dreistufigen Theorieeinstieg und grenzt den Erkenntnisgegenstand der Neuen Institutionenökonomie ab. Abschnitt 5.2 schließt den Theorieeinstieg ab und nimmt eine Konstruktion des Informationsmanagements als spezielles Erkenntnisobjekt der Neuen Institutionenökonomie vor. Davon ausgehend kann im Abschnitt 5.3 die eigentliche Theoriebildung erfolgen. Die Überlegungen orientieren sich dabei an den Phasen des Leistungsaustausches, dessen Koordination im Mittelpunkt dieses Wissenschaftsprogramms steht. In Abschnitt 5.4 schließlich erfolgt eine kritische Würdigung der Aussagekraft des entwickelten Ansatzes für eine Theorie des Informationsmanagements. Es werden einerseits die Lösungsansätze für offene Problemstellungen in der Praxis und andererseits die Erkenntnisgrenzen des Ansatzes beleuchtet.

\subsection{Der Ansatz der Neuen Institutionenökonomie}

Bei der Neuen Institutionenökonomie handelt es sich um ein Wissenschaftsprogramm zur Erforschung ökonomischer Aktivitäten, das nicht eindeutig der Betriebswirtschaftslehre zuzurechnen ist, sondern vielmehr im Überschneidungsbereich zwischen Betriebswirtschaftslehre und Volkswirtschaftslehre angesiedelt ist. ${ }^{1}$ Unter der Bezeichnung „Neue Institutionenökonomie“ werden mehrere methodisch verwandte Ansätze zusammengefasst, die sich gegenseitig überlappen, ergänzen und teilweise aufeinander beziehen. ${ }^{2}$ Ein einheitlicher Oberbegriff hat sich für diese Ansätze bisher nicht durchsetzen können. Neben der hier verwendeten Bezeichnung „Neue Institutionenökonomie" finden sich in der Literatur u.a. auch die Begriffe „Theorie der Verfugungsrechte $^{\text {“3 }}$, „Neue Institutionenökonomik“4 und „Neuer Institutionalismus ${ }^{\text {“5 }}$ als Gesamtbezeichnung. ${ }^{6}$

Die Wurzeln der Neuen Institutionenökonomie liegen in der anglo-amerikanischen, volkswirtschaftlich ausgerichteten Theorie der Unternehmung. Mittlerweile werden die Inhalte und die Methodik aber auch im deutschsprachigen Raum durch die Betriebswirtschaftslehre aufgegriffen. ${ }^{7}$ Dabei kommt es zu einer Verknüpfung der Mikroökonomie als volkswirtschaftlicher Teildisziplin mit der betriebswirtschaftlichen

\footnotetext{
Vgl. Picot (1982), S. 268.

Vgl. Picot/Reichwald/Wigand (1998), S. 37

Budäus/Gerum/Zimmermann/ (1988), S. 9.

Vgl. u.a. Picot/Reichwald/Wigand (2001), S. 39; Richter/Furubotn (1999), S. V.

Vgl. u.a. Schanz (1997a), S. 140; Edeling (1999), S. 7.

6 In der englischsprachigen Diskussion werden u.a. die Begriffe „Theory of the Firm“, „PropertyRights-Paradigm", „Agency Theory“, „Institutional Economics" und "Transaction-Cost Economics“- verwendet. Diese beziehen sich zum Teil nur auf Ausschnitte des gesamten Aussagesystems, zum Teil erheben sie jedoch Anspruch darauf, als Oberbegriff des gesamten Ansatzes zu gelten. Auch dort konnte sich bisher kein einheitlicher Oberbegriff durchsetzen.

Vgl. Schanz (1997a), S. 140.
} 
Organisationstheorie, insbesondere der verhaltenswissenschaftlichen Entscheidungstheorie. ${ }^{8}$ Gerade diese Verbindung von zentralen Annahmen der verhaltenswissenschaftlichen Entscheidungstheorie mit klassischen ökonomischen Kategorien machte die Rezeption der anglo-amerikanischen Konzepte für die deutschsprachige Betriebswirtschaftslehre attraktiv. ${ }^{9}$ Der von dieser Grundidee ausgehende Ansatz kann als Versuch gesehen werden, die Forderung nach einer Synthese von ökonomisch orientierter, traditioneller Betriebswirtschaftslehre und verhaltenswissenschaftlicher Managementlehre umzusetzen, die im Anschluss an die verhaltenswissenschaftliche Öffnung der Betriebswirtschaftslehre in den 1970er Jahren immer wieder erhoben wurde.

\subsubsection{Erkenntnisinteressen der Neuen Institutionenökonomie}

Die grundsätzliche Gemeinsamkeit der verschiedenen Ansätze im Bereich der Neuen Institutionenökonomie liegt in der Absicht, den Fokus bei der Analyse wirtschaftlichen Handelns auf „Institutionen“ als Einflussgrößen und Gestaltungsvariablen zu richten. ${ }^{10}$ Institutionen stellen ein „System formgebundener (formaler) und formungebundener Regeln einschließlich der Vorkehrungen zu deren Durchsetzung " dar. ${ }^{11}$ Sie funktionieren als „sozial sanktionierbare Erwartungen, die sich auf die Handlungs- und Verhaltensweisen eines oder mehrerer Individuen beziehen". ${ }^{12}$ Dieser weiten Fassung des Begriffs können so unterschiedliche Gegenstände wie Verträge, organisatorische Regeln, Rechtsnormen, Sprache und Geld zusammengefasst werden.

Institutionen stabilisieren die Erwartungen der am Wirtschaftsgeschehen beteiligten Akteure und kanalisieren darüber ihr Verhalten, sodass ein arbeitsteiliges Zusammenwirken möglich wird. Die Neue Institutionenökonomie betont den gegenüber den Akteuren exogenen Charakter von Institutionen, die als "constraints" und „opportunities" Handlungen der eigennützig handelnden Individuen von außen steuern. ${ }^{13}$ Institutionen setzen Anreize und bereiten Kosten und machen auf diesem Wege bestimmte Handlungen erwartbar oder unwahrscheinlich. Sie strukturieren das wirtschaftiche Geschehen und verringern dessen Unsicherheit, ohne sie zusammen mit der Wahlfreiheit der Akteure aufzuheben. ${ }^{14}$

Die Vertreter der Neuen Institutionenökonomie verbleiben weitgehend auf dem Boden der neoklassischen Analyse ${ }^{15}$ und verstehen ihre Arbeiten als Erweiterung der traditionellen Perspektive. In der neoklassischen Analyse wird die Entstehung, Wirkungsweise und Effizienz von Institutionen nicht thematisiert. Die Neue Institu-

8 Vgl. Ebers/Gotsch (1999), S. 199.

9 Vgl. Witt (1995), S. 11. Die Aussage bezieht sich dort auf die Transaktionskostenanalyse als Teilbereich der Neuen Institutionenökonomie. Sie kann jedoch wegen der methodischen und inhaltlichen Gemeinsamkeiten auf das gesamte Wissenschaftsprogramm ausgedehnt werden.

${ }^{10} \mathrm{Vgl.} \mathrm{Budäus/Gerum/Zimmermann} \mathrm{(1988),} \mathrm{S.} 9$.

11 Vgl. Richter/Furubotn (1999), S. 7, die sich selbst auf Schmoller (1900), S. 61 beziehen. Über den Begriff der Institution besteht allerdings auch innerhalb der Neuen Institutionenókonomie keine endgültige Einigkeit. Für einen Überblick über verschiedene Begriffsvarianten vgl. z.B. Elsner (1986), S. 199ff.; Richter/Furubotn (1999), S. 7ff.

12 Dietl (1991), S. 37.

13 Vgl. Edeling (1999), S. 9.

14 Vgl. Richter (1994), S. 2; Edeling (1999), S. 9.

15 Vgl. Göhler/Kühn (1999), S. 23. 
tionenökonomie zielt darauf ab, dieses, „institutionelle Vakuum“16 der Neoklassik zu überwinden und auf diese Weise die Aussagekraft ${ }^{17}$ der Theorie zu erhöhen. Den Ausgangspunkt bilden dabei zwei Grundannahmen: ,institutions (1) matter and (2) are susceptible to analysis“"18. Das Erkenntnisinteresse der Neuen Institutionenökonomie richtet sich darauf basierend auf die beiden folgenden Grundfragestellungen: ${ }^{19}$

- Welche (alternativen) Institutionen haben bei welchen Arten von Koordinationsproblemen des ökonomischen Austausches die relativ geringsten Kosten und die größte Effizienz zur Folge?

- Wie wirken sich die Koordinationsprobleme, die Kosten und die Effizienz von Austauschbeziehungen auf die Gestaltung und den Wandel von Institutionen aus?

Den Ursprung der Überlegungen in den einzelnen Teilbereichen der Neuen Institutionenökonomie stellen zwei Veröffentlichungen von COASE dar, ${ }^{20}$ die sich mit der zweckmäßigen Form der Arbeitsteilung bei der Herstellung verschiedener Arten von Gütern in ökonomischen Systemen beschäftigen. Es können (mindestens) drei verschiedene Theoriestränge identifiziert werden, die sich ausgehend von den dort vorgestellten Ideen mit der ökonomischen Analyse von Institutionen beschäftigen. ${ }^{21}$ Dabei handelt es sich um die Theorie der Verfügungsrechte (auch als Property-RightsTheorie bezeichnet), die Transaktionskostentheorie und die Agency-Theorie (auch als Principal-Agent-Theorie bezeichnet). ${ }^{22}$

Die Theorie der Verfügungsrechte befasst sich mit dem Einfluss der Spezifizierung und Verteilung von Verfügungsrechten (property rights) an Gütern auf wirtschaftliches Handeln. Verfugungsrechte legen fest, wie ihr Inhaber in legitimer Weise über Güter verfugen kann, und institutionalisieren somit Verhaltensnormen in Bezug auf die Nutzung von Ressourcen, deren Einhaltung allgemein erwartet und deren Verletzung sanktioniert werden kann. ${ }^{23}$ Die Theorie der Verfügungsrechte untersucht zum einen die Auswirkungen unterschiedlicher Gestaltungs- und Verteilungsformen von Verfugungsrechten und zum anderen ihre historische Entstehung und ihren Wandel im Zeitablauf. Sie fand zunächst bei der Analyse bestimmter Eigentumsformen Anwendung. ${ }^{24}$ So wurde sie für den Vergleich von Privat- und Gemeinschaftseigentum und den Vergleich von Eigentumsregelungen in verschiedenen Wirtschaftssystemen

${ }^{16}$ Schanz (1997a), S. 142.

$17 \mathrm{Vgl}$. Budäus/Gerum/Zimmermann (1988), S. 9. Die genannten Autoren sprechen nicht von der „Aussagekraft“, sondern vom „Realitätsgehalt" der Theorie. Diese Begriffswahl ist jedoch nicht mit der im zweiten Kapitel verdeutlichten radikal-konstruktivistischen Grundposition dieser Arbeit vereinbar. Entsprechend des im zweiten Kapitel beschriebenen Vorgehens handelt es sich genau genommen um Abstraktionen bei der vom Erfahrungsobjekt ausgehenden Konstruktion des Erkenntnisobjekts auf der zweiten Stufe der Theoriebildung.

${ }^{18}$ Matthews (1986), S. 903.

$19 \mathrm{Vgl}$. Ebers/Gotsch (1999), S. 199.

${ }^{20} \mathrm{Vgl}$. Coase (1937), S. 386ff.; Coase (1960), S. 1ff.

${ }^{21}$ Vgl. Schanz (1997a), S. 142; Göhler/Kühn (1999), S. 24f;; Sydow (1999), S. 165.

${ }^{22}$ Zum Teil wird auch die Vertragstheorie als eigener Untersuchungsbereich der Neuen Institutionenökonomie genannt, vgl. z.B. Picot/Reichwald/Wigand (1998), S. 5lff. Diese stellt jedoch in den Arbeiten von Williamson, auf die sich die meisten spăteren Autoren beziehen, eine der Grundlagen der eigenen Theoriebildung dar (,Some Contracting Background“, Williamson (1979), S. 235). Die Vertragstheorie wird hier deshalb nicht als eigener Forschungszweig der Neuen Institutionenökonomie behandelt.

${ }^{23} \mathrm{Vgl}$. Ebers/Gotsch (1999), S. 200.

${ }^{24} \mathrm{Vgl}$. zum folgenden Ebers/Gotsch (1999), S. 204ff. 
eingesetzt. Außerdem ermöglichte sie eine Untersuchung des Entscheidungsverhaltens in öffentlichen und staatlich regulierten Unternehmungen. Später wurde der Anwendungsbereich auf die Analyse von Unternehmungsverfassungen und auf das Innovationsverhalten in Unternehmungen ausgedehnt. Der historisch orientierte Zweig der Theorie der Verfügungsrechte befasst sich mit wirtschaftshistorischen Betrachtungen hinsichtlich der Entstehung und des Wandels besonderer Verfügungsrechtsstrukturen.

Mit der Übertragung von Verfügungsrechten, die im Rahmen von Transaktionen erfolgt und mit Kosten verbunden ist, beschäftigt sich die Transaktionskostentheorie. Die einzelne Transaktion bildet dabei die Grundeinheit zur Analyse ökonomischer Aktivitäten. ${ }^{25}$ Es werden verschiedene institutionelle Arrangements zur Regelung (governance) von Transaktionen dargestellt und auf ihre Eignung zur Koordination arbeitsteiliger Aktivitäten untersucht. Als Maßstab für die Vorteilhaftigkeit einer Koordinationsform ${ }^{26}$ werden die Kosten zur Erstellung des Endproduktes, die sich aus der Summierung von Produktions- und Transaktionskosten ergeben, angesehen. ${ }^{27}$ Dabei stehen sich Märkte und Hierarchien sowie ein Kontinuum institutioneller Koordinationsmöglichkeiten zwischen diesen beiden Extrempunkten als potenzielle Koordinationsformen gegenüber. ${ }^{28}$

Die Agency-Theorie schließlich widmet sich der Untersuchung von Auftraggeber/ Auftragnehmer-Beziehungen und geht im Kern ebenfalls auf COASE zurück. ${ }^{29}$ Eine Agency-Beziehung zwischen zwei oder mehreren Parteien besteht immer dann, „.. when one, designated as the agent, acts for, on behalf of, or as representative for the other, designated the principal, in a particular domain of decision problems" ${ }^{30}$ Durch die Agency-Theorie werden die diesen Beziehungen zu Grunde liegenden Verträge analysiert, in denen der Auftraggeber Entscheidungskompetenzen an den Auftragnehmer delegiert, der für seine Dienste eine Vergütung erhält. Im Rahmen der AgencyTheorie lassen sich eine normative und eine deskriptive Richtung unterscheiden. ${ }^{31}$ Während der normative Zweig mit mathematisch-formalen Modellen die Möglichkeiten einer optimalen Vertragsgestaltung beleuchtet, widmet sich der deskriptive Zweig der positiven Analyse empirisch vorfindbarer Auftraggeber/Auftragnehmer-Beziehungen und weist eine größere Nähe zu den anderen Teilgebieten der Neuen Institutionenökonomie auf.

\footnotetext{
${ }^{25}$ Vgl. Williamson (1990), S. 322.

${ }^{26}$ Als Oberbegriff für die verschiedenen Vertragsmuster zur Abstimmung der Akteure wird in diesem Kapitel der Terminus „Koordinationsform“ verwendet. Im Umfeld der Neuen Institutionenökonomie wird (teilweise synonym dazu) auch eine Reihe ähnlicher Begriffsvarianten wie etwa "Koordinationsinstrument", „Koordinationsmethode“, „Koordinationsprinzip“, „Koordinationsstruktur“ oder „Koordinationsmechanismus“ gebraucht. Zur Beziehung dieser Begriffe vgl. Alt (1997), S. 68ff.

27 Vgl. Williamson (1985), S. 22.

${ }^{28}$ Vgl. Picot (1982), S. 273.

${ }^{29}$ Vgl. Schanz (1997a), S. 143.

${ }^{30}$ Ross (1973), S. 134.

${ }^{31}$ Vgl. Elschen (1991), S. 1006.
} 


\subsubsection{Inhaltliche Leitideen der Neuen Institutionenökonomie}

Die verschiedenen Teilgebiete der Neuen Institutionenökonomie zeichnen sich nicht nur durch die gemeinsame Fokussierung von Institutionen und deren Einfluss auf wirtschaftliches Handeln aus, sondern auch durch gemeinsame Grundannahmen über das menschliche Verhalten im Wirtschaftsgeschehen. ${ }^{32}$ Es wird unterstellt, dass Menschen in ihrem Selbstinteresse handeln, um ihren Nutzen zu erreichen, dass sie sich dabei begrenzt rational verhalten und dass die Gefahr opportunistischen Verhaltens besteht. Begrenzte Rationalität heißt, dass die Individuen den für sie günstigsten Handlungserfolg vor dem Hintergrund unvollständiger Information anstreben. In einem solchen Fall kann sich ihr Verhalten von demjenigen unterscheiden, das sie bei einem vollständigen Informationsstand gewählt hätten. ${ }^{33}$ Opportunistisches Verhalten stellt den Versuch dar, eigene Interessen gegebenenfalls auch zum Nachteil anderer und unter Missachtung sozialer Normen zu verwirklichen ${ }^{34}$ Es wird zwar nicht davon ausgegangen, dass Opportunismus den Normalfall menschlichen Verhaltens darstellt, aber die Möglichkeit zu Opportunismus sollte beim Abschluss von Verträgen stets einkalkuliert werden. ${ }^{35}$ Neben den beschriebenen, gemeinsamen inhaltlichen Leitideen grenzen sich die einzelnen Teilgebiete der Neuen Institutionenökonomie durch ihre jeweils eigenen Untersuchungsobjekte voneinander $a b$, über die sie bestimmte inhaltliche Grundannahmen treffen.

Die Theorie der Verfügungsrechte geht davon aus, dass nicht das Eigentum oder der Besitz eines Gutes ökonomisch interessant sind, sondern die faktische Ausübung der auf das Gut bezogenen Rechte. ${ }^{36}$ Es werden vier Arten von Verfügungsrechten an einem Gut unterschieden: ${ }^{37}$

- Das Recht, das Gut zu nutzen,

- das Recht, sich die aus der Nutzung des Gutes entstehenden Gewinne anzueignen (verbunden mit der Pflicht, die entstehenden Verluste zu tragen),

- das Recht, die Form oder Substanz des Gutes zu ändern und

- das Recht, alle oder einzelne der vorstehenden Rechte auf andere zu übertragen, also das Recht zur Veräußerung.

$\mathrm{Da}$ aus jedem dieser Verfügungsrechte ein eigener Nutzen erzielbar ist, setzt sich der gesamte, aus der Verfügung über das Gut entstehende Nutzen aus den verschiedenen Nutzenkomponenten durch die Ausübung der einzelnen Rechte zusammen. Bei gegebenen institutionellen Rahmenbedingungen werden die Akteure das Gut in den

${ }^{32}$ Vgl. Picot/Reichwald/Wigand (1998), S. 37.

${ }^{33}$ Die Bezeichnung „begrenzte“ Rationalität ist eigentlich irreführend, denn genau genommen verhält sich das Individuum ausgehend von seinem Informationsstand vollkommen rational. $\mathrm{Da}$ die Ausdehnung des Informationsstandes unvermeidlich mit Kosten und einem ex-ante nicht bekannten Nutzen verbunden ist, verstőßt es nicht gegen das Rationalitătsprinzip, wenn Entscheidungen unter unvollkommener Information von denen abweichen, die bei vollkommener Information getroffen worden wären.

$34 \mathrm{Vgl}$. Picot/Reichwald/Wigand (2001), S. 45.

${ }^{35}$ Vgl. Williamson (1985), S. $47 \mathrm{ff}$.

${ }^{36} \mathrm{Vgl}$. Budäus/Gerum/Zimmermann (1988), S. 10. Aus diesem Grund ist eine Übersetzung des englischen Ausdrucks Property Right mit dem deutschen Wort „Eigentumsrecht“ auch nicht adäquat, da ein Verfuigungsrecht nicht an das Eigentum an dem betreffenden Gut gebunden ist.

37 Vgl. Furubotn/Pejovich (1972), S. 1140. 
Grenzen ihrer Verfügungsrechte so verwenden, dass sie ihren persönlichen Nutzen maximieren. Wenn die Verfügungsrechte an einem Gut auf mehrere Individuen aufgeteilt sind, kommt es zu einer Verdünnung der Rechte als Folge von Interessenkonflikten der Beteiligten. ${ }^{38}$ Der aus der Verfügung über das Gut erzielbare NettoNutzen sinkt in einem solchen Fall, da es zu nicht entschädigten Nebenwirkungen des individuellen Verhaltens auf das Nutzenniveau der jeweils anderen Beteiligten kommt, die als externe Effekte bezeichnet werden. Die Theorie der Verfügungsrechte empfiehlt daher, zur Herstellung von Allokationseffizienz die Verfügungsrechte an einem Gut möglichst vollständig einer einzigen Person zuzuordnen, damit externe Effekte vermieden und Anreize für eine effiziente Ressourcenverwendung geschaffen werden. $^{39}$

Die Bestimmung, Übertragung und Durchsetzung ${ }^{40}$ der aufgeführten Rechte ist mit Kosten verbunden, die von der Theorie der Verfügungsrechte als Transaktionskosten bezeichnet werden. Die Theorie der Verfügungsrechte stellt als zweite Kernaussage die Hypothese auf, dass der aus dem Verfügungsrecht erzielbare Nettonutzen um so geringer ist, je höher diese Transaktionskosten im jeweiligen Einzelfall ausfallen. ${ }^{41}$ Bei hohen Transaktionskosten kommt es zu (positiven oder negativen) externen Effekten, da auch solche Akteure von der Verfügung über das Gut tangiert werden, die formal nicht durch Verfügungsrechte an dem Gut beteiligt sind. Vor diesem Hintergrund erscheint nicht nur die möglichst umfassende Bündelung von Verfügungsrechten, sondern auch deren möglichst vollständige Ausformulierung zum Zwecke der Internalisierung externer Effekte in allokativer Hinsicht vorteilhaft.

Die zunächst von WILLIAMSON entwickelte Transaktionskostentheorie befasst sich mit den bei der Abwicklung von Transaktionen entstehenden Kosten der Koordination des Leistungsaustausches. Obwohl es sich bei dem Konstrukt der Transaktionskosten um das zentrale Element des Ansatzes handelt, wird der Transaktionskostenbegriff - ebenso wie auch der Begriff der Transaktion selbst - allerdings nur unzulänglich definiert und operationalisiert. ${ }^{42}$ Die Operationalisierung von Transaktionskosten macht insbesondere deshalb erhebliche Schwierigkeiten, ${ }^{43}$ weil die beschriebenen Aktivitäten überwiegend nicht eindeutig abgrenzbar und für Planungs- und Kontrollzwecke abbildbar sind. Tatsächlich erfolgt ein intuitiver Zugang zum Begriff der Transaktionskosten, indem exemplarisch Kostenarten aufgeführt werden, die bei der Abwicklung von Transaktionen anfallen.

Dennoch lohnt eine weitere Auseinandersetzung mit Aktivitäten, die zur Entstehung von Transaktionskosten führen, da die Operationalisierung als noch zu lösende Aufgabe der Forschung begriffen werden kann und die Relevanz der Transaktionskosten trotz der zum größten Teil fehlenden Operationalisierbarkeit nicht vernach-

${ }^{38} \mathrm{Vgl}$. Ebers/Gotsch (1999), S. 202.

39 Vgl. Furubotn/Pejovich (1972), S. 1141.

${ }^{40}$ Hier ist nicht die Durchsetzung eines Verfügungsrechts gegenüber den Inhabern der anderen Verfügungsrechte, sondern gegenüber formal nicht beteiligten Dritten gemeint.

${ }^{41} \mathrm{Vgl}$. Ebers/Gotsch (1999), S. 202.

${ }^{42}$ Vgl. Picot (1982), S. 270.

${ }^{43}$ Vgl. Picot (1982), S. 271; Walker/Weber (1984), S. 378. 
lässigbar ist. ${ }^{44}$ Die Transaktionskostentheorie unterscheidet Ex ante-Transaktionskosten, die das Zustandekommen von Verträgen zur Durchführung von Transaktionen erst ermöglichen, und Ex post-Transaktionskosten, die furr die nachträgliche Durchsetzung und eventuelle Anpassung von Vereinbarungen entstehen. ${ }^{45}$ Insbesondere der Untersuchung von Ex post-Transaktionskosten, die bis dahin kaum Beachtung fanden, wird erhebliche Aufmerksamkeit gewidmet. In diesem Bereich werden Kosten der Überwachung und Absicherung der Einhaltung von vertraglichen Vereinbarungen, Kosten der Lösung von Konflikten über die Interpretation und Erfüllung von Vereinbarungen und Kosten von Nachverhandlungen auf Grund von Unsicherheiten zum Zeitpunkt des Vertragsabschlusses unterschieden. ${ }^{46}$

Die Höhe der Transaktionskosten hängt im konkreten Fall zum einen von den Charakteristika der Transaktion und zum anderen von den Eigenschaften des zur Koordination der Transaktion gewählten institutionellen Arrangements ab. Besondere Probleme treten aus Sicht der Transaktionskostentheorie immer dann auf, wenn opportunistisch handelnde, mit begrenzter Rationalität ausgestattete Wirtschaftssubjekte spezifische und mit Unsicherheit behaftete Transaktionsbeziehungen eingehen und es dabei zusätzlich zu einer Informationsverkeilung, also der asymmetrischen Verteilung von Information zwischen den Transaktionspartnern, kommt. Die Transaktionskostentheorie untersucht die Leistungsfähigkeit alternativ möglicher institutioneller Arrangements bei der Abwicklung unterschiedlicher Arten von Transaktionen. ${ }^{47} \mathrm{Zu}$ diesem Zweck sind eine genaue Charakterisierung der institutionellen Arrangements sowie der besonderen Eigenschaften der abzuwickelnden Transaktionen erforderlich.

Für die Beschreibung der institutionellen Rahmenbedingungen greift WILLAMSON auf vertragstheoretische Erkenntnisse ${ }^{48}$ zurück und unterscheidet klassische, neoklassische und relationale Verträge. ${ }^{49}$ Klassische Verträge entsprechen der ursprünglichen juristischen Vorstellung von Verträgen, da bei ihnen einmalige Leistung und einmalige Gegenleistung eindeutig bestimmt sind. Lassen sich im Falle längerfristiger Vertragsbeziehungen auf Grund von Unsicherheiten nicht alle Eventualitäten eindeutig beim Abschluss des Vertrages regeln, so können neoklassische Verträge abgeschlossen werden. Diese zeichnen sich dadurch aus, dass sie durch Dritte nachprüfbare Mechanismen zur späteren Lösung von Konflikten vorsehen. Noch dauerhaftere Beziehungen und Situationen, die tiefgreifendem Wandel unterliegen, stellen den Anwendungsbereich von relationalen Verträgen dar. Referenzpunkt für die situationsabhängige Verhaltensanpassung der Vertragspartner sind in solchen Fällen nicht mehr ursprünglich fixierte Vereinbarungen - sofern diese überhaupt vorliegen -, sondern "[the] entire relation as it has developed ... [through] time."

\footnotetext{
${ }^{44}$ Picot weist in diesem Zusammenhang darauf hin, dass das Problem einer fehlenden schlüssigen Operationalisierung in vergleichbarer Weise fur den Begriff der Produktionskosten besteht, der bereits viel länger Gegenstand wissenschaftlicher Untersuchungen ist. Vgl. Picot (1982), S. 270.

${ }^{45} \mathrm{Vgl}$. Williamson (1985), S. $20 \mathrm{ff}$.

${ }^{46}$ Vgl. Williamson (1985), S. 21; Ebers/Gotsch (1999), S. 225.

${ }^{47}$ Vgl. Picot/Reichwald/Wigand (2001), S. 50

48 Vgl. dazu Macneil (1974), Macneil (1978).

${ }^{49} \mathrm{Vgl}$. Williamson (1979), S. $235 \mathrm{ff}$.

${ }^{50}$ Macneil (1978), S. 890.
} 
Die nähere Analyse von Transaktionen bezieht sich einerseits auf die Eigenschaften der Transaktion selbst und andererseits auf die Transaktionsatmosphäre. Bei den Transaktionseigenschaften werden drei kritische Dimensionen unterschieden, die maßgeblich für die Höhe der Transaktionskosten und die Effizienz des institutionellen Arrangements zur Abwicklung der Transaktion sind. Dabei handelt es sich um die Häufigkeit, mit der die Transaktion aufritt, um das Ausmaß, in dem transaktionsspezifische Investitionen zu tätigen sind, und um die Unsicherheit der Transaktion. ${ }^{51}$ Von diesen Einflussfaktoren stellt der Spezifitätsgrad die wichtigste Einflussgröße auf die Höhe der Transaktionskosten dar. Der Spezifitätsgrad drückt den Wertverlust aus, der entsteht, wenn die zur Aufgabenerfüllung erforderlichen Ressourcen nicht in der angestrebten Verwendung eingesetzt, sondern ihrer nächstbesten Verwendung zugeführt werden. ${ }^{52}$ Die Transaktionsatmosphäre umfasst rechtliche, technische und soziale Rahmenbedingungen, die bei der Abwicklung der Transaktion eine Rolle spielen. ${ }^{53}$ Abbildung 5/1 zeigt diese Klassifikation von Einflussgrößen auf die Höhe der Transaktionskosten.

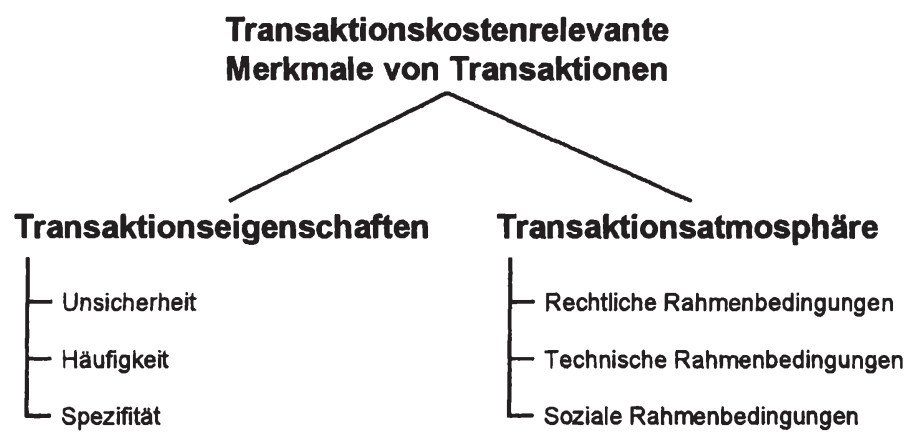

Abb. 5/1: Einflussgrößen auf die Höhe der Transaktionskosten

Ausgehend von den beschriebenen Grundbausteinen strebt die Transaktionskostentheorie Empfehlungen für die Auswahl effizienter institutioneller Arrangements in Abhängigkeit von den jeweils vorliegenden Transaktionseigenschaften an. Für kurzfristig orientierte, klassische Verträge wird die Koordinationsform des Marktes als vorteilhaft angesehen, während sehr langfristig orientierte, relationale Verträge normalerweise im Rahmen der hierarchischen Koordination innerhalb einer Unternehmung zur Anwendung kommen. ${ }^{54}$ Zwischen diesen beiden Polen Markt und Unternehmung ist ein vielfältiges Spektrum an gemischten Formen zur Koordination denkbar, die sowohl marktliche als auch hierarchische Elemente miteinander kombinieren. ${ }^{55}$ Grund-

${ }^{51}$ Vgl. Williamson (1979), S. 239. Diese Charakterisierung von Transaktionen wurde in der Folge weiter differenziert, vgl. z.B. Picot (1982), S. 271ff. Diese Erweiterungen gehören jedoch nicht mehr zu den originären Leitideen des Wissenschaftsprogramms der Neuen Institutionenökonomie, sondern zu deren ausformuliertem Aussagesystem, sodass hier nicht naher darauf eingegangen wird.

52 Vgl. Picot/Reichwald/Wigand (2001), S. 51.

${ }^{53}$ Vgl. Picot (1982), S. 271.

${ }^{54}$ Vgl. Williamson (1979), S. 248ff. Williamson räumt zwar die Möglichkeit ein, dass auch relationale Verträge zwischen Marktpartnern geschlossen werden können, ohne dass es zu einer vertikalen Integration kommt. Sie sind jedoch die einzig geeignete Vertragsform für die interne Koordination innerhalb einer Unternehmung.

55 Vgl. Picot/Reichwald/Wigand (2001), S. 53. 
sätzlich steigen die Transaktionskosten unabhängig vom geltenden institutionellen Arrangement mit dem Spezifitätsgrad der Transaktion. Die Leistungsfähigkeit klassischer Verträge wird für einen geringen Spezifitätsgrad als besonders hoch angenommen, während relationale Verträge bei hohen Spezifitätsgraden im Hinblick auf die entstehenden Transaktionskosten vorteilhafter sind. Von neoklassischen Verträgen wird vermutet, dass sie ihre höchste Leistungsfähigkeit bei einem mittleren Spezifitätsgrad erreichen. ${ }^{56}$

Die Höhe der insgesamt zur Herstellung eines Produktes entstehenden Kosten ergibt sich aus der Summe von Produktions- und Transaktionskosten. Aus diesem Grund sind nicht alleine die Transaktionskosten für die Wahl des geeigneten institutionellen Arrangements ausschlaggebend, sondern auch die Produktionskosten, sofern diese in Abhängigkeit vom institutionellen Arrangement variieren. Zur differenzierteren Berücksichtigung von Produktionskosten wird daher die Transaktionsfrequenz in die Überlegungen mit einbezogen. Von dieser hängt es $a b$, ob spezifische Investitionen zur eigenen Produktion des betrachteten Gutes lohnenswert sind. ${ }^{57}$ Für mittel und hoch spezifische Transaktionen mit einer geringen Frequenz schlägt die Transaktionskostentheorie langfristige, neoklassische Verträge vor. ${ }^{58}$ Diese stellen eine Koordinationsform zwischen den Extrempolen Markt und Hierarchie dar und werden deshalb als hybride bezeichnet. Hybride Koordinationsformen haben in den letzten Jahren verstärkt Beachtung gefunden und die scheinbar einfache Wahl zwischen unternehmungsinterner und unternehmungsexterner Koordination $\mathrm{zu}$ einer schwierigen Optimierungsaufgabe innerhalb eines breiten Spektrums an Koordinationsmöglichkeiten erweitert. ${ }^{59}$ Abbildung 5/2 fasst diese Annahmen über den Zusammenhang zwischen Transaktionsspezifität, Transaktionsfrequenz sowie die jeweils vorteilhafteste Koordinationsform zusammen.

\footnotetext{
${ }^{56} \mathrm{Vgl}$. Picot/Reichwald/Wigand (2001), S. 45

57 Vgl. Williamson (1979), S. 249.

58 Vgl. Williamson (1979), S. 253.

59 Vgl. Picot/Reichwald/Wigand (2001), S. 54.
} 


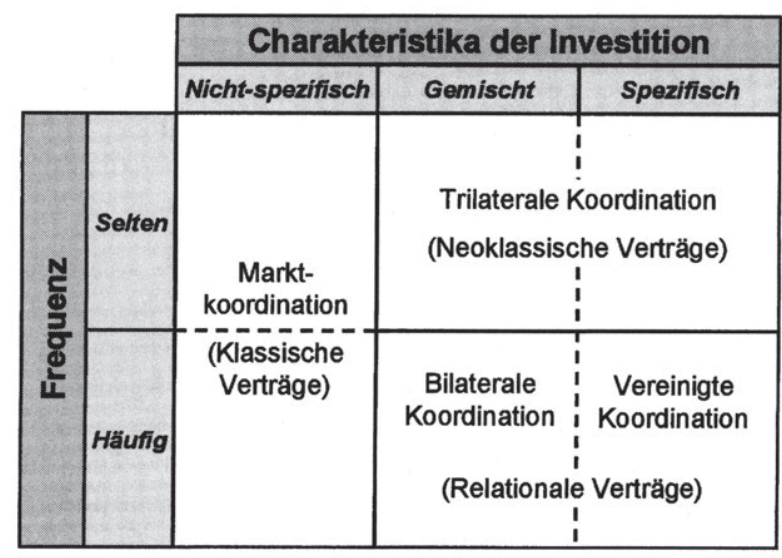

Abb. 5/2: Zusammenhang zwischen Transaktionseigenschaften und Koordinationsform

Quelle: Williamson (1979), S. 247.

Die Agency-Theorie als dritter Zweig der Neuen Institutionenökonomie untersucht die Gestaltung der vertraglichen Beziehung zwischen einem Auftraggeber und einem Auftragnehmer unter den Bedingungen ungleicher Informationsverteilung unter Unsicherheit. ${ }^{60}$ Aus der Perspektive der Agency-Theorie wird die Unternehmung als ein Netz von Verträgen aufgefasst, aus denen auf Grund des ungleichen Informationsstands und der Unsicherheit der Zukunft nur unvollständige Handlungsanweisungen an die beteiligten Individuen hervorgehen. Diese Unvollständigkeiten sind zwar beabsichtigt, sie eröffnen jedoch dem Auftragnehmer (Agent) die Möglichkeit zu eigennützigem Verhalten auf Kosten der Interessen des Auftraggebers (Principal). In der beschriebenen Informationssituation entstehen Kosten aus der Abweichung vom fiktiven Idealzustand des vollkommenen Tausches. Diese Kosten werden als AgencyKosten bezeichnet und sind bei der Gestaltung des Vertragswerkes zu berücksichtigen. Die Agency-Theorie beschreibt die Auftraggeber/Auftragnehmer-Beziehung, typologisiert Informationsasymmetrien und entwickelt Vorschläge für institutionelle Arrangements zur Senkung der Agency-Kosten. ${ }^{61}$

Die Auftraggeber/Auftragnehmer-Beziehung ist durch die eingangs bereits angesprochenen Verhaltensannahmen (Eigeninteresse, begrenzte Rationalität, Opportunismus) über die Akteure und durch eine asymmetrische Informationsverteilung zwischen Principal und Agent gekennzeichnet. Die ungleiche Informationsverteilung bietet häufig gerade den Anlass für das Zustandekommen eines Vertrages, da der Agent über bessere Kenntnisse, Fähigkeiten und/oder Erfahrungen im betreffenden Delegationsbereich verfugt. Der Informationsvorsprung des Agent wird in vielen Fällen noch durch die mangelnde Beobachtbarkeit seines Verhaltens für den Principal erhöht. Der Principal ist häufig nicht in der Lage zu beurteilen, ob ein erzieltes Ergebnis dem Leistungsverhalten des Agent oder nicht beeinflussbaren

\footnotetext{
${ }^{60} \mathrm{Vgl}$. stellvertretend für viele andere Wenger/Terberger (1988), S. 506; Spremann (1990), S. 562; Elschen (1991), S. 1004; Hartmann-Wendels (1992), Sp. 72; Ebers/Gotsch (1999), S. 209.

$61 \mathrm{Vgl}$. Picot/Reichwald/Wigand (2001), S. 56f.
} 
Umwelteinflüssen zuzurechnen ist. ${ }^{62}$ Durch die fehlende Möglichkeit zur Beurteilung des Verhaltens entstehen Handlungsspielräume für den Agent, die dieser zu seinem persönlichen Vorteil nutzen kann. ${ }^{63}$

Innerhalb der Agency-Theorie sind eine normative und eine positive Forschungsrichtung zu unterscheiden. Die normative Variante verfolgt das Ziel, formalanalytisch begründete Empfehlungen für die Gestaltung von Vertragsbedingungen zu geben, mit denen eine Steuerung des Auftragnehmerverhaltens erreicht werden kann. ${ }^{64}$ Die Untersuchungen in diesem Bereich stützen sich auf die Erkenntnisse und Methoden der traditionellen Mikroökonomik und behandeln im Prinzip Probleme, in denen individuelle Nutzenfunktionen einer Maximierung unter Nebenbedingungen unterzogen werden. ${ }^{65}$ Von den empirischen Gegebenheiten, unter denen Verträge geschlossen und erfullt werden, wird dabei weitgehend abstrahiert. ${ }^{66}$ Demgegenüber weist die positive Variante der Agency-Theorie einen stärker empirischen Bezug auf und versucht, in der Realität gegebene Organisations- und Vertragsformen zu erklären sowie Gestaltungsempfehlungen zur Erhöhung ihrer Effizienz zu entwickeln. ${ }^{67}$ Die positive Variante der Agency-Theorie weist auf Grund dieses stärkeren empirischen Bezugs eine größere Nähe zur konkreten Gestaltung und Steuerung von Unternehmungen auf als die normative Variante, deren Modelle häufig sehr restriktive Annahmen voraussetzen. Deshalb steht die positiv ausgerichtete Forschungsrichtung im Mittelpunkt der weiteren Untersuchungen dieses Kapitels.

Als Formen der Informationsasymmetrie unterscheidet die positiv ausgerichtete Agency-Theorie drei Arten der ungleichen Informationsverteilung, die einen Informationsvorteil des Agent gegenüber dem Principal begründen können. Dabei handelt es sich um hidden characteristics, hidden intention und hidden action. ${ }^{68}$ Hidden characteristics treten bereits im Vorfeld des Vertragsabschlusses auf, wenn dem Principal nicht alle vertragsrelevanten Eigenschaften und Leistungsfähigkeiten bekannt sind. Auf Grund von hidden characteristics besteht die Gefahr, dass der Principal unter den verfügbaren Vertragspartnern nicht den leistungsfähigsten und vertrauenswürdigsten auswählt. Dieses Phänomen wird als adverse selection bezeichnet. Nutzt der Agent nach Abschluss des Vertrages seinen Handlungsspielraum eigennützig zu Ungunsten des Principal aus (moral hazard), so wird von hidden action gesprochen. Der Principal erkennt in vielen Fällen nur das Ergebnis von Handlungen des Agent, kann die Handlungen selbst aber nicht beobachten oder nicht beurteilen. Schließlich besteht die Gefahr von hidden intention als drittem Typ asymmetrischer Informationsverteilung. Dabei sind dem Principal nicht alle Interessen des Agent bekannt, sodass er keine Vorkehrungen dagegen treffen kann, dass der Agent seine Handlungen an diesen Interessen ausrichtet. Nach Abschluss des Vertrages ist es eventuell nicht mehr möglich, Gegenmaßnahmen zu ergreifen, da der Principal in eine Abhängigkeitssituation vom Agent geraten ist.

\footnotetext{
${ }^{62} \mathrm{Vgl}$. Ebers/Gotsch (1999), S. 211.

$63 \mathrm{Vgl}$. Picot/Reichwald/Wigand (2001), S. 59.

${ }^{64}$ Vgl. Mikus (1998), S. 452.

${ }^{65}$ Vgl. Richter/Furubotn (1999), S. $165 f$.

${ }^{66} \mathrm{Vgl}$. Ebers/Gotsch (1999), S. 209.

$67 \mathrm{Vgl}$. ähnlich Mikus (1998), S. 452.

${ }^{68} \mathrm{Vgl}$. zum Folgenden Mikus (1998), S. $453 \mathrm{ff}$.
} 
Als Folge der beschriebenen Informationsunvollkommenheiten weicht die Situation in einem Auftraggeber/Auftragnehmer-Verhältnis normalerweise von der Idealsituation des vollkommenen Tausches ab. Es entstehen Agency-Kosten, die sich aus drei Komponenten zusammensetzen: ${ }^{69}$ (1) Kontrollkosten des Principal durch den Aufbau von Überwachungssystemen, (2) Garantiekosten des Agent z.B. in Form von Schadenersatzgarantien oder Rechenschaftsleistungen und (3) verbleibenden Wohlfahrtsverlusten, die sich aus dem verbleibenden Handlungsspielraum des Agent oder aus der Unterlassung von Handlungen durch den Agent ergeben können. Die Agency-Theorie entwickelt Empfehlungen, auf welche Weise diese Kosten möglichst weit gesenkt werden können. Diese betreffen das Verhalten von Principal und Agent vor und nach Vertragsschluss sowie die Gestaltung des Vertrages.

\subsubsection{Methodische Leitideen der Neuen Institutionenökonomie}

Die Arbeiten der Neuen Institutionenökonomie bewegen sich in methodischer Hinsicht weitgehend auf dem Fundament der neoklassischen Theorie. ${ }^{70}$ Die Zielsetzung der betreffenden Vertreter liegt nicht etwa darin, einen radikalen Bruch mit der Neoklassik einzuleiten und diese abzulösen. Methodischer Ausgangspunkt der Theoriebildung ist vielmehr die Feststellung, dass die Prämissen der neoklassischen Theorie viele Probleme ausblenden, die besonders charakteristisch für das Wirtschaftsgeschehen in der realen Welt sind. ${ }^{71}$ Diese Prämissen vereinfachen die Analyse und machen die neoklassischen Modelle handhabbar. Die zu Grunde liegenden Vereinfachungen führen jedoch auch zur Vernachlässigung wichtiger Aspekte und verstellen damit die Sicht auf weitere fruchtbare Erkenntnisse. Durch die Einbeziehung von Institutionen soll das institutionelle Vakuum der neoklassischen Theorie überwunden und auf diese Weise ihre Aussagekraft und damit ihre Leistungsfähigkeit für die Erklärung und Gestaltung ökonomischer Sachverhalte erhöht werden. ${ }^{72}$

$\mathrm{Ob}$ diese Aufhebung von einschränkenden Prämissen eine echte Revolution des ökonomischen Denkens darstellt oder nicht, ist umstritten. ${ }^{73}$ In neueren Arbeiten wird zunehmend in Frage gestellt, ob sich das gängige neoklassische Modell genügend abändern lässt, um auf eine reale Welt kostspieliger Transaktionen und unvollkommener Entscheidungssubjekte anwendbar zu sein. ${ }^{74}$ Infolgedessen wird die Forderung nach einem eigenen Instrumentarium der Neuen Institutionenökonomie jenseits dem der neoklassischen Theorie erhoben. ${ }^{75}$ Tatsächlich handelt es sich bei der Entwicklung eines eigenen Instrumentariums um eine Herausforderung für zukünftige Forschungsanstrengungen. Der Status quo der Neuen Institutionenökonomie, der den Referenzpunkt für die weiteren Überlegungen in diesem Kapitel darstellt, ist dagegen vornehmlich durch die Verwendung von Hybridmodellen gekennzeichnet. In diesen Hybridmodellen werden einzelne neoklassische Annahmen durch die Einführung zusätzlicher Nebenbedingungen modifiziert, während an anderen Annahmen jedoch in unveränderter Form festgehalten wird.

\footnotetext{
${ }^{69}$ Vgl. Jensen/Meckling (1976), S. 308.

${ }^{70} \mathrm{Vgl}$. Arrow (1986), S. 1184; Richter/Furubotn (1999), S. 478.

11 Vgl. Erlei/Leschke/Sauerland (1999), S. 51.

72 Vgl. Budäus/Gerum/Zimmermann (1988), S. 9, Schanz (1997a), S. 142.

${ }^{73}$ Vgl. Richter/Furubotn (1999), S. 477.

74 Vgl. Richter/Furubotn (1999), S. 481.

${ }^{75} \mathrm{Vgl}$. Richter/Furubotn (1999), S. 505ff.
} 
Im Vergleich zur zwischenzeitlich in den Vordergrund getretenen verhaltensorientierten Strömung in der Betriebswirtschaftslehre neigt der Ansatz der Neuen Institutionenökonomie wieder mehr einer deduktiv-nomologischen Vorgehensweise bei der Theoriebildung $\mathrm{zu}^{76}$ Zwar fasst die Neue Institutionenökonomie wirtschaftliches Handeln als sozialen Tatbestand auf und trifft Annahmen über das Verhalten der beteiligten Individuen. Bei der Entwicklung von Aussagen wird jedoch keine interpretative Perspektive im verhaltenstheoretischen Sinne eingenommen, sondern davon ausgegangen, dass soziales Handeln Gesetzmäßigkeiten folgt, die durch eine erklärende Theorie erforscht werden können. Wie die neoklassische Theorie geht auch die Neue Institutionenökonomie bei der Abbildung des menschlichen Verhaltens vom Bild des homo oeconomicus aus, ${ }^{77}$ bei dem die menschliche Persönlichkeit für die Zwecke der Modellbildung auf das Streben nach der Erhöhung des persönlichen Nutzens reduziert wird. Die Forschungsbemühungen zielen darauf $a b$, unabhängig von der konkreten Situation, in der sich die beteiligten Individuen befinden, allgemeingültige Empfehlungen für die Gestaltung von Institutionen als Rahmen wirtschaftlichen Handelns zu geben.

Der methodologische Individualismus, der in der Tradition ökonomischen Denkens in der klassischen und neoklassischen Theorie fest verankert ist, liegt auch den einzelnen Teilbereichen der Neuen Institutionenökonomie zu Grunde. ${ }^{78}$ Im Zentrum der Analyse steht das einzelne Entscheidungssubjekt, nicht die Organisation oder das Kollektiv. ${ }^{79}$ So versteht die Theorie der Verfügungsrechte wirtschaftliches Handeln als die Ausübung von Verfügungsrechten durch Individuen. Kollektives Handeln wird in dieser Sichtweise stets auf individuelle Entscheidungen, die sich an der Maximierung des persönlichen Nutzens orientieren, reduziert. ${ }^{80}$ Die Transaktionskostentheorie konzentriert sich zwar nicht primär auf die an einer Transaktion beteiligten Vertragspartner, die als „Parteien ${ }^{\text {(81 }}$ bezeichnet werden, sondern auf die Transaktion und die rahmenbildende Vertragsstruktur selbst. Aus den getroffenen Verhaltensannahmen (Nutzenmaximierung, begrenzte Rationalität und opportunistisches Verhalten) geht aber hervor, dass die Analyse der Transaktion ebenfalls an den einzelnen, in eine Vertragsbeziehung involvierten Individuen anknüpft. Die Agency-Theorie fasst die Unternehmung als ein Netz von Verträgen auf ${ }^{82}$ und geht zum Zwecke der Analyse davon aus, dass sich die gesamte Unternehmung und ihre Umweltbeziehungen zu Vertragsbeziehungen zwischen Individuen desaggregieren lassen. ${ }^{83}$ Auch dabei wird also eine prinzipiell reduktionistisch ausgerichtete Vorgehensweise verfolgt, die methodisch am Verhalten des Einzelnen ansetzt.

Von der neoklassischen Theorie übernimmt die Neue Institutionenökonomie ferner nicht nur das Merkmal des methodologischen Individualismus, sondern sie setzt auch die marginalistische Tradition als wesentliches methodisches Merkmal neoklassischer Theoriebildung fort. Die formalen Analysen in allen Teilbereichen der neuen

\footnotetext{
${ }^{76}$ Zur deduktiv-nomologischen Position vgl. Kieser (1993), S. 7ff. sowie die dort zitierten Quellen.

$71 \mathrm{Vgl}$. Erlei/Leschke/Sauerland (1999), S. 51.

78 Vgl. Schanz (1997a), S. 156; Erlei/Leschke/Sauerland (1999), S. 51.

79 Vgl. Richter/Furubotn (1999), S. 479.

${ }^{80} \mathrm{Vgl}$. Budảus/Gerum/Zimmermann (1988), S. 11.

${ }^{81}$ Williamson (1979), S. 236, S. 251.

${ }^{82} \mathrm{Vgl}$. Fama (1980), S. 289.

${ }^{83}$ Vgl. Ebers/Gotsch (1999), S. 210.
} 
Institutionenökonomie führen Grenzwertbetrachtungen durch ${ }^{84}$ und unterstellen damit die Möglichkeit zur Realisierung von durch die Theorie ermittelten Optimalpunkten. Dieses Vorgehen impliziert die Annahme, dass durch geeignete institutionelle Arrangements Kräfte erzeugt werden können, die zu stabilen Gleichgewichten in den Optimalpunkten führen. ${ }^{85}$ Die Lage derartiger Gleichgewichte kann durch die Angabe von Marginalbedingungen beschrieben werden, die sich auf analytische Weise bestimmen lassen.

Diese Überlegungen zeigen, dass die Neue Institutionenökonomie sich zwar in inhaltlicher Hinsicht durch die Fokussierung von Institutionen als Rahmen wirtschaftlichen Handelns von der neoklassischen Theorie unterscheidet. In methodischer Hinsicht besteht jedoch weitgehend Kontinuität zu deren Gedankenwelt. Von dieser lösen sich die Vertreter der Neuen Institutionenökonomie nur sehr langsam, indem sie sich von dem formalistischen Denken, das zu den ,adretten Regelmäßigkeiten des neoklassischen Modells ${ }^{\text {(86 }}$ geführt hat, abwenden.

\subsection{Konstruktion des Informationsmanagements als Erkenntnisgegen- stand aus der Perspektive der Neuen Institutionenökonomie}

Analog zur Vorgehensweise bei der Entwicklung des Produktionsorientierten und des Systemorientierten Ansatzes für eine Theorie des Informationsmanagements in den vorangegangenen Kapiteln nehmen die nachfolgenden Ausführungen eine Konstruktion des Informationsmanagements als speziellem Erkenntnisgegenstand der Neuen Institutionenökonomie vor. Diese Konstruktion stellt die dritte Stufe des Theorieeinstiegs dar und baut auf den Erkenntnisinteressen und Leitideen der Neuen Institutionenökonomie als Wissenschaftsprogramm der Betriebswirtschafslehre auf. Im Folgenden wird insbesondere überprüf, inwiefern die Größen, die im Fokus dieses Ansatzes stehen, durch das Informationsmanagement tangiert werden können. Auf der Basis der in diesem Abschnitt vorgenommenen Abgrenzung des speziellen Erkenntnisobjekts erfolgt im nächsten Abschnitt 5.3 die eigentliche Theoriebildung für das Informationsmanagement.

In Abschnitt 5.2.1 werden zunächst die grundsätzlichen Problemstellungen des Informationsmanagements aus der Perspektive der Neuen Institutionenökonomie näher beschrieben. Die weiteren Überlegungen dieses Kapitels werden auf diejenigen Arbeitsgebiete der Neuen Institutionenökonomie eingegrenzt, die für die in dieser Arbeit untersuchten Fragestellungen die größten Erkenntnispotenziale besitzen. Davon ausgehend wird im nachfolgenden Abschnitt 5.2.2 das Informationsmanagement als betriebliches Aufgabenfeld mit den Begriffen und Konzepten der Transaktionskostenanalyse betrachtet. Die Analyse von Auftraggeber/Auftragnehmer-Beziehungen mit den Mitteln der Agency-Theorie bildet einen weiteren geeigneten Ausgangspunkt fur die Entwicklung theoretisch fundierter Aussagen zum Informationsmanagement und

\footnotetext{
${ }^{84}$ Vgl. für die Pionierarbeiten zur Neuen Institutionenökonomie Coase (1937), S. 394, für die Theorie der Verfügungsrechte Alchian/Demsetz (1972), S. 782, für die Transaktionskostentheorie Williamson (1985), S. 90ff., für die Agency-Thorie Fama (1980), S. 295.

${ }^{85}$ Zur Gleichgewichtsvorstellung, die mit der Anwendung marginalistischer Betrachtungen verbunden ist, vgl. auch die Ausführungen zur Rezeption neoklassischer Vorgehensweisen im Abschnitt über die methodischen Leitideen des Faktortheoretischen Ansatzes.

${ }^{86}$ Richter/Furubotn (1999), S. 503.
} 
steht daher im Mittelpunkt des Abschnittes 5.2.3. Abschnitt 5.2.4 stellt die Verbindung zwischen den beiden hier referenzierten Forschungsrichtungen der Neuen Institutionenökonomie her und arbeitet die grundlegenden Bezugseinheiten eines institutionenökonomisch orientierten Informationsmanagements heraus.

\subsubsection{Grundfragestellungen des Informationsmanagements aus Sicht der Neuen Institutionenökonomie}

Im Fokus der Neuen Institutionenökonomie steht die Analyse von Institutionen, in deren Rahmen der Austausch von Leistungen vollzogen wird. Wie im vorigen Abschnitt bereits angesprochen, strukturieren Institutionen als sozial sanktionierbare Erwartungen das wirtschaftliche Geschehen und reduzieren dadurch dessen Unsicherheit. ${ }^{87}$ Auf diese Weise wird das individuelle Handeln der am Leistungsaustausch Beteiligten oft über lange Zeit hinweg in eine bestimmte Richtung gelenkt. Die Neue Institutionenökonomie richtet sich als Forschungsprogramm zum einen auf die Frage der Vorteilhaftigkeit unterschiedlicher Arten von Institutionen unter bestimmten Bedingungen und zum anderen auf die Frage, wovon diese Vorteilhaftigkeit abhängt und wie sie sich im Zeitablauf verändern kann. Dabei werden einerseits die konkrete Ausgestaltung einer Institution und andererseits die Kosten, die mit der Nutzung der Institution für bestimmte Formen des Austausches verbunden sind, bzw. die Allokationseffizienz der Institution betrachtet. Wie in Abbildung 5/3 dargestellt, fasst die Neue Institutionenökonomie je nach Untersuchungsschwerpunkt entweder die erstgenannte oder die zweitgenannte Größe als abhängige bzw. als unabhängige Variable auf. ${ }^{88}$

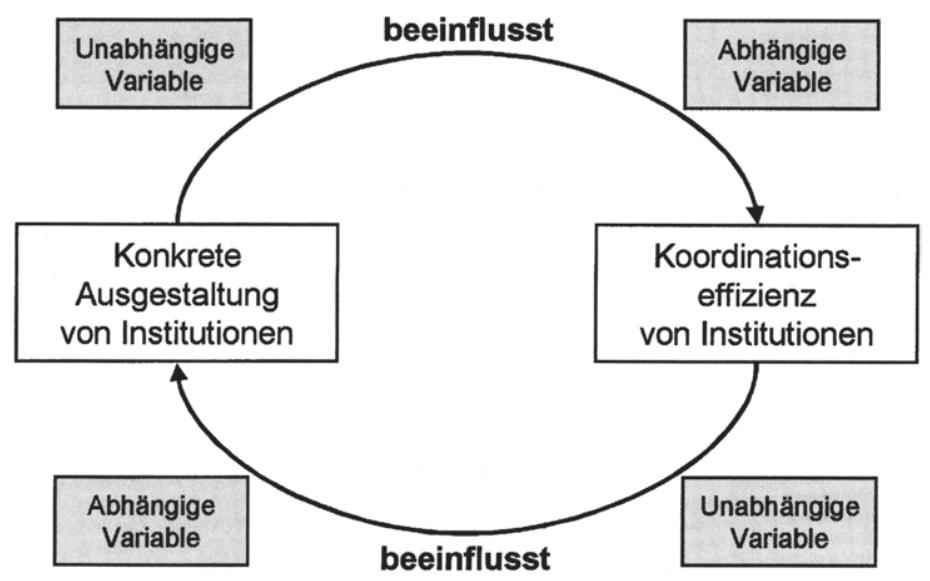

Abb. 5/3: Grundfragestellungen der Neuen Institutionenökonomie

Im Rahmen dieses Wissenschaftsprogramms werden also weniger die eigentlichen Produktionsvorgänge bei der Herstellung und Distribution einer Leistung untersucht.

${ }^{87}$ Vgl. Richter/Bindseil (1995), S. 133.

${ }^{88} \mathrm{Vgl}$. Ebers/Gotsch (1999), S. 199. 
Vielmehr wird die Aufmerksamkeit auf die Koordination dieser Vorgänge von der Beschaffung der Einsatzstoffe über die Erstellung des Produktes bis hin zur Verwendung der Leistung durch die Nutzer am Ende der Leistungskette gelenkt. Diesem grundsätzlichen Vorgehen folgend muss ein Institutionenökonomischer Ansatz für eine Theorie des Informationsmanagements ebenfalls am Konstrukt der Institution ansetzen. Institutionenökonomische Aussagen zum Informationsmanagement betreffen demnach nicht primär die eigentlichen Herstellungsprozesse, obwohl auch diese Aktivitäten der Informationsverarbeitung beinhalten können. Untersucht wird vielmehr die Koordination der Herstellungsprozesse durch entsprechende begleitende Informationsverarbeitungsprozesse.

$\mathrm{Zu}$ diesem Zweck sind die einleitend in Abschnitt 5.1.1 angesprochenen zwei Grundfragestellungen der Neuen Institutionenökonomie aufzugreifen. Einerseits ist zu untersuchen, welche Gestaltungsoptionen innovative informationstechnische Lösungen für das Informationsmanagement eröffnen und wie diese zur Steigerung der Effizienz der einzelnen institutionellen Arrangements und damit zur Reduzierung der jeweiligen Koordinationskosten genutzt werden können. Andererseits ist zu überprüfen, inwiefern die aufgezeigten Gestaltungsoptionen zur Veränderung der institutionellen Arrangements selbst und damit zu einem institutionellen Wandel führen können. Die Abgrenzung dieser beiden Gestaltungsrichtungen des Informationsmanagements, die in Abbildung 5/4 dargestellt sind, ist allerdings als vereinfachende Abstraktion für analytische Zwecke zu sehen, da bei konkreten Gestaltungsproblemen weniger von einer zeitlichen Abfolge als von einer simultanen Wirkung der jeweiligen Maßnahmen auszugehen ist. ${ }^{89}$

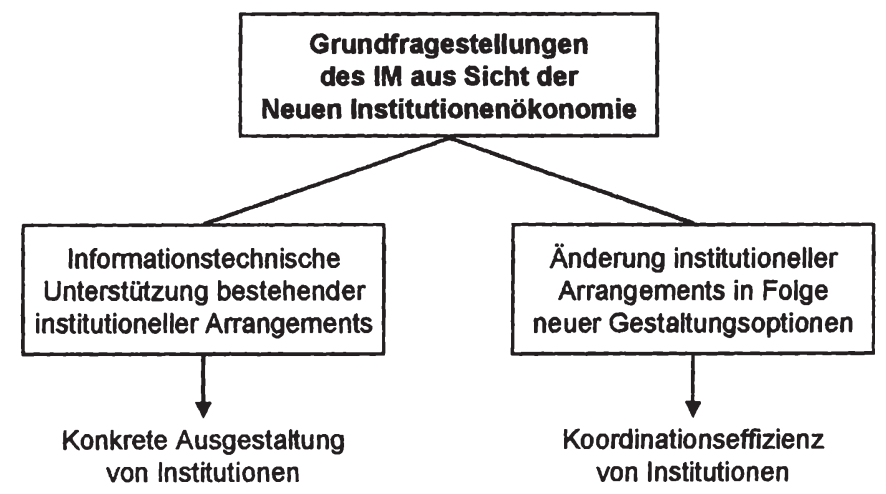

Abb. 5/4: Grundfragestellungen des Informationsmanagements aus Sicht der Neuen Institutionenökonomie

Die verschiedenen Forschungszweige der Neuen Institutionenökonomie unterscheiden sich bei der Spezifizierung der gemeinsamen Grundfragestellungen sowie der jeweils betrachteten Variablen. Im Folgenden werden die verschiedenen Spezifizierungen der im vorigen Abschnitt beschriebenen drei Theoriestränge der Neuen Institutionenökonomie erläutert und deren Eignung für die Ableitung von Aussagen zur Gestaltung und Steuerung technikunterstützter Informationssysteme von Unternehmungen überprüft.

${ }^{89}$ Vgl. dazu allgemein Ebers/Gotsch (1999), S. $199 f$. 
Verfuggungsrechte als Institutionstyp werden durch die gleichnamige Theorie der Verfügungsrechte behandelt. Dieses Arbeitsgebiet der Neuen Institutionenökonomie befasst sich, wie im vorangegangenen Abschnitt dargestellt, mit der Spezifizierung und Verteilung von Verfügungsrechten unter dem Aspekt der allokationseffizienten Verwendung von Gütern. Dabei werden Wohlfahrtseffekte betrachtet, die mit unterschiedlichen Graden der Definierbarkeit und Durchsetzbarkeit von Verfügungsrechten verbunden sind. Der Dreh- und Angelpunkt der Argumentation ist in diesem Zusammenhang die Entstehung externer Effekte in Folge eingeschränkter Möglichkeiten zur Ausübung einzelner Verfügungsrechte an einem Wirtschaftsgut. Solche Verdünnungen von Verfügungsrechten können einerseits auf (gesetzliche) Rahmenbedingungen (z.B. Bauordnungen oder Gewerbevorschriften), oder andererseits auf Transaktionskosten bei der Ausübung einzelner Rechte zurückzuführen sein.

Die verfuggungsrechtlichen Rahmenbedingungen sind durch das Informationsmanagement in einer einzelnen Unternehmung nicht beeinflussbar. Dagegen ist der zweite Untersuchungsbereich, der die Transaktionskosten bei der Ausübung von Verfügungsrechten beinhaltet, im Prinzip als Ausgangspunkt für die Ableitung theoretischer Aussagen zum Informationsmanagement geeignet. So wäre es vorstellbar zu untersuchen, inwiefern das Informationsmanagement in der Lage ist, die Formulierung, Durchsetzung und Übertragung von Verfügungsrechten zu verbessern und durch die Senkung von Transaktionskosten der Verdünnung von Verfügungsrechten entgegenzuwirken. Auf diese Weise könnten nicht nur gesamtwirtschaftiche Allokationsverbesserungen, sondern potenziell auch einzelwirtschaftliche Erfolgssteigerungen erzielt werden. Diese Argumentationslinie wird hier jedoch aus zwei Gründen nicht weiter verfolgt: Zum einen liegt der Hauptanwendungsbereich dieses Forschungszweiges in der Untersuchung gesamter Wirtschaftssysteme, wie beispielsweise der Analyse unterschiedlicher Eigentumsformen oder dem Vergleich von öffentlichem und privatem Eigentum an Unternehmungen. ${ }^{90}$ Zum anderen ist die Theorie der Verfügungsrechte in den aus einzelwirtschaftlicher Sicht interessanteren Punkten, nämlich der Übertragung von Verfügungsrechten beim Austausch ökonomischer Leistungen sowie der Trennung von Verfügungsrechten durch das Eingehen von Auftraggeber/Auftragnehmer-Beziehungen, weniger ausdifferenziert als die beiden anderen großen Theoriestränge der Neuen Institutionenökonomie. Die Transaktionskostentheorie und die Agency-Theorie greifen bei ihren Überlegungen zum Teil auf die Theorie der Verfügungsrechte zurück, sodass diese auch als Grundlage für die beiden anderen Forschungsrichtungen aufgefasst werden kann. ${ }^{91}$ Die weiteren Ausführungen in diesem Kapitel behandeln daher nur noch die Transaktionskostentheorie und die Agency-Theorie und greifen in diesem Rahmen punktuell auf die Theorie der Verfügungsrechte zurück.

Bei dem durch die Transaktionskostentheorie untersuchten Institutionstyp handelt es sich um Verträge, innerhalb derer sich der Austausch wirtschafticher Güter und Leistungen vollzieht. ${ }^{92}$ Der Prozess der Klärung und Vereinbarung von derartigen Verträgen wird als Transaktion bezeichnet ${ }^{93}$ und stellt den grundlegenden Untersuchungsgegenstand dieses Teilgebiets der Neuen Institutionenökonomie dar. ${ }^{94}$ Es

${ }^{90} \mathrm{Vgl}$. Ebers/Gotsch (1999), S. 204ff.

${ }^{91} \mathrm{Vgl}$. Gersch (1998), S. 18f.

${ }^{92} \mathrm{Vgl}$. Ebers/Gotsch (1999), S. 225

${ }^{93}$ Vgl. Picot (1982), S. 269.

${ }^{94}$ Vgl. Picot/Reichwald/Wigand (2001), S. 50. 
werden die Zusammenhänge zwischen der Vertragsform, den Transaktionseigenschaften und den bei der Abwicklung der Transaktion entstehenden Transaktionskosten beleuchtet. Ursprünglich wurden dabei die Transaktionseigenschaften als unabhängige Größe aufgefasst, von der je nach gewählter Vertragsform unterschiedlich hohe Transaktionskosten abhängen. ${ }^{95}$ Durch die Wahl einer „passenden“" Vertragsform wird bei diesem Konzept ein Fit zwischen Transaktionseigenschaften und Koordinationsform hergestellt, das die entstehenden Transaktionskosten minimiert. Neuere Untersuchungen gehen dagegen davon aus, dass auch die Eigenschaften der Transaktion durch die Beteiligten beeinflussbar sind und somit zu Aktionsvariablen im Rahmen der Unternehmungsführung werden. ${ }^{96}$

Die Argumentationslinie der Transaktionskostentheorie bietet mehrere vielversprechende Ansatzpunkte für die Entwicklung von theoretischen Aussagen zum Informationsmanagement. Aus der Perspektive dieses Forschungszweiges ergibt sich für das Informationsmanagement die Zielsetzung, die mit der Erstellung von Leistungen durch die Unternehmung verbundenen Transaktionskosten so weit wie möglich zu reduzieren. Als Aktionsvariablen des Informationsmanagements kommen dabei die verschiedenen Einflussgrößen in Frage, von denen die Transaktionskostentheorie annimmt, dass sie maßgeblich für die Höhe der Transaktionskosten sind. Das Informationsmanagement kann die Effizienz des Leistungsaustausches demzufolge auf der einen Seite durch eine Vereinfachung der Koordinationsaktivitäten erreichen, die eine Reduzierung der bei den einzelnen Vertragstypen anfallenden Transaktionskostenarten bewirkt. Auf der anderen Seite kann es auf eine informationstechnisch bedingte Veränderung der Transaktionsmerkmale hinwirken und damit den Wechsel zwischen verschiedenen Koordinationsformen ermöglichen, durch den ebenfalls das Potenzial zur Reduzierung von Transaktionskosten eröffnet wird. Abbildung $5 / 5$ zeigt diese beiden denkbaren Ansatzpunkte des Informationsmanagements, die in den nachfolgenden Überlegungen diese Kapitels im Einzelnen untersucht werden.

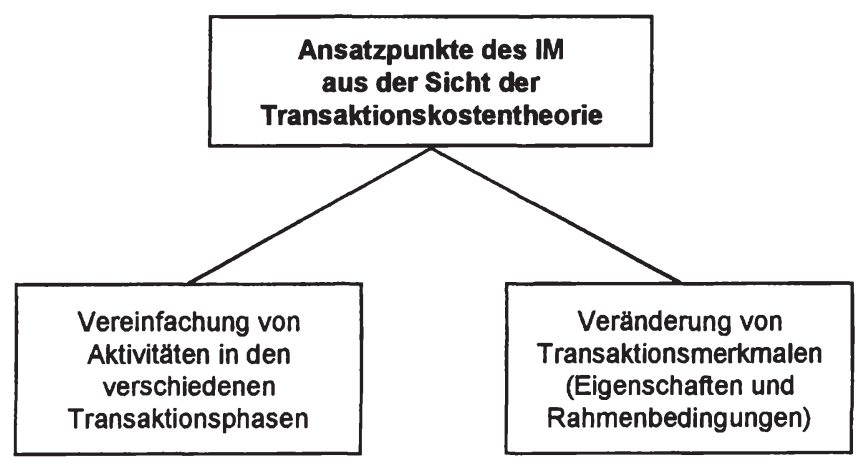

Abb. 5/5: Ansatzpunkte des Informationsmanagements aus Sicht der Transaktionskostentheorie

\footnotetext{
${ }_{95}$ Für dieses Vorgehen vgl. exemplarisch Williamson (1979), S. $233 \mathrm{ff}$.

${ }^{96}$ Für dieses Vorgehen vgl. exemplarisch Gersch (1998), S. 82ff.
} 
Im Mittelpunkt der Agency-Theorie steht ebenfalls die Institution des Vertrags. ${ }^{97}$ Der Ansatz konzentriert sich jedoch nicht wie die Transaktionskostentheorie auf Fragen im Zusammenhang mit der Übertragung von Verfügungsrechten, sondern auf Probleme, die sich aus der Trennung von Verfügungsrechten durch die Errichtung von Auftraggeber/Auftragnehmerbeziehungen ergeben. Charakteristisch ist dabei, dass der Agent für den Principal Entscheidungen trifft bzw. Handlungen durchführt und dabei über einen Vorsprung an Kenntnissen oder Fähigkeiten gegenüber dem Principal verfugt. ${ }^{98}$ In solchen Situationen treten Wohlfahrtsverluste auf, die als Agency-Kosten bezeichnet werden. Die Agency-Theorie beschäftigt sich mit den Ursachen für das Auftreten von Agency-Kosten sowie mit der Empfehlung von Gestaltungsmaßnahmen, die auf deren Reduzierung abzielen. ${ }^{99}$ Der normative Zweig der Agency-Theorie zielt dabei auf eine Interessenangleichung von Principal und Agent durch die Schaffung geeigneter Anreizsysteme. Der positive Zweig dagegen konzentriert sich auf die Reduzierung von Verhaltensspielräumen und Informationsasymmetrien und somit auf die Beseitigung der für den Agent bestehenden Möglichkeiten zu eigennützigem Verhalten.

Auch die Agency-Theorie eignet sich als Ausgangspunkt für die Ableitung theoretischer Aussagen zur Gestaltung und Steuerung technikunterstützter Informationssysteme in Unternehmungen. Dies gilt allerdings weniger für die normative Forschungsrichtung als für die positiv ausgerichtete Variante. Das Konzept der normativ ausgerichteten Agency-Theorie läuft auf eine Angleichung der Interessen von Principal und Agent als Ergebnis mathematischer Kalküle unter Bezugnahme auf die Mikroökonomie hinaus. ${ }^{100}$ Eine derartige Interessenangleichung lässt sich durch die vertragliche Vereinbarung von Anreizstrukturen erzielen, die bewirken, dass die Erfüllung von Zielen des Agent zugleich der Erfüllung von Zielen des Principal dient. ${ }^{101}$ Aus dieser Perspektive müsste das Informationsmanagement an der Schaffung von Anreizsystemen zur Beseitigung des Interessenkonflikts zwischen Principal und Agent mitwirken. Die Setzung von Anreizen für den Agent ist insbesondere durch die Kopplung der Entlohnung an das erzielte Ergebnis möglich. Dabei werden Zielkonflikte reduziert, indem die Erträge beider Vertragsparteien auf die gleiche Leistung zurückführbar werden. ${ }^{102} \mathrm{Da}$ es sich hier um eine formale Problemstellung ohne direkten Zusammenhang zu Fragen der technikunterstützten Informationsverarbeitung handelt, besitzt die normative Variante der Agency-Theorie keine geeigneten Anknüpfungspunkte für Gestaltungs- und Steuerungsmaßnahmen des Informationsmanagements in der Praxis.

Dagegen weist die positive Theorievariante auf Grund ihres stärkeren empirischen Bezugs ein größeres Erkenntnispotenzial für das Informationsmanagement auf. Die Leitideen der positiven Theorievariante legen für die weiteren Überlegungen folgende Argumentationslinie nahe: Das Informationsmanagement sollte darauf abzielen, durch die Entwicklung und den Einsatz entsprechender informationstechnisch unterstützter Lösungen die Verhaltensspielräume und Informationsasymmetrien, die für das Entstehen von Agency-Kosten ursächlich sind, zu vermindern und auf diese Weise die Agency-Kosten zu reduzieren. Im Einzelnen muss die Ableitung von Aussagen für das

\footnotetext{
${ }^{97}$ Vgl. Ebers/Gotsch (1999), S. 209.

98 Vgl. Mikus (1998), S. 452.

${ }^{99} \mathrm{Vgl}$. Picot/Reichwald/Wigand (2001), S. 57ff.

${ }^{100}$ Vgl. Hess (1999), S. 1505.

${ }^{101}$ Vgl. Laux (1990), S. 24.

${ }^{102}$ Vgl. Ebers/Gotsch (1999), S. 214.
} 
Informationsmanagement in diesem Bereich an den verschiedenen Komponenten der Agency-Kosten und den von der Agency-Theorie erkannten Möglichkeiten zur Reduzierung der jeweiligen Kostenarten ansetzen. Abbildung 5/6 fasst die Ansatzpunkte des Informationsmanagements aus Sicht der positiv ausgerichteten Variante der AgencyTheorie zusammen.

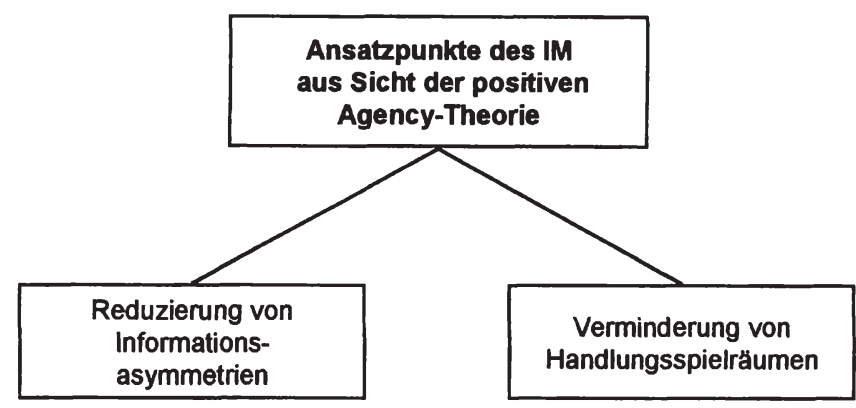

Abb. 5/6: Ansatzpunkte des Informationsmanagements aus Sicht der AgencyTheorie

\subsubsection{Informationsmanagement aus Sicht der Transaktionskostentheorie}

Entsprechend den erläuterten Erkenntnisinteressen liegt die besondere Funktion des durch das Informationsmanagement gestalteten und gesteuerten technikunterstützten Informationssystems der Unternehmung aus Sicht der Transaktionskostentheorie in der Koordination des Güter- und Leistungsaustausches. Das Informationsmanagement unterstützt durch seine Aktivitäten diese Koordination, einerseits als Koordination zwischen der Unternehmung und ihren Lieferanten bzw. Kunden und andererseits als Koordination zwischen den verschiedenen Beteiligten innerhalb der Unternehmung.

Die nachfolgenden Ausführungen präzisieren in Abschnitt 5.2.2.1 zunächst die Bedeutung und die Funktion der technikunterstützten Informationsverarbeitung in den einzelnen Transaktionsphasen. Abschnitt 5.2.2.2 beleuchtet anschließend die Anknüpfungspunkte des Informationsmanagements zur Einflussnahme auf die Transaktionseigenschaften. Zuletzt wird in Abschnitt 5.2.2.3 der Zusammenhang von Gestaltungsmaßnahmen des Informationsmanagements zu dem für die Koordination der Transaktion gewählten Vertragsdesign betrachtet.

\subsubsection{Informationsverarbeitung in den verschiedenen Transaktionsphasen}

Für eine genauere Beschreibung der Aktionsmöglichkeiten, über die das Informationsmanagement aus Sicht der Transaktionskostentheorie verfügt, ist eine detailliertere Betrachtung der einzelnen Transaktionskostenarten erforderlich. $\mathrm{Zu}$ den Transaktionskosten zählen alle Aufwendungen, die zur Erzielung und Verwirklichung einer Vereinbarung über den Austausch von Gütern und Leistungen erbracht werden müssen. ${ }^{103}$ Dabei handelt es sich in erster Linie um Informationskosten, die zur

${ }^{103}$ Vgl. Picot (1982), S. 270; Ouchi (1980), S. 130. 
Beseitigung bzw. Reduzierung unvollkommener Information über die Eigenschaften, Absichten und Verhaltensweisen der jeweils anderen Vertragsseite anfallen. Ferner fallen Transaktionskosten im Rahmen der Abwicklung der Transaktion fur die Koordination der Aktivitäten der Vertragspartner an. Die in Abschnitt 5.1.2 angesprochene Unterscheidung zwischen Ex ante- und Ex post-Transaktionskosten kann für eine nähere Untersuchung einzelner Transaktionskostenarten weiter untergliedert werden. Entsprechend den einzelnen Phasen einer Transaktion lassen sich dann als Ex anteKostenarten Anbahnungskosten und Verhandlungskosten sowie als Ex postKostenarten Abwicklungs- und Kontrollkosten sowie Anpassungskosten unterscheiden. ${ }^{104}$ Abbildung 5/7 visualisiert die verschiedenen Transaktionsphasen mit ihren jeweiligen Kostenarten.

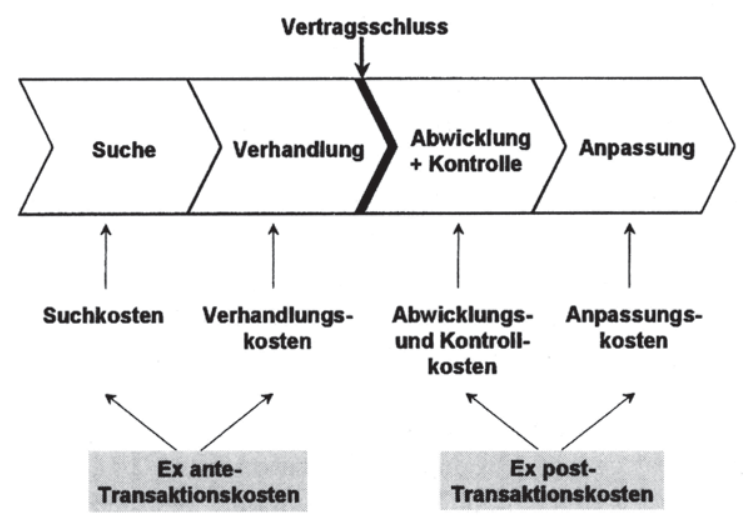

\section{Abb. 5/7: Kostenarten in den verschiedenen Transaktionsphasen}

Im Folgenden werden die Aktivitäten, die zur Entstehung dieser Kostenarten führen, näher betrachtet. Es werden allgemeine Anforderungen an die informationstechnische Unterstützung dieser Aktivitäten, mit der das Informationsmanagement auf eine Senkung der jeweiligen Kostenarten hinwirken kann, formuliert. Weiter unten werden in den Abschnitten 5.3.1 und 5.3.2 Anwendungssysteme betrachtet, mit deren Hilfe das Informationsmanagement die genannten Anforderungen erfüllen kann.

Anbahnungskosten umfassen Kosten im Vorfeld von bilateralen Vertragsverhandlungen, etwa Kosten für Aktivitäten der Beschaffung von Informationen über potenzielle Transaktionspartner und deren generelle Konditionen oder über allgemeine Qualitäts- und Preiskriterien auf dem Markt für die betreffende Leistung. Durch den allgemeinen Trend zur Internationalisierung des Wirtschaftsgeschehens erhöht sich tendenziell die Zahl der für eine Transaktion in Frage kommenden Anbieter und Nachfrager sowie die Zahl der angebotenen Produktvarianten. Ohne eine zielgerichtete informationstechnische Unterstützung steigt folglich der Umfang der für die Auswahl des geeignetesten Vertragspartners notwendigen Suchaktivitäten. Zur Reduzierung der Transaktionskosten müssen sich Gestaltungs- und Steuerungsmaßnahmen des Informationsmanagements in dieser Transaktionsphase auf eine Erhöhung der Markttransparenz richten. Dazu ist die Bereitstellung geeigneter Anwendungssysteme erforderlich,

\footnotetext{
${ }^{104}$ Für eine nähere Erläuterung der einzelnen Kostenarten vgl. Picot (1982), S. $270 \mathrm{f}$.
} 
die durch eine Zusammenführung unternehmungsexterner und unternehmungsinterner Informationsquellen eine umfassende Beobachtung des Marktgeschehens ermöglichen. Zur Anbahnung einer konkreten Transaktion ist ferner eine unkomplizierte Zusammenführung von konkreten Angebots- und Nachfrageaktivitäten wünschenswert.

Verhandlungskosten als zweite Ex ante-Transaktionskostenart entstehen, wenn nach Abschluss der allgemeinen Anbahnungsphase einer oder mehrere potenzielle Vertragspartner ausgewählt und konkrete Verhandlungen zum Abschluss eines Transaktionsvertrages aufgenommen werden. In Abhängigkeit von der jeweils auszutauschenden Leistung kann der Nachfrager die Anbieter in dieser Phase auffordern, genauere Angaben über bestimmte Leistungseigenschaften $\mathrm{zu}$ machen oder spezielle Angebote fur bestimmte Produktkonfigurationen abzugeben. ${ }^{105}$ Ferner sind die allgemeinen Rahmenbedingungen der Vertragsabwicklung, wie z.B. Termine und Zahlungskonditionen, abzustimmen. Die Höhe der in diesem Zusammenhang entstehenden Transaktionskosten richtet sich nach der Intensität und zeitlichen Ausdehnung von Verhandlungen, Vertragsformulierung und schließlich Einigung. Grundsätzlich sind bei standardisierten Verträgen, die für die marktliche Koordination typisch sind, weniger umfangreiche Verhandlungsaktivitäten als bei hochgradig individuell ausgestalteten Verträgen, deren Erfüllung typischerweise hierarchisch koordiniert wird. Das Informationsmanagement kann Verhandlungsaktivitäten durch die Bereitstellung hard- und softwaretechnischer Hilfsmittel unterstützen, die bei räumlicher und zeitlicher Trennung der potenziellen Vertragspartner einen einfachen und schnellen Austausch von Angebots- und Nachfrageinformationen ermöglichen. Dazu zählt einerseits die automatisierte Aushandlung von Vertragsdetails bei Standardverträgen (z.B. in Bezug auf die genaue Produktkonfiguration o.ä.) und andererseits die allgemeine Unterstützung von Kommunikationsaktivitäten, die sich auf den Entwurf und Abschluss eines größeren Vertragswerks richten.

Ex post-Transaktionskosten fallen nach Erzielung einer Einigung und Abschluss des Austauschvertrages an. Wie bereits erwähnt handelt es sich dabei um Kontroll- und Abwicklungskosten sowie um Anpassungskosten. Kontrollkosten entstehen für die Überwachung und Absicherung von Vereinbarungsbestandteilen, z.B. Termin-, Qualitäts-, Mengen-, Preis- oder auch Geheimhaltungsvereinbarungen. ${ }^{106}$ Das Informationsmanagement kann den Aufwand für derartige Kontrollaktivitäten durch die Einrichtung von Qualitätssicherungssystemen sowie durch die Bereitstellung informationstechnisch unterstützter Berichts- und Überwachungssysteme verringern. Dabei ist es sinnvoll, die durch solche Systeme erfassten Kontrollinformationen beiden Vertragsparteien zugänglich zu machen, um die Einigkeit über die Vertragseinhaltung zu gewährleisten. Dadurch wird der Interpretationsspielraum über die Vertragseinhaltung verringert und der Klärungsbedarf beim Auftreten von Abweichungen reduziert.

Wenn die Erstellung der Leistung integrativ, d.h. unter Einbeziehung des Nachfragers erfolgt, ${ }^{107}$ dann kommt es im Zeitraum nach Abschluss der ursprünglichen Vertragsvereinbarung außerdem zu einer weiteren Art des Informationsaustausches

\footnotetext{
${ }^{105}$ Vgl. ähnlich Gersch (1998), S. 209.

${ }^{106}$ Vgl. Picot (1982), S. 270.

${ }^{107}$ Für einen Überblick über das Konzept der integrativen Leistungserstellung vgl. Engelhardt/Freiling (1995a), S. 37ff.
} 
zwischen den Vertragsparteien. Typisch für integrativ erstellte Leistungen ist, dass die eigentliche Leistungserstellung erst nach Abschluss des zu Grunde liegenden Transaktionsvertrages erfolgt. ${ }^{108}$ Die Integrativität kann beispielsweise darin liegen, dass der Leistungsbezieher einzelne Teilleistungen innerhalb eines vertraglich vereinbarten Rahmens nach und nach abruft. Das Informationsmanagement kann diese Art der Leistungserstellung durch die Einrichtung von Lieferabrufsystemen zur quantitativen und zeitlichen Konkretisierung von Rahmenverträgen unterstützen. ${ }^{109} \mathrm{Zur}$ noch weiteren Senkung von Koordinationskosten ist ferner eine über den reinen Informationsaustausch hinausgehende organisatorische Abstimmung zwischen der liefernden und der beziehenden Unternehmung und eine unternehmungsübergreifende Prozesssteuerung vorstellbar.

Vertragsanpassungen werden dann notwendig, wenn im Falle einer längerfristigen Zusammenarbeit zwischen den Transaktionspartnern die vertraglich fixierten Vereinbarungen unvollständig sind und sich nachträglicher Klärungsbedarf herausstellt. Solche Anpassungen sind einerseits beim Auftreten unterschiedlicher Interpretationen über einzelne Vereinbarungsbestandteile und andererseits beim Eintritt unvorhergesehener Umstände im Umfeld der Transaktion erforderlich. ${ }^{110}$ Anpassungskosten entstehen in derartigen Situationen auf Grund von Aktivitäten zur Vereinbarung und Durchsetzung geänderter Vereinbarungen und zur Beseitigung von Interpretationsspielräumen in Bezug auf einzelne Vereinbarungsbestandteile. Die Anforderungen dieser Aktivitäten an die technikunterstützte Informationsverarbeitung betreffen in erster Linie die informationstechnische Unterstützung der Kommunikation zwischen den Vertragsparteien. Aus Sicht des Informationsmanagements besteht also eine hohe Ähnlichkeit zur Verhandlungsphase von Transaktionen. Daher werden Anwendungssysteme zur Unterstützung von Anpassungsaktivitäten im Weiteren nicht mehr gesondert betrachtet.

\subsubsection{Berührungspunkte zwischen den Charakteristika der Transaktion und der technikunterstützten Informationsverarbeitung}

Der Umfang und die Intensität von Aktivitäten, die zur Entstehung der soeben betrachteten Kostenarten bei der Durchführung einer Transaktion führen, hängt nach Auffassung der Transaktionskostentheorie einerseits von den Charakteristika der Transaktion und andererseits von den Eigenschaften des institutionellen Arrangements $a b$, das zur Abwicklung der Transaktion gewählt wird. ${ }^{111}$ Im Folgenden werden zunächst die Charakteristika der Transaktion und ihre Beeinflussbarkeit durch das Informationsmanagement näher betrachtet.

$\mathrm{Zu}$ den Charakteristika der Transaktion, die sich auf die Höhe der Transaktionskosten auswirken, gehören wie bereits angesprochen einerseits Eigenschaften, die in der Transaktion selbst liegen, und andererseits Eigenschaften, die die Rahmenbedingungen der Transaktion beschreiben. Die Eigenschaften der Transaktion umfassen die Unsicherheit, die Häufigkeit und die Spezifität der Transaktion, ${ }^{112}$ während die

\footnotetext{
${ }^{108} \mathrm{Vgl.} \mathrm{Gersch} \mathrm{(1998),} \mathrm{S.} 210$.

${ }^{109}$ Vgl. Gersch (1998), S. 211 sowie die dort zitierten Quellen

${ }^{110}$ Vgl. Ebers/Gotsch (1999), S. $225 f$.

${ }^{111} \mathrm{Vgl}$. Ebers/Gotsch (1999), S. $227 \mathrm{f}$.

${ }^{112}$ Vgl. Williamson (1979), S. 239.
} 
Gesamtheit der Rahmenbedingungen, die auch als Transaktionsatmosphäre bezeichnet wird, rechtliche, technische und soziale Rahmenbedingungen enthält. ${ }^{113}$ In den ursprünglichen Arbeiten zur Transaktionskostentheorie wurden diese Einflussgrößen nicht weiter hinterfragt und als fixer Ausgangspunkt für den Entwurf geeigneter vertraglicher Arrangements zur Minimierung der Transaktionskosten genommen. ${ }^{114}$ In neueren Arbeiten erfolgt dagegen eine Abwendung von dieser eingeschränkten Perspektive, und es wird davon ausgegangen, dass die Charakteristika einer Transaktion zumindest bis zu einem gewissen Grad Gegenstand von Entscheidungen der Unternehmungsführung sein können. ${ }^{115}$ Sie werden damit prinzipiell auch für das Informationsmanagement zu Aktionsvariablen. Dieser Sichtweise wird hier gefolgt.

\section{a) Transaktionsunsicherheit}

Ein steigender Grad an Unsicherheit als Transaktionseigenschaft führt generell zu zunehmenden Schwierigkeiten bei der Findung von Entscheidungen und damit zu einer Intensivierung der Koordinationsaktivitäten in allen Phasen einer Transaktion. ${ }^{116} \mathrm{Als}$ Formen der Unsicherheit werden in der Transaktionskostentheorie einerseits die parametrische Unsicherheit und anderseits die Verhaltensunsicherheit unterschieden. ${ }^{117}$ Parametrische Unsicherheit bezieht sich auf die situativen Bedingungen der Transaktion und ihre zukünttige Entwicklung, während Verhaltensunsicherheit die Möglichkeit des Transaktionspartners zu opportunistischem Verhalten betrifft. Mit zunehmender parametrischer Unsicherheit sind mehr Eventualitäten (Kontingenzen) vorstellbar, sodass es immer schlechter möglich wird, die gegenseitigen Rechte und Pflichten der Beteiligten vollständig vertraglich zu vereinbaren. ${ }^{118}$ Die Unsicherheit der Umweltbedingungen wird allerdings erst in Verbindung mit der unterstellten begrenzten Rationalität der Akteure untereinander zum Problem. ${ }^{119}$ Wenn zur parametrischen Unsicherheit Verhaltensunsicherheit hinzukommt, dann besteht die Möglichkeit, dass einer der Beteiligten Informationsvorsprünge erlangt und diese opportunistisch ausnutzt.

Da eine stark ausgeprägte Unsicherheit stets mit hohen Transaktionskosten verbunden ist, ${ }^{120}$ handelt es sich um ein Transaktionsmerkmal, dessen Intensität durch ein an der Reduzierung von Transaktionskosten orientiertes Informationsmanagement möglichst weit zu reduzieren ist. Dabei ist allerdings davon auszugehen, dass die begrenzte Rationalität der Beteiligten im Prinzip erhalten bleibt, da auch durch eine verbesserte Informationsverarbeitung der Zustand der vollkommenen Information nicht wirtschaftlich sinnvoll hergestellt werden kann. Parametrische Unsicherheit kann teilweise durch den Einsatz von Anwendungssystemen zur Planungsunterstützung, die sich auf eine bessere Prognose künftiger Entwicklungen richten, verringert werden. Dies schafft die Voraussetzungen für eine exaktere Fixierung der gegenseitigen Ansprüche zum Zeitpunkt des Vertragsschlusses. Der Aspekt der Verhaltensunsicherheit stellt eine

\footnotetext{
${ }^{113}$ Vgl. Picot/Reichwald/Wigand (2001), S. 53.

${ }^{114}$ Vgl. Kronen (1994), S. 61.

${ }^{115}$ Vgl. z.B. Ebers (1994), S. 27ff; Kronen (1994), S. 61ff.; Gersch (1998), S. $90 \mathrm{ff}$.

${ }^{116}$ Vgl. Ebers/Gotsch (1999), S. 229.

${ }^{117}$ Vgl. dazu Williamson (1985), S. $57 \mathrm{ff}$.

${ }^{118} \mathrm{Vgl}$. Ebers/Gotsch (1999), S. $229 \mathrm{f}$.

${ }^{119} \mathrm{Vgl}$. Picot/Reichwald/Wigand (2001), S. $52 \mathrm{f}$.

${ }^{120}$ Vgl. Picot (1982), S. 272; Ebers/Gotsch (1999), S. 229.
} 
Schnittstelle von Transaktionskostentheorie und Agency-Theorie, die im Detail Möglichkeiten zur Verringerung der Verhaltensunsicherheit analysiert, dar. ${ }^{121}$ Daher wird dieser Bereich hier im Rahmen der Transaktionskostentheorie nicht weiter betrachtet.

\section{b) Transaktionshäufigkeit}

Eine steigende Häufigkeit gleicher oder ähnlicher Transaktionen wirkt sich unter sonst gleichen Umständen generell kostensenkend auf die Durchschnittskosten der einzelnen Transaktion aus. ${ }^{122}$ Durch die Wiederholung der Transaktion können Fixkostendegressionseffekte erzielt werden, wenn Vereinbarungsbestandteile aus der Erstvereinbarung für die folgenden Transaktionen übernommen werden. Economies of Scale und Lerneffekte treten in diesem Fall auf, wenn durch Spezialisierung auf das betreffende Transaktionsproblem und die Sammlung von Erfahrungen eine Routinisierung des Verfahrens stattfindet und zudem vereinfachte Abwicklungsmöglichkeiten entdeckt werden. Wenn das Kriterium der Häufigkeit auch ursprünglich nur auf die Vertragspartei des Nachfragers abstellte, ${ }^{123}$ ist eine Ausdehnung auf die Partei des Anbieters möglich, da auch bei diesem im Falle des wiederholten Austausches der gleichen oder einer ähnlichen Leistung transaktionskostensenkende Effekte auftreten können.

Das Informationsmanagement verfugt nur über sehr indirekte Möglichkeiten zur Einflussnahme auf die Häufigkeit als Transaktionsmerkmal. So kann es für den Anbieter beispielsweise durch eine geeignete informationstechnische Unterstützung möglich werden, die angebotene Leistung präziser auf die Bedürfnisse auszurichten und so eine stärkere Kundenbindung zu erreichen, die sich in einer erhöhten Transaktionshäufigkeit mit dem gleichen Vertragspartner niederschlägt. In ähnlicher Weise kann auf der Seite des Nachfragers der Beschaffungsprozess durch innovative informationstechnische Unterstützung so gestaltet werden, dass eine größere Standardisierung erreicht wird, die häufig mit einer Verringerung der Anzahl der Transaktionspartner auf einen oder wenige Anbieter verbunden ist (Single Sourcing). Derartige Maßnahmen setzen jedoch originär bei dem nachfolgend behandelten Merkmal der Transaktionsspezifität an und bewirken nur mittelbar eine Erhöhung der Transaktionshäufigkeit. Die Transaktionshäufigkeit als eigenständige Gestaltungsvariable des Informationsmanagements wird daher im Folgenden nicht mehr weiter untersucht.

\section{c) Transaktionsspezifität}

Die Untersuchung der Spezifität als Transaktionseigenschaft stellt ein besonders charakteristisches Merkmal der Transaktionskostentheorie dar, da die bisher betrachteten Merkmale Unsicherheit und Häufigkeit auch von anderen Forschungsansätzen als relevant erkannt und mit den jeweils verwendeten Mitteln untersucht werden. Dagegen wird in Arbeiten zur Transaktionskostentheorie übereinstimmend die Spezifität einer Transaktion als Haupteinflussgröße der Transaktionskosten bezeichnet. ${ }^{124}$ Unter Spezifität wird dabei die Ausrichtung der zur Durchführung einer Transaktion erforderlichen

\footnotetext{
${ }^{121} \mathrm{Vgl}$. Picot/Reichwald/Wigand (2001), S. 53.

${ }^{122}$ Vgl. Picot (1982), S. 272; Ebers/Gotsch (1999), S. 230.

${ }^{123} \mathrm{Vgl}$. Williamson (1979), S. 247.

${ }^{124}$ Vgl. z.B. Williamson (1979), S. 239; Picot/Reichwald/Wigand (1998), S. 43; Gersch (1998), S. 90
} 
Ressourcen auf diesen bestimmten Transaktionstyp verstanden. Durch einen hohen Spezifitätsgrad lassen sich im Allgemeinen Produktivitätsvorteile erzielen, da die verwendeten Inputfaktoren über Spezialisierungsvorteile bei der Herstellung der ausgetauschten Leistung verfugen. ${ }^{125}$ Diese Spezialisierungsvorteile werden jedoch dadurch erkauft, dass die Vertragsparteien stark an den betrachteten Transaktionstyp gebunden werden, da der Wechsel des Vertragspartners oder der betreffenden Leistung mit großen Opportunitätskosten verbunden ist. ${ }^{126}$ Die Existenz transaktionsspezifischer Investitionen begründet somit eine größere Abhängigkeit vom jeweiligen Vertragspartner und erhöht das Interesse an einer dauerhaften Austauschbeziehung. Daraus resultiert die Gefahr, dass der jeweils andere Vertragspartner diese Abhängigkeit opportunistisch ausnutzt.

Das Informationsmanagement kann auf die Spezifität einer Transaktion durch eine entsprechende Ausgestaltung des technikunterstützten Informationssystems der Unternehmung und der darin stattfindenden Prozesse zielgerichtet Einfluss nehmen. Für eine genauere Darstellung der Gestaltungsmöglichkeiten, die sich dem Informationsmanagements in diesem Bereich bieten, ist eine nähere Betrachtung des Spezifitätsphänomens erforderlich. Obwohl es sich bei der Spezifität um eines der zentralen Konstrukte der Transaktionskostentheorie handelt, wird häufig keine systematische Analyse dieser Transaktionseigenschaft vorgenommen. An dieser Stelle wird der Arbeit von GERSCH gefolgt, der verschiedene Dimensionen der Spezifität unterscheidet, die nachfolgend näher erläutert werden. In den einzelnen Dimensionen können die Gestaltungsmaßnahmen des Informationsmanagements die Spezifität des technikunterstützten Informationssystems der Unternehmung im Prinzip sowohl erhöhen als auch senken.

Nach dem Objekt der Spezifität sind spezifische Leistungsergebnisse (Produkte), spezifische Leistungserstellungsprozesse und spezifische Leistungsfähigkeiten (Potenziale) zu unterscheiden. ${ }^{127}$ Für alle diese Objekttypen kann ein Bezug zum Informationsmanagement hergestellt werden. Die technikunterstützte Informationsverarbeitung kann beispielsweise zur Erhöhung der Spezifität der von der Unternehmung angebotenen Leistungsergebnisse beitragen, indem sie eine verstärkten Anpassung des individuellen Angebots an die Bedürfnisse einzelner Kunden ermöglicht. Sofern die Leistungsergebnisse selbst informationelle Bestandteile beinhalten, ist diesbezüglich ebenfalls eine mehr oder weniger ausgeprägte Individualisierung bzw. Standardisierung denkbar. Auch die Prozesse der Informationsverarbeitung als Teil der gesamten Leistungserstellungsprozesse der Unternehmung können mehr oder weniger speziell etwa auf die Erstellung spezifischer Produkte, den Austausch mit einem bestimmten Partner oder die Kombination mit anderen spezifischen Prozessen ausgerichtet sein. Die Leistungsfähigkeiten der Unternehmung werden durch ihr technikunterstütztes Informationssystem ebenfalls tangiert, da einerseits die verwendete Hard- und Software einen mehr oder weniger hohen Spezifitätsgrad aufweist und andererseits das bei den Aufgabenträgern der Informationsverarbeitung vorhandene Know-how sich auf mehr oder weniger transaktionsspezifische Sachverhalte beziehen kann. Die Transaktions-

\footnotetext{
${ }^{125} \mathrm{Vgl}$. Ebers/Gotsch (1999), S. 228. Derartige Ersparnisse können nicht nur auf der Anbieterseite, sondern auch auf der Nachfragerseite auftreten, wenn der Nachfrager sich bei Bezug und Verwendung der Leistung in besonderem Maße auf die betrachtete Transaktion einstellt.

${ }^{126} \mathrm{Vgl}$. Williamson (1979), S. 240.

${ }^{127}$ Zur Unterscheidung zwischen diesen Leistungsdimensionen vgl. Engelhardt/Freiling (1995a), S. 900ff.; Engelhardt/Freiling (1995b), S. 39f.
} 
spezifität der verschiedenen Objekttypen und die Beziehungen der verschiedenen Spezifitätsobjekte untereinander werden als Gestaltungsvariablen des Informationsmanagements in Abschnitt 5.3.3 ausführlich betrachtet.

Die Ausprägung der Spezifität kann qualitativ, quantitativ, räumlich oder zeitlich sein. ${ }^{128} \mathrm{Da}$ sich die Spezifität eines Objekts in mindestens einer dieser Ausprägungen manifestiert, stellen die verschiedenen Ausprägungen der soeben genannten Objekttypen im konkreten Einzelfall Aktionsvariablen des Informationsmanagements dar. Der Bezug der Spezifität beschreibt, woran genau die Unternehmung durch die einzelnen Ausprägungen der verschiedenen Spezifitätsobjekte gebunden wird. Die Spezifität muss sich nicht stets - wie in der Literatur häufig angenommen - auf den Transaktionspartner beziehen, ${ }^{129}$ sondern sie kann sich auch auf die auszutauschende Leistung beziehen und somit möglicherweise einen Wechsel des Transaktionspartners zulassen, wenn die erstellte Leistung im Prinzip ebenfalls durch andere Nachfrager nutzbar ist. Das durch das Informationsmanagement gestaltete und gesteuerte technikunterstützte Informationssystem der Unternehmung und die darin stattfindenden Prozesse können sowohl partnerspezifische als auch leistungsspezifische Elemente aufweisen.

Der Spezifitätsgrad als weitere Dimension des Spezifitätsphänomens betriff die Stärke der Bindung an den Bezugsgegenstand und kann sowohl in statischer als auch in dynamischer Hinsicht betrachtet werden. Zur Messung des Spezifitätsgrads verwendet die Transaktionskostentheorie das gedankliche Konstrukt der Quasi-Rente, die einen Maßstab für die Differenz des Nutzens eines Gutes in seiner tatsächlichen besten Verwendung und der hypothetischen zweitbesten Verwendung darstellt. ${ }^{130}$ Besondere Probleme ergeben sich daraus, dass der Spezifitätsgrad sich im Zeitablauf verändern kann, sodass die Abhängigkeitsbeziehung zwischen den Vertragspartnern einem dynamischen Wandel unterliegt. Kommt es im Zeitablauf bei einem Transaktionstyp zu einer Veränderung des Spezifitätsgrads, so kann dies die Beziehung zwischen den Transaktionspartnern sowohl stärken als auch schwächen. ${ }^{131}$

Für das Informationsmanagement besteht also die Chance, durch entsprechende Gestaltungsmaßnahmen in Bezug auf das technikunterstützte Informationssystem der Unternehmung den Spezifitätsgrad im Verlauf einer Vertragsbeziehung gezielt zu verändern. Während im Hinblick auf mögliche Abhängigkeiten und hohe Wechselkosten generell ein niedrigerer Spezifitätsgrad des technikunterstützten Informationssystems der Unternehmung vorzuziehen ist, ist im Hinblick auf die Effizienz der Koordination in der Abwicklungs- und Kontrollphase generell eine stärker spezifische Ausrichtung des Systems zu bevorzugen. Eine Transaktionskostensenkung als Ziel des Informationsmanagements kann demnach am besten durch eine Flexibilisierung des Systems erreicht werden. Bei einer hohen Anpassungsfähigkeit des technikunterstützten Informationssystems der Unternehmung treten zum einen geringe Lock-in

\footnotetext{
${ }^{128}$ Vgl. Gersch (1998), S. 96.

${ }^{129}$ Vgl. Gersch (1998), S. 96f.

${ }^{130}$ Vgl. dazu Klein/Crawford/Alchian (1978), S. 298; Alchian/Woodward (1988), S. 67; Aufderheide/Backhaus (1995), S. 52. Das Konzept der Quasi-Rente stößt in der Realität auf erhebliche Operationalisierungsschwierigkeiten, sodass eine Messung im konkreten Einzelfall bei der Wahl von Alternativen durch das Informationsmanagement nicht möglich erscheint. Vgl. Gersch (1998), S. 101.

${ }^{131}$ Vgl. Gersch (1998), S. 103.
} 
Effekte während der Abwicklung der Transaktion auf. Zum anderen besteht trotz der geringen Bindung die Möglichkeit zur flexiblen Ausrichtung des Systems auf die jeweiligen transaktionsspezifischen Anforderungen.

\section{d) Transaktionsatmosphäre}

Während die bisherigen Überlegungen sich auf die Transaktion selbst bezogen, ist abschließend noch kurz auf die Transaktionsatmosphäre einzugehen. Zur Transaktionsatmosphäre gehören die rechtlichen, technischen und sozialen Rahmenbedingungen einer Transaktion oder eines Transaktionstyps. Grundsätzlich ist davon auszugehen, dass derartige gesamtwirtschaftliche Rahmenbedingungen nicht durch intendierte einzelwirtschaftliche Maßnahmen, also auch nicht durch Einflussnahmen des Informationsmanagements einer Unternehmung veränderbar sind. In Bezug auf die technischen Rahmenbedingungen besitzt allerdings die umgekehrte Einflussrichtung Relevanz für das Informationsmanagement, da die (informations-) technischen Rahmenbedingungen, innerhalb derer die Geschäftstätigkeit der Unternehmung erfolgt, Auswirkungen auf das Vorgehen in den einzelnen Transaktionsphasen und damit auf die Transaktionskosten haben. Die generelle Verfuggarkeit innovativer hard- und softwaretechnischer Lösungen für die verschiedenen Akteure kann Klärungsprozesse beschleunigen, den Kreis potenzieller Transaktionspartner erweitern und sich damit letztlich (reduzierend) auf die Such-, Verhandlungs-, Kontroll- und Anpassungskosten auswirken. ${ }^{132}$ Es kann deshalb ein äußerer Druck durch die technischen Rahmenbedingungen im Umfeld der Unternehmung entstehen, aus dem sich unter dem Aspekt der Wettbewerbsfahigkeit nahezu zwingende Notwendigkeiten furr das Informationsmanagement zur Ausnutzung der Potenziale innovativer informationstechnischer Möglichkeiten ergeben. ${ }^{133}$

\subsubsection{Zusammenhang zwischen technikunterstützter Informationsverarbeitung und institutioneller Koordination von Transaktionen}

Die Höhe der für die Abwicklung einer Transaktion anfallenden Transaktionskosten hängt nicht nur von der Ausprägung der beschriebenen Transaktionseigenschaften, sondern auch von der Art und Weise ab, in der die Koordination des Leistungsaustausches zwischen den Transaktionspartnern stattfindet. Die Transaktionskostentheorie nimmt an, dass für einen gegebenen Transaktionstyp der Wechsel zwischen verschiedenen Formen der Koordination signifikante Auswirkungen auf die Transaktionskosten haben kann. ${ }^{134}$ Zwischen den im vorigen Abschnitt beschriebenen Transaktionseigenschaften und der gewählten Koordinationsform ist daher eine Passung herzustellen. Im Folgenden werden die verschiedenen Koordinationsformen und die zu Grunde liegenden Vertragsformen kurz erläutert. Anschließend wird aufgezeigt, wie

\footnotetext{
${ }^{132}$ Vgl. ähnlich Picot (1982), S. 272f.

${ }^{133}$ Damit ist die Frage des Zusammenhangs zwischen informationstechnischen Möglichkeiten und organisatorischen Lösungen angesprochen. Grundsätzlich lassen sich in diesem Punkt die Perspektive des technologischen Imperativs, die Perspektive des organisatorischen Imperativs und die Perspektive der Interaktion unterscheiden. Vgl. dazu Markus/Robey (1988), S. 583ff. Die hier beschriebene Wirkung der Rahmenbedingungen ist tendenziell der Perspektive des technologischen Imperativs zuzuordnen, da sich das Informationsmanagement dem äußeren Druck der Transaktionsbedingungen nicht entziehen kann.

${ }^{134}$ Vgl. Williamson (1979), S. 246.
} 
sich die Gestaltungsmaßnahmen des Informationsmanagements auf die Passung zwischen Transaktionseigenschaften und Vertragsdesign auswirken können.

Als Gegenpole bei der Ausgestaltung des Vertrags stehen sich die marktliche und die hierarchische Koordinationsform gegenüber. Die Koordination des Marktes erfolgt ausschließlich auf der Grundlage von Preisen, die sämtliche transaktionsrelevanten Informationen übermitteln, während die Koordinationsleistung der Hierarchie auf Weisungen durch entsprechend befugte Personen beruht. ${ }^{135}$ Die Annahme einer Dichotomie zwischen diesen beiden Koordinationsformen ist allerdings irreführend, da zur Abwicklung einer Transaktion häufig sowohl marktliche als auch hierarchische Koordinationselemente verwendet werden. ${ }^{136}$ Zwischen den idealtypischen Extremen Markt und Hierarchie besteht ein großes Spektrum an Hybridformen, die durch ein gemischtes Vertragsdesign gekennzeichnet sind und sowohl marktliche als auch hierarchische Vereinbarungsbestandteile beinhalten. Die Transaktionskostentheorie überprüft unterschiedliche institutionelle Arrangements auf ihre Koordinationseffizienz bei verschiedenen Transaktionstypen. ${ }^{137}$ Die von WILLIAMSON gewählte Klassifikation von Vertragsformen geht in diesem Bereich auf eine Analyse des amerikanischen Vertragsrechts durch den Rechtswissenschaftler MACNEIL zurück. ${ }^{138}$ Wie bei der Erläuterung der Leitideen der Transaktionskostentheorie in Abschnitt 5.1.2 bereits angesprochen, werden dabei klassische, neoklassische und relationale Verträge als drei grundsätzliche Vertragstypen zur Koordination des Leistungsaustausches unterschieden.

Klassische Verträge sind durch eine kurze Dauer, die präzise Bestimmung des Gegenstands und der Konditionen der Transaktion sowie eine begrenzte persönliche Interaktion der Vertragspartner gekennzeichnet. ${ }^{139}$ Der Vertrag wird ex ante vollständig spezifiziert und die Beziehung zwischen den Transaktionspartnern ist nicht auf eine dauerhafte Zusammenarbeit angelegt. Die institutionelle Koordinationsform des marktlichen Leistungsaustausches basiert typischerweise auf der Vertragsform des klassischen Vertrages zwischen autonomen Transaktionspartnern. ${ }^{140}$

Im Gegensatz dazu werden beim Abschluss neoklassischer Verträge nicht sämtliche Verpflichtungen ex ante festgelegt, da zum Zeitpunkt des Vertragsabschlusses nicht alle Bedingungen, die die Transaktion beeinflussen, vollständig bekannt sind. ${ }^{141} \mathrm{Die}$ Vertragspartner rechnen also bereits beim Abschluss des Vertrages mit der Notwendigkeit zur Vertragsanpassung. Im Gegensatz zu klassischen Verträgen beschränkt sich die Beziehung zwischen den Transaktionspartnern bei neoklassischen Verträgen nicht auf einen bestimmten Zeitpunkt (spot contracting), sondern sie erstreckt sich über einen gewissen Zeitraum, innerhalb dessen sich die Anpassungsnotwendigkeit ergeben

\footnotetext{
${ }^{135}$ Vgl. Sydow (1993), S. 98.

${ }^{136}$ Dies hebt bereits Richardson (1972), S. 895, hervor. Auf der einen Seite stoppt die hierarchisch geplante Koordination häufig nicht an den Grenzen der Unternehmung, auf der anderen Seite bestehen auch innerhalb von Unternehmungen marktliche Koordinationselemente. Insbesondere sind hierarchische Sanktionsmechanismen nur in Verbindung mit einer langfristigen Exit-Option durchsetzbar, die typisch für den Markt als Koordinationsform sind. Vgl. dazu Sydow (1993), S. 101.

${ }^{137} \mathrm{Vgl}$. Ebers/Gotsch (1999), S. 231.

${ }^{138}$ Vgl. dazu MacNeil (1974), S. 691ff.; MacNeil (1978), S. 854ff. und MacNeil (1987), S. 272ff.

${ }^{139} \mathrm{Vgl}$. MacNeil (1987), S. 275.

${ }^{140} \mathrm{Vgl}$. Williamson (1979), S. 248

${ }^{141}$ Vgl. Ebers/Gotsch (1999), S. 231
} 
kann. Bei Konflikten ist die Hinzuziehung einer dritten Partei, des Schlichters, vorgesehen, die in der Lage ist, Interpretationsspielräume zu beseitigen und Leistungsminderungen eines Vertragspartners zu erkennen.

Relationale Verträge als von Anfang an langfristig angelegte Vertragsform sind durch ein noch höheres $\mathrm{Maß}$ an Unbestimmtheit zum Zeitpunkt des Vertragsabschlusses gekennzeichnet, und die einzelnen Vereinbarungsbestandteile entwickeln sich erst nach und nach im Laufe der Vertragszeit. Entgegen der üblichen Annahme, dass institutionelle Regelungen reibungslos funktionieren, ist das Konzept des relationalen Vertrages auf „Unannehmlichkeiten“ aller Art eingestellt. ${ }^{142}$ Die ex ante vertraglich vereinbarten Regeln bilden nur den Rahmen für die verschiedenen Austauschvorgänge im Laufe der Geschäftsbeziehung. ${ }^{143}$ Damit stellen sie die Grundlage für die unternehmungsintern koordinierte Erstellung von Leistungen dar, bei der die Koordination des Austausches von (Teil-) Leistungen im Rahmen von festen Beschäftigungsverhältnissen erfolgt, in denen zwar die Weisungskompetenzen ex ante festgelegt sind, nicht jedoch die einzelnen Weisungen, die im Laufe der Vertragszeit gegeben werden.

Die tatsächlich vorfindbaren Formen der Koordination des Leistungsaustausches lassen sich als ein Kontinuum zwischen dem reinen Marktbezug, der durch die Form des klassischen Vertrages koordiniert wird, und dem vollständig unternehmungsinternen Bezug, dessen Koordination hierarchisch durch die Institution des relationalen Vertrages erfolgt, denken. ${ }^{144}$ Das vielfältige Spektrum zwischen diesen beiden Extremen reicht von generellen oder funktionsbezogenen Unternehmungskooperationen, über strategische Allianzen, Joint Ventures, Franchisingsysteme, Lizenzvergabe an Dritte, dynamische Netzwerke bis zu langfristigen Abnahme- und Belieferungsverträgen. ${ }^{145}$ Die Transaktionskostentheorie widmet sich auf der Basis der herausgearbeiteten grundsätzlichen Vertragsformen besonders dem Problem der vertikalen Integration, also Fragen der Fertigungstiefe, der Entscheidung zwischen Eigenfertigung und Fremdbezug und den verschiedenen Formen der Koordination, die zwischen Markt und Unternehmung angesiedelt sind. ${ }^{146}$

Die Gestaltungsmaßnahmen des Informationsmanagements weisen in zweierlei Hinsicht Berührungspunkte zur Wahl der für die Abwicklung einer Transaktion verwendeten Koordinationsform und dem damit verbundenen Vertragsdesign auf. Durch die Bereitstellung geeigneter Anwendungssysteme können zunächst einmal Koordinationsaktivitäten, die im Rahmen von Leistungserstellung und Leistungsaustausch durchzuführen sind, vereinfacht werden. Dies führt dazu, dass sich die absoluten und relativen Kostenstrukturen der verschiedenen Arten von vertraglichen Arrangements verändern. Auf diese Weise lässt sich das Transaktionskostenniveau innerhalb bestehender Koordinationsformen reduzieren, sodass die Koordinationseffizienz der einzelnen Vertragstypen verbessert wird. Darüber hinaus wurde im vorigen Abschnitt aufgezeigt, dass Berührungspunkte zwischen den Charakteristika der Transaktion und dem Bereich der technikunterstützten Informationsverarbeitung

\footnotetext{
${ }^{142}$ Vgl. Richter (1994), S. 59; Schanz (1997a), S. 145.

${ }^{143}$ Vgl. Ebers/Gotsch (1999), S. 231.

${ }^{144}$ Vgl. Picot (1982), S. 273.

${ }^{145}$ Vgl. Picot/Reichwald/Wigand (2001), S. 53.

${ }^{146}$ Vgl. Schanz (1997a), S. 146.
} 
bestehen. Aus diesem Grund ist vorstellbar, dass entsprechende Gestaltungsmaßnahmen des Informationsmanagements die Merkmale der Transaktion dahingehend verändern, dass ein Wechsel zwischen unterschiedlichen Arten des Leistungsaustausches sinnvoll wird.

Der auf die Senkung von Transaktionskosten zielende Einsatz moderner Informationstechnik kann also einerseits bestehende Koordinationsformen ändern und andererseits bei bestimmten Leistungen die Realisierung völlig neuer Formen der Koordination ermöglichen. Die Verändenungen betreffen dabei allerdings weniger die zu Grunde liegenden abstrakten Muster des klassischen, neoklassischen oder relationalen Vertrags als deren Spezifizierung im Rahmen konkreter Transaktionen bzw. Transaktionstypen. Diese Wirkungsrichtung des Informationsmanagements wird im Abschnitt 5.3.3 näher untersucht.

\subsubsection{Informationsmanagement aus Sicht der Agency-Theorie}

Die nachfolgenden Ausfuhrungen konstruieren das Informationsmanagement als Erkenntnisobjekt der Agency-Theorie. Zu diesem Zweck werden Möglichkeiten untersucht, über die das Informationsmanagement zur Unterstützung der vertraglichen Gestaltung von Auftraggeber/Auftragnehmerbeziehungen in und zwischen Unternehmungen verfügt. Abschnitt 5.2.3.1 überprüft zunächst, welcher Zusammenhang sich überhaupt zwischen der positiven Forschungsvariante der Agency-Theorie und dem Informationsmanagement als betrieblichem Aufgabenfeld herstellen lässt. Davon ausgehend untersuchen die Abschnitte 5.2.3.2, 5.2.3.3 und 5.2.3.4 Ansatzpunkte des Informationsmanagements in den verschiedenen Vertragsphasen von Auftraggeber/Auftragnehmer-Beziehungen.

\subsubsection{Anknüpfungspunkte für eine Theorie des Informationsmanagements in der Agency-Theorie}

In Abschnitt 5.2.1 wurde die weitere Untersuchung bereits auf die positive Variante der Agency-Theorie beschränkt, da diese einen stärkeren empirischen Bezug besitzt und somit besser geeignet ist, Hinweise für die konkrete Gestaltung technikunterstützter Informationssysteme in der Praxis zu liefern. Diese Forschungsrichtung befasst sich mit der Reduzierung von Informationsunvollkommenheiten in der Beziehung zwischen Principal und Agent. Der Principal auf der einen Seite kann Anstrengungen zur besseren Beobachtung des Agent unternehmen, während es für den Agent auf der anderen Seite lohnenswert sein kann, Garantien in Bezug darauf zu erbringen, dass er bestimmte Verhaltensweisen nicht ergreifen wird. ${ }^{147}$ Solche Maßnahmen zielen darauf $\mathrm{ab}$, das institutionelle Arrangement, das der Agency-Beziehung zu Grunde liegt, der idealen, pareto-optimalen Lösung in der neoklassischen Theorie anzunähern. Das Pareto-Optimum besitzt in diesem Zusammenhang den Charakter einer fiktiven Richtgröße, deren Realisierung nur bei vollständiger Beseitigung aller Informationsunvollkommenheiten zu erreichen wäre. ${ }^{148}$ Tatsächlich entstehen durch die Anstrengungen sowohl des Principal als auch des Agent Kosten. Diese ergeben zusammen mit dem verbleibenden Effizienzverlust, der wegen der nicht vollständig zu beseitigenden

\footnotetext{
${ }^{147} \mathrm{Vgl}$. Jensen/Meckling (1976), S. 308.

${ }^{148}$ Vgl. Ross (1973), S. 138; Jensen/Meckling (1976), S. 327f.; Wenger/Terberger (1988), S. 508.
} 
Informationsunvollkommenheiten auftritt, die Agency-Kosten des vertraglichen Arrangements.

Für die Konstruktion des Informationsmanagements als Erkenntnisgegenstand der positiven Agency-Theorie ist zunächst darauf einzugehen, welche Arten von Auftraggeber/Auftragnehmer-Beziehungen grundsätzlich durch Maßnahmen des Informationsmanagements beeinflusst werden können. Ursprünglich untersuchte die Agency-Theorie Fragen der Eigentümerstruktur, wobei der Zusammenhang zwischen Erfolgsanspruch und Handlungsverantwortung im Vordergrund stand. ${ }^{149}$ Tatsächlich kann jedoch festgestellt werden, dass es sich bei Auftraggeber/Auftragnehmer-Beziehungen um eine Art universellen Interaktionstyp handelt, der an sehr vielen Stellen im Wirtschaftsleben Bedeutung besitzt. ${ }^{150}$ Vertragsbeziehungen mit den skizzierten Charakteristika bestehen innerhalb von Unternehmungen sowohl im Über/Unterordnungsverhältnis zwischen Personen unterschiedlicher hierarchischer Ebenen als auch zwischen formal gleichberechtigten Unternehmungsmitgliedern, wenn eine Person eine Leistung erbringt, die eine andere im weiteren Leistungserstellungsprozess verwendet. ${ }^{151}$ Ferner kann auch das Verhältnis zwischen der Unternehmung und ihren Lieferanten, sowie zwischen der Unternehmung und ihren Kunden als AgencyBeziehung aufgefasst werden. ${ }^{152}$ In der Beziehung zu den Lieferanten befindet sich die Unternehmung in der Rolle des Auftraggebers, während sie gegenüber ihren Kunden die Position des Auftragnehmers einnimmt. ${ }^{153}$ Bei allen diesen Typen von Beziehungen kommt es zu einer informationellen Beziehung zwischen dem Auftraggeber und dem Auftragnehmer, sodass das jeweils zu Grunde liegende vertragliche Arrangement potenziell zum Gegenstand von Maßnahmen des Informationsmanagements werden kann.

Aus der Sicht der Agency-Theorie ergibt sich für das Informationsmanagement die Zielsetzung, durch geeignete Gestaltungs- und Steuerungsmaßnahmen in Bezug auf das technikunterstützte Informationssystem der Unternehmung auf eine Reduzierung der Agency-Kosten hinzuwirken. Gelingt dies, so wird die Effizienz des vertraglichen Arrangements erhöht und die Situation dem Pareto-Optimum als fiktiver Orientierungsgröße angenähert. Grundsätzlich sind drei verschiedene Maßnahmentypen vorstellbar, um die Höhe der Agency-Kosten zu verringern. ${ }^{154}$ Dabei handelt es sich um die Setzung von Anreizen, die direktive Verhaltenssteuerung (bzw. die Reduzierung von

\footnotetext{
${ }^{149}$ Vgl. Jensen/Meckling (1976), S. 306.

${ }^{150}$ Vgl. Arrow (1986), S. 1183.

${ }^{151}$ Auftraggeber/Auftragnehmer-Beziehungen treten je nach organisatorischer Struktur der Unternehmung auch über mehrere Ebenen auf. Im Folgenden wird vereinfachend von nur einer Betrachtungsebene ausgegangen. Auch Beziehungstypen, bei denen ein Auftraggeber mehreren Auftragnehmern gegenübersteht, werden im Folgenden nicht betrachtet.

${ }^{152}$ Vgl. Mikus (1998), S. 455.

${ }^{153}$ Die Unternehmung nimmt zugleich die jeweils umgekehrte Position ein, wenn die den realwirtschaftlichen Strömen gegenläufigen Zahlungsströme betrachtet werden. Diese Teilbeziehungen werden im Folgenden nicht weiter betrachtet, da sie für die Zwecke des Informationsmanagements keine speziellen Charakteristika gegenüber den realwirtschaftlichen Teilbeziehungen aufweisen.

${ }^{154} \mathrm{Vgl}$. zum Folgenden Ebers/Gotsch (1999), S. $214 \mathrm{f}$.
} 
Verhaltensspielräumen des Auftragnehmers ${ }^{155}$ ) und die Verbesserung des Informationsstands auf Seiten des Principals.

Durch eine entsprechende Anreizgestaltung soll eine Angleichung der Interessen von Principal und Agent erzielt werden, für die insbesondere die Ergebnisbeteiligung des Agent als geeignet angesehen wird. Dieser Maßnahmetyp ist an der Schnittstelle zur normativen Variante der Agency-Theorie, die auf die Entwicklung von Anreizsystemen abzielt, angesiedelt und wird hier nicht im Detail betrachtet. Die Anreizsituation wird im Folgenden nur noch insofern berücksichtigt, als auf Grund der Gestaltungsmaßnahmen des Informationsmanagements ein Wechsel zwischen marktlicher und hierarchischer Koordination, die mit unterschiedlichen Anreizwirkungen verbunden sind, möglich werden kann. Die direktive Verhaltenssteuerung als zweiter Maßnahmetyp setzt voraus, dass das Verhalten des Agent im Einzelnen durch den Principal gesteuert werden kann. Für diese Art von Maßnahmen sieht die Agency-Theorie üblicherweise nur geringe Erfolgsaussichten, da annahmegemäß das Verhalten des Agent durch den Principal nicht beobachtbar ist oder hinsichtlich der Eignung zur Erzielung des vereinbarten Ergebnisses nicht beurteilt werden kann. ${ }^{156} \mathrm{Im}$ Folgenden ist zu untersuchen, inwiefern eine Modifikation dieser Situation durch das Informationsmanagement erreichbar ist, sodass die Möglichkeiten zur direktiven Verhaltenssteuerung ausgedehnt werden. Die Verbesserung des Informationsstands beim Auftraggeber als dritter Maßnahmetyp erfolgt durch Überwachungssysteme, mit denen das Wissen über das Leistungsverhalten und die Handlungssituation des Agent verbessert wird. Die Einrichtung solcher Systeme ist selbst mit Kosten verbunden, sodass es teilweise zu einer Substitution von Kostenarten kommt, da verbleibende Wohlfahrtsverluste durch Kontrollkosten ersetzt werden. In diesem Zusammenhang ist zu untersuchen, inwiefern das Informationsmanagement durch eine Verringerung von Kosten der Beobachtung eine Auflösung dieses Verdrängungseffekts erreichen kann, sodass sich insgesamt eine Effizienzsteigerung erreichen lässt.

Zur genaueren Analyse der vertraglichen Beziehung zwischen Principal und Agent werden in der Agency-Theorie mehrere Vertragsphasen unterschieden, in denen jeweils unterschiedliche Formen von Agency-Problemen auftreten können. ${ }^{157}$ Bei diesen Phasen handelt es sich um die Auswahl des Vertragspartners, die Vereinbarung mit dem eigentlichen Vertragsabschluss, die Entscheidung bzw. Handlung des Agent und die Entlohnung des Agent. ${ }^{158}$ Wie bei der Darstellung der inhaltlichen Leitideen der Agency-Theorie erläutert, nimmt dieser Ansatz eine Differenzierung in drei idealtypische Arten von Problemsituationen vor, die sich auf die Ursachen der zu beobachtenden Informationsunvollkommenheiten in diesen Phasen bezieht. Dabei handelt es sich um hidden characteristics, hidden intention und hidden action. Die folgenden Überlegungen zu den Einflussmöglichkeiten des Informationsmanagements orientieren sich vom Aufbau her an diesen drei Problemsituationen, zu deren Bewältigung die oben aufgeführten allgemeinen Maßnahmetypen zu spezifizieren sind.

\footnotetext{
${ }^{155}$ Die Reduzierung von Verhaltensspielräumen und die direktive Verhaltenssteuerung können als spiegelbildliche Maßnahmen aufgefasst werden und werden im Folgenden gemeinsam behandelt. ${ }^{156}$ Vgl. Ebers/Gotsch (1999), S. $214 \mathrm{f}$.

${ }^{157}$ Vgl. Mikus (1998), S. 453.

${ }^{158}$ Vgl. Buscher (1997), S. 112.
} 


\subsubsection{Hidden characteristics: Informationsunvollkommenheiten vor Vertrags- abschluss}

Bei der Auswahl des Vertragspartners als frühester Phase einer Auftraggeber/Auftragnehmer-Beziehung geht es für den Auftraggeber darum, einen Auftragnehmer zu finden, der sich für die Erstellung der auszutauschenden Leistung besonders gut eignet. In dieser Phase spielen die Qualitätseigenschaften der potenziellen Vertragspartner eine besondere Rolle. ${ }^{159}$ Die Qualitätseigenschaften beziehen sich auf die Person des Agent selbst und sind folglich nicht willentlich durch diesen beeinflussbar. Eine Einflussnahme des Principals durch Motivation oder Zwang in späteren Phasen der Vertragsbeziehung hat daher keine Aussicht auf Erfolg. ${ }^{160}$ Die Gefahr, die aus dem Auftreten asymmetrischer Information in dieser Vertragsphase resultiert und als hidden characteristics bezeichnet wird, besteht in der Auswahl schlechter Vertragspartner. ${ }^{161}$ Auf dem Markt für die betreffende Leistung insgesamt kann es bei intensiven hidden characteristics zu einem Prozess der adverse selection kommen, in dem die besseren Anbieter durch die schlechteren vom Markt verdrängt werden.

Sowohl Auftraggeber als auch Auftragnehmer verfuigen vor Vertragsschluss über verschiedene Optionen zur Verringerung der bestehenden Informationsdefizite. ${ }^{162} \mathrm{Er}$ kann zusätzliche Informationen über die potenziellen Vertragspartner beschaffen und damit seine Entscheidungssituation bei der Auswahl des Auftragnehmers verbessern. Dieser Maßnahmentyp wird als Screening bezeichnet. Probleme ergeben sich in diesem Zusammenhang daraus, dass die Kosten der Beschaffung weiterer Informationen über die potenziellen Vertragspartner gegenüber dem ex ante unbekannten Nutzen eines verbesserten Informationsstands abzuwägen sind. ${ }^{163}$ Umgekehrt hat der Auftragnehmer die Möglichkeit, dem potenziellen Auftraggeber durch die gezielte Preisgabe von Informationen seine Leistungsfähigkeit und Leistungsbereitschaft zu signalisieren. Dieser Maßnahmetyp wird als Signalling bezeichnet. Schließlich steht dem Auftraggeber auch die Verwendung von Selbstwahlverfahren offen, bei denen der Agent seine Qualitätseigenschaften implizit offenbart. ${ }^{164}$ Dabei wird die Entscheidungssituation für den Auftragnehmer so gestaltet, dass sich aus dessen Entscheidung die individuellen Qualitätseigenschaften erkennen lassen. ${ }^{165}$

Diese genannten Möglichkeiten, die die Agency-Theorie zur Reduzierung des Risikos von Hidden Characteristics vorschlägt, stellen die Ansatzpunkte dar, aus denen sich theoretische Aussagen für das Informationsmanagement ableiten lassen. Sowohl Screening- als auch Signalling-Aktivitäten zur Offenbarung der Qualitätseigenschaften des Agent können durch eine entsprechende Gestaltung der verwendeten Anwendungssysteme unterstützt werden. Dadurch kann das Informationsmanagement bereits in der Auswahlphase von Auftraggeber/Auftragnehmer-Beziehungen den Informationsnachteil auf der Seite des Principals verringern und auf diese Weise zur Reduzierung von Agency-Kosten beitragen. Die Einrichtung von Selbstwahlmechanismen stellt dagegen

\footnotetext{
${ }^{159}$ Vgl. Spremann (1990), S. 565; Mikus (1998), S. 453.

${ }^{160}$ Vgl. Spremann (1990), S. 567.

${ }^{161}$ Vgl. Picot/Reichwald/Wigand (2001), S. 57f;; Mikus (1998), S. 453.

${ }^{162}$ Vgl. dazu Picot/Reichwald/Wigand (2001), S. 58f.; Mikus (1998), S. 453ff.

${ }^{163}$ Vgl. Spremann (1990), S. 568.

${ }^{164}$ Vgl. zu diesen Möglichkeiten zur Verringerung von Informationsasymmetrien Mikus (1990), S

453f.; Spremann (1990), S. 578f;; Arrow (1986), S. 1183ff.

${ }^{165}$ Vgl. Spremann (1990), S. 578; Picot/Reichwald/Wigand (1996), S. 50.
} 
im Kern ein formales Problem dar. ${ }^{166}$ Der formale Aspekt von Selbstwahlmechanismen, der keinen unmittelbaren Zusammenhang zum Gestaltungsbereich des Informationsmanagements aufweist, wird hier (analog zu der Behandlung des formalen Zweigs der Agency-Theorie) nicht weiter betrachtet.

Auf der Beschaffungsseite befindet sich die Unternehmung in der Situation des Auftraggebers, der zur besseren Beurteilung der Qualitätseigenschaften potenzieller Lieferanten Screening-Aktivitäten ergreift. Die Möglichkeiten des Informationsmanagements im Bereich des Screening liegen in der Erschließung neuer sowie der verbesserten Ausnutzung bestehender Informationsquellen über die Anbieter der zu beziehenden Leistungen. Häufig liegen innerhalb einer Unternehmung bereits Informationsquellen über in Frage kommende Lieferanten vor, die jedoch nicht effizient genutzt werden. Dies ist etwa in Unternehmungen mit stark dezentralisierten Organisationsstrukturen der Fall, bei denen auf eine zentrale Beschaffung verzichtet wurde, um eine möglichst vollständige Selbstständigkeit der einzelnen Organisationseinheiten zu gewährleisten. Bei derartigen Konstellationen können innovative informationstechnische Lösungen den Informationsaustausch zwischen den verschiedenen Beschaffungsstellen erleichtern und die Verfügbarkeit von Kenntnissen über Qualitätseigenschaften und Konditionen in Frage kommender Lieferanten innerhalb der Unternehmung erhöhen. Entsprechende Anwendungssysteme im Beschaffungsbereich bieten zudem die Möglichkeit zur Erschließung von Synergiepotenzialen, indem sie durch ein Pooling der Beschaffungsaktivitäten (unter Beibehaltung der dezentralisierten Entscheidungskompetenzen) die Verhandlungsposition der Unternehmung gegenüber den Lieferanten verbessern, sodass sich bessere Lieferkonditionen erzielen lassen. Das Informationsmanagement kann darüber hinaus den Zugang zu neuen Informationsquellen außerhalb der Unternehmung möglich machen, die ebenfalls in Frage kommende Lieferanten betreffen, bei denen die Unternehmung bisher nicht Kunde ist.

Auf der Absatzseite dagegen befindet sich die Unternehmung in der Situation des Auftragnehmers, ${ }^{167}$ dem sich durch die freiwillige Offenbarung von Qualitätseigenschaften gegenüber den Nachfragern die Möglichkeit zur Erzielung von Wettbewerbsvorteilen bietet. Dazu ist es erforderlich, ein Signal zu etablieren, das die andere Marktseite kostenlos oder zu geringen Kosten wahrnehmen kann. ${ }^{168}$ Das Signal muss so geartet sein, dass Anbieter, die nicht über die entsprechenden Qualitätseigenschaften verfügen, auf das Signalisieren verzichten, da sie mit zu hohen Kosten konfrontiert werden, wenn die von ihnen erbrachte Leistung nicht die erwarteten Qualitätseigenschaften aufweist. Von Vertretern der Agency-Theorie wird in diesem Zusammenhang häufig exemplarisch die freiwillige Erbringung von Garantien genannt, die sich für den Anbieter nur dann lohnt, wenn die von ihm angebotene Leistung tatsächlich von hoher Qualität ist. Das Informationsmanagement kann SignallingAktivitäten unterstützen, indem es Anwendungssysteme bereitstellt, die es interessierten Nachfragern ermöglichen, sich über die Qualität der von der Unternehmung angebotenen Leistungen zu informieren. Eine noch weitergehende Unterstützungs-

\footnotetext{
${ }^{166} \mathrm{Vgl}$. dazu Arrow (1986), S. 1185ff; Hartmann-Wendels (1989), S. $726 \mathrm{ff}$.

${ }^{167}$ In einigen Quellen wird im Zusammenhang mit der Analyse von Selbstwahlverfahren auch auf der Absatzseite die Unternehmung als Principal und der Kunde als Agent aufgefasst, vgl. z.B. Spremann (1990), S. 578f.; Arrow (1986), S. 1185ff. Diese Sichtweise wird hier nicht weiter verfolgt, da sie Zahlungsströme betrifft, die oben bereits aus der weiteren Untersuchung ausgeklammert wurden.

${ }^{168} \mathrm{Vgl}$. Spremann (1990), S. 579.
} 
option des Informationsmanagements in diesem Bereich liegt darin, potenziellen Kunden mit Hilfe entsprechender informationstechnischer Lösungen die Möglichkeiten zu bieten, die Qualitätseigenschaften der gewünschten Leistung entsprechend ihrer persönlichen Bedürfnisse individuell zusammenzustellen.

Die bisherigen Überlegungen bezogen sich auf Sachleistungen, die die Unternehmung von ihren Lieferanten bezieht bzw. ihren Kunden anbietet. Vergleichbare Probleme treten auch bei der Auswahl des Vertragspartners für unternehmungsinterne Auftraggeber/Auftragnehmer-Verhältnisse, bei denen nicht Sach- sondern Arbeitsleistungen ausgetauscht werden, ${ }^{169}$ auf. Bei der Suche nach Vertragspartnern für diese Arten von Agency-Beziehungen handelt es sich im Wesentlichen um ein Problem der Personalauswahl auf unterschiedlichen Ebenen der Unternehmungshierarchie. Screening-Aktivitäten in diesem Zusammenhang stellen die Beobachtung und Auswertung des allgemeinen Arbeitsmarktes sowie des Marktes für Führungskräfte und die Beschaffung zusätzlicher Informationen bei konkreten Bewerbungsbzw. Einstellungssituationen dar. Ansatzpunkte des Informationsmanagements in diesem Bereich liegen in der Verbesserung der Kommunikation mit (potenziellen) Bewerbern und der Herstellung von Schnittstellen zwischen den Anwendungssystemen im Personalbereich mit Personalvermittlungsagenturen oder elektronischen Job-Börsen. Signalling-Aktivitäten erfolgen bei diesem Beziehungstyp durch die Bewerber bzw. die Personalvermittler selbst und sind nicht durch das Informationsmanagement gestaltbar.

\subsubsection{Hidden intention: Informationsunvollkommenheiten nach Vertragsschluss mit Ex post-Kenntnis des Principal}

Beim Vorliegen von hidden intention verfügt der Agent über einen Informationsvorsprung, der sich auf den Zeitpunkt nach Vertragsabschluss und vor der Durchführung von Handlungen zur Erfüllung der Vertragspflichten bezieht. ${ }^{170}$ Diese Situation ist dadurch gekennzeichnet, dass der Principal bereits Vorleistungen mit der Folge von sunk costs erbracht hat, die ihn nun in eine Abhängigkeit vom Agent bringen, weil er auf dessen Gegenleistungen angewiesen ist. ${ }^{171}$ Beim Austausch von Sachleistungen zwischen Unternehmungen treten Vorleistungen beispielsweise in Form der Anschaffung spezieller Maschinen, des Treffens von Standortentscheidungen oder (bezogen auf den Gestaltungsbereich des Informationsmanagements) in Form des Aufbaus spezifischer Anwendungssysteme zur unternehmungsübergreifenden Informationsverarbeitung auf. Beim Austausch von Arbeitsleistungen sind Vorleistungen beispielsweise durch die Kosten des Einstellungsverfahrens und (insbesondere bei Führungskräften) durch die Preisgabe unternehmungsinterner Informationen, die der Auftragnehmer bei Beendung des Vertragsverhältnisses gegen die Interessen der Unternehmung nutzen kann, gegeben. Die Gefahr der opportunistischen Ausnutzung solcher Abhängigkeiten wird als holdup bezeichnet. Übersetzt bedeutet der Begriff „holdup“ Überfall. Im vorliegenden Zusammenhang bezeichnet er eine Situation, in der das absichtliche Verhalten eines Individuums deutlich zu Tage tritt und einem anderen Schaden zufügt. ${ }^{172}$ Im Rahmen von Agency-Beziehungen meint holdup die Ausnutzung von

\footnotetext{
${ }^{169}$ Vgl. Sydow (1992), S. 161.

${ }^{170}$ Vgl. Mikus (1998), S. 454, die diese Situation allerdings mit dem allgemeinen Begriff hidden information bezeichnet.

${ }^{171} \mathrm{Vgl}$. Picot/Reichwald/Wigand (2001), S. 60.

${ }^{172}$ Vgl. Spremann (1990), S. 568.
} 
Handlungsspielräumen bei der Erbringung der vertraglich vereinbarten Gegenleistung durch den Agent. ${ }^{173}$

Diese Art des opportunistischen Verhaltens bleibt dem Principal nicht verborgen, sondern tritt offen zu Tage. Die Agency-Theorie geht nicht davon aus, dass der Agent sich in jedem Fall opportunistisch verhält (also gewissermaßen „unfair" spielt), die alleinige Existenz von Verhaltenspielräumen reicht aber aus, um eine unerwünschte Abhängigkeit des Principals zu begründen. Das Problem liegt bei dieser Konstellation darin, dass der Principal zu dem Zeitpunkt, zu dem er irreversible Investitionen tätigt, nicht präzisieren kann, wie der Agent sich verhalten soll, während er dies im weiteren Verlauf der Zusammenarbeit sehr wohl könnte. ${ }^{174}$ Wenn der Agent sich unter diesen Bedingungen opportunistisch verhält, dann kommt es dazu, dass die ausgetauschte Leistung nicht den qualitativen Ansprüchen des Principals entspricht, ohne dass dies im engeren Sinne gegen den vertraglich vereinbarten Rahmen verstoßen würde. Der Principal kann den Agent nicht zu der gewünschten Gegenleistung zwingen, da formal gesehen kein Vertragsverstoß vorliegt. ${ }^{175}$ Motive für ein derartiges Verhalten können einerseits in der bewussten oder unbewussten Ausnutzung opportunistischer Spielräume und andererseits in der Abwälzung von Risiko auf die andere Vertragspartei liegen. ${ }^{176}$ Die Agency-Theorie sieht zwei Möglichkeiten zur Verringerung der hidden intention-Problematik vor, deren Relevanz für das Informationsmanagement im Folgenden überprüft wird. Dabei handelt es sich zum einen um die Verringerung von Unsicherheit und zum anderen um die Verbesserung der Anreizsituation.

Der erste potenzielle Ansatzpunkt furr einen Beitrag des Informationsmanagements zur Bewältigung der hidden intention-Problematik liegt demnach in der Reduzierung von Unsicherheit zu dem Zeitpunkt, zu dem der Principal beziehungsspezifische Vorleistungen erbringt. Hohe Unsicherheit schlägt sich in nicht vorhersehbaren, häufigen Änderungen qualitativer, quantitativer, terminlicher, politischer oder technischer Einflussgrößen der Leistungsbeziehung nieder. ${ }^{177}$ Bei Auftraggeber/AuftragnehmerBeziehungen mit hoher Unsicherheit ist der Verhaltensspielraum des Agent besonders hoch. Dies führt typischerweise dazu, dass der Auftraggeber über Ansprüche gegenüber dem Auftragnehmer verfügt, die nicht explizit fixiert worden sind, sondern implizite Natur besitzen. ${ }^{178}$ Der Auftragnehmer kann die Ausnutzung von Verhaltensspielräumen unter diesen Bedingungen häufig mit dem Eintritt unvorhergesehener externer Entwicklungen nach Vertragsschluss begründen, ohne dass dies durch den Auftraggeber widerlegbar ist. Durch eine Erhöhung der Planungssicherheit könnten die Voraussetzungen dazu geschaffen werden, die gegenseitigen Ansprüche der Vertragspartner genauer zu fixieren, sodass die Grundlage für diskretionäre Verhaltensspielräume des Auftragnehmers während der Vertragserfüllung teilweise wegfallt.

Das Informationsmanagement kann einen Beitrag zur Erhöhung der Planungssicherheit leisten, indem es analyseorientierte Anwendungssysteme zur Verfugung stellt, die Funktionalitäten zur Unterstützung von Planungsaufgaben beinhalten und dabei sowohl

\footnotetext{
${ }^{173}$ Vgl. Alchian/Woodward (1988), S. 67f.

${ }^{174}$ Vgl. Spremann (1990), S. 580.

${ }^{175} \mathrm{Vgl}$. Spremann (1990), S. 569

${ }^{176}$ Vgl. Spremann (1990), S. 570.

${ }^{177}$ Vgl. Picot/Reichwald/Wigand (2001), S. 295.

${ }^{178}$ Vgl. Spremann (1990), S. 569.
} 
unternehmungsinterne als auch unternehmungsexterne Einflussgrößen einbeziehen. Mit Hilfe solcher Anwendungssysteme wird eine genauere Prognose der zukünttigen Entwicklungen im Umfeld der Vertragsbeziehung und die Erarbeitung detaillierter Szenarien unterstützt, für die die jeweils präferierte Verhaltensalternative des Auftragnehmers verbindlich vorgegeben werden kann. Auf dieser Basis wird es möglich, eine Einengung der Verhaltensspielräume des Auftragnehmers durch eine Umwandlung impliziter in vertraglich explizierte Gegenansprüche des Principals vorzunehmen. Das Potenzial dieses Maßnahmetyps zur Senkung von Agency-Kosten ist allerdings begrenzt, da auch bei verbesserter Planungsgrundlage nicht zu erwarten ist, dass sich in einem immer dynamischer werdenden wirtschaftlichen Umfeld zukünftige Überraschungen völlig ausschließen lassen.

Das grundsätzliche Problem bei der Reduzierung von Unsicherheit als Maßnahmetyp des Informationsmanagements zur Bewältigung der hidden intention-Problematik liegt also darin, dass der Idealzustand der vollkommenen Information stets unerreichbar bleibt. Für langfristig angelegte Auftraggeber/Auftragnehmer-Verhältnisse ist die Gefahr des Holdup durch die Verbesserung der Planungssicherheit zwar reduzierbar, sie bleibt jedoch im Prinzip erhalten und wächst mit der Entfernung des Planungshorizonts. Für praktisch relevante Zwecke ist deshalb anzunehmen, dass sich das Ausmaß an Sicherheit nicht so weit erhöhen lässt, dass die Reaktion auf sämtliche vorstellbaren Zukunftsentwicklungen im Vertragswerk fixiert werden kann. Ferner ist darauf hinzuweisen, dass ein verbesserter Informationsstand des jeweiligen Principals nicht per se zur Reduzierung der hidden intention-Problematik führt. Vielmehr ist es erst die Umwandlung von zuvor impliziten Ansprüchen an den Agent in vertraglich fixierte explizite Ansprüche, die die diskretionären Verhaltensspielräume des Auftragnehmers reduziert. Dieses Vorgehen wiederum ist selbst mit Verhandlungskosten zum Zeitpunkt des Vertragsschlusses verbunden und grenzt zudem die Möglichkeiten zu flexiblem Reagieren beim Eintreten unvorhergesehener Entwicklungen im Laufe der Zusammenarbeit ein.

Aus diesen Gründen sind flankierende Maßnahmen zur Bewältigung der hidden intention-Problematik erforderlich. Von der Agency-Theorie wird dabei vorgeschlagen, den Auftragnehmer durch die Schaffung von Anreizen davon abzuhalten, verbleibende Verhaltensspielräume im Laufe der Zusammenarbeit zu seinen Gunsten auszunutzen. $\mathrm{Zu}$ diesem Zweck ist der Principal mit Sanktionsmöglichkeiten auszustatten, indem der Agent ebenfalls in eine Abhängigkeitssituation gebracht wird. ${ }^{179}$ Als geeignetes Mittel dazu wird häufig die Verwendung eines Pfandes genannt, das dem Agent nur nach erfolgreicher Zusammenarbeit zufällt. Diese Form der Absicherung wird auch als „Geiseltausch" bezeichnet. ${ }^{180}$ Bei Verträgen, bei denen Arbeitsleistungen ausgetauscht werden, kann es sich dabei beispielsweise um finanzielle Prämien und Abfindungen beim Erreichen bestimmter Vertragsdauern oder um die entsprechende Gestaltung von Betriebsrenten, Sparplänen u.ä. handeln. Derartige Instrumente werden im Folgenden nicht weiter betrachtet, da sie nicht in den Gestaltungsbereich des Informationsmanagements fallen.

Auch bei Verträgen zum Austausch von Sachleistungen wird (neben einer möglichst frühen Übertragung von Eigentumsrechten an den Auftraggeber) die Herstellung einer

\footnotetext{
${ }^{179}$ Vgl. Spremann (1990), S. 580; Picot/Reichwald/Wigand (1998), S. 50.

${ }^{180} \mathrm{Vgl}$. Picot/Reichwald/Wigand (1998), S. 50.
} 
Abhängigkeit als geeignetes Mittel zur Angleichung der Interessensituation gesehen. ${ }^{181}$ Es ist deshalb zu überprüfen, inwiefern das Informationsmanagement durch eine geeignete Gestaltung von Prozessen der technikunterstützten Informationsverarbeitung die gegenseitige Abhängigkeit von Auftraggeber und Auftragnehmer beeinflussen kann. Dies betrifft insbesondere den Bereich der unternehmungsübergreifenden Informationsverarbeitung, in dem eine spezielle Ausgestaltung der verwendeten Anwendungssysteme erhebliche Abhängigkeiten zwischen Leistungsanbieter und Leistungsnachfrager nach sich ziehen kann.

Eine Abhängigkeit des Lieferanten als Auftragnehmer entsteht, wenn es der nachfragenden Unternehmung als Auftraggeber gelingt, den Aufbau der Zusammenarbeit an die Umsetzung individueller Anforderungen in diesem Bereich zu knüpfen, wie es beispielsweise in der Automobilindustrie üblicherweise der Fall ist. Der Lieferant wird dann gezwungen, bei Vertragsschluss spezifische Vorleistungen zu erbringen, um sich überhaupt als geeigneter Vertragspartner zu qualifizieren. Umgekehrt ist auch vorstellbar, dass der Lieferant individuelle Anforderungen in Bezug auf die unternehmungsübergreifende Informationsverarbeitung durchsetzt, um eine hohe Kundenbindung zu erreichen. Unabhängig davon, auf welche Partei die Initiative zur individuellen Ausgestaltung der Informationsverarbeitung zurückgeht, werden während der Vertragslaufzeit fur beide Vertragsparteien Anreize zur Fortsetzung der Zusammenarbeit wirksam, da der Wechsel des Vertragspartners nicht friktionsfrei möglich ist.

Zumeist wird angenommen, dass zur Verbesserung der im Rahmen einer Auftraggeber/Auftragnehmer-Beziehung bestehenden Anreizsituation eine Erhöhung der gegenseitigen Abhängigkeit erforderlich ist. Im Gestaltungsbereich des Informationsmanagements ist jedoch auch eine entgegengesetzte Einflussnahme vorstellbar, die darauf abzielt, durch eine Reduzierung von Bindungen Anreize zu effizientem Verhalten zu schaffen. Entscheidend aus Sicht der Agency-Theorie ist nämlich nicht die Intensität der Abhängigkeit, sondern die Symmetrie der gegenseitigen Bindungen. Daraus folgt, dass auch eine starke Standardisierung der unternehmungsübergreifenden Informationsverarbeitung dazu beitragen kann, den Auftragnehmer von der Ausnutzung diskretionärer Verhaltensspielräume abzuhalten. Wenn die betreffenden Prozesse nur in geringem Maße partnerspezifisch ausgerichtet sind, dann brauchen beide Parteien bei Vertragsschluss lediglich geringe Vorleistungen zu erbringen, sodass während der Zusammenarbeit ein geringes Interesse an der Fortsetzung der Zusammenarbeit besteht. Die Möglichkeit zum Wechsel des Vertragspartners stellt in diesem Fall eine ernstzunehmende Bedrohung dar, die Anreize für eine hohe Qualität der ausgetauschten Leistungen setzt.

\subsubsection{Hidden action: Informationsunvollkommenheiten nach Vertragsabschluss ohne Ex post-Kenntnis des Principal}

Bei dem dritten Grundtyp von Informationsasymmetrien in Auftraggeber/Auftragnehmer-Beziehungen verfuggt der Agent gegenüber dem Principal zum Zeitpunkt nach Vertragsabschluss über Informationen, die dem Principal auch ex post nicht bekannt werden. ${ }^{182}$ Diese Art der Informationsunvollkommenheit wird als hidden action

\footnotetext{
${ }^{181}$ Vgl. Spremann (1990), S. 580; Picot/Reichwald/Wigand (1998), S. 50.

${ }^{182}$ Vgl. Spremann (1990), S. 571.
} 
bezeichnet und ist dadurch gekennzeichnet, dass der Principal auch nachträglich nicht beurteilen kann, ob sich der Agent bei der Erbringung der Leistung vollständig an die Vertragsvereinbarungen gehalten hat oder ob er zumindest partiell gegen die Interessen des Principals verstoßen hat. ${ }^{183}$ In dieser Situation bestehen für den Agent Verhaltensspielräume, deren opportunistische Ausnutzung als moral hazard bezeichnet wird. Das Problem des moral hazard tritt in den späteren Phasen von Agency-Beziehungen auf, wenn die Entscheidungen und Handlungen des Agent den Interessen des Principal möglicherweise zuwiderlaufen und dieser nicht in der Lage ist, eine angemessene Entlohnung für den Agent zu ermitteln. Zur näheren Analyse der Einflussmöglichkeiten des Informationsmanagements sind zunächst die Ursachen der dieser Konstellation zu Grunde liegenden Informationsasymmetrie zu betrachten.

Der Informationsnachteil des Principal bei der Erstellung der Leistung setzt sich aus zwei Komponenten zusammen. Die erste Komponente der Informationsasymmetrie bezieht sich auf die Beobachtbarkeit des Auftragnehmerverhaltens. Der Principal besitzt in den meisten Fällen keine genaue Kenntnis über die Handlungsmöglichkeiten des Agent. ${ }^{184}$ Selbst wenn er die Möglichkeit hat, das Verhalten des Agent zu beobachten, fehlt ihm typischerweise das erforderliche Fachwissen, um die Handlungen des Agent adäquat beurteilen zu können. ${ }^{185}$ In diesem Fall ist grundsätzlich ein Ausweichen auf die Beobachtung des Ergebnisses als Beurteilungsmaßstab möglich. Aus der alleinigen Beobachtung des Ergebnisses lassen sich jedoch ebenfalls keine sicheren Rückschlüsse auf die Leistungsanstrengungen des Agent ziehen, da das Ergebnis außer von dem Verhalten des Agent von weiteren Einflussfaktoren abhängig ist. $^{186}$

Diese Einflussgrößen werden in der Agency-Theorie zu einer Gesamtgröße, dem exogenen Risiko, als zweiter Ursache für das hidden action-Problem zusammengefasst. ${ }^{187}$ Das exogene Risiko beschreibt die Beeinflussbarkeit des Ergebnisses durch den Agent. Bei hohem exogenen Risiko besteht nur ein sehr mittelbarer Zusammenhang zwischen den Anstrengungen des Agent und dem mit Hilfe dieser Anstrengungen erzielten Ergebnis. Es wird angenommen, dass der Principal die Realisation des exogenen Risikos nicht beobachten kann. Folglich hat er keine Möglichkeit, das Verhalten des Agent allein auf Grund der Beobachtung des Gesamtergebnisses zu beurteilen, da unklar bleibt, inwiefern das Handlungsergebnis eine Folge des Arbeitseinsatzes oder der Umwelteinflüsse ist. ${ }^{188}$

Die Gefahr des moral hazard entsteht allerdings nicht allein aus der Unsicherheit des Principals über das Verhalten des Agent, sondern erst in Verbindung mit dem bereits mehrfach angesprochenen Freiraum, mit dem die Herstellung der durch den Agent zu erbringenden Leistung verbunden ist. In vielen Fällen ist die Art der Nutzung der Ressourcen, die der Agent bei der Herstellung der Leistung verwendet, nicht eindeutig durch technische Bedingungen vorgegeben. Ressourcen, die vielseitig nutzbar sind,

\footnotetext{
${ }^{183}$ Vgl. Mikus (1998), S. 454f.; Picot/Reichwald/Wigand (2001), S. 59.

${ }^{184}$ Vgl. Ebers/Gotsch (1999), S. 213.

${ }^{185} \mathrm{Vgl}$. Picot/Reichwald/Wigand (2001), S. 59.

${ }^{186} \mathrm{Vgl}$. Ebers/Gotsch (1999), S. 213.

${ }^{187}$ Vgl. Spremann (1990), S. 571.

${ }^{188}$ Vgl. Mikus (1998), S. 454f. sowie Spremann (1990), S. 571, der diesen Sachverhalt plakativ beschreibt: „Durch Glück bei Faulheit kann aber dieselbe Gegenleistung zustande kommen wie durch Pech und Fleiß.“
} 
sodass ihre sinnvolle Verwendung im Einzelfall nur schwer beurteilt werden kann, werden als plastisch bezeichnet. ${ }^{189}$ Bei Ressourcen, die nur ein geringes Plastizitätsniveau aufweisen (z.B. bei Fertigungsstraßen in der Automobilindustrie), ist der diskretionäre Verhaltensspielraum des Agent gering, da er bei der Herstellung der Leistung eng an technische Vorgaben gebunden ist. Die Gefahr des moral hazard ist höher, wenn bei der Herstellung der Leistung Ressourcen mit einem hohen Plastizitätsniveau eine große Rolle spielen (z.B. Fachwissen und finanzielle Budgets bei F+E-Projekten), da die Art der Nutzung dann nicht ex ante durch den Principal vorgegeben werden kann.

Damit sind die Anknüpfungspunkte beschrieben, an denen das Informationsmanagement mit dem Ziel einer Reduzierung der hidden action-Problematik ansetzen kann. Die Gestaltungs- und Steuerungsmaßnahmen des Informationsmanagements können sich im Prinzip einerseits auf eine Verbesserung der Beobachtbarkeit des Auftragnehmerverhaltens durch den Auftragnehmer richten und dadurch die zu Grunde liegende Informationsasymmetrie reduzieren. Sie können andererseits an den Ursachen für den Verhaltensspielraum des Auftragnehmers ansetzen und sich auf die Plastizität von Ressourcen, die der Auftragnehmer bei der Herstellung der Leistung verwendet, richten. Eine Verringerung der Plastizität kann selbst bei unverändertem Informationsstand eine Reduzierung von Agency-Kosten bewirken. Abbildung 5/8 zeigt diese Einflussmöglichkeiten, die im Folgenden für die verschiedenen Arten von AgencyBeziehungen in und zwischen Unternehmungen spezifiziert werden.

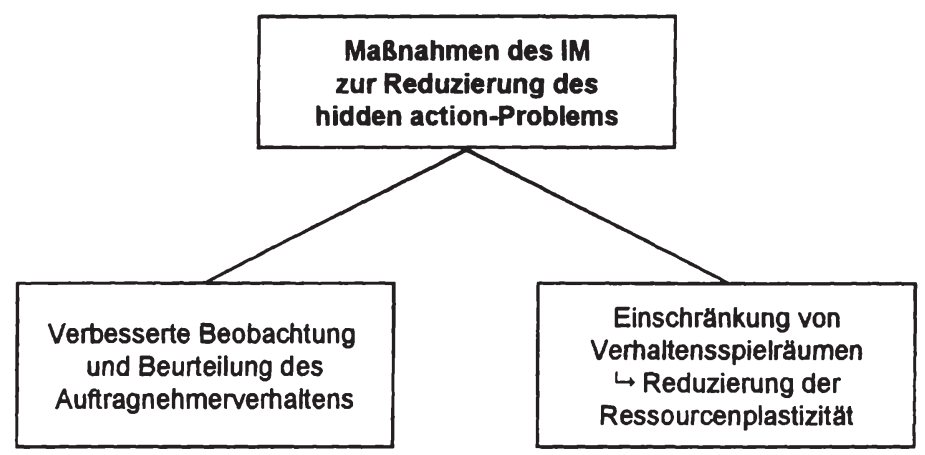

\section{Abb. 5/8: Maßnahmen des Informationsmanagements zur Reduzierung des hidden action-Problems}

Die Verringerung von Informationsasymmetrien, die zu Lasten des Auftraggebers bestehen, läuft grundsätzlich auf die Konzeption informationstechnisch unterstützter Berichtssysteme hinaus, die eine bessere Überwachung des Auftragnehmerverhaltens durch den Auftraggeber ermöglichen. Derartige Berichtssysteme machen den Handlungsbereich des Auftragnehmers für den Auftraggeber transparenter und können sich beispielsweise auf eine Ausweitung der Rechenschaftspflichten, die Einbeziehung von Leistungsvergleichen oder die Schaffung von Kostentransparenz richten. ${ }^{190}$ Das

\footnotetext{
${ }^{189}$ Das Konzept der Ressourcenplastizităt wurde von Alchian und Woodward entwickelt, vgl. dazu Alchian/Woodward (1988), S. 69f.

${ }^{190} \mathrm{Vgl}$. Ebers/Gotsch (1999), S. 215.
} 
Informationsmanagement kann diesen Maßnahmetyp durch die Konzeption und den Einsatz analyseorientierter Anwendungssysteme, die auch als Management Support Systeme bezeichnet werden, ${ }^{191}$ unterstützen. Der Einsatz derartiger Systeme erfordert eine verbindliche Definition der Berichtsinhalte (z.B. der Zusammensetzung einzelner Kennzahlen, die mit Unterstützung des Systems geplant, gesteuert und kontrolliert werden). Damit wird die Basis für die Festlegung konkreter Zielvereinbarungen geschaffen, an denen die Leistung des Auftragnehmers bei der Vertragserfüllung gemessen werden kann. Die Bereitstellung der Ist-Daten durch diesen Systemtyp ermöglicht die Kontrolle der Zielerreichung, auf deren Basis wiederum die Zahlung leistungsbezogener Entlohnungsbestandteile möglich ist.

Die Möglichkeit, die Beobachtung des Auftragnehmerverhaltens zu verbessern, ist in erster Linie bei unternehmungsinternen Agency-Beziehungen, also beim Austausch von Arbeitsleistungen gegeben. Unternehmungsübergreifende Auftraggeber/Auftragnehmer-Beziehungen, bei denen Sachleistungen ausgetauscht werden, sind dadurch gekennzeichnet, dass auf Grund der Vertragsstruktur im Regelfall keine Gelegenheit zur Beobachtung des Auftragnehmerverhaltens besteht. Der Einsatz von Management Support Systemen als Mittel des Informationsmanagements zur Reduzierung der Informationsasymmetrie kommt unter diesen Bedingungen in der Regel nicht in Frage. Die hidden action-Problematik wird bei diesem Beziehungstyp normalerweise durch eine Kopplung der Entlohnung an die durch den Auftraggeber wahrgenommene Qualität des Leistungsergebnisses bewältigt. Im Rahmen einer dauerhaft angelegten Zusammenarbeit kann es allerdings auch im Interesse des Lieferanten liegen, interne Informationen freiwillig $\mathrm{zu}$ offenbaren, um längerfristig eine bessere Teilung des externen Risikos zwischen Auftragnehmer und Auftraggeber zu erreichen.

Die Reduzierung der Ressourcenplastizität als zweiter Ansatzpunkt des Informationsmanagements zur Verringerung der hidden action-Problematik erfordert es, den Standardisierungsgrad von Prozessen der Leistungserstellung mit Hilfe entsprechender informationstechnischer Lösungen zu erhöhen. Dadurch werden die Verhaltensfreiheiten der Aufgabenträger innerhalb dieser Prozesse und somit deren Möglichkeit zur opportunistischen Ausnutzung von Informationsvorsprüngen eingeschränkt. Die Erfolgsaussichten dieses Maßnahmetyps hängen von der Art der durch den jeweiligen Auftragnehmer zu erfullenden Aufgabe, insbesondere von deren Strukturiertheit, ab. Bei unternehmungsinternen Agency-Beziehungen variieren sie typischerweise mit der jeweils betrachteten hierarchischen Ebene innerhalb der Unternehmung.

Je schlechter die jeweils vorliegende Problemstellung strukturiert ist, um so größere Freiräume bestehen bei ihrer Lösung und um so schlechter ist ex post durch den Auftraggeber die sinnvolle Nutzung der Ressourcen zu beurteilen. Dieses Merkmal steht deshalb in Zusammenhang zur hierarchischen Position des betrachteten Auftragnehmers, weil der Anteil an schlecht strukturierten Aufgabenstellungen im jeweiligen Zuständigkeitsbereich mit der hierarchischen Position in der Regel steigt. ${ }^{192}$ Auf der Ebene der Unternehmungsleitung übertragen die Anteilseigner den (Top-) Managern mit Teilen der Verfügungsrechte am Eigenkapital der Unternehmung eine besonders plastische Ressource. Das Handlungsfeld der Auftragnehmer ist bei diesem speziellen Beziehungstyp besonders groß, da eine sinnvolle Verwendung des Eigenkapitals zur

${ }^{191}$ Vgl. Gluchowski/Gabriel/Chamoni (1997), S. 1.

${ }^{192}$ Vgl. Nippa (1988), S. 125ff. 
Steigerung des shareholder value in sehr unterschiedlicher Weise erfolgen kann. Infolgedessen bestehen für das Informationsmanagement praktisch keine Möglichkeiten zur Standardisierung der Aktivitäten des Top-Managements durch eine entsprechende Gestaltung des technikunterstützten Informationssystems der Unternehmung.

Da die Strukturiertheit der Aufgabenstellung mit sinkender Hierarchieebene der Auftragnehmer tendenziell zunimmt, nehmen die Freiheitsgrade bei der Erstellung der vertraglich vereinbarten Leistung zugleich ab. Aus diesem Grund verringert sich das hidden action-Problem auf den niedrigeren hierarchischen Ebenen der Unternehmung. Für das Informationsmanagement sind zudem bessere Voraussetzungen gegeben, die Handlungsfreiheit der Aufgabenträger durch eine verstärkte Automatisierung der Prozesssteuerung weiter einzuschränken. Durch eine verstärkte Standardisierung und Formalisierung der Leistungserstellungsprozesse wird die Ressourcenplastizität reduziert, da das Vorgehen bei der Nutzung der durch die Auftragnehmer verwendeten Ressourcen präziser ex ante durch die Auftraggeber vorgegeben wird. Als Instrument zur Realisierung dieser Zielsetzung sind insbesondere Workflow ManagementSysteme geeignet. Neben der Automatisierung der Steuerung dokumentieren derartige Systeme die Aktivitäten der Beteiligten und erhöhen damit die Transparenz der betreffenden Prozesse, sodass zugleich eine Verringerung der bestehenden Informationsasymmetrien möglich ist.

Bei Auftraggeber/Auftragnehmer-Beziehungen zwischen Unternehmungen zum Austausch von Sachleistungen ist grundsätzlich ebenfalls eine Reduzierung der für die hidden action-Problematik ursächlichen Verhaltensspielräume des Auftragnehmers durch Maßnahmen des Informationsmanagements vorstellbar. Dabei fungieren moderne informations- und kommunikationstechnische Lösungen als Enabler zum Aufbau unternehmungsübergreifender Wertschöpfungsketten. Wenn die zu unterstützenden Prozesse der Leistungserstellung einen hohen Strukturiertheitsgrad aufweisen, dann kann auch unternehmungsübergreifend die Prozesssteuerung automatisiert werden. Der Grad der Formalisierung und Standardisierung der zwischen den beteiligten Unternehmungen ablaufenden Lieferungs- und Leistungsprozesse steigt folglich, sodass die Aktivitäten des Auftragnehmers in verstärktem Maße ex ante vorgegeben werden können. Derartige Maßnahmen reduzieren nicht nur aus Sicht des Auftraggebers die Gefahr des Moral Hazard, sondern sie können auch aus Sicht des Auftragnehmers sinnvoll sein, weil dadurch die Agency-Kosten insgesamt reduziert werden und sich das externe Risiko besser zwischen den Beteiligten aufteilen lässt.

\subsubsection{Berührungspunkte zwischen Transaktionskostentheorie und Agency- Theorie als Ausgangspunkt für die Institutionenökonomische Analyse des Informationsmanagements}

In diesem Abschnitt werden die für das Informationsmanagement relevanten Verbindungen zwischen den bisher getrennt behandelten Teilgebieten der Neuen Institutionenökonomie untersucht. Abschnitt 5.2.4.1 arbeitet zunächst die gemeinsamen Problemkreise von Transaktionskostentheorie und Agency-Theorie, die Anhaltspunkte für den Aufbau der weiteren Untersuchungen in diesem Kapitel bieten, heraus. Anschließend wird in Abschnitt 5.2.4.2 das grundlegende Untersuchungsobjekt, auf das sich institutionenökonomisch fundierte Aussagen zum Informationsmanagement im nachfolgenden Abschnitt 5.3 beziehen müssen, erläutert. 


\subsubsection{Gemeinsame Problemkreise des Informationsmanagements aus Sicht von Transaktionskostentheorie und Agency-Theorie}

Bei der Konstruktion des allgemeinen Erkenntnisgegenstands der Neuen Institutionenökonomie wurde verdeutlicht, dass deren verschiedene Teilgebiete auf gemeinsamen Grundannahmen basieren, jedoch unterschiedliche Untersuchungsschwerpunkte verfolgen. Allerdings ist das Verhältnis zwischen Transaktionskostentheorie und Agency-Theorie als den beiden hier ausgewählten Teilgebieten mit besonderer Relevanz für das Informationsmanagement insgesamt nicht unumstritten. In der Frühzeit der modernen Transaktionskostentheorie, Anfang der 1970er Jahre, standen die beiden Ansätze in einem eher substitutionalen Verhältnis zueinander, wobei die Meinungsverschiedenheiten der jeweiligen Vertreter insbesondere am Aspekt von Weisungsbefugnis und Autorität in marktwirtschaftlichen Systemen ansetzten. ${ }^{193}$ Später wich die Rivalität zwischen den beiden Forschungsrichtungen dagegen der Einsicht, dass sich die Ansätze im Prinzip sehr gut ergänzen. Trotz unterschiedlicher Schwerpunktsetzung und zum Teil auch abweichender Begriffswahl überschneiden sich Agency-Theorie und Transaktionskostentheorie in ihrer Analyse der Austauschsituation. ${ }^{194}$ Folgt man dieser Ansicht, so lässt sich als Synthese beider Teilgebiete „die Unternehmung im Hinblick auf zwei Eigenschaften definieren: die Beobachtbarkeit der Leistung der Inputfaktoren und die Möglichkeit der Enteignung von Quasirenten transaktionsspezifischer Vermögenswerte.“" ${ }^{195}$

Im nachfolgenden Abschnitt 5.3 wird bei der Theoriebildung für das Informationsmanagement von dieser Synthese ausgegangen. Die Überlegungen stützen sich auf beide Forschungszweige der Neuen Institutionenökonomie, die in den bisherigen Abschnitten getrennt behandelt wurden, gemeinsam. Auf dieser Basis wird die Koordination des Leistungsaustausches untersucht, die durch entsprechende Gestaltungsmaßnahmen des Informationsmanagements zu unterstützen ist. Im Falle der Bezugnahme auf die positiv ausgerichtete Variante Agency-Theorie steht dabei die Beseitigung von Informationsasymmetrien im Vordergrund, während im Falle der Bezugnahme auf die Transaktionskostentheorie die Effizienz des vertraglichen Arrangements unter Berücksichtigung von Unsicherheit und Spezifität des Leistungsaustausches fokussiert werden.

Die Theoriebildung für das Informationsmanagement orientiert sich vom Aufbau her an den verschiedenen Phasen, die beim Austausch von Leistungen typischerweise durchlaufen werden. Bereits im vorangegangenen Abschnitt wurden im Rahmen der Konstruktion des Informationsmanagements als Erkenntnisgegenstand der Transaktionskostentheorie und der Agency-Theorie verschiedene Vertragsphasen identifiziert und voneinander abgegrenzt. Mit der Anbahnungs-, der Verhandlungs-, der Kontroll- bzw. Abwicklungsphase und der Anpassungsphase wurden unter Bezug-

\footnotetext{
${ }^{193} \mathrm{Vgl}$. dazu Erlei/Leschke/Sauerland (1999), S. 190f.

${ }^{194}$ Vgl. Ebers/Gotsch (1999), S. 250.

${ }^{195}$ Alchian (1984), S. 39. Auch Williamson erkennt die Komplementarităt der Ansätze an und ordnet den Schwerpunkt der Agency-Theorie, insbesondere ihrer normativen Variante, bei den Ex anteAktivităten des Leistungsaustausches, ein, während der Schwerpunkt der Transaktionskostentheorie bei den Ex post-Aktivităten liege. Vgl. Williamson (1985), S. 26ff. Dieser Sichtweise wird hier nicht gefolgt, da sie vornehmlich für den normativen Zweig der Agency-Theorie zutreffend ist. Die im vorigen Kapitel als relevanter für das Informationsmanagement eingestufte positive Variante der Agency-Theorie befasst sich sowohl mit Informationsasymmetrien vor Vertragsschluss als auch nach Vertragsschluss.
} 
nahme auf die Transaktionskostentheorie vier Phasen unterschieden, in denen verschiedene Arten von Transaktionskosten anfallen. Eine sehr ähnliche Einteilung liegt der Abgrenzung von hidden characteristics, hidden intention und hidden action als unterschiedlichen Ausprägungen von Informationsasymmetrien durch die AgencyTheorie zu Grunde, da die zugehörigen Problemstellungen in unterschiedlichen Phasen der Zusammenarbeit ihre spezielle Bedeutung besitzen.

Im Folgenden wird die Betrachtung auf drei Problemkreise mit Bedeutung für das Informationsmanagement beschränkt, die im Rahmen eines Leistungsaustausches prinzipiell aufeinander folgen. Die Abgrenzung dieser Problemkreise entspricht einer Einteilung in Transaktionsphasen auf der höchsten Abstraktionsebene. Dabei handelt es sich um die Auswahl eines geeigneten Vertragspartners, um die Festlegung des Vertragsdesigns und um die Erfüllung der vertraglich vereinbarten Leistungen. ${ }^{196}$ Auf die explizite Betrachtung einer Anpassungsphase wird im Weiteren verzichtet, da oben bereits erläutert wurde, dass sich bei der Anpassung eines Vertrages für das Informationsmanagement keine grundsätzlich neuen Problemstellungen im Vergleich zur Verhandlungsphase ergeben. Durch diese Beschränkung auf nur drei Phasen ergibt sich eine Analogie zu der im Rahmen der positiv ausgerichteten Agency-Theorie vorgenommenen Unterscheidung dreier Arten von Informationsasymmetrien. Diese Dreiteilung ist in Abbildung 5/9 dargestellt. Sie kann für die Untersuchung des Informationsmanagements als gemeinsamer Nenner beider Forschungszweige aufgefasst werden und wird daher als Ausgangspunkt für die weiteren Ausführungen dieses Kapitels gewählt.

\footnotetext{
${ }^{196}$ Eine entsprechende Dreiteilung findet sich auch bei Richter/Furubotn (1999), S. 318, die sich mit ihren Ausfuhrungen ebenfalls auf einer sehr hohen Abstraktionsebene bewegen und zwischen Aktivitäten vor Vertragsschluss (Suche, Inspektion), Vertragserstellung (Aushandlung) und Aktivitäten nach Vertragsschluss (Erfüllung, Kontrolle, Durchsetzung) unterscheiden. Eine phasenbezogene Betrachtungsweise mit der Unterscheidung dieser drei Vertragsphasen hat sich auch für die Analyse interorganisatorischer Informationssysteme etabliert, die einen wichtigen Untersuchungsbereich der nachfolgenden Überlegungen dieses Kapitels darstellen. Vgl. z.B. Schmid (1993), S. 476f.; Klein (1996), S. 54; Alt (1997), S. 106; Tan/Shaw/Fulkerson (2000), S. 467; Buxmann/König (2000), S. 36ff.
} 


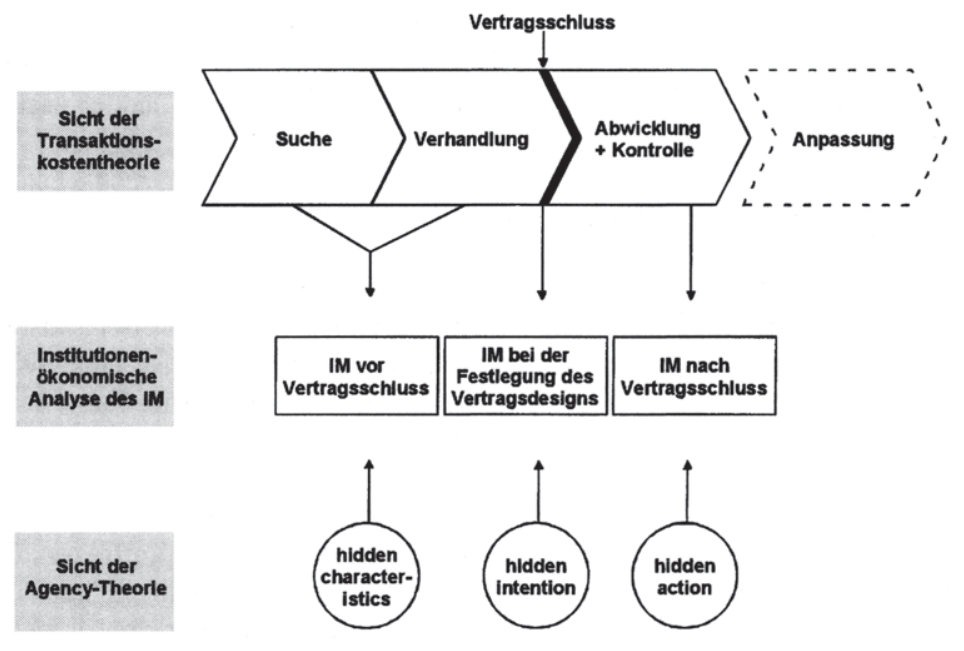

Abb. 5/9: Gemeinsame Problemkreise von Transaktionskostentheorie und AgencyTheorie

Vor der Durchführung weiterer Überlegungen ist zunächst noch darauf einzugehen, welche Arten von Aussagen von einem Institutionenökonomischen Ansatz für eine Theorie des Informationsmanagements erwartet werden können. Entsprechend den Erkenntnisinteressen und Leitideen der Neuen Institutionenökonomie muss sich die nachfolgende Analyse auf der pragmatischen Ebene der Informationsverarbeitung bewegen. Die diesem Forschungsprogramm zu Grunde liegende Denkweise erfordert es, dass anwendungsbezogene Problemstellungen in den Mittelpunkt eines Institutionenökonomischen Ansatzes für eine Theorie des Informationsmanagements gestellt werden. Dementsprechend behandeln die weiteren Überlegungen dieses Kapitels insbesondere funktionale Anforderungen, die sich aus institutionenökonomischer Sicht an das technikunterstützte Informationssystem der Unternehmung und seine verschiedenen Teilsysteme ergeben. Durch die Gestaltung und Steuerung von Systemen zur Erfüllung dieser Anforderungen kann das Informationsmanagement die Koordination von Aktivitäten der beteiligten Vertragsparteien verbessern und auf diesem Wege die Effizienz des Leistungsaustausches erhöhen. Aussagen über die technische Umsetzung dieser Anforderungen, also die materielle Ebene der Informationsverarbeitung, kann ein Institutionenökonomischer Ansatz für eine Theorie des Informationsmanagements dagegen nur in sehr begrenztem Maße liefern. Die Neue Institutionenökonomie beschäftigt sich mit dem Handeln von menschlichen Akteuren im Wirtschaftsgeschehen, nicht jedoch mit der Funktionsweise hard- und softwaretechnischer Komponenten der Informationsverarbeitung.

\subsubsection{Transaktionen als grundlegende Bezugseinheiten des Informations- managements}

Nachfolgend wird der gemeinsame Ansatzpunkt von dem Transaktionskostentheorie und Agency-Theorie bei der Entwicklung von Aussagen zum Informationsmanagement ausgehen, herausgearbeitet. Die Transaktionskostentheorie stellt als allgemeine 
Analyseeinheit Transaktionen in den Mittelpunkt der Betrachtung und untersucht, wie sich verschiedene idealtypische Vertragsmuster auf die Koordinationseffizienz bei der Abwicklung einer Transaktion auswirken. ${ }^{197}$ Die zentrale Untersuchungseinheit, auf die sich die Agency-Theorie bezieht, ist der einer Auftraggeber/Auftragnehmer-Beziehung zu Grunde gelegte Vertrag. ${ }^{198}$ Die betreffenden Vertragsverhältnisse werden genau genommen jedoch ebenfalls dadurch begründet, dass ein Austausch von Leistungen, transaktionskostentheoretisch formuliert also eine Transaktion, zwischen den Vertragspartnern stattfindet. Daher ist es gerechtfertigt, Transaktionen als gemeinsame Bezugseinheit der beiden hier in die Analyse einbezogenen Arbeitsgebiete der Neuen Institutionenökonomie aufzufassen.

Mit dem abstrakten Konstrukt der Transaktion wird dabei ein sehr grundsätzlicher Sachverhalt fokussiert. Dem Transaktionsbegriff können so unterschiedliche Vorgänge wie z.B. der Austausch von Rohstoffen zwischen Unternehmungen, der Verkauf von Endprodukten an private Konsumenten, die Erbringung von Querschnittsfunktionen durch unternehmungsinterne Dienstleistungsbereiche oder die Inanspruchnahme von Arbeitsleistungen der Beschäftigten durch Vorgesetzte innerhalb einer Abteilung subsumiert werden. Transaktionen können also sehr unterschiedliche Formen annehmen und sind nicht an eine bestimmte Koordinationsform gebunden. Das gemeinsame Merkmal aller Transaktionstypen stellt lediglich der Austausch von Leistungen dar, wobei jeweils (mindestens) ein Leistungsanbieter und (mindestens) ein Leistungsnachfrager beteiligt sind.

Den Erkenntnisinteressen und Leitideen von Transaktionskostentheorie und AgencyTheorie folgend müssen sich auch die Aussagen eines Institutionenökonomischen Ansatzes für eine Theorie des Informationsmanagements auf Transaktionen als grundlegende Bezugseinheit beziehen. Die weiteren Überlegungen dieses Kapitels fokussieren daher Gestaltungsoptionen des Informationsmanagements zur Unterstützung von Transaktionen durch das Informationsmanagement und deren Auswirkungen auf die Effizienz der verwendeten vertraglichen Arrangements. Dabei werden keine grundsätzlichen Einschränkungen, etwa in Form von Annahmen über die Art der auszutauschenden Leistung oder die an der Transaktion beteiligten Vertragspartner, vorgenommen.

Jeder Austausch von - materiellen wie auch immateriellen - Leistungen ist mit einem dazu gehörigen Informationsaustausch verbunden, der den eigentlichen Güteraustausch koordiniert. ${ }^{199}$ Koordinierende Maßnahmen, durch die die Abstimmung von Sachverhalten hinsichtlich eines definierten Ziels erfolgt, sind deshalb notwendig, weil zwischen den Aktivitäten der verschiedenen Beteiligten Interdependenzen entstehen, sodass die Handlungen eines Akteurs Veränderungen in den Handlungsfeldern der anderen Akteure verursachen. ${ }^{200}$ Diese Interdependenzen haben zur Folge, dass die erfolgreiche Durchfürung einer Transaktion stets den Austausch von Informationen

\footnotetext{
${ }^{197}$ Vgl. Alt (1997), S. 79.

${ }^{198} \mathrm{Vgl}$. Picot/Reichwald/Wigand (2001), S. 56

${ }^{199} \mathrm{Vgl}$. Alt (1997), S. 27; Buxmann/König (2000), S. 29f., die sich bei ihren Überlegungen allerdings auf die Güterlogistik beziehen. Die Aussagen sind jedoch generell auf den Austausch von Leistungen, die sowohl immaterieller als auch materieller Art sein können, übertragbar.

${ }^{200} \mathrm{Vgl}$. dazu allgemein Reif-Mosel (2000), S. 68.
} 
zum Zwecke der Abstimmung zwischen den Beteiligten erfordert. Dieser Zusammenhang ist in Abbildung 5/10 symbolisch dargestellt.

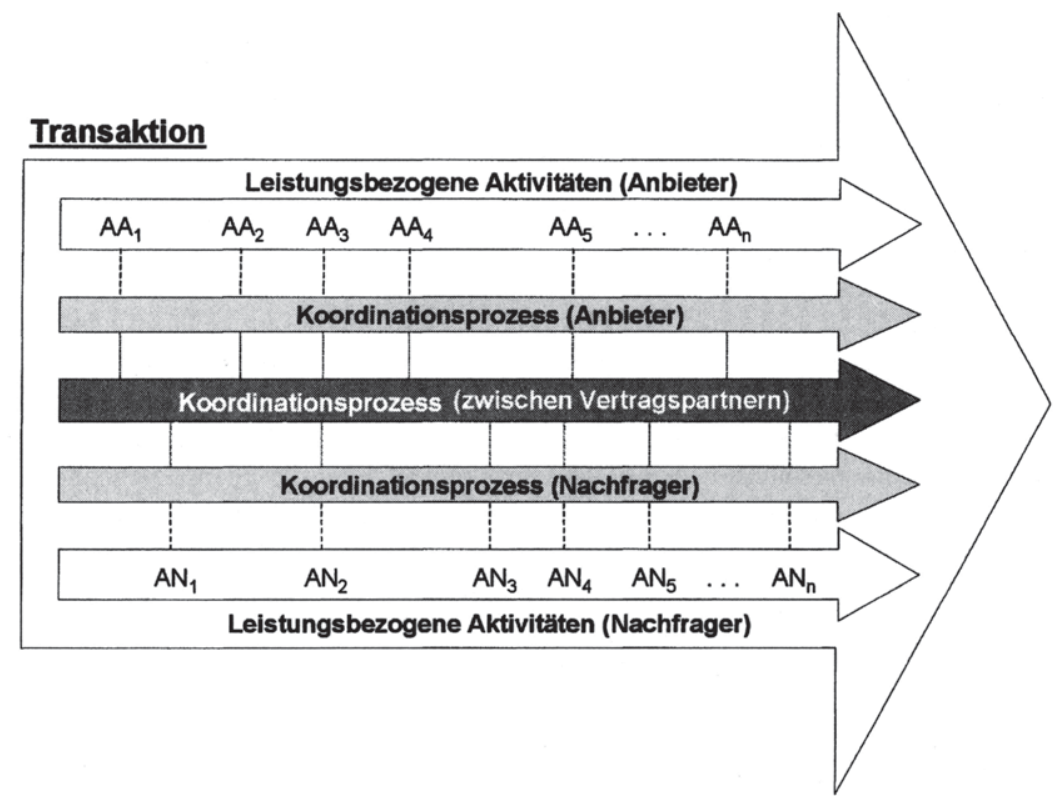

Abb. 5/10: Begleitung von Transaktionen durch koordinierende Informationsverarbeitungsprozesse

Die unmittelbar auf die Leistung bezogenen Aktivitäten des Anbieters sind in der Abbildung als $\mathrm{AA}_{\mathrm{i}}$, diejenigen des Nachfragers als $\mathrm{AN}_{\mathrm{i}}$ bezeichnet. Die leistungsbezogenen Aktivitäten werden durch einen begleitenden Koordinationsprozess aufeinander abgestimmt, der sich - wie die Abbildung zeigt - selbst wiederum aus mehreren Teilbereichen zusammensetzt. Da es sich bei den koordinierenden Prozessen um Informationsverarbeitungsprozesse handelt, die definitionsgemäß durch das Informationsmanagement gestaltet und gesteuert werden, ist jede Transaktion immer zugleich auch Gestaltungsgegenstand des Informationsmanagements. Die hier gewählte Art der Darstellung zeigt, dass in der Regel keine sequentielle Abfolge von Aktivitäten zunächst des Leistungsanbieters und anschließend des Leistungsnachfragers vorliegt. Gerade bei umfangreichen, langfristig angelegten Transaktionen mit hoher Integrativität der Leistungserstellung finden die Aktivitäten von Anbieter und Nachfrager überlappend statt. Ferner wird verdeutlicht, dass die Akteure nicht nur ihre jeweils eigenen Aktivitäten aufeinander abstimmen müssen, sondern dass zusätzlich noch eine Koordination mit den Aktivitäten der jeweils anderen Partei erforderlich ist. Je nach Art der Transaktion kann die Herausforderung für das Informationsmanagements in diesem Zusammenhang darin liegen, die verschiedenen Bestandteile dieses begleitenden Prozesses zusammenzuführen, um eine möglichst hohe Koordinationseffizienz zu erreichen.

Insgesamt ist davon auszugehen, dass die Koordinationseffizienz einer Transaktion in entscheidendem Maße von der Ausgestaltung der Informationsverarbeitung der 
beteiligten Vertragsparteien abhängt. ${ }^{201}$ Dabei können die Gestaltungsmaßnahmen des Informationsmanagements zum einen die Effizienz der Transaktion innerhalb der bestehenden Koordinationsform verbessern und zum anderen Auswirkungen auf die Vorteilhaftigkeit des vertraglichen Arrangements insgesamt haben. Die weiteren Ausführungen in diesem Kapitel wenden sich zunächst der erstgenannten Fragestellung zu. Es wird also untersucht, wie das Informationsmanagement eine möglichst hohe Effizienz der Aktivitäten in den einzelnen Phasen einer Transaktion sicherstellen kann. Das für die Abwicklung der Transaktion verwendete vertragliche Arrangement wird dabei nicht grundsätzlich hinterfragt. Es wird also davon ausgegangen, dass die Entscheidung für ein bestimmtes vertragliches Arrangement auf Grund der für die Wahl der Koordinationsform relevanten Eigenschaften des Leistungserstellungs- und des Koordinationsprozesses bereits getroffen wurde.

Anschließend wird diese Beschränkung aufgehoben, um zu überprüfen, inwiefern es auf Grund innovativer Lösungen des Informationsmanagements zu einer Veränderung der Vorteilhaftigkeit von Vertragsdesigns kommen kann. Die Vorteilhaftigkeit der Koordinationsform hängt einerseits von der relativen Effizienz der alternativ wählbaren Vertragsmuster und andererseits von den Eigenschaften der Transaktion ab. Die Eigenschaften der Transaktion wiederum setzen sich aus den Eigenschaften des eigentlichen Leistungserstellungsprozesses und des koordinierenden Informationsverarbeitungsprozesses zusammen. Die relative Bedeutung der verschiedenen Komponenten hängt dabei davon $a b$, wie hoch der Anteil der Kosten für den Koordinationsprozess an den gesamten Kosten der Transaktion ist. Bei Leistungen mit hohen Produktionskosten fallen die Koordinationskosten relativ weniger ins Gewicht als bei solchen Leistungsarten, die durch vergleichsweise geringe Kosten der Produktion gekennzeichnet sind. Wenn die auszutauschende Leistung selbst informationelle Anteile enthält, dann erstreckt sich der Einflussbereich des Informationsmanagements zudem auch auf die Eigenschaften der eigentlichen Leistungserstellung. Abbildung 5/11 visualisiert den beschriebenen Aufbau der institutionenökonomischen Analyse des Informationsmanagements im folgenden Abschnitt.

\footnotetext{
${ }^{201}$ Vgl. Stiglitz (1985), S. 36.
} 


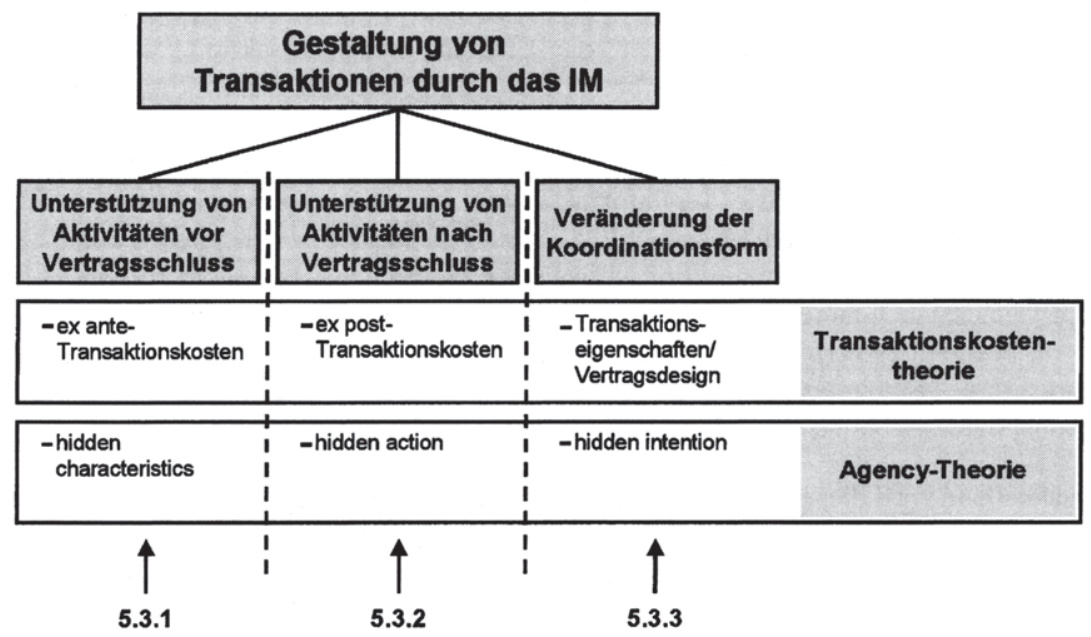

Abb. 5/11: Aufbau der institutionenökonomischen Analyse des Informationsmanagements

Es ist davon auszugehen, dass die einzelnen Transaktionsphasen je nach Art der auszutauschenden Leistung und des angestrebten vertraglichen Arrangements relativ unterschiedlich ausgestaltet sein können. ${ }^{202}$ Diese Heterogenität erfordert es, dass bei einer konkreten Transaktion bereits vor Beginn der Vertragsanbahnung die Entscheidung für die angestrebte Koordinationsform getroffen wird. So erfordern z.B. stärker hierarchisch orientierte Koordinationsformen mit unvollständigeren Verträgen durch die Notwendigkeit der bilateralen Absprache typischerweise intensivere Abstimmungen und weisen deshalb eine hohe Intensität bei den Verhandlungskosten vor dem eigentlichen Abschluss des Vertrages auf. ${ }^{203}$ Marktliche Transaktionen werden dagegen über weitgehend standardisierte Vertragsdesigns abgewickelt, die ein geringeres Maß an Verhandlungen notwendig machen. Bei dieser Transaktionsform kommt jedoch typischerweise eine höhere Zahl von Akteuren auf der Marktgegenseite als Vertragspartner in Frage, sodass eine größere Intensität von Aktivitäten bei der Vertragspartnersuche anzunehmen ist.

Die hier unterschiedenen Phasen der Auswahl des Vertragspartners, der Festlegung des Vertragsdesigns und der Erfüllung der vertraglich vereinbarten Verpflichtungen werden jedoch von Transaktionen aller Koordinationsformen durchlaufen. Diese Phasen sind wie erläutert durch phasenspezifische, idealtypische Problemstellungen (hidden characteristics, hidden intention bzw. hidden action) gekennzeichnet. Aus diesen Problemstellungen ergeben sich unabhängig von der Koordinationsform spezielle Anforderungen an die durch das Informationsmanagement $\mathrm{zu}$ gestaltende, informationstechnische Unterstützung der phasenspezifischen Aktivitäten.

${ }^{202}$ Vgl. Gersch (1998), S. 206.

${ }^{203}$ Vgl. Schüppler (1998), S. $16 f$. 


\subsection{Institutionenökonomische Analyse des Informationsmanagements}

Die nachfolgende Theoriebildung setzt an der Konstruktion des Informationsmanagements als speziellem Erkenntnisobjekt der Neuen Institutionenökonomie im vorausgegangenen Abschnitt 5.2 an. Es wird herausgearbeitet, über welche Gestaltungsoptionen das Informationsmanagement verfügt und wie die Erkenntnisse von Transaktionskostentheorie und Agency-Theorie genutzt werden können, um die Koordination des Leistungsaustausches effizienter zu machen. Wie im vorigen Abschnitt bereits erläutert, orientiert sich der Aufbau der Untersuchung an den aufeinander folgenden Phasen des Leistungsaustausches. Zunächst wird von einer gegebenen Koordinationsform der Transaktion ausgegangen, für die in Abschnitt 5.3.1 Gestaltungsoptionen des Informationsmanagements vor Vertragsschluss und in Abschnitt 5.3.2 die Gestaltungsoptionen des Informationsmanagements nach Vertragsschluss beleuchtet werden. In Abschnitt 5.3.3 wenden sich die Überlegungen schließlich der Frage zu, inwiefern es durch Maßnahmen des Informationsmanagements zu einer veränderten Vorteilhaftigkeit von alternativ möglichen Koordinationsformen kommen kann.

\subsubsection{Informationsmanagement vor Vertragsschluss}

Im Folgenden wird untersucht, welche Ansatzpunkte sich dem Informationsmanagement zur Unterstützung der Koordinationsaktivitäten vor Vertragsschluss bieten. Abschnitt 5.3.1.1 nimmt zunächst eine kurze allgemeine Charakterisierung der Situation vor Vertragsschluss und der Bedeutung der technikunterstützten Informationsverarbeitung in dieser Phase vor. Abschnitt 5.3.1.2 nimmt die Perspektive des Leistungsnachfragers ein und betrachtet Möglichkeiten zur informationstechnischen Unterstützung von Beschaffungsvorgängen. Abschnitt 5.3.1.3 wendet sich anschließend der Perspektive des Leistungsanbieters zu und analysiert institutionenökonomisch begründete Anforderungen an die informationstechnische Unterstützung von Absatzvorgängen.

\subsubsection{Charakterisierung der Informationsverarbeitung vor Vertragsschluss}

In der ersten Phase einer Transaktion geht es darum, einen Vertragspartner zu finden, der in der Lage ist, die auszutauschende Leistung mit den gewünschten Qualitätseigenschaften zu einem angemessenen Preis zu erbringen. Die Identifikation der jeweiligen Marktgegenseite stellt die Hauptaufgabe der Beteiligten in dieser Phase dar. ${ }^{204}$ Dabei fallen für die Nachfrager Ex ante-Transaktionskosten an, die auf Grund von Aktivitäten für die Informationssuche und die Inspektion der angebotenen Leistungen entstehen. ${ }^{205}$ Das Ziel der Anbieter dagegen ist es zu diesem Zeitpunkt, in das „Evoked Set“ möglicher Lösungsalternativen des Nachfragers aufgenommen zu werden. $^{206} \mathrm{Zu}$ diesem Zweck unternehmen die Anbieter Anstrengungen zur Bekanntmachung und Präsentation der von ihnen angebotenen Leistung, die sich ebenfalls in Ex ante-Transaktionskosten für die Erschließung des Marktzugangs niederschlagen.

\footnotetext{
${ }^{204} \mathrm{Vgl}$. Alt/Cathomen (1995), S. 14.

${ }^{205}$ Vgl. Richter/Furubotn (1999), S. 318.

${ }^{206}$ Vgl. Gersch (1998), S. 208. Zum Konzept des Evoked Set vgl. Backhaus (1999), S. 124ff.
} 
In dieser Vertragsphase stehen bei den Aktivitäten der Akteure auf beiden Marktseiten also explizit Informationsprobleme im Vordergrund. Der angestrebte Informationsaustausch zwischen den potenziellen Vertragspartnern ist dabei zunächst als Kommunikation zwischen ex ante anonymen Akteuren auf einem anonymen Markt aufzufassen. Gedanklich ist sowohl bei hierarchisch als auch bei marktlich koordinierten Transaktionen ursprünglich von einer Konkurrenzsituation der Akteure auf einer Marktseite auszugehen. ${ }^{207}$ Kommt es allerdings zu einer Wiederholung der gleichen oder einer ähnlichen Transaktion, so können die Beteiligten in der Anbahnungsphase auf Erfahrungen aus vorangegangenen Transaktionen zurückgreifen, sodass die Anonymität der Marktgegenseite zumindest teilweise aufgehoben wird. Wie die weiteren Ausführungen dieses Abschnitts zeigen werden, spielen in diesen Fällen die bei vorausgegangenen Transaktionen gewonnenen Erfahrungen mit der anderen Vertragsseite und den von ihr angebotenen Leistungen eine erhebliche Rolle. ${ }^{208}$

Bei hierarchisch koordinierten Transaktionen findet eine fundamentale Transformation der Beziehung zwischen den Beteiligten statt, sobald diese sich zum Abschluss eines längerfristigen Vertrags entschließen und spezifische Investitionen für die Realisierung der entsprechenden Transaktion tätigen. ${ }^{209}$ Durch diese fundamentale Transformation wird die ursprünglich marktliche Beziehung der Vertragsparteien in ein bilaterales Verhältnis umgewandelt, das die Züge eines beidseitigen Monopols trägt. Die dauerhafte Gültigkeit des Vertrages gewährleistet, dass nicht für jede einzelne (Teil-) Leistung ein neuer Vertragspartner gesucht werden braucht. Allerdings bleibt auch bei längerfristig angelegten, neoklassischen oder relationalen Verträgen grundsätzlich die Möglichkeit zum Wechsel des Vertragspartners erhalten, wenn auch je nach Höhe der getätigten spezifischen Investitionen mehr oder weniger bedeutende Wechselkosten in Kauf zu nehmen sind. Bei diesem Transaktionstyp ist es deshalb ebenfalls für jeden Akteur erforderlich, sich über die Situation der eigenen und der gegenüberliegenden Marktseite zu informieren, um neu auftretende Akteure oder neu angebotene Leistungen möglichst unverzüglich wahrzunehmen.

Die Kenntnisnahme von Alternativen und die Aushandlung der konkreten Verpflichtungen erfolgen also bei jeder Koordinationsform vor dem Abschluss eines Vertrages zum Austausch von Leistungen. Für das Informationsmanagement ergibt sich aus den oben beschriebenen Zielsetzungen der Marktteilnehmer zum Zeitpunkt vor Vertragsschluss die Aufgabe, Anwendungssysteme bereitzustellen, die die Such- und Verhandlungsaktivitäten der eigenen Unternehmung und der Marktgegenseite vereinfachen und dadurch den Marktzugang erleichtern. Anwendungssysteme mit einer speziell auf diese Problemstellung zugeschnittenen Funktionalität, die auf die wechselseitige Abstimmung von anbietenden und nachfragenden Wirtschaftssubjekten durch Preisbildungs- und Wettbewerbsprozesse abzielen, werden derzeit in Theorie und Praxis unter dem Oberbegriff der Elektronischen Märkte diskutiert. ${ }^{210}$ Dabei handelt es sich um interorganisatorische Informationssysteme, die den teilnehmenden Käufern und Verkäufern

\footnotetext{
${ }^{207}$ Vgl. Erlei/Leschke/Sauerland (1999), S. 182.

${ }^{208}$ Bei der Gestaltung und Pflege längerfristiger Geschäftsbeziehungen geht es sogar gerade darum, eine Kontingenz zwischen den Geschäftspartnern herzustellen. Vgl. dazu Gersch (1998), S. 14; Schütze (1992), S. 29f.

${ }^{209} \mathrm{Vgl}$. Williamson (1985), S. 61.

${ }^{210} \mathrm{Vgl}$. z.B. Malone/Yates/Benjamin (1987), S. 484ff.; Zbornik (1996), S. 60ff.; Picot/Reichwald/Wigand (2001), S. 335ff.
} 
ermöglichen, Informationen über Preise und Produktangebote auszutauschen. ${ }^{211}$ Elektronische Märkte realisieren somit den Begegnungsraum, in dem sich die Akteure bzw. ihre elektronischen Stellvertreter treffen und austauschen können. ${ }^{212}$ Ihr wesentliches Charakteristikum liegt darin, dass sich mehrere rechtlich unabhängige Anbieter und Nachfrager gegenüberstehen und über einen elektronisch unterstützten Preisbildungsmechanismus Leistungen frei austauschen. ${ }^{213}$ Ein Vertrag zwischen den Beteiligten kommt nur dann zu Stande, wenn diese sich freiwillig dazu entschließen, die Transaktion durchzuführen. ${ }^{214}$

Um technisch zu funktionieren, müssen Elektronische Märkte als interorganisatorische Informationssysteme vier Kernelemente beinhalten. Dabei handelt es sich um Kommunikationskanäle zwischen den Marktteilnehmern, um eine gemeinsame Marktsprache auf der semantischen Kommunikationsebene, um Marktapplikationen der Teilnehmer sowie um elektronische Handelsdienste, die die Funktion von Handelsmittlern erfüllen. ${ }^{215}$ Einzelheiten dieser technischen Komponenten, die das Funktionieren Elektronischer Märkte gewährleisten, werden im Rahmen der weiteren Überlegungen dieses Abschnitts nur insoweit betrachtet, wie sie aus institutionenökonomischer Perspektive Relevanz für das Informationsmanagement besitzen. Die nachfolgenden Ausführungen befassen sich stattdessen mit betriebswirtschaftichen Fragen der durch das Informationsmanagement gestalteten und gesteuerten Nutzung elektronischer Märkte in der Transaktionsphase vor Erzielung eines endgültigen Vertragsabschlusses. Sowohl für den Leistungsanbieter als auch für den Leistungsnachfrager wird untersucht, inwiefern eine Anbindung an elektronische Märkte aus Sicht von Agency-Theorie und Transaktionskostentheorie sinnvoll erscheint und welche Gestaltungsoptionen das Informationsmanagement dabei anstreben sollte.

\subsubsection{Anwendungssysteme bei der Auswahl von Vertragspartnern: Die Sicht des Leistungsnachfragers}

Als Nachfrager befindet sich die Unternehmung in der Auswahlphase auf der Suche nach einem geeigneten Lieferanten für die auszutauschende Leistung. ${ }^{216}$ Sie nimmt damit die Position des Auftraggebers ein, der eine zutreffende Beurteilung der potenziellen Auftragnehmer anstrebt, um nachträglich nicht zu beseitigende Qualitätsmängel des Vertragspartners und der von ihm erbrachten Leistung rechtzeitig zu

\footnotetext{
${ }^{211}$ Vgl. Strader/Shaw (2000), S. 78. Die Begriffswahl „interorganisatorische Informationssysteme“ in dieser Definition ist genau genommen dahingehend abzuändern, dass auch dann von Elektronischen Märkten gesprochen wird, wenn es sich bei den Marktteilnehmern auf der Käuferseite um private Endkunden handelt (Business-to-Consumer-Markets). Entscheidend ist die Koordination der Akteure über den Preismechanismus.

${ }^{212} \mathrm{Vgl}$. Schmid (1999), S. 40.

${ }^{213}$ Vgl. Zbornik (1996), S. 61; Alt (1997), S. 107. Fasst man den Begriff des Elektronischen Marktes etwas weiter und geht allein von der Koordination über den Preismechanismus aus, so wird auch dann schon von einem Elektronischen Markt gesprochen, wenn auf nur einer Marktseite mehrere Marktteilnehmer beteiligt sind. Vgl. zu dieser Auffassung z.B. Picot/Reichwald/Wigand (2001), S. $342 \mathrm{ff}$.

${ }^{214}$ Vgl. Schmid (1999), S. 35

${ }^{215}$ Vgl. zum Folgenden die ausführlichen Erläuterungen bei Schmid (1992), S. 99ff.; Zbornik (1996), S. 69ff.; Alt (1997), S. 100ff.

${ }^{216}$ Entsprechend der im Abschnitt 5.2.4.2 erläuterten Annahmen kann es sich bei dieser Leistung sowohl um materielle oder immaterielle Ressourcen als auch um Arbeitsleistungen potenzieller Beschäftigter der Unternehmung handeln.
} 
erkennen. Im Wesentlichen besteht das Problem des Principal in dieser Situation darin, gute und passende Leistungsanbieter von schlechten und unpassenden $\mathrm{zu}$ trennen. ${ }^{217}$ Wie in Abschnitt 5.2.3 erläutert, sieht die Agency-Theorie als Maßnahme des Principal zur Reduzierung dieser Art von Informationsasymmetrie die als Screening bezeichnete zusätzliche Beschaffung von Informationen vor. Screening-Aktivitäten ermöglichen es dem Principal, eventuelle hidden characteristics der potenziellen Vertragspartner aufzudecken. Sie sind jedoch mit (Transaktions-) Kosten verbunden, die dem Wert der zusätzlich erhaltenen Informationen gegenüberstehen. ${ }^{218}$ Die nachfolgenden Überlegungen befassen sich mit Gestaltungsoptionen des Informationsmanagements zur Unterstützung des Screening. Durch die Unterstützung des Screening lässt sich die Effizienz der Aktivitäten vor Vertragsschluss erhöhen, sodass sich die Transaktionskosten in dieser Phase senken lassen und/oder der Informationsstand des Auftragnehmers verbessert werden kann.

\section{a) Mediatisierung der Vertragsanbahnung}

Die informationstechnische Unterstützung von Screening-Aktivitäten erfordert die elektronische Abbildung der Kommunikationsbeziehungen zwischen den Marktteilnehmern. Diese wird nachfolgend als Mediatisierung bezeichnet. ${ }^{219}$ Allgemein wird erwartet, dass sich durch die Mediatisierung der Vertragsanbahnung Such- und Verhandlungsaktivitäten vereinfachen und somit die anfallenden Ex ante-Transaktionskosten signifikant reduzieren lassen. ${ }^{220}$ Dabei handelt es sich um den so genannten Electronic Communication Effect. ${ }^{221}$ Der Electronic Communication Effect resultiert daraus, dass der Einsatz moderner Informationstechnik zum einen eine Beschleunigung des Informationsaustausches ermöglicht, sodass in der Auswahlphase mehr Informationen in einem gegebenen Zeitraum übertragen werden können (oder der gleiche Informationsumfang in kürzerer Zeit). Zum anderen kann erwartet werden, dass die Kosten dieser Kommunikation gleichzeitig sinken. Diese allgemeine Erwartung einer Senkung von Suchkosten kann in der Praxis jedoch nur dann erfüllt werden, wenn es gelingt, den auf Grund abstrakter, theoretischer Überlegungen vermuteten Electronic Communication Effect durch konkrete informationstechnische Lösungen tatsächlich zu realisieren. Dazu ist die Einrichtung von interorganisatorischen Informationssystemen erforderlich, die einen unternehmungsübergreifenden Austausch von Angebots- und Nachfrageinformationen möglich machen.

Anwendungssysteme, die eine Realisierung des Electronic Communication Effect ermöglichen, müssen die nachfragende Partei mit mehreren in Frage kommenden Anbietern der zu beschaffenden Leistung verbinden. Auf diese Weise wird die Gelegenheit zum Vergleich der verschiedenen Angebote geschaffen. Im vorigen Abschnitt wurde bereits erwähnt, dass der entsprechende Anwendungssystemtyp mit dem Begriff des Elektronischen Marktes bezeichnet wird. Elektronische Märkte besitzen aus Sicht der Nachfrager potenziell erhebliche Suchkostenvorteile gegenüber traditionellen Märkten, da sie die Sammlung und den Vergleich von Leistungs- und Preisangeboten

\footnotetext{
${ }^{217}$ Vgl. Spremann (1990), S. 567.

${ }^{218}$ Vgl. Mikus (1998), S. 453.

${ }^{219}$ Vgl. zu dieser Begriffswahl Picot/Reichwald/Wigand (2001), S. 338.

${ }^{220}$ Vgl. z.B. Gurbaxani/Whang (1991), S. 64; Benjamin/Wigand (1995), S. 64; Zbornik (1996), S. 63; Mattes (1999), S. 64; Sauter (1999), S. 102.

${ }^{221}$ Vgl. Malone/Yates/Benjamin (1987), S. 488; Picot/Reichwald/Wigand (1998), S. 332.
} 
erleichtern. ${ }^{222}$ Durch die Zusammenführung von Suchaktivitäten beider Marktseiten auf einem elektronischen Markt entsteht neben dem Electronic Communication Effect ein weiterer Effekt, der sogenannte Electronic Brokerage Effect. Der Electronic Brokerage Effect bewirkt, dass die Zahl der potenziellen Vertragspartner anwächst und die Wahrscheinlichkeit, einen Anbieter zu finden, der den Qualitätsansprüchen des Nachfragers entspricht, steigt. ${ }^{223}$ Elektronische Märkte können als zentrale oder dezentrale Gebilde konstruiert sein und unterschiedliche Mechanismen für die Koordination von Angebots- und Nachfrageseite vorsehen. ${ }^{224}$ Im Folgenden wird untersucht, welche Gestaltungsoptionen und Nutzungsmöglichkeiten sich aus Sicht der Neuen Institutionenökonomie für Elektronische Märkte als vorteilhaft erweisen.

\section{b) Voraussetzungen für die Nutzbarkeit von Elektronischen Märkten aus Sicht des Leistungsnachfragers}

Es ist zunächst zu überprüfen, an welche Voraussetzungen die Verlagerung der Vertragsanbahnung auf Elektronische Märkte geknüpft ist. Aus der Agency-Theorie lassen sich Aussagen über die Nutzbarkeit Elektronischer Märkte ableiten, indem auf die Qualitätseigenschaften der ausgetauschten Leistungen Bezug genommen wird. Dabei ist zwischen Sucheigenschaften, Erfahrungseigenschaften und Vertrauenseigenschaften zu unterscheiden. ${ }^{225}$ Sucheigenschaften können von den Kunden bereits vor dem Abschluss des Vertrags eindeutig festgestellt werden. ${ }^{226}$ Erfahrungseigenschaften sind dagegen dadurch gekennzeichnet, dass sich die Qualität erst bei Inanspruchnahme der Leistung zweifelsfrei feststellen lässt. Vertrauenseigenschaften wiederum zeichnen sich dadurch aus, dass selbst nach dem Erwerb und der Nutzung der Leistung die tatsächliche Qualität nicht zweifelsfrei festgestellt werden kann. Die nachfolgenden Überlegungen konzentrieren sich auf die beiden Typen von Qualitätseigenschaften, bei denen der Auftraggeber überhaupt in der Lage zu einer zuverlässigen Beurteilung ist.

Das Problem der hidden characteristics, aus dem die Gefahr der adverse selection resultiert, tritt immer dann in besonders hohem Maße auf, wenn die auf dem Markt angebotene Produktqualität heterogen ist und bei der Beurteilung der Qualität insbesondere Erfahrungseigenschaften ausschlaggebend sind. Unter diesen Voraussetzungen ist der Principal erst nach Abschluss des Vertrages in der Lage, die Qualitätseigenschaften des Agent zu beurteilen. In dieser Situation kann es sinnvoll sein, bei wiederholten Transaktionen auf den Wechsel des Vertragspartners zu verzichten, wenn die bisherigen Leistungen des Agent den Anforderungen des Principal entsprochen haben. ${ }^{227}$ Dagegen besteht in den Fällen, in denen insbesondere Sucheigenschaften für die Beurteilung der Qualität einer Leistung ausschlaggebend sind, für den Principal die Chance, durch eine Intensivierung der Suchaktivitäten seine Entscheidungssituation bei der Auswahl des Vertragspartners zu verbessern. Elek-

\footnotetext{
${ }^{222} \mathrm{Vgl}$. Strader/Shaw (2000), S. 87.

${ }^{223} \mathrm{Vgl}$. Malone/Yates/Benjamin (1987), S. 488.

${ }^{224}$ Vgl. Alt (1997), S. 106.

${ }^{225}$ Diese Unterscheidung geht zurück auf Nelson (1970), S. 311ff.; Darby/Karni (1973), S. $68 f$.

${ }^{226} \mathrm{Vgl}$. dazu und zum Folgenden Neus (1998), S. 252ff.

${ }^{227}$ Der normative Zweig der Agency-Theorie hat zu dieser Problematik eine Reihe von formalen Modellen entwickelt, die Such- und Stop-Verfahren auf der Seite des Principals untersuchen. Vgl. dazu Spremann (1990), S. 567f., der sich auf Pionierarbeiten von Stigler (1961), McCall (1970) sowie Salop/Stiglitz (1977) bezieht.
} 
tronische Märkte können gerade Suchaktivitäten essenziell vereinfachen. Folglich bietet dieser Systemtyp besonders beim Austausch von solchen Leistungen hohe Effizienzsteigerungspotenziale, bei denen die kritischen Qualitätsmerkmale Sucheigenschaften darstellen.

Die Klassifikation einer Eigenschaft als Sucheigenschaft bedeutet, dass diese Eigenschaft bereits vor Vertragsabschluss eindeutig festgestellt werden kann. ${ }^{228}$ Die Ex anteFeststellbarkeit als Kriterium für die Einordnung als Sucheigenschaft basiert in der Regel $^{229}$ auf einer vollständigen Beschreibbarkeit der entsprechenden Eigenschaft. Wenn die Sucheigenschaften eines Produkts zugleich auch beschreibbar sind, dann ist eine persönliche Begutachtung des Produkts durch den Kaufinteressenten nicht erforderlich. Die Handelbarkeit eines Produkts auf Elektronischen Märkten ist deshalb dann gegeben, wenn es gelingt, auf elektronischem Wege eine aus Sicht der Nachfrager hinreichende Beschreibung der Qualitätseigenschaften zu geben.

Zur Vereinfachung der Vertragsanbahnung durch eine verbesserte Leistungsbeschreibung kann (insbesondere bei hoher Heterogenität der auf dem Markt angebotenen Leistungen) eine Standardisierung von Qualitätsmerkmalen dienen. Eine solche Standardisierung erleichtert die Übermittlung der jeweiligen Merkmalsausprägung zwischen Principal und Agent und ermöglicht eine intersubjektive Überprüfbarkeit der Qualitätseigenschaften. Die Standardisierung der für die Kaufentscheidung ausschlaggebenden Produktbeschreibungsmerkmale stellt deshalb aus Sicht der Agency-Theorie eine hinreichende Voraussetzung für die Handelbarkeit eines Produkts auf Elektronischen Märkten dar. ${ }^{230}$ Die Produktbeschreibung kann im Prinzip sowohl durch die anbietende als auch durch die nachfragende Marktseite (und auch durch den Marktbetreiber als Intermediär) erfolgen. Bei komplizierten, zusammengesetzten Produkten und Sonderbedarfen ist eine Mediatisierung der Vertragsanbahnung also dann möglich, wenn die Produktbeschreibung mit ausreichender Genauigkeit durch den Nachfrager (etwa auf dem Wege von Ausschreibungsverfahren) vorgenommen werden kann. $^{231}$

Diese auf der Agency-Theorie basierenden Überlegungen können durch die Bezugnahme auf die Transaktionskostentheorie ergänzt werden. Eine Kernaussage der Transaktionskostentheorie stellt die These dar, dass der marktliche Koordinationsmechanismus bei gelegentlichen und bei häufig wiederkehrenden Transaktionen mit geringer Spezifität die höchste Effizienz aufweist. ${ }^{232}$ Die transaktionskostentheoretischen Schlussfolgerungen, die zu diesem Ergebnis füren, basieren auf Annahmen, die das Verhalten der Beteiligten, die Eigenschaften der Transaktion sowie das verwendete

\footnotetext{
${ }^{228}$ Vgl. Neus (1998), S. 238.

${ }^{229}$ Eine Sucheigenschaft muss allerdings nicht zwingend auch beschreibbar sein. So stellt etwa der exakte Sitz eines Kleidungsstücks eine Sucheigenschaft dar, die sich nicht vollständig durch die Angabe von Konfektionsgröße, Farbe usw. beschreiben lässt. Vgl. zu diesem Beispiel Neus (1998), S. 238.

${ }^{230}$ Die hohe Bedeutung von Standards für die Handelbarkeit von Produkten auf Elektronischen Märkten betonen u.a. auch Alt (1997), S. 129; Picot/Reichwald/Wigand (1998), S. 169. Zbornik (1996), S. 117, weist darauf hin, dass die Standardisierung von Produktbeschreibungen nicht zwingend impliziert, dass auch die Produkte selbst standardisiert sein müssen. Die Standardisierung von Produkten ist allerdings immer dann unabdingbar, wenn Angebot und Nachfrage elektronisch zum Ausgleich gebracht werden sollen.

${ }^{231}$ Vgl. dazu Zbornik (1996), S. 111; Mattes (1999), S. 62; Buxmann/König (2000), S. 37.

${ }^{232}$ Vgl. dazu Williamson (1979), S. 248f; Williamson (1985), S. $73 \mathrm{ff}$.
} 
vertragliche Arrangement betreffen. Der allgemeine Zusammenhang zwischen Spezifität und Koordinationsform wird von der Möglichkeit zur informationstechnischen Unterstützung der Vertragsanbahnung mit Hilfe Elektronischer Märkte nicht tangiert. Die Annahmen der Transaktionskostentheorie sind nicht allein schon wegen der Möglichkeit zur Mediatisierung des Marktgeschehens zu modifizieren. Daraus kann geschlossen werden, dass der Anwendungsbereich Elektronischer Märkte dem traditioneller Märkte entspricht und aus Sicht der Transaktionskostentheorie insbesondere nicht-spezifische Leistungen umfasst.

Die Mediatisierung von Beschaffungsprozessen für sogenannte C-Teile (auch: indirekte Teile) wird derzeit in Theorie und Praxis unter dem Stichwort des E-Procurement diskutiert. ${ }^{233}$ Aus den Überlegungen dieses Abschnitts ergibt sich allerdings, dass für den Aufbau von E-Procurement-Prozessen weniger die Unterscheidung zwischen A-, B- und C-Teilen ausschlaggebend ist als die Merkmale Spezifität, Beschreibbarkeit und Standardisierbarkeit. Diese Voraussetzungen sind häufig gleichzeitig gegeben, ohne dass die entsprechenden Leistungsmerkmale jedoch zwingend miteinander verknüpft sind. ${ }^{234}$ Die aktuell verstärkte Fokussierung auf die Beschaffung von C-Teilen hängt insbesondere damit zusammen, dass typischerweise gerade für diesen Transaktionstyp die Prozesskosten im Vergleich zu den Einstandspreisen der beschafften Produkte besonders hoch sind, während die Effizienz von Beschaffungsprozessen für direkte Teile bereits in der Vergangenheit stark verbessert wurde. ${ }^{235}$ Mit institutionenökonomischen Notwendigkeiten ist diese Fokussierung dagegen nicht begründbar.

\section{c) Anforderungen an die Funktionalität Elektronischer Märkte aus Sicht des Leistungsnachfragers}

Sind die Voraussetzungen für eine Mediatisierung der Vertragsanbahnung gegeben, so lassen sich bei der informationstechnischen Unterstützung von Beschaffungsprozessen mit Hilfe Elektronischer Märkte unternehmungsinterne und unternehmungsexterne Prozessschritte unterscheiden. Die unternehmungsinternen Prozessschritte werden durch sogenannte Marktapplikationen auf der Seite des Leistungsnachfragers unterstützt. Diese Marktapplikationen, die einleitend als eines der vier Kernelemente elektronischer Märkte genannt wurden, ermöglichen den in der Unternehmung eingesetzten betriebswirtschaftichen Anwendungssystemen den Zugang zu elektronischen Handelsdiensten. ${ }^{236}$ Elektronische Handelsdienste wiederum bieten eine informationstechnische Unterstützung der unternehmungsexternen Schritte eines Beschaffungsprozesses.

Betrachtet man die internen Prozessschritte, so kann die durch die Nutzung von Marktapplikationen ermöglichte Verlagerung der Beschaffungsaktivitäten auf Elektronische Märkte eine Vereinfachung des Vorgehens bewirken und damit die Transaktionskosten der betreffenden Beschaffungsprozesse senken. Gerade bei indirekten Teilen ist die interne Seite von Beschaffungsprozessen bisher häufig in hohem Maße

\footnotetext{
${ }^{233} \mathrm{Vgl}$. dazu z.B. Nenninger/Gerst (1999), S. 283ff;; Mattes (1999), S. 59ff; Baron/Shaw/Bailey (2000), S. 385ff.

${ }^{234} \mathrm{Vgl}$. Malone/Yates/Benjamin (1987), S. 486, die Beispiele für Produkte anführen, die durch eine hohe Spezifităt bei leichter Beschreibbarkeit bzw. durch eine geringe Spezifităt bei komplizierter oder unvollständiger Beschreibbarkeit gekennzeichnet sind.

${ }^{235}$ Vgl. Nenninger/Gerst (1999), S. 288; Mattes (1999), S. 60, Shaw (2000), S. 12.

${ }^{236}$ Vgl. Zbornik (1996), S. 143.
} 
ineffizient ausgestaltet, da unternehmungsintern zahlreiche Stellen kontaktiert werden müssen und zentral vorliegende Informationen nicht für die dezentralen Bedarfsstellen verfugbar sind. ${ }^{237}$ Die Mediatisierung dieser Schritte eröffnet zugleich Potenziale zur Senkung der direkten Bezugskosten, da durch ein Pooling von zuvor vollständig dezentral durchgeführten Beschaffungsaktivitäten bessere Konditionen (Preise, Zahlungsbedingungen $u$.ä.) bei den Lieferanten erzielt werden können.

Die Reduzierung von Auswahlkosten im unternehmungsinternen Bereich basiert insbesondere auf der Automatisierung von Aktivitäten, die bis dahin durch die Mitarbeiter der Unternehmung manuell durchgeführt wurden. ${ }^{238}$ Diese wird durch die Einrichtung von Schnittstellen zwischen den betrieblichen Anwendungssystemen und den Marktapplikationen sowie durch die Automatisierung von Genehmigungsverfahren ermöglicht. Als exemplarische Marktapplikation bei der Vereinfachung der internen Prozesse zur Beschaffung von Standardprodukten seien hier elektronische Kataloge genannt, für die durch die zentrale Beschaffung der Unternehmung bereits eine Vorauswahl hinsichtlich des bestellbaren Leistungsangebotes getroffen wurde. ${ }^{239}$ Die Realisierung dieses Applikationstyps erfolgt derzeit häufig durch Intranet-Lösungen, die eine dynamische Generierung von Katalogseiten aus den in einer Datenbank abgelegten Produktinformationen ermöglichen und über Schnittstellen mit den logistischen Systemen der Unternehmung verbunden sind. ${ }^{240}$ Elektronische Kataloge reduzieren die Zahl der im Rahmen einer einzelnen Transaktion zu berücksichtigenden Alternativen und verringern dadurch den Umfang der für einen einzelnen Beschaffungsvorgang erforderlichen Suchaktivitäten. Ferner ermöglichen sie unter Beibehaltung der dezentralen Beschaffungsverantwortung bei den Bedarfsstellen eine unternehmungsweite Zusammenführung der Einzelbedarfe und schaffen dadurch ein Potenzial zur Durchsetzung niedrigerer Beschaffungspreise bei den Lieferanten. Eine zu restriktive Vorauswahl durch die zentrale Beschaffungsstelle der Unternehmung bzw. eine zu starke Einbindung dieser Stelle in den Beschaffungsprozess ist aus Sicht der Neuen Institutionenökonomie allerdings abzulehnen, da sie zu neuen AgencyProblemen zwischen der Bedarfs- und der Beschaffungsstelle führt. Die verwendete technische Lösung ist dagegen aus institutionenökonomischer Sicht weitgehend irrelevant, solange eine geeignete Produktbeschreibung die Möglichkeit zur Beurteilung der angebotenen Produkte durch die Bedarfsstelle gewährleistet.

Auch bei den unternehmungsexternen Prozessschritten führt die Mediatisierung zu einer Vereinfachung des Vorgehens. Elektronische Handelsdienste stellen diejenige Komponente elektronischer Märkte dar, die im unternehmungsübergreifenden Bereich zur Vereinfachung von Beschaffungsprozessen beiträgt. Sie erbringen verschiedenste Dienstleistungen für die Teilnehmer und können deshalb auch als Mehrwertdienste aufgefasst werden, die auf der Nutzung von Übertragungskanälen als rein technischen Komponenten Elektronischer Märkte basieren. ${ }^{241}$ In der Phase vor Vertragsschluss besteht die Leistung der Mehrwertdienste insbesondere in dem Auffinden von handelsrelevanten Informationen über die Marktteilnehmer der Markt-

\footnotetext{
${ }^{237} \mathrm{Vgl}$. Mattes (1999), S. 61.

${ }^{238}$ Vgl. Baron/Shaw/Bailey (2000), S. 387.

${ }^{239} \mathrm{Vgl}$. Mattes (1999), S. 112. Zur Funktionalität elektronischer Kataloge vgl. Baron/Shaw/Bailey (2000), S. 385ff.

${ }^{240}$ Vgl. Mattes (1999), S. 112; Lincke/Zimmermann (1999), S. $204 f$.

${ }^{241}$ Vgl. Zbornik (1996), S. 146.
} 
gegenseite und die von ihnen angebotenen Leistungen. Dies betrifft etwa Verfügbarkeitsabfragen bei einzelnen Lieferanten oder den Vergleich von Lieferkonditionen. Die durch die Einrichtung von Marktinformationsdiensten angestrebte Verbesserung der Markttransparenz orientiert sich am neoklassischen (und folglich auch institutionenökonomischen) Ideal vollkommener Märkte, ${ }^{242}$ die die ökonomisch effizienteste Herstellung und Verwendung der auszutauschenden Leistungen gewährleisten. Neben den Marktinformationsdiensten stellen sogenannte Vermittlungsdienste die zweite Kategorie von Elektronischen Handelsdiensten dar. Vermittlungsdienste erbringen ihre Funktionalität im Anschluss an die allgemeinen Suchaktivitäten zur Verbesserung des Marktüberblicks. Ihre Aufgabe besteht darin, Anbieter und Nachfrager an Hand ihrer komplementären Marktaktivitäten konkret zusammenzubringen. ${ }^{243}$

Vermittlungsdienste müssen in Abhängigkeit von der strukturellen Konfiguration des jeweiligen Elektronischen Marktes, die entweder zentral oder dezentral ausgelegt sein kann, ${ }^{244}$ unterschiedliche Funktionalitäten erbringen. Bei dezentralen Marktstrukturen ist jeder Anbieter mit jedem Nachfrager direkt verbunden. ${ }^{245}$ Ein automatisierter Ausgleich von Angebot und Nachfrage durch elektronisch abgebildete Preisbildungsmechanismen ist in diesem Fall nicht möglich. Für die Vermittlungsdienste folgt daraus, dass lediglich die Vermittlung der Produktbeschreibung sowie die Präsentation konkreter Angebote bzw. Nachfragen zu unterstützen sind. Die Abschlusspreise werden bilateral zwischen den Vertragsparteien ausgehandelt, wobei die Berücksichtigung partnerspezifischer Aspekte möglich ist. Bei zentralen Elektronischen Märkten dagegen wird die Kommunikation der Marktteilnehmer über einen zentralen Knoten vermittelt. Die Preisbildung wird bei dieser Konfiguration direkt informationstechnisch unterstützt oder sogar vollständig automatisiert. ${ }^{246}$ Bei dieser Marktstruktur ergibt sich (im Falle der automatisierten Preisbildung) die Anforderung, dass die Vermittlungsdienste eine sogenannte Matching-Funktion erfüllen müssen, d.h. dass sie übereinstimmende Angebots- und Nachfrageaufträge finden und einen automatischen Abgleich dieser Aufträge bewirken. ${ }^{247}$ Wenn auf dem betreffenden Markt homogene Güter gehandelt werden, dann erfolgt die Vermittlung von Vertragspartnern an Hand ex ante festgelegter Verhaltensregeln, die für alle Marktteilnehmer in gleicher Weise gelten. $^{248}$

Wenn auf dem betreffenden Markt jedoch nicht homogene, sondern differenzierte Güter mit verschiedenen Angebotsvarianten und heterogenen Präferenzen auf Seiten der Nachfrager gehandelt werden, dann ist der Preis nicht das alleinige Kriterium für die Auswahl des Vertragspartners. Vielmehr sind vor dem erfolgreichen zu Stande kommen eines Vertragsabschlusses die Einzelheiten der Transaktion zwischen den Beteiligten abzustimmen. Es sind also Verhandlungen über die genauen Vertrags-

\footnotetext{
${ }^{242}$ Vgl. Zbornik (1996), S. 148; Schmid (1999), S. 32; Rißmann et al. (1999), S. 142.

${ }^{243}$ Vgl. Zbornik (1999), S. 150.

${ }^{244} \mathrm{Vgl}$. Zbornik (1999), S. $61 \mathrm{ff}$

${ }^{245} \mathrm{Vgl}$. Picot/Reichwald/Wigand (1998), S. 340.

${ }^{246}$ Dies gilt auch für Elektronische Marktformen, bei denen nur auf einer Marktseite mehrere Marktteilnehmer beteiligt sind. Diese Marktformen werden z.T. auch als einzelbetriebliche Marktveranstaltungen bezeichnet. Vgl. Zbornik (1996), S. 108; Picot/Reichwald/Wigand (1998), S. 320. Derartige Marktformen erfüllen allerdings nicht vollständig die oben genannten Anforderungen an Elektronische Mărkte, die verlangen, dass auf beiden Marktseiten mehrere autonome Akteure teilnehmen.

${ }^{247} \mathrm{Vgl}$. dazu Malone/Yates/Benjamin (1987), S. 488; Alt (1997), S. 112ff.

${ }^{248}$ Vgl. Zbornik (1996), S. 150.
} 
bedingungen erforderlich, die beispielsweise die Produktkonfiguration, Lieferkonditionen oder Zahlungsmodalitäten betreffen. Elektronische Märkte boten bisher nur wenig Unterstützung für die in dieser Transaktionsphase erforderlich werdenden Aktivitäten. ${ }^{249}$ Dieser Anwendungsbereich steht jedoch im Mittelpunkt aktueller Forschungsaktivitäten, die sich unter dem Stichwort der „Kontraktoptimierung“ mit der Aushandlung von Vertragsdetails auf Elektronischen Märkten befassen. ${ }^{250}$ Die betriebswirtschaftliche Fundierung der in diesem Zusammenhang entwickelten Gestaltungsempfehlungen zur Automatisierung der entsprechenden Aktivitäten stützt sich allerdings in erster Linie auf spieltheoretische Konzepte. ${ }^{251}$ Aus der hier verfolgten Perspektive der Neuen Institutionenökonomie können dazu kaum Anhaltspunkte abgeleitet werden.

Im Gegensatz zur bisher betrachteten marktlichen Koordination steht bei der hierarchischen Koordination typischerweise kein allgemeines, weitgehend standardisiertes Vertragsmuster zur Verfügung, auf dessen Basis im Rahmen einer einzelnen Transaktion eine schnelle Einigung möglich ist. ${ }^{252}$ Unter diesen Bedingungen ist die Automatisierung der jeweiligen Verhandlungsaktivitäten ausgeschlossen. Dem Informationsmanagement bleibt dann nur die Möglichkeit, die von den Beteiligten persönlich durchgeführten Verhandlungen durch allgemeine Informations- und Kommunikationstechniken zur Unterstützung eher schlecht strukturierter, multipersoneller Aktivitäten zu erleichtern. Die als Computer Supported Cooperative Work (CSCW) bezeichnete Nutzung solcher Techniken richtet sich auf die Erleichterung der Kommunikation zwischen den Akteuren, die Koordination der Einzelaktivitäten und/oder die Verarbeitung gemeinsamer Objekte (hier z.B. Vertragsentwürfe). ${ }^{253}$ Electronic Mail-Systeme, Coautorensysteme, Sitzungsunterstützungssysteme und Videokonferenzsysteme stellen Beispiele für Techniken im CSCW-Bereich dar. Der Einsatz derartiger Anwendungssysteme, deren Funktionalitäten von Elektronischen Märkten in der Regel nicht geboten werden, kann den bei hierarchisch koordinierten Transaktionen in besonderem Maße ergebnisoffenen Prozess des Aushandelns von Vertragsdetails erleichtern. Er stellt damit einen Beitrag des Informationsmanagements zur Senkung der vor Abschluss des Vertrages entstehenden Koordinationskosten bei diesem Transaktionstyp dar.

\section{d) Strukturelle Konfiguration Elektronischer Märkte}

Es wurde bereits erwähnt, dass Elektronische Märkte sowohl zentral als auch dezentral ausgestaltet sein können. Die Auswahl einer Alternative für die strukturelle Konfiguration fällt ebenfalls in den Gestaltungsbereich des Informationsmanagements. Auch zu dieser Problemstellung sind einige Anhaltspunkte aus der Neuen Institutionenökonomie ableitbar. Zentral und dezentral strukturierte Elektronische Märkte

\footnotetext{
${ }^{249}$ Vgl. Zbornik (1996), S. 139f.; Picot/Reichwald/Wigand (2001), S. 349.

${ }^{250}$ Vgl. dazu Blanning/Bui (2000), S. 62; Peters (2000), S. $413 \mathrm{ff}$.

${ }^{251}$ Vgl. dazu Peters (2000), S. 417ff.

${ }^{252}$ Als Ausnahme von dieser Tendenzaussage seien hier Branchen-Tarifverträge genannt, die ein standardisiertes Vertragsmuster für den hierarchisch koordinierten Austausch von Arbeitsleistungen in der betreffenden Branche zur Verfügung stellen und dadurch die Aushandlung von Beschåttigungsverträgen stark vereinfachen. Typisch für spezifische Transaktionen, die über ein relationales Vertragsmuster abgewickelt werden, ist jedoch, dass vor dem zu Stande kommen einer Einigung viele Einzelheiten zwischen den Beteiligten zu klären sind und dennoch ein hoher Spielraum für nachträgliche Anpassungen des Vertrages offen bleibt.

${ }^{253}$ Vgl. Reif (2000), S. 87ff.
} 
weisen jeweils spezifische Kosten- und Nutzengefüge auf, die bei der Mediatisierung von Beschaffungsprozessen gegeneinander abzuwägen sind. Der Nutzen liegt in der Verfügbarkeit der jeweils marktstrukturspezifisch ausgestalteten Elektronischen Handelsdienste als Mehrwertdienste. Die Kosten dagegen setzen sich aus indirekten Transaktionskosten für das Vorhandensein eines Zugangs $\mathrm{zu}$ den entsprechenden Handelsdiensten und deren rein informative Inanspruchnahme sowie aus direkten Transaktionskosten, die nur bei dem erfolgreichen Abschluss eines Vertrages entstehen, ${ }^{254}$ zusammen. Dabei fallt die Kategorie der direkten Transaktionskosten im Allgemeinen nur auf Elektronischen Märkten mit zentralen Strukturen an, die von einem Marktbetreiber als Handelsmittler getragen werden.

Tendenziell ist daher davon auszugehen, dass die Kosten der Marktbenutzung auf zentralen Elektronischen Märkten höher sind als auf dezentral strukturierten Elektronischen Märkten. Die Nutzung zentral strukturierter Elektronischer Märkte im Rahmen von Beschaffungsprozessen ist vor diesem Hintergrund nur dann vorteilhaft, wenn der zusätzliche Nutzen, den die vom Marktbetreiber bereitgestellten Marktinformations- und Vermittlungsdienste stiften, die zusätzlichen Kosten für die Einschaltung dieses Intermediärs übersteigt. Da zentrale Elektronische Märkte dem Idealbild des vollkommenen Marktes am nächsten kommen, ${ }^{255}$ liegt der Nutzen der Einschaltung eines Intermediärs vor allem in einer gegenüber Elektronischen Märkten mit dezentralen Strukturen noch höheren Markttransparenz und einer noch leichter möglichen Automatisierung der Preisfindung. Eine generelle Vorteilhaftigkeit der einen oder anderen Strukturalternative kann nicht begründet werden. Im Folgenden wird jedoch die These aufgestellt, dass durch die weitere Entwicklung der Informationstechnik in diesem Bereich die Nutzenvorteile der zentralen Marktstruktur zukünttig sinken werden.

Neben den bisher erläuterten, allein auf transaktionskostentheoretische Gesichtspunkte abstellenden Überlegungen sind auch aus der Sicht der Agency-Theorie Anhaltspunkte für die Wahl der Marktstruktur ableitbar. Die Entscheidung zur Teilnahme am Marktgeschehen hat zur Folge, dass es zwischen dem Marktbetreiber und dem jeweiligen Marktteilnehmer zu einer eigenständigen Auftraggeber/AuftragnehmerBeziehung kommt. Durch die Einschaltung des Intermediärs entstehen folglich zusätzliche Agency-Probleme, aus denen zusätzliche Agency-Kosten resultieren. Dabei besteht die Gefahr, dass der Marktbetreiber Informationen, die er durch die Beobachtung des Marktverhaltens gewinnt (z.B. Informationen über die individuelle Zahlungsbereitschaft oder über individuelle Präferenzen), gegen die Interessen der Marktteilnehmer verwendet. Ferner ist denkbar, dass der Marktbetreiber die Marktergebnisse in irgendeiner Weise manipulativ beeinflusst. ${ }^{256}$ Diese Gefahren sind insbesondere dann gegeben, wenn der Marktbetreiber selbst zugleich Marktteilnehmer

\footnotetext{
${ }^{254}$ Die Betreiber Elektronischer Marktplätze vereinnahmen in der Regel eine Provision in Höhe von fünf bis zehn Prozent des Marktvolumens. Vgl. Mattes (1999), S. 78.

${ }^{255}$ Vgl. Zbornik (1996), S. 62.

${ }^{256}$ Ein fast schon klassisches Beispiel für die Einflussnahme des Marktbetreibers auf das Marktgeschehen stellen die in den 80er Jahren etablierten Flugreservienungssysteme der Linien United und American Airlines dar. Diese Systeme ließen zwar die Buchung von Flügen konkurrierender Anbieter zu, sie listeten jedoch die eigenen Angebote für die jeweils nachgefragte Strecke zuerst auf und konnten dadurch einen erheblichen Marktanteil gewinnen. Vergleichbare Manipulationen sind auf zentralen Elektronischen Märkten auch heute nicht auszuschließen.
} 
ist oder eng mit einem der Marktteilnehmer zusammenarbeitet. ${ }^{257}$ Eine Ausnutzung der Informationsvorsprünge durch den Marktbetreiber kann aus der hier eingenommenen Perspektive des Leistungsnachfragers $\mathrm{zu}$ ungerechtfertigt hohen Beschaffungspreisen oder zu Qualitätsminderungen bei den nachgefragten Leistungen führen. Der Ausschluss des Marktbetreibers vom Marktgeschehen kann diese Problematik verringern, ohne jedoch die allein durch die Einschaltung eines Intermediärs entstehenden Agency-Probleme vollständig aufzuheben.

Auf Grund der dargestellten transaktionskostentheoretischen und agency-theoretischen Überlegungen wird hier folgende These aufgestellt: Es ist davon auszugehen, dass eine dezentrale Marktstruktur effizienter ist, wenn es gelingt, eine annähernd gleiche Markttransparenz wie auf zentralen Elektronischen Märkten herzustellen. Dazu ist es erforderlich, durch entsprechende softwaretechnische Hilfsmittel die Vermittlungsdienstleistungen des Marktbetreibers zu substituieren. Das Informationsmanagement kann auf der Seite des Leistungsnachfragers zur Verwirklichung dieser Zielsetzung beitragen, indem es Anwendungssysteme für die Beschaffung bereitstellt, die unter Verzicht auf die Einschaltung einer zentralen Instanz den Vergleich von Leistungsangeboten unterschiedlicher Anbieter, die Aushandlung von Vertragsdetails sowie den Abschluss von Verträgen mit dem ausgewählten Vertragspartner zulassen. Der Aufbau einer derart umfassenden Funktionalität wird durch die Entwicklung sogenannter Intelligenter Software-Agenten zur Unterstützung marktlicher Transaktionen angestrebt.

Allgemein handelt es sich bei Intelligenten Software Agenten um Programme, die selbstständig die Auftäge menschlicher Benutzer ausführen. ${ }^{258}$ Im Zusammenhang mit der Nutzung Elektronischer Märkte können Intelligente Software Agenten auf der Basis mehr oder weniger restriktiver Angaben über die zu beschaffende Leistung Angebotsinformationen unterschiedlicher Marktteilnehmer sammeln und Einkaufstransaktionen ausführen. ${ }^{259}$ Kennzeichnende Merkmale solcher Agenten sind Autonomie, persönliche Anpassbarkeit und Kommunikationsfähigkeit bzw. Mobilität. ${ }^{260}$ Autonomie bedeutet hier die weitgehende Unabhängigkeit vom menschlichen Anwender und die selbstständige Bestimmung des Vorgehens bei der Bearbeitung des Auftrages durch den Agenten. Persönliche Anpassbarkeit heißt, dass die Software in der Lage ist, auf die speziellen Präferenzen des Nutzers entweder durch explizite Eingabe dieser Präferenzen oder durch entsprechende Techniken des maschinellen Lernens abgestimmt zu werden. Unter Kommunikationsfähigkeit bzw. Mobilität wird die Möglichkeit zur Koordination des Vorgehens mit anderen Agenten (hier: den Agenten der anderen Marktteilnehmer) verstanden. Bei anhaltender Weiterentwicklung dieser Technik zu einem verlässlichen und leicht benutzbaren Hilfsmittel, werden Intelligente Software-Agenten zukünttig in der Lage sein, ein hohes Maß an Markttransparenz herzustellen und Verträge verbindlich abzuschließen. Intelligente Software-Agenten besitzen daher auf dem Gebiet der Elektronischen Märkte das

\footnotetext{
${ }^{257}$ In diesem Fall wird von „biased markets“ (Malone/Yates/Benjamin, (1987), S. 492) bzw. von „dominierten Märkten“ (Picot/Reichwald/Wigand (1998), S. 332) gesprochen.

${ }^{258} \mathrm{Vgl}$. Tolle/Chen (2000), S. 366.

${ }^{259}$ Vgl. O'Connor/O'Keefe (2000), S. 128.

${ }^{260}$ Vgl. dazu Weld/Etzioni (1995), S. 44ff.; Tolle/Chen (2000), S. 368f. Die übrigen dort genannten Aspekte (Risk and Trust, Temporal Continuity, Domain/Expectations, Cooperation) sind weniger ausschlaggebend für die Untersuchung elektronisch unterstützter Marktaktivitäten.
} 
Potenzial, den Weg für die Dezentralisierung der Marktstrukturen freizumachen und die etablierten Intermediäre weitgehend zu verdrängen. ${ }^{261}$

Für bestimmte Transaktionen stellt die Umgehung eines zentralen Knotenpunktes für den Ausgleich von Angebot und Nachfrage allerdings kein erfolgversprechendes Szenario dar. Dies betrifft insbesondere den Austausch stark standardisierter Leistungen, die durch eine vergleichsweise kurze Haltbarkeit gekennzeichnet sind (z.B. zeitlich genau festgelegte Stromkapazitäten oder leicht verderbliche Rohstoffe). ${ }^{262}$ Für diesen Transaktionstyp ist anzunehmen, dass zentral ablaufende Auktionsverfahren, bei denen die Preisfindung zeitnah über ein sogenanntes multilaterales Matching erfolgt, den transaktionskostengünstigsten Koordinationsmechanismus darstellen. ${ }^{263}$ Entsprechende Auktionssysteme verfügen zumeist weder über elektronische Produktkataloge noch über redaktionelle Inhalte. ${ }^{264}$ Sie sind weniger auf einen umfassenden Marktüberblick für die Marktteilnehmer als auf die schnelle und effiziente Allokation der gehandelten Güter ausgerichtet. ${ }^{265}$ Eine Dezentralisierung des Marktgeschehens durch den Einsatz Intelligenter Software-Agenten im Rahmen von Beschaffungsprozessen ist bei diesem Transaktionstyp auch aus Sicht der Transaktionskostentheorie nicht sinnvoll, da für das multilaterale Matching eine zentrale Koordinationsinstanz zwingend erforderlich ist.

\subsubsection{Anwendungssysteme bei der Auswahl von Vertragspartnern: Die Sicht des Leistungsanbieters}

Auf der Absatzseite nimmt die Unternehmung bei der Durchführung von Transaktionen als Leistungsanbieter die Position des Auftragnehmers ein, der seine Leistungen potenziellen Auftraggebern anbietet und bei erfolgreichem Vertragsabschluss in deren Auftrag tätig wird. Die Gefahr der hidden characteristics, die die erste Phase von Auftraggeber/Auftragnehmer-Beziehungen dominiert, weist aus Sicht des Auftragnehmers spiegelbildliche Merkmale zur Sicht des Auftraggebers auf. Die Unterstützung dieser Vertragsphase durch das Informationsmanagement kann deshalb weitgehend analog zu den vorangegangenen, die Sicht des Leistungsnachfragers betreffenden Überlegungen untersucht werden.

In Abschnitt 5.2.3.2 wurde erläutert, dass die Agency-Theorie zur Lösung des hidden characteristics-Problems neben Screening-Aktivitäten auf der Seite des Principal auch die Offenbarung von Qualitätseigenschaften durch Signalling (oder durch die hier nicht mehr betrachteten Selbstwahlverfahren) auf der Seite des Agent vorsieht. SignallingAktivitäten verursachen wie auch Screening-Aktivitäten Transaktionskosten, die dem Wert einer erfolgreich angebahnten Transaktion gegenüberstehen. Es ist daher zu untersuchen, inwiefern das Informationsmanagement diese Transaktionskosten senken kann, indem es Signalling-Aktivitäten erleichtert und damit das Finden von Vertragspartnern auf der Absatzseite der Unternehmung unterstützt. Das Problem des

\footnotetext{
${ }^{261} \mathrm{Vgl}$. ähnlich O'Connor/O'Keefe (2000), S. 144.

${ }^{262} \mathrm{Vgl}$. Mattes (1999), S. 84.

${ }^{263}$ Vgl. dazu Alt (1997), S. 116. Die klassische Analogie zur Feststellung des Preises bei Auktionssystemen ist der Walrasianische Auktionator. Auf die unterschiedlichen Typen von Auktionsverfahren kann hier nicht năher eingegangen werden. Vgl. dazu Klein (2000), S. $628 \mathrm{ff}$.

${ }^{264}$ Vgl. Mattes (1999), S. 84.

${ }^{265}$ Vgl. Alt (1997), S. 117
} 
Agent besteht in dieser Phase aus zwei Teilen. Zum einen ist der Auftraggeber als potenzieller Vertragspartner grundsätzlich über die Existenz und Leistungsbereitschaft des Agent zu informieren. Zum anderen ist er zu überzeugen, dass die angebotene Leistung die vom Auftraggeber geforderten Qualitätsmerkmale aufweist bzw. den Leistungsangeboten der Konkurrenten überlegen ist.

\section{a) Voraussetzungen für die Nutzbarkeit von Elektronischen Märkten aus Sicht des Leistungsanbieters}

Der in Abschnitt 5.3.1.2 bereits angesprochene Electronic Communication Effect, der allgemein durch die Mediatisierung von Suchaktivitäten entsteht, kann sich auch auf der Absatzseite positiv auf die Anbahnungsaktivitäten der Unternehmung auswirken. Dazu muss das Informationsmanagement Anwendungssysteme bereitstellen, mit denen auf einfache Weise der Kontakt zu möglichst vielen potenziellen Kunden aufgebaut werden kann. Die Anbindung an Elektronische Märkte kann in diesem Zusammenhang nicht nur für die Nachfrager, sondern auch für die Anbieter zu einer Reduktion von Transaktionskosten im Vergleich zur Nutzung traditioneller Märkte führen. ${ }^{266}$ Durch den Anschluss an Elektronische Märkte gewinnt die Unternehmung Zugang zu einer Vielzahl von Nachfragern, sodass der Kreis der für den Austausch der angebotenen Leistung in Frage kommenden Vertragspartner erheblich ausgeweitet wird. Allerdings ist vorstellbar, dass die verbesserte Markttransparenz eine Stärkung der Marktmacht auf der Seite der Leistungsnachfrager und eine zunehmende Bedeutung des Preises als entscheidendes Kriterium für den Vertragsabschluss nach sich zieht. ${ }^{267}$ Aus agency-theoretischer Sicht entsteht gerade dadurch die Gefahr einer Verschärfung des Preiskampfs, der einen Prozess der adverse selection auslösen kann. Daraus ergibt sich für das Informationsmanagement (in Abhängigkeit von der Unternehmungsstrategie) ${ }^{268}$ die Anforderung, durch eine entsprechende informationstechnische Unterstützung einerseits eine Ausdehnung des potenziellen Kundenkreises zu ermöglichen und andererseits zugleich dem Druck zur Verschärfung des Preiswettbewerbs entgegenzuwirken.

Analog zum Vorgehen bei der Untersuchung der Nachfrageseite ist auch bei der Betrachtung der Angebotsseite zunächst zu überprüfen, welche Leistungen sich überhaupt für einen Austausch über Elektronische Märkte eignen. Die allgemeine Antwort der Transaktionskostentheorie, dass die marktliche Koordination für Transaktionen mit einem geringen Spezifitätsgrad die höchste Effizienz aufweist, stellt auch hier den Ausgangspunkt für die weiteren Überlegungen dar. Auf der Absatzseite besitzt diese Aussage allerdings noch weitreichendere Bedeutung als auf der Beschaffungsseite, da die Vermarktbarkeit der angebotenen Leistungen die Grundvoraussetzung für den Bestand der Unternehmung darstellt. Mit anderen Worten: Nur wenn es der Unternehmung überhaupt gelingt, Produkte mit einem geringen Spezifitätsniveau herzustellen, ist sie aus Sicht der Transaktionskostentheorie als autonomer Akteur am

\footnotetext{
${ }^{266}$ Vgl. Zbornik (1996), S. 63; Strader/Shaw (2000), S. 88.

${ }^{267}$ Vgl. Choi/Whinston (2000), S. 36; Strader/Shaw (2000), S. 83.

${ }^{268}$ Eine Verschärfung des Preiskampfs ist aus Sicht des Anbieters nur dann nicht abzulehnen, wenn die Unternehmung die Kostenfihrerschaft auf dem Markt innehat oder anstrebt. Allgemein erscheint es fraglich, ob durch eine Verlagerung des Absatzes auf Elektronische Märkte generell eine Kostenführerschaft angestrebt werden kann. Vgl. Hermanns (1999), S. 94. Im Gegenteil dürfte eine Strategie der Differenzierung zukünftig verstärkt an Bedeutung gewinnen. Vgl. dazu Strauß/Schoder (1999), S. 67f.; Figueiredo (2000), S. 44ff.
} 
Markt auf Dauer existenzfähig. Besitzen die angebotenen Produkte dagegen ein höheres Spezifitätsniveau, so ist aus Sicht der Transaktionskostentheorie eine hierarchische oder hybride Koordination des dauerhaften Leistungsaustausches vorteilhaft. Die grundlegenden Annahmen, die innerhalb der Transaktionskostentheorie zu diesen Schlussfolgerungen führen, werden durch die Mediatisierung des Marktgeschehens im Prinzip nicht beeinflusst. Die Schlussfolgerungen sind daher im Wesentlichen auch für Elektronische Märkte aufrecht zu erhalten.

Die Nutzung Elektronischer Märkte für den Absatz nicht-spezifischer Leistungen stellt für den Leistungsanbieter zunächst einmal nichts anderes als die Erschließung eines neuen Vertriebskanals dar. ${ }^{269}$ Die im Folgenden zu untersuchende Fragestellung ist, für welche Art von Produkten unter institutionenökonomischen Gesichtspunkten eine Verlagerung des Absatzes auf Elektronische Märkte besonders vorteilhaft ist und wie diese durch das Informationsmanagement gestaltet werden sollte. Bei der Beantwortung dieser Frage ist erneut eine Bezugnahme auf die Behandlung des hidden characteristics-Problem durch die Agency-Theorie hilfreich. Im Rahmen der Analyse der Nachfrageseite wurde bereits erläutert, dass die Unterscheidung zwischen Such-, Erfahrungs- und Vertrauenseigenschaften eine besondere Bedeutung für das hidden characteristics-Problem spielt. Die Nutzung Elektronischer Märkte vereinfacht für die Nachfrager das Auffinden geeigneter Vertragspartner insbesondere für solche Leistungen essenziell, bei denen für eine Beurteilung der Qualität in erster Linie Sucheigenschaften ausschlaggebend sind ${ }^{270}$ Bei diesem Transaktionstyp besitzt die Kommunikationspolitik für den Anbieter ein besonders großes Wirkungspotenzial, ${ }^{271}$ da durch entsprechende Signalling-Aktivitäten ex ante die Möglichkeit gegeben ist, die Qualität der angebotenen Leistung zu offenbaren.

Wenn die Handelbarkeit eines Produkts auf Elektronischen Märkten verbessert werden soll, ist es also erforderlich, den Anteil solcher Qualitätseigenschaften, die in die Kategorie der Sucheigenschaften einzustufen sind, zu erhöhen. Es stellt sich daher die Frage, inwiefern die ex ante-Beurteilbarkeit von Qualitätseigenschaften zum Gestaltungsgegenstand des Informationsmanagements gemacht werden kann. Ein Erreichen dieser Zielsetzung ist im Prinzip auf zwei Wegen vorstellbar. Zum einen ist Ergänzung bisheriger Leistungsbündel durch die Hinzufügung von Teilleistungen möglich, bei denen die Beschreibbarkeit auf Elektronischen Märkten gegeben ist. Dabei kann es sich beispielsweise um digitale Zusatzleistungen wie etwa individualisierte Informationsangebote oder produktbegleitende Dienstleistungen (z.B. die Sendungsverfolgung bei der Distributionslogistik) handeln, ${ }^{272}$ bei denen auch eine physische Distribution der Leistung auf elektronischem Wege möglich ist. Zum anderen ist es denkbar, die Voraussetzungen für eine Handelbarkeit des Produktes auf Elektronischen Märkten durch eine Umwandlung von Qualitätseigenschaften, die wegen ihrer besonderen Charakteristika bisher Erfahrungseigenschaften darstellten, in Sucheigenschaften

\footnotetext{
${ }^{269}$ Dies betonen auch Strader/Shaw (2000), S. 88. Eine stärkere Verknüpfung der extern orientierten Aktivitäten auf Elektronischen Märkten mit den internen Prozessen der Unternehmung, die weit über die Erschließung eines neuen Vertriebkanals hinaus das gesamte Geschäftssystem der Unternehmung betrifft, ist grundsätzlich möglich. Im Kontext der hier betrachteten Auswahl von Vertragspartnern bei der Anbahnung von Transaktionen stehen derartige Maßnahmen jedoch nicht im Zentrum des Interesses.

${ }^{270} \mathrm{Vgl}$. dazu die Ausfuihrungen in Abschnitt 5.3.1.2.

${ }^{271}$ Vgl. Neus (1998), S. 238.

${ }^{272}$ Vgl. Hermanns (1999), S. 94.
} 
herzustellen. Zu diesem Zweck müssten die entsprechenden Eigenschaften bereits vor dem Abschluss des Vertrages „erfahrbar" gemacht werden. Vor diesem Hintergrund entstehen auf Elektronischen Märkten neue Anwendungsfelder für Techniken der Virtual Reality, für die sich im Internet der Standard VRML (Virtual Reality Markup Language) herausgebildet hat. ${ }^{273}$ Durch den Einsatz von Virtual Reality-Techniken können die Erfahrungseigenschaften eines Produktes simuliert werden, ohne dass es erforderlich ist, das Produkt tatsächlich auszutauschen. Da die Nachfrager dadurch in die Lage versetzt werden, die betreffenden Eigenschaften zu überprüfen, bevor sie sich zum Abschluss des Kaufvertrags entscheiden, findet eine Umwandlung von Erfahrungs- in Sucheigenschaften statt.

Wenn trotz der erweiterten Möglichkeiten zur Leistungsbeschreibung ex ante keine vollständige Offenbarung der Leistungsqualität möglich ist, dann bleiben Erfahrungseigenschaften für die Beurteilung der Leistung bedeutend. Bei Folgekäufen ist die Qualität jedoch bekannt, sofern der Kunde als Principal nicht den Wechsel zu einem anderen Produkt bzw. Anbieter vorzieht. ${ }^{274}$ Wenn Leistungen dieses Qualitätstyps wiederholt gehandelt werden und das Preis/Leistungsverhältnis der bisher gelieferten Leistungen den Ansprüchen des Nachfragers entsprach, kann es deshalb für den Principal vorteilhaft sein, den Leistungsgeber nicht $\mathrm{zu}$ wechseln. Durch dieses Verhalten werden Suchkosten reduziert, da bei der Wiederholung der Transaktion der Vertragspartner und die von ihm angebotene Leistung bereits bekannt sind. ${ }^{275}$ Diese Situation bietet dem in diesem Abschnitt betrachteten Leistungsanbieter als Agent die Chance, eine Beziehung zum Nachfrager aufzubauen, die den Nachfrager vom Wechsel des Vertragspartners abhält. Als geeignetes Mittel dazu wird in der Literatur die Individualisierung der angebotenen Leistung vorgeschlagen. ${ }^{276}$ Das Informationsmanagement kann diese Zielsetzung vor Vertragsschluss durch die Bereitstellung von Anwendungssystemen unterstützen, die es ermöglichen, das Angebot entsprechend den jeweiligen Nachfragerpräferenzen zu verbessern und damit den vom Nachfrager empfundenen Nutzen zu erhöhen. Damit würde auch die Gefahr von Preiskämpfen, bei denen die Kaufentscheidung des Kunden sich allein am Preis orientiert, weitgehend entschärt und somit das Risiko der adverse selection reduziert. Grundsätzlich sind zwei Alternativen für die informationstechnische Unterstützung vorstellbar. Einerseits kann eine individuelle Konfiguration der Leistung durch den Kunden selbst erfolgen und andererseits kann der Leistungsanbieter auf der Basis von ihm bekannten Präferenzen des einzelnen Kunden eine Individualisierung des Angebotes vornehmen. Im Folgenden wird näher untersucht, welche Funktionalität derartige Systeme aus Sicht der Neuen Institutionenökonomie aufweisen sollten.

\footnotetext{
${ }^{273}$ Vgl. Shaw (2000), S. 9; Submaraniam/Shaw/Gardner (2000), S. 166.

${ }^{274}$ Vgl. Neus (1998), S. 239.

${ }^{275}$ Diese Überlegungen machen deutlich, dass die Einordnung einer Qualitătseigenschaft als Suchoder Erfahrungseigenschaft nicht absolut ist, sondern vom subjektiven Kenntnisstand des einzelnen abhängt.

${ }^{276}$ Vgl. z.B. Engelhardt/Gersch (1995) S. 201ff.; Hildebrand (1997), passim; Reichwald/Piller (2000), S. 359ff.; Piller/Zanner (2001), S. 88.
} 


\section{b) Anforderungen an die Funktionalität Elektronischer Märkte aus Sicht des Leistungsanbieters}

Das Problem der Bekanntmachung des Produktangebotes ${ }^{277}$ stellt sich bei der Verlagerung des Vertriebsgeschehens auf Elektronische Märkte nur dann, wenn eine dezentrale Struktur des Elektronischen Marktes mit direkter Verbindung zwischen Anbietern und Nachfragern vorliegt, wie sie beispielsweise beim direkten Vertrieb ohne Handelsmittler über das Internet gegeben ist. Im Gegensatz dazu kann auf Grund der Besonderheiten des Koordinationsprozesses auf zentral strukturierten Elektronischen Märkten, auf denen eine automatische Preisfindung etwa im Rahmen eines Auktionsverfahrens stattfindet, die Wahrnehmbarkeit des eigenen Angebotes für alle potenziellen Kunden als gegeben gesehen werden. In Abschnitt 5.3.1.2 wurde aus Sicht des Leistungsnehmers bereits erläutert, dass unter institutionenökonomischen Gesichtspunkten eine zentrale Marktkonfiguration nur bei besonders stark standardisierten Produkten mit vergleichsweise kurzer Haltbarkeit vorteilhaft ist. Auch aus Sicht des Leistungsanbieters besteht ein Effizienzvorteil zentraler Elektronischer Märkte in erster Linie in den Fällen, in denen eine Preis/Mengen-Strategie zur Absetzung stark standardisierter Produkte, bei denen Sucheigenschaften für die Qualitätsbeurteilung entscheidend sind, verfolgt wird. Dagegen ist auf Märkten mit dezentraler Struktur für den Anbieter eher die Möglichkeit zur Differenzierung gegeben, da zum einen die Präsentation der Leistungsqualität sowie das Angebot von Zusatzleistungen leichter möglich sind und zum anderen die Markttransparenz für die Nachfrager tendenziell geringer ${ }^{278}$ ist. Die weiteren Ausführungen gehen daher von einer dezentralen Konfiguration des Marktes aus und betreffen die Anbahnung von Transaktionen mit Hilfe von Marktapplikationen und Elektronischen Handelsdiensten aus Sicht des Leistungsanbieters.

Zur Bekanntmachung der eigenen Unternehmung und ihres Angebotes können auf dezentralen Elektronischen Märken (insbesondere im Internet) im Prinzip alle gängigen Instrumente der Kommunikationspolitik in mediengerechter Form eingesetzt werden: Dazu zählen beispielsweise Werbung, Public Relations, Verkaufsforderung, Messebeteiligungen (bei virtuellen Messen), Sponsoring, Placement, insbesondere Information Placement, Kundenclubs, (Internet-)Events und Product Publicity. ${ }^{279}$ Betrachtet man von diesen Werbeinstrumenten diejenigen genauer, die einen konkreten Bezug zur Anbahnungsphase einer einzelnen Transaktion haben, so ist zwischen Pushund Pull-Kommunikation zu unterscheiden ${ }^{280}$ Die Pull-Kommunikation wird durch den Kunden selbst angestoßen, der sich entscheiden kann, ob er an den angebotenen Informationen interessiert ist oder nicht. Darunter fallt im Internet hauptsächlich die Platzierung von Links zur eigenen Internet-Site in Form von Bannerwerbung, Buttons,

\footnotetext{
${ }^{277}$ Die in diesem Abschnitt angesprochenen Möglichkeiten stellen eine Ergänzung der traditionellen Kommunikationskanäle zur Bekanntmachung des eigenen Angebotes dar. Sie erweitern damit das Handlungsrepertoire des Anbieters in der Anbahnungsphase, ohne jedoch die bisherigen Kommunikationsmechanismen zu verdrängen.

${ }^{278}$ Wie im vorigen Abschnitt erläutert kann auch auf dezentralen Elektronischen Märkten durch den Einsatz Intelligenter Software-Agenten eine hohe Markttransparenz für die Nachfrager hergestellt werden. Wenn die Leistungsanbieter allerdings eine Differenzierungsstrategie verfolgen, steht den Suchaktivitäten der Nachfrager eine sinkende Vergleichbarkeit der einzelnen Angebote entgegen.

${ }^{279}$ Vgl. dazu Hermanns (1999), S. 94.

${ }^{280}$ Vgl. Submaraniam/Shaw/Gardner (2000), S. 154.
} 
Advertorials, u.ä. ${ }^{281}$ auf anderen Internet-Seiten. Auch das Erscheinen des eigenen Angebotes in der Ergebnisliste von Suchmaschinen kann eine Pull-Kommunikation durch den Kunden auslösen. Bei der Push-Kommunikation wird der Übertragungsprozess dagegen durch den Absender gestartet. Dazu gehören insbesondere die direkte Ansprache des potenziellen Vertragspartners durch E-MailNewsletters sowie durch sogenannte „Webcasting"-Systeme, die sich im Internet etabliert haben. ${ }^{282}$ Wenn die Anbahnung einer Transaktion auf dem Wege der Ausschreibung erfolgt, nimmt die anbietende Unternehmung zwar zunächst eine passive Rolle ein, indem sie auf Initiative des Nachfragers über die Ausschreibung unterrichtet wird. ${ }^{283}$ Bei diesem Transaktionstyp kann es jedoch ebenfalls erforderlich sein, informationstechnische Unterstützungskonzepte zu entwickeln, die (z.B. mit Hilfe von Intelligenten Software-Agenten, deren Grundkonzept bereits im vorangegangenen Abschnitt beschrieben wurde) die Kenntnisnahme neu initiierter Ausschreibungsverfahren gewährleisten.

Nachdem die Aufmerksamkeit des Kunden gewonnen wurde, besteht die nächste Aufgabe darin, das eigene Angebot möglichst einfach zugänglich zu machen und überzeugend zu präsentieren, um in das Evoked Set von Alternativen des Kunden aufgenommen zu werden. Die Präsentation des Angebots erfolgt in sogenannten „Virtual Storefronts“, die auf eine bequeme und effiziente Unterstützung von Anbahnungsaktivitäten (des Kunden) innerhalb des eigenen Angebotes ausgerichtet sind. ${ }^{284}$ In Virtual Storefronts sind die Sucheigenschaften der angebotenen Leistung möglichst präzise zu beschreiben, um das hidden characteristics-Problem für den Auftraggeber so weit wie möglich zu reduzieren. Der Einsatz multimedialer Informationstechnik kann dabei zur Vervollkommnung der Produktbeschreibung beitragen und die Erfolgschancen der Vertragsanbahnung vergrößern. ${ }^{285}$ Mit steigendem Umfang der multimedialen Produktbeschreibung (z.B. durch Hinzufügung von Bewegtbild- oder Virtual Reality-Präsentationen) wachsen allerdings die Ansprüche an die Breite des Übertragungskanals. Das Vorhandensein einer entsprechenden kommunikationstechnischen Infrastruktur stellt deshalb die Voraussetzung für eine derartige Ausdehnung der informationstechnisch unterstützten Produktbeschreibung dar.

Neben der reinen Präsentation, die die Bekanntmachung des Angebotes für den Principal auf dezentralen Elektronischen Märkten unterstützt und damit die Sucheigenschaften der Leistung fokussiert, bieten Virtual Storefronts noch weitere Funktionalitäten. Diese sind verstärkt auf eine Individualisierung des Angebotes gerichtet. Sie ermöglichen damit den Aufbau einer Beziehung zum Kunden, die, wie im vorigen Abschnitt aus Sicht der Agency-Theorie erläutert, die Effizienz eines wiederholten Austausches von solchen Leistungen erhöht, deren Qualitätsbeurteilung in erster Linie von Erfahrungseigenschaften ${ }^{286}$ abhängt. Transaktionskostentheore-

\footnotetext{
${ }^{281} \mathrm{Zu}$ den verschiedenen Varianten in diesem Zusammenhang vgl. z.B. Submaraniam/Shaw/Gardner (2000), S. 154f.

${ }^{282} \mathrm{Vgl}$. dazu Submaraniam/Shaw/Gardner (2000), S. 156

${ }^{283}$ Dies kann beispielsweise über entsprechende Online-Datenbank- und Informationssysteme erfolgen, mit denen Ausschreibende die Bieter schnell und effizient über neue Vorhaben informieren, vgl. Mattes (1999), S. $62 \mathrm{f}$.

${ }^{284}$ Vgl. dazu Shaw (2000), S. 8f.; Submaraniam/Shaw/Gardner (2000), S. 158.

${ }^{285}$ Vgl. Zbornik (1996), S. 118.

${ }^{286}$ Eine Individualisierung der Leistung mit dem Ziel einer höheren Kundenbindung ist grundsătzlich auch für Produkte möglich, deren Qualitätsbeurteilung von Sucheigenschaften abhängt. Die
} 
tisch gesehen wird mit einer derartigen Individualisierung des Leistungsbündels die Idee verbunden, die Spezifität der Transaktion aus Anbieter- und aus Nachfragersicht zu entkoppeln. Dadurch soll es für den Anbieter möglich werden, nachfragerspezifische Leistungen $\mathrm{zu}$ erbringen und dabei nachfragerunspezifische Leistungserstellungsprozesse einzusetzen. ${ }^{287}$ Auf diese Weise entstehen Anreize gegen einen Anbieterwechsel durch den Nachfrager. Da für den Anbieter der Abschluss von Verträgen mit einer Vielzahl unterschiedlicher Vertragspartner möglich bleibt, wird zugleich die üblicherweise für die Hersteller spezifischer Leistungen bestehende Gefahr reduziert, dass der Nachfrager seine starke Machtposition opportunistisch ausiuutzt.

Diejenigen Funktionalitäten von Virtual Storefronts, die zur Individualisierung des Angebots beitragen können, werden im Folgenden vor dem Hintergrund der Besonderheiten, die die Anbahnung von Transaktionen dieses Typs auf Elektronischen Märkten kennzeichnen, näher betrachtet. Dazu gehören insbesondere Suchdienste, Konfigurationsdienste und Katalogdienste. ${ }^{288}$

Die Suchfunktionalität gibt den Nachfragern die Möglichkeit, spezielle Detailinformationen über die auszutauschende Leistung zu erhalten. Sie erleichtert dadurch besonders bei einem heterogenen, komplizierten und/oder erklärungsbedürftigen Produktangebot die Vertragsanbahnung. Leistungen mit diesen Beschreibungsmerkmalen sind dadurch gekennzeichnet, dass eine vollständige Präsentation des Angebotes sehr umfangreich werden kann, wenn sie detaillierte Erklärungen zu einzelnen Teileigenschaften und Beschreibungen der verschiedenen Produktvarianten beinhalten soll. In der Anbahnungsphase für Transaktionen dieser Art findet typischerweise ein ausführlicher Informationsprozess über das einzelne Angebot auf der Seite des Kunden statt. Dabei besitzen die verschiedenen Kunden als potenzielle Vertragspartner nicht nur zu Beginn des Anbahnungsprozesses unterschiedliche Kenntnisse, sondern auch unterschiedliche Interessen und fragen daher unterschiedliche Informationen nach. Der Suchdienst einer Virtual Storefront versetzt die Kunden in die Lage, die von ihnen gewünschte Information gezielt nachzufragen. Dadurch wird eine Individualisierung des Suchprozesses möglich, die die Effizienz der Vertragsanbahnung für den einzelnen Nachfrager deutlich erhöhen kann.

Bei Leistungen dieses Typs sehen sich die Agents jedoch nicht nur individuellen Suchprozessen der Principals gegenüber, sondern auch die letztlich durch die Kunden entfaltete Nachfrage weist im Regelfall ein hohes Heterogenitätsniveau auf. Daher wird die Effizienz des Leistungsaustausches bei diesem Transaktionstyp nicht nur durch eine Individualisierung des Suchprozesses, sondern auch durch eine kundenspezifische Anpassung des Leistungsangebotes erhöht. ${ }^{289}$ Virtual Storefronts können zu einer derartigen Individualisierung beitragen, indem sie eine Konfigurationsfunktionalität

Vorteilhaftigkeit einer Beibehaltung des Vertragspartners bei wiederholten Leistungstauschen ist jedoch agency-theoretisch besonders für Güter mit hoher Bedeutung von Erfahrungseigenschaften begründbar.

${ }^{287}$ Vgl. dazu Mayer (1993), S. 153; Gersch (1998), S. 130ff.

${ }^{288}$ Vgl. dazu Submaraniam/Shaw/Gardner (2000), S. 157.

${ }^{289}$ Ein vollständiger Überblick über Möglichkeiten und Grenzen einer kundenspezifischen Individualisierung der angebotenen Leistung, die derzeit unter den Stichworten One-to-One Marketing und Mass Customization erhöhte Aufmerksamkeit finden, kann hier nicht gegeben werden. Vgl. dazu z.B. Piller (2001), S. 200ff.; Gilmore/Pine (2000), S. 7ff.; Wolf/Scherer (1999), S. 19ff. Stattdessen erfolgt hier eine Beschränkung auf die informationstechnischen Aspekte dieser Problemstellung. 
anbieten, die dem Kunden die Möglichkeit gibt, die nachgefragte Leistung speziell auf seine individuellen Präferenzen abzustimmen. ${ }^{290}$ Dazu gehört nicht nur die Auswahl von Leistungsmerkmalen, die im Prinzip (allerdings bei höheren Suchkosten) auch über traditionelle Vertriebskanäle erhältlich sind, sondern auch die Anreicherung des Angebotes mit informationellen Zusatzleistungen, die erst durch die Mediatisierung des Leistungsaustausches ermöglicht wird. Dies betrifft in erster Linie begleitende, informationelle Leistungen, die mit Hilfe von kundenindividuellen Zugriffen auf größere Datenbestände (z.B. transaktionsspezifische Auftragsdaten) ermöglicht werden. ${ }^{291}$ Wenn die hier im Vordergrund stehenden Erfahrungseigenschaften Gegenstand der Leistungsindividualisierung sind, dann kann die Konfigurationsfunktionalität einer Virtual Storefront Folgetransaktionen fördern und somit zum Aufbau einer längerfristigen Kundenbeziehung beitragen.

Allerdings ist der Konfigurationsdienst einer Virtual Storefront dadurch gekennzeichnet, dass die anbietende Unternehmung als Auftragnehmer im Vorfeld einer konkreten Transaktion mehr oder weniger passiv bleibt und dem Kunden als Auftraggeber die Individualisierung der Leistung weitgehend überlässt. Noch einen Schritt weiter gehen Bemühungen, durch Initiative des Anbieters die Präferenzen des Nachfragers aktiv zu erkennen und das kundenindividuelle Angebot diesen Präferenzen entsprechend anzupassen. Vor diesem Hintergrund sollten Virtual Storefronts über eine so genannte Katalogfunktionalität verfügen, die in der Lage ist, eine auf den einzelnen Kunden abgestimmte, flexible Präsentation des Angebotes vorzunehmen. ${ }^{292}$ Zur Umsetzung einer aktiven Abgabe individualisierter Angebote ist allerdings mehr als nur der Einsatz eines für diese Zwecke geeigneten Katalogmoduls als Teil einer Virtual Storefront notwendig.

Vielmehr erfordert die Abgabe individuell angepasster Leistungsangebote die Wiedererkennung des Kunden und die Kenntnis der speziellen Kundenwünsche. Der bereits in Abschnitt 5.3.1.2 angesprochene Electronic Brokerage Effekt, der die Intermediationsfunktion der Informationstechnik bei der Verlagerung von Anbahnungsaktivitäten auf Elektronische Märkte beschreibt, führt zu einer Intensivierung des direkten Kundenkontaktes und erfüllt damit eine wichtige Voraussetzung für eine aktive Individualisierung des Angebotes. Elektronische Märkte bieten in diesem Zusammenhang vielfältige Möglichkeiten, Informationen über die Kunden und ihre jeweiligen Präferenzen $\mathrm{zu}$ erfassen, zusammenzutragen und davon ausgehend Kundenprofile zu erstellen. ${ }^{293}$ Es sei allerdings darauf hingewiesen, dass Vorschriften im Bereich des Datenschutzes eine Realisierung des technisch Machbaren einschränken können. Die Datenschutzproblematik wird in dieser Arbeit jedoch nicht näher betrachtet.

$\mathrm{Zu}$ den Ansatzpunkten gehören z.B. die Identifizierung des Kunden durch die Einrichtung von Anmeldeverfahren und die Verwendung so genannter „Cookies“, die

\footnotetext{
${ }^{290} \mathrm{Vgl}$. Submaraniam/Shaw/Gardner (2000), S. 157. Wenn für die Individualisierung eine ausgeprägte Interaktion zwischen Anbieter und Nachfrager erforderlich ist, dann besitzt die Konfiguration durch den Kunden vorbereitenden Charakter. Die im direkten Kontakt vorgenommene, endgültige Anpassung des Angebots wird dadurch effizienter. Vgl. Piller/Zanner (2001), S. 91.

${ }^{291}$ Vgl. ähnlich Hermanns (1999), S. 94; Choi/Whinston (2000), S. 41.

${ }^{292}$ Vgl. ăhnlich Submaraniam/Shaw/Gardner (2000), S. 157.

${ }^{293}$ Vgl. Sauter (1999), S. 113 ; Piller/Zanner (2001), S. 89.
} 
Erfassung und Speicherung des Informationsverhaltens in Log-Dateien ${ }^{294}$ und die Analyse der erhobenen Daten mit Hilfe von Data Mining-Verfahren ${ }^{295}$. Ein noch weitergehendes Gesamtkonzept zur Erhebung und Nutzung von Informationen über individuelle Kundenpräferenzen kann zudem weit über die auf Elektronischen Märkten gewonnenen Daten hinausgehen. Im Rahmen eines umfassenden Customer Relationship Managements sind weitere Erkenntnisse zu berücksichtigen, die an verschiedenen weiteren Kontaktpunkten mit dem Kunden (z.B. im Rechnungswesen oder im Logistikbereich) entstehen. ${ }^{296}$ Dies läuft auf den Aufbau eines unter-

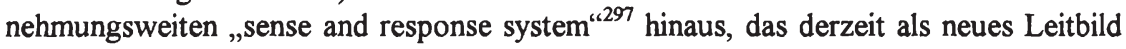
der Gestaltung des technikunterstützten Informationssystems der Unternehmung diskutiert wird.

Die bisher in diesem Abschnitt erläuterten Anwendungen zur Unterstützung der Anbahnung von Verträgen fokussieren den Austausch von Leistungen mit unternehmungsexternen Transaktionspartnern und sind in die Kategorie der Marktapplikationen und Elektronischen Handelsdienste auf dezentralen Elektronischen Märkten einzuordnen. Abschließend sei noch darauf hingewiesen, dass diese Aktivitäten auf der Seite des Anbieters mit der unternehmungsinternen Informationsverarbeitung zu verknüpfen sind. Eine reibungslose Ausführung der durch die Kunden abgegebenen Aufträge erfordert die Weiterverarbeitung der auf Elektronischen Märkten angenommenen Bestellungen durch die unternehmungsintern orientierten betriebswirtschaftlichen Anwendungssysteme (ERP-Systeme) der Unternehmung. Diese Problemstellung betriff in jedem Fall technische Aspekte, sie kann allerdings auch auf betriebswirtschaftliche Fragen ausgedehnt werden. Dann ist zu untersuchen, wie das gesamte Geschäft der Unternehmung zu verändern ist, um die potenziellen Vorteile der Mediatisierung der Koordination von Transaktionen in möglichst großem Umfang auszunutzen. ${ }^{298}$ Dabei geht es jedoch um die Erfüllung der vertraglichen Verpflichtungen. Diese Problemstellung hat nicht unmittelbar mit dem hier betrachteten Problem der Auswahl eines geeigneten Transaktionspartners und den Möglichkeiten zur Unterstützung von Signalling-Aktivitäten vor Vertragsschluss zu tun.

Ferner ist darauf hinzuweisen, dass die technikunterstützte Informationsverarbeitung bei der Abgabe individualisierter Leistungsangebote als Gestaltungsgegenstand des Informationsmanagements nur einen Teilaspekt der Gesamtproblemstellung darstellt. Der Aufbau und die Pflege von Kundenbeziehungen für den Austausch komplexer Leistungen mit hoher Bedeutung von Erfahrungseigenschaften betreffen nicht nur die hier betrachteten Aktivitäten vor Vertragsschluss, sondern Aktivitäten in allen Transaktionsphasen. Zudem gehen sie weit über informationstechnische Konzepte hinaus. So hängt die Möglichkeit zur Individualisierung von Leistungen nicht nur von informationstechnischen, sondern beispielsweise auch von (hier nicht näher betrachteten) logistischen und produktionstechnischen Fähigkeiten ab. ${ }^{299}$ Sie erfordert

\footnotetext{
${ }^{294}$ Vgl. Gluchowski/Müller (2000), S. 18ff.

${ }^{295}$ Zum Konzept des Data Mining vgl. z.B. Bissantz/Hagedorn/Mertens (1998), S. 445ff.

${ }^{296}$ Das „Customer Relationship Management" wird derzeit in Theorie und Praxis intensiv diskutiert und beinhaltet neben informationstechnischen Aspekten grundsätzliche Probleme, die den Aufbau und die Pflege von Geschäftsbeziehungen betreffen. Vgl. dazu z.B. Piller/Zanner (2001), S. 89; Hettich/Hippner/Wilde (2000), S. 136ff.

${ }^{297}$ Shaw (2000), S. 16.

${ }^{298}$ Vgl. Mattes (1999), S. 29.

${ }^{299}$ Vgl. Piller/Zanner (2001), S. 89.
} 
außerdem eine damit einhergehende Individualisierung der Preis- und Kontrahierungspolitik. ${ }^{300}$ Den in diesem Abschnitt beschriebenen Anwendungsmöglichkeiten der Informationsverarbeitung auf Elektronischen Märkten kommt dabei die Rolle eines „Enabler" $\mathrm{zu}^{301}$ der die Unternehmung dem Ideal eines Dialogs mit jedem einzelnen Kunden als Voraussetzung für die individuelle Befriedigung seiner speziellen Bedürfnisse ein erhebliches Stück näher bringt. ${ }^{302}$

\subsubsection{Informationsmanagement nach Vertragsschluss}

Nachdem die Vertragspartner eine Einigung über die tatsächliche Durchführung der Transaktion erzielt und den Vertrag geschlossen haben, kann die betreffende Leistung ausgetauscht werden. Die nachfolgenden Überlegungen richten sich auf Anforderungen, die aus Sicht der Neuen Institutionenökonomie an die durch das Informationsmanagement gestaltete Funktionalität der Anwendungssysteme in dieser Transaktionsphase zu stellen sind. Nach einer grundsätzlichen Beschreibung der Informationsverarbeitung nach Vertragsschluss (Abschnitt 5.3.3.1) folgt eine Betrachtung transaktionsorientierter (Abschnitt 5.3.3.2) und analyseorientierter (Abschnitt 5.3.3.3) Informationssysteme bei der Erfullung der vertraglich vereinbarten Verpflichtungen.

\subsubsection{Charakterisierung der Informationsverarbeitung nach Vertragsschluss}

In der Phase nach Abschluss des Vertrages erfullen die Vertragsparteien ihre vertraglich vereinbarten Verpflichtungen. Je nach Art der übergebenen Leistung ist dazu eine mehr oder weniger genaue Abstimmung der Aktivitäten der Beteiligten erforderlich. Ferner findet nach der Lieferung eine Kontrolle der Qualität durch den Auftraggeber statt. Für das Informationsmanagement geht es in diesem Zusammenhang darum, durch den Einsatz geeigneter Anwendungssysteme eine möglichst hohe Effizienz der Vertragsabwicklung zu gewährleisten und die Kontrolle des Auftragnehmers zu unterstützen. Die genaue Ausgestaltung der technikunterstützten Informationsverarbeitung in dieser Vertragsphase hängt von der gewählten Koordinationsform $a b$.

Beim marktlich koordinierten Austausch von solchen Produkten, bei denen eine Einbeziehung externer Faktoren in den Leistungserstellungsprozess unbedeutend ist, kann vom rein klassischen Vertragsmuster ausgegangen werden. Dieses zeichnet sich dadurch aus, dass Leistung und Gegenleistung unmittelbar nach Vertragsschluss erfolgen und als zeitlich zusammenfallend aufgefasst werden können. ${ }^{303}$ Die Realisierungsphase ist typischerweise sehr kurz und verursacht kaum Transaktionskosten. Die Informationsverarbeitung spielt dabei nur insoweit eine Rolle, als die Distribution von (digitalen) Leistungsbestandteilen auf Elektronischem Wege abgewickelt werden kann. Eine aufwändige Koordination der Aktivitäten von Leistungsgeber und Leistungsnehmer, die im Mittelpunkt institutionenökonomischer Analysen steht, ist nach Vertragsschluss normalerweise nicht mehr erforderlich. Bei dieser Koordinationsform

\footnotetext{
${ }^{300} \mathrm{Vgl}$. Choi/Whinston (2000), S. 42.

${ }^{301}$ Vgl. Sauter (1999), S. 112 sowie die dort zitierten Quellen.

${ }^{302}$ Vgl. ähnlich Submaraniam/Shaw/Gardner (2000), S. 166.

${ }^{303} \mathrm{Vgl}$. Picot/Reichwald/Wigand (1998), S. 51.
} 
ist keine besondere Möglichkeit zur Überwachung des Auftragnehmerverhaltens gegeben, sodass sich die Kontrolle allein auf das Leistungsergebnis beziehen kann. Da es sich bei der Überprüfung des Leistungsergebnisses im Kern nicht um ein Problem der technikunterstützten Informationsverarbeitung handelt, wird die rein marktliche Koordinationsform im Folgenden nicht näher betrachtet.

Charakteristisch für integrativ erstellte Leistungen ist dagegen der Umstand, dass die eigentliche Leistungserstellung erst nach dem Zustandekommen eines konkreten Vertrages erfolgt. ${ }^{304}$ Der Leistungsgeber integriert bei der Erstellung der Leistung externe Faktoren, die entweder Gegenstand der Leistungserstellungsprozesse sind oder dispositiven Charakter haben, also der Abstimmung und Koordination der Aktivitäten der Vertragspartner dienen. ${ }^{305}$ Die Realisierung der Transaktion weicht unter diesen Umständen vom rein klassischen Vertragsmuster $a b$, da ein von Null verschiedener Zeitraum zur Erfüllung des Vertrages angenommen werden muss. Bei diesem Leistungstyp ist folglich stets von einer nicht vernachlässigbaren Notwendigkeit zur Koordination von Aktivitäten nach Vertragsschluss auszugehen, deren Effizienz durch geeignete informationstechnische Lösungen erhöht werden kann. Ferner ist auch hier die Übergabe von Prozessergebnissen auf elektronischem Wege vorstellbar. Die relative Bedeutung der Realisierungsphase ist daher im Vergleich zum oben betrachteten Fall des „spot contracting“ weitaus höher. Wenn die Leistungserstellung integrativ erfolgt, bietet sich dem Informationsmanagement also auch bei eher marktlich koordinierten Transaktionen die Möglichkeit zur Einflussnahme auf die ex post anfallenden Transaktionskosten.

Die größte relative Bedeutung besitzt die Phase nach Vertragsschluss im Rahmen hierarchisch koordinierter Transaktionen. Wie in Abschnitt 5.3.1.1 bereits angesprochen, findet mit dem Abschluss des Vertrages eine fundamentale Transformation der Beziehung zwischen den Vertragspartnern statt, die ex post die Züge eines bilateralen Monopols aufweist. Bei der Regelung durch relationale Verträge sind zwar vergleichsweise umfangreiche Verhandlungsaktivitäten zur anfänglichen Festlegung der Vertragskonditionen erforderlich. $\mathrm{Da}$ die Vertragspartner jedoch eine längerfristige vertragliche Bindung eingehen, kommt es bei Transaktionsvorgängen, die nach dem Abschluss der ursprünglichen Vereinbarung stattfinden, zu einem Verschwinden der Such- und Verhandlungsphase. ${ }^{306}$ Statt auf dem Wege der Vertragspartnersuche und der Aushandlung von Einzelverträgen erfolgt die Koordination der Aktivitäten zur Vertragserfüllung über Anweisungen. ${ }^{307}$ Die Legitimation zur Erteilung solcher Anweisungen ist Inhalt des zwischen den Transaktionspartnern geschlossenen relationalen Vertragsarrangements. Generell ist davon auszugehen, dass die Bedeutung der Ex post-Aktivitäten für die erfolgreiche Abwicklung der Transaktion um so höher ist, je stärker vom klassischen Vertragsmuster abgewichen wird. Die nach Vertragsschluss bestehenden Einflussmöglichkeiten des Informationsmanagements zur Reduzierung von Transaktionskosten wachsen also mit zunehmendem Anteil hierarchischer Koordinationselemente innerhalb des vertraglichen Arrangements.

\footnotetext{
${ }^{304}$ Vgl. Gersch (1998), S. 210.

${ }^{305}$ Vgl. Gersch (1998), S. 210.

${ }^{306}$ Vgl. ähnlich Nenninger/Gerst (1999), S. 289.

${ }^{307} \mathrm{Vgl}$. Malone/Yates/Benjamin (1987), S. 485.
} 
Anwendungssysteme, die die Realisierung einer Transaktion unterstützen und dabei von der eine hierarchische Koordination kennzeichnenden Anweisungs- und Lenkungsfunktion einer übergeordneten Instanz ausgehen, werden in der Literatur im Allgemeinen als Elektronische Hierarchien bezeichnet. ${ }^{308}$ Unter diesen Begriff werden sowohl konventionelle betriebliche Anwendungssysteme zur unternehmungsinternen Koordination der Leistungserstellung als auch unternehmungsübergreifende Anwendungssysteme zur Abstimmung der Aktivitäten formal selbstständiger Unternehmungen gefasst. ${ }^{309}$ Die Ausdehnung der Begriffsverwendung auf zwischenbetriebliche Transaktionsvorgänge geht auf MALONE/YATES/BENJAMIN zurück. Diese sprechen dann von (Elektronischen) Hierarchien, wenn im Rahmen eines einzelnen Tauschvorgangs nicht mehr zwischen alternativ möglichen Vertragspartnern gewählt wird. ${ }^{310}$ Eine derart weite Begriffsfassung ist insofern gerechtfertigt, als bei allen Vertragsverhältnissen, die vom puren klassischen Vertragsmuster mit dem Zusammenfall von Leistung und Gegenleistung abweichen, zu einem gewissen Teil auch hierarchische Koordinationselemente verwendet werden. Die Möglichkeit zur engeren Verknüpfung der technikunterstützten Informationssysteme getrennter Unternehmungen mit Hilfe moderner Informations- und Kommunikationstechnik wird als Electronic Integration Effect bezeichnet. Der Electronic Integration Effect ergänzt die beiden oben angesprochenen Effekte, also den Electronic Communication Effect und den Electronic Brokerage Effect. Elektronische Hierarchien können sowohl operative als auch analytische Funktionalitäten bereitstellen. ${ }^{311}$

Eine überschneidungsfreie Abgrenzung zwischen Elektronischen Märkten und Elektronischen Hierarchien lässt sich allerdings nur theoretisch vornehmen. Diese Abgrenzung würde erfordern, dass Elektronische Märkte ausschließlich die mit Hilfe rein klassischer Vertragsarrangements abgewickelte Form des Leistungsaustausches ohne Ex post-Aktivitäten unterstützen, während der Anwendungsbereich Elektronischer Hierarchien allein in der Koordination auf Dauer angelegter, relationaler Vertragsverhältnisse liegt. In der Praxis ist stattdessen eine Entwicklung zu beobachten, die auf eine Ausweitung der Funktionalität Elektronischer Märkte, die immer mehr auch spätere Transaktionsphasen und damit hierarchische Koordinationselemente unterstützen, zusteuert. ${ }^{312}$ Umgekehrt bieten viele Anwendungssysteme in der Praxis, die entsprechend ihres funktionalen Schwerpunktes als Elektronische Hierarchien zu bezeichnen sind, in zunehmendem Maße auch Unterstützung für die Anbahnungsaktivitäten von Markttransaktionen. Die nachfolgend zu Grunde gelegte Begriffsabgrenzung geht vom Hierarchiebegriff aus und betrachtet unter dem Oberbegriff der Elektronischen Hierarchie solche Anwendungssysteme, die sich auf die weisungsgebundene Koordination von Aktivitäten mehrerer Vertragspartner in der hier betrachteten Transaktionsphase nach Abschluss des Vertrages richten.

\footnotetext{
${ }^{308}$ Vgl. ähnlich Hubmann (1989), S. 165; Alt (1997), S. 106.

${ }^{309}$ Vg. Zbornik (1996), S. 59.

${ }^{310}$ Vgl. Malone/Yates/Benjamin (1987), S. 485.

${ }^{311}$ So nennt etwa Zbornik Buchhaltungs-, Lagerverwaltungs- und Managementinformationssysteme als Beispiele fuir Elektronische Hierarchien. Vgl. Zbornik (1996); S. 59. Zur allgemeinen Unterscheidung zwischen operativen Anwendungssystemen, die auch als transaktionsorientierte Systeme bezeichnet werden, und analytischen (dispositiven) Anwendungssystemen vgl. Chamoni/Gluchowski (1998), S. 10ff; Alpar et al. (2000), S. 31ff.

${ }^{312} \mathrm{Vgl}$. Strader/Shaw (2000), S. 79.
} 
Aus Sicht der Agency-Theorie wird diese letzte Vertragsphase durch das Problem der hidden action mit der daraus resultierenden Gefahr des Moral Hazard dominiert. Im Abschnitt 5.2.3.4 wurde bereits erläutert, dass sich dem Informationsmanagement grundsätzlich zwei verschiedene Ansatzpunkte zur Reduzierung dieser Problemstellung bieten. Einerseits bietet die Verbesserung der Beobachtbarkeit des Auftragnehmerverhaltens einen Ansatzpunkt für Maßnahmen des Informationsmanagements. Andererseits kann als Zielsetzung die Einschränkung von Handlungsfreiheiten des Auftragnehmers bzw. die direktive Verhaltenssteuerung bei der Erfüllung der vertraglichen Pflichten verfolgt werden, zu der insbesondere die Reduzierung der Ressourcenplastizität beiträgt.

Allgemein gesprochen kann das Informationsmanagement die Effizienz der Vertragsabwicklung durch die Bereitstellung von Anwendungssystemen erhöhen, die einerseits die Entscheidungssituation der jeweils zuständigen hierarchischen Stelle verbessern und anderseits die Koordination von Aktivitäten zur Umsetzung der getroffenen Entscheidungen unterstützen. Die Verbesserung der Entscheidungssituation erfordert geeignete analytische Funktionalitäten der verwendeten Anwendungssysteme, während die Koordination von Durchführungsaktivitäten durch entsprechende operative Funktionalitäten unterstützt werden kann. Operative und analytische Funktionalitäten betriebswirtschaftlicher Anwendungssysteme können als logische Komplemente aufgefasst werden, die unterschiedliche Zielsetzungen bei der Unterstützung der jeweiligen Aufgabenträger verfolgen. ${ }^{313}$ Diese Ansatzpunkte des Informationsmanagements zur Reduzierung von Koordinationskosten nach Vertragsschluss sind in Abbildung $5 / 12$ dargestellt.

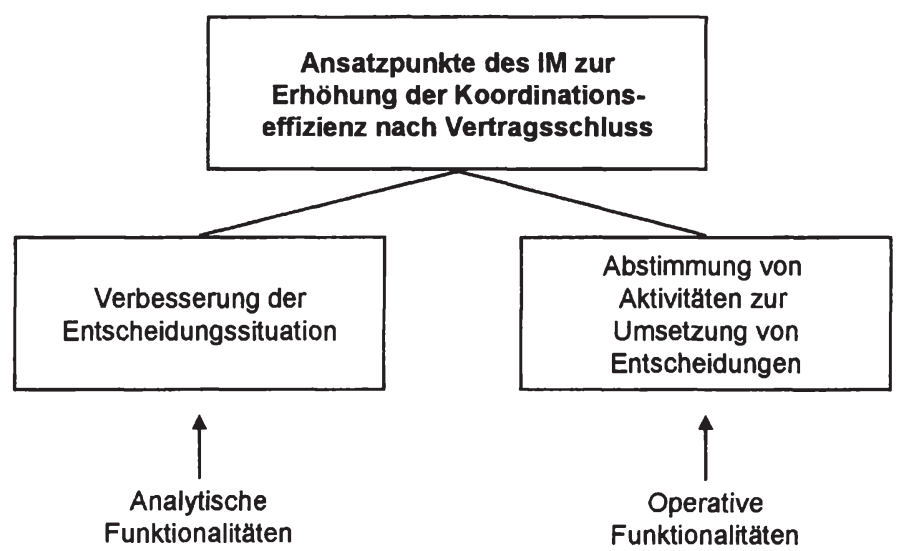

\section{Abb. 5/12: Ansatzpunkte des Informationsmanagements zur Erhöhung der Koordinationseffizienz nach Vertragsschluss}

Es sei darauf hingewiesen, dass die in der Praxis verwendeten Anwendungssysteme typischerweise nicht auf einen dieser beiden Funktionsbereiche beschränkt sind, sondern sowohl analytische als auch operative Funktionalitäten bieten. Die in der

\footnotetext{
${ }^{313}$ Vgl. Gabriel (1999), S. 420.
} 
Theorie weit verbreitete Unterscheidung zwischen diesen beiden Systemtypen ${ }^{314}$ erfolgt daher allein aus konzeptionellen Gründen. Die Ausführungen im nächsten Abschnitt konzentrieren sich zunächst auf die operativen Funktionalitäten, während im Vordergrund des darauf folgenden Abschnitts institutionenökonomisch begründete Anforderungen an analyseorientierte Anwendungssysteme stehen.

\subsubsection{Operative Funktionalitäten von Anwendungssystemen bei der Erfüllung vertraglicher Pflichten}

Vertragliche Arrangements, die im Rahmen der Vertragserfüllung den Einsatz hierarchischer Koordinationselemente vorsehen, zeichnen sich dadurch aus, dass zum Zeitpunkt des Vertragsschlusses die auszutauschende Leistung nicht vollständig fertiggestellt ist. Für den erfolgreichen Abschluss der Transaktion ist daher nach Vertragsschluss nicht nur die Übergabe der Leistung, sondern auch die Durchführung von Leistungserstellungsprozessen, an denen der Leistungsnehmer in irgendeiner Form beteiligt ist, erforderlich. In Abschnitt 5.2.4.2 wurde erläutert, dass die eigentlichen Leistungserstellungsprozesse, die selbst sowohl physische Güter als auch informationelle Leistungsbestandteile zum Gegenstand haben können, stets durch einen koordinierenden Informationsverarbeitungsprozess begleitet werden. Entsprechend dem Erkenntnisinteresse der Neuen Institutionenökonomie richten sich die nachfolgenden Überlegungen auf den zuletzt genannten, begleitenden Koordinationsprozess. Von diesem hängt die Höhe der entstehenden Ex post-Transaktionskosten und der AgencyKosten ab.

Operative Anwendungssysteme ${ }^{315}$ als Ausprägungsform Elektronischer Hierarchien gehen von festen Zielvorgaben innerhalb eines gegebenen Entscheidungsrahmens aus. In den Grenzen dieses Rahmens werden einerseits die an der Leistungserstellung mitwirkenden Aufgabenträger bei der Erfüllung ihrer Aufgaben unterstützt und andererseits die Aktivitäten der verschiedenen Beteiligten koordiniert. Grundsätzlich stehen sich dabei mit der Ex ante-Koordination und der Ad hoc-Koordination zwei Koordinationsalternativen gegenüber. ${ }^{316}$ Bei der Ex ante-Koordination findet bereits vor Durchführung der Prozesse eine vollständige Antizipation der erforderlichen Aktivitäten statt, während bei der Ad hoc-Koordination die Abstimmung der Aktivitäten erst zum Zeitpunkt der tatsächlichen Prozessdurchführung erfolgt. Die Anwendbarkeit dieser Koordinationsalternativen hängt von den Eigenschaften der zu unterstützenden Leistungserstellungsprozesse $\mathrm{ab}$. Die Entscheidung für eine der Alternativen ist mit sehr unterschiedlichen Anforderungen an die informationstechnische Unterstützung der Koordination verbunden. ${ }^{317}$

\footnotetext{
${ }^{314}$ Für einen Überblick dazu vgl. Gluchowski/Gabriel/Chamoni (1997), S. 44ff.

${ }^{315}$ Dieser Systemtyp wird zum Teil auch als transaktionsorientierte Systeme oder als Transaktionssysteme bezeichnet. Vgl. Gluchowski/Gabriel/Chamoni (1997), S. 46. Dieser Begriffsverwendung wird hier nicht gefolgt, da zu starke Verwechselungsmöglichkeiten mit dem Begriff der Transaktion und der Transaktionskosten im Sinne der Neuen Institutionenökonomie bestehen.

${ }^{316}$ Vgl. ähnlich Reif-Mosel (2000), S. 70. Diese bezeichnet die Ad hoc-Koordination allerdings als ex post-Koordination. Dieser Begriffswahl wird hier nicht gefolgt, da die entsprechenden Koordinationsaktivitäten nicht nach, sondern während der Prozessdurchfuihrung stattfinden.

${ }^{317} \mathrm{Zu}$ diesen Alternativen vgl. Rolf (1998), S. 178.
} 


\section{a) Ex ante-Koordination}

Die Ex ante-Koordination ist dadurch gekennzeichnet, dass den koordinierten Akteuren vor Aufnahme der Leistungserstellung konkrete Anweisungen darüber gegeben werden, wie auf das Eintreten bestimmter Situationen reagiert werden soll. Während der tatsächlichen Durchführung von Prozessen der Leistungserstellung entstehen somit keine Transaktionskosten mehr für die Aushandlung von Verhaltenselementen. Die Wahl dieser Koordinationsalternative ist an bestimmte Voraussetzungen gebunden, die die zu koordinierenden Leistungserstellungsprozesse betreffen. Eine ex ante vorgenommene, vollständige Festlegung der zur Herstellung einer Leistung durchzuführenden Aktivitäten ist dann möglich, wenn der betreffende Prozess immer den gleichen, gut strukturierbaren und formalisierbaren Ablauf aufweist und die Kommunikationsbeziehungen und Entscheidungskompetenzen dauerhaft festgelegt sind. ${ }^{318}$ Für Prozesse mit diesen Eigenschaften ist eine standardisierte Ex anteKoordination vorteilhaft, da die stets wiederkehrende Aushandlung von Routinen während des Ablaufes unnötig Ressourcen binden würde und daher mit ineffizient hohen Transaktionskosten verbunden wäre. ${ }^{319}$

Für einen schnelle und effiziente Erstellung der entsprechenden Leistung kommt es unter diesen Bedingungen allein darauf an, die beteiligten Personen über das Vorliegen von Handlungsvoraussetzungen $\mathrm{zu}$ informieren und dadurch die vorgegebenen Aktivitätsfolgen abzurufen. Ex post-Transaktionskosten entstehen bei dieser Koordinationsalternative lediglich für die Steuerung des Prozessablaufes und die Versorgung der an der Leistungserstellung beteiligten Aufgabenträger mit den zur adäquaten Erfüllung ihrer Aufgaben benötigten Informationen. Dort liegen auch die Ansatzpunkte des Informationsmanagements zur Reduzierung von Transaktionskosten fur diese Art von Leistungserstellungsprozessen. Dementsprechend muss sich die technische Unterstützung des begleitenden Koordinationsprozesses auf die Automatisierung der Ablaufsteuerung und die Bereitstellung der zur Durchführung der einzelnen Prozessschritte jeweils benötigten Informationen beziehen. Konzepte zur Realisierung dieser Zielsetzung werden in der Literatur unter dem Oberbegriff „Workflow Management" zusammengefasst. ${ }^{320}$

Der Bereich des Workflow Managements stellt ein weites und facettenreiches Forschungsgebiet dar, das vom Problem der grundsätzlichen Formalisierbarkeit von Geschäftsprozessen über Ansätze zu deren Modellierung und Vorgehensweisen zur Einführung von Workflow Management-Anwendungen bis hin zu Fragen der Veränderung der Arbeitswelt durch neue Formen der Arbeitsteilung reicht. ${ }^{321}$ Entsprechend den Grundüberlegungen zum Aussagebereich eines Institutionenökonomischen Ansatzes für eine Theorie des Informationsmanagements in Abschnitt 5.2.4.2 konzentrieren sich die Ausführungen an dieser Stelle auf einen Teilaspekt dieses Forschungsgebietes. Untersucht wird, welche Anforderungen sich an die Funktionalität von Workflow Management-Systemen in Bezug auf die Koordination der beteiligten Akteure ableiten lassen. Die Automatisierbarkeit der Ablaufsteuerung, die an die Voraussetzung

\footnotetext{
${ }^{318} \mathrm{Vgl}$. Reif/Knittel (1996), S. 49.

${ }^{319} \mathrm{Vgl}$. ähnlich Rolf (1998), S. 178

${ }^{320} \mathrm{Vgl}$. für einen allgemeinen Überblick über das Workflow Management und die in diesem Bereich entwickelten Systeme z.B. Jablonski/Böhm/Schulze (1997), S. 5ff.; Weiß/Krcmar (1996), S. $503 \mathrm{ff}$. Heilmann (1994), S. 8ff.

${ }^{321} \mathrm{Vgl}$. Jablonski/Bbhm/Schulze (1997), S. 5; Rolf (1998), S. 80 i.V.m. S. 176ff.
} 
gebunden ist, dass die Folge der Teilprozesse und Tätigkeiten durch grundlegende Ablaufstrukturen (Sequenz, Nebenläufigkeit, Alternative) beschreibbar ist, ${ }^{322}$ wird dabei als gegeben angenommen.

Die Reduzierung von Ex post-Transaktionskosten mittels Workflow Management beruht auf der Zerlegung der Leistungserstellungsprozesse und der anschließenden Fixierung von Handlungsabfolgen. ${ }^{323}$ Diese Handlungsfolgen werden in Workflow Management-Systemen abgebildet, sodass die Koordination während der tatsächlichen Prozessdurchführung durch das Anwendungssystem erfolgen kann. Das Workflow Management-System legt den einzelnen Aufgabenträgern den konkreten Vorgang ${ }^{324}$ zur Abarbeitung der ihnen zugeteilten Prozessschritte vor und gewährleistet anschließend die unverzügliche Weiterleitung an die nächste beteiligte Stelle. ${ }^{325}$ Die Effizienz des Leistungsaustausches steigt dabei, da die Abwicklung der Leistungserstellungsprozesse schneller, sicherer und zuverlässiger wird. ${ }^{326}$ Um die Notwendigkeit externer Eingriffe in das Geschehen möglichst weit zu reduzieren, sollte in einem Workflow Management-System nicht nur der routinemäßig stattfindende Prozessablauf abgebildet werden. Vielmehr sollte das System auch in der Lage sein, Ausnahmeregelungen vorzunehmen. So muss beispielsweise die Gefahr von Verzögerungen und Unterbrechungen des üblichen Ablaufs bei einzelnen Vorgängen frühzeitig erkannt werden, um unerwünschte Entwicklungen mit Hilfe von standardisierten Reaktionsmustern weitgehend $\mathrm{zu}$ vermeiden. ${ }^{327}$ Dazu sind Funktionalitäten wie eine permanente Terminüberwachung und die Möglichkeit zur Einrichtung einfacher Vertretungsregelungen vorzusehen. ${ }^{328}$

Eine reibungslose Prozessabwicklung erfordert nicht nur die bisher betrachtete Automatisierung der Abläufe, sondern auch eine möglichst umfassende Versorgung der jeweils zuständigen Stellen mit den zur Ausführung der ihnen zugewiesenen Prozessschritte benötigten Informationen. ${ }^{329}$ Diese Problemstellung wird im Rahmen des Workflow Managements durch das Konzept der Applikationsintegration gelöst. ${ }^{330}$ Applikationsintegration bedeutet, dass das Workflow Management-System mit den verschiedenen Anwendungssystemen in der Unternehmung, mit denen die eigentliche Bearbeitung der jeweiligen Vorgänge erfolgt, über Softwareschnittstellen verknüpft wird. Dabei kann es sich beispielsweise um operative, transaktionsorientierte Anwendungssysteme oder um Systeme zur Dokumentenerstellung und -bearbeitung handeln. Je nach Art der zu bearbeitenden Aufgabe werden ein oder mehrere Applikationen verwendet. ${ }^{331}$ Durch die Möglichkeit zum Aufruf dieser Anwendungen können die Übergabe und Weiterleitung von Teilergebnissen sowie der Zugriff auf vom Benutzer angeforderte zusätzliche Informationen über den Prozessstatus realisiert werden. Auf diese Weise werden Medienbrüche im Laufe der Prozessdurchführung verringert

\footnotetext{
${ }^{322} \mathrm{Vgl}$. Reif/Knittel (1996), S. 49, die sich auf Kreifelts (1983), S. 221ff. beziehen.

${ }^{323}$ Vgl. Paetau (1991), S. 145.

${ }^{324}$ Als Vorgang wird ein einzelnes Prozessexemplar bezeichnet, das die konkrete Ausprägung eines

Prozesstyps darstellt. Vgl. zu dieser begrifflichen Differenzierung Reif-Mosel (2000), S. 114.

${ }^{325} \mathrm{Vgl}$. Picot/Reichwald/Wigand (1998), S. 152.

${ }^{326}$ Vgl. Kirn (1995), S. 101.

${ }^{327}$ Vgl. Jablonski/Böhm/Schulze (1997), S. 220

${ }^{328} \mathrm{Vgl}$. Picot/Reichwald/Wigand (1998), S. 153.

${ }^{329}$ Vgl. ähnlich Weiß (1998), S. 27.

${ }^{330} \mathrm{Vgl}$. Jablonski/Böhm/Schulze (1997), S. 221.

${ }^{331} \mathrm{Vgl}$. Jablonski/Böhm/Schulze (1997), S. 365.
} 
und die Voraussetzungen zur Reduzierung von Liege- und Transportzeiten geschaffen. Ferner entstehen mit der integrierten Unterstützung der Prozessabwicklung im Vergleich zur traditionellen Form der Arbeitsteilung neue Möglichkeiten zur Parallelisierung von Prozessschritten, da mehrere Aufgabenträger zugleich auf die entsprechenden Statusinformationen zugreifen können. ${ }^{332}$ Infolgedessen lässt sich im Bereich der Bearbeitungszeiten ebenfalls eine Beschleunigung der Prozesse erreichen.

Auch ohne vollständige Automatisierung der Ablaufsteuerung eröffnet die Integration von Anwendungssystemen neue Potenziale zur Reduzierung von Koordinationskosten. Durch den Aufbau einer integrierten Vorgangsbearbeitung mit weitgehend selbstständigen Einzelarbeitsplätzen lässt sich die Arbeitsteilung innerhalb der Leistungserstellungsprozesse teilweise aufheben. ${ }^{333}$ Bei der integrierten Vorgangsbearbeitung entfält ein Großteil der Interdependenzen zwischen den an einem Prozess beteiligten Arbeitsplätzen, sodass die Notwendigkeit zur arbeitsplatzübergreifenden Abstimmung von Aktivitäten in erheblichem Umfang verringert wird. Ferner wird eine verstärkte Ausrichtung der Leistungserstellung auf die speziellen Anforderungen der jeweiligen Auftraggeber möglich. Der jeweils zuständige Aufgabenträger kann Änderungswünsche flexibler berücksichtigen und ist auf Grund seines vollständigeren Überblicks über den Bearbeitungsstand besser in der Lage, diesbezügliche Anfragen zu beantworten. Diese organisatorische Lösung kann durch die Automatisierung von Teilprozessen ergänzt werden, um eine weitere Reduzierung von Ex post-Transaktionskosten und eine möglichst hohe Koordinationseffizienz zu erreichen.

Betrachtet man die Ex ante-Koordination von Leistungserstellungsprozessen nach Vertragsschluss aus der Perspektive der Agency-Theorie, so ist der Erfolg von Gestaltungsmaßnahmen des Informationsmanagements an den Auswirkungen auf die hidden action-Problematik mit der Gefahr des Moral Hazard zu messen. Es ist zu überprüfen, inwiefern Anwendungssysteme zur Automatisierung der Ablaufsteuerung bestehende diskretionäre Verhaltensspielräume beseitigen und/oder den Informationsstand des Auftraggebers verbessern können. Workflow Management-Systeme wirken unmittelbar handlungssteuernd für die einzelnen Aufgabenträger, indem sie diese aktiv zur Durchführung bestimmter Aktivitäten auffordern. Folglich kommt es zu einer informationstechnisch mediierten Ausweitung der Möglichkeiten des Principals, direktiv Einfluss auf die Leistungsabgabe durch den Agent zu nehmen. Der Auftraggeber wird indirekt in die Lage versetzt, den Leistungserstellungsprozess über detaillierte Anweisungen zu koordinieren, für die ihm ohne die Verwendung dieses Systemtyps die erforderlichen Informationen fehlen würden. Diskretionäre Handlungsspielräume der an der Leistungserstellung beteiligten Aufgabenträger werden durch diese Ausweitung der direktiven Verhaltenssteuerung eingeschränkt. ${ }^{334}$ Die Ressourcenplastizität sinkt, da die Art der Nutzung der bei der Leistungserstellung verwendeten Ressourcen an enge technische Vorgaben gebunden wird.

Neben dieser Vermittlungsfunktion bieten Workflow Management-Systeme auch Möglichkeiten zur besseren Beobachtung des Geschehens, die eine weitere Maßnahme zur Beseitigung der hidden action-Problematik darstellt. Die Reduzierung von Informationsasymmetrien zu Lasten des Auftraggebers wird durch die Monitoring-

\footnotetext{
${ }^{332}$ Vgl. Picot/Reichwald/Wigand (1998), S. 153.

${ }^{333}$ Vgl. Rolf (1998), S. 81; Picot/Reichwald/Wigand (1998), S. $226 f$.

${ }^{334}$ Vgl. Rolf (1998), S. 80
} 
Funktionalitäten derartiger Systeme erreicht, die die Überwachung der laufenden Arbeitsabläufe und die Zurückverfolgung der im Rahmen einzelner Vorgänge durchgeführten Aktivitäten ermöglichen. ${ }^{335}$ Diese Funktionalitäten sind zwar primär auf die Leistung selbst bezogen und streben kurzfristig eine rechtzeitige Reaktion auf Verzögerungen bei einzelnen Vorgängen sowie langfristig eine Optimierung der Ablauforganisation insgesamt an. Sie lassen jedoch auch die Ermittlung und den Vergleich der Produktivitäten einzelner Mitarbeiter zu und geben somit Anhaltspunkte für die Beurteilung der individuellen Anstrengungen bei der Leistungsabgabe. Damit werden die Voraussetzungen zur Realisierung eines personenbezogenen Performance Measurement geschaffen, mit dem die Gefahr des von den Auftraggeberinteressen stark abweichenden Auftragnehmerverhaltens weitgehend beseitigt werden kann.

Das Konzept der integrierten Vorgangsbearbeitung ist dagegen aus Sicht der AgencyTheorie ambivalent zu beurteilen. Einerseits wird die Position des Auftragnehmers bei der ganzheitlichen Prozessabwicklung durch die Zusammenfassung von Aktivitäten gestärkt, da bei dieser organisatorischen Lösung vergrößerte Handlungsspielräume bei der Leistungserstellung bestehen. Infolgedessen verbessern sich die Möglichkeiten des Auftragnehmers zur Durchführung verdeckter Handlungen gegen die Interessen des Auftraggebers. Auf der anderen Seite ist bei der integrierten Vorgangsbearbeitung durch die Reduzierung der am Prozess beteiligten Personen eine weitaus bessere Beeinflussbarkeit des erzielten Ergebnisses durch den Einzelnen gegeben. Eine umfassende Beobachtung des Geschehens ist im Idealfall gar nicht nötig, da der Einsatz des Auftragnehmers an Hand der abgelieferten Leistungen erheblich besser als bei stark arbeitsteiligen Prozessen beurteilt werden kann. Die relative Bedeutung dieser beiden gegenläufigen Effekte hängt vom Ausmaß und von der Beobachtbarkeit des exogenen Risikos im Einzelfall ab.

Die bisherigen Überlegungen richteten sich auf eine Automatisierung von Abläufen für unternehmungsinterne Leistungserstellungsprozesse mit Hilfe Elektronischer Hierarchien. Diese ist bereits seit längerem Gegenstand des Informationsmanagements in Theorie und Praxis. ${ }^{336}$ Auch unternehmungsübergreifende Leistungserstellungsprozesse nach Vertragsschluss können jedoch unter dem Aspekt der möglichst effizienten Ablaufsteuerung untersucht werden. Derartige Prozesse finden im Rahmen von Transaktionen statt, deren Koordination sowohl marktliche als auch hierarchische Elemente enthält. Wenn bei unternehmungsübergreifenden Prozessen Workflow Management-Systeme isoliert in den einzelnen Unternehmungen eingesetzt werden, dann handelt es sich lediglich um Insellösungen, die noch ein erhebliches Potenzial zur weiteren Steigerung der Koordinationseffizienz aufweisen. ${ }^{337}$

Die Ausdehnung der Ablaufsteuerung auf mehrere beteiligte Unternehmungen ist als zwischenbetriebliche Elektronische Hierarchie ${ }^{338}$ zur Unterstützung einer integrativen Leistungserstellung zwischen formal eigenständigen Organisationseinheiten $\mathrm{zu}$ klassifizieren. Bei dieser Form des Leistungsaustausches wird während der Vertragslaufzeit der betreffenden Transaktion die Erstellung von Leistungsbestandteilen abgerufen, die im Rahmen der Vertragsvereinbarung nur unvollständig spezifiziert wurden.

\footnotetext{
${ }^{335} \mathrm{Vgl}$. dazu Jablonski/Böhm/Schulze (1997), S. 201ff

${ }^{336}$ Vgl. ähnlich Zbornik (1996), S. 59.

${ }^{337}$ Vgl. Jablonski/Böhm/Schulze (1997), S. 349.

${ }^{338}$ Zbornik (1996), S. 59.
} 
Typische Beispiele für diesen Transaktionstyp stellen sogenannte Single SourceChannels und längerfristige Rahmenverträge zur Lieferung bestimmter Einsatzmaterialien dar. Zum Austausch der einzelnen Teilleistungen innerhalb des vereinbarten Vertragsrahmens ist eine qualitative, quantitative, räumliche und zeitliche Abstimmung der durch die Vertragspartner durchgeführten Teilprozesse vorzunehmen. ${ }^{339}$ Eine vollständige Automatisierung der Ablaufsteuerung erfordert dabei die Kopplung der technikunterstützten Informationssysteme der beteiligten Unternehmungen auf den in Abbildung 5/13 dargestellten Untersuchungs- und Gestaltungsebenen.

Leistungsanbieter

(Unternehmung 1)

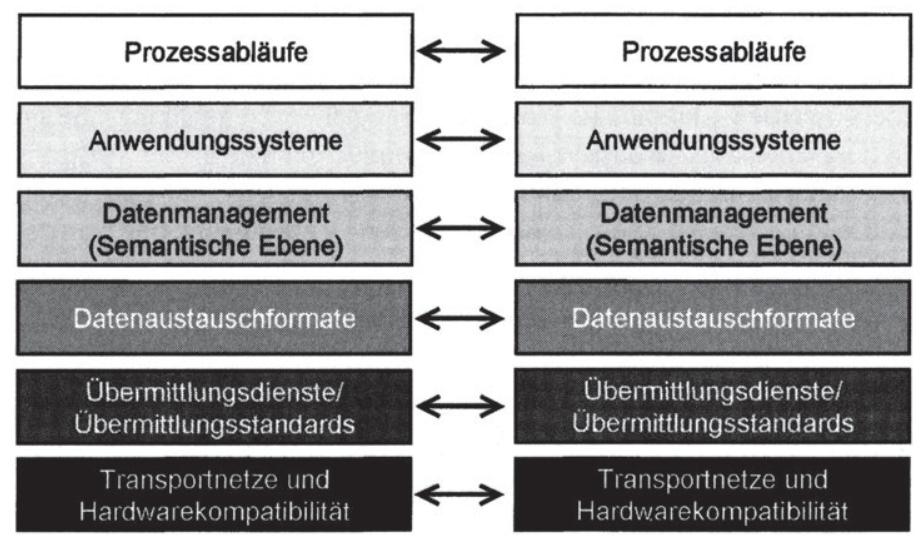

Abb. 5/13: Verknüpfungsebenen der unternehmungsübergreifenden Informationsverarbeitung Quelle: in Anlehnung an Gersch (1998), S. 255.

Diese Ebenen betreffen unterschiedliche Gestaltungsaspekte, die zwischen den beteiligten Unternehmungen beim Aufbau einer unternehmungsübergreifenden Informationsverarbeitung abzustimmen sind. Je höher eine Ebene in diesem Klassifikationsschema angesiedelt ist, um so stärker stehen bei der Untersuchung und Gestaltung der jeweiligen Prozesse anwendungsbezogene Aspekte im Vordergrund. Auf der untersten Ebene bezieht sich die Analyse auf die physische Kompatibilität der Netzwerk-Komponenten, während auf der obersten Ebene betriebswirtschaftlichorganisatorische Fragestellungen betrachtet werden. Dazwischen liegen verschiedene Stufen mit nach oben hin abnehmendem Technikbezug. Die Herstellung einer Verknüpfung zwischen den Informationssystemen der beteiligten Unternehmungen erfolgt über Schnittstellen und erfordert auf einer bestimmten Gestaltungsebene die Kompatibilität der Systeme auf allen darunter liegenden Ebenen. Dementsprechend kann die Verknüpfung auf einer Betrachtungsebene als Voraussetzung für die Integration der Systeme auf der jeweils nächsten darüber liegenden Ebene angesehen werden.

\footnotetext{
${ }^{339}$ Vgl. Gersch (1998), S. 203.
} 
Die Ex ante-Koordination von unternehmungsübergreifenden Leistungserstellungsprozessen mit automatisierter Ablaufsteuerung erfordert ein betriebswirtschaftliches Gesamtkonzept, das auf der höchsten der in Abbildung 5/13 genannten Untersuchungsund Gestaltungsebenen anzusiedeln ist. Wegen der erläuterten Zusammenhänge zwischen den Ebenen macht die Realisierung einer zwischenbetrieblichen Elektronischen Hierarchie also die Integration der technikunterstützten Informationssysteme auf sämtlichen dieser Ebenen erforderlich. Auf der Basis eines unternehmungsübergreifenden Workflow Managements können Leistungserstellungsprozesse auch dann einheitlich geplant, gesteuert und kontrolliert werden, wenn die Zuständigkeiten für die einzelnen Aktivitäten über mehrere Unternehmungen in einer Wertschöpfungskette verteilt sind. ${ }^{340}$ Dem Auftraggeber werden dadurch erweiterte Möglichkeiten zur direktiven Verhaltenssteuerung gegeben, auch wenn der Auftragnehmer nicht in der eigenen Unternehmung beschäftigt ist und somit formal kein Über/Unterordnungsverhältnis zwischen Auftraggeber und Auftragnehmer besteht. Die Kontrollmöglichkeiten des Auftraggebers werden durch die Einrichtung einer unternehmungsübergreifenden Ablaufsteuerung ebenfalls verbessert, da Unregelmäßigkeiten in der Auftragsabwicklung leichter wahrgenommen und sanktioniert werden können. Dies trägt auch zu einer besseren Aufteilung des externen Risikos zwischen Auftraggeber und Auftragnehmer bei.

\section{b) Ad hoc-Koordination}

Die Ad hoc-Koordination ist dadurch gekennzeichnet, dass die nach Vertragsschluss stattfindenden Aktivitäten der koordinierten Akteure erst während der Durchfuhrung des entsprechenden Leistungserstellungsprozesses abgestimmt werden. Bei dieser Koordinationsstrategie können umfangreiche Ex post-Transaktionskosten entstehen, da das genaue Vorgehen zum Austausch der Leistung nicht vollständig mit der Vertragsvereinbarung festgelegt wird. Der Einsatz der Ad hoc-Koordination wird dann erforderlich, wenn auf Grund der Eigenschaften der zu übergebenden Leistung und des dazugehörigen Leistungserstellungsprozesses keine transaktionskostengünstige Ex ante-Koordination der Aktivitäten möglich ist. Dies ist der Fall, wenn komplizierte Leistungen ausgetauscht werden, deren Eigenschaften zum Zeitpunkt des Vertragsschlusses noch nicht bis in alle Einzelheiten bestimmt werden können. Bei der Erstellung derartiger Leistungen handelt es sich typischerweise um schwächer strukturierte Problemstellungen, die eine kreative Problemlösung notwendig machen und keine exakte Formalisierung des Vorgehens zulassen. ${ }^{341}$ Häufig nutzen die Beteiligten dabei nicht festlegbare bzw. zusätzliche, medienungebundene Kommunikationskanäle. ${ }^{342}$

Wegen der fehlenden Formalisierbarkeit erfordert der Einsatz der Ad hoc-Koordination einen personenbezogenen Koordinationsmechanismus, also die Koordination durch

\footnotetext{
${ }^{340}$ Vgl. dazu Tan/Shaw/Fulkerson (2000), S. 469ff.

${ }^{341}$ Ein Beispiel für diesen Leistungstyp stellt die erstmalige Entwicklung einer Markteinführungsstrategie für ein bestimmtes Produkt auf einem bisher nicht bearbeiteten Markt, etwa im fernöstlichen Raum, dar. An dieser Aufgabe sind Mitarbeiter mehrerer Organisationseinheiten der Unternehmung beteiligt, zwischen deren Handlungen umfangreiche Interdependenzen bestehen. Der genaue Inhalt der Strategie steht erst nach Abschluss des Planungsprozesses fest. Weder das Ergebnis dieses Prozesses noch der - typischerweise nicht lineare - Verlauf des Planungsprozesses können im Voraus exakt bestimmt werden.

${ }^{342} \mathrm{Vgl}$. Weiß/Krcmar (1996), S. 510f.
} 
Anweisung oder durch Selbstabstimmung. ${ }^{343}$ Die Koordination durch Selbstabstimmung wird üblicherweise nicht als hierarchisch eingeordnet, da kein Über/Unterordnungsverhältnis zwischen den Beteiligten besteht. Die informationstechnische Unterstützung der Selbstabstimmung fällt deshalb nicht unmittelbar in den Bereich der Elektronischen Hierarchien, die definitionsgemäß von der Anweisungs- und Lenkungsfunktion einer übergeordneten Instanz ausgehen. Sie wird dennoch an dieser Stelle behandelt, da sie sich genau wie "echte“ Elektronische Hierarchien auf die Koordination von Akteuren richtet, die zuvor die Erstellung einer bestimmten Leistung vereinbart haben. Die betreffenden Systeme sind folglich ebenfalls in die Transaktionsphase nach Vertragsschluss einzuordnen. Agency-Beziehungen bestehen bei der Koordination durch Selbstabstimmung nicht nur zwischen den an der Leistungserstellung beteiligten Personen als Gesamtheit und dem Empfänger der erstellten Leistung, sondern auch zwischen den an der Leistungserstellung mitwirkenden Personen untereinander. Denkbar ist ferner, dass die Empfänger der Leistung zugleich dem Kreis der Leistungsersteller angehören.

Bei schlecht strukturierten Problemstellungen ist es nicht sinnvoll, auf eine Automatisierung der Ablaufsteuerung für die betreffenden Prozesse abzuzielen. Vielmehr geht es für das Informationsmanagement um die Bereitstellung einer Infrastruktur, die die flexible Abstimmung der Aktivitäten zum Zeitpunkt der Prozessabwicklung unterstützt. ${ }^{344}$ Bei der Koordination durch Anweisung steht lediglich die Übermittlung der in der aktuellen Situation abgegebenen Handlungsaufträge, also die Kommunikation zwischen Leistungsgeber und Leistungsnehmer, im Vordergrund. Bei der Koordination durch Selbstabstimmung ist es dagegen notwendig, dass die Aufgabenträger im Rahmen der Leistungserstellung gemeinsam mit Informationen umgehen, sie sammeln, kommentieren, verteilen usw. ${ }^{34}$ Die entsprechende Arbeitskonstellation wird als Computer Supported Cooperative Work (CSCW) und die Anwendungssysteme in diesem Bereich als CSCW-Systeme bezeichnet. ${ }^{346}$ CSCW-Systeme stellen Funktionalitäten zur Verfügung, die sich auf die Unterstützung der Kommunikation, der Koordination und/oder der inhaltlichen Erfüllung der Aufgaben richten. ${ }^{347}$ Die Schwerpunkte sind bei den verschiedenen Systemtypen unterschiedlich ausgeprägt, wobei aus Sicht der Neuen Institutionenökonomie weniger die inhaltliche Unterstützung der eigentlichen Aufgaben als die Koordination der arbeitsteiligen Aktivitäten interessiert, die bei diesem Leistungstyp wiederum die Kommunikation der Beteiligten voraussetzt. In dem Maße, in dem die Prognostizierbarkeit der Ergebnisse eines Leistungserstellungsprozesses sinkt, steigt die Notwendigkeit zur Kommunikation der an dem Prozess beteiligten Aufgabenträger. ${ }^{348}$

Die Kommunikationsunterstützung spielt also sowohl bei der Koordination durch Anweisung als auch bei der Koordination durch Selbstabstimmung eine zentrale Rolle. Die Ausführungen konzentrieren sich im Folgenden auf die Selbstabstimmung, sind jedoch analog auf die Koordination durch Anweisung anwendbar. Im Rahmen der Kommunikation können sowohl räumliche als auch zeitliche Barrieren bestehen, durch

\footnotetext{
${ }^{343}$ Vgl. Reif-Mosel (2000), S. 70ff;; Kieser/Kubicek (1992), S. 104.

${ }^{344} \mathrm{Vgl}$. Reif/Knittel (1996), S. 50.

${ }^{345}$ Vgl. Hansen (1992), S. 50.

${ }^{346} \mathrm{Vgl}$. Reif-Mosel (2000), S. 9ff. sowie die zahlreichen dort angegebenen Quellen.

${ }^{347} \mathrm{Vgl}$. Jablonski/Böhm/Schulze (1997), S. 377.

${ }^{348}$ Vgl. Reif-Mosel (2000), S. 118.
} 
deren Überwindung eine gemeinsame Arbeitsumgebung für die an der Leistungserstellung mitwirkenden Personen geschaffen wird. ${ }^{349}$ Die verschiedenen CSCWSystemtypen lassen sich danach klassifizieren, von welchen räumlichen und zeitlichen Gegebenheiten sie bei der Unterstützung der Kommunikation ausgehen. ${ }^{350}$ Es sind dann entsprechend den Ausprägungen in den beiden Dimensionen Raum (an einem Ort/an verschiedenen Orten) und Zeit (synchron/asynchron) vier Anwendungssystemklassen zu unterscheiden. Abbildung 5/14 zeigt Beispiele für Systemtypen in den einzelnen Klassen dieses Klassifikationsschemas, auf deren jeweilige Funktionalität hier nicht im Einzelnen einzugehen ist. Die allgemeine technische Entwicklung geht allerdings dahin, die bei dieser Klassifikation voneinander abgegrenzten Anwendungssystemklassen zu integrieren und mit einzelnen Systemen eine umfassende Kommunikationsunterstützung zu bieten, die sowohl die Überwindung zeitlicher als auch räumlicher Differenzen zulässt. ${ }^{351}$

\begin{tabular}{|c|c|c|}
\hline Raum Zeit & $\begin{array}{l}\text { Zur gleichen Zeit } \\
\text { (synchron) }\end{array}$ & $\begin{array}{l}\text { Zu verschiedenen Zeiten } \\
\text { (asynchron) }\end{array}$ \\
\hline An einem Ort & $\begin{array}{l}\text {-Sitzungsunterstützungs- } \\
\text { systeme } \\
\text {-Group Decision Support } \\
\text { Systeme }\end{array}$ & $\begin{array}{l}\text { - Coautorensysteme } \\
\text { - Terminmanagementsysteme } \\
\text { - Projektmanagementsysteme } \\
\text { - Prozessunterstiutzungs- } \\
\text { systeme }\end{array}$ \\
\hline $\begin{array}{c}\text { An } \\
\text { verschiedenen } \\
\text { Orten }\end{array}$ & $\begin{array}{l}\text {-Telekonferenzsysteme } \\
\text { (Audio- und } \\
\text { Videokonferenzsysteme) } \\
\text {-Screen-sharing-Systeme }\end{array}$ & $\begin{array}{l}\text { - Group Decision Support } \\
\text { Systeme } \\
\text { - Electronic Mall Systeme } \\
\text { - Computerkonferenzsysteme }\end{array}$ \\
\hline
\end{tabular}

Abb. 5/14: Raum-Zeit-Klassifikation von CSCW-Techniken Quelle: Reif-Mosel (2000), S. 88.

Hinsichtlich der die Kommunikation der Beteiligten voraussetzenden Koordinationsunterstützung sind $\mathrm{CSCW}-$ Systeme eher passiv ausgelegt. ${ }^{352}$ Die Zielsetzung liegt darin, einen flexiblen Verlauf der zur Erstellung der Leistung erforderlichen Prozesse möglich zu machen. Auf Grund der schwach ausgeprägten Strukturiertheit der Problemstellung hängt der tatsächliche Verlauf eines konkret stattfindenden Prozesses in hohem Maße von informellen Aktivitäten ab und ist ex ante nicht prognostizierbar. Je nach der Ebene, an der die Koordination der Aktivitäten im Rahmen von Leistungserstellungsprozessen ansetzt, können grundsätzlich zwei Bezugspunkte der Koordination unterschieden werden. ${ }^{353}$ Während die oben erläuterten Workflow Management-Systeme sich auf die Aktivitäten selbst beziehen und diese in eine bestimmte Ordnung bringen, setzen CSCW-Systeme bei den zu erstellenden Objekten als angestrebtem Prozessergebnis an. Es wird festgelegt, über welche

\footnotetext{
${ }^{349}$ Vgl. Borghoff/Schlichter (1995), S. 79.

${ }^{350} \mathrm{Vgl}$. dazu Reif-Mosel (2000), S. 87ff. sowie die dort zitierten Quellen.

${ }^{351}$ Vgl. Reif-Mosel (2000), S. 87.

${ }^{352}$ Vgl. Jablonski/Böhm/Schulze (1997), S. 379.

${ }^{353}$ Vgl. zum Folgenden Jablonski/Böhm/Schulze (1997), S. 378.
} 
Möglichkeiten zur Veränderung dieser Objekte die einzelnen Aufgabenträger während des Prozessablaufs prinzipiell verfügen. Die betreffenden Möglichkeiten werden als Rechte bezeichnet. Dieses Konzept ermöglicht ein ergebnisoffenes Vorgehen, bei dem den verschiedenen Akteuren in den Grenzen der ihnen zugewiesenen Rechte weitgehende Freiheiten zur Beeinflussung des Leistungsergebnisses verbleiben. Innerhalb dieses Rahmens bieten die Systeme ferner sogenannte Gegenwärtigkeitsinformationen, die dem einzelnen Aufgabenträger einen Überblick über den aktuellen Stand der Dinge und die bereits an dem Objekt ausgeführten Aktivitäten geben. $^{354}$

Aus Sicht der Agency-Theorie können CSCW-Systeme zur Verringerung der hidden action-Problematik beitragen, wenn sie eine Einschränkung von Verhaltensspielräumen des Auftragnehmers und/oder eine Verbesserung der Beobachtbarkeit der Leistungserstellungsprozesse durch den Auftraggeber ermöglichen. Es ist deshalb zu überprüfen, welche Potenziale dieser Anwendungssystemtyp nach Vertragsschluss einerseits zur Ausweitung der direktiven Verhaltenssteuerung durch den Auftraggeber und andererseits zur besseren Kontrolle der Aktivitäten des Auftragnehmers aufweist. Das erwähnte Zugriffs- und Rechtekonzept für verändernde Maßnahmen an den zu erstellenden Objekten schränkt den Handlungsspielraum der Aufgabenträger bei der Leistungserstellung ein. Entsprechend der passiven Ausrichtung von CSCW-Systemen bei der Koordinationsunterstützung bewirkt es jedoch keine wirkliche direktive Verhaltenssteuerung, da die verschiedenen Rechte im Rahmen der Selbstabstimmung nicht als konkrete Handlungsvorgaben formuliert sind. Es wird lediglich festgelegt, welche Aktivitäten im Rahmen der Leistungserstellungsprozesse grundsätzlich erlaubt bzw. nicht erlaubt sind, ohne jedoch unmittelbar Direktiven für die Wahl von Verhaltensalternativen vorzugeben. Die Ressourcenplastizität wird also nur geringfügig gesenkt, da die Nutzung der von den Aufgabenträgern verwendeten Ressourcen nach wie vor nicht eindeutig durch technische Bedingungen vorgegeben wird. Eine echte direktive Verhaltenssteuerung würde als Ex post-Koordinationsmechanismus die Koordination durch Anweisungen erfordern.

Die Koordination durch Anweisungen setzt einen entsprechenden Kenntnisstand des Anweisenden über die aktuelle Situation voraus, der jedoch nicht im Fokus der hier betrachteten operativen Anwendungssysteme steht. Operative Anwendungssysteme können diesen Koordinationsmechanismus lediglich durch die Übermittlung der jeweils situationsgebunden abgegebenen Handlungsanweisungen unterstützen. Beschränkungen bei der Übermittlung von Anweisungen stellen jedoch nicht das typische Problem dar, das nach Vertragsschluss zum Auftreten von moral hazard führt. Tatsächlich werden - insbesondere bei den hier betrachteten schlecht strukturierten Problemstellungen - Auftraggeber/Auftragnehmer-Beziehungen häufig gerade deswegen eingegangen, weil der Auftraggeber nicht über ausreichende Fachkenntnisse verfügt, um die betreffende Problemstellung selbst zu lösen und die gewünschte Leistung selbst zu erstellen. Der für die Entstehung von Agency-Kosten ursächliche, informationelle Engpass liegt dabei weniger in der Übermittlung von Handlungsanweisungen als in der fehlenden Entscheidungsgrundlage des Auftraggebers. Dieser ist typischerweise nicht in der Lage, die Vorteilhaftigkeit von Handlungsalternativen zu beurteilen. Bei Leistungserstellungsprozessen mit geringem Strukturiertheitsgrad und

\footnotetext{
${ }^{354}$ Vgl. Jablonski/Böhm/Schulze (1997), S. 379.
} 
hohem Singularitätscharakter sind häufig große Freiräume des Auftragnehmers und damit ein hohes Plastizitätsniveau der verwendeten Ressourcen gewollt, da die Fähigkeit zur flexiblen Reaktion auf aktuelle Situationsänderungen angestrebt wird. Die Potenziale operativer Anwendungssysteme zur Ausweitung der direktiven Verhaltenssteuerung sind daher als gering und nur in untypischen Sonderfällen bestehend einzustufen.

Die Beobachtung des Auftragnehmerverhaltens als zweiter Ansatzpunkt zur Senkung von Agency-Kosten gestaltet sich bei schlecht strukturierten Problemstellungen besonders schwierig. Verbesserungen in diesem Bereich besitzen sowohl für die Koordination durch Anweisungen als auch für die Koordination durch Selbstabstimmung Relevanz und können potenziell zur Reduzierung von Agency-Kosten beitragen. Die zeit- und raumübergreifenden Kommunikationsfunktionalitäten von CSCW-Systemen ${ }^{355}$ sind prinzipiell als Medium zur Erleichterung der Kontrolle des Auftragnehmers durch den Auftraggeber geeignet. Durch die Vermittlung synchroner Kommunikationsvorgänge werden Möglichkeiten zur ausführlicheren, interaktiven Berichterstattung geschaffen, bei der die Gelegenheit zu Rückfragen durch den Auftraggeber besteht. Die Unterstützung im Bereich der asynchronen Kommunikation ist dagegen weniger interaktiv ausgerichtet und erfordert eine zwischenzeitliche Speicherung der Kommunikationsinhalte. Auf Grund dieser Funktionsweise können die gespeicherten Inhalte zugleich zur Dokumentation von Aktivitäten genutzt werden, auf die der Auftraggeber bei Bedarf zugreifen kann, um das Verhalten des Auftragnehmers einer eingehenderen Überprüfung zu unterziehen.

Auch die Funktionalitäten zur Bereitstellung von Gegenwärtigkeitsinformationen in CSCW-Systemen können dazu genutzt werden, das Auftragnehmerverhalten zu beobachten. Diese Funktionalitäten machen die Speicherung von historischen Informationen über die prozessbezogenen Aktivitäten der Beteiligten notwendig. Durch die Auswertung dieser ohne weitere Maßnahmen zur Verfügung stehenden Informationen lässt sich der Verlauf der jeweiligen Leistungserstellungsprozesse rekonstruieren. Für eine zutreffende Beurteilung des Auftragnehmerverhaltens ist ferner - entsprechend den allgemeinen Aussagen der Agency-Theorie - zusätzlich die Berücksichtigung von Informationen erforderlich, die die Realisierung des externen Risikos betreffen. Bei schlecht strukturierten Aufgabenstellungen ist eine vollständige Beseitigung der hidden action-Problematik allerdings auch von einem noch so umfangreichen Angebot an situationsbezogenen Informationen nicht generell zu erwarten. Die angesprochenen Defizite bei den Fachkenntnissen des Auftraggebers lassen sich dadurch nicht überwinden.

Ebenso wie die Ex ante-Koordination kann sich auch die Ad hoc-Koordination nicht nur auf Transaktionen innerhalb von Unternehmungen, sondern auch auf Transaktionen zwischen Unternehmungen beziehen, deren Koordination sowohl marktliche als auch hierarchische Elemente umfasst. Bei dieser Form des Leistungsaustausches sind die gegenseitigen Ansprüche ebenfalls zum Zeitpunkt des Vertragsschlusses noch nicht vollständig spezifizierbar. Wegen des geringeren Strukturiertheitsgrades der Aufgabenstellung können die Ansprüche der hier betrachteten Ad hoc-Koordination zwischen Unternehmungen tendenziell in noch geringerem Ausmaß expliziert werden

\footnotetext{
${ }^{355}$ Wie oben erläutert können die Kommunikationsfunktionalitäten von CSCW-Systemen im Prinzip auch im Rahmen der Koordination durch Anweisung genutzt werden.
} 
als bei der Ex ante-Koordination. Es sind keine generellen Tätigkeitsfolgen definierbar, die im Laufe der Zusammenarbeit nur noch abgerufen zu werden brauchen. Ein typisches Beispiel für diesen Transaktionstyp stell die gemeinsame Durchführung umfangreicher Projekte unter Beteiligung mehrerer Unternehmungen, etwa im Bereich der Forschung und Entwicklung oder beim Aufbau gemeinsamer Vertriebs- oder Beschaffungskapazitäten, dar. Im Vergleich zum bisher erläuterten, unternehmungsinternen Einsatz von CSCW-Systemen sind dabei tendenziell noch größere zeitliche und räumliche Diskontinuitäten sowie eine höhere Heterogenität der verwendeten Hard- und Software zu überbrücken. ${ }^{356}$

Bei Systemen zur unternehmungsübergreifenden Unterstützung schlecht strukturierter Prozesse können grundsätzlich die gleichen Ebenen der Integration unterschieden werden wie bei Systemen zur unternehmungsübergreifenden Automatisierung der Ablaufsteuerung. Auch hier erfordert die Verknüpfung der technikunterstützten Informationssysteme der beteiligten Unternehmungen auf einer bestimmten Betrachtungs- und Gestaltungsebene die Integration der Systeme auf allen darunter liegenden Ebenen. $\mathrm{Da}$ eine vollständige Vorauskoordination der Prozesse bei diesem Leistungstyp definitionsgemäß nicht möglich ist, sind auf der betriebswirtschaftlichen Ebene weniger umfangreiche Absprachen für die erfolgreiche Abwicklung der Transaktion erforderlich. Die Notwendigkeit zur Abstimmung der technikunterstützten Informationsverarbeitung reduziert sich vielmehr auf technische Aspekte, die Raum für eine flexible inhaltliche Erfüllung der Aufgaben lassen. Da die beteiligten Unternehmungen nur für einen begrenzten Zeithorizont zusammenarbeiten, kommt es im Vergleich zum unternehmungsinternen Einsatz von CSCW-Systemen verstärkt darauf an, die entsprechenden Verknüpfungen der technikunterstützten Informationsverarbeitung schnell auf- und abbauen zu können, sodass der Verwendung von Standards, die in Abschnitt 5.3.4.1 noch näher betrachtet wird, erhöhte Bedeutung zukommt.

Die beschriebenen Möglichkeiten zur Verringerung von Agency-Kosten bei Einsatz der Ad hoc-Koordination im unternehmungsinternen Bereich sind grundsätzlich auf unternehmungsübergreifende Transaktionen übertragbar. Verbesserte Kommunikationsmöglichkeiten schaffen in den oben beschriebenen Grenzen Möglichkeiten zur Ausweitung der direktiven Verhaltenssteuerung auch zwischen Unternehmungen, falls diese im Rahmen der Vertragsvereinbarung vorgesehen ist. Auch die unternehmungsübergreifende Vergabe von Rechten zur Kenntnisnahme und zur Veränderung von Eigenschaften der zu erstellenden Leistung reduziert die Möglichkeiten des Auftragnehmers zur Durchführung von Handlungen, die den Interessen der auftraggebenden Unternehmung zuwiderlaufen. Schließlich können die Auswertungsmöglichkeiten von CSCW-Systemen ebenfalls unternehmungsübergreifend genutzt werden, um die Beobachtbarkeit des Auftragnehmerverhaltens zu verbessern. Durch den gezielten Einsatz von CSCW-Systemen kann das Informationsmanagement deshalb auch bei unternehmungsübergreifenden Prozessen zur Senkung von Transaktions- und Agency-Kosten beitragen und damit in manchen Bereichen eine zwischenbetriebliche Zusammenarbeit überhaupt erst möglich machen.

\footnotetext{
${ }^{356}$ Vgl. Picot/Reichwald/Wigand (2001), S. 319.
} 


\subsubsection{Analytische Funktionalitäten von Anwendungssystemen bei der Erfüllung vertraglicher Pflichten}

Analytisch orientierte Anwendungssysteme stellen neben operativen Systemen die zweite grundsätzliche Ausprägungsform Elektronischer Hierarchien dar. Im Gegensatz zu den bisher behandelten operativen Systemen steht bei diesem Systemtyp die Zielsetzung im Vordergrund, die Entscheidungssituation der auf Grund der Vertragsvereinbarung zuständigen hierarchischen Instanz zu verbessern. Analytische Informationssysteme dienen den Entscheidungsträgern zum zielgerichteten Zugriff auf und zur Auswertung der gespeicherten Informationen im Planungs- und Entscheidungsprozess. ${ }^{357}$ Der Anwendungsschwerpunkt liegt also in der entscheidungsorientierten Versorgung betrieblicher Fach- und Führungskräfte mit Informationen. ${ }^{358}$ Für ein institutionenökonomisch ausgerichtetes Informationsmanagement geht es in diesem Zusammenhang darum, die effiziente Erfüllung von Verträgen zu gewährleisten, die dem relationalen Vertragsmuster entsprechen und während der Vertragslaufzeit eine Koordination durch Anweisungen vorsehen. Aus Sicht der Transaktionskostentheorie sind dabei allgemein die Potenziale analytischer Anwendungssysteme zur Reduzierung von Ex post-Koordinationskosten zu beleuchten. Bei Bezugnahme auf die Agency-Theorie sind dagegen stärker die persönliche Beziehung zwischen Auftraggeber und Auftragnehmer sowie die Reduzierung des hidden action-Problems als Anwendungsschwerpunkt dieses Systemtyps zu fokussieren.

Häufig wird gefordert, den Einsatzbereich analytischer Anwendungssysteme nicht auf die höchsten Führungsebenen der Unternehmung zu beschränken, sondern alle Ebenen der Unternehmung in allen Funktionsbereichen zu unterstützen. ${ }^{359}$ Dieser weite Anwendungsbereich ist auch durch die Neue Institutionenökonomie begründbar, da das relationale Vertragsmuster als Voraussetzung der informationstechnischen Unterstützung durch Elektronische Hierarchien typischerweise auf allen Ebenen des unternehmungsinternen Leistungsaustausches verwendet wird. Folgt man der oben begründeten Auffassung, dass das abstrakte Konstrukt der Transaktion aus Sicht der Neuen Institutionenökonomie die grundlegende Bezugseinheit des Informationsmanagements darstellt, dann ist sogar eine noch weitere Ausdehnung des Anwendungsbereiches anzustreben. Analytische Anwendungssysteme können aus dieser Perspektive auch die Effizienz von solchen Formen des Leistungsaustausches verbessern, bei denen die Leistung zwischen Unternehmungen ausgetauscht wird und die Koordination der Transaktion nicht nur rein marktliche, sondern auch hierarchische Elemente enthält.

Die nachfolgenden Ausführungen orientieren sich vom Aufbau her an der weit verbreiteten Klassifizierung von analytisch orientierten Systemen zur Unterstützung von Führungsaufgaben. ${ }^{360}$ Dabei wird zwischen datenorientierten sowie modell- und methodenorientierten Systemen unterschieden. Datenorientierte Systeme richten sich auf die umfassende Versorgung von Entscheidungsträgern mit den zur Erfüllung ihrer Aufgaben erforderlichen, entscheidungsbezogen aufbereiteten Informationen. Modellund methodenorientierte Systeme dagegen zielen auf die Verbesserung des Urteilsvermögens bei den für die Entscheidung zuständigen Personen. Beide Systemtypen

\footnotetext{
${ }^{357}$ Vgl. Gabriel (1999), S. 418.

${ }^{358}$ Vgl. Chamoni/Gluchowski (1999), S. 4.

${ }^{359} \mathrm{Vgl}$. Gabriel/Chamoni/Gluchowski (2000), S. 88.

${ }^{360}$ Vgl. dazu z.B. Gabriel/Gluchwoski (1997), S. 422ff.; Hess (1999), S. $1503 f$.
} 
besitzen aus Sicht der Neuen Institutionenökonomie unterschiedliche Potenziale zur Steigerung der Effizienz des Leistungsaustausches, die im Folgenden genauer herauszuarbeiten sind.

\section{a) Potenziale modell- und methodenorientierter analytischer Systeme}

Bei der modell- und methodenorientierten Erscheinungsform analytischer Anwendungssysteme steht die Zielsetzung im Vordergrund, in eher schlecht-strukturierten Entscheidungssituationen die Qualität der Entscheidungen durch die informationstechnisch unterstützte Anwendung theoretisch fundierten Fachwissens zu verbessern. Dabei bildet die Problemstrukturierung, Alternativengenerierung und Alternativenbewertung bei erkanntem Problemlösungsbedarf und weniger die Problemerkennung und Wahrnehmung von Signalen den Schwerpunkt des Funktionsumfangs. ${ }^{361}$ Die erkannte Ausgangssituation wird in ein explizites Modell überführt und dann mit einer Methode gelöst, deren Modellaussage schließlich in die Realität zurückzutransformieren ist. Diese Ausprägung analytischer Informationssysteme wird auch als Decision Support Systeme bezeichnet. ${ }^{362}$

Durch den Einsatz dieses Systemtyps lassen sich Ex post-Transaktionskosten reduzieren, indem die Koordinationsaktivitäten der zuständigen hierarchischen Instanz im Laufe der Vertragserfüllung bezüglich des Vorgehens vereinfacht und insbesondere inhaltlich verbessert werden. Das Urteilsvermögen der Entscheidungsträger wird gestärkt. Die Nutzung des Systems versetzt die betreffenden Personen in die Lage, angemessenere Anweisungen an die verschiedenen Aufgabenträger zu geben und dadurch die nach Vertragsschluss stattfindenden Prozesse der Leistungserstellung besser zu koordinieren. Dadurch wird die Fähigkeit zur Reaktion auf aktuelle Entwicklungen, die bei relationalen Verträgen durch die Unvollständigkeit der ursprünglich geschlossenen Vereinbarung systematisch vorgesehen ist, gesteigert. In Bezug auf die Phasen des Managementzyklusses liegt der Anwendungsbereich dieses Systemtyps zwar in erster Linie in der Planungs- und Entscheidungsphase. ${ }^{363}$ Die Systeme können jedoch auch in der Kontrollphase, der aus Sicht der Neuen Institutionenökonomie nach Vertragsschluss eine besonders hohe Bedeutung zukommt, angewendet werden und methodische Unterstützung bei der Analyse von Abweichungen von den ursprünglich geplanten Größen leisten.

Aus Sicht der Agency-Theorie liegt das besondere Potenzial analyseorientierter Anwendungssysteme nach Vertragsschluss im Bereich der Kontrolle von Anstrengungen des Auftragnehmers durch den Auftraggeber. Bei der modell- und methodenorientierten Variante dieses Systemtyps geht es in diesem Zusammenhang weniger um die Bereitstellung von Informationen zur genaueren Beobachtung des Auftragnehmers als um die Beurteilung der betreffenden Beobachtungen. Der für Agency-Beziehungen typische Vorsprung an Fachkenntnissen auf der Seite des Auftragnehmers lässt sich mittels dieser Systeme reduzieren. Die situationsbezogene Anwendung der durch die Systeme zur Verfügung gestellten Modelle und Methoden gibt dem Auftraggeber auch

\footnotetext{
${ }^{361}$ Vgl. Gluchowski/Gabriel/Chamoni (1997), S. 170

${ }^{362} \mathrm{Im}$ Bereich der Analytischen Informationssysteme konnte sich wie in vielen anderen Bereichen auch bisher keine allgemein akzeptierte Begriffsverwendung durchsetzen. Vgl. dazu z.B. Gabriel/Gluchowski (1997), S. 308; Mertens (1998), S. 9f. Die hier verwendeten Begriffe folgen der Terminologie von Gluchowski/Gabriel/Chamoni (1997), S. 147ff.

${ }^{363}$ Vgl. Gluchowski/Gabriel/Chamoni (1997), S. 165.
} 
bei relativ geringen eigenen Fachkenntnissen Anhaltspunkte über die Angemessenheit des Auftragnehmerverhaltens. ${ }^{364}$ Damit werden die Voraussetzungen für eine leistungsgerechte Entlohnung des Auftragnehmers geschaffen. Für eine zutreffende Beurteilung kommt es dabei darauf an, mit Hilfe der verwendeten Modelle und Methoden den Beitrag des Auftragnehmers zum erzielten Ergebnis zu bewerten. Dafür ist es erforderlich, die Realisierung des externen Risikos zu erfassen und deren Auswirkungen auf das letztlich entstandene Ergebnis korrekt abzuschätzen. Gelingt dies, so verbessern die Systeme die Möglichkeiten zur Kontrolle des Agent und verringern auf diese Weise dessen Möglichkeiten zur opportunistischen Ausnutzung von Verhaltensspielräumen.

Auch der durch die Agency-Theorie vorgesehene zweite Typ von Maßnahmen zur Reduzierung der hidden action-Problematik, nämlich die Einschränkung der für die Problematik ursächlichen diskretionären Handlungsspielräume, lässt sich durch den Einsatz modell- und methodenorientierter analytischer Anwendungssysteme unterstützen. Die Voraussetzung dafür ist, dass die im Rahmen der Leistungserstellung zu lösenden Probleme auf Grund ihres Strukturiertheitsgrades durch die Anwendung der bereitgestellten Modelle und Methoden zuverlässig lösbar sind. Wenn unter diesen Bedingungen die Systemnutzung durch den Auftragnehmer erfolgt und dieser verbindlich an die Anwendung gebunden wird, dann kann eine erfolgreiche Einschränkung der Handlungsfreiheit erzielt werden. Bei einem solchen Vorgehen kommt es zu einer mittelbaren Ausweitung der direktiven Verhaltenssteuerung durch den Auftraggeber, da die Reaktion auf das Eintreten bestimmter Situationen während der Vertragslaufzeit durch das System entschieden wird. Dies entspricht konzeptionell einer Reduzierung der Plastizität der vom Auftragnehmer verwendeten Ressourcen, für die die Art der Nutzung mit Hilfe des analytischen Anwendungssystems in verstärktem Maße technisch vorgegeben wird. Die Grenzen, innerhalb derer sich der Auftragnehmer trotz dieser Vorgaben opportunistisch verhalten kann, werden also durch diesen Typ von $\mathrm{Maßnahmen} \mathrm{ebenfalls} \mathrm{eingeengt.}$

\section{b) Potenziale datenorientierter analytischer Anwendungen}

Bei der datenorientierten Erscheinungsform analytischer Anwendungssysteme steht die Zielsetzung im Vordergrund, dem Entscheidungsträger eine umfassende Versorgung mit den für eine möglichst gute Entscheidung benötigten Informationen zu bieten. Die Funktionalitäten der älteren Variante dieses Systemtyps, die auch als Management Information Systeme bezeichnet wird, umfassen vorrangig die Bereitstellung standardisierter, periodisch generierter Berichte, die aus der vorhandenen Datenbasis der Unternehmung gewonnen werden. $^{365}$ Die nachfolgend schwerpunktmäßig betrachtete neuere Variante dieses Systemtyps wird auch als Executive Information Systeme bezeichnet. Gegenüber den Management Information Systemen sind die Executive Information Systeme stärker interaktiv ausgerichtet und bieten Funktionalitäten für die Bereitstellung aktueller entscheidungsrelevanter interner und

\footnotetext{
${ }^{364}$ Dies setzt allerdings voraus, dass der Benutzer die Möglichkeit hat, die Anwendung des Fachwissens durch das System mit Hilfe geeigneter Erklärungskomponenten nachzuvollziehen. Dadurch kann nicht nur das Vertrauen in die ermittelten Ergebnisse auf Seiten des Benutzers gestärkt, sondern auch dessen eigene Urteilsfähigkeit in Bezug auf die jeweilige Problemstellung verbessert werden.

${ }^{365}$ Vgl. Chamoni/Gluchowski (1999), S. $6 f$.
} 
externer Informationen zur Selektion und Analyse über intuitiv benutzbare und individuell anpassbare Benutzungsoberflächen. ${ }^{366} \mathrm{Im}$ Vergleich zu modell- und methodenorientierten analytischen Anwendungssystemen geht es hier weniger um die Anwendung theoretisch fundierter Fachkenntnisse als um einen „explorativen Data Support ${ }^{، 367}$, mit dem frühzeitig Entwicklungstendenzen erkannt und Analysen initiiert werden sollen. Auch die Kontrollphase des Managementzyklusses wird bei diesem Systemtyp weniger durch die Anwendung von Methoden zur Abweichungsanalyse als durch die Gegenüberstellung detaillierter Plan-, Soll- und Ist-Informationen unterstützt.

Wegen der steigenden unternehmungsexternen und unternehmungsinternen Dynamik im Wirtschaftsgeschehen wird die Lösung betriebswirtschaftlicher Probleme immer stärker von der Verfügbarkeit aktueller Informationen abhängig. ${ }^{368}$ Die erfolgreiche Verwendung datenorientierter analytischer Anwendungssysteme wirkt sich vor diesem Hintergrund unmittelbar auf die im Rahmen der Leistungserstellung nach Vertragsschluss entstehenden Transaktionskosten aus, da die Unsicherheit der zuständigen hierarchischen Instanzen bei der Entscheidungsfindung reduziert wird. Damit wird die allgemeine These, dass es durch den Einsatz moderner Informationstechnik zu einer Reduzierung der unternehmungsinternen Koordinationskosten kommt, ${ }^{369}$ unterstützt. Bereits Anfang der 90er Jahre konnte festgestellt werden, der Technologieschub der vorherigen Dekade habe völlig neue Präsentationsformen und Zugriffe auf Informationen ermöglicht, die dem Management eine neue Qualität und Aktualität der Informationsaufbereitung versprechen. ${ }^{370}$ Diese Entwicklung setzte sich auch in den 90er Jahren in ähnlicher Weise fort. Tendenziell wird die Effizienz der Koordination durch relationale Verträge erhöht, wenn mittels datenorientierter analytischer Anwendungssysteme als Ausprägungsform Elektronischer Hierarchien eine höhere Verfügbarkeit aktueller und zielorientiert aufbereiteter Informationen bei den zuständigen hierarchischen Instanzen realisiert werden kann.

Der potenzielle Anwendungsbereich analyseorientierter Informationssysteme wird in der Literatur üblicherweise überall dort verortet, wo dispositive bzw. analytische Aufgaben in Organisationen zu lösen sind. ${ }^{371}$ In diesem Abschnitt wurde bereits einleitend im Gegensatz dazu gefordert, den Anwendungsbereich analytischer Anwendungssysteme nicht auf unternehmungsintern koordinierte Transaktionen zu beschränken, sondern diesen Systemtyp auch bei unternehmungsübergreifenden Transaktionen mit einer Kombination marktlicher und hierarchischer Koordinationselemente einzusetzen. Eine derartige Ausdehnung des Einsatzgebietes erfordert entsprechende inhaltliche Ergänzungen des Datenbestands. Die Aufbereitung der Daten in analyseorientierten Anwendungssystemen orientiert sich bisher auf Grund der unternehmungsinternen Fokussierung typischerweise am Absatzbereich oder an internen Verantwortungsbereichen der Unternehmung. ${ }^{372}$ Bei der Planung, Steuerung und Kontrolle dauerhafter, unternehmungsübergreifender Transaktionsbeziehungen steht dagegen die Beurteilung von Lieferanten im Vordergrund. Es sind deshalb Auswertungsmöglichkeiten zu schaffen, die sich auf den Beschaffungsbereich beziehen und die

\footnotetext{
${ }^{366}$ Vgl. Gluchowski/Gabriel/Chamoni (1997), S. 203.

${ }^{367}$ Jahnke (1993), S. 31.

${ }^{368}$ Vgl. Gabriel (1999), S. 418.

${ }^{369} \mathrm{Vgl}$. Gurbaxani/Whang (1991), S. 68.

${ }^{370}$ Vgl. Miksch (1991), S. 12.

${ }^{371} \mathrm{Vgl}$. Gabriel/Chamoni/Gluchowski (2000), S. 87f. Hervorhebung durch den Verfasser.

${ }^{372}$ Vgl. Gabriel/Chamoni/Gluchowski (2000), S. 88.
} 
Analyse des Lieferantenverhaltens ermöglichen. Um eine möglichst hohe Transparenz zu erreichen, kann es vorteilhaft sein, wenn der Lieferant als Auftragnehmer dem Kunden als Auftraggeber die eigenen Planungs- und Kontrolldaten zur Übernahme in dessen Datenbestand zugänglich macht. Dies ist bei längerfristigen Kooperationen auch aus Sicht des Lieferanten sinnvoll, da durch eine freiwillige Offenbarung interner Informationen zum einen das Vertrauen des Kunden steigt und zum anderen eine bessere Aufteilung des externen Risikos auf Auftraggeber und Auftragnehmer erreicht werden kann.

Moderne Varianten dieses Systemtyps sollten neben unternehmungsinternen auch unternehmungsexterne Daten sowie neben quantitativen auch qualitative Daten integrieren. ${ }^{373}$ Viele Auswertungen und Analysen erlangen erst durch den Vergleich mit unternehmungsexternen Informationen signifikante Bedeutung und ermöglichen zieladäquate Entscheidungen. ${ }^{374}$ Durch die Erfüllung dieser Anforderungen kann der potenzielle Anwendungsbereich datenorientierter analytischer Systeme auch auf besonders schlecht strukturierte Probleme ausgedehnt werden, bei denen die im vorigen Abschnitt erläuterten modell- und methodenorientierten analytischen Anwendungen nur begrenzt einsetzbar sind. Unter transaktionskostentheoretischen Gesichtspunkten ergeben sich somit neue Möglichkeiten für das Informationsmanagement, die Informationsversorgung mittels analytischer Anwendungssysteme auf die Ebene des TopManagements auszudehnen und dessen Koordinationsaufgaben effektiv zu unterstützen. Gerade die Aufgaben des Top-Managements sind durch einen geringen Strukturiertheitsgrad und eine hohe Bedeutung von Informationen aus der Unternehmungsumwelt, die häufig nicht quantifizierbar sind, gekennzeichnet. ${ }^{375}$

Aus der Perspektive der Agency-Theorie betrachtet können datenorientierte analytische Anwendungssysteme zunächst einmal den Auftragnehmer bei der Erstellung der Leistung unterstützen und somit eine Verringerung des verbleibenden Wohlfahrtsverlustes bewirken. Ferner sind sie geeignet, das Potenzial zur besseren Nachverfolgung der Aktivitäten des Auftragnehmers zu erschließen, das dem Einsatz moderner Informationstechnik ebenfalls in allgemeiner Form zugeschrieben wird. ${ }^{376}$ Durch die Verwendung dieser Systeme wird insbesondere das Monitoring während der Vertragslaufzeit, also die laufende Überwachung des Auftragnehmers durch den Auftraggeber, vereinfacht. ${ }^{377}$ Dabei wird die hidden action-Problematik mit der Gefahr des moral hazard reduziert, indem der Auftraggeber in die Lage versetzt wird, die Aktivitäten des Auftragnehmers leichter zu beobachten. Auf diese Weise verringert sich für den Auftragnehmer die Möglichkeit zur opportunistischen Ausnutzung bestehender Verhaltensspielräume bei der Erfüllung der vertraglich vereinbarten Verpflichtungen.

Im Vergleich zur Sichtweise der Transaktionskostentheorie, die die Unterstützung der hierarchisch übergeordneten Instanz in den frühen Phasen des Managementzyklusses betont, verschiebt sich aus Sicht der Agency-Theorie der Anwendungsschwerpunkt dieses Systemtyps. Unter agency-theoretischen Gesichtspunkten steht bei der Unterstützung des Auftraggebers durch datenorientierte analytische Anwendungs-

\footnotetext{
${ }^{373}$ Vgl. Meier (1998), S. 105f; Mertens (1998), S. $10 f$.

${ }^{374} \mathrm{Vgl}$. Behme/Mucksch (1998), S. 85.

${ }^{375}$ Vgl. Gluchowski/Gabriel/Chamoni (1997), S. 18.

${ }^{376}$ Vgl. Gurbaxani/Whang (1991), S. 67.

${ }^{377}$ Vgl. Hess (1999), S. 1506.
} 
systeme die Kontrollphase im Vordergrund. Dies entspricht der grundsätzlichen Annahme der Agency-Theorie, dass die vollständige Übernahme von Entscheidungszuständigkeiten durch den Auftraggeber in vielen Fällen auf Grund des spezifischen Qualifikationsvorteils des Auftragnehmers gar nicht gewünscht ist. Bei einer weitgehenden Delegation von Entscheidungskompetenzen, die wegen dieses Qualifikationsvorteils häufig als vorteilhaft anzusehen ist, kann die eigentliche Anwendung des Systems in der frühen Planungsphase durch den Auftragnehmer auf der hierarchisch untergeordneten Ebene der Unternehmung erfolgen. Der Auftraggeber auf der hierarchisch übergeordneten Ebene nutzt dann in erster Linie die Dokumentationsfunktionalität des Systems, die nicht nur den Zugriff auf Informationen zur eingetretenen Ist-Situation, sondern auch auf Informationen zur ursprünglichen Planung des Auftragnehmers ermöglicht.

Die vergleichsweise geringe Strukturiertheit der Aufgabenstellungen im Anwendungsbereich datenorientierter analytischer Anwendungssysteme erfordert große Handlungsfreiräume bei der Erfüllung der betreffenden Aufgabenstellungen. Eine Einengung der diskretionären Handlungsspielräume des Auftragnehmers, die generell den zweiten von der Agency-Theorie vorgeschlagenen Ansatzpunkt zur Reduzierung der hidden action-Problematik darstellt, kommt unter diesen Voraussetzungen nicht in Frage. Deshalb bleibt, wie Abbildung 5/15 zeigt, als einziger erfolgversprechender Weg zur Senkung der Agency-Kosten nach Vertragsschluss die Beobachtung des Auftragnehmers.

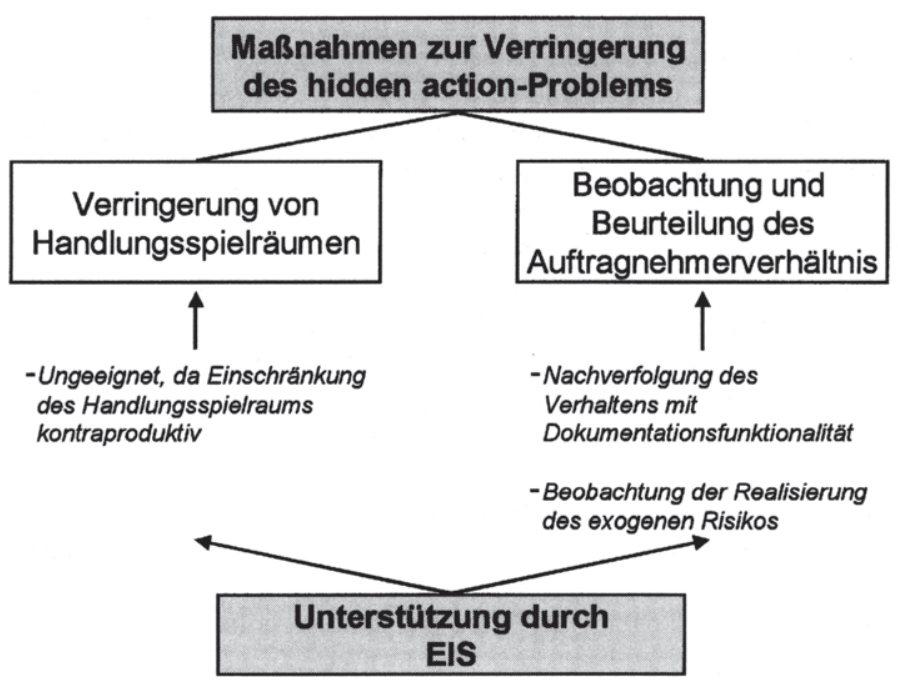

Abb. 5/15: Reduzierung des hidden action-Problems mit Hilfe von Executive Information Systemen

Auf Grund der beschriebenen typischen Eigenschaften der durch datenorientierte analytische Anwendungssysteme unterstützten Aufgabenstellungen ist bei diesem Leistungstyp davon auszugehen, dass das durch den Auftragnehmer produzierte Leistungsergebnis in besonderem Maße dem Einfluss des exogenen Risikos ausgesetzt ist. Eine rein auf das Verhalten im Rahmen der Leistungserstellung beschränkte 
Beobachtung reicht in solchen Fällen nicht aus, um die Leistung des Auftragnehmers adäquat beurteilen zu können. Vielmehr werden zusätzlich die Informationen zur Realisierung des exogenen Risikos benötigt, die durch das jeweilige Anwendungssystem ebenfalls bereitzustellen sind.

Wie im Abschnitt 5.2.3.4 erläutert, hängt von der Höhe des exogenen Risikos die Beeinflussbarkeit des Leistungsergebnisses durch den Auftragnehmer ab. Wenn datenorientierte analytische Anwendungssysteme zusätzlich zu den Informationen über das Verhalten des Auftragnehmers auch Informationen über die Realisierung des exogenen Risikos verfügbar machen, dann wird der Auftraggeber in die Lage versetzt, ein differenzierteres Urteil über die Anstrengungen des Auftragnehmers zu fallen. Infolgedessen erscheint es gerechtfertigt, die in der Agency-Theorie üblicherweise getroffene Annahme, dass die Beobachtung des exogenen Risikos durch den Auftraggeber nicht möglich ist, zu relativieren. Das Informationsmanagement kann auf eine Überwindung der zu Gunsten des Auftragnehmers bestehenden Informationsasymmetrien hinwirken und damit dessen Möglichkeiten reduzieren, durch opportunistisches Verhalten gegen die Interessen des Auftraggebers zu verstoßen. Die Voraussetzung für eine Umsetzung dieser Maßnahme ist allerdings, dass dem Auftraggeber die Größen, von denen das erzielte Leistungsergebnis im konkreten Einzelfall abhängt, bekannt sind. Im Hinblick auf die bereits angesprochenen systematischen Qualifikationsvorsprünge des Auftragnehmers ist daher davon auszugehen, dass in vielen Fällen keine vollständige Aufhebung der für die Gefahr des Moral Hazard ursächlichen Informationsasymmetrie erreicht werden kann.

Grundsätzlich kann das exogene Risiko einer Agency-Beziehung sowohl von Entwicklungen, die zwar unternehmungsintern sind, jedoch nicht im Verantwortungsbereich des Auftragnehmers liegen, als auch von unternehmungsexternen Einflussgrößen abhängen. Je stärker der strategische Charakter der im Rahmen der Leistungserstellung zu treffenden Entscheidungen ausgeprägt ist, umso größere Bedeutung besitzen unternehmungsexterne Entwicklungen für den letztendlich erzielten Erfolg. ${ }^{378}$ Es ist in solchen Fällen erforderlich, die unternehmungsindividuelle Entwicklung der unternehmungsexternen, gesamtwirtschaftlichen oder branchenbezogenen Entwicklung gegenüberzustellen. ${ }^{379}$ Für eine effiziente Unterstützung der Planung, Steuerung und Kontrolle von strategischen Maßnahmen müssen Executive Information Systeme deshalb über Schnittstellen verfügen, die die Übernahme externer Informationen, die nicht bereits innerhalb der Unternehmung vorliegen, in den eigenen Datenbestand erlauben. Dabei ist zu beachten, dass unternehmungsexterne Daten im Gegensatz zu unternehmungsinternen Daten, die üblicherweise mit relativ einfachen Datenbank- und Rechenoperationen aus den operativen Anwendungssystemen extrahiert werden können, typischerweise ein nach Quelle und Art deutlich höheres Heterogenitätsniveau aufweisen. $^{380}$

In der Praxis enthalten datenorientierte analytische Anwendungssysteme bisher in erster Linie unternehmungsinterne Informationen. ${ }^{381}$ Als Quellen für externe Informationen kommen beispielsweise Fachpublikationen und Berichte von Marktforschungsinstituten

\footnotetext{
${ }^{378} \mathrm{Vgl}$. ähnlich Meier (1998), S. 105.

${ }^{379}$ Vgl. Mertens (1998), S. 16ff;; Schönert (1998), S. 74; Uhr/Kosilek/Schaffrath (1998), S. 53.

${ }^{380}$ Vgl. Meier (1998), S. 107; Behme/Mucksch (1998), S. 91.

${ }^{381}$ Vgl. Meier (1998), S. 105.
} 
in Frage, die zum Teil manuell in den Datenbestand übernommen werden müssen. ${ }^{382}$ Ein exponentielles Wachstum der Quellen für externe Informationen ist ferner im Internet, insbesondere im World Wide Web zu beobachten, das langfristig bei der Beschaffung externer Informationen im Vordergrund stehen dürfe. ${ }^{383}$ Die systematische Nutzbarmachung von Inhalten des World Wide Web zur Verbesserung der eigenen Entscheidungssituation wird derzeit unter dem Schlagwort Web Farming propagiert. ${ }^{384}$ Ein Web Farming-System stellt ein Vorsystem für Executive Information Systeme dar, das analog zu den operativen Datenbanksystemen Input für den eigenen Datenbestand liefert. ${ }^{385}$ Der Prozess des Web Farming kann in vier Phasen unterteilt werden. Dazu gehören die Suche nach verfügbaren Quellen für die benötigten Inhalte im World Wide Web, die Extraktion der Daten, die Aufbereitung der Daten entsprechend der Strukturierung des eigenen Datenbestandes sowie die Bekanntmachung der aktualisierten Informationen bei den Nutzern des Systems. ${ }^{386}$

Die mit dem Web Farming verbundenen Problemstellungen betreffen in erster Linie technische Fragen und können daher nicht mit den Mitteln der Neuen Institutionenökonomie und insbesondere der Agency-Theorie analysiert werden. Für die AgencyTheorie stehen vielmehr anwendungsbezogene Fragen der Nutzung der entsprechenden Systeme im Vordergrund. Wegen der Vielschichtigkeit der Entscheidungsprobleme im Anwendungsbereich datenorientierter analytischer Anwendungssysteme setzt sich das $\mathrm{zu}$ beobachtende exogene Risiko typischerweise wiederum aus einer Reihe von Einflussgrößen zusammen, die erst in ihrer Gesamtheit eine zutreffende Beurteilung des Auftragnehmerverhaltens zulassen. Ähnlich wie im Bereich der Früherkennung liegt die zentrale Problemstellung deshalb in der Zusammenfügung einzelner Entwicklungen, die jeweils durch Kennzahlen und Indikatoren erfasst werden, zu einem komplexen, realitätsnäheren Gesamtbild. ${ }^{387}$ Für eine verlässliche Beurteilung des Leistungsergebnisses ist ferner ein Verständnis der Wirkungszusammenhänge zwischen den Vorgängen im Verantwortungsbereich des Auftragnehmers und den externen Einflussgrößen erforderlich, die durch die datenorientierte Variante analytischer Anwendungssysteme nicht gewährleistet werden kann. Für eine möglichst vollständige Beseitigung der Informationsasymmetrie zu Gunsten des Auftragnehmers sind deshalb datenorientierte mit modell- und methodenorientierten Funktionalitäten analytischer Anwendungssysteme zu kombinieren. ${ }^{388}$

\subsubsection{Informationsmanagement bei der Festlegung des Vertragsdesigns}

Entsprechend dem in Abschnitt 5.2.4 skizzierten Vorgehen haben die bisherigen Überlegungen sich auf die Gestaltungsmöglichkeiten des Informationsmanagements in

\footnotetext{
${ }^{382} \mathrm{Vgl}$. Holten/Ehlers (1998), S. 124.

${ }^{383} \mathrm{Vgl}$. Behme/Mucksch (1998), S. 85; Holten/Ehlers (1998), S. 124.

${ }^{384}$ Der Begriff des Web Farming geht auf Hackathorn (1996), passim, zurück.

${ }^{385} \mathrm{Vgl}$. Hackathorn (1999), information flow.

${ }^{386}$ Vgl. Hackathorn (1999), refining information.

${ }^{387}$ Vgl. dazu Schönert (1998), S. 79.

${ }^{388}$ Die Kombination der beiden Varianten analytischer Anwendungssysteme wird als Executive Support System bezeichnet. Vgl. Gluchowski/Gabriel/Chamoni (1997), S. 241ff. Bei diesem Systemtyp handelt es sich bisher mehr um einen konzeptionellen Ansatz als um eine bereits verfügbare Klasse von Softwaresystemen. Zukünttige Weiterentwicklungen in diesem Bereich eroffnen aus Sicht der Agency-Theorie große Potenziale zur erheblichen Verringerung der hidden action-Problematik.
} 
den verschiedenen Transaktionsphasen gerichtet, ohne das für die Abwicklung der Transaktion verwendete vertragliche Arrangement selbst zu hinterfragen. Diese Beschränkung wird im Folgenden aufgehoben und die Ausführungen wenden sich der Frage zu, inwiefern es durch die Maßnahmen des Informationsmanagements zu einer Veränderung der Vorteilhaftigkeit von Vertragsdesigns kommen kann.

Bei der Untersuchung des Vertragsdesigns werden die Berührungspunkte zwischen der Transaktionskostentheorie und der Agency-Theorie besonders deutlich, da die theoretischen Konzepte, mit denen diese beiden Zweige der Neuen Institutionenökonomie den Problemkreis analysieren, eine sehr hohe Ähnlichkeit aufweisen. Der Ausgangspunkt der Überlegungen innerhalb der Transaktionskostentheorie liegt in dem Phänomen der Spezifität. Das Vorhandensein von Spezifität begründet Abhängigkeiten von einmal getroffenen Entscheidungen und verursacht Wechselkosten, wenn das jeweilige Spezifitätsobjekt einem anderen Verwendungszweck zugeführt werden soll. ${ }^{389}$ Der Spezifität einer Transaktion kommt deshalb kritische Bedeutung bei der Festlegung des Vertragsdesigns zu. Die Agency-Theorie dagegen stellt auf die Erbringung von Vorleistungen durch eine oder beide Vertragsparteien ab. Da das Vorhandensein von Vorleistungen wiederum eine mögliche Ursache (neben weiteren) für die Spezifität einer Transaktion darstellt, ist das Untersuchungsfeld der Transaktionskostentheorie in diesem Punkt etwas allgemeiner abgegrenzt als das der Agency-Theorie. Die Folgeprobleme, die sich aus dem Auftreten von Spezifität bzw. aus der Existenz von Vorleistungen ergeben, sind jedoch bei beiden Forschungsrichtungen gleichartig. Sie liegen in einer Abhängigkeit vom jeweils anderen Vertragspartner, die durch ein entsprechendes Vertragsdesign zu berücksichtigen ist.

Im Folgenden werden diese allgemeinen Überlegungen auf den Bereich der technikunterstützten Informationsverarbeitung übertragen. In Abschnitt 5.3.3.1 ist zunächst zu überprüfen, inwiefern sich durch entsprechende Gestaltungsmaßnahmen des Informationsmanagements Einfluss auf die Spezifität einer Transaktion, die die bedeutendste Transaktionseigenschaft bei der Festlegung des Vertragsdesigns darstellt, nehmen lässt. Dabei steht insbesondere die Höhe der Vorleistungen im Vordergrund, die im Gestaltungsbereich des Informationsmanagements für die erfolgreiche Durchfuihrung einer Transaktion zu erbringen sind. Anschließend werden in Abschnitt 5.3.3.2 die verschiedenen Vertragsdesigns betrachtet. Es wird untersucht, inwiefern die Effizienz der Vertragsmuster selbst durch die in diesem Kapitel beschriebenen Gestaltungsoptionen des Informationsmanagements berührt wird. Aus der gemeinsamen Betrachtung beider Entwicklungen können in Abschnitt 5.3.3.3 Aussagen zur Veränderung der Vorteilhaftigkeit alternativer Koordinationsformen gewonnen werden.

\subsubsection{Gestaltung von Vorleistungen und Transaktionsspezifität durch das Infor- mationsmanagement}

Die folgenden Betrachtungen richten sich zunächst auf die Objekte im Bereich der technikunterstützten Informationsverarbeitung, die durch das Informationsmanagement mehr oder weniger spezifisch ausgestaltet werden können. Danach werden verschiedene Ebenen unterschieden, auf denen die Gestaltung der Spezifität stattfindet. Abschließend wird untersucht, inwiefern die traditionellen Annahmen der Neuen

${ }^{389} \mathrm{Vgl}$. Gersch (1998), S. 91. 
Institutionenökonomie über den Zusammenhang zwischen Spezifität und Produktivität für den Gestaltungsbereich des Informationsmanagements zu modifizieren sind.

\section{a) Spezifitätsobjekte im Gestaltungsbereich des Informationsmanagements}

Im Abschnitt 5.2.2.2 wurden die verschiedenen Typen von Spezifitätsobjekten im Bereich der technikunterstützten Informationsverarbeitung angesprochen. Dabei handelt es sich um Leistungsergebnisse, Leistungserstellungsprozesse sowie Leistungsfähigkeiten im Zusammenhang mit der Herstellung informationeller Produkte. Diese Objekte können mehr oder weniger stark auf die Durchführung einer speziellen Transaktion bzw. eines Transaktionstyps ausgerichtet sein.

Die von der Agency-Theorie fokussierten Vorleistungen betreffen solche Objekte, deren Spezifität bereits vor der endgültigen Durchführung der Transaktion mit dem Ziel der Einrichtung einer längerfristigen Zusammenarbeit zwischen den Vertragspartnern aufgebaut wird. Geht man davon aus, dass bei dauerhaften Kooperationen zwischen den Vertragspartnern die eigentliche Erstellung der Leistung integrativ und somit erst nach Vertragsabschluss erfolgt, dann betreffen Vorleistungen generell nicht die Leistungsergebnisse, da diese zum Zeitpunkt des Vertragsschlusses noch nicht vorliegen. Die Leistungsergebnisse als Spezifitätsobjekte im Bereich der Informationsverarbeitung werden wegen dieses fehlenden Vorleistungscharakters im Folgenden nicht weiter untersucht. Vorleistungen im engeren Sinne betreffen vielmehr ausschließlich die Leistungsfähigkeiten im Bereich der Informationsverarbeitung. Wie die folgenden Überlegungen zeigen, können auf indirektem Wege jedoch auch die Leistungserstellungsprozesse zu Vorleistungen führen. Aus diesem Grund bezieht sich die nachfolgende Betrachtung der Spezifitätsgestaltung durch das Informationsmanagement auf diese beiden Objekttypen.

Bei den Leistungsfähigkeiten im Gestaltungsbereich des Informationsmanagements können Vorleistungen sowohl die menschlichen als auch die technischen Elemente des technikunterstützten Informationssystems der Unternehmung betreffen. Unternehmungsinterne Vorleistungen im technischen Bereich können etwa in der Anschaffung spezifischer hardwaretechnischer Komponenten oder in der individuellen Entwicklung spezieller Anwendungssoftware, die zur Erstellung einer bestimmten Leistung genutzt werden soll, bestehen. Im Bereich des Humankapitals liegen korrespondierende Vorleistungen beispielsweise in der Aneignung von Fachkenntnissen über die Nutzung der für die betreffende Transaktion eigens angeschafften technischen Komponenten, die nicht direkt auf andere Transaktionen übertragbar sind.

Auch zum Aufbau einer unternehmungsübergreifenden Informationsverarbeitung im Rahmen einer längerfristigen Zusammenarbeit sind häufig spezifische Investitionen in die hard- und softwaretechnische Infrastruktur notwendig, aus denen sowohl Einstiegsals auch Umstiegskosten resultieren. ${ }^{390}$ Zur Errichtung einer derartigen Infrastruktur müssen die beteiligten Mitarbeiter die jeweiligen systemtechnischen Infrastrukturen der Vertragspartner miteinander verknüpfen. Dies erfordert neben der Anschaffung der entsprechenden technischen Komponenten auch den Erwerb detaillierter transaktionsbezogener Kenntnisse über die Situation der kooperierenden Unternehmungen durch die beteiligten Mitarbeiter. Dementsprechend werden beim

\footnotetext{
${ }^{390}$ Vgl. Picot/Reichwald (1991), S. 296, ăhnlich auch Schüppler (1998), S. 18.
} 
Aufbau einer unternehmungsübergreifenden Informationsverarbeitung auch für das der Unternehmung zur Verfügung stehende Humankapital spezielle Leistungsfähigkeiten aufgebaut, die nicht ohne weiteres für andere Transaktionstypen verwendbar sind.

Die Durchführung spezifischer Prozesse der Informationsverarbeitung findet bei integrativer Leistungserstellung erst nach dem erfolgreichen Abschluss eines Vertrages im Rahmen der Abwicklung der Transaktion statt. Dabei handelt es sich nicht unmittelbar um Vorleistungen, da vor der Durchführung der Transaktion keine einseitigen Investitionen zu tätigen sind, deren Rückflüsse durch opportunistisches Verhalten der anderen Vertragsseite gefährdet werden können. Bei einer längerfristigen Zusammenarbeit können jedoch im Laufe der Zeit ebenfalls Bindungswirkungen entstehen, wenn die Prozesse der Informationsverarbeitung immer stärker auf den Leistungsaustausch mit dem speziellen Vertragspartner ausgerichtet werden. Die menschlichen Aufgabenträger der Informationsverarbeitung erwerben dabei mehr und mehr partnerspezifisches Wissen, dessen Wert ganz wesentlich von der Fortsetzung der Zusammenarbeit abhängt. Eine ähnliche Entwicklung ist auch in Bezug auf die technischen Komponenten des technikunterstützten Informationssystems der Unternehmung zu erwarten, da zur Verbesserung der Prozesse im Laufe der Kooperation zahlreiche Parametrisierungen sowie kleinere und größere funktionale Anpassungen der Hard- und Software vorgenommen werden. Eine Beendung der Zusammenarbeit vermindert hier ebenfalls die Nutzbarkeit der Ressourcen und verursacht Umstellungskosten.

Indirekt kann die Einrichtung transaktions- oder partnerspezifischer Prozesse also ebenfalls den Charakter einer Vorleistung aufweisen, da sie im Laufe der Zeit eine vergleichbare Bindungswirkung entfaltet. Im Rahmen der Durchführung derartiger Prozesse im laufenden Betrieb können - bewusst oder unbewusst - spezifische Leistungsfähigkeiten entstehen, die ein Interesse an der Beibehaltung des Vertragspartners begründen und dadurch eine mehr oder weniger starke Abhängigkeitssituation verursachen. Daher ist davon auszugehen, dass der Spezifitätsgrad der Leistungsfähigkeiten im Bereich der Informationsverarbeitung weitgehend dem Spezifitätsgrad der Leistungserstellungsprozesse folgt. Auf Gund dieser Wirkungskette stellt die Spezifität von Prozessen der Informationsverarbeitung eine kritische Gestaltungsvariable dar, die die Festlegung des Vertragsdesigns beeinflussen kann. Im Folgenden wird die Frage untersucht, inwiefern durch das Informationsmanagement eine aktive Gestaltung dieser Parameter möglich ist und welche Gestaltungsrichtung aus institutionenökonomischer Sicht Vorteile für die Koordination des Leistungsaustausches aufweist.

\section{b) Ebenen der Spezifitätsgestaltung}

Die in der Literatur häufig vertretene Annahme eines zwingenden Kausalzusammenhangs zwischen spezifischen Leistungsergebnissen, spezifischen Leistungserstellungsprozessen und spezifischen Leistungsfahigkeiten der Unternehmung ist in dieser pauschalen Form abzulehnen. ${ }^{391}$ Eine spezifische Leistung muss nicht zwingend durch in gleichem Maße spezifische Prozesse und Ressourcen erbracht werden, wenn die Spezifität des Ergebnisses durch die Kombination von standardisierten Modulen

${ }^{39}$ Vgl. Gersch (1998), S. 143ff. 
erreicht wird. ${ }^{392}$ Demzufolge können die zur Erstellung nachfragerspezifischer Teilleistungen verwendeten Prozesse und Potenziale einen mehr oder weniger hohen Spezifitätsgrad aufweisen und damit zum Gegenstand von Effektivitäts- und Effizienzkalkülen werden. Allgemein wird in diesem Zusammenhang im Rahmen der Transaktionskostentheorie eine höhere Produktivität von spezifischen Prozessen und Potenzialen vermutet, die Kosten- und/oder Qualitätsvorteile gegenüber unspezifischen Prozessen und Potenzialen ermöglicht. ${ }^{393}$ Diese größere Effizienz wird jedoch durch eine im Vergleich zu unspezifischen Prozessen und Potenzialen geringere Flexibilität erkauft, die die Übertragung der Ressourcen auf andere Verwendungsarten erschwert.

Nachfolgend wird untersucht, inwiefern diese Annahmen im Gestaltungsbereich des Informationsmanagements zutreffend sind. In Bezug auf die Leistungsfähigkeiten im Bereich der Informationsverarbeitung wird auf Grund der oben erläuterten Überlegungen angenommen, dass deren Spezifitätsgrad dem Spezifitätsgrad der Leistungserstellungsprozesse weitgehend folgt. Demnach stellen in erster Linie die Leistungserstellungsprozesse im Bereich der Informationsverarbeitung einen Ansatzpunkt des Informationsmanagements zur Trennung der Spezifität von Leistungsergebnis und eingesetzten Ressourcen dar. Für eine nähere Untersuchung ist die Bezugnahme auf die in Abschnitt 5.3.2.2 unterschiedenen Betrachtungs- und Gestaltungsebenen, auf denen eine Abstimmung der Informationsverarbeitung zwischen den Transaktionspartnern erforderlich ist, hilfreich. Der Fokus liegt dabei auf dem Leistungsaustausch zwischen Unternehmungen und somit auf der unternehmungsübergreifenden Informationsverarbeitung, eine vergleichbare Abstimmung ist jedoch auch im Falle der unternehmungsinternen Durchfürung der betreffenden Transaktion erforderlich.

Wenn die beschriebenen Annahmen der Transaktionskostentheorie auch fur den Gestaltungsbereich des Informationsmanagements zutreffen, dann müsste bei der unternehmungsübergreifenden Informationsverarbeitung ebenfalls ein Verdrängungseffekt zwischen der Flexibilität und der Produktivität der verwendeten Ressourcen zu beobachten sein. Spezifische Anwendungssysteme zur Unterstützung des unternehmungsübergreifenden Informationsaustausches im Rahmen einer längerfristigen Zusammenarbeit müssten eine höhere Qualität und/oder niedrigere Kosten bieten als standardisierte Anwendungssysteme. Auf der anderen Seite müssten standardisierte Anwendungssysteme eine höhere Flexibilität aufweisen und dadurch einen leichteren Wechsel des Vertragspartners zulassen.

Die Möglichkeit zum Wechsel des Vertragspartners setzt im Gestaltungsbereich des Informationsmanagements die technische und organisatorische Kompatibilität der im Rahmen der unternehmungsübergreifenden Informationsverarbeitung eingesetzten Ressourcen voraus. Kompatibilität bedeutet wörtlich „Vereinbarkeit ${ }^{\text {(394 }}$ und erfordert allgemein, dass die Anwendungssysteme von Sender und Empfänger furr die erfolgreiche Durchführung eines Informationsaustausches aufeinander abgestimmt sind. $^{395}$ In Bezug auf die unternehmungsübergreifende Informationsverarbeitung

\footnotetext{
${ }^{392}$ Vgl. dazu Gersch (1998), S. 130ff.; Engelhardt/Freiling (1995b), S. 42. Als grundsätzliche Formen der Leistungsindividualisierung kommen die Individualisierung der Grundleistung, die Individualisierung durch Zusatzleistungen und die Individualisierung durch ein Baukastensystem in Frage. Vgl. dazu im Einzelnen Gersch (1998), S. 128f.

${ }^{393}$ Vgl. z.B. Williamson (1979), S. 241; Ebers/Gotsch (1999), S. 228; Gersch (1998), S. 146.

${ }^{394} \mathrm{Vgl}$. Voss/Raabe (1997), S. 380.

${ }^{395}$ Vgl. Picot/Reichwald/Wigand (1996), S. 301.
} 
bezeichnet Kompatibilität den Sachverhalt, dass die technikunterstützten Informationssysteme der beteiligten Unternehmungen miteinander verknüpft werden können. Inkompatibilitäten zwischen den verwendeten Systemen potenzieller alternativer Vertragspartner führen zu Bindungen an den bisherigen Vertragspartner (LockinEffekte), da sie den reibungslosen Übergang von einem Vertragspartner zum nächsten verhindern oder zumindest erschweren. Umgekehrt reduziert ein hohes allgemeines Kompatibilitätsniveau des technikunterstützten Informationssystems der Unternehmung die Notwendigkeit von Anpassungsmaßnahmen beim Wechsel des Vertragspartners. Die in Abschnitt 5.3.2.2 erläuterten Untersuchungs- und Gestaltungsebenen des unternehmungsübergreifenden Informationsaustausches können im hier untersuchten Zusammenhang als Kompatibilitäts- bzw. Spezifitätsstufen aufgefasst werden, auf denen jeweils eigene Vereinbarungen zwischen den Transaktionspartnern zu treffen sind.

Das bei der Entscheidung für ein nicht-marktliches Vertragsdesign verfolgte Ziel der Erhöhung von Produktivität und Qualität der Informationsverarbeitung erfordert eine möglichst weitgehende Integration der technikunterstützten Informationssysteme. ${ }^{396}$ Je höher die Ebene, auf der die Systeme der beteiligten Unternehmungen miteinander verknüpft sind, angesiedelt ist, desto mehr Potenzial bietet sich zur Erzielung von Effizienzvorteilen $^{397}$ gegenüber einer marktlichen Koordination des Leistungsaustausches. Im Rahmen einer längerfristigen Zusammenarbeit werden die Vertragspartner daher bestrebt sein, eine Integration ihrer technikunterstützten Informationssysteme auf einem möglichst hohen Niveau zu erreichen. Allerdings wird bei der Verknüpfung der Systeme auf der jeweiligen Gestaltungsebene Spezifität geschaffen, wenn durch die Entwicklung individueller Lösungen die Kompatibilität des eigenen Systems mit den Systemen potenzieller anderer Vertragspartner aufgegeben wird. Der als allgemeingültig angenommene Zusammenhang zwischen Produktivität und Flexibilität bestätigt sich daher grundsätzlich auch für den Bereich der unternehmungsübergreifenden Informationsverarbeitung. Dieses Ergebnis ist in Abbildung 5/16 grafisch dargestellt.

\footnotetext{
${ }^{396} \mathrm{Vg}$. Buxmann/König (2000), S. 50.

${ }^{397}$ Derartige Effizienzvorteile können beispielsweise durch die Vermeidung von Mehrfacheingaben, die Beseitigung von inkonsistenten Datenbeständen, die Beschleunigung der Prozesssteuerung u.v.m. erreicht werden. Vgl. Mertens/Griese/Ehrenberg (1998), S. 69.
} 

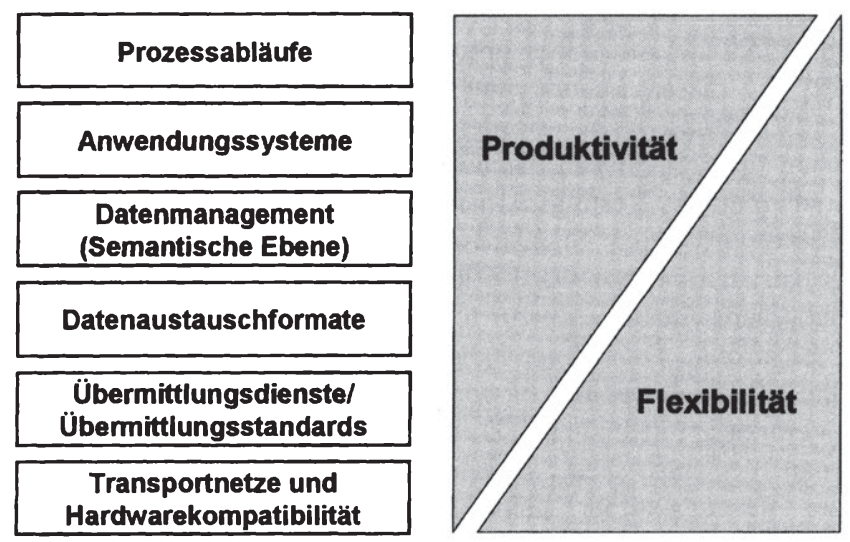

Abb. 5/16: Verdrängungseffekt zwischen Produktivität und Flexibilität im Bereich der unternehmungsübergreifenden Informationsverarbeitung

c) Auflösung des traditionellen Zusammenhangs zwischen Effektivität und Flexibilität durch moderne Standards der Informationsverarbeitung

Die allgemeine Entwicklung im Bereich der am Markt verfügbaren Informations- und Kommunikationstechniken führt zu einer teilweisen Auflösung des soeben erläuterten Zusammenhangs. Die Verwendung innovativer Informations- und Kommunikationstechnik ermöglicht eine schnellere und kostengünstigere, raum- und zeitübergreifende Übertragung von Informationen und erleichtert dadurch die Voraussetzungen für den Aufbau einer unternehmungsübergreifenden Informationsverarbeitung. ${ }^{398}$ Diese Entwicklung eröffnet neue Möglichkeiten zur Integration der technikunterstützten Informationssysteme unter Verwendung standardisierter technischer Lösungen. Infolgedessen ergeben sich Spielräume, die angestrebten Produktivitäts- und Qualitätssteigerungen zu erzielen, ohne zugleich den Spezifitätsgrad der unternehmungsübergreifend durchzufürenden Informationsverarbeitungsprozesse in gleichem Maße wie bisher zu erhöhen. Die Kompatibilität der technikunterstützten Informationssysteme der beteiligten Unternehmungen mit den Systemen potenzieller anderer Vertragspartner bleibt dann in stärkerem Maße erhalten. Folglich verringert sich die Bindungswirkung der im Rahmen einer Transaktion durchzuführenden Prozesse der Informationsverarbeitung, sodass die Abhängigkeit von einer Fortsetzung der Zusammenarbeit mit dem einmal gewählten Vertragspartner reduziert wird.

Das Ausmaß dieser Senkung des Spezifitätsgrads hängt davon ab, bis zu welcher der in Abbildung 5/16 dargestellten Betrachtungs- und Gestaltungsebenen standardisierte Lösungen eingesetzt werden können. Bei den informations- und kommunikationstechnischen Rahmenbedingungen ist in diesem Zusammenhang eine Verlagerung der Entwicklung von den unteren auf höhere Ebenen zu beobachten. Auf der untersten Ebene, auf der rein übertragungstechnische Probleme der Verknüpfung von Systemen bisher getrennt arbeitender Unternehmungen zu lösen sind, stehen bereits seit längerem

\footnotetext{
${ }^{398}$ Vgl. Gurbaxani/Whang (1991), S. 66; Picot/Reichwald/Wiegand (2001), S. 71.
} 
leistungsfähige Standards zur Verfügung, die zudem permanent durch die Telekommunikationsanbieter weiter entwickelt werden. Die Entwicklung spezifischer Lösungen ist auf dieser Ebene zum Aufbau einer unternehmungsübergreifenden Informationsverarbeitung nicht erforderlich. Bei der Integration der verschiedenen Teilsysteme auf diesem Niveau bleibt also die Kompatibilität mit den Systemen potenzieller alternativer Vertragspartner erhalten. Auch auf den beiden darüber liegenden Ebenen existieren Standards, die für den unternehmungsübergreifenden Informationsaustausch genutzt werden können. Hier sind insbesondere die Standards zum automatischen Austausch von Geschäftsdaten zu nennen, die unter dem Oberbegriff Electronic Data Interchange (EDI) entwickelt wurden. ${ }^{399}$ Die EDIStandards sind allerdings wenig anpassungsfreundlich und haben darüber hinaus keine umfassende Verbreitung gefunden. ${ }^{400}$ Die Integration der technikunterstützten Informationssysteme auf der Basis dieser Standards engt daher den zukünftigen Handlungsspielraum der Vertragspartner auch bei weitgehendem Verzicht auf individuelle Lösungen in beträchtlichem Maße ein.

Auf diesen Ebenen kommt es auf Grund der angesprochenen allgemeinen informationsund kommunikationstechnischen Entwicklungen jedoch zu einer Veränderung der Entscheidungssituation. Die durch die Internet-Technik verwendeten Standards, die ein hohes Flexibilitätsniveau aufweisen, und in der jüngsten Vergangenheit weltweit außerordentliche Verbreitung gefunden haben, sind bis hinauf zur vierten Ebene des oben dargestellten Klassifikationsschemas einzuordnen. ${ }^{401}$ Der Internet-Technik wird deshalb das Potenzial zugesprochen, bestehende Inkompatibilitäten aufzulösen und die proprietären Bindungswirkungen der bisher (z.B. auf Basis der EDI-Standards) realisierten Lösungen $\mathrm{zu}$ beseitigen. ${ }^{402}$ Entscheiden sich die Vertragspartner für eine unternehmungsübergreifende Integration der Informationsverarbeitung auf der Basis dieser neuen Standards, so wird die Option zum Ausstieg aus der Zusammenarbeit für die beteiligten Vertragspartner gegenüber der bisherigen Situation erleichtert. Dabei wird die Verringerung der Bindungswirkung dadurch erreicht, dass die allgemeine Kompatibilität der technikunterstützten Informationssysteme der beteiligten Unternehmungen bis auf die Ebene der Datenaustauschformate ausgedehnt wird.

Auf den darüber liegenden, noch stärker anwendungsbezogenen Ebenen liegen vergleichbare Standards, durch deren Übernahme eine weitere Erhöhung des Kompatibilitätsniveaus erzielt werden kann, nicht unmittelbar vor. Gerade die inhaltliche Verknüpfung der Informationsverarbeitung auf diesen Betrachtungsebenen ist jedoch als wesentliches Element der unternehmungsübergreifenden Integration der Informa-

\footnotetext{
${ }^{399}$ Vgl. dazu die Übersichten bei Klein (1996), S. 61ff.; Gersch (1998), S. 48ff. Auf der syntaktischen Ebene handelt es sich dabei beispielsweise um EDIFACT in Europa und ANSI X.12 in Nordamerika. Vgl. Lockamy (1995), S. 175f.; Hess (1999), S. 192, Diese nutzen die Standards auf der Übermittlungsebene wie X.400, X.25, FTAM. Vgl. Klein (1996), S. 61; Gersch (1998), S. 255f; Hess (1999), S. 193.

${ }^{400}$ Vgl. Lötsch (1997), S. 122; Schüppler (1998), S. 33; Tan/Shaw/Fulkerson (2000), S. 458.

${ }^{401}$ Mertens/Griese/Ehrenberg ordnen die Protokollfamilie TCP/IP der dritten und vierten Ebene des ISO/OSI-Referenzmodells zu. Vgl. Mertens/Griese/Ehrenberg (1998), S. 70. Über diesen sind die Protokolle der verschiedenen Internet-Dienste wie z.B. FTP und HTTP anzuordnen. Neuere Erweiterungen wie etwa der Datenstandard XML besitzen noch stärkeren semantischen Bezug und können daher die vierte Ebene des in Abbildung 5/16 dargestellten Klassifikationsschema vollständig abdecken.

${ }^{402}$ Vgl. Mattes (1999), S. 64f.; Tan/Shaw/Fulkerson (2000), S. 468f.
} 
tionsverarbeitung zu sehen. ${ }^{403}$ Mit steigendem Anwendungsbezug steigt die Heterogenität der Anforderungen an die informationstechnische Unterstützung der Transaktion, sodass die Effizienzvorteile individueller Lösungen auf diesen Ebenen tendenziell stärker ins Gewicht fallen.

Allerdings gibt es auch in diesem Bereich Ansätze zur Bildung von Standards, die die Flexibilität der Informationsverarbeitung weiter erhöhen sollen. Dazu gehören beispielsweise die Anstrengungen der Hersteller betriebswirtschaftlicher Standardanwendungssoftware, die darauf abzielen, branchenunspezifische und branchenspezifische Software-Module anzubieten, die sich für einen reibungslosen Informationsaustausch mit den Anwendungssystemen von Geschäftspartnern eignen. Viele dieser Lösungen machen jedoch strenge Schnittstellenvorgaben und können nicht universell mit anderen Anwendungssystemen verknüpft werden. Deshalb entstehen bei der Übernahme der durch den Softwarehersteller vorgesehenen Lösung immer noch Lockin-Effekte, die einen zukünftigen Wechsel des Transaktionspartners erschweren können. Auf der höchsten Ebene des oben dargestellten Klassifikationsschemas sind betriebswirtschaftliche Referenzmodelle angesiedelt. Derartige Referenzmodelle, die häufig branchenspezifischen Charakter haben und in gewissen Grenzen Konfigurationsmaßnahmen im Einzelfall zulassen, streben eine möglichst hohe Effizienz bei gleichzeitiger Standardisierung der im Rahmen einer Transaktion durchzuführenden Prozesse der Informationsverarbeitung an. ${ }^{404}$ Die Ansätze auf diesen Ebenen werden im Folgenden näher betrachtet.

Die Reduzierung von Vorleistungen, die die Möglichkeit zum Wechsel des Transaktionspartners vereinfacht, basiert auf der Standardisierung von Schnittstellen zwischen den technikunterstützten Informationssystemen der beteiligten Unternehmungen. Je höher diese Standardisierung in dem oben dargestellten Klassifikationsschema angesiedelt ist, um so geringer wird der Umstellungsaufwand bei einer Beendung der Zusammenarbeit mit dem bisherigen Vertragspartner. Auf der Ebene der Anwendungssysteme stellt das Objektorientierte Software-Design und dabei insbesondere das Komponentenkonzept den Schlüssel zur Verknüpfung der verschiedenen Teilsysteme dar. ${ }^{405}$ Bei Verfolgung des Objektorientierten Ansatzes werden umfangreiche Software-Systeme aus kleineren, überschaubaren Komponenten zusammengesetzt, die miteinander nur über klar definierte Schnittstellen verknüpft sind. ${ }^{406}$ Die einzelnen Komponenten als Objekte werden gekapselt, sodass die internen Zustände und Prozesse nicht von außen erkennbar sind. ${ }^{407}$ Die Objekte können sich gegenseitig durch das Versenden von Nachrichten aktivieren, sodass ein Objekt auf die Funktionen des anderen Objekts zugreifen kann. ${ }^{408}$ Softwaretechnisch gesehen ermöglicht die Anwendung dieses Konzeptes die Komposition umfangreicher

\footnotetext{
${ }^{403} \mathrm{Vgl}$. Thome (1999), S. 178.

${ }^{404}$ Für eine allgemeine Darstellung von Referenzmodellen vgl. Schütte (1998), S. 69ff., sowie insbesondere für interorganisatorische Referenzmodelle Schüppler (1998), S. 167ff.

${ }^{405} \mathrm{Vgl}$. dazu Tan/Shaw/Fulkerson (2000), S. 463ff.

${ }^{406}$ Vgl. Erler (2000), S. $120 f$.

${ }^{407}$ Vgl. Wirfs-Brock/Wilkerson/Wiener (1993), S. 17; Schader/Rundshagen (1996), S. $15 f$.

${ }^{408}$ Im ERP-System des Anbieters SAP erfolgt die Umsetzung dieses Konzeptes beispielsweise über sogenannte Business Application Programming Interfaces (BAPIs). Die Anwendungssysteme verschiedener Unternehmungen können über BAPIs miteinander verknüpft werden. BAPIs werden technisch als Funktionsbausteine realisiert, die per Remote Function Call (RFC) von anderen Anwendungsmodulen aufgerufen werden können. Vgl. dazu Buxmann/König (2000), S. 84.
} 
Softwaresysteme durch die Verknüpfung besser überschaubarer Teilsysteme, die unabhängig voneinander entwickelt und angepasst werden können. ${ }^{409}$ Aus institutionenökonomischer Perspektive ist dabei der Aspekt der Individualisierung durch die Kombination standardisierter Einzelbestandteile interessant. Letzten Endes handelt es sich hier um eine Möglichkeit zur Erzielung eines erhöhten Spezifitätsgrads, die auf dem Prinzip eines Baukastensystems aufbaut. Die Objektorientierung stellt damit auf der Ebene der Anwendungssysteme ein Mittel des Informationsmanagements zur Trennung der Spezifität von Leistungsergebnis und Leistungserstellungsprozess dar.

Eine noch weitere Reduzierung der Transaktionsspezifität lässt sich durch die Ausdehnung der Objektorientierung auf die Ebene der betriebswirtschaftlichen Prozesse als höchste Betrachtungs- und Gestaltungsebene der unternehmungsübergreifenden Informationsverarbeitung erreichen. In diese Richtung gehen aktuelle Bestrebungen, die unter dem Schlagwort Business Objects darauf abzielen, objektorientierte Ansätze aus dem Bereich der Software-Entwicklung auf betriebswirtschaftliche Problemstellungen zu übertragen. ${ }^{410}$ Dadurch sollen die Vorteile, die die Objektorientierung auf der Software-Ebene bringt, auch für die Ebene der betriebswirtschaftlichen Prozesse der Informationsverarbeitung nutzbar gemacht werden. Die objektorientierte Gestaltung der Prozesse ermöglicht ein hohes Ausmaß an Flexibilität, da Veränderungen in der Realität durch das Entfernen, Hinzufügen oder Ändern einzelner Objekte leicht in den zu Grunde gelegten Prozessmodellen abgebildet werden können. ${ }^{411}$ Der Wechsel des Transaktionspartners ist in diesem Fall ohne aufwendige Anpassungsmaßnahmen möglich. Dies gilt allerdings nur dann, wenn der neue Vertragspartner seine Prozesse ebenfalls als Business Objects gestaltet und die gleichen Schnittstellenspezifikationen vorgenommen hat. Da das Konzept der Business Objects bisher noch keine umfassende Verbreitung gefunden hat, ermöglicht die Einrichtung von Business Objects zwar von der Grundidee her eine noch höhere Flexibilität bzw. eine noch weitergehende Absenkung des Spezifitätsgrads. Im konkreten Einzelfall ist jedoch immer noch zu erwarten, dass sich die Bindungswirkung auf der Prozessebene mangels alternativer Vertragspartner durch den Aufbau von Business Objects kaum reduzieren lässt.

Aus institutionenökonomischer Sicht ist eine Verringerung der Bindungswirkung auf der Ebene der betriebswirtschaftlichen Prozesse der Informationsverarbeitung im Prinzip auch ohne strenge Einhaltung des Objektorientierten Paradigmas möglich. Entscheidende Voraussetzung dafür ist, dass eine Standardisierung der Prozessschnittstellen $^{412}$ zwischen den Transaktionspartnern realisiert wird, die die Umstellungskosten

\footnotetext{
${ }^{409}$ Vgl. Baldwin/Clark (1997), S. 85f.

${ }^{410}$ Vgl. dazu Erler (2000), passim. Die vollstăndige Übertragung von Grundkonzepten der Objektorientierung auf die betriebswirtschaftliche Ebene erfordert ein semantisch objektorientiertes Business Engineering. Vgl. Erler (1998), S. 15.

${ }^{411}$ Vgl. Erler (1998), S. 29. Shaw dehnt die Grundidee des Komponenten-Konzeptes noch weiter aus. Er fordert eine „component-based economy“, in der interoperable Schnittstellen und operationale Standards es möglich machen, Komponenten von Wissen, Geschäftsprozessen, Geschäftssystemen, Erfahrungen, Software und weiterer Ressourcen, in unterschiedlichster Weise an unterschiedlichsten Stellen miteinander zu verknüpfen. Vgl. Shaw (2000), S. 21.

${ }^{412}$ Die Standardisierung der Schnittstellen erfordert auch eine Standardisierung der betreffenden Prozesse. Die nachfolgenden Überlegungen gelten daher nur für solche Prozesse, die die oben erläuterten Voraussetzungen für die Anwendung der Ex ante-Koordination nach Vertragsschluss erfuillen. Diese Prozesse sind durch eine hohe Gleichformigkeit gekennzeichnet und laufen in
} 
für die Beteiligten im Falle einer Beendung der Zusammenarbeit reduziert. Allein schon die Orientierung am Komponentenkonzept schafft also gute Voraussetzungen zur Überwindung der bisher bestehenden Defizite im Bereich der unternehmungsübergreifenden Ablaufsteuerung durch Workflow Management-Systeme. ${ }^{413}$ Durch die Übernahme der bereits angesprochenen Referenzmodelle für Geschäftsprozesse, die von Software-Herstellern und Beratungshäusern entwickelt wurden, ${ }^{414}$ kann der Spezifitätsgrad der unternehmungsübergreifenden Informationsverarbeitung auf der Prozessebene reduziert werden. Damit wird zunächst einmal die direkte Umsetzbarkeit der betriebswirtschaftlichen Anforderungen durch am Markt verfugbare Standardanwendungssoftware gewährleistet. Darüber hinaus wird zugleich auch auf dieser höchsten Betrachtungs- und Gestaltungsebene die Kompatibilität mit den Systemen potenzieller alternativer Vertragspartner geschaffen, sofern diese ebenfalls die in den Referenzmodellen vorgesehenen Schnittstellenvorgaben einhalten.

Die vollständige Umsetzung der betreffenden Referenzmodelle zur Abwicklung der unternehmungsinternen Teilprozesse, die die Unterstützbarkeit durch Standardanwendungssoftware gewährleisten soll, ist dabei nicht zwingend erforderlich. Die Möglichkeit zum Wechsel des Vertragspartners wird vielmehr allein dadurch gegeben, dass die Schnittstellenspezifikationen zwischen den beteiligten Unternehmungen den entsprechenden Standards genügen. Diese Standardisierung der Prozessschnittstellen ermöglicht institutionenökonomisch gesehen eine Übertragung des Baukastenprinzips auf die betriebswirtschaftliche Ebene von Informationsverarbeitungsprozessen. Auch auf dieser höchsten Ebene lässt sich somit eine Aufhebung des Kausalzusammenhangs zwischen spezifischem Leistungsergebnis und spezifischem Leistungserstellungsprozess erreichen, indem die Individualisierung des Leistungsergebnisses auf dem Wege einer flexiblen Verknüpfung standardisierter Prozessbausteine erfolgt.

Die beschriebenen Entwicklungen führen dazu, dass sich der oben in genereller Form postulierte Crowding-out-Effekt zwischen Effizienz und Flexibilität der Leistungserstellungsprozesse im Bereich der technikunterstützten Informationsverarbeitung teilweise auflöst. Dem Informationsmanagement bieten sich dadurch neue Möglichkeiten für den Aufbau einer unternehmungsübergreifenden Integration der Informationsverarbeitung im Rahmen einer längerfristigen Zusammenarbeit zwischen den Vertragspartnern. Durch die Übernahme am Markt verfugbarer Standards lassen sich die für den Aufbau des Informationsaustausches notwendigen Vorleistungen reduzieren und dennoch die Voraussetzungen für die Steigerung der Effizienz der Leistungserstellungsprozesse schaffen. Die Flexibilität des technikunterstützten Informationssystems der Unternehmung bleibt dabei weitgehend erhalten.

Der Effizienzvorsprung spezifischer Lösungen sinkt infolgedessen tendenziell, sodass sich der von einer Individualisierung der Leistungserstellungsprozesse im Bereich der Informationsverarbeitung zu erwartende Vorteil verringert. Eine vollständige Beseitigung des Zusammenhangs zwischen Spezifität und Effizienz ist allerdings nicht $\mathrm{zu}$

immer wieder gleicher Weise ab. Schlechter strukturierte Prozesse mit einem hohen Neuigkeitsgrad (z.B. im Bereich von Forschungs- und Entwicklungskooperationen) lassen sich nicht in dieser Weise standardisieren.

${ }^{413}$ Vgl. dazu Jablonski/Böhm/Schulze (1997), S. 355.

${ }^{414}$ Als Beispiel für derartige Referenzmodelle sei hier das Referenzmodell der Firma SAP zu ihrem Produkt R/3 genannt. Vgl. dazu Buck-Emden/Galimow (1996), S. 5ff. Rolf (1998), S. 103ff. 
erwarten, da durch die Übernahme von Marktstandards immer nur zum Wettbewerb aufgeschlossen werden kann. ${ }^{415}$ Durch die Einhaltung von Standards werden die Freiheitsgrade zum Aufbau unternehmungseigener Kernkompetenzen reduziert. ${ }^{416}$ Strategische Vorsprünge sind dagegen allein durch individuelle Lösungen $\mathrm{zu}$ erzielen, ${ }^{417}$ sodass im Einzelfall immer zu überprüfen bleibt, inwiefern es sich bei den betreffenden Prozessen um kritische Einflussfaktoren für den Markterfolg handelt. Trotz des Vorhandenseins leistungsfähiger Standardlösungen sind in einem solchen Fall nach wie vor Spielräume zur Erzielung von Wettbewerbsvorteilen durch eine Individualisierung von unternehmungsübergreifenden Prozessen der Informationsverarbeitung zu erwarten. Der Spezifitätsgrad dieser Prozesse wird dann zur Aktionsvariablen, die ausgehend von Effektivitäts- und Effizienzüberlegungen des Informationsmanagements zu gestalten ist.

\subsubsection{Veränderte Kostenstrukturen der Koordination des Leistungsaustausches}

Die Transaktionskostentheorie nimmt an, dass die bei der Abwicklung einer Transaktion entstehenden Transaktionskosten nicht nur von den Transaktionseigenschaften, sondern auch von der Art des gewählten vertraglichen Arrangements abhängen. ${ }^{418}$ Für die transaktionskosteneffiziente Durchführung einer Transaktion ist dieser Auffassung zu Folge ein Fit zwischen den Charakteristika der Transaktion und dem verwendeten Vertragsmuster herzustellen. Nachdem im vorigen Abschnitt die Möglichkeiten des Informationsmanagements zur Gestaltung der Transaktionseigenschaften, insbesondere der Spezifität, beleuchtet wurden, wenden sich die Ausführungen in diesem Abschnitt den verschiedenen Vertragsdesigns zu. Es erfolgt zunächst eine kurze Bestandsaufnahme der unterschiedlichen Kostenstrukturen, durch die alternativ mögliche Koordinationsformen typischerweise gekennzeichnet sind. Anschließend wird überprüft, inwiefern sich diese Kostenstrukturen durch die Nutzung der in diesem Kapitel herausgearbeiteten Gestaltungsoptionen des Informationsmanagements vor und nach Vertragsschluss ändert.

\section{a) Kostenstrukturen unterschiedlicher Koordinationsformen}

Zur Untersuchung von Veränderungen der Kostenstrukturen alternativer Vertragsdesigns ist zunächst die Ausgangssituation zu betrachten. Auf dieser Basis können Modifikationen, die durch Maßnahmen des Informationsmanagements entstehen, präziser verdeutlicht werden. Im Folgenden wird auf die Kostenstrukturen der marktlichen und der hierarchischen Koordinationsform als Extrempole Bezug genommen. Dies geschieht unter der Annahme, dass mittlere, hybride Koordinationsformen (in unterschiedlichem Ausmaß) gleichzeitig sowohl marktliche als auch hierarchische Koordinationselemente verwenden, ${ }^{419}$ sodass ihre jeweiligen Kostenstrukturen von den Kostenstrukturen und von der Gewichtung der einzelnen Koordinationselemente abhängen.

\footnotetext{
${ }^{415}$ Vgl. Oesterle (1990), S. 21.

${ }^{416}$ Vgl. Schüppler (1998), S. 36.

${ }^{417}$ Griese (1990), S. 646.

${ }^{418} \mathrm{Vgl}$. Ebers/Gotsch (1999), S. $227 \mathrm{f}$.

${ }^{419} \mathrm{Vgl}$. dazu Sydow (1993), S. 98ff. der eine ausführliche Würdigung des Für und Wider dieser Sichtweise vornimmt.
} 
Die Transaktionskostentheorie geht üblicherweise davon aus, dass die verschiedenen vertraglichen Arrangements unterschiedliche Transaktionskostenstrukturen aufweisen, wobei ein trade-off zwischen den einzelnen Kostenkomponenten besteht. ${ }^{420}$ Die rein marktliche Koordinationsform mit klassischen Verträgen zeichnet sich dadurch aus, dass bei jeder Transaktion ein neuer Vertragspartner gefunden und der konkrete Vertrag ausgehandelt werden muss. Demzufolge fallen bei marktlich koordinierten Transaktionen die Ex ante-Transaktionskosten anteilig besonders stark ins Gewicht. Die Gefahr opportunistischen Verhaltens ist allerdings relativ gering, da bei nachfolgenden Transaktionen ein Wechsel zu anderen Vertragspartnern leicht möglich ist. Diese Möglichkeit zur Beendung der Zusammenarbeit, auf der die starke Anreizwirkung der marktlichen Koordinationsform basiert, wird auch als Exit-Option bezeichnet. ${ }^{421}$ Die hierarchische Koordination mit relationalen Verträgen auf der anderen Seite ermöglicht, ausgehend von einer einmal gefundenen Einigung, vergleichsweise leichte Vertragsanpassungen ohne die Notwendigkeit ständig neuer Suchund Verhandlungsaktivitäten. Allerdings ist die Gefahr opportunistischen Verhaltens höher, da der Wechsel des Vertragspartners weniger leicht realisiert werden kann. Demzufolge entsteht der größte Teil der Transaktionskosten bei der hierarchischen Koordinationsform erst nach Vertragsschluss. Gleichzeitig bestehen bei der hierarchischen Koordinationsform jedoch bessere Möglichkeiten zur Kontrolle des Vertragspartners.

Die Agency-Theorie verfügt nicht über ein vergleichbar ausdifferenziertes Vertragskonzept wie die Transaktionskostentheorie, sondern sie belässt es bei einer weitaus abstrakteren Berücksichtigung von Verträgen. ${ }^{422}$ Aus Sicht der Agency-Theorie weisen die von der Transaktionskostentheorie unterschiedenen, alternativ möglichen Vertragsarrangements zur Abwicklung des Leistungsaustausches jedoch ebenfalls verschiedene Kostenstrukturen auf. Wie bei der allgemeinen Darstellung der AgencyTheorie erläutert, setzen sich die im Rahmen einer Auftraggeber/AuftragnehmerBeziehung entstehenden Agency-Kosten aus drei Komponenten zusammen. Dabei handelt es sich um Kontrollkosten auf Seiten des Principals furr den Aufbau von Überwachungssystemen, Garantiekosten auf Seiten des Agent und verbleibende Wohlfahrtsverluste durch nicht beseitigtes opportunistisches Verhalten. ${ }^{423}$ Die verschiedenen Typen vertraglicher Arrangements richten sich schwerpunktmäßig entweder eher auf die Setzung von Anreizen oder eher auf die Kontrollierbarkeit des Verhaltens der Vertragspartner. ${ }^{424}$ Die rein marktliche Koordination mit klassischen Verträgen basiert stärker auf dem Instrument der Anreizgestaltung, während die rein hierarchische Koordination mit relationalen Verträgen sich stärker auf die Kontrolle des Verhaltens konzentriert. ${ }^{425}$ Aus diesen Gründen resultieren bei eher am Markt orientierten vertraglichen Arrangements tendenziell geringere Wohlfahrtsverluste als bei eher an der hierarchischen Koordination orientierten Vertragsdesigns. Auf der anderen Seite ermöglicht die hierarchische Koordinationsform, die auf relationalen Verträgen basiert, eine leichtere Kontrolle des Agent durch den Principal und damit verringerte Kontroll-

\footnotetext{
${ }^{420}$ Vgl. dazu z.B. Richter/Furubotn (1999), S. 50ff.; Picot (1982), 273ff., Williamson (1979), S. $233 \mathrm{ff}$.

Diese Annahmen lassen sich bis hin zu Coase (1937), S. 390ff. zurückverfolgen.

${ }^{421}$ Vgl. Sydow (1993), S. 101; Hirschman (1970), S. 4.

${ }^{422}$ Vgl. Ebers/Gotsch (1999), S. 250.

${ }^{423} \mathrm{Vgl}$. dazu die Erläuterungen in Abschnitt 5.1.2.

${ }^{424}$ Vgl. Ebers/Gotsch (1999), S. 232.

${ }^{425}$ Vgl. Ebers/Gotsch (1999), S. 233.
} 
kosten. Auf Grund des wachsenden Vertrauens im Laufe der Zusammenarbeit ${ }^{426}$ ist auch bei den Garantiekosten tendenziell ein Vorteil der hierarchischen gegenüber der marktlichen Koordination zu vermuten.

Abbildung 5/17 fasst die erläuterten Annahmen, die Transaktionskostentheorie und Agency-Theorie über die jeweiligen koordinationsformenspezifischen Kostenstrukturen der verschiedenen Vertragstypen treffen, zusammen.

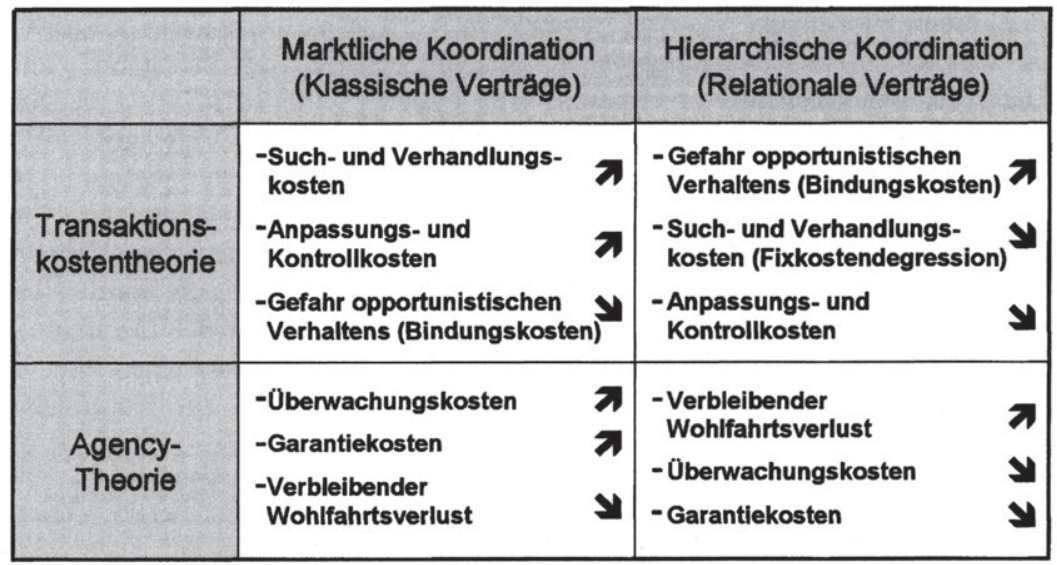

Abb. 5/17: Kostenstrukturen verschiedener Koordinationsformen aus Sicht von Transaktionskostentheorie und Agency-Theorie

\section{b) Veränderung der Kostenstrukturen durch Gestaltungsmaßnahmen des Informationsmanagements}

Die soeben erläuterten, „traditionellen“ Annahmen der Neuen Institutionenökonomie über die unterschiedlichen Effizienzmerkmale verschiedener Koordinationsformen sind in Folge der in diesem Kapitel dargelegten neuen Gestaltungsmöglichkeiten des Informationsmanagements in den verschiedenen Vertragsphasen zu modifizieren. Generell ist davon auszugehen, dass die Verfügbarkeit moderner Informations- und Kommunikationstechnik unabhängig vom gewählten Vertragsdesign das Potenzial zu einer allgemeinen Senkung der Transaktionskosten bzw. der Agency-Kosten eröffnet. ${ }^{427}$ Der Informationsaustausch zwischen tatsächlichen und potenziellen Vertragspartnern lässt sich durch die aufgezeigten Gestaltungspotenziale des Informationsmanagements in mehrfacher Hinsicht vereinfachen. Diese These wird im Folgenden genauer überprüft.

Wie erläutert, kann das Informationsmanagement Aktivitäten vor Vertragsschluss durch die Verlagerung der Vertragsanbahnung auf Elektronische Märkte unterstützen. Durch diese Mediatisierung kommt es zu einer Reduzierung der ex ante entstehenden Kostenarten als Kostenkomponenten der Transaktionskosten. Zum einen unterstützen leistungsfähige Elektronische Märkte sowohl Screening- als auch Signalling-Aktivi-

\footnotetext{
${ }^{426} \mathrm{Vgl}$. Picot/Reichwald/Wigand (1996), S. 273. Bei längerfristigen Transaktionsbeziehungen ist opportunistisches Verhalten zur Erzielung kurzfristiger Vorteile auf Kosten des Vertragspartners kontraproduktiv. Eine vertrauensvolle Transaktionsatmosphäre wirkt sich daher vorteilhaft aus.

${ }^{427} \mathrm{Vgl}$. dazu Malone/Yates/Benjamin (1987), S. 489 sowie die Ausführungen in Abschnitt 5.3.1.2.
} 
täten, sodass die Markttransparenz bei der Suche prinzipiell geeigneter Vertragspartner insgesamt erhöht wird. Zum anderen vereinfachen Elektronische Märkte die Aushandlung von Vertragsdetails in der an die Suche anschließenden Verhandlungsphase, wenn für den endgültigen Abschluss des Vertrages lediglich die geringfügige Anpassung standardisierter Vertragsmuster erforderlich ist. Wie gesehen, ist die Transaktionsphase vor Vertragsschluss bei der marktlichen Form des Leistungsaustausches, die durch stärker am klassischen Vertragsmuster orientierte Verträge koordiniert wird, besonders transaktionskostenintensiv. Von der Verlagerung der Vertragsanbahnung auf Elektronische Märkte profitieren also in erster Linie klassische Verträge und damit die marktliche Koordinationsform.

In Abschnitt 5.3.1 wurde jedoch erläutert, dass Elektronische Märkte grundsätzlich auch bei hierarchischen Transaktionen die Auswahl des Vertragspartners unterstützen können. Allerdings hat eine Mediatisierung von Anbahnungsaktivitäten bei dieser Koordinationsform, bei der die ex ante entstehenden Transaktionskosten ohnehin einen vergleichsweise kleinen Anteil an den gesamten Transaktionskosten einnehmen, nur geringere Auswirkungen. Dies ist auf die unterschiedliche Ausgestaltung der Vertragsanbahnung bei den verschiedenen Koordinationsformen zurückzuführen. Im Gegensatz zur marktlichen Koordination ist bei hierarchischen Transaktionen typischerweise die Aushandlung eines umfangreicheren Vertragswerks notwendig, das nicht lediglich die Spezifikation eines allgemeinen Standards darstellt. Eine Automatisierung der Vereinbarungsaktivitäten ist dabei kaum möglich. Die Transaktionskosten für die Anbahnung relationaler Verträge sind deshalb durch das Informationsmanagement nur in geringerem Umfang als bei klassischen Verträgen reduzierbar.

Wie ebenfalls in diesem Kapitel erläutert, kann das Informationsmanagement durch den Einsatz Elektronischer Hierarchien auch einen Beitrag zur Reduzierung der nach Vertragsschluss entstehenden Transaktionskosten leisten. Geht man vom Idealtypus des reinen klassischen Vertrags aus, dann fallen Leistung und Gegenleistung in einem Zeitpunkt zusammen und ex post treten keinerlei Transaktionskosten mehr auf. Tatsächlich sind jedoch sowohl die marktliche als auch die hierarchische Koordination in ihrer Idealform kaum von empirischer Relevanz. ${ }^{428}$ Je größeres Gewicht bei einer Transaktion, die auf einer Kombination hierarchischer und marktlicher Koordinationselemente beruht, der Anteil der nicht vollständig explizierten Verpflichtungen der Vertragspartner besitzt, um so stärker kann sich eine Transaktionskostensenkung mittels Elektronischer Hierarchien auswirken. Bei stärker marktlich orientierten Transaktionen betrifft dies insbesondere den Bereich der operativen Abwicklung des Leistungsaustausches, während bei stärker hierarchisch orientierten Transaktionen zusätzlich die Vermeidung opportunistischen Verhaltens durch verbesserte Kontrollmöglichkeiten unterstützt wird.

Damit kann zunächst einmal festgehalten werden, dass durch die in diesem Kapitel aufgezeigten Gestaltungsoptionen des Informationsmanagements grundsätzlich die Effizienz aller Vertragsformen zur Koordination des Leistungsaustausches gesteigert werden kann. Gegenwärtig ist in diesem Zusammenhang in vielen Arbeiten eine Konzentration der Analyse auf den Bereich der unternehmungsübergreifenden Informationsverarbeitung $\mathrm{zu}$ beobachten, die durch sogenannte interorganisatorische

\footnotetext{
${ }^{428}$ Vgl. Sydow (1993), S. 101.
} 
Informationssysteme realisiert wird. ${ }^{429}$ Dabei handelt es sich um einen Anwendungssystemtyp, der bei der hybriden und der marktlichen Koordinationsform zum Einsatz kommt. Diese starke Fokussierung birgt allerdings die Gefahr einer sachlich unangemessenen Verkürzung der Untersuchung, da die beschriebenen Maßnahmen des Informationsmanagements auch für die unternehmungsinterne Koordination erhebliche Effizienzsteigerungspotenziale besitzen. Diese Einschränkung der Betrachtung hängt möglicherweise damit zusammen, dass gerade die internen Koordinationskosten von Vertretern der Transaktionskostentheorie, die ursprünglich als eine Theorie des Marktversagens konzipiert wurde, systematisch unterschätzt werden. ${ }^{430}$ Folglich werden die Potenziale des Einsatzes moderner Informations- und Kommunikationstechnik zu einseitig der als besonders transaktionskostenintensiv klassifizierten marktlichen Koordination zugeordnet.

\subsubsection{Auswirkungen auf die Vorteilhaftigkeit der Koordinationsform}

In den beiden letzten Abschnitten wurden durch das Informationsmanagement induzierte Veränderungen untersucht, die die für die Wahl der Koordinationsform ausschlaggebenden Einflussgrößen betreffen. Auf dieser Basis wenden sich die nachfolgenden Überlegungen der Fragestellung zu, welche Auswirkungen sich aus dem gleichzeitigen Auftreten der erläuterten Entwicklungen auf die Vorteilhaftigkeit der Koordinationsform ergeben. Es wird untersucht, inwiefern sich die relative Effizienz alternativ wählbarer Vertragsdesigns zur Durchführung einer Transaktion verändert. ${ }^{431}$ Grundsätzlich ist wie erwähnt davon auszugehen, dass der Einsatz moderner Informationstechnik eine schnellere und kostengünstigere raum- und zeitüberbrückende Nachrichtenübertragung möglich macht (Electronic Commuication Effect). ${ }^{432} \mathrm{Als}$ allgemeine Schlussfolgerung kann die These aufgestellt werden, dass eine erleichterte Kommunikation zu einer kommunikationsintensiveren Koordination führt. ${ }^{433}$ Die Auswirkungen dieser Entwicklung auf die Effizienz der Koordinationsform sind in Abbildung 5/18 dargestellt. In Folge der gezeigten Kurvenverschiebung ist von einer Tendenz zur verstärkten Nutzung marktlich orientierter Koordinationsformen auszugehen. ${ }^{434}$ Die Spezifitätsniveaus $S_{1}$ und $S_{2}$, bei denen jeweils ein Wechsel der Koordinationsform die Effizienz der Transaktion erhöht, verschieben sich nach $S_{1}$ ' und $\mathrm{S}_{2}$.

\footnotetext{
${ }^{429}$ Vgl. dazu z.B. die Arbeiten von Klein (1996), Alt (1997).

${ }^{430}$ Vgl. Sydow (1993), S. 147.

${ }^{431}$ Diese relative Hơhe der Kosten wird in der Transaktionskostentheorie als ausschlaggebend für die Festlegung des Vertragsdesigns angesehen. Die einzelnen Kostenarten müssen dabei nicht zwingend exakt quantifizierbar sein. Vgl. Williamson (1985), S. 21f;; Sauter (1985), S. 62f.; Gersch (1998), S. 87. Als Beurteilungsmaßstab wird wie erläutert die Summe von Produktions- und Transaktionskosten zu Grunde gelegt.

${ }^{432} \mathrm{Vgl}$. Picot/Reichwald/Wigand (2001), S. 70f.

${ }^{433}$ Die These wurde in dieser allgemeinen Form von Malone/Rockart (1991), S. 92ff., aufgestellt.

${ }^{434}$ Vgl. Picot/Reichwald/Wigand (2001), S. 71f.; Voß/Gutenschwager (2001), S. 48. Die Annahme geht in dieser Form auf Malone/Yates/Benjamin (1987), S. 484, zurück.
} 


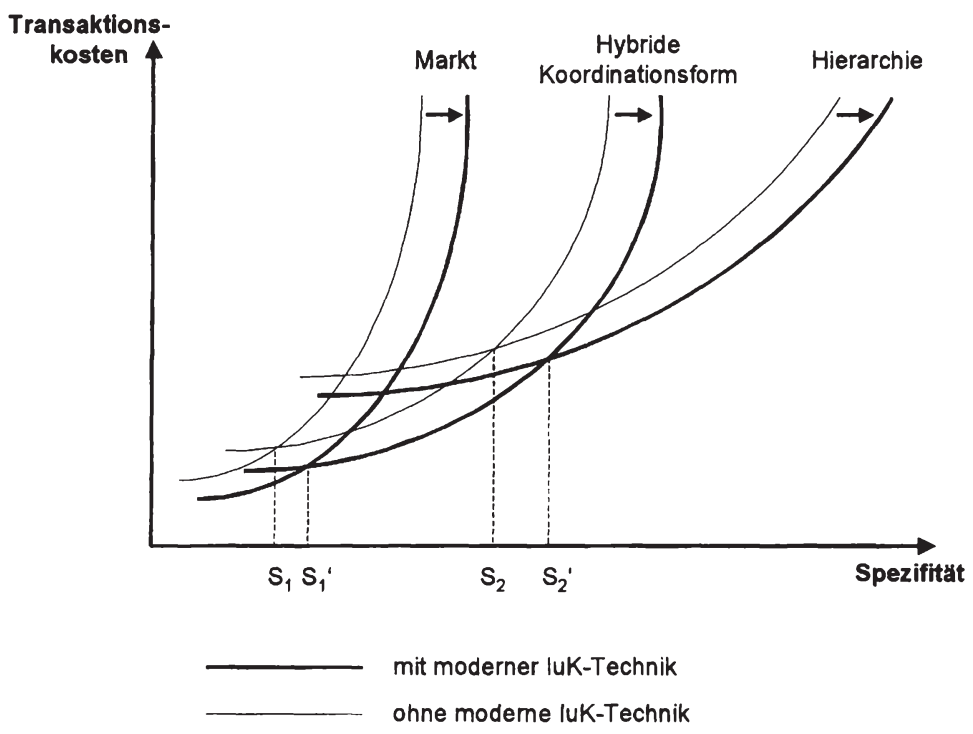

Abb. 5/18: Einfluss moderner Informations- und Kommunikationstechnik auf den Verlauf der Transaktionskosten Quelle: Picot/Reichwald/Wigand (2001), S. 72.

Die Art der Abbildung macht allerdings nicht deutlich, dass durch die Nutzung moderner Informations- und Kommunikationstechniken zugleich ein weiterer Effekt eintritt, der die Spezifität der Transaktion betrifft. Wie in Abschnitt 5.3.3.1 beschrieben, wird es möglich, eine höhere Spezifität des Ergebnisses und eine höhere Produktivität der Prozesse zu erreichen, ohne die Ressourcen in gleichem Maße spezifisch auszugestalten. Die aus der Abbildung hervorgehende Schlussfolgerung ist daher in dieser Form zu pauschal, um daraus im konkreten Fall Anhaltspunkte für Gestaltungsmaßnahmen des Informationsmanagements ableiten zu können. ${ }^{435}$ Für den weiteren Ausbau des hier entwickelten Institutionenökonomischen Ansatzes einer Theorie des Informationsmanagements ist deshalb eine genauere Untersuchung der Zusammenhänge erforderlich.

\section{a) Sicht der Transaktionskostentheorie}

Der in der transaktionskostentheoretisch ausgerichteten Literatur häufig vorzufindende Argumentationsweg postuliert eine zunehmende Abkehr von der traditionellen, integrierten Unternehmung zu Gunsten mittlerer und marktlicher Koordinationsformen. ${ }^{436}$ Diese Entwicklung sei um so stärker ausgeprägt, je mehr der Einsatz moderner Informations- und Kommunikationstechnik im konkreten Einzelfall die Kosten der Koordination bei der arbeitsteiligen Leistungserstellung und -übertragung

\footnotetext{
${ }^{435}$ Das gilt auch für die etwas weniger allgemeine These, es könne „in vielen Fällen die Koordinationsarbeit auf den Markt abgewälzt werden. “ (Schmid (1999), S. 46). Dadurch könne die Struktur des Betriebes unter Nutzung der generischen Marktdienste vereinfacht und auf die Kernkompetenzen ausgerichtet werden.

${ }^{436}$ Vgl. Reichwald/Möslein (2000), S. 120.
} 
reduzieren kann. Das Ergebnis - so die weitere Argumentation - ist ein reduzierter Transaktionskostenvorteil der vertikalen Integration von Aktivitäten. ${ }^{437}$ Als Folge der Senkung von Koordinationskosten lohne sich die Integration der Leistungserstellung innerhalb der Unternehmung tendenziell erst bei Leistungen höheren Spezifitätsgrads. Marktliche und mittlere Koordinationsformen erweisen sich deshalb für immer weiterreichende Wirtschaftsbereiche als ökonomisch vorteilhaft. Das Resultat sei eine in zunehmenden Maße zu beobachtende Auflösung von Unternehmensgrenzen, der eine Ausweitung der unternehmungsübergreifenden Zusammenarbeit gegenüberstehe. ${ }^{438}$ Dabei konkurriert die „Move-to-the-Market-Hypothese“, die in der Konsequenz von einer dominanten Vorteilhaftigkeit des Marktes ausgeht, mit der „Move-to-theMiddle-Hypothese“, die letztendlich eine Dominanz mittlerer Koordinationsformen erwartet. ${ }^{439}$

Die „Move-to-the-Market-Hypothese“ und die „Move-to-the-Middle-Hypothese“ stützen sich zentral auf die Annahme, dass die unternehmungsinterne Koordination generell weniger transaktionskostenintensiv ist als die externe Koordination über den Markt. Dies entspricht der oben geschilderten Beobachtung, dass bei Vertretern der Transaktionskostentheorie in vielen Fällen eine systematische Unterschätzung der internen Transaktionskosten zu beobachten ist. Tragfähiger scheint im Vergleich dazu die Annahme, dass die hierarchische Koordination sich von der marktlichen nicht allgemeingültig durch die absolute Höhe der anfallenden Transaktionskosten, sondern lediglich durch den Zeitpunkt der Kostenentstehung relativ zum Abschluss des Vertrages unterscheidet. Unter diesen Bedingungen ist im Prinzip auch eine umgekehrte Entwicklung hin zu einer verstärkten Internalisierung von Transaktionen denkbar. ${ }^{440}$ Aus dieser Perspektive ermöglicht insbesondere der Einsatz analyseorientierter Anwendungssysteme eine Senkung der internen Koordinationskosten. ${ }^{441}$ Damit werden die Voraussetzungen für eine interne, hierarchisch koordinierte Leistungserstellung mit erhöhter Produktivität durch eine spezifische Ausgestaltung der im Rahmen der Leistungserstellung verwendeten Ressourcen geschaffen.

Ferner liegt den beiden Hypothesen eine kontingenztheoretische, also sehr stark situativ orientierte Sichtweise zu Grunde, nach der die Wahl der Koordinationsform direkt von der Ausprägung von Transaktionseigenschaften abhängig ist. ${ }^{442}$ Dieser deterministische Ansatz macht eine Relativierung durch eine stärker konfigurationstheoretisch ausgerichtete Argumentation erforderlich. An die Stelle der funktionalen Abhängigkeit tritt dabei die Möglichkeit zur aktiven Gestaltung der für die Höhe der Transaktionskosten entscheidenden Einflussgrößen. In diesem Rahmen ist die Zielsetzung zu verfolgen, einen möglichst guten Fit zwischen den verschiedenen Gestaltungsparametern herzustellen. ${ }^{443}$ Geht man von der Gestaltbarkeit der Variablen durch das Informationsmanagement aus, so bietet der Einsatz Elektronischer Märkte

\footnotetext{
${ }^{437} \mathrm{Vgl}$. Malon/Yates/Benjamin (1989), S. 166.

${ }^{438} \mathrm{Vgl}$. dazu insbesondere auch Picot/Reichwald/Wigand (2001), S. 2ff.

${ }^{439} \mathrm{Vgl}$. Reichwald/Möslein (2000), S. 120.

${ }^{440}$ Diese Annahme findet sich in der Literatur nur relativ selten. Ein Beispiel stellt Antonelli (1988),

S. 7, dar, der einen - allerdings nur vorubergehenden Trend - zur Funktionsinternalisierung erwartet.

${ }^{441} \mathrm{Vgl}$. auch Gurbaxani/Whang (1991), S. 66ff.

${ }^{442}$ Vgl. Klein (1996), S. 152.

${ }^{443}$ Vgl. Klein (1996), S. 152.
} 
und Elektronischer Hierarchien erweiterte Möglichkeiten zur Realisierung neuer Koordinationsformen und erhöht zugleich die Flexibilität bei ihrer Gestaltung. ${ }^{44}$

Die Vorteilhaftigkeit des Vertragsdesigns hängt nicht nur von den bisher betrachteten Kostenstrukturen der verschiedenen Koordinationsformen, sondern auch von den im Abschnitt 5.3.3.1 betrachteten Transaktionseigenschaften ab. Dort wurde die in der Transaktionskostentheorie üblicherweise zu Grunde gelegte Annahme, dass durch die spezifische Ausgestaltung von Ressourcen eine höhere Effizienz der Leistungserstellung erreicht werden kann, überprüft und auf Grund der allgemeinen Entwicklung der Rahmenbedingungen für den Gestaltungsbereich des Informationsmanagements relativiert. Wegen der Verfügbarkeit der in Abschnitt 5.3.3.1 genannten technischen und betriebswirtschaftlichen Standards der Informationsverarbeitung sinkt im Einflussbereich des Informationsmanagements der durch die spezifische Ausgestaltung der verwendeten Ressourcen erzielbare Effizienzvorteil. Die teilweise ${ }^{445}$ Auflösung des allgemein vermuteten Verdrängungseffekts zwischen Effizienz und Flexibilität erweitert die Gestaltungsoptionen im Bereich der Spezifität als Transaktionseigenschaft.

Als Folge der beschriebenen Entwicklungen werden neue Handlungsstrategien vorstellbar, die darauf abzielen, die Vorteile der marktlichen Koordination und der hierarchischen Koordination miteinander zu verknüpfen. ${ }^{446}$ Gelingt dies, so können einerseits marktlich orientierte vertragliche Arrangements zu verringerten Ex ante-Transaktionskosten genutzt werden. Der relative Anreizvorteil der marktlichen gegenüber der hierarchischen Koordinationsform wird dabei genutzt und der relative Nachteil stets neu zu vereinbarender, klassischer Vertragsarrangements gegenüber längerfristig gültigen, relationalen Vertragsdesigns durch den Einsatz Elektronischer Märkte reduziert. Andererseits wird es auf der Basis der oben angesprochenen Standards auch möglich, im unternehmungsübergreifenden Bereich eine dauerhafte Zusammenarbeit mit hierarchisch orientierten Vertragsarrangements aufzubauen, ohne eine zu starke Bindung an die einmal getroffene Wahl des Vertragspartners einzugehen. Durch unternehmungsübergreifende Elektronische Hierarchien, die auf den genannten Standards basieren, wird eine weitreichende Integration der Informationsverarbeitung mit hoher Effizienz der betreffenden Prozesse realisiert. Dabei wird der relative Vorteil leicht anpassbarer relationaler Verträge mit der starken Anreizwirkung kombiniert, die aus der Möglichkeit zum Wechsel des Vertragspartners resultiert.

\footnotetext{
${ }^{444}$ Vgl. Klein (1996), S. 159.

${ }^{445}$ Es ist zu beachten, dass die Kosten der Informationsverarbeitung nur einen Teil der gesamten Kosten der Leistungserstellung darstellen. Da sich die Untersuchung in dieser Arbeit auf den Bereich der Informationsverarbeitung beschränkt, wird davon ausgegangen, dass der vielfach empirisch belegte Effizienzvorteil einer spezifischen Ressourcengestaltung im Bereich der sonstigen Ressourcen unverändert bestehen bleibt. Es kommt durch die beschriebenen Gestaltungsoptionen des Informationsmanagements also nicht zu einer vollstăndigen Aufhebung des Verdrängungseffektes, sondern zu einer Abmilderung, deren Bedeutung vom relativen Kostenanteil der Informationsverarbeitung an den gesamten Kosten der Leistungserstellung abhăngt.

${ }^{446}$ Die Relevanz dieser Option wurde bereits 1987 von Malone/Yates/Benjamin erkannt, die schon damals auf das Potenzial der Informationstechnologie aufmerksam machten, „the best of both worlds" miteinander zu vereinen. Dies geschah, ohne dass seinerzeit viele der heute bestehenden Standards, insbesondere der Internet-Standard, verfügbar gewesen wären. Vgl. dazu Malone/Yates/Benjamin (1987), S. 494f.
} 
Das Ziel, „the best of both worlds“ miteinander zu vereinen, erfordert Konzepte, die sich darauf richten, zugleich einen höheren Anteil von Leistungen unternehmungsextern $\mathrm{zu}$ beziehen und die Zahl der Lieferanten $\mathrm{zu}$ reduzieren. ${ }^{447}$ Eine derartige Form des Leistungsaustausches macht es für die beteiligten Unternehmungen möglich, sich auf die Erstellung spezialisierter Produkte zu konzentrieren und dabei eng mit ihren Lieferanten bzw. Abnehmern zusammenzuarbeiten. ${ }^{448}$ Die dazu notwendige unternehmungsübergreifende Integration der Informationsverarbeitung findet im Prinzip bereits seit Mitte der 1980 er Jahre starke Beachtung in Theorie und Praxis. ${ }^{449}$ Die Integration zwischen den Vertragspartnern betrifft beispielsweise Informationen über die allgemeine Marktnachfrage, konkrete Produktionsaufträge, Lagerbestände, logistische Aktivitäten und ähnliches. ${ }^{450}$

Neu ist in diesem Zusammenhang die verstärkte Zuwendung zu dynamischeren Formen der Integration von Geschäftsprozessen kooperierender Organisationseinheiten. ${ }^{451}$ Während der hohe Spezifitätsgrad der unternehmungsübergreifenden Informationsverarbeitung bisher Kooperationen mit eher statischem Charakter verlangte, wird durch die inzwischen verfugbaren, neuen Standards eine kurzfristigere Ausrichtung der Zusammenarbeit möglich. Gerade der Internet-Technik wird zugesprochen, besonders geeignet für Partnerschaften zu sein, bei denen die Vertragspartner keine dauerhafte Bindung eingehen wollen. ${ }^{452}$ Aus Sicht der Transaktionskostentheorie wird die Effizienz des Leistungsaustausches durch diese Neuorientierung weiter verbessert, da die im Rahmen der Transaktion wirksamen Anreize unter weitgehender Vermeidung von Produktivitätsverlusten erhöht werden. Als Bezeichnung für flexible und zeitlich begrenzte Kooperationen dieser Art hat sich in der jüngsten Vergangenheit der Begriff des Virtuellen Unternehmens etabliert. ${ }^{453}$ Das Konzept des Virtuellen Unternehmens wird insbesondere für den Mittelstand als interessant gewertet, da kleinere Unternehmungen typischerweise nicht über genügend interne Ressourcen zur Abwicklung größerer Aufträge oder Projekte verfügen. ${ }^{454}$

Im Ergebnis wird hier also der „Move-to-the-Middle-Hypothese“ zugestimmt. Diese kann jedoch durch die Bezugnahme auf die für die Vorteilhaftigkeit der Koordinationsform ausschlaggebenden Einflussgrößen differenzierter begründet werden. Die Verknüpfung der Vorzüge von marktlicher und hierarchischer Koordination bedeutet vertragstheoretisch gesprochen einerseits, dass mehr Transaktionsbestandteile über eine eigene, am klassischen Vertragsmuster orientierte Vereinbarung abgewickelt werden. Andererseits bleiben die Grundzüge der Zusammenarbeit über einen längeren Zeitraum unverändert und können damit zum Gegenstand eines relationalen Vertragsarrangements gemacht werden. Dieses Vorgehen zielt darauf ab, die auf Grund der Exit-Option bestehende hohe Anreizwirkung klassischer Verträge mit der für unvollständige Verträge nach relationalem Vertragsmuster typischen, besseren Anpassbarkeit der Vereinbarung zu kombinieren. Dabei wird der Umstand ausgenutzt, dass die beiden

\footnotetext{
${ }^{447} \mathrm{Vgl}$. Malone/Yates/Benjamin (1987), S. $494 \mathrm{f}$.

${ }^{448}$ Vgl. Shaw (2000), S. 17.

${ }^{449}$ Vgl. Mertens (1985), S. 81 ff.

${ }^{450}$ Vgl. Shaw (2000), S. 20.

${ }^{451}$ Vgl. Buxmann/König (2000), S. 5.

${ }^{452}$ Vgl. Tan/Shaw/Fulkerson (2000), S. 469; Mattes (1999), S. 65; Sieber (1997), S. 217ff.

${ }^{453}$ Vgl. z.B. Wolter/Wolff/Freund (1998), S. 1ff.; Mertens/Griese/Ehrenberg (1998), S. 3; Sieber (1997), S. 200.

${ }^{454} \mathrm{Vgl}$. Wolter/Wolff/Freund (1998), S. 1ff.
} 
Vertragstypen trotz der oben angesprochenen Tendenz zur Nivellierung reziproke Kostenstrukturen aufweisen, da sie in den verschiedenen Vertragsphasen unterschiedlich transaktionskostenintensiv sind. Die hohen Ex ante-Transaktionskosten der marktlichen Koordinationsform werden durch die langfristig orientierte Konzentration auf feste Vertragspartner vermieden, während die hohen Ex post-Transaktionskosten der hierarchischen Koordinationsform durch die verringerte Bindung an die bisherige Vertragspartnerwahl reduziert werden.

Die bisherigen Überlegungen bezogen sich auf eine aggregierte Betrachtung der Koordinationsform ohne explizite Berücksichtigung der einzelnen Vertragsparteien. Tatsächlich hängt es allerdings von der konkreten Aufteilung der erzielbaren Effizienzgewinne ab, inwiefern eine aus der Gesamtsicht vorteilhafte Änderung des vertraglichen Arrangements auch für die einzelnen Vertragspartner erstrebenswert ist. Abschließend ist deshalb zu überprüfen, inwiefern die Mediatisierung der Koordination auch für die einzelnen Parteien eine Bewegung zu mittleren Koordinationsformen zwischen Markt und Hierarchie sinnvoll macht.

Aus Sicht des Leistungsanbieters ist eine zu starke Betonung marktlicher Koordinationselemente abzulehnen, wenn auf der Absatzseite (etwa wegen des hohen Erfolgsbeitrags eines Kunden) ein Interesse an der Fortsetzung der Geschäftsbeziehung besteht. In einem solchen Fall liegt es im Interesse des Anbieters, gegenüber dem Nachfrager die Möglichkeit zum Wechsel des Vertragspartners durch die Verwendung spezifischer Ressourcen, z.B. proprietärer elektronischer Kataloge, einzuengen. ${ }^{455}$ Die Verwendung eines rein klassischen Vertragsdesigns, also eine vollständige Vermarktlichung der Koordination und eine sehr starke Absenkung des Spezifitätsgrads, steht der Bindung von Kunden fur nachfolgende Transaktionen entgegen. Der Anbieter wird in diesem Fall eine lediglich unvollständige Vermarktlichung der Koordinationsform bevorzugen. Die These einer Entwicklung zu mittleren Koordinationsformen wird also auch aus Sicht des Anbieters bestätigt. Die beschriebene Zielsetzung des Anbieters dürfte jedoch wegen der Verfügbarkeit konkurrierender Angebote von potenziellen alternativen Vertragspartnern ohne ein komplementäres Interesse des Nachfragers nur schwer durchsetzbar sein.

Aus Sicht des Leistungsnachfragers stimmen bei einer vollständigen Vermarktlichung der Koordination die Gesamtbeurteilung und die persönliche Nutzenbeurteilung tendenziell besser überein, da der Nachfrager häufig in stärkerem Maße von einer Vermarktlichung profitiert. Auf der Beschaffungsseite bietet ein marktliches Vertragsarrangement $\mathrm{zu}$ (durch Maßnahmen des Informationsmanagements) reduzierten Ex ante-Transaktionskosten verbesserten Schutz vor opportunistischem Verhalten des Lieferanten. Deshalb ist unter Anreiz- und Kostengesichtspunkten von einem Interesse des Nachfragers an einer stärker marktlichen Koordination auszugehen. Beim Nachfrager kann jedoch eine zu den Bindungsinteressen des Anbieters komplementäre Interessensituation entstehen, wenn auch die Unsicherheit als Transaktionseigenschaft in die Betrachtung einbezogen wird. In diesem Fall können aus Sicht des Nachfragers die speziellen Vorteile eines unvollständigen vertraglichen Arrangements diejenigen Transaktionskostenvorteile, die sich durch den Wechsel der Koordinationsform potenziell erzielen lassen, überwiegen. Mit steigender Unsicherheit der Transaktion

\footnotetext{
${ }^{455}$ Vgl. Baron/Shaw/Bailey (2000), S. 390, ähnlich Gersch (1998), S. 173ff.
} 
wachsen die Schwierigkeiten einer ex ante vollständigen Festlegung der gegenseitigen Vertragsansprüche, wie sie ein klassisches Vertragsdesign erfordert. Die Möglichkeit zur nachträglichen Anpassung des Vertrages gewinnt dann an Bedeutung. Da bei klassischen Verträgen stets neue Vereinbarungen auszuhandeln sind, kann es bei wachsender Unsicherheit auch für den Anbieter vorteilhaft sein, auf eine vollständige Vermarktlichung der Koordination zu verzichten, selbst wenn diese unter dem Aspekt sinkender Spezifität durch das Informationsmanagement ermöglicht wird.

Zu dem gleichen Ergebnis gelangt man, wenn man die Betrachtung um Nutzengesichtpunkte erweitert. Die Spezifität einer Transaktion und die tendenziell höheren Transaktionskosten eines hierarchischen Vertragsarrangements lassen sich aus Sicht der beschaffenden Unternehmung dann rechtfertigen, wenn sie ihr zu Wettbewerbsvorteilen auf ihrem eigenen Absatzmarkt verhelfen. Auch in diesem Fall kann ein Verzicht auf die vollständige Ausnutzung der durch die Mediatisierung der Koordination eröffneten Vermarktlichungspotenziale sinnvoll sein, da auf dem eigenen Absatzmarkt eine Vergütung der höheren Koordinationskosten stattfindet. Eine stärker hierarchisch ausgerichtete Koordination mit einer größeren Bindung zwischen den Vertragspartnern erhöht dann den im Rahmen des Leistungsaustausches erzielbaren Gesamtnutzen.

\section{b) Sicht der Agency-Theorie}

Flankierende Erkenntnisse zu den oben aus der Perspektive der Transaktionskostentheorie abgeleiteten Aussagen können durch eine Bezugnahme auf die AgencyTheorie gewonnen werden. Als Vorteilhaftigkeitskriterium ist aus Sicht der AgencyTheorie die Frage zu überprüfen, ob sich durch einen eventuellen Wechsel der Koordinationsform die Summe der im Rahmen des Leistungsaustausches entstehenden Agency-Kosten reduzieren lässt. Dazu sind die drei Komponenten der Agency-Kosten, also die Garantiekosten, die Kontrollkosten sowie die verbleibenden Wohlfahrtsverluste, näher zu betrachten. Auf Grund des beschriebenen Crowding-out zwischen Kontroll- und Garantiekosten auf der einen Seite sowie verbleibenden Wohlfahrtsverlusten auf der anderen Seite führt ein Wechsel des vertraglichen Arrangements stets zu einer Veränderung der Agency-Kostenstruktur. ${ }^{456} \mathrm{Ob}$ es insgesamt $\mathrm{zu}$ einer Effizienzsteigerung oder einer Effizienzverringerung kommt, hängt ähnlich wie aus Sicht der Transaktionskostentheorie auch aus der Perspektive der Agency-Theorie von den Eigenschaften der Leistung und des Leistungserstellungsprozesses ab. Bei gut beurteilbaren Leistungen sind wegen der vergleichsweise niedrigen Garantie- und Kontrollkosten tendenziell marktliche Arrangements auf Grund der starken Anreizwirkungen vorzuziehen. Dagegen fallen bei nur schlecht zu beurteilenden Leistungen die Vorteile, die die hierarchischen Koordination im Bereich der Garantie- und Kontrollkosten aufweist, stärker ins Gewicht. Je bedeutender ferner die für die Erstellung der Leistung zu erbringenden Vorleistungen sind, um so effizienter erscheinen auf Grund der hidden intention-Problematik dauerhafte Kooperationen mit starken hierarchischen Vertragselementen. Auslöser für einen Wechsel des vertraglichen Arrangements können also einerseits Veränderungen bei den mit den einzelnen

\footnotetext{
${ }^{456}$ Produktionsbezogene Gesichtspunkte, die zwingend eine Internalisierung der Leistungserstellung erfordern könnten, werden hier aus der Analyse ausgeklammert. Die Überlegungen beziehen sich an dieser Stelle nur auf den Austausch solcher Leistungstypen, bei denen eine Wahl zwischen alternativen Koordinationsformen überhaupt realistisch ist.
} 
Vertragsdesigns verbundenen Kostenarten und andererseits Veränderungen der Leistungseigenschaften und der notwendigen Vorleistungen sein.

Geht man zunächst davon aus, dass die Leistungseigenschaften und die notwendigen Vorleistungen gegeben sind, so kann die Untersuchung auf die Kostenstruktur der verschiedenen Vertragsdesigns beschränkt werden. Die in diesem Kapitel behandelten Gestaltungsoptionen des Informationsmanagements eröffnen bei allen Koordinationsformen Potenziale zur Senkung von Agency-Kosten. Elektronische Märkte auf der einen Seite unterstützen vor dem Hintergrund der hidden characteristics-Problematik Screening- und Signalling-Aktivitäten und reduzieren dadurch Garantie- und Kontrollkosten der marktlichen Koordinationsform. Elektronische Hierarchien auf der anderen Seite reduzieren ebenfalls in erster Linie Garantie- und Kontrollkosten, ihr Anwendungsbereich liegt jedoch bei den nach Abschluss des ursprünglichen Vertrages entstehenden Kostenarten und damit bei der hidden action-Problematik. Wie erläutert lassen einige Ausprägungen dieses Systemtyps, etwa im Bereich der Workflow Management-Systeme und zum Teil auch der methodenorientierten analytischen Anwendungssysteme, in begrenztem Umfang auch die Verringerung von Verhaltensspielräumen zu und ermöglichen dadurch zusätzlich die Reduzierung der verbleibenden Wohlfahrtsverluste. Die Anreizwirkungen der verschiedenen Vertragstypen werden dagegen durch den Einsatz Elektronischer Märkte und Elektronischer Hierarchien nicht berührt.

Wie Abbildung 5/19 verdeutlicht, lässt sich argumentativ nachweisen, dass die Senkung von Agency-Kosten durch den Einsatz Elektronischer Märkte und Elektronischer Hierarchien nicht einfach zu einer allgemeinen Senkung des Kostenniveaus bei allen Vertragsformen ohne Veränderung der Vorteilhaftigkeit führt. Komparativ gesehen ist vielmehr ein stärkerer Effekt bei der marktlichen Koordinationsform zu erwarten. Diese Annahme ist damit zu begründen, dass das Kostensenkungspotenzial bei marktlich orientierten Formen des Leistungsaustausches die kostenintensiveren Komponenten der Agency-Kosten betrifft. Bei hierarchisch orientierten Formen des Leistungsaustausches sind dagegen diejenigen Kostenarten betroffen, die anteilig an den Gesamtkosten ohnehin weniger ins Gewicht fallen. ${ }^{457}$ Selbst wenn man annimmt, dass auch die jeweils verbleibenden Wohlfahrtsverluste zum Teil durch verbesserte Garantie- und Kontrollsysteme reduziert werden können, dürfte die relative Kostensenkung wegen der koordinationsformspezifischen Anreizsituation dennoch bei der marktlichen Koordination stärker ins Gewicht fallen.

\footnotetext{
${ }^{457}$ Mit einer ăhnlichen Argumentation begründen Malone/Yates/Benjamin, dass sich der Einsatz Elektronischer Märkte und Elektronischer Hierarchien relativ stärker auf die Koordinationskosten der marktlichen Koordination auswirkt. Vgl. Malone/Yates/Benjamin (1987), S. 489. Sie stützen sich dabei allerdings auf transaktionskostentheoretische Argumente, die später auf Grund der oben angesprochenen systematischen Unterschătzung der internen Transaktionskosten kritisiert wurden. Eine Begründung auf Basis der Agency-Theorie kommt unter Vermeidung dieses Kritikpunkts zum gleichen Ergebnis.
} 
Markt

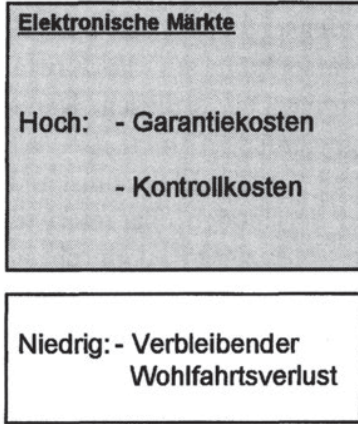

Hierarchie

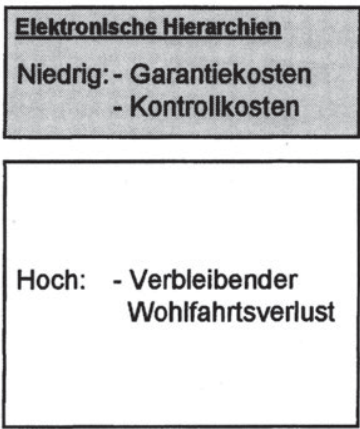

Abb. 5/19: Asymmetrische Reduzierung von Agency-Kosten durch die Mediatisierung von Koordinationsprozessen

Aus diesen Überlegungen folgt, dass bei einem Wechsel von der hierarchischen zur marktlichen Koordination kostensenkende Anreizeffekte entstehen, während der kostensteigernde Verlust der Kontroll- und Garantiemöglichkeiten durch die Leistungsmerkmale Elektronischer Märkte abgemildert wird. Aus der Perspektive der AgencyTheorie ist deshalb ceteris paribus davon auszugehen, dass sich die Vorteilhaftigkeit der alternativ möglichen Koordinationsformen dahingehend verändert, dass ein verstärkter Einsatz marktlicher Koordinationselemente die Effizienz des Leistungsaustausches fördert. Dabei wird eine detailliertere Explizierung von bisher auf relationalen Vertragsarrangements basierenden Ansprüchen möglich, die auf Grund ihres impliziten Charakters im Konfliktfall nur schwer durchzusetzen sind.

Ausgehend von dieser These, die ansonsten unveränderte Transaktionsbedingungen voraussetzt, wird im Folgenden die Annahme aufgehoben, dass die Eigenschaften der auszutauschenden Leistungen exogen gegeben sind. Wenn im Gegenteil von der Möglichkeit zur Gestaltung der Leistungseigenschaften durch das Informationsmanagement ausgegangen wird, dann ist aus Sicht der Agency-Theorie eine Verbesserung der Beurteilbarkeit der Leistung anzustreben. Je bessere Gelegenheiten der Auftraggeber hat, die Qualitätseigenschaften der vom Auftragnehmer erbrachten Leistung zu beobachten und zu beurteilen, um so geringer sind die opportunistischen Verhaltensspielräume des Auftragnehmers. Bei schlecht beobachtbaren Leistungen empfiehlt sich im Allgemeinen eine Internalisierung der Leistungserstellung zur Verbesserung der Kontrollmöglichkeiten, während gut beobachtbare Leistungen zur Verbesserung der Anreizsituation tendenziell eher über marktlich orientierte Vertragsarrangements ausgetauscht werden können. Vor diesem Hintergrund müssten sich effizienzsteigernde Maßnahmen darauf richten, die Handelbarkeit der Leistung auf Märkten, bezogen auf den Gestaltungsbereich des Informationsmanagements also Elektronischen Märkten, zu fördern.

Die Möglichkeiten, über die das Informationsmanagements in diesem Zusammenhang verfügt, wurden bereits in Abschnitt 5.3.1 beleuchtet. Zum einen kann der Anteil an Sucheigenschaften durch verbesserte Beschreibungsmöglichkeiten sowie das Angebot digitaler Zusatzleistungen erhöht werden. Zum anderen können durch eine Individualisierung des Angebots Folgekäufe angeregt werden, sodass indirekt eine Umwand- 
lung von Erfahrungs- in Sucheigenschaften stattfindet. Wenn durch die Mediatisierung der Koordination die Möglichkeit zur Vermarktung der Leistung neu geschaffen wird, dann ist zu erwarten, dass unter agency-theoretischen Gesichtspunkten in vielen Fällen die Gesamteffizienz des Leistungsaustausches erhöht werden kann, indem ein Wechsel von relationalen zu stärker am klassischen Vertragsmuster orientierten, marktnäheren Arrangements vorgenommen wird.

$\mathrm{Da}$ es auch durch die Gestaltung der Leistungserstellungsprozesse zu einer veränderten Effizienz der alternativ möglichen Vertragsdesigns kommen kann, sind abschließend noch die Auswirkungen der zunehmenden Verfügbarkeit von Standards im Bereich der unternehmungsübergreifenden Informationsverarbeitung zu betrachten. Durch die Nutzung der in Abschnitt 5.3.4.1 genannten technischen und betriebswirtschaftlichen Standards wird es in vielen Fällen möglich, das Ausmaß der für den Aufbau einer unternehmungsübergreifenden Zusammenarbeit zu erbringenden Vorleistungen erheblich zu reduzieren. Diese Gestaltungsoption ist besonders im Hinblick auf die bei der Festlegung des Vertragsdesigns auftretende hidden intentionProblematik relevant. Die bei Nutzung solcher Standards erleichterte Möglichkeit zum Wechsel des Vertragspartners erhöht im Rahmen hierarchisch koordinierter Formen des Leistungsaustausches den Anreiz zu effizientem Verhalten.

Damit ergeben sich Auswirkungen auf die Vorteilhaftigkeit der Koordinationsform, die den bisher behandelten Entwicklungen im Bereich der Effizienz von Vertragsarrangements und der Leistungseigenschaften entgegen gerichtet sind. Es kommt zu einer einseitigen Verbesserung der Anreizsituation bei unternehmungsübergreifenden, dauerhaften Kooperationen mit starken hierarchischen Koordinationselementen Bei Leistungen, die bisher über marktlich orientierte Vertragsarrangements ausgetauscht wurden, kann infolgedessen eine Intensivierung der Zusammenarbeit zwischen den Vertragspartnern sinnvoll erscheinen. Dadurch lassen sich Garantie- und Kontrollkosten unter weitgehender Vermeidung der negativen Anreizwirkungen einer vollständigen Internalisierung der Leistungserstellung erzielen. Umgekehrt ergeben sich ausgehend von einer bisher internen Koordination der Leistungserstellung neue Optionen zur Externalisierung, die eine Verbesserung der Anreizsituation unter weitgehender Vermeidung von Kostensteigerungen bei den Garantie- und Kontrollkosten möglich macht. Im Gegensatz zu den oben behandelten Gestaltungsoptionen läuft dies allerdings nicht auf eine vollständige Vermarktlichung mit klassischen Vertragsdesigns, sondern auf eine dauerhaft ausgerichtete Kooperation mit einer Mischung klassischer und relationaler Vertragselemente hinaus.

Auch aus der Perspektive der Agency-Theorie werden also durch die in diesem Kapitel behandelten Gestaltungsoptionen des Informationsmanagements neue Handlungsstrategien vorstellbar, die darauf abzielen, die Vorteile der marktlichen und der hierarchischen Koordinationsform miteinander zu verknüpfen.

\subsection{Aussagekraft des Institutionenökonomischen Ansatzes für eine Theorie des Informationsmanagements}

Die Neue Institutionenökonomie lehnt die Vereinfachungen des neoklassischen Modells, auf dem der in Kapitel 3 dieser Arbeit referenzierte Faktortheoretische Ansatz der Betriebswirtschaftslehre beruht, ab und konzentriert sich auf eine andere, „empi- 
risch robustere ${ }^{\text {4458 }}$ Abbildung des Untersuchungsbereichs. Durch die Anerkennung von begrenzter Rationalität, Marktunvollkommenheiten sowie der internen Koordinationsprobleme in Organisationen wird ein erheblicher konzeptioneller Fortschritt erreicht. ${ }^{459}$ Der Ansatz ist durch eine einfache und präzise Theoriekonstruktion gekennzeichnet und erreicht in Bezug auf klar definierte Problemstellungen mit einer geringen Anzahl von Grundkonzepten und erklärenden Variablen einen hohen Erklärungsgehalt. ${ }^{460}$ Diese Einfachheit und die Zielsetzung einer Verbindung von Markt- und Organisationstheorie lassen die Neue Institutionenökonomie als attraktive Theorie der Koordination ökonomischer Aktivitäten erscheinen. ${ }^{461}$

Genau genommen handelt es sich bei den verschiedenen Zweigen der Neuen Institutionenökonomie allerdings nicht um wirklich neue Ideen. So kombiniert die Transaktionskostentheorie zentrale Annahmen der verhaltenswissenschaftichen Entscheidungstheorie mit der bis in die 1930er Jahre zurückreichenden, eher im disziplinären Kontext der Volkswirtschaftslehre geführten Diskussion um die Abgrenzung von Märkten und Unternehmungen als zentrale Institutionen der Wirtschaft. ${ }^{462}$ Auch in Deutschland ist das institutionelle Denken in der Wirtschaftswissenschaft keineswegs neu. Es hat im Gegenteil eine lange Tradition. In der Volkswirtschaftslehre findet es sich etwa in der historischen Schule und dem Ordoliberalismus und auch in Teilgebieten der Betriebswirtschaftslehre, wie z.B. im Rechnungswesen, der Wirtschaftsprüfung oder der Steuerlehre wird die Bedeutung von Institutionen seit langem in die Analyse einbezogen. ${ }^{463}$ Die Bildung von Theorien wurde davon allerdings nicht dominant beeinflusst. Allerdings kann auch in der Ex post-Systematisierung von Sachverhalten und Gestaltungsmöglichkeiten eine hoch einzuschätzende Leistung wissenschaftlicher Aktivitäten liegen. ${ }^{464}$ Positiv formuliert liegt gerade eine Stärke der Neuen Institutionenökonomie und insbesondere der Transaktionskostentheorie darin, dass sie die Ergebnisse anderer betriebswirtschaftlicher Theorien zu integrieren und auf deren Ergebnisse zurückzugreifen vermag. ${ }^{465}$

Dennoch sind die Neue Institutionenökonomie und ihre verschiedenen Teilgebiete nicht ohne Kritik geblieben, die sich zum Teil auf den Ansatz als Ganzes und zum Teil auf einen noch unzureichenden Ausbau der Theorie bezieht. Die nachfolgenden Überlegungen beleuchten vor diesem Hintergrund die Aussagekraft des hier entwickelten Institutionenökonomischen Ansatzes für eine Theorie des Informationsmanagements. In Abschnitt 5.4.1 wird zunächst untersucht, welche Ansätze zur Lösung der im zweiten Kapitel beschriebenen idealtypischen Probleme der Informationsverarbeitung in der Praxis sich mit den Mitteln der Neuen Institutionenökonomie ableiten lassen. Darauf folgt in Abschnitt 5.4.2 eine kritische Würdigung, die ausgehend von der an der Neuen Institutionenökonomie geübten Kritik die Erkenntnisgrenzen des Institutionenökonomischen Ansatzes für eine Theorie des Informationsmanagements überprüft.

\footnotetext{
${ }^{458}$ Richter/Furubotn (1999), S. 477.

${ }^{459} \mathrm{Vgl}$. Sydow (1992), S. 145.

${ }^{460}$ Vgl. Ebers/Gotsch (1999), S. 251.

${ }^{461}$ Vgl. Sydow (1992), S. 145.

${ }^{462} \mathrm{Vgl}$. Witt (1995), S. 11.

${ }^{463}$ Vgl. Budäus/Gerum/Zimmermann (1988), S. 9.

${ }^{464} \mathrm{Vgl}$. Schanz (1997), S. 149.

${ }^{465}$ Vgl. Ebers/Gotsch (1999), S. 242.
} 


\subsubsection{Lösungsansätze für offene Probleme in der Praxis}

Das Erkenntnisinteresse der Neuen Institutionenökonomie richtet sich auf die Koordination des Leistungsaustausches. Prozesse der Informationsverarbeitung können dementsprechend unter zwei Gesichtspunkten betrachtet werden. Einerseits koordinieren sie als begleitende Prozesse die eigentliche Leistungserstellung, andererseits handelt es sich um Prozesse, die selbst Gegenstand von koordinierenden Aktivitäten sind. Diese beiden Aspekte stellen die Ansatzpunkte zur Ableitung von Lösungsbeiträgen für die im zweiten Kapitel dargesteliten ungelösten Problemstellungen in der Praxis dar, die ein Institutionenökonomischer Ansatz für eine Theorie des Informationsmanagements leisten kann. Im folgenden Abschnitt 5.4.1.1 wird zunächst überprüft, welche Anhaltspunkte sich aus dem hier entwickelten Ansatz ableiten lassen, um die Effektivität der Informationsverarbeitung zu gewährleisten. Wegen der Besonderheiten des Institutionenökonomischen Ansatzes werden im anschließenden Abschnitt 5.4.1.2 die Problemkreise Effizienz der Informationsverarbeitung und organisatorische Zuordnung informationswirtschaftlicher Aufgaben gemeinsam betrachtet. Abschnitt 5.4.1.3 untersucht die Rolle des Menschen bei der Informationsverarbeitung aus Sicht der Neuen Institutionenökonomie.

\subsubsection{Effektivität der Informationsverarbeitung}

Die Frage, wie die technikunterstützte Informationsverarbeitung auf die allgemeinen Unternehmungsziele ausgerichtet werden kann, steht nicht im Mittelpunkt des hier entwickelten Institutionenökonomischen Ansatzes. Strategische Fragen werden in der Neuen Institutionenökonomie aus der Betrachtung ausgeklammert, sodass die unternehmungsstrategische Bedeutung, die der Erstellung einer (informationellen) Leistung zukommt, nicht explizit berücksichtigt wird. ${ }^{466}$ Der Nutzen des Austausches von Leistungen wird also nicht zum Gegenstand der Analyse gemacht, sondern die Untersuchungen fokussieren Kosten- und Effizienzgesichtspunkte. Aus diesem Grund können nur indirekt Anhaltspunkte darüber gewonnen werden, wie das Informationsmanagement die Effektivität der Informationsverarbeitung erhöhen kann.

Anders als der im vorigen Kapitel behandelte Systemorientierte Ansatz der Betriebswirtschaftslehre, der mit dem Überleben des Systems Unternehmung von einer sehr abstrakten Zielsetzung ausgeht, macht die Neue Institutionenökonomie konkretere Angaben über die durch die Unternehmung zu verfolgenden Ziele. Im Gegensatz zu anderen Wissenschaftsprogrammen der Betriebswirtschaftslehre ist die Neue Institutionenökonomie bzw. die Transaktionskostentheorie als eines ihrer Teilgebiete in der Lage, die Existenz von Unternehmungen überhaupt zu erklären. ${ }^{467}$ Unternehmungen realisieren die hierarchische Koordinationsform des Leistungsaustausches und werden entsprechend der spezifischen Sichtweise dieses Ansatzes gegründet, um Marktversagen, das aus hohen Kosten der Nutzung des Preismechanismus resultiert, zu überwinden. Demzufolge besteht die Zielsetzung von Unternehmungen darin, die Erstellung und den Austausch einer bestimmten Leistung zu niedrigeren Gesamtkosten als die marktliche und hybride Koordinationsformen zu gewährleisten, wobei die Gesamtkosten die Summe aus Transaktions- und Produktionskosten darstellen. Aus Sicht der

\footnotetext{
${ }^{466}$ Vgl. Gersch (1998), S. 88; Picot (1991), S. 346f.

${ }^{467}$ Vgl. Ebers/Gotsch (1999), S. 241.
} 
Neuen Institutionenökonomie ist die interne Erstellung einer Leistung innerhalb der Unternehmung also kein Selbstzweck, sondern sie ist allein auf Grund der relativ höheren Effizienz gegenüber allen anderen Vertragsformen zu rechtfertigen.

Aus dieser Zielsetzung der Gesamtunternehmung lässt sich die Zielsetzung der technikunterstützten Informationsverarbeitung ableiten. Das Kriterium, an dem die Effektivität der Informationsverarbeitung zu messen ist, ist die Koordinationseffizienz der begleiteten Transaktion. Die übliche Unterscheidung zwischen Effektivität und Effizienz, bei der unter Effektivität der Grad der Zielerreichung und unter Effizienz die Einhaltung des Wirtschaftlichkeitsprinzips beim Vorgehen zur Zielerreichung verstanden wird, kann auch unter diesen Bedingungen aufrecht erhalten werden. Maßnahmen des Informationsmanagements sind dementsprechend dann effektiv, wenn sie die Effizienz der Koordination erhöhen. Sie sind jedoch nicht zwingend zugleich auch effizient, sondern erst dann, wenn sie bei der Steigerung der Effizienz selbst das Wirtschaftlichkeitsprinzip einhalten. Diesen Zusammenhang verdeutlicht Abbildung $5 / 20$.

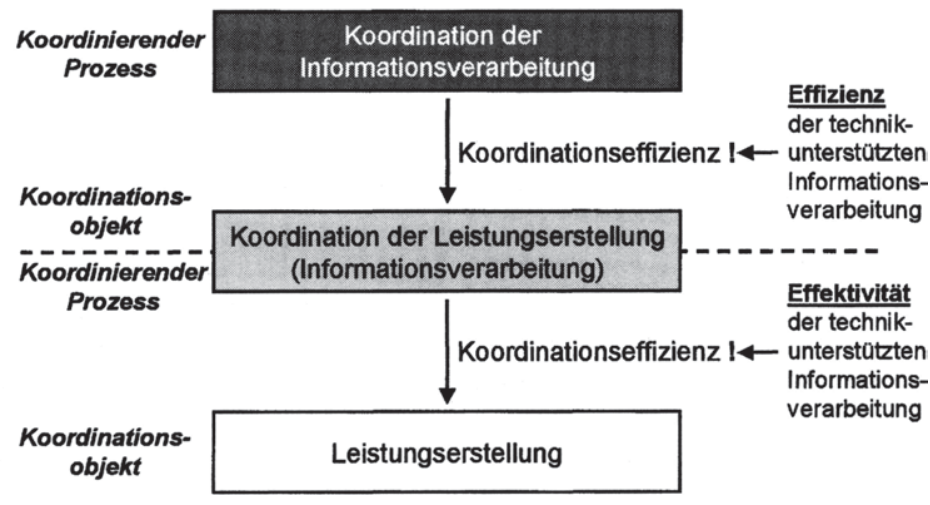

Abb. 5/20: Effektivität und Effizienz der technikunterstützten Informationsverarbeitung aus Sicht der Neuen Institutionenökonomie

Wie im Abschnitt 5.4.2 bei der Betrachtung der Erkenntnisgrenzen noch näher erläutert werden wird, kann die Koordinationseffizienz auf Grund methodischer Probleme im Rahmen der Neuen Institutionenökonomie nicht absolut bestimmt werden. Wegen der informationellen Beschränkungen in der realen Welt können alle in der Praxis verwendeten Koordinationsformen im Vergleich zum friktionslosen, neoklassischen Modell niemals mehr als Second Best-Lösungen sein. ${ }^{468}$ Das Kriterium für die Effizienz einer Koordinationsform ist deshalb, dass zur Erstellung und zum Austausch einer Leistung relativ weniger Ressourcen verbraucht werden als bei allen anderen vertraglichen Arrangements, die im konkreten Fall als Koordinationsalternative in Frage kommen. Für das Informationsmanagement kann daraus folgende Effektivitätsbedingung abgeleitet werden: Die Effektivität der technikunterstützten Informationsverarbeitung ist dann gegeben, wenn der die eigentliche Leistungserstellung begleitende Koordinationsprozess so ausgestaltet ist, dass unter den gegebenen technischen

${ }^{468}$ Vgl. Eggertson (1990), S. 21; Richter/Furubotn (1999), S. $488 f$. 
Rahmenbedingungen kein Wechsel des Vertragsdesigns für die betreffende Transaktion sinnvoll ist. Umgekehrt liegt Ineffektivität der Informationsverarbeitung vor, wenn andere, ebenfalls zur Wahl stehende Koordinationsformen eine höhere relative Effizienz aufweisen, sodass entweder ein Wechsel der Koordinationsform vorteilhaft erscheint oder weitere Effizienzsteigerungen innerhalb des bisherigen Vertragsarrangements erforderlich sind.

Die bisherigen Überlegungen betrafen die Effektivität des technikunterstützten Informationssystems der Unternehmung als Ganzem. Betrachtet man einzelne Anwendungssysteme innerhalb dieses Gesamtsystems, so ist deren Effektivität daran zu messen, ob den Nutzern tatsächlich die zur Lösung ihrer Aufgaben benötigten Informationen in adäquater Form zur Verfugung gestellt werden. Die Transaktionskostentheorie und insbesondere die Agency-Theorie bieten ebenfalls Anhaltspunkte zur Lösung dieser Fragestellung. Diese beziehen sich auf die verschiedenen Arten von Informationsasymmetrien, die in den aufeinanderfolgenden Phasen des Leistungsaustausches auftreten. Die Neue Institutionenökonomie identifiziert nicht nur diese Arten von Informationsasymmetrien, sondern sie sieht auch Maßnahmen vor, mit denen daraus resultierende Ineffizienzen reduziert werden können. Softwaresysteme, deren Anwendungsbereich in der Koordination der eigentlichen Leistungserstellungsprozesse liegt, müssen Funktionalitäten zur Unterstützung dieser Maßnahmen bieten. Die Effektivität einzelner Anwendungssysteme kann also hergestellt werden, indem das Informationsmanagement bei ihrer Gestaltung und Steuerung vorsieht, die in diesem Kapitel erläuterten Einflussmöglichkeiten zur Verringerung der im Verlauf einer Transaktion auftretenden Informationsasymmetrien zu unterstützen.

Problematisch an der von der Neuen Institutionenökonomie verwendeten Effektivitätsbzw. Effizienzbedingung sind die damit einhergehenden Messbarkeitsprobleme. Diese betreffen zunächst einmal die fehlende Quantifizierbarkeit der Transaktionskosten an sich. ${ }^{469}$ Wenn die fur die Abwicklung einer Transaktion im Rahmen eines bestimmten vertraglichen Arrangements entstehenden Kosten der Koordination nicht exakt quantifiziert werden können, dann ist auch ein relativer Vergleich alternativer Koordinationsformen nicht zuverlässig möglich. Folglich ist das Effizienzkonzept der Transaktionskostentheorie so elastisch, dass ex post im Prinzip jede Koordinationsform als effizient erklärt werden kann. ${ }^{470}$ Ferner bedeutet das Fehlen eines absoluten Effizienzpunkts, das nur nach einer „effizienten“, nicht aber nach der „effizientesten“ Lösung gesucht werden kann. Wenn eine Situation erreicht ist, in der die verwendete Koordinationsform besser als alle alternativ möglichen Vertragsdesigns ist, so ist die Effizienzbedingung bereits erfullt, und es besteht kein Anlass, die Suche nach weiteren Effizienzsteigerungen fortzusetzen. Tatsächlich bezieht sich die Effizienz immer auf den gegebenen Möglichkeitsbereich eines Individuums, bei dessen Abgrenzung jedoch unter Umständen nicht alle relevanten Nebenbedingungen berücksichtigt wurden. ${ }^{471}$ Aus diesem Grund kann jedes realisierte Ergebnis als effizient (oder auch als ineffizient) gedeutet werden. Die Koordinationseffizienz als Kriterium für die Effektivität der Informationsverarbeitung kann für das Informationsmanagement in der Praxis also nicht mehr als eine Näherungsgröße darstellen.

\footnotetext{
${ }^{469}$ Vgl. dazu Picot (1982), S. 270; Williamson (1985), S. $21 \mathrm{f}$.

${ }^{470}$ Vgl. Sydow (1992), S. 152.

${ }^{471}$ Vgl. Richter/Furubotn (1999), S. 489.
} 


\subsubsection{Effizienz der Informationsverarbeitung und organisatorische Zuordnung informationswirtschaftlicher Aufgaben}

Die im vorigen Abschnitt angestellten Überlegungen zur Effektivität betrachteten die technikunterstützte Informationsverarbeitung unter dem Aspekt der Koordination von Prozessen der eigentlichen Leistungserstellung. Dagegen ist bei der Untersuchung der Effizienz der Gesichtspunkt zu fokussieren, dass die technikunterstützte Informationsverarbeitung ebenfalls ein Objekt von Koordinationsmaßnahmen darstellt. Während also bisher die Koordinationseffizienz der Transaktion als Kriterium für die Effektivität von Maßnahmen des Informationsmanagements analysiert wurde, ist nun die Wirtschaftlichkeit zu untersuchen, mit der das Informationsmanagement auf die Herstellung der Transaktionseffizienz hinwirkt. Damit rückt die Frage in den Mittelpunkt, wie die Prozesse der Informationsverarbeitung zu koordinieren sind, um selbst dem Effizienzkriterium zu genügen.

Um die Effizienz der technikunterstützten Informationsverarbeitung mit den Mitteln von Transaktionskostentheorie und Agency-Theorie analysieren zu können, sind die entsprechenden Prozesse selbst als Transaktionen aufzufassen, die im Hinblick auf eine möglichst hohe Koordinationseffizienz zu gestalten sind. Dabei findet eine Verlagerung der Untersuchung auf die Meta-Ebene statt, da betrachtet wird, wie die eine Transaktion koordinierenden Prozesse selbst koordiniert werden. Darüber hinaus sind die folgenden Überlegungen in analoger Weise auf solche Prozesse der Informationsverarbeitung anwendbar, die nicht der Koordination dienen, sondern der Herstellung informationeller Produktbestandteile, die Teil der an den Nachfrager übergebenen Leistung sind. Da die Effizienz von Transaktionen unter den Annahmen der Neuen Institutionenökonomie nicht nur von den Eigenschaften der Transaktion, sondern auch von der gewählten Koordinationsform abhängt, kann die Effizienz der technikunterstützten Informationsverarbeitung nicht unabhängig von der organisatorischen Zuordnung informationswirtschafticher Aufgaben analysiert werden. Beide Problemkreise werden daher im Folgenden gemeinsam behandelt.

Die Transaktionskostentheorie und die Agency-Theorie liefern Konzepte zur Analyse und Gestaltung des Austausches von Leistungen, die ein hohes Maß an Allgemeingültigkeit aufweisen. Das bedeutet, dass die in genereller Form formulierten Aussagen dieser beiden Teilgebiete der Neuen Institutionenökonomie im Prinzip auch auf den Bereich der technikunterstützten Informationsverarbeitung übertragen werden können. Dabei sind grundsätzlich die gleichen Einflussgrößen zu berücksichtigen, die bisher in diesem Kapitel allgemeingültig für jeden Transaktionstyp betrachtet wurden. Wenn Prozesse der Informationsverarbeitung also selbst als Transaktionen aufgefasst werden, dann lassen sich ohne größere Modifikationen Aussagen darüber gewinnen, wie eine möglichst hohe Effizienz dieses speziellen Transaktionstyps hergestellt werden kann. Diese Aussagen betreffen einerseits die Vorteilhaftigkeit alternativ möglicher Koordinationsformen der Informationsverarbeitung und andererseits den Umgang mit Auftraggeber/Auftragnehmer-Problemen, die in den einzelnen Transaktionsphasen zwischen den verschiedenen Trägern informationswirtschaftlicher Aufgaben bestehen.

Die nachfolgenden Überlegungen beginnen bei der Frage, welche Gestaltungsempfehlungen sich mit Hilfe der Neuen Institutionenökonomie für die Wahl der Koordinationsform von Prozessen der Informationsverarbeitung ableiten lassen. Damit wird von dem Vorgehen in Abschnitt 5.3, in dem zunächst die verschiedenen 
Transaktionsphasen und anschließend das Vertragsdesign untersucht wurden, abgewichen. Dies entspricht der dort einleitend gemachten Anmerkung, dass bei konkreten Transaktionen bereits vor der Aufnahme von Suchaktivitäten Vorstellungen darüber bestehen müssen, welches Vertragsmuster zur Koordination der Transaktion angestrebt wird. Die Wahl der Koordinationsform stellt sich im Bereich der technikunterstützten Informationsverarbeitung nur dann als eigenständige Problemstellung, wenn die betreffenden Aktivitäten überhaupt von den eigentlichen Prozessen der Leistungserstellung losgelöst werden können. Bei unmittelbar zur Koordination der Leistungserstellung dienenden Aktivitäten (z.B. im Bereich des Workflow Managements) ist dies nicht der Fall, da diese untrennbar mit den entsprechenden Prozessen verbunden sind. Dagegen ist bei Aktivitäten, die selbst zur Unterstützung der unmittelbaren Koordinationsprozesse dienen (z.B. im Bereich der Netzwerkpflege oder des Benutzer-Service), eine eigenständige organisatorische Lösung vorstellbar, die von derjenigen im Bereich der eigentlichen Leistungserstellung abweichen kann.

Entsprechend den oben erläuterten Annahmen von Transaktionskostentheorie und Agency-Theorie sind die Transaktionseigenschaften, insbesondere die Spezifität bzw. die zu erbringenden Vorleistungen, näher zu betrachten, um Aussagen über die Wahl der Koordinationsform zu gewinnen. Bei Bezugnahme auf diese Einflussgrößen können für das Informationsmanagement folgende Anhaltspunkte für die Gestaltung und Steuerung von unterstützenden informationswirtschaftichen Prozessen abgeleitet werden: Das Ausmaß an Vorleistungen ist möglichst weit zu reduzieren, um durch die realistische Möglichkeit zum Austritt aus der Zusammenarbeit eine geringe Abhängigkeit des Auftraggebers vom Auftragnehmer zu erreichen. Bei hoher Spezifität des Leistungsergebnisses ist deshalb zu überprüfen, ob (z.B. mit Hilfe des Baukastenprinzips) eine Trennung der Spezifität des Ergebnisses von der Spezifität der erforderlichen Leistungserstellungsprozesse vorgenommen werden kann.

Wenn dies möglich ist oder wenn eine relativ niedrige Spezifität ohnehin vorliegt, dann kann sich das Vertragsdesign stärker am klassischen oder neoklassischen Muster orientieren, um durch einen relativ hohen Anteil an marktlichen Koordinationselementen eine gute Anreizsituation zu realisieren. Das würde bedeuten, dass die betreffenden Prozesse aus der Unternehmung ausgelagert und durch externe Partner erbracht werden könnten. Beispiele für derartige Prozesse, die auf der Meta-Ebene die Koordination der eigentlichen Leistungserstellung unterstützen und dabei typischerweise ein niedriges bis mittleres Spezifitätsniveau aufweisen, sind etwa der Betrieb von Großrechnern oder die Durchführung von Benutzerschulungen für Standardsoftware. Aus Sicht der Neuen Institutionenökonomie sind für diese Leistungstypen vertragliche Arrangements anzustreben, die einen möglichst reibungslosen Wechsel des Vertragspartners ermöglichen. Dies gilt insbesondere dann, wenn die Kontrolle des Auftragnehmers durch den Auftraggeber auf das Leistungsergebnis beschränkt werden kann, weil dieses zum einen relativ leicht zu beobachten und zum anderen durch den Auftragnehmer zu beeinflussen ist. Häufig sind unter diesen Voraussetzungen hybride Arrangements interessant, bei denen die Vertragspartner sich einerseits im Rahmen einer dauerhaft Kooperation (teilweise) spezialisieren können, während andererseits zugleich die gegenseitige Bindung begrenzt bleibt.

Wenn eine Trennung der Spezifität von Ergebnis und Prozess dagegen nicht möglich ist, wenn die speziellen Eigenschaften der Transaktion also eine hohe Spezifität der Leistungserstellungsprozesse zwingend erforderlich machen, dann verhindert dies auch 
einen friktionslosen Wechsel des Vertragspartners. Bei derartigen Transaktionen bestehen höhere Anreize zu opportunistischem Verhalten, denen das vertragliche Arrangement durch eine stärkere Betonung hierarchischer Koordinationselemente Rechnung tragen muss. Beispiele für Transaktionen dieses Typs auf der Meta-Ebene der Informationsverarbeitung sind etwa fachkonzeptbezogene Beratungsleistungen bei der Entwicklung von Anwendungssystemen oder strategische Planungsaktivitäten für den Bereich der technikunterstützten Informationsverarbeitung. Die Anforderungen an die spezifische Kenntnis der Unternehmungssituation sind für die jeweiligen Träger dieser Aufgaben so hoch, dass die Wahl der Exit-Option zumindest für den Auftraggeber häufig mit hohen Wechselkosten verbunden ist. Die Vorleistung des Auftraggebers liegt dabei in der intensiven Einarbeitung des Auftragnehmers zu Beginn der Vertragslaufzeit. Eine starke gegenseitige Bindung von Auftraggeber und Auftragnehmer lässt sich bei diesem Leistungstyp nicht vermeiden. Unter diesen Bedingungen ist es erforderlich, eine längerfristige Zusammenarbeit aufzubauen und zur Vermeidung von Ineffizienzen die durch die Neue Institutionenökonomie vorgesehenen Möglichkeiten zur Disziplinierung des Auftragnehmers zu nutzen.

Erweitert man die Untersuchung um weitere Transaktionseigenschaften, so sind zusätzlich die Unsicherheit und die Häufigkeit der Transaktion zu berücksichtigen. Wenn eine Transaktion nicht vollkommen unspezifische Merkmale aufweist, dann lohnt sich der verstärkte Einsatz hierarchischer Elemente um so eher, je größer die Häufigkeit der Transaktion ist. ${ }^{472}$ Dies wird damit begründet, dass die Produktivitätsvorteile, die im Falle der Internalisierung durch eine Spezialisierung von Ressourcen erreicht werden können, bei hoher Transaktionsfrequenz die Nachteile der gegenüber der marktlichen Koordinationsform schlechteren Anreizsituation überwiegen. In ähnlicher Weise wirkt sich eine wachsende Unsicherheit der Transaktion auf die Vorteilhaftigkeit der Koordinationsform aus. ${ }^{473}$ Je stärker die Unsicherheit ausgeprägt ist, um so stärker fällt die leichtere Anpassbarkeit relationaler Verträge ins Gewicht, sodass bei hoher Unsicherheit die hierarchische Koordinationsform im Vergleich zu anderen Vertragsdesigns die relativ höchste Koordinationseffizienz aufweist.

Überträgt man diese Überlegungen auf die oben angesprochenen Leistungstypen auf der Meta-Ebene der technikunterstützten Informationsverarbeitung, so können die bisher abgeleiteten Gestaltungsempfehlungen für das Informationsmanagement ergänzt werden. Bei Aufgaben mittlerer Spezifität wie etwa der Durchführung von Schulungen für betriebswirtschaftliche Standardsoftware, die durch ein Customizing an die individuellen Anforderungen der Unternehmung angepasst wurde, wird die Häufigkeit der Transaktion zur relevanten Größe für die Vorteilhaftigkeit der Koordinationsform. Ist eine häufige Wiederholung dieser Aufgaben gegeben, so erscheint der Aufbau von Eigenerstellungskapazitäten attraktiv, während bei nur geringer Wiederholungsrate die mit dem externen Bezug verbundene bessere Anreizstruktur zu einer höheren Effizienz führt.

Auch die Unsicherheit als Transaktionseigenschaft kann bei Aufgaben mittlerer Spezifität auf der Meta-Ebene der Informationsverarbeitung die Effizienz der Koordinationsform beeinflussen. Typischerweise sind anspruchsvolle, schlecht strukturierte Aufgaben durch eine hohe Unsicherheit sowie hohes externes Risiko gekennzeichnet,

${ }^{472}$ Vgl. Picot/Reichwald/Wigand (2001), S. 53.

${ }^{473}$ Vgl. dazu Williamson (1979), S. 254ff. 
während besser strukturierte Aufgaben eine relativ niedrige Transaktionsunsicherheit aufweisen. So ist beispielsweise bei Beratungsleistungen generell mit relativ starken Änderungen der Anforderungen im Zeitablauf zu rechnen. Die Qualität des Leistungsergebnisses hängt zudem von einer Vielzahl von Größen ab, die zum großen Teil nicht im Einflussbereich des einzelnen Aufgabenträgers liegen. Unter diesen Bedingungen kann auch bei mittlerer Spezifität (etwa bei stärkerem technischen Bezug der Beratung) die Wahl eines eher hierarchisch orientierten Vertragsdesigns vorteilhaft sein. Dieses geht schon zum Zeitpunkt des Vertragsschlusses von der später auftretenden Notwendigkeit zu Anpassungen aus und bietet gute Kontrollmöglichkeiten für den Auftraggeber. Dagegen ist es möglich, bei besser strukturierten Aufgaben wie etwa der Wartung und Pflege des Netzwerks zum Zeitpunkt des Vertragsschlusses die gegenseitigen Ansprüche der Vertragspartner weitgehend zu explizieren, sodass ein stärker marktlich orientiertes Vertragsarrangement auch bei hoher Wiederholungshäufigkeit vorteilhaft erscheint. Trotz der aufgezeigten prinzipiellen Relevanz von Transaktionshäufigkeit und Transaktionsunsicherheit sind diese Eigenschaften allerdings als nachrangig für die Wahl der Koordinationsform zu sehen. Auch auf der Meta-Ebene der Informationsverarbeitung besitzt die Transaktionsspezifität die größten Auswirkungen auf die Vorteilhaftigkeit des Vertragsarrangements.

Unabhängig von der letztlich gewählten Koordinationsform werden bei Prozessen der Informationsverarbeitung als speziellem Transaktionstyp die gleichen Phasen durchlaufen, und es treten die gleichen phasenspezifischen Probleme auf, die in diesem Kapitel in allgemeiner Form für alle Transaktionstypen erläutert wurden. Dies gilt sowohl für Prozesse, die die eigentliche Leistungserstellung begleiten und koordinieren, als auch für die Unterstützung dieser Prozesse auf der Meta-Ebene der Informationsverarbeitung. Maßnahmen des Informationsmanagements zur Steigerung der Effizienz können sich nicht nur auf die Wahl des geeignetesten Vertragsdesigns für Prozesse der Informationsverarbeitung, sondern auch auf die einzelnen Aktivitäten in den verschiedenen Vertragsphasen richten, wobei vor Vertragsschluss hidden characteristics- und nach Vertragsschluss hidden action-Probleme auftreten. Dabei steht im Prinzip das gesamte Maßnahmenspektrum zur Verfügung, das die AgencyTheorie zur Disziplinierung von Auftragnehmern vorsieht. Demzufolge kann Koordinationseffizienz von Prozessen der Informationsverarbeitung, wie die jedes anderen Transaktionstyps auch, durch die Gestaltung der Anreizsituation, die direktive Verhaltenssteuerung und die Verringerung von Informationsasymmetrien zu Gunsten des Auftragnehmers gesteigert werden. Die Problemstellungen und der von der Neuen Institutionenökonomie entwickelte Maßnahmenkatalog zu ihrer Lösung erfüllen für das Informationsmanagement also in doppelter Hinsicht eine Orientierungsfunktion. Einerseits dienen sie als Maßstab für die Effektivität, indem sie Aufschluss darüber geben, was die technikunterstützte Informationsverarbeitung eigentlich leisten soll. Andererseits lassen sich zugleich Aussagen darüber ableiten, wie diese Leistung erbracht werden soll, sodass das Informationsmanagement auf dieser Basis auch die Effizienz der technikunterstützten Informationsverarbeitung sicherstellen kann.

Die spezifischen Probleme in den jeweiligen Vertragsphasen sowie die verschiedenen Arten von Maßnahmen, die die Neue Institutionenökonomie zur Bewältigung dieser Probleme vorsieht, wurden in diesem Kapitel ausfürlich behandelt. Die Darstellung braucht hier nicht im Einzelnen wiederholt zu werden. In diesem Kapitel wurde herausgearbeitet, über welche Ansatzpunkte das Informationsmanagement in den verschiedenen Transaktionsphasen verfugt, um Aktivitäten der Koordination zu 
unterstützen und somit die Effizienz des Leistungsaustausches zu fördern. Diese Ansatzpunkte sind prinzipiell auch dann gegeben, wenn es sich bei der betrachteten Transaktion selbst um einen Prozess der Informationsverarbeitung handelt. Die Potenziale der technikunterstützten Informationsverarbeitung zur Steigerung der Transaktionseffizienz werden dabei gewissermaßen rekursiv auf die technikunterstützte Informationsverarbeitung selbst bezogen. Die erläuterten Funktionalitäten von Anwendungssystemen unterstützen dann vor Vertragsschluss die Auswahl von Aufgabenträgern im Bereich der Informationsverarbeitung. Nach Vertragsschluss erleichtern sie mit operativen und analytischen Funktionalitäten die Koordination der verschiedenen Aufgabenträger. Beispiele für Anwendungssysteme in diesem Bereich sind datenorientierte analytische Systeme, die Planungs- und Kontrollaktivitäten für informationswirtschaftliche Abteilungen unterstützen, oder CSCW-Systeme, die bei Projekten zur Entwicklung von Individualsoftware die Kommunikation und Koordination der Projektmitglieder vereinfachen.

\subsubsection{Rolle des Menschen in der Informationsverarbeitung}

Die Neue Institutionenökonomie ist von ihrem Erkenntnisinteresse sowie von ihren inhaltlichen und methodischen Leitideen her grundsätzlich geeignet, die einleitend in dieser Arbeit kritisierte Vorstellung des „Konstruktionskorridors“ zu überwinden und zu einem angemesseneren Menschenbild bei der Gestaltung und Steuerung technikunterstützter Informationssysteme beizutragen. Das Konstrukt der Institution, das als Betrachtungs- und Gestaltungsgegenstand im Mittelpunkt dieses Wissenschaftsprogramms der Betriebswirtschaftslehre steht, bezieht sich auf das menschliche Zusammenleben im Allgemeinen sowie auf die arbeitsteilige Leistungserstellung im Besonderen. ${ }^{474}$ Menschen werden innerhalb dieses Ansatzes als Akteure im Wirtschaftsgeschehen aufgefasst, und es werden explizite Annahmen über ihr Verhalten gemacht. Technische Aspekte dagegen spielen nur in indirekter Form eine Rolle. Sie gehen als Bestandteil der Transaktionsatmosphäre in die Modellbildung ein, ohne dass eine explizite Untersuchung ihrer Zusammenhänge erfolgt. Die Bezugnahme auf die Neue Institutionenökonomie als Referenzdisziplin schafft daher prinzipiell die Voraussetzungen für die geforderte Abkehr von einer rein technikzentrierten Sichtweise und die Zuwendung zu einer verstärkten Akteursorientierung durch das Informationsmanagement in der Praxis.

Das Verhalten der Aufgabenträger im Bereich der Informationsverarbeitung ist aus institutionenökonomischer Sicht nicht anders abzubilden als das der übrigen Akteure im Wirtschaftsgeschehen. Bei der Gestaltung und Steuerung des technikunterstützten Informationssystems der Unternehmung ist deshalb zu erwarten, dass die an den verschiedenen Prozessen beteiligten Personen ein möglichst hohes individuelles Nutzenniveau anstreben. Dabei treffen sie begrenzt rationale Entscheidungen, und es besteht zumindest die Gefahr, dass sie eventuell auftretende opportunistische Handlungsspielräume $z u$ ihrem eigenen Vorteil ausnutzen. ${ }^{475}$ Die funktionalen Anforderungen an die technikunterstützte Informationsverarbeitung, die in diesem Kapitel für die einzelnen Transaktionsphasen abgeleitet wurden, resultieren aus den genannten

\footnotetext{
${ }^{474} \mathrm{Vgl}$. Picot/Reichwald/Wigand (2001), S. 39.

${ }^{475}$ Begrenzte Rationalität und Opportunismus sind die zentralen Verhaltensannahmen der Neuen Institutionenökonomie, vgl. Williamson (1990), S. 50; Schanz (1997a), S. 165.
} 
Verhaltensannahmen und den von der Neuen Institutionenökonomie entwickelten Instrumenten zur Lösung der damit verbundenen Probleme. Die Erfulllung dieser Anforderungen soll dazu beitragen, die Einschränkungen im Bereich der Rationalität zu vermindern sowie die Gelegenheiten und Interessen zu opportunistischen Handlungen einzudämmen. Wenn das Verhalten von Aufgabenträgern der Informationsverarbeitung nicht von dem Verhalten anderer Akteure abweicht, dann sind vergleichbare Instrumente auch für den informationswirtschaftlichen Bereich von Unternehmungen zu entwickeln.

Daraus ergeben sich sowohl für den Betrieb als auch für die Entwicklung von Anwendungssystemen Konsequenzen. Das Informationsmanagement muss aus Sicht der Neuen Institutionenökonomie davon ausgehen, dass die Benutzer nicht von sich aus für einen reibungslosen und produktiven Systembetrieb sorgen werden. Vielmehr besitzen die Beteiligten eigene Interessen (z.B. die Minimierung ihres persönlichen Arbeitsaufwands oder die Nutzung von Internet-Zugängen und E-Mail Accounts für private Zwecke), die im Regelfall von den durch den Arbeitgeber verfolgten Zielen des Systemeinsatzes abweichen. Es sind deshalb Vorkehrungen zu treffen, durch die die Anwender von der Verfolgung dieser Interessen abgehalten werden. Den Erkenntnissen der Agency-Theorie entsprechend sind dabei grundsätzlich die Schaffung von Anreizen, die Einschränkung von diskretionären Verhaltensspielräumen und der Aufbau von Monitoring-Systemen vorstellbar.

Die konkreten Maßnahmen können im Einzelfall sehr unterschiedlich ausgestaltet werden und sind an dieser Stelle nicht im Detail zu betrachten. Exemplarisch seien hier die Einführung variabler Entlohnungsbestandteile, die an das Erreichen von ex ante vereinbarten, persönlichen Zielsetzungen des jeweiligen Aufgabenträgers gebunden sind, oder die Einschränkung von Berechtigungen zur Nutzung bestimmter Softwarefunktionalitäten genannt. Entscheidend ist, dass die Neue Institutionenökonomie explizit die Gefahr von Konflikten zwischen den verschiedenen Aufgabenträgern der technikunterstützten Informationsverarbeitung anerkennt. Der Ansatz benennt die Ursachen dieser Konflikte, verdeutlicht mit hidden characteristics, hidden intention und hidden action ihre Auswirkungen und entwickelt Konzepte zur Lösung der resultierenden Problemstellungen. Ein institutionenökonomisch fundiertes Informationsmanagement kann diese Erkenntnisse in der Praxis nutzen, um die Verkürzungen einer zu starken Fokussierung technischer Aspekte beim Betrieb von Anwendungssystemen vermeiden.

Auch im Rahmen der Entwicklung von Anwendungssystemen treten vielschichtige Auftraggeber/Auftragnehmer-Probleme auf. Es ist damit zu rechnen, dass sowohl die mit der Leitung des Entwicklungsprojekts betraute Person als auch die in der Projektgruppe mitwirkenden Akteure eigene Interessen verfolgen, die von den offiziellen Projektzielen abweichen. Solche individuellen Zielsetzungen können beispielsweise in der Erhöhung des Projektbudgets, der Ausdehnung der eigenen Einflusssphäre oder der Durchsetzung von Abteilungsinteressen zu Lasten anderer Fachabteilungen liegen. Die Hinzuziehung externer Berater zu unternehmungseigenen Projekten ist in diesem Zusammenhang ebenfalls kritisch zu sehen. Der Interessenkonflikt ist bei diesem Beziehungstyp besonders stark ausgeprägt, da für die externe Person vollkommen andere Anreize (wie z.B. die Ausdehnung der im Rahmen des betrachteten Projekts erbrachten Beratungsleistungen oder die Generierung von Anschlussaufträgen) wirksam sind als für die Auftraggeber des Projekts. 
$\mathrm{Da}$ innerhalb von Projekten typischerweise ein hoher Anteil an schlecht strukturierten Aufgabenstellungen mit hohem externen Risiko zu bearbeiten ist, sind die von der Agency-Theorie empfohlenen Maßnahmen nur begrenzt anwendbar. Unter diesen Bedingungen sind die zwischen den verschiedenen Beteiligten auftretenden Asymmetrien an Situationskenntnissen auch durch die Einführung von computergestützten Monitoring-Systemen kaum zu überbrücken. Ferner fehlen dem Auftraggeber selbst bei gutem Überblick über die aktuelle Situation häufig die Fachkenntnisse zur Realisierung der direktiven Verhaltenssteuerung oder zur adäquaten Beurteilung des Auftragnehmerverhaltens. Die Schaffung von Anreizen als weiteres Instrument der Agency-Theorie kann zwar dazu beitragen, die Interessen der Projektbeteiligten den offiziellen Projektzielen anzugleichen. Bei hohem externen Risiko, das bei größeren Projekten den Regelfall darstellen dürfte, sind die erreichten Projektergebnisse jedoch nur zum Teil auf die Anstrengungen der Projektmitarbeiter zurückführbar. Eine starke Ergebnisbezogenheit der Entlohnung führt dann dazu, dass das externe Risiko vorwiegend durch den Auftragnehmer getragen wird, bei dem die Agency-Theorie jedoch eine höhere Risikoaversion vermutet als beim Auftraggeber. ${ }^{476}$

Die Neue Institutionenökonomie schafft also Voraussetzungen für eine angemessenere Berücksichtigung der Rolle des Menschen durch das Informationsmanagement in der Praxis. Die an der Entwicklung und dem Betrieb von Anwendungssystemen beteiligten Personen werden als Akteure aufgefasst, die aktiv die Erhöhung ihres individuellen Nutzenniveaus anstreben und dabei auch gegen die Interessen anderer Akteure verstoßen können. Allerdings steht die Analyse menschlichen Verhaltens selbst nicht unmittelbar im Vordergrund institutionenökonomischer Untersuchungen. Vielmehr stellt das menschliche Verhalten aus Sicht dieses Ansatzes eine von mehreren Größen dar, die für die Höhe der entstehenden Transaktionskosten bzw. Agency-Kosten maßgeblich sind. Das Menschenbild der Neuen Institutionenökonomie ist nicht ohne Kritik geblieben. Diese bezieht sich in erster Linie auf die Reduktion der menschlichen Persönlichkeit auf rein ökonomische Aspekte und die Annahme, dass Opportunismus eine generelle Verhaltensmaxime darstellt. ${ }^{477}$ Die genannten Kritikpunkte werden im folgenden Abschnitt noch detaillierter betrachtet. Hier ist zunächst festzuhalten, dass die Maßnahmen der Neuen Institutionenökonomie zur Erhöhung der Koordinationseffizienz ebenfalls an rein ökonomischen Gesichtspunkten ansetzen. Andere Möglichkeiten zur Motivation von Mitarbeitern im informationswirtschaftichen Bereich werden nicht in Betracht gezogen.

\subsubsection{Erkenntnisgrenzen}

Die Neue Institutionenökonomie lenkt den Blick auf Unvollkommenheiten des Erfahrungsbereichs, die durch die neoklassische Theorie nicht erfasst werden. Die starken Vereinfachungen, die den Hauptkritikpunkt an der Neoklassik sowie dem darauf beruhenden Faktortheoretischen Ansatz der Betriebswirtschaftslehre, dessen Erkenntnispotenziale für das Informationsmanagement im dritten Kapitel dieser Arbeit untersucht wurden, darstellen, sollen dadurch überwunden werden. Der Neuen Institutionenökonomie liegt jedoch ebenfalls eine spezifische Sicht des Wirtschaftsgeschehens zu Grunde, aus der sich die Grenzen des mit Hilfe dieses Ansatzes erzielbaren Erkennt-

\footnotetext{
${ }^{476}$ Vgl. Ebers/Gotsch (1999), S. 211.

${ }^{477}$ Vgl. dazu z.B. Schanz (1997a), S. 165ff.
} 
nisgewinns ergeben. Innerhalb dieser Grenzen liegen auch die Erkenntnisgrenzen des in diesem Kapitel entwickelten Institutionenökonomischen Ansatzes für eine Theorie des Informationsmanagements. Im folgenden Abschnitt 5.4.2.1 werden zunächst einige Kritikpunkte aufgegriffen, die sich bei genauerer Betrachtung nicht auf konzeptionelle Defizite, sondern auf den bisherigen Stand der Erkenntnis beziehen und durch weitere Forschungsanstrengungen potenziell beseitigt werden können. Die anschließenden Abschnitte 5.4.2.2 und 5.4.2.3 behandeln mit der Verwendung von Hybridmodellen und der Reduzierung des menschlichen Verhaltens auf ökonomische Gesichtspunkte prinzipielle Probleme, die sich innerhalb des konzeptionellen Bezugsrahmens der Neuen Institutionenökonomie nicht lösen lassen.

\subsubsection{Defizite beim bisherigen Theorieausbau}

Die Neue Institutionenökonomie sieht sich selbst nach wie vor als relativ junge Disziplin, deren theoretischer Ausbau bei Weitem noch nicht abgeschlossen ist. ${ }^{478}$ Ein Teil der gegen dieses Wissenschaftsprogramm vorgebrachten Einwände ist mehr auf diesen fehlenden Theorieausbau zurückzuführen, als auf generelle Konstruktionsdefizite, die auch durch weitere Forschungsanstrengungen nicht zu überwinden sind. Diese "heilbaren" Probleme werden im Folgenden näher betrachtet und in ihren Auswirkungen auf den hier entwickelten Institutionenökonomischen Ansatz für eine Theorie des Informationsmanagements untersucht.

\section{a) Operationalisierungsprobleme}

Der erste Kritikpunkt, der sich nicht auf tiefergehende konzeptionelle Defizite, sondern auf den bisher erreichten Stand der Erkenntnis bezieht, betrifft ungelöste Operationalisierungsprobleme bei der genauen Erfassung der zu analysierenden Einflussgrößen. ${ }^{479}$ Innerhalb der Transaktionskostentheorie treten solche Probleme bei allen verwendeten Typen von Variablen, also sowohl bei der Beschreibung verschiedener Koordinationsformen als auch bei der Bestimmung von Transaktionskosten als auch bei der Erfassung der Transaktionseigenschaften auf. ${ }^{480}$ Dies stellt gerade bei den Transaktionskosten, bei denen es sich die zentrale Untersuchungsgröße des Ansatzes mit hoher Bedeutung für das gesamte entwickelte Aussagesystem handelt, ein schwerwiegendes Defizit dar. ${ }^{481}$ Auch das Verhältnis von Transaktionskosten zu Produktionskosten und Kapitalkosten bleibt ungeklärt, sodass Zurechnungsprobleme zwischen den verschiedenen Kostenarten bestehen. ${ }^{482}$ Vergleichbare Probleme mit der Operationalisierung treten ebenfalls im Rahmen der Agency-Theorie auf, bei der insbesondere die präzise Erfassung des verbleibenden Wohlfahrtsverlusts als Komponente der Agency-Kosten Schwierigkeiten bereitet. ${ }^{483}$

Wegen dieser Schwierigkeiten erweist sich die direkte Ableitung von Handlungsempfehlungen für die Gestaltung von konkreten Auftraggeber/AuftragnehmerBeziehungen oder für die Wahl der geeignetesten Koordinationsform als problematisch.

\footnotetext{
${ }^{478}$ Vgl. Schanz (1997a), S. 140f;; Richter/Furubotn (1999), S. 477ff.; Ebers/Gotsch (1999), S. 243.

${ }^{479}$ Vgl. Sydow (1992), S. 146.

${ }^{480}$ Vgl. Picot (1982), S. 281.

${ }^{481}$ Vgl. Windsperger (1998), S. $269 f$.

${ }^{482} \mathrm{Vgl}$. Sydow (1992), S. 146.

${ }^{483}$ Vgl. Ebers/Gotsch (1999), S. 224; Schanz (1997a), S. 149.
} 
Von einer unmittelbaren, gar rechnerischen Umsetzbarkeit in Planungsaktivitäten und von einer umfassenden empirischen Überprüfung sind die Transaktionskostentheorie und die Agency-Theorie deshalb noch vergleichsweise weit entfernt ${ }^{484}$ Es besteht im Gegenteil die Gefahr, dass aus willkürlichen Operationalisierungsversuchen in Bezug auf die von der Neuen Institutionenökonomie verwendeten Variablen zirkuläre und damit tautologische Argumentationen resultieren und dass ex post jede realisierte Koordinationsform als effizient gewertet wird. ${ }^{485}$

Dieser Kritikpunkt trifft grundsätzlich ebenfalls auf den hier entwickelten Institutionenökonomischen Ansatz für eine Theorie des Informationsmanagements zu. Für die erläuterten Gestaltungsoptionen des Informationsmanagements in den verschiedenen Vertragsphasen kann also weder exakt bestimmt werden, in welchem Ausmaß die mit dem Austausch der Leistung verbundenen Transaktionskosten bzw. AgencyKosten reduziert werden, noch, welche Höhe der verbleibende Kostenbetrag aufweist. Der Vergleich unterschiedlicher Maßnahmen des Informationsmanagements, die potenziell zur Steigerung der Koordinationseffizienz geeignet sind, wird dadurch erschwert. Weil das verwendete Effizienzkriterium nur eine Näherungsgröße darstellt, ist auch die relative Vorteilhaftigkeit alternativ möglicher Vertragsdesigns nicht in jedem Fall eindeutig zu ermitteln. Das bedeutet beispielsweise, dass im Einzelfall nicht immer genau klar sein muss, ob eine Externalisierung des Leistungsbezugs tatsächlich angebracht ist, wenn das Informationsmanagement durch geeignete Gestaltungsmaßnahmen die Spezifität einer Transaktion reduziert. Die Positionierung einer Koordinationsform im Spektrum zwischen Markt und Hierarchie bereitet ebenfalls Schwierigkeiten, sodass die Vergleichbarkeit alternativer, für die Abwicklung der Transaktion in Frage kommender Vertragsdesigns nicht immer gegeben ist.

Unzulänglichkeiten bei der Operationalisierung der theoretischen Konstrukte sind allerdings kein spezifisches Problem der Neuen Institutionenökonomie. Tatsächlich bestehen etwa bei der Operationalisierung betriebswirtschaftlicher oder volkswirtschaftlicher Produktionskosten, die bereits viel länger Gegenstand wissenschaftlicher Untersuchungen sind als die Transaktionskosten und Agency-Kosten, vergleichbare Mängel. ${ }^{486}$ Die Verbesserung der Operationalisierbarkeit kann deshalb als vordringliche Aufgabe zukünftiger Forschungsaktivitäten begriffen werden, die den bisher erreichten Stand der Theorie weiter ausbauen. Durch eine präzisere Operationalisierung könnte auch das mehrfach in diesem Kapitel angesprochene Problem gelöst werden, dass die Komplexität der internen Koordination von Vertretern der Neuen Institutionenökonomie häufig unterschätzt wird, sodass die internen Koordinationskosten nicht angemessen berücksichtigt werden. Wenn eine genauere Messung von Transaktionskosten möglich ist, dann lässt sich deren absolute und relative Höhe im konkreten Einzelfall eindeutig bestimmen. Damit könnte die systematische Fehleinschätzung der mit einem bestimmten Vertragstyp verbundenen Koordinationskosten vermieden werden.

Die angesprochenen Zurechnungsprobleme zwischen den verschiedenen Kostenarten, die gleichfalls auf Grund von Operationalisierungsschwierigkeiten entstehen, sind außerdem weniger gravierend, da - wie dargestellt - die Summe aller im Rahmen des

\footnotetext{
${ }^{484}$ Vgl. Picot (1982), S. 281, der sich allerdings nur auf die Transaktionskostentheorie bezieht.

${ }^{485}$ Vgl. Kieser (1988), S. 317.

${ }^{486}$ Vgl. Picot (1982), S. 270; Sauter (1985), S. 63.
} 
Leistungsaustausches entstehenden Kostenarten und nicht allein die Höhe der Transaktionskosten als Effizienzkriterium verwendet wird. Der große heuristische Wert, der einer expliziten Berücksichtigung von Transaktionskosten und AgencyKosten auch für das Informationsmanagement zukommt, wird durch die bisher unvollständige Möglichkeit zur Operationalisierung dieser Größen jedoch nicht beeinträchtigt.

\section{b) Vernachlässigung von Nutzengesichtspunkten}

Die Transaktionskostentheorie ist durch eine sehr einseitige Kostenorientierung gekennzeichnet, die die Erlösseite des Leistungsaustausches stark vernachlässigt. ${ }^{487}$ Genau genommen müsste die Vorteilhaftigkeit einer Transaktion jedoch an ihrem insgesamt erzielbaren Nutzen gemessen werden. Neben den entstehenden Kosten müssten also auch die Erlöse berücksichtigt werden, da höhere Kosten durch einen um so höheren Erlös gerechtfertigt sein können. In ähnlicher Weise wie die Transaktionskostentheorie geht auch die Agency-Theorie von einer gegebenen Leistung aus und untersucht, wie diese zu möglichst geringen Agency-Kosten hergestellt und zwischen Auftragnehmer und Auftraggeber übergeben werden kann. Die Eigenschaften der Leistung selbst sowie der Zusammenhang zwischen Leistungseigenschaften, Erlösen und Agency-Kosten sind dabei nicht explizit Gegenstand der Betrachtung. Zugleich werden auch strategische Gesichtspunkte aus der Untersuchung ausgeklammert, sodass die Bedeutung, die der Erstellung der Leistung für die Wettbewerbsposition der Unternehmung zukommt, in der Analyse nicht berücksichtigt wird. ${ }^{488}$

Diese Einseitigkeit institutionenökonomischer Betrachtungen hat Konsequenzen für den in diesem Kapitel entwickelten Ansatz für eine Theorie des Informationsmanagements, der auf den Erkenntnisinteressen und Leitideen von Transaktionskostentheorie und Agency-Theorie basiert. Die aufgezeigten Gestaltungsoptionen des Informationsmanagements in den verschiedenen Vertragsphasen und die mit den Mitteln der Neuen Institutionenökonomie abgeleiteten Aussagen über Auswirkungen auf die Vorteilhaftigkeit der Koordinationsform, bedürfen vor diesem Hintergrund der Ergänzung. So sind beispielsweise Instrumente zu entwickeln, die Auskunft darüber geben, wann und in welchem Ausmaß sich die spezifische Ausgestaltung von Prozessen der Informationsverarbeitung trotz der tendenziell schlechteren Anreizstruktur und der hohen Bindung an den jeweiligen Vertragspartner durch eine Verbesserung der Wettbewerbssituation rechtfertigen lässt. Ferner wurden die Erlöswirkungen der Mediatisierung von Ex ante-Aktivitäten, durch die die Bedeutung des Preises als Kaufkriterium ansteigt, nur am Rande in die Untersuchung einbezogen. Im Einzelfall ist eine veränderte Beurteilung von Gestaltungsmaßnahmen des Informationsmanagements vorstellbar, wenn neben Kosten- auch Erlösgesichtspunkte berücksichtigt werden. Ähnliche Schwachpunkte gelten für die aus der Agency-Theorie abgeleiteten Aussagen. So werden beispielsweise ausschließlich die Kostenwirkungen diskretionärer Verhaltensspielräume hervorgehoben, ohne auf den Nutzen einzugehen, der bei hohen Handlungsspielräumen durch den Fähigkeitsvorsprung des Auftragnehmers erzielt werden kann.

\footnotetext{
${ }^{487}$ Vgl. Gersch (1998), S. 88; Kaas/Fischer (1993), S. 693.

${ }^{488}$ Vgl. Gersch (1998), S. 88; Benkenstein (1994), S. 487.
} 
Bei der Vernachlässigung von Nutzengesichtspunkten handelt es sich ebenfalls um eine Problemstellung, die innerhalb des konzeptionellen Bezugsrahmens der Neuen Institutionenökonomie durch einen weiteren Theorieausbau lösbar erscheint. Die einseitige Beschränkung der Analyse auf Kostenwirkungen unterschiedlicher Formen des Leistungsaustausches ist nicht zwingend durch das Untersuchungsdesign vorgegeben, sondern erfolgte bisher eher willkürlich durch die Forschenden. Da sich der Ansatz in methodischer Hinsicht auf die Instrumente der neoklassischen Theorie, die sich nicht allein auf die Untersuchung von Kosten beschränken, stützt, ist bei einer Verlagerung des Forschungsschwerpunkts eine Ergänzung der bisherigen Erkenntnisse möglich. Tatsächlich wird in den meisten Arbeiten explizit die Annahme getroffen, dass das menschliche Verhalten durch individuelles Streben nach Nutzenmaximierung gekennzeichnet ist. Daher dürfte auch unter Vertretern der Neuen Institutionenökonomie die grundsätzliche Relevanz von Nutzenaspekten für den Austausch von Leistungen unbestritten sein.

\section{c) Einseitige Konzentration auf die Auftraggeberperspektive und fehlende Betrachtung multipersoneller Aspekte}

Die Vielschichtigkeit realer Austauschsituationen wird bisher nicht angemessen durch die Agency-Theorie und die Transaktionskostentheorie berücksichtigt. So erfolgt die Analyse von Auftraggeber/Auftragnehmer-Beziehungen zumeist einseitig aus Sicht des Auftraggebers. ${ }^{489}$ In diesem Fall wird lediglich dem Auftragnehmer unterstellt, opportunistisch gegen die Interessen des Auftraggebers zu verstoßen. Diese Vorgehensweise ist nicht konsistent mit den grundsätzlichen Verhaltensannahmen der Neuen Institutionenökonomie, die allen Beteiligten, also im Prinzip auch dem Auftraggeber, das Streben nach Erhöhung des persönlichen Nutzens unterstellen. Ein weiteres Defizit stellt die unzureichende Berücksichtigung der Tatsache dar, dass sich bei der Erstellung und dem Austausch von Leistungen typischerweise nicht nur ein Auftraggeber und ein Auftragnehmer, sondern häufig eine Vielzahl von Personen gegenüberstehen. ${ }^{490}$ Dabei treten zusätzliche Probleme durch die mögliche Mehrstufigkeit von Austauschbeziehungen sowie durch die kollektive Erstellung einer Leistung durch mehrere Beteiligte auf. ${ }^{491}$ Mit den vorliegenden Mitteln des positiven Zweigs der Agency-Theorie ist infolgedessen nur eine rudimentäre Analyse netzwerkartiger hybrider Koordinationsformen möglich. ${ }^{492}$ Die Transaktionskostentheorie ist zwar weniger stark durch die einseitige Fokussierung einer einzelnen Vertragspartei gekennzeichnet. ${ }^{493}$ Sie ist jedoch ebenfalls nur begrenzt in der Lage, multipersonelle Transaktionsbeziehungen in die Analyse einzubeziehen. Somit fehlt es auch hier an einem überzeugenden Ansatz zur Berücksichtigung netzwerkartiger Koordinations-

\footnotetext{
${ }^{489}$ Vgl. Perrow (1986), S. 14f.; Ebers/Gotsch (1999), S. 222.

${ }^{490}$ Vgl. Föhr (1991), S. 141ff.; Ebers/Gotsch (1999), S. 223.

${ }^{491}$ Die normativen Forschungsrichtung der Agency-Theorie hat inzwischen Modelle vorgelegt, die sich auf die Gestaltung multipersoneller Auftraggeber/Auftragnehmer-Beziehungen richten. Vgl. z.B. Erlei/Leschke/Sauerland (1999), S. 136ff. Der in diesem Kapitel betrachtete positive Zweig der Agency-Theorie ist jedoch nach wie vor durch eine mangelnde Berïcksichtigung dieses Sachverhalts gekennzeichnet.

${ }^{492}$ Vgl. ähnlich Sydow (1992), S. 172.

${ }^{493}$ So hebt Williamson hervor, dass spezifische Investitionen Bindungswirkungen entfalten, die nicht auf eine der Vertragsparteien beschränkt sind. Vgl. Williamson (1985), S. 52ff. Problematische Abhängigkeitssituationen entstehen insbesondere dann, wenn die spezifischen Investitionen asymmetrisch zwischen den Vertragspartnern verteilt sind. Diese Überlegungen sind nicht auf die Perspektive einer Vertragspartei begrenzt.
} 
formen. Diese werden, wie weiter oben in diesem Kapitel gesehen, als Kombination marktlicher und hierarchischer Koordinationselemente und nicht als eigenständiger weiterer Weg zur Abwicklung des Leistungsaustausches konzeptionalisiert. ${ }^{494}$

Die genannten Vereinfachungen finden sich auch in dem hier entwickelten Institutionenökonomischen Ansatz für eine Theorie des Informationsmanagement wieder. Die herausgearbeiteten Gestaltungsoptionen richten sich auf die Disziplinierung des Auftragnehmers, die durch geeignete informationstechnische Lösungen unterstützt werden soll. Bei einer Umkehrung der Perspektive wäre zu überlegen, wie stattdessen der Auftragnehmer informationstechnisch unterstützt werden könnte, um einen Schutz vor opportunistischem Verhalten des Auftraggebers zu erreichen. Die Untersuchung von Ansatzpunkten des Informationsmanagements wurde ferner auf die Beziehung zwischen jeweils nur einer Vertragspartei beschränkt. Folglich wurden keine Probleme erfasst, die erst dadurch entstehen, dass die erstellte Leistung das Gemeinschaftsprodukt einer Vielzahl von Beteiligten darstellt, deren individuelle Ergebnisbeiträge nicht eindeutig erfasst werden können. Die Entwicklung neuer Instrumente der Agency-Theorie zur Lösung dieser Problematik ließe die Definition zusätzlicher Anforderungen $\mathrm{zu}$, durch deren Erfüllung das Informationsmanagement die Effizienz des Leistungsaustausches weiter steigern könnte. Eine eigenständige Betrachtung von Netzwerken, an denen mehrere Unternehmungen beteiligt sind, müsste zudem Gestaltungsempfehlungen in Bezug auf die Verwendung multilateraler interorganisatorischer Anwendungssysteme zur unternehmungsübergreifenden Informationsverarbeitung mit einem geschlossenen Teilnehmerkreis entwickeln.

Insgesamt erscheint auch dieser Kritikpunkt innerhalb des konzeptionellen Bezugsrahmens der Neuen Institutionenökonomie durch eine Weiterentwicklung des Aussagesystems überwindbar. Da jede Transaktion eine Leistung und eine Gegenleistung (zumeist die Bezahlung der eigentlichen Leistung) umfasst, nimmt der Auftraggeber der Leistung im Hinblick auf die Gegenleistung stets zugleich die Rolle des Auftragnehmers ein. ${ }^{495}$ Für den Austausch der Gegenleistung bestehen dabei spiegelbildliche Probleme zum Austausch der eigentlichen Leistung. Das bedeutet, dass die von der Agency-Theorie entwickelten Instrumente bei einer Umkehrung der Positionen im Prinzip auch auf den Austausch der Gegenleistung angewendet werden können. Damit ließe sich die Aussagekraft des Ansatzes unter Beibehaltung der bisherigen Leitideen weiter erhöhen.

\subsubsection{Verwendung von Hybridmodellen}

Viele Arbeiten zur Neuen Institutionenökonomie gehen davon aus, dass die Modellwelt der neoklassischen Theorie nicht grundlegend zu verwerfen ist, um eine angemessenere Abbildung des Untersuchungsbereichs und eine höhere Erklärungs- und Prognosekraft des bestehenden Aussagesystems zu erreichen. ${ }^{496}$ Vertreter dieser Auffassung argumentieren, es sei nicht erforderlich, die Prämissen der Neoklassik als realitätsfern zu kritisieren, da Modelle und mithin auch ihre Prämissen nicht realitätsnah, sondern

\footnotetext{
${ }^{494}$ Die Transaktionskostentheorie geht davon aus, dass die hybride Koordination bei einer einzelnen Transaktion in der Regel instabil ist, da Kräfte zur Bewegung in Richtung rein marktlicher oder rein hierarchischer Arrangements auftreten. Vgl. Sydow (1993), S. 134.

${ }^{495} \mathrm{Vgl}$. Spremann (1990), S. 564f.

${ }^{496}$ Vgl. Richter/Furubotn (1999), S. 482.
} 
problemadäquat sein müssen. ${ }^{497}$ Die Modelle und Prämissen der neoklassischen Theorie seien zu ihrer Zeit problemadäquat gewesen, aus heutiger Sicht würden jedoch wichtige Probleme vernachlässigt. Folgt man dieser Auffassung, so stellt die Einführung zusätzlicher Nebenbedingungen ein geeignetes Mittel dar, um die Qualität der neoklassischen Theorie unter Beibehaltung ihres methodischen Fundaments zu erhöhen. ${ }^{498}$ Es geht aus dieser Sicht um eine Verallgemeinerung der neoklassischen Theorie, ${ }^{499}$ deren Ergebnisse nur unter ganz speziellen Bedingungen Gültigkeit besitzen.

Die zusätzlichen Nebenbedingungen, die von der Neuen Institutionenökonomie zur Ergänzung des bestehenden Theoriegebäudes eingeführt wurden, betreffen in erster Linie die im Rahmen des Leistungsaustausches bestehende Informationssituation. Die von der Neuen Institutionenökonomie analysierten Problemstellungen treten deswegen auf, weil den Akteuren im Wirtschaftsgeschehen weder die Aktivitäten und Eigenschaften der anderen Akteure noch die zukünttigen Entwicklungen im Umfeld von Transaktionen vollständig bekannt sind. Der Ansatz untersucht, wie die bestehenden Informationsunvollkommenheiten durch die adäquate Ausgestaltung von Institutionen bewältigt werden können. Den Referenzpunkt der Gestaltung bilden dabei die von der neoklassischen Theorie identifizierten Optimalgleichgewichte, deren genaue Lage mit Hilfe von Marginalbedingungen angegeben werden kann.

Die in diesem Zusammenhang auftretende Problematik der Verwendung von Hybridmodellen wird am Beispiel der Effizienzanalyse im Rahmen der Transaktionskostentheorie als Teilgebiet der Neuen Institutionenökonomie besonders deutlich. Die Transaktionskostentheorie führt die Evolution institutioneller Arrangements auf ökonomische Effizienzvorteile einzelner Koordinationsformen zurück. ${ }^{500}$ Das der Mikroökonomie entlehnte Effizienzkonzept besitzt bei genauer Betrachtung jedoch allein unter den in der neoklassischen Theorie angenommen Bedingungen vollkommener Märkte seine determinierende Kraft. ${ }^{501}$ Sobald dieses friktionslose Universum verlassen wird und zur Durchfürung von Transaktionen knappe Ressourcen aufgewendet werden müssen, ist das neoklassische Effizienzkonzept kein realistisch erreichbarer Referenzpunkt mehr. Dies bedeutet, dass gemessen am idealisierten neoklassischen Maßstab alle Lösungen der wirklichen Welt allenfalls zweitbeste Lösungen sein könnten. ${ }^{502}$ Bisher ist jedoch kein alternatives Analyseschema zum neoklassischen Effizienzkriterium verfügbar. Die Effizienzanalyse der Neuen Institutionenökonomie beschränkt sich deshalb zumeist auf eine relative Betrachtung, die einen Vergleich unterschiedlicher institutioneller Arrangements vornimmt. Das Effizienzkriterium bildet dann der möglichst sparsame Einsatz knapper Ressourcen unterschiedlicher Koordinationsalternativen für den Austausch der betrachteten Leistung. ${ }^{503}$

Ähnliche Inkonsistenzen sind auch in Bezug auf andere Teile des institutionenökonomischen Aussagesystems zu beobachten. An verschiedenen Stellen schafft die

\footnotetext{
${ }^{497}$ Vgl. Erlei/Leschke/Sauerland (1999), S. 48.

${ }^{498}$ Vgl. ahhnlich Richter/Furubotn (1999), S. 482.

${ }^{499}$ Vgl. de Alesi (1983), S. 66.

${ }^{500}$ Vgl. Sydow (1992), S. 152.

${ }^{501} \mathrm{Vgl}$. Sydow (1992), S. 152.

${ }^{502} \mathrm{Vgl}$. Richter/Furubotn (1999), S. 489.

${ }^{503}$ Vgl. Ebers/Gotsch (1999), S. 225.
} 
Mischung alter und neuer Annahmen bei der Theoriebildung Aussagesysteme, die in sich nicht schlüssig sind. ${ }^{504}$ So wird beispielsweise nicht immer mit konsequenten Annahmen über den Informationsstand der Akteure gearbeitet. Im Extremfall wird davon ausgegangen, dass dieselben Organisationsmitglieder bei hoher Komplexität und Unsicherheit der Transaktion auf Grund ihrer begrenzten Rationalität und Tendenz zu opportunistischem Verhalten einer Kontrolle zu unterwerfen sind, während sie zugleich - etwa gegenüber hierarchisch untergeordneten Personen - als Transaktionskostenminimierer auftreten, die keinerlei kognitiven Beschränkungen unterliegen. ${ }^{505}$ Das gleichzeitige Vorhandensein von begrenztem und unbegrenztem Informationsstand in Bezug auf verschiedene Bereiche des Leistungsaustausches impliziert ein Nebeneinander von positiven Transaktionskosten und solchen von Null. ${ }^{506}$ Demnach werden Transaktionskosten selektiv in das neoklassische Modell eingefuihrt.

Diese methodischen Schwächen der Neuen Institutionenökonomie gelten auch für den hier entwickelten Institutionenökonomischen Ansatz einer Theorie des Informationsmanagements. In Abschnitt 5.4.1 wurde bereits darauf hingewiesen, dass die Koordinationseffizienz der Transaktion als Effektivitätskriterium für die Maßnahmen des Informationsmanagements nur eine Näherungsgröße darstellt. Wegen der Unvollkommenheiten des realen Wirtschaftsgeschehens existiert in keinem konkret vorstellbaren Fall ein marginalistisch bestimmbares Effizienzgleichgewicht, das als Orientierungsgröße für das Informationsmanagement dienen könnte. Zur Bestimmung eines solchen Gleichgewichts müsste zwischen vermeidbaren und unvermeidbaren Unvollkommenheiten getrennt werden können. ${ }^{507}$ Während die unvermeidbaren Unvollkommenheiten für das Informationsmanagement Erwartungsvariablen darstellen und als Nebenbedingungen die Grenzen des Erreichbaren markieren würden, könnten die vermeidbaren Unvollkommenheiten durch Gestaltungsmaßnahmen des Informationsmanagements beeinflusst werden und würden damit Aktionsvariablen darstellen.

Tatsächlich lassen sich viele für das Informationsmanagement relevante Größen nicht eindeutig einer dieser beiden Kategorien zuordnen, da ihre Beeinflussbarkeit gerade wegen der Informationsunvollkommenheiten nicht bekannt ist. Wissenschaftstheoretisch gesehen läuft dies auf eine mangelnde Trennbarkeit abhängiger und unabhängiger Variablen hinaus. Die Folge ist nicht allein, dass die in diesem Kapitel herausgearbeiteten Gestaltungsoptionen des Informationsmanagements in den verschiedenen Transaktionsphasen eine nicht erreichbare Zielsetzung verfolgen. Vielmehr muss darüber hinaus angenommen werden, dass der für die Beurteilung von Maßnahmen des Informationsmanagements verwendete Qualitätsmaßstab in dieser Form ungeeignet ist. Im konkreten Fall kann die Vorteilhaftigkeit von Entscheidungsalternativen somit nicht endgültig mit Hilfe dieses Maßstabs entschieden werden.

Entsprechend der oben geübten Kritik, dass die Neue Institutionenökonomie zum Teil mit inkonsequenten Annahmen über den Informationsstand der menschlichen Akteure arbeitet, betrifft eine weitere Inkonsistenz des in diesem Kapitel entwickelten Ansatzes die Aufgabenträger im Bereich des Informationsmanagements. Die Nutzung der erläuterten Gestaltungsoptionen des Informationsmanagements zur Steigerung der

\footnotetext{
${ }^{504}$ Vgl. Richter/Furubotn (1999), S. 483.

${ }^{505}$ Vgl. Sydow (1992), S. 163.

${ }^{506} \mathrm{Vgl}$. Richter/Furubotn (1999), S. 484.

${ }^{507}$ Vgl. dazu Richter/Furubotn (1999), S. 489.
} 
Koordinationseffizienz setzt zwei Dinge voraus: Zum einen müssen die für das Informationsmanagement zuständigen Personen tatsächlich an einer solchen Effizienzsteigerung interessiert und zum anderen müssen sie fähig sein, die in der jeweiligen Situation vorteilhafteste Gestaltungsalternative auszuwählen. Betrachtet man diese Voraussetzungen genauer, so muss festgestellt werden, dass sie nicht mit den allgemeinen Annahmen der Neuen Institutionenökonomie vereinbar sind.

Vielmehr ist davon auszugehen, dass die Träger von Aufgaben des Informationsmanagements normalerweise selbst in einem Auftraggeber/Auftragnehmer-Verhältnis stehen, in dem sie gegenüber ihren Vorgesetzten die Rolle des Auftragnehmers einnehmen. ${ }^{508}$ Von einem Auftragnehmer vermutet die Agency-Theorie jedoch, dass dieser nicht an einer möglichst effizienten Abwicklung der Transaktion, sondern an seiner persönlichen Nutzenerhöhung interessiert ist. Die Annahme, dass das Informationsmanagement die aufgezeigten Gestaltungsoptionen zur Erhöhung der Koordinationseffizienz tatsächlich nutzt, impliziert also, dass es sich bei den zuständigen Aufgabenträgern gewissermaßen um gespaltene Persönlichkeiten handelt. Diese verhalten sich in ihrer Rolle als Auftragnehmer opportunistisch, während sie sich zugleich in ihrer Rolle als Auftraggeber allein an Effizienzgesichtspunkten orientieren. Ähnliche Vorbehalte betreffen den Informationsstand, über den die Träger von Aufgaben des Informationsmanagements bei ihren Entscheidungen verfügen. Einerseits unterstützen sie mit ihren Gestaltungsmaßnahmen die Reduzierung von Informationsasymmetrien im Rahmen der Erstellung und des Austausches von Leistungen, während sie andererseits vollständig über ihre eigenen Einflussmöglichkeiten und die Auswirkungen ihrer Maßnahmen informiert sind. Damit werden auch hier Informationsunvollkommenheiten selektiv in das neoklassische Modell eingeführt.

Insgesamt hängen die Ergebnisse, zu denen die Hybridmodelle der Neuen Institutionenökonomie kommen, entscheidend davon ab, welche Annahmen der neoklassischen Theorie modifiziert und welche beibehalten werden. ${ }^{509}$ Diese Variation der Modellergebnisse mit den verschiedenen Modellvarianten ist hoch problematisch, da die Beurteilung der Ausgestaltung einer Institution nicht ausschließlich von den Zusammenhängen im Untersuchungsbereich, sondern auch von der Auswahl der Modellvariante abhängt. ${ }^{510}$ Folglich können aus der Theorie keine eindeutigen Gestaltungsempfehlungen für die Praxis abgeleitet werden. Die Forderung nach einer konzeptionellen Weiterentwicklung der Neuen Institutionenökonomie, die durch ein neues Instrumentarium das Arbeiten mit Hybridmodellen überflüssig macht, hat bisher jedoch nicht mehr als visionären Charakter. Ob eine Umsetzung dieser Idee innerhalb des bestehenden Bezugsrahmens erreicht werden kann, ist fraglich. Methodisch sauberer erscheint ein radikalerer Schnitt. Dieser würde den Aufbau eines gänzlich neuen Ansatzes erfordern, der die bestehenden Informationsunvollkommenheiten im Wirtschaftsgeschehen in angemessenerer Weise erfasst und auf der Basis eines eigenen Instrumentariums Erklärungs- und Gestaltungsaussagen erarbeitet.

\footnotetext{
${ }^{508}$ Wenn die Träger von Aufgaben des Informationsmanagements nicht über Vorgesetzte innerhalb der Unternehmung verfügen, weil sie selbst zum Top-Management auf der obersten Hierarchieebene gehören, so nehmen sie nicht gegenüber den Vorgesetzten, sondern gegenüber den Anteilseignern die Rolle des Auftragnehmers ein.

${ }^{509}$ Vgl. Richter/Furubotn (1999), S. 485.

${ }^{510}$ Vgl. Erlei/Leschke/Sauerland (1999), S. 166.
} 


\subsubsection{Reduzierung des menschlichen Verhaltens auf ökonomische Gesichtspunkte}

Ein weiterer Kritikpunkt, der auf generelle Konstruktionsdefizite zurückgeht und daher nicht durch eine Weiterentwicklung von Transaktionskostentheorie und AgencyTheorie überwunden werden kann, betrifft das menschliche Verhalten, über das nur rudimentäre Annahmen getroffen werden. ${ }^{511}$ Während die Neue Institutionenökonomie explizit auf die Überwindung des institutionellen Vakuums der neoklassischen Theorie abzielt, werden keine Anstrengungen unternommen, auch das gleichfalls vorliegende kognitiv-motivationale Vakuum zu überwinden. ${ }^{512}$ Stattdessen wird eine enge Sichtweise verfolgt, die vernachlässigt, dass neben dem Streben nach Nutzenmaximierung auch andere Einflussgrößen für das individuelle Verhalten eine Rolle spielen. ${ }^{513}$ Die Neue Institutionenökonomie ist also durch eine Art „Tunnelblick “514 auf das Wirtschaftsgeschehen gekennzeichnet, der bestimmte - ökonomische - Verhaltensmerkmale sehr stark betont, andere jedoch vollständig ausblendet. Verhaltenswissenschaftliche Erkenntnisse, wie etwa neuere Motivationstheorien, die nicht nur die Vielfalt unterschiedlicher Bedürfnisstrukturen und Wertorientierungen hervorheben, sondern auch die Notwendigkeit zu deren Berücksichtigung bei der Gestaltung des Arbeitslebens betonen, werden weitgehend ignoriert. ${ }^{515}$ Die Folge dieser „Neigung zum partout-Ökonomischen “" ${ }^{\text {(16 }}$ ist eine Tendenz zur Verfremdung der Wirklichkeit, die eine zutreffende Analyse des individuellen Verhaltens im Wirtschaftsgeschehen nicht fördert, sondern im Gegenteil dem Verständnis der Hintergründe eher entgegen steht. Diese Kritik richtet sich insbesondere gegen das Konzept des Opportunismus, das sowohl in der Transaktionskostentheorie als auch in der Agency-Theorie den Drehund Angelpunkt der Argumentation darstellt. ${ }^{517}$

Betrachtet man das zu Grunde liegende Menschenbild genauer, so zeigt sich, dass die Fähigkeit der Transaktionskostentheorie sowie der Neuen Institutionenökonomie insgesamt, die sozialen Zusammenhänge nicht-vertraglicher Art in die Analyse von institutionellen Arrangements einzubeziehen, beschränkt ist. Sie geht nicht über die innerhalb des Ansatzes genannten Alternativen über die Fähigkeiten und das Verhalten von Individuen sowie die zur Erstellung von Leistungen und deren Verteilung notwendigen Faktoren hinaus. ${ }^{518}$ Damit werden die tatsächlichen sozialen Verhältnisse, in denen wirtschaftliches Handeln stattfindet, nicht sachgerecht abgebildet und Verhaltensweisen, die auf Macht, normativen Bindungen, Identitätsansprüchen oder intrinsischer Motivation beruhen, bleiben unberücksichtigt. ${ }^{519}$ Die Vertreter der Neuen Institutionenökonomie sehen sich infolgedessen nicht selten gezwungen, auf theorieexogene Erklärungsfaktoren sozialer, kultureller, politischer oder technischer Natur

\footnotetext{
${ }^{511}$ Vgl. Sydow (1992), S. 163; S. 173.

${ }^{512}$ Vgl. Schanz (1997a), S. 167.

${ }^{513}$ Vgl. GoshalMoran (1996), S. 13ff.; Noorderhaven (1996), S. 105ff.

${ }^{514}$ Ebers/Gotsch (1999), S. 249.

${ }^{515} \mathrm{Vgl}$. Sydow (1992), S. 163.

${ }^{516}$ Schanz (1997a), S. 147.

${ }^{517}$ Die Opportunismus-Annahme wird zwar dadurch relativiert, dass die Vertreter des Ansatzes selbst anerkennen, dass sich Menschen nicht immer und überall opportunistisch verhalten. Vgl. z.B. Williamson (1990), S. 73. Gerade wegen dieser Relativierung ist die starke Konzentration auf die Möglichkeiten und Folgen des Opportunismus jedoch kritisch zu sehen, da eine Verhaltensannahme in den Mittelpunkt der Analyse gestellt wird, die das menschliche Verhalten offensichtlich nicht zentral auszeichnet.

${ }^{518} \mathrm{Vgl}$. Witt (1995), S. 236.

${ }^{519}$ Vgl. Ebers/Gotsch (1999), S. 251.
} 
zurückzugreifen. Aus dieser Einschränkung der Analyse folgt letztlich die These, dass die verschiedenen Teilgebiete der Neuen Institutionenökonomie nicht die jeweiligen zentralen Ursachen der historischen Entstehung von Institutionen und der Wahl der Koordinationsform arbeitsteiliger Prozesse erfassen. ${ }^{520}$ Der selbst erklärte Anspruch, die verhaltenswissenschaftlich ausgerichtete Organisationstheorie und die rein ökonomisch ausgerichtete neoklassische Theorie integrieren $\mathrm{zu}$ wollen, wird aus diesen Gründen nicht erreicht. ${ }^{521}$

Für den hier entwickelten Institutionenökonomischen Ansatz für eine Theorie des Informationsmanagements bedeutet diese Beschränkung der Untersuchung, dass ebenfalls nicht alle Aspekte menschlichen Verhaltens berücksichtigt werden, die grundsätzlich Relevanz für die technikunterstützte Informationsverarbeitung besitzen. Daraus ergeben sich sowohl Auswirkungen auf die Effektivität als auch auf die Effizienz der Informationsverarbeitung. $\mathrm{Da}$ als Maßstab für die Effektivität allein auf die Koordinationseffizienz der Transaktion Bezug genommen wird, wird die Unterstützung von Aktivitäten die die Mitarbeiter der Unternehmung zur Verfolgung anderer Zielsetzungen durchführen, vollständig ausgeblendet. Dies ist deshalb problematisch, weil davon auszugehen ist, dass ein ökonomisch relevanter Zusammenhang zwischen individuellem Leistungsverhalten und individueller Arbeits(un)zufriedenheit besteht. ${ }^{522}$ Die informationstechnische Unterstützung von nicht rein ökonomisch motivierten Tätigkeiten könnte demnach potenziell die Leistungsbereitschaft der Beschäftigten erhöhen und dadurch einen eigenen Beitrag zur Gewährleistung einer möglichst hohen Effizienz des Leistungsaustausches erbringen. Diese Feststellung wirf weitere Fragen auf. So wäre etwa zu untersuchen, welche anderen Motive genau für die Mitarbeiter der Unternehmung Bedeutung besitzen, in welchem Zusammenhang diese zum individuell gezeigten Leistungsverhalten stehen und wie die Verfolgung dieser Motive informationstechnisch unterstützt werden kann. Diese Fragestellungen werden hier nicht weiter untersucht, da sie nicht mit den Mitteln der Neuen Institutionenökonomie beantwortet werden können.

Auch diejenigen Maßnahmen des Informationsmanagements, die sich auf die Steigerung der Effizienz richten, sind von der Kritik am vereinfachten Menschenbild der Neuen Institutionenökonomie betroffen. Wie erläutert, ist zur Sicherstellung der Effizienz aus Sicht der Neuen Institutionenökonomie die technikunterstützte Informationsverarbeitung selbst als Transaktion aufzufassen und deren Transaktionseffizienz zu untersuchen. $\mathrm{Da}$ innerhalb des Ansatzes keine differenzierte Betrachtung der Aufgabenträger im Bereich der Informationsverarbeitung stattfindet, besteht die Gefahr, dass die abgeleiteten Maßnahmen zur Effizienzsteigerung dysfunktional wirken. So laufen die Einschränkung diskretionärer Verhaltensspielräume und die Reduzierung von Informationsasymmetrien, die von der Agency-Theorie als Möglichkeiten zur Disziplinierung des Auftragnehmers vorgesehen werden, letztlich auf eine starke Gängelung und Überwachung der Beschäftigten durch die jeweiligen Vorgesetzten hinaus. Die Agency-Theorie vernachlässigt die Auswirkungen, die von diesen Instrumenten auf die Leistungsbereitschaft der Auftragnehmer ausgehen. Es kann daher nicht ausgeschlossen werden, dass unerwünschte Nebeneffekte auftreten, und die genannten Maßnahmen alles in allem sogar kontraproduktive Effekte zur Folge haben. Die Kritik,

\footnotetext{
${ }^{520}$ Vgl. Kieser (1988), S. 314.

${ }^{521}$ Vgl. ähnlich Sydow (1992), S. 164.

${ }^{522}$ Vgl. Schanz (1997a), S. 166.
} 
die allgemein gegen die Verhaltensannahmen der Neuen Institutionenökonomie geübt wird, kann und muss auf Grund dieser Überlegungen auch auf den in diesem Kapitel entwickelten Institutionenökonomischen Ansatz für eine Theorie des Informationsmanagements übertragen werden.

Abschließend ist auf die Frage einzugehen, ob eine Lösung der Probleme, die sich aus der nur rudimentären Berücksichtigung des menschlichen Verhaltens ergeben, innerhalb des konzeptionellen Bezugsrahmens der Neuen Institutionenökonomie möglich ist. WILLIAMSON selbst weist darauf hin, dass sein reduziertes Modell ausreiche, um zu korrekten Prognosen über die relative Vorteilhaftigkeit alternativer institutioneller Arrangements zu kommen. ${ }^{523}$ Dieser Feststellung ist zum einen entgegenzuhalten, dass von der Korrektheit der Prognose nicht auf die Korrektheit der Annahmen geschlossen werden kann. ${ }^{524}$ Zum anderen ist gerade die empirische Prognosekraft des Ansatzes umstritten, da häufig lediglich empirisch vorgefundene Koordinationsformen ex post mit institutionenökonomischen Argumenten legitimiert werden. Zum Teil wird argumentiert, dass sich durch die Einbeziehung von Vertrauen als zusätzlicher Einflussgröße ein Gegengewicht gegen die Opportunismus-Annahme herstellen ließe, ohne dass neue, die Argumentation verkomplizierende Motivationstheorien in die Analyse einbezogen werden müssten. ${ }^{525}$ Insgesamt verhindert das undifferenzierte Menschenbild der Neuen Institutionenökonomie jedoch den Zugang zu nichtökonomischen Phänomenen, ${ }^{526}$ sodass unangemessene Verkürzungen letzten Endes trotz der Möglichkeit zu kosmetischen Verbesserungen unvermeidlich bleiben.

\footnotetext{
${ }^{523} \mathrm{Vgl}$. Williamson (1985), S. $391 \mathrm{f}$.

524 Vgl. Ebers/Gotsch (1999), S. 244.

${ }^{525} \mathrm{Vgl}$. Ebers/Gotsch (1999), S. 244.

${ }^{526}$ Vgl. Schanz (1997a), S. 166.
} 
Dirk Beier - 978-3-631-75500-6

Downloaded from PubFactory at 01/11/2019 04:07:38AM 


\section{Zusammenführung der entwickelten Theorieansätze am Beispiel Mobile Business}

Bei der Erläuterung der wissenschaftstheoretischen Grundposition dieser Arbeit in Abschnitt 2.2 wurde zur Überwindung bestehender Theoriedefizite eine multiperspektivische Analyse des Informationsmanagements gefordert. Durch die Bezugnahme auf mehrere Theoriekonzeptionen kann ein differenzierteres Bild mit mehr Details und Facetten des Erfahrungsgegenstands gezeichnet werden, als dies im Falle der Beschränkung auf nur eine Untersuchungsperspektive möglich ist. Um zu einer derartigen multiperspektivischen Analyse beizutragen, wurden in den anschließenden Kapiteln drei ausgewählte Wissenschaftsprogramme der Betriebswirtschaftslehre referenziert. Diese Wissenschaftsprogramme basieren zum Teil auf voneinander abweichenden Annahmen und nähern sich dem gemeinsamen Erfahrungsgegenstand mit verschiedenen Erkenntnisinteressen. Folglich haben auch die mit den jeweiligen Mitteln abgeleiteten Ansätze für eine Theorie des Informationsmanagements ein sehr unterschiedliches Aussehen.

Zur Vervollständigung des Gesamtbilds ist abschließend eine Zusammenführung der in dieser Arbeit entwickelten Aussagesysteme erforderlich. Dabei ist es nicht sinnvoll, diese Zusammenführung durch eine Synthese der verschiedenen Ansätze zu einer übergreifenden Theorie des Informationsmanagements vorzunehmen. Die partielle Inkonsistenz der Prämissen, die den einbezogenen Wissenschaftsprogrammen zu Grunde liegen, müsste bei einer derartigen Synthese zwangsläufig zu einer partiellen Inkonsistenz der erhaltenen Aussagen führen. Die Zusammenfürung der Ansätze in diesem Kapitel erfolgt stattdessen durch ihre Anwendung auf einen gemeinsamen, exemplarischen Gestaltungsbereich des Informationsmanagements in der Praxis. Für diese Zwecke wird hier die aktuelle Entwicklung im Bereich des Mobilfunks aufgegriffen, der mehr und mehr mit der traditionellen Informationsverarbeitung konvergiert und zunehmend Bedeutung für die Realisierung innovativer betriebswirtschaftlicher Konzepte in der Praxis gewinnt. Es wird untersucht, welche Anhaltspunkte die verschiedenen Ansätze für eine Theorie des Informationsmanagements zum Aufbau und Betrieb von Mobile Business-Anwendungen in der Praxis geben können.

Abschnitt 6.1 nimmt zunächst eine Bestandsaufnahme des Mobile Business vor. Es werden technische Hintergründe, Erscheinungsformen und Anwendungspotenziale der angesprochenen Konvergenzentwicklung dargestellt. Anschließend arbeitet Abschnitt 6.2 die Berührungspunkte dieser Entwicklung mit dem Informationsmanagement als betrieblichem Aufgabenfeld heraus. Der abschließende Abschnitt 6.3 beleuchtet die Beziehungen zwischen den in den vorangegangenen Kapiteln entwickelten Theorieansätzen für das Informationsmanagement. Dies geschieht, indem die ableitbaren Aussagen zur Lösung (bzw. Vermeidung) der idealtypischen ungelösten Problemstellungen des Informationsmanagements in der Praxis auf das Mobile Business bezogen werden und das Verhältnis dieser Aussagen untereinander überprüft wird.

\subsection{Mobile Business: Abgrenzung, technische Hintergründe und Anwendungspotenziale}

In diesem Abschnitt erfolgt zunächst eine Bestandsaufnahme der aktuellen Situation im Umfeld des Mobile Business. Abschnitt 6.1.1 beschreibt die Konvergenz von mobiler und leitungsgebundener Informationsverarbeitung als Fortsetzung der Internet- 
Revolution. In Abschnitt 6.1.2 wird davon ausgehend eine begriffliche Abgrenzung des Mobile Business und eine Einordnung unterschiedlicher Erscheinungsformen vorgenommen. Eine kurze Erläuterung der technischen Hintergründe ist Gegenstand von Abschnitt 6.1.3. Abschnitt 6.1.4 wendet sich den innovativen Leistungsmerkmalen und Anwendungspotenzialen der nächsten Mobilfunkgenerationen $\mathrm{zu}$, die aus betriebswirtschafticher Sicht besondere Relevanz für das Informationsmanagement besitzen.

\subsubsection{Mobile Business als Fortsetzung der „Internet-Revolution“}

Die nachfolgenden Ausführungen nehmen zunächst eine allgemeine Einordnung des Mobile Business vor. Dabei handelt es sich um ein betriebswirtschaftliches Konzept, das die ökonomische Ausnutzung von aktuell stattfindenden Entwicklungen im Bereich der Informations- und Kommunikationstechnik anstrebt. Abschnitt 6.1.1.1 beschreibt die bereits heute $z u$ beobachtenden Veränderungen, $z u$ denen die Diffusion des neuen Kommunikationsmediums Internet gefüht hat. Abschnitt 6.1.1.2 skizziert die weiteren Entwicklungsperspektiven, die durch die Erweiterung der Internet-Technik um mobile Funktionalitäten entstehen.

\subsubsection{Das Internet als Auslöser wirtschaftlichen Wandels}

Die Verbreitung des Internet hat in den vergangenen Jahren weltweit tiefgreifende Auswirkungen auf Wirtschaft und Gesellschaft gehabt. Seit der Entwicklung des World Wide Web, das im Jahre 1991 durch das Europäische Kernforschungszentrum CERN in Genf vorgestellt wurde, ist das Internet weltweit zum Medium mit der schnellsten Diffusion geworden. ${ }^{1}$ Wurde dieses Medium vor den 1990er Jahren in erster Linie im militärischen Bereich und von Forschungseinrichtungen als Kommunikationsmittel eingesetzt, so schufen die erweiterten Darstellungsmöglichkeiten und verbesserten Benutzungsoberflächen des World Wide Web die Voraussetzungen für einen verstärkten zivilen Gebrauch der Internet-Technik. ${ }^{2}$ In der Folge kam es zu starken Veränderungen sowohl der Nutzerstruktur als auch der Nutzungszwecke. Ein breites Spektrum unterschiedlichster Aktivitäten wird mittlerweile über das Internet abgewickelt, das sowohl im privaten als auch im beruflichen Bereich tief in das tägliche Leben eingedrungen ist. Vor diesem Hintergrund erscheint es gerechtfertigt, von einer gesellschaftlichen und ökonomischen Revolution zu sprechen, die durch das neue Medium ausgelöst wurde. ${ }^{3}$

Diese Situation fordert Unternehmungen aller Größen in nahezu allen Wirtschaftszweigen heraus. Zahlreiche Untersuchungen prognostizieren für die nächsten Jahre ein exponentielles Anwachsen des über das Internet abgewickelten Geschäftsvolumens. ${ }^{4}$ Darüber hinaus kann auch die Bedeutung dieser Technik als Enabler für neue organisatorische Lösungen kaum überschätzt werden. Die immer noch wachsende Verbreitung der Internet-Technik hat also nicht nur Auswirkungen auf die Handlungsmöglichkeiten im Bereich der unternehmungsinternen Leistungserstellung, sondern in

1 Vgl. Zerdick (2001), S. 152.

2 Vgl. Loebbecke (2001), S. 93.

3 Vgl. Shaw (2000), S. 3.

4 Vgl. Shaw (2000), S. 4. 
vielen Branchen besitzt sie das Potenzial, das gesamte Geschäft grundlegend zu verändern. ${ }^{5}$ Neue Produkte und Märkte entstehen, die Beziehungen zwischen Unternehmungen und Kunden bzw. Lieferanten werden auf eine neue Basis gestellt und traditionelle Geschäftsmodelle verlieren an Bedeutung. ${ }^{6}$ Dementsprechend ist die Entwicklung von Konzepten erforderlich, die es den Unternehmungen ermöglichen, die ökonomischen Chancen der Internet-Revolution zu nutzen und zugleich die ebenfalls vorhandenen Risiken zu mindern bzw. zu vermeiden.

In der Betriebswirtschaftslehre und ihren Nachbardisziplinen wie auch in der Unternehmungspraxis findet seit etwa Mitte der 1990er Jahre eine intensive Auseinandersetzung mit derartigen Konzepten statt. Die Mediatisierung von wertschöpfenden Aktivitäten wird dabei sehr häufig mit dem Präfix „E-“ als Abkürzung für das englische Wort „Electronic“ zum Ausdruck gebracht. Die Begriffe „E-Commerce“, „EBusiness", „E-Shopping“, „E-Procurement" sowie die im füften Kapitel bereits ausführlich erläuterten Elektronischen Märkte (,E-Markets") und Elektronischen Hierarchien („E-Hierarchies") stellen nur eine kleine Auswahl aus der Vielzahl von Wortkreationen dar, die diesem Schema folgen. Ohne Übertreibung kann festgestellt werden, dass das „E-“ über den jeweiligen Bedeutungszusammenhang im Einzelfall hinaus zum übergreifenden Symbol für eine neues Zeitalter geworden ist, in dem weltweit nicht nur potenziell jeder mit jedem kommunizieren, sondern auch jeder mit jedem Geschäfte machen kann.

Im Verlauf des Jahres 2000 war ein deutliches Abflauen der bis dahin im Umfeld der Internet-Branche vorherrschenden Euphorie zu beobachten. Die Korrektur überspannter Geschäftsprognosen bei vielen in diesem Bereich tätigen Unternehmungen und die darauf folgenden, zum Teil dramatischen Einbrüche der jeweiligen Aktienkurse sind als symptomatisch für diese Entwicklung anzusehen. Die zuvor überhitzte Stimmung machte einer allgemeinen Ernüchterung Platz, und es stand die Frage im Raum, ob das Ende des Internet-Zeitalters möglicherweise bereits gekommen war. Ein solcher Stimmungsumschwung hatte sich allerdings schon länger abgezeichnet, da die sehr optimistischen Erwartungen der Beteiligten im konkreten Fall häufig nicht sachlich zu begründen waren. Tatsächlich erscheinen zu vorsichtige Prognosen über die weitere Entwicklung des Internet jedoch ebenso wenig gerechtfertigt wie die übertriebenen Erwartungen vor dem Rückschlag im Jahr 2000. Die grundlegenden ökonomischen Potenziale dieses Mediums sind nach wie vor unverändert vorhanden. Es ist deshalb davon auszugehen, dass lediglich eine realistischere Einschätzung der zukünftigen Entwicklungsperspektiven, nicht jedoch eine vollständige Abkehr von der Nutzung der Internet-Technik angemessen ist. Dem entspricht auch, dass die allgemeine Erwartungshaltung sich nach dem zwischenzeitlichen Einbruch zumindest teilweise erholen konnte.

\subsubsection{Veränderung der Internet-Welt durch mobile Anwendungen}

Vieles spricht dafur, dass die wirtschaftliche Nutzung des Internet sich nach wie vor in einem frühen Stadium befindet und dass dieses Medium das Geschäftsleben zukünftig noch sehr viel stärker als bisher durchdringen und verändern wird. Unterdessen

\footnotetext{
Vgl. Gersch (2000), S. 41.

6 Vgl. Hermanns/Sauter (1999), S. 5.
} 
zeichnet sich allerdings bereits ein neuer, ebenfalls durch den technischen Fortschritt getriebener Trend $a b$, der die Kraft besitzt, das Internet und seine Anwendungsmöglichkeiten selbst zu revolutionieren. Die Entwicklung der Mobiltelefonie hat in der zweiten Hälfte der 1990er Jahre alle Erwartungen übertroffen. ${ }^{7}$ Die Verbreitung von Endgeräten, die einen Zugang $\mathrm{zu}$ den momentan im Betrieb befindlichen Mobilfunknetzen der zweiten Generation erlauben, übertrifft inzwischen in vielen Ländern die Zahl der Internet-Anschlüsse oder wird dies in absehbarer Zeit tun. ${ }^{8}$ Im Herbst 2000 wurden die Lizenzen für den Aufbau von Mobilfunknetzen der dritten Generation vergeben, deren Funktionalität weit über die Vermittlung gesprochener Sprache hinausgehen wird. Dabei tätigten die künftigen Netzbetreiber in einigen Ländern exorbitant hohe Investitionen allein für den Lizenzerwerb.

Die Nutzungspotenziale des Mobilfunks werden durch die neuen Netze essenziell erweitert, sodass die Mobilkommunikation derzeit dabei ist, die Wertschöpfungsketten in der Telekommunikations- und der Internetwelt völlig neu zu gestalten. ${ }^{9}$ Zum Teil wird das Internet sogar schon als "traditionelle" Technik bezeichnet, die durch den Mobilitätsaspekt einen neuen Schub bekommen und „zukunftsfähig“ gemacht werden soll. ${ }^{10}$ Dies geht bis hin zu der These, dass nur wenige E-Business-Unternehmungen überleben werden, wenn sie nicht auch mobile E-Business-Unternehmungen werden. ${ }^{11}$ Das oben angesprochene Präfix „E-“ wird in der Diskussion mehr und mehr durch das „M-“ als Abkürzung für „Mobile“ abgelöst, und es werden Begriffe wie „Mobile Commerce“, „Mobile Business“, „Mobile Banking“ und viele weitere ins Gespräch gebracht.

Durch den technischen Fortschritt im Bereich der Mobilfunks kommt es zu einer allgemeinen Konvergenz leitungsgebundener und mobiler Informations- und Kommunikationstechniken. Es ist zu erwarten, dass das Internet und die Mobilfunknetze zu einem gemeinsamen, überall verfügbaren Netzwerk verschmelzen werden. ${ }^{12}$ Diese Konvergenz bezieht sich zum einen auf die jeweils verwendeten Endgeräte und zum anderen auf die innerhalb der Netze angebotenen Dienste. ${ }^{13}$ Die Vision für den zukünttigen Zugang zu Informationen über das zusammengewachsene Netz basiert auf der Leitidee: „anywhere, anytime, on any device“14. Das mobile Internet bringt die bisher weitgehend an stationäre Endgeräte gebundene Internet-Nutzung zu den Anwendern hin und dehnt damit die Möglichkeiten zur Interaktion auf Situationen aus, in denen derzeit noch eine Trennung von Netz und Benutzer gegeben ist. Häufig wird darauf hingewiesen, dass diese neue Technik auf einen schon länger latent vorhandenen Bedarf trifft, der durch aktuelle gesellschaftliche Entwicklungen noch erheblich verstärkt wird. ${ }^{15}$ Die ständig steigende Mobilität sowohl in der beruflichen als auch in der privaten Sphäre sowie die zunehmende Technik-Verbundenheit in großen Teilen der

7 Vgl. Gentner/Legler (2000), S. 14.

8 Vgl. z.B. Pflug (2001), S. 21; Zobel (2001), S. 14; Kamp/Velthuis (2000), S. 1, die sich jeweils auf Prognosen von führenden Marktforschungsinstituten und von Telekommunikationsunternehmungen beziehen.

9 Vgl. Laartz/Hjartar (2000), S. 29.

10 Vgl. Mobilocity (2001), S. 2

11 Vgl. Zobel (2001), S. 11.

12 Vgl. Lucent Technologies (2001), Einleitung; Kamp/Velthuis (2000), S. 2.

13 Vgl. Flätgen/Selle (2000), S. 8.

${ }^{14}$ Dies ist das von vielen Seiten aufgegriffene Motto der .NET-Strategie, die von Microsoft zur CeBIT 2001 vorgestellt wurde. Vgl. Microsoft (2001b), Introduction.

$15 \mathrm{Vgl}$. Borowicz/Scherm (2000), S. 471; Wirtz (2001), S. 9. 
Bevölkerung schaffen insgesamt gute Voraussetzungen für eine rasche Diffusion des Mobile Business.

Bereits auf der Basis der zur Zeit genutzten zweiten Mobilfunkgeneration wurden erste Schritte unternommen, die bislang im Mittelpunkt stehenden Sprachdienste um Anwendungen der mobilen Datenübertragung zu ergänzen und damit den Aufbau des Mobile Business einzuleiten. Europa und Japan haben dabei im weltweiten Vergleich eine Vorreiterposition eingenommen, die sich jedoch noch auf relativ niedrigem Niveau bewegt. ${ }^{16}$ Allerdings konnten insbesondere mit dem i-Mode Service, mit dem es NTT DoCoMo in Japan gelang, innerhalb von nur 18 Monaten mehr als 10 Millionen Kunden zu akquirieren, erste wirtschaftliche Erfolge erzielt werden. ${ }^{17}$ Dennoch befinden sich Mobile Business und Mobile Commerce gegenwärtig bestenfalls auf einer Anfangsstufe, da die verfügbare Infrastruktur von Endgeräten und Netzen der zweiten Generation zumindest in Europa noch keine Realisierung in größerem Umfang zulassen. $^{18}$

Die ausgesprochen hohen Wachstumsraten, die von den Untersuchungen der großen Marktforschungsinstitute prognostiziert werden, sind mit denen vergleichbar, die vor dem Einknicken der Internet-Euphorie für Electronic Business und Electronic Commerce verkündet wurden. ${ }^{19}$ Selbst wenn man unterstellt, dass diese Vorhersagen ähnlich wie die Prognosen für den „traditionellen" Internet-Bereich sehr hoch gegriffen sind, kann es als fast sicher gelten, dass Mobile Business und Mobile Commerce zum Erfolg werden. ${ }^{20}$ Nur in wenigen Beiträgen zur öffentlichen Diskussion wird angenommen, dass die Mobilfunktechnik insgesamt die hohen Erwartungen enttäuschen wird. ${ }^{21}$ Auch wenn sich die Geschwindigkeit der weiteren Entwicklung nicht exakt voraussagen lässt: Auf Grund des unbestreitbar hohen grundsätzlichen Innovationspotenzials ist die Frage nicht, ob die mobile Datenübertragung sich überhaupt durchsetzen wird. Die Frage ist vielmehr allein, wie schnell dies der Fall sein wird. ${ }^{22}$

\subsubsection{Begriffliche Abgrenzung und Erscheinungsformen des Mobile Business}

Dieser Abschnitt enthält die begrifflichen Grundlagen für die weitere Untersuchung des Mobile Business. In Abschnitt 6.1.2.1 erfolgt zunächst eine Definition des Mobile Business, während Abschnitt 6.1.2.2 eine Klassifikation unterschiedlicher Anwendungsgebiete vornimmt.

\subsubsection{Definition von Mobile Business}

Wenn ein bestimmter Untersuchungsgegenstand von vielen Seiten aufgegriffen und in unterschiedlichen Zusammenhängen untersucht wird, ist häufig zu beobachten, dass sich keine klare Begriffsbildung durchsetzen kann. Personen, die mit den gleichen

\footnotetext{
${ }^{16} \mathrm{Vgl}$. Heng (2001), S. 26; Kamp/Velthuis (2000), S. 2.

17 Vgl. dazu Mizukoshi/Okino/Tardy (2000), S. 1ff.; Zobel (2001), S. $107 \mathrm{ff}$.

18 Vgl. Plesmann (2000), S. 9.

${ }^{19}$ Vgl. z.B. die Übersichten dazu bei Schreiber (2000), S. 68ff.; Wiedmann (2000), S. 684; Zobel (2001), S. 16ff. Varshney/Vetter/Kalatoka (2001), Einleitung.

20 Vgl. Plesman (2000), S. 10.

${ }^{21}$ Vgl. für diese Position z.B. Heng (2001), S. 27.

${ }^{22}$ Vgl. ähnlich Kamp/Velthuis (2000), S. 2; Mobilocity (2000), S. 6.
} 
Begriffen arbeiten, verbinden mit diesen nicht selten ein mehr oder weniger stark voneinander abweichendes inhaltliches Verständnis, da die Begriffe semantisch nicht eindeutig belegt sind. Diese Beschreibung kennzeichnet auch die Begriffswelt im Umfeld der mobilen Datenübertragung, obwohl das Mobile Business und die damit verwandten Begriffsvarianten in Theorie und Praxis gerade erst neu in die Diskussion gebracht wurden. Nahezu jeder Diskussionsbeitrag, ob von Beratungshäusern oder von wissenschaftlicher Seite, verwendet eine eigene Definition, wobei die verschiedenen Abgrenzungen sich im Allgemeinen relativ stark ähneln, im Detail jedoch zum Teil deutliche Differenzen aufzeigen. Ein kurzer Blick auf verschiedene Ansätze zur Definition der Begriffe Mobile Commerce und Mobile Business verdeutlicht diese Problematik:

„Mobile Commerce soll hier als eine spezielle Form des Electronic Commerce aufgefasst werden. Bei dem Electronic Commerce wird die Geschäftsabwicklung elektronisch über offentliche und private Netze unterstützt. Kommen hierbei Mobilfunknetze oder Satelliten sowie mobile Endgerăte zum Einsatz, soll von Mobile Commerce die Rede sein. .23

„Die elektronisch gestützte Abwicklung von Online-Geschăftsvorfallen auf Basis der Nutzung mobiler Endgeräte wird als Mobile Commerce (kurz: M-Commerce) bezeichnet." ${ }^{\text {"24 }}$

„Mobile Commerce refers to any transaction with monetary value that is conducted via a mobile telecommunications network. “25

"Mobile Commerce is the use of mobile handheld devices to communicate, interact via an always-on, high-speed connection to the Internet." 26

"Mobile Commerce is the use of wireless technologies to provide convenient, personalized and location-based services to your customers, employees and partners."27

„Mobile Commerce bezeichnet Ausprägungen der wirtschaftlichen Nutzung von mobilen Endgeräten, vor allem von Mobiltelefonen und PDAs. [...] Bei Mobile Commerce kommuniziert das System des Anwenders drahtlos mit anderen Systemen, welche „im Auftrag“ des Mobilgerăts ihrerseits mit Rechnern über das Internet in Verbindung treten können. ${ }^{\text {“28 }}$

„Unter Mobile Business versteht man Geschăftsmodelle, bei denen Geschăftsprozesse wie zum Beispiel Daten- und Informationsaustausch durch die Integration von mobilen Endgerăten wie Handys oder PDAs unabhăngig von Ort und Zeit abgefragt, beeinflusst oder sogar gesteuert werden können. “29

„Unter Mobile Business verstehe ich alle auf mobilen Geräten („Devices") ausgetauschten Dienstleistungen, Waren sowie Transaktionen. [...] Als M-Business gilt eine Transaktion dabei auch, wenn sie letztlich im klassischen Offline-Bereich zu Ende geführt wurde. [...] Ich möchte im Folgenden den Begriff M-Commerce vermeiden, weil

${ }^{23}$ Borowicz/Scherm (2000), S. 469.

${ }^{24}$ Wiedmann/Buxel/Buckler (2000), S. 684.

${ }^{25}$ Durlacher Research, zitiert nach Mobilocity (2000), S. 4.

${ }^{26}$ Forrester Research, zitiert nach Mobilocity (2000), S. 4.

27 Mobilocity (2000), S. 4.

28 Schmitzer/Butterwegge (2000), S. 355.

${ }^{29}$ Hartmann/Dirksen (2001), S. 16. 
er von der Begrifflichkeit her zu stark auf die Durchführung von Transaktionen, den Kauf von Waren und Ähnliches hindeutet. “30

Grundsätzlich sollen Definitionen dem jeweils verfolgten Erkenntnisinteresse dienen, sodass keine der hier aufgeführten Begriffsabgrenzungen generell als richtig oder falsch gewertet werden kann. Die Gegenüberstellung der verschiedenen Begriffsbildungen macht jedoch deutlich, dass sehr unterschiedliche Aspekte des Mobile Business bzw. Mobile Commerce hervorgehoben werden. Es fällt zunächst auf, dass in den meisten Fällen der Begriff Mobile Commerce fokussiert wird und keine explizite Unterscheidung zwischen Mobile Commerce und Mobile Business stattfindet. Bei näherer Betrachtung wird ferner offenkundig, dass die verschiedenen Begriffsvarianten zumindest in Teilen nicht miteinander vereinbar sind. Diese Inkompatibilitäten sind in erster Linie auf die unterschiedliche Begriffsreichweite der verschiedenen Abgrenzungen zurückzuführen. Im Folgenden werden die Gemeinsamkeiten und Unterschiede der aufgeführten Definitionsansätze untersucht, um zu überprüfen, wie eine Definition für die Zwecke des Informationsmanagements die verschiedenen Teilaspekte sinnvoll behandeln sollte. Die Betrachtungen konzentrieren sich hier zunächst auf den Begriff des Mobile Business, der im nächsten Abschnitt gegenüber dem Mobile Commerce und anderen Begriffsvarianten abgegrenzt wird. Dabei werden folgende Kriterien betrachtet:

- Art der Kommunikationssituation

- Zweck der Nutzung

- Objekt der Übertragung

- Organisatorische Zugehörigkeit der Nutzer

- Anbindung an das Internet

Mobile Telekommunikation zeichnet sich allgemein durch eine spezielle Kommunikationssituation aus: Mindestens einer der Kommunikationsteilnehmer befindet sich zum Zeitpunkt der Kommunikationsaufnahme an einem prinzipiell beliebigen Ort, an den er nicht gebunden ist, und er kann während des Kommunikationsprozesses seinen Standort verändern. ${ }^{31}$ Allen oben aufgeführten Definitionsansätzen ist gemeinsam, dass sie in irgendeiner Form an diese spezielle Kommunikationssituation anknüpfen. Der Fokus liegt dabei entweder stärker auf dem genutzten Mobilfunknetz oder auf dem Endgerät, das für den Netzzugang verwendet wird. Wenn die Definition allein auf den Einsatz von Funknetzen abstellt, dann besteht allerdings eine starke Nähe zu anderen Konzepten drahtloser Kommunikation wie etwa dem derzeit ebenfalls stark diskutierten Wireless LAN. ${ }^{32}$ Orientiert man sich dagegen an dem im vorigen Abschnitt angesprochenen Leitbild „anywhere, anytime, on any device“, so erscheint es zweckmäßiger, bei der Definition des Mobile Business auf die Verwendung mobiler Endgeräte, die den Benutzer (mit Ausnahme der Netzverfügbarkeit) in keiner Weise an irgendwelche lokalen Gegebenheiten binden, abzustellen. Die Frage, inwiefern diese Endgeräte sich mit bestehenden Netzwerken von Unternehmungen verbinden lassen,

\footnotetext{
30 Zobel (2001), S. 3.

31 Vgl. Taday (1998), S. 40

${ }^{32}$ Vgl. dazu Henke (2001), S. $28 \mathrm{ff}$.
} 
besitzt für das Informationsmanagement besondere Relevanz und ist daher explizit mit in die weitere Betrachtung einzubeziehen.

Die meisten oben aufgeführten Definitionen stellen ferner auf die Nutzung der betreffenden Endgeräte für wirtschaftliche Zwecke ab. So ist beispielsweise von der Durchführung von Transaktionen mit monetärem Wert oder von der Bereitstellung bestimmter Dienste und Leistungsmerkmale für Benutzer der Endgeräte die Rede. Tatsächlich stellt die wirtschaftliche Nutzung auch aus Sicht des Informationsmanagements ein konstitutives Merkmal des Mobile Business dar. Die explizite Nennung einzelner Leistungsmerkmale wie Personalisierung oder Lokalisierung ist im Rahmen der Definition jedoch nicht zweckmäßig. Zum einen müssen die vielfältigen Nutzungsformen mobiler Endgeräte nicht stets alle aufgelisteten Leistungsmerkmale beinhalten und zum anderen würden weitere innovative Leistungsmerkmale, deren zukünftige Entwicklung bisher noch nicht absehbar ist, durch eine solche Definition nicht erfasst.

Auch das Kriterium der wirtschaftlichen Nutzung stellt an sich nur ein notwendiges, nicht jedoch ein hinreichendes Merkmal dar. Die gegenwärtig verbreiteten Endgeräte der zweiten Mobilfunkgeneration, deren Hauptfunktionalität in der Regel die Übertragung gesprochener Sprache darstellt, werden bereits für geschäftliche Zwecke genutzt, ohne dass dies allein schon als Mobile Business oder Mobile Commerce zu bezeichnen wäre. ${ }^{33}$ Zur Erschließung der im vorigen Abschnitt angesprochenen Innovationspotenziale ist es vielmehr notwendig, dass die Art der Anwendung über die reine Sprachvermittlung hinaus geht. Erst in Verbindung mit der Übertragung von nicht-sprachlichen Daten wird die wirtschaftliche Nutzung mobiler Endgeräte im Folgenden als Mobile Business bezeichnet.

Hinsichtlich der Annahmen über die Benutzer der Endgeräte unterscheiden sich die oben aufgeführten Definitionen deutlich. Dieser Punkt hängt eng mit der Frage zusammen, welche Art von wirtschaftlicher Nutzung der Geräte stattfindet. Einige Definitionen sind daran geknüpft, dass es sich bei den Geschäftsvorgängen um externe Transaktionen handeln muss, während andere auch die unternehmungsinterne Nutzung der Endgeräte im Rahmen der Erstellung von Leistungen mit in die Begriffsbildung einbeziehen. Im nächsten Abschnitt wird dieses Kriterium verwendet, um verschiedene Erscheinungsformen des Mobile Business zu unterscheiden und auf diese Weise unter anderem auch die Begriffe Mobile Business und Mobile Commerce voneinander abzugrenzen. Der hier zunächst fokussierte Begriff Mobile Business wird in dieser Arbeit so weit gefasst, dass keine Einschränkungen in Bezug auf die organisatorische Zugehörigkeit der Endgerätebenutzer vorgenommen werden. Dieser weiten Begriffsfassung lassen sich sämtliche Formen der mobilen Datenübertragung für wirtschaftliche Zwecke subsumieren.

In einigen Definitionen wird schließlich noch die Anbindung der Mobilfunknetze an das Internet explizit hervorgehoben. Diesem Vorgehen wird hier nicht gefolgt. Die Verschmelzung von Internet und Mobilkommunikation stellt zwar, wie im vorigen Abschnitt erläutert, den Motor der Entwicklung dar. Grundsätzlich sind jedoch auch andere Realisierungsformen des Mobile Business vorstellbar, die nicht zwingend die

${ }^{33}$ Diese Auffassung vertritt auch Gerpott (2001), S. 36. 
Einbeziehung der Internet-Technik vorsehen. Die Art der Verknüpfung von mobiler und standortgebundener Informationsverarbeitung sollte also nicht ausdrücklich erwähnt werden, um den Geltungsbereich der Definition nicht unnötig einzuengen.

Aus den vorstehenden Ausführungen ergibt sich folgende Definition, die den weiteren Überlegungen dieses Kapitels zu Grunde gelegt wird:

Mobile Business bezeichnet die Nutzung von mobilen Endgeräten zur Übertragung sprachlicher und nicht-sprachlicher Daten für wirtschaftliche Zwecke, die sowohl unternehmungsintern als auch unternehmungsextern orientiert sein können.

\subsubsection{Klassifizierung unterschiedlicher Nutzungsformen des Mobile Business}

Die hier vorgenommene Abgrenzung des Mobile Business umfasst eine Reihe unterschiedlicher Erscheinungsformen, die sich alle dem Oberbegriff Mobile Business subsumieren lassen. Plakativ formuliert wird Mobile Business sowohl die Art der angebotenen Leistungen verändern als auch die Art und Weise, wie diese Leistungen erstellt werden. Auf der obersten Stufe ist deshalb zwischen der unternehmungsinternen und der unternehmungsexternen Nutzung der mobilen Datenübertragung zu unterscheiden. In den Fällen, in denen der Benutzer des Endgeräts nicht der Unternehmung angehört, wird im Folgenden von Mobile Commerce gesprochen. ${ }^{34}$ Dadurch ergibt sich eine Analogie zu der in der Literatur häufig vorgenommenen Abgrenzung der Begriffe Electronic Business und Electronic Commerce. Dabei stellt Electronic Business ebenfalls den Oberbegriff dar und Electronic Commerce wird als spezielle Erscheinungsform des Electronic Business aufgefasst, bei der eine geschäftliche Transaktion zwischen selbstständig agierenden Wirtschaftseinheiten stattfindet. ${ }^{35}$ Die unternehmungsinterne Nutzung mobiler Endgeräte als spezielle Erscheinungsform des Mobile Business wird im Weiteren als Mobile Work bezeichnet. Abbildung 6/1 verdeutlicht diese Unterscheidung und nimmt eine weitergehende Systematisierung der verschiedenen Varianten von Mobile Work und Mobile Commerce auf der darunter liegenden Betrachtungsebene vor.

\footnotetext{
${ }^{34}$ Es sei angemerkt, dass in der Literatur bisher häufig auch der Begriff Mobile Commerce als Oberbegriff verwendet wird, der dann sowohl die unternehmungsinterne als auch die unternehmungsexterne Form der mobilen Datenübertragung einschlieBt. Vgl. z.B. Schmitzer/Butterwegge (2000), S. 357; Mobilocity (2001), S. 3. Dieses lässt jedoch keine klare Unterscheidung zwischen Mobile Commerce und Mobile Business zu.

${ }^{35}$ Vgl. Gersch (2000), S. 2.
} 


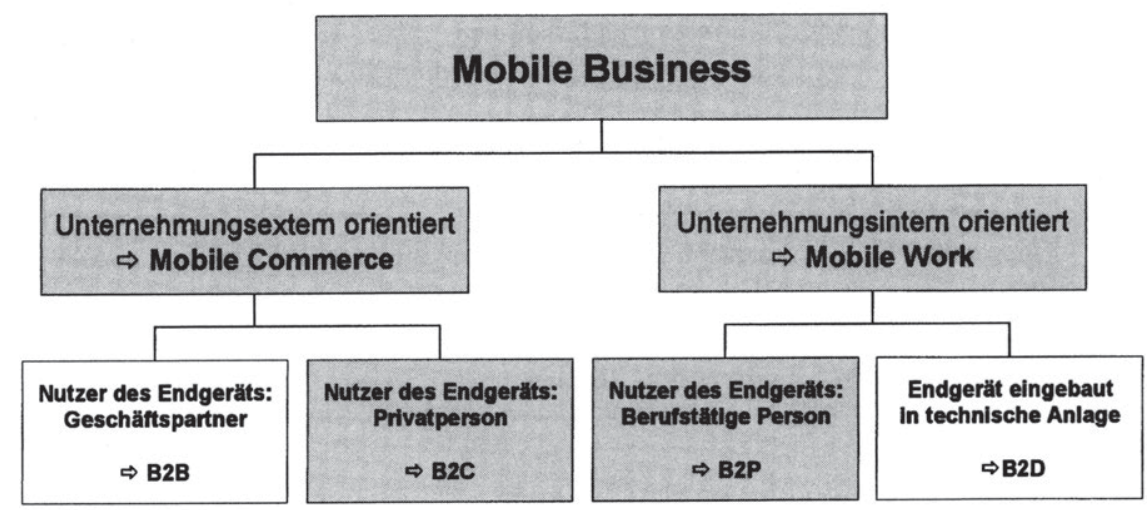

Abb. 6/1: Erscheinungsformen des Mobile Business

Je nachdem, ob es sich bei dem unternehmungsexternen Kommunikationspartner um einen privaten Konsumenten oder um einen Geschäftspartner der Unternehmung handelt, ist zwischen Business-to-Consumer (B2C) und Business-to-Business (B2B) als Varianten des Mobile Commerce zu unterscheiden. Auch diese Unterscheidung erfolgt analog zu der entsprechenden Systematisierung im Bereich des "traditionellen“ Electronic Commerce. Während der Großteil des Electronic Commerce bisher auf das Geschäft zwischen Unternehmungen (B2B) entfällt, ${ }^{36}$ ist für den Mobile Commerce die Bedeutung des B2B umstritten. Es wird sowohl die Ansicht geäußert, dass die Bedeutung des Mobile Commerce für den B2B-Bereich und den B2C-Bereich sehr ähnlich ist, ${ }^{37}$ als auch die Ansicht, dass das B2B-Geschäft im Mobile Commerce nur eine untergeordnete Rolle spielen wird. ${ }^{38}$ Tatsächlich erscheinen die speziellen Innovationspotenziale des Mobile Commerce, die in Abschnitt 6.1.4 noch näher betrachtet werden, insbesondere für Privatkunden interessant. Komplizierte Transaktionen zwischen Unternehmungen dürtten in der Regel weiterhin stationär über den jeweiligen PC am Arbeitsplatz der Mitarbeiter abgewickelt werden. ${ }^{39}$ Im Hinblick auf die jeweiligen Anforderungen an Gestaltungsmaßnahmen des Informationsmanagements weisen beide Varianten des Mobile Commerce allerdings keine größeren Unterschiede auf. Die weiteren Untersuchungen in diesem Kapitel konzentrieren sich dennoch vorwiegend auf die B2C-Variante des Mobile Commerce, die aus diesem Grund in Abbildung 6/1 optisch hervorgehoben ist.

Die unternehmungsintern orientierte Ausprägung des Mobile Business, die hier als Mobile Work bezeichnet wird, kann einerseits die Form des Business-to-Professional (B2P) und andererseits die Form des Business-to-Device (B2D) haben. Beim B2P verwenden berufstätige Personen ${ }^{40}$ das Endgerät, um Zugang zu den standortbezo-

${ }^{36} \mathrm{Vgl}$. Gersch (2000), S. 6.

37 Vgl. Arthur D. Little (2000), S. 3.

${ }^{38}$ Vgl. Heng (2001), S. 27.

$39 \mathrm{Vgl}$. Dean/Ketterer/Thiel (2000), S. 35f.

${ }^{40}$ Diese Variante des Mobile Business wird zum Teil auch als Business-to-Employee (B2E) bezeichnet. Vgl. z.B. Mobilocity (2001), S. 14. Diesem Vorschlag wird hier nicht gefolgt, da der Benutzer des Endgerăts nicht zwingend eine abhăngig beschäftigte Person (Employee) sein muss, sondern 
genen Netzen der Unternehmung zu erhalten. Damit wird es vorstellbar, die Verbindung zu mobilen Mitarbeitern unabhängig von ihrem Aufenthaltsort ununterbrochen aufrecht zu erhalten und diesen Personen jederzeit synchronisierte Informationen aus den Anwendungssystemen der Unternehmung zur Verfügung zu stellen. ${ }^{41}$ Diese Möglichkeit zwingt Unternehmungen dazu, das traditionelle Konzept des Büros als Ort der physischen Präsenz grundlegend zu überdenken. ${ }^{42}$ B2D dagegen bedeutet, dass technische Geräte für die mobile Datenübertragung ausgerüstet und mit den leitungsgebundenen Netzen der Unternehmung verknüpft werden. Anwendungen liegen hier etwa auf dem Gebiet des Flottenmanagements von Speditionen oder der automatischen Übermittlung von Messergebnissen aller Art (z.B. Lagerbeständen u.ä.). ${ }^{43}$ Bei der Entwicklung von B2D-Lösungen handelt es sich in erster Linie um ein technisches Problem, für das die in dieser Arbeit entwickelten betriebswirtschaftlichen Theorieansätze des Informationsmanagements keine Lösungshinweise liefern können. Die weiteren Untersuchungen zur unternehmungsintern orientierten Erscheinungsform des Mobile Business konzentrieren sich deshalb auf den B2P-Bereich, der entsprechend in Abbildung 6/1 ebenfalls optisch hervorgehoben ist.

Abschließend seien einige weitere Nutzungsformen der mobilen Datenübertragung, an deren Entwicklung derzeit intensiv gearbeitet wird, kurz erwähnt. Dazu gehört beispielsweise der Einbau von Mobilfunkmodulen in Konsumgüter wie Kühlschränke, Mikrowellengeräte, Waschmaschinen o.ä., der als Device-to-Consumer (D2C) zu klassifizieren ist. Ferner ist in diesem Zusammenhang die unter dem Stichwort Deviceto-Device (D2D) diskutierte Datenübertragung zwischen verschiedenen technischen Geräten, etwa zwischen Fahrzeugen zur Vermeidung von Unfällen, zu nennen. ${ }^{44}$ Auch die Kommunikation zwischen Privatpersonen (C2C) stellt ein Anwendungsfeld dar, auf dem die dritte Mobilfunkgeneration ganz neue Möglichkeiten (z.B. die EchtzeitÜbertragung von Farbbildern) bieten wird. Die genannten Nutzungsformen weisen keinen oder nur einen sehr mittelbaren Zusammenhang zum Informationsmanagement als betrieblichem Aufgabenfeld auf und werden deshalb im Folgenden nicht näher betrachtet.

\subsubsection{Technische Grundlagen des Mobile Business}

Die folgenden Ausführungen erläutern die technischen Hintergründe des Mobile Business. Diese stellen die Rahmenbedingungen für die später in diesem Kapitel untersuchten Gestaltungsoptionen des Informationsmanagements beim Aufbau von Mobile Business Lösungen dar. Im Abschnitt 6.1.3.1 werden zunächst kurz die Übertragungsstandards im Bereich des Mobilfunks behandelt. Der Schwerpunkt liegt dort auf den technischen Weiterentwicklungen, die mit den nächsten Netzgenerationen gegenüber der derzeit im Betrieb befindlichen zweiten Generation eingeführt werden. Abschnitt 6.1.3.2 geht auf die verwendeten Sprachen und ihre Darstellungsmöglichkeiten ein. In Abschnitt 6.1.3.3 schließlich wird die Situation im Endgerätebereich

auch auf der Basis von anderen Vertragsverhältnissen, etwa als Firmeninhaber oder als unabhăngiger Berater, tätig sein kann.

${ }^{41} \mathrm{Vgl}$. ähnlich Kamp/Velthuis (2000), S. 3f.

${ }^{42}$ Vgl. Mobilocity (2001), S. 2.

${ }^{43} \mathrm{Vgl}$. Borowicz/Scherm (2000), S. 472, die diese Anwendungen allerdings als B2B M-Commerce klassifizieren.

44 Vgl. Zobel (2001), S. 196. 
beschrieben, in dem verschiedene Gerätetypen und Betriebssysteme miteinander konkurrieren.

\subsubsection{1 Übertragungsstandards}

Im Folgenden werden die zur mobilen Datenübermittlung verwendeten Übertragungsstandards erläutert. Die Darstellung von Einzelheiten beschränkt sich dabei auf die für ein betriebswirtschaftlich orientiertes Informationsmanagement relevanten Kernpunkte. Entsprechend der historischen Abfolge sind bisher drei Generationen mobiler Netze zu unterscheiden. Die erste Generation basierte auf analoger Übertragung und wurde bereits in den 1970er Jahren entwickelt. ${ }^{45}$ In Deutschland zählt dazu das von der Telekom eingesetzte C-Netz, das erst vor kurzem außer Betrieb genommen wurde. Die aktuell verfuggbaren Mobilfunknetze werden als zweite Generation bezeichnet und basieren auf digitaler Übertragungstechnik. Im Vergleich zur ersten Generation weist die zweite Generation eine Reihe von Vorteilen auf, etwa die Unempfindlichkeit gegenüber Störungen, eine effiziente Fehlerkorrektur, die flexible Anknüpfung an leitungsgebundene Netzwerke und eine höhere Kapazität hinsichtlich der Zahl der angeschlossenen Endgeräte. ${ }^{46}$ Als zukunftsträchtigster Standard für die Etablierung der dritten Mobilfunkgeneration wird das Universal Mobile Telecommunications System (UMTS) gesehen ${ }^{47}$ UMTS-Netze werden gegenwärtig in vielen Ländern der Welt aufgebaut. Bis die Netze der dritten Generation flächendeckend verfügbar sind, wird jedoch noch geraume Zeit verstreichen. Aus diesem Grund finden derzeit bestimmte Standards, mit denen sich die Bandbreite von bestehenden Netzen der zweiten Generation erhöhen lässt, in verstärktem Maße Beachtung. In diesem Zusammenhang wird auch von der Mobilfunkgeneration 2,5 gesprochen. $^{48}$

\section{a) Die zweite Mobilfunkgeneration}

Bei der derzeit in Betrieb befindlichen zweiten Mobilfunkgeneration konnte sich kein weltweit einheitlicher Übertragungsstandard durchsetzen. Am weitesten verbreitet ist der Standard GSM (Global System for Mobile Communication), der in mehr als 300 Netzen in über 100 Ländern eingesetzt wird und den Frequenzbereich um $900 \mathrm{MHz}$ nutzt. ${ }^{49}$ In Deutschland bildet GSM die Basis für die beiden D-Netze. Eine Weiterentwicklung von GSM stellt DCS 1800 dar, das außer in Europa auch in Asien, Lateinamerika und Afrika genutzt wird und auf dem in Deutschland die E-Netze aufbauen. DCS arbeitet im Frequenzbereich um $1800 \mathrm{MHz}$. In den USA (CDMA) und Japan (PCS) werden jeweils andere Standards verwendet, die um $1900 \mathrm{MHz}$ operieren.

Ein wesentlicher Unterschied zwischen GSM und dem in den USA verbreiteten Übertragungsstandard liegt darin, dass unterschiedliche Verfahren für die Zuteilung der verfügbaren Frequenzen zu den in einer Funkzelle angemeldeten Endgeräten verwendet werden. $^{\text {s0 }}$ Während bei CDMA (Code Division Multiple Access) in den USA ein

${ }^{45} \mathrm{Vgl}$. Schreiber (2000), S. 29

${ }^{46} \mathrm{Vgl}$. Schreiber (2000), S. 30.

${ }^{47}$ Vgl. Schreiber (2000), S. 41.

${ }^{48}$ Vgl. Gerpott/Böhm (2001), S. 5.

${ }^{49}$ Vgl. Herold (2001), S. 18.

${ }^{50}$ Vgl. dazu Schreiber (2000), S. $30 \mathrm{ff}$. 
Frequenzband gleichzeitig mehreren Nutzern in einer Zelle zugeteilt wird und die Frequenzen in benachbarten Funkzellen wiederverwendet werden können, liegt dem GSM-Standard das sogenannte TDMA-Verfahren (Time Division Multiple Access) zu Grunde. Dabei stehen für jede Frequenz acht verschiedene Time-Slots zur Verfügung, die zwischen den Nutzern aufgeteilt werden.

Wenn in einem TDMA-Netz eine Funkverbindung zwischen einem Endgerät und der Basisstation aufgebaut ist, dann wird der entsprechende Time-Slot exklusiv fur diese Verbindung reserviert. Dieses Vorgehen wird auch als „circuit switching“ bezeichnet $^{51}$ und stellt die Hauptursache für die insgesamt geringe Bandbreite von GSMNetzen dar. GSM arbeitet mit maximal 22,8 KBit/s Übertragungsgeschwindigkeit, von denen jedoch durchschnittlich nur $9,6 \mathrm{Kbit} / \mathrm{s}$ für die mobile Datenübertragung zur Verfügung stehen. Diese Übertragungsraten sind nicht ausreichend für echte multimediale Dienstleistungsangebote. ${ }^{52}$ Das Netz, das NTT DoCoMo in Japan für den imode Service verwendet, arbeitet zwar ebenfalls nur mit 9,6 Kbit/s, es handelt sich allerdings bereits um ein Paketvermittlungsnetz, in dem always-on-Verbindungen der Endgeräte möglich sind. ${ }^{53}$ Die Paketvermittlungstechnik wird in Europa erst mit der Mobilfunkgeneration 2,5 eingeführt.

\section{b) Die Mobilfunkgeneration 2,5}

Als Zwischenschritt, der die Phase bis zur flächendeckenden Verfügbarkeit von Mobilfunkgenerationen der dritten Generation überbrücken soll, rüsten die GSM-Netzbetreiber gegenwärtig ihre bestehenden Netze technisch auf. ${ }^{54}$ Durch die Aufrüstung sollen die Kapazitäten zur mobilen Datenübertragung erheblich erhöht werden, ohne dass ein vollständig neuer Netzaufbau notwendig ist. Es handelt sich im Wesentlichen um eine software- und hardwarebezogene Ergänzung, die für die Netzbetreiber relativ leicht $\mathrm{zu}$ implementieren ist. ${ }^{55}$

Seit Ende 1999 ist der Übertragungsstandard HSCSD (High Speed Circuit Switched Data) verfügbar, der die Bandbreite der einzelnen Time-Slots von 9,6 KBit/s auf 14,4 $\mathrm{KBit} / \mathrm{s}$ erhöht. Dies wird durch den Wegfall eines Teils der Fehlerbeseitigungsprotokolle erreicht. ${ }^{56}$ Zusätzlich werden bis zu acht Time-Slots gebündelt, sodass sich eine maximale Übertragungskapazität von $115,2 \mathrm{KBit} / \mathrm{s}$ ergibt. Die Verbindungen erfolgen allerdings weiterhin „circuit switched“, sodass für die Verbindung reservierte Übertragungskapazitäten ungenutzt bleiben, wenn augenblicklich keine Daten zwischen Basisstation und Endgerät übertragen werden. Problematisch bei HSCSD ist, dass zum einen durch den Wegfall von Fehlerbeseitigungsroutinen die Übertragungsqualität leidet und dass zum anderen die ohnehin in Ballungsräumen bestehende Knappheit verfügbarer Frequenzen zusätzlich verschärft wird.

Ein anderes Konzept zur Erhöhung der Bandbreite verfolgt der Standard GPRS (General Packet Radio Services), mit dem die Paketvermittlung in die GSM-Netze

51 Vgl. Dean/Ketterer/Thiel (2000), S. 34.

${ }^{52} \mathrm{Vgl}$. Gerpott/Böhm (2001), S. 5.

53 Vgl. Zobel (2001), S. 258.

${ }_{55}^{54}$ Vgl. Gerpott/Böhm (2001), S. 5.

55 Vgl. Zobel (2001), S. 259.

${ }^{56}$ Vgl. Schreiber (2000), S. 38. 
eingeführt wird. ${ }^{57}$ Dieses Vermittlungsverfahren wird auch als "packet switching“ bezeichnet und ermöglicht es, die Kapazitäten von Telekommunikationsnetzwerken möglichst vollständig auszunutzen. ${ }^{58}$ Die zu übertragende Datenmenge wird in einzelne Pakete zerlegt und für jedes Paket wird diejenige Route vom Sender zum Empfänger durch das Netzwerk gewählt, die zum Zeitpunkt der Festlegung die höchsten Übertragungsraten bietet. Im Gegensatz zum „circuit switching“ werden die Frequenzen dabei nur dann belegt, wenn tatsächlich Daten durch das Netz versendet werden. Durch den gleichzeitigen Zugriff auf mehrere Time-Slots kann die Übertragungsgeschwindigkeit bei GPRS theoretisch bis zu 171,2 KBit/s erreichen. Die Paketvermittlung ermöglicht für die Endgeräte eine always-on-Funktionalität, also die ständige Verbindung zum Netz, ohne dass ein erneutes Einwählen erforderlich ist. ${ }^{59}$

Mit dem Übertragungsstandard EDGE (Enhanced Data Rates for GSM-Evolution) soll die Bandbreite von GSM-Netzen noch weiter erhöht werden. Wie GPRS ist auch EDGE ein paketorientiertes Vermittlungsverfahren. ${ }^{60}$ Dabei wird die Bandbreite der einzelnen Übertragungskanäle gegenüber GPRS bis zum Faktor drei effizienter ausgenutzt, sodass je Kanal Übertragungsraten von bis zu $48 \mathrm{KBit} / \mathrm{s}$ erreicht werden. ${ }^{61}$ Durch die Zusammenfassung von acht Kanälen ergibt sich eine maximale theoretische Übertragungsgeschwindigkeit von $384 \mathrm{KBit} / \mathrm{s}$. Diese Technik erlaubt damit bereits echte multimediale Applikationen mit Musik- und Bildübertragungen. Dennoch wird EDGE nur als Übergangstechnik zwischen der zweiten und der dritten Generation angesehen, da auch hier die Bündelung von Kanälen die Verknappung der verfügbaren Netzressourcen verschärft und hohe Kosten für die Anwender erwartet werden. ${ }^{62}$

Es ist darauf hinzuweisen, dass die hier genannten theoretisch möglichen Übertragungsraten normalerweise nicht den im praktischen Einsatz realisierten Geschwindigkeiten entsprechen. So ist etwa bei GPRS zunächst mit einer tatsächlichen Bandbreite von ungefähr $28 \mathrm{KBit} / \mathrm{s}$ zu rechnen ${ }^{63}$ und auch bei den für EDGE genannten Werten handelt es sich lediglich um theoretische Orientierungsgrößen. Dies hängt insbesondere damit zusammen, dass typischerweise eine Vielzahl von Nutzern um die Übertragungskanäle konkurrieren, sodass sich die erforderliche Zusammenfassung von acht Kanälen zur vollständigen Ausschöpfung der theoretischen Obergrenze in der Praxis kaum umsetzen lassen wird. Da die im Folgenden behandelte Technik der dritten Mobilfunkgeneration jedoch noch nicht sofort flächendeckend, d.h. auch außerhalb von Ballungsräumen, verfuggbar sein wird, ist es wahrscheinlich, dass die erläuterten Standards der Generation 2,5 insbesondere in ländlichen Gebieten dennoch über einen längeren Zeitraum eingesetzt werden. Die verwendeten Endgeräte müssten dann in der Lage sein, je nach Verfugbarkeit zwischen mehreren Übertragungsstandards hin- und herzuschalten. ${ }^{64}$

${ }^{57} \mathrm{Vgl}$. Herold (2001), S. 18.

58 Vgl. Dean/Ketterer/Thiel (200), S. 34.

59 Vgl. Zobel (2001), S. 258f.

${ }^{60} \mathrm{Vgl}$. Schreiber (2000), S. 41.

61 Vgl. Herold (2001), S. 19.

${ }^{62}$ Vgl. Schreiber (2000), S. 41.

63 Vgl. Zobel (2001), S. 259.

64 Vgl. Herold (2001), S. 20. 


\section{c) Die dritte Mobilfunkgeneration}

Mit der dritten Mobilfunkgeneration werden sich die Bandbreiten erneut erheblich erhöhen. Bei dem Standard UMTS (Universal Mobile Telecommunications System) handelt es sich um eine neue Übertragungstechnik im Frequenzbereich zwischen 1900 und $2200 \mathrm{MHz}$. Im Vergleich zur Generation 2,5 ist der Schritt für die Netzbetreiber wesentlich bedeutender, da ein vollkommen neues Funknetz mit eigener Infrastruktur errichtet werden muss. ${ }^{65}$ Wegen dieses hohen erforderlichen Aufwands ist damit zu rechnen, dass die Netze der dritten Generation über längere Zeit als Insellösung nur Industriegebiete und größere Städte abdecken werden. ${ }^{66}$ Hinsichtlich ihrer Topologie gleichen UMTS-Netze den bisherigen GSM-Netzen, da ein mehrstufiges Zellenkonzept verwendet wird. ${ }^{67}$

Auch bei UMTS-Netzen erfolgt die Datenübertragung paketvermittelt, sodass die insgesamt verfügbare Übertragungskapazität dynamisch und abhängig von der für den jeweiligen Dienst erforderlichen Bandbreite zwischen den Nutzern aufgeteilt wird. ${ }^{68}$ Die Aufteilung erfolgt im Gegensatz zum GSM-Standard nicht nach dem TDMA-, sondern nach dem CDMA-Verfahren, bei dem zwischen Sender und Empfänger ein variabler Code vereinbart wird, an Hand dessen die Datenpakete innerhalb einer bestimmten Frequenzbreite identifiziert werden können. ${ }^{69}$ Zum Teil werden beide Verfahren miteinander kombiniert, um die Übertragungskapazität weiter zu erhöhen, so z.B. in dem von der Firma Ericsson vorgestellten TDCDMA-Standard (Time Division Code Division Multiple Access). ${ }^{70}$

Insgesamt wird bei UMTS eine maximale theoretische Datenübertragungskapazität von $2 \mathrm{MBit} / \mathrm{s}$ erreicht. Diese gilt allerdings zunächst für städtische Gebiete und im quasistationären Betrieb. ${ }^{71}$ Für Nutzer im Freien, die sich mit geringer Geschwindigkeit fortbewegen, stehen $384 \mathrm{KBit} / \mathrm{s}$ zur Verfügung und in Fahrzeugen können bis zu 144 $\mathrm{KBit} / \mathrm{s}$ erreicht werden. Auch im quasi-stationären Betrieb sind die Maximalgeschwindigkeiten in der Praxis kaum zu realisieren, da die tatsächlich verfugbare Bandbreite um so kleiner ist, je mehr Nutzer sich in der betreffenden Funkzelle aufhalten. ${ }^{72}$ Dennoch eignen sich die hohen Bandbreiten von UMTS zur Realisierung von im Vergleich zur Generation 2,5 noch umfangreicheren multimedialen Anwendungen, die z.B. die Übertragung von Bewegtbildern vorsehen. Über InternetGateways sind ferner alle auf dem Internet-Standard TCP/IP basierenden Applikationen auch in UMTS-Netzen möglich, sodass die Voraussetzungen für eine Fortsetzung der Konvergenz von leitungsgebundener und mobiler Informationsverarbeitung geschaffen werden.

Inzwischen finden erste Entwicklungen für Netze der vierten Generation statt, die die Übertragungsgeschwindigkeiten mutmaßlich weiter erhöhen werden. Durch die unmittelbare Integration von IP in mobile Endgeräte könnte es zu Bandbreiten von bis

${ }^{65} \mathrm{Vgl}$. Zobel (2001), S. 259.

${ }^{66} \mathrm{Vgl}$. Herold (2001), S. 20.

${ }^{67} \mathrm{Vgl}$. Schreiber (2000), S. 42.

68 Vgl. Gerpott (2001), S. 36.

${ }^{69} \mathrm{Vgl}$. Gentner/Legler (2000), S. 15.

${ }^{70} \mathrm{Vgl}$. Schreiber (2000), S. 42.

${ }^{71}$ Vgl. Herold (2001), S. 20.

${ }^{72} \mathrm{Vgl}$. Gerpott (2001), S. 36. 
zu $20 \mathrm{MBit} / \mathrm{s} \mathrm{kommen.}^{73}$ Allerdings liegen bisher kaum konkrete Ergebnisse in dieser Richtung vor, sodass die hier beschriebene gegenwärtige Situation für einige Jahre stabil bleiben dürfte. ${ }^{74}$

\subsubsection{Verbindungs- und Darstellungsstandards}

Die bisher betrachteten Übertragungsstandards des Mobilfunks betrafen allein den Transport von Daten durch die jeweiligen Netze. Die logische Abwicklung der Übertragungsvorgänge sowie die zu übertragenden Daten und die Möglichkeiten zu ihrer Darstellung wurden dagegen noch nicht betrachtet. Folglich sind die im vorigen Abschnitt erläuterten Übertragungsstandards den rein technisch orientierten Ebenen 1 bis 4 des ISO/OSI-Referenzmodells ${ }^{75}$ zuzuordnen. Ähnlich wie im leitungsgebundenen Internet, wo die Übertragungsprotokolle TCP/IP, Diensteprotokolle wie HTTP und FTP sowie Anwendungs- und Darstellungssprachen wie HTML und Java benutzt werden, ist es für die Übertragung von Inhalten auf mobile Endgeräte zusätzlich erforderlich, Regeln für den Aufbau von Verbindungen, die Darstellung der Daten und die Realisierung von Anwendungen festzulegen.

Derzeit ist zu beobachten, dass sich auf der Anwendungsebene das Wireless Application Protocol (WAP) mehr und mehr als weltweiter Standard durchsetzt. ${ }^{76}$ Eine Vielzahl von Firmen, die im Umfeld des Mobilfunks operieren (Hersteller von Endgeräten, Netzbetreiber usw.) haben sich mit dem Ziel, neue und innovative Dienste anbieten zu können, zu einer Interessengemeinschaft, dem WAP-Forum, zusammengeschlossen. ${ }^{77}$ Dem WAP-Forum gehören mittlerweile über 500 Mitglieder an. Dabei ist die Mitgliedschaft von NTT DoCoMo besonders hervorzuheben, da der erfolgreiche i-Mode-Dienst in Japan nicht auf WAP, sondern auf dem proprietären cHTML (compact HTML) basiert. Bei cHTML handelt es sich um eine vereinfachte Version der Internet-Sprache HTML, die es ermöglicht, multimediale Inhalte wie Sound und farbige Bilder auf mobile Endgeräte mit im Vergleich zu stationären Rechnern kleineren Displays und geringeren Rechenkapazitäten zu übertragen. ${ }^{78}$ Mittelfristig ist $\mathrm{zu}$ erwarten, dass WAP und der i-Mode-Standard konvergieren und eine gemeinsame Lösung auf der Basis von Internet-Standards, die zugleich auch für das leitungsgebundene Internet verwendet werden, erreicht wird. ${ }^{79}$

Genau genommen handelt es sich bei WAP selbst nicht um einen einzelnen Standard, sondern um eine Sammelbezeichnung für mehrere Übertragungsebenen mit jeweils eigenen Standards. Bestehende Standards wurden durch das WAP-Forum vereinheitlicht und auch in ihrer Bezeichnung angepasst. Dazu gehören Standards auf folgenden Schichten: ${ }^{80}$

${ }^{73}$ Vgl. Plesmann (2000), S. 2; Zobel (2001), S. 260.

${ }^{74}$ Vgl. Plesmann (2000), S. 2.

75 Auf eine Erläuterung des weithin bekannten ISO/OSI-Referenzmodells wird hier verzichtet. Vgl. dazu z.B. Gabriel et al. (1994), S. 63ff.

76 Vgl. Kamp/Velthuis (2000), S. 3.

77 Vgl. Schreiber (2000), S. 52.

${ }^{78}$ Vgl. Zobel (2001), S. 108.

79 Vgl. Varshney/Vetter/Kalatoka (2000), Emerging W3C standards; Born (2001) S. 24.

${ }^{80} \mathrm{Vgl}$. Schmitzer/Butterwegge (2001), S. 357f. 
- Transportschicht mit Standard WDP/UDP (Wireless/User Datagram Protocol)

- Sicherheitsschicht mit Standard WTLS (Wireless Transport Layer Security)

- Transaktionsschicht mit Standard WTP (Wireless Transaction Protocol)

- Sitzungsschicht mit Standard WSP (Wireless Session Protocol)

- Anwendungsschicht mit Standard WAE (Wireless Application Environment)

Grundsätzliches Ziel bei der Entwicklung von WAP war es, so wenig wie möglich geräteinterne Ressourcen zu verwenden und gleichzeitig die Beschränkungen der Geräte durch erweiterte Funktionalitäten des Netzwerks auszugleichen. ${ }^{81}$ Im November 1999 kamen die ersten WAP-fähigen Endgeräte auf den Markt, und inzwischen sind über $70 \%$ aller neuen Mobiltelefone WAP-fähig ausgestattet. ${ }^{82}$ Allerdings war die Technik zum Zeitpunkt der Markteinführung noch nicht sehr ausgereift und konnte die (unter anderem durch unrealistische Werbebotschaften geweckten) Erwartungen bei den Kunden nicht erfüllen. ${ }^{83}$ Umständliche Einwählprozeduren und das anfängliche Fehlen von nützlichen Anwendungsmöglichkeiten haben dem Wireless Application Protocol in Europa zwischenzeitlich eine schlechte Reputation eingetragen. WAP wird daher von einigen Seiten nur noch als Übergangslösung bezeichnet, die schon bald durch einen neuen Standard abgelöst werden könnte und für die Mobilfunknetze der dritten Generation keine Bedeutung mehr haben dürfte. ${ }^{84}$

Tatsächlich hängt die schlechte Reputation jedoch weniger mit der begrenzten Leistungsfähigkeit von WAP als mit der begrenzten Leistungsfähigkeit der vorhandenen Endgeräte und der darin implementierten Software sowie insbesondere auch den übertragungstechnischen Limitationen von „circuit switched"-Netzen der zweiten Mobilfunkgeneration zusammen. ${ }^{85}$ Der am Markt weitaus erfolgreichere i-Mode-Dienst verfügt mit 9,6 KBit/s zwar nicht über eine größere Bandbreite als die GSM-Netze, die Datenübertragung erfolgt jedoch bereits paketorientiert. Die Endgeräte, die in Japan im Gegensatz zu den in Europa verwendeten Geräten mit größeren Displays ausgestattet sind und die Darstellung farbiger Grafiken ermöglichen, können dadurch always-on an das Netz angeschlossen werden. Dies stellt die entscheidende Voraussetzung für die Entwicklung neuer Applikationen mit einem im Vergleich zu den ersten WAPAngeboten weitaus größeren potenziellen Kundenkreis dar. Da inzwischen auch einflussreiche Unternehmungen, die bisher auf abweichende Lösungen gesetzt hatten, auf das Wireless Application Protocol umschwenken ${ }^{86}$ ist eine Ablösung von WAP durch einen neuen Standard in naher Zukunft nicht mehr sehr wahrscheinlich.

In technischer Hinsicht verwendet das Wireless Application Protocol einen Microbrowser als Client Software und unterstützt Text, grafische Darstellungen und standardisierte Web-Inhalte. ${ }^{87}$ In naher Zukunft wird die Übertragung weiterer multimedialer Daten wie z.B. Fotos, Sound und später auch Bewegtbildern möglich sein. Auf der Datendarstellungsebene wird derzeit die Sprache WML (Wireless Markup

\footnotetext{
${ }^{81}$ Vgl. Schreiber (2000), S. 53.

${ }^{82} \mathrm{Vgl}$. Kamp/Velthuis (2000), S. 3.

${ }^{83} \mathrm{Vgl}$. Zobel (2001), S. 38f.

${ }^{84}$ Vgl. z.B. Wirtz (2001), S. 8; Rochlitz (2001), S. 11.

${ }^{85}$ Vgl. Kamp/Velthuis (2000), S. 3.

${ }^{86} \mathrm{Vgl}$. z.B. Microsoft (2001b), Microsoft Mobility Initiatives.

${ }^{87} \mathrm{Vgl}$. Varshney/Vetter/Kalatoka (2000), Wireless and Mobile Middleware.
} 
Language) verwendet, die als Seitenbeschreibungssprache ähnlich wie die im leitungsgebundenen Internet etablierte Sprache HTML angelegt ist. ${ }^{88}$ Der Prozess der Datenübertragung ist dabei so aufgebaut, dass ein Internet-Gateway (der sich typischerweise in der mehrere Funkzellen bedienenden Vermittlungsstelle befindet) als Proxy Server fungiert und die Datenanforderungen der mobilen Clients in Internet-Anfragen übersetzt. In umgekehrter Übertragungsrichtung werden die Daten des Informationsanbieters durch das Gateway in kompakte Formate mit reduzierten Dateigrößen transformiert, die anschließend durch das Mobilfunknetz an die Endgeräte versendet werden. Dieses Vorgehen ist schematisch in Abbildung 6/2 dargestellt.

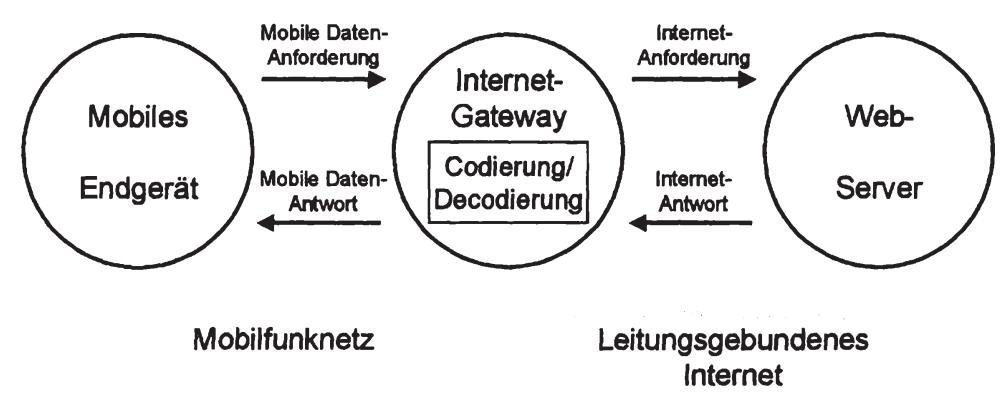

Abb. 6/2: Verknüpfung des leitungsgebundenen Internet mit Mobilfunknetzen Quelle: in Anlehnung an VarshneyNetter/Kalakota (2000), Wireless and Mobile Middleware.

Es zeichnet sich ab, dass zukünftig (wahrscheinlich durch eine Annäherung des bisher genutzten WML an den Internet-Standard XML (Extended Markup Language)) eine Trennung von Content und Darstellung erfolgen wird, sodass die Inhalte endgeräteunabhängig vorgehalten werden können. ${ }^{89}$ Die Internet-Daten lassen sich dann ohne weitere Umwandlung an den Microbrowser übertragen, der diese flexibel in einer furr das jeweilige Endgerät geeigneten Form aufbereitet. Abbildung 6/3 verdeutlicht die verschiedenen Grundkonzepte und die zu erwartende Entwicklung.

${ }^{88}$ Vgl. dazu Schreiber (2000), S. 56ff.

${ }^{89}$ Vgl. Pflug (2001), S. 21. 


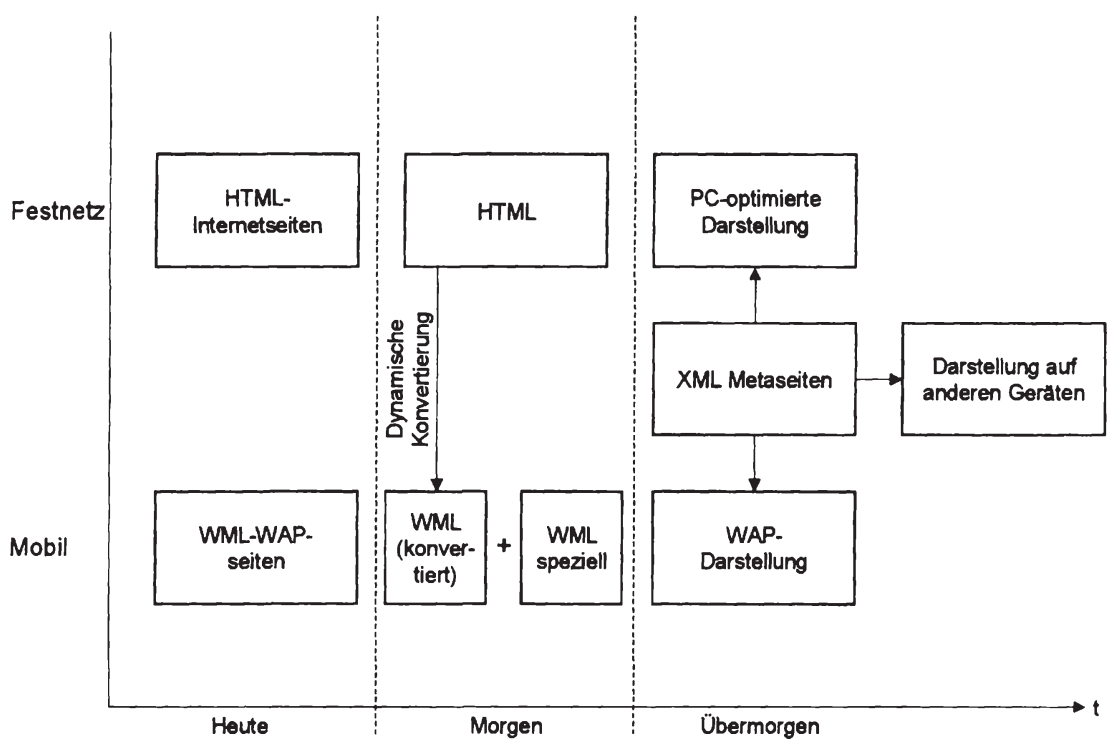

Abb. 6/3: Entwicklungsperspektiven für die Zusammenführung von leitungsgebundenem und mobilem Internet

Quelle: Zobel (2001), S. 275.

\subsubsection{Endgeräte}

Die Endgeräte der zweiten Mobilfunkgeneration sind in erster Linie für die Übertragung von gesprochener Sprache konstruiert. Zwar werden zum Teil Zusatzfunktionalitäten wie Kalender, To Do-Listen oder E-Mail angeboten, diese sind jedoch umständlich $\mathrm{zu}$ handhaben und in ihrer Leistungsfähigkeit stark beschränkt. ${ }^{90}$ Eine erfolgreiche Etablierung von Mobile Business-Konzepten erfordert daher die Entwicklung neuer Endgeräte. Diese müssen nicht nur die beschriebenen Mobilfunkstandards und Darstellungsmöglichkeiten beherrschen, sondern darüber hinaus über leistungsfähige Benutzungsoberflächen verfügen, die neben dem traditionellen Telefonieren auch ein komfortables Arbeiten mit datenorientierten Applikationen ermöglichen.

Im Vergleich zu den Mobiltelefonen der zweiten Generation sind derartige Endgeräte mit größeren Speichern und Displays sowie erweiterten Rechenkapazitäten auszustatten. Mit der wachsenden Leistungsfähigkeit und dem steigenden Funktionsumfang dieser Geräte werden zudem Betriebssysteme benötigt, die eine effiziente Ausnutzung der verfügbaren Ressourcen gewährleisten. Auf kleinstem Raum müssen immer mehr Funktionen bedient und Applikationen ausgeführt werden. ${ }^{91}$ Die bisher am Markt angebotenen Geräte sind entweder stärker auf die Durchführung von Kommunikationsprozessen oder stärker auf die Bereitstellung von der für Office-Anwendungen erforderlichen Rechenleistung ausgelegt. ${ }^{92}$ Die verschiedenen Lösungen orientieren sich

\footnotetext{
90 Vgl. Zobel (2001), S. 277.

9] Vgl. Gross/Koch (2001), S. 80.

92 Vgl. Varshney/Vetter/Kalakota (2000), Wireless User Infrastructure.
} 
an unterschiedlichen Kundenbedürfnissen und streben spezialisierte Angebote zur Befriedigung dieser Bedürfnisse an. ${ }^{93}$

Auf dem Markt für mobile Endgeräte sind gegenwärtig zwei gleichzeitig stattfindende Konkurrenzkämpfe zu beobachten. ${ }^{94}$ Der eine Konkurrenzkampf findet zwischen den Herstellern von Mobiltelefonen statt, die zur besseren Unterstützung der mobilen Datenübertragung an der Weiterentwicklung der vorhandenen Telefone der zweiten Generation arbeiten und dabei untereinander im Wettbewerb stehen. Solche Mobiltelefone mit erweiterter Funktionalität werden auch als Smart Phones bezeichnet. ${ }^{95}$ Der zweite Konkurrenzkampf, der hier näher betrachtet werden soll, betrifft den Wettbewerb zwischen unterschiedlichen mobilen Plattformen. Dabei konkurriert das Konzept des Smart Phone mit dem des Personal Digital Assistant (PDA) und dem des Pocket PC. Auf dieser Ebene bilden die Hersteller von Mobiltelefonen eine Gruppe, die sich insgesamt im Wettbewerb mit den Herstellergruppen anderer Gerätetypen befindet.

Grundsätzlich ist ein mobiler Zugang zum Internet auch über Notebook-PCs, deren Leistungsfähigkeit an die stationärer Rechner heranreicht, möglich. Diese sind allerdings nur begrenzt mit den oben genannten, echten mobilen Endgeräten vergleichbar, da sie ohne Stromanschluss über geringere Nutzungsdauern verfuggen und höhere Anforderungen an die örtliche Umgebung (Sitzgelegenheiten, Abstellmöglichkeiten usw.) stellen. ${ }^{96}$ Zudem werden diese Geräte nicht jederzeit mitgeführt, sodass die Verfügbarkeit des Netzzugangs nur in eingeschränktem Maße ausgeweitet wird. Ähnliches gilt für so genannte In-Car-Systeme, die aus Automobilen heraus einen Netzzugang möglich machen. Hier ist die Verfügbarkeit des Netzzugangs an den Aufenthalt in dem betreffenden Fahrzeug gebunden. Die nachfolgende Betrachtung beschränkt sich daher auf Smart Phones, PDAs und Pocket PCs als echte mobile Endgerätetypen.

Der angesprochene Konkurrenzkampf zwischen den verschiedenen mobilen Plattformen ist zugleich ein Wettbewerb zwischen unterschiedlichen Betriebssystemen. Die Smart Phones verwenden das Betriebssystem EPOC, das von der Symbian-Gruppe, zu der Mitglieder wie Nokia, Ericsson und Motorola gehören, weiterentwickelt wird und etwa $1 \mathrm{MB}$ Hauptspeicherplatz benötigt. ${ }^{97}$ Die Benutzungsoberfläche dieses Betriebssystems orientiert sich an der aus der Welt der stationären Arbeitsplatzrechner bekannten Schreibtisch-Metapher. ${ }^{98}$ Analog zum Vorgehen bei Arbeitsplatzrechnern erfolgt die Interaktion des Benutzers mit dem System über Fenster, Symbole, Menüs und Zeiger. Die Eingabe von Daten geschieht dabei über eine dem QWERTY- bzw. QWERTZ-Standard entsprechende Tastatur. Der Benutzer arbeitet in erster Linie dokumentenorientiert und verfügt über schnellen Zugriff auf unterschiedliche Anwendungen, zwischen denen durch Tastendruck gewechselt werden kann. Auch die Benutzungsoberfläche der Pocket PCs ist ähnlich konstruiert und verwendet typischerweise eine abgespeckte Version der entsprechenden PC-Betriebssysteme mit einer Art Verkleinerung der Schreibtischmetapher. ${ }^{99}$ Hier ist insbesondere das Betriebssystem

93 Vgl. Kamp/Velthuis (2000), S. 3.

94 Vgl. Plesman (2000), S. 3.

95 Vgl. Microsoft (2001b), Microsoft Mobility Intitiatives.

96 Vgl. Scheer et al. (2001), S. 9.

97 Vgl. Gross/Koch (2001), S. 77; Varshney/Vetter/Kalakota (2001), Wireless User Infrastructure.

$98 \mathrm{Vgl}$. dazu Gross/Koch (2001), S. $75 \mathrm{ff}$.

99 Vgl. Gross/Koch (2001), S. 75. 
Windows CE zu nennen, das mit $2 \mathrm{MB}$ einen größeren Speicherbedarf hat und etwas langsamer arbeitet als EPOC, jedoch umfangreichere Features furr die Entwicklung von Anwendungen bietet. ${ }^{100}$ Dieser Plattform bietet unmittelbare Kompatibilität mit herkömmlichen Arbeitsplatzrechnern, sodass Daten besonders einfach zwischen mobilen und stationären Geräten ausgetauscht werden können. Den Personal Digital Assistants liegt dagegen ein abweichendes Konzept zur Gestaltung der Benutzungsoberfläche zu Grunde. Als bekannteste Vertreter dieser Kategorie sind die Palm Pilots der Firma 3 Com zu nennen, die als Betriebssystem PalmOS verwenden, das nur ca. 300 KByte Speicherplatz benötigt. Bei der Gestaltung der Benutzungsoberfläche wurde hier zu Gunsten einer stärkeren grafischen Orientierung auf einige Bestandteile der klassischen Schreibtischmetapher verzichtet. ${ }^{101}$ Das Positionieren des Zeigers sowie das Auswählen und Aktivieren von Symbolen erfolgt mit einem Stift direkt über einen berührungssensitiven Bildschirm. Auch die Eingabe von Text geschieht berührungssensitiv, entweder über eine On-Screen-Tastatur oder über eine spezielle Schreibfläche zur Erkennung handschriftlicher Zeichen.

Momentan wird darüber hinaus daran gearbeitet, die Interaktion zwischen Gerät und Benutzer durch die Einführung von Spracherkennungssystemen weiter zu vereinfachen. Die Spracherkennung bietet gerade im mobilen Einsatz große Vorteile, da die Benutzer die Hände frei haben und nicht ständig den Blick auf die Benutzungsschnittstelle richten müssen. ${ }^{102} \mathrm{Da}$ als Hardware nur ein Mikrofon und ein Lautsprecher notwendig sind, können zudem wesentlich kleinere Geräte entwickelt werden. Allerdings erfordern natürlichsprachliche Benutzungsoberflächen relativ große Rechenleistung. Dies stand der Realisierung solcher Systeme bisher noch im Wege.

Insgesamt ist auch im Endgerätebereich mit einer Konvergenz unterschiedlicher Lösungen zu rechnen, sodass die beschriebenen Unterschiede zwischen den verschiedenen Gerätetypen zukünftig immer kleiner werden dürften. ${ }^{103}$ Die Geräte werden immer mehr zu Universalgeräten, ${ }^{104}$ sodass die momentan noch zu beobachtende Kommunikations- oder Applikationsspezialisierung in künftigen Endgerätegenerationen weitaus weniger stark ausgeprägt sein wird.

\subsubsection{Innovative Leistungsmerkmale und Anwendungspotenziale des Mobile Business}

Nachdem im vorigen Abschnitt die technischen Hintergründe des Mobile Business erläutert wurden, untersuchen die folgenden Ausführungen die Leistungsmerkmale und Anwendungspotenziale von Mobilfunknetzen der dritten Generation und der Generation 2,5. In Abschnitt 6.1.4.1 wird zunächst ein Überblick über die in der aktuellen Diskussion genannten Anwendungsbereiche gegeben. Davon ausgehend erörtert Abschnitt 6.1.4.2 begleitende Merkmale, die zwar einen Fortschritt gegenüber bisherigen Möglichkeiten der zweiten Mobilfunkgeneration darstellen, die für sich genommen jedoch keine originären Anwendungen des Mobile Business ermöglichen. In

\footnotetext{
${ }^{100}$ Vgl. Varshney/Vetter/Kalakota (2000), Wireless User Infrastructure.

${ }^{101} \mathrm{Vgl}$. Gross/Koch (2001), S. 77.

${ }^{102}$ Vgl. Gross/Koch (2001), S. $75 f$.

${ }^{103} \mathrm{Vgl}$. Varshney/Vetter/Kalakota (2001), Wireless User Infrastructure.

${ }^{104}$ Vgl. Zobel (2001), S. $277 f$.
} 
Abschnitt 6.1.4.3 schließlich werden echte innovative Leistungsmerkmale beschrieben, auf deren Basis innovative Anwendungen aufgebaut werden können.

\subsubsection{1 Überblick}

Im Vordergrund der folgenden Untersuchungen steht die Frage, inwiefern die mobile Datenübertragung bisher ungekannte Nutzungsmöglichkeiten eröffnet, die die in Abschnitt 6.1.1.2 geäußerte Erwartung rechtfertigen, dass es durch das Mobile Business zu einer Fortsetzung der Internet-Revolution kommen wird. Mobile BusinessKonzepte werden nur dann erfolgreich sein, wenn sie einen erkennbaren neuen Kundennutzen stiften. ${ }^{105}$ In vielen Diskussionsbeiträgen wird deshalb die Ansicht geäußert, dass es sich nicht lediglich um mobile Varianten bereits bestehender EBusiness-Lösungen handeln dürfte. ${ }^{106}$ So einig sich die verschiedenen Autoren jedoch in dieser Grundaussage sind, so heterogen sind die Erwartungen darüber, welches genau die Leistungsmerkmale und Anwendungspotenziale sind, mit denen sich die optimistischen Prognosen fur Mobile Business und Mobile Commerce in der Praxis umsetzen lassen.

Zum Teil wird hervorgehoben, dass bisher noch eine „Killer-Applikation“ für die Netze der nächsten Mobilfunkgenerationen fehle, die - vergleichbar mit dem SMS-Service bei den Netzen der zweiten Generation - dem Mobile Business zum endgültigen Durchbruch verhelfen könnte. ${ }^{107}$ Diesem Vorbehalt wird andererseits mit dem Hinweis begegnet, der Nutzen ergebe sich nicht aus einer einzelnen „Killer-Applikation“, sondern aus dem Zusammenspiel unterschiedlicher Leistungsmerkmale, die in den bisherigen Netzen nicht in ähnlicher Form verfügbar waren. ${ }^{108}$ Durch diese Leistungsmerkmale eröffnen sich Möglichkeiten zur Realisierung originärer neuer Anwendungen mit einem entsprechenden Zusatznutzen. Ein kurzer Überblick über verschiedene in der Literatur genannte Leistungsmerkmale und Anwendungsgebiete, die einen solchen zusätzlichen Nutzen bewirken sollen, verdeutlicht die Heterogenität der Vorstellungen und gibt zugleich einen Eindruck von der Vielschichtigkeit der Problemstellung:

\footnotetext{
${ }^{105} \mathrm{Vgl}$. Zobel (2001), S. 12.

${ }^{106}$ Vgl. exemplarisch Schmitzer/Butterwegge (2000), S. 355; Dean/Ketterer/Thiel (2000), S. 34; Borowicz/Scherm (2000), S. 469.

${ }^{107} \mathrm{Vgl}$. z.B. Borowicz/Scherm (2000), S. 472; Kollmann (2001), S. 59.

${ }^{108}$ Vgl. z.B. Seiler/Gruber (2000), S. 47; Wirtz (2001), S. 9.
} 


\begin{tabular}{|l|l|}
\hline $\begin{array}{l}\text { Innovative Leistungsmerkmale des Mobile } \\
\text { Business }\end{array}$ & Quelle \\
\hline $\begin{array}{l}\text { Ubiquität, Kontextspezifität, Datenproaktivität, Abschluss/ } \\
\text { Zahlung, Interaktion, Integration Unterhaltung, Remote } \\
\text { Control }\end{array}$ & Zobel (2001), S. 43ff. \\
\hline $\begin{array}{l}\text { Ortsunabhängigkeit/Mobilität, Personalisierung, Lokalisie- } \\
\text { rung, Multimedialität }\end{array}$ & Gerpott (2001), S. 37f. \\
\hline $\begin{array}{l}\text { Lokalisierung, Ubiquität und Instant Connectivity, } \\
\text { Personalisierung }\end{array}$ & Wohlfahrt (2001), S. 50f. \\
\hline $\begin{array}{l}\text { Mobilität, Ubiquität, Kontextsensitivität, Erreichbarkeit, } \\
\text { Remote Control, Sicherheit }\end{array}$ & Scheer et al. (2001), S. 11f. \\
\hline $\begin{array}{l}\text { Kundenseite: Ortsunabhängigkeit, Verfügbarkeit, Benut- } \\
\text { zungsfreundlichkeit, Interaktivität, Sicherheit } \\
\text { Anbieterseite: Ortsbestimmung, Erreichbarkeit, } \\
\text { Standardisierung, Personalisierung }\end{array}$ & $\begin{array}{l}\text { Schmitzer/Butterwegge (2000), } \\
\text { S. 356. }\end{array}$ \\
\hline $\begin{array}{l}\text { Convenience, Sofortige Verfügbarkeit, Sicherheit, Orts- } \\
\text { unabhängigkeit, Erreichbarkeit, Personalisierung, } \\
\text { Lokalisierbarkeit, Kostengünstigkeit }\end{array}$ & $\begin{array}{l}\text { Wiedmann/Buxel/Buckler (2000), } \\
\text { S. 685ff. }\end{array}$ \\
\hline $\begin{array}{l}\text { Ubiquity, Convenience and Accessibility, Localization, } \\
\text { Personalization }\end{array}$ & Mobilocity (2000), S. 4 \\
\hline
\end{tabular}

Abb. 6/4: Leistungsmerkmale und Anwendungspotenziale des Mobile Business

Bei näherer Betrachtung der Zusammenstellung in Abbildung 6/4 zeigt sich, dass sich die in den verschiedenen Fachbeiträgen aufgeführten Listen mit Leistungsmerkmalen und daraus folgenden Anwendungspotenzialen stark ähneln, im Detail jedoch nicht unerhebliche Unterschiede aufweisen. Vor einer Behandlung der einzelnen Punkte ist zunächst eine Konsolidierung dieser Listen erforderlich.

Es ist einerseits ein Kern von Leistungsmerkmalen erkennbar, die übereinstimmend in allen Publikationen als innovativer Technologiewertbeitrag der nächsten Mobilfunkgenerationen genannt werden. Dazu gehören die Aspekte Ubiquität, Personalisierung und Lokalisierung. Die Merkmale Personalisierung und Lokalisierung werden dabei zum Teil unter dem Oberbegriff der Kontextspezifität bzw. Kontextsensitivität behandelt. Neben persönlichem und lokalem Kontext können ferner auch zeitliche Gesichtspunkte zum Ansatzpunkt kontextspezifischer Angebote gemacht werden. ${ }^{109}$ Anstelle von Ubiquität wird in einigen Beiträgen von Ortsunabhängigkeit/Mobilität gesprochen. Diese Begriffe stellen allerdings keine vollständigen Synonyme dar. Ihre Beziehung wird weiter unten bei der näheren Betrachtung des betreffenden Leistungsmerkmals erläutert. Bei den aufgeführten Kernmerkmalen handelt es sich um die Basis für die Entwicklung originärer innovativer Applikationen, die einen echten Zusatznutzen des Mobile Business gegenüber den bisherigen technischen Möglichkeiten begründen können.

Neben den Kernmerkmalen werden andererseits eine Reihe von Merkmalen nur in einzelnen oder einem Teil der Quellen aufgeführt. Dazu gehören Aspekte wie Datenproaktivität, Erreichbarkeit, Convenience, Multimedialität, Abschluss/Zahlungsmöglichkeit, Sicherheit und Remote Control. Durch die Hervorhebung dieser Punkte setzen die Autoren individuelle Untersuchungsschwerpunkte und nähern sich dem Mobile Business aus unterschiedlichen Perspektiven. Bei den betreffenden Merkmalen handelt es sich allerdings eher um begleitende Aspekte, die zwar ebenfalls einen

\footnotetext{
${ }^{109}$ Vgl. Plesman (2000), S. 4.
} 
Beitrag zum Nutzen der nächsten Netzgenerationen leisten. Dieser Nutzenbeitrag ist jedoch entweder nur in Verbindung mit den oben genannten originären Leistungsmerkmalen realisierbar, oder er könnte prinzipiell auch durch andere, bereits existierende technische Lösungen erreicht werden. Einige der begleitenden Merkmale stellen Nebenbedingungen bzw. Voraussetzungen für die Realisierung originärer innovativer Anwendungen dar. Abbildung 6/5 zeigt die hier vorgenommene Einteilung von Leistungsmerkmalen des Mobile Business in Kernmerkmale und begleitende Merkmale.

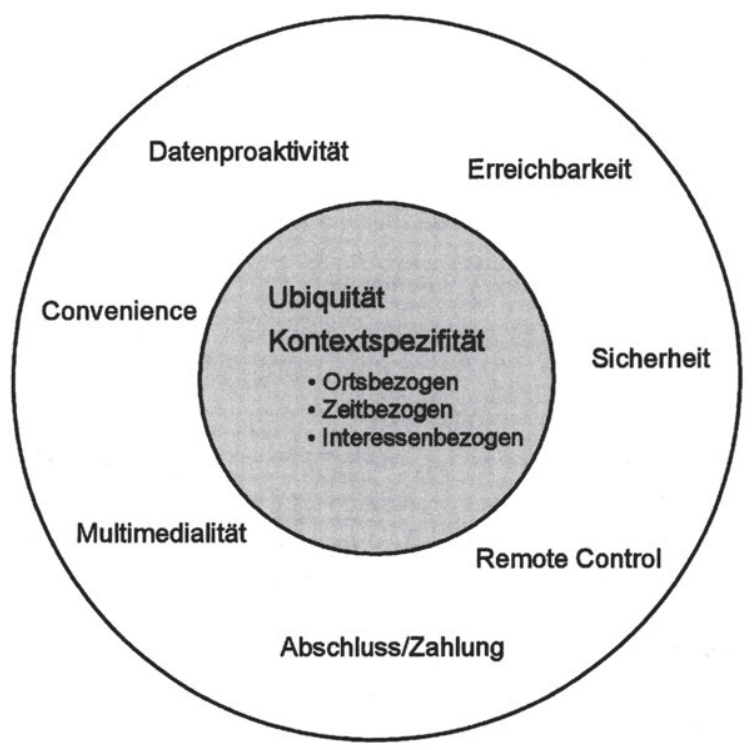

Abb. 6/5: Kernmerkmale und begleitende Merkmale des Mobile Business

Die nachfolgenden Ausführungen wenden sich zunächst den begleitenden Merkmalen zu. Anschließend werden die zum Kern gehörenden, echten innovativen Leistungsmerkmale und Anwendungspotenziale des Mobile Business näher erläutert.

\subsubsection{Begleitende Leistungsmerkmale der nächsten Mobilfunkgenerationen}

Das Merkmal Erreichbarkeit bedeutet, dass der Benutzer des Endgeräts jederzeit und überall kontaktiert und somit zeitnah über aktuelle Ereignisse und Entwicklungen informiert werden kann. ${ }^{110}$ Ähnliche Inhalte werden mit dem Schlagwort Datenproaktivität verbunden, das speziell auf die Möglichkeit zum Aufbau von PushDiensten in paketvermittelten Mobilfunknetzen mit always-on-Funktionalität abstellt. ${ }^{111}$ Tatsächlich stellen diese Anwendungsmöglichkeiten einen echten Fortschritt gegenüber den gegenwärtig noch in Betrieb befindlichen Netzen der zweiten Generation dar. Allerdings besteht eine starke Nähe zum Leistungsmerkmal Ubiquität, das genau genommen die Erreichbarkeit und die Datenproaktivität einschließt. Ubiquität ist erst

${ }^{110} \mathrm{Vgl}$. Wiedmann/Buxel/Buckler (2000), S. 687.

${ }^{111}$ Vgl. dazu Zobel (2001), S. 54ff. 
dann gegeben, wenn der Benutzer bzw. das Endgerät stets erreichbar ist und jederzeit Daten aus dem Netz empfangen kann. Das Merkmal Ubiquität ist darüber hinaus jedoch nicht nur passiv orientiert, sondern es umfasst zusätzlich auch die Möglichkeit für den Benutzer, jederzeit selbst aktiv zu werden und auf die im Netz verfügbaren Informationen zuzugreifen. Aus diesem Grund werden Erreichbarkeit und Datenproaktivität im Folgenden nicht mehr als eigenständige Punkte betrachtet, sondern unter dem Oberbegriff Ubiquität zusammengefasst.

Convenience und Multimedialität beziehen sich auf die erhöhte Leistungsfähigkeit zukünttiger mobiler Endgeräte. Während unter Convenience die Einfachheit der Bedienung über benutzungsfreundliche Schnittstellen verstanden wird, ${ }^{112}$ bezeichnet Multimedialität die Möglichkeit, durch Integration von Text, Stand- und Bewegtbildern sowie Sound aussagekräftige Inhalte zu vermitteln. ${ }^{113}$ Diese beiden Leistungsmerkmale stellen zwar ebenfalls wesentliche Weiterentwicklungen gegenüber der zweiten Mobilfunkgeneration dar. Im Vergleich zu anderen Informations- und Kommunikationstechniken handelt es sich jedoch nicht um einzigartige Charakteristika der mobilen Datenübertragung. Aussagekräftige multimediale Inhalte konnten auch bisher schon unter Einsatz leitungsgebundener Kommunikationstechniken mit komfortablen Benutzungsoberflächen von einem Sender zu einem Empfänger übertragen werden. Durch die begleitenden Merkmale Convenience und Multimedialität allein kann folglich kein zusätzlicher Kundennutzen gestiftet werden, der nicht auch durch herkömmliche technische Lösungen erreichbar wäre.

Die Möglichkeit zum verbindlichen Abschluss von Geschäftsvorgängen, die direkt mit dem mobilen Endgerät bezahlt werden können, wird an einigen Stellen ebenfalls als innovatives Anwendungsfeld des Mobile Commerce bzw. Mobile Business genannt. ${ }^{114}$ Durch die Zahlungsfunktion könnten mobile Endgeräte zum Substitut für die Verwendung von Kreditkarten bei traditionellen Kaufvorgängen (z.B. an der Tankstelle, im Supermarkt usw.) werden. Darüber hinaus lassen die Merkmale Abschlussmöglichkeit und Zahlung auch die friktionsfreie Abwicklung von entgeltpflichtigen Transaktionen zu, die eigens für mobile Nutzer konzipiert worden sind (z.B. Informationsdienstleistungen). Diese Funktionalitäten haben allerdings nur ergänzenden Charakter bei der Stiftung von innovativem Kundennutzen. Die Zahlungsmöglichkeit als solche stellt kein originäres Anwendungsfeld der Mobilfunktechnik dar, und bei der Zahlung von eigens für mobile Nutzer entwickelten Dienstleistungen handelt es sich nur um einen nebensächlichen Aspekt des betreffenden Angebots. Die Möglichkeit zur direkten Bezahlung wird zwar häufig Voraussetzung für die erfolgreiche Etablierung der betreffenden Angebote sein, der zusätzliche Nutzen für den Kunden basiert jedoch in der Regel auf speziellen Leistungsbestandteilen im Bereich der Kontextspezifität oder der Ubiquität solcher Angebote.

Die Sicherheit und der (seltener explizit hervorgehobene) Schutz der übertragenen Daten sind in allen Bereichen des Mobile Business eine unbedingt notwendige Voraussetzung für den Aufbau innovativer Applikationen. ${ }^{115}$ Für den Abschluss verbindlicher Rechtsgeschäfte muss nicht nur die Möglichkeit zur Authentifizierung von Personen,

\footnotetext{
${ }^{112}$ Vgl. Wiedmann/Buxel/Buckler (2000), S. 686; Mobilocity (2000), S. 4.

${ }^{113} \mathrm{Vgl}$. Gerpott (2001), S. 37f.

${ }^{114}$ Vgl. z.B. Schreiber (2000), S. 111ff; Huthmacher (2000), S. 14. Zobel (2001), S. $56 f$.

${ }^{115}$ Vgl. Pflug (2001), S. 21.
} 
sondern auch ein Schutz vor unerwünschten Zugriffen auf die übertragenen Daten und den Datenbestand gegeben sein. ${ }^{116}$ Die Übertragung personenbezogener oder sonstiger vertraulicher Daten durch mobile Netze unterliegt besonderen Risiken, die aus den Spezifika der Funktechnik resultieren. ${ }^{117}$ Anders als bei leitungsgebundener Kommunikation können die Signale nicht physikalisch gegen unbefugtes Mithören und unbefugte Aufzeichnung abgeschirmt werden. End-to-End-Sicherheit, Verschlüsselung sowie der Aufbau von Trust Centern und eine Public Key-Infrastruktur werden deshalb verstärkt an Bedeutung gewinnen. ${ }^{118}$ Obwohl das Leistungsmerkmal Sicherheit für die Etablierung innovativer Applikationen absolut unerlässlich ist, handelt es sich lediglich um eine (allerdings erfolgskritische) Nebenbedingung, die selbst keinen zusätzlichen Nutzen für die Kunden stiftet.

Einige Beiträge schreiben auch dem Bereich Remote Control einen eigenen Technologiewertbeitrag von Mobilfunknetzen der nächsten Generationen zu. ${ }^{119}$ Bisherige Konzepte zur drahtlosen Steuerung von Geräten funktionierten nur über geringe Entfernungen (z.B. über Infrarot-Lösungen). Bei der leitungsgebundenen Steuerung von Geräten über größere Entfernungen (z.B. der Fernwartung von Rechnern via Internet) muss sich das zu steuernde Gerät bisher an einem genau vorgegebenen Ort befinden. Diese technischen Einschränkungen entfallen, wenn die betreffenden Geräte mit Mobilfunkempfängern ausgestattet sind und durch den Benutzer über mobile Endgeräte angesteuert werden können. Damit werden neue Lösungen zur Steuerung von Haushaltsgeräten und technischen Anlagen interessant, bei denen sich der Benutzer zuküntig nicht mehr am gleichen Ort wie das Gerät aufhalten muss. Tatsächlich stellt der Bereich Remote Control ein Anwendungsfeld dar, in dem küntig ein echter zusätzlicher Kundennutzen zu erwarten ist. Dieser bezieht sich allerdings in erster Linie auf die private Nutzung, bei der kein Zusammenhang zum Informationsmanagement als betrieblichem Aufgabenfeld besteht. In den Fällen, in denen das Leistungsmerkmal Remote Control geschäftlich genutzt wird, betrifft es weniger betriebswirtschaftliche als rein technische Fragestellungen. Aus diesem Grund werden veränderte Möglichkeiten zur Steuerung von Geräten über größere Entfernungen im Folgenden nicht mehr näher betrachtet.

\subsubsection{Kernmerkmale für den Aufbau innovativer Anwendungen mit zusätzlichem Kundennutzen}

In diesem Abschnitt werden mit der allgegenwärtigen Verfügbarkeit und der kontextspezifischen Übermittlung von Daten die Kernmerkmale der nächsten Mobilfunkgenerationen näher erläutert. Durch die Möglichkeit zum Aufbau ubiquitärer und/oder kontextspezifischer Applikationen bieten sich originäre, bisher ungekannte Nutzungsmöglichkeiten, die die Erwartung, dass es zu einer Fortsetzung der Internet-Revolution kommen wird, gerechtfertigt erscheinen lassen. Da der potenzielle zusätzliche Nutzen für die Kunden in diesen Anwendungsfeldern als ausgesprochen hoch angesehen wird, ist hierin der Motor für das weitere Zusammenwachsen von mobiler und leitungsgebundener Informations- und Kommunikationstechnik zu erkennen.

\footnotetext{
${ }^{116}$ Vgl. Wiedmann/Buxel/Buckler (2000), S. 686.

${ }^{117}$ Vgl. Hülsmann/Mörs/Schaar (2001), S. 196.

${ }^{118}$ Vgl. Pflug (2001), S. 21.

${ }^{119}$ Vgl. Zobel (2001), S. 62f.; Scheer et al. (2001), S. 11.
} 


\section{a) Ubiquität}

Für die Benutzer von mobilen Endgeräten ist unabhängig von ihrem Aufenthaltsort jederzeit ein Netzzugang verfügbar. Tatsächlich ist der naheliegendste Wertbeitrag einer mobilen Technologie die durch deren Anwendung gewonnene Bewegungsfreiheit. ${ }^{120}$ Dabei handelt es sich allerdings um ein Leistungsmerkmal, das im Prinzip auch bei den Netzen der zweiten Mobilfunkgeneration gegeben ist. Es reicht daher nicht aus, bei der Untersuchung innovativer Anwendungspotenziale des Mobile Business allein am Aspekt der Ortsunabhängigkeit bzw. Mobilität anzuknüpfen, wie dies in einigen der in Abbildung 6/4 aufgeführten Beiträge geschieht. ${ }^{121}$ Entscheidend ist vielmehr das weiter abzugrenzende Merkmal der Ubiquität. Entsprechend der wörtlichen Übersetzung des Begriffs bezeichnet Ubiquität die Allgegenwärtigkeit von Informations- und Kommunikationssystemen. ${ }^{122}$ Diese schließt neben der Ortsunabhängigkeit die permanente Erreichbarkeit sowie weitere Teilaspekte, die im Folgenden erläutert werden, ein.

Im Vergleich zu den Netzen der zweiten Generation bezieht sich die Ortsunabhängigkeit nicht nur auf die Möglichkeit zur Durchführung von Telefongesprächen, sondern auch auf die breitbandige Übertragung von Daten. Das Internet verfugt in seiner bisherigen leitungsgebundenen Form nur über wenige Kontaktpunkte, an denen die Benutzer Zugang zu den internetspezifischen Leistungsangeboten erhalten können. Mit dem Rechner am Arbeitsplatz und dem heimischen Computer nutzt eine einzelne Person in der Regel nur zwei Kontaktpunkte, um eine Verbindung zum Internet aufzubauen. ${ }^{123}$ Viele zukünttige Nutzer von Mobile Business-Lösungen befinden sich dagegen typischerweise nicht an einem bestimmten Ort, sondern sie benutzen ihre Endgeräte an wechselnden Standorten oder während sie selbst in Bewegung sind (z.B. im Zug). ${ }^{124}$ Dies entspricht dem bereits angesprochenen, übergreifenden Trend zu mehr Mobilität in Wirtschaft und Gesellschaft. Mit den Endgeräten und Übertragungsstandards der nächsten Netzgenerationen wird den Benutzern die Möglichkeit gegeben, jederzeit und unabhängig von ihrem aktuellen Standort auf größere Datenbestände zuzugreifen und Transaktionen durchzuführen. ${ }^{125}$

Da die Nutzer ihr jeweiliges Endgerät stets mit sich führen, weisen Anwendungen des Mobile Business eine noch höhere Verfügbarkeit auf als bisherige Electronic Business-Lösungen und ermöglichen damit eine noch höhere Reaktionsgeschwindigkeit. ${ }^{126}$ Durch die Einführung der Paketvermittlung mit always-on-Funktionalität in den nächsten Netzgenerationen entfällt zudem die Zeit für den Aufbau von Verbindungen, die beim Netzzugang in der gegenwärtigen Form z.B. für das Starten des Rechners oder für das Einwählen in das mobile Internet benötigt wurde. Die allgegenwärtige Verfügbarkeit des Netzzugangs führt sowohl bei der unternehmungsextern orientierten Variante des Mobile Business als auch bei dessen unternehmungsintern orientierter Variante zu neuen Anwendungspotenzialen.

\footnotetext{
${ }^{120}$ Vgl. Scheer et al. (2001), S. 11.

${ }^{121}$ Für dieses Vorgehen vgl. z.B. Wiedmann/Buxel/Buckler (2000), S. 687; Schmitzer/Butterwegge (2000), S. 356.

${ }^{122}$ Vgl. Scheer et al. (2001), S. 11.

${ }^{123} \mathrm{Vgl}$. Scheer et al. (2001), S. 11.

${ }^{124} \mathrm{Vgl}$. Schmitzer/Butterwegge (2001), S. 356.

${ }^{125}$ Vgl. Wohlfahrt (2001), S. 50.

${ }^{126}$ Vgl. Schmitzer/Butterwegge (2000), S. 356.
} 
Im Falle des unternehmungsextern orientierten Mobile Commerce erfolgt ein Zugriff auf öffentliche Informationsangebote, die beispielsweise von Portalen oder von Content Providern offeriert werden. ${ }^{127}$ Das Leistungsmerkmal Ubiquität ermöglicht beim Mobile Commerce ein im Vergleich zum „traditionellen“ Electronic Commerce spontaneres Verhalten der Nachfrager. Damit werden die Voraussetzungen für impulsivere Transaktionen geschaffen, da für den Kunden keine Verschiebung von Käufen bis zum nächsten Netzzugang mehr erforderlich ist. ${ }^{128}$ Vor dem Hintergrund dieser Entwicklung kommt es zu einer steigenden Bedeutung von Nischenzeiten als „beschäftigungsbedingten Stillstandszeiten “129, die bisher weitgehend ungenutzt blieben. Die durchschnittliche Nutzungsdauer von i-mode-Diensten in Japan beträgt beispielsweise weniger als drei Minuten. ${ }^{130}$ Dementsprechend sind die Angebote so zu konzipieren, dass sie gerade bei kurzen Verweilzeiten sinnvoll genutzt werden können. ${ }^{131}$ Aber auch außerhalb der Nischenzeiten eröffnet der ubiquitäre Netzzugang das Potenzial zu einem tiefgreifenden Wandel des Kaufverhaltens. ${ }^{132} \mathrm{Da}$ die Kaufinteressenten sich nicht mehr an den Ort des Angebots bzw. des Netzzugangs begeben müssen, werden Preisvergleiche, die bereits durch die Verbreitung des Internet vereinfacht wurden, noch einmal wesentlich erleichtert. Der Kunde wird dabei in die Lage versetzt, während des Einkaufs vor Ort alternative Angebote zu überprüfen. Auch für den Sender im Kommunikationsprozess ergeben sich neue Aktionsmöglichkeiten. Es wird ohne Zeitverzögerung möglich, den Empfänger zu kontaktieren und diesen jederzeit und überall mit Echtzeit-Informationen $\mathrm{zu}$ versorgen. ${ }^{133}$ Im unternehmungsextern orientierten Bereich ist dies insbesondere für kostenpflichtige, push-basierte Informationsdienstleistungen sowie für die Übermittlung aktueller Angebotsinformationen interessant.

Auch im unternehmungsintern orientierten Bereich des Mobile Work ergeben sich durch die Ubiquität des Netzzugangs als innovativem Leistungsmerkmal der nächsten Mobilfunkgenerationen neue Anwendungsfelder. Bei dieser Erscheinungsform des Mobile Business erfolgt über entsprechende Gateways ein Zugriff auf die in den unternehmungseigenen Informations- und Kommunikationssystemen vorgehaltenen Datenbestände. Wenn das mobile Endgerät always-on mit dem Mobilfunknetz verbunden ist, kann eine automatische Synchronisierung zwischen dem Endgerät und den sonstigen Systemen der Unternehmung vorgenommen werden. ${ }^{134}$ Dies betriff z.B. den Abgleich von E-Mails, Kalendereinträgen u.ä. Die mobil arbeitenden Beschäftigten werden dadurch stets über neueste Entwicklungen benachrichtigt. Es wird außerdem möglich, einen mobilen Zugang $\mathrm{zu}$ den bisher nur leitungsgebunden genutzten betriebswirtschaftlichen Anwendungssystemen einzurichten. Unabhängig vom aktuellen Aufenthaltsort der Mitarbeiter können damit nicht nur Transaktionen vorgenommen werden (z.B. Erfassung neuer Kundenaufträge), sondern es werden auch aktuelle Statusinformationen (z.B. Bearbeitungsstand einzelner Aufträge) und Auswertungen

\footnotetext{
${ }^{127}$ Zur Rolle von Portalen und Content Providern in der Wertschöpfungskette des Mobile Business vgl. Abschnitt 6.2.1.

${ }^{128} \mathrm{Vgl}$. Zobel (2001), S. 45ff.

${ }^{129}$ Wiedmann/Buxel/Buckler (2000), S. $686 \mathrm{f}$.

${ }^{130} \mathrm{Vgl}$. Mizukoshi/Okino/Tardy (2000), S. lf.

${ }^{131}$ In Japan waren beispielsweise 30-Sekunden-Spiele sehr erfolgreich, mit denen die Nutzer spontan kurze Wartezeiten aller Art überbrücken konnten. Vgl. Muzukoshi/Okino/Tardy (2000), S. 2.

${ }^{132} \mathrm{Vgl}$. Zobel (2001), S. 44ff.

${ }^{133}$ Vgl. Wohlfahrt (2001), S. 50 f.

${ }^{134}$ Vgl. Pflug (2001), S. $22 \mathrm{f}$.
} 
von analytischen Informationssystemen (z.B. Kundenanalysen) ubiquitär verfügbar. Diese Überlegungen machen deutlich, dass gerade der unternehmungsintern orientierte Bereich des Mobile Business eine weitere Konvergenz leitungsgebundener und mobiler Techniken erfordert, da für die Realisierung der angesprochenen Anwendungen eine möglichst umfassende Kompatibilität der verschiedenen Systeme gegeben sein muss.

\section{b) Kontextspezifität}

Die Einführung der nächsten Mobilfunkgenerationen hebt technische Limitationen, die bislang in vielen Fällen einer noch stärkeren Individualisierung von Leistungsangeboten entgegen standen, auf. Die Ubiquität des Netzzugangs führt dazu, dass der Benutzer künftig in unterschiedlichsten Situationen oder Umgebungen in der Lage ist, mit dem Endgerät Transaktionen zu tätigen. Dadurch können Leistungen offeriert werden, die zu seiner Person, seiner gesellschaftlichen Situation, seinem aktuellen Aufenthaltsort und den dort herrschenden Verhältnissen sowie zu zeitlichen Gegebenheiten passen. ${ }^{135}$ Dieses Leistungsmerkmal, das als Kontextspezifität bezeichnet wird, ${ }^{136}$ macht es möglich, den Nutzer permanent zu begleiten und ihm in Abhängigkeit von der aktuellen Situation spezifische Optionen zur Auswahl anzubieten. Zum Aufbau kontextspezifischer Anwendungen ist es dabei notwendig, das Umfeld des Benutzers zu erfassen und auszuwerten, um die für den Benutzer relevanten Dienste einzugrenzen und aktiv anzubieten. ${ }^{137}$ Davon ausgehend kann sich eine individuelle Angebotsanpassung auf örtliche, zeitliche und/oder persönliche Gesichtspunkte stützen. ${ }^{138}$ Das Merkmal Kontextspezifität ist insgesamt eher für den unternehmungsextern orientierten Bereich, also für den Aufbau von Anwendungen des Mobile Commerce interessant. Innovationspotenziale für den unternehmungsintern orientierten Bereich basieren tendenziell eher auf dem Kernmerkmal Ubiquität.

Die Möglichkeit zur Bezugnahme auf den ortsbezogenen Kontext basiert auf verbesserten Methoden zur Lokalisierung der Nutzer. Bei der Ortsbestimmung in den GSM-Netzen der zweiten Mobilfunkgeneration wird ohne Endgeräteunterstützung mit Hilfe des sogenannten Cell-of-Origin-Verfahrens (COO) lediglich eine Genauigkeit von mehreren hundert Metern (ca. $300 \mathrm{~m}$ ) erreicht. ${ }^{139}$ Zukünftig wird die Lokalisierung indessen bis auf weniger als 30 Meter möglich sein, ${ }^{140}$ wobei in technischer Hinsicht drei Verfahren miteinander konkurrieren. ${ }^{141}$ Die genauesten Ergebnisse liefert derzeit die Ortsbestimmung mit Hilfe des Global Positioning Systems (GPS), für dessen Einsatz die Endgeräte allerdings mit entsprechenden GPS-Empfängern ausgestattet sein müssen. Die beiden anderen Verfahren stützen sich dagegen allein auf Signale innerhalb des jeweiligen Mobilfunknetzes. Bei E-OTD (Enhanced Observed Time Difference)

\footnotetext{
${ }^{135}$ Vgl. ăhnlich Borowicz/Scherm (2000), S. 473.

${ }^{136}$ Als Synonym für „Kontextspezifität" wird zum Teil auch der Begriff „Kontextsensitivităt“ verwendet. Vgl. z.B. Scheer et al. (2001), S. 11. Zwischen diesen beiden Begriffsvarianten wird im Folgenden nicht unterschieden.

${ }^{137} \mathrm{Vgl}$. Scheer et al. (2001), S. 11.

${ }^{138}$ In einigen Beitrăgen wird der aktionsbezogene Kontext als vierte Bezugsgröße genannt (z.B. Tanken, Einkaufen, Tennis spielen u.ä.). Vgl. z.B. Zobel (2001), S. 51; Scheer et al. (2001), s. 11. Dieser stellt jedoch genau genommen keine eigene Kategorie dar, da aktionsbezogene Angebote von durch den Anbieter erfassbaren örtlichen, zeitlichen und persönlichen Einflussgrößen ausgehen müssen. So werden etwa mit bestimmten Orten spezielle Aktivitäten verknüpft.

${ }^{139} \mathrm{Vgl}$. Gerpott/Böhm (2001), S. 6; Wohlfahrt (2001), S. 50.

${ }^{140} \mathrm{Vgl}$. Kamp/Velthuis (2000), S. 3.

${ }^{141}$ Vgl. dazu Zobel (2001), S. 268ff.; Wohlfahrt (2001), S. 50.
} 
werden die relativen Laufzeiten von Funksignalen zwischen nahe gelegenen Basisstationen und dem Endgerät gemessen und miteinander verglichen. Dies ist relativ aufwändig und erfordert ebenfalls eine zusätzliche Ausstattung der Endgeräte. Die Triangulationsmethode kommt ohne Aufrüstung der Endgeräte aus und beruht darauf, dass die Endgeräte in der Regel mit mehreren Basisstationen in Kontakt sind. Mit Hilfe der an den verschiedenen Stationen empfangenen Signalstärke kann die Position des Endgeräts ermittelt werden.

Das Nutzungspotenzial der Verfahren zur Positionsbestimmung, die die Grundlage zur Erstellung ortsspezifischer Dienste, sogenannter Location Based Services, darstellen, kann kaum überschätzt werden. Wenn der Aufenthaltsort der Endgeräteinhaber bekannt ist, dann sind Konzepte vorstellbar, die vom Aufenthaltsort auf die wahrscheinlichen aktuellen Bedürfnisse des Kunden schließen und entsprechende Lösungsangebote vorschlagen. In der Literatur werden zahlreiche Anwendungsbeispiele der Lokalisierungstechnik von Navigationshilfen über Standort-Verfolgung, lokale Reiseführer bis hin zu ortsspezifischen Shopping-Assistenten genannt. ${ }^{142}$ Auch im Bereich der Werbung entstehen neue Möglichkeiten zur präzisen Fokussierung von Zielgruppen. Dabei ist beispielsweise an Produktinformationen zu denken, die nur an solche Personen versendet werden, die sich augenblicklich in der Nähe befinden und das Angebot tatsächlich nutzen können. ${ }^{143}$

Unter Bezugnahme auf den zeitspezifischen Kontext können Leistungen offeriert werden, die sich auf den aktuellen Zeitpunkt oder den Zeitraum beziehen. ${ }^{144}$ Wenn die entsprechende Bezugnahme sich an größeren Zeiträumen, z.B. Jahreszeiten oder Monaten orientiert, liegt allerdings kein echter Fortschritt gegenüber den bereits existierenden technischen Möglichkeiten vor. Innovativ sind vielmehr solche Angebote, die tagesaktuell konzipiert oder sogar auf spezielle Tageszeiten abgestimmt sind. Hierzu könnten der zeitliche und der lokale Kontext miteinander verknüpft werden, indem beispielsweise eine dynamische Zuordnung von Inhalten zu bestimmten Orten vorgenommen wird. Auf dieser Basis lassen sich etwa Veranstaltungen ankündigen oder potenzielle Kunden über kurzfristig gültige Sonderangebote informieren. Durch die ubiquitäre Erreichbarkeit der Endgeräteinhaber wird dabei eine verzögerungslose Bekanntmachung der betreffenden Angebote realisiert.

Ein weiterer Aspekt, an dem kontextspezifische Maßnahmen ansetzen können, betrifft die persönlichen Interessen der Nachfrager. Angesichts der auch zukünftig relativ hohen Verbindungskosten und der gegenüber stationären Rechnern eingeschränkten Darstellungs- und Interaktionsmöglichkeiten mobiler Endgeräte, kommt es entscheidend darauf an, das Angebot möglichst personenspezifisch zu konzipieren und insbesondere auch zu präsentieren. ${ }^{145}$ Zum einen sollten Inhalte, die für den einzelnen Kunden nicht interessant sind, so weit wie möglich vermieden werden, etwa durch spezielle Voreinstellungen oder durch aktive Filterfunktionen. Zum anderen können Angebote entwickelt werden, die individuell auf die Präferenzen der verschiedenen Kunden ausgerichtet sind. Dabei ist von Vorteil, dass es sich bei mobilen Endgeräten in der Regel um Gebrauchsgegenstände handelt, die nicht zwischen mehreren Benutzern

\footnotetext{
${ }^{142}$ Vgl. z.B. Gerpott (2001), S. 38; Zobel (2001), S. 50ff.; Schmitzer/Butterwegge (2000), S. 356.

${ }^{143} \mathrm{Vgl}$. ähnlich Wiedmann/Buxel/Buckler (2000), S. 687.

${ }^{144} \mathrm{Vgl}$. Zobel (2001), S. 51; Scheer et al. (2001), S. 11.

${ }^{145}$ Vgl. Lai/Mohan/Gustafson, (2000), S. 1. Schmitzer/Butterwegge (2000), S. 356.
} 
geteilt werden. ${ }^{146}$ Verglichen mit dem Electronic Business, bei dem stationäre Rechner bzw. IP-Adressen nicht selten von wechselnden Personen benutzt werden, sodass eine zuverlässige Identifikation die aktive Mitarbeit der Nutzer erfordert, ist deshalb eine erheblich bessere Erkennung der Kunden an Hand der Mobilfunknummer möglich. ${ }^{147} \mathrm{Je}$ stärker die Individualisierung des Angebots ausgeprägt ist, um so höhere Anforderungen bestehen an die Kenntnis der spezifischen Kundeninteressen auf der Seite des Anbieters. Durch die Personalisierung im Bereich des Mobile Commerce wächst also die Bedeutung des Customer Relationship Management, das mit dem mobilen Kanal zum Kunden eine neue Dimension erhält. ${ }^{148}$

Bei der Konzipierung kontextspezifischer Angebote können die erläuterten örtlichen, zeitlichen und interessenbezogenen Ansatzpunkte miteinander kombiniert werden, um eine noch stärkere Individualisierung der Leistung zu erreichen. Dazu müssen die verwendeten Anwendungssysteme in der Lage sein, die verschiedenen Kontextebenen miteinander zu verknüpfen, um für den individuellen Nutzer das jeweils passende Angebot zu generieren. ${ }^{149}$

Es sei darauf hingewiesen, dass vor der erfolgreichen Etablierung kontextspezifischer Anwendungen mit größeren Kundenkreisen noch erhebliche Akzeptanzprobleme zu überwinden sind. Dies hängt insbesondere damit zusammen, dass zum einen die Vorteile dieser Konzepte den Kunden noch weitgehend unbekannt sind und zum anderen in Bezug auf den Aufbau umfangreicher Kundendatenbanken als Grundlage kontextspezifischer Angebote Befurchtungen hinsichtlich der Verletzung der Privatsphäre bestehen. ${ }^{150}$ Erst wenn die Kunden von dem erzielbaren innovativen Nutzen überzeugt sind und ein ausreichendes $\mathrm{Ma} ß$ an Vertrauen aufgebaut wurde, kann es zu einer umfassenden Verbreitung kontextspezifischer Anwendungen kommen.

\subsection{Berührungspunkte zwischen Mobile Business und Informations- management}

Die im vorangegangenen Abschnitt vorgenommene Bestandsaufnahme verdeutlicht, dass die zu erwartende Verbreitung des Mobile Business eine übergreifende Entwicklung mit hoher Bedeutung fur die gesamte Unternehmung darstellt. Der Aufbau von mobilen Anwendungen und die Ansprache von Kunden über mobile Kanäle betreffen verschiedene Funktionsbereiche (z.B. Marketing, Logistik, Informationswirtschaft) und sollten folglich aus unterschiedlichen Perspektiven untersucht werden. ${ }^{151}$ Die Ausführungen in diesem Abschnitt analysieren die Berührungspunkte zwischen dem Mobile Business und dem im Mittelpunkt dieser Arbeit stehenden Informationsmanagement als speziellem Teilgebiet der Unternehmungsfuhrung. Dabei stehen insbesondere die

\footnotetext{
${ }^{146}$ Vgl. Lai/Mohan/Gustafson, (2000), S. 1; Garcia/Petit/Mialaret (2000), S. 6.

${ }^{147} \mathrm{Vgl}$. Wohlfahrt (2001), S. 51.

${ }^{148}$ Vgl. ähnlich Kamp/Velthuis (2000), S. 4.

${ }^{149}$ Vgl. Zobel (2001), S. 53.

${ }^{150}$ Vgl. Wiedmann/Buxel/Buckler (2000), S. 687.

${ }^{151}$ Dies spiegelt sich auch in den unterschiedlichen Lösungen bei der organisatorischen Zuordnung von Zuständigkeiten in der Praxis wider. Laut einer Studie der Unternehmensberatung Arthur D. Little, an der sich 344 Unternehmen beteiligten, liegt die Verantwortung für Mobile Commerce in $37 \%$ der Fälle bei der Geschäftsführung, in $26 \%$ beim Marketing und nur in ca. $9 \%$ der Fälle ist der Bereich IT/Informationsmanagement zuständig. Vgl. Arthur D. Little (2000), S. 19.
} 
Auswirkungen im Vordergrund, die sich aus der Konvergenz von leitungsgebundener und mobiler Informations- und Kommunikationstechnik ergeben. Die Konvergenzentwicklung führt dazu, dass die Einführung von Mobile Business-Anwendungen nicht isoliert $\mathrm{zu}$ sehen ist, sondern auch bisherige Lösungen im leitungsgebundenen Bereich betrifft und dort zum Teil Modifikationen erforderlich macht.

Im nachfolgenden Abschnitt 6.2.1 werden zunächst idealtypisch die Wertschöpfungsaktivitäten im Bereich des Mobile Business und die beteiligten Akteure betrachtet. Dies dient zur Abgrenzung des weiteren Untersuchungsfelds und zur Identifikation von Schnittstellen, die durch das Informationsmanagement beim Aufbau von Anwendungssystemen des Mobile Business zu berücksichtigen sind. Die anschließenden Überlegungen nehmen die Perspektive der Anwender ein. Abschnitt 6.2.2 erläutert die Gestaltungsfelder des Informationsmanagements beim Aufbau von unternehmungsextern orientierten Mobile Commerce-Anwendungen. In Abschnitt 6.2.3 schließlich werden die Gestaltungsfelder des Informationsmanagements beim Aufbau von unternehmungsintern orientierten Mobile Work-Anwendungen betrachtet.

\subsubsection{Wertschöpfungsaktivitäten und Akteure des Mobile Business}

Trotz der zum Teil exorbitanten Investitionen in die Netzinfrastruktur gibt es noch keine vollkommene Transparenz bezüglich möglicher Wettbewerbsrollen, Geschäftsmodelle und Erfolgsfaktoren der nächsten Mobilfunkgenerationen. ${ }^{152}$ Die bisherige Marktsituation bei der Nutzung der zweiten Netzgeneration war dadurch gekennzeichnet, dass die Netzbetreiber hauptsächlich darauf bedacht waren, ihre Netzabdeckung auszubauen, Neukunden zu akquirieren und die Geschäftsprozesse für die Sprachübermittlung zu optimieren. ${ }^{153}$ Ein Teil der Netzvermarktung erfolgte unterdessen durch sogenannte Service-Provider, die nicht über ein eigenes Funknetz verfügten, sondern als virtuelle Netzbetreiber auftraten. Den Netzbetreibern vorgelagert war die Wertschöpfungsstufe der Infrastrukturanbieter, die den Netzbetreibern die notwendige technische Ausstattung lieferten. Abbildung 6/6 zeigt die entsprechende Wertschöpfungskette, an der lediglich eine überschaubare Anzahl von Akteuren beteiligt war.

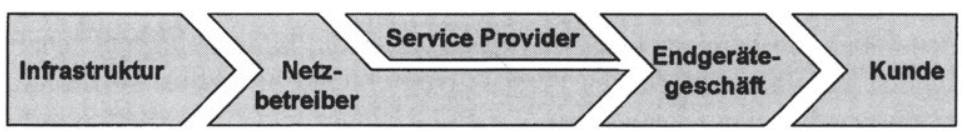

Abb. 6/6: Wertschöpfungskette der zweiten Mobilfunkgeneration Quelle: in Anlehnung an Gentner/Legler (2000), S. 16.

Im Gegensatz zu der dargestellten, relativ klar definierten Rollenverteilung, müssen sich die Marktstrukturen für die nächsten Netzgenerationen erst noch herausbilden. Da die Einführung der breitbandigen mobilen Datenübertragung die Anwendungsmöglichkeiten der Mobilfunktechnik erheblich erweitert, ist damit zu rechnen, dass die Zahl der am Wertschöpfungsprozess beteiligten Akteure insgesamt steigen wird. Die nächsten

${ }^{152} \mathrm{Vgl}$. Laartz/Hjartar (2000), S. 29.

${ }^{153}$ Vgl. Gentner/Legler (2000), S. 15. 
Mobilfunkgenerationen eröffnen neue Geschäftsoptionen für Portalbetreiber, Content Provider und Anwender des Mobile Business. Häufig wird deshalb vorgeschlagen, die Wertschöpfungskette der zweiten Mobilfunkgeneration zu modifizieren und in erweiterter Form als Wertschöpfungskette des Mobile Business bzw. der nachfolgenden Mobilfunkgenerationen aufzufassen. ${ }^{154}$ Abbildung 6/7 zeigt zwei Möglichkeiten für eine Konfiguration der in diesem Sinne veränderten Wertschöpfungskette.

a)

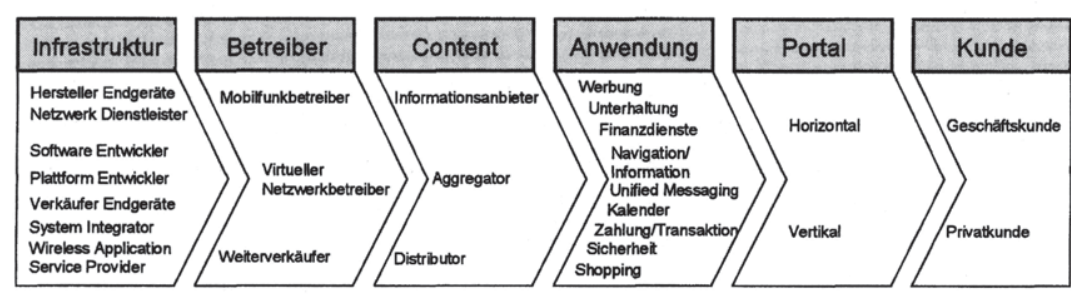

b)

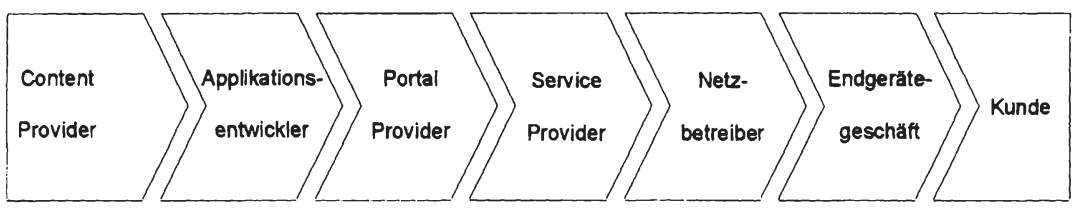

Abb. 6/7: Mögliche Wertschöpfungsketten im Mobile Business

Quelle: a) Zobel (2001), S. 122.

Quelle: b) Gentner/Legler (2000), S. 17.

Die neu hinzukommenden Akteure werden in den in Abbildung 6/7 dargestellten Vorschlägen an unterschiedlichen Stellen in der Wertschöpfungskette, die sich wegen der steigenden Komplexität des Geschäfts insgesamt verlängert, eingeordnet. Es ist allerdings zu erwarten, dass die Grenzen zwischen den verschiedenen Wertschöpfungsstufen immer mehr verschwinden und die Betätigungsfelder der jeweiligen Beteiligten mehr und mehr ineinander übergehen. So sind beispielsweise die Content Provider gefordert, auch portaltypische Angebote zu offerieren, während es furr die Netzbetreiber sinnvoll erscheint, ihre Aktivitäten auch auf das Anwendungsgeschäft auszudehnen. Darüber hinaus sind nicht stets alle Stufen der in der Abbildung dargestellten Wertschöpfungsketten an der Erstellung einer bestimmten Leistung beteiligt. Vor diesem Hintergrund erscheint es problematisch, bei der Untersuchung des vielschichtigen Mobile Business-Umfelds überhaupt mit einer derartigen Darstellung mit Anspruch auf Allgemeingültigkeit zu arbeiten.

Eine Alternative liegt in der Konzipierung eines flexibler verwendbaren Bezugsrahmens, der unterschiedliche Betätigungsfelder umfasst, in denen die verschiedenen Akteure im Rahmen des Mobile Business Leistungen erbringen und zur Wertschöpfung beitragen können. Ein derartiger Bezugsrahmen ermöglicht den Akteuren eine bessere Orientierung, indem er Anhaltspunkte zur Entwicklung von Strategien und zur Abgren-

${ }^{154}$ Vgl. z.B. Gentner/Legler (2000), S. 17; Scheer et al. (2001), S. 8; Zobel (2001), S. 122. 
zung geschäftlicher Aktivitäten bietet. ${ }^{155}$ Dabei ist es nicht notwendig, dass die Unternehmungen ihre jeweiligen Handlungsfelder auf einzelne Teilbereiche des Bezugsrahmens beschränken. Vielmehr ist es einer einzelnen Unternehmung möglich, mehrere Ebenen des Bezugsrahmens anzusprechen und auf den betreffenden Gebieten Leistungen anzubieten. Andererseits ist es jedoch auch nicht erforderlich, dass alle Leistungen von einem einzelnen Akteur selbst erbracht werden, da auf externe Anbieter, die mit ihrem Geschäft andere Ebenen des Bezugsrahmens adressieren, zurückgegriffen werden kann. ${ }^{156}$ Der Sinn der Verwendung eines solchen Bezugsrahmens liegt also in der klaren Positionierung der eigenen Unternehmung gegenüber den Kunden und gegenüber den Geschäftsfeldern anderer Unternehmungen. Abbildung 6/8 enthält den hier gewählten Bezugsrahmen zur Einordnung von Mobile Business-Aktivitäten, der mit der Art der Leistung und der Art des Leistungsnehmers zwei Analysedimensionen enthält. Es werden die verschiedenen Merkmalsausprägungen in den beiden Analysedimensionen aufgeführt und jeweils Beispiele gegeben.

\begin{tabular}{|c|c|c|c|c|}
\hline & \multirow{3}{*}{$\begin{array}{l}\text { Mobile } \\
\text { Business- } \\
\text { Anbieter } \\
\text { Vorleistung) }\end{array}$} & \multicolumn{3}{|c|}{ Mobile Business-Nachfrager } \\
\hline & & \multicolumn{2}{|c|}{$\begin{array}{l}\text { Unternehmungsexterne Nutzung } \\
\text { (Mobile Commerce) }\end{array}$} & \multirow{2}{*}{$\begin{array}{c}\text { Unternehmungs- } \\
\text { interne Nutzung } \\
\text { (Mobile Work) }\end{array}$} \\
\hline & & Geschäftskunde | & Privatkunde & \\
\hline Infrastruktur & $\begin{array}{l}\text { Aufbau von Basis- } \\
\text { stationen }\end{array}$ & - & - & $\begin{array}{l}\text { WAN-Verbindung zu } \\
\text { Netzbetreiber }\end{array}$ \\
\hline Netzbetrieb & $\begin{array}{l}\text { Netzbetrieb für } \\
\text { Service Provider }\end{array}$ & $\begin{array}{l}\text { Netzbetrieb für } \\
\text { Geschäftskunden }\end{array}$ & $\begin{array}{l}\text { Service Providing für } \\
\text { Privatperson }\end{array}$ & Carrier-Leistungen \\
\hline Endgerăte & Smart Phone zum & $\begin{array}{l}\text { PDÄ fur geschäft- } \\
\text { lichen Nutzer }\end{array}$ & Smart Phone für & $\begin{array}{l}\text { Pocket } \mathrm{PC} \text { für inter- } \\
\text { nen Einsatz }\end{array}$ \\
\hline Portal & $\begin{array}{l}\text { Portalbetrieb für } \\
\text { Netzanbieter }\end{array}$ & $\begin{array}{l}\text { Finanzportal zur ge- } \\
\text { schäftlichen Nutzung }\end{array}$ & $\begin{array}{l}\text { Spieleportal zur } \\
\text { privaten Nutzung }\end{array}$ & $\begin{array}{l}\text { Multi Access-Portal } \\
\text { fur Mitarbelter }\end{array}$ \\
\hline Conten & $\begin{array}{l}\text { Zulieferung an } \\
\text { Portalbetreiber }\end{array}$ & $\begin{array}{l}\text { WAP-basierte Börsen- } \\
\text { informationen }\end{array}$ & $\begin{array}{l}\text { WAP-basierte Sport- } \\
\text { informationen }\end{array}$ & $\begin{array}{l}\text { Bereitsteillung von } \\
\text { Intranet-Inhalten }\end{array}$ \\
\hline Anwendung & $\begin{array}{l}\text { Location Based } \\
\text { Service fur Portal }\end{array}$ & $\begin{array}{l}\text { Mobile Buchung von } \\
\text { Flugtickets }\end{array}$ & $\begin{array}{l}\text { Information aber aktu- } \\
\text { elle lokale Angebote }\end{array}$ & Mobiler ERP-Zugriff \\
\hline
\end{tabular}

Abb. 6/8: Bezugsrahmen des Mobile Business und Beispiele

Abbildung 6/8 zeigt, dass es sich bei dem Leistungsnehmer entweder um einen echten Endkunden (Mobile Business-Nachfrager) oder um eine Unternehmung handeln kann, die selbst als Anbieter im Bereich des Mobile Business tätig ist, und die Leistung als Vorleistung zur Erstellung ihres eigenen Angebots verwendet. Der zuletzt genannte Fall betriff brancheninterne Transaktionen zur Zusammenstellung integrierter Leistungsangebote an die Benutzer mobiler Endgeräte, die beispielsweise von Portalen offeriert werden. Der hier vorgestellte Bezugsrahmen kann auch Leistungen beinhalten, die durch den Leistungsnehmer selbst erstellt werden. So kann die Leistung beispielsweise bei Aktivitäten im Bereich des Mobile Work prinzipiell entweder durch die leistungsnehmende Unternehmung intern erbracht oder von einem externen Anbieter bezogen werden.

Die nachfolgenden Überlegungen in diesem Kapitel beschränken sich auf bestimmte Teilbereiche des hier verwendeten Bezugsrahmens. Diese sind in Abbildung 6/8 optisch hervorgehoben. Brancheninterne Transaktionen mit Mobile Business-Anbietern als Leistungsnehmern werden aus der weiteren Untersuchung ausgeschlossen. Dies entspricht dem Gesamtkonzept der Arbeit, da die in den vorangegangenen Kapiteln ent-

${ }^{155}$ Vgl. Varshney/Vetter/Kalakota (2000), Mobile Commerce Framework.
${ }^{156}$ Vgl. Varshney/Vetter/Kalakota (2000), Mobile Commerce Framework. 
wickelten Ansätze für Theorien des Informationsmanagements so angelegt sind, dass die Aussagen unabhängig von einer bestimmten Art des Geschäfts der zu betrachtenden Unternehmung sind. In gleicher Weise werden auch Leistungsarten nicht mehr weiter in die Betrachtung einbezogen, die insbesondere auf der Anbieterseite hohen Branchenbezug besitzen (Infrastruktur, Endgeräte, Netzbetrieb, Portalgeschäft, Content Providing). Die hier entwickelten Theorieansätze sind nicht speziell genug, um in diesem Zusammenhang auftretende, leistungsspezifische Problemstellungen zu analysieren.

Stattdessen konzentrieren sich die nachfolgenden Untersuchungen auf den Bereich der Anwendungen. Diese Leistungsart kann in so vielgestaltiger Form auftreten, dass kein eindeutiger Branchenbezug vorliegt. Durch die Entwicklung von ubiquitären und/oder kontextspezifischen Mobile Business-Anwendungen können Unternehmungen ihre bisherige Geschäftstätigkeit ergänzen oder ganz neue Geschäftsmodelle realisieren. Die weiteren Überlegungen in diesem Kapitel untersuchen die Frage, wie das Informationsmanagement diese Zielsetzung (unabhängig vom konkret vorliegenden Geschäftstyp) durch die Anpassung bestehender und die Bereitstellung neuer Informations- und Kommunikationssysteme unterstützen kann. Bei der unternehmungsinternen Variante des Mobile Business sind die Leistungsarten Content Providing und Portal in die Überlegungen einzubeziehen, für die beim Aufbau von Mobile Work-Anwendungen ebenfalls Lösungen unter Zuständigkeit des Informationsmanagements zu entwickeln sind. Aus diesem Grund sind in Abbildung 6/8 bei der unternehmungsinternen Nutzung auch die Bereiche Content Providing und Portal leicht hervorgehoben.

\subsubsection{Gestaltungsfelder des Informationsmanagements beim Aufbau von Mobile Commerce-Anwendungen}

In diesem Abschnitt werden die Gestaltungsmöglichkeiten des Informationsmanagement beim Aufbau von Anwendungen im Bereich des unternehmungsextern orientierten Mobile Commerce betrachtet. In Theorie und Praxis besteht große Einigkeit darüber, dass die unreflektierte Übertragung von Erkenntnissen des Electronic Commerce auf den Bereich des Mobile Commerce nur wenig Aussicht auf Erfolg besitzt. ${ }^{157}$ Die Anwendungsmöglichkeiten von Electronic Commerce und Mobile Commerce unterscheiden sich so sehr, dass Erfolgsmodelle des E-Commerce bei der Einführung des M-Commerce nicht einfach kopiert werden können. ${ }^{158}$ Wie in Abschnitt 6.1 .4 verdeutlicht, reichen die Nutzungspotenziale der mobilen Datenübertragung weit über einen ortsunabhängigen Zugang zum traditionellen Internet hinaus. Folglich geht es für die Unternehmungen um mehr als nur um eine Ausweitung ihrer bisherigen WebPräsenz. ${ }^{159}$ Für die Zwecke des Informationsmanagements erscheint es deshalb sinnvoll, Mobile Commerce als eigenständigen Gestaltungsbereich neben dem Electronic Commerce aufzufassen. Dieser Gestaltungsbereich ermöglicht zum einen vollkommen neue Anwendungen und besitzt zum anderen das Potenzial, bestehende Lösungen im Bereich des E-Commerce teilweise zu substituieren. ${ }^{160}$

\footnotetext{
${ }^{157} \mathrm{Vgl}$. Borowicz/Scherm (2000), S. 469.

${ }^{158} \mathrm{Vgl}$. Dean/Ketterer/Thiel (2000), S. 34.

${ }^{159} \mathrm{Vgl}$. Mobilocity (2000), S. 3.

${ }^{160} \mathrm{Vgl}$. ähnlich Zobel (2001), S. 3f.
} 
Bei der allgemeinen Darstellung innovativer Anwendungspotenziale des Mobile Business in Abschnitt 6.1.4 wurde bereits erläutert, dass ein originärer Kundennutzen durch Anknüpfung an die Kernmerkmale Ubiquität und Kontextspezifität erreicht werden kann. Die folgenden Überlegungen untersuchen deshalb, welche Anforderungen sich aus diesen Leistungsmerkmalen der mobilen Datenübertragung an Gestaltungsmaßnahmen des Informationsmanagements ergeben. Es wird überprüft, inwiefern das Informationsmanagement die Erstellung ubiquitärer und kontextspezifischer Angebote unterstützen kann. Die Abschnitte 6.2.2.1, 6.2.2.2 und 6.2.2.3 befassen sich mit den verschiedenen Arten von Kontext (ortsbezogen, zeitbezogen und interessenbezogen), während Abschnitt 6.2.2.4 auf die Erstellung ubiquitärer Angebote eingeht.

Für die anwendenden Unternehmungen stellt die Einführung mobiler Applikationen in der Regel den nächsten Schritt nach der Realisierung von Lösungen im Bereich des leitungsgebundenen Electronic Commerce dar. In Ausnahmefällen sind allerdings auch Geschäftsmodelle vorstellbar, die entweder ausschließlich auf die mobile Verbindung zum Nachfrager der Leistung setzen oder die das traditionelle Leistungsangebot um einen mobilen Kanal zum Kunden ergänzen, ohne bisher Konzepte des Electronic Commerce adaptiert zu haben. ${ }^{161}$ Die nachfolgenden Überlegungen konzentrieren sich insbesondere auf den zuerst genannten Fall, in dem die Unternehmungen bereits über eine bestehende Systemarchitektur mit elektronischer Schnittstelle zum Kunden über das leitungsgebundene Internet verfügen. Auf diese Architektur können und müssen neu entwickelte Anwendungskonzepte des Mobile Commerce aufbauen.

\subsubsection{Unterstützung ortsbezogener Leistungsangebote durch das Informations- management}

Mit der Technik der nächsten Mobilfunkgenerationen wird, wie gesehen, eine im Vergleich zu bisherigen Möglichkeiten weitaus präzisere Bestimmung des Aufenthaltsorts von mobilen Endgeräten und damit auch ihrer Benutzer möglich. Folglich bietet sich die Gelegenheit, dem Kunden spezifische Leistungen zu offerieren, die besonders auf seinen aktuellen ortsbezogenen Kontext abgestimmt sind. Die praktische Umsetzung dieses allgemeinen Anwendungspotenzials ist jedoch an zwei Voraussetzungen gebunden: Die Unternehmung muss zum einen Kenntnis über den aktuellen Aufenthaltsort besitzen und sie muss zum anderen in der Lage sein, ihr Angebot in Abhängigkeit von dieser Lokalisierungsinformation $\mathrm{zu}$ variieren und individuell $\mathrm{zu}$ konfigurieren.

Die Lokalisierungsinformationen befinden sich in der Regel zunächst einmal im Besitz des Netzbetreibers, in dessen Netz die mobilen Endgeräte eingebucht sind. Grundsätzlich sind mehrere Alternativen vorstellbar, wie diese Informationen für die Anwender verfügbar gemacht werden können. Dabei ist es nicht unbedingt erforderlich, dass die betreffenden Daten tatsächlich an die Anwender übergeben werden. Eine Lösung, bei der die Lokalisierungsinformationen nicht an die Leistungsanbieter übermittelt werden, besteht im Aufbau zentralisierter Datenbanken, die ortsbezogene Angebote bündeln und gegenüber den Benutzern der mobilen Endgeräte als Intermediär fungieren. Derartige Datenbanken bieten den Nachfragern einen einheitlichen Zugang

${ }^{161}$ Vgl. Schmitzer/Butterwegge (2001), S. 356f. 
zum Angebot von Location Based Services. Sie sind als offene Systeme zu entwerfen, in die die Anwender ihre jeweiligen Leistungsangebote eintragen und jederzeit ändern können. Abbildung 6/9 zeigt den prinzipiellen Aufbau dieser Lösung:

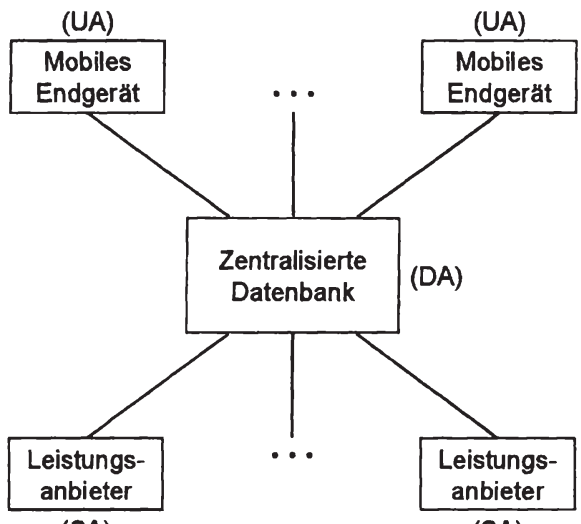

(SA)

(SA)

Abb. 6/9: Zentralisierte Datenbank für ortsbezogene Leistungsangebote

Das Service Location Protocol (SLP), das von der Service Location Protocol Working Group, einer Arbeitsgruppe der Internet Engineering Task Force (IETF) entwickelt wurde und derzeit im Rahmen des OpenSLP-Projekts weiterentwickelt wird, strebt eine Umsetzung des in Abbildung 6/9 dargestellten Konzepts auf der Basis von Internet-Standards an. Dieses Protokoll ist sprachunabhängig und stellt daher keine speziellen Anforderungen an die hard- und softwaretechnische Ausstattung der zu verbindenden Akteure. ${ }^{162}$ Mit dem User Agent (in der Abbildung abgekürzt als UA), dem Service Agent (SA) und dem Directory Agent (DA) werden drei Arten von Software Agenten verwendet, die SLP-Nachrichten verarbeiten können. ${ }^{163}$ Dabei verwaltet der Directory Agent das zentrale Verzeichnis der verfügbaren Dienste, der Service Agent stellt Informationen über die Lokalisierung bestimmter Dienste bereit und der User Agent sucht nach der Lokalisierung der von den Benutzern angeforderten Dienste.

Für die Anbieter beinhaltet diese Lösung, dass die ortsbezogenen Leistungen ex ante vollständig zu konfigurieren sind, da keine situationsbezogene Anpassung (etwa unter Berücksichtigung interessenspezifischer Kontexte) möglich ist. Die jeweiligen Angebote sind mit den Mitteln des Service Location Protocol, das spezielle Attribute zur Klassifikation von Leistungen verwendet, zu beschreiben und an die zentralisierte Datenbank zu übermitteln. Der dazu nötige technische Aufwand hängt davon ab, wie häufig die in die zentralisierte Datenbank eingetragenen Informationen verändert werden sollen. Wenn es sich lediglich um Stammdaten handelt (z.B. die Adressen der in der Umgebung liegenden Hotels oder Restaurants, die bestimmten Ketten angeschlossen sind), dann ist es nicht erforderlich, automatisierte Schnittstellen einzurichten. Im Gegensatz dazu liegt der für die Zwecke des Informationsmanagements relevantere Fall dann vor, wenn die in die zentralisierte Datenbank eingetragenen Infor-

\footnotetext{
${ }^{162}$ Vgl. Chen (2001), Service Location Protocol.

${ }^{163}$ Vgl. SLP-Project (2001), Agents.
} 
mationen regelmäßig aktualisiert werden sollen (z.B. Preisinformationen für bestimmte Produkte).

Bei hoher Änderungsfrequenz und großem Umfang der zu übermittelnden Daten ist eine manuelle Pflege der ortsbezogenen Angebote regelmäßig nicht sinnvoll. Es ist vielmehr eine Schnittstelle zwischen den unternehmungsintern verwendeten Anwendungssystemen und der öffentlich zugänglichen Angebotsdatenbank einzurichten, über die eine reibungslose Synchronisation von internem und externem Datenbestand erfolgen kann. Diese Schnittstelle muss so gestaltet sein, dass zur Beschreibung der Leistungen standardisierte Bezeichnungen verwendet werden. Erst auf der Basis solcher standardisierter Beschreibungen wird für den Kunden eine komfortable Nutzung der ortsbezogenen Datenbank und ein Vergleich der von unterschiedlichen Anbietern offerierten Leistungen möglich. ${ }^{164}$ Es ist daher zu erwarten, dass die Betreiber der Datenbank entsprechende Anforderungen an die angeschlossenen Leistungsanbieter stellen. Die anbietenden Unternehmungen müssen deshalb Lösungen dafür finden, wie die in der unternehmungsinternen Produktdatenbank enthaltenen Angaben über Leistungsmerkmale ohne manuellen Eingriff in die Sprache des Service Location Protocols oder anderer möglicherweise zukünftig etablierter Standards, transformiert werden können. ${ }^{165}$ Nach der Transformation können die Daten des Anbieters über Exportschnittstellen an die zentralisierte Datenbank übertragen werden.

Wenn die ortsbezogenen Angebote unter Umgehung der zentralisierten Datenbank direkt an die Kunden übermittelt werden können, dann entfält die Notwendigkeit zur Standardisierung der Leistungsbeschreibung. In diesem Fall benötigen die Unternehmungen selbst die Lokalisierungsinformationen, um für ihr Angebot kontextspezifisch den Bezug zum Aufenthaltsort herzustellen. Die Ortsangabe kann entweder manuell durch eine entsprechende Eingabe des Kunden, während dieser sich über das Angebot informiert, oder automatisch durch Einsatz eines der in Abschnitt 6.1.4.3 genannten Lokalisierungsverfahren unter Hinzuziehung des Netzbetreibers erfolgen. Da die Übermittlung von Lokalisierungsinformationen einen Mehrwertdienst des Netzbetreibers darstellt, ist davon auszugehen, dass die zuletzt genannte Variante (auch im Vergleich zur oben beschriebenen Anbindung an eine zentralisierte Datenbank) relativ teuer sein wird. Diesem Nachteil steht allerdings der Vorteil gegenüber, dass über den eigenen Mobile Internet-Server der Unternehmung eine individuellere Leistungsbeschreibung und die Berücksichtigung weiterer kontextspezifischer Merkmale bei der Abgabe des Angebots möglich ist.

Unabhängig davon, auf welche Weise die Lokalisierungsinformationen und die Angebotsinformationen zusammengebracht werden, stellt sich auf der Seite des Anbieters das Problem, eine ortsspezifische Konfiguration von Leistungen vorzunehmen. Möglichkeiten zur ortsbezogenen Variation von Angebotsmerkmalen bestehen z.B. in der lokalen Anpassung des Preises oder der Leistungsausstattung. So könnten etwa bei einer Hotelkette der Übernachtungspreis von Stadt zu Stadt oder bei einer Einzel-

\footnotetext{
${ }^{164} \mathrm{Vgl}$. Varshney/Vetter/Kalakota (2000), Product Location.

${ }^{165}$ Vorstellbar ist hier einerseits der Einsatz von Übersetzungstools, die die erforderliche Transformation automatisiert bewerkstelligen. Andererseits ist zu überprüfen, inwieweit die betreffenden Standards auch für den unternehmungsinternen Gebrauch zur Leistungsbeschreibung übernommen werden können. In diesem Fall würde der aufwändige und möglicherweise nicht immer eindeutig mögliche Transformationsprozess entfallen.
} 
handelskette die Ladenpreise von Filiale zu Filiale variieren. Auch spezielle Aktionsangebote für einzelne Regionen, die in anderen Regionen nicht erhältlich sind, sind vorstellbar. Derartige Angebotsvariationen hängen nicht originär mit speziellen Mobile Commerce-Lösungen zusammen und sind daher normalerweise bereits in den ERPSystemen der Unternehmung abgebildet. Die Aufgabe des Informationsmanagements liegt in diesem Zusammenhang also nicht darin, ortsspezifische Angebotskonfigurationen überhaupt vorzunehmen, sondern die vorliegenden Angebotskonfigurationen für den mobilen Zugang verfügbar zu machen. Dazu ist eine dynamische Verknüpfung der über das mobile Internet an potenzielle Kunden übermittelten Informationen mit den innerhalb der ERP-Systeme vorhandenen Angebotsdaten erforderlich.

Die erweiterten multimedialen Darstellungsmöglichkeiten der nächsten Mobilfunkgenerationen lassen erwarten, dass digitale Angebote, bei denen die Leistung ausschließlich aus den auf das Endgerät übertragenen Inhalten besteht, mehr und mehr an Bedeutung gewinnen. Gerade bei digitalisierten Leistungen bietet die Verknüpfung von Inhalten mit Lokalisierungsinformationen ein großes Anwendungspotenzial. Beispiele für derartige Leistungsarten stellen ortsspezifische Umgebungsinformationen (mobile Reiseführer), Routenbeschreibungen, die sich auf den aktuellen Aufenthaltsort als Startposition beziehen, oder ortsbezogene Wettervorhersagen dar. Auch in diesen Fällen liegt die Problematik für das Informationsmanagement weniger in der Konfiguration des Angebots als in der Verknüpfung von Lokalisierungsinformationen mit bereits bestehenden inhaltlichen Informationen. Bezogen auf das Beispiel des mobilen Reiseführers bedeutet dies etwa, dass die an den Kunden übermittelten Inhalte nicht erst zum Zeitpunkt der Übertragung generiert werden. Sie liegen vielmehr bereits in den unternehmungsinternen Systemen vor und müssen nur noch aus dem vorhandenen Datenbestand selektiert und in mobilfunkgerechter Form aufbereitet werden.

\subsubsection{Unterstützung zeitbezogener Leistungsangebote durch das Informations- management}

Eine Bezugnahme auf zeitbezogene Merkmale als Kriterium für die Entwicklung kontextspezifischer Angebote stellt dann eine relevante Handlungsoption dar, wenn in Abhängigkeit von der Zeit eine individuelle Konfiguration von Leistungen möglich ist. Dies könnte beispielsweise bei Frischeprodukten oder Hotels, die noch nicht vermietete Zimmer ab einer bestimmten Uhrzeit zu reduzierten Preisen anbieten, der Fall sein. Je nach angebotener Leistung kann es auch sinnvoll sein, bei der Konfiguration ortsbezogene und zeitbezogene Gesichtspunkte zu kombinieren. Eine Kombination beider Aspekte würde etwa darin bestehen, aktuelle Veranstaltungsangebote nur solchen Personen anzubieten, die den Veranstaltungsort ausgehend von ihrem aktuellen Aufenthaltsort tatsächlich erreichen können.

Aus Sicht des Informationsmanagements unterscheiden sich ortsbezogene und zeitbezogene Angebote nur unwesentlich. Wie ortsbezogene liegen auch zeitbezogene Angebotsinformationen in der Regel bereits unternehmungsintern vor und sind lediglich gezielt zu selektieren, um sie an die Benutzer mobiler Endgeräte zu übermitteln. Die dabei entstehenden Probleme weisen ebenfalls analoge Züge auf. Während dauerhafte zeitliche Differenzierungen des Angebots keine Automatisierung der Übermittlung an zentrale Datenbanken erfordern, ist die Schaffung von Schnittstellen unumgänglich, 
wenn wiederholt aktuelle, zeitlich befristete Angebote generiert werden sollen. Werden zentrale Datenbanken durch eine direkte Verbindung zwischen dem Interessenten und dem Anbieter umgangen, dann sind die übertragenen Angebotsinformationen wie auch im Bereich der ortsbezogenen Informationen dynamisch mit dem unternehmungsinternen Datenbestand zu verknüpfen.

Im Hinblick darauf, dass der Aspekt der Ubiquität ein Kernmerkmal der nächsten Mobilfunkgenerationen darstellt und potenzielle Käufer leichter als bisher zu erreichen sind, könnte es dazu kommen, dass das Ausmaß zeitspezifischer Angebote zukünftig insgesamt steigt. Die Realisierung solcher Angebote erfordert zunächst einmal entsprechende Anpassungen der unternehmungsintern eingesetzten Anwendungssysteme im Vertrieb, die auf eine flexible Zuschneidung von Leistungen ausgerichtet werden müssen. Bei der Übermittlung solcher individuell konfigurierten Angebote an die Nutzer von mobilen Endgeräten handelt es sich um eine nachgelagerte Problemstellung, die sich nicht von der Übermittlung sonstiger Angebote unterscheidet.

\subsubsection{Unterstützung interessenbezogener Leistungsangebote durch das Informa- tionsmanagement}

Neben ortsbezogenen und zeitbezogenen können auch interessenbezogene Merkmale als Kriterium für die Entwicklung kontextspezifischer Angebote im Bereich des Mobile Commerce verwendet werden. Unabhängig von eventuellen Vorhaben zur Einführung von Mobile Commerce-Anwendungen sind schon jetzt häufig starke Anstrengungen zu beobachten, den Kunden individuelle Angebote $\mathrm{zu}$ offerieren, die speziell auf ihre persönlichen Interessen zugeschnitten sind. Vor dem Hintergrund eines sich verschärfenden Wettbewerbs streben viele Unternehmungen an, die Absatzmärkte weniger als anonyme, weitgehend homogene, allenfalls segmentierte Masse zu begreifen, sondern den Einzelkunden mit seinen individuellen Bedürfnissen und Wünschen ins Zentrum der Überlegungen zu stellen. ${ }^{166}$ Durch die maßgeschneiderte Anpassung der Leistung im Rahmen eines One-to-One-Marketing soll ein höherer Kundennutzen erreicht und damit ein Wettbewerbsvorteil gegenüber der Konkurrenz realisiert werden. Dies erfordert nicht nur die Fähigkeit zur flexiblen Zusammenstellung des Leistungsbündels, sondern auch die Kenntnis der individuellen Kundeninteressen im Einzelfall.

Die Individualisierung der Kundenbeziehung wird in Theorie und Praxis derzeit insbesondere unter dem Schlagwort Customer Relationship Management (CRM) diskutiert. Beim Customer Relationship Management handelt es sich um einen Ansatz, der darauf abzielt, mit Hilfe moderner Informations- und Kommunikationstechnik auf lange Sicht profitable Kundenbeziehungen durch ganzheitliche und individuelle Marketing-, Vertriebs- und Servicekonzepte aufzubauen und zu festigen. ${ }^{167} \mathrm{Da}$ moderne IuKTechnik eine Vielzahl von Ansatzpunkten für die Analyse und Pflege von Kundenbeziehungen bietet, ${ }^{168}$ ergeben sich Anforderungen an das Informationsmanagement, die durch eine entsprechende Gestaltung der in der Unternehmung verwendeten Anwendungssysteme zu erfüllen sind. Dabei geht es zum einen um die Zusammenführung aller in der Unternehmung vorliegenden kundenbezogenen Informationen, die

\footnotetext{
${ }^{166}$ Vgl. Picot/Reichwald/Wigand (2001), S. 461.

${ }^{167} \mathrm{Vgl}$. Hettich/Hippner/Wilde (2000), S. 1346.

${ }^{168} \mathrm{Vgl}$. Schmid/Bach/Österle (2000), S. 13.
} 
für ein erfolgreiches CRM in ganzheitlicher Form zur Verfügung stehen müssen (One Face of the Customer). Zum anderen geht es um eine differenzierte Ansprache des Kunden, die ebenfalls auf einem vereinheitlichten Informationsstand basiert (One Face to the Customer). ${ }^{169}$

Software-Lösungen im Umfeld des Customer Relationship Managements richten sich zunächst einmal auf die Konsolidierung bisher unverbundener Datenbestände. Dieses Problem stellt sich immer dann, wenn verschiedene Anwendungssysteme isoliert voneinander eingesetzt werden und jeweils spezielle Informationen über die Kunden enthalten. Trotz der fortschreitenden Verbreitung von ERP-Systemen kommen typischerweise nach wie vor sowohl im Front-End- als auch im Back-End-Bereich eine Reihe unterschiedlicher Anwendungssysteme zum Einsatz, die nicht vollständig integriert sind. Beim Aufbau eines über die verschiedenen Anwendungen hinweg konsolidierten Kundendatenbestands ist neben der eindeutigen Identifizierung der Kunden festzulegen, welche Eigenschaften der Kunden für die Unternehmung von Interesse sind und in welcher Weise diese in die Datenbank aufgenommen werden sollen. ${ }^{170}$ Ferner ist zu überprüfen, welche Interdependenzen zwischen den Funktionsbereichen der verschiedenen Anwendungssysteme bestehen. Auf dieser Basis lassen sich sowohl operative als auch analytische Aufgabenstellungen als Teilbereiche eines umfassenden CRM unterstützen.

Das operative CRM umfasst alle Funktionalitäten, bei denen es zu einem unmittelbaren Kontakt zwischen dem Kunden und der Unternehmung kommt. Der Einsatz von CRM-Systemen ermöglicht eine individuellere Ausgestaltung solcher direkten Kundenkontakte und bietet somit die Gelegenheit, die angebotenen Leistungen besser auf die Interessen des Einzelnen abzustimmen. Ferner wird suboptimales Verhalten, das aus einem unvollständigen Bild des Kunden resultiert, vermieden. Die Herausforderung für das Informationsmanagement liegt in diesem Bereich darin, die Kundeninformation in geeigneter Form aufzubereiten und den Mitarbeitern vor, während und nach dem direkten Kundenkontakt einen schnellen und unkomplizierten Zugriff darauf zu ermöglichen. Analytisches CRM dagegen betrifft die systematische Auswertung von Kundendaten, die bei unterschiedlichen Kontakten an verschiedenen Stellen der Unternehmung angefallen sind (z.B. Stammdaten, Kaufhistorien, Aktionsdaten, Reaktionsdaten). Die differenzierte Ansprache der Kunden verlangt eine Segmentierung der in sich heterogenen Kundschaft. ${ }^{171}$ Gegenstand analytischer CRMAktivitäten können entweder aggregierte Daten einer Vielzahl von Kunden oder desaggregierte Daten des Einzelnen sein. Wenn sich die Auswertung auf aggregiertem Niveau bewegt, werden die Daten in der Regel in ein analyseorientiertes Data Warehouse, das zusätzlich aus unternehmungsexternen Quellen gefüllt werden kann, extrahiert und anschließend mit dem Ziel der Erkennung von Mustern und der darauf basierenden Bildung von Clustern ausgewertet. ${ }^{172}$ Auf desaggregiertem Niveau zielt die Analyse typischerweise darauf, das Verhalten einzelner Kunden zu betrachten, um diese bestimmten Clustern zuordnen zu können. Damit wird ein besseres Verständnis der Kundenpräferenzen angestrebt, das die Unternehmung in die Lage versetzt,

\footnotetext{
${ }^{169}$ Vgl. Hettich/Hippner/Wilde (2000), S. 1346.

${ }^{170}$ Vgl. Egan (1999), Customer Information Scope; Kurtyka (2000), Customer Knowledge Management.

${ }^{171} \mathrm{Vgl}$. Hettich/Hippner/Wilde (2000), S. 1356.

${ }^{172}$ Vgl. Brown (2000), S. 220; Schmid/Bach/Österle (2000), S. 16.
} 
interessenspezifische Anpassungen der offerierten Leistungen auch aktiv vorzunehmen. Es sei erwähnt, dass bei der individuellen Analyse personenbezogener Daten zumindest in Deutschland enge datenschutzrechtliche Restriktionen zu beachten sind, die in dieser Arbeit jedoch aus der Untersuchung ausgeklammert werden.

Die Verbreitung des Electronic Commerce im leitungsgebundenen Internet hat bereits zu neuen Möglichkeiten für eine Individualisierung der Kundenbeziehung geführt, da die Anbieter über ihre Website in den Besitz umfangreicher neuer Kundeninformationen gelangten. ${ }^{173}$ Entsprechend der zu Beginn dieses Kapitels angesprochenen großen Beliebtheit des Präfix „E-“ wird in diesem Zusammenhang zumeist von ECRM gesprochen. ${ }^{174}$ Die Einführung von Mobile Commerce-Anwendungen bedeutet für das Customer Relationship Management nun, dass erneut ein zusätzlicher Kontaktpunkt zum Kunden entsteht. Die Erweiterung der CRM-Idee auf den mobilen Kanal stellt eine Ergänzung des bestehenden Konzepts dar und wird auch als Mobile Customer Relationship Management (M-CRM) ${ }^{175}$ bezeichnet. Das Informationsmanagement kann das M-CRM in zweierlei Hinsicht unterstützen. Zum einen sind die im Rahmen des CRM bereits verfügbaren Informationen für die Gestaltung des mobilen Kommunikationskanals zu nutzen. Diese Aufgabe ist dem operativen M-CRM zuzuordnen und erfordert, den Aspekt des One Face to the Customer auf den mobilen Internet-Zugang auszudehnen. Mit Hilfe des vorhandenen Kundenprofils, das sich auf unterschiedlichste Informationsquellen stützen kann, sind die auf das Endgerät übertragenen Inhalte so anzupassen, dass sie möglichst präzise den individuellen Interessen des Benutzers entsprechen.

Zum anderen gewinnt die Unternehmung durch den mobilen Kommunikationskanal zusätzliche Informationen, die für eine weitere Ausdifferenzierung des Kundenbilds genutzt werden können. Diese Aufgabe ist dem analytischen M-CRM zuzuordnen und beinhaltet, dass auch der Aspekt des One Face of the Customer mit dem mobilen Kommunikationskanal verknüpt wird. Als Basis für eine Verfeinerung von Kundenprofilen stehen hier insbesondere Protokolldateien zur Verfügung, die das Interaktionsverhalten der mobilen Endgerätebenutzer dokumentieren. ${ }^{176}$ Zur Analyse dieser Datenbasis können beispielsweise Data Mining-Verfahren, die aus einer umfangreichen Datenmenge implizit vorhandene, aber bisher unentdeckte nützliche Informationen extrahieren, eingesetzt werden. Dieser gerade neu entstehende Ansatz wird auch als WAP Log Mining bezeichnet ${ }^{177}$ und richtet sich einerseits auf die Verbesserung des mobilen Web-Auftritts sowie andererseits auf die Untersuchung von Verhaltensmustern der Endgerätebenutzer.

Das Auswertungspotenzial der WAP Log-Dateien geht jedoch weit über die anonymisierte Analyse im Rahmen von Data Mining-Verfahren hinaus. Da die Endgeräte über ihre Rufnummer eindeutig identifiziert werden können und in der Regel nur von einer Person genutzt werden, lässt sich das Interaktionsverhalten jedes einzelnen Kunden beobachten und für das gesamte CRM nutzen. Durch die genauere Kenntnis der Interessen wird es im Rahmen des operativen M-CRM möglich, mit automatischen Fil-

\footnotetext{
${ }^{173}$ Vgl. Brown (2000), S. xiii ff.; Wohlfahrt (2001), S. 49.

${ }^{174} \mathrm{Vgl}$. Kurtyka (2000), Customer Knowledge Management.

${ }^{175}$ Vgl. Kamp/Velthuis (2000), S. 4.

${ }^{176} \mathrm{Vgl}$. Bensberg/Weich (2001), S. 55.

${ }^{177}$ Vgl. Bensberg/Weich (2001), S. 55.
} 
tern für die im mobilen Internet angebotenen Inhalte zu arbeiten, die kundenindividuell aktiviert bzw. deaktiviert werden können. Im Hinblick auf die vergleichsweise wenig leistungsfähigen Benutzungsoberflächen mobiler Endgeräte stellt dies einen entscheidenden Schritt zur kontextspezifischen Anpassung des Leistungsangebots im Mobile Commerce dar. Die Kenntnisse über individuelle Kundenpräferenzen, die durch die Auswertung des mobilen Interaktionsverhaltens gewonnen wurde, können darüber hinaus auch im Rahmen des „traditionellen“ CRM für die persönliche Ausgestaltung von Kommunikationsvorgängen an anderen Kundenkontaktpunkten genutzt werden. Ferner ist vorstellbar, bei Untersuchungen im Bereich des analytischen CRM, die über den mobilen Kanal gewonnenen Kundeninformationen mit weiteren Informationen, die aus anderen Quellen innerhalb der Unternehmung stammen, zu kombinieren. ${ }^{178}$ Abbildung 6/10 zeigt die Einbettung des M-CRM in das Gesamtkonzept eines umfassenden Customer Relationship Management. Bei der dort im Zentrum stehenden konsolidierten Kundendatenbank muss es sich nicht zwingend um eine eigene physische Datenbank handeln. Entscheidend ist vielmehr die logische Vereinheitlichung der Kundensicht, die auch auf Basis der bestehenden physischen Datenbanken erreicht werden kann.

\footnotetext{
${ }^{178}$ Es sei darauf hingewiesen, dass dabei ebenfalls datenschutzrechtliche Restriktionen zu beachten sein werden, die nicht Gegenstand dieser Arbeit sind.
} 


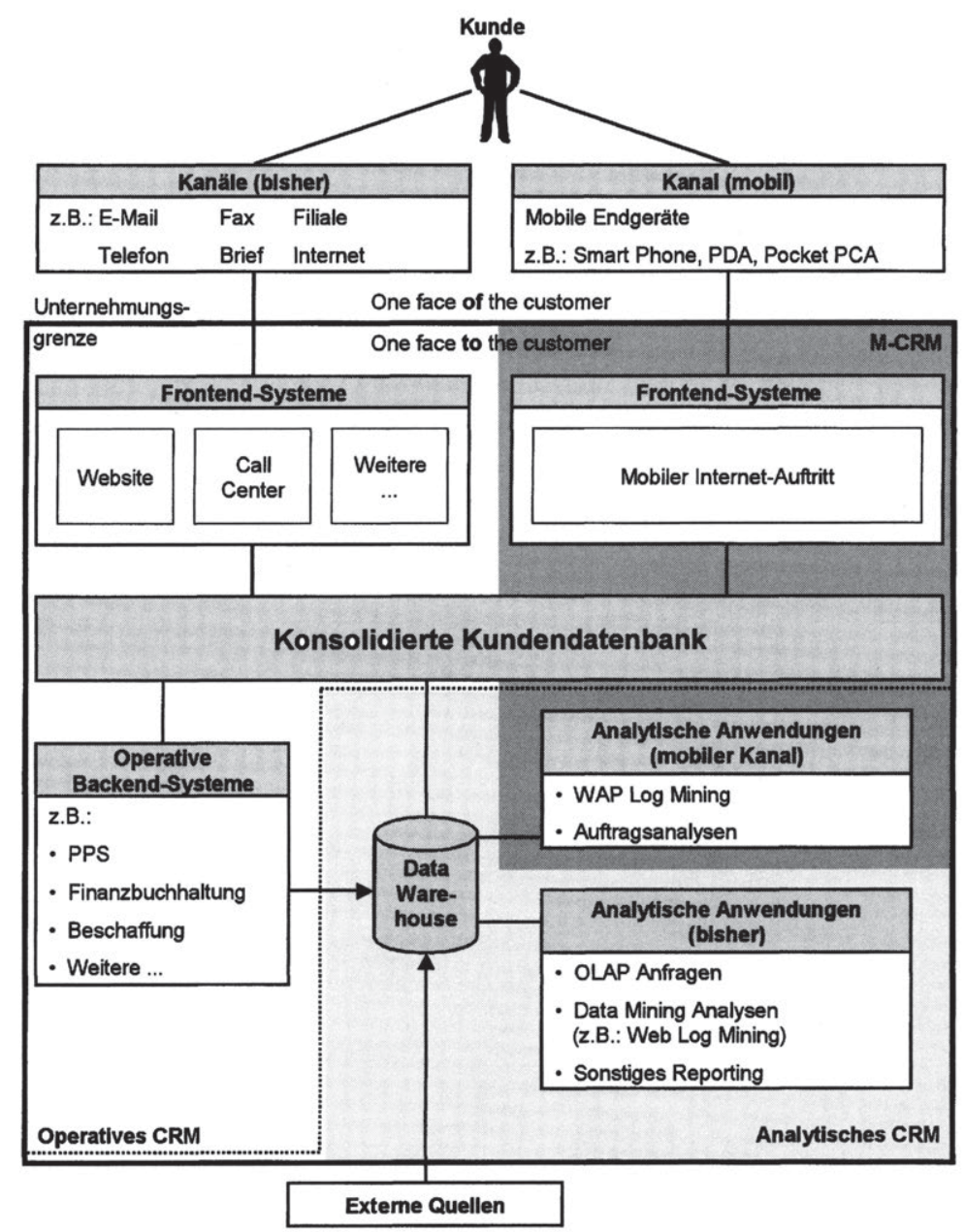

Abb. 6/10: M-CRM als Teil eines umfassenden Customer Relationship Managements

\subsubsection{Unterstützung ubiquitärer Leistungsangebote durch das Informations- management}

Der Aspekt der Ubiquität als innovatives Leistungsmerkmal der nächsten Mobilfunkgenerationen kann seinen Nutzen nur dann voll entfalten, wenn eine konsequente Verknüpfung des mobilen Netzzugangs mit den bestehenden leitungsgebundenen Systemen stattfindet. Daraus ergeben sich unmittelbar Anforderungen an das Informationsmanagement, das für die bestehenden Anwendungssysteme die Möglichkeit zu lesenden und schreibenden Zugriffen über mobile Endgeräte schaffen muss. Der Zugriff kann dabei entweder auf Initiative des Kunden (Pull) oder auf Initiative der Unternehmung (Push) erfolgen. 


\section{a) Pull-basierte Kontakte}

Wachsende Mobilität und Flexibilität im Alltag führen dazu, dass bei vielen Kunden der Wunsch entsteht, bei der Kontaktaufnahme mit dem Anbieter einer Leistung zwischen mehreren Kommunikationskanälen auswählen zu können. ${ }^{179}$ Vor diesem Hintergrund bedeutet die Einführung von Mobile Commerce-Anwendungen, dass für die Kunden eine zusätzliche Möglichkeit geschaffen wird, mit Unternehmungen, die eine bestimmte Leistung anbieten, in Kontakt zu treten. Die Kunden dürften diesen neuen Kontaktpunkt allerdings nur dann nutzen, wenn die mobil verfügbaren Inhalte mit denjenigen Inhalten, die über „traditionelle“ Kanäle kommuniziert werden, vollständig abgestimmt sind. Die Zielsetzung muss daher darin liegen, dass über unterschiedliche Kanäle und Endgeräte (z.B. PC, PDA, Telefon) auf die gleichen Inhalte zugegriffen werden kann. ${ }^{180}$ Dies betrifft nicht allein den unmittelbaren Abschluss einer Transaktion, sondern auch die vorher und nachher stattfindenden Aktivitäten. ${ }^{181}$ Dementsprechend ist auf Seiten der Unternehmung eine umfassende Integration erforderlich, die sowohl das über den mobilen Kanal offerierte Leistungsspektrum als auch das After Sales-Serviceangebot (z.B. die mobile Abfrage des aktuellen Status einer Bestellung) einbezieht.

Den Berührungspunkt, an dem die mobile Anfrage des Kunden den Leistungsanbieter erreicht, stellt bei Mobile Commerce-Anwendungen der Mobile Internet-Server der Unternehmung dar. Dieser muss folglich mit den unternehmungsintern eingesetzten Anwendungssystemen, insbesondere denen im Vertrieb und Service, verknüpft sein. Als Vorbild für die Realisierung solcher Verknüpfungen können Lösungen im leitungsgebundenen Electronic Commerce dienen, bei denen die via Internet eingehenden Seitenaufrufe innerhalb der Unternehmung Datenbankabfragen auslösen. Die durch den Internet-Server generierten Antworten werden als dynamische HTML-Seiten onthe-fly auf der Basis des jeweiligen Abfrageergebnisses generiert. Die Lösungen für den Mobile Commerce müssten ähnlich konstruiert sein. Anfragen, die von Kaufinteressenten im Vorfeld einer Transaktion an die Unternehmung herangetragen werden, betreffen das verfügbare Leistungsangebot und sind dementsprechend mit der Produktdatenbank der Unternehmung zu verknüpfen. Das erfolgreiche Zustandekommen einer Transaktion setzt neben dem lesenden Zugriff des Kunden auf die Produktdatenbank voraus, dass über den Mobile Internet-Server ein schreibender Zugriff zur Generierung von Bestellungen möglich ist. Wenn der Kunde zudem in der After Sales-Phase Kontakt mit der Unternehmung aufnimmt, sind stets spezielle Aufträge betroffen, sodass auch die Möglichkeit geschaffen werden muss, über den Mobile Internet-Server auftragsbezogene Anfragen an den unternehmungsinternen Datenbestand auszulösen.

Im Vergleich zum Electronic Commerce werden beim Mobile Commerce höhere Ansprüche an die endgerätegerechte Aufbereitung der an den mobilen Kunden übertragenen Information gestellt. Dabei handelt es sich um eine Problemstellung, die im engen Zusammenhang mit der im vorigen Abschnitt behandelten Frage der Unterstützung personenbezogener Leistungsangebote durch das Informationsmanagement steht. Es ist daher entscheidend, dass bei jeder über den Mobile Internet-Server

\footnotetext{
${ }^{179}$ Vgl. Röver (2000), S. 51f.

${ }^{180}$ Vgl. Dean/Ketterer/Thiel (2000), S. 36. Dies lässt die Möglichkeit zur Steuerung der Kanalwahl durch den Kunden z.B. mit Hilfe differenzierter Preismodelle, unberührt. Vgl. dazu Schmid/Bach/Österle (2000), S. 42.

${ }^{181}$ Vgl. Röver (2000), S. 52.
} 
eingehenden Kontaktaufnahme ein Abgleich mit der konsolidierten Kundendatenbank stattfindet. Bei Bekanntheit des Kunden können die dort vorgehaltenen Informationen dazu verwendet werden, die an das mobile Endgerät versandten Informationen mit Hilfe geeigneter Filtereinstellungen kundenindividuell anzupassen.

Die individuelle Anpassung der übertragenen Inhalte ist besonders wichtig, wenn der Kunde die ubiquitäre Verfügbarkeit des Netzzugangs zur Kontaktaufnahme in eng begrenzten Nischenzeiten nutzt, in denen der Schnelligkeit des Zugriffs kritische Bedeutung zukommt. Für eine aufwändige Suche nach den gewünschten Inhalten besteht unter diesen Voraussetzungen keine Zeit. Auf Grund der hohen Anforderungen an die schnelle Verfügbarkeit sind die betreffenden Anwendungen so zu konfigurieren, dass bei ihrem Aufruf keine langwierigen Einstellungen durch den Kunden mehr vorgenommen werden müssen, da dessen Identität erkannt wird und eine automatische Anpassung stattfindet.

\section{b) Push-basierte Kontakte}

Die allgegenwärtige Verfügbarkeit von Mobile Commerce-Anwendungen eröffnet für die Leistungsanbieter die Möglichkeit, jederzeit selbst die Initiative zu ergreifen und Kontakt zu den Kunden aufzunehmen. ${ }^{182}$ Auf Grund der always-on-Funktionalität des paketvermittelten Mobilfunks werden die Unternehmungen in die Lage versetzt, ihr Leistungsspektrum flexibler zu gestalten und mit hohem Aktualitätsgrad Informationen an ihre Kunden zu übermitteln. Die stetige Erreichbarkeit der Kunden macht die Verknüpfung push-basierter Kontakte mit kontextspezifischen Aspekten besonders interessant. Dadurch lassen sich bestimmte Entwicklungen, die beispielsweise mit dem Aufenthaltsort oder der Interessensituation des Endgerätebenutzers zusammenhängen, beobachten und eine konkrete Kontaktaufnahme an das Eintreten bestimmter Ereignisse (etwa das Erreichen bestimmter Aktienkurse, das Vorliegen bestimmter Sportergebnisse oder das Aufsuchen bestimmter Orte durch den Kunden) binden. ${ }^{183}$ Ziel des Informationsmanagements ist es in diesem Zusammenhang, die Verknüpfung von Beobachtung und Kontaktaufnahme zu automatisieren. Dies lässt sich insbesondere mit Hilfe von intelligenten Agenten realisieren. ${ }^{184}$

Es sind zahlreiche Anwendungen vorstellbar, bei denen die push-basiert übermittelten Inhalte selbst die eigentliche Leistung für den Endgerätebenutzer darstellen (z.B. bei den oben genannten Aktienkurs- oder Sportinformationen). Der Aspekt der Datenproaktivität ermöglicht es den anwendenden Unternehmungen darüber hinaus, Kontakt zu potenziellen Kunden aufzunehmen, um Informationen über aktuelle Leistungsangebote zu übertragen. Auf der Seite der Anbieter kommt es folglich zu einer Erweiterung der Werbemöglichkeiten, wobei in der Regel das zuvor gegebene Einverständnis des Kunden Voraussetzung für die Kontaktaufnahme ist. Für eine systematische Nutzung dieses Funktionsmerkmals ist es notwendig, die Kontaktaufnahme via Mobilfunk in ein Gesamtkonzept für die Ansprache der Kunden einzubinden, das verschiedene Kanäle einbezieht. Es besteht also eine enge Verbindung zu dem im vorigen Abschnitt erläuterten Konzept des Customer Relationship Management, das

\footnotetext{
${ }^{182} \mathrm{Vgl}$. Wohlfahrt (2001), S. 50f.

${ }^{183}$ Vgl. Pflug (2001), S. 22.

${ }^{184} \mathrm{Vgl}$. Scheer et al. (2001), S. 11.
} 
den Einsatz moderner Informations- und Kommunikationstechnik zum Aufbau und zur Festigung langfristig profitabler Kundenbeziehungen anstrebt.

Die ubiquitäre Erreichbarkeit der Kunden eröffnet insbesondere für den Bereich des Kampagnenmanagements als Teil des operativen CRM große Anwendungspotenziale. Das Kampagnenmanagement zielt darauf ab, dem richtigen Kunden das richtige Informations- und Leistungsangebot im richtigen Kommunikationsstil über den richtigen Kommunikationskanal zum richtigen Zeitpunkt zu übermitteln. ${ }^{185}$ Bei der Entwicklung einer Kampagne ist zu entscheiden, welche Produkte einbezogen werden, wie die Preisstellung für diese Produkte ist, welche Werbemaßnahmen ergriffen werden, wie lang die Aktion läuft und wem die Produkte angeboten werden. ${ }^{186}$ Grundlage dieser Entscheidungen sind Kundenanalysen, aus denen konkrete Aktionsfolgen abgeleitet werden. ${ }^{187}$ Die Möglichkeit zur Kundenansprache beschränkte sich für das Kampagnenmanagement bisher hauptsächlich auf die schriftliche Kommunikationsform (herkömmliche Post und E-Mail) und - nach Einwilligung des Kunden - auf telefonische Kontakte. ${ }^{188}$

Mit der mobilen Datenübertragung tritt nun ein neuer Kanal hinzu, über den sich die „Streuverluste" bei der Ausgestaltung von Kampagnen durch die Verknüpfung mit kontextspezifischen Merkmalen erheblich reduzieren lassen. Dabei stellt insbesondere die Bezugnahme auf ortsbezogene Aspekte eine echte Neuerung dar. ${ }^{189}$ Der Zeitpunkt der Kontaktaufnahme kann bei Berücksichtigung des aktuellen Aufenthaltsorts so abgestimmt werden, dass der Kunde unmittelbar danach die Gelegenheit hat, die Transaktion abzuschließen bzw. die Leistung in Anspruch zu nehmen. Beispiele für derartige Push-basierte Kontakte sind etwa die Benachrichtigung registrierter Kunden über aktuelle Angebote oder über die Verfügbarkeit bestimmter Produkte, wenn diese sich in der Nähe einer Verkaufsstelle der Unternehmung befinden, sowie die Information von Reisenden über die nächstgelegene Möglichkeit zur Inanspruchnahme bestimmter Leistungen. Aus Sicht des Informationsmanagements liegt die besondere Herausforderung in diesem Zusammenhang darin, aufenthaltsortsbezogene Informationen und allgemeine Angebotsinformationen miteinander zu verbinden. Während sich die Aufenthaltsinformationen im Besitz der Netzbetreiber befinden und für die Anwender nicht kostenlos verfugbar sind ${ }^{190}$ werden die Angebotsinformationen unternehmungsintern vorgehalten. Die Effizienz zukünftiger Lösungen in diesem Bereich wird somit nicht allein von technischen Gesichtspunkten abhängen, sondern in erheblichem Maße auch von den zwischen Anwendern und Netzbetreibern auszuhandelnden Preismodellen beeinflusst werden.

\subsubsection{Gestaltungsfelder des Informationsmanagements beim Aufbau von Mobile Work-Anwendungen}

Mobile Work-Anwendungen unterstützen mobil arbeitende Beschäftigte durch den direkten Zugang zu unternehmungsintern vorgehaltenen Datenbeständen verschieden-

\footnotetext{
${ }^{185} \mathrm{Vgl}$. Hettich/Hippner/Wilde (2000), S. 1354.

${ }^{186}$ Vgl. Brown (2000), S. 9.

${ }^{187} \mathrm{Vgl}$. Hettich/Hippner/Wilde (2000), S. 1354.

${ }^{188}$ Vgl. Brown (2000), S. 10.

${ }^{189}$ Vgl. Lai/Mohan/Gustafsson (2000), S. 2.

${ }^{190}$ Vgl. Gerpott (2001), S. 40.
} 
ster Art (z.B. Nachrichten, Kunden- und Auftragsdaten, Terminpläne usw.). ${ }^{191} \mathrm{Im}$ Vergleich zum Mobile Commerce resultieren die Nutzenpotenziale der mobilen Datenübertragung im Bereich des Mobile Work sehr viel stärker aus dem Leistungsmerkmal Ubiquität. Der Nutzen einer allgegenwärtigen Verfügbarkeit des Netzzugangs lässt sich allerdings durch eine kontextspezifische Ausgestaltung der übertragenen Inhalte noch erhöhen. Die Gestaltungsaufgaben des Informationsmanagements beim Aufbau von Mobile Work-Anwendungen betreffen sowohl technische als auch organisatorische Gesichtspunkte. Abschnitt 6.2.3.1 wendet sich zunächst den Anforderungen an technikbezogene Gestaltungsmaßnahmen $\mathrm{zu}$, mit denen das Informationsmanagement die Verfügbarkeit unternehmungsintern vorliegender Informationen für mobil arbeitende Beschättigte herstellen kann. Abschnitt 6.2.3.2 untersucht davon ausgehend, welche neuen Möglichkeiten sich durch die Techniken zur mobilen Datenübertragung für veränderte organisatorische Lösungen bei der Gestaltung von Geschäftsprozessen ergeben.

\subsubsection{Technische Aspekte: Ubiquitärer und kontextspezifischer Zugang der Beschäftigten zu unternehmungsinternen Informationen}

In technischer Hinsicht liegt der Ausgangspunkt beim Aufbau von Mobile WorkAnwendungen in der Konvergenz von mobilen und leitungsgebundenen Informationsund Kommunikationstechniken. Diese Konvergenz sowie das gleichzeitige Zusammenwachsen bisher getrennter Anwendungssysteme im leitungsgebundenen Bereich eröffnen dem Informationsmanagement die Möglichkeit, eine Reihe von Restriktionen zu überwinden, durch die die informationswirtschaftliche Situation in Unternehmungen bisher typischerweise gekennzeichnet ist. ${ }^{192}$ So können Mitarbeiter, die zu Hause oder insbesondere auch unterwegs arbeiten möchten, nur bedingt Anwendungen und Daten, die sich auf den Rechnern der Unternehmung befinden, nutzen. Ferner kann auf Daten, die für ein spezielles Gerät bestimmt sind, z.B. einen PC, einen PDA oder ein Mobiltelefon, in der Regel nicht ohne weiteres mittels anderer Geräte zugegriffen werden. Eine unvollständige Integration der bestehenden Anwendungssysteme führt außerdem dazu, dass Beschäftigte, die an mehreren Standorten mit verschiedenen Anwendungssystemen arbeiten, häufig nicht über eine kohärente Sicht der von ihnen verwendeten Daten verfügen. Auch benutzerspezifische Einstellungen sind zumeist lokal, allenfalls im lokalen Netz, gespeichert und können nicht standortunabhängig genutzt werden.

Durch die Beseitigung der angesprochenen Mängel und eine bessere Verknüpfung bisher isolierter Anwendungen lassen sich umfassendere und noch leistungsstärkere Lösungen entwickeln, mit denen die Effizienz der technikunterstützten Informationsverarbeitung erheblich gesteigert werden kann. ${ }^{193}$ Das Potenzial zur Leistungssteigerung basiert dabei insbesondere auf dem Aspekt der Unabhängigkeit. Die Konvergenz der verschiedenen Techniklinien und die vollständigere Integration von bisher getrennt betriebenen Software-Systemen versetzt die Mitarbeiter in die Lage, unternehmungsinterne Daten und Anwendungen in vielerlei Hinsicht unabhängiger als bisher zu nutzen. Die Unabhängigkeit bezieht sich beispielsweise auf den aktuellen

\footnotetext{
${ }^{191}$ Vgl. Plesman (2000), S. 6.

${ }^{192}$ Vgl. zum Folgenden Microsoft (2001a), Einleitung.

${ }^{193}$ Vgl. ähnlich Microsoft (2001a), Einleitung.
} 
Aufenthaltsort, auf das zur Übermittlung von Informationen verwendete Übertragungsmedium sowie auf die Art des Endgeräts und auf dessen Benutzungsoberfläche. Die Realisierung dieser Unabhängigkeitspotenziale erfordert eine technische Architektur, bei der Inhalte, die den Mitarbeitern übermittelt werden, zunächst plattformunabhängig vorgehalten und erst "spät“ in gerätespezifische Informationen umgesetzt werden. ${ }^{194}$ Darüber hinaus sind die in der Unternehmung verwendeten Anwendungssysteme und Datenbestände für einen ubiquitären Zugriff vorzubereiten sowie unternehmungsweit zu konsolidieren, um eine kohärente Sicht für die Benutzer herzustellen und logische Inkonsistenzen zu vermeiden.

Der Bedarf nach Plattformunabhängigkeit macht so genannte „Multi AccessPortale" wünschenswert, die den Benutzern über verschiedenste Endgeräte und Übertragungsmedien Zugang zu speziell auf sie zugeschnittenen (personalisierten) Inhalten ermöglichen ${ }^{195}$ Diese Portale fungieren im Idealfall als zentrale Intermediäre, die auf der einen Seite über Schnittstellen mit den verschiedenen Anwendungssystemen und Datenbeständen innerhalb der Unternehmung verknüpft sind und auf der anderen Seite differenzierte Benutzermodelle verwenden, um die übertragenen Inhalte gezielt zu individualisieren. Im Folgenden steht zunächst die Verbindung zwischen Multi AccessPortalen und den Benutzern im Vordergrund. Die Verknüpfung mit anderen Systemen der Unternehmung wird anschließend näher betrachtet. Jede Verbindung zwischen Benutzer und Portal macht situationsspezifisch eine formale und inhaltliche Anpassung der übertragenen Informationen notwendig.

Die Form der Darstellung ist je nach Benutzungsoberfläche des gerade genutzten Endgeräts zu konfigurieren. Grundsätzlich sind dazu zwei Alternativen vorstellbar: ${ }^{196}$ Es kann entweder mit mehreren jeweils plattformspezifischen Portalversionen gearbeitet werden oder die endgerätespezifische Anpassung der Darstellungsform kann dynamisch während der Verbindung stattfinden. Zur Vermeidung redundanter Datenhaltung erscheint die zuletzt genannte Alternative vorteilhaft. Standardisierte Lösungen liegen in diesem Bereich allerdings noch nicht vor. Die Anpassung der übermittelten Inhalte wiederum erfolgt in Abhängigkeit von der Person des Benutzers mit Hilfe des individuellen Benutzermodells. Für eine Ausdifferenzierung des Benutzermodells kann beim unternehmungsintern orientierten Mobile Work im Vergleich zum unternehmungsextern orientierten Mobile Commerce auf weitaus umfangreichere Informationen zurückgegriffen werden, die bereits aus anderen Zusammenhängen vorliegen. Dabei handelt es sich z.B. um Informationen über das fachliche Arbeitsgebiet, die hierarchische Position der beschättigten Person oder den aktuellen Status von Projekten, an denen diese Person beteiligt ist. Zusätzlich ist vorstellbar, die Systeme zur Benutzermodellierung mit adaptiven Fähigkeiten auszustatten, die es ermöglichen, aus der Beobachtung des einzelnen Benutzers zu lernen und das Benutzermodell aktiv an dessen Verhalten anpassen. ${ }^{197}$

Die Verknüpfung des Portals mit den anderen Systemen der Unternehmung vereinfacht für die Benutzer die Zugangsprozedur und stellt die endgeräteunabhängige, ubiquitäre

\footnotetext{
${ }^{194} \mathrm{Vgl}$. Dean/Ketterer/Thiel (2000), S. 36.

${ }^{195}$ Vgl. Garcia/Ribas (2000), S. 1.

${ }^{196} \mathrm{Vgl}$. Garcia/Ribas (2000), S. 6.

${ }^{197} \mathrm{Vgl}$. Mertens/Höhl (1999), S. 3. Zur detaillierten Darstellung von Möglichkeiten zur Benutzermodellierung vgl. Mertens/Höhl (1999), passim.
} 
Verfügbarkeit von Anwendungen und Daten sicher. Vor diesem Hintergrund ist mit einem erhöhten Kapazitätsbedarf bei den unternehmungsinternen Netzen, die den Datenfluss zwischen der bestehenden Systemlandschaft und dem neu hinzukommenden Multi Access-Portal abwickeln, zu rechnen. ${ }^{198}$ Für eine möglichst umfassende Integration sollten unterschiedlichste Anwendungssystemtypen in das Konzept der Ubiquität einbezogen werden. Im Folgenden werden die Einbeziehung von so genannten Basissystemen zur allgemeinen Unterstützung der Kommunikation und Koordination sowie von operativ und analytisch ausgerichteten Anwendungssystemen im betriebswirtschaftlichen Bereich näher betrachtet.

Alle Formen von Anwendungen, welche die Kommunikation für die Mitarbeiter der Unternehmung einfacher, schneller oder bequemer gestalten, sind potenziell für eine Zusammenführung, die sich an der zu Beginn dieses Kapitels angesprochenen Leitidee "anywhere, anytime, on any device“ orientiert, interessant. ${ }^{199}$ Die Integration unterschiedlicher Nachrichtenformate (z.B. E-Mail, SMS, Fax, Voice Mail) findet derzeit starke Beachtung in Theorie und Praxis und wird auch als Unified Messaging bezeichnet. Unified Messaging, für das sich ebenfalls noch kein allgemeiner Standard herausgebildet hat, ermöglicht einen vom ursprünglichen Sendeformat unabhängigen Zugang zu Nachrichten. Es erlaubt dem Benutzer somit das Senden und Empfangen von Nachrichten über eine Vielzahl von Endgeräten, von denen jeweils das in der aktuellen Situation geeigneteste gewählt werden kann. Da alle technikunterstützten Kommunikationsvorgänge beim Unified Messaging zentralisiert über nur einen Server abgewickelt werden, wird dem Benutzer eine vereinheitlichte Gesamtsicht und ein vollständiger Überblick über das Kommunikationsgeschehen geboten.

Wenn mobil arbeitende Beschäftigte bei der Erfüllung ihrer Aufgaben umfassend unterstützt werden sollen, muss auch ein ubiquitärer Zugang zu Anwendungen im betriebswirtschaftlichen Bereich sowie zu den darin verfuggaren Datenbeständen hergestellt werden. ${ }^{200}$ Dazu ist es notwendig, sowohl für operativ als auch für analytisch ausgerichtete Systeme eine softwaretechnische Schnittstelle zu dem oben beschriebenen Multi Access-Portal einzurichten. Über diese Schnittstelle müssen für berechtigte Personen sowohl lesende als auch schreibende Zugriffe mit mobilen Endgeräten möglich sein. Von den im ERP-Geschäft tätigen Anbietern wurden inzwischen erste Konzepte zur Einrichtung von mobilen Schnittstellen vorgelegt, die allerdings nur zum Teil als Online-Lösungen konstruiert sind. ${ }^{201}$ Die Online-Varianten sehen in der Regel vor, dass über Portale auf der Grundlage von DHTML (Dynamic HTML) und/oder Java eine Verbindung zu den mobilen Endgeräten aufgebaut wird. Werden Daten oder die Anwendungslogik über den Browser des Endgeräts aufgerufen, so generiert die Software dynamisch HTML-Seiten on-the-fly. ${ }^{202}$ Dieses Verfahren konnte bisher jedoch keine weite Verbreitung finden, da kaum DHTML-fähige mobile Endgeräte am Markt verfügbar sind. WAP-basierte Lösungen werden indessen derzeit noch durch die eingeschränkte Leistungsfähigkeit der Benutzungsoberfläche WAPfähiger Endgeräte behindert. Sollte sich $\mathrm{XML}$ als plattformunabhängiger gemeinsamer

\footnotetext{
${ }^{198} \mathrm{Zu}$ den Auswirkungen, die durch die Einführung von Mobile Business auf unternehmungsinterne Netze entstehen, vgl. Suppan (2001), S. 34ff.

${ }^{199}$ Vgl. ähnlich Zobel (2001), S. 193.

${ }^{200}$ Vgl. ähnlich Zobel (2001), S. 193.

${ }^{201} \mathrm{Vgl}$. Ehrenwirth (2001), S. 10ff.

${ }^{202}$ Vgl. Ehrenwirth (2001), S. 11.
} 
Standard für unterschiedliche Gerätetypen etablieren, so ist zu erwarten, dass auch die Anbieter von ERP-Software zukünttig entsprechende Konverter als Middleware anbieten werden. ${ }^{203}$

Für ein komfortables Arbeiten mit mobilen Endgeräten ist es wünschenswert, dass auch der Zugang zu den betriebswirtschaftlichen Anwendungssystemen mit dem Benutzermodell des Multi Access-Portals verknüpft wird. Damit könnte eine Reihe von benutzerspezifischen Eingaben wie etwa der explizite Aufruf bestimmter Transaktionsmasken beim Einwählen oder die umständliche Eingabe häufig angeforderter Datenbankabfragen vereinfacht werden. Zu Beginn dieses Abschnitts wurde ferner die Herstellung einer kohärenten Gesamtsicht für den Benutzer durch eine Konsolidierung von bisher getrennten Datenbeständen gefordert. Dieses Problem ist allerdings nicht speziell dem Bereich des Mobile Business zuzuordnen und wird daher an dieser Stelle nicht weiter behandelt.

\subsubsection{Organisatorische Aspekte: Neugestaltung von Prozessen auf Basis der mobilen Datenübertragung}

Die Leistungspotenziale eines nahezu allgegenwärtig für die Beschäftigten zur Verfügung stehenden mobilen Netzzugangs lassen sich nur dann ausschöpfen, wenn neue organisatorische Konzepte entwickelt werden, die auf den veränderten technischen Möglichkeiten aufbauen. Die Konvergenz von mobiler und leitungsgebundener Informations- und Kommunikationstechnik muss sich folglich auch in der Gestaltung des Arbeitslebens niederschlagen. Die Mitarbeiter der Unternehmung werden in die Lage versetzt, jederzeit und entsprechend ihren individuellen Bedürfnissen Informationen zu senden und zu empfangen. Dabei sind sie nicht mehr an ihren stationären Rechner gebunden, wie es bisher in der Regel der Fall war. Diese Entwicklung stellt die bisherige Vorstellung vom Büro als Ort der physischen Präsenz in Frage, da bei funktionierenden Mobile Work-Anwendungen im Prinzip überall, wo sich die Beschäftigten aufhalten, zugleich ihr Büro sein kann. ${ }^{204}$

Es ist daher zu erwarten, dass der allgemeine Trend zu einer zunehmenden Verschmelzung von beruflicher und privater Sphäre durch die Verbreitung von Mobile Work-Anwendungen noch verstärkt wird. ${ }^{205}$ Berufstätige können nicht nur von zu Hause aus, sondern auch während sie sich an entlegenen Orten oder unmittelbar auf der Reise in Bewegung befinden, dringende Aufgaben erfullen und an der Lösung aktueller Problemstellungen mitwirken. Die Kontaktaufnahme ist dabei nicht nur pull-basiert durch die Beschäftigten, sondern auch push-basiert möglich, sodass zeitsensitive Informationen auch auf Initiative der zentralen Stelle an die Beschäftigten weitergeleitet werden können. ${ }^{206}$ Der primäre Gestaltungsschwerpunkt des Informationsmanagements dürfte sich daher mit der Einführung von Mobile Work-Anwen-

\footnotetext{
${ }^{203}$ Zur Zeit wird lediglich von Baan entsprechende Middleware zur XML- und HTML-Konvertierung bereitgestellt. Als angeschlossene Endgeräte sind dabei allerdings nur Laptop-PC's und PDA's vorgesehen. Vgl. Ehrenwirth (2001), S. 12. Eine vereinfachte Mobile-Version der Anwendungssoftware wird bisher nicht angeboten. Im Idealfall wäre diese jedoch auch gar nicht erforderlich, weil die endgerätespezifische Anpassung erst nach der XML-Konvertierung erfolgen könnte.

${ }^{204} \mathrm{Vgl}$. Mobilocity (2001), S. 2.

${ }^{205} \mathrm{Vgl}$. ähnlich Microsoft (2001b), Introduction.

${ }^{206}$ Vgl. Mobilocity (2001), S. 8.
} 
dungen verschieben. Während die informationstechnische Unterstützung von Geschäftsprozessen bisher in erster Linie auf die organisatorischen Erfordernisse der Prozesse selbst ausgerichtet war, kommt im Umfeld des Mobile Work eine neue Gestaltungsorientierung hinzu und löst die alte zum Teil ab. Es tritt verstärkt die Sichtweise des mobil arbeitenden, wechselnde Kommunikationskanäle nutzenden, einzelnen Mitarbeiters in den Mittelpunkt der Bestrebungen um eine flexible Unterstützung durch das Informationsmanagement. Dies führt sowohl bei gut strukturierten als auch bei weniger gut strukturierten Prozessen zu einem Bedarf nach veränderten Organisationskonzepten.

Im Bereich der gut strukturierten Prozesse ist im ersten Schritt zu erwarten, dass die Möglichkeit zum Zugriff auf allgemeine, nicht speziell auf die mobile Nutzung zugeschnittene Anwendungen geschaffen wird. ${ }^{207}$ Wegen der anfangs noch bestehenden Restriktionen bei Benutzungsoberflächen und Browsern stehen dabei in erster Linie unidirektionale Datenflüsse mit lesenden Zugriffen im Vordergrund. Die Realisierung des Zugangs erfolgt durch Funktionsergänzungen (add-ons) bei den vorhandenen Anwendungssystemen, ${ }^{208}$ und eine Einbettung der betreffenden Aktivitäten in die bestehenden Prozesse ist zunächst nur punktuell gegeben. Beispiele sind hier der Abruf von Lagerbeständen oder Dispositionsdaten durch Außendienstmitarbeiter. ${ }^{209}$ Erst im nächsten Schritt ist mit einer echten konzeptionellen Integration mobiler Aktivitäten und davon ausgehend einer organisatorischen Anpassung der Geschäftsprozesse zu rechnen. Durch die bidirektionale Ausgestaltung der Informationsflüsse können Daten von jedem Ort aus erfasst und in Echtzeit an die in der Unternehmung verwendeten Systeme übermittelt werden. ${ }^{210}$ Mehrfacheingaben können somit beseitigt und die Abwicklung von Kundenaufträgen beschleunigt werden. Beispiele in diesem Bereich sind die Erfassung neuer Aufträge über mobile Endgeräte oder die Abwicklung von Reisekostenabrechnungen vor Ort.

Da bei gut strukturierten Prozessen die Voraussetzungen für eine Ex ante-Koordination der Tätigkeiten gegeben sind, kann die Möglichkeit zu mobil durchführbaren Aktivitäten bei der Gestaltung der Prozesse berücksichtigt und systematisch in Konzepte zum Workflow Management einbezogen werden. Dies ermöglicht nicht nur einen schnelleren Start der Prozessabwicklung infolge der mobilen Auftragserfassung, sondern es verspricht auch Effizienzsteigerungen durch eine beschleunigte Steuerung des Prozessverlaufs. Während bei push-basiert ausgelösten Prozessschritten bisher die Anwesenheit der zuständigen Mitarbeiter an ihrem Arbeitsplatz erforderlich war, könnten die betreffenden Aktivitäten (z.B. Prüfungsaktivitäten) zukünftig auch mobil ausgeführt werden. Insgesamt ist bei gut strukturierten Prozessen somit eine Ausweitung des Anteils an mobil abgewickelten Prozessschritten zu erwarten.

Auch im Bereich von eher schlecht strukturierten Prozessen eröffnet die mobile Datenübertragung Potenziale zur Einführung veränderter organisatorischer Konzepte. Eine vollständige Ex ante-Koordination ist bei diesem Prozesstyp grundsätzlich nicht zu erreichen und stellt somit kein relevantes Zielkriterium für Gestaltungsmaßnahmen des Informationsmanagements dar. Aus diesem Grund zielte die informationstechnische

\footnotetext{
${ }^{207} \mathrm{Vgl}$. ähnlich Hartmann/Dirksen (2001), S. 17.

${ }^{208}$ Vgl. Ehrenwirth (2001), S. 10.

${ }^{209}$ Vgl. Hartmann/Dirksen (2001), S. 17f.

${ }^{210}$ Vgl. Mobilocity (2001), S. 4f.
} 
Unterstützung schlecht strukturierter Prozesse durch Konzepte des Workgroup Computing bereits in der Vergangenheit im Wesentlichen auf eine möglichst hohe Flexibilität der Prozessabwicklung. Mit der Einführung von Mobile Work-Anwendungen ergeben sich neue Möglichkeiten zur Vereinfachung der Ad hoc-Koordination während der Prozessdurchfuhrung. Die ubiquitäre Verfugbarkeit des Netzzugangs er.ıöht die Erreichbarkeit der Prozessbeteiligten, sodass auch bei räumlich getrenntem Arbeiten noch leichter eine Kontaktaufnahme möglich wird. Demzufolge dürfte die synchrone Form der Koordination zunehmend an Bedeutung gewinnen, sodass die Flexibilität der Prozessabwicklung weiter gesteigert werden kann. Die räumliche Trennung von zusammenarbeitenden Beschäftigten wird weniger problematisch, da sich auf technischem Wege eine gemeinsame Arbeitsumgebung schaffen lässt. In diesem Zusammenhang stiftet das Unified Messaging einen erheblichen Nutzen, da bisher getrennte Kommunikationsarten verknüpft werden, sodass medienunabhängig die Erreichbarkeit der Kommunikationspartner erhöht wird.

Die inhaltliche Unterstützung bei der Erfüllung von Aufgaben in schlecht strukturierten Prozessen erfolgt in erster Linie durch analytische Funktionalitäten der in der Unternehmung eingesetzten betriebswirtschaftlichen Anwendungssysteme. Durch die Einrichtung eines mobilen Zugangs zu Analyseinformationen verfügen die Entscheidungsträger auch unterwegs über einen aktuelleren Informationsstand als bisher. Somit können kurzfristige Entwicklungen auch dann berücksichtigt werden, wenn gerade kein leitungsgebundener Zugang $\mathrm{zu}$ den unternehmungsinternen Anwendungssystemen möglich ist. Damit steigt die - häufig als kritische Größe bezeichnete Reaktionsgeschwindigkeit auf unvorhergesehene Ereignisse. Da die Mobilität des Arbeitens gerade für Entscheidungsträger in schlecht strukturierten Prozessen eher die Regel als die Ausnahme darstellt, fallt die Ubiquität von Mobile Work-Anwendungen in diesem Bereich besonders stark ins Gewicht. Beispiele sind hier der mobile Zugang zu neuesten Berichtszahlen (etwa Umsatzzahlen, die mehrdimensional ausgewertet werden können) oder zu Kundendatenanalysen, die als Planungsgrundlage bei der Verhandlung über weitere Geschäftsabschlüsse dienen können. Auch bei diesem Prozesstyp dürte damit der Anteil an mobil abgewickelten Prozessschritten, der bereits bisher vergleichsweise hoch war, steigen, da die Entscheidungsträger unabhängig von ihrem Aufenthaltsort mit detaillierten und aktuellen Informationen versorgt werden können.

\subsection{Anwendung der entwickelten Theorieansätze und Untersuchung ihrer Beziehungen}

Im Folgenden wird untersucht, inwiefern die in dieser Arbeit entwickelten Ansätze für eine Theorie des Informationsmanagements untereinander im Zusammenhang stehen. $\mathrm{Zu}$ diesem Zweck werden diese Ansätze auf den Aufbau und Betrieb von Mobile Business-Anwendungen als speziellen Gestaltungsbereich des Informationsmanagements angewendet. Es werden die Erkenntnispotenziale betrachtet, die die verschiedenen Ansätze zur Lösung der idealtypischen ungelösten Problemstellungen des Informationsmanagements in der Praxis aufweisen. $\mathrm{Da}$ die hier betrachteten Mobile Business-Anwendungen erst noch zu entwickeln sind, beziehen sich die folgenden Überlegungen mehr auf den Aspekt der präventiven Problemvermeidung als auf die Beseitigung bestehender Probleme bei im Betrieb befindlichen Anwendungen. Es wird untersucht, welches Verhältnis zwischen den jeweils ableitbaren Aussagen besteht. Da 
die zu Grunde liegenden Wissenschaftsprogramme von zum Teil sehr verschiedenen Annahmekomplexen ausgehen, sind sowohl komplementäre, neutrale als auch konkurrierende Beziehungen zwischen den jeweiligen Aussagesystemen vorstellbar. Für die Lösung von konkreten Gestaltungsproblemen in der Praxis bedeutet das, dass auch eine Überwindung der im zweiten Kapitel konstatierten Theoriedefizite nicht immer zu eindeutigen Handlungsempfehlungen für das Informationsmanagement führt.

Überwiegend sind allerdings einander ergänzende Aussagen zu erwarten, da die Ansätze unterschiedliche Erkenntnisinteressen bei der Analyse des Mobile Business verfolgen und dementsprechend abweichende Untersuchungsschwerpunkte setzen. So setzt der Produktionsorientierte Ansatz des Informationsmanagements in erster Linie bei den eigentlichen Aktivitäten der Informationsverarbeitung auf der objektbezogenen Ebene an, während der Systemorientierte Ansatz sich auf die Lenkung der betreffenden Anwendungssysteme unabhängig von der Art und Weise der darin konkret stattfindenden Aktivitäten bezieht. Der Institutionenökonomische Ansatz wiederum fokussiert im Wesentlichen die inhaltlichen Anforderungen an Mobile Business-Prozesse und weniger deren Umsetzung, also mehr das was als das wie bei der Gestaltung und Steuerung von Mobile Business-Anwendungen.

Die nachfolgenden Abschnitte beleuchten nacheinander die verschiedenen idealtypischen Problemstellungen des Informationsmanagements in der Praxis. Abschnitt 6.3.1 geht zunächst auf die Effektivität der technikunterstützten Informationsverarbeitung im Bereich des Mobile Business ein und untersucht die Interdependenzen der jeweils ableitbaren Aussagen. Anschließend wendet sich Abschnitt 6.3.2 der Effizienz der mobilen Informationsverarbeitung sowie den aus den verschiedenen Theorieansätzen folgenden Anhaltspunkten zur Effizienzsteigerung zu. In Abschnitt 6.3.3 wird die organisatorische Zuordnung informationswirtschaftlicher Aufgaben im Bereich des Mobile Business betrachtet. Abschnitt 6.3.4 schließlich untersucht, inwiefern die verschiedenen Theorieansätze eine angemessene Berücksichtigung der Rolle, die die beteiligten Menschen beim Aufbau und Betrieb von Mobile Business-Anwendungen spielen, zulassen.

\subsubsection{Effektivität der technikunterstützten Informationsverarbeitung im Bereich des Mobile Business}

Während die allgemeine Notwendigkeit zur Abstimmung der technikunterstützten Informationsverarbeitung mit den Zielen der Gesamtunternehmung an keiner Stelle prinzipiell in Frage gestellt wird, bieten die Ansätze im Einzelnen sehr verschiedene Anhaltspunkte, um die Effektivität der Informationsverarbeitung sicherzustellen. Dies ist zum einen darauf zurückzuführen, dass Effektivitätsgesichtspunkte nicht immer in gleichem Maße im Vordergrund der Untersuchung stehen. Zum anderen bestehen unterschiedliche inhaltliche Vorstellungen über die durch die Unternehmung zu verfolgenden Ziele, aus denen sich entsprechend unterschiedliche Vorgaben für die Ziele der technikunterstützten Informationsverarbeitung ergeben. Dies gilt auch furr die hier betrachteten Ziele von Mobile Business-Anwendungen, die sich wiederum aus den übergeordneten Zielen des technikunterstützten Informationssystems der Unternehmung als Ganzem ableiten.

Der Faktortheoretische Ansatz und insbesondere dessen hier referenzierter Teilbereich, der sich auf die Leistungserstellung als Teil der güterwirtschaftlichen Sphäre 
von Unternehmungen bezieht, behandeln Fragen der Effektivität nur am Rande. Die Unternehmungsziele stellen aus dieser Perspektive inhaltliche Vorgaben dar, die selbst nicht zum Gestaltungs- und Steuerungsbereich des Informationsmanagements gehören und somit als gegeben aufzufassen sind. Die Schnittstelle zwischen allgemeiner Unternehmungsführung und Informationsmanagement liegt in der (operativen) Produktionsprogrammplanung. Die Ziele für Mobile Business-Anwendungen ergeben sich aus dieser Perspektive durch eine Auflösung der Gesamtprogrammplanung für das übergeordnete technikunterstützte Informationssystem der Unternehmung. Dabei werden Art und Anzahl der unter Nutzung der mobilen Datenübertragung herzustellenden informationellen Produkte ermittelt. Effektivität von Mobile Business-Anwendungen liegt nach dieser Auffassung dann vor, wenn der geplante Gewinn der im Betrachtungszeitraum jeweils herzustellenden Produkte maximiert wird. Zur Bestimmung dieses Optimums müssen die technisch determinierten Produktionskoeffizienten, die zur Verfügung stehenden Elementarfaktorkapazitäten und die Stückgewinne je Produktart bekannt sein.

In Abschnitt 3.4.1.1 wurde festgestellt, dass diese Voraussetzungen nur bei bestimmten Prozesstypen der technikunterstützten Informationsverarbeitung erfüllt sind. Die betreffenden Prozesse müssen zum einen gut strukturiert sein, da sonst keine stabilen Produktionskoeffizienten vorliegen. Sie müssen zum anderen unternehmungsextern orientiert sein, damit sich der Stückgewinn für die herzustellenden Produkte ermitteln lässt. Daraus folgt, dass der Produktionsorientierte Ansatz für eine Theorie des Informationsmanagements lediglich für gut strukturierte Prozesse Anhaltspunkte zur Gewährleistung der Effektivität bietet. Diese Anhaltspunkte beziehen sich zudem nicht auf das gesamte Anwendungsgebiet Mobile Business, sondern sie beschränken sich auf den Bereich des Mobile Commerce, in dem die hergestellten informationellen Produkte als einzelne Wirtschaftsgüter ausgetauscht und vergütet werden. Allerdings dürfte bei unternehmungsextern orientierten Prozessen wegen der Unwägbarkeiten des Absatzbereichs die Unsicherheit der Planung so groß sein, dass eine modellmäßige Erfassung des Entscheidungsproblems im konkreten Einzelfall kaum möglich ist. In der Praxis ist daher auch für gut strukturierte Prozesse des Mobile Commerce eine zuverlässige Ermittlung des gewinnmaximalen Produktionsprogramms nur in Ausnahmefällen erreichbar.

Der Systemorientierte Ansatz für eine Theorie des Informationsmanagements weist einen engeren Bezug zur Effektivität der Informationsverarbeitung auf als der Produktionsorientierte Ansatz. Es wird explizit die Abstimmung des Systemgeschehens, bezogen auf den hier betrachteten Systemtyp also des Geschehens in Mobile Business-Anwendungen, mit den zu verfolgenden Zielen des Systems fokussiert. Zentraler Argumentationspunkt ist dabei die Kapazität der Lenkungsinstanz, deren Maßnahmen das Überleben des Systems sicherstellen sollen. Es werden Möglichkeiten untersucht, wie diese Instanz in die Lage versetzt werden kann, das Systemverhalten zu beeinflussen und dadurch das Ausmaß der Zielerreichung, also die Effektivität des Systems, zu verbessern. Allerdings werden keine normativen Vorgaben darüber gemacht, welche Ziele durch das System zu verfolgen sind und auf welche Weise diese definiert werden sollen. Dies impliziert im Vergleich zum Produktionsorientierten Ansatz des Informationsmanagements eine breitere Orientierung, die nicht allein auf das Gewinnziel beschränkt ist. 
Effektivitätseinbußen schlagen sich aus dieser Sicht in Abweichungen vom SollZustand des Systems nieder und werden durch äußere und innere Störungen verursacht. Zur Vermeidung solcher Abweichungen ist die Lenkbarkeit des Systems so weit wie möglich zu erhöhen. Dies lässt sich insbesondere durch die Schaffung zusätzlicher Entscheidungsstellen erreichen, die nach dem Subsidiaritätsprinzip für die Lenkung einzelner Systemteile zuständig sind. Anwendungssysteme im Bereich des Mobile Business sollten also zur Steigerung der Effektivität in weitere Subsysteme zerlegt und jeweils einzeln von einer eigenen Instanz mit Hilfe von Mischlenkungskonzepten auf die Systemziele ausgerichtet werden. Die übergeordneten Instanzen sollten ihre Einflussnahme dagegen auf die Meta-Ebene beschränken und lediglich allgemeine Vorgaben in Bezug auf den Soll-Zustand der einzelnen Teilsysteme sowie auf deren äußere Schnittstellen machen. Diese Empfehlungen lassen sich sowohl durch die Kybernetik I als auch die Kybernetik II begründen.

Aus der längerfristig orientierten Perspektive der Kybernetik II erhält die dauerhafte Überlebensfähigkeit des Systems selbst normativen Charakter und wird somit zum eigenständigen Meta-Ziel, aus dem sich Anforderungen an die Effektivität des Systems ergeben. Anwendungssysteme im Bereich des Mobile Business sind daher in die Lage $\mathrm{zu}$ versetzen, erfolgreiche Lernprozesse höherer Ordnung durchzuführen, auf deren Basis Anpassungen an veränderte System/Umweltsituationen stattfinden können. Neben einer kreativen allgemeinen Atmosphäre, die die Entstehung und Verbreitung von innovativen Impulsen fördert, eignet sich dazu auch die Institutionalisierung von Gelegenheiten zum Austausch neuer Ideen. Dies betrifft zum einen fachbezogene unternehmungsexterne Kontakte der Aufgabenträger im Bereich des Mobile Business (z.B. den Besuch von Konferenzen oder Tagungen zur Nutzung der mobilen Datenübertragung) und zum anderen einen funktions- und bereichsübergreifenden Gedankenaustausch innerhalb des Systems.

Da die geforderte starke Dezentralisierung von Entscheidungszuständigkeiten die Gelegenheiten zur zentralen Einflussnahme reduziert, resultiert die Gefahr, dass die Stabilität des Gesamtsystems durch einen sinkenden inneren Systemzusammenhalt beeinträchtigt wird. Daher kommt der Unternehmungskultur flankierende Funktion für die Sicherstellung einer hohen Effektivität von Mobile Business-Anwendungen zu. Auf der symbolischen Ebene kann das Informationsmanagement den dezentralen Entscheidungsträgern Orientierungshilfen bei der Auswahl ihrer Lenkungsmaßnahmen bieten und dadurch indirekt deren Verhalten beeinflussen. Die Einflussnahme erfolgt in diesem Fall dadurch, dass sich die Entscheidungsträger im Bereich des Mobile Business bei ihren Handlungen von den durch die Kultur vermittelten Werten und Normen leiten lassen.

Der Institutionenökonomische Ansatz für eine Theorie des Informationsmanagements macht konkretere inhaltliche Angaben über die zu verfolgenden Ziele und verwendet als Effektivitätskriterium die Koordinationseffizienz der zu begleitenden Transaktion. Strategische Ziele und Problemstellungen werden hier allerdings ebenfalls nicht im Einzelnen betrachtet. Gestaltungsmaßnahmen des Informationsmanagements bei der Nutzung der mobilen Datenübertragung sind aus dieser Perspektive effektiv, wenn sie dazu beitragen, die Effizienz der Koordination zu erhöhen. Wie in Abschnitt 5.4.1.1 erläutert, ist eine Messung der Koordinationseffizienz nicht absolut, sondern nur relativ möglich, da in der Praxis lediglich Second Best-Lösungen erreicht werden können, die eine geringere Effizienz als das theoretisch erzielbare Optimum bei vollkommenem 
Informationsstand aller Beteiligten aufweisen. Die Effektivität der die Transaktion begleitenden Informationsverarbeitungsprozesse ist dann gegeben, wenn bei der Erstellung und dem Austausch der Leistung in der vorliegenden Form relativ weniger Ressourcen verbraucht werden als bei sämtlichen alternativ möglichen Vertragsarrangements.

Folgt man der Institutionenökonomischen Auffassung, so ist die Effektivität einzelner Anwendungssysteme danach zu beurteilen, ob diese die durch den Ansatz vorgesehenen Maßnahmen zur Steigerung der Koordinationseffizienz unterstützen. Daraus ergeben sich im Vergleich zu den anderen Ansätzen des Informationsmanagements präzisere Anforderungen an die Funktionalität von Mobile Business-Anwendungen. Die Gestaltung der betreffenden Systeme sollte von den verschiedenen Arten von Informationsasymmetrien und den daraus resultierenden Gefahren ausgehen, die in den aufeinanderfolgenden Phasen der Transaktion auftreten. Für das Informationsmanagement geht es darum, durch die Nutzung der mobilen Datenübertragung bessere Möglichkeiten zur Beobachtung von Eigenschaften und Verhalten des jeweiligen Vertragspartners (insbesondere des Auftragnehmers) zu schaffen sowie eventuell auftretende diskretionäre Handlungsspielräume einzuschränken.

Die Konvergenz von mobiler und leitungsgebundener Informationsverarbeitung eröffnet vor Vertragsschluss die Möglichkeit, die Mediatisierung von Koordinationsprozessen auszudehnen. Neben den bisher verwendeten stationären Rechnern können die Nachfrager künftig auch mobile Endgeräte dazu nutzen, einen Überblick über die Marktsituation zu gewinnen und geeignete Vertragspartner zu identifizieren. Die Ubiquität des Netzzugangs wird in vielen Bereichen die Markttransparenz sprunghaft erhöhen, da die Benutzer mobiler Endgeräte jederzeit - also beispielsweise auch dann, wenn sie sich in den Verkaufsräumen eines Anbieters befinden und ein Produkt persönlich in Augenschein nehmen - die Angebote alternativer Anbieter überprüfen können. Durch die Berücksichtigung kontextspezifischer Merkmale lassen sich allein die ernsthaft in Frage kommenden Anbieter herausfiltern, um unnötige Suchaktivitäten zu vermeiden. Auch die Anbieter können kontextbezogene Filterfunktionen nutzen, um eine Individualisierung zu erreichen und ihre Leistung besser auf die Bedürfnisse der Nachfrager abzustimmen.

In der Phase nach Vertragsschluss ist die Effektivität der technikunterstützten Informationsverarbeitung am Beitrag zur Lösung der hidden action-Problematik zu messen. Hier ermöglicht der mobile Zugang zu den Anwendungssystemen im leitungsgebundenen Bereich eine verstärkte Anwendung von Workflow-Konzepten. Dadurch werden die Möglichkeiten zur direktiven Einflussnahme erneut ausgedehnt und die bisherigen Handlungsspielräume der Auftragnehmer noch mehr eingeschränkt. Ferner können die Systeme die Beobachtbarkeit des Auftragnehmers erweitern, da eine Kontrolle des Mitarbeiterverhaltens auch in Situationen möglich wird, in denen sich diese nicht unmittelbar an ihrem Arbeitsplatz befinden. Dies gilt nicht nur für gut strukturierte Prozesse, sondern auch für schlechter strukturierte Aufgabenstellungen, bei denen die mobile Datenübertragung im Rahmen von CSCW-Konzepten eingesetzt werden kann. Ein hohes Potenzial kommt ferner dem push- und pull-basierten mobilen Zugriff auf datenorientierte analytische Systeme zu, der dem Auftraggeber eine noch zeitnähere und detailliertere Kontrolle des Auftragnehmerverhaltens ermöglicht. 
Da die Koordinationseffizienz bei konkreten Gestaltungsproblemen nicht gemessen werden kann, lässt sich auch für Anwendungen im Bereich des Mobile Business nicht exakt feststellen, inwiefern die Effektivität des Systems gegeben ist. Die fehlende Möglichkeit zur präzisen Quantifizierung von Transaktions- und Agency-Kosten verhindert zum einen den zuverlässigen Vergleich zwischen verschiedenen, zur Abwicklung der Transaktion in Frage kommenden Koordinationsalternativen. Das NichtVorhandensein eines absoluten Nullpunkts bewirkt zum anderen, dass im Rahmen des gewählten und als vorteilhaft angenommenen Vertragsarrangements nicht alle Potenziale zur weiteren Effizienzsteigerung ausgenutzt werden. Das Effizienzkonzept bei begrenzter Rationalität bezieht sich immer auf den von einem einzelnen Akteur erkannten Möglichkeitsbereich, der jedoch unter Umständen nicht alle relevanten Handlungsalternativen enthält. Aus diesen Gründen kann im Prinzip jede realisierte Lösung im Bereich des Mobile Business nachträglich kaum widerlegbar als effizient erklärt werden, sodass die Effektivität der betreffenden Systeme nicht mehr als eine Näherungsgröße darstellt.

Diese Ausführungen zeigen, dass die verschiedenen Ansätze für eine Theorie des Informationsmanagements ganz unterschiedliche normative Vorgaben hinsichtlich der durch den Einsatz von Mobile Business-Anwendungen zu verfolgenden Ziele machen. Aus Sicht des Produktionsorientierten Ansatzes stehen die Produktivität von Prozessen, in denen Techniken zur mobilen Datenübertragung für die Herstellung informationeller Produkte genutzt werden, sowie der mit den betreffenden Produkten erzielbare Gewinn im Vordergrund der Betrachtung. Hier werden also die Mobile Business-Anwendungen selbst mit ihren jeweiligen Prozessen fokussiert. Dagegen hebt der Institutionenökonomische Ansatz den begleitenden Charakter der mobilen Datenübertragung und deren Bedeutung für die Abwicklung der eigentlichen Transaktion hervor. Die Betrachtung beschränkt sich hier ferner allein auf die Kostenseite der betreffenden Transaktion. Da sich diese beiden Ansätze auf rein ökonomische Gesichtspunkte unter Beachtung des Rationalprinzips konzentrieren, sind sie von der normativen Grundausrichtung her miteinander vereinbar. Die jeweils abgeleiteten Aussagen können sich daher im Prinzip ergänzen, ohne dass die gleichzeitige Bezugnahme auf beide Theorieansätze zu Inkonsistenzen in der Argumentation führen muss.

Der Produktionsorientierte Ansatz für eine Theorie des Informationsmanagements geht im Rahmen der Effektivitätsanalyse nicht über die Betrachtung der durchzuführenden Mobile Business-Prozesse hinaus, während der Institutionenökonomische Ansatz zusätzlich die zu begleitenden Leistungserstellungsprozesse als Koordinationsobjekt in die Betrachtung einbezieht. Er gibt darüber hinaus inhaltliche Hinweise für die Entwicklung von Konzepten zur Steigerung der Effektivität durch das Informationsmanagement, während der Produktionsorientierte Ansatz lediglich Anhaltspunkte zu deren Messung bietet. Tatsächlich ist die durch den Produktionsorientierten Ansatz geforderte Produktivität von Mobile Business-Prozessen allerdings auch aus Sicht des Institutionenökonomischen Ansatzes vorteilhaft, der als Vorteilhaftigkeitskriterium die gesamten Kosten, die bei der Abwicklung der Transaktion anfallen, verwendet. In diesem Sinne schließt der Institutionenökonomische Ansatz die normativen Vorgaben des Produktionsorientierten Ansatzes ein und erweitert dessen Betrachtungsfeld um die Zusammenhänge zwischen Mobile Business-Prozessen und anderen Aktivitäten der Leistungserstellung innerhalb der Unternehmung. 
Das Effektivitätskonzept des Systemorientierten Ansatzes für eine Theorie des Informationsmanagements steht nicht unmittelbar mit den beiden bisher verglichenen Konzepten im Zusammenhang. Da der Systemorientierte Ansatz vollständig auf normative Vorgaben verzichtet, sind die daraus ableitbaren Aussagen zur Erreichung der Ziele von Mobile Business-Anwendungen allgemeiner angelegt als diejenigen des Produktionsorientierten und des Institutionenökonomischen Ansatzes. Somit ist das von diesen Ansätzen vorgegebene Systemziel (Koordinations-) Effizienz als spezieller Fall aufzufassen, für den eine Anwendung des systemtheoretischen Lenkungskonzepts ebenso gut möglich ist wie für gleichfalls vorstellbare alternative Zielsetzungen.

Diese generelle Vereinbarkeit der Konzepte hat für das Informationsmanagement in der Praxis bedeutende Konsequenzen. Bei der Gestaltung von Mobile BusinessAnwendungen können die Aussagen zur Effektivität, die aus den am ökonomischen Rationalprinzip orientierten Ansätzen und dem Systemorientierten Ansatz ableitbar sind, gleichzeitig beachtet werden, ohne dass allein dadurch bereits Widersprüche impliziert werden.

Eine vollständige Komplementarität zwischen den jeweiligen Konzeptionen zur Sicherstellung der Effektivität liegt allerdings nicht vor. So betont der Systemansatz sehr stark die Chancen, die in einer dezentralen Konfiguration von Entscheidungszuständigkeiten für die Erreichung der Systemziele liegen, während der Institutionenökonomische Ansatz stärker das Risiko derartiger Gestaltungsmaßnahmen betont. Dies ist auf eine geringere Fokussierung der Interessensituation und eine optimistischere Beurteilung der Erfolgschancen von symbolischen Lenkungsmaßnahmen durch den Systemorientierten Ansatz zurückzufüren. Nicht gesamtzielkonforme Lenkungsentscheidungen auf dezentraler Ebene erfolgen nach dieser Auffassung eher unbeabsichtigt und können durch die Integrations- und Koordinationsfunktion der Unternehmungskultur weitgehend vermieden werden. Dagegen geht der Institutionenökonomische Ansatz des Informationsmanagements davon aus, dass die Interessen der dezentralen Entscheidungsträger nicht mit den übergeordneten Zielsetzungen des Gesamtsystems übereinstimmen, sodass die Gefahr eines absichtlichen Verstoßes gegen zentrale Vorgaben stärker ins Gewicht fällt. Umgekehrt wiederum sind die durch den Institutionenökonomischen Ansatz vorgeschlagenen Maßnahmen zur Verringerung dieser Gefahr (Einschränkung von Handlungsspielräumen und Reduzierung von Informationsasymmetrien) nach Auffassung des Systemorientierten Ansatzes kontraproduktiv bzw. nicht in ausreichendem Umfang realisierbar. In diesem Punkt besteht also ein teilweise konfliktäres Verhältnis zwischen den systemtheoretisch und den institutionenökonomisch begründeten Aussagen für das Informationsmanagement, sodass sich keine eindeutigen Empfehlungen für konkrete Gestaltungsmaßnahmen in der Praxis ergeben.

In ähnlich konfliktärem Zusammenhang steht das synoptische Planungsideal des Produktionsorientierten Ansatzes mit der durch den Systemorientierten Ansatz geforderten Fähigkeit zur flexiblen Reaktion auf unvorhergesehene Entwicklungen der System/Umweltsituation. Eine detaillierte und verbindliche Produktionsprogrammplanung, die nach Vorstellung des Produktionsorientierten Ansatzes die Effektivität von Mobile Business-Anwendungen gewährleistet, führt aus der Perspektive des Systemorientierten Ansatzes zu unerwünschter Unbeweglichkeit des Systems während des Planungszeitraums. Diese unterschiedlichen Einschätzungen sind auf voneinander abweichende Annahmen über die Sicherheit der Planungssituation zurückzuführen. 
Tatsächlich ist auch aus Sicht des Systemorientierten Ansatzes eine möglichst exakte Vorgabe des zu produzierenden Systemoutput vorteilhaft, wenn die Stabilität der System/Umweltsituation diese zulässt. Es wird jedoch angenommen, dass die äußere und innere Komplexität des Systems in der Regel einer solchen Vorgabe im Wege steht. Auch in diesem Zusammenhang weisen die aus den verschiedenen Theorieansätzen ableitbaren Anhaltspunkte für Maßnahmen des Informationsmanagements zur Sicherstellung der Effektivität von Mobile Business-Anwendungen in der Praxis also nicht eindeutig in die gleiche Richtung.

Die beschriebenen Widersprüche sind allerdings nur relativ geringfügig. Insgesamt sind die hier entwickelten Theorieansätze in Bezug auf die Steigerung der Effektivität von Mobile Business-Anwendungen (und darüber hinaus auch von sonstigen Anwendungssystemen im Gestaltungsbereich des Informationsmanagements) gut miteinander vereinbar. In diesem Bereich bestätigt sich also die allgemeine Erwartung, dass eine multiperspektivische Analyse des Informationsmanagements zu einem differenzierten Verständnis der in der Praxis zu lösenden Problemstellungen führt.

\subsubsection{Effizienz der technikunterstützten Informationsverarbeitung im Bereich des Mobile Business}

Die verschiedenen Ansätze für eine Theorie des Informationsmanagements bieten ebenfalls Möglichkeiten zur Ableitung von solchen Gestaltungsmaßnahmen, die sich auf die Effizienz von Anwendungssystemen des Mobile Business richten. Wie im vorigen Abschnitt dargestellt, stellt das ökonomische Rationalprinzip in normativer Hinsicht sowohl für den Produktionsorientierten Ansatz als auch für den Institutionenökonomischen Ansatz den zentralen Ausgangspunkt der Überlegungen dar. Da die gesamte Argumentation stark von diesem Ausgangspunkt geprägt ist, sind diese beiden Ansätze besonders gut für die Durchführung von Effizienzanalysen geeignet. Der Systemorientierte Ansatz stellt dagegen Effizienzfragen weniger stark in den Mittelpunkt der Betrachtung. Wenn die Effizienz selbst als langfristiges Ziel von Anwendungssystemen im Bereich des Mobile Business begriffen wird, lassen sich allerdings ebenfalls einige Anhaltspunkte zur Erreichung dieses Systemziels ableiten.

Die Aussagen des Produktionsorientierten Ansatzes für eine Theorie des Informationsmanagements beziehen sich in diesem Zusammenhang einerseits auf die Phase der Vorkombination der in Mobile Business-Anwendungen eingesetzten Elementarfaktoren und andererseits auf deren Endkombination. Zielsetzung der Gestaltungsmaßnahmen des Informationsmanagements im Rahmen der Vorkombination ist die Errichtung eines Leistungspotenzials, bei dem die verschiedenen Elementarfaktoren eine optimale Ergiebigkeit aufweisen und einen dementsprechend hohen Potenzialbeitrag leisten. In Abschnitt 3.3.1 wurden Bedingungen für eine größtmögliche Ergiebigkeit der Elementarfaktoren im Bereich der computergestützten Informationsverarbeitung abgeleitet, die auch auf Mobile Business-Anwendungen übertragbar sind.

Bei der Anschaffung von hardwaretechnischen Betriebsmitteln ist in diesem Zusammenhang zu berücksichtigen, dass der quantitative Kapazitätsbedarf nach der Realisierung erster Lösungen mit einer fortgesetzten Diffusion der mobilen Datenübertragung später tendenziell noch steigen wird. Im softwaretechnischen Bereich fällt die Tatsache besonders ins Gewicht, dass sich für viele technische Teilprobleme des Zusammenwachsens von mobilen und leitungsgebundenen Systemen noch keine 
allgemein akzeptierten Standards etabliert haben. In einem derart dynamischen Umfeld ist es aus Sicht des Faktortheoretischen Ansatzes vorteilhaft, in den meisten Anwendungsgebieten auf individuelle Lösungen zu verzichten und stattdessen auf am Markt angebotene Software zurückzugreifen, die im Bedarfsfall leichter auszutauschen ist. Im Rahmen der Vorkombination bietet die mobile Datenübertragung außerdem neue Chancen zur gleichzeitigen Erschließung der mit dem Verrichtungsprinzip und dem Prozessfolgeprinzip verbundenen Effizienzvorteile. Durch eine Verknüpfung dieser Prinzipien lässt sich bei gegebenem Elementarfaktorbestand ein besonders hohes Leistungspotenzial aufbauen.

Dieses Leistungspotenzial wird im Rahmen der Endkombination zur eigentlichen Erstellung der im jeweiligen Planungszeitraum herzustellenden informationellen Produkte genutzt. Das Instrumentarium, das der Produktionsorientierte Ansatz zur Analyse der Endkombination bereitstellt, bietet dem Informationsmanagement in der Praxis lediglich Anhaltspunkte für die Gestaltung von solchen Mobile Business-Anwendungen, die Routine- und Regelprozesse der Informationsverarbeitung unterstützen. Mit Hilfe von Produktions- und Kostenfunktionen kann im konkreten Fall eine effiziente Durchfuhrung von Leistungserstellungsprozessen der genannten Typen geplant werden. Weiterhin lassen sich auf dieser Basis nach Abschluss der Prozesse eventuell aufgetretene Ineffizienzen identifizieren und für die nächste Planungsperiode beseitigen. Darüber hinaus ermöglicht dieses Instrumentarium in der längerfristigen Perspektive einen Vergleich alternativ möglicher Prozessvarianten, die bei der Gestaltung von Mobile Business-Anwendungen in Frage kommen.

Zur Sicherstellung der Effizienz in der Entwicklungsphase von Mobile BusinessAnwendungen sollte sich das Informationsmanagement als Projektmanagement an der generell durch den Produktionsorientierten Ansatz aufgestellten Forderung nach detailgenauer Planung orientieren. Durch eine präzise Planung lässt sich beim Aufbau von Anwendungssystemen zur mobilen Datenübertragung sicherstellen, dass die im Rahmen des betreffenden Projekts verfolgten Ziele erreicht werden und keine überflüssigen Ressourcen verschwendet werden. Der Ansatz bietet allerdings kaum methodische Unterstützung zur Umsetzung dieser allgemeinen Forderung in der Praxis, da der Planungsgegenstand bei der Projektplanung tendenziell schlecht strukturierte Prozesse umfasst, auf die das für Produktionsprobleme konzipierte Planungsinstrumentarium nicht oder nur sehr eingeschränkt anwendbar ist.

Der Systemorientierte Ansatz für eine Theorie des Informationsmanagements weist nicht nur ein insgesamt geringeres Erkenntnispotenzial für die Ableitung von Maßnahmen zur Effizienzsteigerung auf, sondern verdeutlicht im Gegensatz zu den anderen Ansätzen auch Risiken, die mit einer zu starken Effizienzorientierung verbunden sind. Aus Sicht des Systemorientierten Ansatzes bedeutet eine vollständige Ausschöpfung der Ergiebigkeit von Produktionsfaktoren, dass das System über keinerlei Reserven mehr zur Reaktion auf unvorhergesehene äußere Störungen verfügt. Daher sollte die vollständige Auslastung der vorhandenen Kapazitäten lediglich im Ausnahmefall vorkommen. Dies gilt unabhängig davon, ob die faktorspezifischen Kosten sich mit dem Beschäftigungsgrad verändern. Der nicht in Anspruch genommene Kapazitätsanteil fungiert als passiver Schutz, der in dynamischen System/Umweltsituationen zur Vermeidung von Instabilitäten dient und damit die Überlebenschancen des Systems verbessert. Obwohl diese Maßnahme der kurzfristigen Effizienz zuwiderläuft, sollte das System mit um so größeren Kapazitätsreserven ausgestattet werden, je stärker seine 
äußere und innere Komplexität ausgeprägt ist. Für die Ausgestaltung von Anwendungssystemen des Mobile Business in der Praxis bedeutet dies, dass die Fähigkeit zur Bewältigung von unvorhergesehenen Beschäftigungsschwankungen nach Auffassung des Systemorientierten Informationsmanagements Priorität vor einer Optimierung der kurzfristigen Prozesseffizienz hat.

In ähnlicher Weise wirken sich auch andere Maßnahmen aus, die durch den Systemorientierten Ansatz zur Verbesserung der Systemlenkbarkeit vorgeschlagen werden. So wird empfohlen, zur Reduzierung der Komplexität von Mobile BusinessAnwendungen Subsysteme zu bilden, für die jeweils eigene Lenkungsinstanzen einzurichten sind. Damit diese Subsysteme möglichst unabhängig voneinander zur Erfüllung der Gesamtziele beitragen können, sollten sie weitgehend selbstständig fungieren und mit den anderen Subsystemen nur über klar definierte Schnittstellen verbunden werden. Diese Forderung nach Selbstständigkeit impliziert zugleich, dass potenzielle Größenvorteile, die durch die Bündelung von Ressourcen der verschiedenen Subsysteme entstehen könnten, ungenutzt bleiben. Hier ist allerdings anzumerken, dass die übergeordneten Ziele, die durch die Verwendung von Techniken zur mobilen Datenübertragung verfolgt werden (anywhere, anytime, on any device), gerade in der Verknüpfung von mobiler und leitungsgebundener Informationsverarbeitung liegen. Im Bereich des Mobile Business ist folglich nur eine weniger vollständige Isolierung der verschiedenen Subsysteme erreichbar als an anderen Stellen im Gestaltungsbereich des Informationsmanagements.

Auch symbolische Lenkungsmaßnahmen, die dem langfristigen inneren Systemzusammenhalt dienen, führen im Hinblick auf die kurzfristige Effizienz des Systemgeschehens zu vergleichbaren Auswirkungen. Dies gilt beispielsweise für institutionalisierte Zusammenküntte, bei denen sich etwa die Mitglieder von Mobile BusinessProjekten regelmäßig zum Gedankenaustausch treffen. Diese dienen zur Pflege der Kultur und können zugleich auch das Entstehen sowie die Ausbreitung von Lernimpulsen fördern. Diese Zusammenkünfte sollten aus Sicht des innovativ ausgerichteten Informationsmanagements auch dann durchgeführt werden, wenn im Einzelfall keine konkrete Zielsetzung im Hinblick auf aktuell aufgetretene Störungen des Systems vorliegt. Auch in diesen Punkten wird die relativ geringe Priorität deutlich, die das Effizienzziel für ein systemorientiert ausgerichtetes Informationsmanagement besitzt, das mit seinen Maßnahmen auf die längerfristige Überlebensfähigkeit abzielt.

Anhaltspunkte zur Steigerung der Effizienz von Mobile Business-Anwendungen bietet der Systemorientierte Ansatz nur dann, wenn man die Effizienz selbst als übergeordnetes Systemziel auffasst, dessen Nicht-Erreichung das Überleben des Systems nachhaltig bedroht. Die Kenntnis über Möglichkeiten zur effizienten Nutzung von Techniken der mobilen Datenübertragung stellt aus dieser Perspektive einen Teil der Tiefenstruktur der organisationalen Wissensbasis dar, aus dem sich Vorgaben an das Verhalten des jeweiligen Anwendungssystems ergeben. Die Einhaltung dieser Vorgaben bei akuten (äußeren und inneren) Störungen kann das konservativ ausgerichtete Informationsmanagement - wie bei anderen Systemzielen auch - durch passive und aktive Lenkungsmaßnahmen erster Ordnung sicherstellen. Darüber hinaus sind die Voraussetzungen für Lernprozesse höherer Ordnung zu schaffen, um langfristig immer neue Effizienzsteigerungen durch die Realisierung verbesserter technischer und organisatorischer Lösungen zu gewährleisten. Diese Zielsetzung kann - ebenfalls wie bei anderen Systemzielen auch - durch die beschriebenen innovativ ausgerichteten 
Maßnahmen des Informationsmanagements auf der materiellen und der symbolischen Ebene erreicht werden.

Aus der Perspektive des Institutionenökonomischen Ansatzes für eine Theorie des Informationsmanagements ist die Effizienz von Anwendungssystemen im Bereich des Mobile Business durch eine Verlagerung der Analyse auf die Meta-Ebene zu untersuchen. Die betreffenden Systeme, die als Koordinationsmedium zur Durchführung von Transaktionen genutzt werden, sind dabei selbst als Gegenstand von Koordinationsaktivitäten aufzufassen. Prozesse, in denen die Techniken zur mobilen Datenübertragung angewendet werden, stellen dann eigenständige Transaktionen dar, für die es gilt, ein hohes Maß an Koordinationseffizienz zu erreichen. Durch dieses Vorgehen wird es möglich, das von der Transaktionskostentheorie und der Agency-Theorie entwickelte Instrumentarium zur Effizienzsteigerung auf die Gestaltung und Steuerung von Mobile Business-Anwendungen zu übertragen. Die betreffenden Transaktionen werden im Folgenden als Mobile Business-Transaktionen bezeichnet.

Nach Auffassung der Transaktionskostentheorie ist die Effizienz von Mobile Business-Transaktionen einerseits von den Eigenschaften dieses speziellen Transaktionstyps und andererseits von dem zur Abwicklung dieser Transaktion gewählten vertraglichen Arrangement abhängig. Aufgabe des Informationsmanagements ist es, diese beiden Einflussgrößen der Transaktionskosten so aufeinander abzustimmen, dass der Umfang der für die Koordination aufzuwendenden Ressourcen ein Minimum erreicht. Ein echtes Entscheidungsproblem bei der Festlegung des Vertragsdesigns stellt sich allerdings nur dann, wenn überhaupt eine Loslösung der jeweiligen Aktivitäten im Umfeld des Mobile Business von den sonstigen Prozessen innerhalb der Unternehmung vorstellbar und technisch realisierbar ist. In Abschnitt 5.4.1.2 wurde allgemein für den Bereich der technikunterstützten Informationsverarbeitung festgestellt, dass diese Voraussetzungen nur bei solchen Aktivitäten gegeben sind, die nicht unmittelbar zur Koordination der eigentlichen Transaktion dienen, sondern selbst unterstützenden Charakter haben. Im Bereich des Mobile Business kann es sich dabei z.B. um die Entwicklung und Implementierung von technischen Schnittstellenkonzepten für die Verknüpfung der aufzubauenden Systeme mit den im Betrieb befindlichen standortbezogenen Anwendungssystemen der Unternehmung oder um die Wartung und Pflege des Mobile Internet-Servers handeln. Die eigentlichen mobilen Aktivitäten dürften dagegen kaum für eine eigenständige organisatorische Lösung geeignet sein.

Für unterstützende Aktivitäten im Umfeld des Mobile Business ist also zu überprüfen, welche Koordinationsform ein Höchstmaß an Transaktionseffizienz sicherstellt. Von den Transaktionseigenschaften besitzt dabei insbesondere die Spezifität zentrale Bedeutung. Wenn ohnehin ein niedriges Spezifitätsniveau vorliegt, oder wenn es möglich ist, dieses durch eine verstärkte Standardisierung der betreffenden Prozesse herzustellen, dann kann durch die verstärke Nutzung marktlicher Koordinationselemente die Anreizsituation der betreffenden Transaktion verbessert werden.

Generell gilt, dass das Spezifitätsniveau von Mobile Business-Anwendungen zumindest anfänglich tendenziell höher sein wird als das von Prozessen im Bereich der leitungsgebundenen Informationsverarbeitung. Auf den beiden obersten Ebenen des in Abschnitt 5.3.3.1 verwendeten Klassifikationsschemas liegen noch keine standardisierten Konzepte vor. Die Anbieter betriebswirtschaftlicher Anwendungssoftware 
haben zwar erste Konzepte für die Nutzung ihrer Programme durch mobil arbeitende Beschäftigte vorgelegt, von einem allgemein anerkannten Standard ist die derzeitige Situation jedoch noch weit entfernt. Das bedeutet, dass Unternehmungen, die auf dieser Ebene Lösungen zur Realisierung von Mobile Business-Prozessen entwickeln wollen, unvermeidlich eine hohe Bindung an ihr jeweiliges Konzept eingehen müssen. $\mathrm{Da}$ es sich beim Mobile Business um eine vollkommen neue Entwicklung handelt, gilt dies auch für die höchste Stufe des hier verwendeten Klassifikationsschemas, also für die betriebswirtschaftliche Ebene der betreffenden Prozesse.

Diese Überlegungen machen deutlich, dass die vergleichsweise hohe Spezifität von Mobile Business-Anwendungen in erster Linie aus der realisierten betriebswirtschaftlich-organisatorischen Lösung resultiert. Sie gilt also nicht zwangsläufig auch für die hier zu betrachtenden unterstützenden Prozesse wie etwa die Server-Pflege oder die Schnittstellenprogrammierung. Gerade bei Problemen mit stärkerem Technikbezug fallen die unternehmungsspezifischen Besonderheiten in der Regel nicht sehr stark ins Gewicht. Je nach Häufigkeit und Unsicherheit dieser Aktivitäten erscheint es vorteilhaft, auf eine vollständige Internalisierung zu verzichten und eine marktliche oder hybride Form der Koordination zu wählen. Umgekehrt gilt, dass bei unterstützenden Aufgaben mit hoher Spezifität, etwa bei der Entwicklung innovativer organisatorischer Lösungen auf Basis der mobilen Datenübertragung, eine stärkere Betonung hierarchischer Koordinationselemente erforderlich ist, da sich eine intensivere gegenseitige Bindung der Beteiligten nicht vermeiden lässt.

Auf Basis der Agency-Theorie können ergänzende Aussagen zur Ausgestaltung der einzelnen Phasen von Transaktionen im Bereich des Mobile Business abgeleitet werden. Eine möglichst hohe Effizienz von Leistungserstellung und Leistungsaustausch erfordert aus Sicht dieses Forschungszweigs der Neuen Institutionenökonomie die Überwindung der phasenspezifischen Koordinationsprobleme hidden characteristics, hidden intention und hidden action. Zur Bewältigung dieser Probleme, die jeweils durch verschiedene Arten von Informationsasymmetrien entstehen, kann das Informationsmanagement sich auf die durch den Ansatz entwickelten Maßnahmen zur Steigerung der Koordinationseffizienz stützen. Wie bei der technikunterstützten Informationsverarbeitung insgesamt kommt auch bei der Nutzung der mobilen Datenübertragung der gesamte Maßnahmenkatalog zur Disziplinierung von Auftragnehmern in Frage. Das Informationsmanagement kann die Effizienz von Mobile Business-Transaktionen also durch die Gestaltung der Anreizsituation, die Einschränkung von diskretionären Verhaltensspielräumen und durch die Reduzierung von Informationsasymmetrien, die zu Gunsten des Auftragnehmers bestehen, steigern. Diese Maßnahmen werden hier nicht erneut im Einzelnen dargestellt.

In Abschnitt 5.4.1.2 wurde festgestellt, dass die Potenziale zur Steigerung der Effizienz, die eine Mediatisierung der Koordination auf Basis innovativer informationstechnischer Lösungen bietet, auch - gewissermaßen rekursiv - auf Prozesse der technikunterstützten Informationsverarbeitung selbst angewendet werden können. Es ist daher zu überprüfen, inwieweit dies ebenfalls für den Bereich der mobilen Datenübertragung der Fall ist. In der Tat ist eine Mediatisierung von Koordinationsprozessen im Bereich des Mobile Business sowohl vor als auch nach Vertragsschluss vorstellbar und geeignet, einen positiven Beitrag zur Sicherstellung der Effizienz zu leisten. Dazu eigenen sich jedoch im Wesentlichen Anwendungssysteme der leitungsgebundenen Informationsverarbeitung, mit denen sich die Auswahl von Aufgabenträgern für Mobile 
Business-Anwendungen sowie die Koordination der von diesen durchgeführten Aktivitäten unterstützen lässt. Nur punktuell können darüber hinaus auch Lösungen der mobilen Datenübertragung, insbesondere des Mobile Work, zur Koordination von Mobile Business-Transaktionen eingesetzt werden.

Die Ausführungen dieses Abschnitts machen deutlich, dass die verschiedenen Ansätze für eine Theorie des Informationsmanagements in Bezug auf die Effizienz von Mobile Business-Anwendungen lediglich zum Teil miteinander vereinbar sind. Neben komplementären Aussagen beinhalten sie auch solche Aussagen, die untereinander ein konfliktäres Verhältnis aufweisen. Dies gilt insbesondere für den Optimalitätsanspruch des Produktionsorientierten Ansatzes, der im Gegensatz zu den beträchtlichen Kapazitätsreserven und zu einem Großteil der symbolischen Handlungen steht, die durch den Systemorientierten Ansatz zum Schutz vor Instabilitäten vorgeschlagen werden. Zwar wird auch nach den Empfehlungen des Produktionsorientierten Ansatzes die Kapazität der Elementarfaktoren nicht immer vollständig ausgenutzt. Dies hängt jedoch mit den faktorspezifischen Kostenstrukturen bei der intensitätsmäßigen Anpassung sowie der Idee der Optimalkapazität zusammen, deren Bestimmung selbst als Optimierungsproblem aufgefasst wird. Im Rahmen des Systemorientierten Ansatzes wird dagegen von der Unmöglichkeit zur vollständigen Optimierung ausgegangen, und es wird auf die Bedrohung des Systems durch unvorhergesehene Entwicklungen abgestellt. Die Ursache für die fehlende Übereinstimmung zwischen diesen beiden Theorieansätzen liegt also im Wesentlichen in unterschiedlichen Annahmen über den Informationsstand der für die Entscheidung zuständigen Personen begründet. Durch diese Unterschiede kommt es in der Praxis zu einer Konkurrenz zwischen den jeweils ableitbaren Gestaltungsempfehlungen, sodass stets zu überprüfen ist, welches Annahmensystem bezogen auf den konkreten Einzelfall tragfähiger erscheint.

Ein Vergleich zwischen den durch den Systemorientierten und den Institutionenökonomischen Ansatz für eine Theorie des Informationsmanagements gebotenen Anhaltspunkten für Gestaltungsmaßnahmen macht ähnliche Widersprüche deutlich. Die Agency-Theorie schlägt, wie erläutert, unter anderem eine Einschränkung von diskretionären Handlungsspielräumen der Aufgabenträger im Bereich des Mobile Business vor, um eine Disziplinierung dieser Auftragnehmer und somit eine Steigerung der Koordinationseffizienz zu erreichen. Dies steht in diametralem Gegensatz zu der allgemein durch den Systemorientierten Ansatz empfohlenen Dezentralisierung von Entscheidungskompetenzen, mit der eine Steigerung der Lenkungskapazität erzielt und die Überlebensfähigkeit von Mobile Business-Anwendungen verbessert werden soll. Auch in diesem Punkt sind die Abweichungen auf divergierende Annahmen über den Erkenntnisgegenstand zurückzuführen. Während sich die Systemtheorie allein auf die Betrachtung von Informationsunvollkommenheiten konzentriert, stellt die Neue Institutionenökonomie explizit auch Interessengegensätze zwischen Principal und Agent in den Mittelpunkt der Untersuchung. Damit kommt es ebenfalls zu einer Konkurrenzsituation zwischen den jeweils aus den Theorieansätzen ableitbaren Empfehlungen, die es bei konkreten Gestaltungsproblemen notwendig machen, die Angemessenheit der unterschiedlichen Annahmensysteme zu überprüfen. Im Einzelfall ist dabei insbesondere die Frage zu beantworten, inwieweit die jeweilige Unternehmungskultur geeignet ist, die Interessen der verschiedenen Akteure im Umfeld der betreffenden Mobile Business-Anwendung erfolgreich anzugleichen. 
Die Beziehung zwischen dem Systemorientierten Ansatz für eine Theorie des Informationsmanagements und den beiden anderen Ansätzen ist allerdings auch im Bereich der Effizienz nicht ausschließlich konfliktär. Dies betrifft insbesondere diejenigen Aussagen, die aus dem Systemorientierten Ansatz (speziell aus dem Teilbereich, der sich auf die Kybernetik II bezieht), abgeleitet werden können, wenn Effizienzsteigerungen als Lemprozesse aufgefasst werden. Diese Aussagen sind mit dem Produktionsorientierten und dem Institutionenökonomischen Ansatz vereinbar und geeignet, deren eher kurzfristig ausgerichtete Empfehlungen zur Effizienz von Mobile Business-Anwendungen zu ergänzen. Tatsächlich ist es auch aus Sicht der am ökonomischen Rationalprinzip orientierten Ansätze vorteilhaft, die nachhaltige Fähigkeit zur wiederholten Realisierung von Effizienzsteigerungen herzustellen, selbst wenn dadurch kurzfristig nicht alle Kapazitätsreserven vollständig ausgeschöpft werden. Die Empfehlungen zur Auslösung und Förderung von Lernprozessen höherer Ordnung durch das Informationsmanagement sind daher auch aus Sicht der beiden anderen Ansătze akzeptabel, sodass in diesem Punkt eine komplementäre Beziehung zwischen den jeweiligen Aussagesystemen besteht.

\subsubsection{Organisatorische Zuordnung informationswirtschaftlicher Aufgaben im Bereich des Mobile Business}

Die organisatorische Zuordnung informationswirtschaftlicher Aufgaben stellt bei der Nutzung der mobilen Datenübertragung, wie im Bereich der leitungsgebundenen Informationsverarbeitung auch, eine kritische Größe für den Erfolg der verwendeten Anwendungssysteme dar. Aus den hier entwickelten Ansätzen für eine Theorie des Informationsmanagements können zu dieser Problematik ebenfalls Anhaltspunkte für konkrete Gestaltungsmaßnahmen in der Praxis abgeleitet werden. Nach Auffassung des Institutionenökonomischen Ansatzes ist die Frage der organisatorischen Zuordnung von Aufgaben allerdings nicht von der Untersuchung von Effizienzaspekten zu lösen. Deshalb erfolgte bereits im vorangegangenen Abschnitt eine integrierte Analyse beider Problemstellungen aus Sicht dieses Ansatzes. Dabei wurde in erster Linie die Koordinationsform, über die Leistungserstellung und Leistungsaustausch bei Mobile Business-Transaktionen abgewickelt werden, insgesamt fokussiert. Weniger betrachtet blieb dagegen die unternehmungsinterne Organisation der entsprechenden Prozesse. Dagegen liefern der Produktionsorientierte und der Systemorientierte Ansatz solche Gestaltungsempfehlungen, die sich stärker auf interne Organisationsfragen von Mobile Business-Anwendungen beziehen.

Der Produktionsorientierte Ansatz für eine Theorie des Informationsmanagements stellt in diesem Zusammenhang insbesondere den Delegationsaspekt in den Vordergrund der Untersuchung. Die Zuordnung von Anordnungs- und Entscheidungsbefugnissen im Bereich des Mobile Business sollte sich an der Tragweite der Entscheidung sowie an der Interdependenz zu anderen Unternehmungsteilbereichen orientieren. Wenn nur ein geringes Delegationsrisiko vorliegt, dann kann eine dezentrale Lösung realisiert werden, um die übergeordneten Instanzen zu entlasten. Dies ist regelmäßig bei Entscheidungszuständigkeiten für gut strukturierte Prozesse zur Erfüllung von Routine- und Regelaufgaben der mobilen Informationsverarbeitung der Fall. Dagegen sollten Entscheidungszuständigkeiten für Projekt- und Einzelfallaufgaben im Bereich des Mobile Business, bei denen tendenziell ein höheres Delegationsrisiko wegen größerer Tragweite sowie intensiverer Interdependenzen zu anderen Unternehmungs- 
bereichen vorliegt, zentraler zugeordnet werden. Dies gilt auch für richtungsweisende Entscheidungen beim Aufbau von Mobile Business-Anwendungen, denen zum einen hohe Bedeutung für die Wettbewerbsposition der Unternehmung zukommt und die zum anderen erhebliche Auswirkungen auf bestehende Prozesse in der gesamten Unternehmung haben können.

Die Leitungsspanne und Gliederungstiefe organisatorischer Einheiten, zu deren Konfiguration der Faktortheoretische Ansatz ebenfalls Anhaltspunkte liefert, richtet sich nur dann allein nach Gesichtspunkten des Informationsmanagements, wenn diese Einheiten ausschließlich im Bereich der technikunterstützten Informationsverarbeitung tätig sind. Bezogen auf das Mobile Business triff dies im Wesentlichen auf unterstützende Funktionen wie die Wartung und Pflege eines Multi Access-Portals oder interne Beratungsleistungen zur Entwicklung innovativer Anwendungslösungen zu. Für diese Aufgabenstellungen sollte aus Sicht des Faktortheoretischen Ansatzes so weit wie möglich die Einheit der Auftragserteilung gewährleistet werden. Eine hohe Leitungsspanne (verbunden mit geringer Gliederungstiefe) ist für die entsprechenden Organisationseinheiten dann vorteilhaft, wenn ein hoher Anteil an generellen Regelungen realisierbar ist und die übergeordnete Instanz sich auf disponierende Tätigkeiten konzentrieren kann. Der mögliche Anteil genereller Regelungen wiederum hängt im konkreten Fall von der Gleichförmigkeit des Geschehens ab. Wenn die betrachteten Aufgaben im Umfeld der mobilen Datenübertragung nur eine geringe Gleichformigkeit aufweisen, sind fallweise Regelungen unvermeidbar. Unter diesen Bedingungen wird die übergeordnete Instanz in stärkerem Ausmaß mit fachlichen Problemen belastet und kann folglich nur eine vergleichsweise geringe Leitungsspanne bewältigen.

Es kann angenommen werden, dass die Dynamik des Geschehens beim Aufbau und Betrieb von Mobile Business-Anwendungen zumindest anfänglich besonders stark ausgeprägt ist. Die Begründung für diese Annahme liegt in dem hohen Innovationsgrad der verwendeten Technik. Da viele technische Standards im Umfeld der mobilen Datenübertragung erst noch entwickelt werden müssen, ist eine Konkurrenz zwischen alternativen Konzepten zu erwarten, die bei den anwendenden Unternehmungen die Fähigkeit zu flexiblem Reagieren auf veränderte Marktsituationen notwendig macht. Darüber hinaus liegen auf der Anwendungsebene zunächst keinerlei oder kaum Erfahrungen mit der Durchführung von Mobile Business-Prozessen vor, sodass etwa potenzielle Fehlerquellen, Möglichkeiten zur Beseitigung akut aufgetretener Probleme oder vorteilhafte fachliche Lösungen nicht als bekannt vorausgesetzt werden können. Die Gleichförmigkeit des Geschehens dürfte daher weitaus geringer ausgeprägt sein als bei der leitungsgebundenen Informationsverarbeitung und an vielen anderen Stellen in der Unternehmung. Der Produktionsorientierte Ansatz für eine Theorie des Informationsmanagements kommt daher zu dem Ergebnis, dass im Bereich des Mobile Business insgesamt mit einer vergleichsweise niedrigeren Leitungsspanne und einer entsprechend hohen Gliederungstiefe gearbeitet werden sollte.

Der Systemorientierte Ansatz für eine Theorie des Informationsmanagements setzt bei der Untersuchung der organisatorischen Zuordnung von Aufgaben am Gesetz der erforderlichen Varietät an. Aus dieser Perspektive beeinträchtigt eine vollständige Zentralisierung von Zuständigkeiten die Stabilität des Systems, da Mobile BusinessAnwendungen einer so hohen Störvarietät ausgesetzt sind, dass eine einzelne Instanz mit der Lenkung dieses Anwendungssystemtyps überfordert ist. Stattdessen, so die weitere Argumentation, sind Mobile Business-Anwendungen in Subsysteme zu unter- 
teilen, um die Lenkungskapazität relativ zur äußeren und inneren Störvarietät zu erhöhen und damit die Voraussetzungen zum Überleben des Systems zu verbessern. Mit der Dezentralisierung von Entscheidungszuständigkeiten wird hier also weniger das Risiko der Delegation als die Chance zur schnelleren und angemesseneren Reaktion auf unvorhergesehene Entwicklungen verbunden. Zur Ausschöpfung dieses Potenzials sind die dezentralen Instanzen mit einem hohen $\mathrm{Ma}$ an Entscheidungsfreiheit auszustatten.

Die Bildung von Subsystemen sollte aus Sicht der Kybernetik I so weit fortgesetzt werden, bis die Lenkungsinstanz in der Lage ist, die Komplexität des jeweiligen Subsystems zu bewältigen und die Stabilität des Systems dauerhaft zu gewährleisten. Die Kombination von aktiven und passiven Lenkungsmaßnahmen sowie die Überlagerung von Regelkreisen machen es möglich, den Output der einzelnen Subsysteme so exakt zu bestimmen, dass eine präzise Lenkung erreicht wird. Dagegen kann nach Auffassung der Kybernetik II auch bei fortgesetzter Subsystembildung keine ausreichende Komplexitätsreduktion erreicht werden, um eine vollständige Beherrschbarkeit von Anwendungssystemen im Bereich des Mobile Business herzustellen. Die Reaktion auf unvorhergesehene Entwicklungen erfolgt aus dieser Perspektive nicht mit Hilfe von Lenkungsmaßnahmen einer einzelnen Entscheidungsinstanz, sondern sie kann nur selbstständig innerhalb der einzelnen Teilsysteme stattfinden. Daraus ergibt sich die Empfehlung, die Handlungsfreiheit der Aufgabenträger in Mobile Business-Anwendungen noch weiter zu erhöhen und Entscheidungskompetenzen noch weiter zu dezentralisieren als dies aus Sicht der Kybernetik I vorteilhaft erscheint.

Dies bedeutet, dass zentrale Einflussnahmen auf das Geschehen in Mobile BusinessAnwendungen nur insoweit vorzunehmen sind, wie es nötig ist, um eine ausreichende Erwartbarkeit des Subsystemverhaltens für die anderen Subsysteme in der Unternehmung herzustellen. Die zentralen Instanzen sollten ihre Interventionen folglich auf die Bildung und Kopplung von Subsystemen beschränken. Sie sollten zum einen die Ziele der einzelnen Subsysteme im Bereich des Mobile Business vorgeben und mit den übergeordneten Zielen der Unternehmung abstimmen. Zum anderen ist es ihre Aufgabe, die Schnittstellen zwischen diesen Subsystemen sowie zwischen der mobilen und der leitungsgebundenen Informationsverarbeitung insgesamt festzulegen. Eine Einflussnahme bei konkreten Lenkungsproblemen ist dagegen nicht wünschenswert, da die dezentralen Lenkungsinstanzen über einen besseren Informationsstand verfügen und somit in der Lage sind, angemessener auf akute Störungen des Subsystems zu reagieren. Diese Informationsasymmetrien zu Gunsten der dezentralen Stellen lassen sich auch durch innovative informations- und kommunikationstechnische Lösungen nicht vollständig beseitigen.

Neben den bisher betrachteten Aussagen zur Zuordnung von Führungsaufgaben bietet der Systemorientierte Ansatz für eine Theorie des Informationsmanagements auch Anhaltspunkte für die organisatorische Zuordnung von Durchführungsaufgaben im Bereich des Mobile Business. Die Empfehlung, kleine, weitgehend selbstständig funktionierende Subsysteme zu bilden, um die Lenkbarkeit des Gesamtsystems zu erhöhen, kann nur dann konsequent umgesetzt werden, wenn sie durch eine geeignete organisatorische Konfiguration der Durchführungsaufgaben begleitet wird. Eine zentrale Ansiedlung dieser Aufgaben würde der geforderten Selbstständigkeit der Subsysteme entgegenlaufen, da sie die dezentralen Einheiten in höherem Ausmaß von Entwicklungen, die nicht in ihrem Einflussbereich liegen, abhängig macht. Aus diesem Grund ist 
aus Sicht des Systemorientierten Ansatzes eine dezentrale Ansiedlung vorzuziehen, da sich dadurch die Interdependenzen zwischen den verschiedenen Einheiten reduzieren lassen, sodass die äußere Varietät der einzelnen Subsysteme reduziert wird. Das bedeutet, dass die dezentralen Einheiten im Bereich des Mobile Business mit ausreichenden Mitteln ausgestattet werden sollten, um ihre Aufgaben ohne größere Unterstützung des Gesamtsystems zu lösen. Dies könnte im konkreten Fall beispielsweise Mitarbeiterkapazitäten im Bereich des Web-Design oder der Hardware-Wartung und Pflege betreffen, bei denen Anwendungssysteme des Mobile Business nicht auf zentrale Ressourcen angewiesen sein sollten. Eine dezentrale Ansiedlung von Zuständigkeiten für diese Aufgabenfelder erhöht zugleich die langfristige Anpassungsfähigkeit der jeweiligen Anwendungssysteme. Dies schafft nicht nur die Voraussetzungen zur schnelleren Erkennung von Anpassungsnotwendigkeiten, sondern die erforderlichen Veränderungsprozesse lassen sich auch leichter durchführen als bei zentralisierten Lösungen mit starken Interdependenzen zu anderen Systemteilen.

In Bezug auf die organisatorische Zuordnung informationswirtschafticher Aufgaben im Bereich des Mobile Business bestehen also ebenfalls sowohl komplementäre als auch konkurrierende Beziehungen zwischen den aus den verschiedenen Theorieansätzen ableitbaren Gestaltungsempfehlungen. Komplementarität ist zunächst einmal deshalb gegeben, weil sich die Aussagen des Institutionenökonomischen Ansatzes gegenüber denen der beiden anderen Ansätze auf eine abweichende Fragestellung beziehen. Die unternehmungs- bzw. systeminterne organisatorische Konfiguration von Mobile Business-Anwendungen kann weitgehend unabhängig davon untersucht werden, ob die erstellte Leistung innerhalb der gleichen Unternehmung verwendet oder an einen anderen selbstständigen Akteur im Wirtschaftsgeschehen übergeben wird. Tatsächlich liefert der Institutionenökonomische Ansatz für eine Theorie des Informationsmanagements, der sich hauptsächlich mit der zuletzt genannten Fragestellung befasst, kaum Hinweise zum internen organisatorischen Aufbau von Mobile BusinessAnwendungen. Er kann somit die beiden anderen Ansätze ergänzen und bedarf seinerseits der Ergänzung durch deren intern ausgerichtete Aussagen.

Die Beziehung zwischen den aus dem Produktionsorientierten und den aus dem Systemorientierten Ansatz ableitbaren Anhaltspunkten für die organisatorische Zuordnung von Aufgaben des Mobile Business in der Praxis hat allerdings überwiegend konkurrierenden Charakter. Dies ist insbesondere auf die unterschiedliche Beurteilung einer dezentralen Ansiedlung von Entscheidungs- bzw. Lenkungskompetenzen zurückzuführen. Während der Produktionsorientierte Ansatz in diesem Zusammenhang stärker das mit der Delegation verbundene Risiko hervorhebt, betont der Systemorientierte Ansatz die Chance einer besseren und schnelleren Entscheidung durch die dezentralen Lenkungsinstanzen. Vor diesem Hintergrund kommen beide Ansätze zu unterschiedlichen Ergebnissen hinsichtlich der im Bereich des Mobile Business vorteilhaften organisatorischen Ansiedlung von Zuständigkeiten.

Wegen des besonders dynamischen Umfelds und der potenziell hohen Bedeutung von Mobile Business-Lösungen für die Gesamtunternehmung empfiehlt der Produktionsorientierte Ansatz, den jeweils übergeordneten Instanzen verstärkt auch fachbezogene Entscheidungen zuzuordnen. Da dies zu einer stärkeren Belastung des dispositiven Faktors führt, sind die Leitungsspanne zu reduzieren und die Gliederungstiefe zu erhöhen. Mit einer sehr ähnlichen Begründung, die sich ebenfalls auf das dynamische Umfeld von Mobile Business-Anwendungen bezieht, kommt der Systemorientierte 
Ansatz zu einem genau entgegengesetzten Ergebnis. Die Empfehlung lautet hier, die Entscheidungszuständigkeiten besonders stark zu dezentralisieren, um die erforderliche, besonders große Lenkungskapazität herzustellen. Umgekehrt ist die Schlussfolgerung zulässig, dass eine hohe Leitungsspanne möglich ist, da die Lenkungsinstanzen kaum durch situative Einflussnahmen in Anspruch genommen werden. Damit ergeben sich für das Informationsmanagement in der Praxis widersprüchliche Gestaltungsempfehlungen, sodass sich nahezu jede realisierte organisatorische Konfiguration von Aufgaben des Mobile Business durch Bezugnahme auf den einen oder den anderen der beiden Ansätze rechtfertigen lässt.

Da nicht von einer generellen Dominanz eines dieser Ansätze ausgegangen werden kann, stellt sich im Einzelfall bei konkreten Gestaltungsproblemen die Frage, welcher Ansatz tragfähigere Gestaltungsempfehlungen hinsichtlich der Zuordnung von Zuständigkeiten im Bereich des Mobile Business liefert. Die Antwort darauf hängt im Wesentlichen davon ab, welche Urteils- und Handlungsfähigkeit den dezentralen Aufgabenträgern zugetraut wird. Ist davon auszugehen, dass die Mitarbeiter nur eine relativ geringe fachliche Qualifikation besitzen, so erscheint die Delegation von Entscheidungen entsprechend der Auffassung des Produktionsorientierten Ansatzes tatsächlich nur als Notlösung zur Entlastung der vorgesetzten Personen. Verfügen die im Bereich des Mobile Business beschäftigten Mitarbeiter der Unternehmung dagegen auf Grund einer hohen fachlichen Qualifikation über ein zuverlässiges Urteilsvermögen, so erscheint die Verlagerung von Entscheidungskompetenzen weitaus erfolgversprechender. In diesem Fall ist tendenziell eher der Auffassung des Systemorientierten Ansatzes zu folgen, der eine verstärkte Dezentralisierung von Lenkungskompetenzen und eine hohe Handlungsfreiheit der dezentralen Aufgabenträger fordert. Den (durch den Institutionenökonomischen Ansatz besonders hervorgehobenen) Interessengegensätzen zwischen zentralen und dezentralen Stellen sollte dabei durch eine Intensivierung der symbolischen Maßnahmen des Informationsmanagements entgegengewirkt werden.

Abschließend ist zu überprüfen, inwiefern aus den verschiedenen Ansätzen für eine Theorie des Informationsmanagements unterschiedliche Empfehlungen zur organisatorischen Zuordnung von Durchführungsaufgaben resultieren. Der Systemorientierte Ansatz schlägt in diesem Zusammenhang eine möglichst umfassende Trennung der einzelnen Subsysteme vor, durch die das zu bewältigende Komplexitätsniveau auf ein handhabbares Maß reduziert werden soll. Dieses Vorgehen weist auch aus Sicht des Institutionenökonomischen Ansatzes Vorteile auf, da die Verringerung von Abhängigkeiten das externe Risiko bei der Erstellung der Leistung durch die jeweiligen Auftragnehmer reduziert. Somit wird es furr den Auftraggeber besser möglich, das Verhalten des Auftragnehmers an Hand der erzielten Ergebnisse zu beurteilen. Eine derartige Konfiguration erfüllt ferner das durch den Produktionsorientierten Ansatz vertretene Postulat der Einheit der Auftragserteilung, da die fachlichen und die disziplinarischen Leitungsstrukturen übereinstimmen. Einschränkend ist allerdings darauf hinzuweisen, dass unter den durch den Produktionsorientierten Ansatz betrachteten Produktivitätsgesichtspunkten im Einzelfall auch die Zusammenfassung gleicher Aufgabenfelder aus unterschiedlichen Abteilungen mit dem Ziel der Erschließung von Größenvorteilen vertretbar sein kann. $\mathrm{Da}$ dies gleichfalls aus Sicht des Institutionenökonomischen Ansatzes die Gesamteffizienz von Leistungserstellung und Leistungsaustausch steigern kann, ergeben sich auch in diesem Punkt keine eindeutigen Pauschalempfehlungen für das Informationsmanagement in der Praxis. 


\subsubsection{Rolle des Menschen bei der Informationsverarbeitung im Bereich des Mobile Business}

Die in dieser Arbeit entwickelten Ansätze für eine Theorie des Informationsmanagements können insgesamt nur einen relativ geringen Beitrag zu einer angemesseneren Berücksichtigung der Rolle des Menschen in der Informationsverarbeitung leisten. Dies ist darauf zurückzuführen, dass keiner der Ansätze eine differenzierte Modellierung des menschlichen Verhaltens vornimmt. Der Produktionsorientierte und der Institutionenökonomische Ansatz betrachten das Handeln von Menschen allein unter dem Aspekt der Rationalität und treffen dabei unterschiedliche Annahmen über den Informationsstand der beteiligten Personen. Der Systemorientierte Ansatz wiederum erkennt die Persönlichkeit der menschlichen Elemente von Systemen zwar an und reduziert deren Verhalten nicht ausschließlich auf ökonomisch motivierte Handlungen. Eine inhaltliche Auseinandersetzung mit der Vielschichtigkeit menschlichen Verhaltens findet jedoch auch hier nicht statt. Nachfolgend wird untersucht, welche Anhaltspunkte zur Gestaltung von Mobile Business-Anwendungen auf dieser vergleichsweise schmalen Basis abgeleitet werden können.

Aus Sicht des Produktionsorientierten Ansatzes sind im Bereich der menschlichen Arbeitsleistungen allgemein einerseits solche mit objektbezogenem Charakter und andererseits dispositiv-anordnende Tätigkeiten zu unterscheiden. Diese Unterscheidung kann auch für Arbeitsleistungen bei der Nutzung der mobilen Datenübertragung vorgenommen werden. Aus dieser Perspektive wird die Gestaltung von Mobile BusinessAnwendungen durch das Informationsmanagement der rationalen und der gestaltendvollziehenden Schicht des dispositiven Faktors zugeordnet. Folglich sollte sich die Analyse dieser Tätigkeiten allein am ökonomischen Prinzip orientieren und von Einflüssen, die mit der Person des Aufgabenträgers zusammenhängen, abstrahieren. Dieser Theorieansatz trifft daher keine Aussagen, die sich auf die Träger von Aufgaben des Informationsmanagements im Umfeld des Mobile Business beziehen. Die Rolle, die diese Akteure beim Aufbau und Betrieb der betreffenden Anwendungssysteme spielen, wird nicht näher betrachtet.

Ein ähnliches Ergebnis ergibt sich auch für die objektbezogenen menschlichen Arbeitsleistungen bei der Nutzung der mobilen Datenübertragung. Diese werden als Elementarfaktoren aufgefasst, die lediglich unter Effizienzgesichtspunkten zu betrachten sind. Einflüsse, die mit der Person der Aufgabenträger im Bereich des Mobile Business zusammenhängen, werden dabei als subjektive Bedingungen der Ergiebigkeit klassifiziert, die in ihrer Gesamtheit das individuelle Eignungspotenzial der einzelnen Person ausmachen. Aufgabe des Informationsmanagements ist es nach dieser Vorstellung, einen Ausgleich zwischen dem benötigten und dem vorhandenen Eignungspotenzial der Mitarbeiter, die der Unternehmung für die Durchfürung von Mobile Business-Prozessen zur Verfugung stehen, herzustellen. Die Notwendigkeit von Qualifikationsmaßnahmen und Personalbeschaffungsaktivitäten (bzw. gegebenenfalls Personalfreisetzungsaktivitäten) ergibt sich dabei aus dem zuvor ermittelten Personalbedarf, an den das vorhandene, gesamte Eignungspotenzial möglichst präzise anzupassen ist. Der Zusammenhang zwischen persönlichen Charaktermerkmalen und der individuellen Eignung für die Erfüllung einer bestimmten Aufgabenstellung im Umfeld des Mobile Business wird durch den Ansatz nicht näher untersucht. 
Diese Überlegungen machen deutlich, dass sich Aussagen, die aus dem Produktionsorientierten Ansatz für eine Theorie des Informationsmanagements zum Aufbau und Betrieb von Mobile Business-Anwendungen ableitbar sind, maßgeblich an der Konstruktionskorridor-Vorstellung orientieren, ohne dass dies explizit zum Ausdruck gebracht wird. Der Ansatz liefert weder Anhaltspunkte dazu, auf welche Weise die späteren Anwender bereits in den Prozess der Entwicklung derartiger Anwendungssysteme einzubeziehen sind, noch dazu, welche potenziellen Konflikte in der Nutzungsphase auftreten und wie diese gelöst werden können. Stattdessen beschränkt er sich auf die Forderung nach Herstellung eines Gleichgewichts zwischen erforderlichen und verfügbaren Qualifikationen.

Der Systemorientierte Ansatz für eine Theorie des Informationsmanagements sieht die Aufgabenträger im Bereich des Mobile Business in erster Linie als Quelle von systeminterner Komplexität. Das Verhalten dieser menschlichen Akteure kann nicht im Einzelnen vorgegeben werden, sodass im Vergleich zu rein technischen Systemen erhöhte Anforderungen an die Kapazität der Lenkungsinstanz bestehen. Die Rolle des Menschen bei der Durchführung von Mobile Business-Prozessen liegt hier also einerseits in der Erbringung von Funktionen zur Erfüllung der Anwendungssystemziele, andererseits jedoch in der Verursachung von Störungen, die zu Zielabweichungen führen können. Nach Auffassung der Kybernetik I sollte ein konservativ ausgerichtetes Informationsmanagement darauf hinwirken, das Ausmaß an unerwünschten Verhaltensabweichungen einzudämmen, um die Stabilität des Systemoutput sicherzustellen. Durch die Bildung von Subsystemen und den Einsatz von Mischlenkungskapazitäten ist die relative Kapazität der Lenkungsinstanzen so weit zu erhöhen, bis sie die jeweils wirkende Störvarietät erfolgreich ausgleichen können.

Die Kybernetik II geht dagegen davon aus, dass die skizzierte Zielsetzung der Kybernetik I wegen der unüberwindbaren Komplexität, die Anwendungssysteme im Bereich des Mobile Business aufweisen, nicht erreichbar ist. In der unvollständigen Möglichkeit der externen Bestimmung menschlichen Handelns wird allerdings auch die Chance zur Entstehung von Lernprozessen gesehen, durch die sich das System an veränderte Situationen anpasst. Ein innovativ orientiertes Informationsmanagement sollte sich daher nicht auf die Beseitigung der durch die Aufgabenträger im Bereich des Mobile Business verursachten inneren Varietät, sondern auf deren Kanalisierung richten. Dieses Konzept zielt im Kern auf die Ausnutzung von Informationsvorsprüngen, die die dezentralen Aufgabenträger innerhalb des Systems gegenüber der zentralen Instanz besitzen. Daraus ergeben sich vergleichsweise hohe Anforderungen an die fachlichen und persönlichen Kompetenzen der betreffenden Mitarbeiter, von deren Lernfähigkeit das langfristige Überleben des gesamten Systems und somit indirekt auch der gesamten Unternehmung abhängt.

Der Systemorientierte Ansatz furr eine Theorie des Informationsmanagements vollzieht also eine Abkehr vom mechanistischen Menschenbild, ohne jedoch eine tiefergehende Analyse des menschlichen Verhaltens vorzunehmen. Obwohl die persönlichen Bedürfnisse von Aufgabenträgern des Mobile Business nicht im Mittelpunkt der Untersuchung stehen, sind die durch den Ansatz gegebenen Gestaltungsempfehlungen geeignet, deren Motivation und Zufriedenheit zu fördern. Dies stellt jedoch lediglich einen willkommenen Nebeneffekt der Maßnahmen eines Systemorientierten Informationsmanagements dar, dessen Absicht allein in der Verbesserung der Lenkungsmöglichkeiten liegt. Wie der Produktionsorientierte Ansatz ist auch der Systemorientierte 
Ansatz nicht in der Lage, eine vollständige Überwindung der KonstruktionskorridorVorstellung zu leisten, da ebenfalls keine inhaltliche Auseinandersetzung mit den Bestimmungsgrößen des menschlichen Verhaltens stattfindet. Daher bieten sich nur wenige Anhaltspunkte für eine angemessenere Berücksichtigung der Rolle des Menschen beim Aufbau und Einsatz von Mobile Business-Anwendungen durch das Informationsmanagement in der Praxis.

Im Gegensatz zu den beiden bisher behandelten Theorieansätzen trifft der Institutionenökonomische Ansatz explizite inhaltliche Annahmen über das Verhalten der am Wirtschaftsgeschehen beteiligten Menschen. Aus diesem Grund bietet der Ansatz bessere Voraussetzungen für das Informationsmanagement, im Rahmen des Aufbaus und Betriebs von Mobile Business-Anwendungen in der Praxis eine verstärkte Akteursorientierung zu Grunde zu legen. Bei den zentralen Verhaltensannahmen dieses Ansatzes handelt es sich um die Ausrichtung des Handelns am individuellen Nutzen, die begrenzte Rationalität der Entscheidungsfindung und die Gefahr des Opportunismus. Geht man davon aus, dass das Verhalten der menschlichen Akteure bei der Nutzung der mobilen Datenübertragung nicht von dem Verhalten anderer Personen im Wirtschaftsgeschehen abweicht, so sind die genannten Verhaltensannahmen auch auf diesen Bereich zu übertragen.

Aus Sicht des Institutionenökonomischen Ansatzes für eine Theorie des Informationsmanagements ist also davon auszugehen, dass die jeweiligen Aufgabenträger eigenen Zielsetzungen nachgehen, die nicht mit den „offiziellen“ Zielen, die durch den Einsatz von Mobile Business-Anwendungen verfolgt werden, übereinstimmen. Dies betrifft nicht nur die für Durchfuhrungsaufgaben im Bereich des Mobile Business zuständigen Personen, sondern auch die Träger von Aufgaben des Informationsmanagements, die selbst als Auftragnehmer für übergeordnete hierarchische Instanzen fungieren. Aus der Annahme, dass alle Beteiligten lediglich eingeschränkt rational handeln, folgt in zweierlei Hinsicht die Gefahr von suboptimalen Handlungsentscheidungen. Diese können einerseits unbeabsichtigt sein und allgemein aus Informationsunvollkommenheiten, die bei der Erfüllung der jeweiligen Aufgabe bestehen, resultieren. Sie können andererseits beabsichtigt sein und auf die opportunistische Ausnutzung von Handlungsspielräumen abzielen. Im Zusammenhang mit der mobilen Datenübertragung können sich die Interessen der Aufgabenträger beispielsweise auf die Nutzung der jeweiligen Endgeräte zu Unterhaltungszwecken oder auf private Aktivitäten beziehen, die erst dadurch möglich werden, dass mit der Realisierung von Mobile Business-Lösungen die erforderliche Anwesenheitszeit am Arbeitsplatz reduziert wird.

Aus diesen Überlegungen ergibt sich die Schlussfolgerung, dass ohne begleitende Koordinationsmaßnahmen kein reibungsloser Betrieb von Mobile Business-Anwendungen gewährleistet werden kann. Dies gilt ferner nicht nur für die Nutzungsphase von Mobile Business-Anwendungen, sondern auch für Projekte zur Entwicklung derartiger Systeme, in denen es ebenfalls zu nicht vernachlässigbaren Interessenkonflikten zwischen den verschiedenen Beteiligten kommen kann. Es ist zu erwarten, dass in solchen Projekten neben den durch die Unternehmungsleitung vorgegebenen Zielen beispielsweise auch Abteilungsinteressen, Karriereinteressen und allgemeine persönlich Überzeugungen eine Rolle spielen. Im Umfeld der mobilen Datenübertragung sind daher, wie an anderen Stellen auch, die Schaffung von Anreizen, die Einschränkung von diskretionären Verhaltensspielräumen und der Aufbau von 
Monitoring-Systemen erforderlich, um die unvermeidlich auftretenden Agency-Kosten zu reduzieren.

Genau genommen nimmt der Institutionenökonomische Ansatz allerdings ebenfalls keine Auseinandersetzung mit der Vielschichtigkeit menschlichen Verhaltens vor, da das Streben nach persönlicher Nutzenmaximierung sehr stark in den Vordergrund gestellt wird. Dieses Vorgehen reduziert soziales Geschehen auf rein ökonomische Gesichtspunkte und blendet weitere Einflüsse, die nicht auf vertraglichen Bindungen zwischen den Beteiligten basieren, aus der Betrachtung aus. Die Anhaltspunkte, die dieser Ansatz zur Überwindung der Konstruktionskorridor-Vorstellung beim Aufbau und Einsatz von Mobile Business-Anwendungen bietet, beschränken sich dementsprechend ausschließlich auf ökonomische Aspekte. Dies wird auch bei einer näheren Betrachtung der vorgeschlagenen Maßnahmen zur Unterstützung der Koordination im Bereich des Mobile Business deutlich, die verhaltenswissenschaftlich fundierte Führungskonzepte und Möglichkeiten zur Motivation der Aufgabenträger außer Acht lassen. Die verstärkte Akteursorientierung eines institutionenökonomisch ausgerichteten Informationsmanagements bleibt daher unvollständig und in Bezug auf die Nutzung der mobilen Datenübertragung auf einen speziellen Teilaspekt des menschlichen Zusammenarbeitens begrenzt.

Die verschiedenen Ansätze für eine Theorie des Informationsmanagements stehen in diesem Problembereich nur in einem sehr schwachen Zusammenhang untereinander. Der Empfehlung des Produktionsorientierten Ansatzes, ein Gleichgewicht zwischen benötigtem und vorhandenem Eignungspotenzial herzustellen, kann auch aus Sicht der beiden anderen Ansätze zugestimmt werden. Nach Auffassung des Systemorientierten Ansatzes ist ergänzend zu berücksichtigen, dass bei der Ermittlung des quantitativen Personalbedarfs ausreichende Kapazitätsreserven eingeplant werden sollten, um die Systemstabilität auch bei unerwarteten Beschäftigungsschwankungen sicherzustellen. Die unter Bezugnahme auf die Kybernetik II vorgeschlagene starke Entscheidungsfreiheit mit hohen fachlichen Anforderungen an die Mitarbeiter wirkt sich in qualitativer Hinsicht auf das benötigte Eignungspotenzial zur Realisierung von Mobile Business-Lösungen aus. Spezielle komplementäre oder konkurrierende Beziehungen zwischen diesen beiden Ansätzen sind darüber hinaus nicht erkennbar.

Obwohl der Systemorientierte Ansatz die Persönlichkeit der Mitarbeiter anerkennt und diese nicht als reine Vollzugsinstanzen bei der Nutzung der mobilen Datenübertragung sieht, thematisiert er potenzielle Interessengegensätze weniger stark als der Institutionenökonomische Ansatz. Die geforderten Entscheidungsfreiheiten der Mitarbeiter im Bereich des Mobile Business werden aus Sicht des Institutionenökonomischen Ansatzes weniger positiv beurteilt, da sie zumindest potenziell auch gegen die Interessen der übergeordneten Instanz ausgenutzt werden können. Allerdings erkennt auch dieser Ansatz die Notwendigkeit zur Gewährung von Handlungsfreiheiten an, da die Auftragnehmer über bessere Fach- und/oder Situationskenntnisse verfügen als die Auftraggeber. Umgekehrt sind die neben der Einschränkung von Handlungsspielräumen vorgeschlagenen Maßnahmen zur Disziplinierung des Auftragnehmers auch aus der Perspektive des Systemorientierten Ansatzes akzeptabel, um den inneren Zusammenhalt von komplexen Anwendungssystemen im Bereich des Mobile Business zu gewährleisten. Folglich liefern beide Ansätze weitgehend komplementäre Erkenntnisse zur angemesseneren Berücksichtigung der Rolle des Menschen bei der Informationsverarbeitung im Bereich des Mobile Business. 


\section{Schlussbetrachtung}

Die in der Einleitung formulierte Zielsetzung dieser Arbeit lag darin, die Erkenntnisse bestehender betriebswirtschaftlicher Wissenschaftsprogramme zu nutzen, um zu einer Überwindung von Theoriedefiziten im Bereich des Informationsmanagements beizutragen. Dabei ging es weniger um die Herstellung eines vollständigen Gesamtbilds als um die Entwicklung einzelner Ansätze für eine Theorie des Informationsmanagements, die jeweils unterschiedliche Facetten des Themas beleuchten und als Bausteine einer multiperspektivischen Analyse aufzufassen sind. Die Zusammenführung der hier entwickelten Theorieansätze sowie eine Konkretisierung der darin enthaltenen Aussagen erfolgte durch ihre exemplarische Anwendung auf die Gestaltung und Steuerung von Anwendungssystemen im Bereich des Mobile Business durch das Informationsmanagement.

Diese Schlussbetrachtung nimmt eine kritische Würdigung des Vorgehens und der erarbeiteten Ergebnisse (Abschnitt 7.1) vor und gibt einen kurzen Ausblick auf mögliche weitere Forschungsaktivitäten (Abschnitt 7.2).

\subsection{Kritische Würdigung von Vorgehen und Ergebnissen}

Im dritten, vierten und fünten Kapitel dieser Arbeit wurde auf den Faktortheoretischen Ansatz, den Systemorientierten Ansatz und die Neue Institutionenökonomie Bezug genommen, um theoretisch fundierte Anhaltspunkte für Gestaltungsmaßnahmen des Informationsmanagements abzuleiten. Es handelt sich dabei um Wissenschaftsprogramme der Betriebswirtschaftslehre, die von einem disziplinenspezifisch abgegrenzten gemeinsamen Erfahrungsbereich ausgehen und folglich einzelwirtschaftliche Problemstellungen zum Ausgangspunkt der Analyse nehmen. Aus dieser fachbezogenen Beschränkung der Untersuchung ergeben sich zugleich bestimmte Einschränkungen hinsichtlich der Art der ableitbaren Aussagen. So waren rein technische, psychologische, soziologische und andere, aus betriebswirtschaftlicher Sicht fachfremde Aspekte des Informationsmanagements von vornherein aus der Betrachtung ausgeschlossen. Dennoch sind die hier ausgewählten Forschungsansätze so unterschiedlich angelegt, dass eine vielschichtige Analyse des Aufgabenfelds erreicht werden konnte.

Der Produktionsorientierte Ansatz und der Institutionenökonomische Ansatz für eine Theorie des Informationsmanagements orientieren sich in erster Linie am ökonomischen Rationalprinzip und treffen unterschiedliche Annahmen über die Informationsund Interessensituation der Beteiligten. Dagegen geht der Systemorientierte Ansatz vom Grundgedanken des Invarianztheorems aus und zielt darauf ab, die Erkenntnisse anderer Wissenschaftsdisziplinen für betriebswirtschaftliche Fragestellungen nutzbar zu machen. Wie die abschließenden Überlegungen des Abschnitts 6.3 zeigen, sind die auf Basis dieser Wissenschaftsprogramme abgeleiteten Ergebnisse weitgehend geeignet, einander bei der Lösung der idealtypischen Problemstellungen des Informationsmanagements in der Praxis zu ergänzen. Dies gilt insbesondere für den Produktionsorientierten und den Institutionenökonomischen Ansatz, die sich auf komplementäre Gesichtspunkte bei der Erstellung und dem Austausch von Leistungen konzentrieren. Die Empfehlungen des Systemorientierten Ansatz stehen dagegen zum Teil im Widerspruch zu den Empfehlungen der beiden anderen Ansätze. In diesen Fällen liefern 
die hier entwickelten Aussagesysteme keine eindeutigen Handlungsempfehlungen, sodass im Einzelfall zu überprüfen ist, welchem Ansatz tragfähigere Annahmen hinsichtlich der konkret vorliegenden Entscheidungssituation zu Grunde liegen.

Insgesamt kann festgestellt werden, dass zahlreiche Anhaltspunkte zur Lösung der verschiedenen idealtypischen Problemstellungen, an denen die Aussagekraft der abgeleiteten Ergebnisse zu messen ist, gewonnen werden konnten. Dies gilt nicht nur für die Effektivität und die Effizienz der technikunterstützten Informationsverarbeitung, sondern auch für die organisatorische Zuordnung informationswirtschaftlicher Aufgaben. Alle drei hier entwickelten Theorieansätze beschäftigen sich (mit unterschiedlichen Schwerpunkten) ausführlich mit diesen Problembereichen. Damit werden der Praxis generelle Orientierungspunkte für eine kritischere Auseinandersetzung mit tagesaktuellen Modeströmungen geboten, die im Bereich der Informationsverarbeitung von verschiedenen Seiten initiiert und verstärkt werden, und deren unreflektierte Übernahme in vielen Fällen nicht vorteilhaft erscheint. Durch die Beachtung dieser Orientierungspunkte lässt sich in der Praxis eine größere Unabhängigkeit von den jeweils neuesten Trends erreichen. Dies erhöht die Stabilität der technikunterstützten Informationsverarbeitung und schafft die Gelegenheit zu einer Konsolidierung der in der Unternehmung realisierten technischen und organisatorischen Lösungen.

Der Beitrag, den die hier entwickelten Ansätze für eine Theorie des Informationsmanagements zu einer angemesseneren Berücksichtigung der Rolle des Menschen liefern, ist dagegen als relativ gering einzustufen. Eine eingehende Untersuchung der Bestimmungsgrößen menschlichen Handelns strebt keiner der Ansätze an, sodass das Verhalten der an Prozessen der technikunterstützten Informationsverarbeitung beteiligten Personen nicht näher analysiert wird. Der Produktionsorientierte Ansatz des Informationsmanagements betrachtet die menschlichen Aufgabenträger allein in ihrer Funktion als Elementarfaktoren und blendet sonstige Verhaltenselemente vollständig aus der Untersuchung aus. Dagegen erkennen die beiden anderen Ansätze die Persönlichkeit der Aufgabenträger zwar an, sie liefern jedoch ebenfalls kaum Hinweise für eine verstärkte Akteursorientierung des Informationsmanagements. Am konkretesten sind in diesem Punkt die Aussagen des Institutionenökonomischen Ansatzes, der zumindest explizite Annahmen über das Verhalten der am Wirtschaftsgeschehen beteiligten Individuen triff. Diese sind allerdings - wie ausführlich erläutert - nicht ohne erhebliche Kritik von den Vertretern anderer Forschungsansätze geblieben.

Die im sechsten Kapitel der Arbeit vorgenommene exemplarische Anwendung der verschiedenen Theorieansätze auf den Bereich des Mobile Business zeigt, dass trotz deren hohem Abstraktionsniveau eine Konkretisierung möglich ist und zu tragfähigen Ergebnissen führt. Mit der Konvergenz von mobilen und leitungsgebundenen Informations- und Kommunikationstechniken wurde ein Entwicklung aufgegriffen, die zwar selbst den Charakter eines aktuellen Modetrends hat, die jedoch auf Grund ihres hohen generellen Innovationspotenzials das Umfeld der technikunterstützten Informationsverarbeitung nachhaltig verändern und die Unternehmungen noch lange Zeit beschäftigen dürfte. Das Zusammenwachsen von mobilen und leitungsgebundenen Informations- und Kommunikationstechniken hat gerade erst begonnen und wird in absehbarer Zukunft tiefgreifende Auswirkungen auf die unterschiedlichsten Bereiche des Wirtschaftsgeschehens haben. Die innovativen Leistungsmerkmale der mobilen Datenübertragung besitzen das Potenzial, an vielen Stellen einen echten zusätzlichen Nutzen zu stiften, der mit den bisherigen leitungsgebundenen Konzepten nicht erreicht werden 
kann. Am langfristigen Erfolg des Mobile Business dürfte vor diesem Hintergrund kein Zweifel bestehen.

Es kann davon ausgegangen werden, dass eine vergleichbare Konkretisierung der hier entwickelten Ansätze für eine Theorie des Informationsmanagements nicht nur für das Mobile Business, sondern auch für andere Problemstellungen in der Praxis vorgenommen werden kann. Die Ansätze sind daher vielseitig nutzbar und können in unterschiedlichen Zusammenhängen zum Einsatz kommen. Andere Anwendungsfelder könnten z.B. die in den 1990er Jahren besonders diskutierte verstärkte Prozessorientierung oder die zunehmende Globalisierung der Geschäftstätigkeit und deren Auswirkungen auf das Informationsmanagement sein.

\subsection{Ausblick}

$\mathrm{Da}$ in dieser Arbeit nur auf eine begrenzte Auswahl an Wissenschaftsprogrammen Bezug genommen wurde, sind die hier entwickelten Theorieansätze nicht in der Lage, ein vollständiges Bild des außerordentlich komplexen Aufgabenfelds Informationsmanagement zu liefern. Zur weiteren Ausdifferenzierung des Verständnisses wäre es daher wünschenswert, den Erfahrungsgegenstand auch aus anderen Perspektiven zu beleuchten. Durch die Bezugnahme auf hier nicht berücksichtigte Wissenschaftsprogramme könnten andere Aspekte aus dem Erfahrungsgegenstand herausgegriffen und einer Analyse zugänglich gemacht werden. Dazu könnten einerseits weitere Forschungsansätze der Betriebswirtschaftslehre aufgegriffen werden, die sich innerhalb der im zweiten Kapitel beschriebenen disziplinären Grenzen des Fachs bewegen. Andererseits ist auch vorstellbar, spezielle Probleme des Informationsmanagements mit Hilfe von Forschungsansätzen benachbarter Wissenschaften zu untersuchen und damit die disziplinäre Begrenzung der vorliegenden Arbeit zu überwinden.

Die kritische Würdigung im vorigen Abschnitt hat deutlich gemacht, dass ein besonderer Bedarf nach weiterer Forschung das menschliche Verhalten im Bereich der technikunterstützten Informationsverarbeitung betrifft. Dies ist darauf zurückzuführen, dass die hier entwickelten Theorieansätze nur wenig zu einer angemesseneren Berücksichtigung der Rolle des Menschen durch das Informationsmanagement beitragen konnten. Durch eine Bezugnahme auf die sozialwissenschaftlichen Nachbardisziplinen der Betriebswirtschaftslehre könnten daher wertvolle Erkenntnisse gewonnen werden, mit denen sich eine verstärkte Akteursorientierung bei der Gestaltung und Steuerung der technikunterstützten Informationsverarbeitung in Unternehmungen erreichen ließe. Diese Erkenntnisse würden sowohl für die Entwicklung von Anwendungssystemen als auch für deren Betrieb Anhaltspunkte liefern, die zu einer noch besseren Zielerreichung des Informationsmanagements in der Praxis beitragen könnten.

Eine ähnlicher Bedarf nach weiterer Forschung besteht auch in Bezug auf die technischen Problemstellungen im Bereich des Informationsmanagements, die durch die hier referenzierten Wissenschaftsprogramme der Betriebswirtschaftslehre ebenfalls nicht näher betrachtet werden. Eine Bezugnahme auf die Informatik sowie auf ingenieurwissenschaftliche Disziplinen könnte zu diesen Problemstellungen wertvolle Gestaltungshinweise liefern, mit denen sich die hier erarbeiteten Ergebnisse ergänzen ließen. So wären beispielsweise Aussagen dazu wünschenswert, wie die geforderten technischen Standards im Bereich der unternehmungsübergreifenden Informations- 
verarbeitung konkret ausgestaltet bzw. verbessert werden sollen, um eine noch höhere Produktivität bei gleichzeitiger Flexibilität der zu unterstützenden Prozesse zu erreichen. Auch bei zahlreichen unternehmungsinternen Problemstellungen erfordert eine Umsetzung betriebswirtschaftlich sinnvoller Konzepte die Nutzung von Erkenntnissen anderer Wissenschaftsdisziplinen, die sich mit den technischen Aspekten der Nutzung von Hard- und Softwarekomponenten beschäftigen. In umgekehrter Einflussrichtung ist ferner vorstellbar, dass technische Fortschritte, die durch Erkenntnisse dieser Disziplinen erreicht werden, die Chance zur Entwicklung neuer betriebswirtschaftlicher Konzepte bieten.

Diese abschließenden Überlegungen bestätigen, dass eine multiperspektivische Analyse des Informationsmanagements, die verschiedene Untersuchungsgesichtspunkte verbindet, wünschenswert ist. Neben dem hier erarbeiteten eigenen Erkenntnisbeitrag, der auf der Einnahme ausgewählter Untersuchungsperspektiven basiert, bietet die vorliegende Arbeit einen Bezugsrahmen für zukünftige Forschungsbestrebungen, die sich ebenfalls auf die Überwindung bislang bestehender Theoriedefizite richten und dabei andere Aspekte für die Analyse auswählen. 


\section{Literaturverzeichnis}

Ackoff, Russell L. (1977): Organisation und interdisziplinäre Forschung, in: Witte, Eberhard; (Hrsg.): Entscheidungstheorie, Wiesbaden 1977, S. 274-289.

Adam, Dietrich (1998): Produktions-Management, 9. Auflage, Wiesbaden 1998.

Albach, Horst (1971): Ansätze zu einer empirischen Theorie der Unternehmung, in: Kortzfleisch, Gert v. (Hrsg.), Wissenschaftsprogramm und Ausbildungsziele der Betriebswirtschaftslehre, Berlin 1971, S. 133-156.

Albach, Horst (1986): Allgemeine Betriebswirtschaftslehre, Zum Gedenken an Erich Gutenberg, in: Zeitschrift für Betriebswirtschaft, 56. 1986, Nr. 7, S. 578-613.

Albach, Horst (1988): ZfB-Editorial, in: Zeitschrift für Betriebswirtschaft, 56. 1988, Nr. 1, S. 3-10.

Albert, Hans (1976): Aufklärung und Steuerung, Hamburg 1976.

Albert, Hans (1977): Individuelles Handeln und soziale Steuerung, in: Lenk, Hans (Hrsg.): Handlungstheorien interdisziplinär, Band IV, München 1977, S. 177225 .

Alchian, Armen A. (1984): Specifity, specialization, and coalitions, in: Journal of Institutional and Theoretical Economics, 140. 1984, Nr. 1, S. 34-49.

Alchian, Armen A.; Woodward, S. (1988): The Firm is Dead. Long Live the Firm. A Review of Oliver E. Williamson's The Economic Institutions of Capitalism, in: Journal of Economic Literature, 26. 1988, Nr. 1 (March), S. 65-79.

Alessi, Louis de (1983): Property Rights, Transaction Costs and X-Inefficiency: An Essay in Economic Theory, in: American Economic Review, 73. 1983, Nr. 2, S. 64-81.

Allport, Floyd Henry (1969): Institutional Behaviour - Essays Toward a ReInterpreting of Contemporary Social Organization. New York 1969.

Alpar, Paul; Grob, Heinz Lothar; Weimann, Peter; Winter, Robert (2000): Anwendungsorientierte Wirtschaftsinformatik, 2. Auflage, Braunschweig/ Wiesbaden 2000.

Alt, Rainer; Cathomen, Ivo (1995): Handbuch Interorganisationssysteme - Anwendungen für die Waren und Finanzlogistik, Braunschweig/Wiesbaden 1995.

Alt, Rainer (1997): Interorganisationssysteme in der Logistik: Interaktionsorientierte Gestaltung von Koordinationsinstrumenten, Wiesbaden 1997.

Altenburger, Otto A. (1979): Potentialfaktoren als derivative Produktionsfaktoren der Dienstleistungsproduktion - Einige Gedanken zur Produktionstheorie der Dienstleistungen, in: Zeitschrift für Betriebswirtschaft, 49. 1979, Nr. 10, S. 863872.

Ammon, Alfred (1927): Objekt und Grundbegriffe der theoretischen Nationalökonomie, Leipzig et al. 1927. 
Angermeyer, Hans Christoph (1990): Informationsmanagement als organisatorische Aufgabe: Aufgabenstellung - Entwicklungsstufen - Elemente - Kompetenz, in: Zeitschrift Führung und Organisation, 59. 1990, Nr. 3, S. 176-180.

Angermeyer, Hans Christoph (1993): Die Einführung von InformationsManagement - eine Führungsaufgabe, in: Zeitschrift Führung und Organisation, 62. 1993, Nr. 4, S. 235-241.

Ansoff, Harry Igor (1984): Implanting Strategic Management, Englewood Cliffs, New York et al. 1984.

Antonelli, Christiano (1988): A new industrial organization approach, in: Antonelli C. (Hrsg.): New information technology and industrial change. Dodrecht et al. 1988, S. 1-12.

Ashby, W. Ross (1974): Einführung in die Kybernetik, Frankfurt a.M. (1974).

Argyris, Chris; Schön, Donald A. (1978): Organizational Learning, Reading (Massachusetts) 1978.

Arrow, Kenneth J. (1986): Agency and the Market, in: Arrow, K. J. Intriligator, M.D. (Hrsg.): Handbook of Mathematical Economics, Vol. III, North Holland Amsterdam, S. 1183-1195.

Arthur D. Little (2000): M-Commerce-Studie, http://www.arthurdlittle.de/services/management_consulting/ e-business/archive/m_commerce_studie.pdf, abgerufen am 15.05.2001.

Aufderheide, Detlef; Backhaus, Klaus (1995): Institutionenökonomische Fundierung des Marketing: Der Geschäftstypenansatz, in: Kaas, Klaus Peter (Hrsg.): Kontrakte, Geschäftsbeziehungen, Netzwerke - Marketing und neue Institutionenökonomik, Düsseldorf 1995, S. 43-60.

Bachinger, Richard (1990): Einführung: Annäherungen an einen schillernden Begriff - Zehn Merkmale - Geschichtslose Unternehmen gibt es nicht, in: Bachinger, Richard (Hrsg.): Unternehmenskultur - Ein Weg zum Markterfolg, Frankfurt a.M. 1990, S. 11-15.

Backhaus, Klaus (1999): Investitionsgüter-Marketing, 6. Auflage, München 1999.

Baetge, Jörg (1974): Betriebswirtschaftliche Systemtheorie - Regelungstheoretische Planungs-Überwachungsmodelle für Produktion, Lagerung und Absatz, Opladen 1974.

Baethge, Martin; Oberbeck, Herbert (1986): Die Zukunft der Angestellten, Frankfurt a.M./New York 1986.

Baldwin, Carliss Y.; Clark, Kim B. (1997): Managing in an age of Modularity, in: Harvard Business Review, 76. 1997, Nr. 5, S. 84-93.

Bamberger, Ingolf (1989): Commitment und Willensbildung, in: Szyperski, Norbert (Hrsg.): Handwörterbuch der Planung, Stuttgart 1989, Sp. 199-209.

Barnard, Chester I. (1938): The Functions of the Executive, Cambridge 1938.

Baron, John P.; Shaw, Michael J.; Bailey, Andrew D. (2000): Electronic Catalogs in the Web-Based Business-to-Business Procurement Process, in: Shaw, Michael J. et al. (Hrsg.): Handbook on Electronic Commerce, Berlin et al. 2000, S. 385410 . 
Bateson, Gregory (1972): Steps to an Ecology of Mind, New York 1972.

Beer, Stafford (1967): Kybernetik und Management, Frankfurt a.M. 1967.

Behme, Wolfgang; Mucksch, Harry (1998): Auswahl und Klassifizierung externer Informationen zur Integration in ein Data Warehouse, in: Uhr, Wolfgang; Breuer, Sven-Einar (Hrsg.): Integration externer Informationen in Management Support Systems, Dresden 1998, S. 85-104.

Beier, Dirk; Gabriel, Roland; Streubel, Frauke (1997): Ziele und Aufgaben des Informationsmanagements, Arbeitsbericht Nr. 97-23 des Lehrstuhls für Wirtschaftsinformatik, Bochum 1997.

Benkenstein, Martin (1994): Die Gestaltung der Fertigungstiefe als wettbewerbsstrategisches Entscheidungsproblem - Eine Analyse aus transaktions- und produktionstheoretischer Sicht, in: Zeitschrift für betriebswirtschaftliche Forschung, 46. 1994, Nr. 6, S. 483-499.

Bensberg, Frank; Weich, Martin (2001): WAP Log Mining als Analyseinstrument für den M-Commerce, in: Information Management \& Consulting, 16. 2001, Nr. 2, S. 55-58.

Berger, Ulrike; Bernhard-Mehlich, Isolde (1993): Die Verhaltenswissenschaftliche Entscheidungstheorie, in: Kieser, Alfred (Hrsg.): Organisationstheorien, Stuttgart et al. 1993, S. 127-159.

Bertalanffy, Ludwig v. (1950): An Outline of General Systems Theory, in: The British Journal for the Philosophy of Science, 1. 1950, S. 134-165.

Bertalanffy, Ludwig v. (1969): General System Theory - Foundations, Development, Applications, New York 1969.

Biethahn, Jörg; Mucksch, Harry; Ruf, Walter (1990): Ganzheitliches Informationsmanagement, Bd. 1: Grundlagen, München/Wien 1990.

Biethahn, Jörg; Mucksch, Harry; Ruf, Walter (1991): Ganzheitliches Informationsmanagement, Bd. 2: Daten- und Entwicklungsmanagement, München/Wien 1991.

Biethahn, Jörg; Mucksch, Harry; Ruf, Walter (1994): Ganzheitliches Informationsmanagement, Bd. 1: Grundlagen, 3. Auflage, München/Wien 1994.

Bissantz, Nicolas; Hagedorn, Jürgen; Mertens, Peter (1998): Data Mining, in: Mucksch, Harry; Behme, Wolfgang (Hrsg.): Das Data Warehouse-Konzept: Architektur - Datenmodelle - Anwendungen, 3. Auflage, Wiesbaden 1998, S. 445-474.

Blankart, Charles (1998): Öffentliche Finanzen in der Demokratie - Eine Einführung in die Finanzwissenschaft, 3. Auflage, München 1998.

Blanning, Robert W.; Bui, Tung X. (2000): Decision Support Systems and Internet Commerce, in: Shaw, Michael J. et al. (Hrsg.): Handbook on Electronic Commerce, Berlin et al. 2000, S. 53-75.

Bleicher, Knut (1986): Die Rolle von Unternehmern und Managern bei der Entwicklung von Unternehmens-Kulturen, in: Arbeitsgemeinschaft zur Förderung der Partnerschaft in der Wirtschaft (Hrsg.): Unternehmenskultur in Deutschland - Menschen machen Wirtschaft, Gütersloh 1986, S. 38-46. 
Bleicher, Knut (1988): Betriebswirtschaftslehre - Disziplinäre Lehre vom Wirtschaften in und zwischen Betrieben oder interdisziplinäre Wissenschaft vom Management?, in: Wunderer, Rolf (Hrsg.): Betriebswirtschaftslehre als Management- und Führungslehre, 2. Auflage, Stuttgart 1988, S. 109-131.

Bode, Jürgen (1993): Betriebliche Produktion von Information, Wiesbaden 1993.

Bode, Jürgen (1997): Der Informationsbegriff in der Betriebswirtschaftslehre, in: Zeitschrift für betriebswirtschaftliche Forschung, 44. 1997, Nr. 5, S. 449-469.

Bode, Jürgen; Zelewski, Stefan (1992): Die Produktion von Dienstleistungen Ansätze zu einer Produktionswirtschaftslehre der Dienstleistungsunternehmen, in: Betriebswirtschaftliche Forschung und Praxis, 49. 1992, Nr. 6, S. 594-607.

Bohr, Kurt; Eberwein, Rolf-Dieter (1989): Die Organisationsform Fertigungsinsel Begriff und Vergleich mit der Werkstattfertigung, in: Wirtschaftswissenschaftliches Studium, 18. 1989, Nr. 5, S. 218-223.

Born, Achim (2001): WAP zwischen Hoffen und Bangen - Die zweite Chance, in: Computerwoche extra, Nr. 3, April 2001, S. 23-25.

Borowicz, Frank; Scherm, Ewald (2000): ZP-Stichwort: Mobile Commerce, in Zeitschrift für Planung, 11. 2000, Nr. 4, S. 469-475.

Boulding, Kenneth E. (1956): General Systems Theory - The Sceleton of Science, in: General Systems, 1. 1956, S. 11-17.

Brenner, Charles (1972): Grundzüge der Psychoanalyse, 6. Auflage, Frankfurt a. M. 1972.

Brenner, Walter (1994): Konzepte des Informationssystem-Managements, Heidelberg 1994.

Brockhaus, Rainer (1992): Informationsmanagement als ganzheitliche, informationsorientierte Gestaltung von Unternehmen - organisatorische, personelle und technologische Aspekte, Göttingen 1992.

Brombacher, Reinhard (1991): Effizientes Informationsmanagement - die Herausforderung für Gegenwart und Zukunft, in: Jacob, Herbert, Becker, Jörg; Krcmar, Helmut (Hrsg.): Integrierte Informationssysteme, Schriften zur Unternehmensführung, Bd. 44, Wiesbaden 1991, S. 111-134.

Brown, Stanley (2000): Customer relationship management - a strategic imperative in the world of e-business, Toronto et al. 2000.

Buck-Emden, Rüdiger; Galimow Jürgen (1996): Die Client-Server-Technologie des SAP-Systems R-3: Basis für betriebswirtschaftliche Standard-Anwendungen, 3. Auflage, Bonn et al. 1996.

Budäus, Dietrich; Gerum, Elmar; Zimmermann, Gebhard (1988): Betriebswirtschaftslehre und Theorie der Verfügungsrechte, Wiesbaden 1988.

Buscher, Udo (1997): Verrechnungspreise aus organisations- und agencytheoretischer Sicht, Wiesbaden 1997.

Busse von Colbe, Walther; Laßmann, Gert (1991): Betriebswirtschaftstheorie, Band 1: Grundlagen, Produktions- und Kostentheorie, 5. Auflage, Berlin et al. 1991. 
Busse von Colbe, Walther; LaBmann, Gert (1990): Betriebswirtschaftstheorie, Band 3: Investitionstheorie, 3. Auflage, Berlin et al. 1990.

Buxmann, Peter; König, Wolfgang (2000): Zwischenbetriebliche Kooperationen auf Basis von SAP-Systemen - Perspektiven für die Logistik und das Servicemanagement, Berlin et. al. 2000.

Capurro, Rafael (1987): Was ist Information?, in: Handbuch der modernen Datenverarbeitung, 24. 1987, Nr. 133, S. 107-114.

Cartwright, Nancy (1983): How the Laws of Physics Lie, New York et al. 1983.

Chalmers, Alan F. (1989): Wege der Wissenschaft, 2. Auflage, Berlin u.a. 1989.

Chamoni, Peter; Gluchowski, Peter (1999): Analytische Informationssysteme Einordnung und Überblick, in: Chamoni, Peter; Gluchowski, Peter (Hrsg.): Analytische Informationssysteme - Data Warehouse, On-Line Analytical Processing, Data Mining, 2. Auflage, Berlin et al. 1999, S. 3-25.

Chen, Harry (2001): Service Discovery in the Future for Mobile Commerce, http://www.cs.umbc.edu/ dchakrl/papers/mcommerce.html, abgerufen am 07.05.2001.

Chmielewicz, Klaus (1994): Forschungskonzeptionen der Wirtschaftswissenschaft, 3. Auflage, Stuttgart 1994.

Choi, Soon-Yong; Whinston, Andrew B. (2000): The Future of the Digital Economy, in: Shaw, Michael J. et al. (Hrsg.): Handbook on Electronic Commerce, Berlin et al. 2000, S. 25-52.

Clark, David L. (1985): Emerging Paradigms in organizational theory and research, in: Lincoln, Yvonna S. (Hrsg.): Organizational Theory and Inquiry. The Paradigm Revolution, Beverly Hills et al. 1985, S. 43-78.

Coase, Ronald H. (1937): The Nature of the Firm, in: Economica, 17. 1937, Nr. 16, S. 386-405.

Coase, Ronald H. (1960): The Problem of Social Cost, in: Journal of Law and Economics, 3. 1960, Nr. 1, S. 1-44.

Coenenberg, Adolf Gerhard (1999): Kostenrechnung und Kostenanalyse, 4. Auflage, Landsberg a.L. 1999.

O'Connor, Gina Golarelli; O'Keefe, Robert (2000): The Internet as a New Marketplace: Implications for Consumer Behaviour and Marketing Management, in: Shaw, Michael J. et al. (Hrsg.): Handbook on Electronic Commerce, Berlin et al. 2000 , S. $123-146$.

Corsten, Hans (1988): Dienstleistungen in produktionstheoretischer Interpretation, in: Das Wirtschaftsstudium, 17. 1988, S. 81-87.

Corsten, Hans (1990): Betriebswirtschaftslehre der Dienstleistungsunternehmungen Einführung, 2. Auflage, München/Wien 1990.

Corsten, Hans (2000): Produktionswirtschaft - Einführung in das industrielle Produktionsmanagement, 9. Auflage, München/Wien 2000.

Coy, Wolfgang (1992): Für eine Theorie der Informatik!, in: Coy, Wolfgang; Nake, Frieder; Pflüger, Jörg-Martin; Rolf, Arno; Seetzen, Jürgen; Siefkes, Dirk; 
Stransfeld, Reinhard (Hrsg.): Sichtweisen der Informatik, Braunschweig/Wiesbaden 1992, S. 17-32.

Cyert, Richard M.; March, James G. (1963): A Behavioural Theory of the Firm, Englewood Cliffs 1963.

Darby, Michael R.; Karni, Edi (1973): Free Competition and the optimal amount of fraud, in: Journal of Law and Economics, 16. 1973, Nr. 2, S. 67-88.

Davenport, Thomas (1993): Process Innovation - Reengineering Work Through Information Technology, Boston 1993.

Dean, David; Ketterer, Hanno; Thiel, Wolfgang (2000): IT-Herausforderungen im M-Commerce, in: Information Management \& Consulting, 15. 2000, Nr. 4, S. 34-37.

Dietl, Helmut (1991): Institutionen und Zeit, München 1991.

Dietz, Albrecht (1997): Reflexionen über die „Grundlagen der Betriebswirtschaftslehre" anlässlich des hundertsten Geburtstages von Erich Gutenberg, in: Zeitschrift für betriebswirtschaftliche Forschung, 49. 1997, Nr. 12, S. 10661083.

Dill, Peter (1987): Unternehmenskultur: Grundlagen und Anknüpfungspunkte für ein Kulturmanagement, Bonn 1987.

Dill, Peter; Hügler, Gert (1987): Unternehmenskultur und Führung betriebswirtschaftlicher Organisationen - Ansatzpunkte für ein kulturbewußtes Management, in: Heinen, Edmund (Hrsg.): Unternehmenskultur - Perspektiven für Wissenschaft und Praxis, München 1987, S. 141-209.

Dobschütz, Leonhard v. (1992): Wirtschaftlichkeitsanalyse von Anwendungssystemen: Prämissen und Praxis, in: Information Management, 7. 1992, Nr. 4, S. $42-47$.

Dormayer, Jürgen; Kettern, Thomas (1987): Kulturkonzepte in der allgemeinen Kulturforschung - Grundlage konzeptioneller Überlegungen zur Unternehmenskultur, in: Heinen, Edmund (Hrsg.): Unternehmenskultur, München 1987, S. 49-66.

Drepper, Christian (1992): Unternehmenskultur - Selbstbeobachtung und Selbstbeschreibung im Kommunikationssystem "Unternehmen", Frankfurt a. M. 1992

Drumm, Hans Jürgen (1992): Personalplanung, in: Gaugler, Eduard; Weber, Wolfgang (Hrsg.): Handwörterbuch des Personalwesens, 2. Auflage, Stuttgart 1992, Sp. 1758-1769.

Earl, Michael J. (1988): IT and Strategic Advantage: A Framework of Frameworks, in: Earl, Michael J. (Hrsg.): Information Management - the Strategic Dimension, Oxford 1988, S. 110-128.

Eberhard, Stefan (1998): Wertorientierte Unternehmungsführung - der modifizierte Stakeholder-Ansatz, Wiesbaden 1998.

Ebers, Mark (1994): Die Gestaltung interorganisationaler Informationssysteme Möglichkeiten und Grenzen einer transaktionskostentheoretischen Erklärung, in: Sydow, Jörg (Hrsg.): Management interorganisationaler Beziehungen, Darmstadt 1994, S. 22-48. 
Ebers, Mark; Gotsch, Wilfried (1993): Institutionenökonomische Theorien der Organisation, in: Kieser, Alfred (Hrsg.): Organisationstheorien, Stuttgart et al. 1993, S. 193-242.

Egan, Nancy T. (1999): Customer Relationship Management: A Collaboration Between Business and IT, in: DM Review, June 1999, http://www.dmreview.com, abgerufen am 10.07.2001.

Ehrenwirth, Marcus (2001): ERP goes mobile, in: Computerwoche extra, Nr. 3, 2001, S. 10-12.

Elschen, Rainer (1991): Gegenstand und Anwendungsmöglichkeiten der AgencyTheorie, in: Zeitschrift für betriebswirtschaftliche Forschung, 43. 1991, Nr. 11, S. 1002-1012.

Elsner, Wolfram (1986): Ökonomische Institutionenanalyse - Paradigmatische Entwicklung der ökonomischen Theorie und der Sinn eines Rückgriffs auf die ökonomische Klassik am Beispiel der Institutionenanalyse, Berlin 1986

Emmrich, Volkhard (1997): Spielregeln in der lernenden Organisation, in: Wieselhuber, Norbert et al. (Hrsg.): Handbuch Lernende Organisation, Wiesbaden 1997, S. 109-116.

Endres, Walter (1991): Der Betrieb - Grundriss der Allgemeinen Betriebswirtschaftslehre, Bergisch Gladbach 1991.

Engelhardt, Werner H. (1997): Das Marketing in der Betriebswirtschaftslehre - Eine paradigmatische Betrachtung, in: Bruhn, Manfred; Steffenhagen, Hartwig (Hrsg.): Marktorientierte Unternehmensführung: Reflexionen - Denkanstöße Perspektiven, Wiesbaden 1997, S. 3-17.

Engelhardt, Werner H.; Gersch, Martin (1995): Informationsmanagement als Instrument zur erfolgreichen Gestaltung von Geschäftsbeziehungen - am Beispiel des deutschen Pharmagroßhandels, in: Trommsdorff, Volker (Hrsg.): Handelsforschung 1995/96, Wiesbaden 1995, S. 201-222.

Engelhardt, Werner H.; Freiling, Jörg (1995a): Die integrative Gestaltung von Leistungspotenzialen, in: Zeitschrift für betriebswirtschaftliche Forschung, 47. 1995, Nr. 10, S. 899-918.

Engelhardt, Werner H.; Freiling, Jörg (1995b): Integrativität als Brücke zwischen Einzeltransaktionen und Geschäftsbeziehung, in: Marketing ZFP, 17. 1995, Nr. 1, S. 37-43.

Engelhardt, Werner Hans; Kleinaltenkamp, Michael (1990): Strategische Planung 1, TV-Lehrbrief, Freie Universität Berlin, Berlin 1990.

Erlei, Mathias; Leschke, Martin; Sauerland, Dirk (1999): Neue Institutionenökonomik, Frankfurt/Münster 1999.

Erler, Thomas (1998): Business Objects - Eine objektorientierte Gestaltungsphilosophie für das Business Engineering, Arbeitsbericht Nr. 98-27 des Lehrstuhls für Wirtschaftsinformatik, Bochum 1998.

Erler, Thomas (2000): Business Objects als Gestaltungskonzept strategischer Informationssystemplanung, Frankfurt a.M. et al. 2000. 
Eulgem, Stefan (1998): Die Nutzung unternehmensinternen Wissens: ein Beitrag aus der Perspektive der Wirtschaftsinformatik, Frankfurt a.M. et al. 1998.

Fama, Eugene F. (1980): Agency Problems and the Theory of the Firm, in: Journal of Political Economy, 88. 1980, Nr. 2, S. 288-307.

Fank, Matthias (1996): Einfürung in das Informationsmanagement - Grundlagen, Methoden, Konzepte, München et al. 1996.

Felderer, Bernhard; Homburg, Stefan (1991): Makroökonomik und neue Makroökonomik, 5. Auflage, Berlin et al. 1991.

Ferstl, Otto, K.; Sinz, Elmar J. (1993): Grundlagen der Wirtschaftsinformatik, München/Wien 1993.

Figueiredo, John M. de (2000): Finding Sustainable Profitability in Electronic Commerce, in: Sloan Management Review, 41. 2000, Nr. 4, S. 41-52.

Finke, Wolfgang F. (1987): Informationsmanagement in Organisationen: Neue Führungsaufgaben beim Einsatz der Informations- und Kommunikationstechnik, in: Zeitschrift Führung und Organisation, 56. 1876, Nr. 6, S. 360-368.

Flory, Markus (1995): Computergestützter Vertrieb von Investitionsgütern. Analyse - Gestaltungsempfehlungen - Perspektiven, Wiesbaden 1995.

Foerster, Heinz v. (1988): Das Konstruieren einer Wirklichkeit, in: Watzlawick, Paul (Hrsg.): Die erfundene Wirklichkeit, 5. Auflage, München 1988, S. 39-60.

Fotilas, Panagiotis (1983): Mikroelektronik im Industriebetrieb Betriebswirtschaftlich-organisatorische Auswirkungen auf Produktentwicklung und Produktionsprozeß, Berlin 1983.

Francella, K. (1983): Information Resource Management: A Brief Overview, in: Data Management, 21. 1983, Nr. 1, S. 15-16.

Franken, Rolf; Frese , Erich (1989): Kontrolle und Planung, in: Szyperski, Norbert: (Hrsg.): Handwörterbuch der Planung, Stuttgart 1989, Sp. 888-898.

Fuchs, Herbert (1973): Systemtheorie und Organisation - Die Theorie offener Systeme als Grundlage zur Erforschung und Gestaltung betrieblicher Systeme, Wiesbaden 1973.

Furubotn, Eirik G.; Pejovich, Svetozar (1972): Property rights and economic theory: A survey of recent literature, in: Journal of Economic Literature, 10. 1972, S. 1137-1162.

Gabriel, Roland (1996): Neue Herausforderungen der Wirtschaftsinformatik Qualitätsanforderungen an betriebliche Informationssysteme, in: Balderjahn, Ingo; Gabriel, Roland (Hrsg.): Aktuelle Aufgaben und Entwicklungen der Betriebswirtschaft Arbeitsbericht Nr. 61 des Instituts für Unternehmungsführung und Unternehmensforschung, Bochum 1996, S. 169-188.

Gabriel, Roland (1999): Strategische Bedeutung der analytischen Informationssysteme, in: Chamoni, Peter; Gluchowski, Peter (Hrsg.): Analytische Informationssysteme - Data Warehouse, On-Line Analytical Processing, Data Mining, 2. Auflage, Berlin et al. 1999, S. 417-426. 
Gabriel, Roland; Begau, Klaus; Knittel, Friedrich; Taday, Holger (1994): Büroinformations- und -kommunikationssysteme - Aufgaben, Systeme, Anwendungen, Heidelberg 1994.

Gabriel, Roland; Beier, Dirk (2000): Informationsmanagement - Grundbegriffe und Gestaltungsgegenstand, Lehrmaterial Nr. 31/00 des Lehrstuhls für Wirtschaftsinformatik, Ruhr-Universität Bochum 2000.

Gabriel, Roland; Chamoni, Peter; Gluchowski, Peter (2000): Data Warehouse und OLAP - analyseorientierte Informationssysteme für das Management, in: Zeitschrift furr betriebswirtschaftliche Forschung, 52. 2000, Nr. 2, S. 74-93.

Gabriel, Roland; Gluchowski, Peter (1997): Management Support Systeme, in: Wirtschaftswissenschaftliches Studium, 26. 1997, Nr. 6, 8, 10, S. 308-313, 422427, 535-540.

Garcia, Ignacio; Petit, Nicolas; Mialaret, Vincent (2000): M-Customer Segmentation: Preparing for the Mobile Internet Wave, http://www.arthurdlittle.de/services/management_consulting/ e-business/articles04/mcsegmentationFinal.pdf, abgerufen am 07.05.2001.

Garcia, Roberto; Ribas, Christine (2000): Multi-Access Portals: Information Whenever, Wherever, However You Want It, http://www.arthurdlittle.de/services/management_consulting/e-business/ articles04/multi-accessportalsFinal.pdf, abgerufen am 07.05.2001.

Gaugler, Eduard (1989): Personalplanung, in: Szyperski, Norbert (Hrsg.): Handwörterbuch der Planung, Stuttgart 1989, Sp. 1350-1362.

Gälweiler, Aloys (1974): Unternehmensplanung - Grundlagen und Praxis, Frankfurt 1974.

Gälweiler, Aloys (1987): Strategische Unternehmensführung, Frankfurt a.M. et al. 1987.

Gebert, Diether (1992): Kommunikation, in: Frese, Erich (Hrsg.): Handwörterbuch der Organisation, 3. Auflage, Stuttgart 1992, Sp. 1110-1121.

Gentner, Andreas; Legler, Steffen (2000): Einstieg in die mobile Datenwelt mit UMTS - mögliche Geschäftsmodelle und Erfolgsaussichten der deutschen Lizenzinhaber, in: Information Management \& Consulting, 15. 2000, Nr. 4, S. 14-20.

Gerpott, Thorsten (2001): Wettbewerbsstrategische Gestaltungsfelder für Mobilfunknetzbetreiber auf Mobile-Business-Märkten, in: Information Management \& Consulting, 16. 2001, Nr. 2, S. 34-42.

Gerpott, Thorsten; Böhm, Stephan (2001): Carrier müssen neues Rollenverständnis entwickeln - Geldquellen gesucht, in: Computerwoche extra, Nr. 3, 2001, S. 4-6.

Gersch, Martin (1998): Vernetzte Geschäftsbeziehungen - die Nutzung von EDI als Instrument des Geschäftsbeziehungsmanagement, Wiesbaden 1998.

Gersch, Martin (2000): E-Commerce - Einsatzmöglichkeiten und Nutzungspotenziale, Arbeitsbericht Nr. 82 des Instituts für Unternehmungsführung und Unternehmensforschung, Ruhr-Universität Bochum 2000. 
Gilmore, James H.; Pine, B. Joseph (2000): Markets of One - Creating CustomerUnique Value through Mass Customization, Boston 2000.

Gipper, Helmut (1986): Sprache als In-Formation (geistige Prägung), in: Folberth, Otto G. (Hrsg.): Der Informationsbegriff in Technik und Wissenschaft, München/Wien 1986, S. 257-280.

Gitt, Werner (1989): Information - die dritte Grundgröße neben Materie und Energie, in: Siemens-Zeitschrift, 63. 1989, Nr. 4, S. 4-9.

Gluchowski, Peter; Gabriel, Roland; Chamoni, Peter (1997): Management Support Systeme: computergestützte Informationssysteme für Führungskräfte und Entscheidungsträger, Berlin et al. 1997.

Gluchowski, Peter; Müller, Jochen (2000): Web-Log-Mining - Auf den Fährten der Internet-Surfer, in: is report, Zeitschrift für betriebswirtschaftliche Informationssysteme, 4. 2000, Nr. 7, S. 18-21.

Göbel, Elisabeth (1998): Theorie und Gestaltung der Selbstorganisation, Berlin 1998.

Gomez, Peter (1998): Zum Tode von Hans Ulrich, in: Zeitschrift für betriebswirtschaftliche Forschung, 50. 1998, Nr. 4, S. 387.

Goshal, S.; Moran, P. (1996): Bad for practice: a critique of the transaction cost theory, in: American Management Review, 21. 1996, Nr. 1, S. 13-47.

Griese, Joachim (1990): Ziele und Aufgaben des Informationsmanagements, in: Kurbel, Karl; Strunz, Horst (Hrsg.): Handbuch Wirtschaftsinformatik, Stuttgart 1990, S. 643-651.

Grochla, Erwin (1964): Planung, betriebliche, in: Beckerath, Erwin v. (Hrsg.): Handwörterbuch der Sozialwissenschaften, Band 8, Stuttgart 1964, S. 314-325.

Gross, Tom; Koch, Thomas (2001): Neue Herausforderungen für die Gestaltung von Benutzungsschnittstellen Mobiler Endgeräte, in: Informationstechnik und Technische Informatik, 43. 2001, Nr. 2, S. 74-82.

Gurbaxani, Vijay; Whang, Seungjin (1991): The Impact of Information Systems on Organizations and Markets, in: Communication of the ACM, 4. 1991, Nr. 1, S. 59-73.

Gussmann, Bernd; Breit, Claus (1987): Ansatzpunkte für eine Theorie der Unternehmenskultur, in: Heinen, Edmund (Hrsg.): Unternehmenskultur - Perspektiven für Wissenschaft und Praxis, München 1987, S. 107-139.

Gutenberg, Erich (1951): Grundlagen der Betriebswirtschaftslehre, Band 1: Die Produktion, Berlin et al. 1951.

Gutenberg, Erich (1952): Planung im Betrieb, in: Zeitschrift für Betriebswirtschaft, 22. 1952 , Nr. 12 , S. 669-684.

Gutenberg, Erich (1956): Offene Fragen der Produktions- und Kostentheorie, in: Zeitschrift für handelswissenschaftliche Forschung, 8. 1956, Nr. 8, S. 429-449.

Gutenberg, Erich (1958): Einführung in die Betriebswirtschaftslehre, Wiesbaden 1958.

Gutenberg, Erich (1979): Grundlagen der Betriebswirtschaftslehre, Band 2: Der Absatz, Berlin et al. 1979. 
Gutenberg, Erich (1980): Grundlagen der Betriebswirtschaftslehre, Band 3: Die Finanzen, 8. Auflage, Berlin et al. 1975.

Gutenberg, Erich (1983): Grundlagen der Betriebswirtschaftslehre, Band 1: Die Produktion, 24. Auflage, Berlin et al. 1983.

Gutenberg, Erich (1984): Rückblick, in: Zeitschrift für Betriebswirtschaft, 58. 1988. Nr. 12, S. 1151-1168.

Haase, Michaela (2000): Institutionenökonomische Betriebswirtschaftstheorie Allgemeine Betriebswirtschaftslehre auf sozial- und institutionenökonomischer Grundlage, Wiesbaden 2000.

Haberstock, Lothar (1999): Kostenrechnung II - (Grenz-) Plankostenrechnung, 8. Auflage, Berlin 1999.

Hackathorn, Richard D. (1996): Web Farming for Data Warehousing, Bolder Technology, October 1996, http://www.bolder.com/web-farming.pdf.

Hackathorn, Richard D. (1999): Farming Web Resources for the Data Warehouse, in: DM Review, June 1999, http://www.dmreview.com.

Hahn, Alois (1987): Identität und Selbstthematisierung, in: Hahn, Alois (Hrsg.): Selbstthematisierung und Selbsterzeugnis: Bekenntnis und Geständnis, Frankfurt a.M. 1987, S. 9-24.

Hahn, Dietger (1997): Controlling in Deutschland - State of the Art, in: Gleich, Ronald; Seidenschwarz, Werner (Hrsg.): Die Kunst des Controlling, München 1997, S. 13-46.

Hahn, Dietger; Lassmann, Gert (1989): Produktionswirtschaft - Controlling industrieller Produktion, Band 2, Heidelberg 1989.

Hansen, Hans-Robert (1996): Wirtschaftsinformatik I, 7. Auflage, Stuttgart 1996.

Hamel, Winfried (1992): Zielsysteme, in: Frese, Erich (Hrsg.): Handwörterbuch der Organisation, 3. Auflage, Stuttgart 1992, Sp. 2634-2652.

Hammer, Michael; Champy, James (1993): Reengineering the Corporation, New York 1993.

Hartmann, Stephan; Dirksen, Volker (2001): Effizienzsteigerungen von unternehmensinternen Prozessen durch die Integration von Komponenten des MBusiness, in: Information Management \& Consulting, 16. 2001, Nr. 2, S. 16-19.

Hartmann-Wendels, Thomas (1992): Agency-Theorie, in: Frese, Erich (Hrsg.): Handwörterbuch der Organisation, 3. Auflage, Stuttgart 1992.

Haussmann, Thomas (1991): Erklären und Verstehen: Zur Theorie und Pragmatik der Geschichtswissenschaft, Frankfurt/M. 1991.

Hayek, Friedrich A. v. (1969): Freiburger Studien, Tübingen 1969.

Hayek, Friedrich A. v. (1972): Die Theorie komplexer Phänomene, Tübingen 1972.

Hayek, Friedrich A. v. (1973): Law, Legislation and Liberty, Bd. 1: Rules and Order, London 1973.

Heckhausen, Heinz (1980): Motivation und Handeln, Berlin et. al. 1980. 
Heinen, Edmund (1969): Zum Wissenschaftsprogramm der entscheidungsorientierten Betriebswirtschaftslehre, in: Zeitschrift für Betriebswirtschaft, 39. 1969, Nr. 4, S. 207-220.

Heinen, Edmund (1971): Der entscheidungsorientierte Ansatz der Betriebswirtschaftslehre, in: Kortzfleisch, Gert v. (Hrsg.): Wissenschaftsprogramm und Ausbildungsziele der Betriebswirtschaftslehre, Berlin 1971, S. 20-37.

Heinen, Edmund (1976): Grundfragen der entscheidungsorientierten Betriebswirtschaftslehre, München 1976.

Heinen, Edmund (1978): Zum Wissenschaftsprogramm der entscheidungsorientierten Betriebswirtschaftslehre, in: Schweitzer, Marcell (Hrsg.): Auffassungen und Wissenschaftsziele der Betriebswirtschaftslehre, Darmstadt, 1978.

Heinen, Edmund (1983): Betriebswirtschaftliche Kostenlehre, 6. Auflage, Wiesbaden 1983.

Heinen, Edmund (1985a): Einführung in die Betriebswirtschaftslehre, 9. Auflage, Wiesbaden 1985.

Heinen, Edmund (1985b): Wandlungen und Strömungen in der Betriebswirtschaftslehre, in: Probst, Gilbert J.B.; Siegwert, Hans (Hrsg.): Integriertes Management, Stuttgart 1985, S. 37-63.

Heinen, Edmund (1987): Unternehmenskultur als Gegenstand der Betriebswirtschaftslehre, in: Heinen, Edmund (Hrsg.): Unternehmenskultur - Perspektiven für Wissenschaft und Praxis, München 1987, S. 1-48.

Heinrich, Lutz J.; Burgholzer Peter (1987): Informationsmanagement, München/Wien 1987.

Heinrich, Lutz J. (1996): Informationsmanagement - Planung, Überwachung und Steuerung der Informationsinfrastruktur, 5. Auflage, München/Wien 1996.

Heinrich, Lutz J. (1999): Informationsmanagement - Planung, Überwachung und Steuerung der Informationsinfrastruktur, 6. Auflage, München/Wien 1999.

Hejl, Peter (1982): Sozialwissenschaft als Theorie selbstreferentieller Systeme, Frankfurt a.M./New York 1982.

Hejl, Peter (1983): Kybernetik 2. Ordnung, Selbstorganisation und Biologismusverdacht - Aus Anlass der Kontroverse um das „Evolutionäre Management“, in: Die Unternehmung, 37. 1983, Nr. 1, S. 41-62.

Heng, Stefan (2001): Mega Business oder Mickey Mouse?, in: Technologie \& Management, 50. 2001, Nr. 3-4, S. 24-27.

Henke, Klaus (2001): Inhouse mobil mit drahtlosen LANs, in: Computerwoche extra, Nr. 3, 2001, S. 28-30.

Hermanns, Arnold (1999): Electronic Commerce - Herausforderungen für das Marketing-Management, in: Hermanns, Arnold; Sauter, Michael (Hrsg.): Management-Handbuch Electronic Commerce, München 1999, S. 87-100.

Herold, Wolf-Eckard (2001): Generationswechsel im Mobilfunknetz, in: Technologie \& Management, 50. 2001, Nr. 3-4, S. 18-20. 
Hess, Thomas (1999): Die Agency-Theorie als Gestaltungshilfe für Führungsinformationssysteme, in: Wirtschaftswissenschaftliches Studium, 28. 1999, Nr. 11, S. 1503-1509.

Hess, Oliver (1999): Internet, Electronic Data Interchange und SAP R/3 - Synergien und Abgrenzungen im Rahmen des Electronic Commerce, in: Hermanns, Arnold; Sauter, Michael (Hrsg.): Management-Handbuch Electronic Commerce, München 1999, S. 185-196.

Hettich, Steffi; Hippner, Hajo; Wilde, Klaus (2000): Customer Relationship Management (CRM), in: Das Wirtschaftsstudium, 29. 2000, Nr. 10, S. 13461366.

Hildebrand, Knut (1995): Informationsmanagement: wettbewerbsorientierte Informationsverarbeitung, München 1995.

Hildebrand, Volker (1997): Individualisierung als strategische Option der Marktbearbeitung, Wiesbaden 1997.

Hill, Wilhelm (1988): Betriebswirtschaftslehre als Managementlehre, in: Wunderer, Rolf (Hrsg.): Betriebswirtschaftslehre als Management- und Führungslehre, 2. Auflage, Stuttgart 1988.

Hirschman, Albert O. (1970): Exit, Voice and Loyalty - Responses to Decline in Firms, Organizations and States, Cambridge 1970.

Hoch, Detlev J.; Schirra, Wolfgang (1993): Entwicklung der Informationstechnologie - Management des Wandels in einer Zeit des Paradigmenwechsels, in: Scheer, August-Wilhelm (Hrsg.): Handbuch Informationsmanagement, Wiesbaden 1993, S. 3-47.

Holten, Roland; Ehlers, Lars (1998): Repository zur Integration externer Informationen in Führungsinformationssysteme, in: Uhr, Wolfgang; Breuer, Sven-Einar (Hrsg.): Integration externer Informationen in Management Support Systems - Wirtschaftsinformatik Fachtagung, Technische Universität Dresden 1998, S. 123-136.

Homans, George Caspar (1978): Theorie der sozialen Gruppe, Opladen 1978.

Hoppen, Dirk (1992): Organisation und Informationstechnologie - Grundlagen für ein Konzept der Organisationssystemgestaltung, Hamburg 1992.

Hruschka, Erich (1958): Die Planung im Leistungsgefüge der Betriebswirtschaft, in: Betriebswirtschaftliche Forschung und Praxis, 10. 1958, Nr. 7/8, S. 423-435.

Huber, Heinrich; Maier, Thomas (1991): Wettbewerbsorientiertes Informationsmanagement, in: Office Management, 39. 1991, Nr. 11, S. 6-12.

Hubmann, Egbert (1989): Elektronisierung von Beschaffungsmärkten und Beschaffungshierarchien, München 1989

Hülsmann, Franz Werner; Mörs, Sven; Schaar, Peter (2001): Mobilkommunikation und Datenschutz, in: Datenschutz und Datensicherheit, 25. 2001, Nr. 4, S. 196-204.

Hummel, Siegfried; Männel, Wolfgang (1986): Kostenrechnung, 4. Auflage, Wiesbaden 1986. 
Hundt, Sönke (1977): Zur Theoriengeschichte der Betriebswirtschaftslehre, Köln 1977.

Huthmacher, Christian (2000): Die mobile Revolution, in: Technologie \& Management, 49. 2000, Nr. 9-10, S. 14-15.

Jacob, Herbert (1996): Produktions- und Absatzprogrammplanung, in: Kern, Werner; Schröder, Horst; Weber, Jürgen (Hrsg.): Handwörterbuch der Produktionswirtschaft, 2. Auflage, Stuttgart 1996, Sp. 1468-1483.

Jacob, Olaf; Mende, Martin (1992): Informatik und Reorganisation: Integrierte Gestaltung von Informationssystemen und Organisation, Arbeitsbericht des Instituts für Wirtschaftsinformatik an der Hochschule St. Gallen, 1992.

Jahnke, Bernd (1993): Einsatzkriterien, kritische Erfolgsfaktoren und Einführungsstrategien für Führungsinformationssysteme, in: Behme, Wolfgang; Schimmelpfeng, Katja (Hrsg.): Führungsinformationssysteme. Neue Entwicklungstendenzen im EDV-gestützten Berichtswesen, Wiesbaden 1993, S. 29-43.

Jehle, Egon (1973): Über Fortschritt und Fortschrittskriterien in betriebswirtschaftlichen Theorien, Stuttgart 1973.

Jensen, Michael C.; Meckling, William H. (1976): Theory of the Firm. Managerial Behavior, Agency Costs and Ownership Structure, in: Journal of Financial Economics, 3. 1976, S. 305-360.

Kaas, Klaus; Fischer, Marc (1993): Der Transaktionskostenansatz, in: Das Wirtschaftsstudium, 22. 1993, Nr. 8-9, S. 686-693.

Kalakota, Ravi; Whinston, Andrew B. (1996): Frontiers of Electronic Commerce, New York 1996.

Kamp, Jannecke van der; Velthuis, Carol (2000): Will the World Become a Mobile Village, http://www.arthurdlittle.de/services/management_consulting/ e-business/articles04/introductoryFinal.pdf, abgerufen am 07.05.2001.

Kasper, Helmut (1990): Symbolisches Management - der Vorgesetzte als „Sinnstifter", in: Bachinger, Richard (Hrsg.): Unternehmenskultur: ein Weg zum Markterfolg, Frankfurt a.M. 1990, S. 19-25.

Kasper, Helmut (1991): Neuerungen durch selbstorganisierende Prozesse, in: Staehle, Wolfgang; Sydow, Jörg (Hrsg.): Managementforschung, Bd. 1, Berlin/New York 1991, S. 1-74.

Kasper, Helmut; Mayrhofer, Wolfgang; Meyer, Michael (1998): Managerhandeln nach der systemtheoretisch-konstruktivistischen Wende, in: Die Betriebswirtschaft, 58. 1998, Nr. 5, S. 603-621.

Kern, Werner (1992): Industrielle Produktionswirtschaft, 5. Auflage, Stuttgart 1992.

Kieser, Alfred (1988): Erklären die Theorie der Verfugungsrechte und der Transaktionskostenansatz historischen Wandel von Institutionen?, in: Budäus, Dietrich; Gerum, Elmar; Zimmermann, Gebhard: Betriebswirtschaftslehre und Theorie der Verfugungsrechte, Wiesbaden 1988, S. 299-323.

Kieser, Alfred (1993): Anleitung zum kritischen Umgang mit Organisationstheorien, in: Kieser, Alfred (Hrsg.): Organisationstheorien, Stuttgart et al. 1993, S. 1-35. 
Kieser, Alfred; Kubicek, Herbert (1978): Organisationstheorien, Bd. 1, Stuttgart 1978.

Kieser, Alfred; Kubicek, Herbert (1992): Organisation, 3. Auflage, Berlin/New York 1992.

Kieser, Alfred; Reber, Gerhard; Wunderer, Rolf (1987): Handwörterbuch der Führung, Stuttgart 1987.

Kirchgässner, Gebhard (2000): Homo Oeconomicus, 2. Auflage, Tübingen 2000.

Kirn, Stefan (1995): Organisatorische Flexibilität durch Workflow-ManagementSysteme?, in: Handbuch der modernen Datenverarbeitung, 32. 1995, Nr. 182, S. $100-112$.

Kirsch, Werner (1971): Entscheidungsprozesse, Bd. 2, Wiesbaden 1971.

Kirsch, Werner (1979): Die verhaltenswissenschaftliche Fundierung der Betriebswirtschaftslehre, in: Raffée, Hans; Abel, Bodo (Hrsg.): Wissenschaftstheoretische Grundfragen der Wirtschaftswissenschaften, München 1979, S. 105-120.

Kirsch, Werner (1992): Kommunikatives Handeln, Autopoiese, Rationalität Sondierungen zu einer evolutionären Führungslehre, München 1992.

Kirsch, Werner; Knyphausen, Dodo zu (1991): Unternehmungen als „autopoietische“ Systeme?, in: Staehle, Wolfgang; Sydow, Jörg (Hrsg.): Managementforschung, Bd. 1, Berlin/New York 1991, S. 75-101.

Klaus, Georg (1965): Kybernetik aus philosophischer Sicht, 4. Auflage, Berlin 1965.

Klaus, Georg (1969): Wörterbuch der Kybernetik, Frankfurt a.M. 1965.

Klaus, Peter (1987): Durch den Strategie-Theorie-Dschungel, in: Die Betriebswirtschaft, 47. 1987, Nr. 1, S. 50-68.

Klein, Benjamin; Crawford Robert G.; Alchian, Armen A. (1978): Vertical Integration, Appropriate Rents and the Competitive Contracting Process, in: Journal of Law and Economics, 21. 1978, Nr. 2, S. 297-326.

Klein, Stefan (1989): Manager und Planer, in: Szyperski, Norbert (Hrsg.): Handwörterbuch der Planung, Stuttgart 1989, Sp. 1000-1006.

Klein, Stefan (1996): Informationstechnologie und Unternehmensnetzwerke, in: Bellmann, Klaus; Hippe, Alan (Hrsg.): Management von Unternehmensnetzwerken - Interorganisationale Konzepte und praktische Umsetzung, Wiesbaden 1996, S. 157-190.

Klein, Stefan (1996): Interorganisationssysteme und Unternehmensnetzwerke Wechselwirkungen zwischen organisatorischer und informationstechnischer Entwicklung, Wiesbaden 1996.

Klein, Stefan (2000): The Emergence of Auctions on the World Wide Web, in: Shaw, Michael J. et al. (Hrsg.): Handbook on Electronic Commerce, Berlin et al. 2000, S. 627-645.

Kleinbeck, Uwe; Quast, Hans-Henning (1992): Motivation, in: Frese, Erich (Hrsg.): Handwörterbuch der Organisation, 3. Auflage, Stuttgart 1992, Sp. 1420-1434.

Klimecki, Rüdiger G.; Probst, Gilbert J.B. (1990): Entstehung und Entwicklung der Unternehmungskultur, in: Lattmann, Charles (Hrsg.): Die Unternehmenskultur: 
ihre Grundlagen und ihre Bedeutung für die Führung der Unternehmung, Heidelberg 1990, S. 41-65.

Kloock, Josef (1969): Betriebswirtschaftiche Input/Output-Modelle, Wiesbaden 1969.

Kloock, Josef (1993): Produktion, in: Vahlens Kompendium der Betriebswirtschaftslehre, Band 1, 3. Auflage, München 1993, S. 253-310.

Klutmann, Lorenz (1992): Integration eines strategischen Informations- und Kommunikationsmanagements in alternative Organisationsformen, Frankfurt a.M. et al. 1992.

Koch, Helmut (1961): Betriebliche Planung - Grundlagen und Grundfragen der Unternehmungspolitik, Wiesbaden 1961.

Koch, Helmut (1977): Aufbau der Unternehmensplanung, Wiesbaden 1977.

Kollmann, Tobias (2001): Ist M-Commerce ein Problem der Nutzungslücke, in: Information Management \& Consulting, 16. 2001, Nr. 2, S. 59-64.

Kosiol, Erich (1961): Erkenntnisgegenstand und methodologischer Standort der Betriebswirtschaftslehre, in: Zeitschrift für Betriebswirtschaft, 31. 1961, Nr. 3, S. 129-136.

Kosiol, Erich (1972): Die Unternehmung als wirtschaftiches Aktionszentrum, Reinbek 1972.

Kosiol, Erich; Szyperski, Norbert; Chmielewicz, Klaus (1965): Zum Standort der Systemforschung im Rahmen der Wissenschaften, in: Zeitschrift für handelswissenschaftliche Forschung, 17. 1965, S. 337-378.

Krcmar, Helmut (1990): Bedeutung und Ziele von InformationssystemArchitekturen, in: Wirtschaftsinformatik, 32. 1990, Nr. 5, S. 395-402.

Krcmar, Helmut (1991): Annäherungen an das Informationsmanagement Management- und oder Technologiedisziplin?, in: Staehle, Wolfgang (Hrsg.): Managementforschung, Bd. 2, 1991, S. 163-203.

Krcmar, Helmut (1997): Informationsmanagement, Berlin et al. 1997.

Krcmar, Helmut (2000): Informationsmanagement, 2. Auflage, Berlin et al. 2000.

Krcmar, Helmut; Federmann, Christina (1990): Informationsmanagement in der Bundesrepublik Deutschland - zum Problembewußtsein der DV-Leiter in Großunternehmen, in: Information Management, 5. 1990, Nr. 4, S. 6-16.

Kreifelts, Thomas (1983): Bürovorgänge: Ein Modell für die Abwicklung kooperativer Arbeitsabläufe in einem Bürosystem, in: Wißkirchen, Peter (Hrsg.): Informationstechnik und Bürosysteme, Stuttgart 1983, S. 215-245.

Krieg, Walter (1971): Kybernetische Grundlagen der Unternehmungsgestaltung, Bern/Stuttgart 1971.

Kronen, Juliane (1994): Computergestützte Unternehmenskooperation, Wiesbaden 1994.

Krüger, Wilfried (1993): Organisation der Unternehmung, 2. Auflage, Stuttgart, Berlin, Köln 1993. 
Kudera, Werner; Ruff, K.; Schmidt, R. (1982): Auswirkungen von Rationalisierungsmaßnahmen auf die betriebliche Situation von kaufmännischen Angestellten, in: Boehm. Ulrich v. (Hrsg.): Rationalisierung der Büroarbeit und kaufmännische Berufsausbildung, Frankfurt a.M./NewYork 1982, S. 135-144.

Kuhn, Thomas S. (1973): Die Struktur wissenschaftlicher Revolutionen, Frankfurt 1973.

Kuhn, Alfred (1990): Unternehmensfuihrung, 2. Auflage, München 1990.

Küpper, Hans-Ulrich; Weber, Jürgen; Zünd, André (1990): Zum Verständnis und Selbstverständnis des Controlling - Thesen zur Konsensbildung, in: Zeitschrift für Betriebswirtschaft, 60. 1990, Nr. 3, S. 281-293.

Küpper, Hans-Ulrich (1979): Dynamische Produktionsfunktion der Unternehmung auf der Basis des Input-Output-Ansatzes, in: Zeitschrift für Betriebswirtschaft, 49. 1979, Nr. 2, S. 93-106.

Kurtyka, Jerry (2000): CRM in the Adaptive Enterprise, in: DM Review, February 2000, http://www.dmreview.com.

Laartz, Andreas; Hjartar, Klemens (2000): Mobile Portale - der Kampf um die Kundenschnittstelle im Zukunftsmarkt M-Commerce hat begonnen, in: Information Management \& Consulting, 15. 2000, Nr. 4, S. 29-33.

Lai, Jeffrey; Mohan, Ajit; Gustafson, Grant (2000): Understanding the Emerging Mobile Commerce Marketplace, http://www.arthurdlittle.de/ services/management_consulting/e-business/articles04/ScenariosFinal.pdf, abgerufen am 07.05.2001.

Lakatos, Imre (1974): Falsifikation und die Methodologie wissenschaftlicher Forschungsprogramme, in: Lakatos, Imre; Musgrave, Alan (Hrsg.): Kritik und Erkenntnisfortschritt, Braunschweig 1974, S. 89-189.

Laux, Helmut (1990): Risiko, Anreiz und Kontrolle - Principal-Agent-Konzept Einführung und Verbindung mit dem Delegationswert-Konzept, Heidelberg 1990.

Lehner, Franz (1993): Informatik-Strategien: Entwicklung, Einsatz und Erfahrungen, München et al. 1993.

Lehner, Franz (1995): Grundfragen und Positionierung der Wirtschaftsinformatik, in: Lehner, Franz; Hildebrand, Knut; Maier, Ronald (Hrsg.): Wirtschaftsinformatik Theoretische Grundlagen, München/Wien 1995, S. 1-72.

Lehner, Franz; Maier, Ronald (1994): Information in Betriebswirtschaftslehre, Informatik und Wirtschaftsinformatik, Forschungsbericht Nr. 11 der Schriftenreihe des Lehrstuhls für Wirtschaftsinformatik und Informationsmanagement, Wissenschaftliche Hochschule für Unternehmensführung - Otto-BeisheimHochschule, Koblenz 1994.

Leonhardt, Kai (2001): M-Commerce zwischen Hype und Realität, in: Computerwoche, 28. 2001, Nr. 8, S. 72-73.

Lincke, David-Michael; Zimmermann, Hand-Dieter (1999): Integrierte Standardanwendungen für Electronic Commerce - Anforderungen und Evaluationskriterien, in: Sauter, Michael; Hermanns, Arnold: Management-Handbuch 
Electronic Commerce - Grundlagen, Strategien, Praxisbeispiele, München 1999, S. $197-210$.

Lindblom, Charles E. (1959: The Science of „Muddling Through“, in: Public Administration Review, 19. 1959, Nr. 2, S. 79-88.

Link, Jörg (1982): Die methodologischen, informationswirtschaftlichen und führungspolitischen Aspekte des Controlling, in: Zeitschrift für Betriebswirtschaft, 52. 1982 , Nr. 3, S. 261-280.

Lockamy, Archie (1995): Global Logistics, managing the Product-Delivery System, Boston 1995.

Loebbecke, Claudia (2001): eCommerce: Begriffsabgrenzung und Paradigmenwechsel, in: Betriebswirtschaftliche Forschung und Praxis, 48. 2001, Nr. 2, S. 93-108.

Lötsch, Bert (1997): Prozeßunterstützung durch moderne Kommunikations- und Planungssysteme, in Zeitschrift für wirtschaftliche Fertigung, 92. 1997, Nr. 3, S. 122-123.

Lucent Technologies (2001): Mobile Internet, http://www.lucent.com/developer/wireless, abgerufen am 02.05.2001.

Lücke, Wolfgang (1973): Qualitätsprobleme im Rahmen der Produktions- und Absatztheorie, in: Koch, Helmut (Hrsg.): Zur Theorie des Absatzes - Festschrift zum 75. Geburtstag von Erich Gutenberg, Wiesbaden 1974, S. 262-299.

Lücke, Wolfgang (1997): Die prägende Wirkung der Lehre Gutenbergs für die Entwicklung der Betriebswirtschaftslehre, in: Zeitschrift für Betriebswirtschaft, 67. 1997 , Nr. 12, S. 1245-1256.

Luhmann, Norbert (1984a): Soziale Systeme - Grundriß einer allgemeinen Theorie, Frankfurt a.M. 1984.

Luhmann, Norbert (1984b): Soziologische Aspekte des Entscheidungsverhaltens, in: Die Betriebswirtschaft, 44. 1984, Nr. 4, S. 591-603.

Luhmann, Norbert (1985): Die Autopoiesis des Bewußtseins, in: Soziale Welt, 36. 1985 , Nr. 5, S. $402-446$.

MacNeil, Ian R. (1974): The Many Futures of Contract, in: Southern California law review, 47. 1974, S. 691-738.

MacNeil, Ian R. (1978): Contracts: Adjustment of Long-Term Economic Relations under Classical, Neoclassical, and Relational Contract Law, in: North Western University Law Review, 1978, Nr. 72, S. 854-905.

MacNeil, Ian R. (1987): Relational contract theory as sociology: A reply to Professors Lindenberg and de Vos, in: Journal of institutional and theoretical economics, 143. 1987, Nr. 2, S. 272-290.

Madhavan, Ravindranath; Shah, Reshma H.; Grover, Rajiv (1994): Relationship Marketing: An Organizational Process Perspective, in: Sheth, Jagdish; Parvatiyar, Atul (Hrsg.): Relationship Marketing: Theory, Methods and Applications, Research Conference Proceedings, Atlanta 1994, S. 1-10.

Mag, Wolfgang (1992): Die Funktionserweiterung der Unternehmungsführung, in: Wirtschaftswissenschaftliches Studium, 21. 1992, Nr. 2, S. 60-64. 
Mag, Wolfgang (1995): Unternehmungsplanung, München 1995.

Maier, Ronald; Lehner, Franz (1995): Daten, Information, Wissen, in: Lehner, Franz; Hildebrand, Knut; Maier, Ronald (Hrsg.): Wirtschaftsinformatik Theoretische Grundlagen, München/Wien 1995, S. 165-272.

Malik, Fredmund (1982): Evolutionäres Management, in: Die Unternehmung, 36. 1982, Nr. 2, S. 91-105.

Malik, Fredmund (1992): Strategie des Managements komplexer Systeme - Ein Beitrag zur Management-Kybernetik evolutionärer Systeme, 4. Auflage, Bern et al. 1992.

Malik, Fredmund (1993): Systemisches Management, Evolution, Selbstorganisation: Grundprobleme, Funktionsmechanismen und Lösungsansätze für komplexe Systeme, Bern et al. 1993.

Malone, Thomas W.; Yates, Joanne; Benjamin, Robert (1987): Electronic Markets and Electronic Hierarchies, in: Communications of the ACM, 30. 1987, Nr. 6, S. 484-487.

Malone, Thomas W.; Yates, Joanne; Benjamin, Robert (1989): The Logic of Electronic Markets, in: Harvard business Review, 68. 1989, Nr. 3, S. 166-172.

Malone, Thomas W.; Rockart, John F. (1991): Computers, Networks and the Corporation, in: Scientific American, September 1991, S. 92-99.

March, James G.; Simon, Herbert A. (1958): Organizations, New York 1958.

Marchand, Donald A. (1989): Information Management: Strategies and tools in Transition, in: Information Management Review, 1989, Nr. 3, S. 27-34.

Markus, Lynne; Robey, Daniel (1988): Information Technology and Organizational Change: Causal Structure in Theory and Research, in: Management Science, 34. 1988, Nr. 5, S. 583-598.

Martiny, Lutz; Klotz, Michael (1989): Strategisches Informationsmanagement: Bedeutung und organisatorische Umsetzung, 3. Auflage, Berlin 1998.

Maruyama, M. (1963): The second cybernetics - Deviation amplifying mutual causal processes, in: American Scientist, 51. 1963, S. 164-179.

Matenaar, Dieter (1983): Organisationskultur und organisatorische Gestaltung - Die Gestaltungsrelevanz der Kultur des Organisationssystems der Unternehmung, Berlin/München 1983.

Mattes, Frank (1999): Electronic Business-to-Business, Stuttgart 1999.

Matthews, Robin C. (1986): The Economics of Institutions and the Sources of Economic Growth, in: Economic Journal, 95. 1986, Nr. 96, S. 903-918.

Maturana, Humberto R. (1981): Autopoiesis, in: Zeleny, Milan (Hrsg.): Autopoiesis: A Theory of Living Organization, New York 1981, S. 21-33.

Maturana, Humberto R.; Varela Francisco J. (1980): Autopoiesis and Cognition, Dordrecht 1980.

Maturana, Humberto R; Varela Francisco J.; Uribe, Ricardo B. (1982): Autopoiese: die Organisation lebender Systeme, ihre nähere Bestimmung und ein 
Modell, in: Maturana, Humberto R. (Hrsg.): Erkennen: Die Organisation und Verkörperung von Wirklichkeit, Braunschweig/Wiesbaden 1982, S. 157-169.

Maturana, Humberto R.; Varela, Francisco J. (1987): Der Baum der Erkenntnis Die biologischen Wurzeln des menschlichen Erkennens, Bern/München 1987.

Mayer, Rainer (1993): Strategien erfolgreicher Produktgestaltung - Individualisierung und Standardisierung, Wiesbaden 1993.

Mc Call, J.J. (1970): Economics of Information and Job Search, in: Quaterly Journal of Economics, 30. 1970, Nr. 2, S. 113-126.

Meier, Marco (1998): Integration von Informationen aus dem Internet in das SAPEIS - Konzeption und prototypische Realisierung eines Redaktions-Leitstands, in: Uhr, Wolfgang; Breuer, Sven-Einar (Hrsg.): Integration externer Informationen in Management Support Systems - Wirtschaftsinformatik Fachtagung, Technische Universität Dresden 1998, S. 105-122.

Mertens, Peter (1985): Zwischenbetriebliche Integration der EDV, in: InformatikSpektrum, ohne Jahrgang, 1985, Nr. 8, S. 81-90.

Mertens, Peter (1998): Integration interner, externer, qualitativer und quantitativer Daten auf dem Weg zum Aktiven MIS, in: Uhr, Wolfgang; Breuer, Sven-Einar (Hrsg.): Integration externer Informationen in Management Support Systems, Dresden 1998, S. 9-30.

Mertens, Peter; Griese, Joachim; Ehrenberg, Dieter (1998): Virtuelle Unternehmen und Informationsverarbeitung, Berlin et al. 1998.

Mertens, Peter; Höhl, Michael (1999): Wie lernt der Computer den Menschen kennen? Bestandsaufnahme und Experimente zur Benutzermodellierung in der Wirtschaftsinformatik, FORWISS-Report, FR-1999-0002, Erlangen 1999.

Meyer, Manfred (1996): Operations Research - Systemforschung, 4. Auflage, Stuttgart 1986.

Microsoft (2001a): Was die .NET-Strategie von Microsoft fur Unternehmen bedeutet, http://www.Microsoft.com/germany/themen/net/bedeutung.htm, abgerufen am 09.05.2001.

Microsoft (2001b): The Microsoft Vision and Strategies for Mobile Technology, http://www.microsoft.com/business/mobility/vision.asp, abgerufen am 09.05.2001.

Miksch, Katharina (1991): Management-Informationssysteme. Der Nutzen steckt im Detail, in: Diebold Management Report, ohne Jahrgang, 1991, Nr. 11, S. 12-16.

Mikus, Barbara (1998): ZP-Stichwort: Principal-Agent-Theorie, in: Zeitschrift für Planung, 9. 1998, Nr. 9, S. 451-458.

Miller, James Grier (1978): Living Systems, New York et al. 1978.

Mirow, Michael (1969): Kybernetik - Grundlage einer allgemeinen Theorie der Organisation, Wiesbaden 1969.

Mirow, Michael (1999): Von der Kybernetik zur Autopoiese - Systemtheoretisch abgeleitete Thesen zur Konzernentwicklung, in: Zeitschrift für Betriebswirtschaft, 69. 1999, Nr. 1, S. 13-27. 
Mirowski, Philip (1990): From Mandelbrot to Chaos in Economic Theory, in: Southern Economic Journal, 57. 1990, Nr. 2, S. 289-307.

Mizukoshi, Yukata; Okino, Kimihide; Tardy, Olivier (2000): Lessons from Japan, http://www.bcg.com/publications/files/Lessons_from_Japan_Oct00.pdf, abgerufen am 15.05.2001.

Mobilocity (2000): Understanding the Fundamentals of M-Commerce, http://www.mobilocity.net/publications/fundamentals_mcom.pdf, abgerufen am 07.05.2001.

Mobilocity (2001): Fundamentals of Mobile Business-to-Employees (B2E) Applications, http://www.mobilocity.net/publications/fundamentals_B2E.pdf, abgerufen am 07.05.2001.

Mönig, Hartmut (1985): Fertigungsorganisation und Wirtschaftlichkeit einer Fertigungsinsel, in: Zeitschrift furr betriebswirtschaftliche Forschung, 37. 1985, Nr. 1, S. 83-101.

Montada, Leo (1970): Die Lernpsychologie Jean Piagets, Stuttgart (1970).

Mössner, Gerd U. (1982): Planung flexibler Unternehmungsstrategien, München 1982.

Müller, Klaus (1992): „Katastrophen“, „Chaos“ und „Selbstorganisation“. Methodologie und sozialwissenschaftliche Heuristik der jüngeren Systemtheorie, in: Prokla. Zeitschrift für kritische Sozialwissenschaft, 22. 1992, Nr. 3, S. 340-373.

Müller, Wolfgang (1987): Zur informationstheoretischen Erweiterung der Betriebswirtschaftslehre - Ein Modell der Informationsproduktion, in: Adam, Dietrich (Hrsg.): Neuere Entwicklungen in der Produktions- und Investitionspolitik, Wiesbaden 1987, S. 119-135.

Nagel, Claudia (1995): Zur Kultur der Organisation - Eine organisationspsychologische Untersuchung in der Automobilindustrie, Frankfurt a.M. 1995.

Nawatzki, Jürgen (1994): Integriertes Informationsmanagement: Die Koordination von Informationsverarbeitung, Organisation und Personalwirtschaft bei der Planung, Durchführung, Kontrolle und Steuerung des Einsatzes neuer Informationstechnologie in der Unternehmung, Bergisch Gladbach/Köln 1994.

Naisbitt, John (1984): Megatrends, 2. Auflage, Bayreuth 1984.

Nebl, Theodor (1996): Einführung in die Produktionswirtschaft, München/Wien 1996.

Nelson, Phillip (1970): Information and Consumer Behaviour, in: Journal of Political Economy, 78. 1970, Nr. 5, S. 311-329.

Nenninger, Michael; Gerst, Martina (1999): Wettbewerbsvorteile durch Electronic Procurement, in: Hermanns, Arnold; Sauter, Michael: Management Handbuch ECommerce, München 1999, S. 283-296.

Neuberger, Oswald (1977): Organisation und Führung, Stuttgart et al. 1977

Neumann, John von; Morgenstern, Oskar (1961): Spieltheorie und wirtschaftiches Verhalten, Würzburg 1961.

Neus, Werner (1998): Einfuhrung in die Betriebswirtschaftslehre aus institutionenökonomischer Sicht, Tübingen 1998. 
Nippa, Michael (1988): Gestaltungsgrundsätze für die Büroorganisation: Konzepte für eine informationsorientierte Unternehmungsentwicklung unter Berücksichtigung neuer Bürokommunikationstechniken, Berlin 1988.

Noorderhaven, Niels G. (1996): Opportunism and trust in transaction cost economics, in: Goenewegen, John (Hrsg.): Transaction Cost Economics and Beyond, Deveneter 1996, S. 105-128.

Nüttgens, Markus (1995): Koordiniert-dezentrales Informationsmanagement: Rahmenkonzept - Koordinationsmodelle - Werkzeug-Shell, Wiesbaden 1995.

Oberschulte, Hans (1994): Organisatorische Intelligenz - Ein integrativer Ansatz des organisatorischen Lernens, Dissertation Universität Saarbrücken, München/ Mering 1994.

Ochsenbauer, Christian; Klofat, Bernhard (1987): Überlegungen zur paradigmatischen Dimension der aktuellen Unternehmenskulturdiskussion in der Betriebswirtschaftslehre, in: Heinen, Edmund (Hrsg.): Unternehmenskultur - Perspektiven für Wissenschaft und Praxis, München 1987, S. 67-106.

Oeser, Eerhard (1986): Der Informationsbegriff in der Philosophie und in der Wissenschaftstheorie, in: Folberth, Otto G.; Hackl, Clemens (Hrsg.): Der Informationsbegriff in Technik und Wissenschaft München/Wien 1986. S. 231-250.

Österle, Hubert (1981): Entwurf betrieblicher Informationssysteme, München/Wien 1981.

Österle, Hubert (1990): Unternehmensstrategie und Standardsoftware, Schlüsselentscheidungen für die 90er Jahre, in: Oesterle, Hubert (Hrsg.): Integrierte Standardsoftware: Entscheidungshilfen für den Einsatz von Softwarepaketen, Bd. 1, Hallbergmoos 1990, S. 11-36.

Ouchi, William G. (1980): Markets, Bureaucracies and Clans, in: Administrative Science Quaterly, 25. 1980, Nr. 2, S. 129-141.

Paetau, Michael (1991): Kooperative Konfiguration. Ein Konzept zur Systemanpassung an die Dynamik kooperativer Arbeit, in: Friedrich, Jürgen; Rödiger, Karl-Heinz (Hrsg.): Computergestützte Gruppenarbeit (CSCW), Reihe: Berichte des German Chapter of the ACM, Bd. 34, Stüttgart 1991, S. 137-151.

Parker, David; Stacey, Ralph (1995): Chaos, Management and Economics - The Implications of Non-Linear Thinking, 2. Auflage, London 1995.

Pautzke, Gunnar (1989): Die Evolution der organisatorischen Wissensbasis Bausteine zu einer Theorie des organisatorischen Lernens, Dissertation Universität München, Herrsching 1989.

Pawlowski, Peter (1992): Betriebliche Qualifikationsstrategien und organisationales Lernen, in: Staehle, Wolfgang; Conrad, Peter (Hrsg.): Managementforschung, Bd. 2, Berlin/New York 1992, S. 177-237.

Perrow, Charles (1986): Economic theories of organization, in: Theory and Society, 15. 1986, Nr. 1, S. 11-45.

Peterhans, Markus (1996): Informationsmanagement - Theoretische Grundlagen und Führungskonzept, Zürich 1996. 
Pfau, Wolfgang (1997): Betriebliches Informationsmanagement: Flexibilisierung der Informationsinfrastruktur, Wiesbaden 1997.

Pfeiffer, Peter (1990): Technologische Grundlage, Strategie und Organisation des Informationsmanagements, Berlin/New York 1990.

Pflug, Volkmar (2001): Die Revolution des Electronic Business, in: Technologie \& Management, 50. 2001, Nr. 3-4, S. 21-23.

Piaget, Jean (1976): Die Äquilibration der kognitiven Strukturen, Stuttgart 1976.

Picot, Arnold (1975): Zur Frage der Ableitung von empirisch überprüfbaren Aussagen aus systemtheoretischen Ansätzen der Organisationsforschung, in: Systemforschung in der Betriebswirtschaftslehre, in: Jehle v. E. (Hrsg.): Tagungsbericht des Arbeitskreises für Wissenschaftstheorie im Verband der Hochschullehrer für Betriebswirtschaft e.V. Stuttgart 1975, S. 87-105

Picot, Arnold (1982): Transaktionskostenansatz in der Organisationstheorie: Stand der Diskussion und Aussagewert, in: Die Betriebswirtschaft, 42. 1982, Nr. 2, S. 267-284.

Picot, Arnold (1990): Der Produktionsfaktor Information in der Unternehmensführung, in: Information Management, 5. 1990, S. 6-14.

Picot, Arnold (1990): Organisation von Informationssystemen und Controlling, in: Controlling, 2. 1990, Nr. 6, S. 296-305.

Picot, Arnold (1991): Ein neuer Ansatz zur Gestaltung der Leistungstiefe, in: Zeitschrift für betriebswirtschaftliche Forschung, 43. 1991, Nr. 4, S. 336-357.

Picot, Arnold; Franck, Egon (1988): Die Planung der Unternehmensressource Information, in: Das Wirtschaftsstudium, 17. 1988, Nr. 10, 11, S. 544-549, 608-614.

Picot, Arnold; Franck, Egon (1993): Aufgabenfelder eines Informationsmanagement, in: Das Wirtschaftsstudium, 22. 1993, Nr. 5+6, S. 433-437, 520-526.

Picot, Arnold; Reichwald, Ralf (1991): Informationswirtschaft, in: Heinen, Edmund (Hrsg.): Industriebetriebslehre: Entscheidungen im Industriebetrieb, 9. Auflage, Wiesbaden 1991, S. 241-393.

Picot, Arnold; Reichwald, Ralf; Wigand, Rolf (1998): Die grenzenlose Unternehmung - Information, Organisation und Management, 3. Auflage, Wiesbaden 1996.

Picot, Arnold; Reichwald, Ralf; Wigand, Rolf (2001): Die grenzenlose Unternehmung - Information, Organisation und Management, 4. Auflage, Wiesbaden 2001.

Piller, Frank T. (2000): Mass Customization - Ein wettbewerbsstrategisches Konzept im Informationszeitalter, Wiesbaden 2000.

Piller, Frank T.; Zanner, Stefan (2001): Mass Customization und Personalisierung im Electronic Business, in: Das Wirtschaftsstudium, 31. 2001, Nr. 1, S. 88-96.

Plesman, Maarten (2000): Serving the mobile customer, http://www.arthurdlittle.de/services/management_consulting/

e-business/archive/MobileCommerce.pdf, abgerufen am 15.05.2001. 
Popper, Karl R. (1995): Auf der Suche nach einer besseren Welt, 8. Auflage, München 1995.

Porter, Michael E.; Millar, Victor E. (1986): Wettbewerbsvorteile durch Information, in: Harvard Manager, ohne Jahrgang 1986, Nr. 1, S. 26-35.

Prigogine, Iliya (1980): From Being to Becoming, San Francisco 1980.

Probst, Gilbert (1987): Selbst-Organisation - Ordnungsprozesse in sozialen Systemen aus ganzheitlicher Sicht, Berlin/Hamburg 1987.

Probst, Gilbert J.B.; Dyllick, Thomas (1982): Begriffe, Analogiebildung und Intention im evolutionären Management, in: Die Unternehmung, 36. 1982, Nr. 2, S. 107-112.

Probst, Gilbert J.B.; Romhardt, Kai (1997): Bausteine des Wissensmanagements ein praxisorientierter Ansatz, in: Wieselhuber, Norbert et al. (Hrsg.): Handbuch Lernende Organisation, Wiesbaden 1997, S. 129-143.

Putnam, Linda L. (1983): The interpretive perspective: an alternative to functionalism, in: Putnam, Linda L.; Pcanowsky, Michael E. (Hrsg.): Communication and Organizations. An Interpretive Approach, Beverly Hills et al. 1983, S. 31-54.

Raffée, Hans (1974): Grundprobleme der Betriebswirtschaftslehre, Göttingen 1974.

Raffée, Hans (1993): Gegenstand, Methoden und Konzepte der Betriebswirtschaftslehre, in: Bitz, Michael et al. (Hrsg.), Kompendium der Betriebswirtschaftslehre, Band 1, 3. Auflage, München 1993, S. 1-46.

Rehberg, Jürgen (1973): Wert und Kosten von Informationen, Frankfurt am Main 1973.

Reichmann, Thomas (1996): Management und Controlling. Gleiche Ziele - unterschiedliche Wege und Instrumente. In: Zeitschrift für Betriebswirtschaft, 66. 1996, Nr. 5, S. 559-585.

Reichwald, Ralf (1997): Neue Arbeitsformen in der vernetzten Unternehmung: Flexibilität und Controlling, in: Picot, Arnold (Hrsg.): Information als Wettbewerbsfaktor, Stuttgart 1997, S. 233-263.

Reichwald, Ralf; Behrbohm, Peter (1983): Flexibilität als Eigenschaft produktionswirtschaftlicher Systeme, in: Zeitschrift für Betriebswirtschaft, 53. 1983, Nr. 9, S. 831-853.

Reichwald, Ralf; Möslein, Kathrin (2000): Nutzenpotentiale und Nutzenrealisierung in verteilten Organisationsstrukturen - Experimente, Erprobungen und Erfahrungen auf dem Weg zur virtuellen Unternehmung, in: Zeitschrift für Betriebswirtschaft, Ergänzungsheft 2/2000, S. 117-136.

Reichwald, Ralf; Piller, Frank (2000): Mass-Customization-Konzepte im Electronic Business, in: Weiber, Rolf (Hrsg.): Handbuch Electronic Business, Wiesbaden 2000, S. 359-382.

Reif, Ane-Kristin; Knittel, Friedrich (1996): Informationsverarbeitung, Koordination und Kooperation im Büro - Konzepte der Computerunterstützung für die Büroarbeit, Arbeitsbericht Nr. 96-20 des Lehrstuhls für Wirtschaftsinformatik, Ruhr-Universität Bochum 1996. 
Reif-Mosel, Ane-Kristin (2000): Computergestützte Kooperation im Büro - Gestaltung unter Berücksichtigung der Elemente Aufgabe, Struktur, Technik und Personal, Frankfurt a.M. 2000.

Richardson, George B. (1972): The Organization of Industry, in: the Economic Journal, 82. 1972, Nr. 327, S. 882-896.

Richter, Rudolf (1994): Institutionen ökonomisch analysiert - zur jüngeren Entwicklung auf einem Gebiet der Wirtschaftstheorie, Tübingen 1994.

Richter, Rudolf; Bindseil, Ulrich (1995): Neue Institutionenökonomik, in: Wirtschaftswissenschaftliches Studium, 24. 1995, Nr. 3, S. 132-140.

Richter, Rudolf; Furubotn, Eirik G. (1999): Neue Institutionenökonomik - Eine Einführung und kritische Würdigung, 2. Auflage, Tübingen 1999.

RiBmann, Martina; Loos, Christoph; Mei-Pochtler, Antonella; Dean, David (1999): Der Kampf um den Konsumenten in der neuen Agorà, in: Hermanns, Arnold; Sauter, Michael: Management Handbuch Electronic Commerce, München 1999, S. 141-155.

Rochlitz, Alexander (2001): An UMTS führt kein Weg vorbei, Interview, in Technologie und Management, 50. 2001, Nr. 3-4, S. 10-12.

Roethlisberger, Fritz Jules; Dickson, William J. (1939): Management and the worker, Cambridge/ Mass. 1939.

Rohrbach, Peter (1999): Electronic Commerce im Business-to-Business-Bereich Herausforderungen, Konzeption und Fallbeispiele, in: Sauter, Michael; Hermanns, Arnold: Management-Handbuch Electronic Commerce - Grundlagen, Strategien, Praxisbeispiele, München 1999, S. 271-282.

Rolf, Arno (1998): Grundlagen der Organisations- und Wirtschaftsinformatik, Berlin et al. 1998.

Rosen, Harvey; Windisch, Rupert (1997): Finanzwissenschaft I, München/Wien 1997.

Rosenstiel, Lutz v. (1978): Arbeitsgruppe, in Mayer, Arthur (Hrsg.): Organisationspsychologie, Stuttgart 1978, S. 236-271.

Rosenstiel, Lutz v. (1992): Organisationspsychologie, in: Frese, Erich (Hrsg.): Handwörterbuch der Organisation, 3. Auflage, Stuttgart 1992, Sp. 1619-1633.

Ross, Stephen A. (1973): The Economic Theory of Agency: The Principal's Problem, in: American Economic Journal, 63. 1973, Nr. 3, S. 134-139.

Röver, Stefan (2000): Mobile Commerce: Von der Vision zur Realität, in: Handbuch der modernen Datenverarbeitung, 37. 2000, Nr. 215, S. 49-56.

Rühli, Edwin (1989): Entscheidungsorientierter Ansatz und Allgemeine Betriebswirtschaftslehre, in: Krisch, Werner; Picot, Arnold (Hrsg.), Die Betriebswirtschaftslehre im Spannungsfeld zwischen Spezialisierung und Generalisierung, Wiesbaden 1989.

Rühli, Edwin (1990): Ein methodischer Ansatz zur Erfassung und Gestaltung von Unternehmenskulturen, in: Lattmann, Charles (Hrsg.): Die Unternehmenskultur, Heidelberg 1990, S. 189-205. 
Sackmann, Sonja A. (1990): Möglichkeiten der Gestaltung von Unternehmenskultur, in: Lattmann, Charles (Hrsg.): Unternehmenskultur: ihre Grundlagen und ihre Bedeutung für die Führung der Unternehmung, Heidelberg 1990, S. 153-188.

Salop, S.; Stiglitz, J.E. (1977): Bargains and Ripoffs - A Model of Monopolistically Competitive Price Dispersion, in: Review of Economic Studies.

Sandner, Karl (1982): Evolutionäres Management - Voraussetzungen und Konsequenzen eines Ansatzes der Steuerung sozialer Systeme, in: Die Unternehmung, 36. 1982, Nr. 2, S. 77-89.

Sauter, Franz (1985): Die Ökonomie von Organisationsformen - Eine transaktionskostentheoretische Analyse, München 1985.

Sauter, Michael (1999): Chancen, Risiken und strategische Herausforderungen des Electronic Commerce, in: Sauter, Michael; Hermanns, Arnold: ManagementHandbuch Electronic Commerce - Grundlagen, Strategien, Praxisbeispiele, München 1999, S. 101-117.

Schader, Martin; Rundshagen, Michael (1996): Objektorientierte Systemanalyse Eine Einführung, 2. Auflage, Berlin et al. 1996.

Schanz, Günther (1997a): Wissenschaftsprogramme der Betriebswirtschaftslehre, in: Bea, Franz Xaver; Dichtl, Erwin; Schweitzer, Marcell (Hrsg.): Allgemeine Betriebswirtschaftslehre, Bd. 1, 7. Auflage, Stuttgart 1997, S. 81-198.

Schanz, Günther (1997b): Wissenschaftsprogramme - Orientierungsrahmen und Bezugspunkte betriebswirtschaftlichen Forschens und Lehrens, in: Wirtschaftswissenschaftliches Studium, 26.1997, Nr. 11, S. 554-561.

Scheer, August-Wilhelm; Feld, Thomas; Göbl, Mark; Hoffmann, Michael (2001): Das Mobile Unternehmen, in: Information Management \& Consulting, 16. 2001, Nr. 2, S. 7-15.

Schellmann, Hartmut (1997): Informationsmanagement - Theoretischer Anspruch und betriebliche Realität, Wiesbaden 1997.

Schierenbeck, Henner (1995): Grundzüge der Betriebswirtschaftslehre, 12. Auflage, München 1995.

Schlögl, Christian (2001): Bestandsaufnahme Informationsmanagement - Eine szientometrische, qualitative und empirische Analyse, Wiesbaden 2001.

Schmid, Beat (1993): Elektronische Märkte, in: Wirtschaftsinformatik, 35. 1993, Nr. 5, S. 465-480.

Schmid, Beat (1999): Elektronische Märkte, in: Hermanns, Arnold; Sauter, Michael: Management Handbuch E-Commerce, München 1999, S. 31-48.

Schmid, Marcel (1992): Kommunikationsmodelle für elektronische Märkte und mögliche Infrastrukturen zu deren Realisierung, Diss. Hochschule St. Gallen 1992.

Schmid, Roland E.; Bach, Volker; Österle, Hubert (2000): Mit Customer Relationship Management zum Prozessportal, in: Bach, Volker; Österle, Hubert (Hrsg.): Customer Relationship Management in der Praxis - Erfolgreiche Wege zu kundenzentrierten Lösungen, Berlin et al. 2000, S. 3-55. 
Schmidt, Siegfrid J. (1982): Unsere Welt - und das ist alles, in: Merkur, Heft 36, 1982, S. 356-365.

Schmitzer, Benno; Butterwegge, Gerald (2000): M-Commerce, in: Wirtschaftsinformatik, 42. 2000, Nr. 4, S. 355-358.

Schmoller, Gustav v. (1900): Grundriß der Allgemeinen Volkswirtschaftslehre, München 1900.

Schneider, Dieter (1990a): Verfehlte Erwartungen an eine Allgemeine Betriebswirtschaftslehre in Lehre und Forschung. Eine Stellungnahme zum Münsteraner Thesenpapier, in: Die Betriebswirtschaft, 50. 1990, Nr. 2, S. 272-280.

Schneider, Dieter (1993): Betriebswirtschaftslehre, Bd. 1: Grundlagen, München 1993.

Schneider, Dieter (1996): Biologische Vorbilder für eine evolutorische Theorie der Unternehmung?, in: Zeitschrift für betriebswirtschaftliche Forschung, 48. 1996, Nr. 12, S. 1098-1114.

Schneider, Dieter (1997): Betriebswirtschaftslehre, Bd. 3: Theorie der Unternehmung, München/Wien 1997.

Schneider, Ursula (1990b): Kulturbewußtes Informationsmanagement - Ein organisationstheoretischer Gestaltungsrahmen für die Infrastruktur betrieblicher Informationsprozesse, München 1990.

Schönert, Olaf (1998): Architekturen rechnergestützter Frühaufklärungssysteme, in: Uhr, Wolfgang; Breuer, Sven-Einar (Hrsg.): Integration externer Informationen in Management Support Systems - Wirtschaftsinformatik Fachtagung, Technische Universität Dresden 1998, S. 71-83.

Schotters, Markus (1992): Informationsmanagement für mittelständische Unternehmungen: Aufgaben, funktionale Anforderungen und Ausgliederungsmöglichkeiten, Bergisch Gladbach/Köln 1992.

Schreiber, Gerhard Andreas (2000): Schlüsseltechnologie Mobilkommunikation: mCommerce - das Handy eröffnet neue Märkte, Köln 2000.

Schreyögg, Georg (1984): Unternehmensstrategie - Grundfragen einer Theorie der strategischen Unternehmensführung, Berlin et al. 1984.

Schreyögg, Georg; Noss, Christian (1997): Zur Bedeutung des organisationalen Wissens furr organisatorische Lernprozesse, in: Wieselhuber, Norbert et al. (Hrsg.): Handbuch Lernende Organisation, Wiesbaden 1997, S. 67-76.

Schüler, Wolfgang (1987): Der Produktionsfaktor Information im Blick der Unternehmensführung, in: Jahrbuch für Betriebswirte, 12. 1987, S. 60-66.

Schüler, Wolfgang (1989): Informationsmanagement: Gegenstand und organisatorische Konsequenzen, in: Spremann, Klaus; Zur, Eberhard (Hrsg.): Informationstechnologie und strategische Führung.

Schüppler, David (1998): Informationsmodelle für überbetriebliche Prozesse: ein Ansatz zur Gestaltung von Interorganisationssystemen, Frankfurt a.M. et al. 1998. 
Schütte, Reinhard (1998): Grundsätze ordnungsmäßiger Referenzmodellierung Konstruktion konfigurations- und anpassungsorientierter Modelle, Wiesbaden 1998.

Schütze, Roland (1992): Kundenzufriedenheit - After Sales Marketing auf industriellen Märkten, Wiesbaden 1992.

Schwarze, Jochen (1990): Betriebswirtschaftiche Aufgaben und Bedeutung des Informationsmanagements, in: Wirtschaftsinformatik, 32. 1990, Nr. 2, S. 104115 .

Schwarze, Jochen (1995): Komplexitätsreduktion durch hierarchische Dekomposition, in Wirtschaftswissenschaftliches Studium, 24. 1995, Nr. 8-9, S. 679-685.

Schwarze, Jochen (1998): Informationsmanagement - Planung, Steuerung, Koordination und Kontrolle der Informationsversorgung im Unternehmen, Herne/Berlin 1998.

Schwarzer, Bettina (1994): Prozessorientiertes Informationsmanagement in multinationalen Unternehmen - eine empirische Untersuchung in der Pharmaindustrie, Wiesbaden 1994.

Schwarzer, Bettina; Krcmar, Helmut (1994): Business Redesign - Implikationen für das Informationsmanagement, in: Krickl, Otto (Hrsg.): Geschäftsprozeßmanagement - Prozeßorientierte Organisationsgestaltung und Informationstechnologie, Heidelberg 1994, S. 79-92.

Schweitzer, Marcell (1997): Gegenstand und Methoden der Betriebswirtschaftslehre, in: Bea, Franz Xaver; Dichtl, Erwin, Schweitzer, Marcell (Hrsg.): Allgemeine Betriebswirtschaftslehre, Bd. 1, 7. Auflage, Stuttgart 1997, S. 1-80.

Schweitzer, Marcell; Friedl, Birgit (1992): Beitrag zu einer umfassenden Controlling-Konzeption, in: Spremann, Klaus; Zur, Eberhard (Hrsg.): Controlling, Grundlagen - Informationssysteme - Anwendungen, Wiesbaden 1992, S. 141-167.

Schweitzer, Marcell; Küpper, Hans-Ulrich (1974): Produktions- und Kostentheorie der Unternehmung, Reinbek 1974.

Schweitzer, Marcell, Küpper, Hans-Ulrich (1986): Systeme der Kostenrechnung, 4. Auflage, Wiesbaden 1986.

Seibt, Dietrich (1990): Informationsmanagement und Controlling, in: Wirtschaftsinformatik, 32. 1990, Nr. 2, S. 116-126.

Seibt, Dietrich (1993): Begriff und Aufgaben des Informationsmanagement - ein Überblick, in: Preßmar, Dieter (Hrsg.): Informationsmanagement, Wiesbaden 1993, S. 3-30.

Seidel, Eberhard (1988): Ökologisches Controlling - Zur Konzeption einer ökologisch verpflichteten Führung von und in Unternehmen, in: Wunderer, Rolf (Hrsg.), Betriebswirtschaftslehre als Management- und Führungslehre, Stuttgart 1982, S. 307-322.

Seiffert, Helmut (1983): Einführung in die Wissenschaftstheorie, Bd. 2, Phänomenologie, Hermeneutik und historische Methode, Dialektik, 8. Auflage, München 1983. 
Seiler, Martin; Gruber, Peter (2000): Bei UMTS-Anwendungen ist Phantasie gefragt - Schicksal der Mobilfunker hängt am M-Commerce, Diskussionsrunde mit Helmut an de Meulen, Yannis Christoforidis, Ernst Fischer, Detlev Kostyra, Reinhold Stammeier, in: Computerwoche, 27. 2000, Nr. 47, S. 47-48.

Selig, Jürgen (1986): EDV-Management - Eine empirische Untersuchung der Entwicklung von Anwendungssystemen in deutschen Unternehmen, Berlin et al. 1986.

Senge, Peter (1990): The Fifth Discipline - The Art and Practice of The Learning Organization, New York 1990.

Seyffert, Rudolf (1956): Betrieb, in: Nicklisch, Heinrich (Hrsg.): Handwörterbuch der Betriebswirtschaft, 3. Auflage, Bd. 1, Sp. 736.

Seyffert, Rudolf, (1957): Über Begriff, Aufgaben und Entwicklung der Betriebswirtschaftslehre, 4. Auflage, Stuttgart 1957.

Shannon, Claude E.; Weaver, Warren (1976): Mathematische Grundlagen der Informationstheorie, München 1976.

Shaw, Michael J. (2000): Electronic Commerce: State of the Art, in: Shaw, Michael J. et al. (Hrsg.): Handbook on Electronic Commerce, Berlin et al. 2000, S. 3-24.

Sieber, Pascal (1997): Die Internet-Unterstützung Virtueller Unternehmen, in: Schreyögg, Georg; Sydow, Jörg (Hrsg.): Managementforschung, Bd. 7, Berlin 1997, S. 199-234.

Simon, Herbert A. (1976): Administrative Behaviour. A Study of Decision-Making Processes in Administrative Organizations, 3. Auflage, New York 1976.

SLP-Project (2001): An Introduction to SLP, http://www.openslp.org/doc/html/IntroductionToSLP/index.html, abgerufen am 03.07.2001.

Soeffner, Hans Georg (1980): Überlegungen zur sozialwissenschaftlichen Hermeneutik am Beispiel der Interpretation eines Textausschnittes in einem freien Interview, in: Heinze, Thomas; Klusemann, Hans Werner; Soeffner, Hans-Georg (Hrsg.): Interpretationen einer Bildungsgeschichte - Überlegungen zur sozialwissenschaftlichen Hermeneutik, Bensheim 1980.

Solaro, Dietrich (1997): Controlling und Controllingphilosophie, in: Gleich, Ronald; Seidenschwarz, Werner (Hrsg.): Die Kunst des Controlling, München 1997.

Sonntag, Karl Heinz (1997): Wege zur Lernkultur und organisationalen Effizienz, in: Wieselhuber, Norbert et al. (Hrsg.): Handbuch Lernende Organisation, Wiesbaden 1997, S. 45-53.

Spremann, Klaus (1990): Asymmetrische Information, in: Zeitschrift fur Betriebswirtschaft, 60. 1990, Nr. 5/6, S. 561-586.

Stachowiak, Herbert (1965): Gedanken zu einer allgemeinen Theorie der Modelle, in: Studium Generale, 18. 1965, Nr. 5, S. 432-463.

Staehle, Wolfgang (1988): Managementwissen in der Betriebswirtschaftslehre Geschichte eines Diffusionsprozesses, in: Wunderer, Rolf (Hrsg.): Betriebswirtschaftslehre als Management- und Führungslehre, 2. Auflage, Stuttgart 1988, S. 3-21. 
Staehle, Wolfgang (1999): Management - eine verhaltenswissenschaftliche Perspektive, 8. Auflage, München 1999.

Staerkle, Robert (1985): Wechselwirkungen zwischen Organisationskultur und Organisationsstruktur, in: Probst, Gilbert J.B. (Hrsg.): Integriertes Management, Bonn/Stuttgart 1985, S. 529-553.

Stein, Johann Heinrich v. (1993): Betriebswirtschaftslehre, Gegenstand der, in: Wittmann, Waldemar (Hrsg.): Handwörterbuch der Betriebswirtschaft, 5. Auflage, Stuttgart 1993, Sp. 470-482.

Steinmann, Horst; Hennemann, Carola (1997): Die lernende Organisation - eine Antwort auf die Herausforderungen der Managementpraxis?, in: Wieselhuber, Norbert et al. (Hrsg.): Handbuch Lernende Organisation, Wiesbaden 1997, S. 33-44.

Steinmann, Horst; Schreyögg, Georg (1993): Management: Grundlagen der Unternehmensführung - Konzepte - Funktionen - Fallstudien, 3. Auflage, Wiesbaden 1993.

Steinmann, Horst; Schreyögg, Georg (2000): Management: Grundlagen der Unternehmensführung - Konzepte - Funktionen - Fallstudien, 5. Auflage, Wiesbaden 2000.

Steinmüller, Wilhelm (1981): Eine sozialwissenschaftliche Konzeption der Informationswissenschaft (Informationstechnologie und Informationsrecht), in: Nachrichten für Dokumentation, 32. 1981, Nr. 2, S. 69-77.

Steven, Marion (1998): Produktionstheorie, Wiesbaden 1998.

Stigler, George J. (1961): The Economics of Information, in: Journal of Political Economy, 69. 1961, Nr. 3, S. 213-225.

Stiglitz, Joseph E. (1985): Information and Economic Analysis: A Perspective, in: Economic Journal, 95. 1985, S. 21-41.

Stogdill, Ralph M. (1974): Handbook of Leadership, New York 1974.

Stonier, Tom (1986): What is information, in: Expert Systems 86 - Proceedings of the 6th technical conference of the British Computer Society Specialist Group on Expert Systems, Cambridge University Press, 1986.

Strader, Troy J.; Shaw, Michael J. (2000): Electronic Markets: Impact and Implications, in: Shaw, Michael J. et al. (Hrsg.): Handbook on Electronic Commerce, Berlin et al. 2000, S. 77-98.

Strauß, Ralf E. (1996): Determinanten und Dynamik des Organizational Learning, Wiesbaden 1996.

StrauB, Ralf E.; Schoder, Detlef (1999): Electronic Commerce - Herausforderungen aus Sicht der Unternehmen, in: Sauter, Michael; Hermanns, Arnold: Management-Handbuch Electronic Commerce - Grundlagen, Strategien, Praxisbespiele, München 1999, S. 61-74.

Streubel, Frauke (1996): Theoretische Fundierung eines ganzheitlichen Informationsmanagements, Arbeitsbericht Nr. 96-21 des Lehrstuhls für Wirtschaftsinformatik, Bochum 1996. 
Streubel, Frauke (2000): Organisatorische Gestaltung und Informationsmanagement in der lernenden Unternehmung - Bausteine eines Managementkonzeptes organisationalen Lernens, Frankfurt a.M. et al. 2000.

Strombach, Werner (1986): Was ist Information? - Versuch einer philosophischen Analyse, Vorlesungsreihe Schering, Heft 12, herausgegeben von der Pharma Forschung Schering, Berlin 1986.

Stünzner, Lilia (1996): Systemtheorie und betriebswirtschaftliche Organisationsforschung - Eine Nutzenanalyse der Theorien auopoietischer und selbstreferentieller Systeme, Berlin 1996.

Subramaniam, Chandrasekar; Shaw, Michael J.; Gardner, David (2000): Product Marketing on the Internet, in: Shaw, Michael J. et al. (Hrsg.): Handbook on Electronic Commerce, Berlin et al. 2000, S. 147-173.

Suppan, Jürgen (2001): UMTS und die Folgen: Netze vor dem Infarkt, in: Computerwoche extra, Nr. 3, 2001, S. 34-35.

Sydow, Jörg (1993): Strategische Netzwerke - Evolution und Organisation, Wiesbaden 1993.

Taday, Holger (1998): Telekommunikation - Standortgebundene und mobile Telekommunikationssysteme, Lehrmaterial Nr. 22/98 des Lehrstuhls für Wirtschaftsinformatik, Ruhr-Universität Bochum 1998.

Tan, Gek Woo; Shaw, Michael J.; Fulkerson, William (2000): Web-based Global Supply Chain Management, in: Shaw, Michael J. et al. (Hrsg.): Handbook on Electronic Commerce, Berlin et al. 2000, S. 457-478.

Terry, George Robert; Franklin, St. G. (1982): Principles of Management, 8. Auflage Homewood 1982.

Thome, Rainer (1999): Electronic Commerce-Plattformen - Grundlagen, Anforderungen und Empfehlungen, in: Sauter, Michael; Hermanns, Arnold: ManagementHandbuch Electronic Commerce - Grundlagen, Strategien, Praxisbeispiele, München 1999, S. 175-183.

Trott zu Solz, Clemens von (1992): Informationsmanagement im Rahmen eines ganzheitlichen Konzeptes der Unternehmensführung, Göttingen 1992.

Uhr, Wolfgang; Kosilek, Ernest; Schaffrath, Michael (1998): Entwicklung von Symptomerkennungsagenten zur Identifizierung von Abweichungen in der Entwicklung von Markt- und Unternehmenskennzahlen, in: Uhr, Wolfgang; Breuer, Sven-Einar (Hrsg.): Integration externer Informationen in Management Support Systems - Wirtschaftsinformatik Fachtagung, Technische Universität Dresden 1998, S. 47-70.

Ulrich, Hans (1970): Die Unternehmung als produktives soziales System - Grundlagen der allgemeinen Unternehmungslehre, 2. Auflage, Bern/Stuttgart 1970.

Ulrich, Hans (1971): Der systemorientierte Ansatz in der Betriebswirtschaftslehre, in: Kortzfleisch, Gert v. (Hrsg.), Wissenschaftsprogramm und Ausbildungsziele der Betriebswirtschaftslehre, Berlin 1971, S. 43-60. 
Ulrich, Hans (1972): Systemorientiertes Management, in: Intuition oder Information?, Separatdruck der UNIVAC 1972, wiederabgedruckt in: Ulrich, Hans: Management, Bern 1984, S. 64-84.

Ulrich, Hans (1988): Von der Betriebswirtschaftslehre zur systemorientierten Managementlehre, in: Wunderer, Rolf (Hrsg.), Betriebswirtschaftslehre als Management- und Führungslehre, Stuttgart 1988, S. 173-190.

Ulrich, Peter (1990): Symbolisches Management - Ethisch-kritische Anmerkungen zur gegenwärtigen Diskussion über Unternehmenskultur, in: Lattmann, Charles (Hrsg.): Die Unternehmenskultur: ihre Grundlagen und ihre Bedeutung für die Führung der Unternehmung, Heidelberg 1990, S. 277-302.

Ulrich, Peter; Hill, Wilhelm (1979): Wissenschaftstheoretische Grundlagen der Betriebswirtschaftslehre, in: Raffée, Hans; Abel, Bodo, Wissenschaftstheoretische Grundfragen der Wirtschaftswissenschaften, München 1979, S. 161-190.

Varshney, Upkar; Vetter, Ronald; Kalakota, Ravi (2000): Mobile Commerce - A New Frontier, http://www.computer.org/computer/articles/october/ Varshney/Varshney.html, abgerufen am 07.05.2001.

Voß, Stefan; Gutenschwager, Kai (2001): Informationsmanagement, Belin et al. 2001.

Walker, G.; Weber, D. (1984): A Transaction Cost-Approach to Make-or-Buy Decision, in: Administrative Sciene Quaterly, 29. 1984, Nr. 4, S. 373-391.

Weber, Jürgen (1993): Controlling, Informations- und Kommunikationsmanagement - Grundsätzliche begriffliche und konzeptionelle Überlegungen, in: Betriebswirtschaftliche Forschung und Praxis, 44. 1993, Nr. 6, S. 628-649.

Weber, Jürgen (1995): Einführung in das Controlling, 6. Auflage, Stuttgart 1995.

Weber, Jürgen (1997): Controllership versus Controlling - A never-ending story, Stellungnahme zum Editorial von Prof. Dr. Péter Horvàth in Heft 4/1996, in: Controlling, 1997, Nr. 3, S. 180-182.

Weber, Jürgen; Brettel, Malte; Großklaus, Armin; Hamprecht, Markus; Rösch, B. E.; Schäffer, Utz (1995): Grundgedanken zur Entwicklung einer Theorie der Unternehmensführung, WHU-Forschungspapier Nr. 30, Koblenz 1995.

Weber, Wolfgang (1993): Einführung in die Betriebswirtschaftslehre, 2. Auflage, Wiesbaden 1993.

Weinert, Ansfried B. (1984): Menschenbilder als Grundlage von Führungstheorien, in: Zeitschrift für Organisation, 53. 1984, Nr. 2, S. 117-123.

Weiß, Dietmar (1998): Prozesskostenrechnung und Workflow Management Konzeption und Umsetzung eines Schnittstellensystems, Wiesbaden 1998.

Weiß, Dietmar; Krcmar, Helmut (1998): Workflow-Management: Herkunft und Klassifikation, in: Wirtschaftsinformatik, 38. 1996, Nr. S. 503-514.

Weld, Daniel; Etzioni, Oren (1995): Intelligent Agents on the Internet: Fact, Fiction and Forecast, in: IEEE Expert, 10. 1995, Nr. 4, S. 44-49.

Wenger, Ekkehard; Terberger, Eva (1988): Die Beziehung zwischen Agent und Prinzipal als Baustein der ökonomischen Theorie der Organisation, in: Wirtschaftswissenschaftliches Studium, 17. 1988, Nr. 10, S. 506-514. 
Wesseler, B. (1992): Der ORG/DV-Leiter - eine Schaltstelle im Management, in: ORG/DV-Leiter-Enquête 1992. Hrsg.: Online. Köln 1992.

Wiedmann, Klaus-Peter; Buxel, Holger; Buckler, Frank (2000): Mobile Commerce, in: Wirtschaftwissenschaftliches Studium, 29. 2000, Nr. 12, S. 685691.

Wiegand, Martin (1996): Prozesse organisationalen Lernens, Wiesbaden 1996.

Wild, Jürgen (1971): Zur Problematik der Nutzenbewertung von Informationen, in: Zeitschrift für Betriebswirtschaft, 41. 1971, Nr. 5, S. 315-334.

Wild, Jürgen (1982): Grundlagen der Unternehmensplanung, 4. Auflage, Opladen 1982.

Williamson, Oliver E. (1979): Transaction Cost Economics: The Governance of Contractual Relations, in: Journal of Law and Economics, 22. 1979, Nr. 2, S. 233-261.

Williamson, Oliver E. (1985): The Economic Institutions of Capitalism. New York 1985.

Williamson, Oliver E. (1990): Die ökonomischen Institutionen des Kapitalismus, Tübingen 1990.

Willke, Helmut (1987a): Systemtheorie: Eine Einführung in die Grundprobleme, Stuttgart/New York 1987.

Willke, Helmut (1987b): Strategien der Intervention in autonome Systeme, in: Baecker, Dirk; Markowitz, Jürgen; Stichweh, Rudolf; Willke, Helmut (Hrsg.): Theorie als Passion: Niklas Luhmann zum 60. Geburtstag, Frankfurt 1987, S. 333-361.

Windsperger, Josef (1998): Ungelöste Probleme der Transaktionskostentheorie, in: Journal für Betriebswirtschaft, 48. 1998, Nr. 5-6, S. 266-276.

Wirfs-Brock, Rebecca; Wilkerson, Brian; Wiener, Lauren: (1993): Objektorientiertes Software-Design, München et al. 1993.

Wirtz, Bernd (2001): Mobile Verheißung - Wunsch und Realität, Interview von Achim Born, in: Computerwoche extra, Nr. 3, 2001, S. 8-9.

Wiswede, Günter (1992): Gruppen und Gruppenstrukturen, in: Frese, Erich (Hrsg.): Handwörterbuch der Organisation, 3. Auflage, Stuttgart 1992, Sp. 735-754.

Witt, Frank H. (1995): Theorietraditionen der Betriebswirtschaftslehre - deutschsprachige Betriebswirtschaftslehre und angloamerikanische Management- und Organisationsforschung, Wiesbaden 1995.

Wittmann, Waldemar (1959): Unternehmung und unvollkommene Information Unternehmerische Voraussicht, Ungewißheit und Planung, Köln 1959.

Wöhe, Günter (1990): Einführung in die Allgemeine Betriebswirtschaftlehre, 17. Auflage, München 1990.

Wöhe, Günter (2000): Einführung in die Allgemeine Betriebswirtschaftslehre, 20. Auflage, München 2000.

Wohlfahrt, Jens (2001): One-to-one Marketing im Mobile Commerce, in: Information Management \& Consulting, 16. 2001, Nr. 2, S. 49-54. 
Wolf, Knut; Scherer, Hardy (1999): Die Herausforderung E-Commerce annehmen, in: Fachzeitschrift für Information Management \& Consulting, Sonderausgabe 1999, S. 19-23.

Wolfram, Gerd (1990): Organisatorische Gestaltung des Informations-Managements: Konzeption und aufbauorganisatorische Aspekte, Bergisch Gladbach/Köln 1990.

Wolfsteiner, Wolf D. (1995): Das Management der Kernfähigkeiten - Ein ressourcenorientierter Strategie- und Strukturansatz, Rosch-Buch/Hallstadt 1995.

Wollnik, Michael (1988): Ein Referenzmodell des Informations-Managements, in: Information Management, 3. 1988, Nr. 3, S. 34-43.

Wollnik, Michael (1992): Organisationstheorie, interpretative, in: Frese, Erich (Hrsg.): Handwörterbuch der Organisation, 3. Auflage, Stuttgart 1992, Sp. 1778-1797.

Wolter, Hans-Jürgen; Wolff, Karin; Freund, Werner (1998): Das virtuelle Unternehmen - Eine Organisationsform für den Mittelstand, Wiesbaden 1998.

Zahn, Erich; Rüttler, Martin (1989): Informationsmanagement. Eine strategische Antwort auf kritische Herausforderungen der Unternehmungsumwelt, in: Controlling, 1. 1989, Nr. 1, S. 34-43.

Zahn, Erich; Rüttler, Martin (1990): Ganzheitliches Informationsmanagement Informationsbereitschaft, Informationspotential, Informationsfähigkeit, in: Heilmann, Heidi (Hrsg.): Informationsmanagement - Aufgabe der Unternehmungsleitung, Stuttgart 1990, S. 1-27.

Zbornik, Stefan (1996): Elektronische Märkte, elektronische Hierarchien und elektronische Netzwerke: Koordination des wirtschaftlichen Leistungsaustausches durch Mehrwertdienste auf der Basis von EDI und offenen Kommunikationssystemen, diskutiert am Beispiel der Elektronikindustrie, Konstanz 1996.

Zelewski, Stephan (1987): Der Informationsbroker, in: Die Betriebswirtschaft, 47. 1987, Nr. 6, S. 737-748.

Zemanek, Heinz (1986): Information und Ingenieurwissenschaft, in: Folberth, Otto G.; Hackl, Clemens (Hrsg.): Der Informationsbegriff in Technik und Wirtschaft, München, Wien 1986, S. 17-44.

Zerdick, Axel (2001): Die Internet-Ökonomie - Strategien für die digitale Wirtschaft, 3. Auflage, Berlin et al. 2001.

Zobel, Jörg (2001): Mobile Business und M-Commerce - Die Märkte der Zukunft erobern, München/Wien 2001. 


\section{Bochumer Beiträge zur Unternehmungsführung und Unternehmensforschung}

Herausgegeben vom Direktorium des Instituts

für Unternehmungsführung und Unternehmensforschung der Ruhr-Universität Bochum

Band 1 Busse von Colbe, Walther/Mattessich, Richard (Hrsg.): Der Computer im Dienste der Unternehmungsführung (1968)

Band 2 Busse von Colbe, Walther/Meyer-Dohm, Peter (Hrsg.): Unternehmerische Planung und Entscheidung (1969)

Band 3 Anthony, Robert N.: Harvard-Fälle aus der Praxis des betrieblichen Rechnungswesens. Herausgegeben von Richard V. Mattessich unter Mitarbeit von Klaus Herrnberger und Wolf Lange (1969)

Band 4 Mattessich, Richard: Die wissenschaftlichen Grundlagen des Rechnungswesens (1970)

Band 5 Schweim, Joachim: Integrierte Unternehmungsplanung (1969)

Band 6 Busse von Colbe, Walther (Hrsg.): Das Rechnungswesen als Instrument der Unternehmungstührung (1969)

Band 7 Domsch, Michel: Simultane Personal- und Investitionsplanung im Produktionsbereich (1970)

Band 8 Leunig, Manfred: Die Bilanzierung von Beteiligungen. Eine bilanztheoretische Untersuchung (1970)

Band 9 Franke, Reimund: Betriebsmodelle. Rechensystem für Zwecke der kurzfristigen Planung, Kontrolle und Kalkulation (1972)

Band 10 Wittenbrink, Hartwig: Kurzfristige Erfolgsplanung und Erfolgskontrolle mit Betriebsmodellen (1975)

Band 11 Lutter, Marcus (Hrsg.): Recht und Steuer der internationalen Unternehmensverbindungen (1972)

Band 12 Niebling, Helmut: Kurzfristige Finanzrechnung auf der Grundlage von Kosten- und Erlösmodellen (1973)

Band 13 Perlitz, Manfred: Die Prognose des Unternehmenswachstums aus Jahresabschlüssen deutscher Aktiengesellschaften (1973)

Band 14 Niggemann, Walter: Optimale Informationsprozesse in betriebswirtschaftlichen Entscheidungssituationen (1973)

Band 15 Reichardt, Harald: Der aktienrechtliche Abhängigkeitsbericht unter ökonomischen Aspekten (1974)

Band 16 Backhaus, Klaus: Direktvertrieb in der Investitionsgüterindustrie - Eine Marketing-Entscheiung (1974)

Band 17 Plinke, Wulff: Kapitalsteuerung in Filialbanken (1975)

Band 18 Steffen, Rainer: Produktionsplanung bei Fließbandfertigung (1977)

Band 19 Kolb, Jürgen: Industrielle Erlösrechnung - Grundlagen und Anwendungen (1978)

Band 20 Busse von Colbe, Walther/Lutter, Marcus (Hrsg.): Wirtschaftsprüfung heute: Entwicklung oder Reform? (1977)

Band 21 Uphues, Peter: Unternehmerische Anpassung in der Rezession (1979) 
Band 22 Gebhardt, Günther: Insolvenzprognosen aus aktienrechtlichen Jahresabschlüssen (1980)

Band 23 Domsch, Michel: Systemgestützte Personalarbeit (1980)

Band 24 Schmied, Volker: Alternativen der Arbeitsgestaltung und ihre Bewertung (1982)

Band 25 Wäscher, Gerhard: Innerbetriebliche Standortplanung bei einfacher und mehrfacher Zielsetzung (1982)

Band 26 Weber, Martin: Entscheidungen bei Mehrfachzielen - Verfahren zur Unterstützung von Individual- und Gruppenentscheidungen (1983)

Band 27 Kroesen, Alfred: Instandhaltungsplanung und Betriebsplankostenrechnung (1983)

Band 28 Plinke, Wulf: Erlösplanung im industriellen Anlagengeschäft (1985)

Band 29 Chamoni, Peter: Simulation störanfälliger Systeme (1986)

Band 30 Arning, Andreas: Die wirtschaftliche Bewertung der Zentrenfertigung - Dargestellt am Beispiel einer Fertigungsinsel (1987)

Band 31 Gebhardt, Günther: Finanzielle Planung und Kontrolle bei internationaler Unternehmenstätigkeit

Band 32 Markiewicz, Michael: Ersatzteildisposition im Maschinenbau - Betriebswirtschaftliche Methoden der Planung und Überwachung (1988)

Band 33 Pellens, Bernd: Der Informationswert von Konzernabschlüssen - Eine empirische Untersuchung deutscher Börsengesellschaften (1989)

Band 34 Mrotzek, Rüdiger: Bewertung direkter Auslandsinvestitionen mit Hilfe betrieblicher Investitionskalküle (1989)

Band 35 Deppe, Joachim: Quality Circle und Lernstatt - Ein integrativer Ansatz (1989, 3. Auflage 1993)

Band 36 Rademacher, Michael: Arbeitszeitverkürzung und -flexibilisierung - Formen und betriebliche Auswirkungen (1990)

Band 37 Kaiser, Klaus: Kosten- und Leistungsrechung bei automatisierter Produktion (1991, 2. Auflage 1993)

Band 38 Müller, Hermann: Industrielle Abfallbewältigung - Entscheidungsprobleme aus betriebswirtschaftlicher Sicht (1991)

Band 39 Schörner, Peter: Gesetzliches Insiderhandelsverbot - Eine ordnungspolitische Analyse (1991)

Band 40 Bentler, Martin: Grundsätze ordnungsmäßiger Bilanzierung für die Equitymethode (1991)

Band 41 Brüggerhoff, Jürgen: Management von Desinvestitionen (1992)

Band 42 Bröker, Erich W.: Erfolgsrechnung im industriellen Anlagengeschäft - Ein dynamischer Ansatz auf Zahlungsbasis - (1993)

Band 43 Frankenberg, Peter: Transnationale Analyse US-amerikanischer und deutscher Jahresabschlüsse - Eine theoretische und empirische Untersuchung (1993)

Band 44 Kleinaltenkamp, Michael: Standardisierung und Marktprozeß - Entwicklungen und Auswirkungen im CIM-Bereich (1993)

Band 45 Pellens, Bernhard: Aktionärsschutz im Konzern - Empirische und theoretische Analyse der Reformvorschläge der Konzernverfassung (1994)

Band 46 Reckenfelderbäumer, Martin: Marketing-Accounting im Dienstleistungsbereich - Konzeption eines prozeßkostengestützten Instrumentariums (1995) 
Band 47 Knittel, Friedrich: Technikgestützte Kommunikation und Kooperation im Büro. Entwicklungshindernisse - Einsatzstrategien - Gestaltungskonzepte (1995)

Band 48 Riezler, Stephan: Lebenszyklusrechnung - Instrument des Controlling strategischer Projekte (1996)

Band 49 Schulte, Jörn: Rechnungslegung und Aktienkursentwicklung - Erklärung und Prognose von Aktienrenditen durch Einzel- und Konzernabschlußdaten (1996)

Band 50 Muhr, Martin: Zeitsparmodelle in der Industrie - Grundlagen und betriebswirtschaftliche Bedeutung mehrjähriger Arbeitszeitkonten (1996)

Band 51 Brotte, Jörg: US-amerikanische und deutsche Geschättsberichte. Notwendigkeit, Regulierung und Praxis jahresabschlußergänzender Informationen (1997)

Band 52 Gersch, Martin: Vernetzte Geschäftsbeziehungen. Die Nutzung von EDI als Instrument des Geschäftsbeziehungsmanagement (1998)

Band 53 Währisch, Michael: Kostenrechnungspraxis in der deutschen Industrie. Eine empirische Studie (1998)

Band 54 Völkner, Peer: Modellbasierte Planung von Geschäftsprozeßabläufen (1998)

Band 55 Fülbier, Rolf Uwe: Regulierung der Ad-hoc-Publizität. Ein Beitrag zur ökonomischen Analyse des Rechts (1998)

\section{Band 1 - 55 erschienen beim Gabler Verlag Wiesbaden}

Band 56 Ane-Kristin Reif-Mosel: Computergestützte Kooperation im Büro. Gestaltung unter Berücksichtigung der Elemente Aufgabe, Struktur, Technik und Personal (2000)

Band 57 Claude Tomaszewski: Bewertung strategischer Flexibilität beim Unternehmenserwerb. Der Wertbeitrag von Realoptionen (2000)

Band 58 Thomas Erler: Business Objects als Gestaltungskonzept strategischer Informationssystemplanung (2000)

Band 59 Joachim Gassen: Datenbankgestützte Rechnungslegungspublizität. Ein Beitrag zur Evolution der Rechnungslegung (2000)

Band 60 Frauke Streubel: Organisatorische Gestaltung und Informationsmanagement in der lemenden Unternehmung. Bausteine eines Managementkonzeptes organisationalen Lernens (2000)

Band 61 Andreas von der Gathen: Marken in Jahresabschluß und Lagebericht (2001)

Band 62 Lars Otterpohl: Koordination in nichtlinearen dynamischen Systemen (2002)

Band 63 Ralf Schremper: Aktienrückkauf und Kapitalmarkt. Eine theoretische und empirische Analyse deutscher Aktienrückkaufprogramme (2002)

Band 64 Peter Ruhwedel: Aufsichtsratsplanungssysteme. Theoretische Grundlagen und praktische Ausgestaltung in Publikumsaktiengesellschaften (2002)

Band 65 Jens Thorn: Taktisches Supply Chain Planning. Planungsunterstützung durch deterministische und stochastische Optimierungsmodelle. 2002.

Band 66 Dirk Beier: Informationsmanagement aus Sicht der Betriebswirtschaftslehre. Theoretische Ansätze und das Beispiel Mobile Business. 2002. 


\section{Informationsmanagement in mittelständischen Unternehmen}

\section{Eine mikroökonomische und empirische Untersuchung}

Frankfurt/M., Berlin, Bern, Bruxelles, New York, Oxford, Wien, 2001.

XXIX, 657 S., zahlr. Abb.und Tab.

Informationsmanagement und strategische Unternehmensführung. Bd. 4 Herausgegeben von Prof. Dr. Franz Schober und Prof. Dr. Johannes Ruhland. ISBN 3-631-38875-6 · br. € 85.90*

Diese Untersuchung setzt sich mit den Spezifika des Informationsmanagements mittelständischer Unternehmen auseinander. Die im Kern ökonomisch orientierte Analyse diskutiert drei Themengebiete: die Potentiale und Risiken der Informationstechnik (IT), die organisationalen Bestimmungsgründe der IT-Nutzung sowie die Möglichkeiten einer an die Besonderheiten mittelständischer Unternehmen adaptierten Ausgestaltung des Informationsmanagements. Die empirische Überprüfung der theoretischen Aussagen liefert ein differenziertes Bild über die Auswirkungen eines adäquaten Informationsmanagements auf den organisationalen Wandel und auf die Wettbewerbsfähigkeit der mittelständischen Unternehmung. Dabei zeigen sich zum Teil Unterschiede zwischen deutschen und französischen Unternehmen.

Aus dem Inhalt: Theorien des Informationsmanagements · Ökonomische Analyse der mittelständischen Unternehmung · Analyse kritischer Erfolgsfaktoren · Organisationale Bestimmungsgründe der IT-Nutzung · Potentiale und Risiken der Informationstechnik - Ausgestaltung des Informationsmanagements - Empirische Ergebnisse zum Informationsmanagement in mittelständischen Unternehmen - Vergleich deutscher und französischer Unternehmen

Frankfurt/M · Berlin · Bern · Bruxelles · New York · Oxford · Wien

Auslieferung: Verlag Peter Lang AG

Moosstr. 1, $\mathrm{CH}-2542$ Pieterlen

Telefax 0041 (0) $32 / 3761727$

*inklusive der in Deutschland gültigen Mehrwertsteuer

Preisänderungen vorbehalten

Homepage http://wuw.peterlang.de 